$\mathrm{DOE} / \mathrm{MC} / 25137--3104$

DE92 001299

\title{
Demonstation Program Performance Test Summary Reports
}

\author{
Topical Report
}

Work Performed Under Contract No.: DE-FC21-89MC25137

For
U.S. Department of Energy

Office of Fossil Energy

Morgantown Energy Technology Center

P.O. Box 880

Morgantown, West Virginia 20507-0880

\author{
By \\ Colorado-Ute Electric Association, Inc. \\ P.O. Box 1149 \\ Montrose, Colorado 81402
}

March 1992 


\section{Performance Test Summary Reports \\ Table of Contents}

Section

1.0 Introduction

2.0 Summary of Phase I and II Test Programs

3.0 Performance Summary Report Contents

4.0 Split Combustor Tests

5.0 Acknowledgements

Rage

Appendix

A Performance Test Summary Reports

Page

$A-1$

Table

1 Fuel Properties

Page

2 Summary of Tests Conducted

3 Performance Test Summary Reports 
DOCUMENT SUMMARY

\subsection{INTRODUCTION}

This volume contains the performance test summary reports generated by the Colorado-Ute Electric Association's Nucla Circulating Fluidized Bed Demonstration Program. These summary reports supplement the performance test results presented in the Demonstration Project Final Technical Report and the Annual Technical Progress Reports covering the periods Start-up through 1988, 1989 and 1990 through Test Completion. Performance test summary reports are presented from both the Phase I and Phase II test programs. The Phase I test program began in February 1987 and was completed in June 1990. This segment was jointly sponsored by the United States Department of Energy (DOE) and the Electric Power Research Institute (EPRI). The Phase $1 . T$ test program commenced in crctober 1991 following a DOE extension agreement, and was completed in January 1991 with sole sponsorship by the DOE. The objectives of these programs were to conduct a cost-shared Clean Coal Technology Project to demonstrate the feasibility of circulating fluidized bed combustion cechnology and to evaluate the economic, environmental, and operational benefits of CFB steam generators on a utility scale.

\subsection{SUMMARY OF PHASE I AND II TEST PROGRAMS}

Detailed planning for a test program was initiated by EPRI in 1985. On-site preparation for the test program commenced in February 1987 and cold-mode shakedown activities were largely completed by October 1988. Detailed performance testing of the Nucla CFB at specified unit operating conditions commenced in March 1989 with the completion of the hot-mode test plan. From April 1989 through the completion of the Phase I test program in June 1990, a total of 45 steady-state performance tests were completed. These tests established the effects of load, excess air, primary to secondary air ratio, unit operating temperatures, coal and limestone feed configurations, and coal type and size distributions on emissions performance and combustion and boiler efficiencies. Data were also collected from these tests to quantify heat transfer in the combustion chambers, tubular air heater effectiveness, and baghouse collection efficiency. Dynamic response and unit start-up data were collected to determine any CFB technology limitations and to optimize unit performance. 
During the Phase II test program between October 1990 and January 1991, an additional 27 steady-state performance tests were conducted. These uditional tests provided new information in areas with limited test results from Phase I. Tests were also completed on Dorchester coal as part of the alternate fuels testing.

In summary, a total of 72 steady-state performance tests were conducted during both phases of testing. Twenty-two tests were performed at half load, six at three-quarter load, two at $90 \%$ load, and forty-two at full load. As part of alternate fuels testing, eight of these tests were conducted on a local Nucla coal (Peabody) and four were conducted on Dorchester coal. Sixty tests were completed on Salt Creek coal, the baseline fuel. The properties of these coals are summarized in Table 1 below. The range of property vaiues in the second column represents the variability of the local Peabody coal burned during 1988 and 1989.

Table 1. Fuel Properties

\begin{tabular}{lccc} 
Property & $\begin{array}{c}\text { Peabody } \\
\text { Coal }\end{array}$ & $\begin{array}{c}\text { Salt Creek } \\
\text { Coal }\end{array}$ & $\begin{array}{c}\text { Dorchester } \\
\text { Coal }\end{array}$ \\
\hline HHV, Btu/lb & $7490-11840$ & 10460 & 9041 \\
Sulfur, \& & $0.51-2.75$ & 0.44 & 1.53 \\
Ash, o & $9.8-42.8$ & 14.6 & 22.8 \\
Moisture, \% & $4.1-14.9$ & 10.0 & 10.97 \\
Fixed C, \% & 43.5 & 43.4 & 35.8 \\
Volatiles, \% & 28.4 & 32.3 & 30.4
\end{tabular}

\subsection{PERFORMANCE SUMMARY REPORT CONTENTS}

Table 2 provides a listing of the performance test summaries available in Appendix A of this volume, as also shown in Table 3. The tests are listed in chronological order, which is also the order that the summary reports are presented in this report. The additional information contained in Table 2 includes the test date, an indication that all required solids samples were obtained, coal type, dry percent $\mathrm{O}_{2}$ during the test period, load, calcium to sulfur ratio, limestone and coal feed configurations, comparative tests, remarks as to test type, and test category.

Information included in the performance summary reports includes:

- Process Operating Summary Report (2 pages): plant output; main steam flow, temperature, pressure; coal and limestone feed rates; bed drain and fly ash flow rates; superficial velocity; bed temperature; flue gas flow rate and composition; air flow rates; fan pressures; bed pressure; 


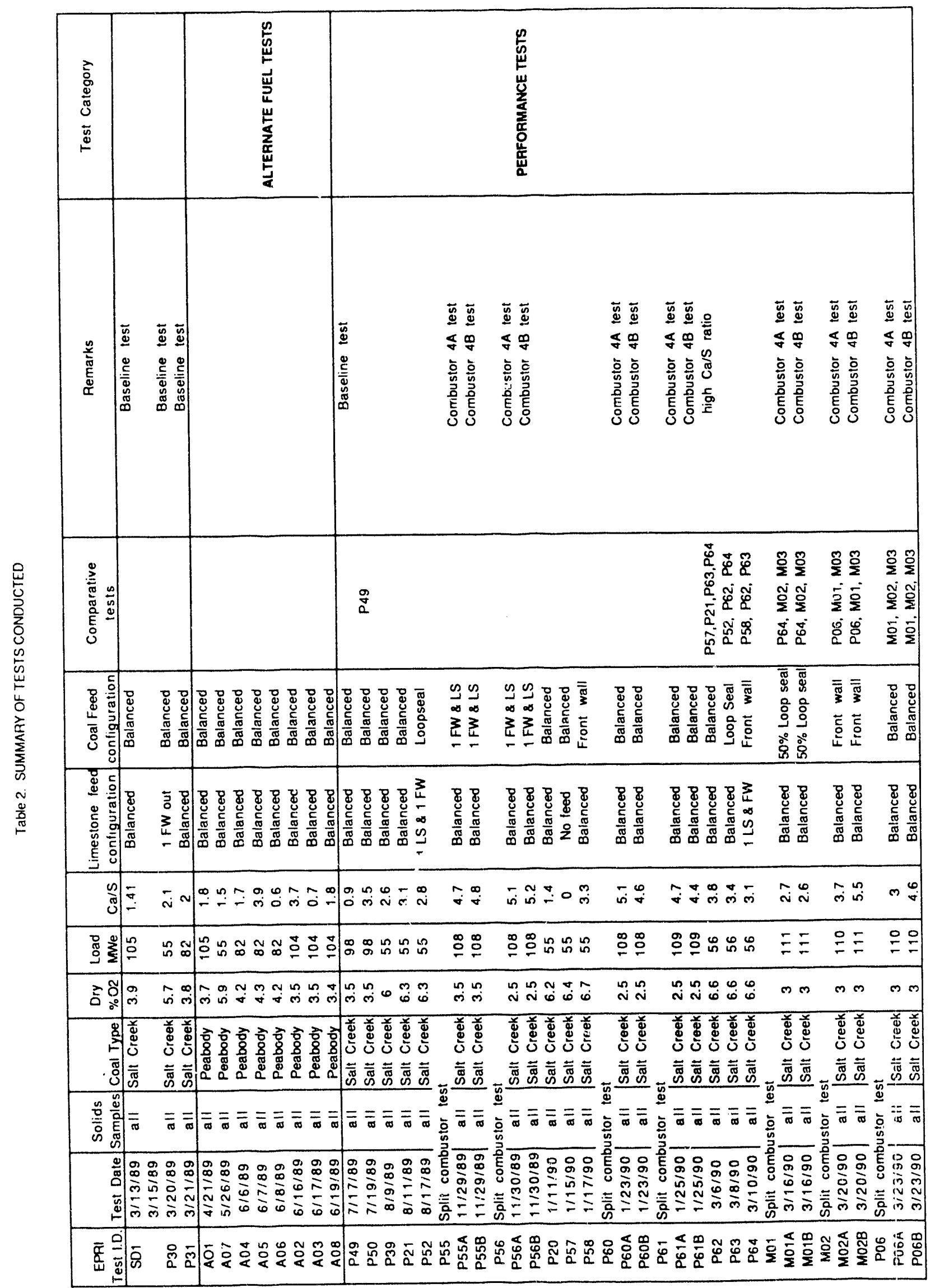




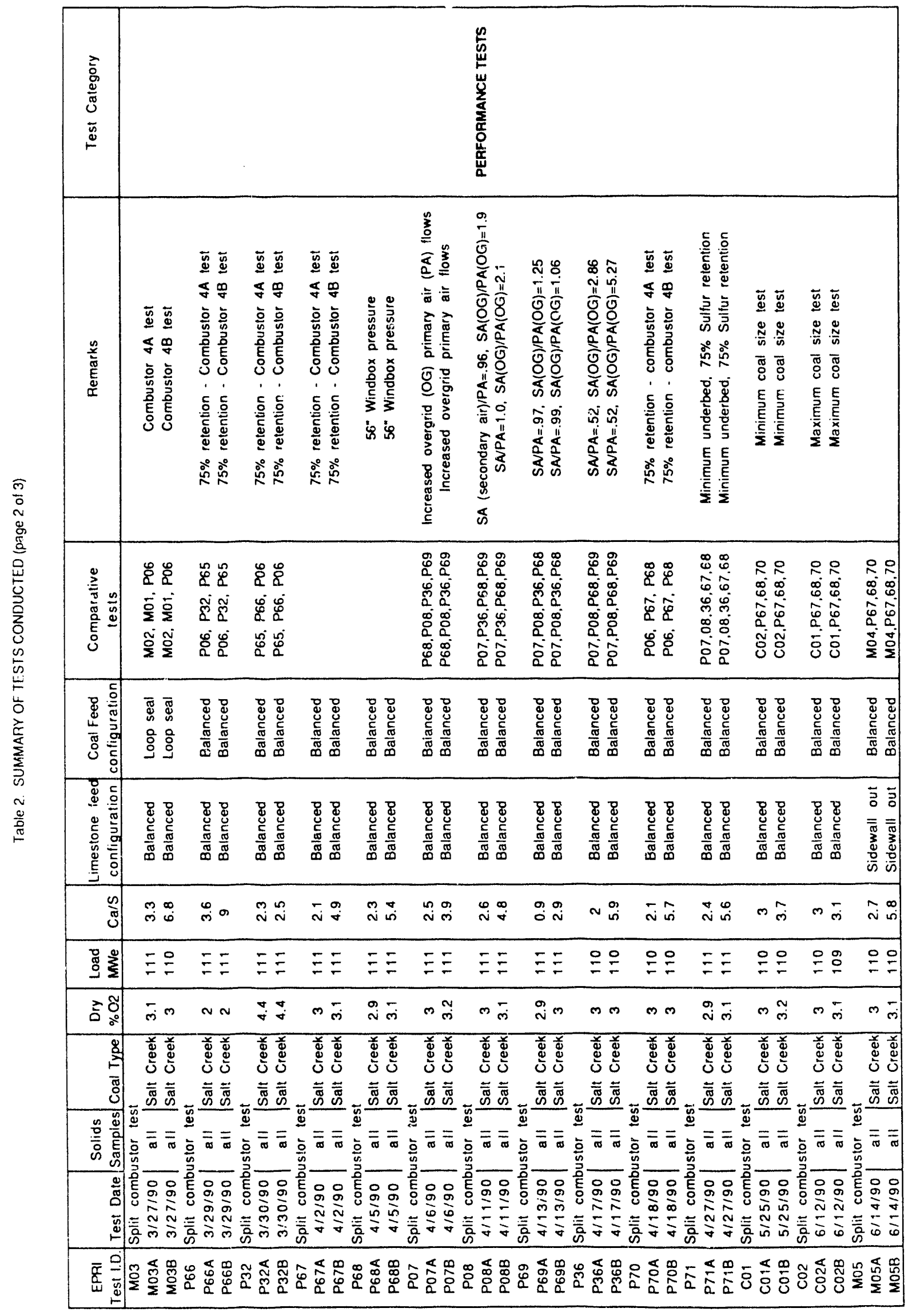




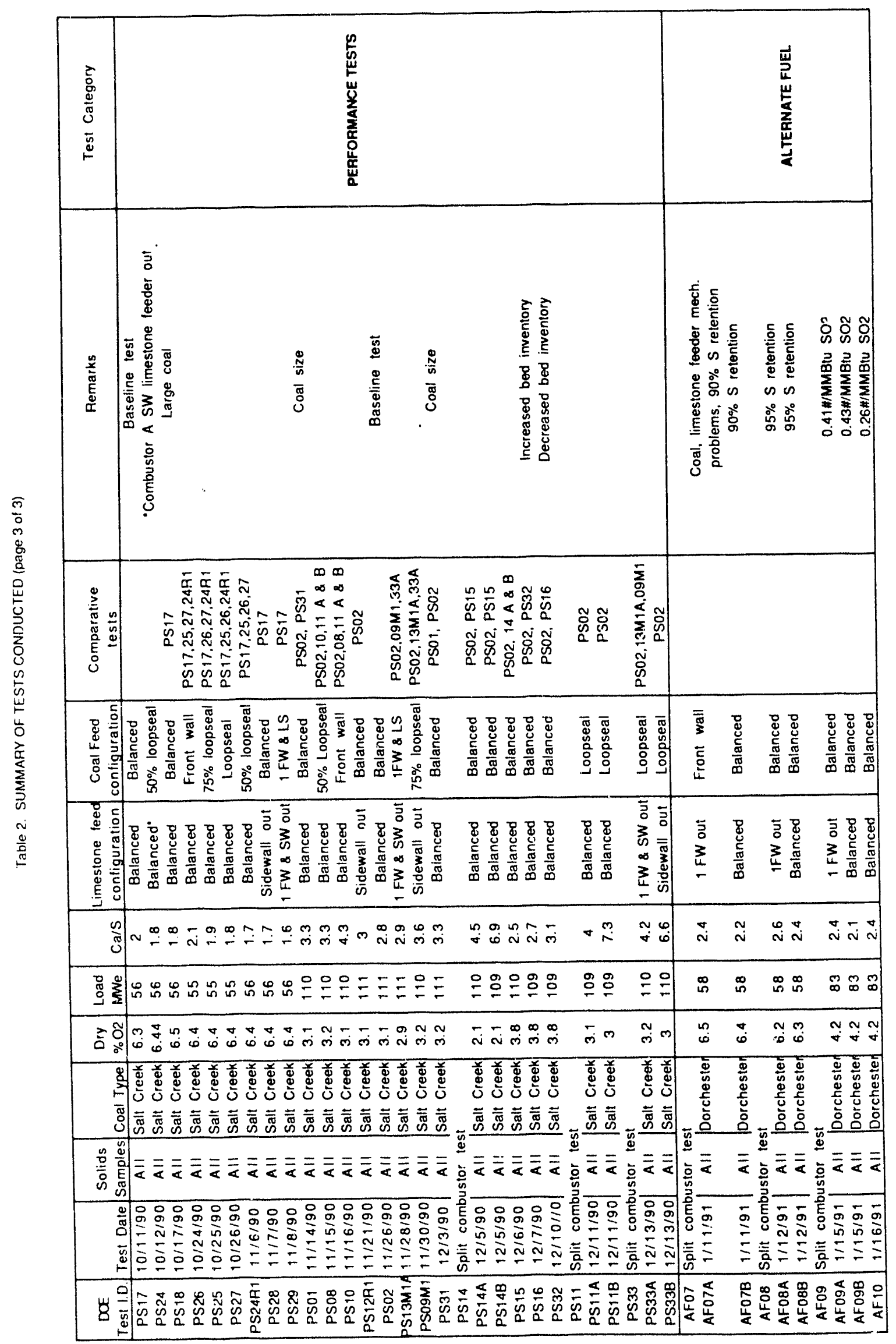

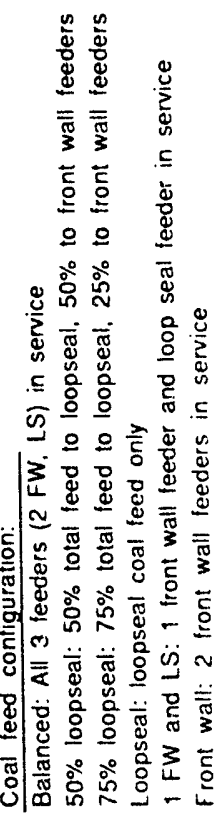


various temperatures in the unit; feed water flow; attemperator flows; ambient conditions

- Performance Summary Report: Ca utilization; $\mathrm{Ca} / \mathrm{S}$; $\mathrm{SO}_{2}$ retention; combustion efficiency; boiler efficiency; excess air; air heater effectiveness; wet flue gas flow rate; materials balance summary; unit heat rate

- Material Balance Report (2 pages): inputs from coal, sorbent and air; outputs from flue gas, fly ash, and bed drain

- Heat Balance Report: boiler efficiency calculation credits and losses

- Chemical Analysis Report: coal, sorbent, fly ash and bed drain analyses

- Physical Analysis Report: coal, sorbent and bed drain size distributions

\subsection{SPLIT COMBUSTOR TESTS}

Because of an operating temperature differential that existed between combustors at full lozd, some of the full-load tests were conducted as "split" combustor tests, in which each combustion chamber was tested separately. Typically, this was done whenever the ash distribution was low (low waterwall differential pressure) and the temperature differential between combustors was high. In addition, some tests were run as split combustor tests due to limestone feeder problems which resulted in different feed configurations for the two combustors. During the first half of a split combustor test, the flue gas analyzer is set up to sample on one combustor only and coal, limestone, and bottom ash samples are taken from that combustor. For the second half of the test, flue gas and solids samples are obtained from the other combustor. It is not possible to take fly ash samples from only one combustor, so calculations that are strongly dependent upon fly ash analyses, such as boiler and combustion efficiency, cannot be made for each combustor. Fly ash samples from the stream common to both combustors are taken throughout the test, making it possible to calculate an averaged combustion and boiler efficiency for both combustors.

Three sets of performance summaries were produced for each split combustor test, as opposed to the one set produced for the combined combustor tests. For example, test P71 was conducted as a split combustor test; the performance summaries begin on page $A-260$. The first data set, P71 (pages A-260 through A-263), provides data and calculated results for the entire boiler, and shows temperature differentials between combustors of $79^{\circ} \mathrm{F}$ at $20 "$ from the distributor plate, $92.6^{\circ} \mathrm{F}$ at $66^{\prime \prime}$, and $82^{\circ} \mathrm{F}$ at $118^{\prime \prime}$. Each of the other two data sets for this test, $\mathrm{P} 71 \mathrm{~A}$ and $\mathrm{P} 71 \mathrm{~B}$ (pages A-264 through A-269), provides data and calculated results for combustors $A$ and $B$, respectively. During the Phase I test program, two of the split combustor tests were concluded 
after data collection from only one combustor was completed. Test MO4 was concluded early (after data collection from combustor A only) due to the inability to maintain $75 \%$ sulfur retention with no loop seal limestone feed. Test P65 was concluded early (after data collection from combustor $B$ only) due to several 4C coal feeder trips. Solids samples were not sent to the lab for analysis, therefore performance test summary reports are not available for these two tests.

\subsection{ACKNOWLEDGEMENTS}

This volume of Performance Test Summary Reports was sponsored and reviewed by the U.S. Department of Energy under Cooperative Agreement DE-FC21-89MC25137. Administration of the cooperative agreement was performed by the DOE's Morgantown Energy Technology Center (METC) in Morgantown, West Virginia.

This report was prepared by Combustion systems Incorporated for the Colorado-Ute Electric Association with assistance and input from CUEA. The following individuals from CUEA are responsible for the implementation of the DOE agreement:

Raymond E. Keith, Acting Project Manager, Business Contact Thomas J. Heller, Technical Contact

Stuart A. Bush, Senior Engineer, Project Coordinator

CUEA, Inc. would like to acknowledge the Electric Power Research Institute (EPRI) for providing use of their test hardware and software in completing this report and for their direct involvement and sponsorship of the phase I test program, of which some data are reported herein. 
Appendix A

PERFORMANCE TEST SUMMARY REPORTS

This appendix contains the performance test summary reports described in sections 1-5. Table 3 provides a listing of the test summaries contained in the appendix and their corresponding page numbers. The "test numbers" on Table 3 represent the following categories of tests:

Test number
SXX
PXX
AXX
YXXA
YXXB
$Y X X$
MXX
CXX
PSXX
YXXR
YXXM
AFXX

Type of test

full-load baseline test performance test, Salt Creek coal, Phase I alternate fuel test, Phase I test on combustor A (split combustor test) test on combustor B (split combustor test) average results from both combustors coal or limestone feed distribution test coal size test performance test, Salt Creek coal, Phase II repeated test modified test alternate fuel test, Pha:e II 
Table 3. Performance Test Summary Reports

\begin{tabular}{|c|c|c|c|c|c|c|c|c|}
\hline & Test & & & Test & & & Test & \\
\hline Number & Number & Page & Number & Number & Page & Number & Number & Page \\
\hline Phase I: & & & & & & & & \\
\hline 1 & SD1 & A- 3 & & P61A & A-113 & 35 & P07 & A-210 \\
\hline 2 & P30 & A. 7 & & P61B & A-116 & & P07A & A- 214 \\
\hline 3 & P31 & A. 11 & 24 & P62 & A.119 & & P07B & A- 217 \\
\hline 4 & A01 & A- 15 & 25 & P63 & A. 123 & 36 & P08 & A. 220 \\
\hline 5 & A07 & A. 19 & 26 & P64 & $A-127$ & & POBA & A. 224 \\
\hline 6 & AO4 & A. 23 & 27 & M01 & A-131 & & P08B & A. 227 \\
\hline 7 & A05 & A. 27 & & M01A & A. 135 & 37 & P69 & A. 230 \\
\hline 8 & A06 & A.31 & & M01B & A. 138 & & P69A & A.234 \\
\hline 9 & AO2 & A. 35 & 28 & M02 & A- 141 & & P69B & A-237 \\
\hline 10 & $\mathrm{AOB}$ & A.39 & & M02A & A. 144 & 38 & P36 & A. 240 \\
\hline 11 & A08 & A- 43 & & M02B & A- 147 & & P36A & A. 244 \\
\hline 12 & P49 & A. 47 & 29 & P06 & A. 150 & & P36B & A. 247 \\
\hline 13 & P50 & A-51 & & P06A & A-154 & 39 & P70 & A. 250 \\
\hline 14 & P39 & A. 55 & & P06B & A. 157 & & P70A & A. 254 \\
\hline 15 & P21 & A. 59 & 30 & M03 & A. 160 & & P70B & A.257 \\
\hline 16 & P52 & A. 63 & & M03A & A. 164 & 40 & P71 & A. 260 \\
\hline 17 & P55 & A. 67 & & M03B & A-167 & & P71A & A-264 \\
\hline & P55A & A-71 & 31 & P66 & A- 170 & & P71B & A. 267 \\
\hline & P55B & A. 74 & & P66A & A- 174 & 41 & $\mathrm{CO1}$ & A. 270 \\
\hline 18 & P56 & A- 77 & & P66B & A. 177 & & C01A & A- 274 \\
\hline & P56A & A- 81 & 32 & P32 & A. 180 & & $\mathrm{C01B}$ & A. 277 \\
\hline & P56B & A. 84 & & P32A & A. 184 & 42 & $\mathrm{CO} 2$ & A- 280 \\
\hline 19 & P20 & A. 87 & & P32B & A- 187 & & $\mathrm{CO} 2 \mathrm{~A}$ & A-284 \\
\hline 20 & P57 & A- 91 & 33 & P67 & A. 190 & & $\mathrm{C} 02 \mathrm{~B}$ & A. 287 \\
\hline 21 & P58 & A. 95 & & P67A & A. 194 & 43 & M05 & A. 290 \\
\hline 22 & P60 & A.99 & & P67B & A. 197 & & M05A & A-294 \\
\hline & P6OA & A-103 & 34 & P68 & A- 200 & & M05B & A.297 \\
\hline & P60B & A. 106 & & P68A & A. 204 & & & \\
\hline 23 & P61 & A. 109 & & P68B & A-207 & & & \\
\hline Phase II: & & & & & & & & \\
\hline 44 & PS17 & A. 300 & 57 & PSO2 & A.352 & 66 & PS33 & A-399 \\
\hline 45 & PS24 & A. 304 & 58 & PS13M1A & A.356 & & PS33A & A. 403 \\
\hline 46 & PS18 & A-308 & 59 & PS09M1 & A. 359 & & PS33B & A. 406 \\
\hline 47 & PS26 & A-312 & 60 & PS31 & A-363 & 67 & AF07 & A. 409 \\
\hline 48 & PS25 & $A-316$ & 61 & PS14 & A. 367 & & AF07A & A- 413 \\
\hline 49 & PS27 & A.320 & & PS14A & A.371 & & AF07B & A. 416 \\
\hline 50 & PS24R1 & A-324 & & PS14B & A-374 & 68 & AF08 & A. 419 \\
\hline 51 & PS28 & A-328 & 62 & PS15 & A.377 & & AF08A & A. 423 \\
\hline 52 & PS29 & A. 332 & 63 & PS16 & $A-381$ & & AF08B & A- 426 \\
\hline 53 & PS01 & A.336 & 64 & PS32 & $A-385$ & 69 & AF09 & A. 429 \\
\hline 54 & PS08 & A. 340 & 65 & PS11 & A-389 & & AF09A & A. 433 \\
\hline 55 & PS10 & A. 344 & & PS11A & A-393 & & AF09B & A. 436 \\
\hline 56 & PS12R1 & A- 348 & & PS11B & A. 396 & 70 & AF 10 & A. 439 \\
\hline
\end{tabular}




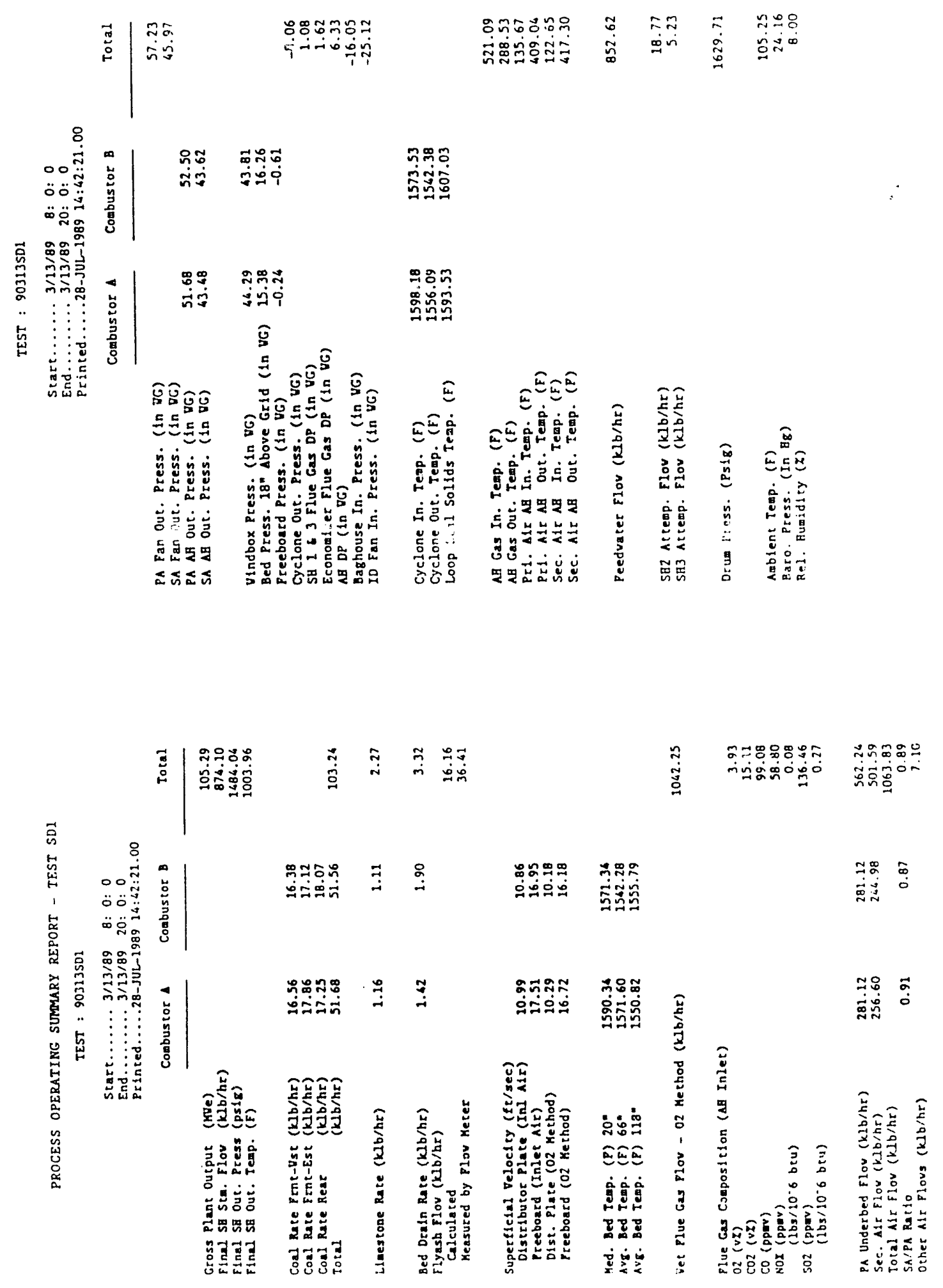




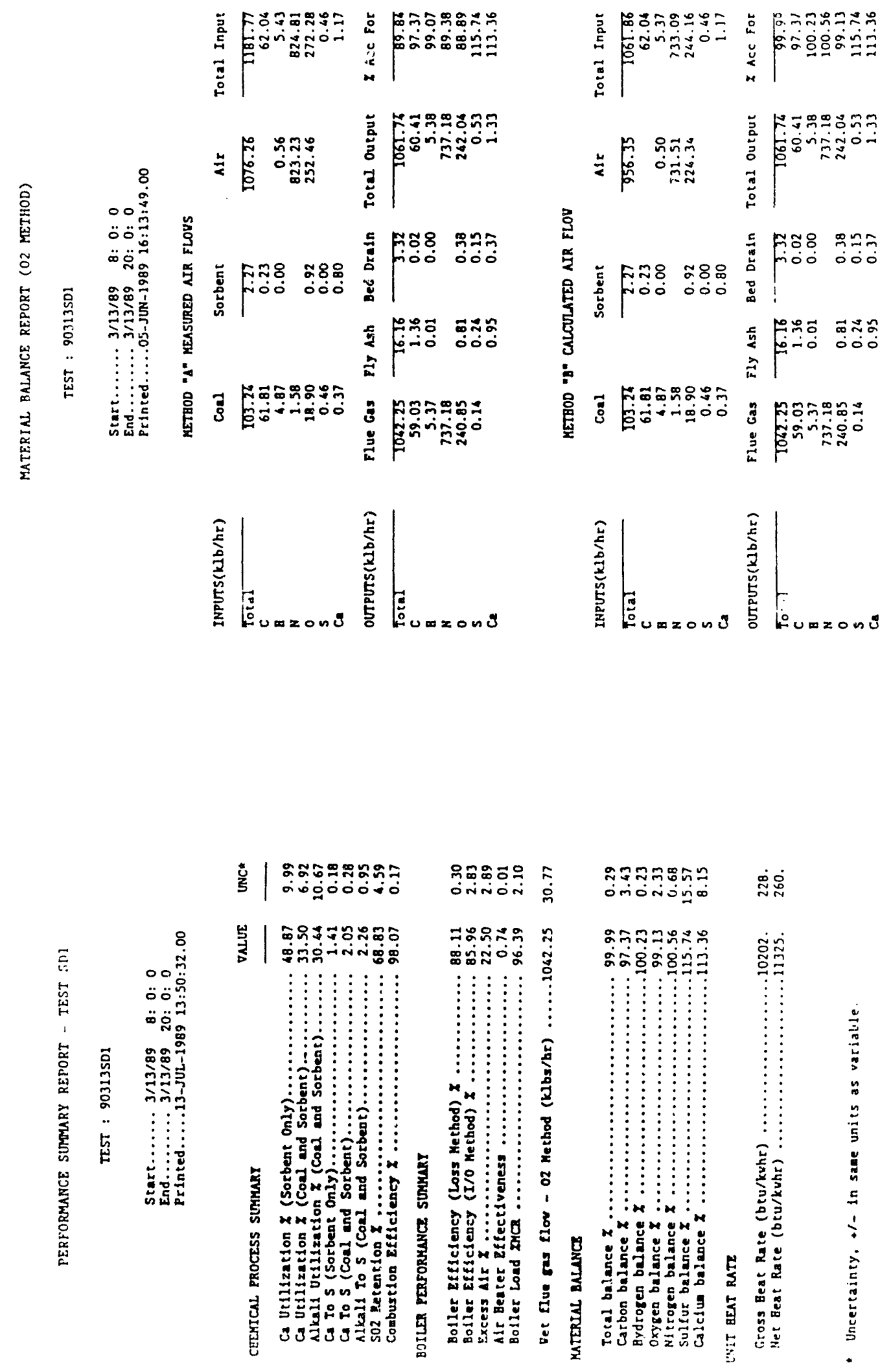




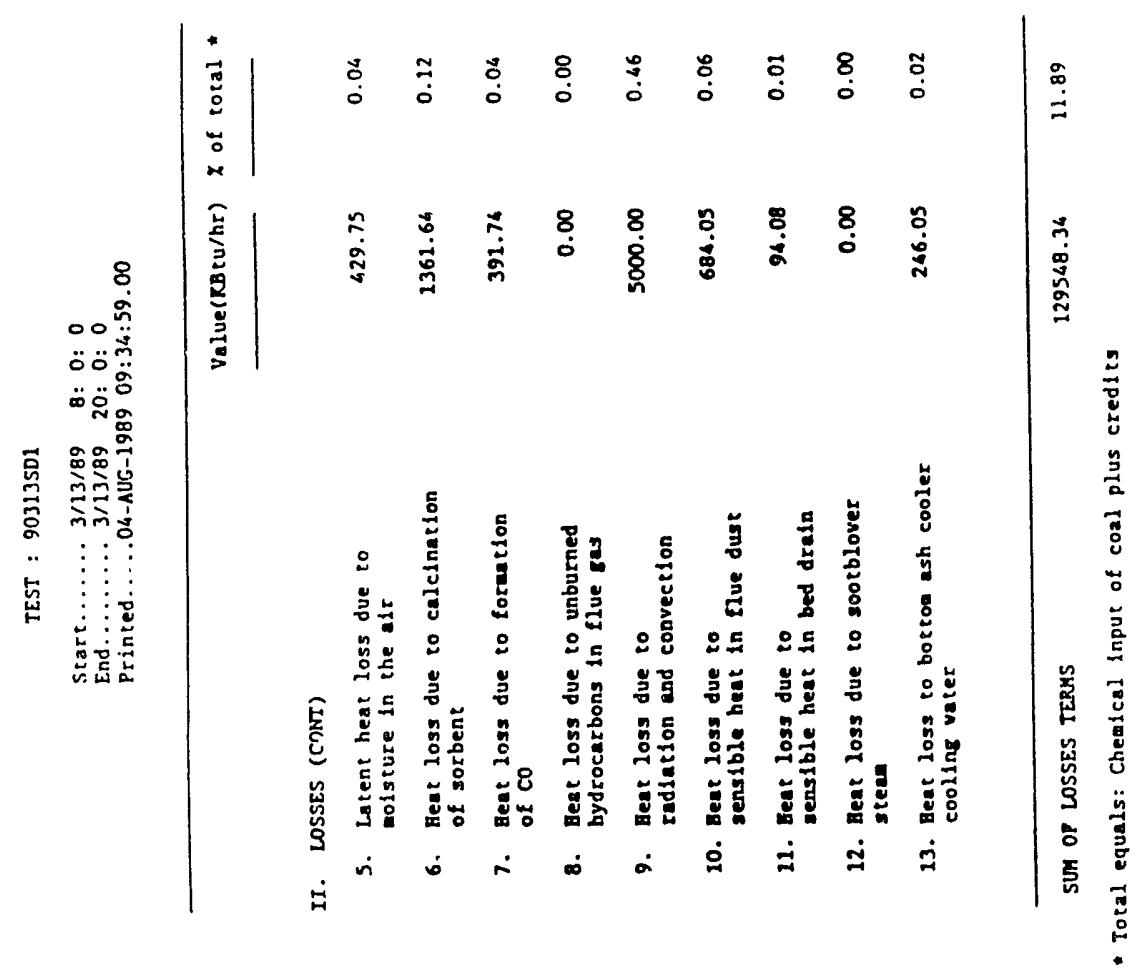

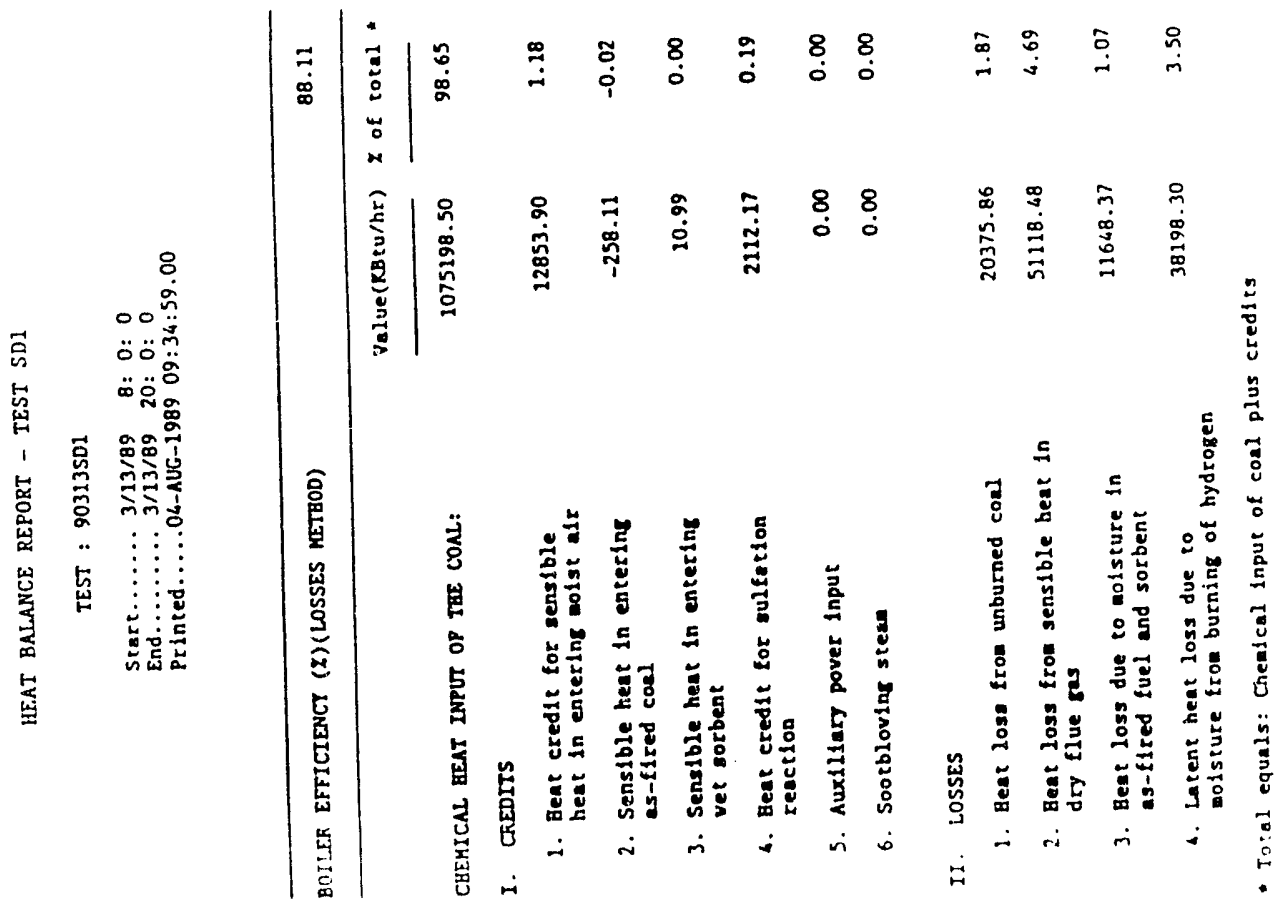



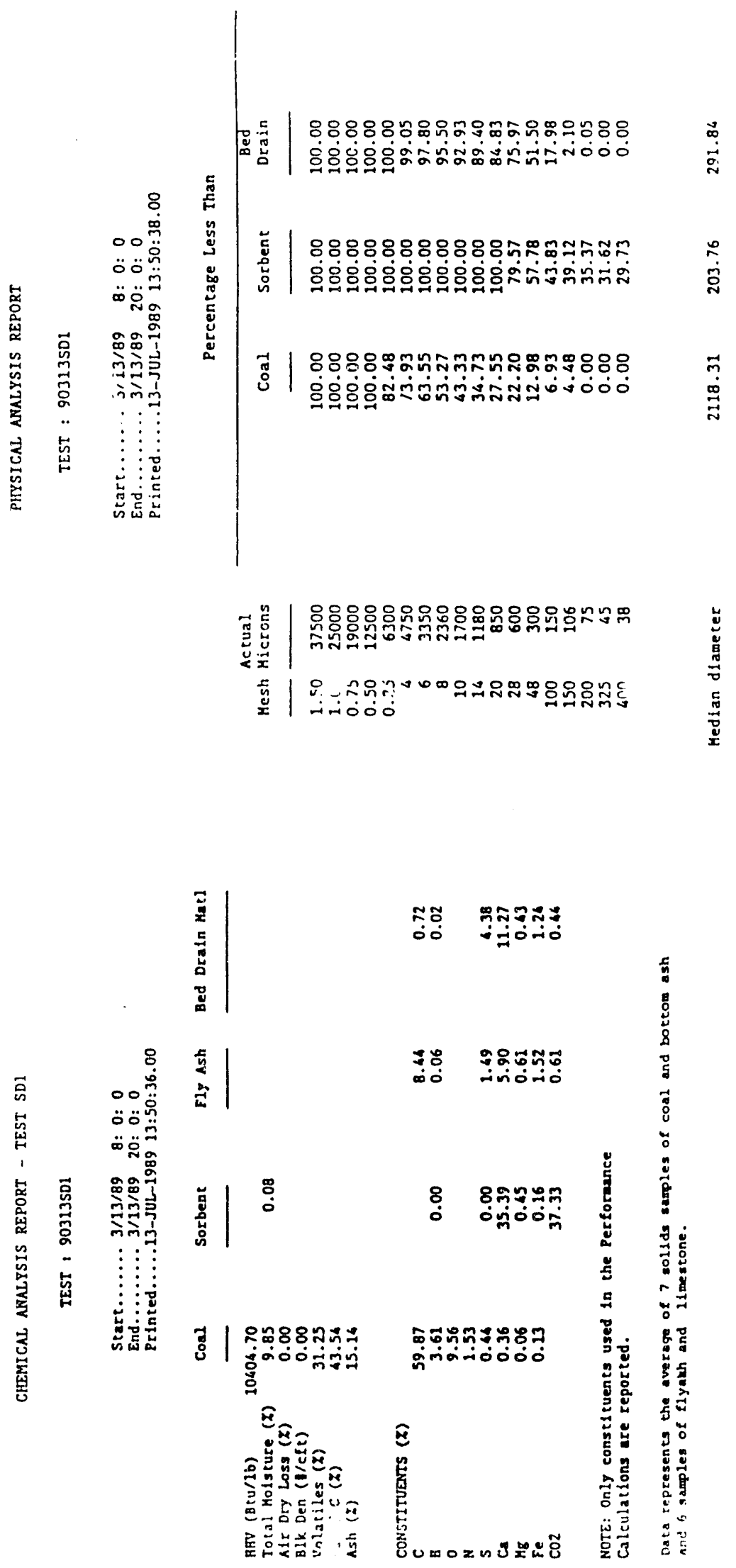

A-6 

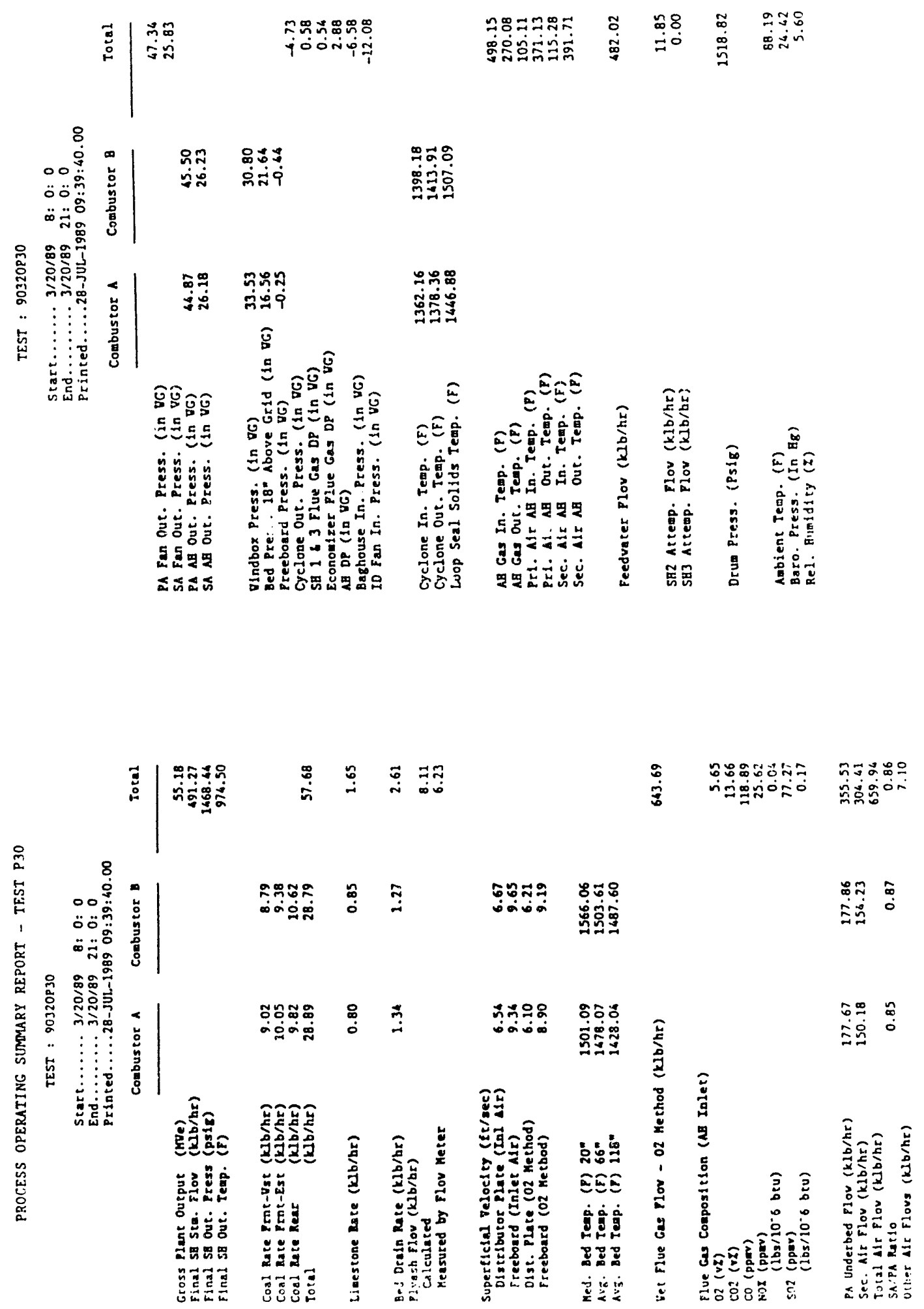

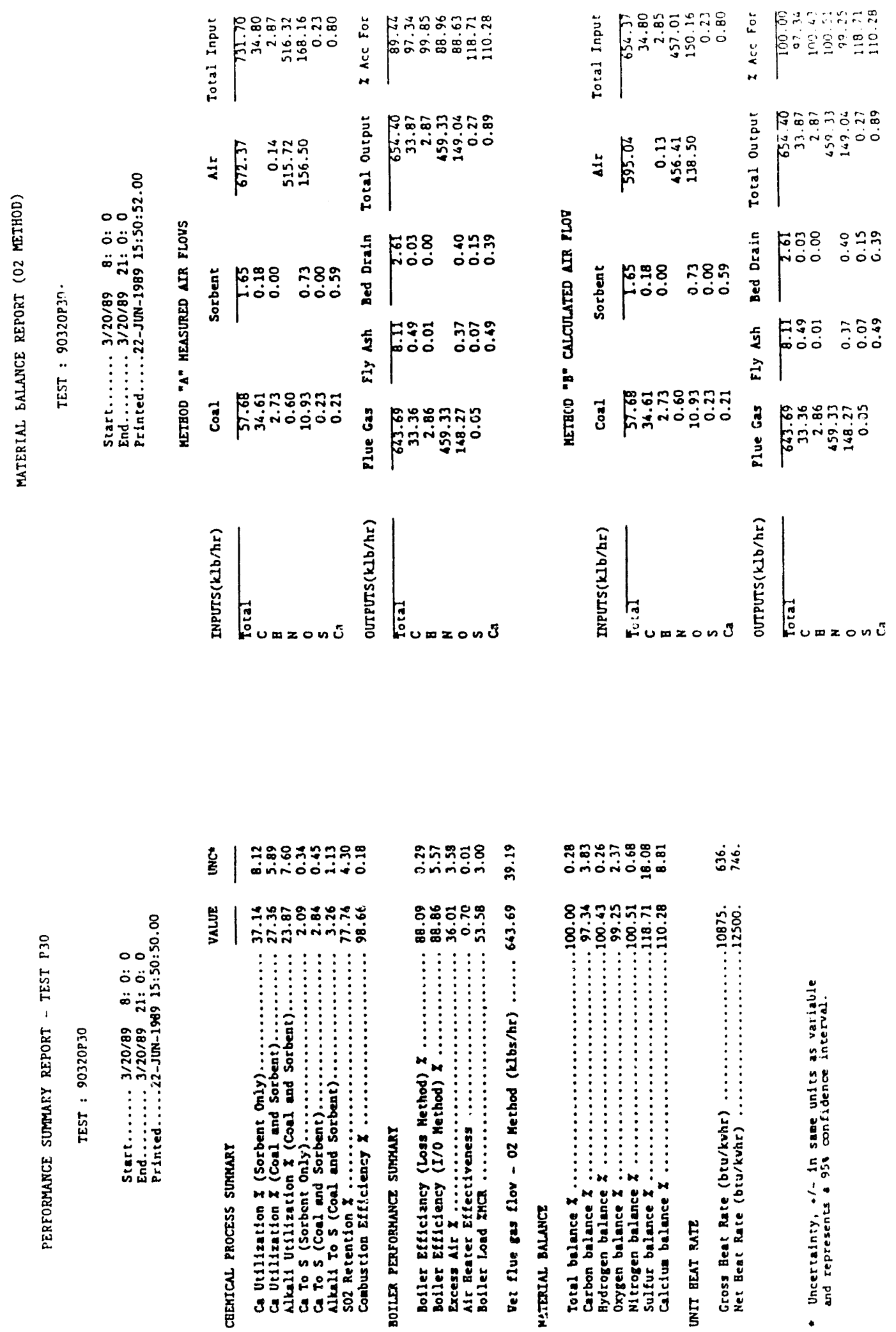


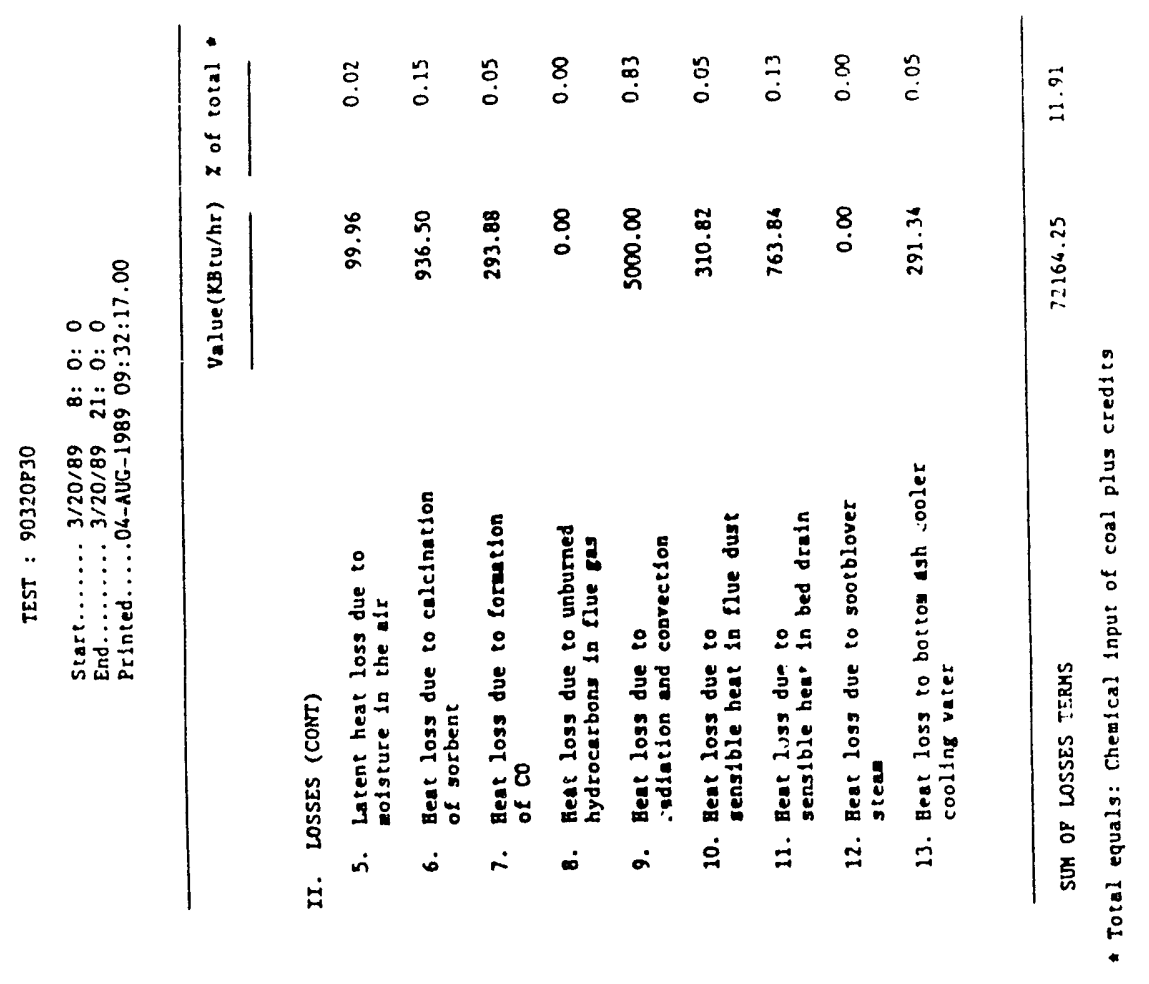

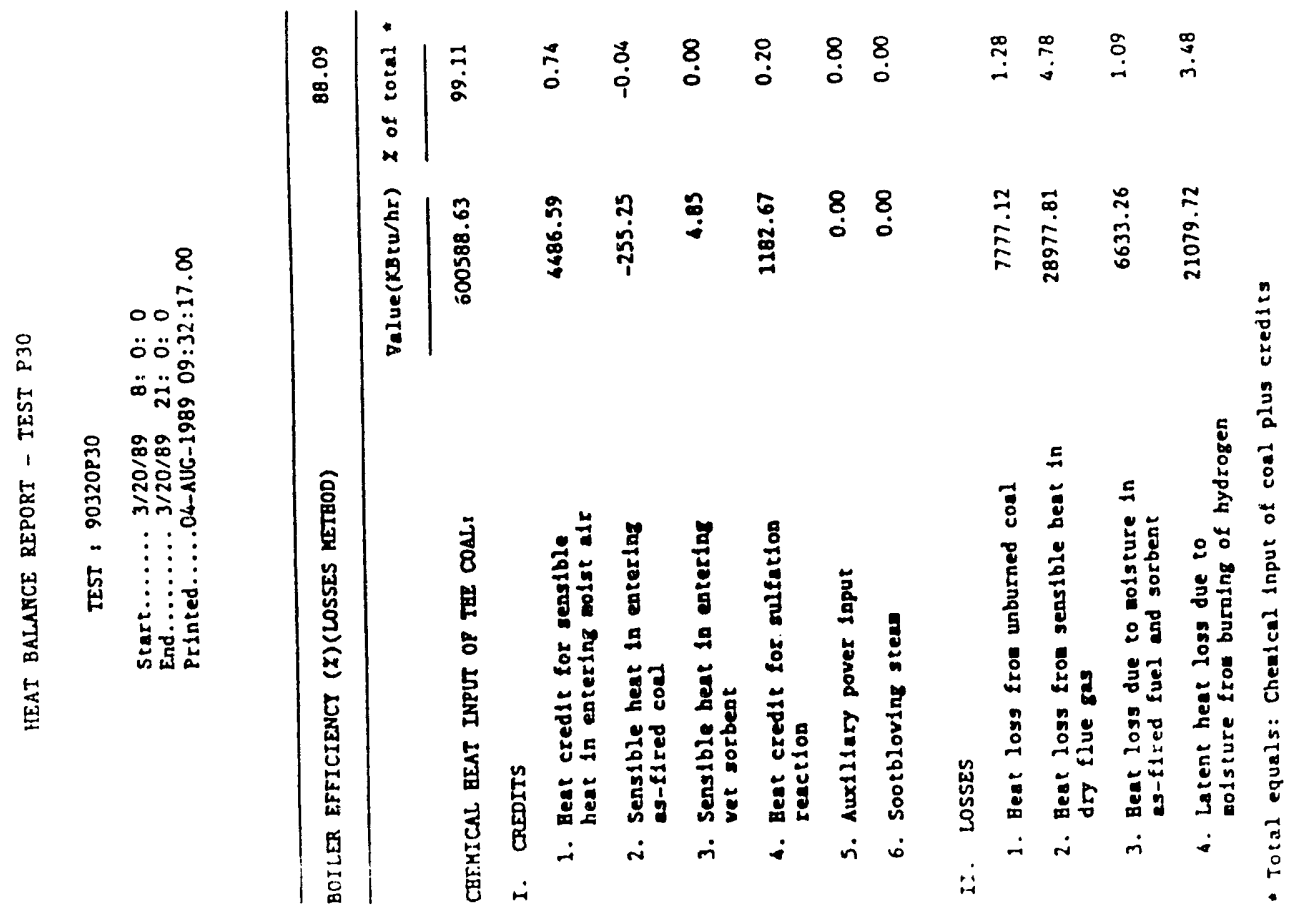



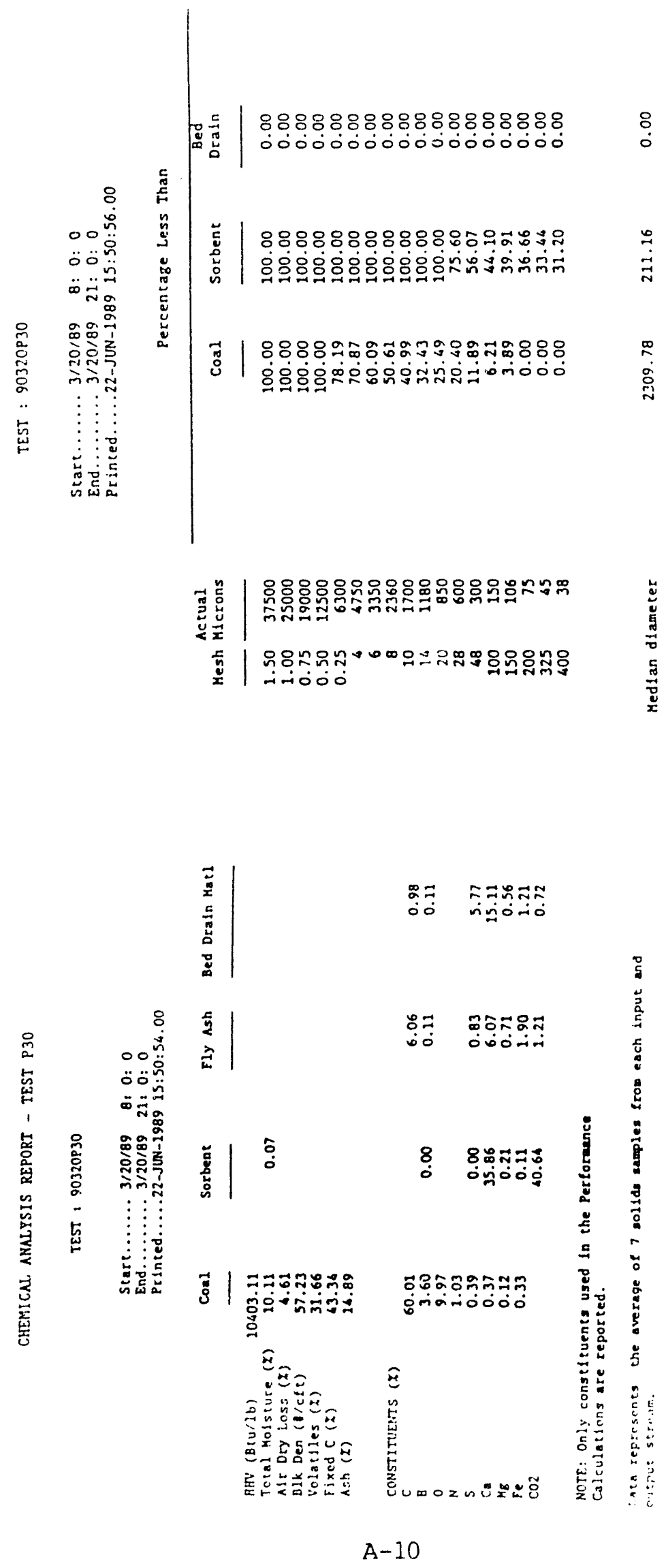

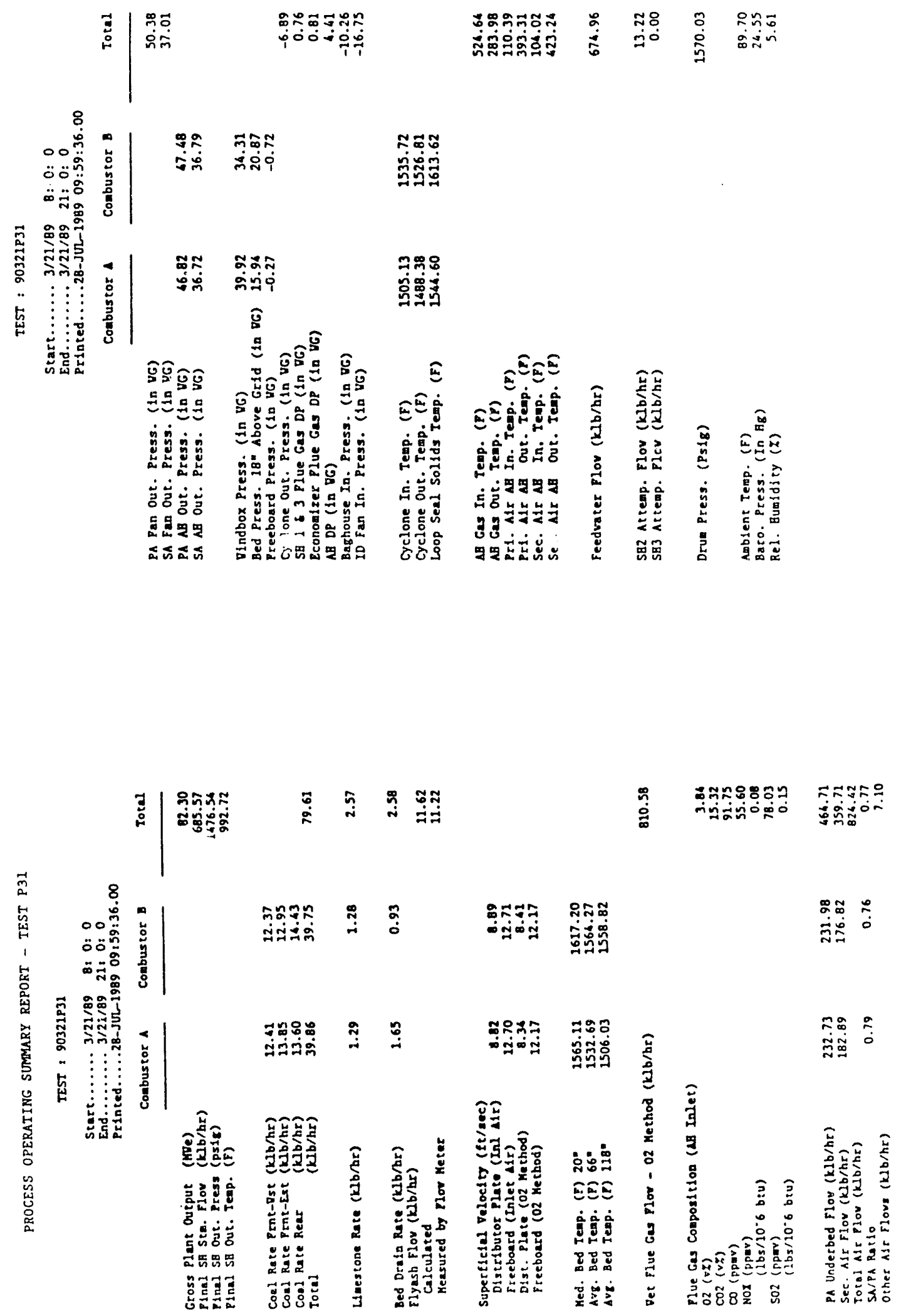


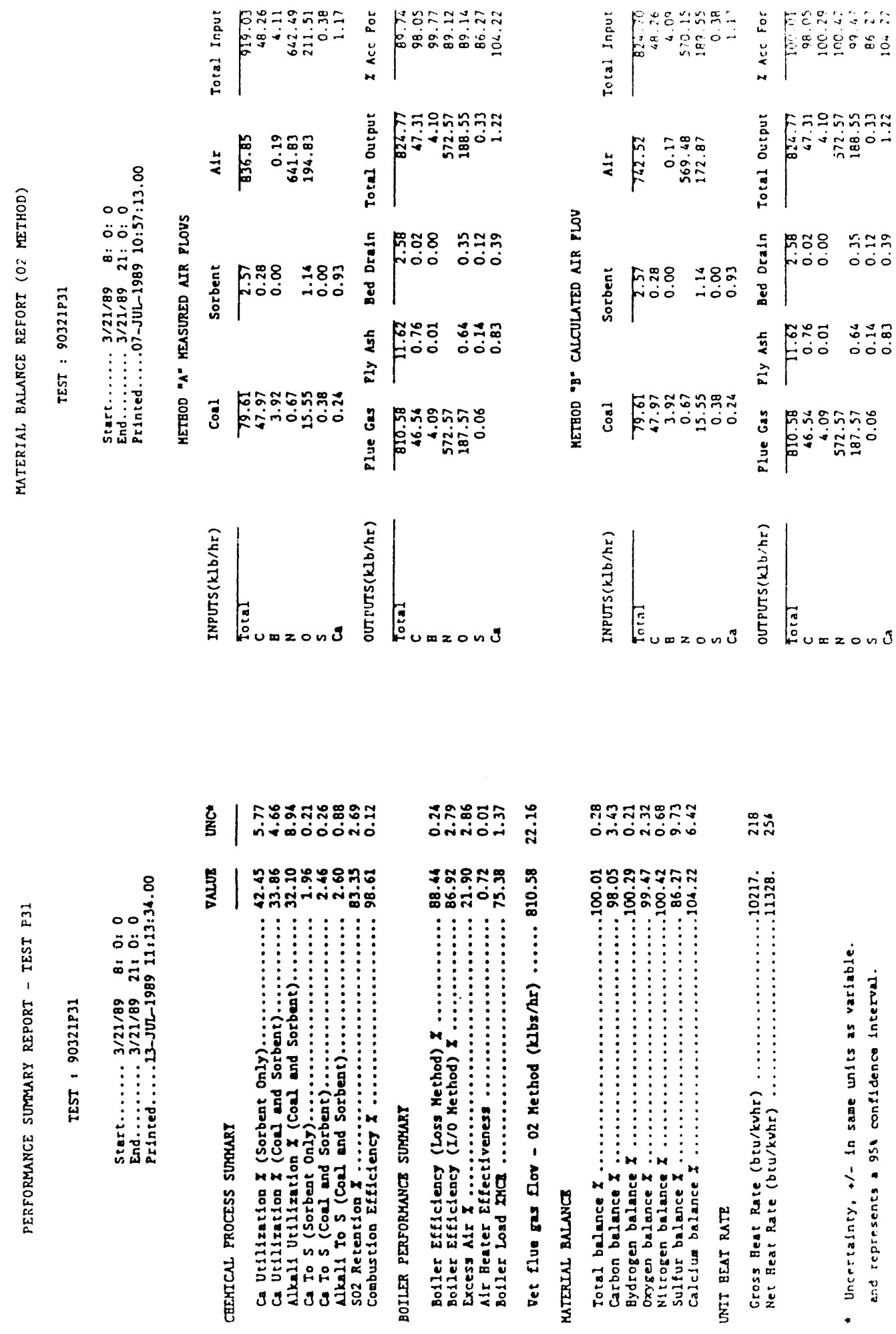




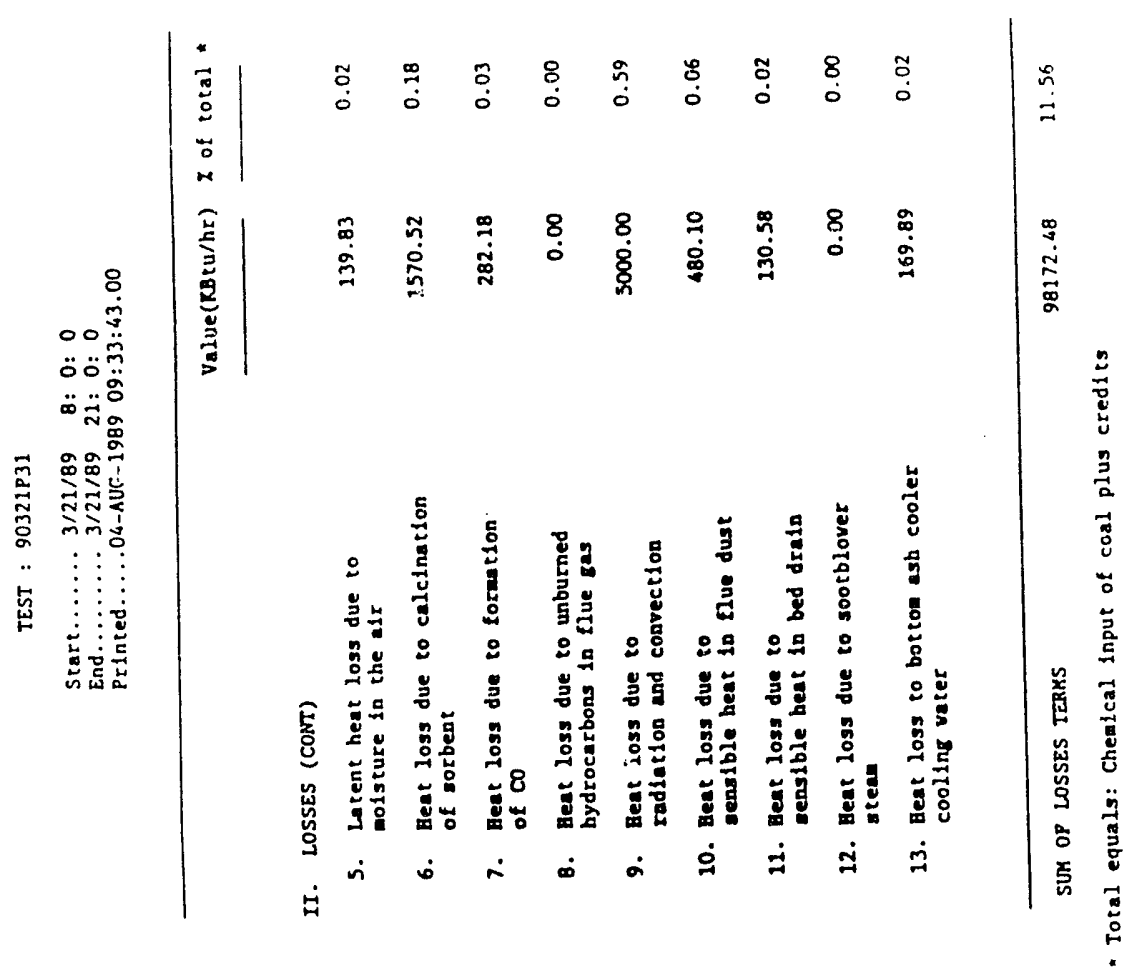

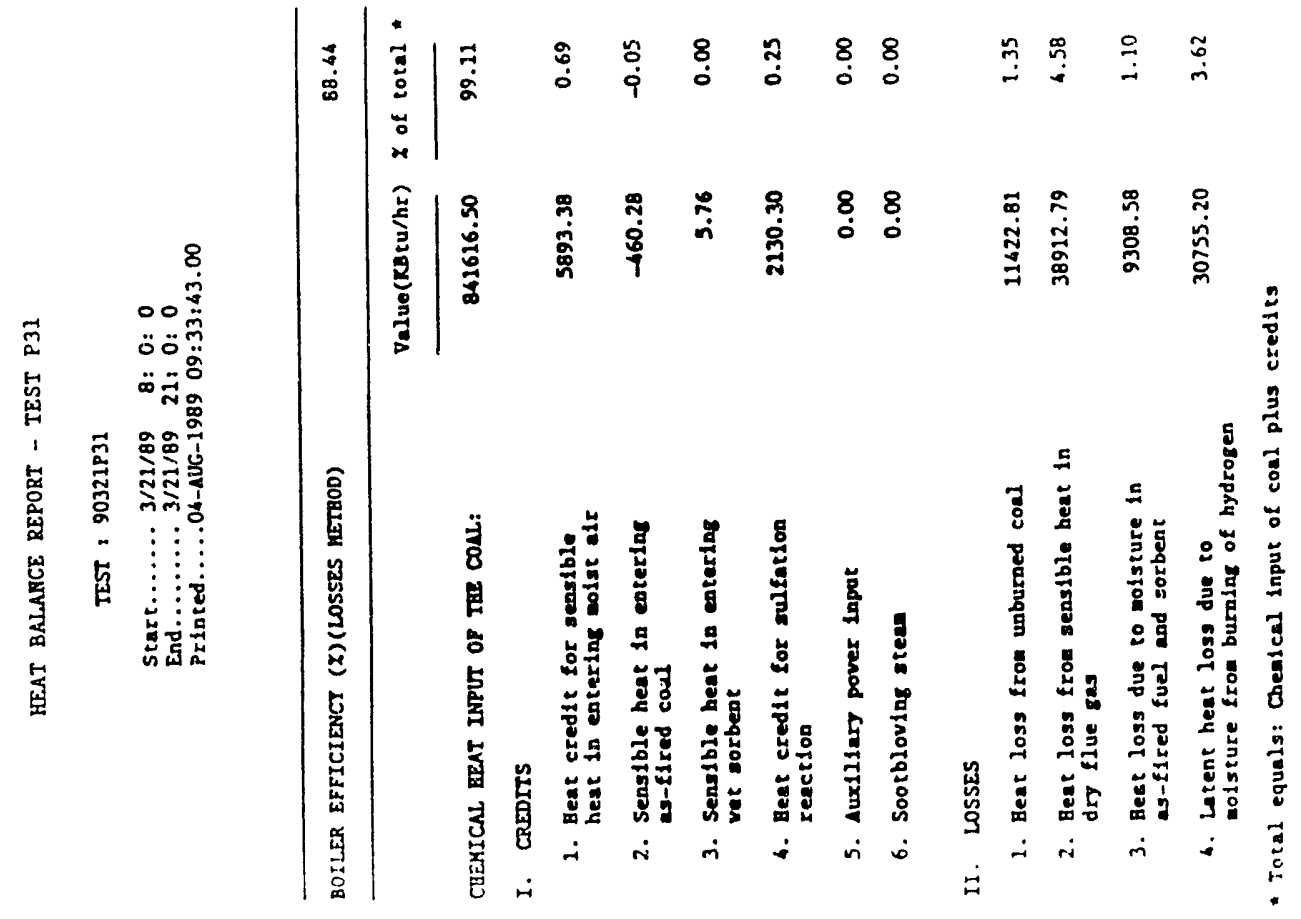



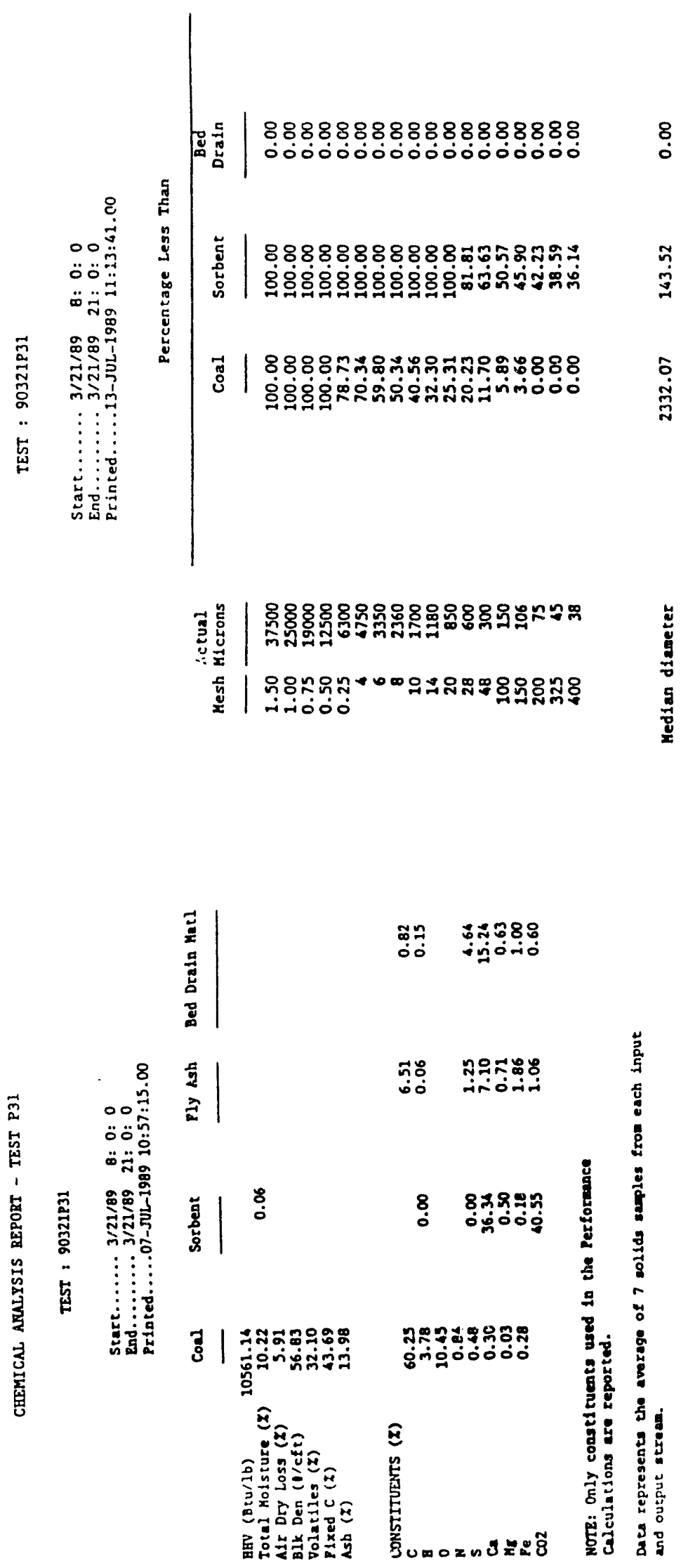

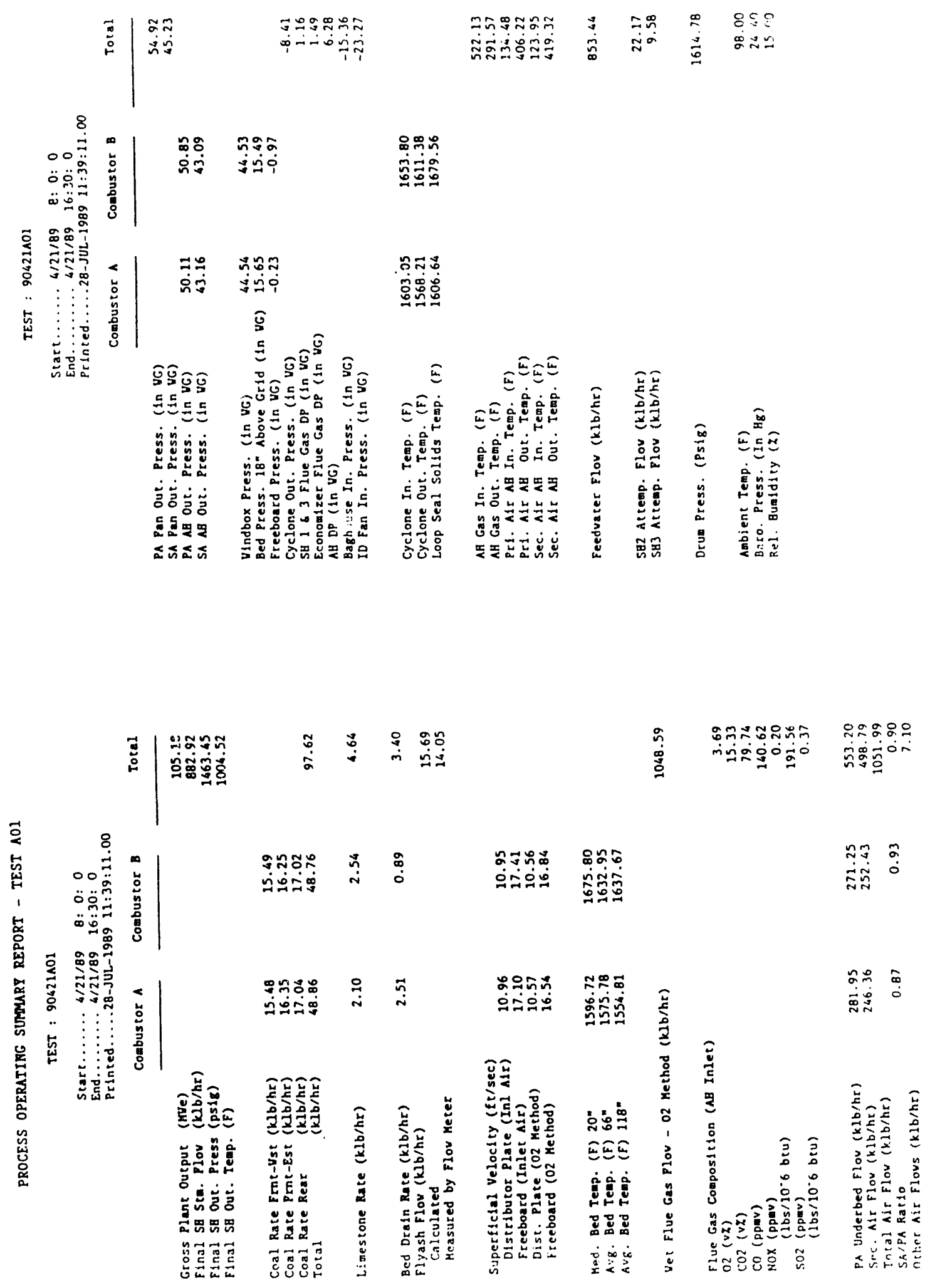

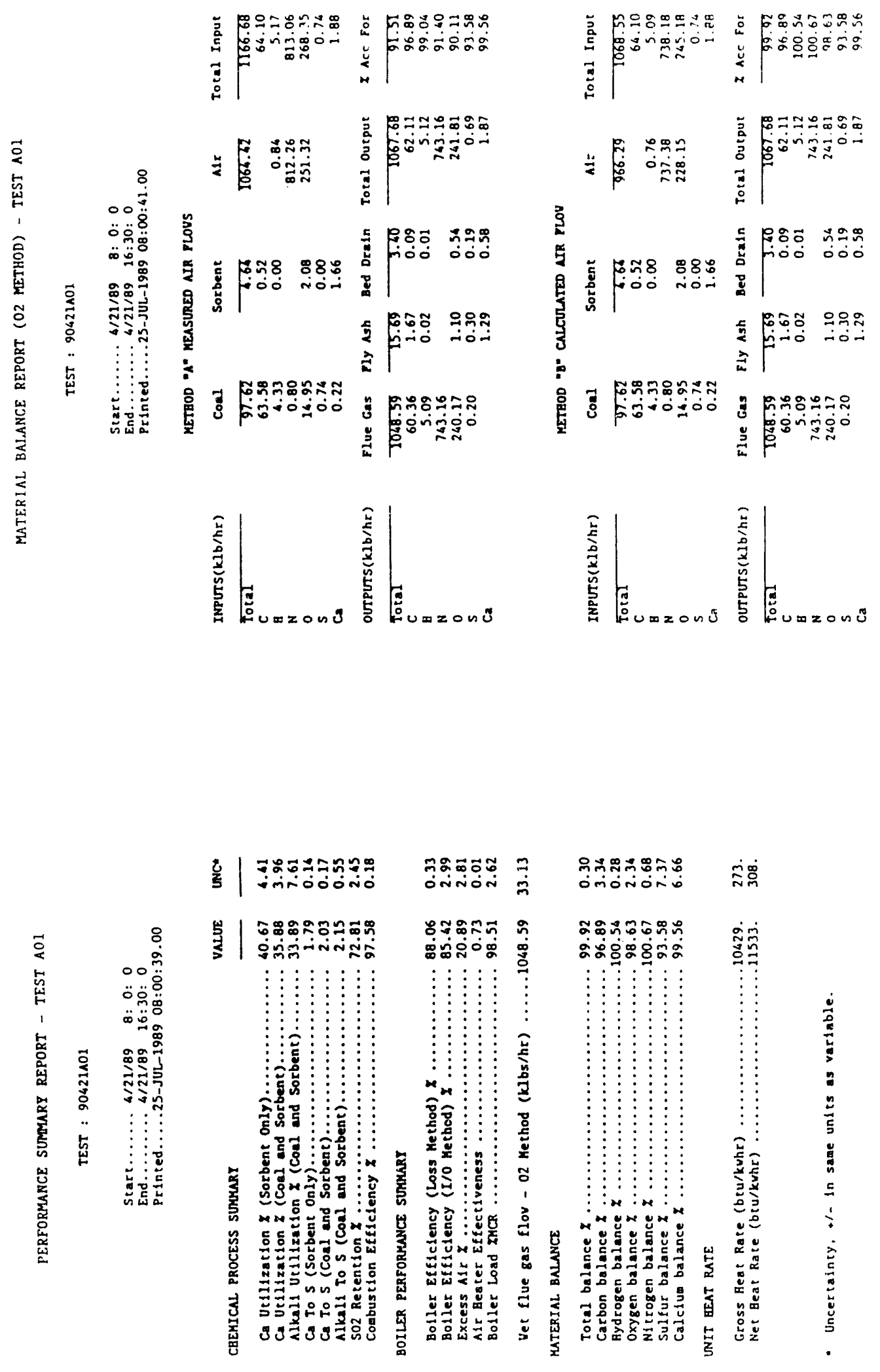


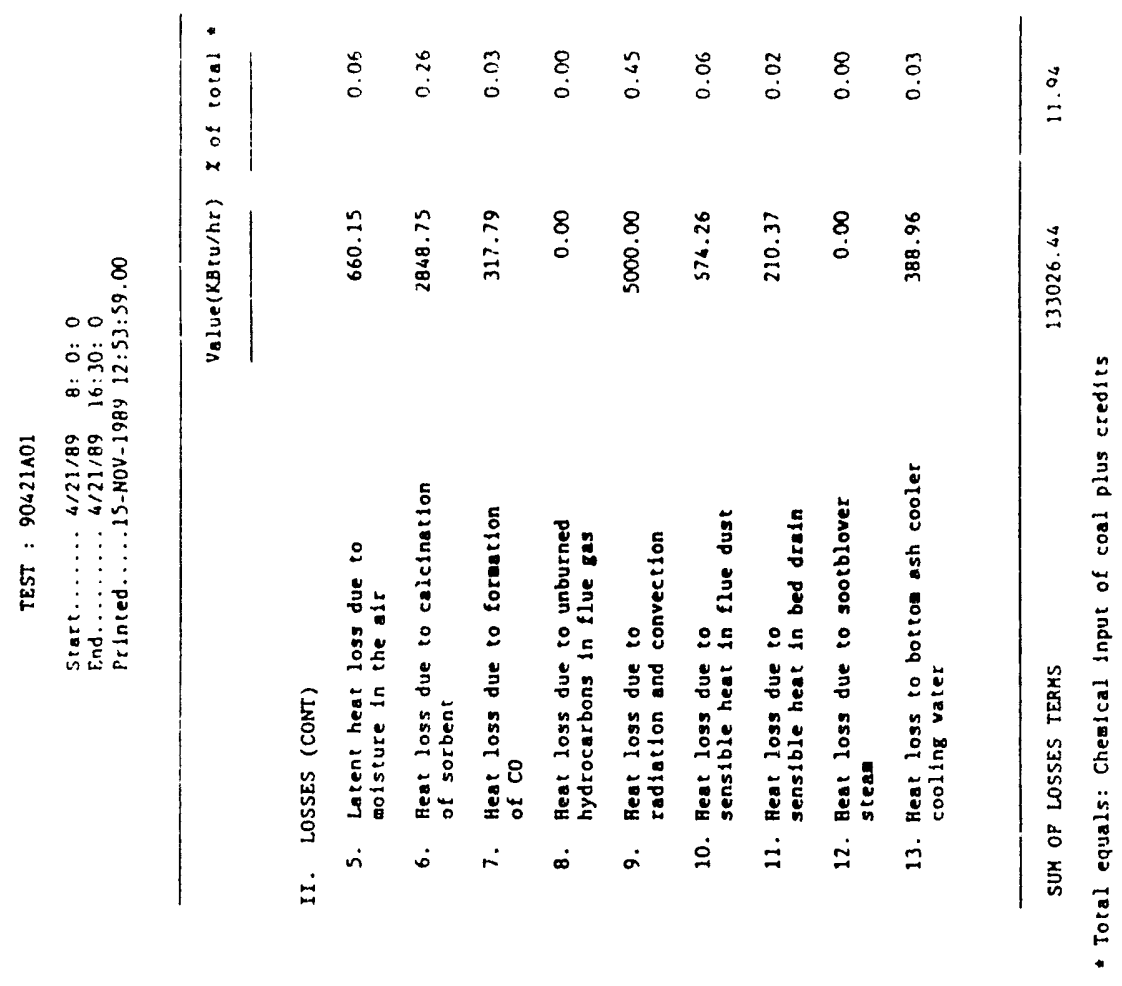

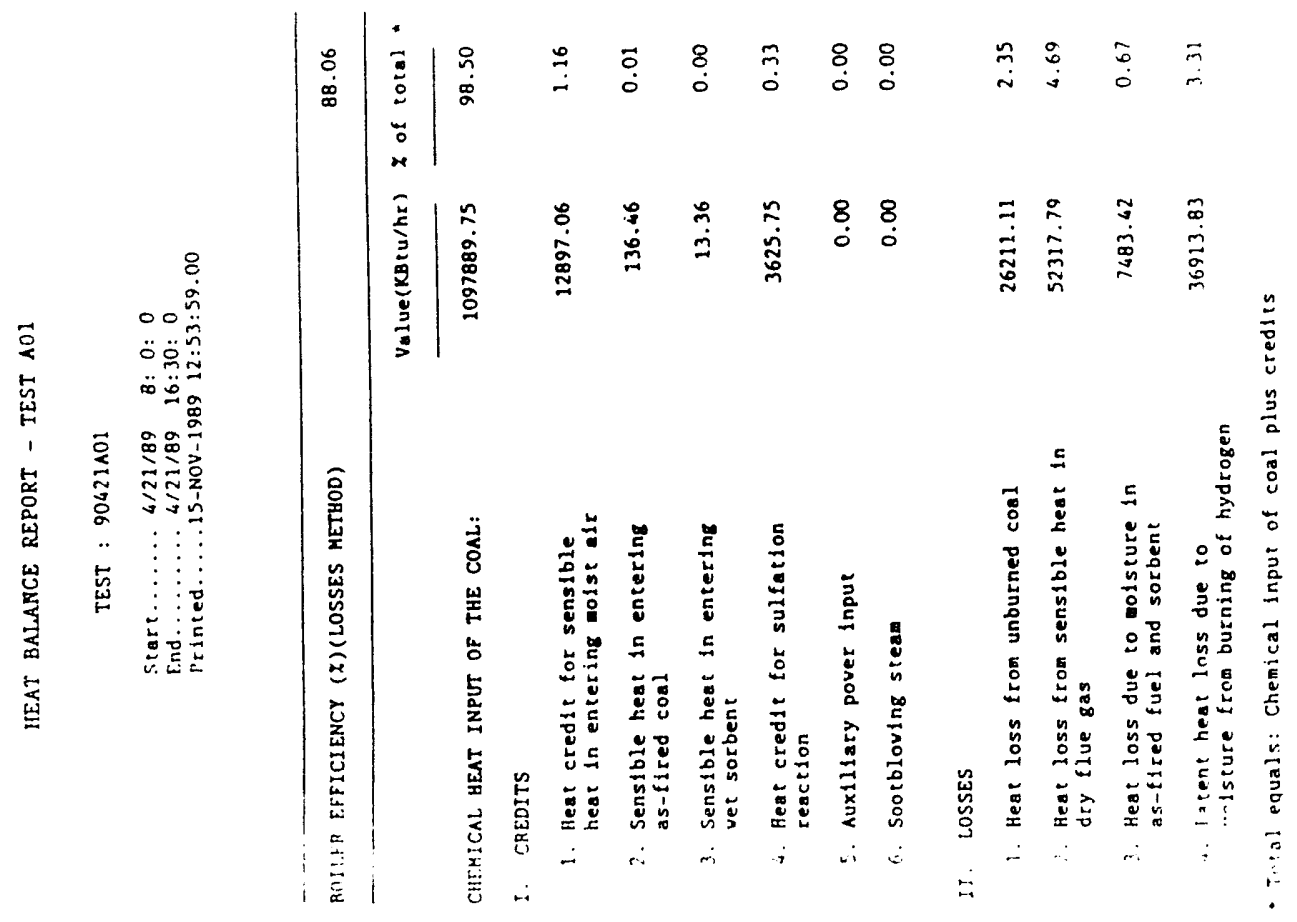



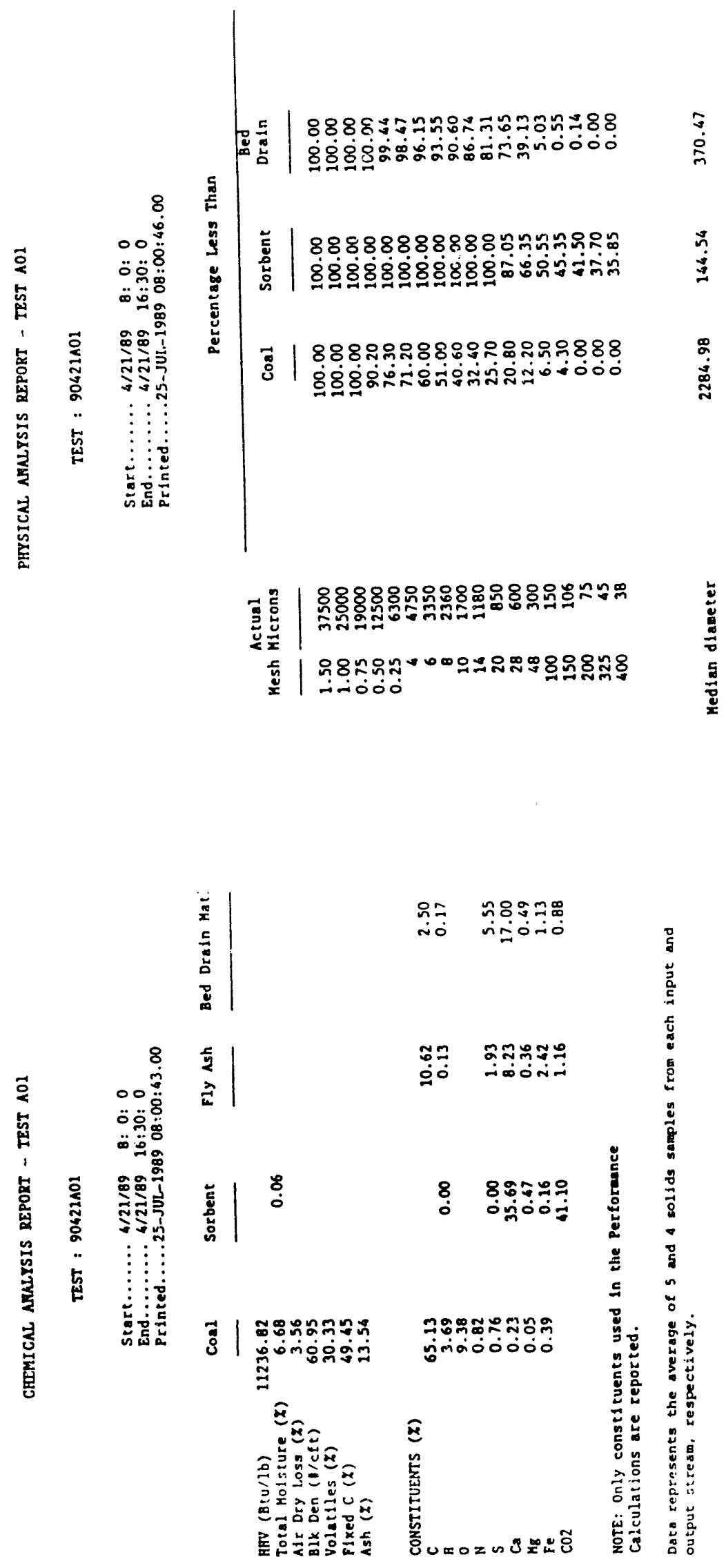

A-18 


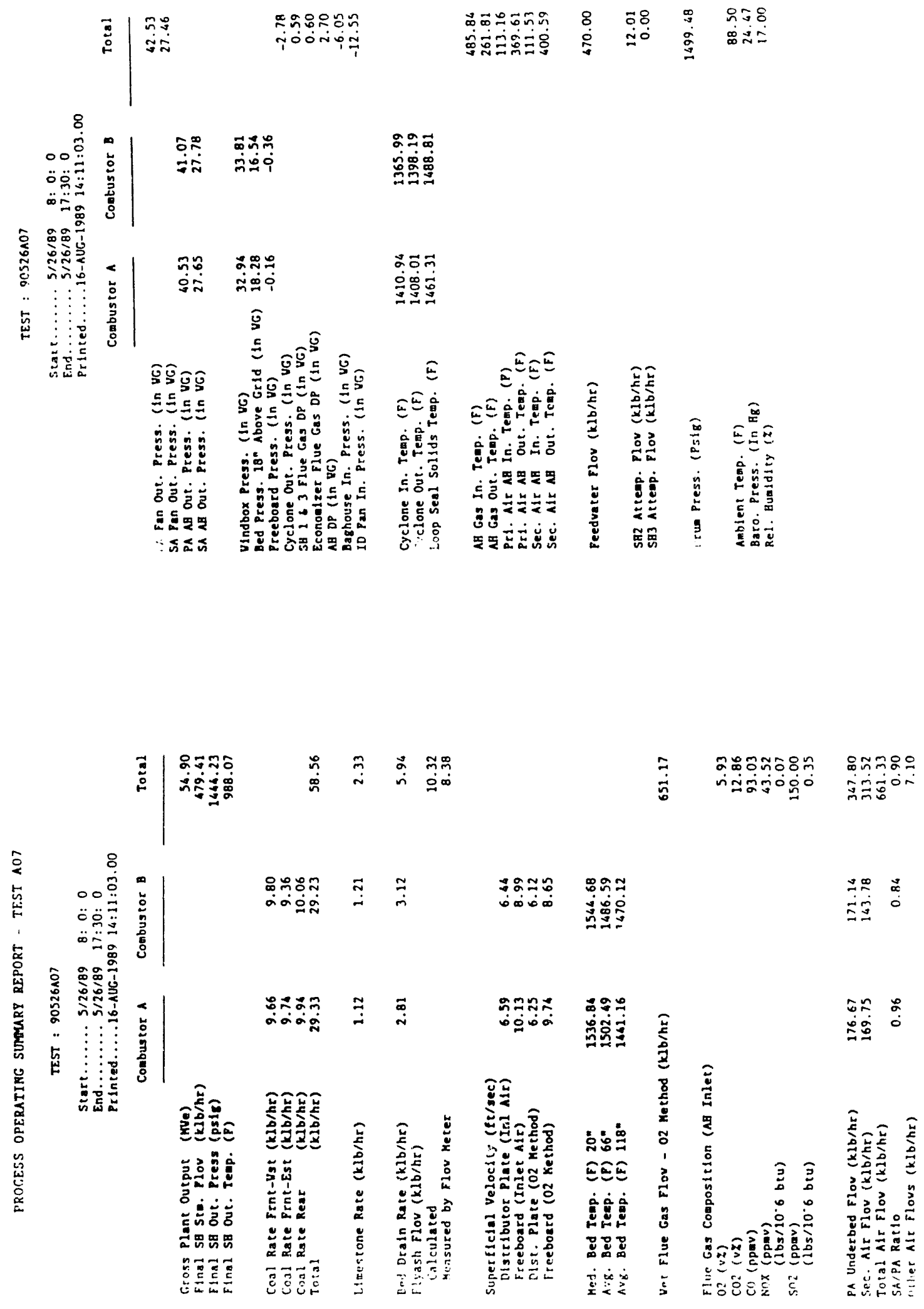




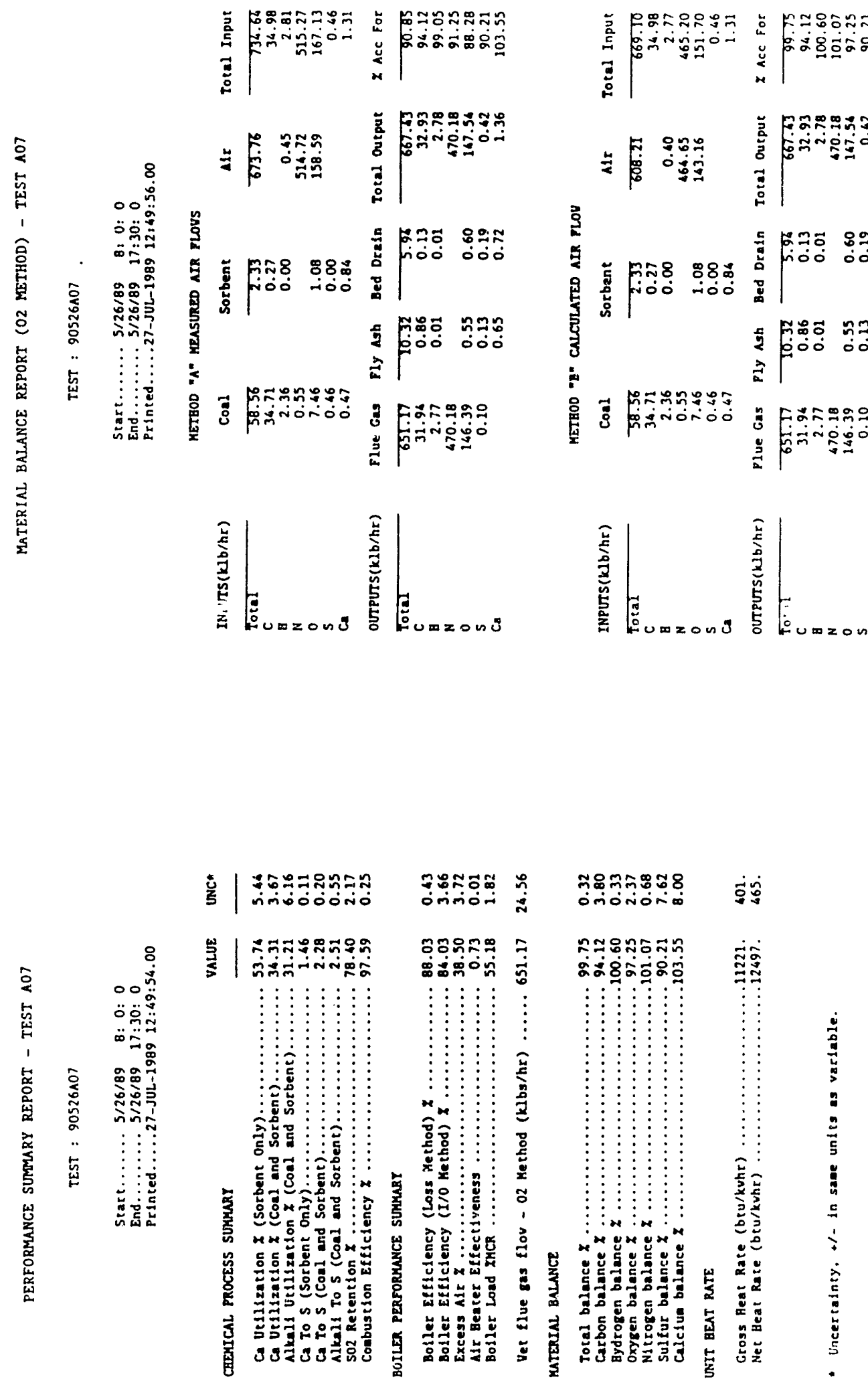




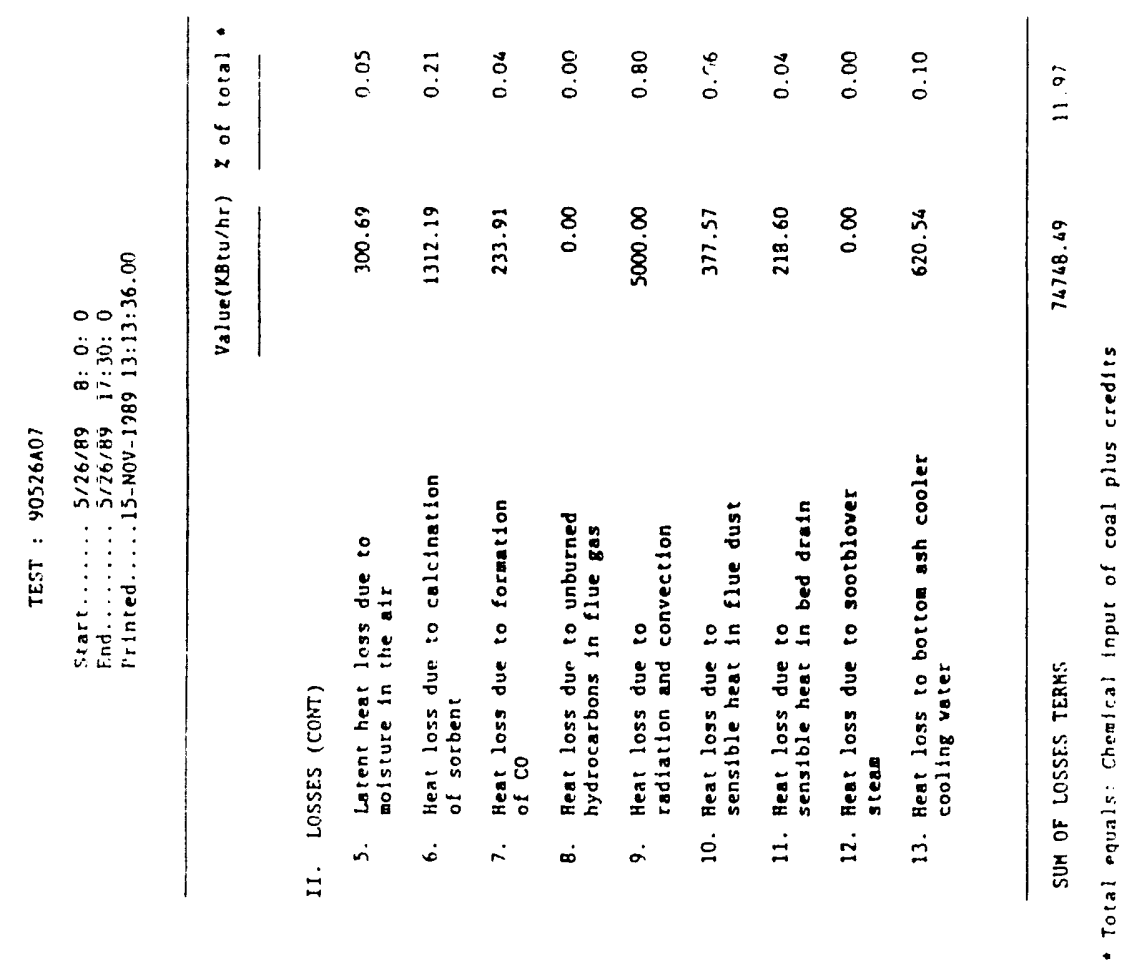

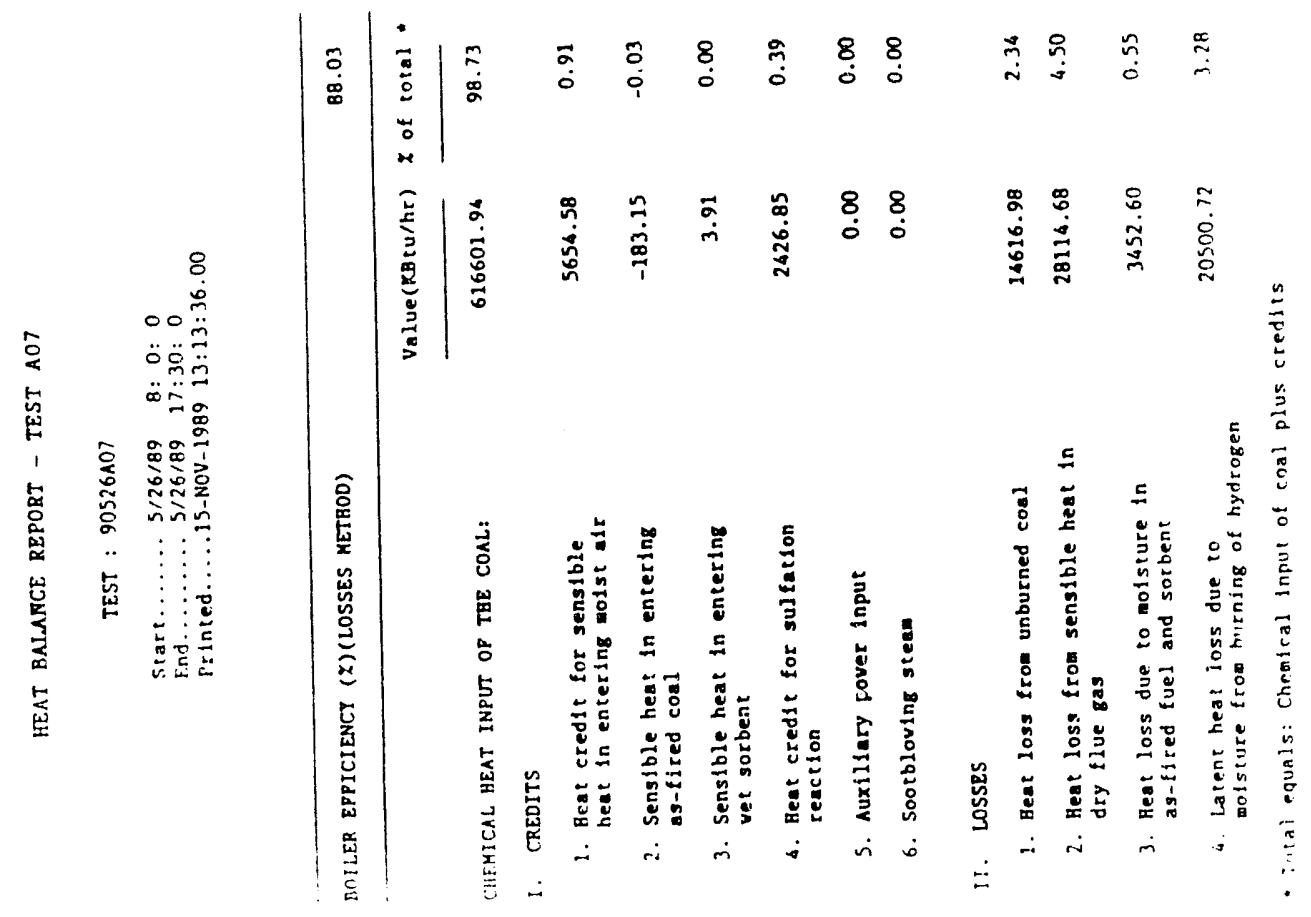



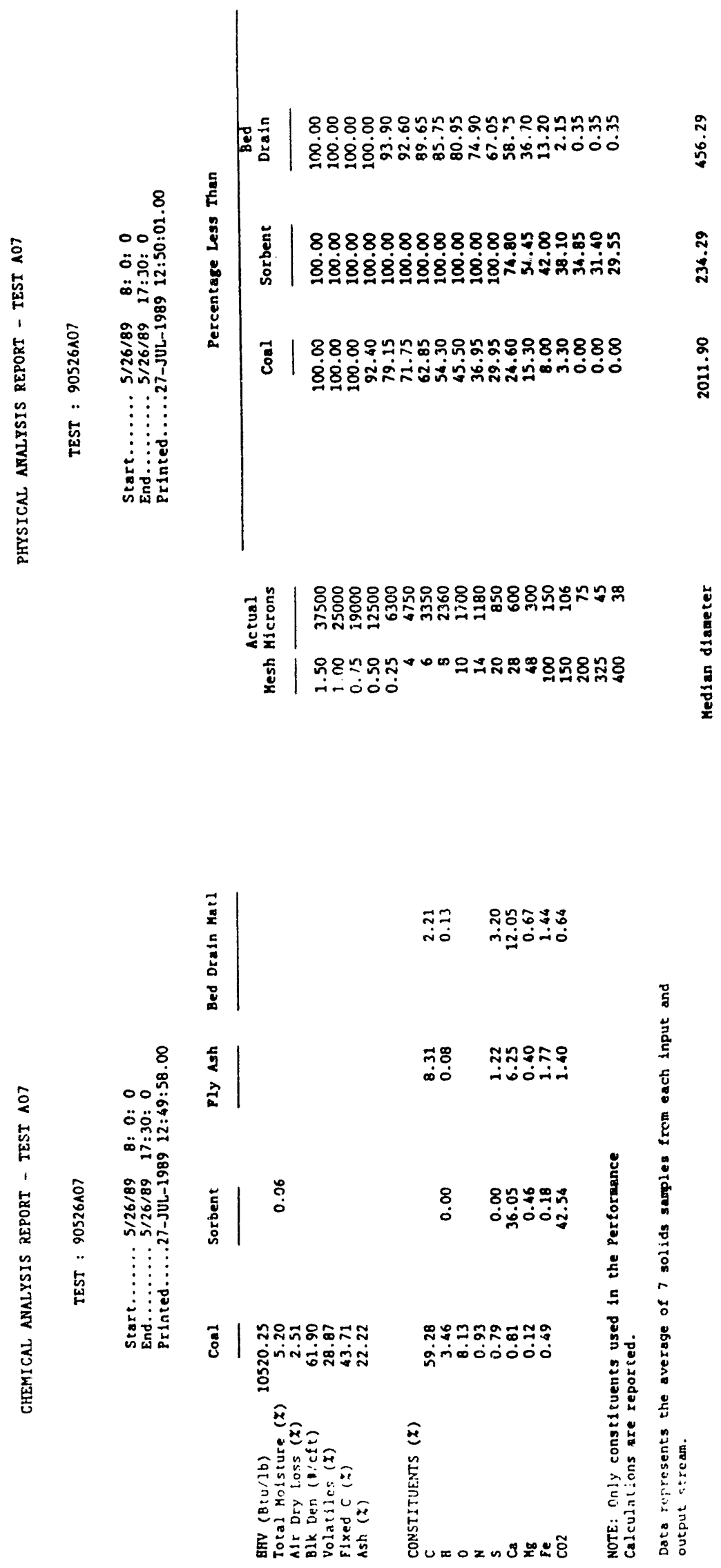

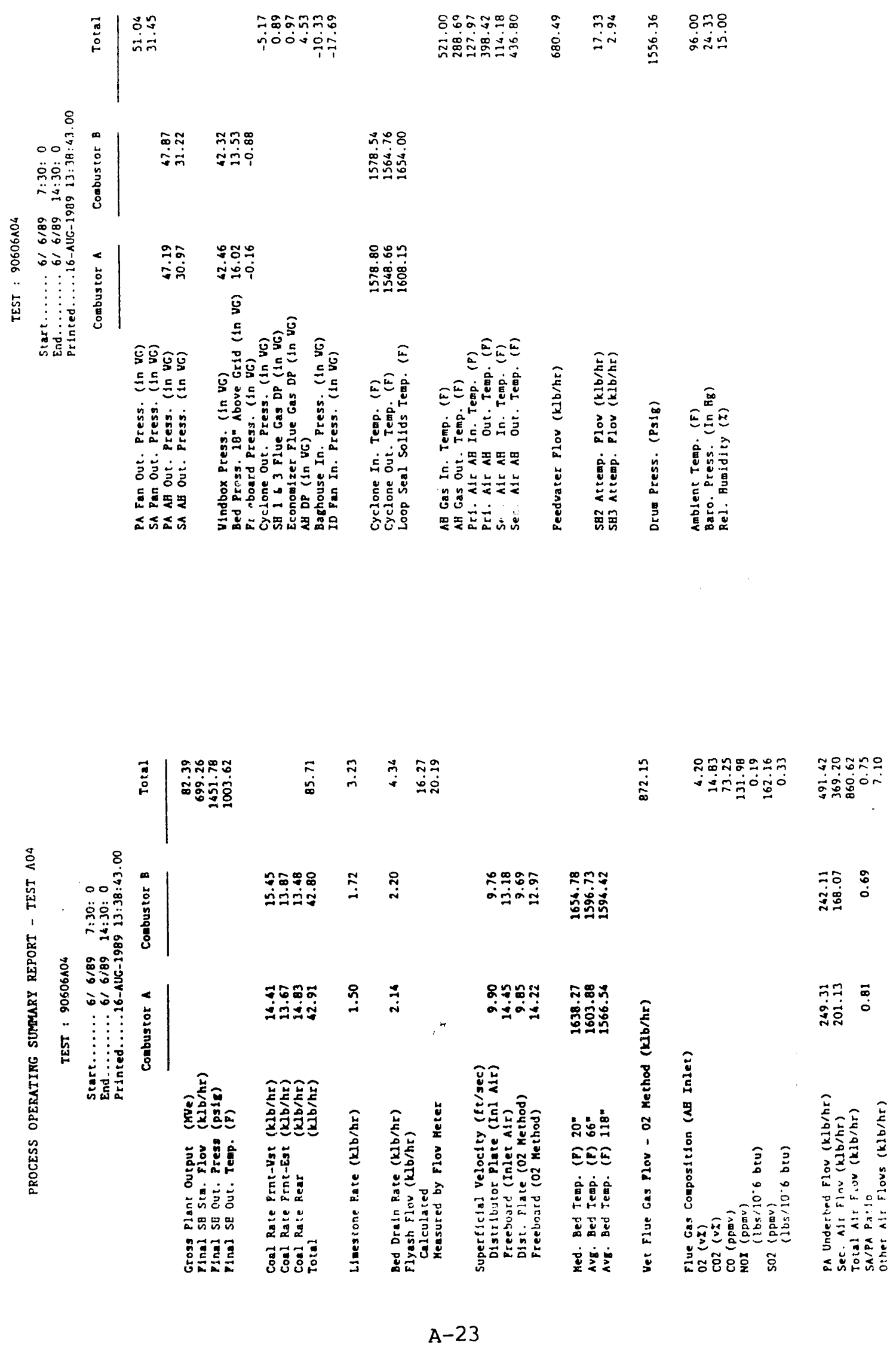

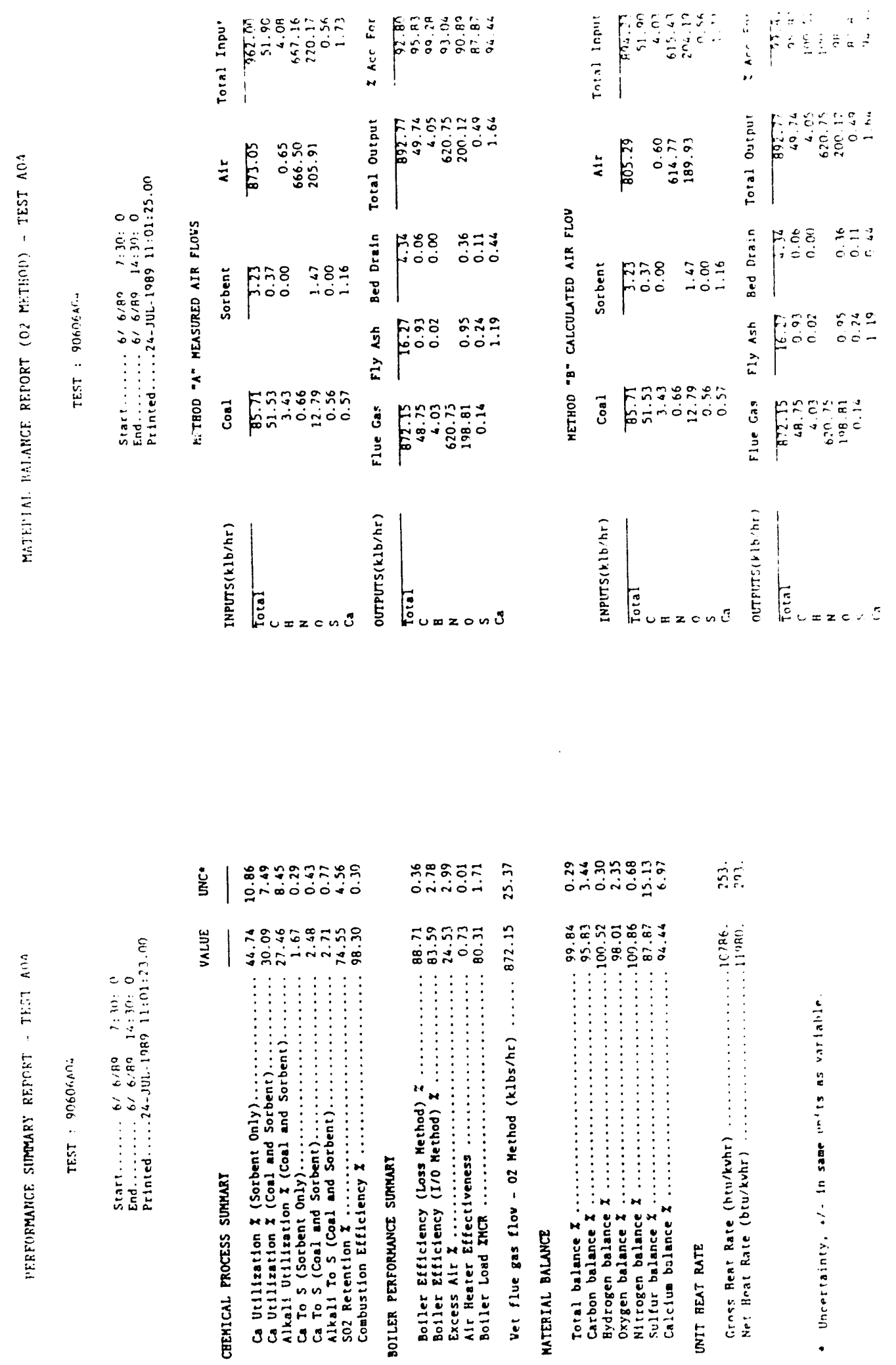


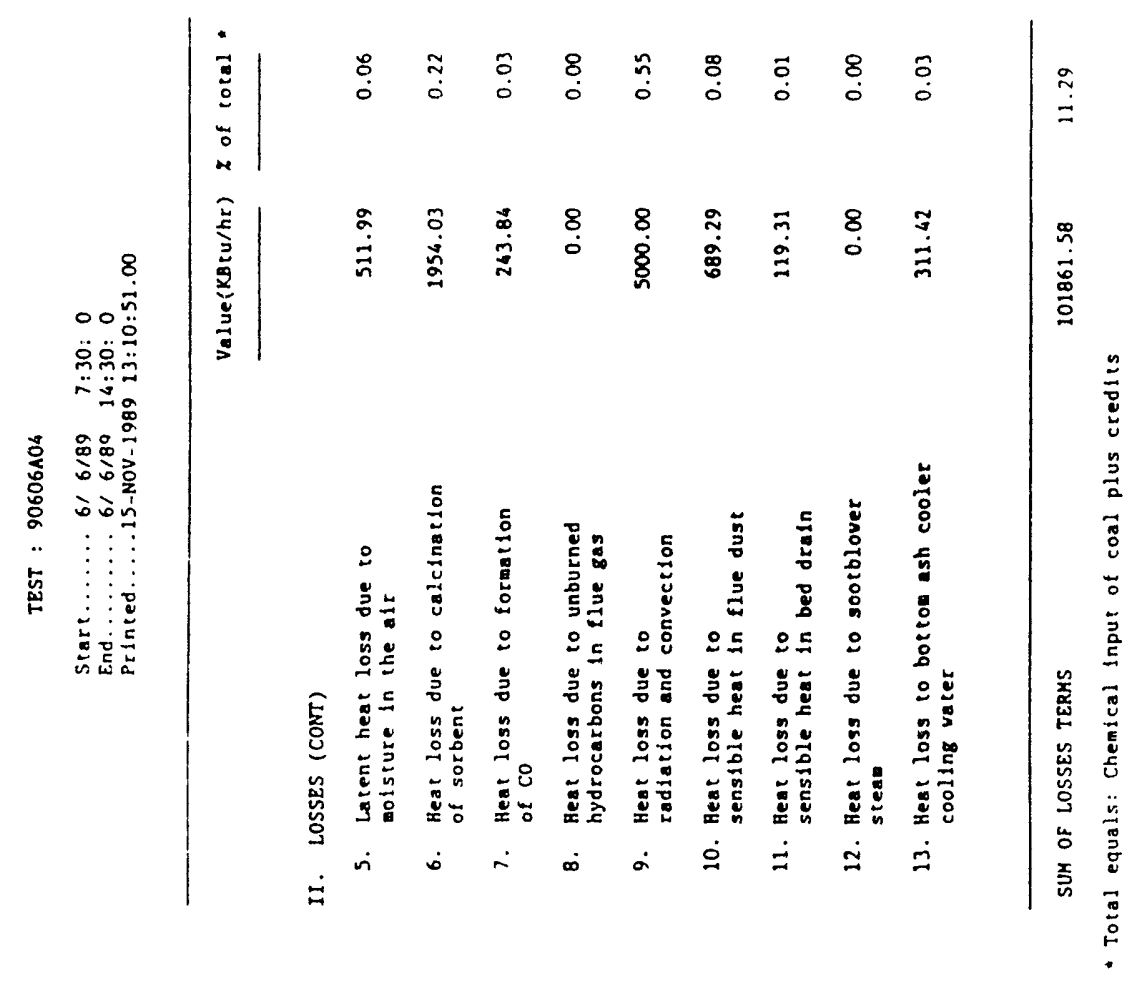

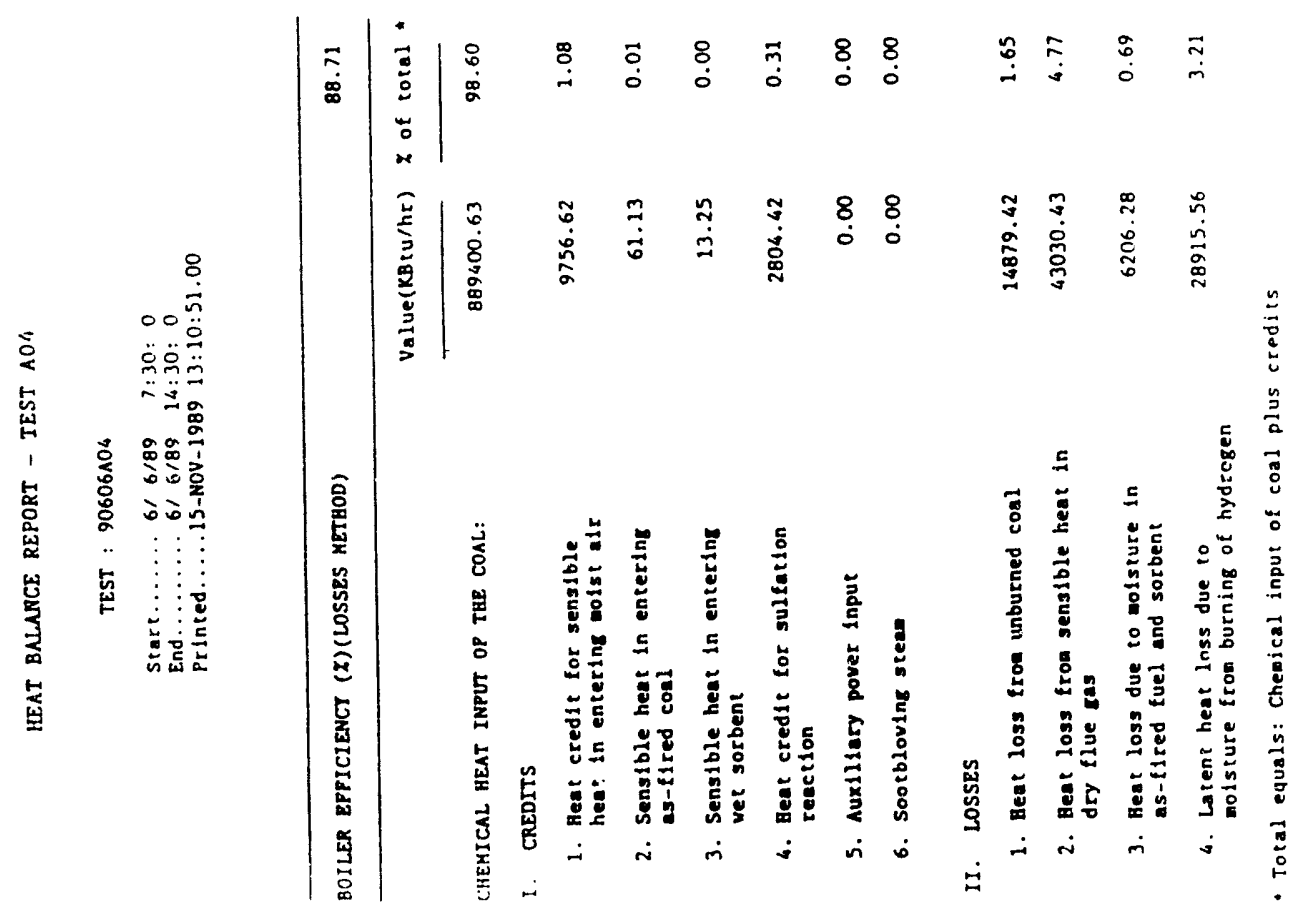



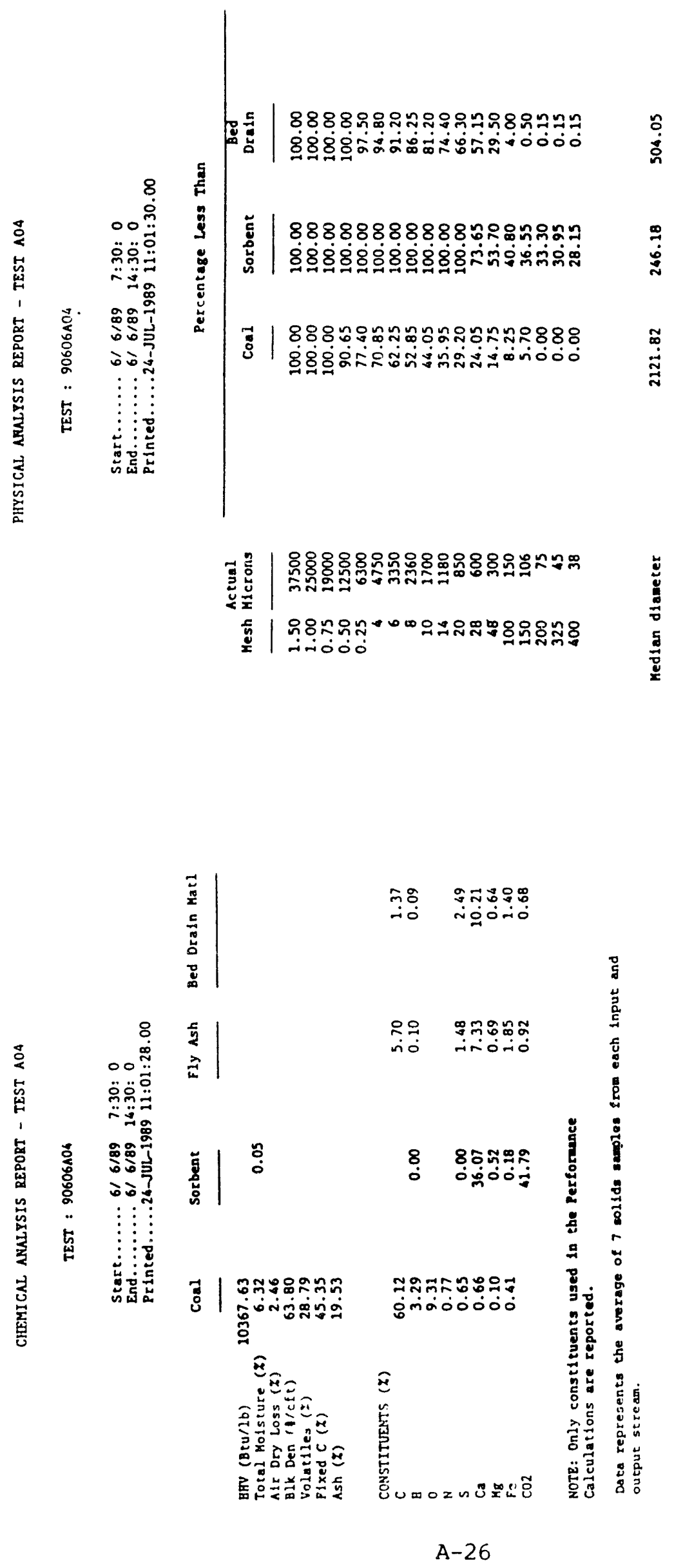

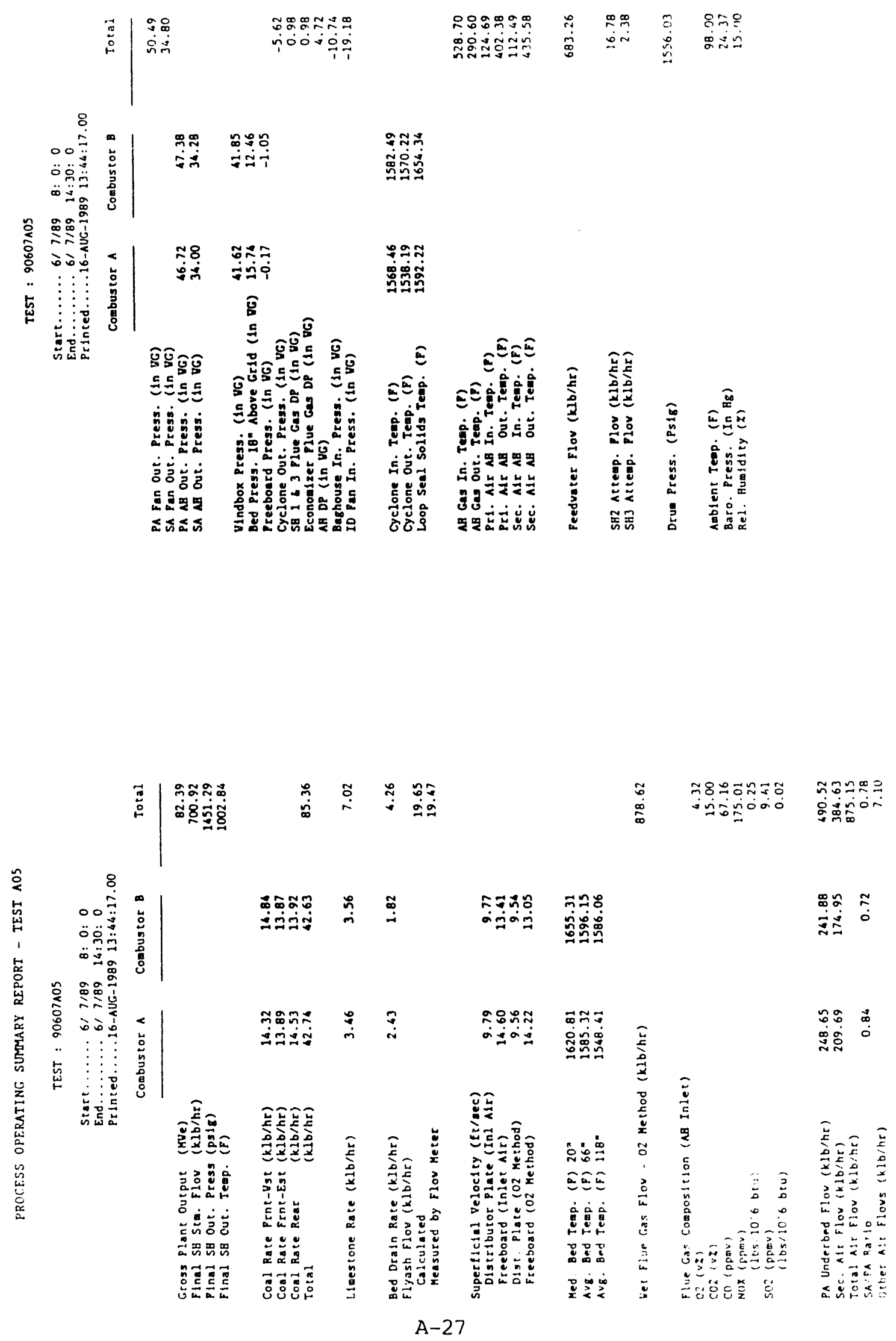


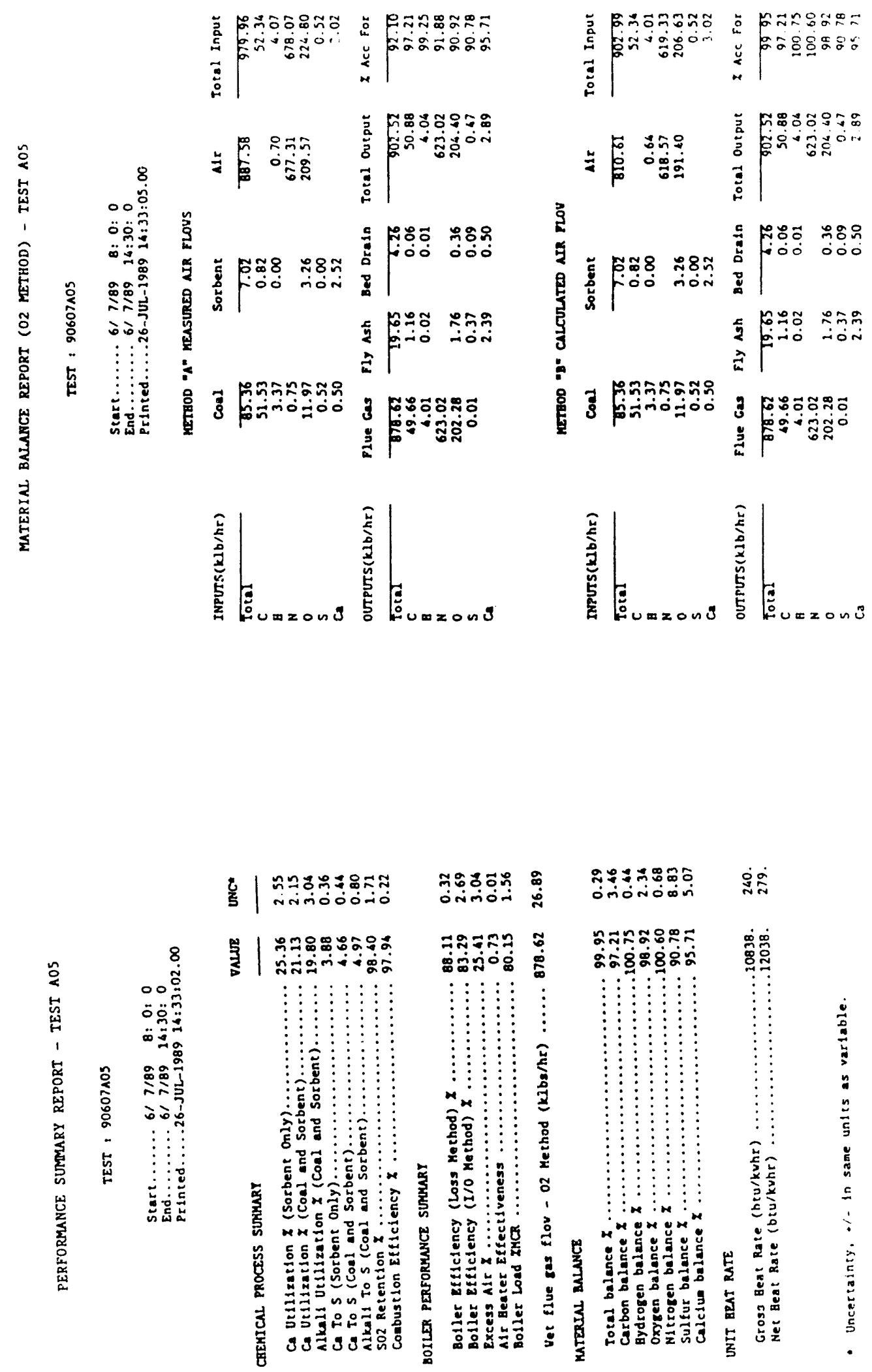




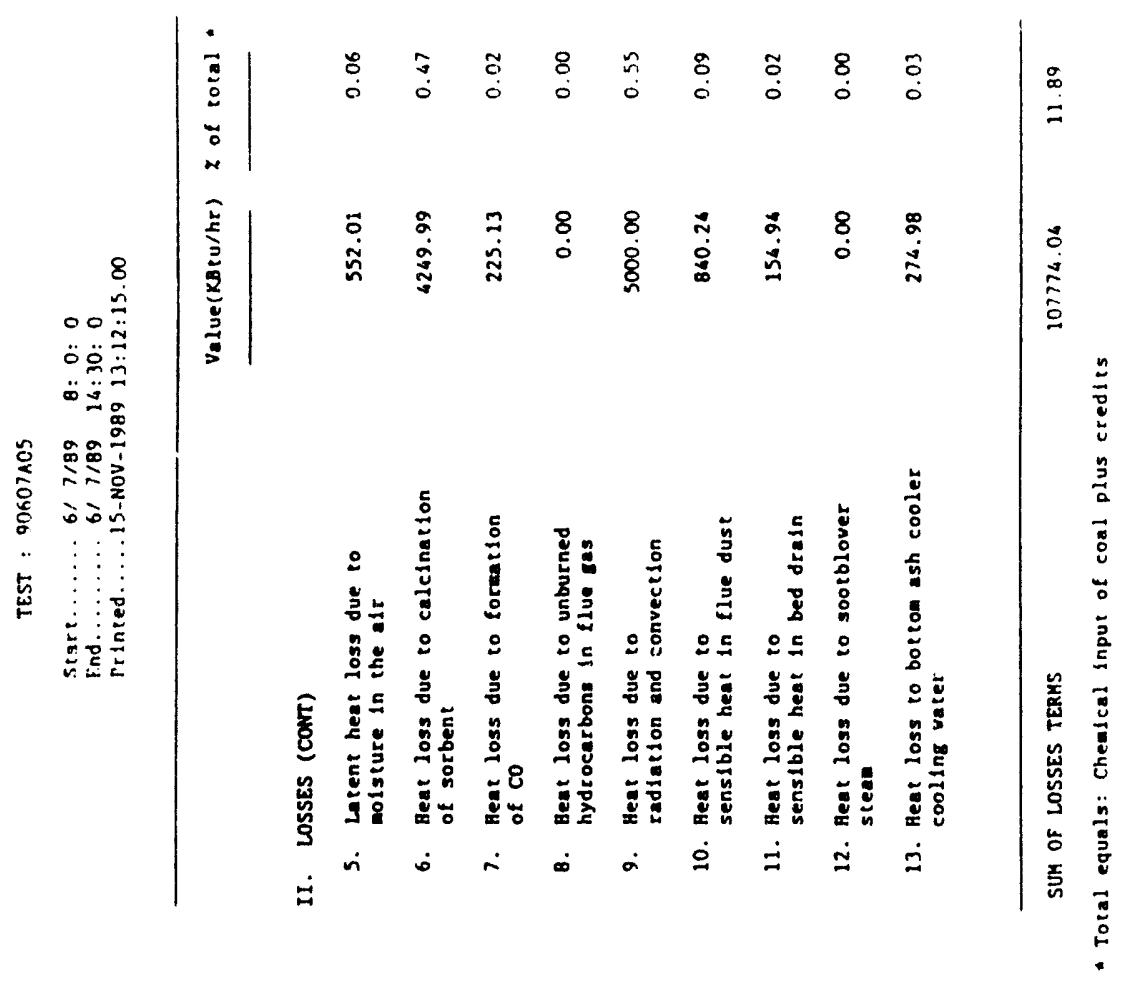

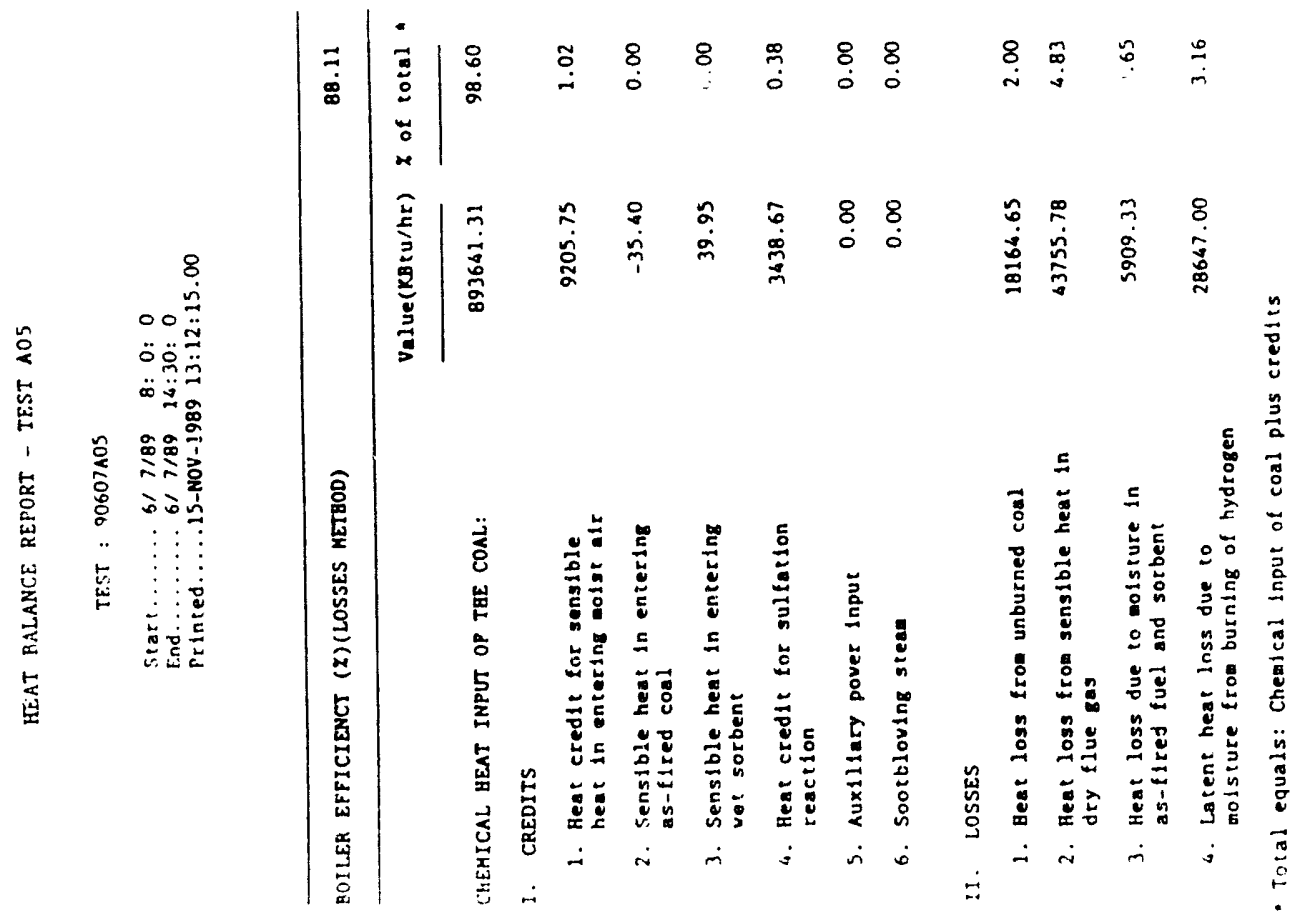



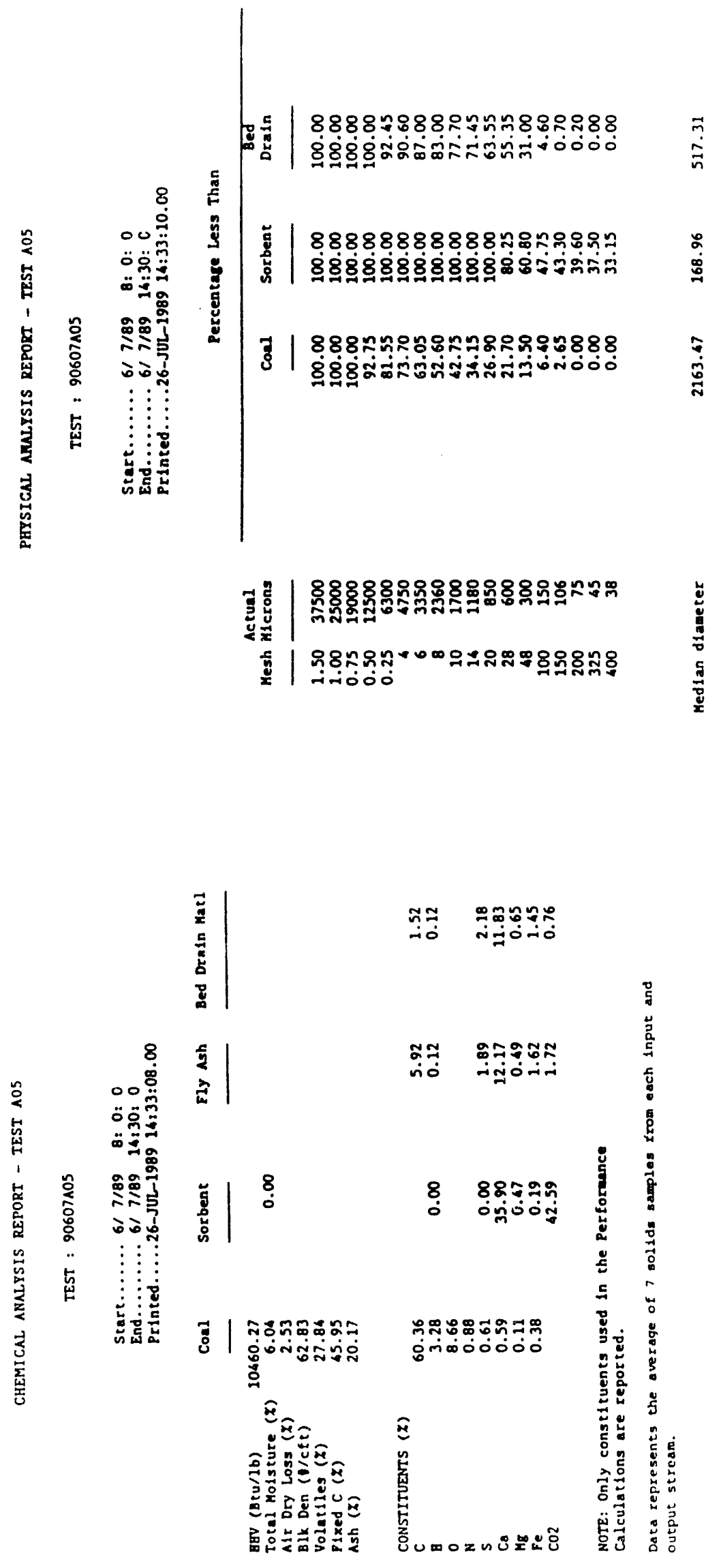

A -30 

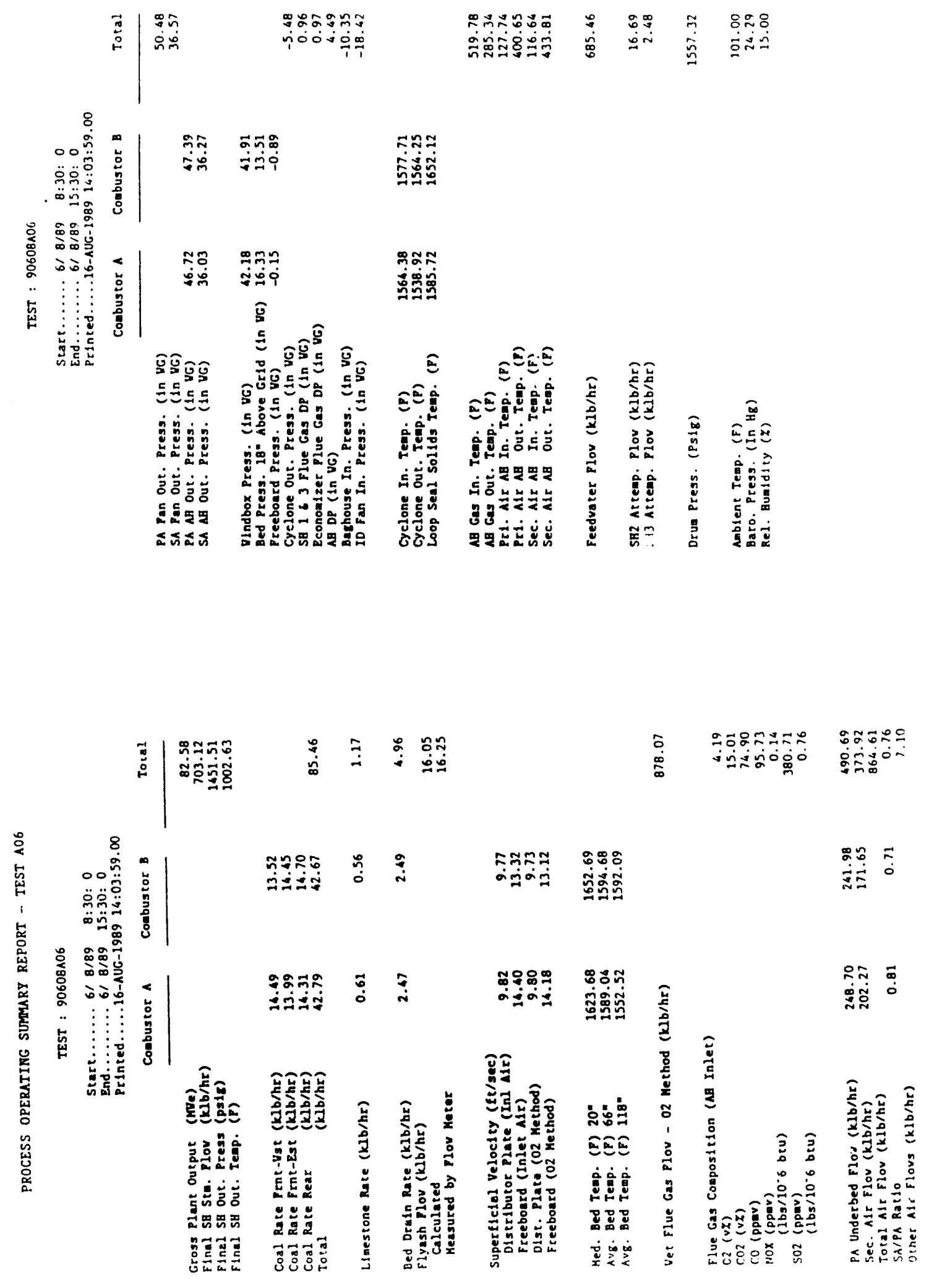

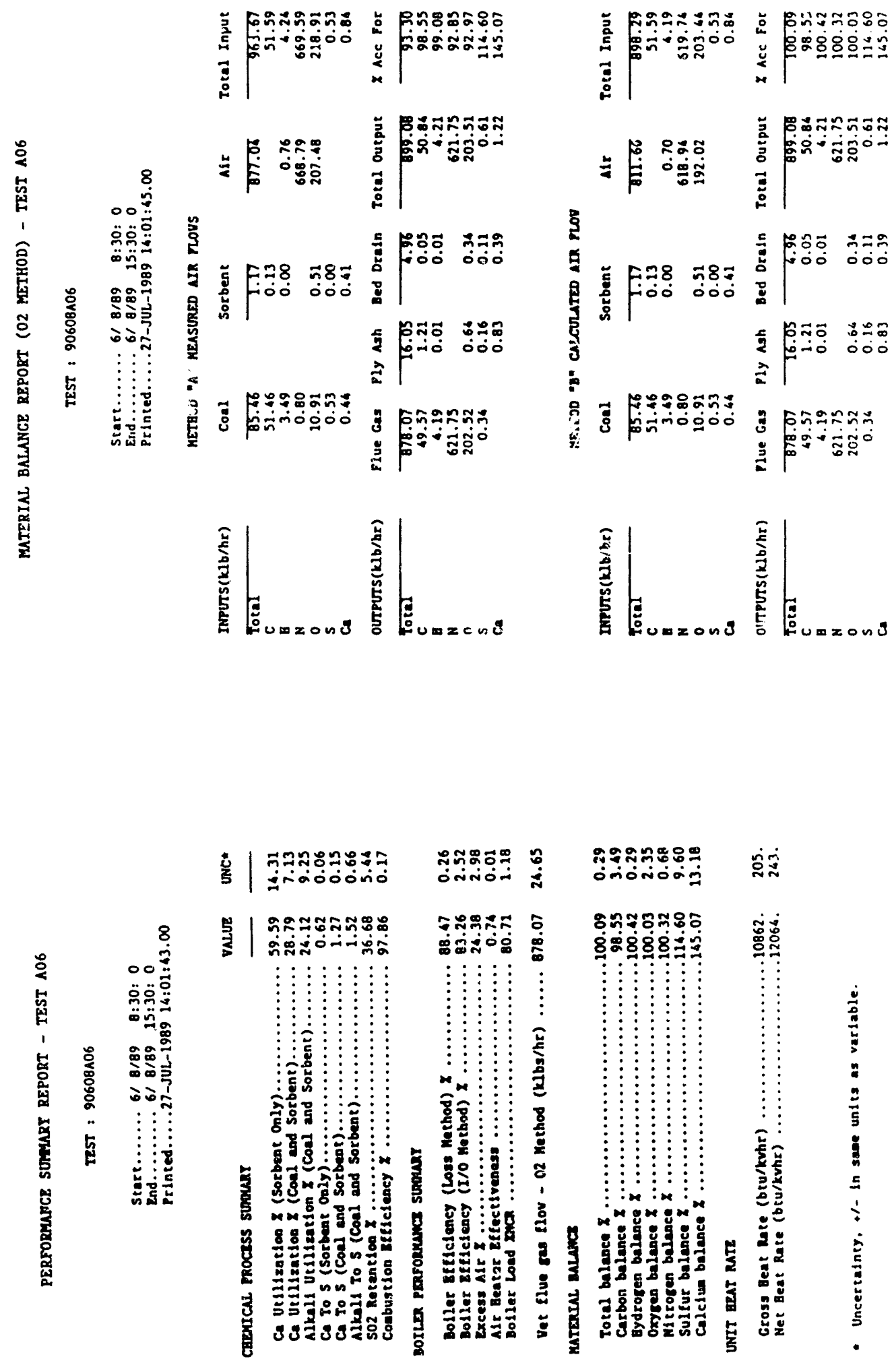


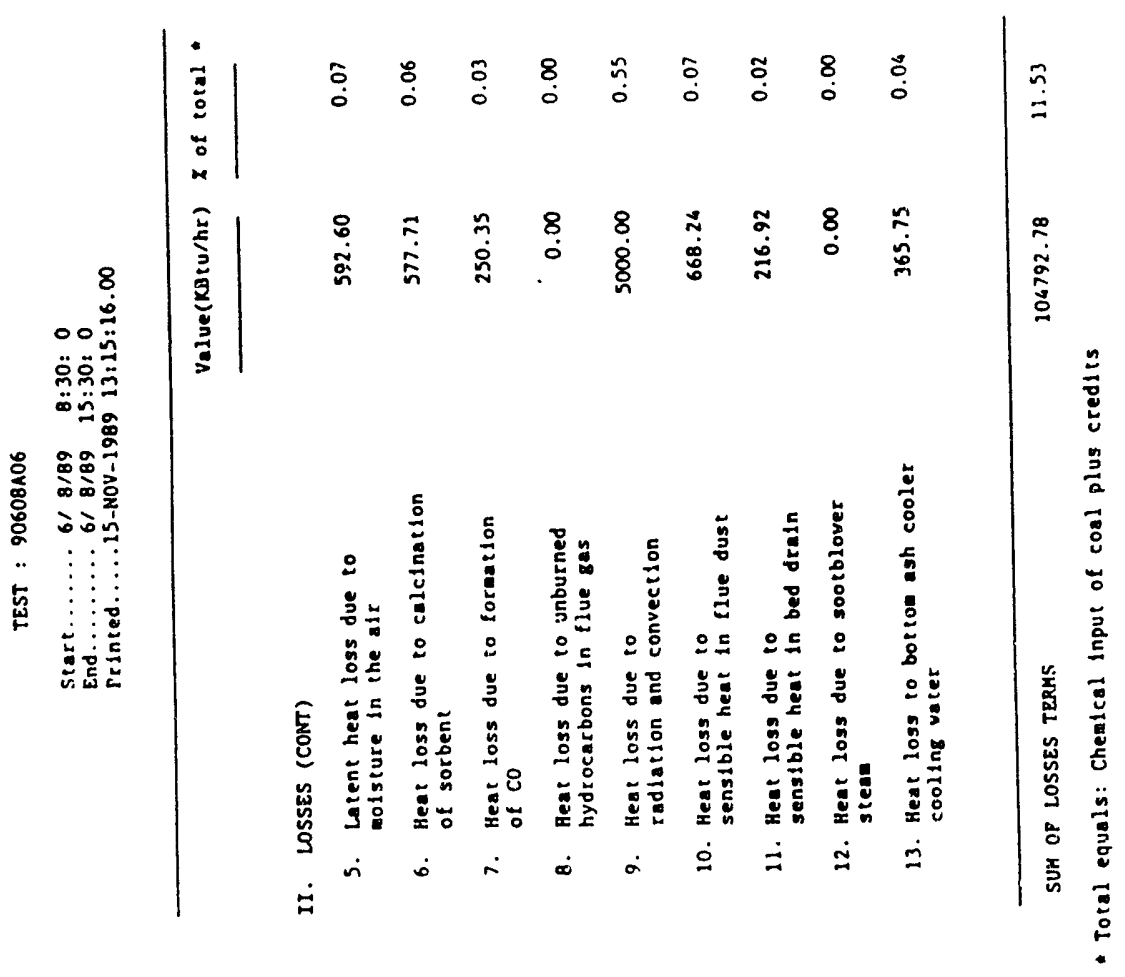

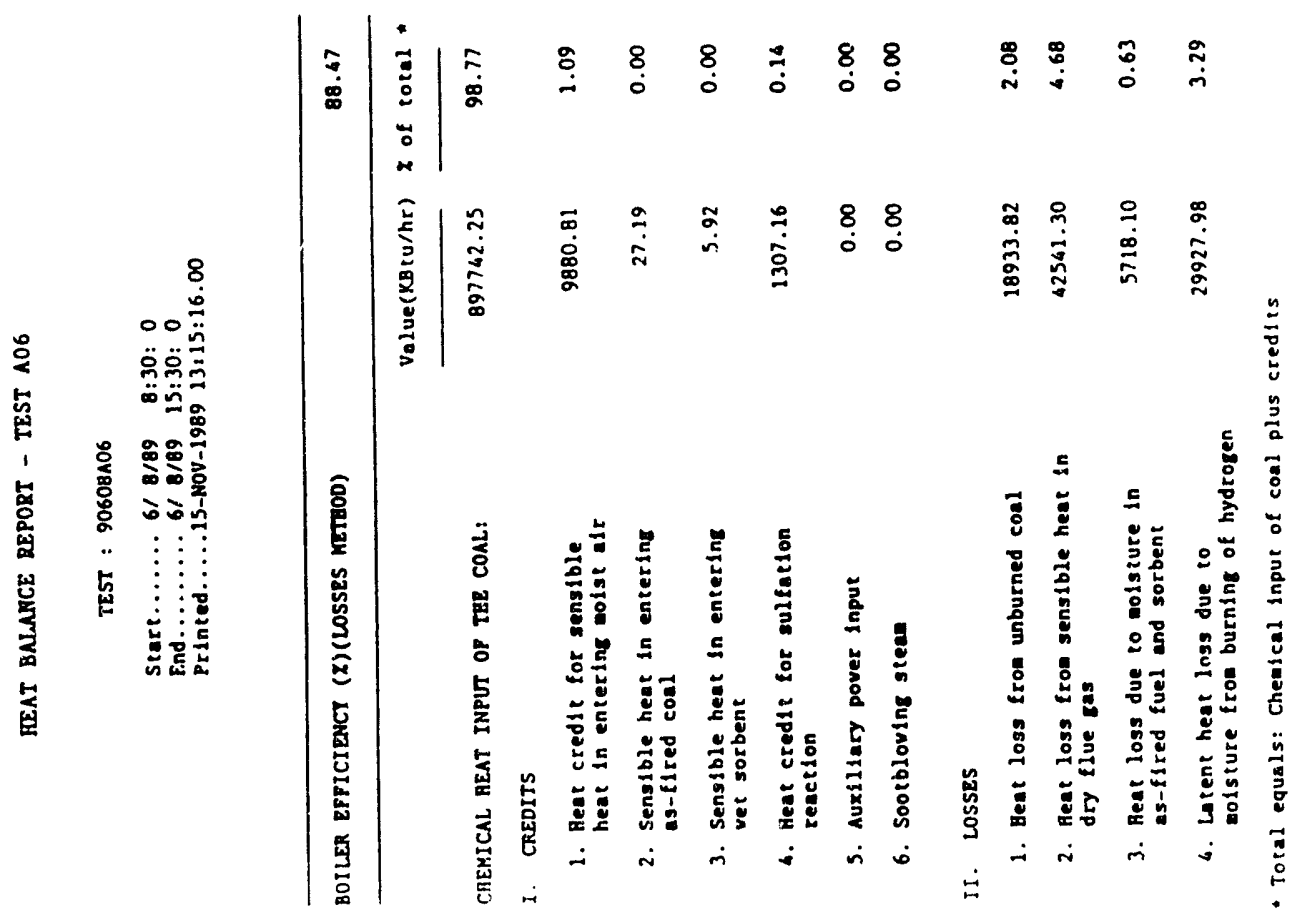



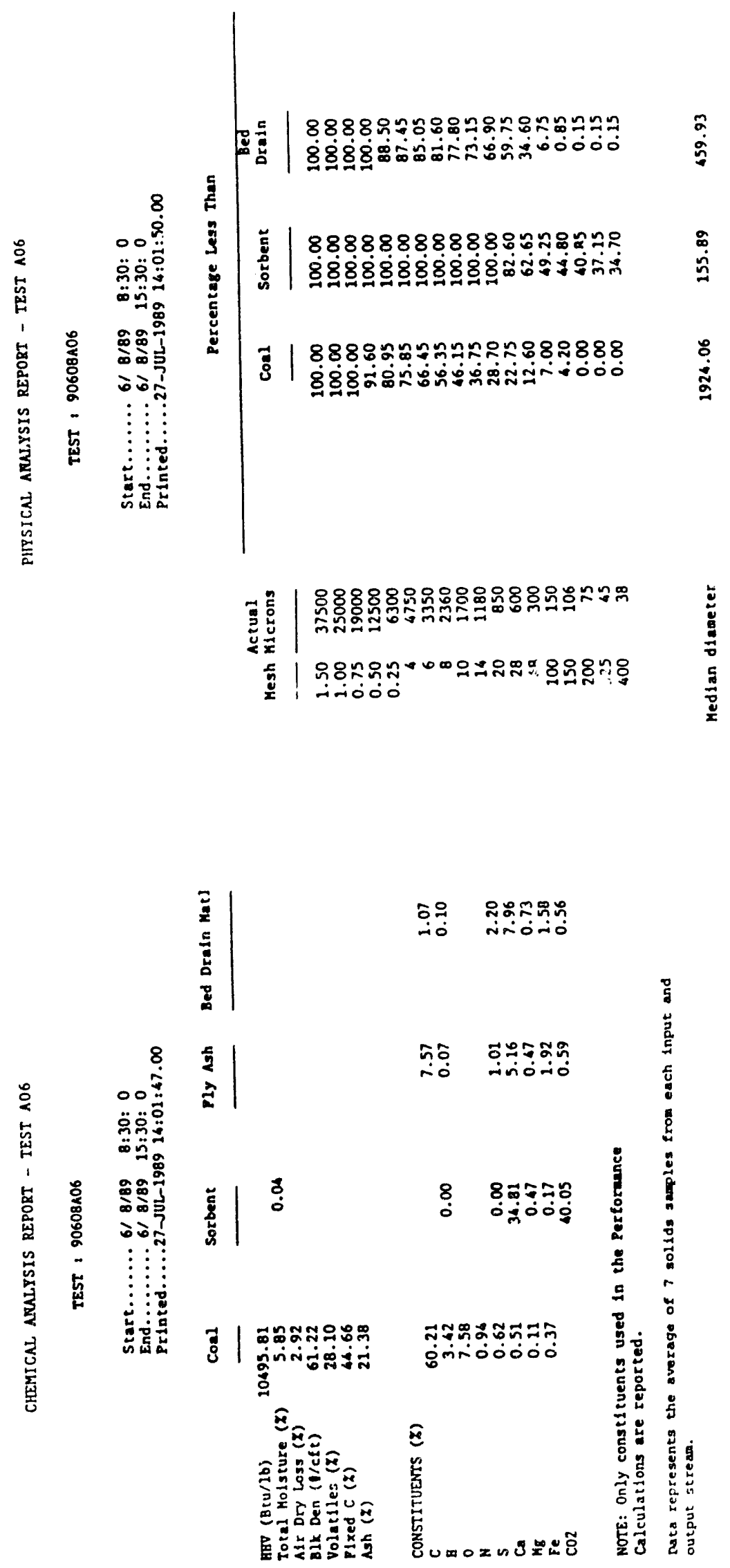

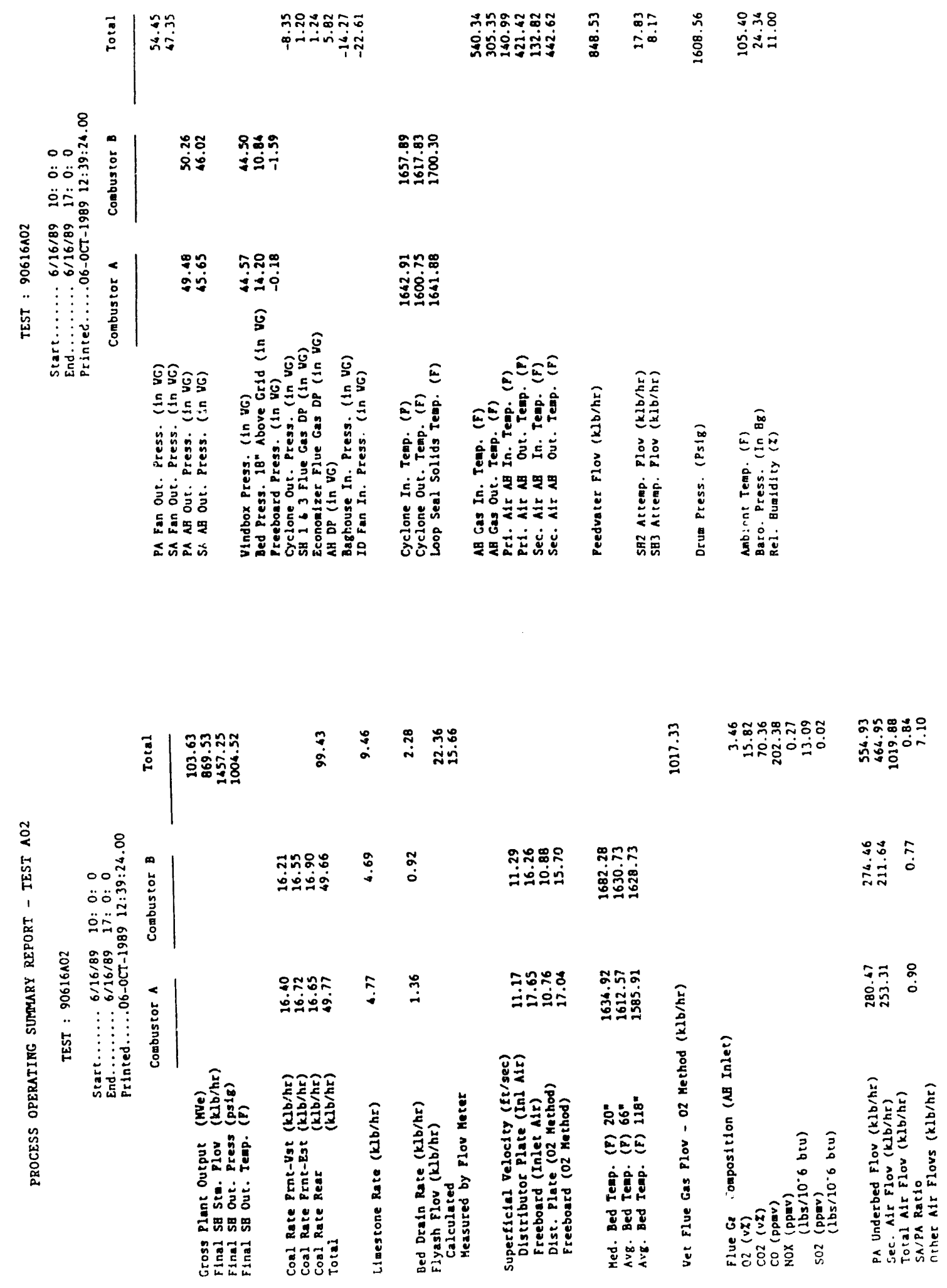

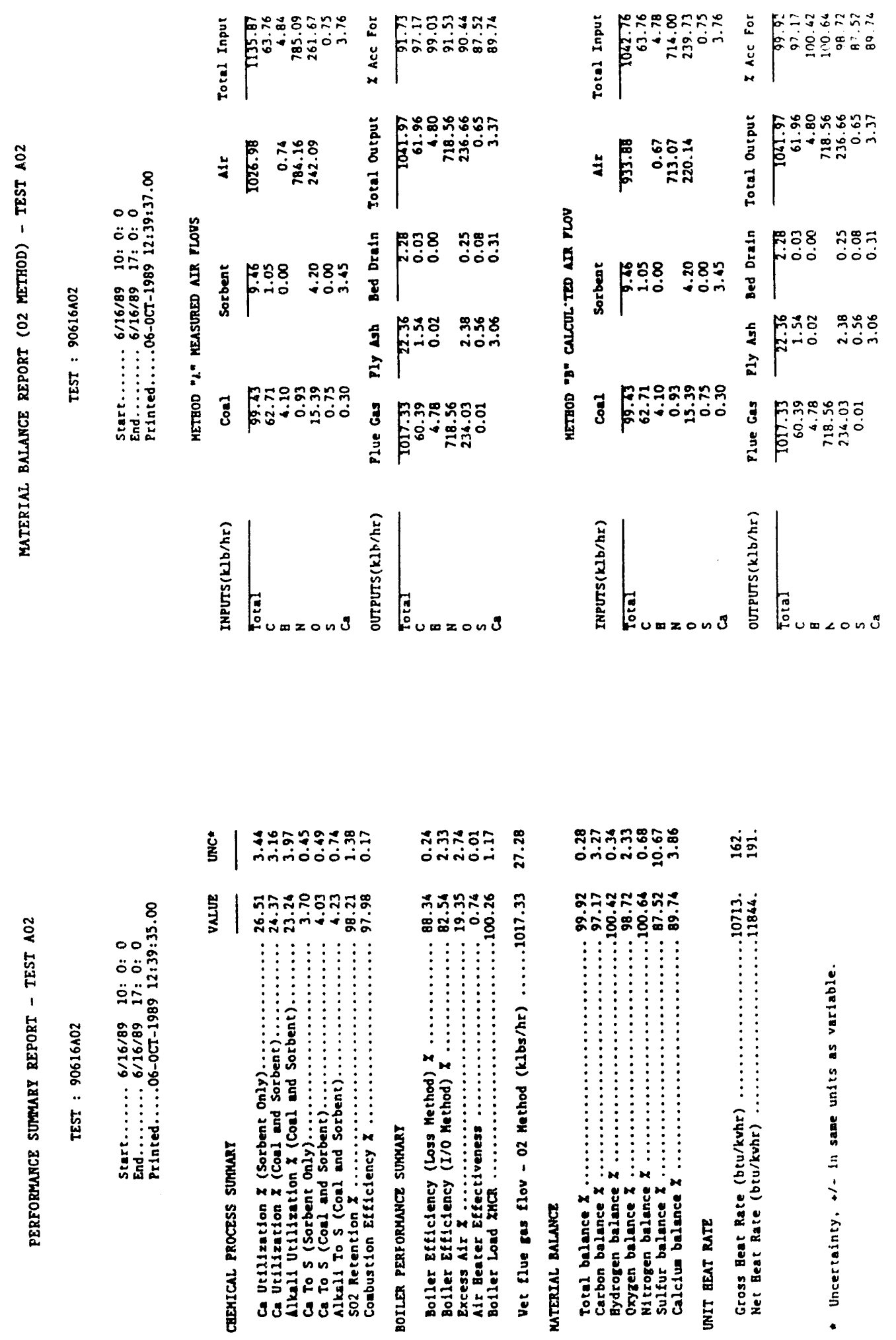

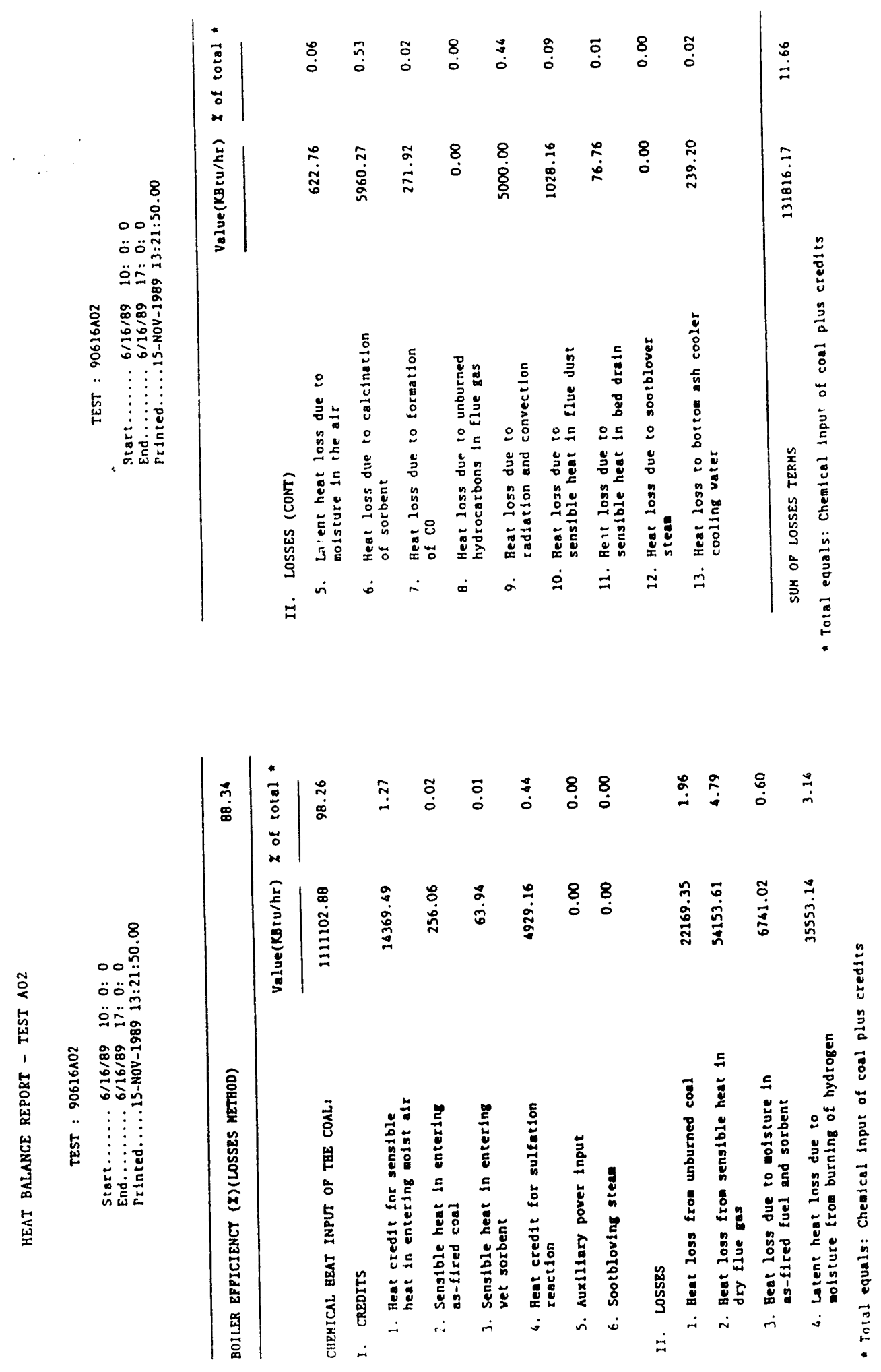

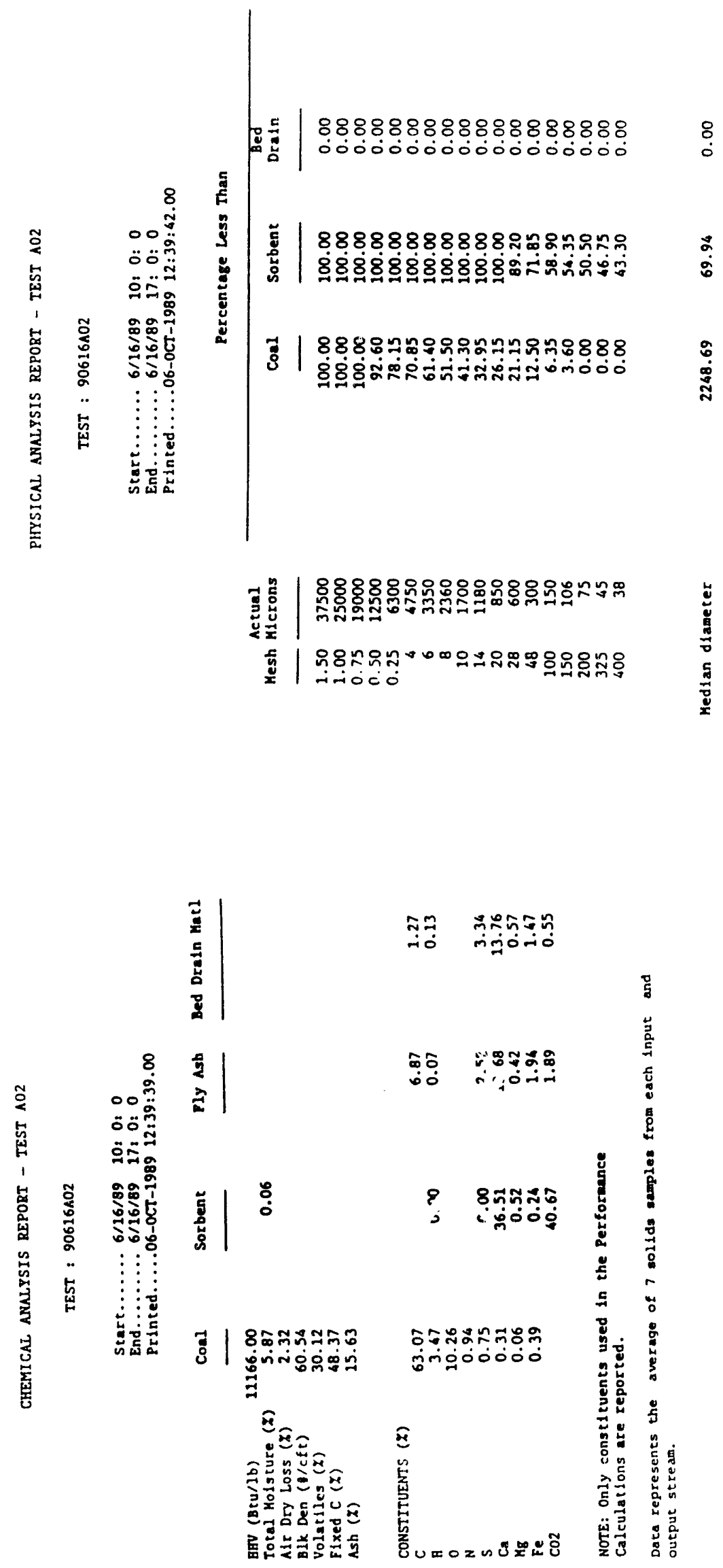

$\lambda-38$ 

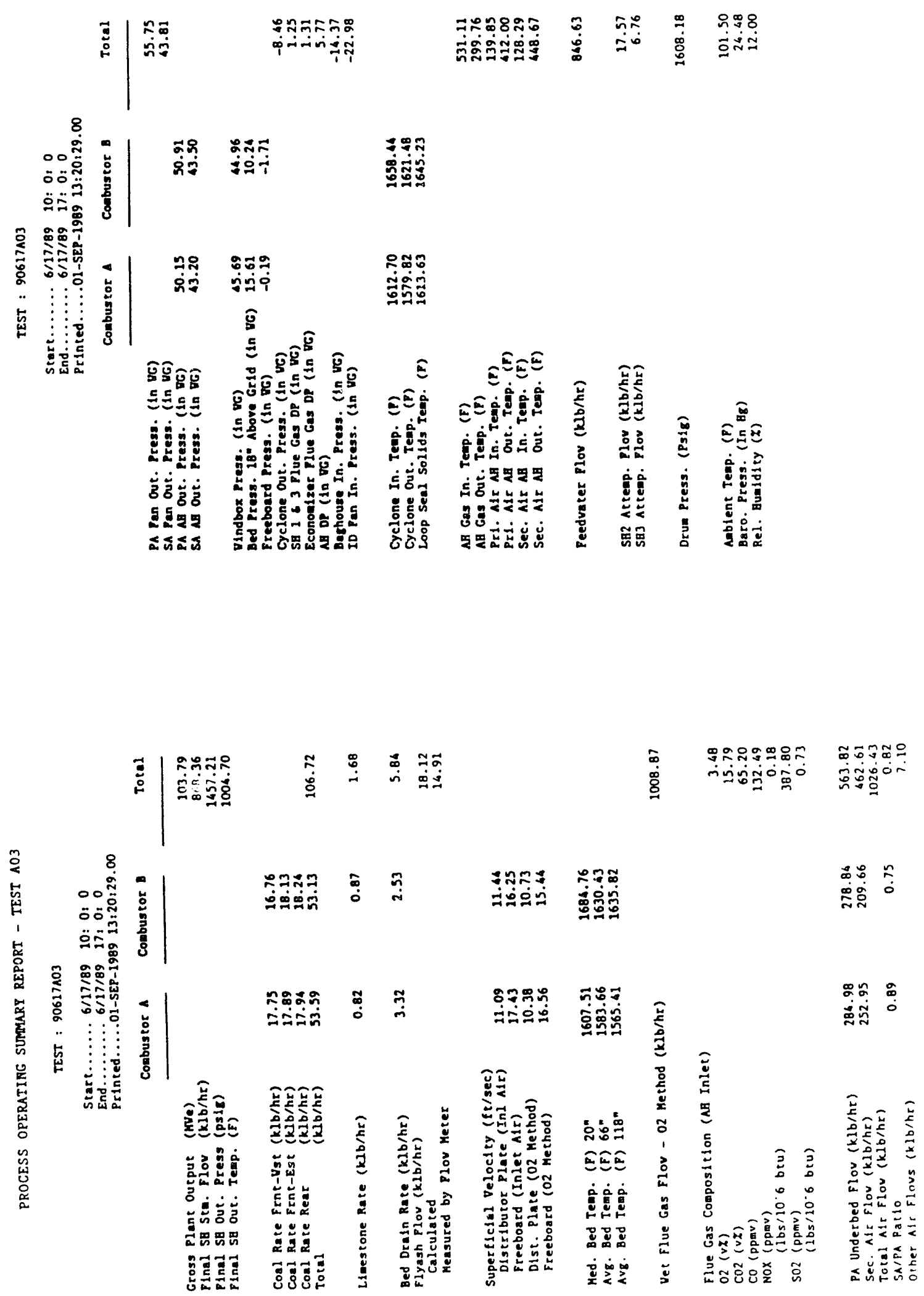

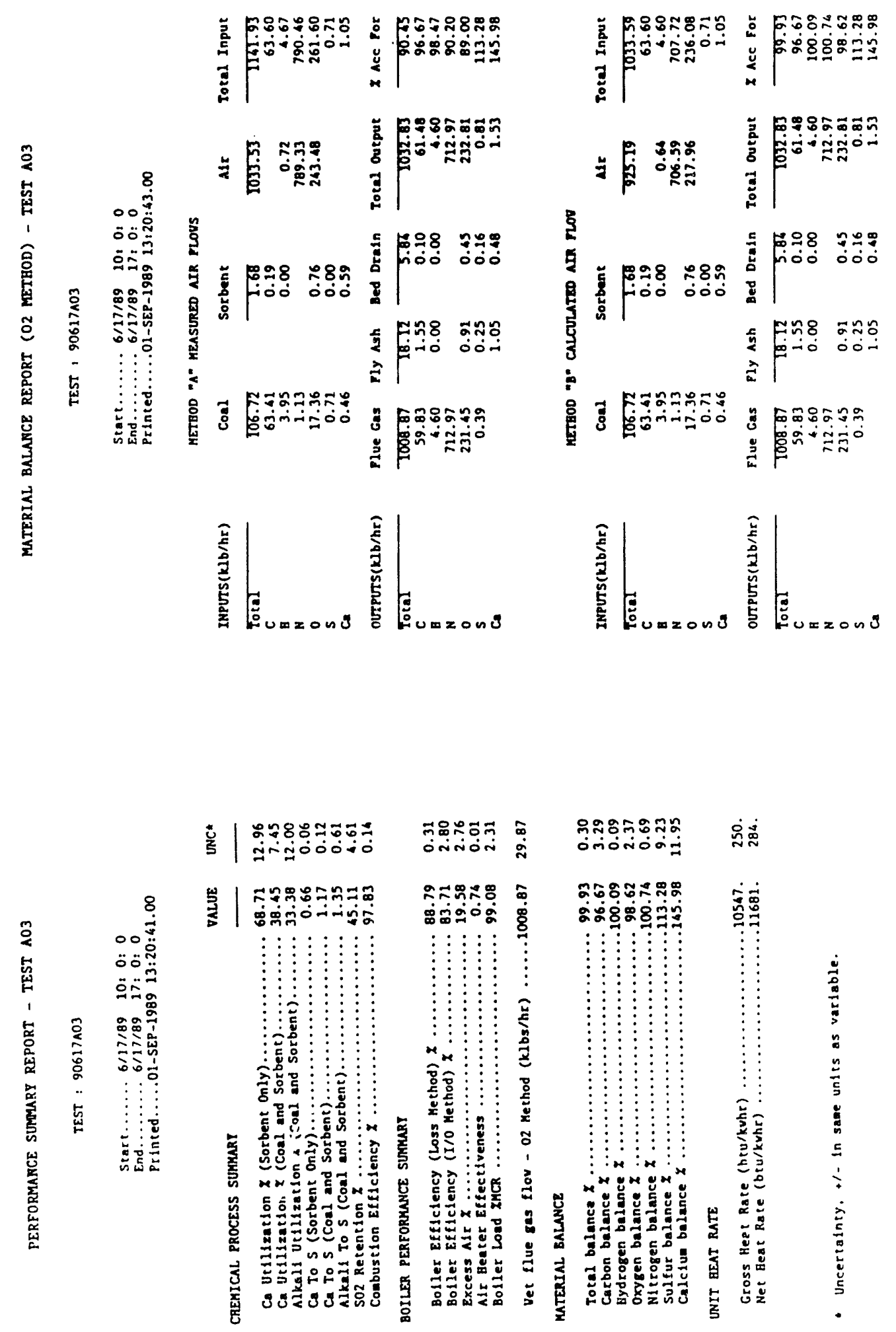

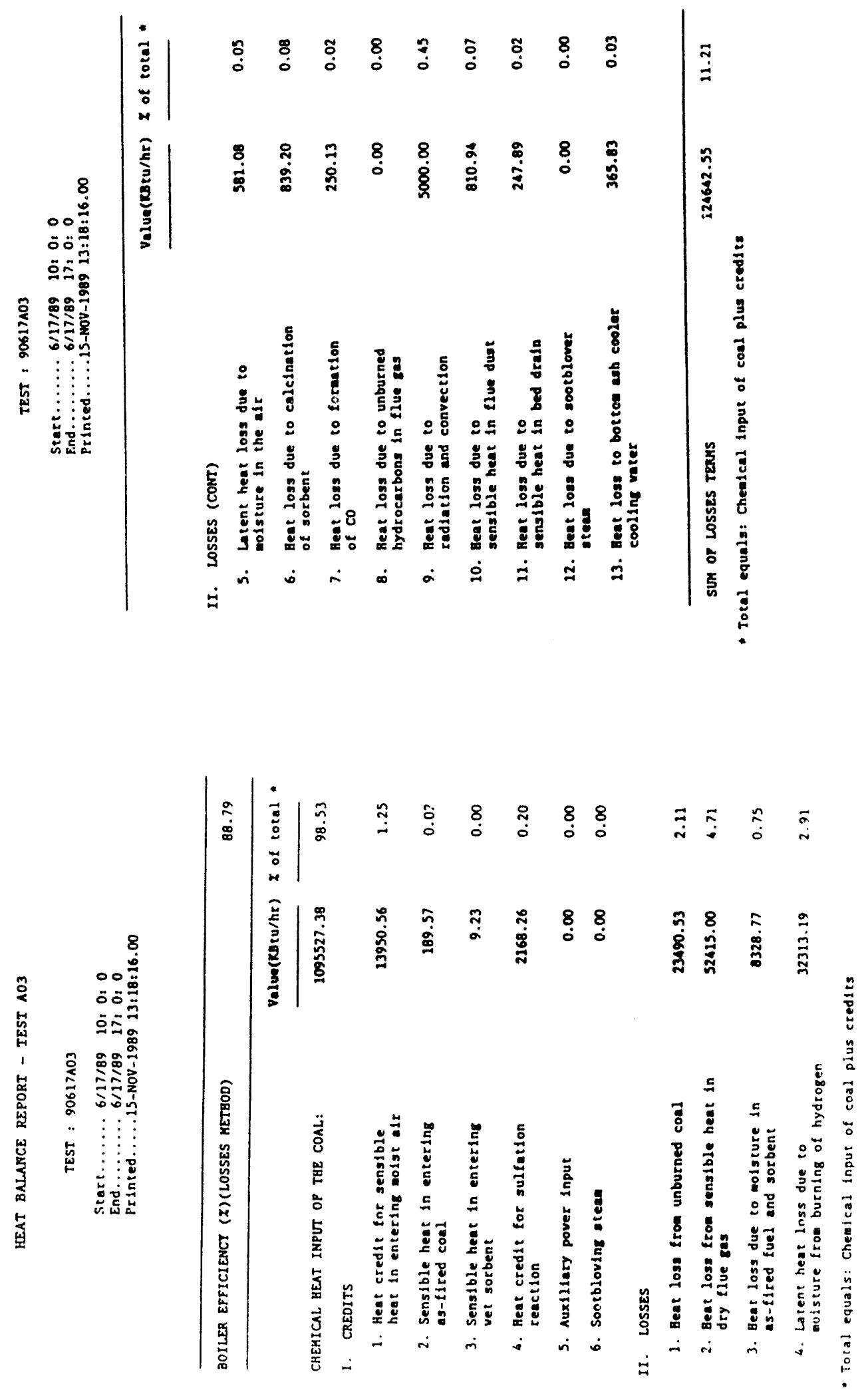

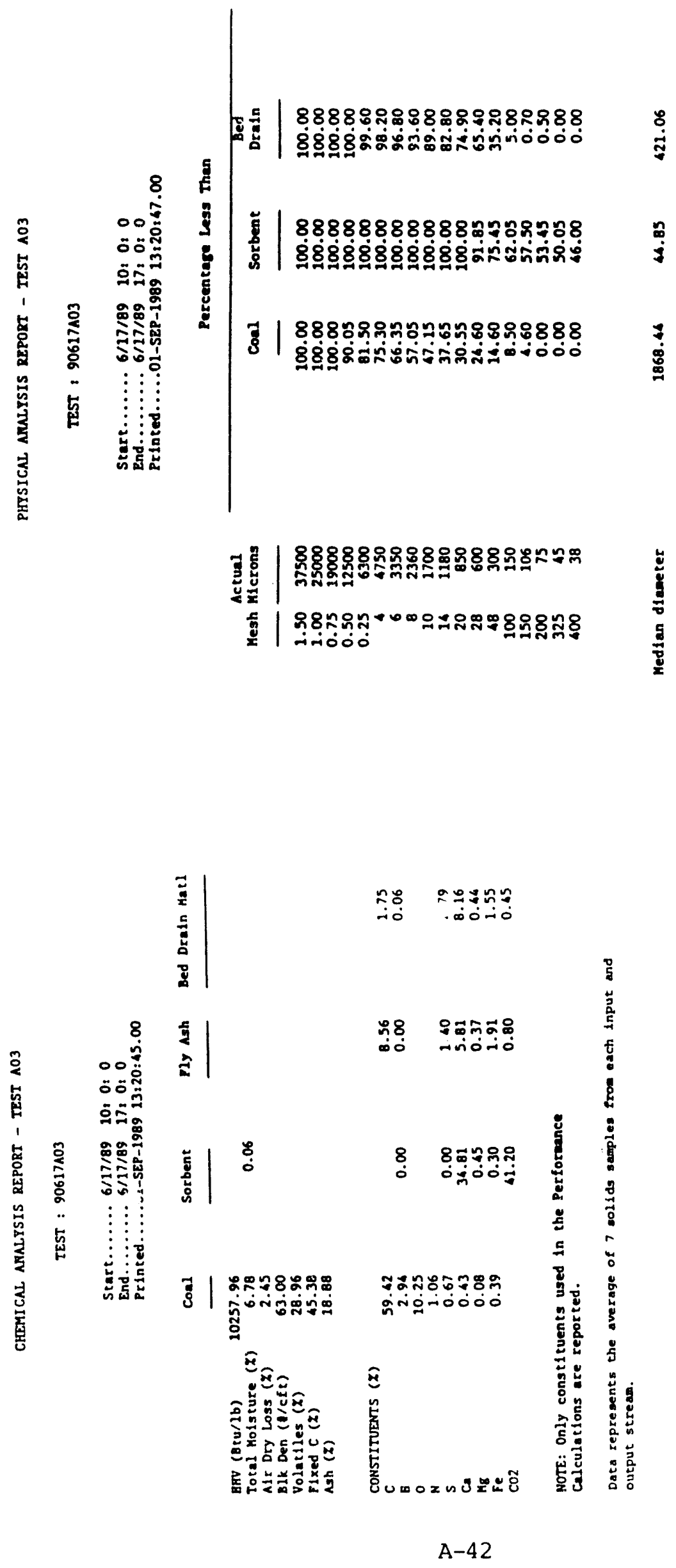

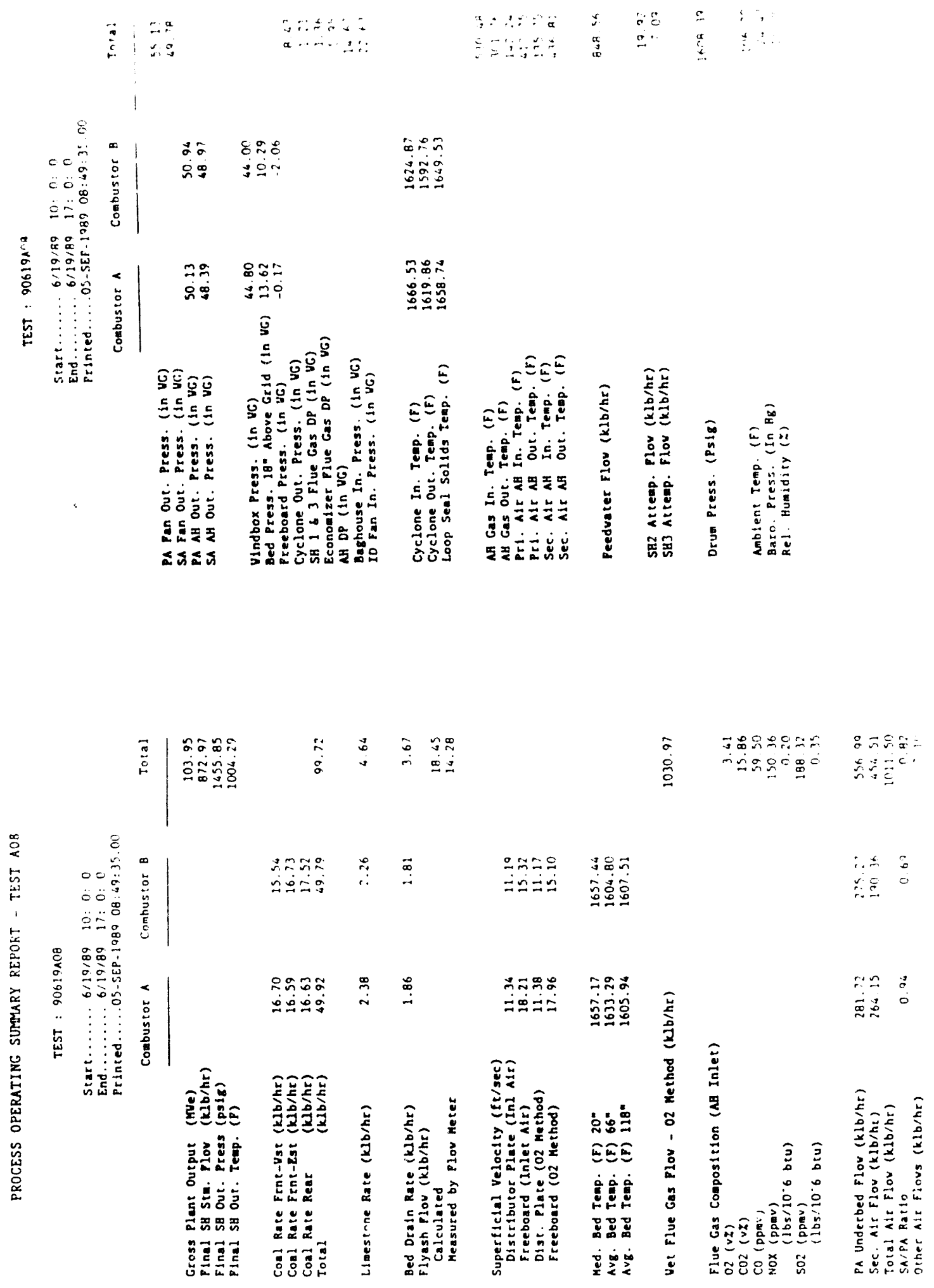


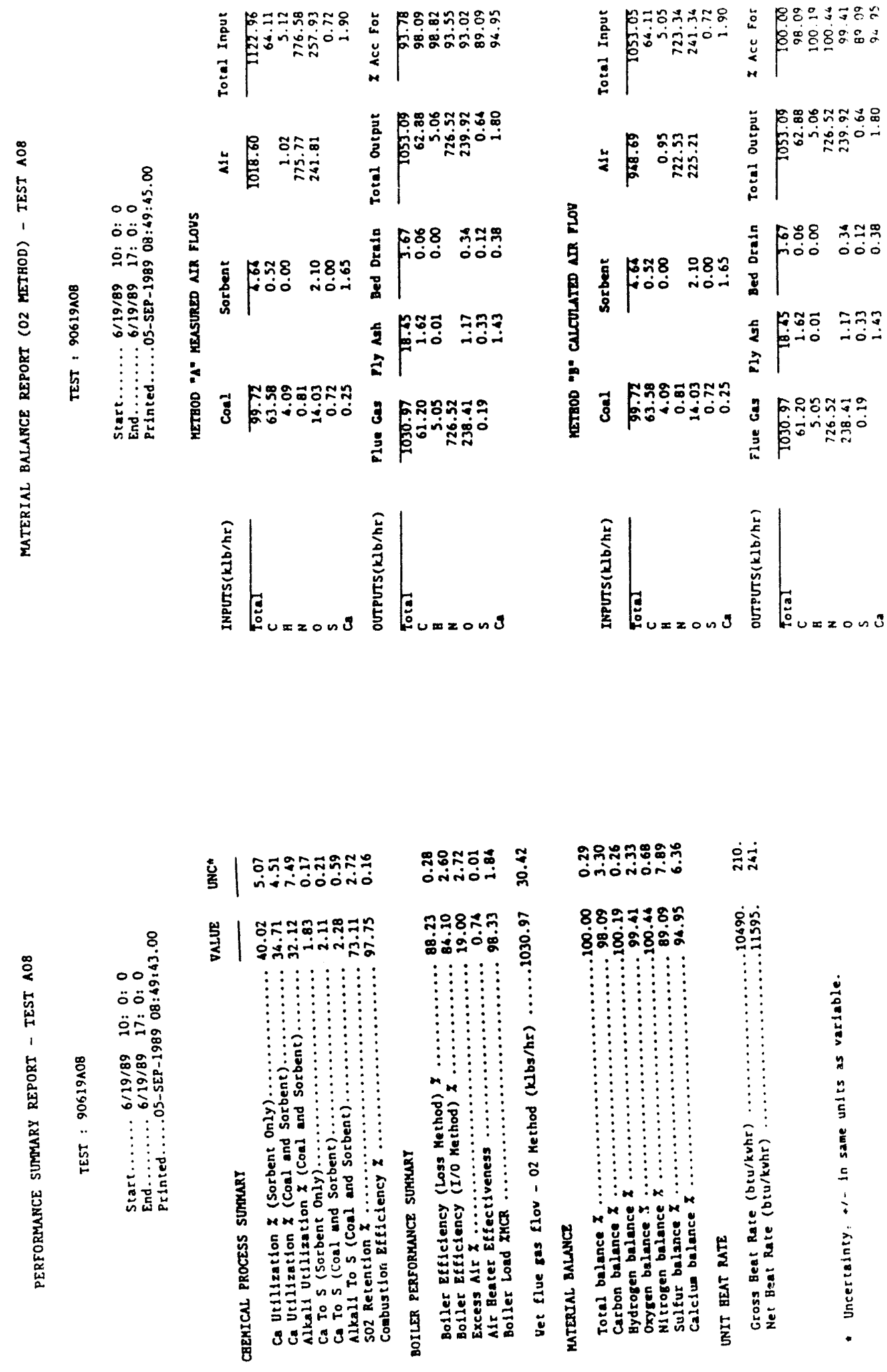




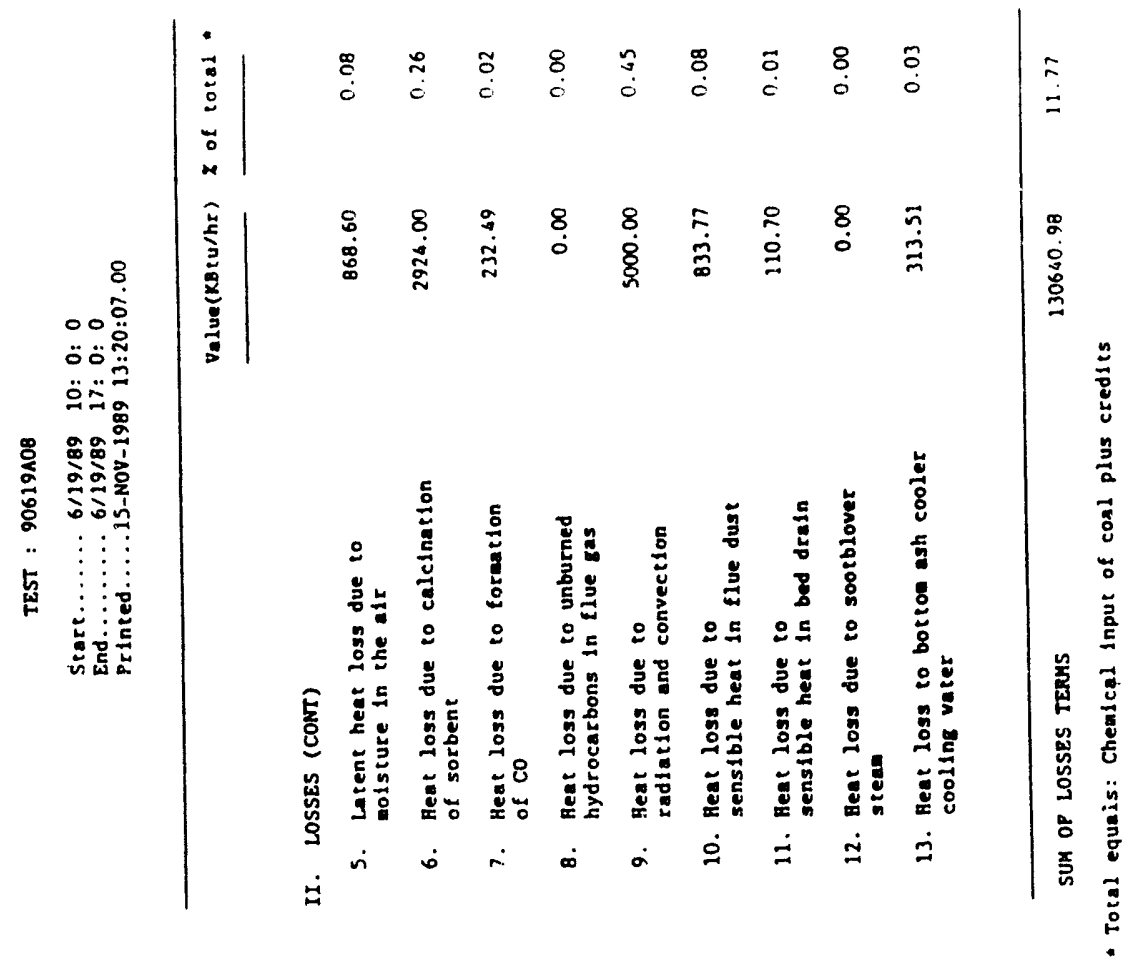

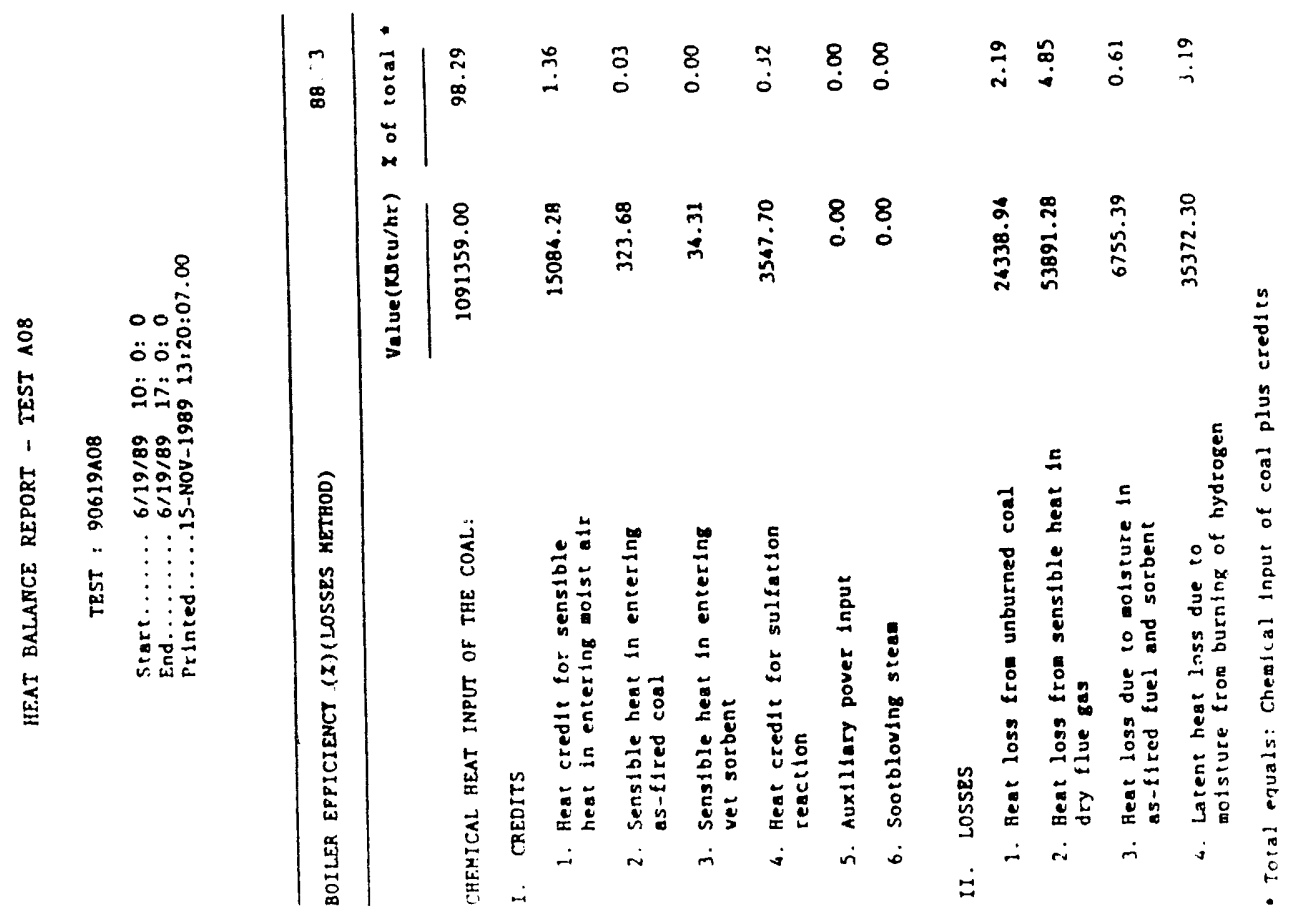



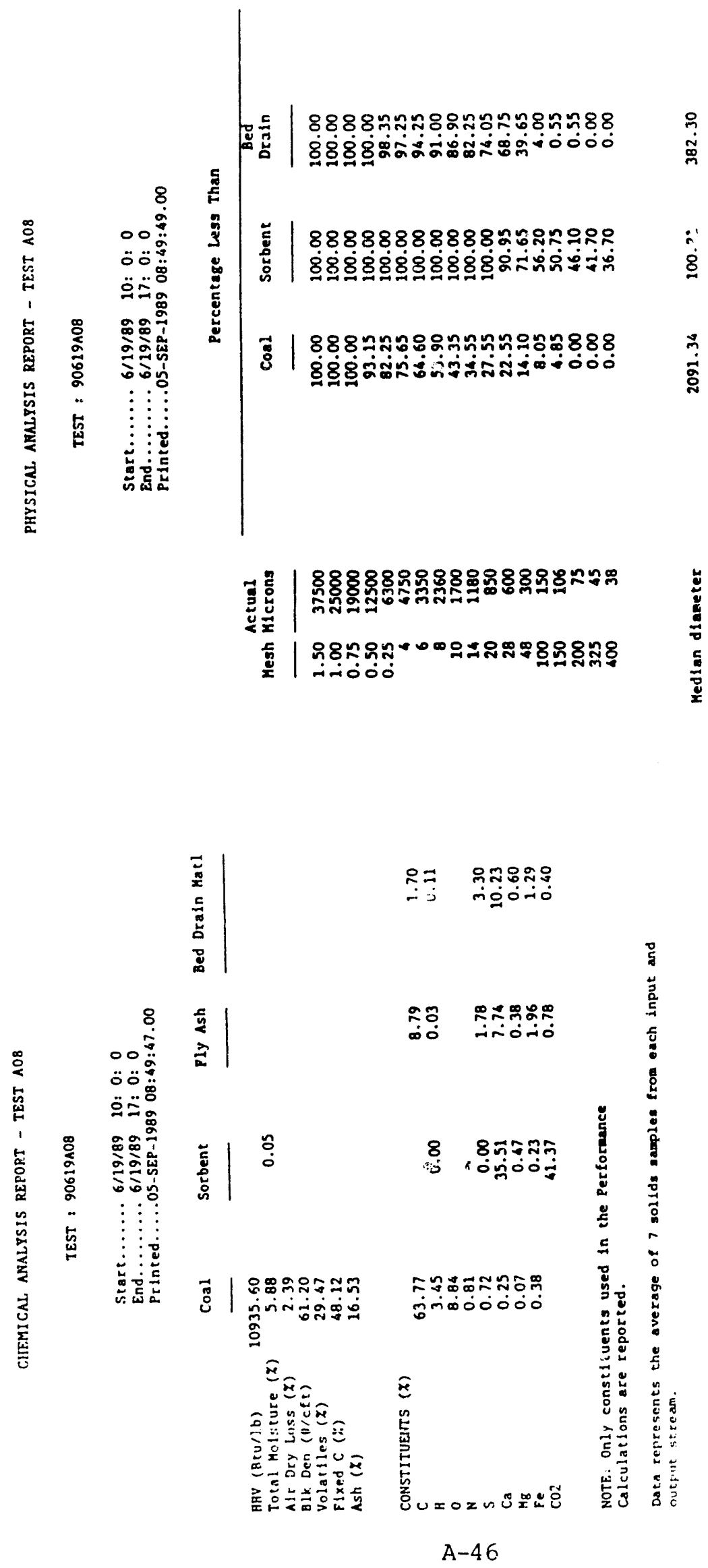


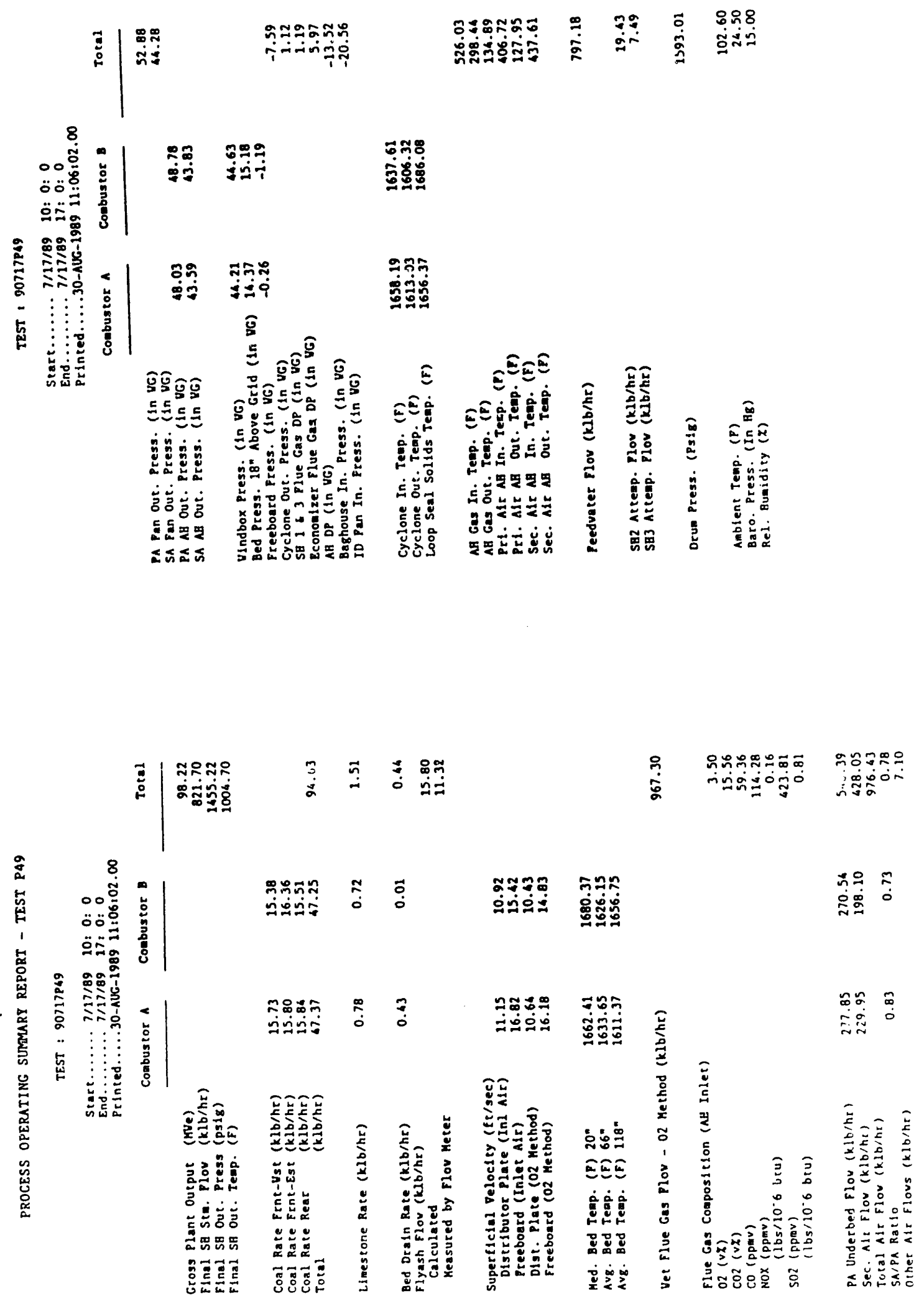



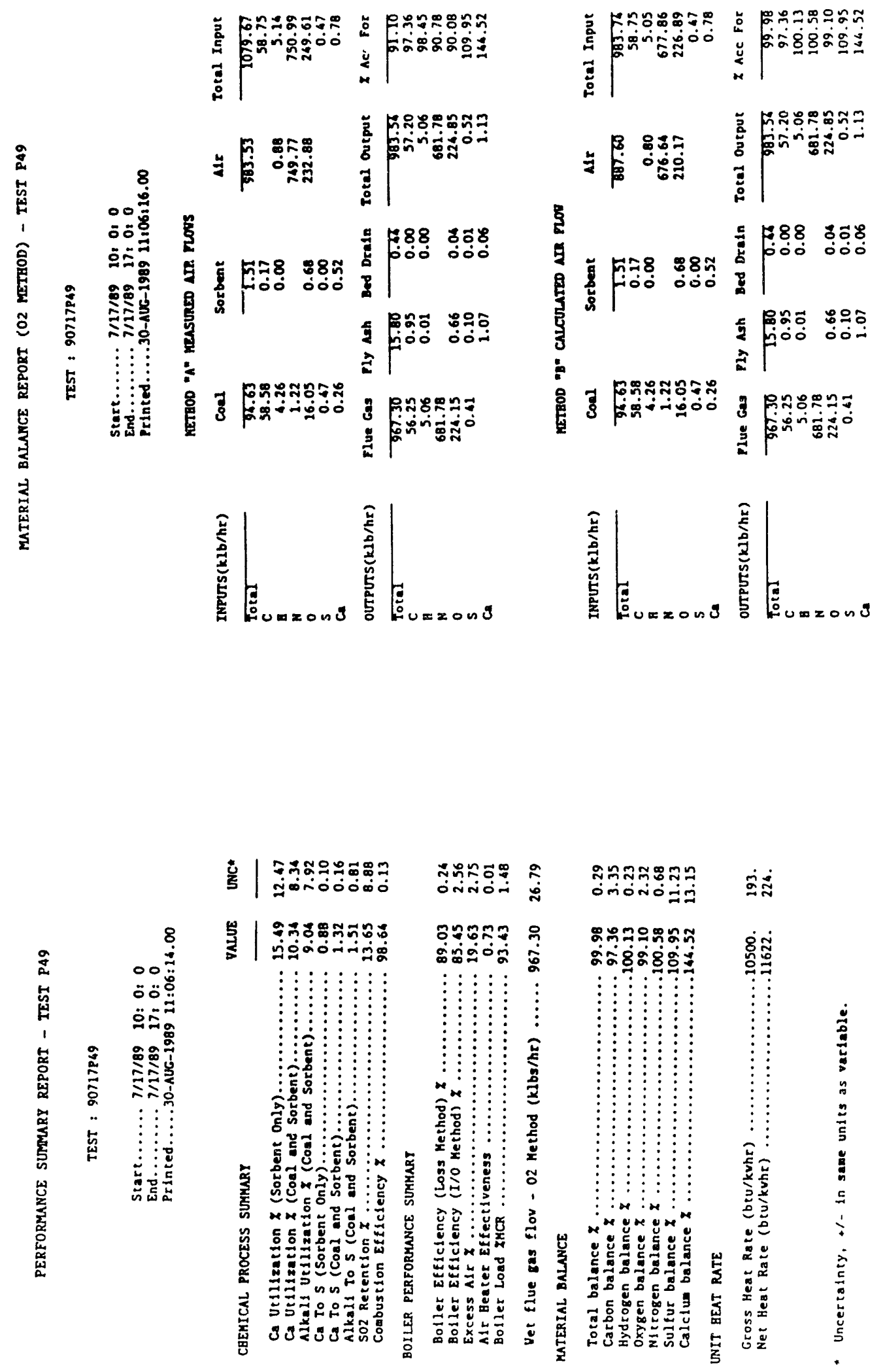


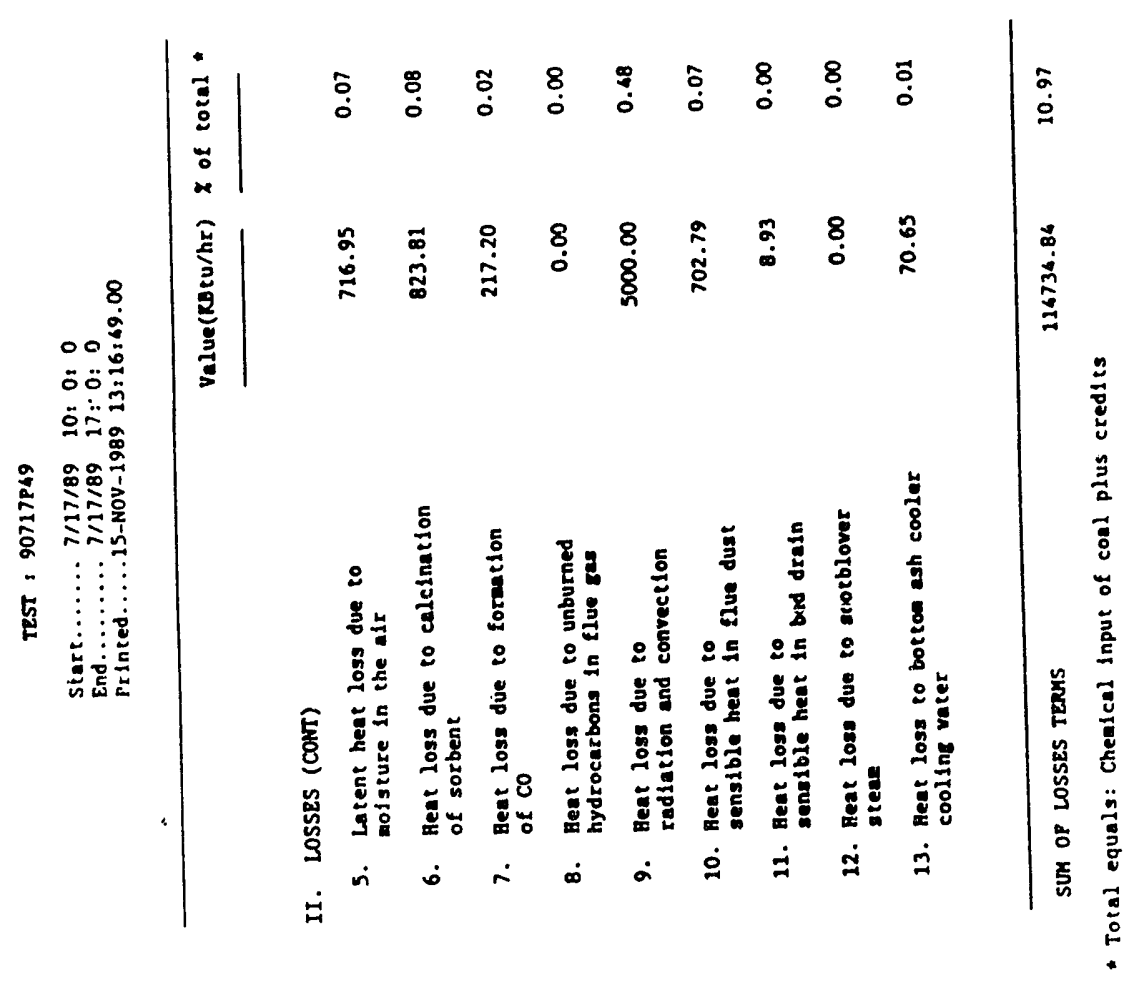

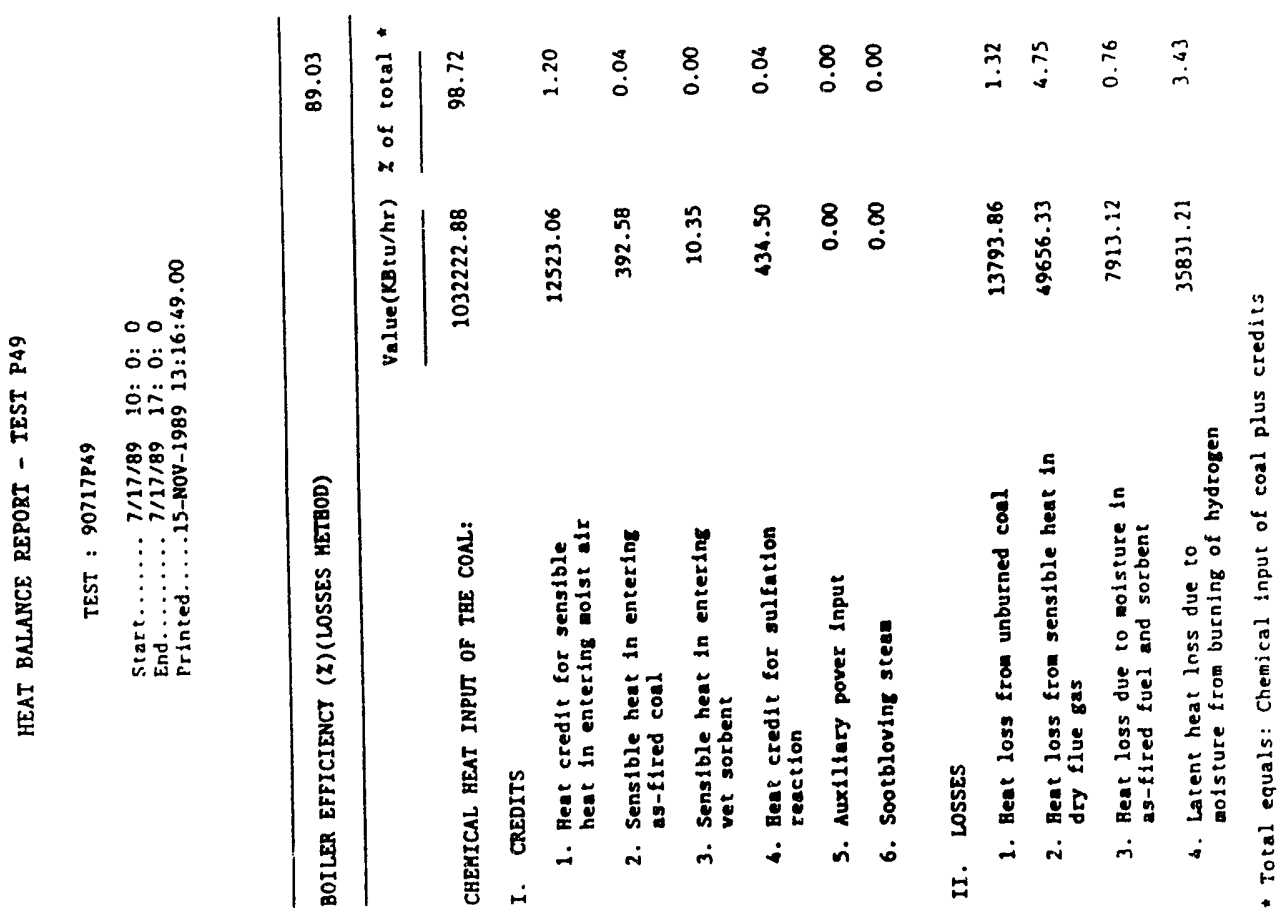



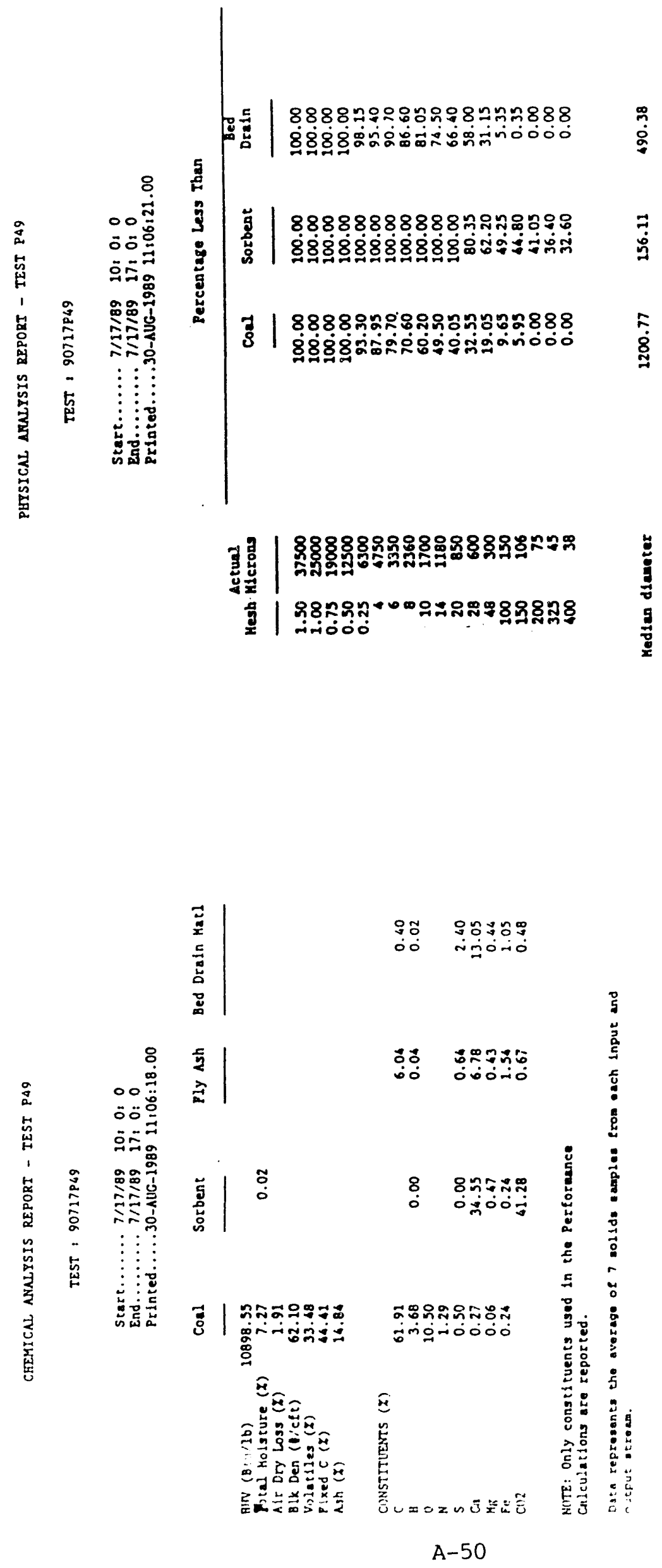

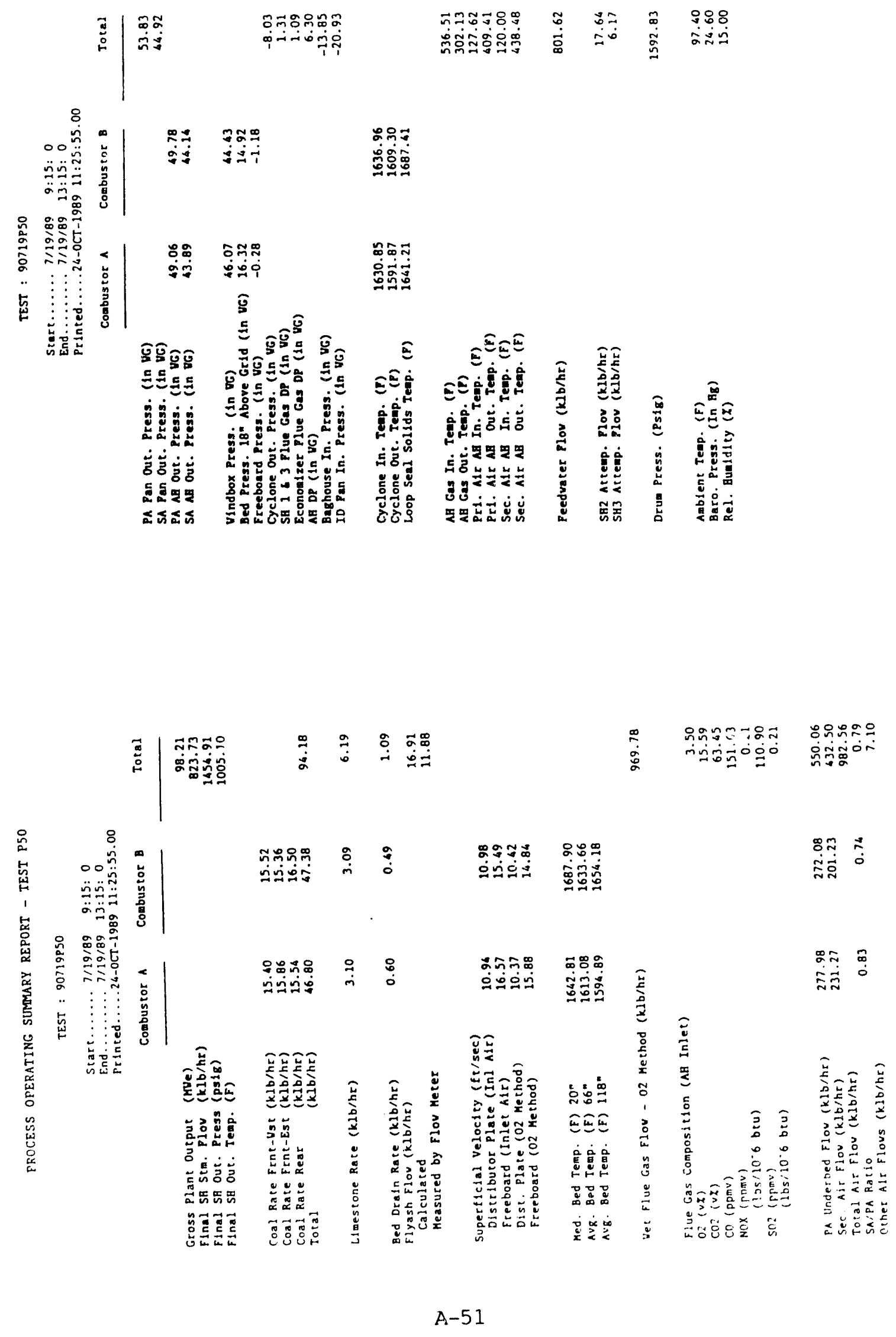

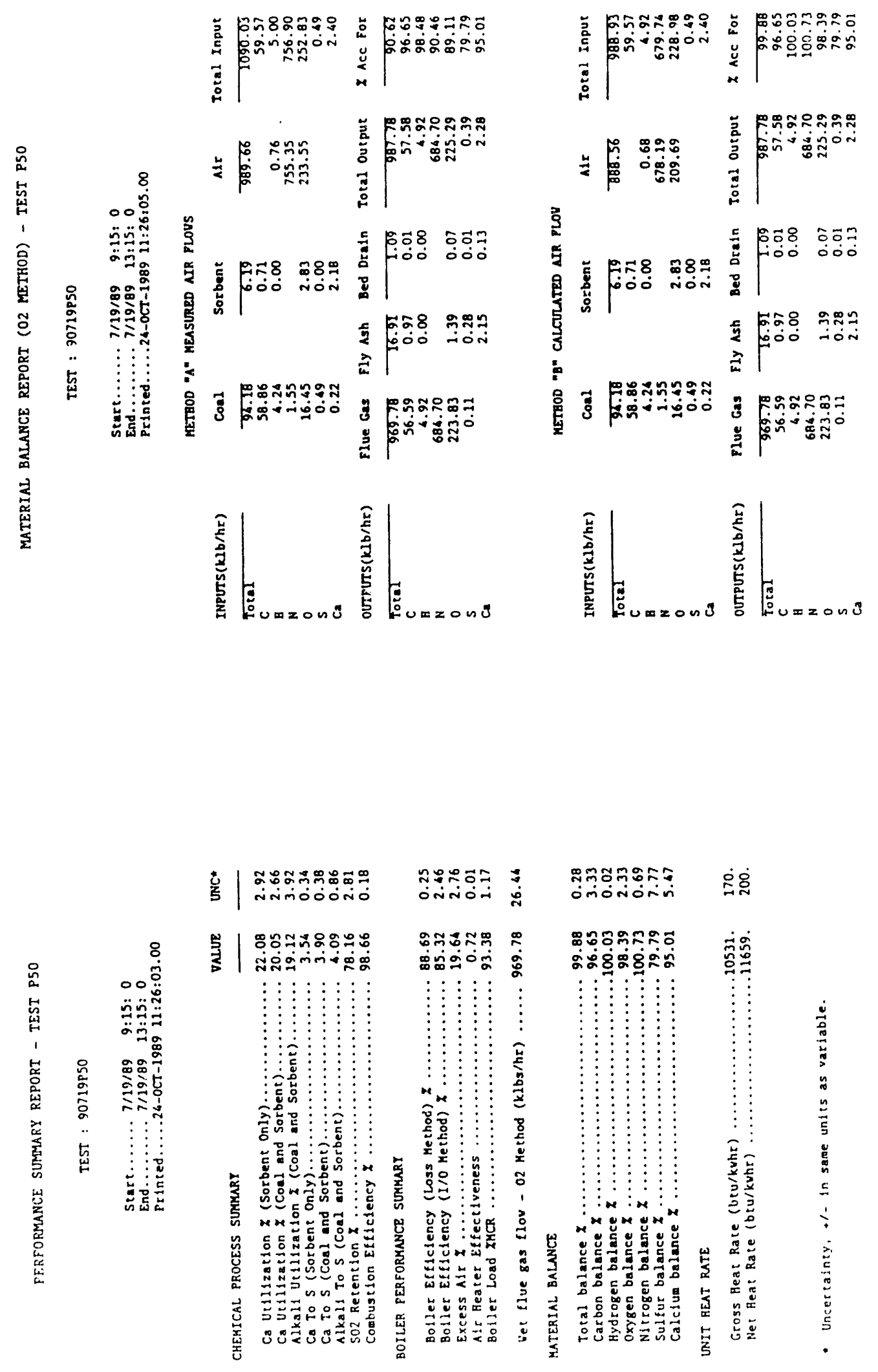


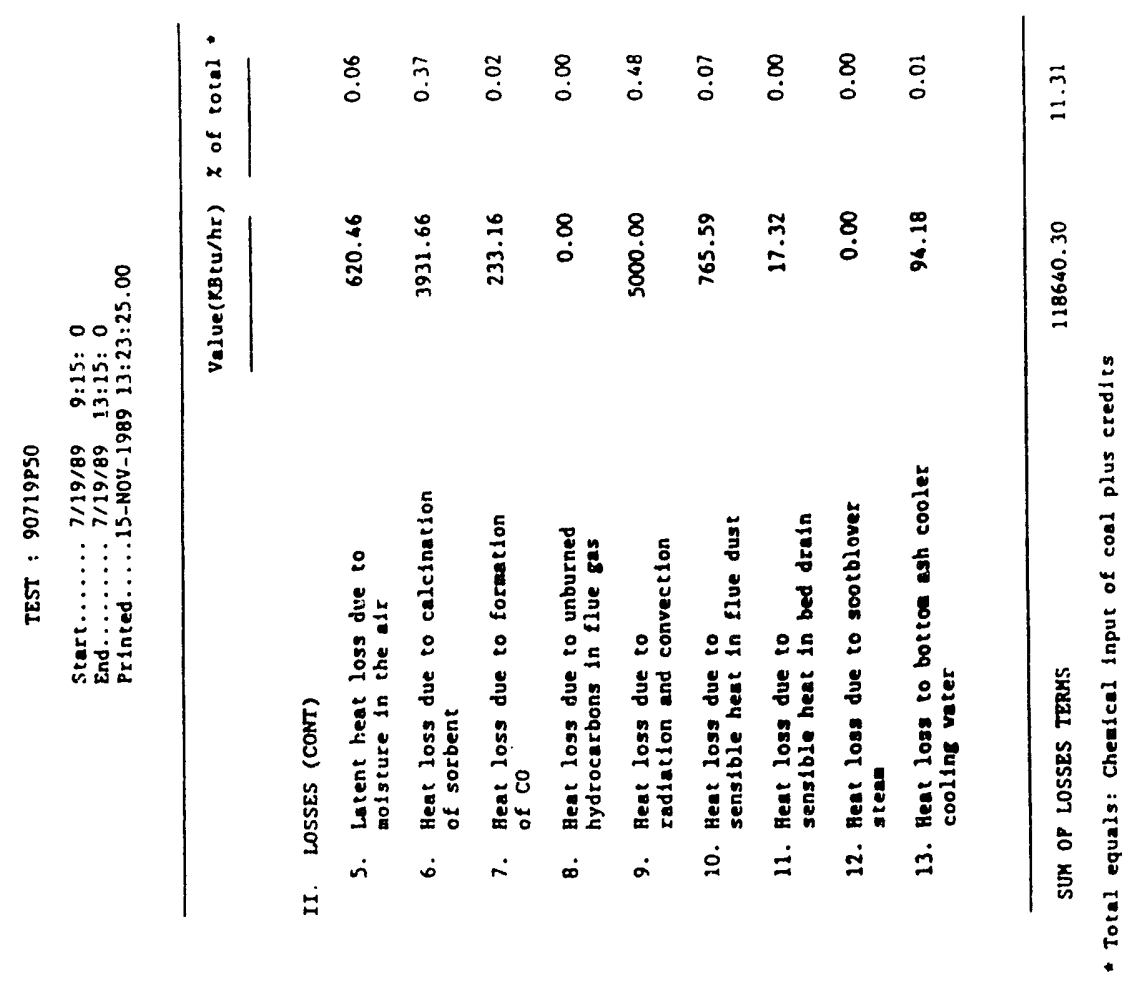

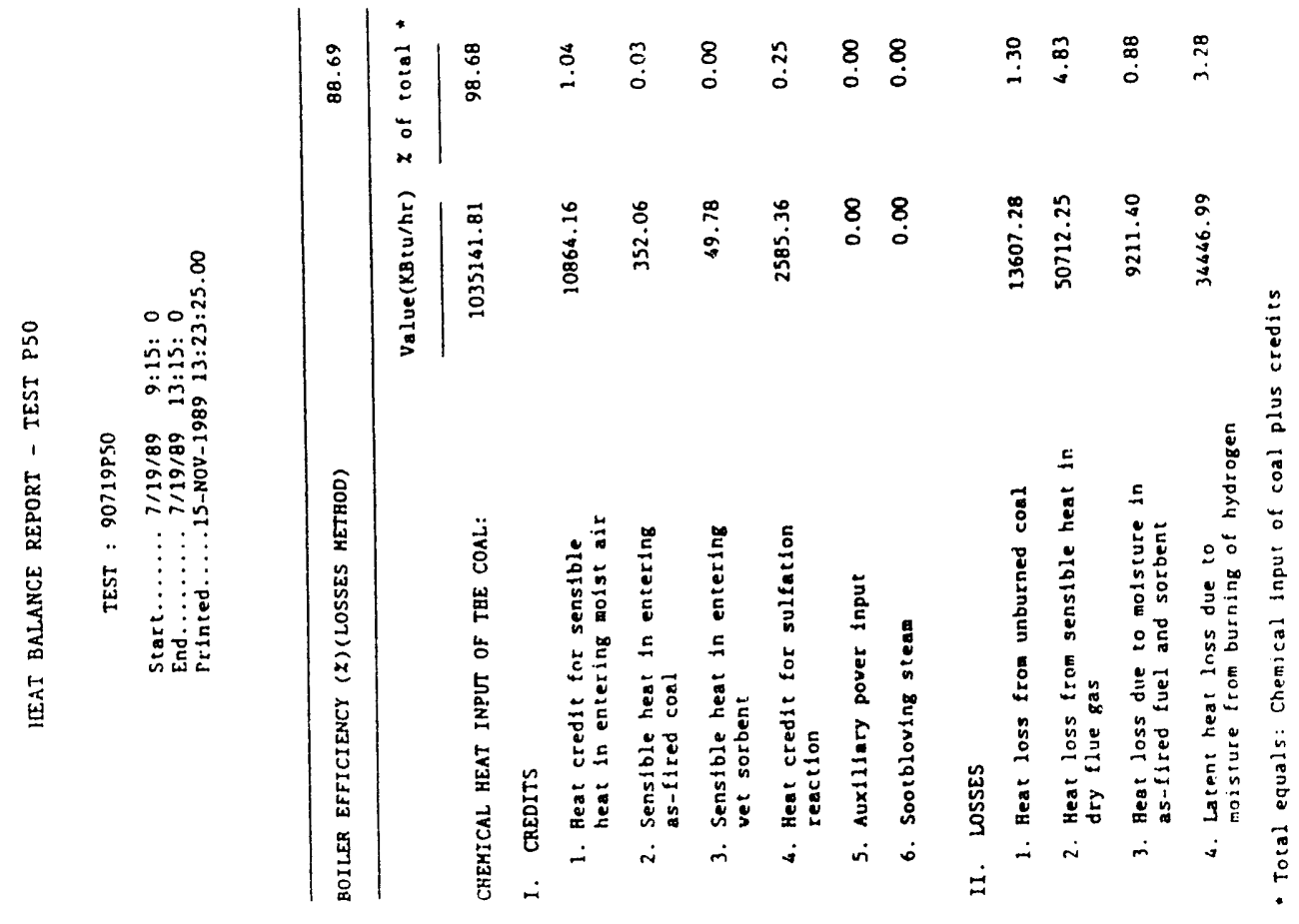



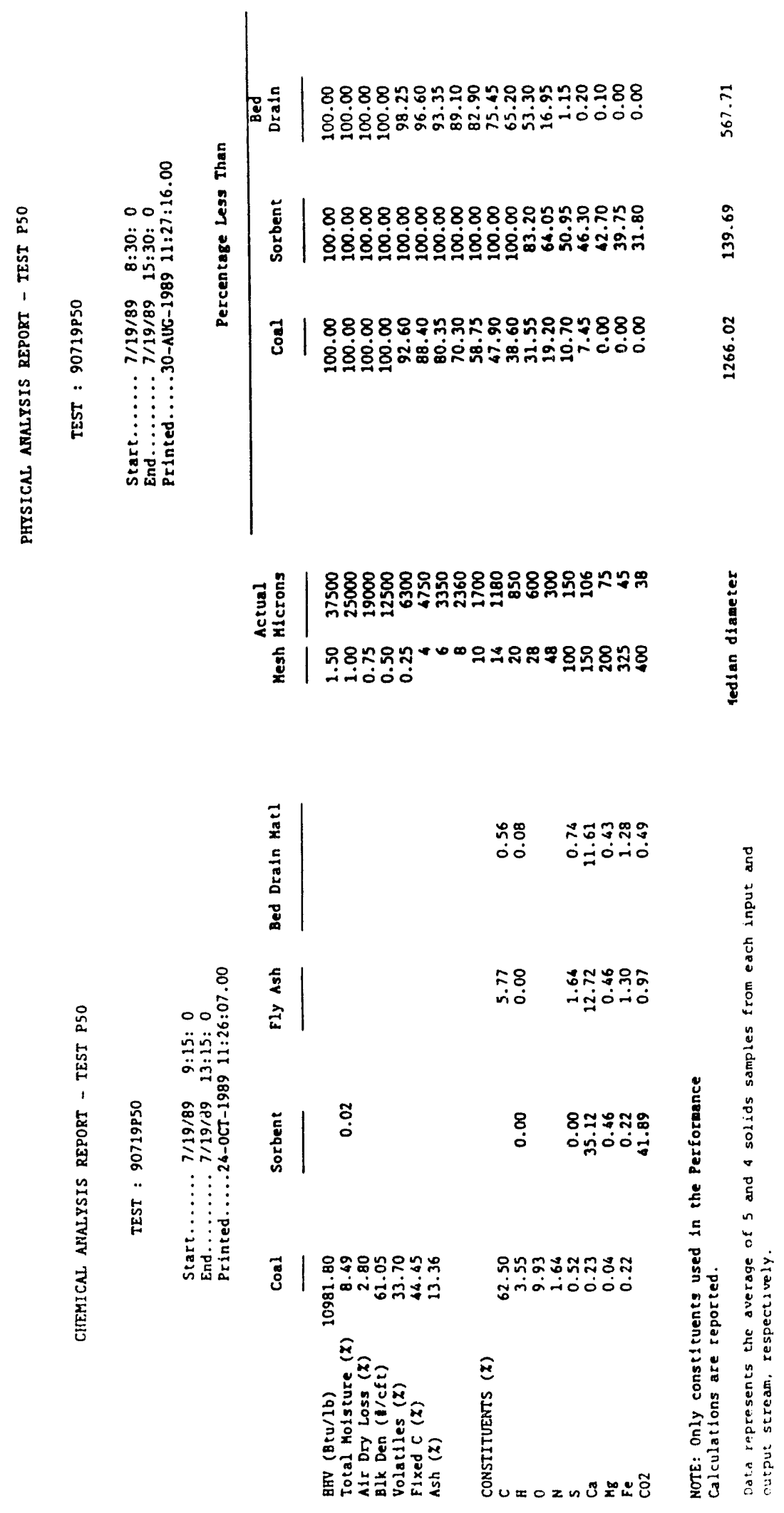

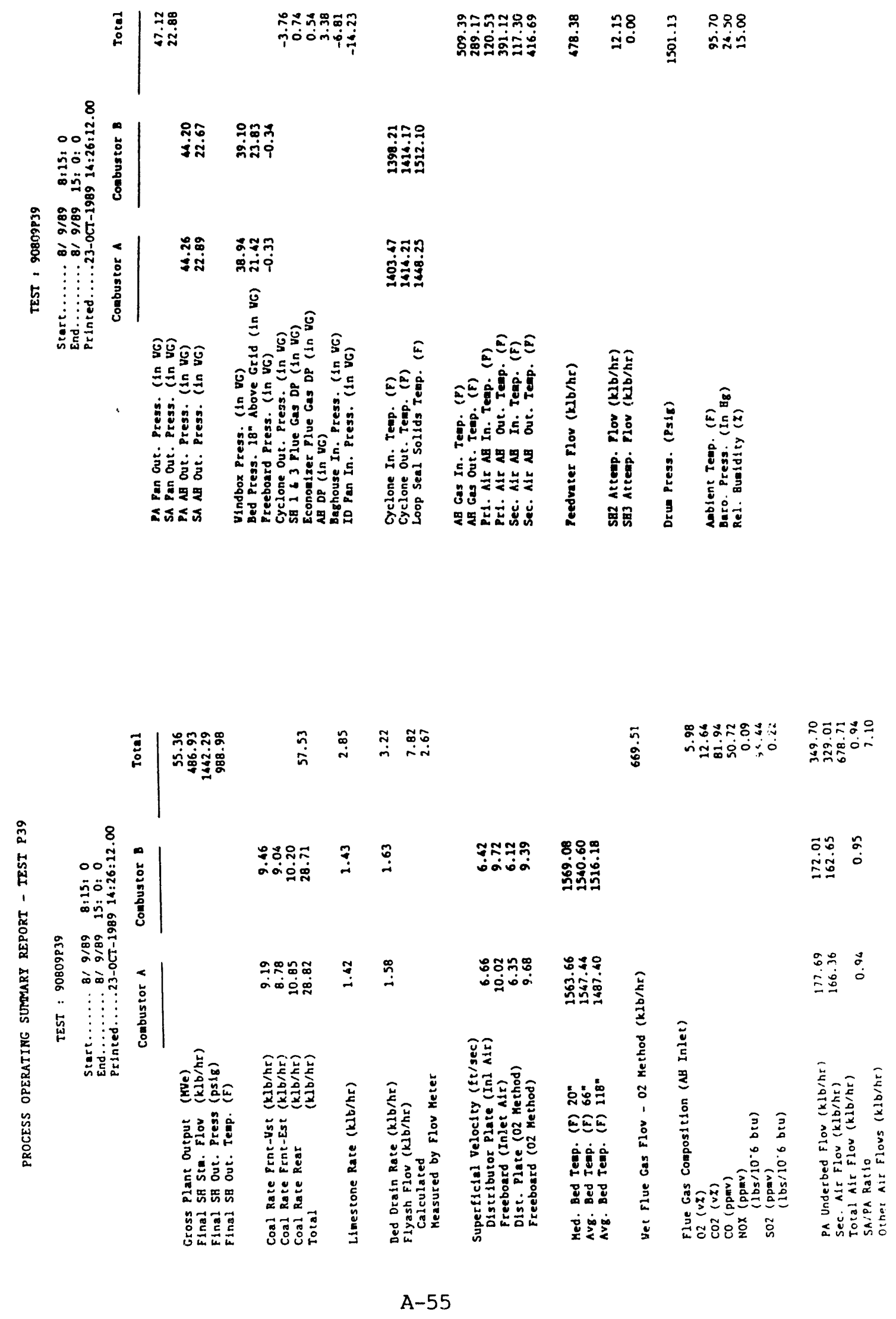


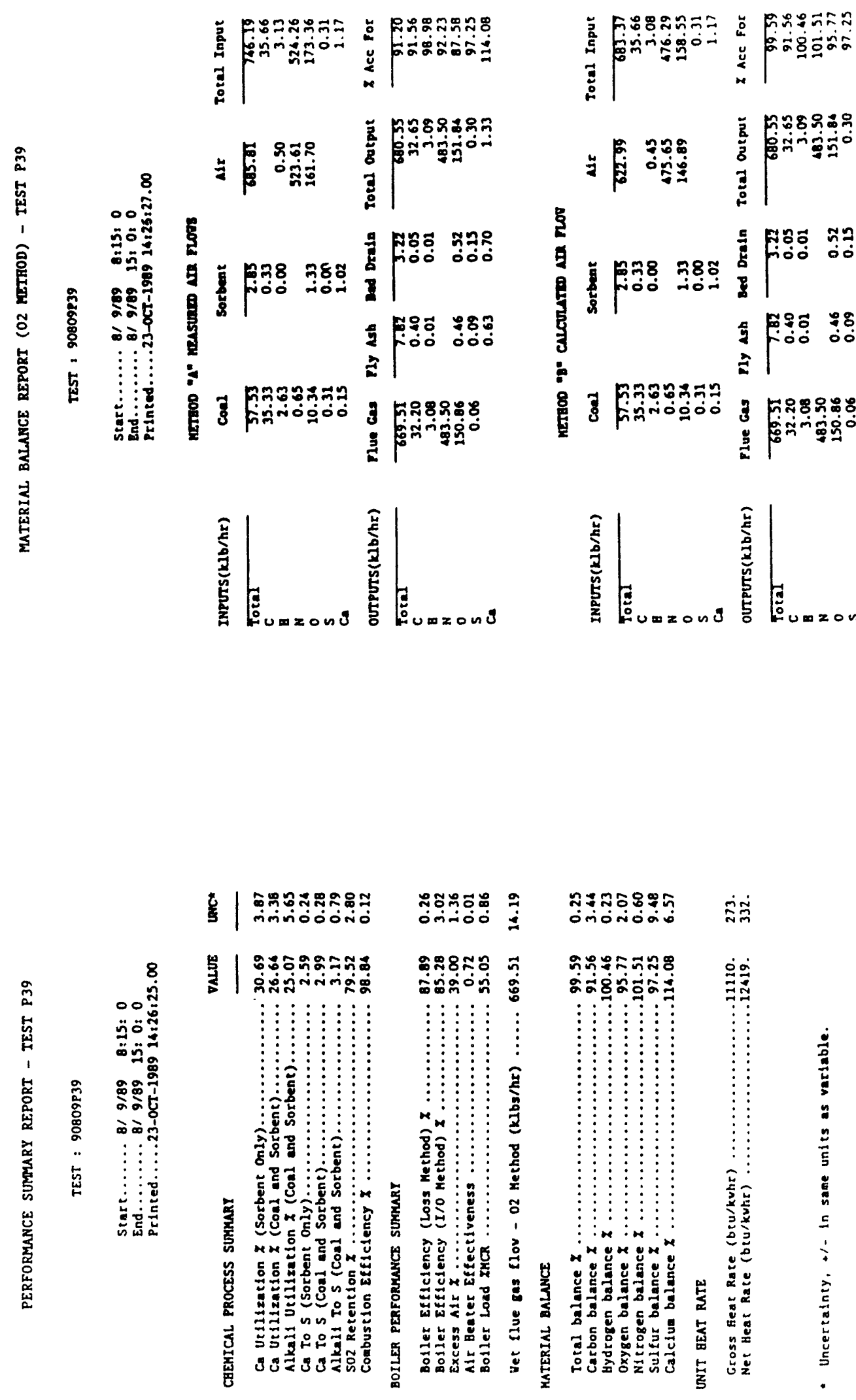




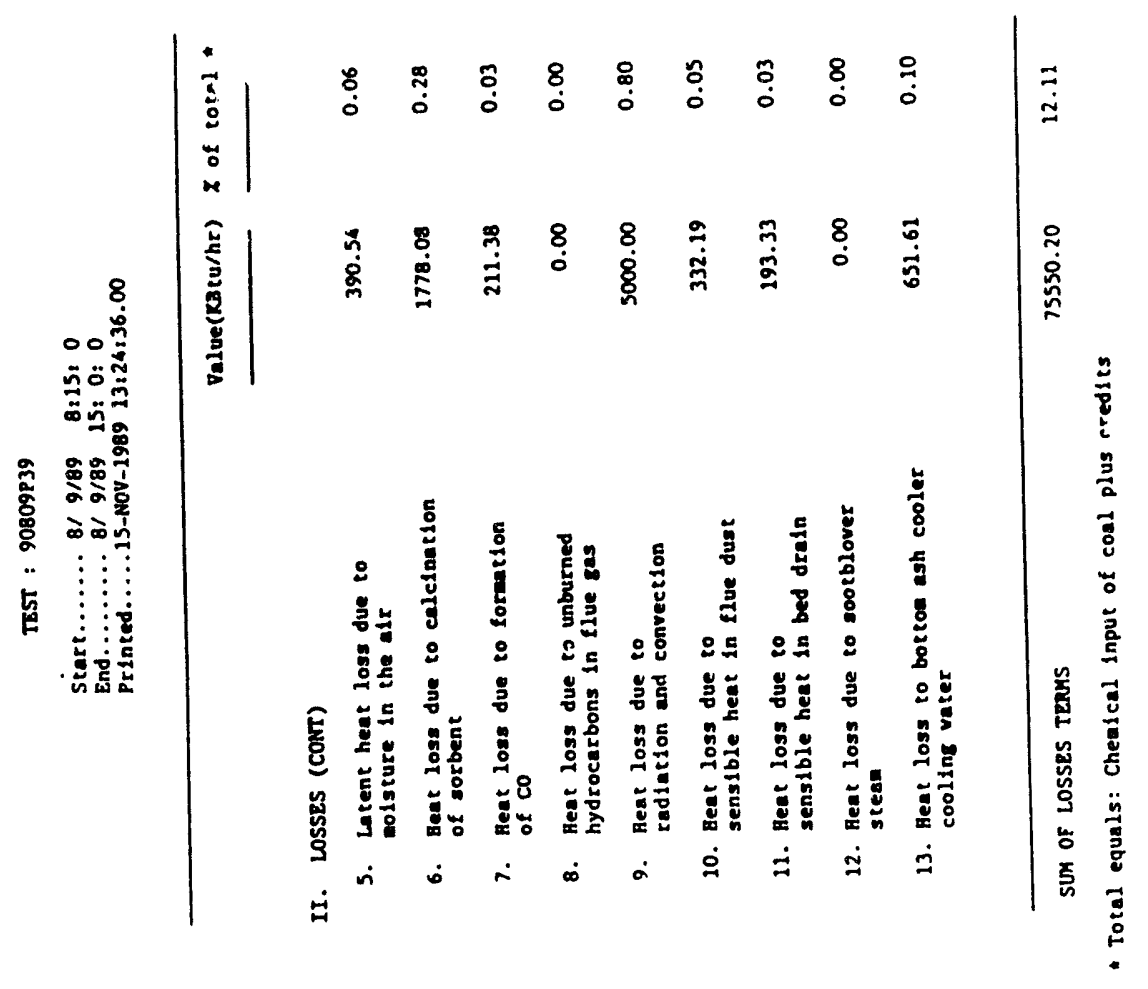

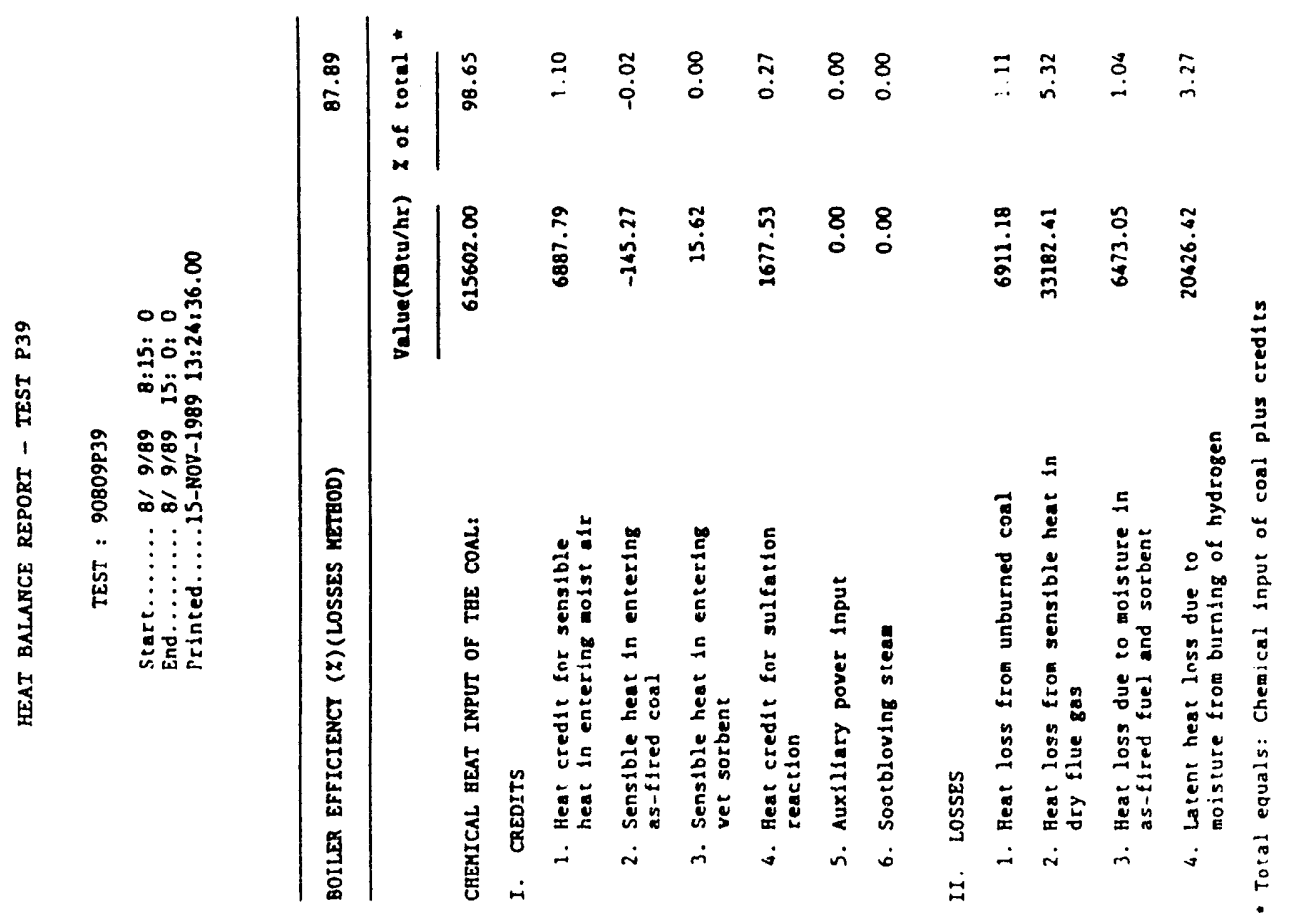



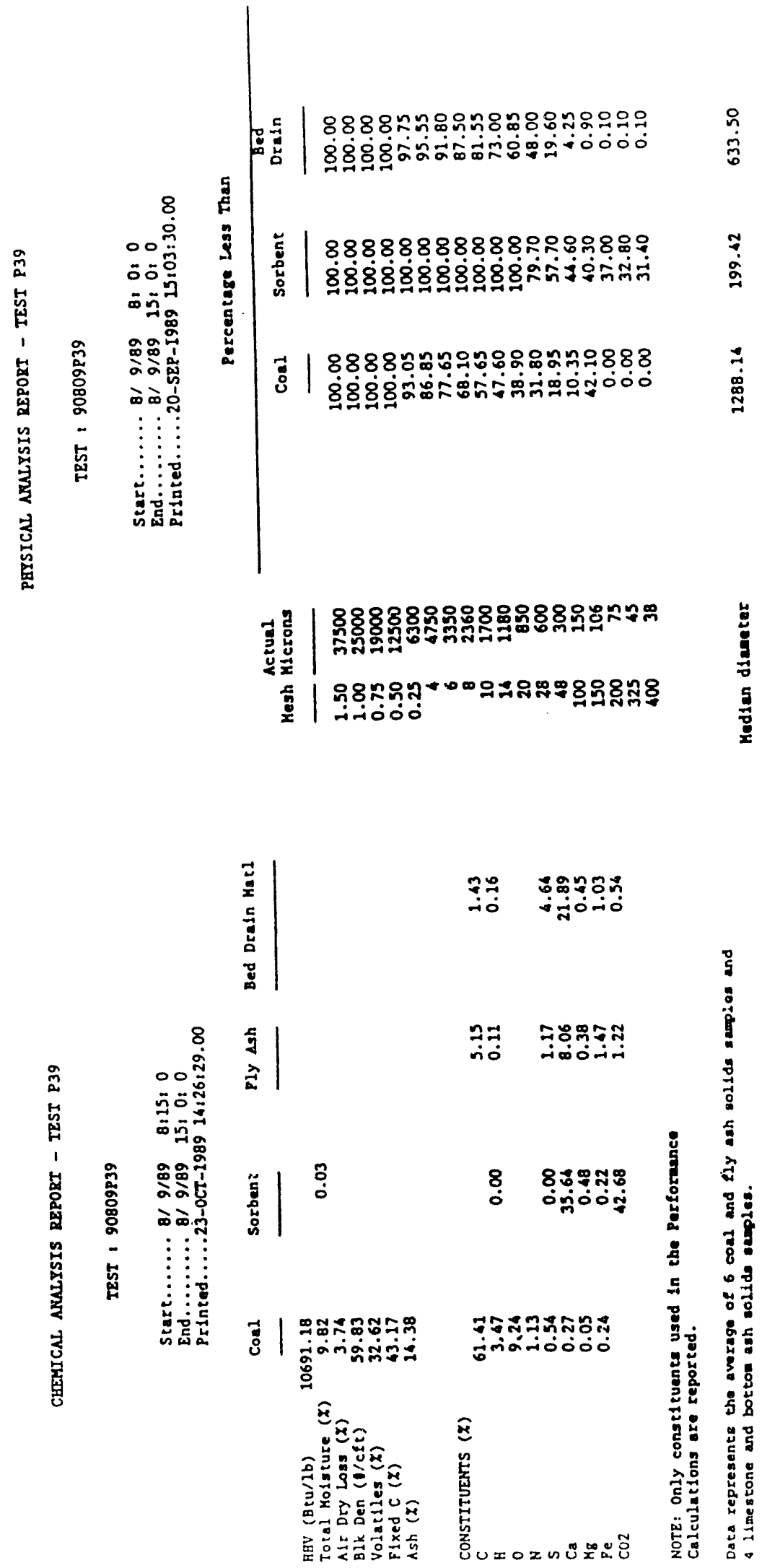

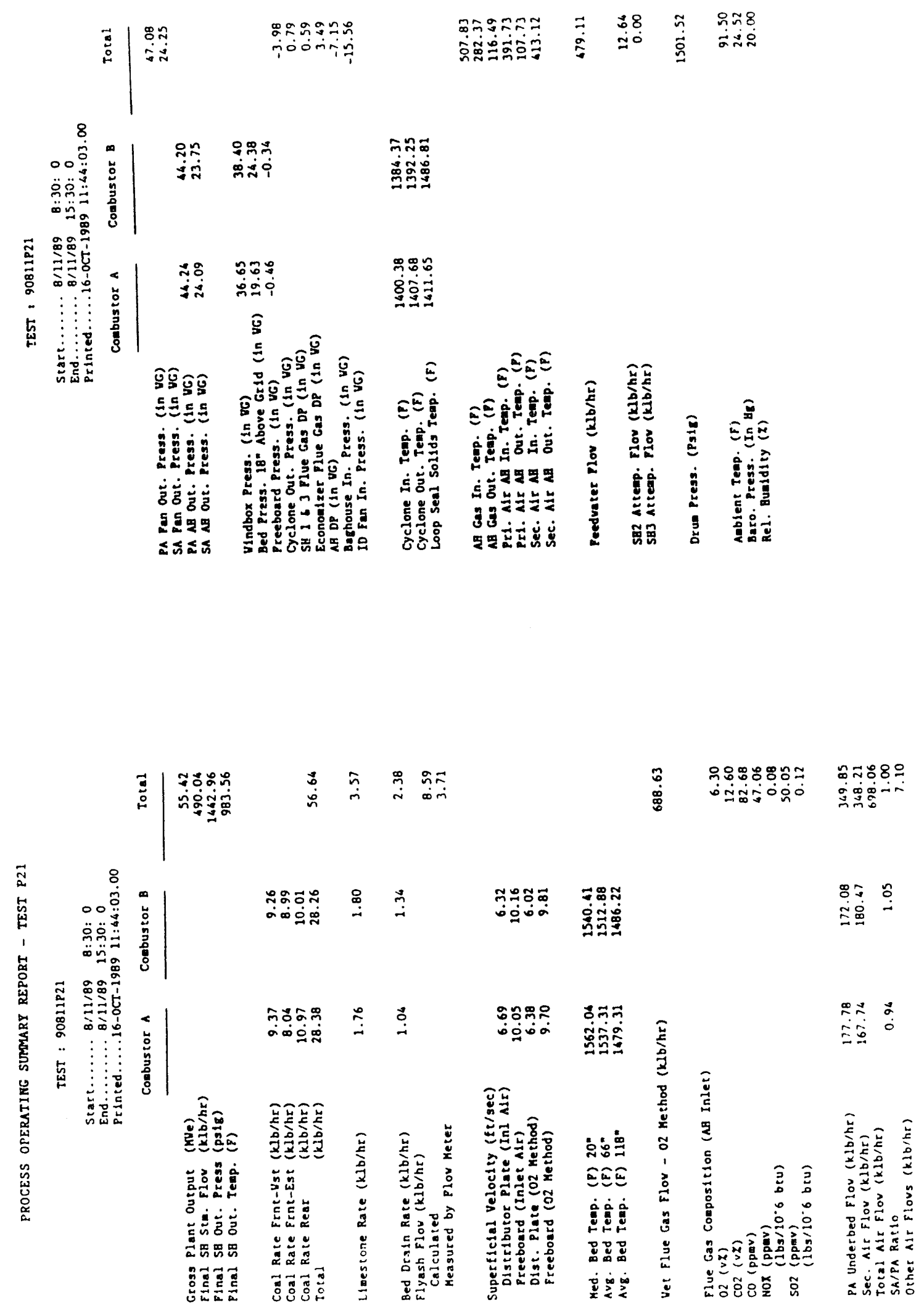


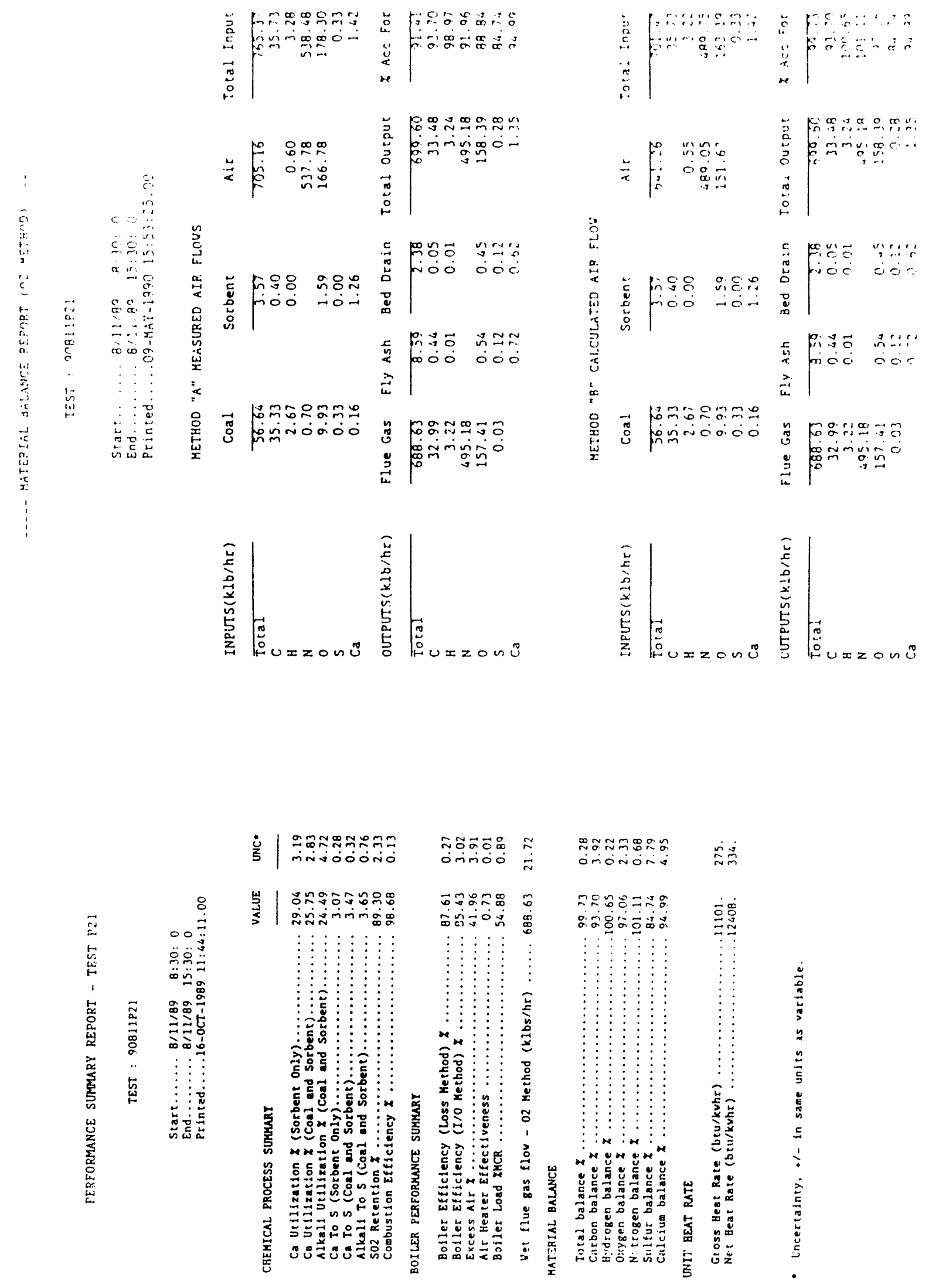




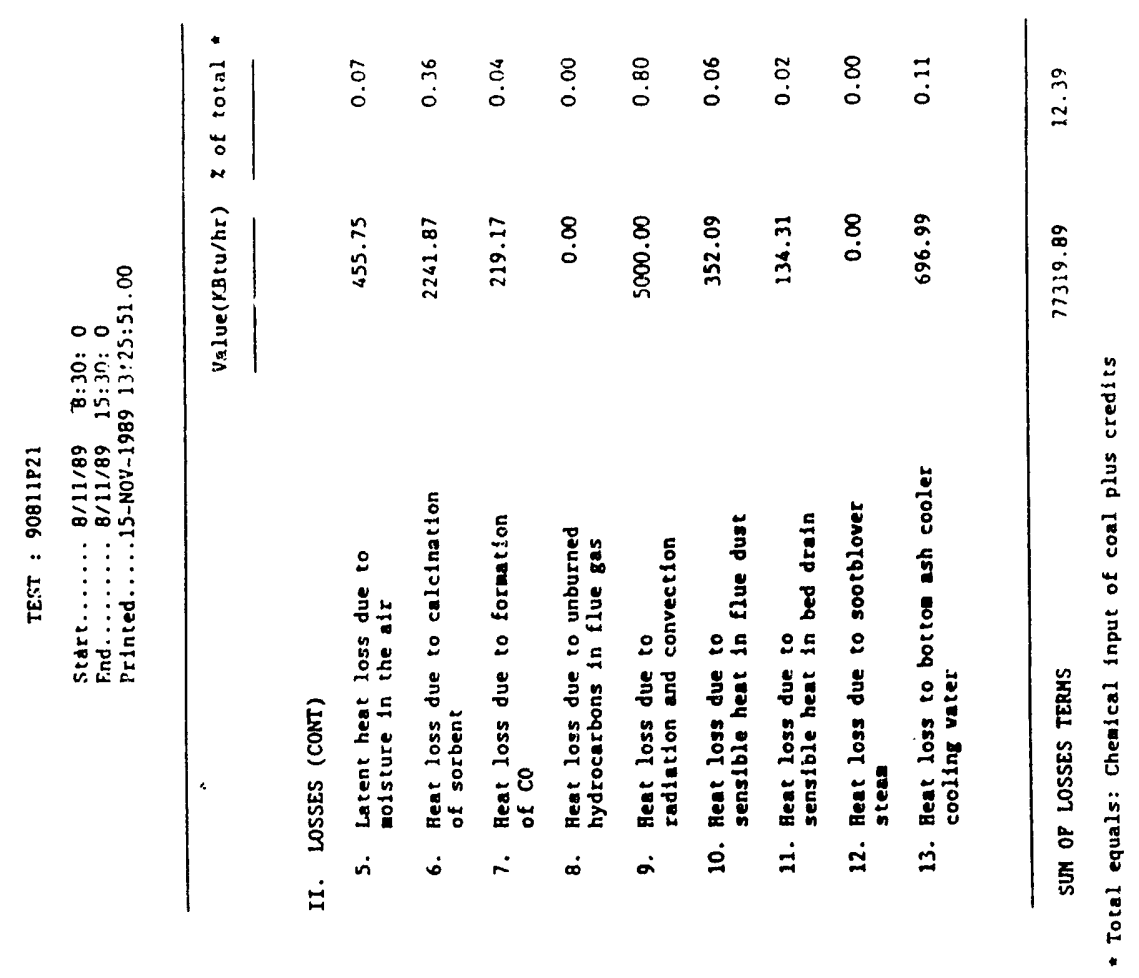

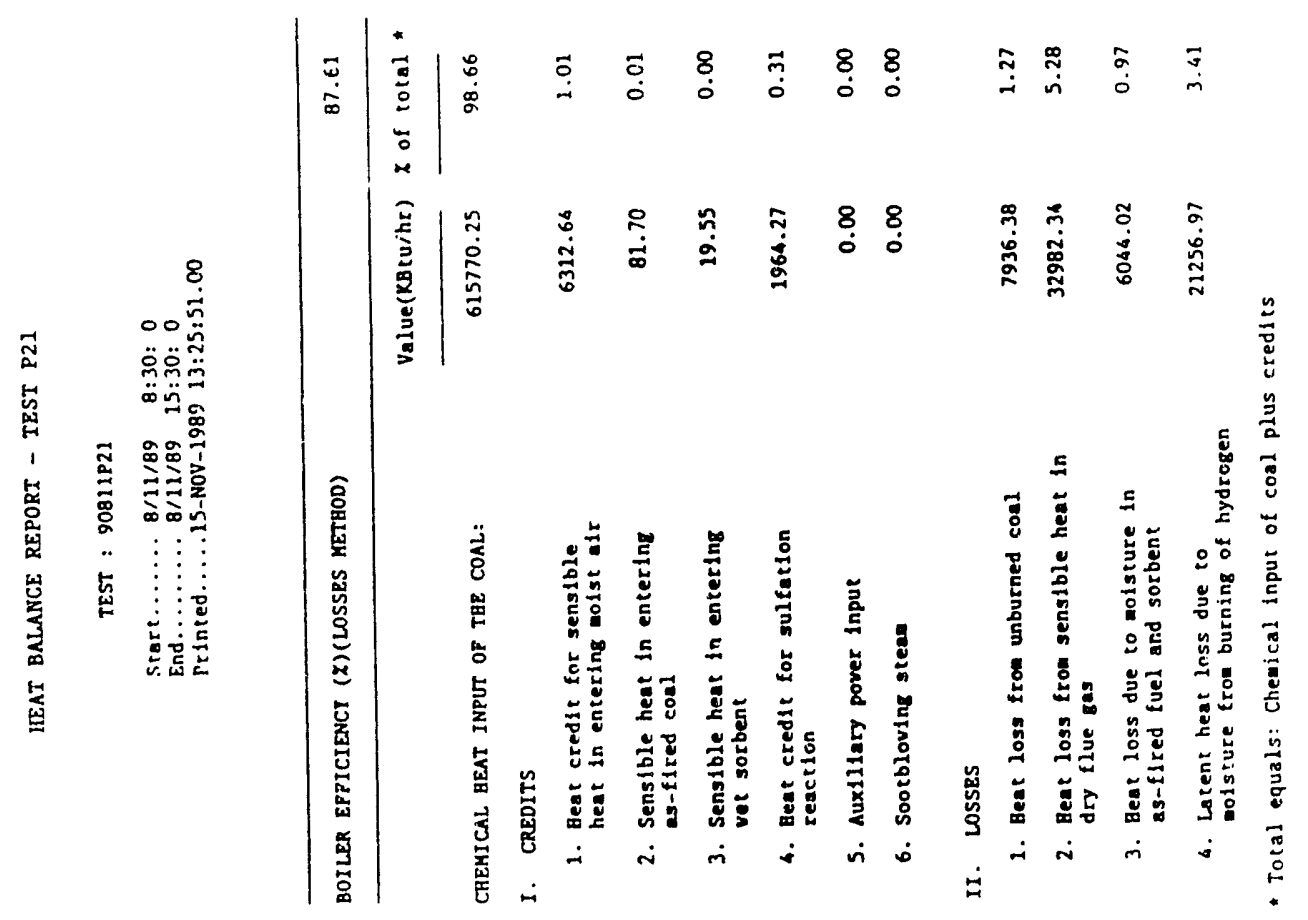



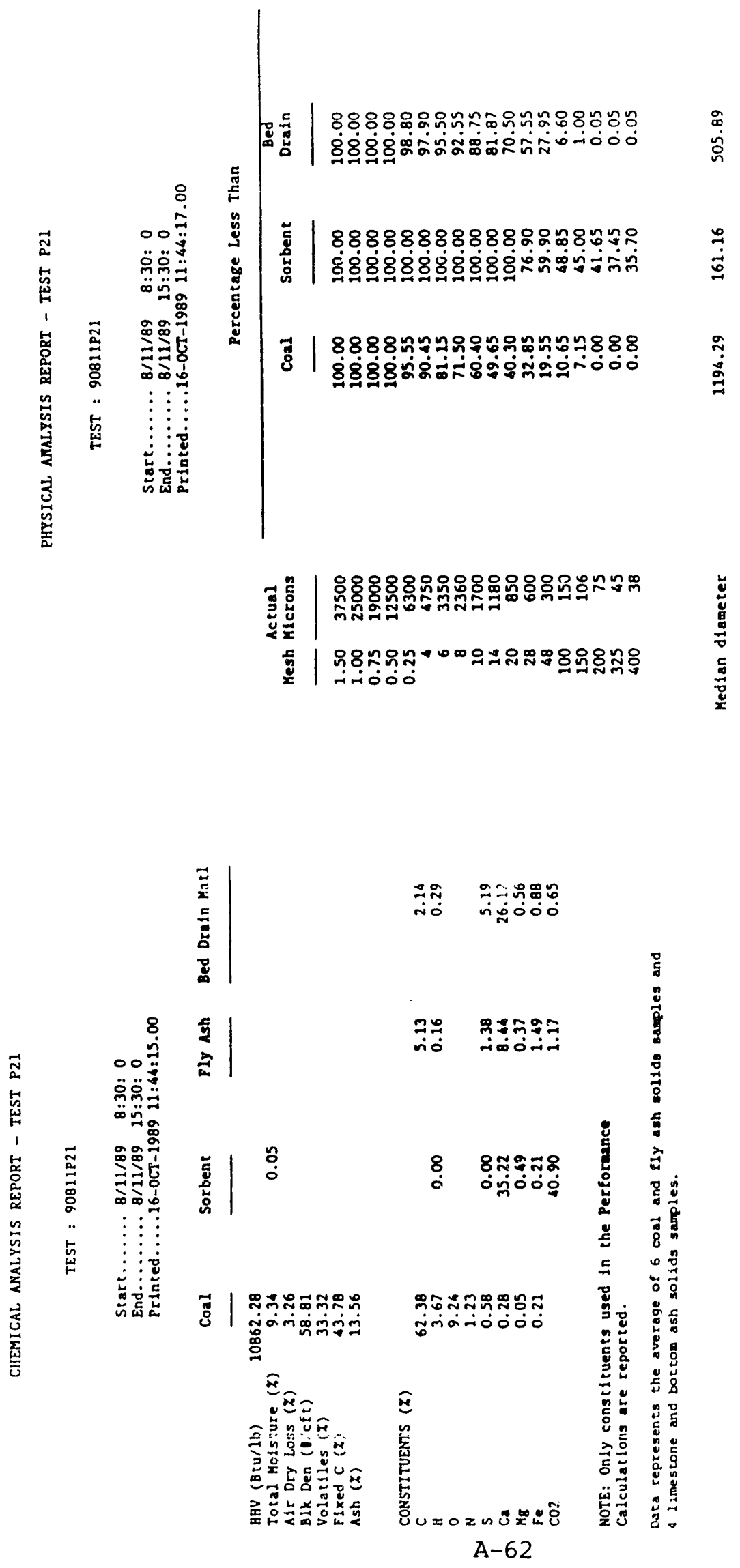

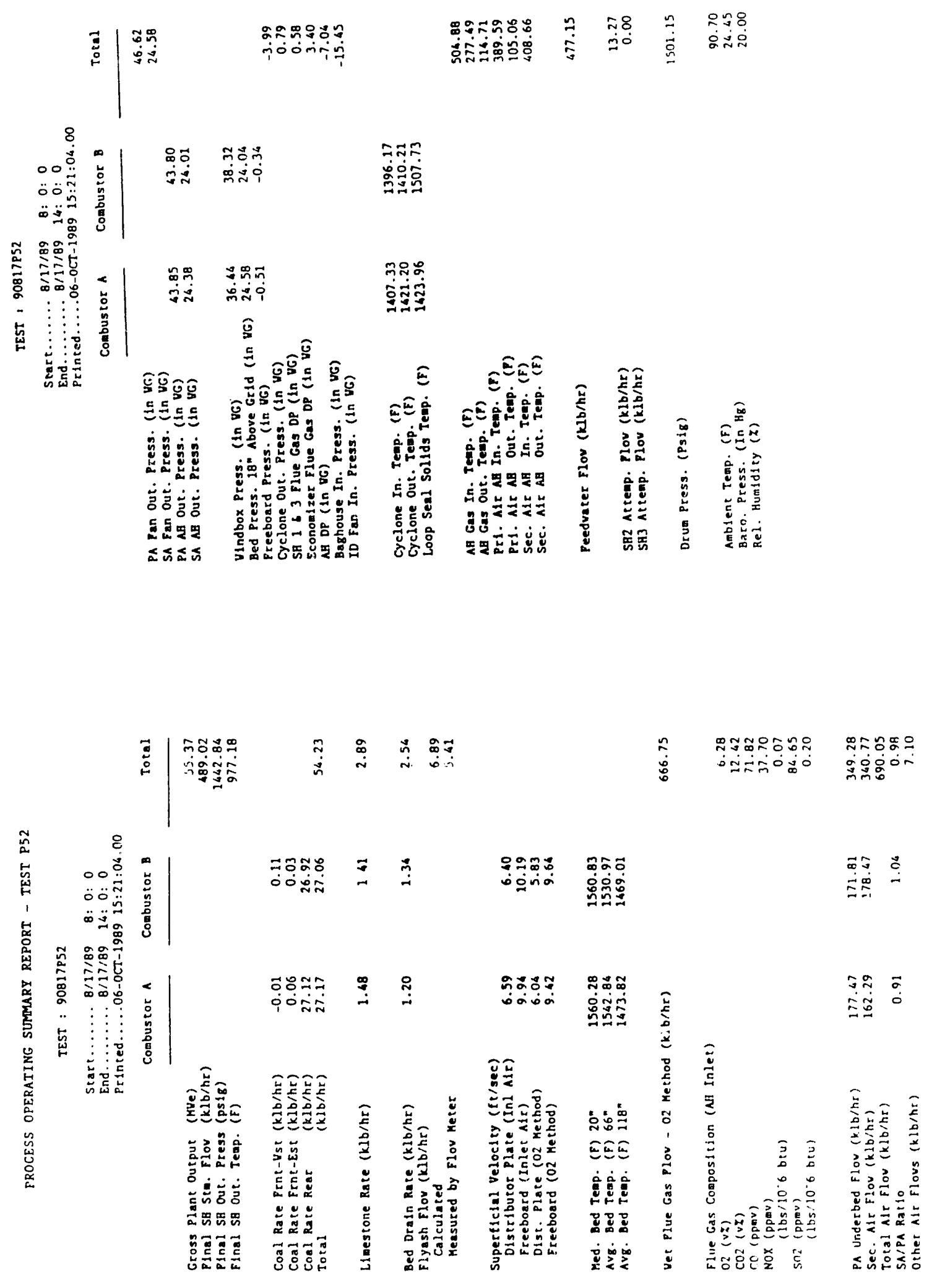


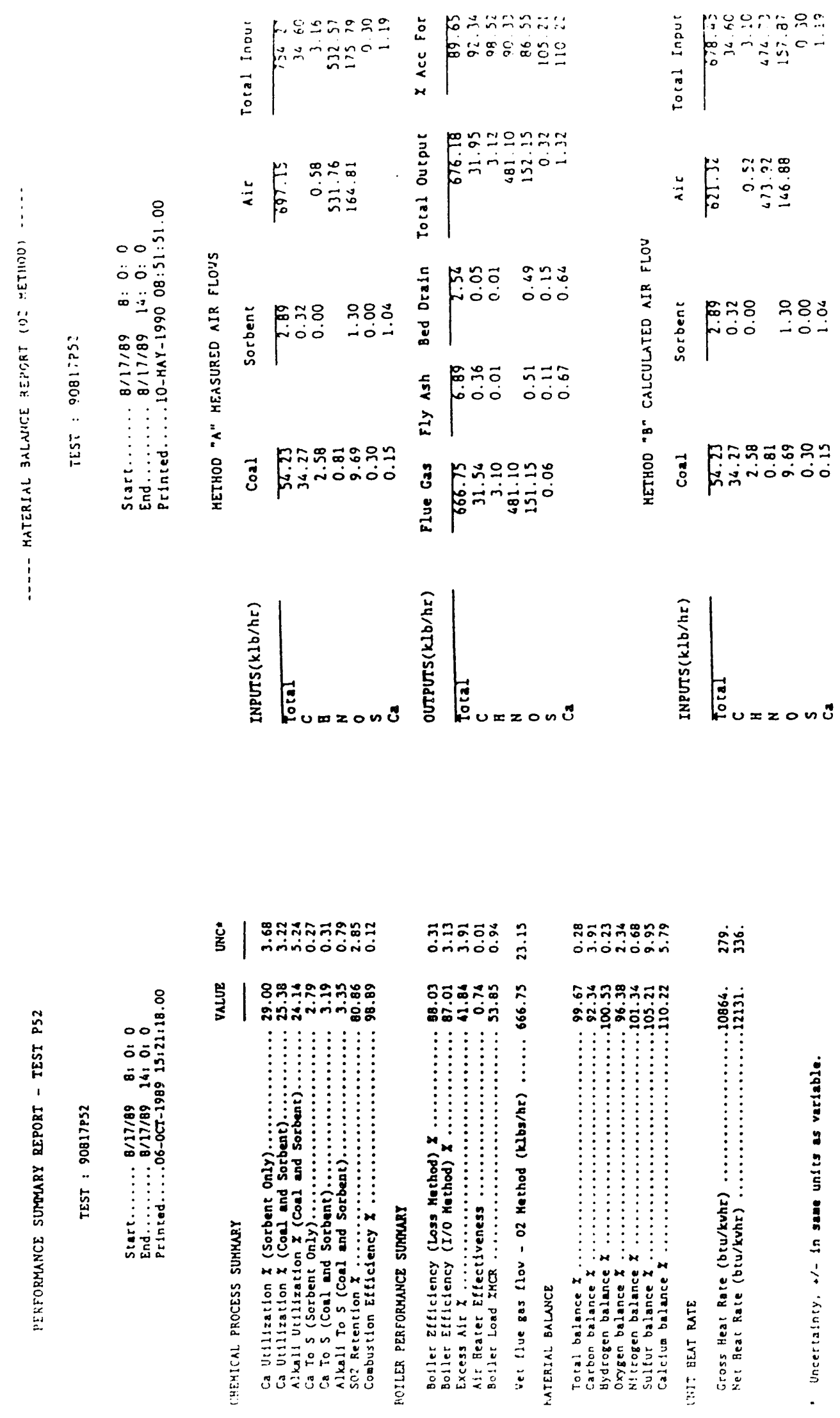




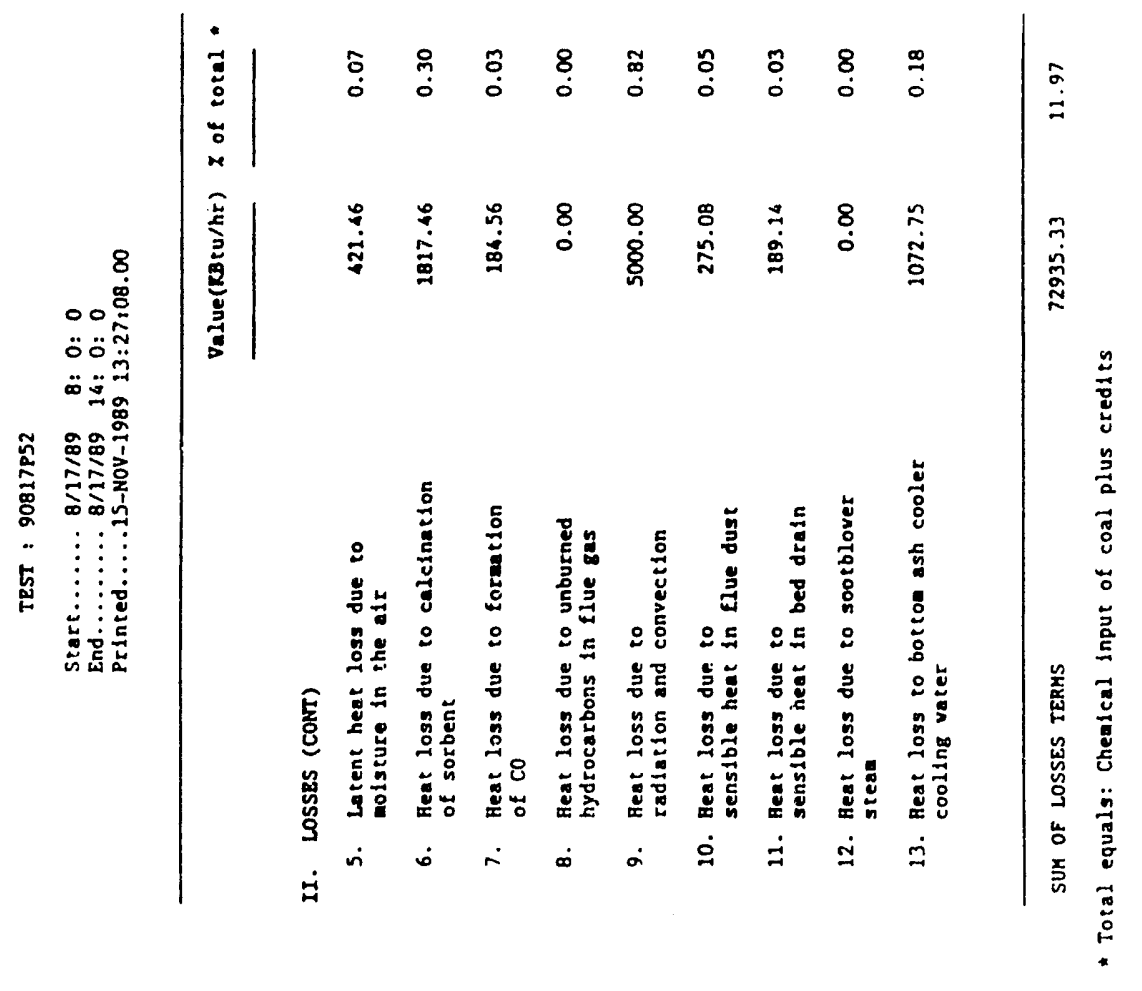

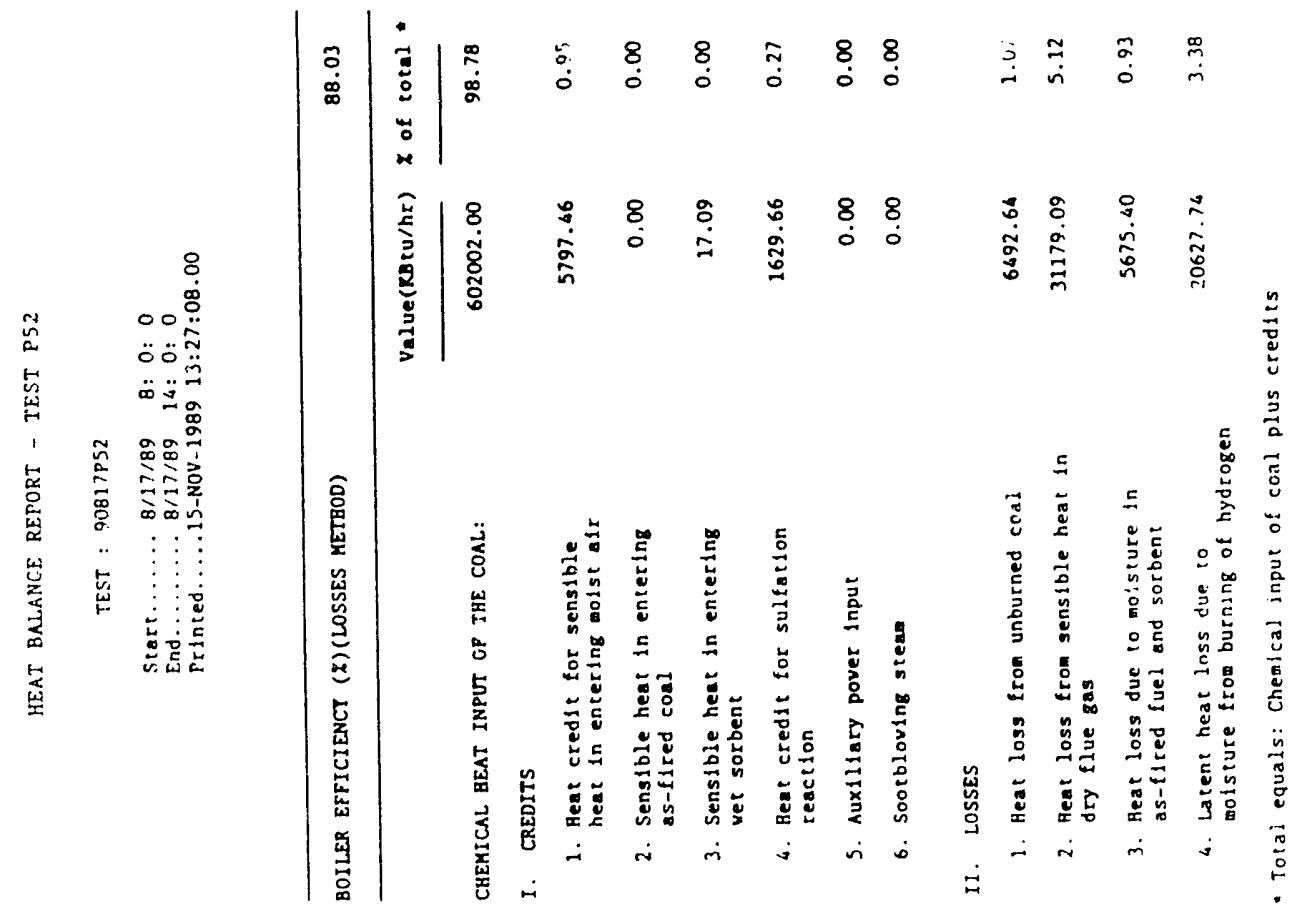



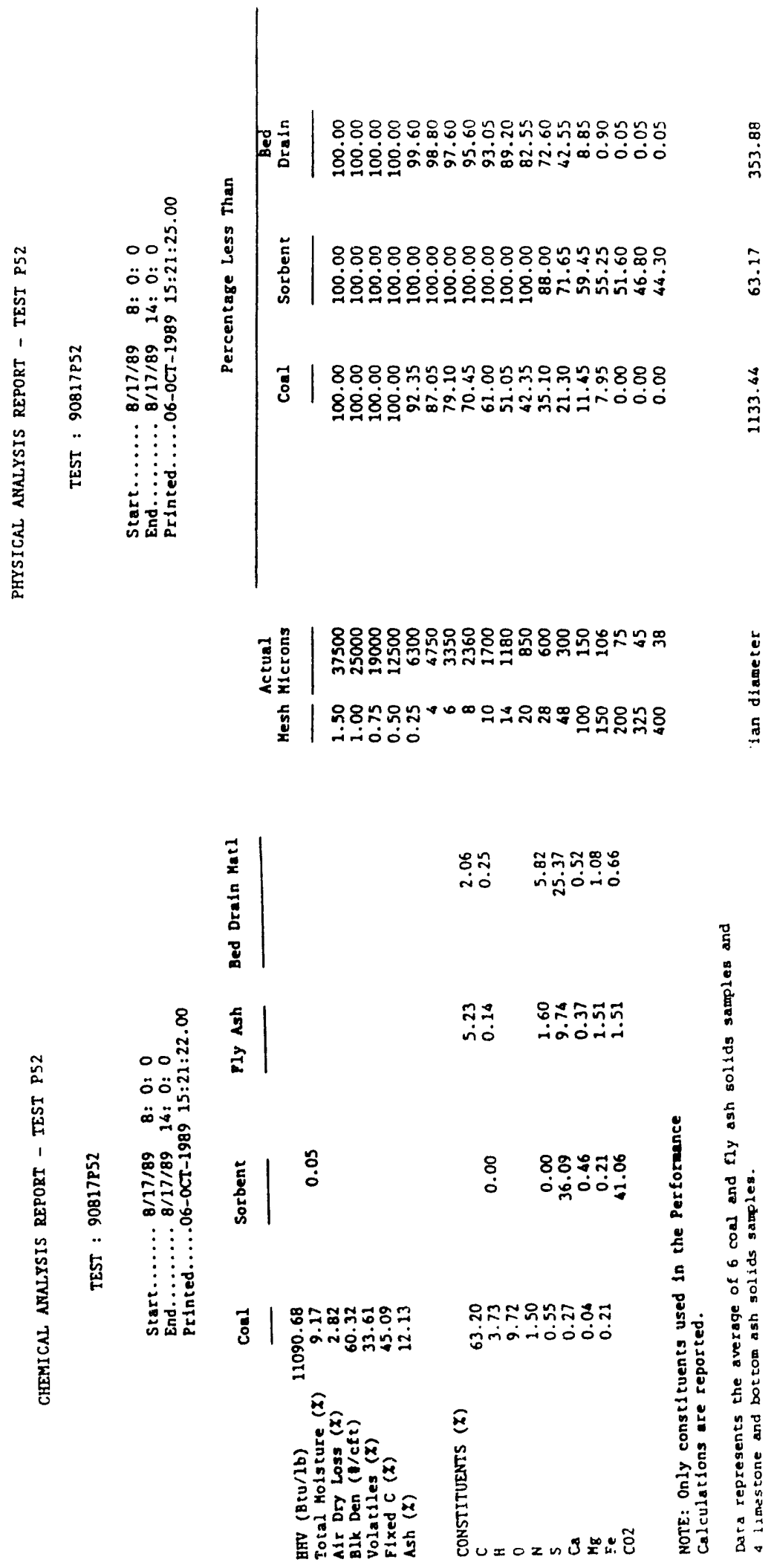


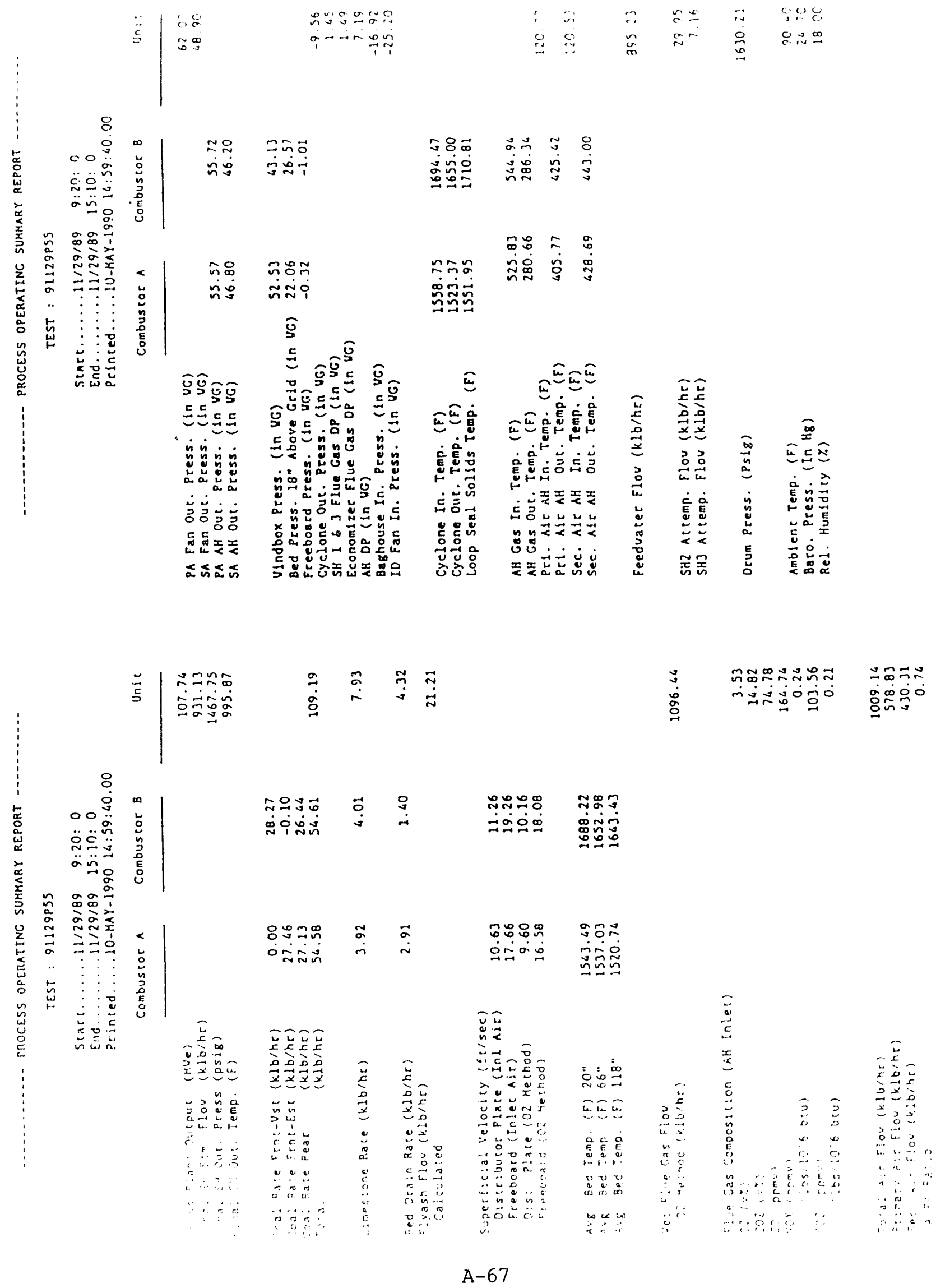




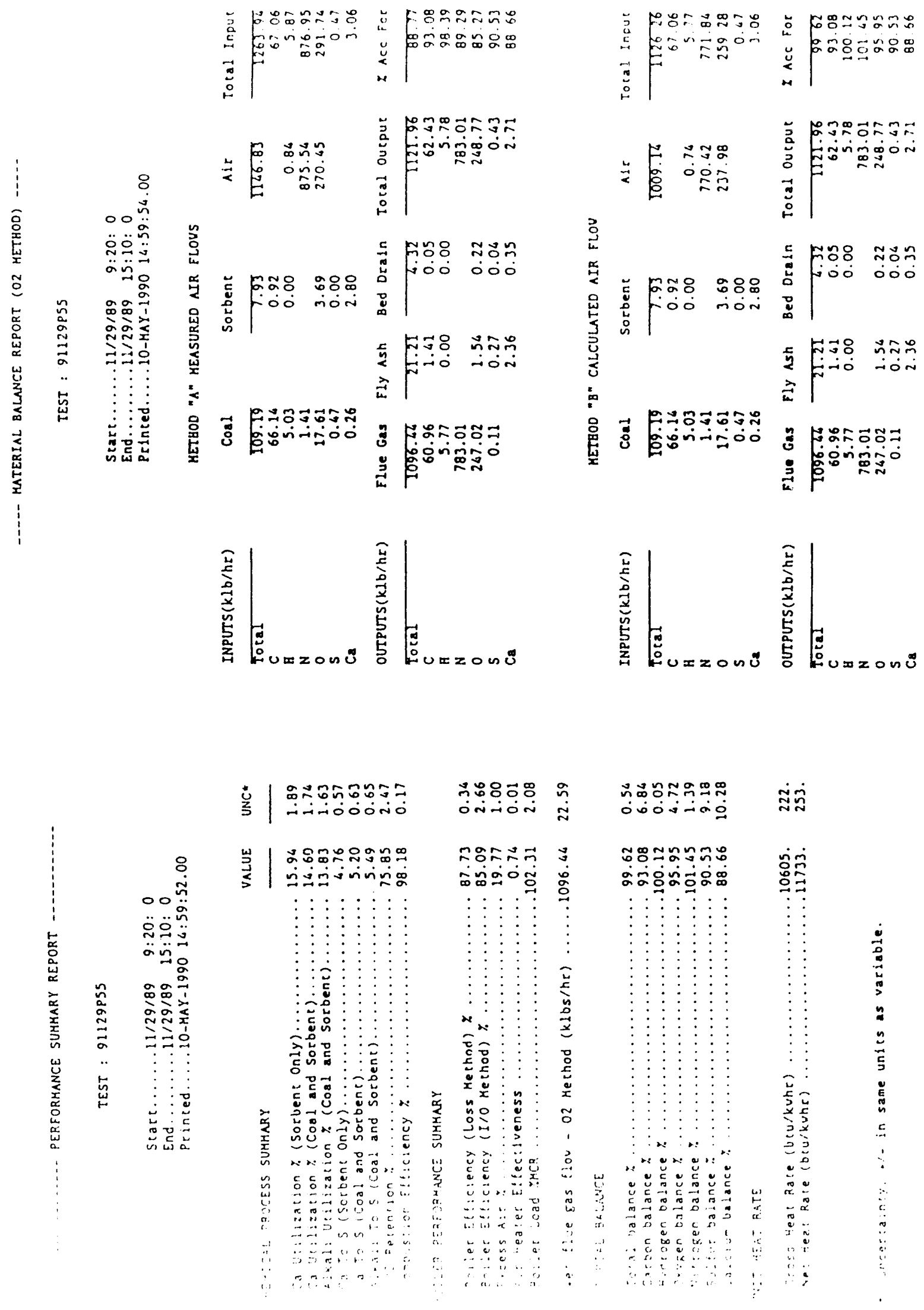




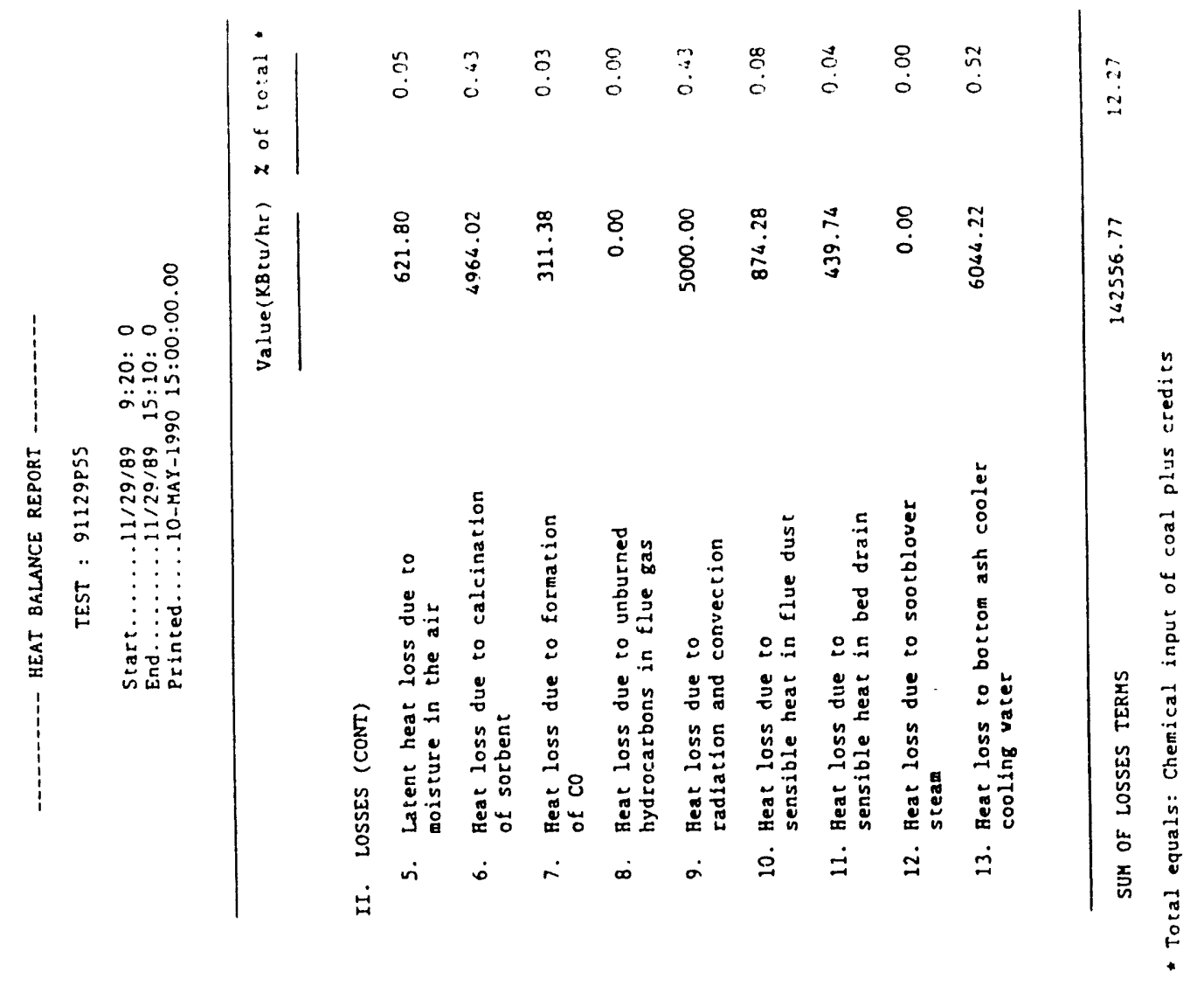

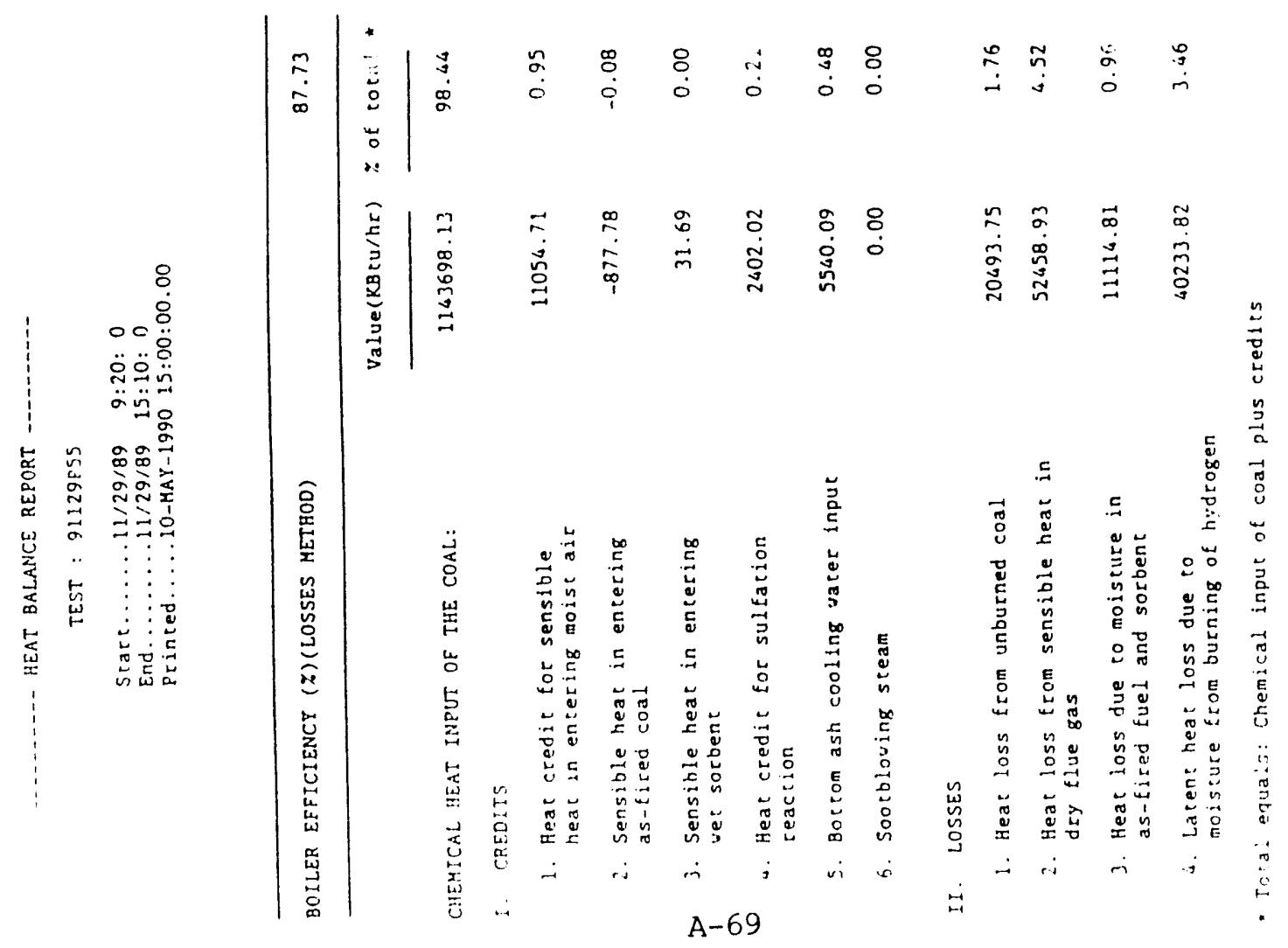



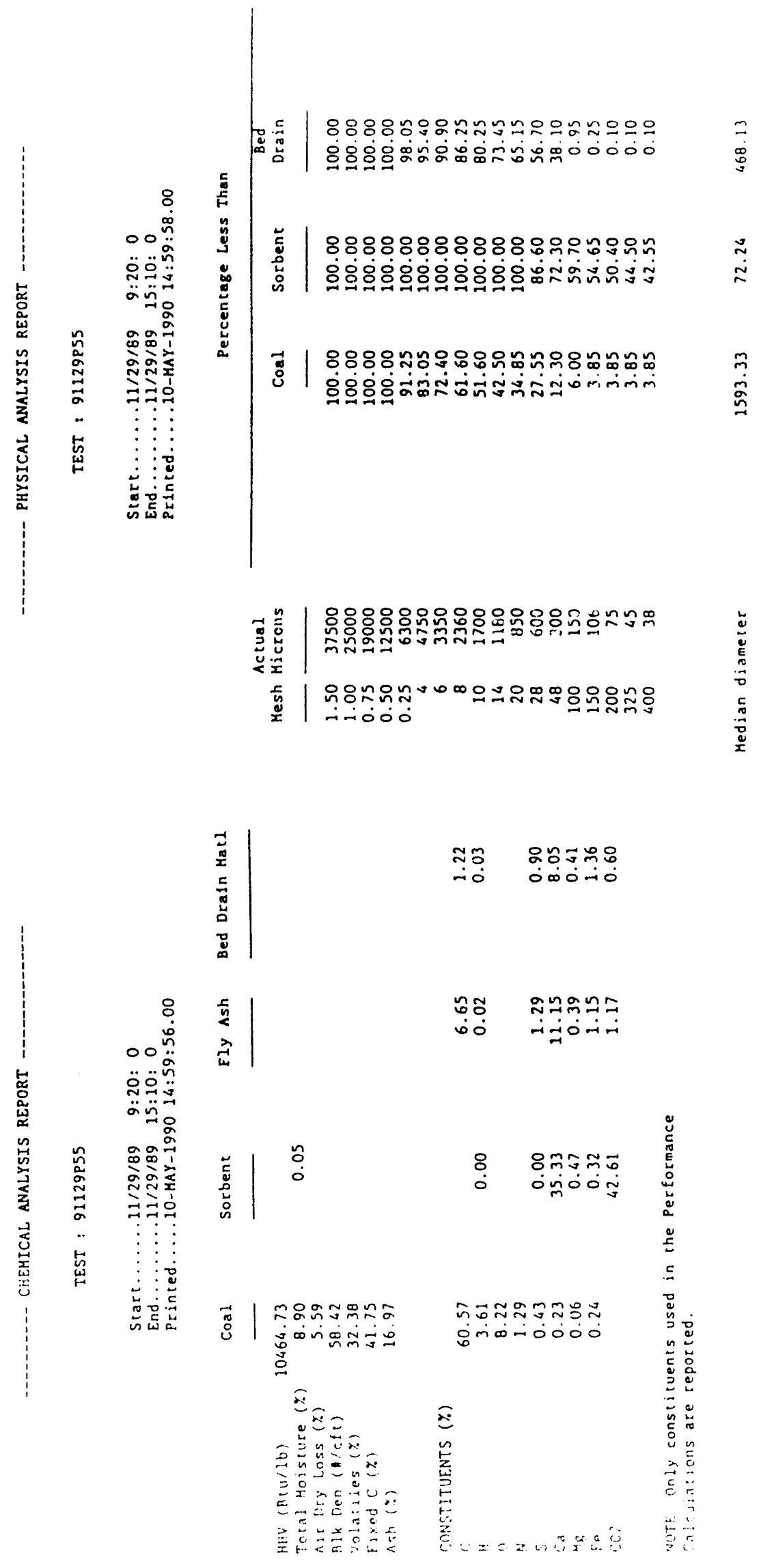

A -70 

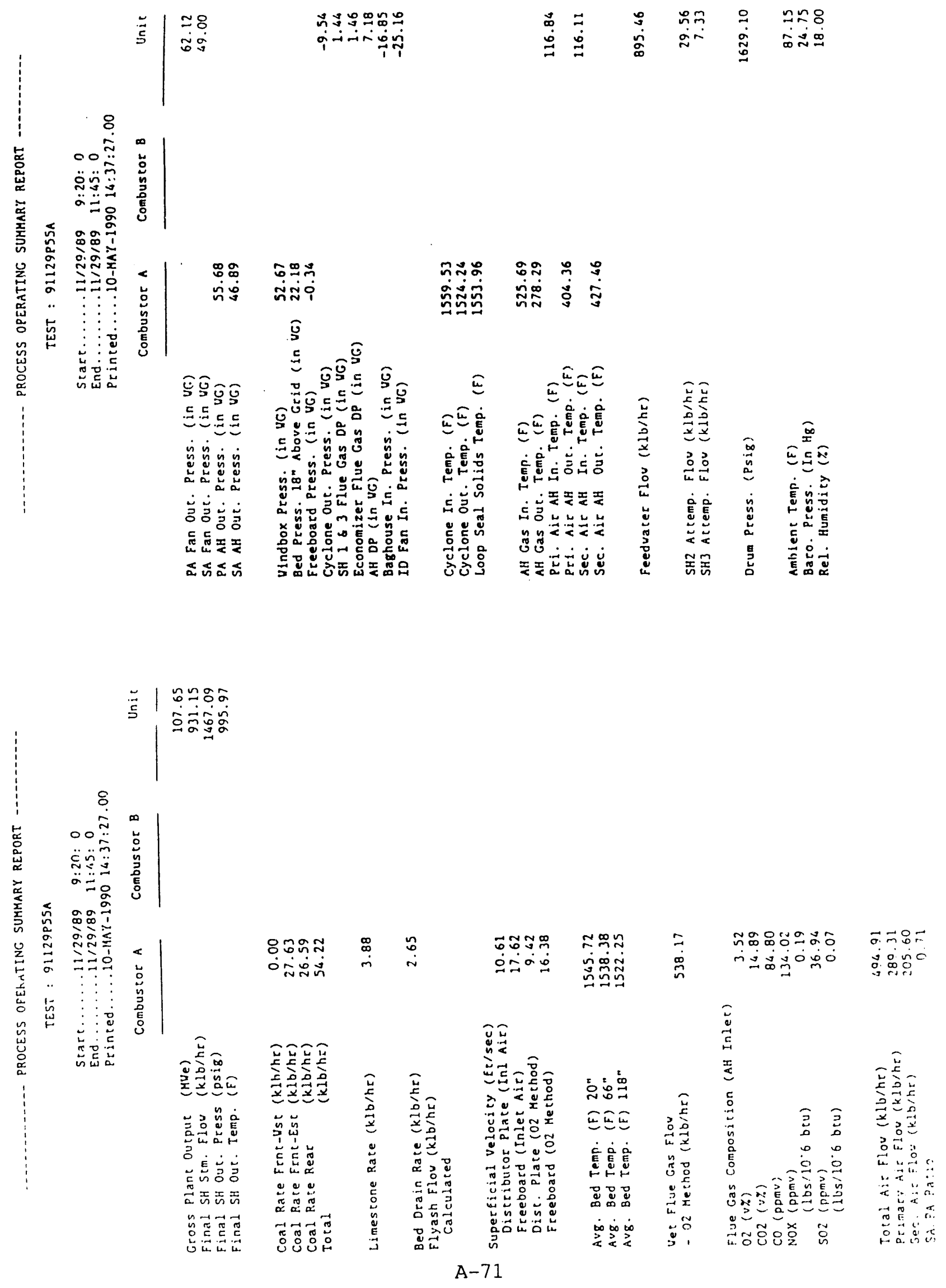


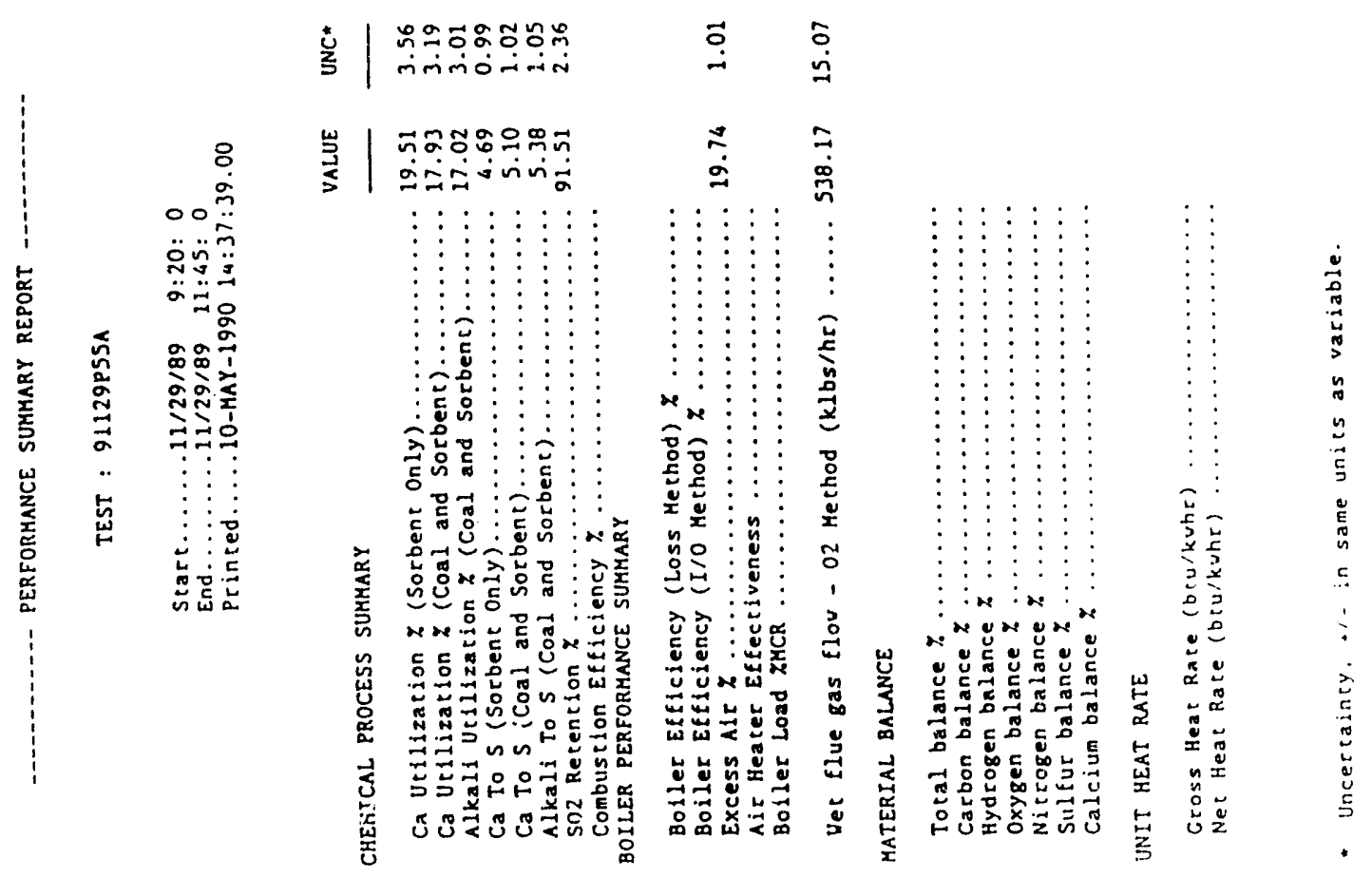




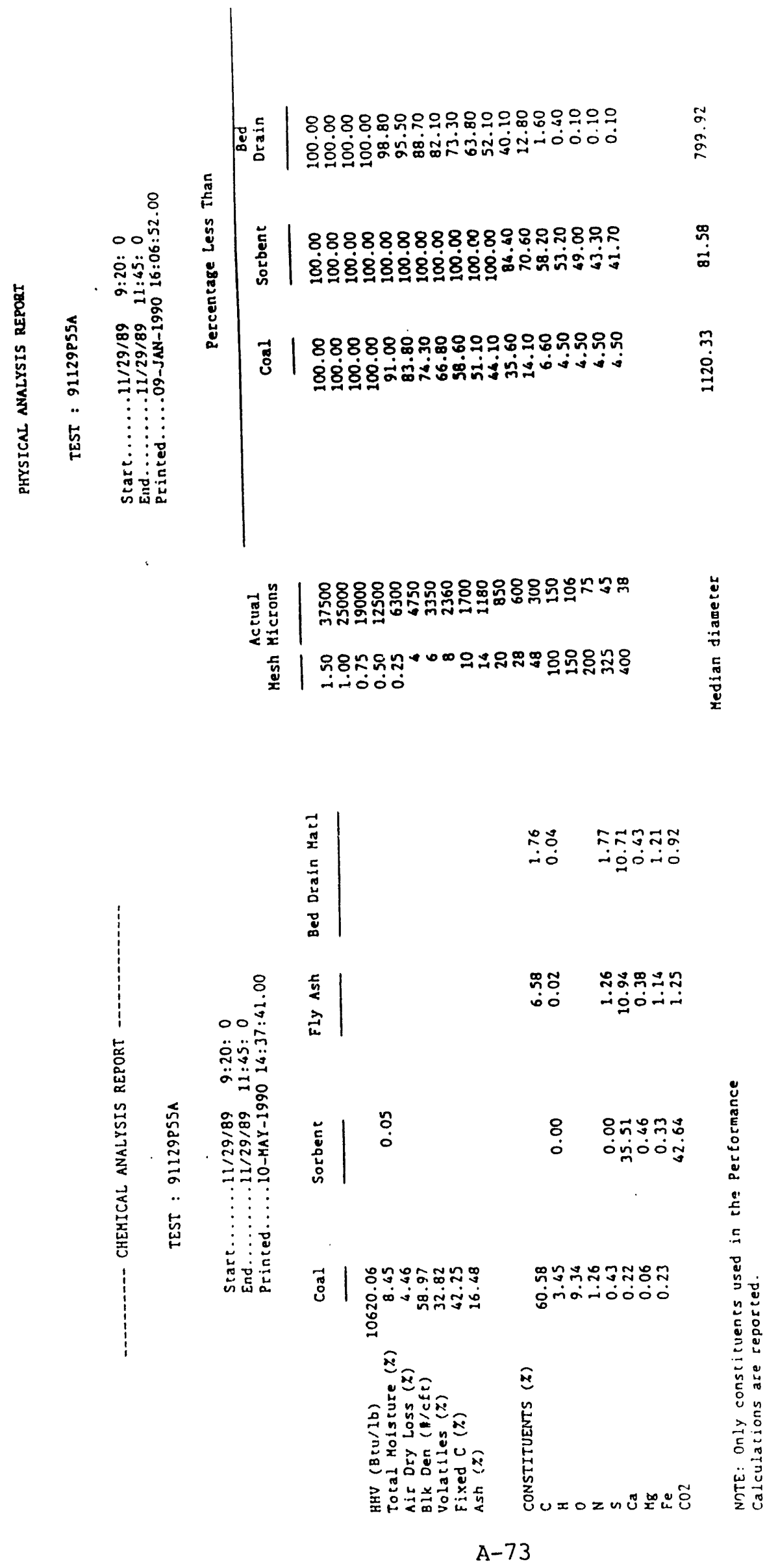



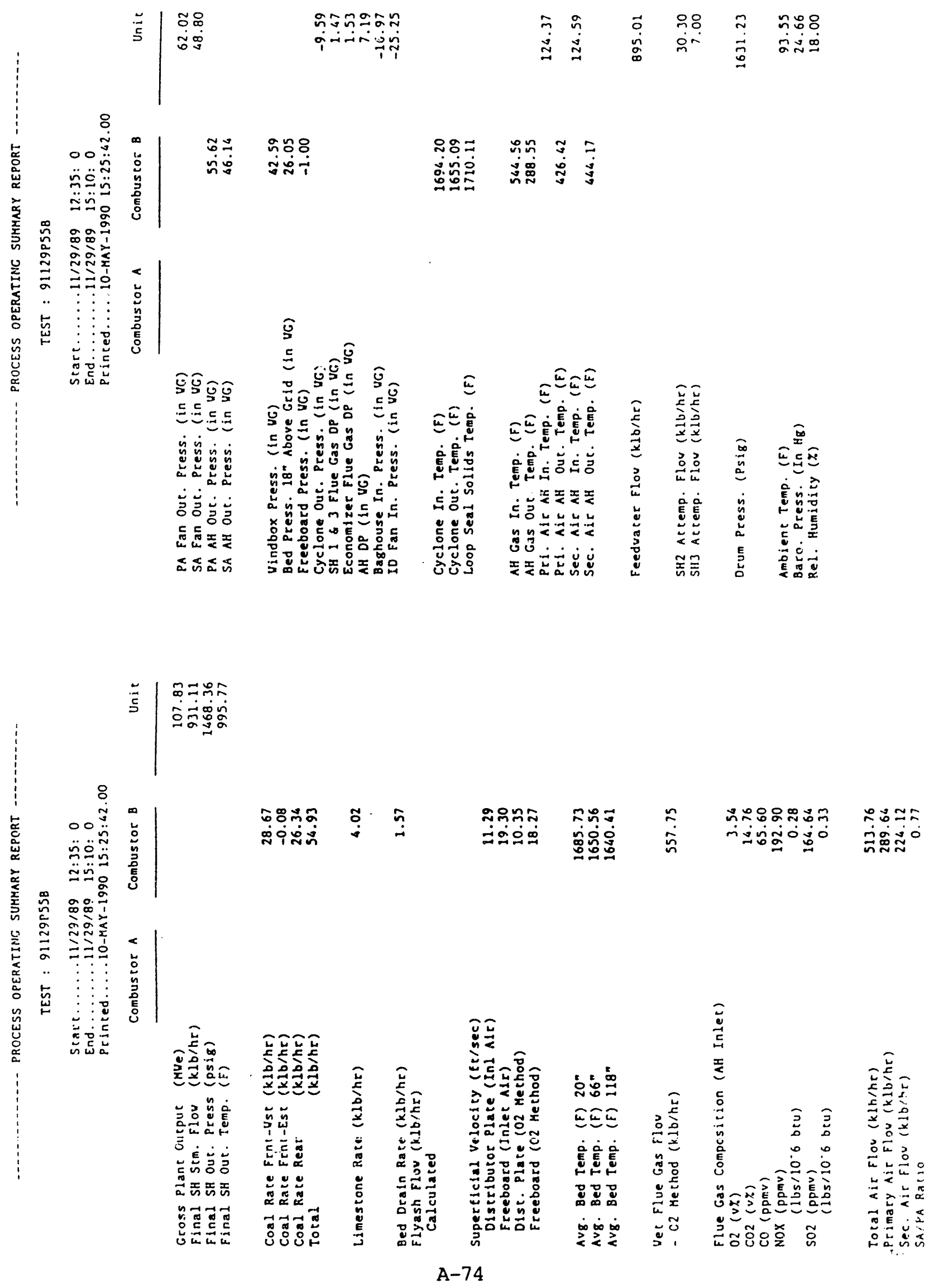


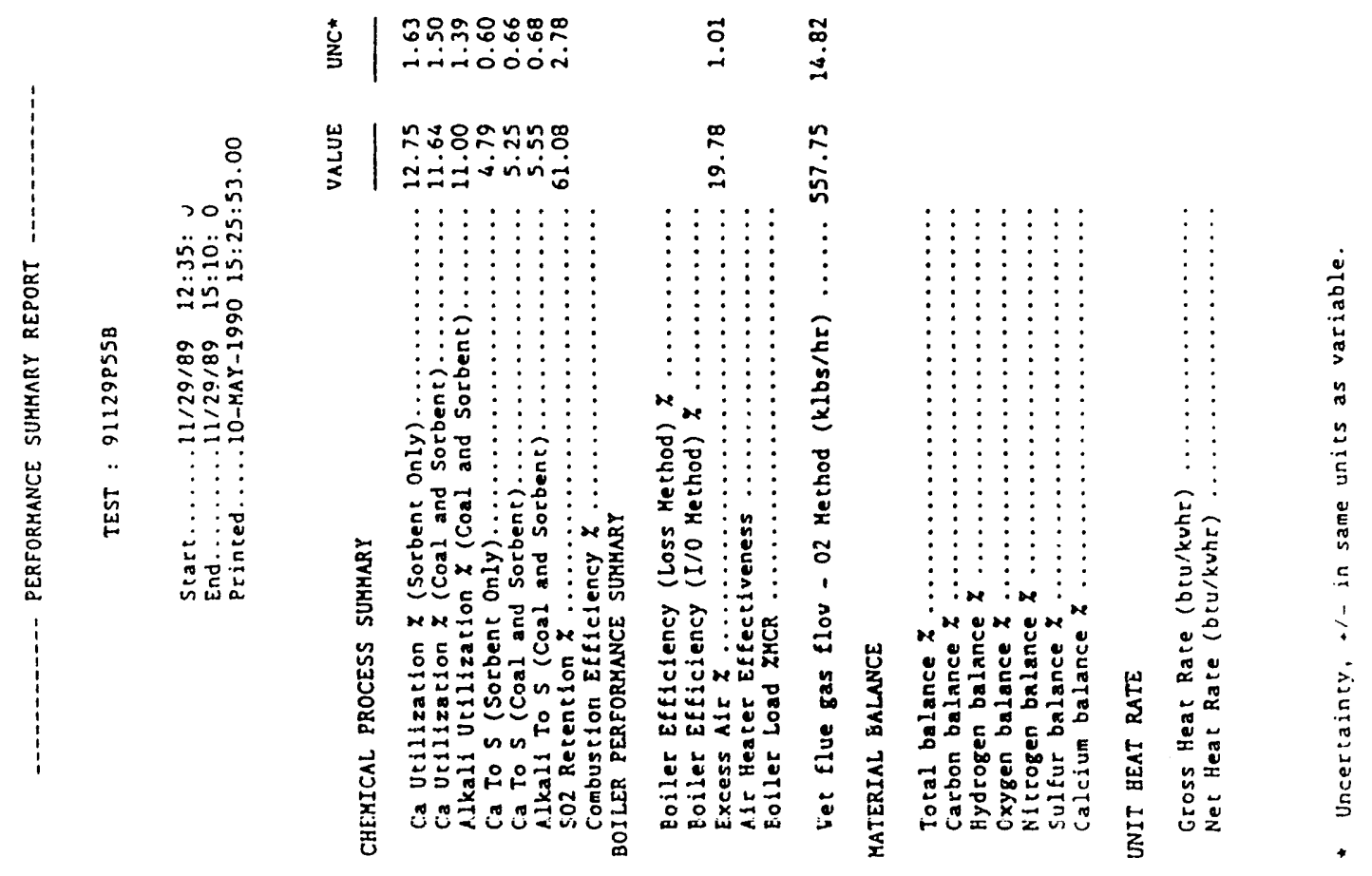

A -75 

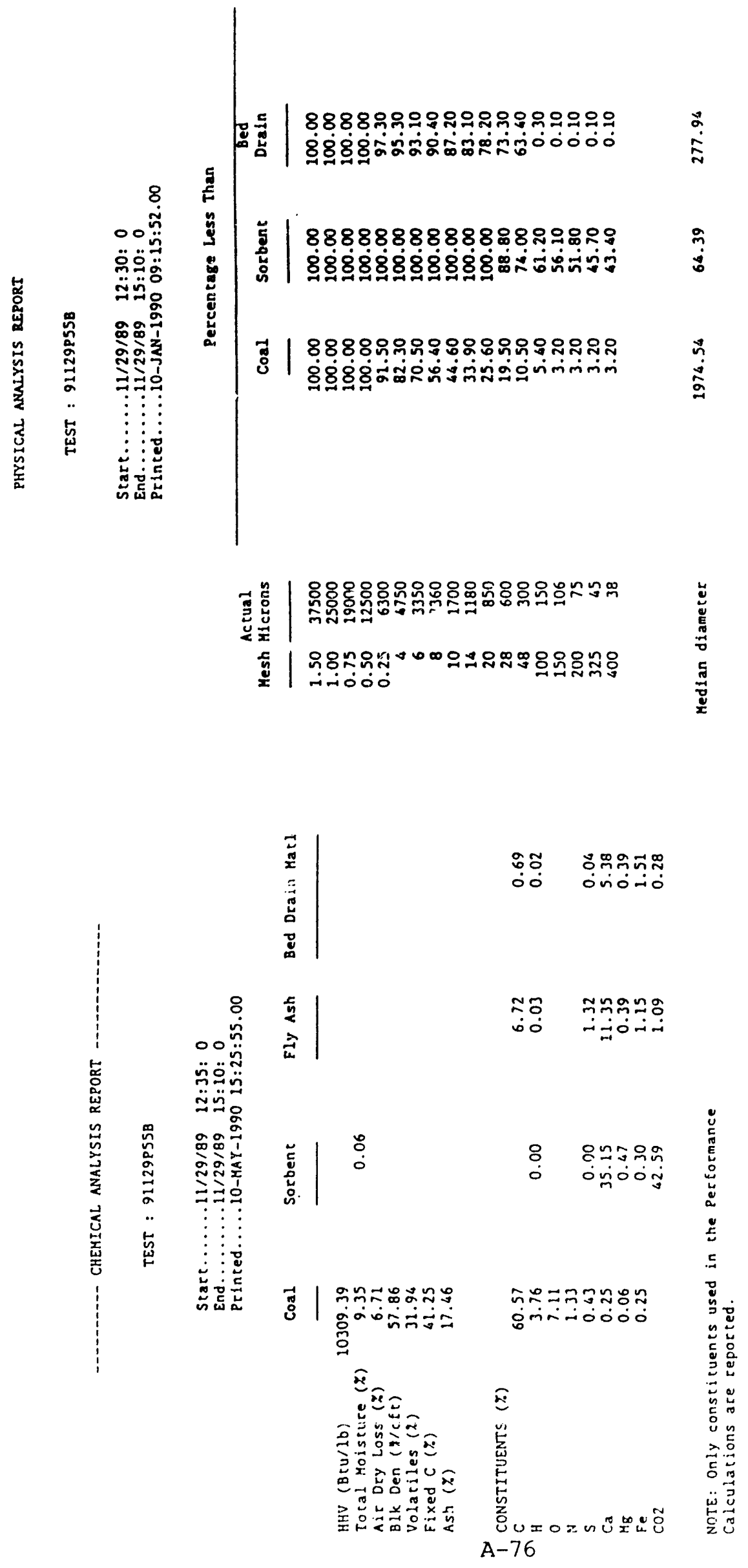

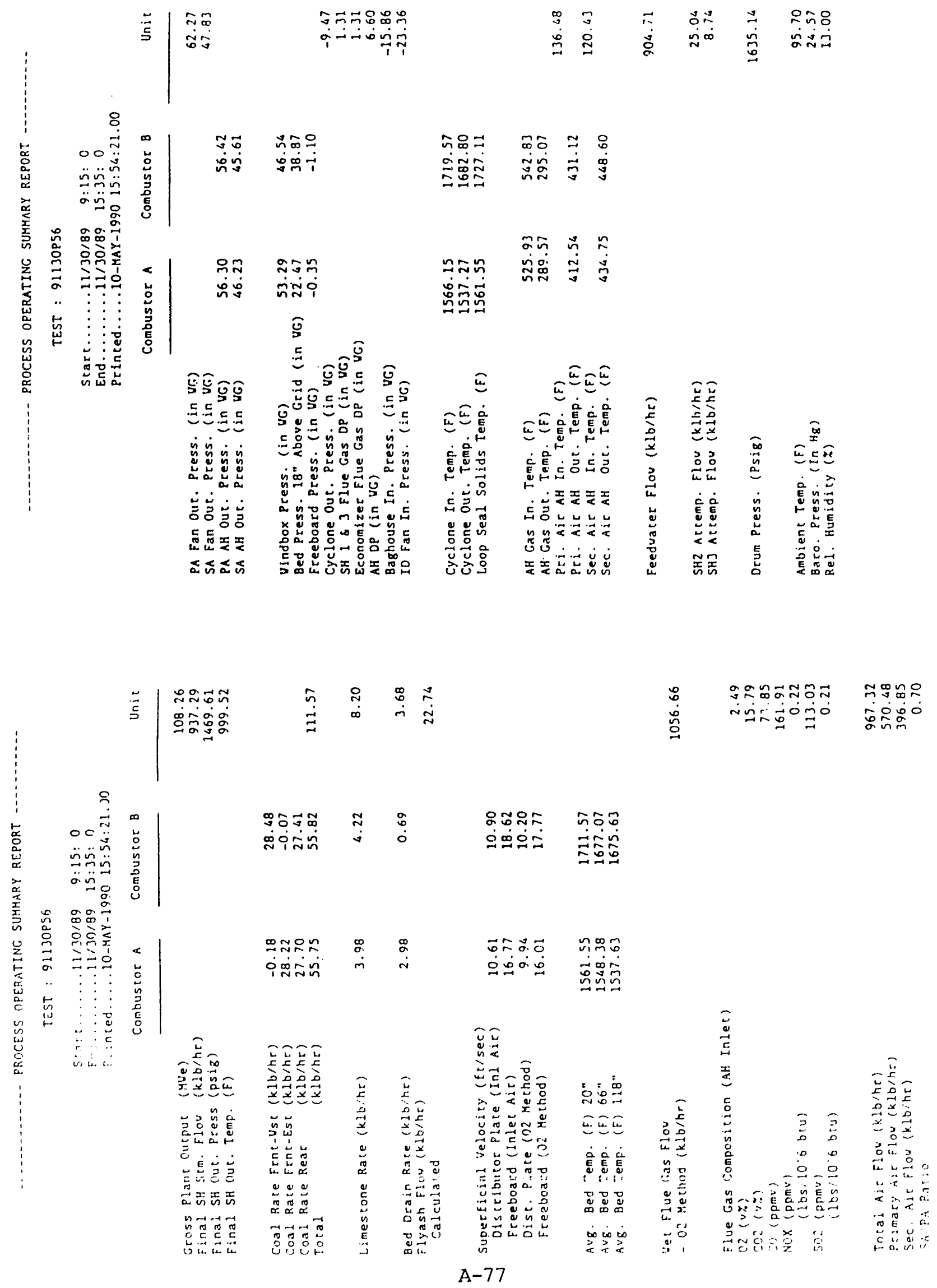


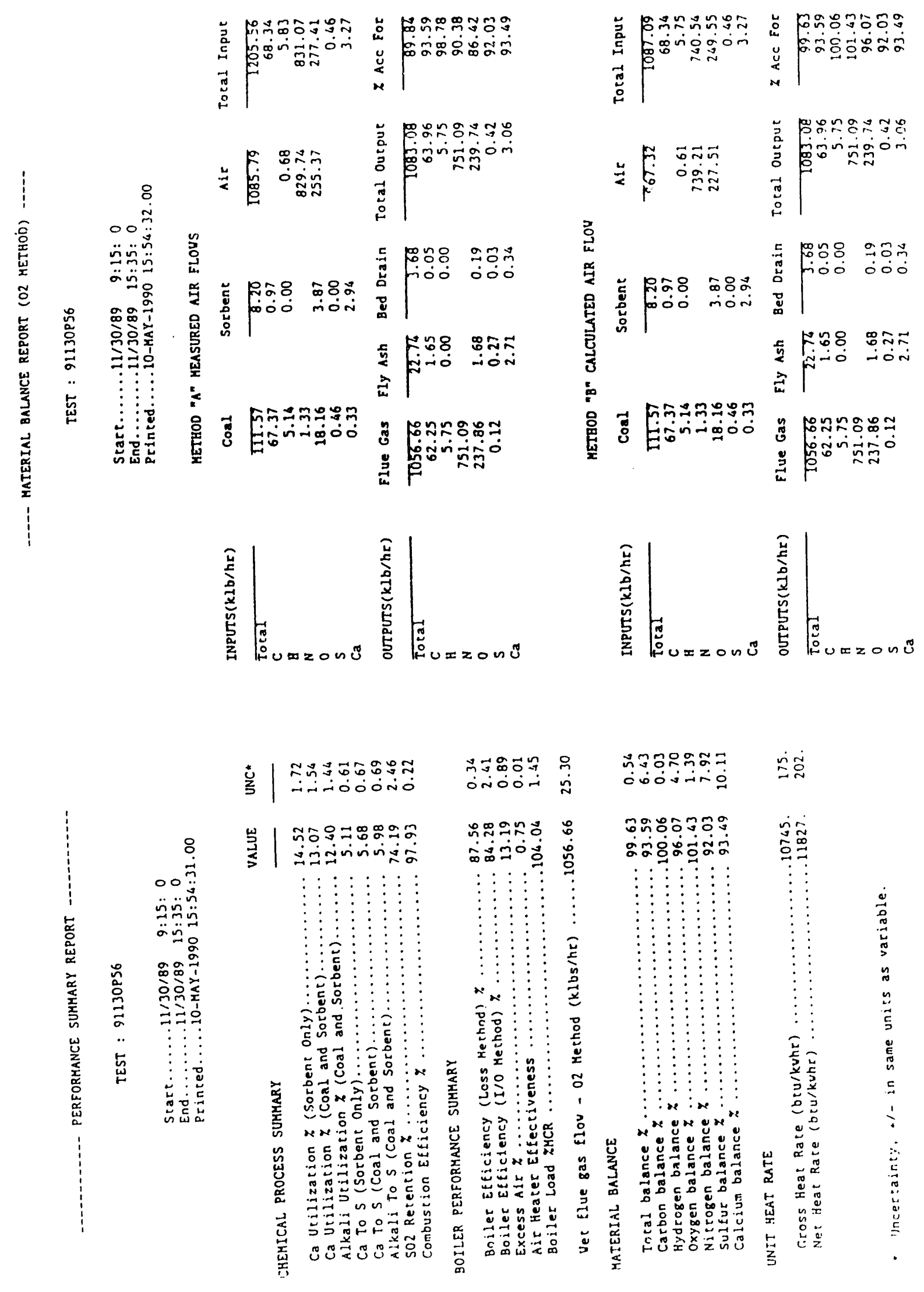



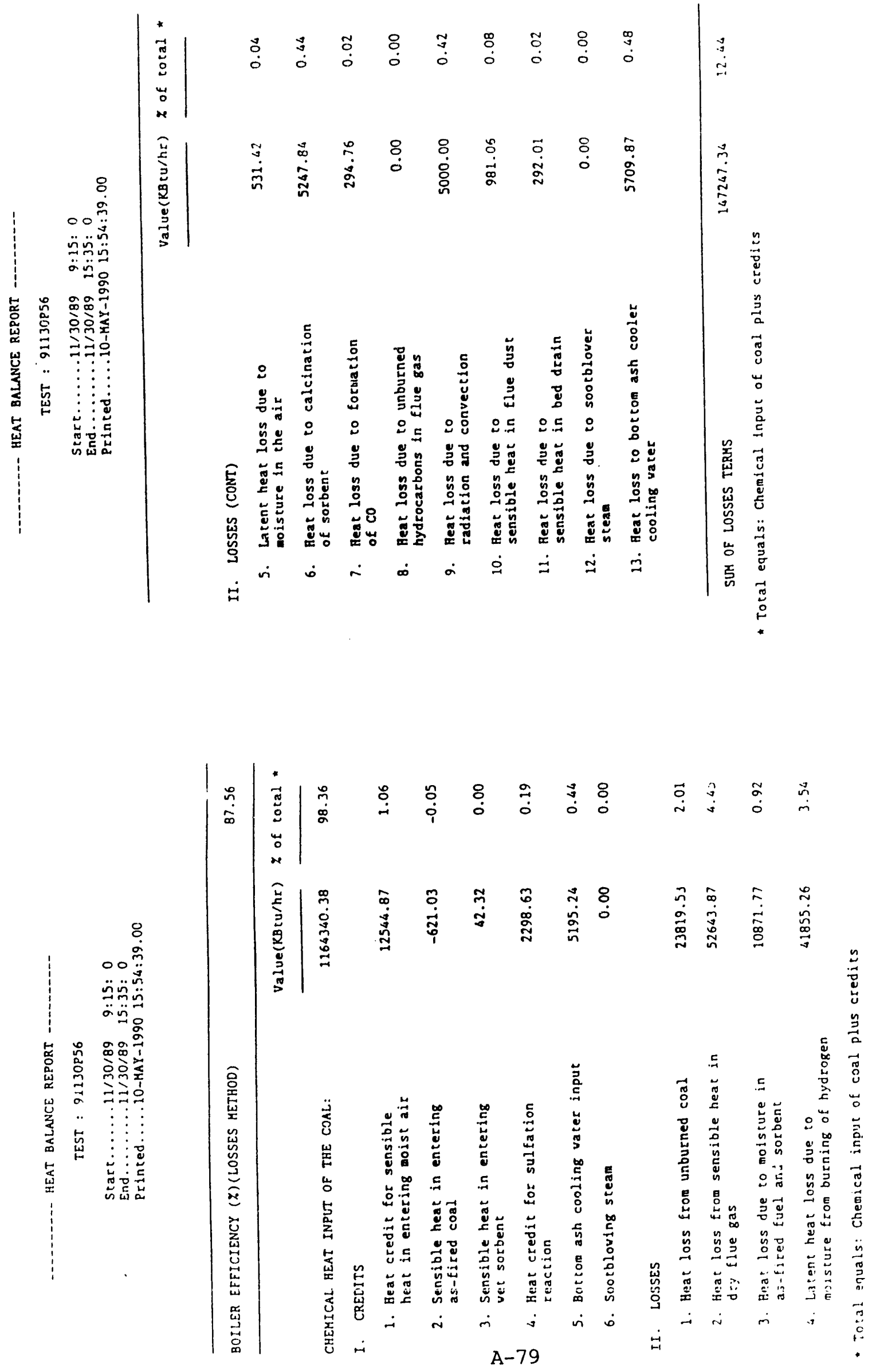

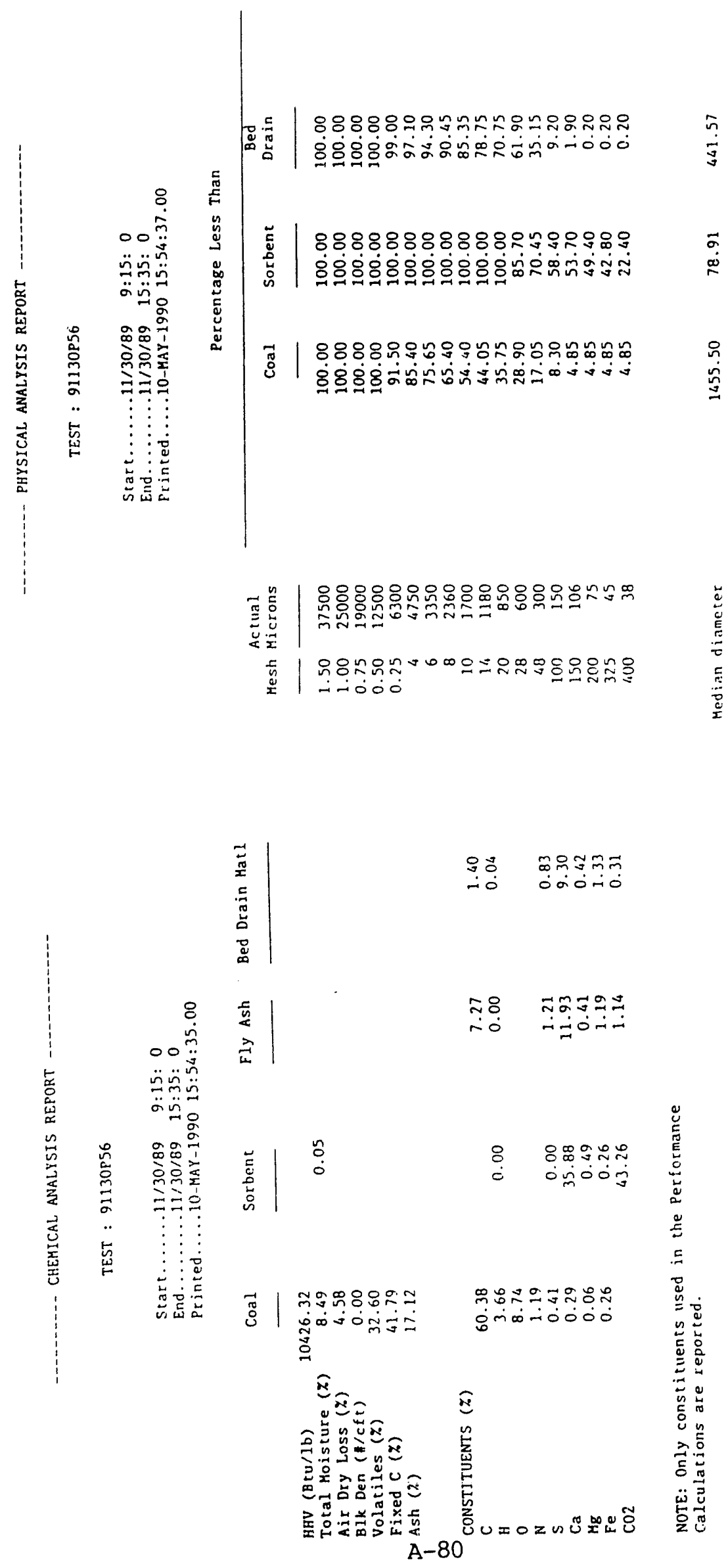

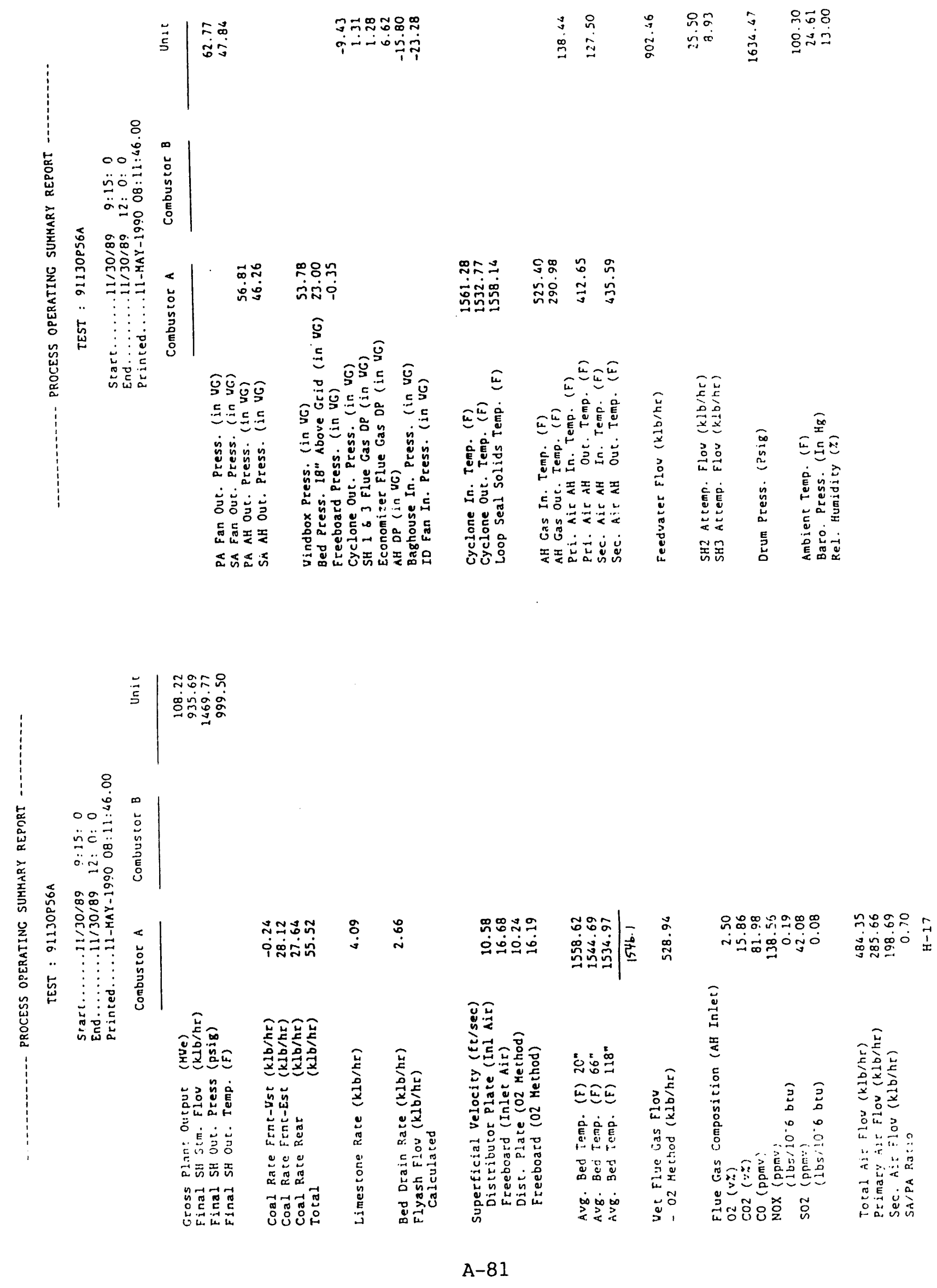


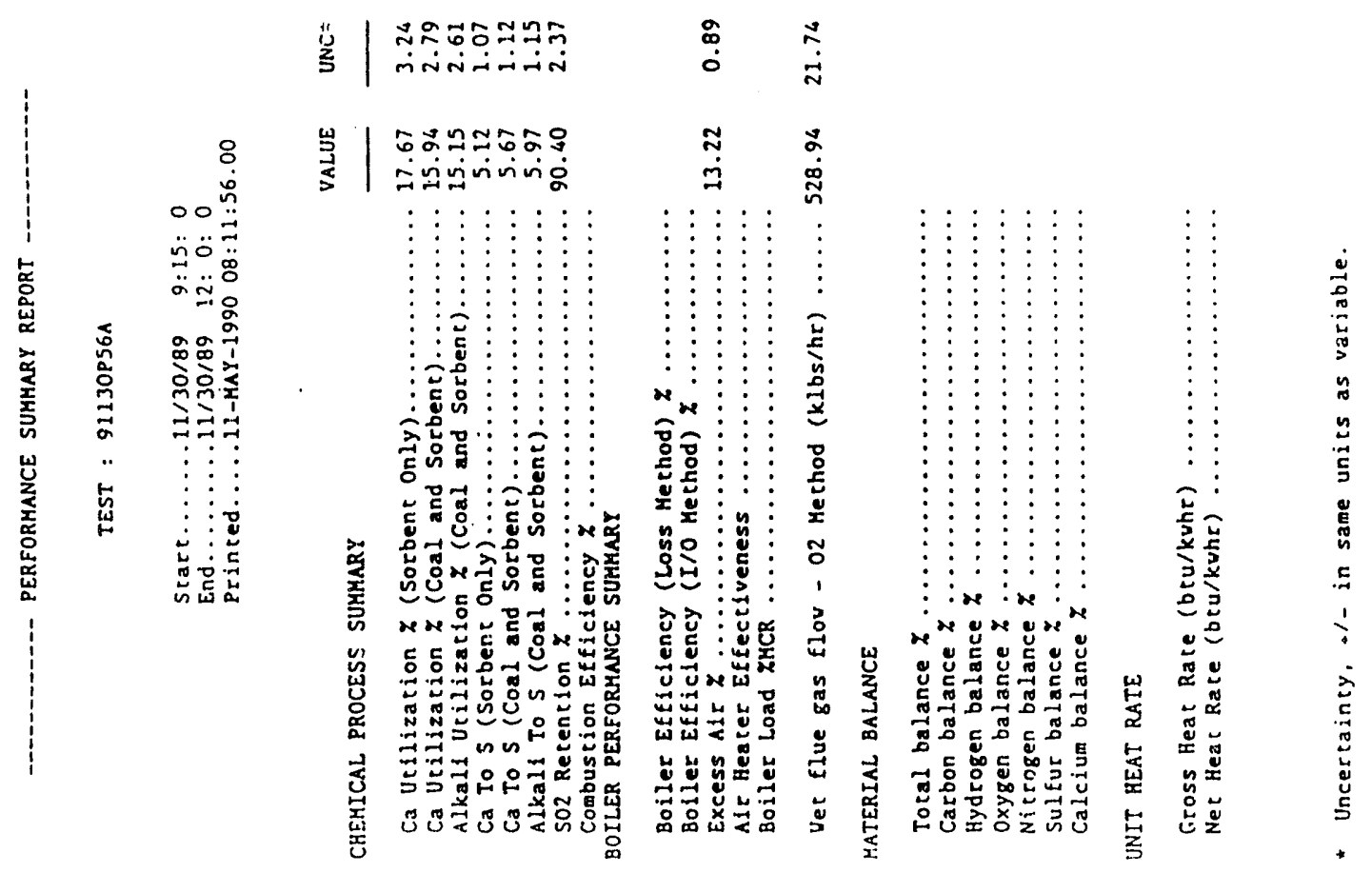

A-82 


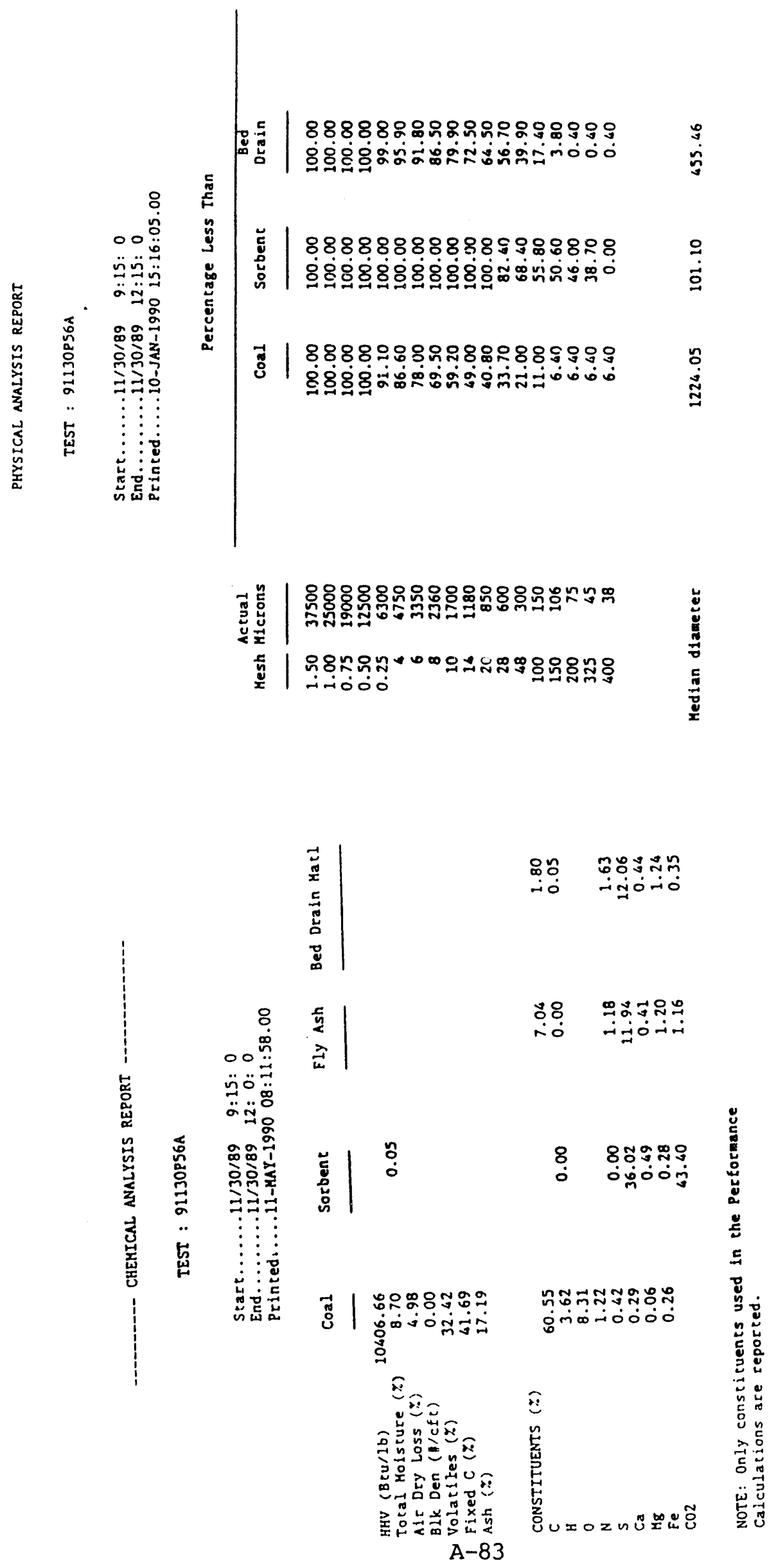



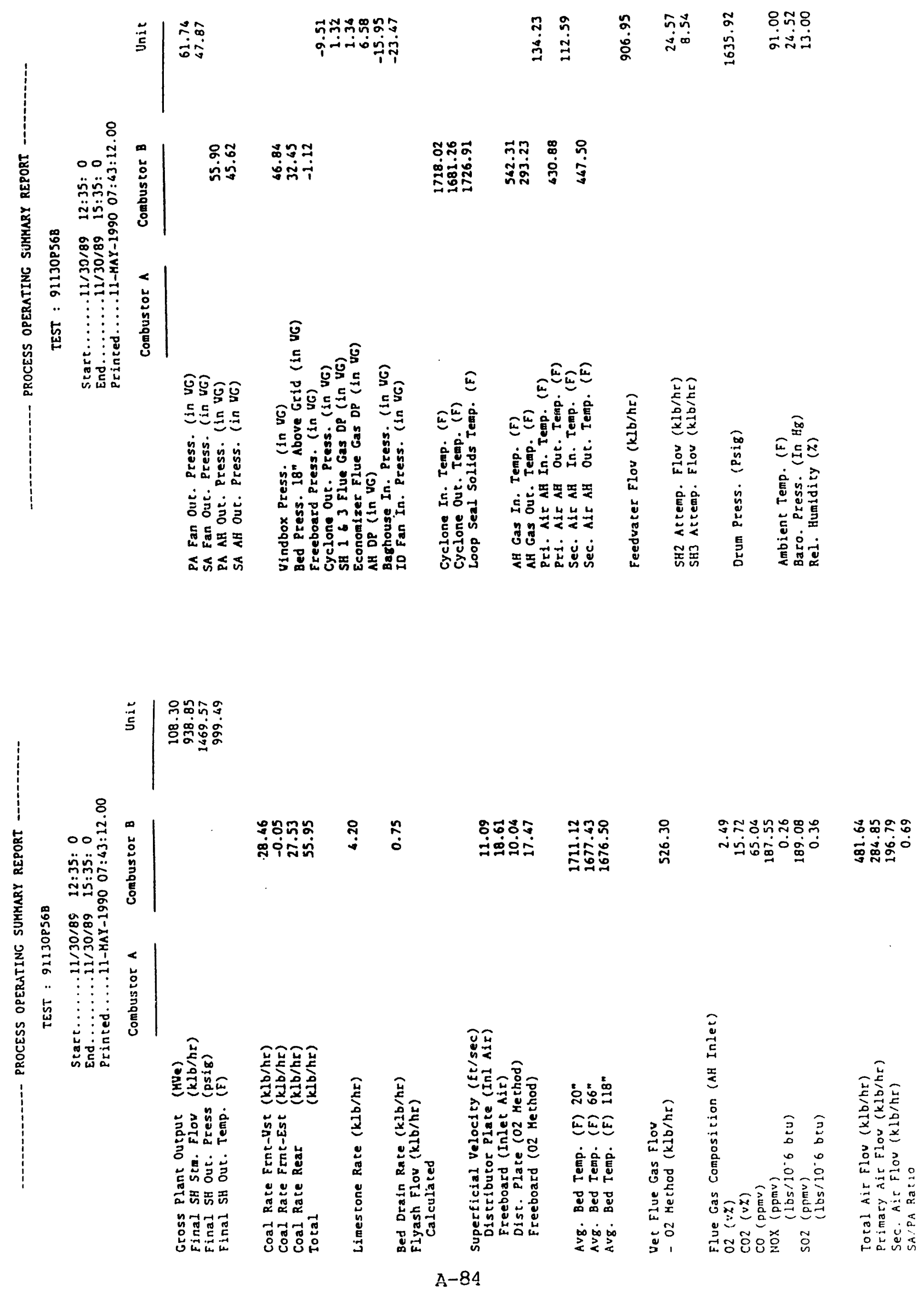


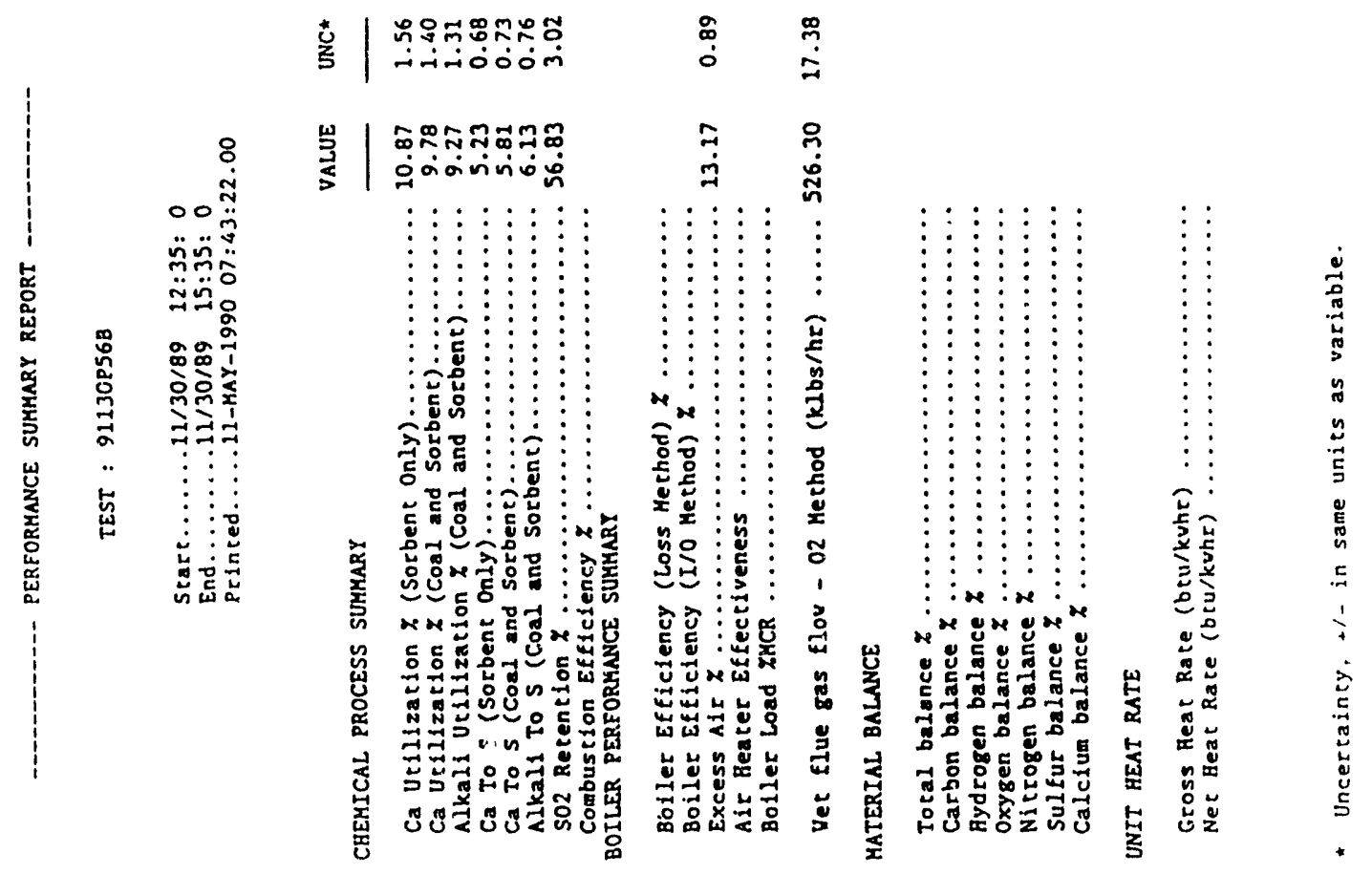

A-85 


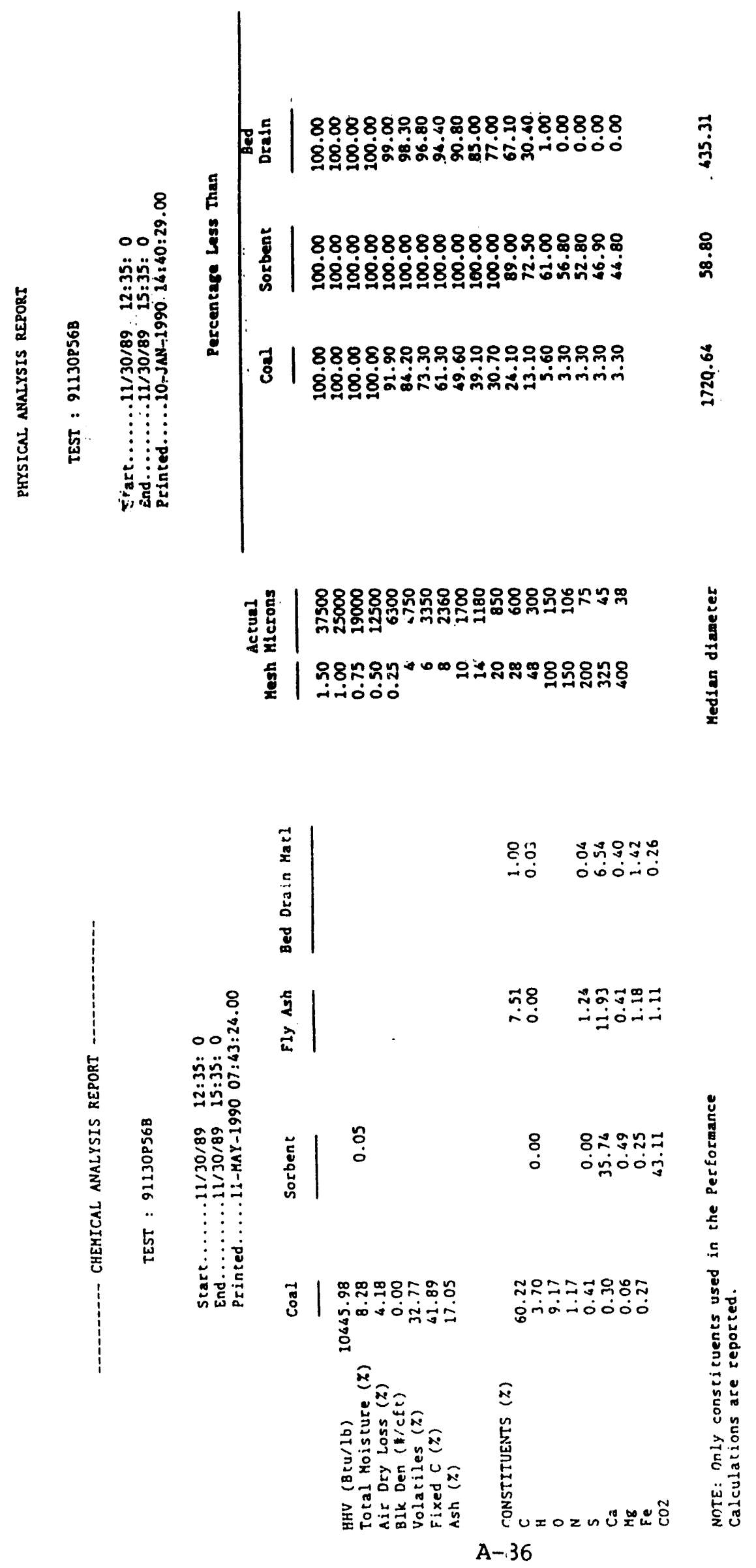



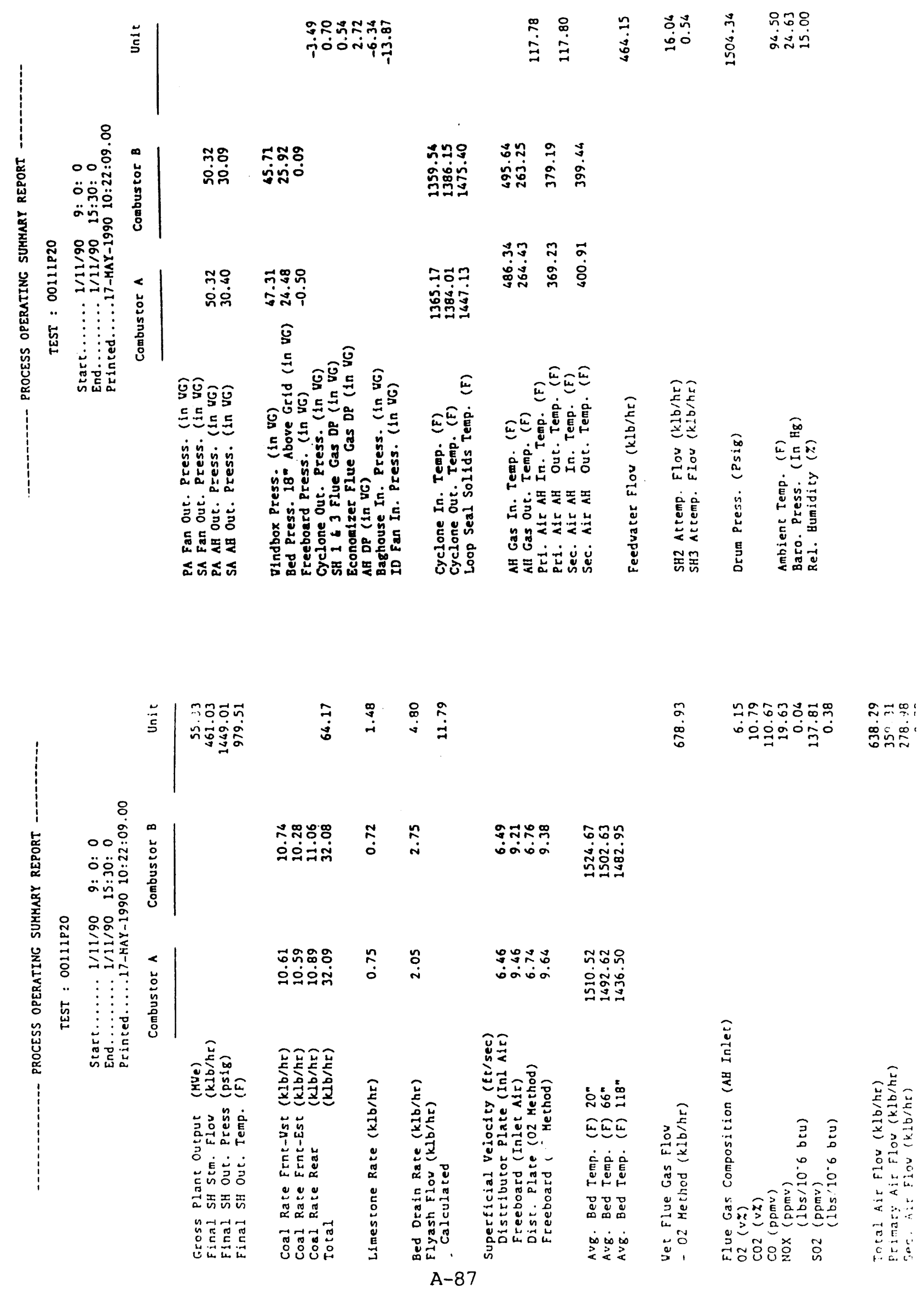


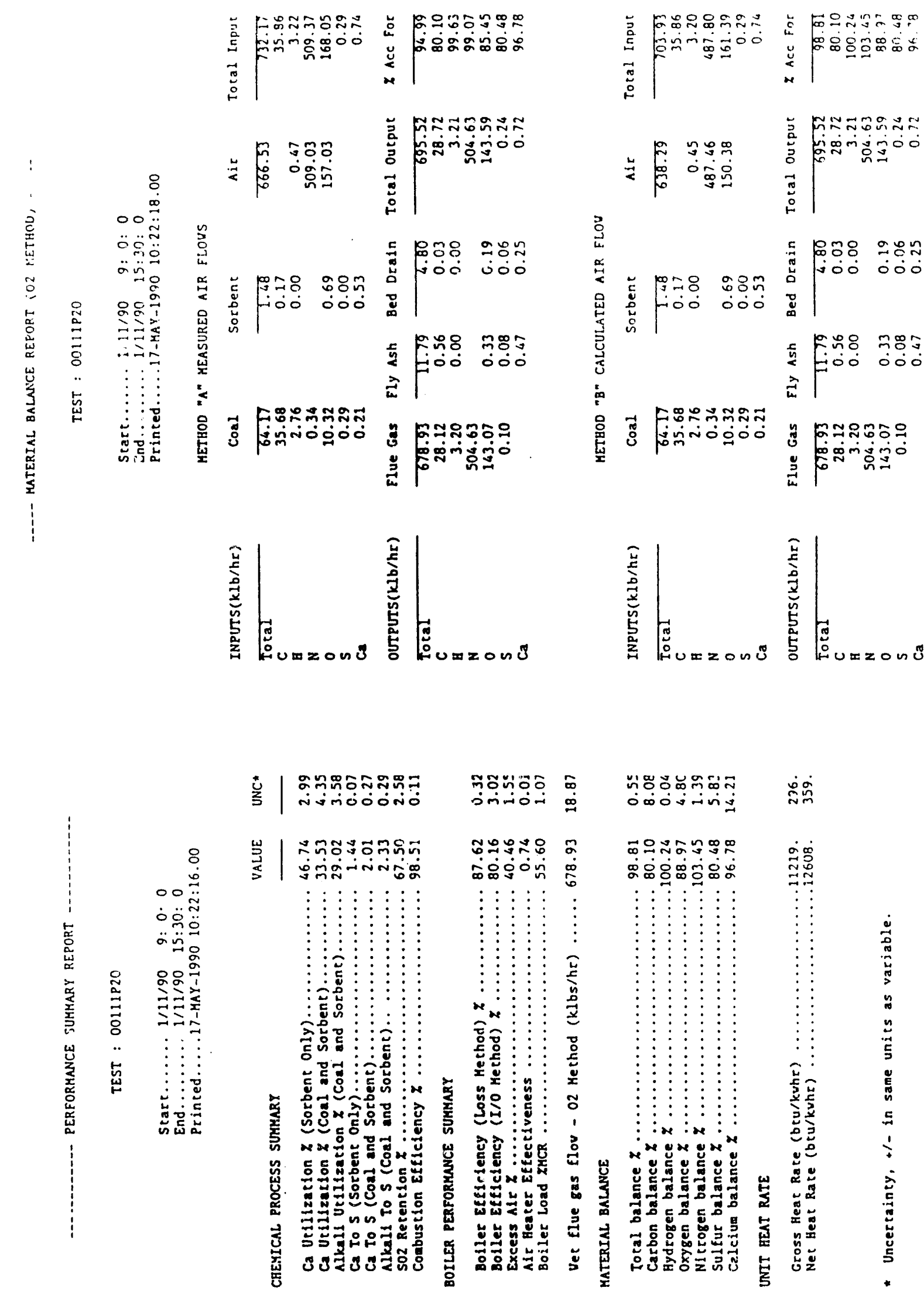



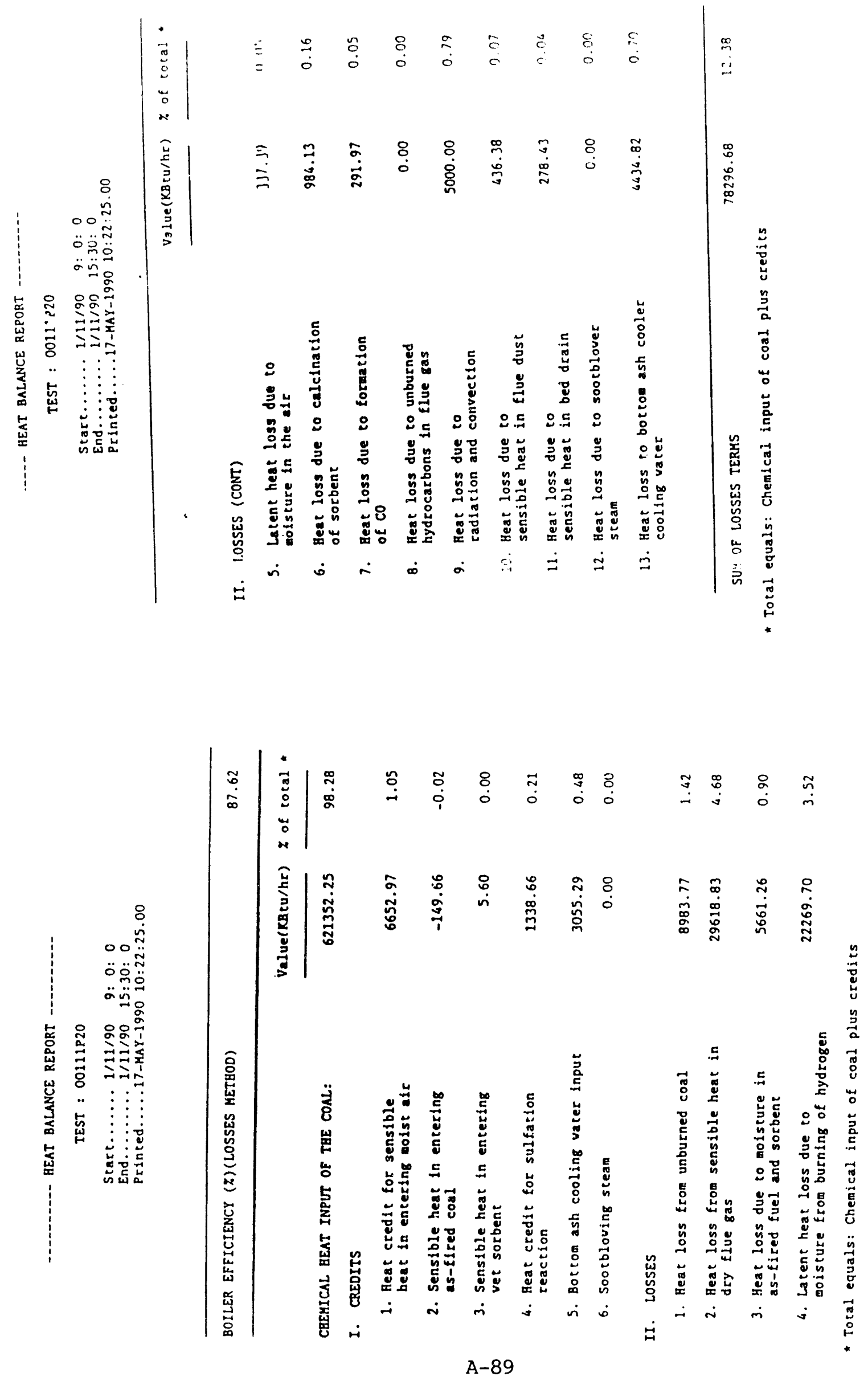

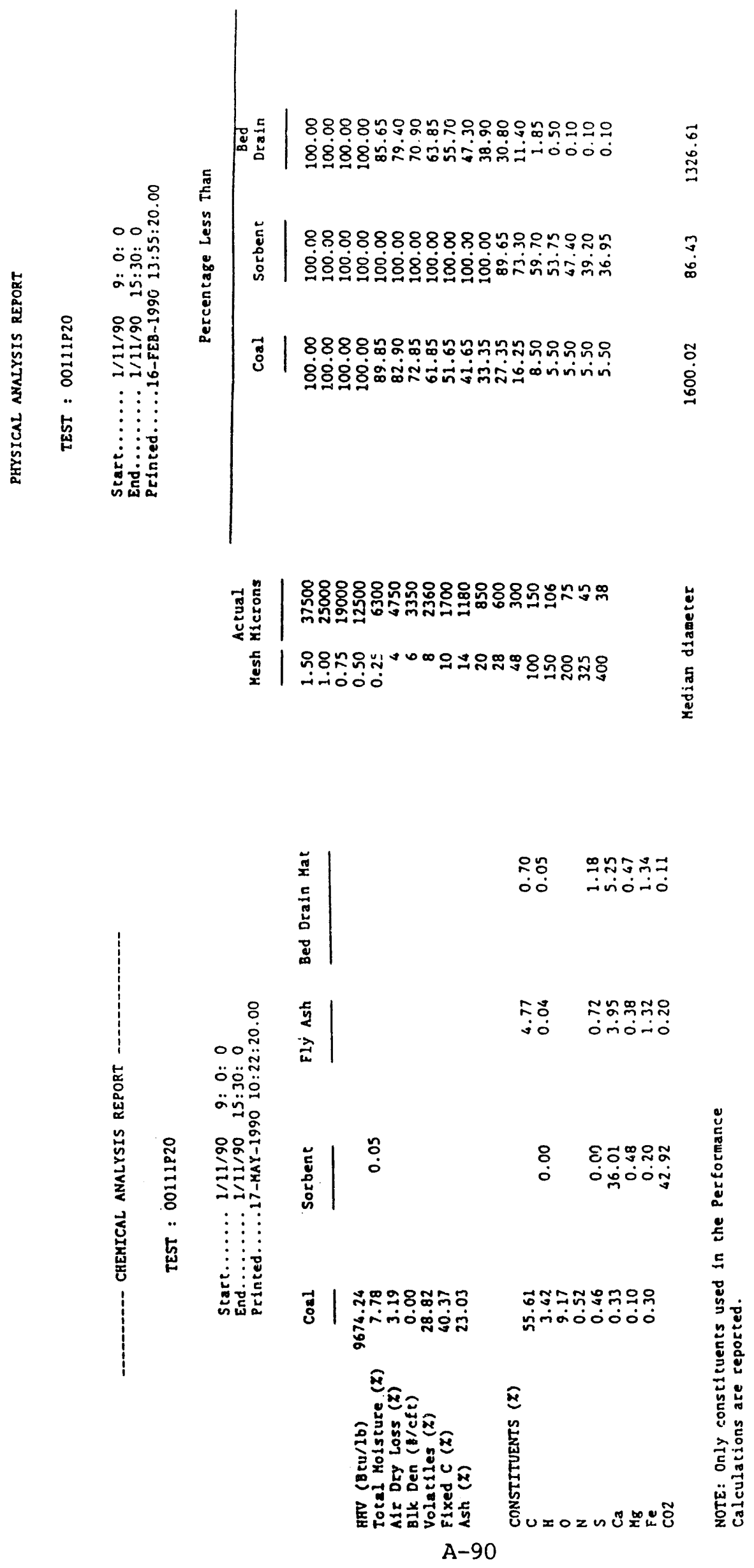

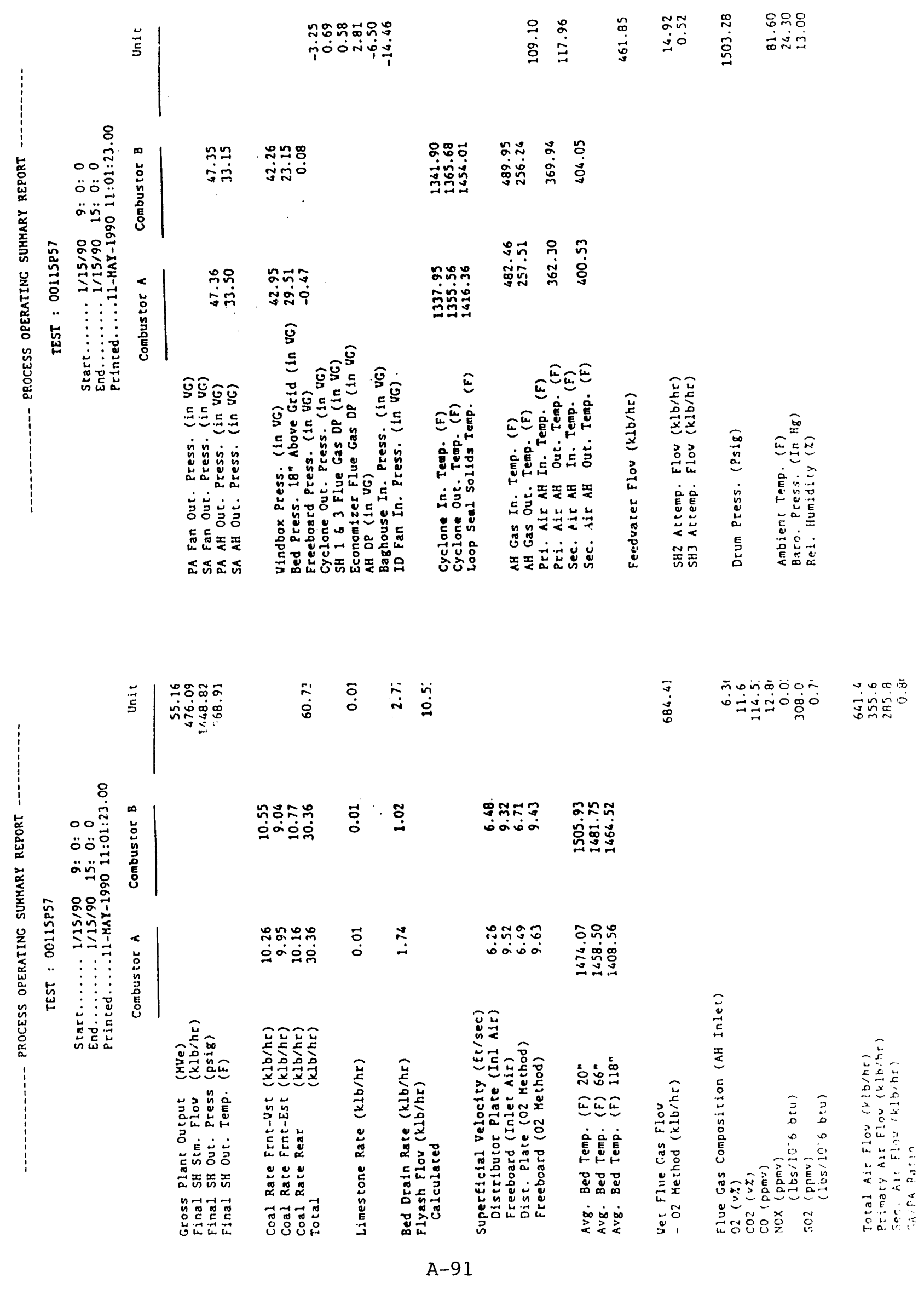


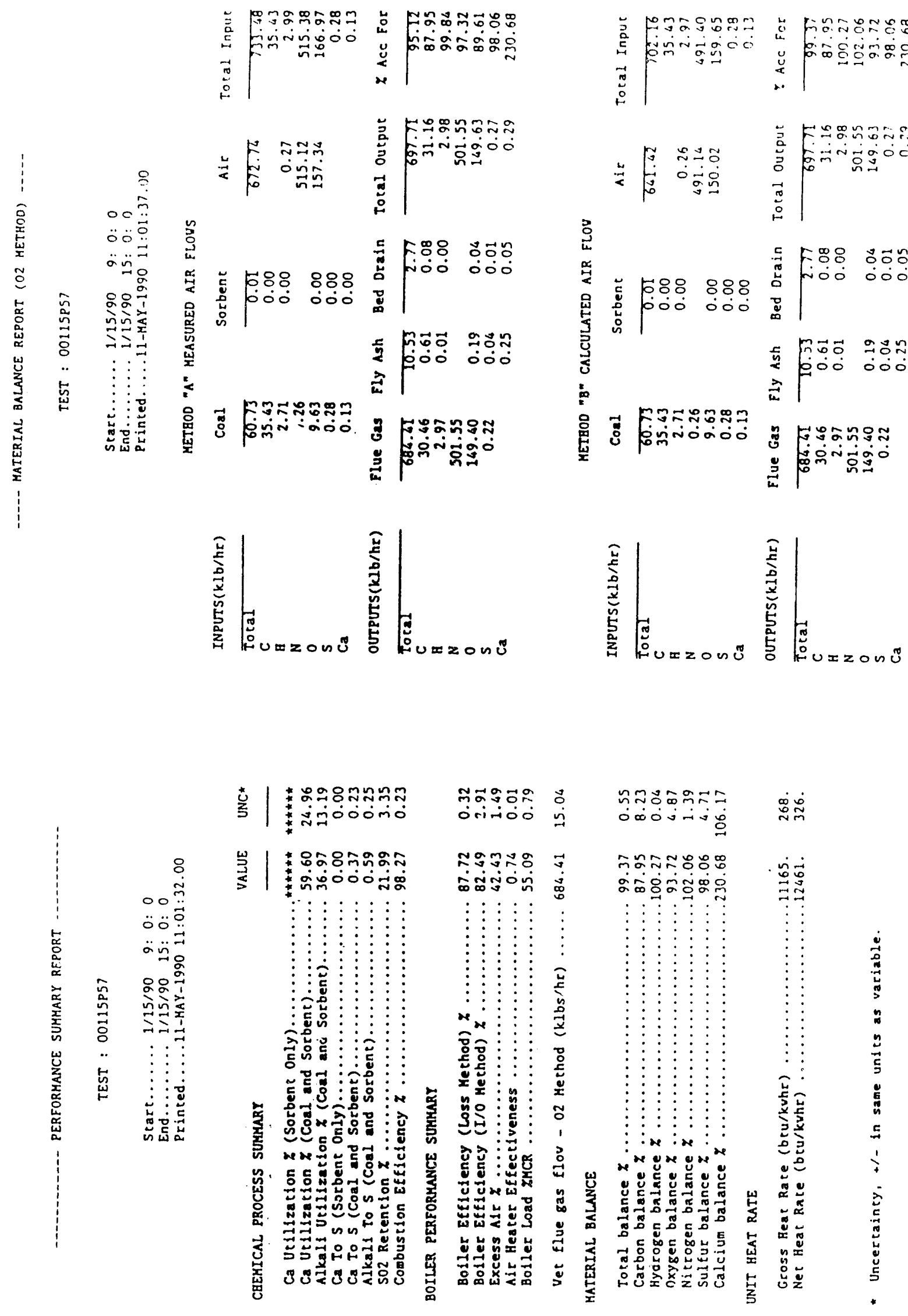



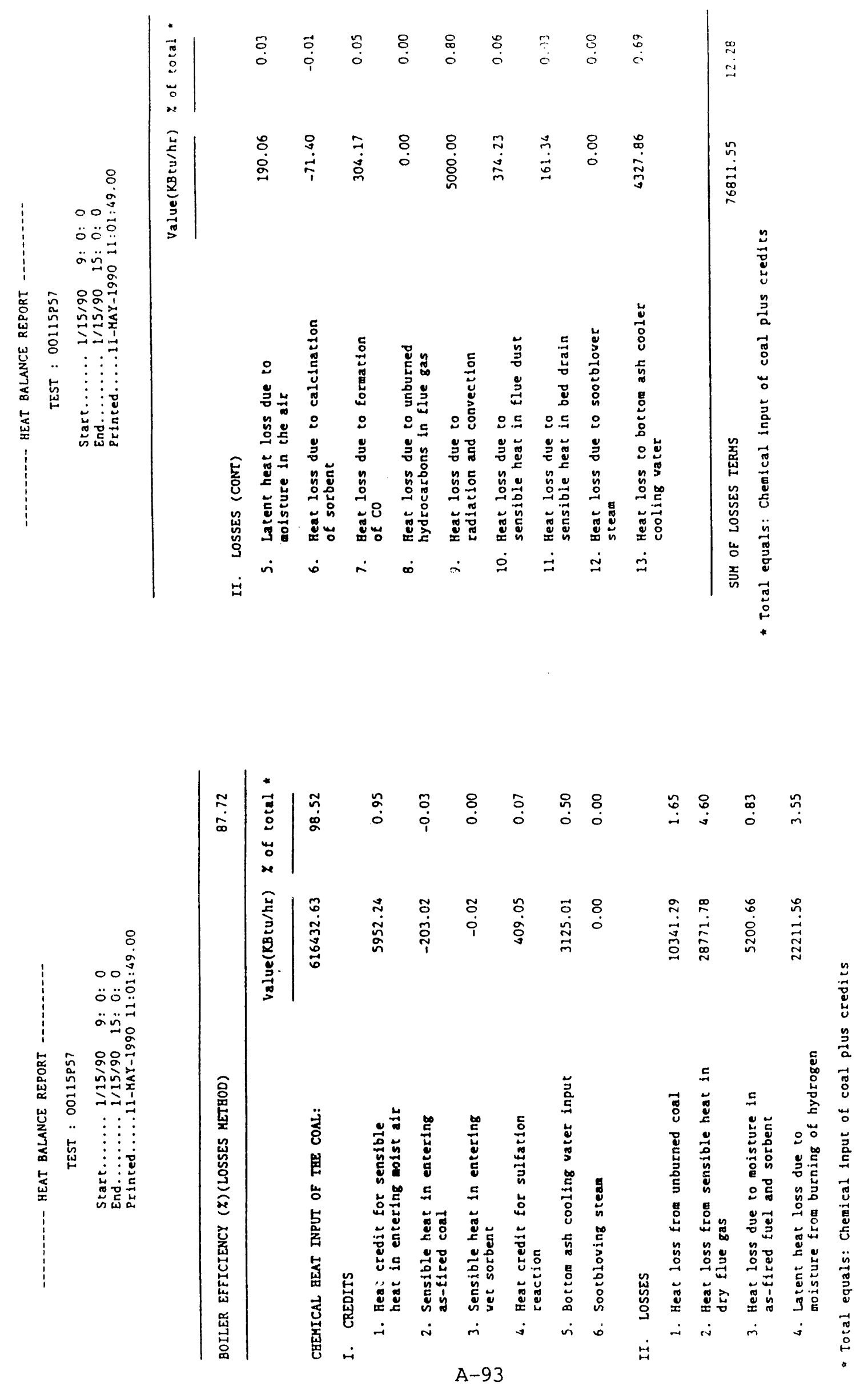


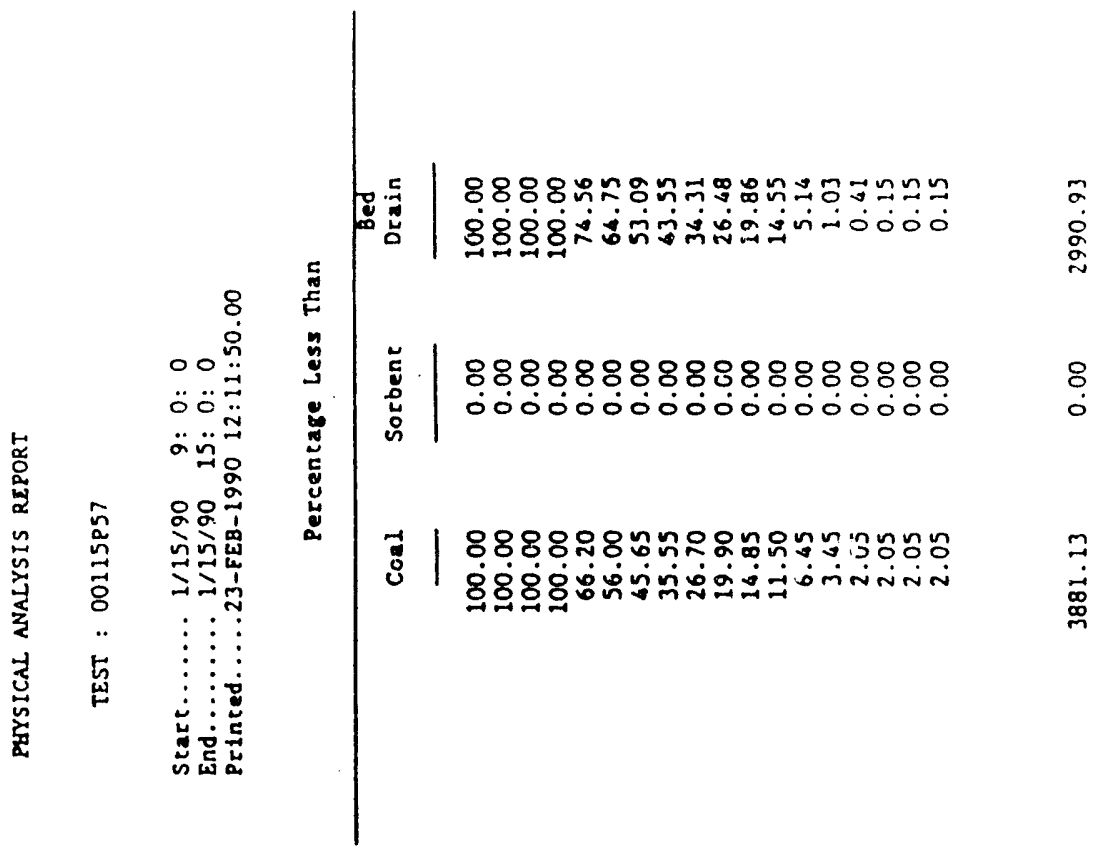

8

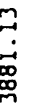
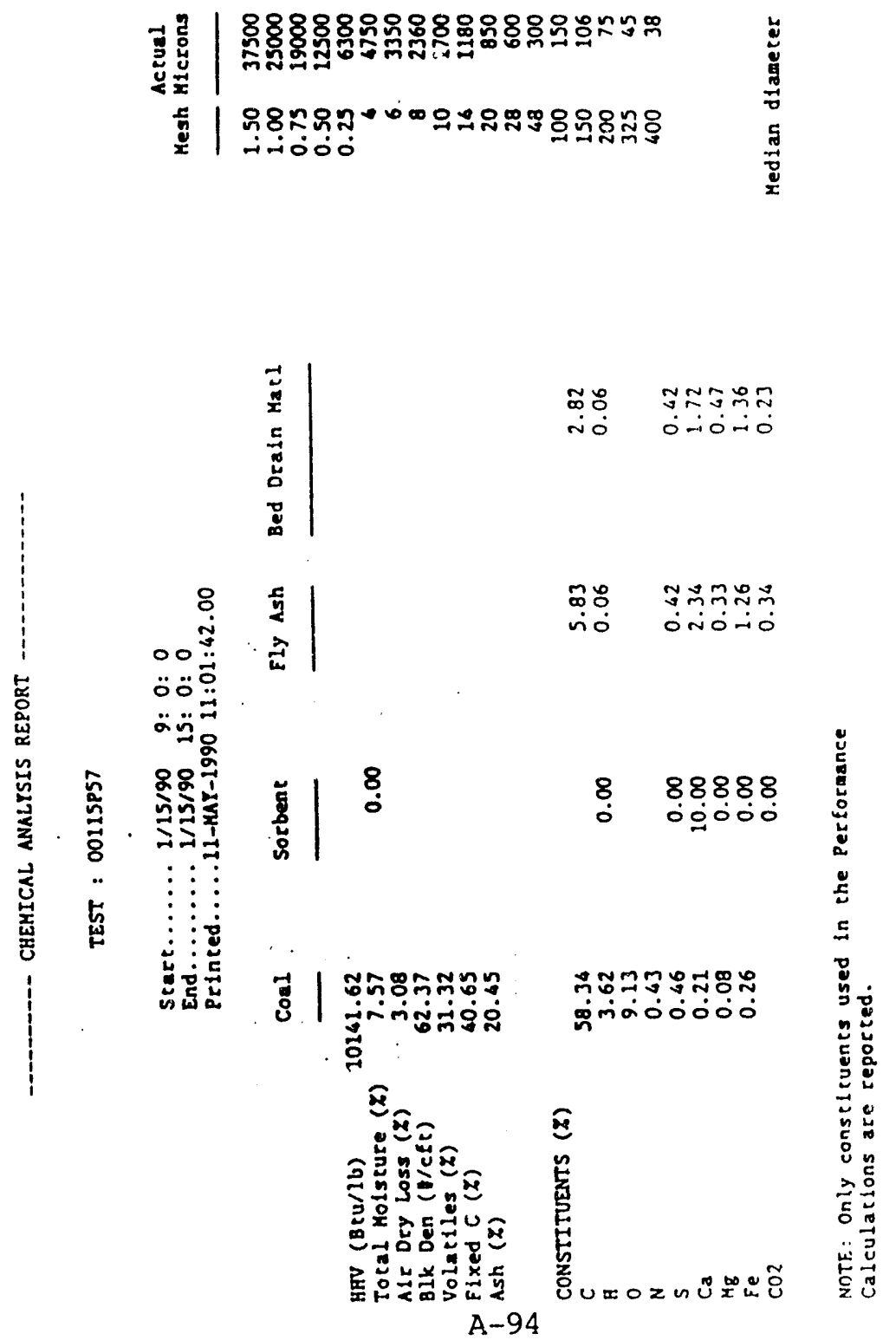


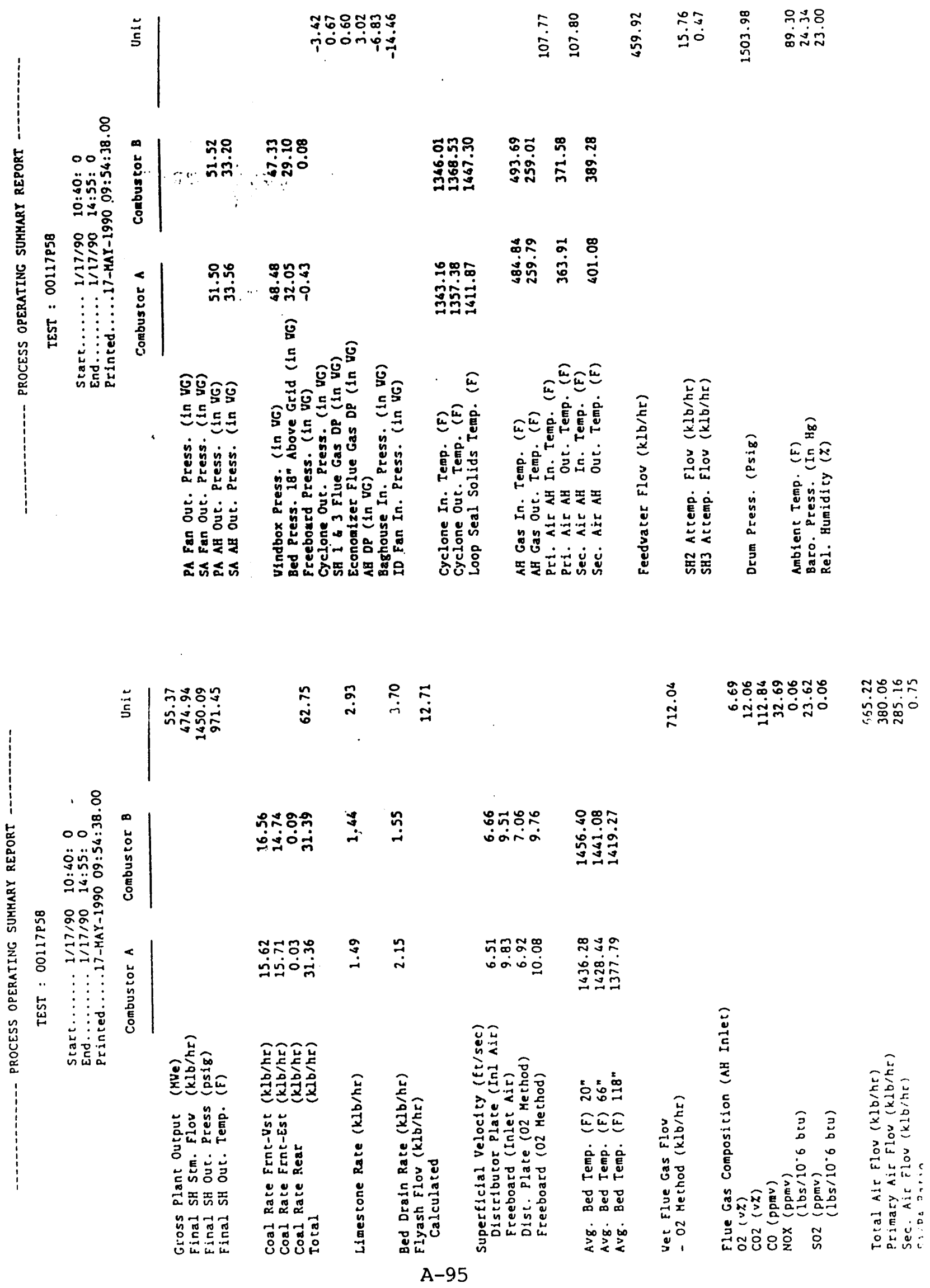




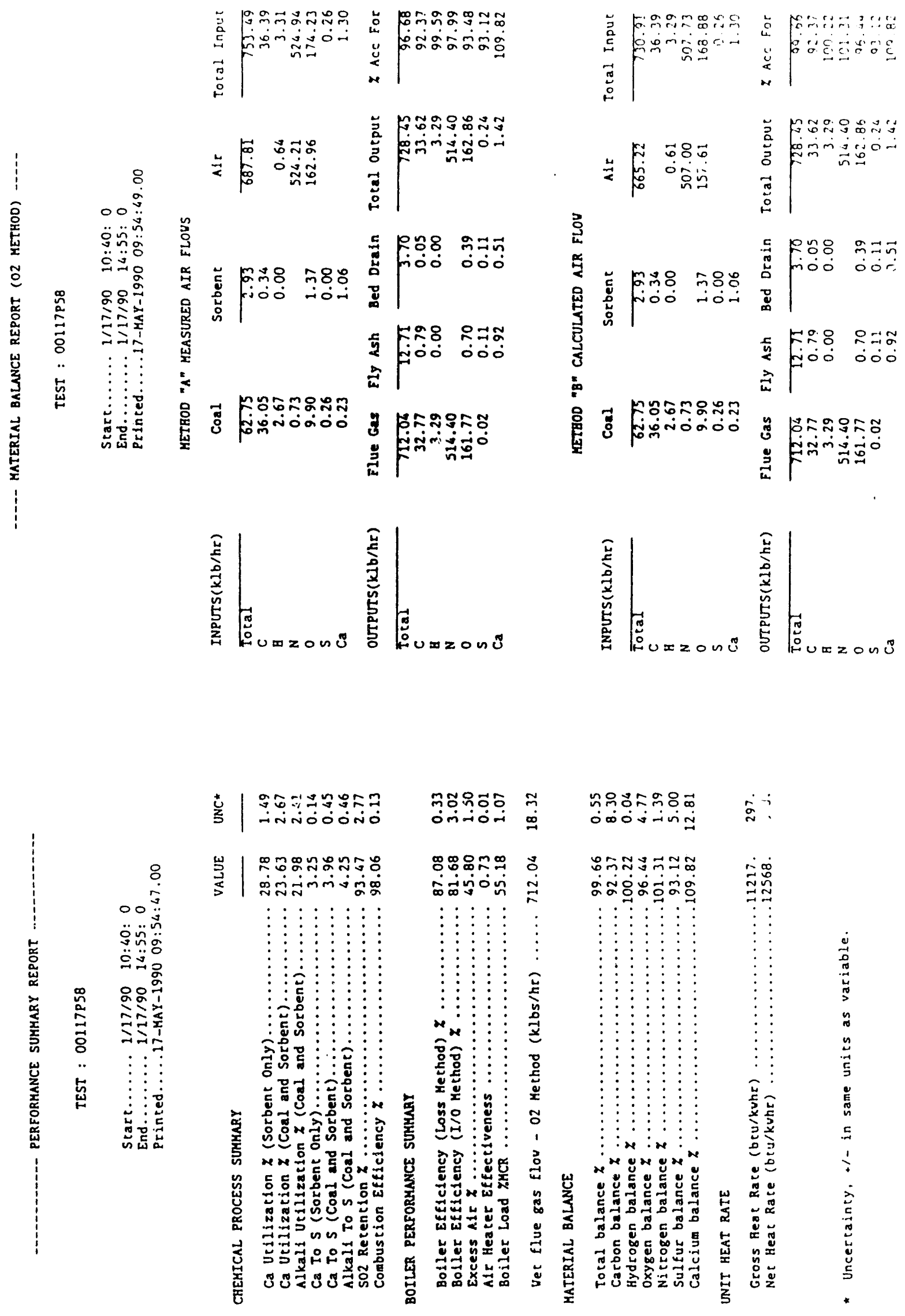



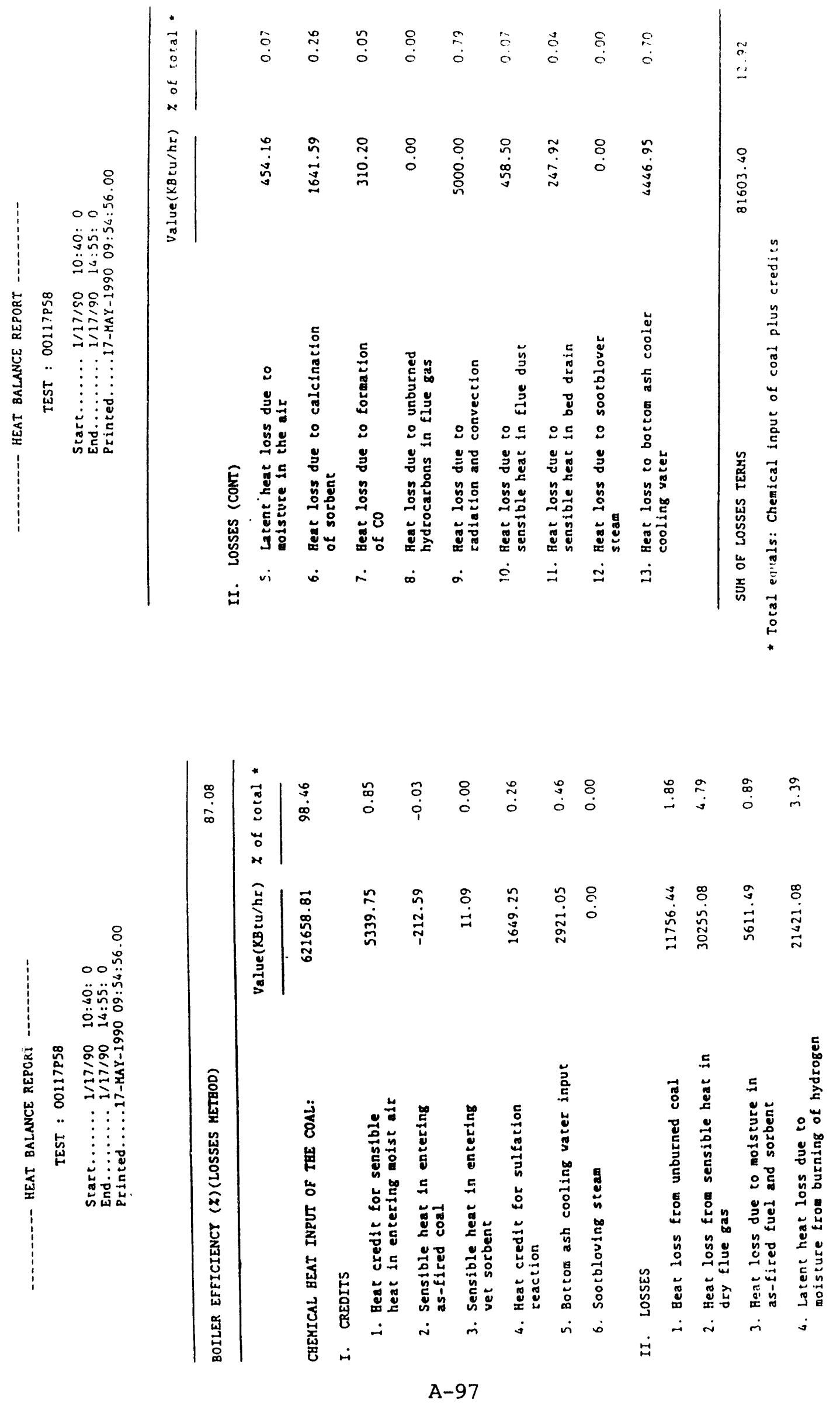


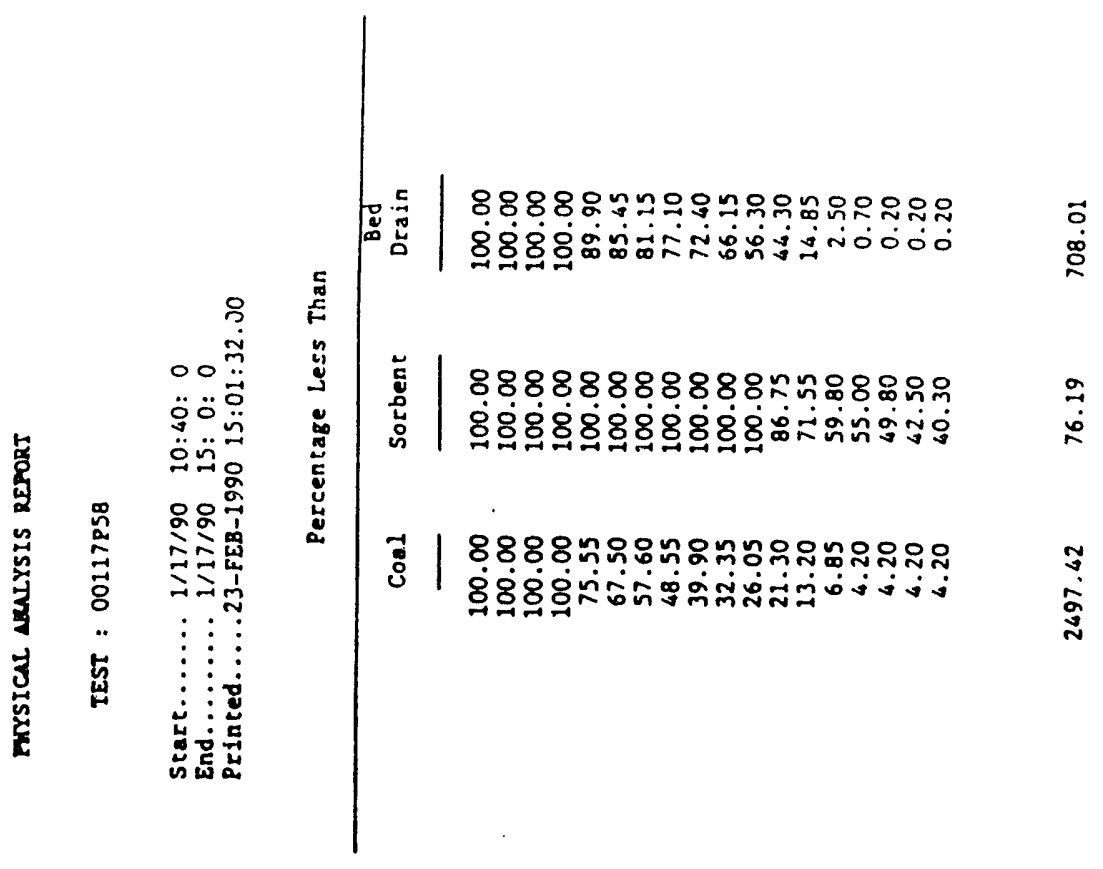

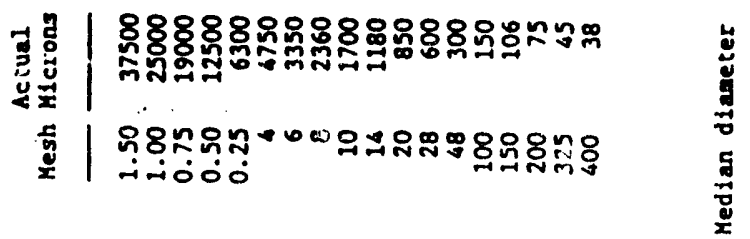

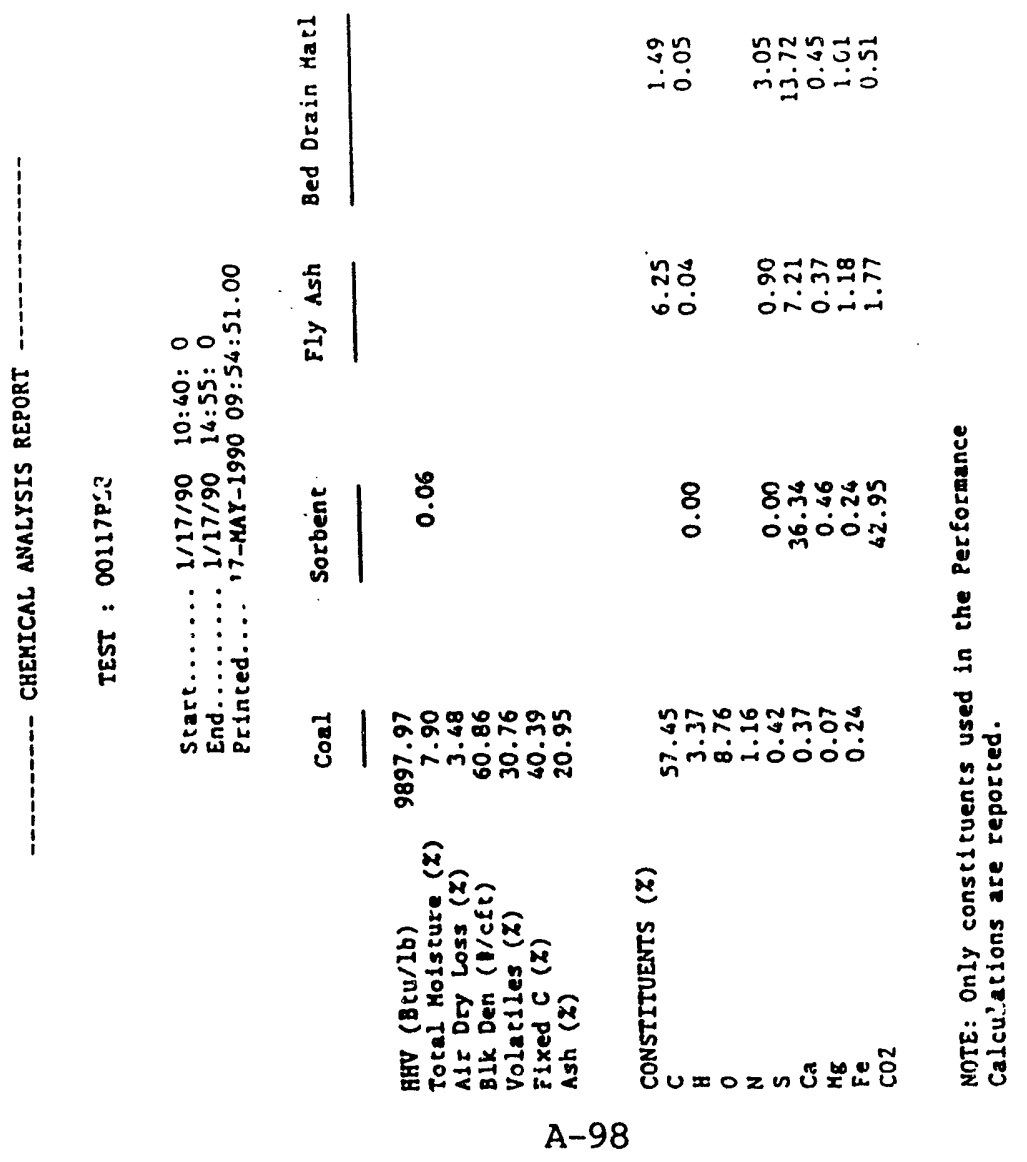



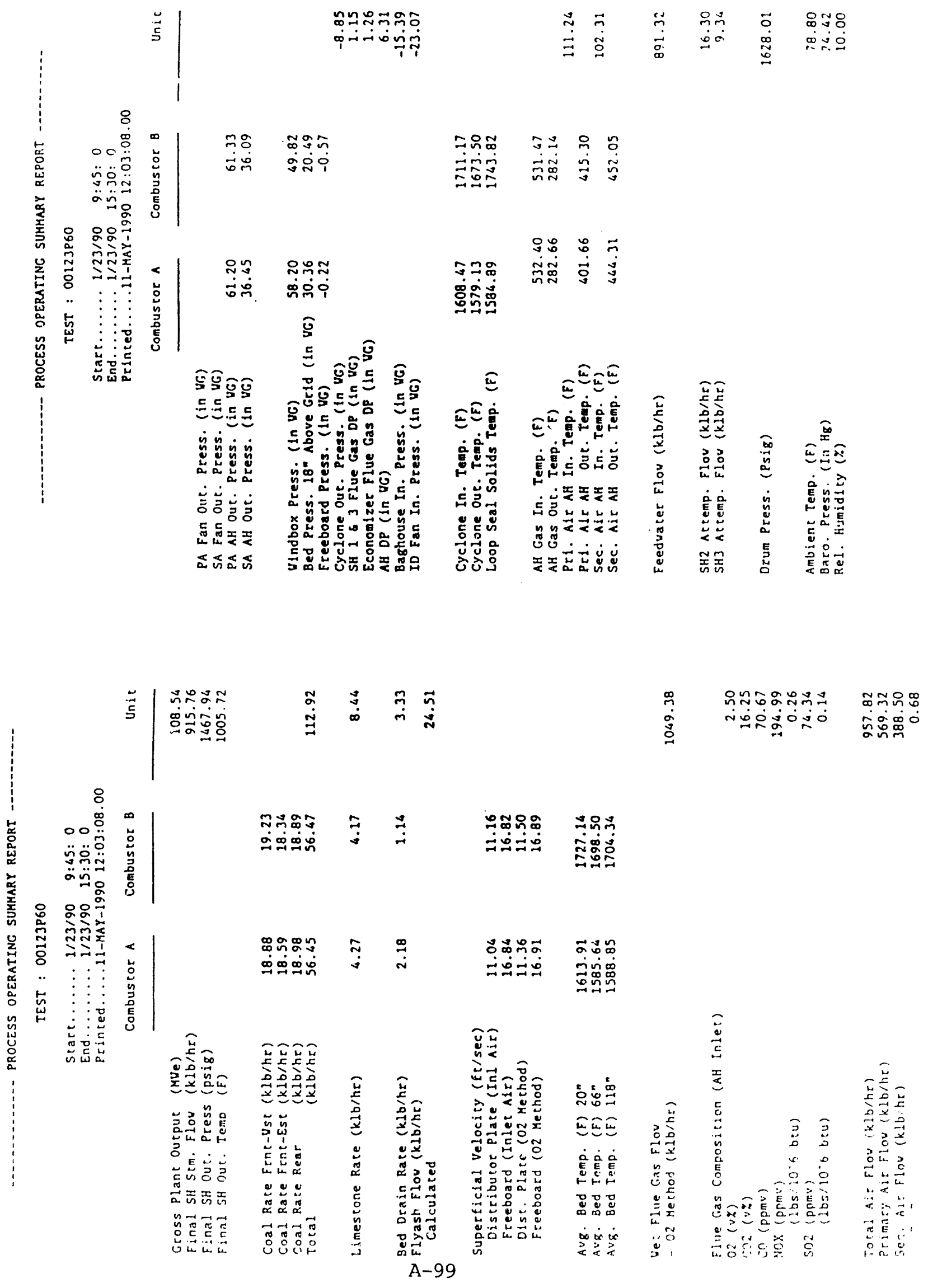


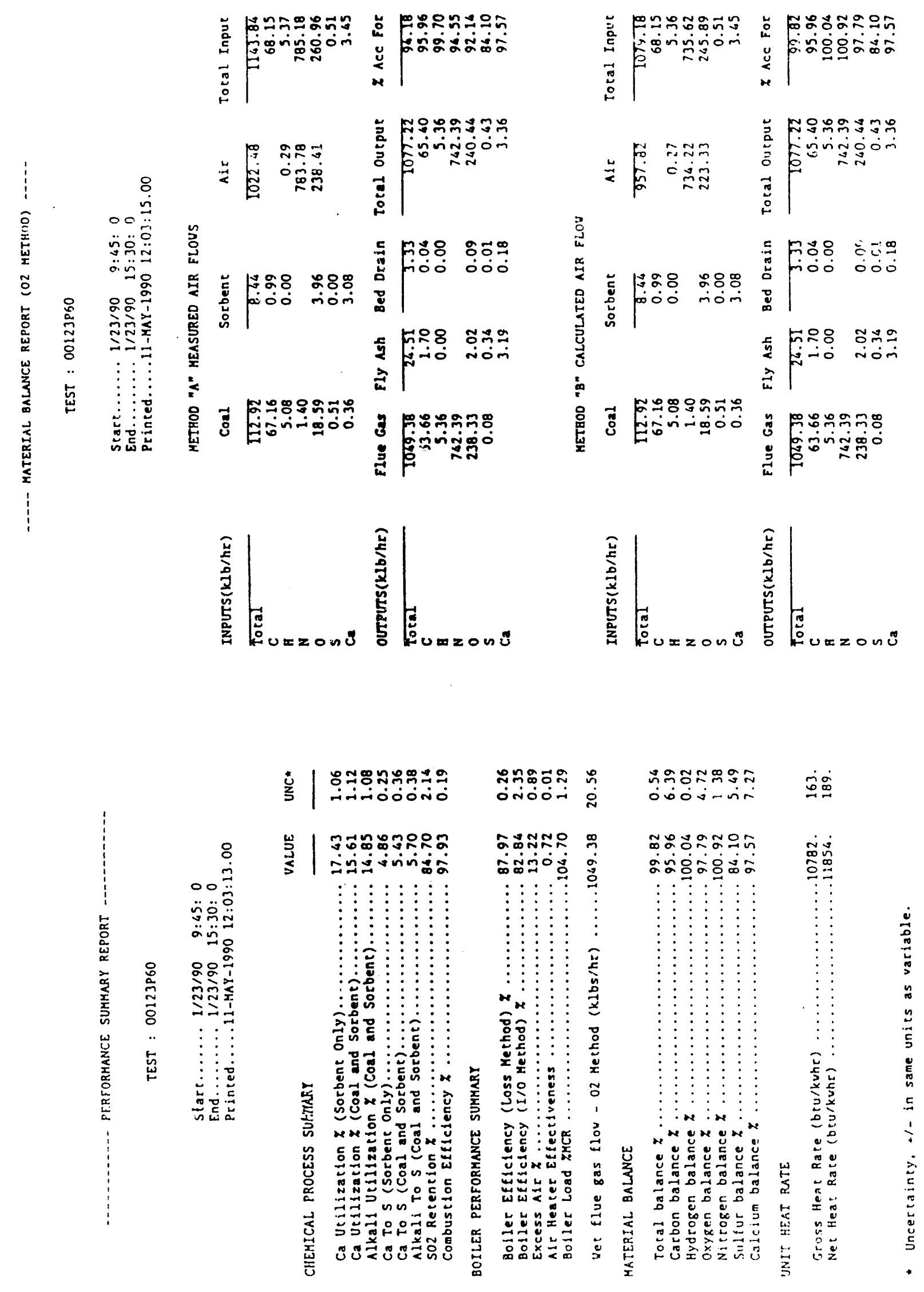



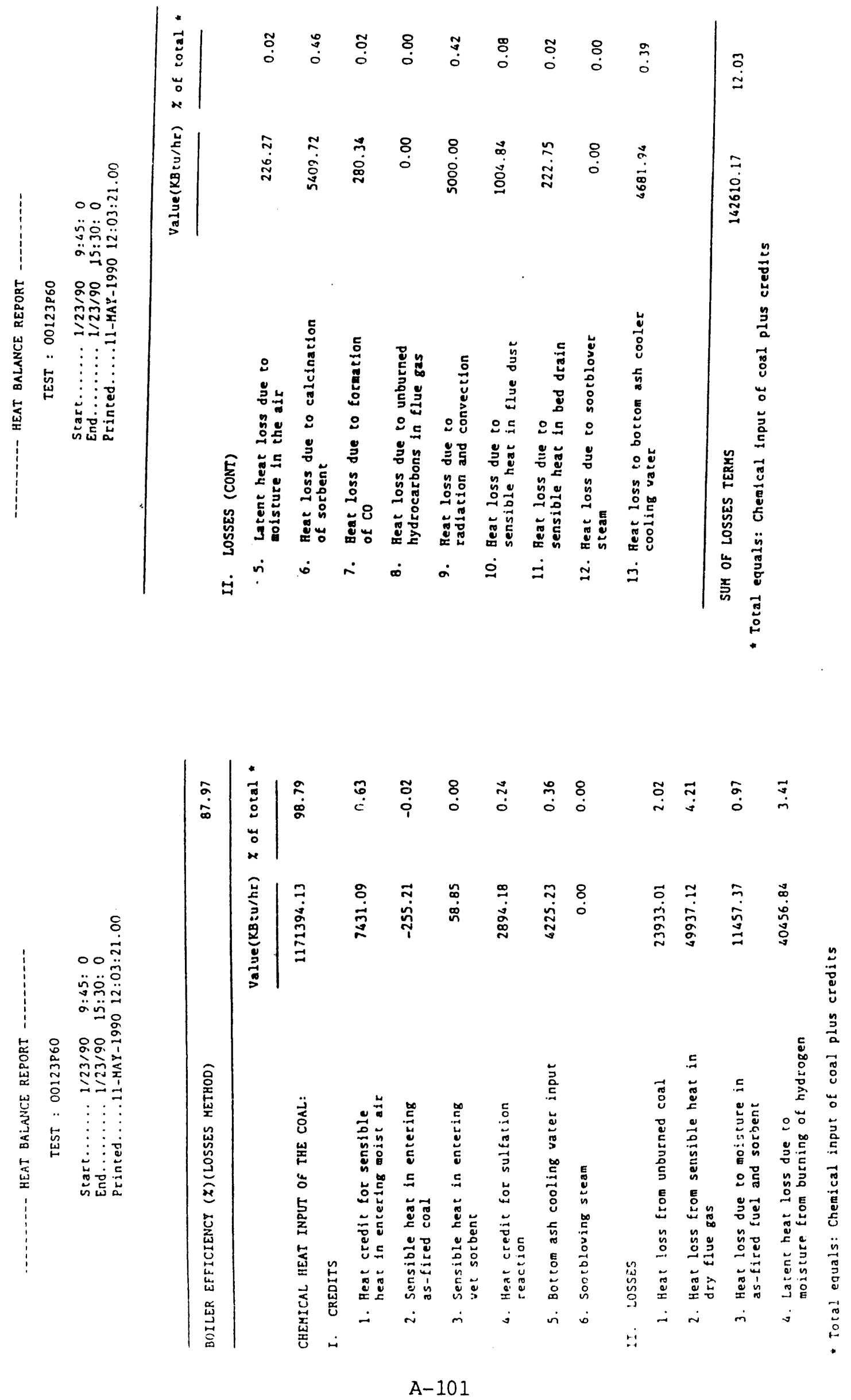


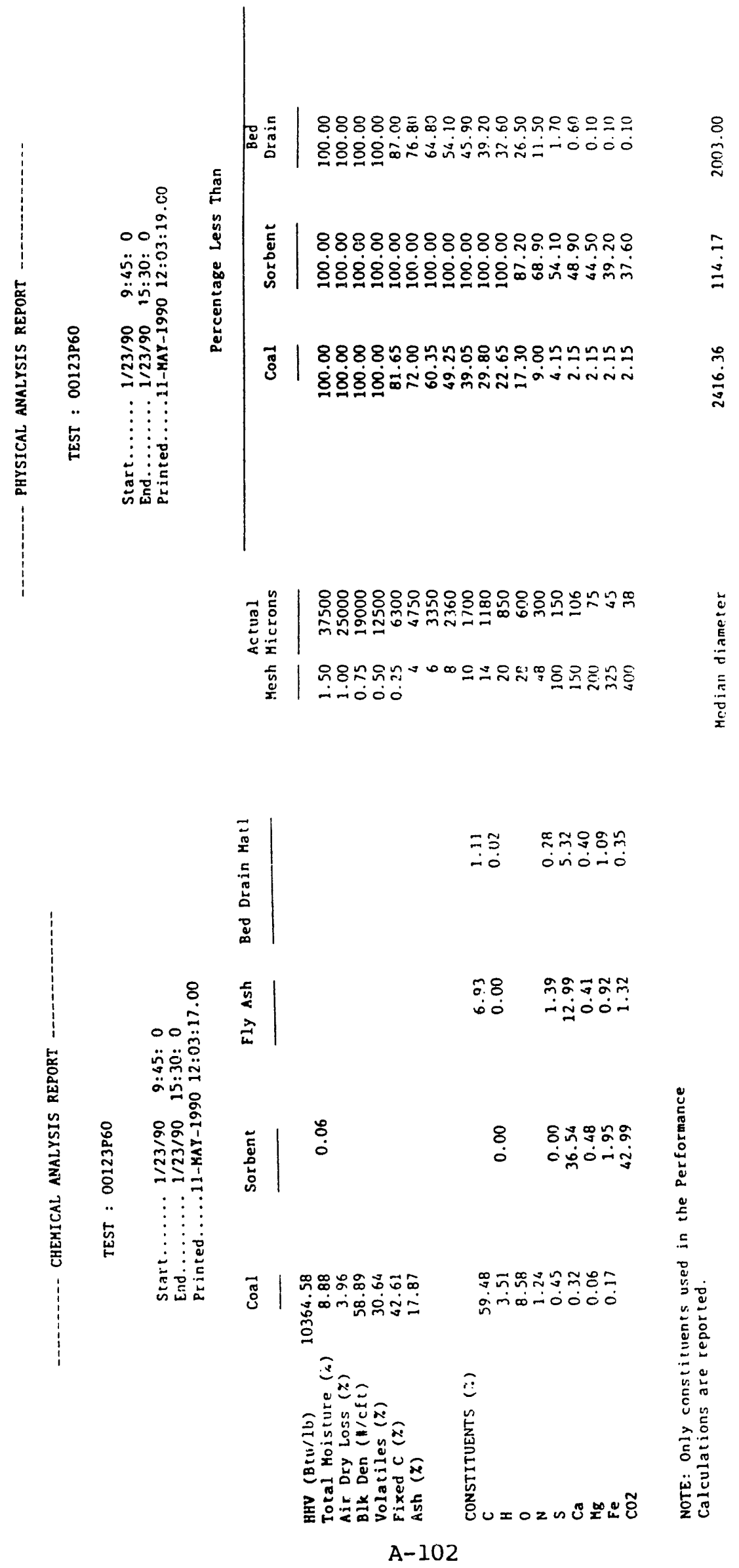



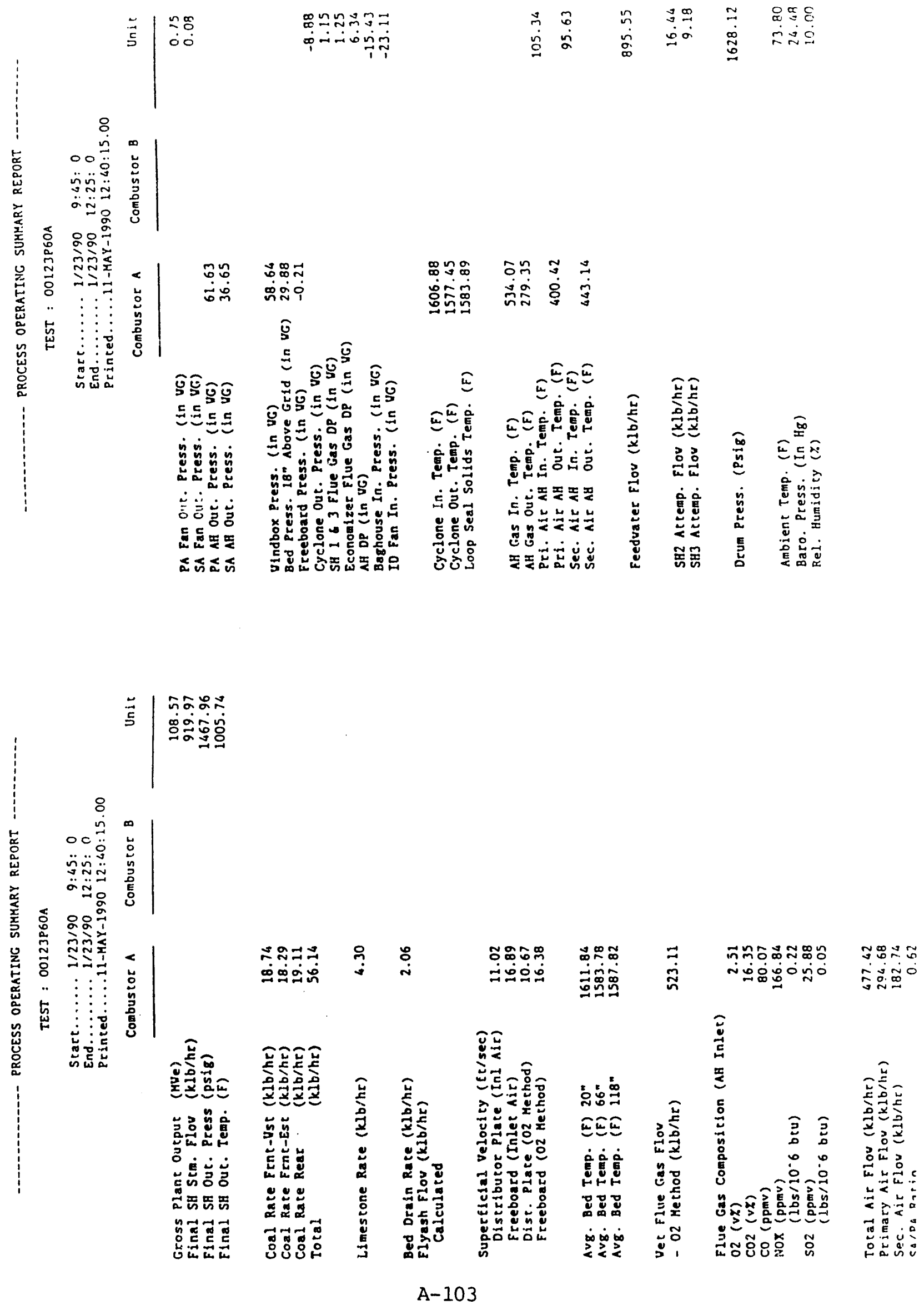


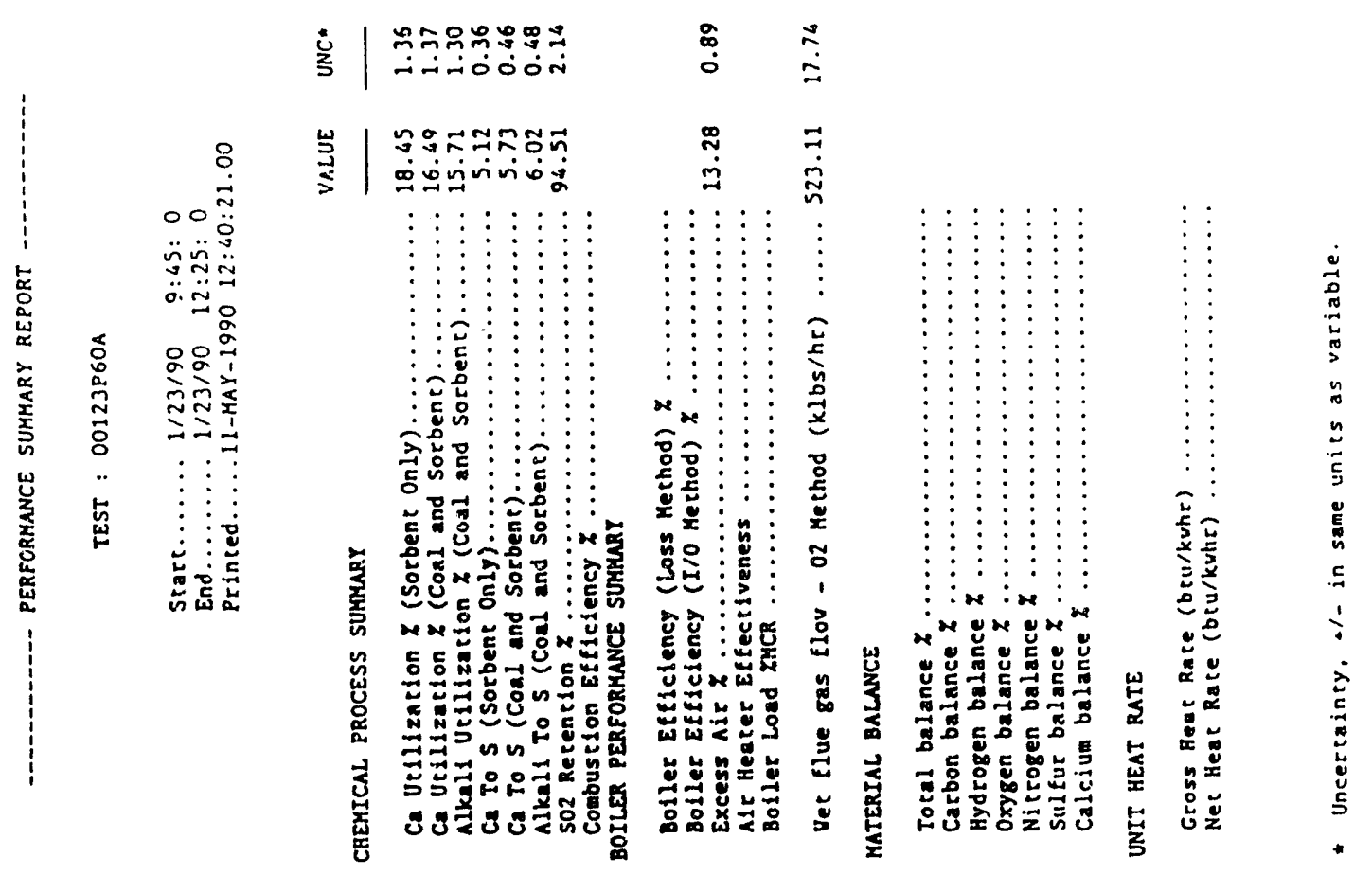



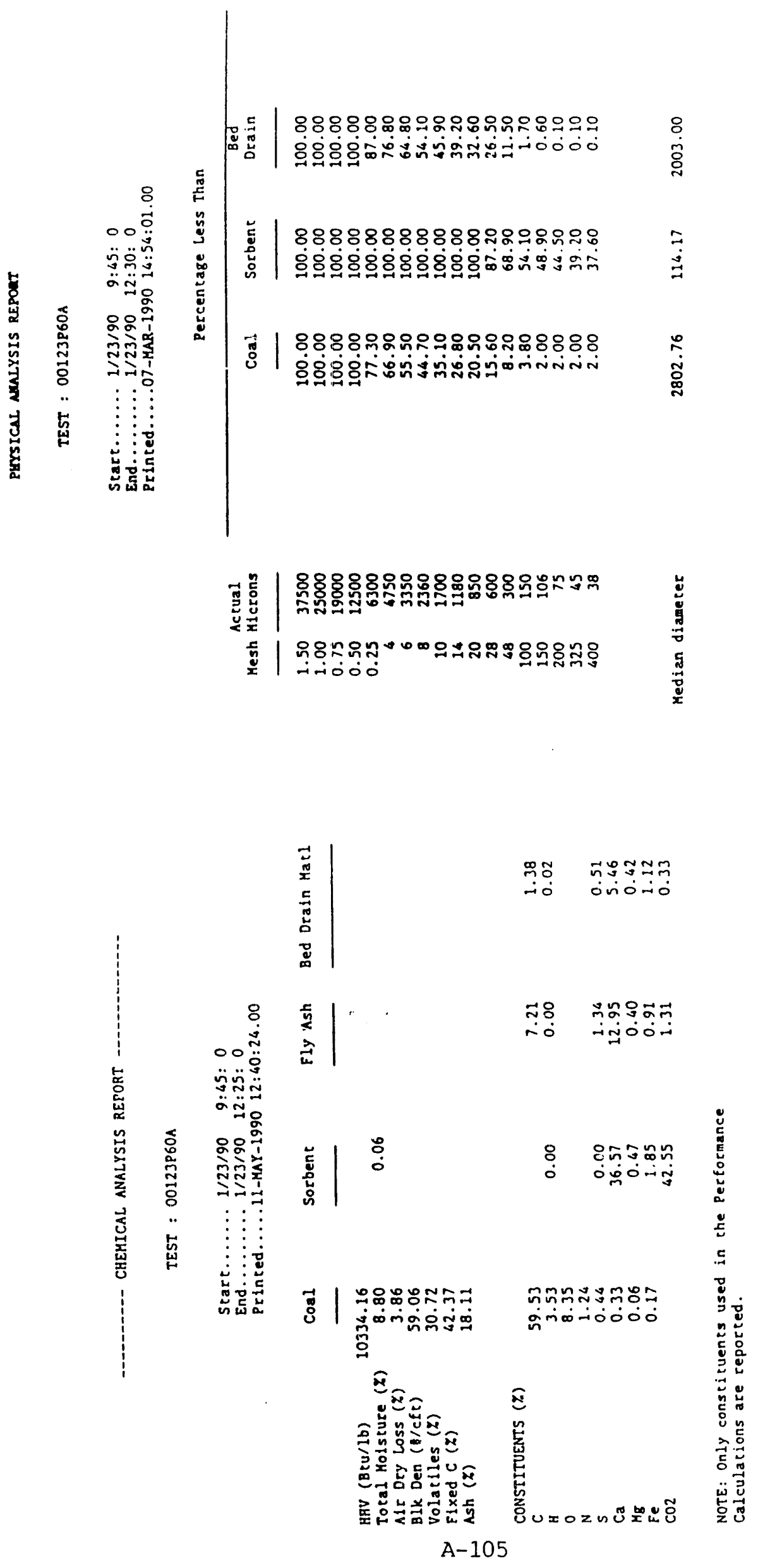

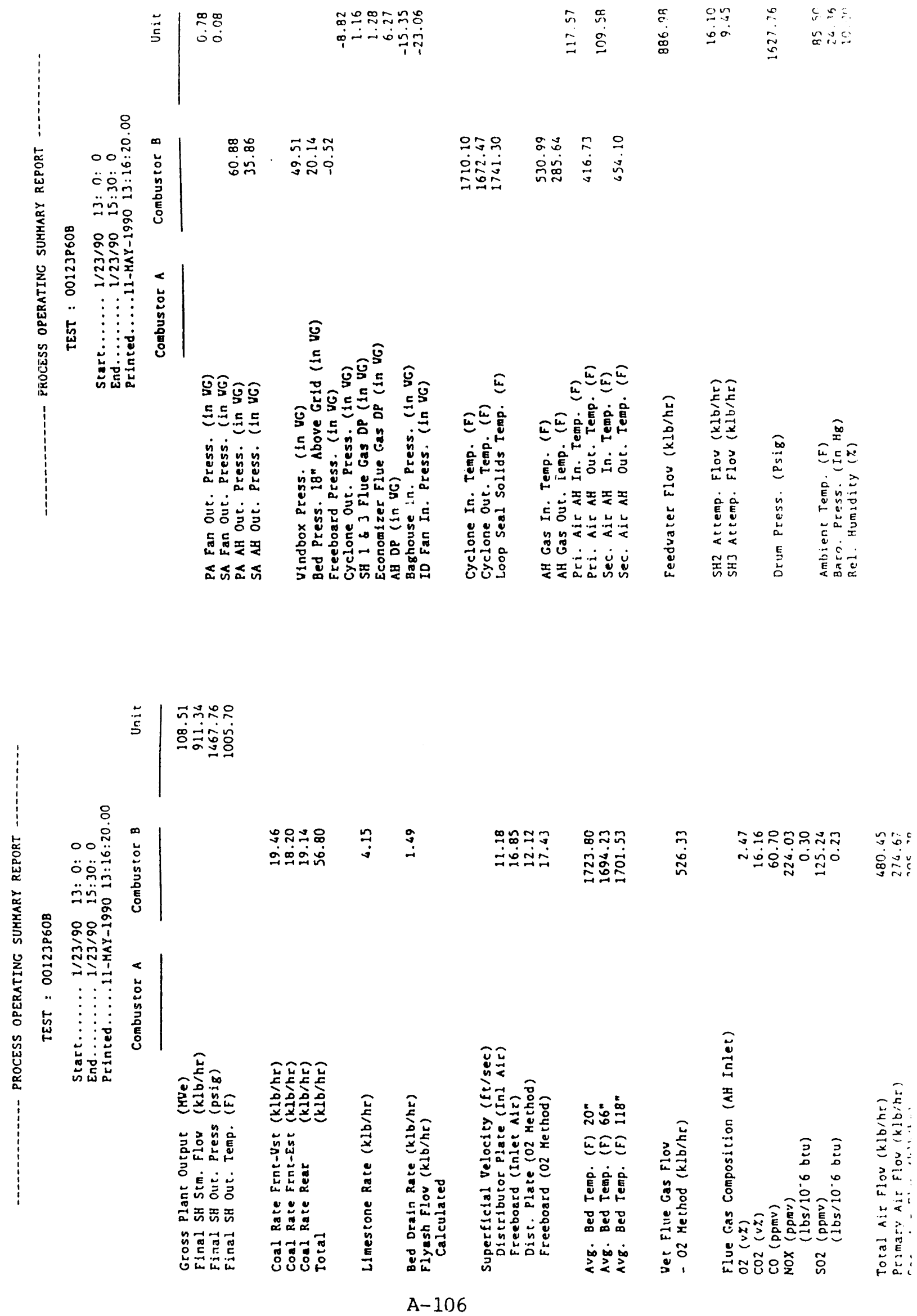


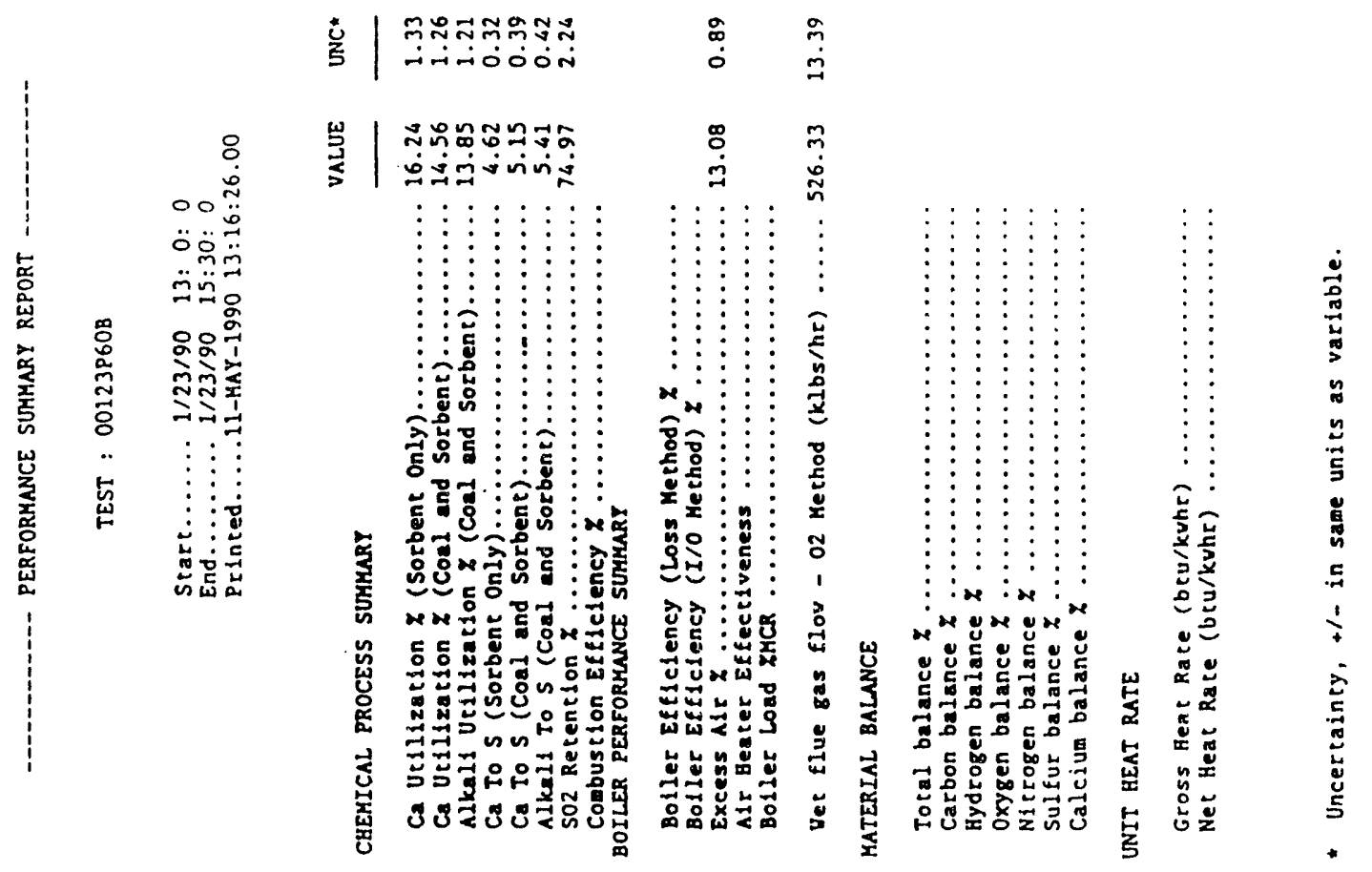

A-107 

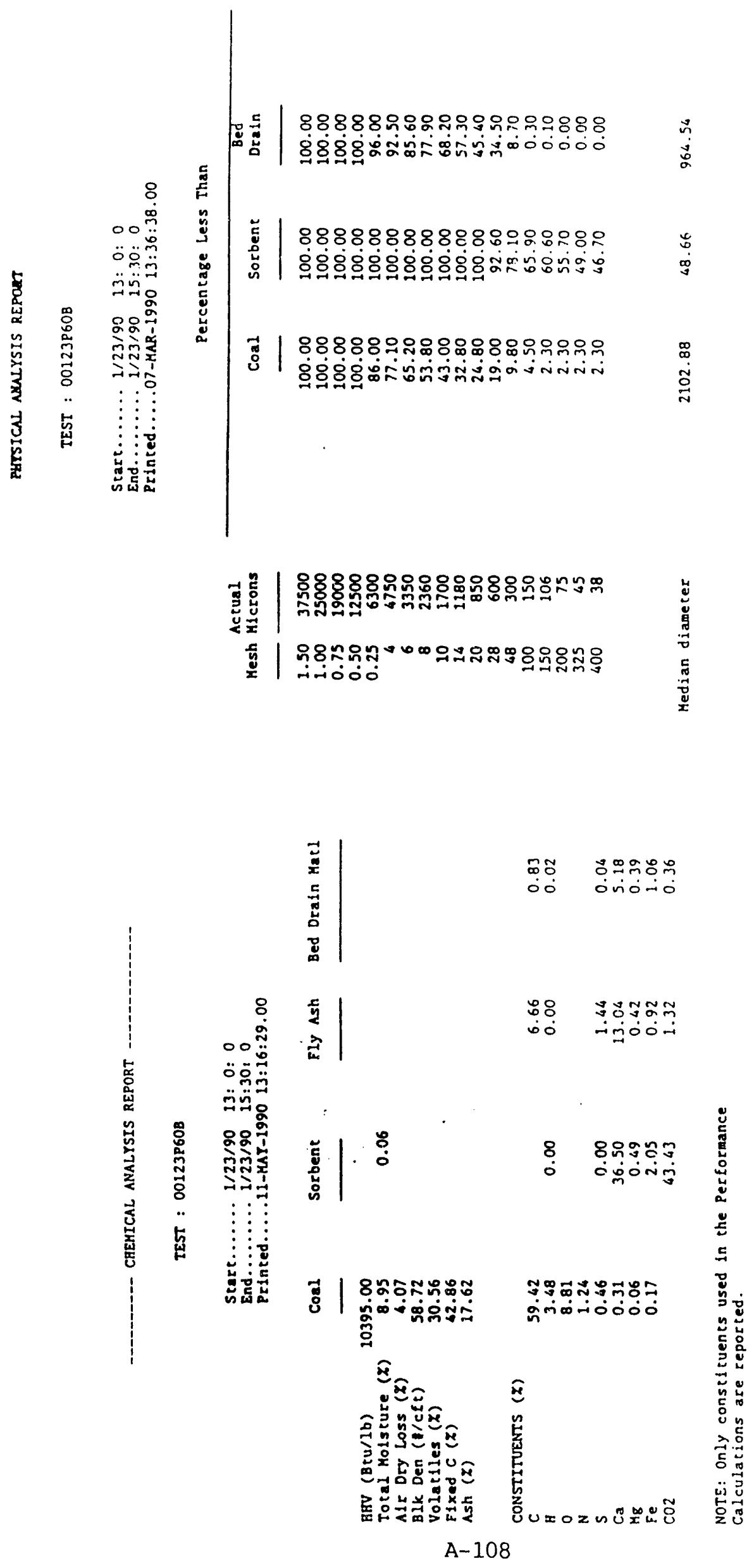

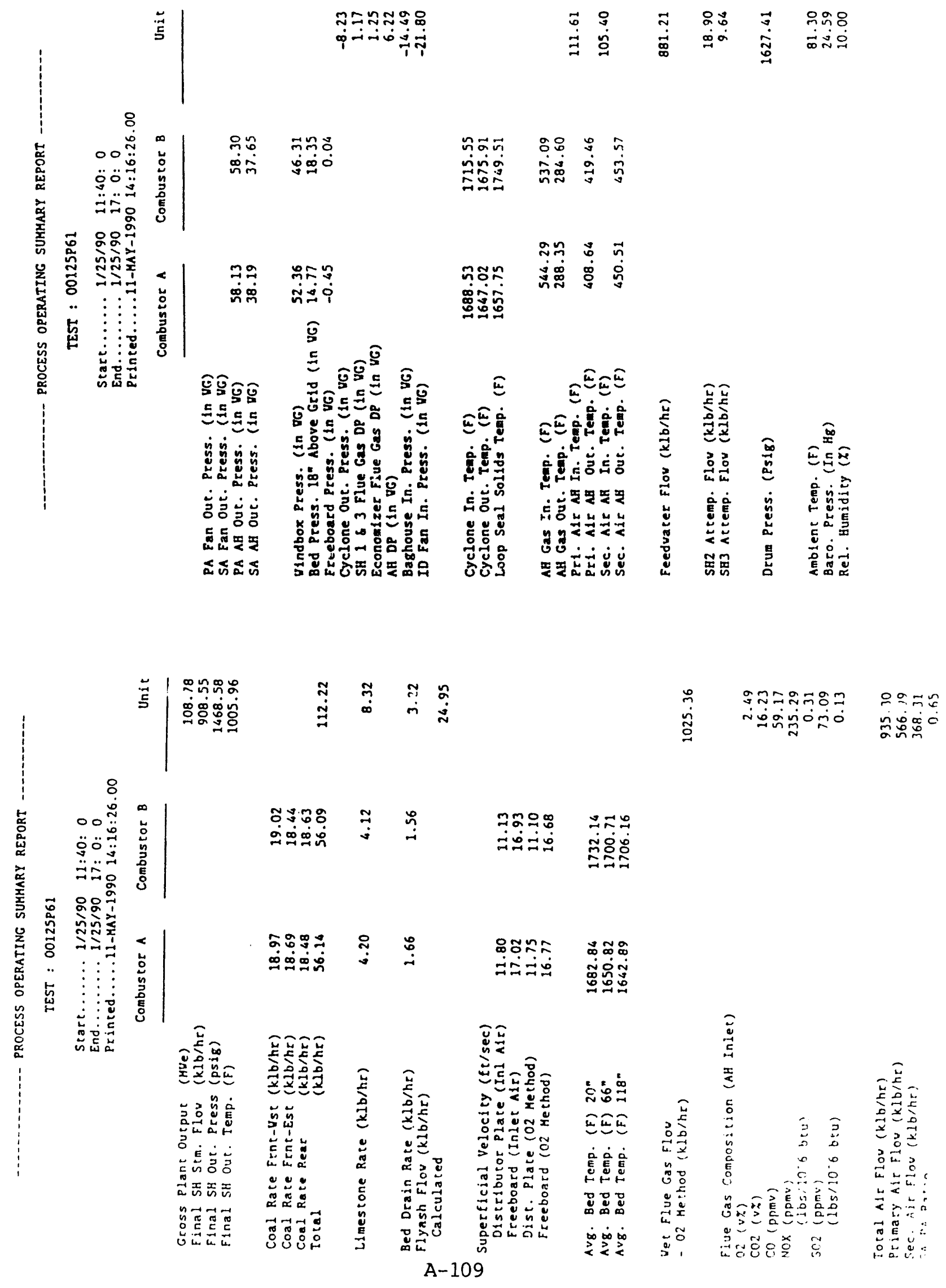


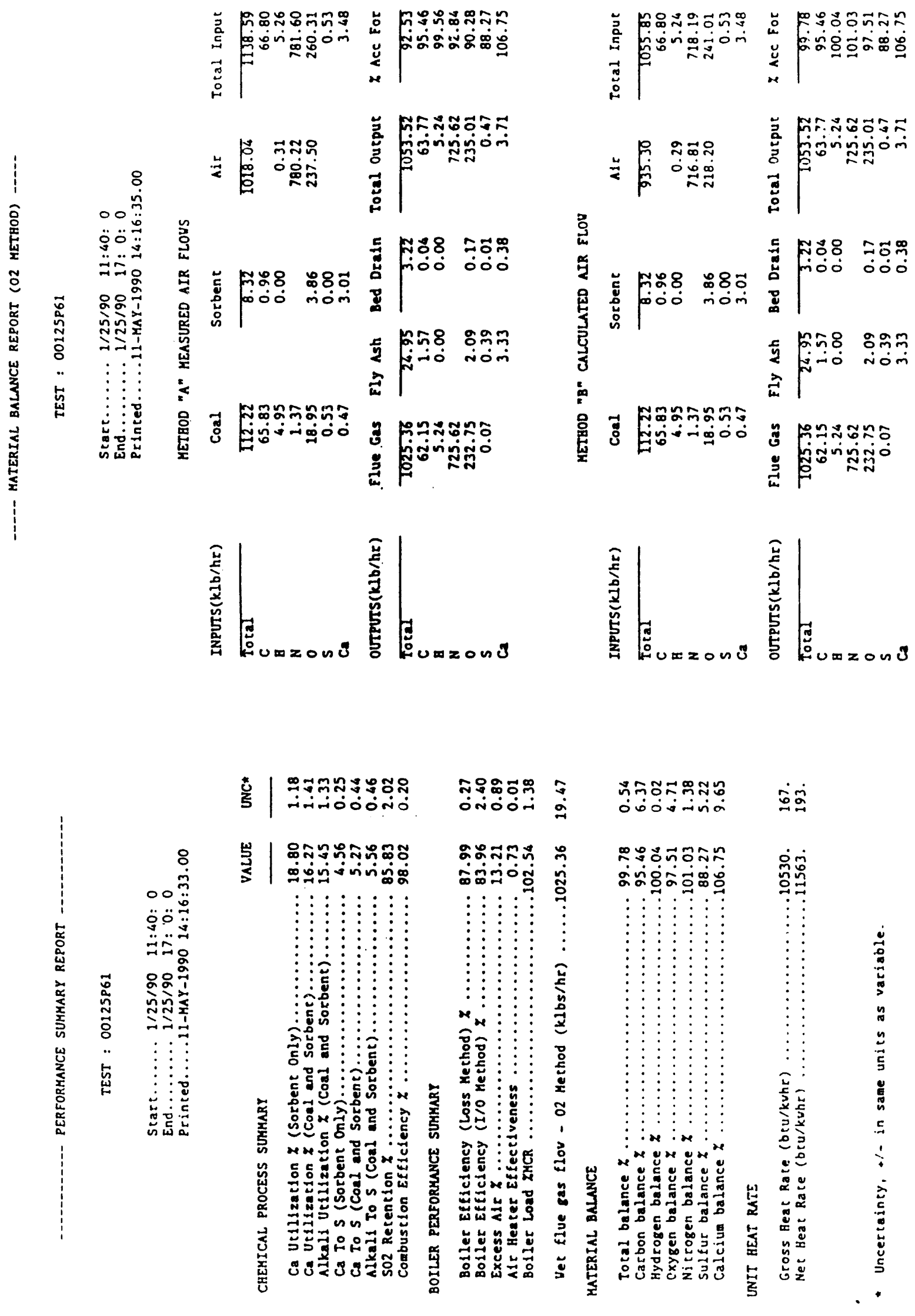




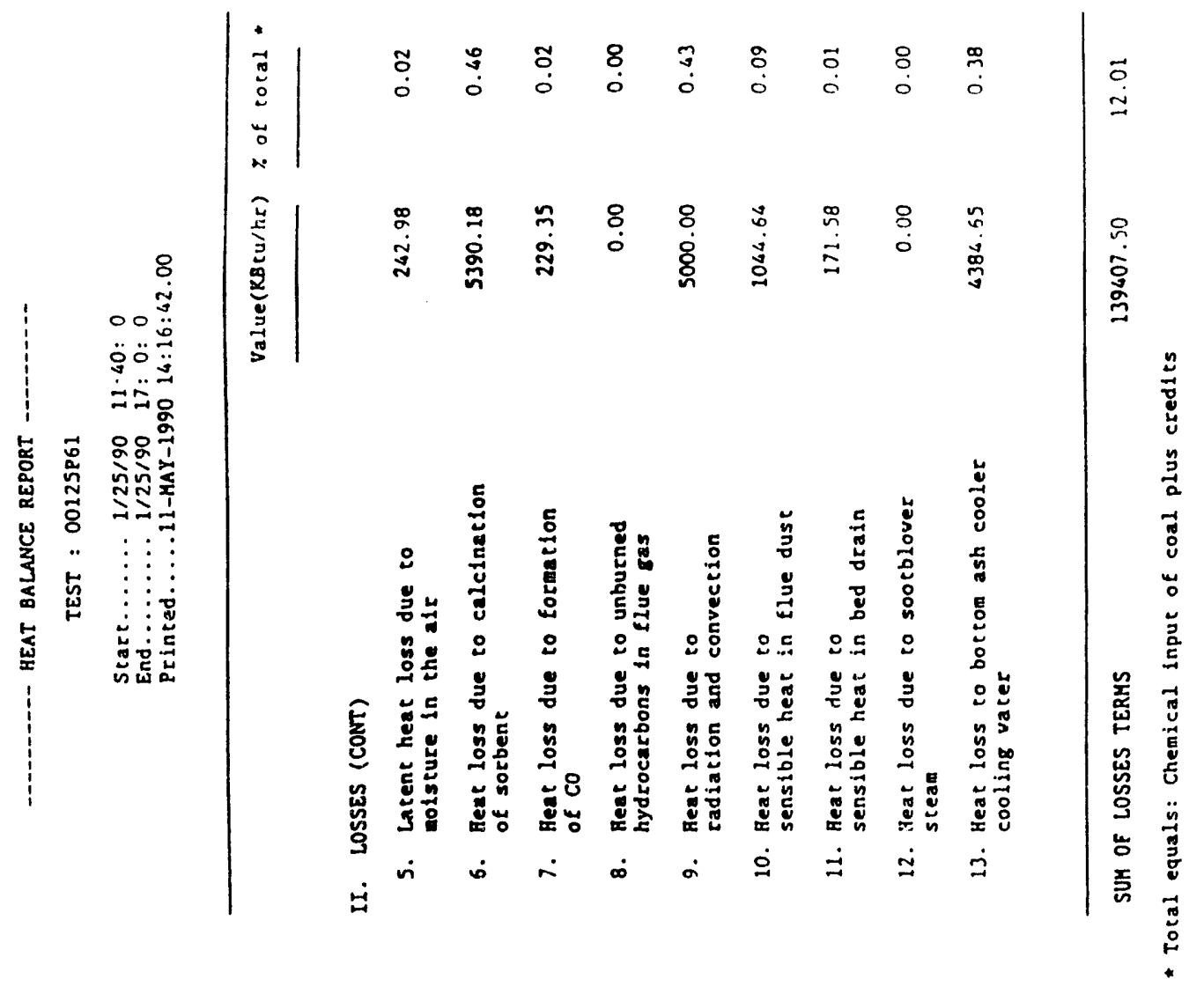

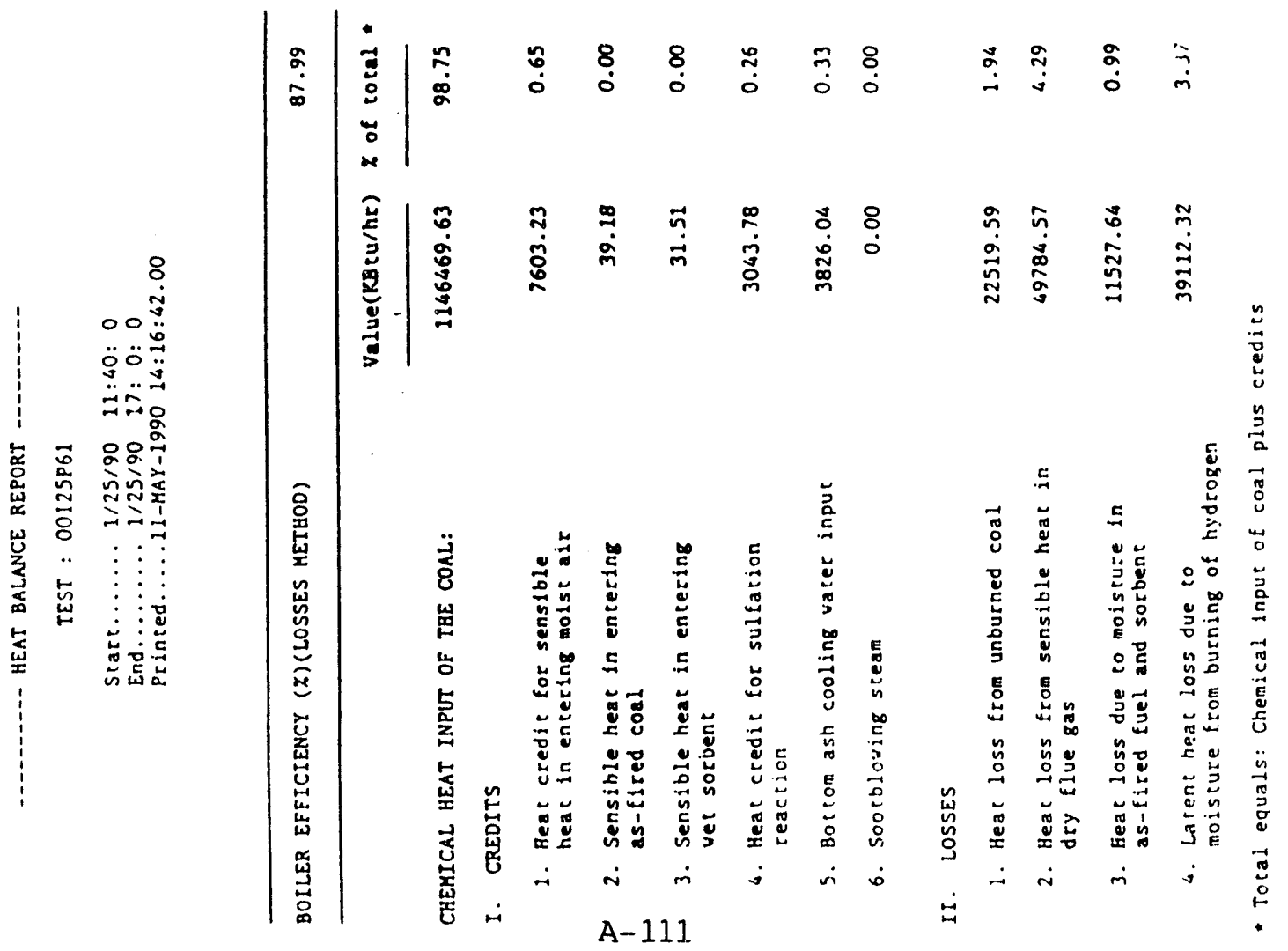



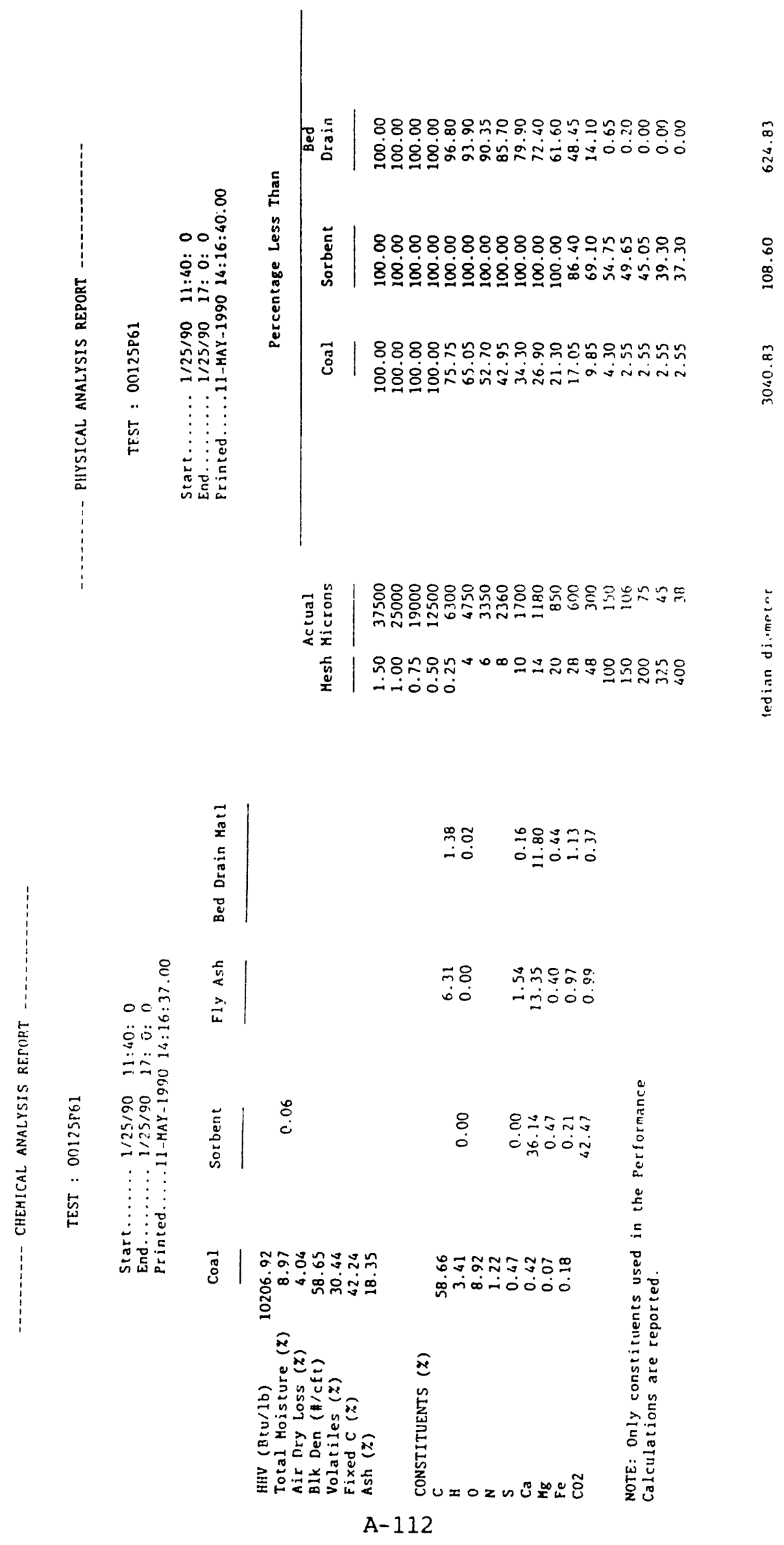

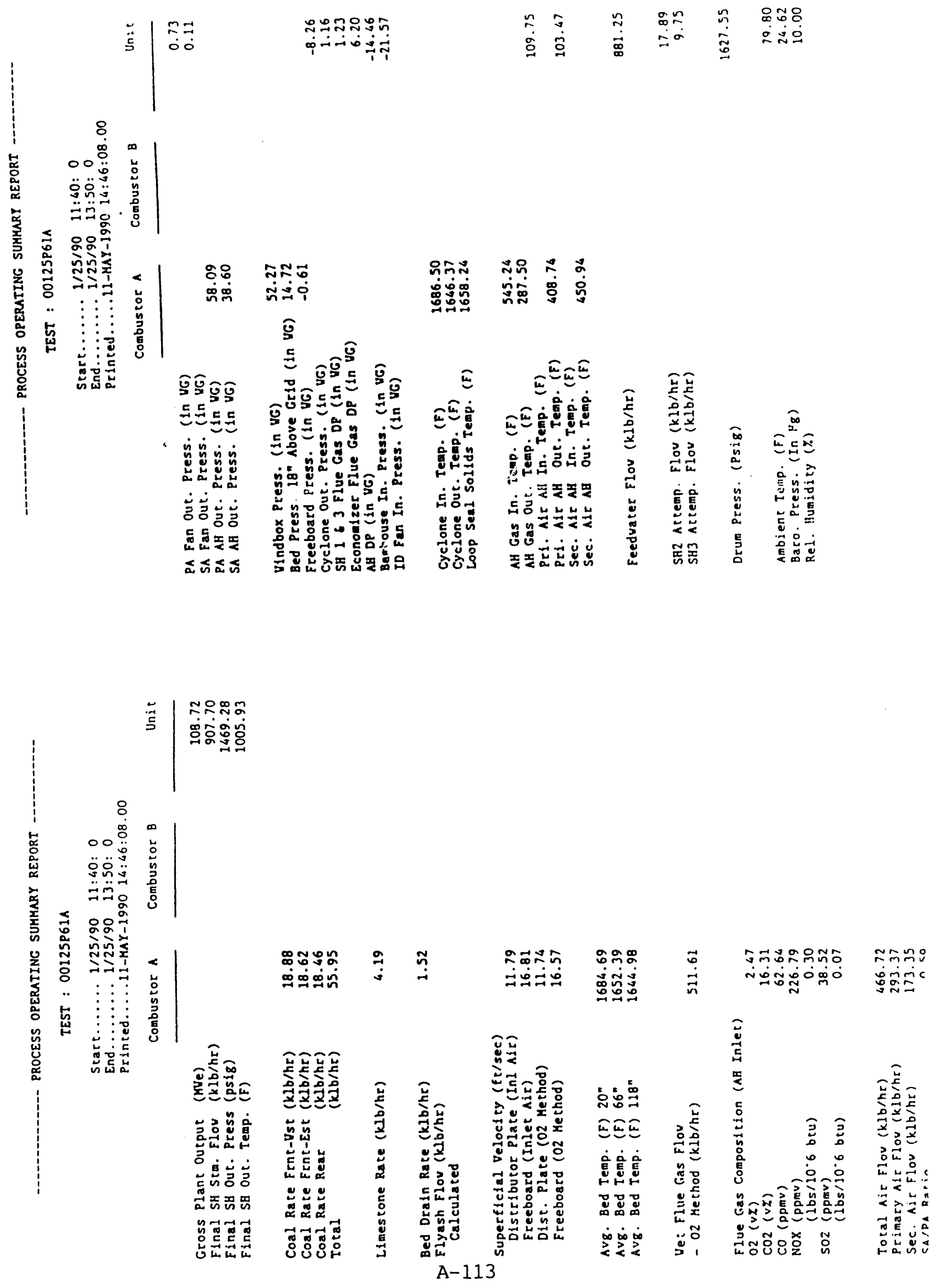


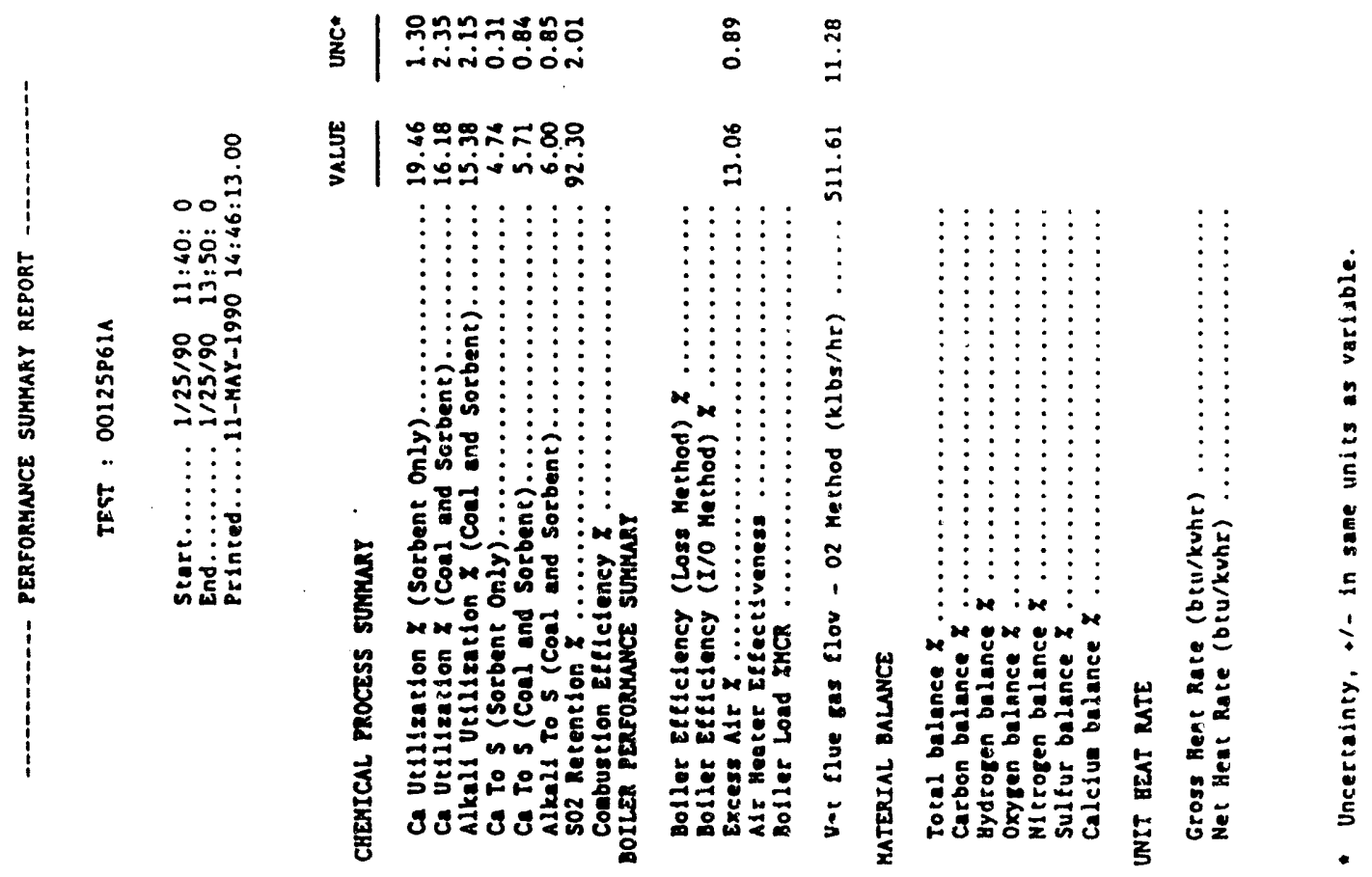



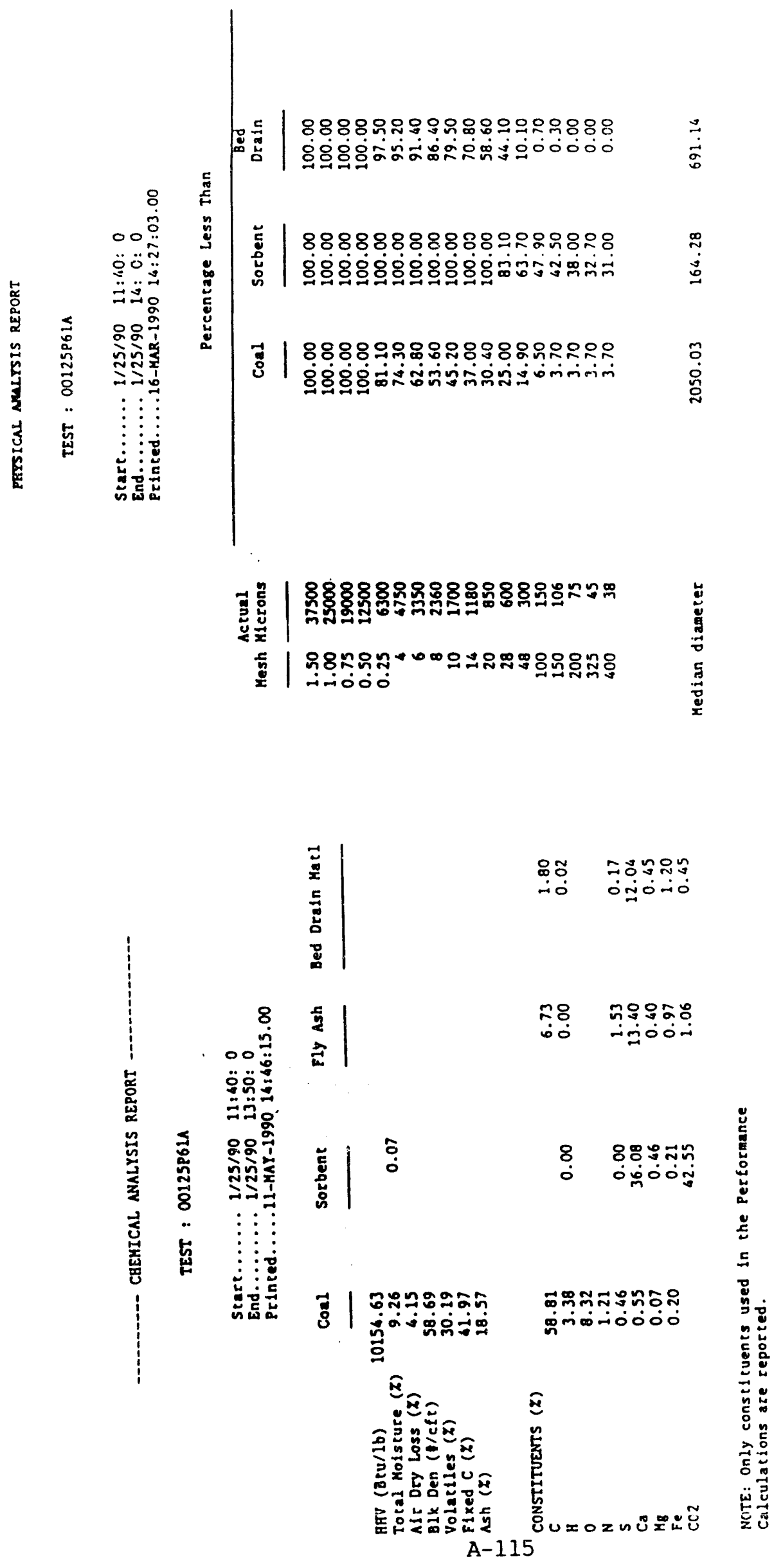

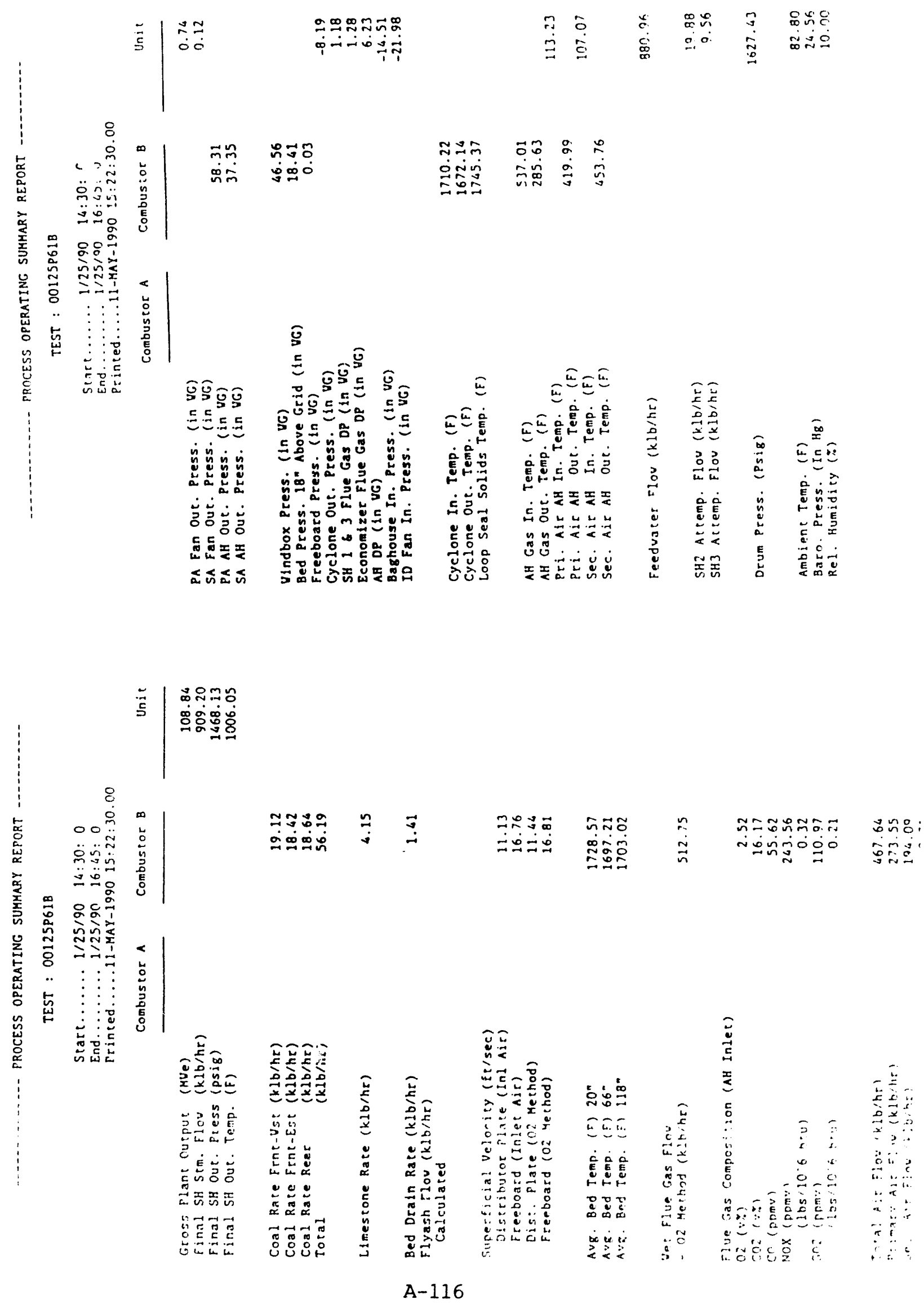


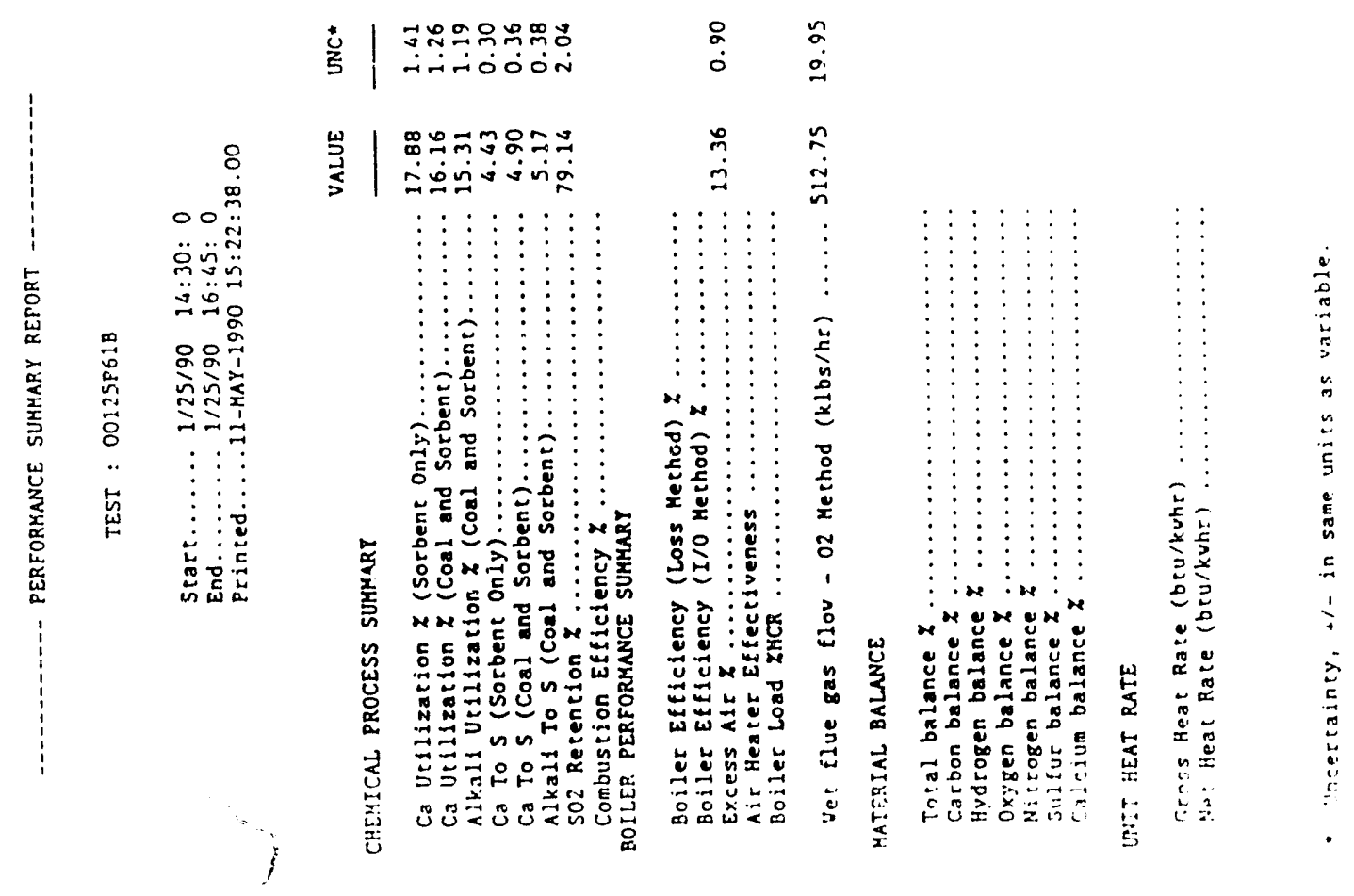



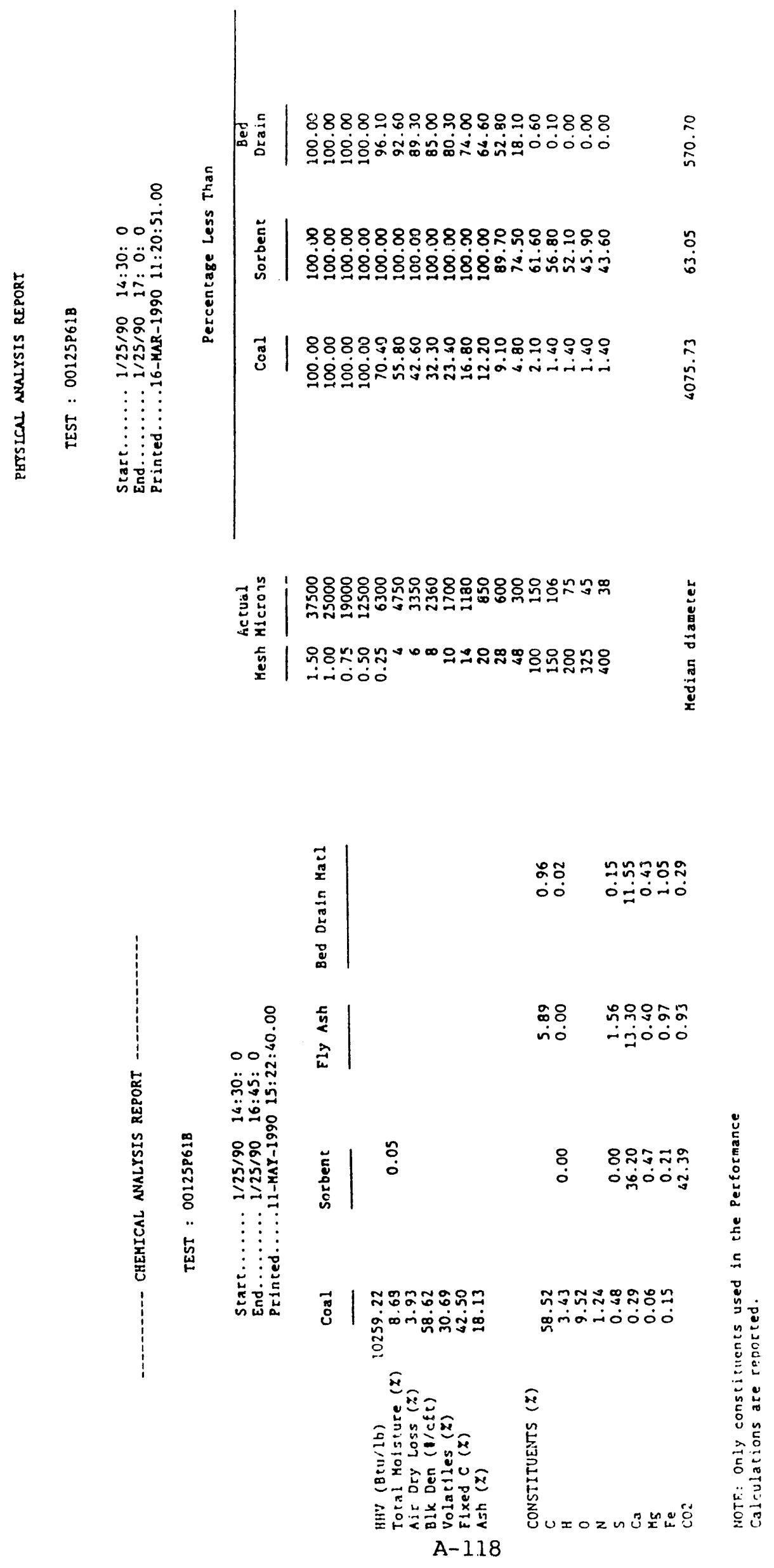

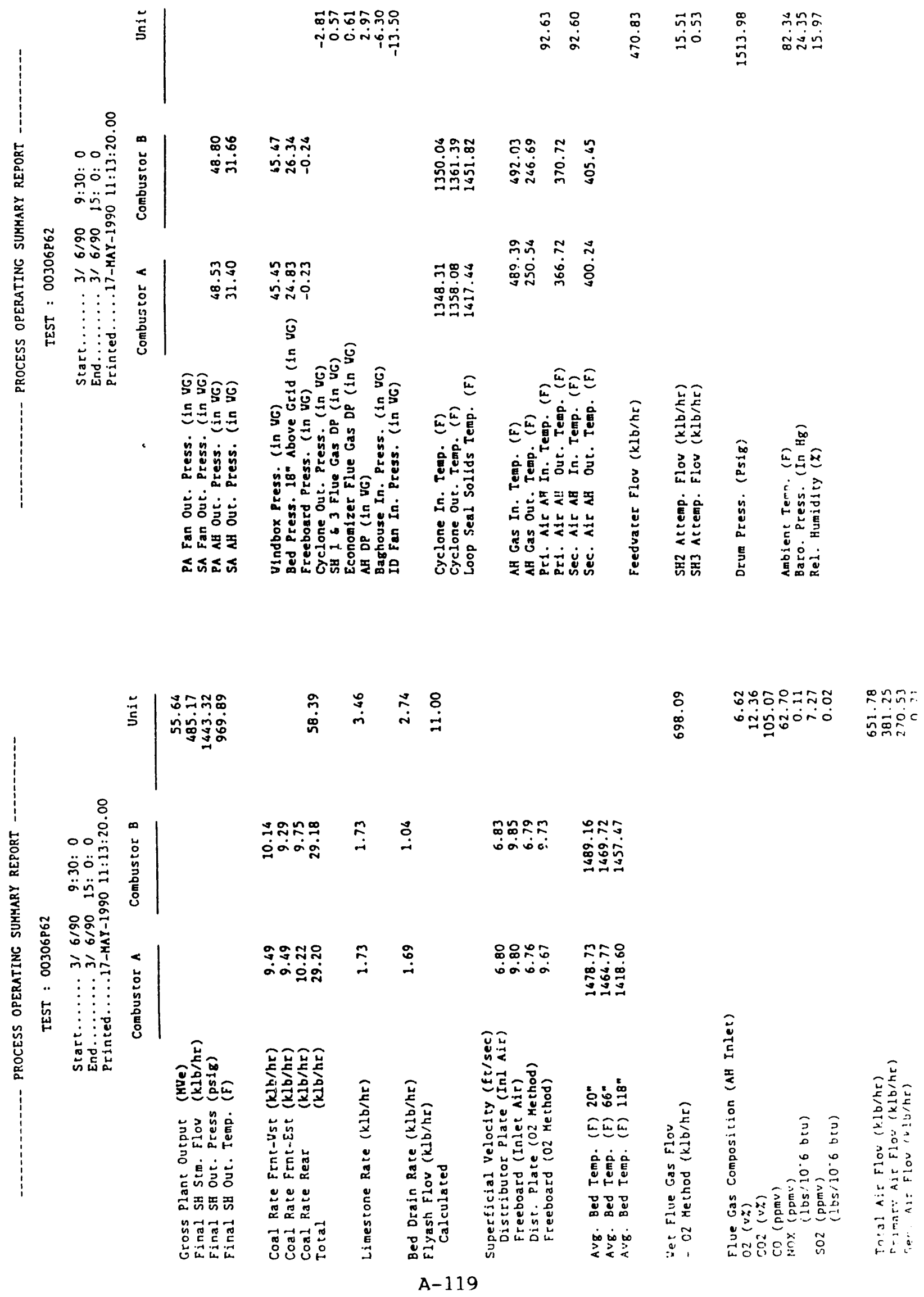


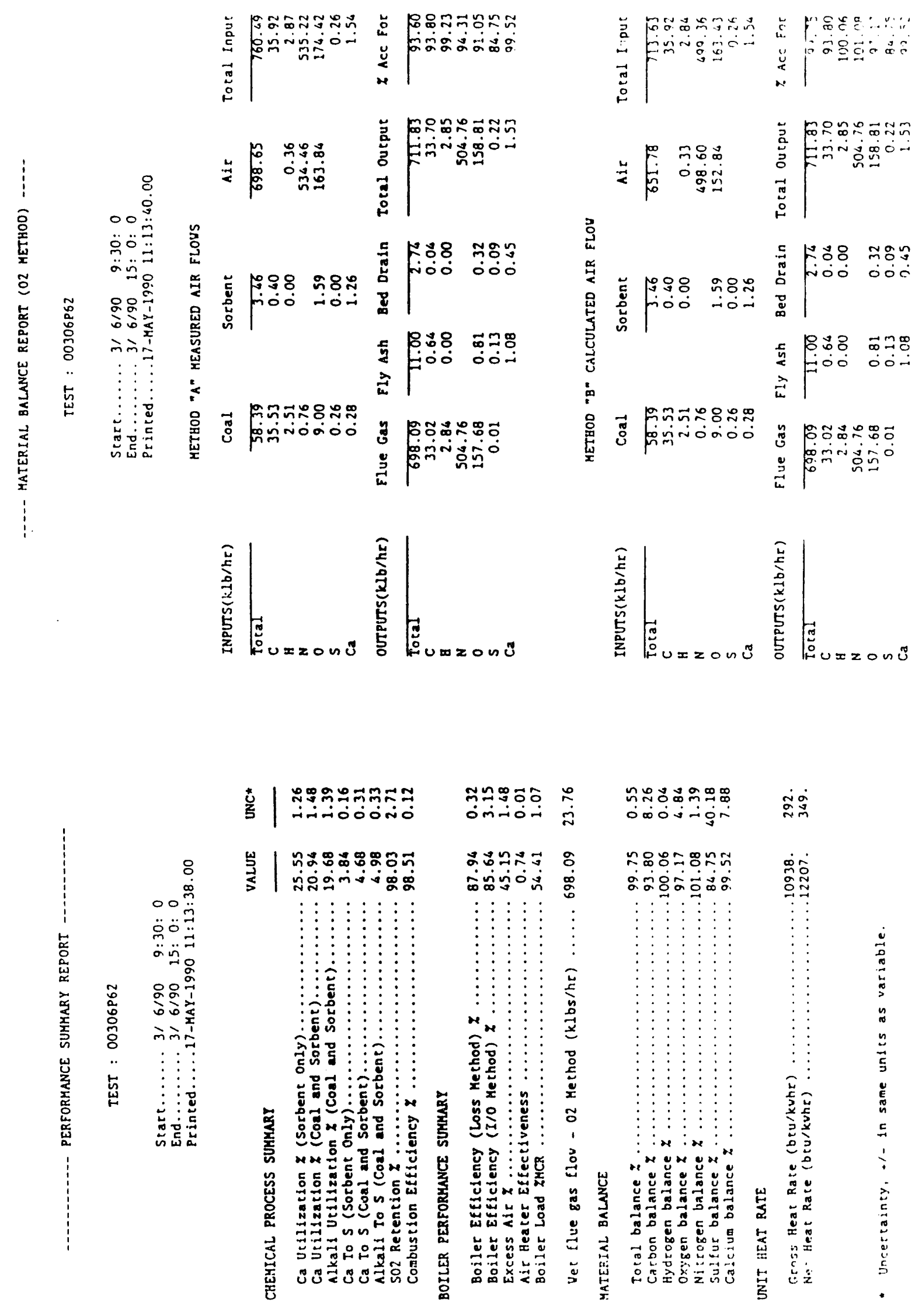



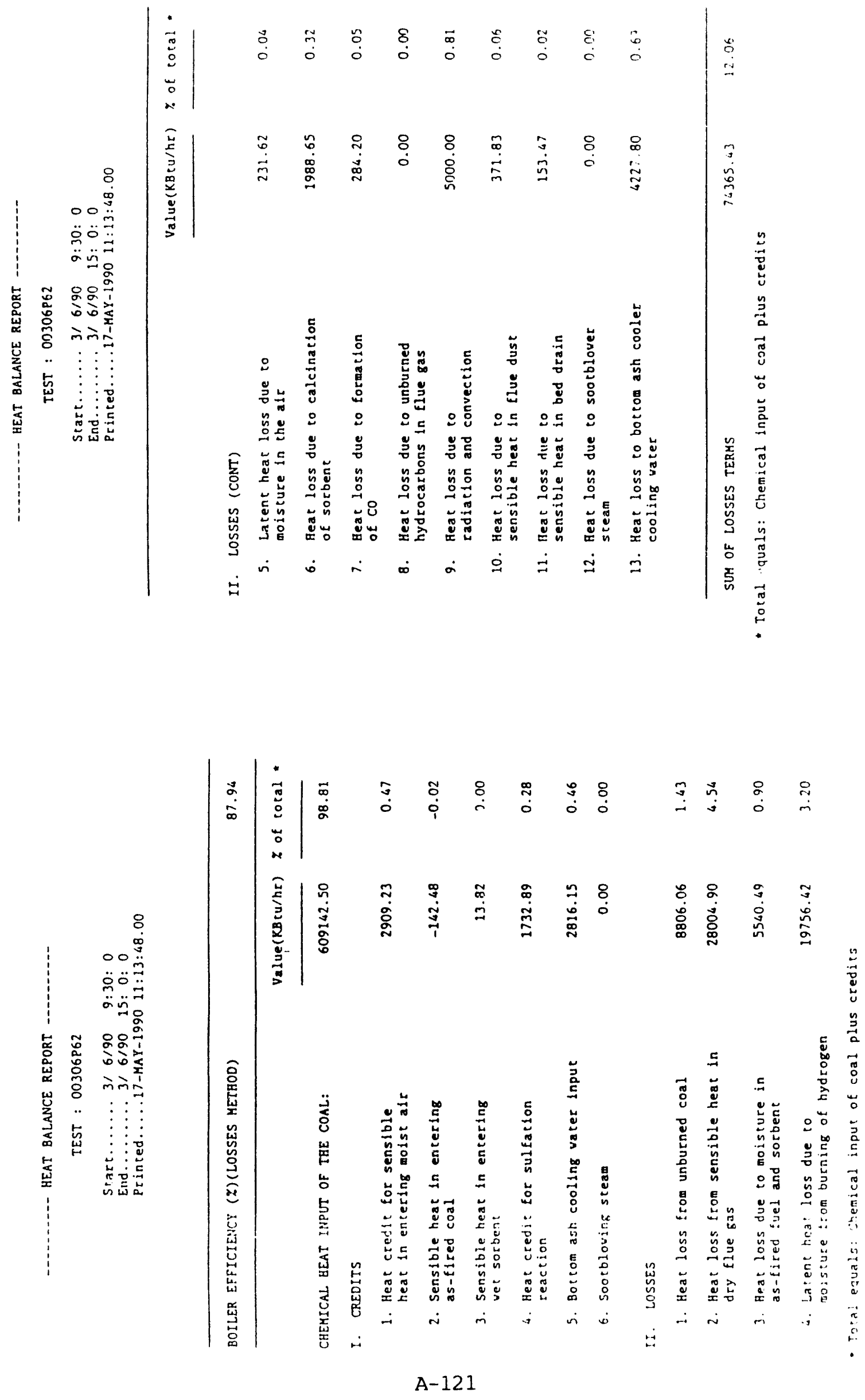

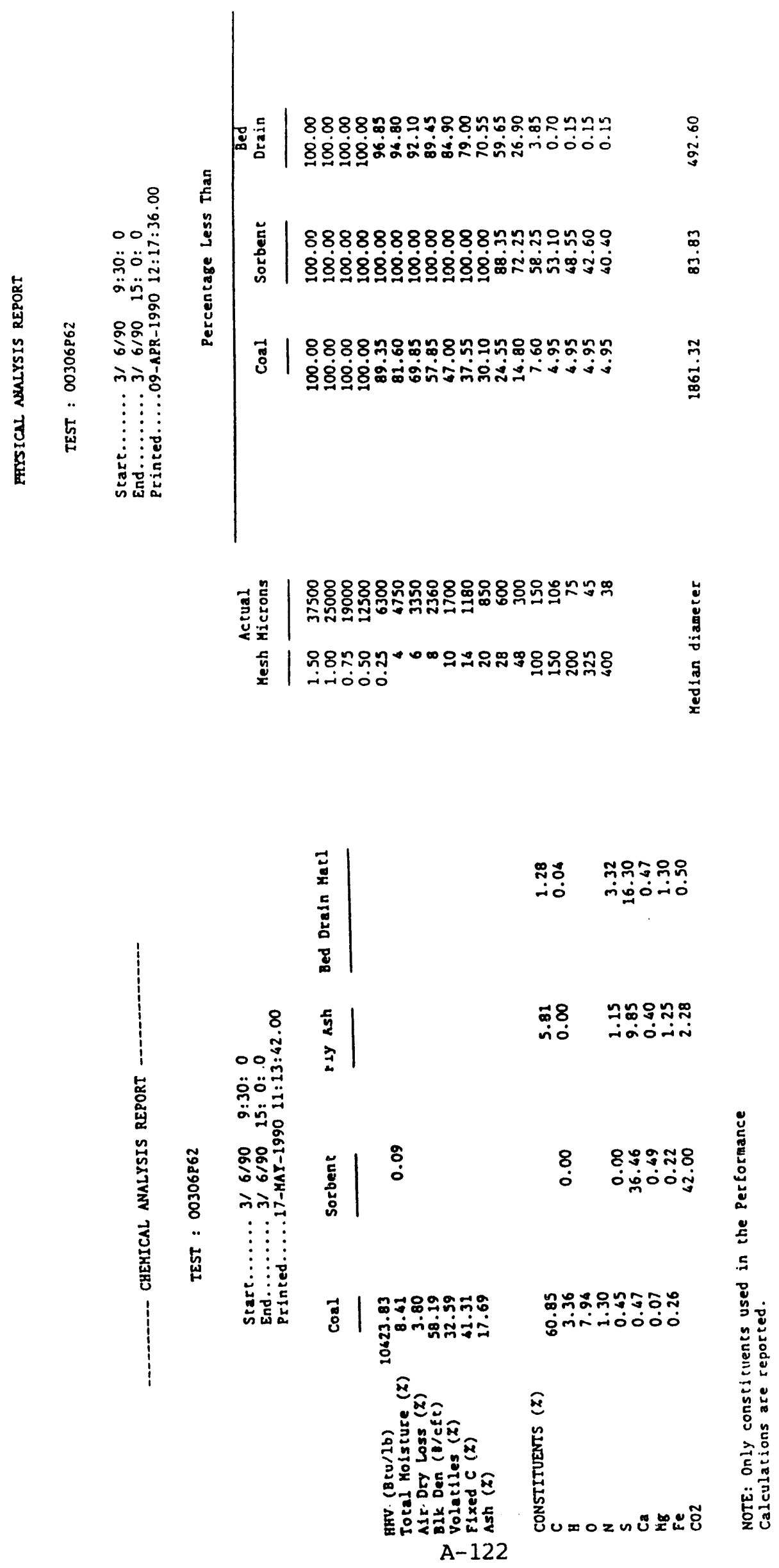


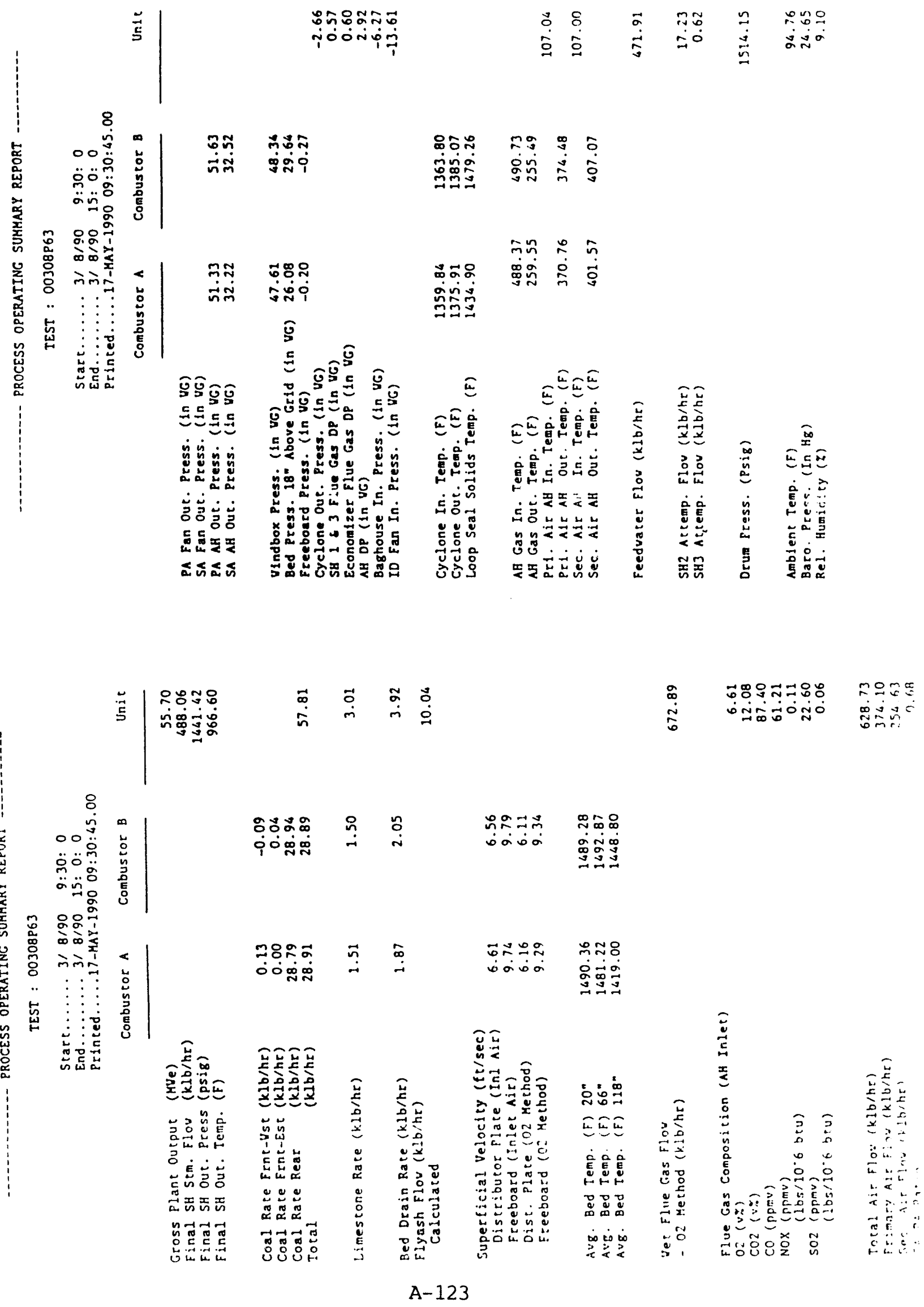




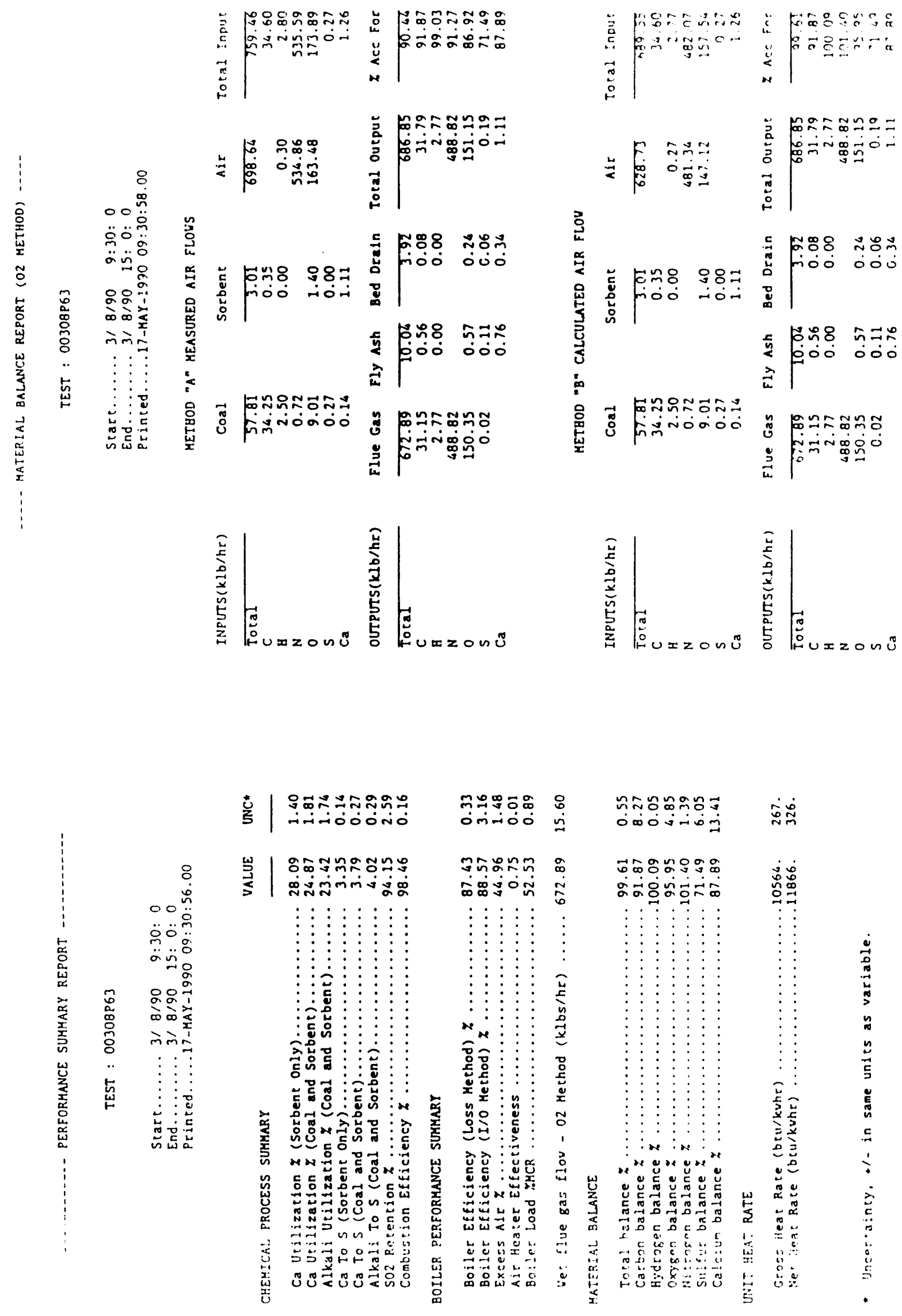



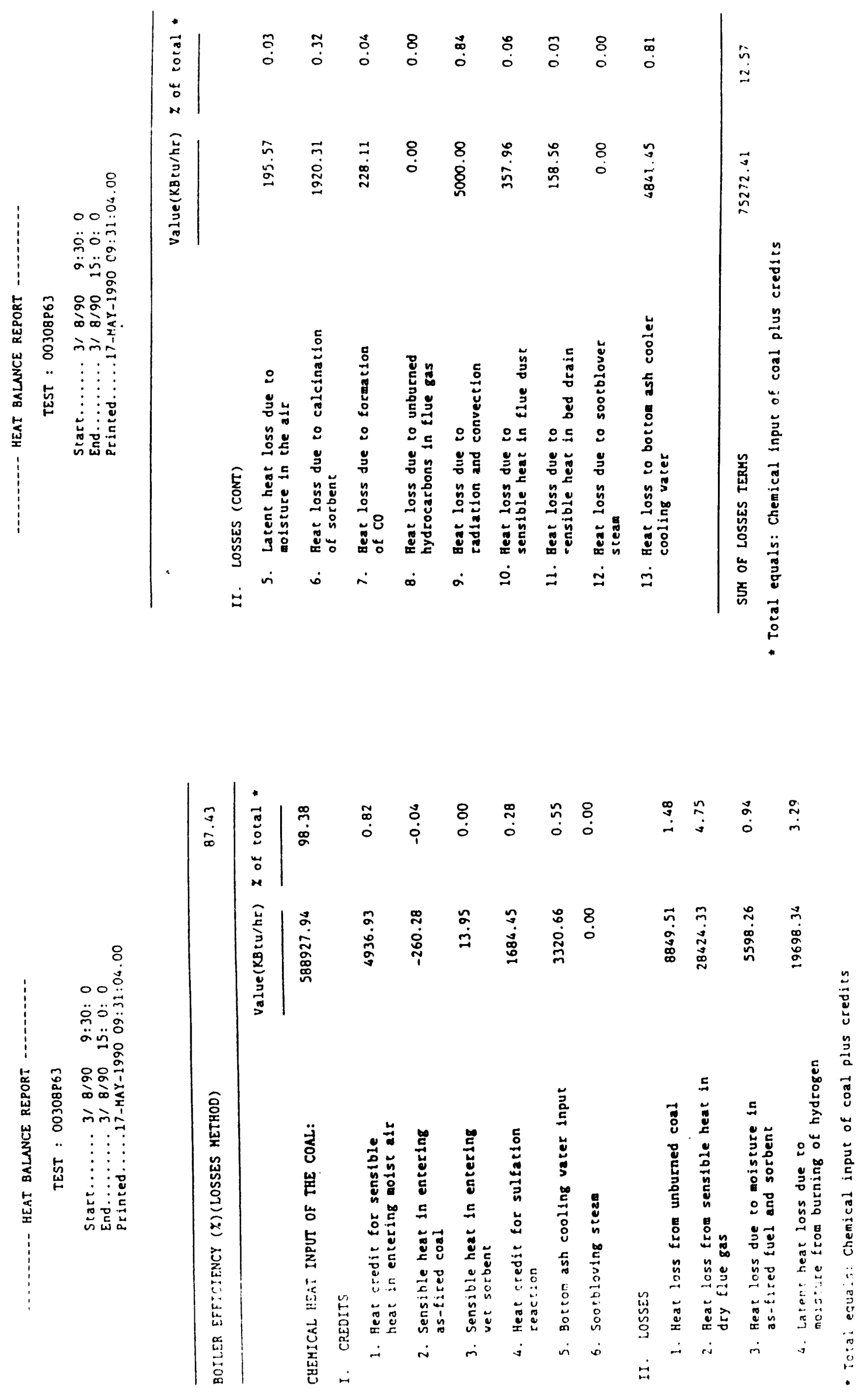

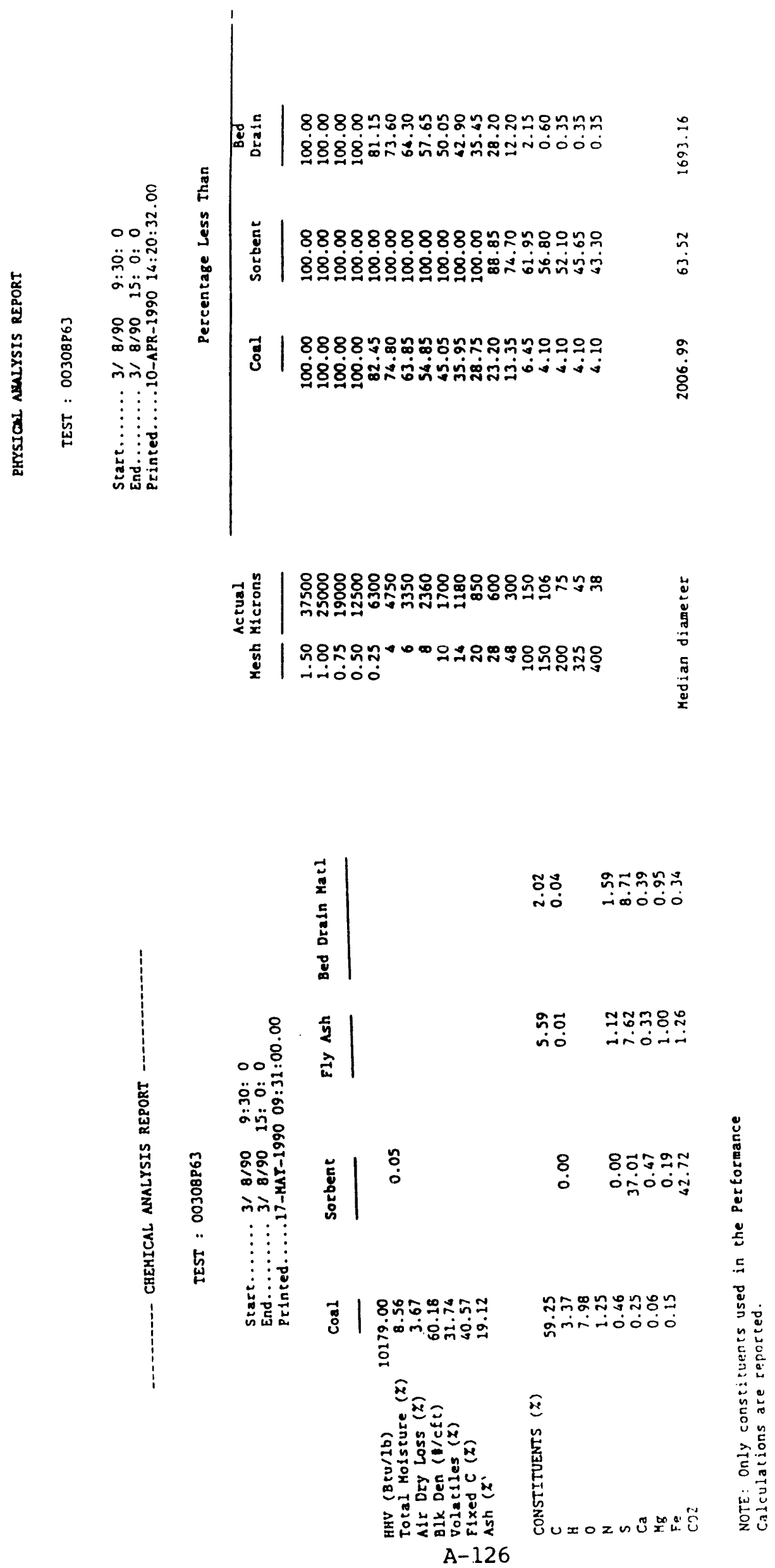

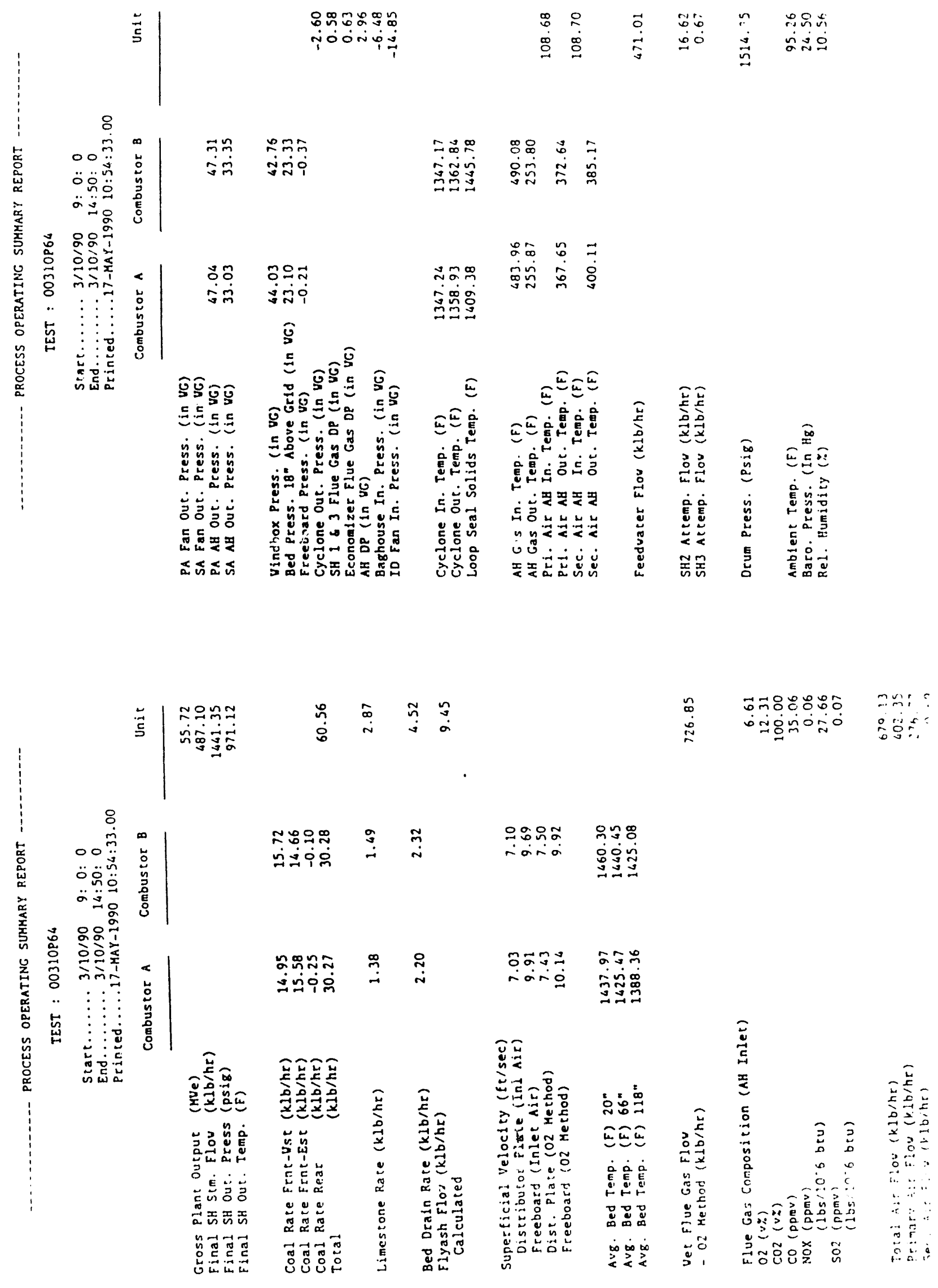


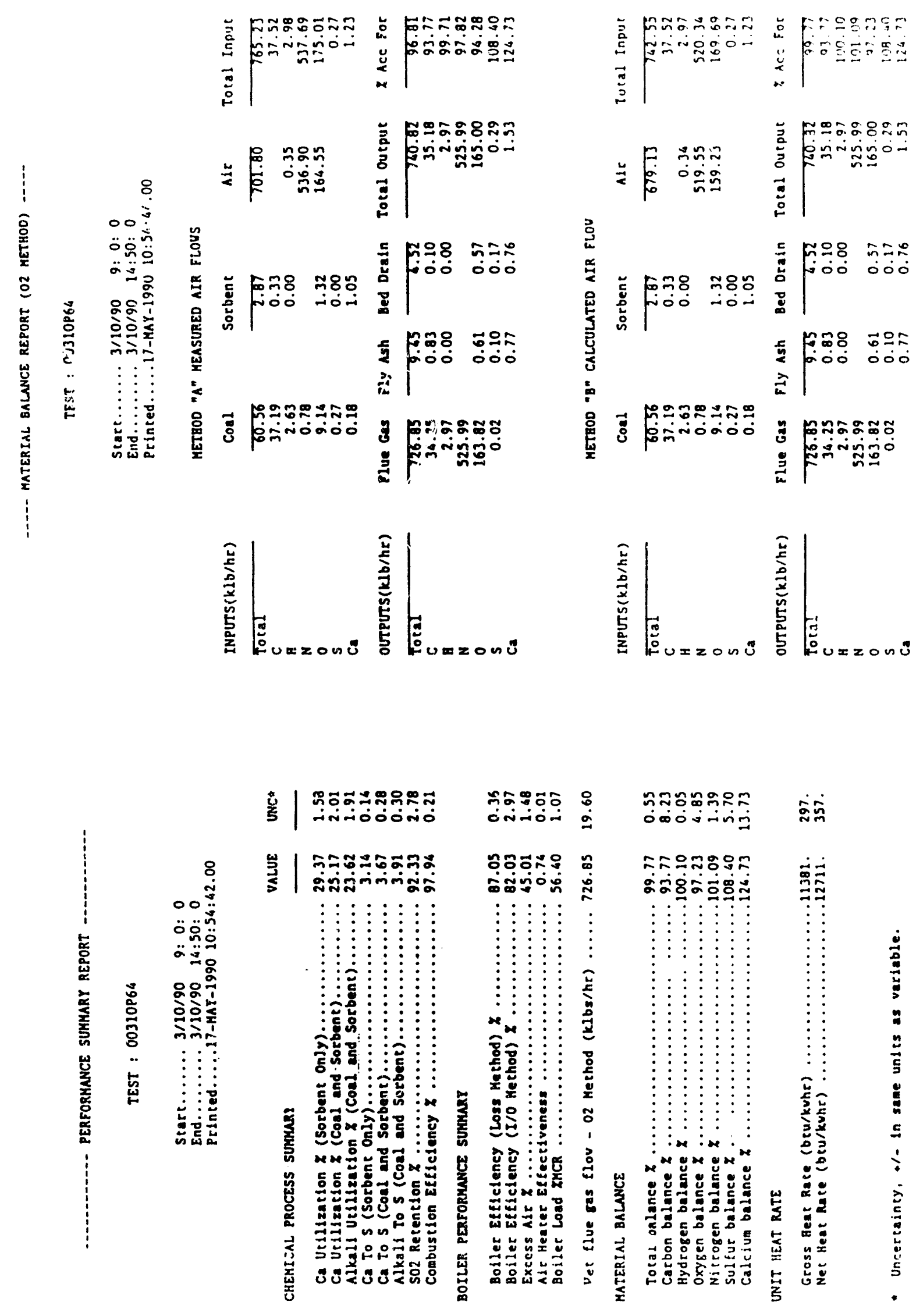



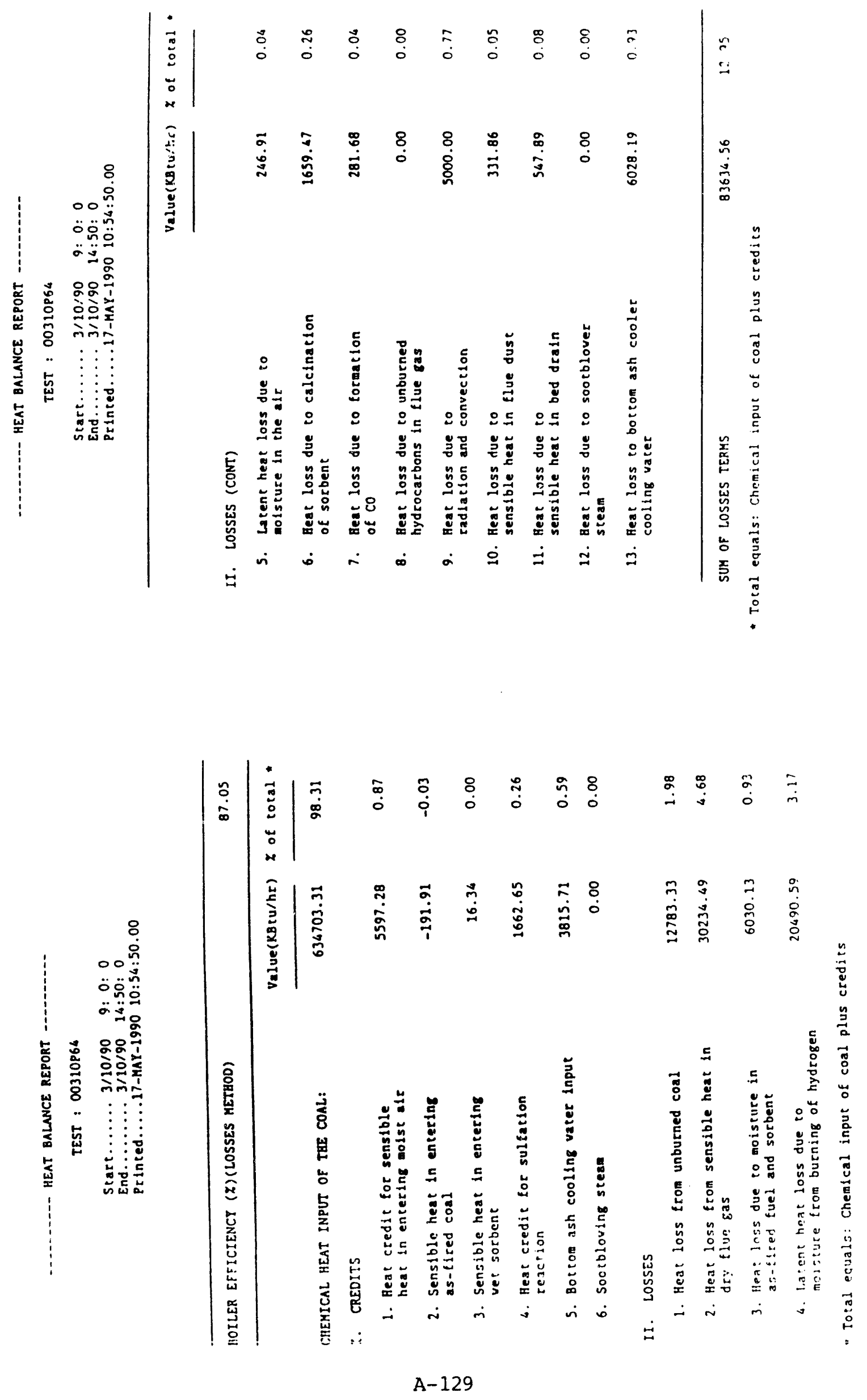


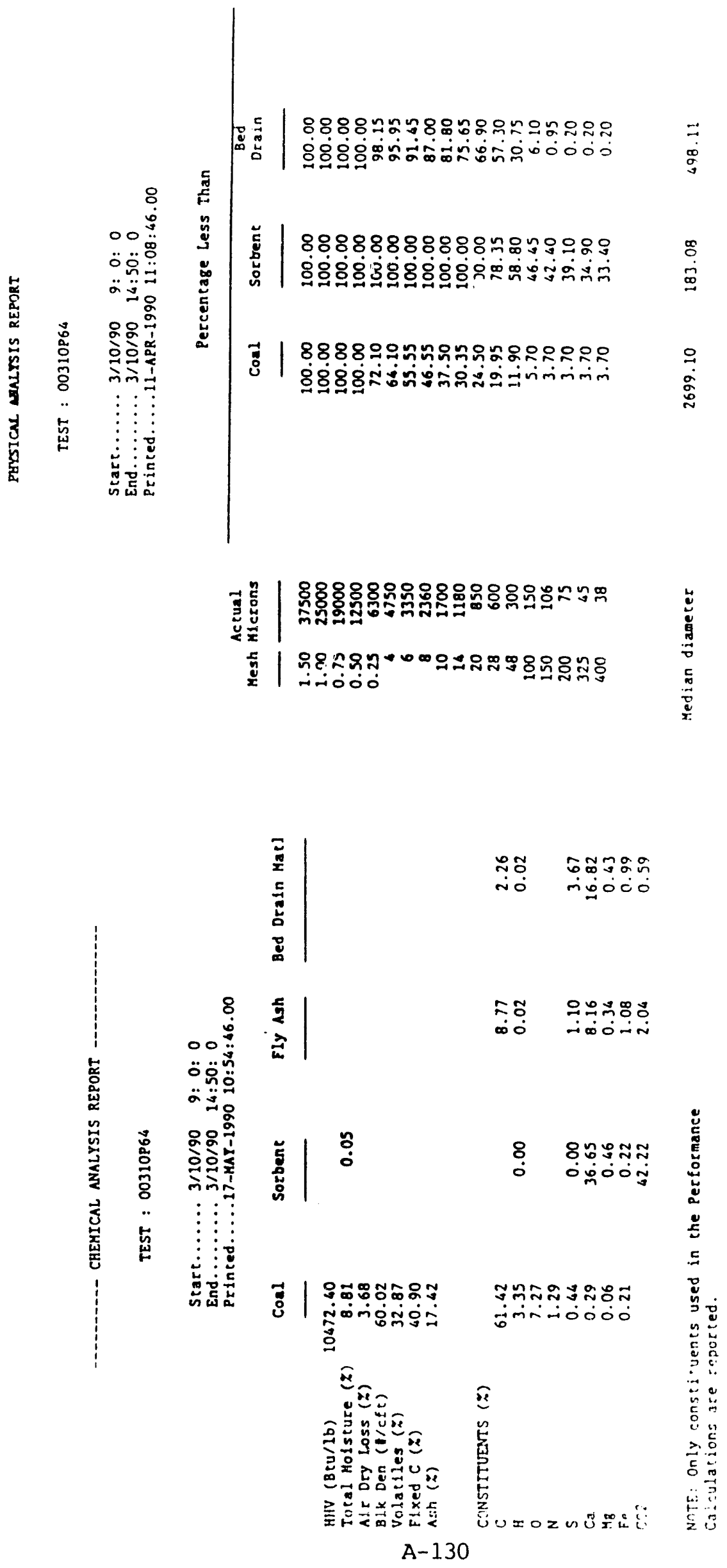



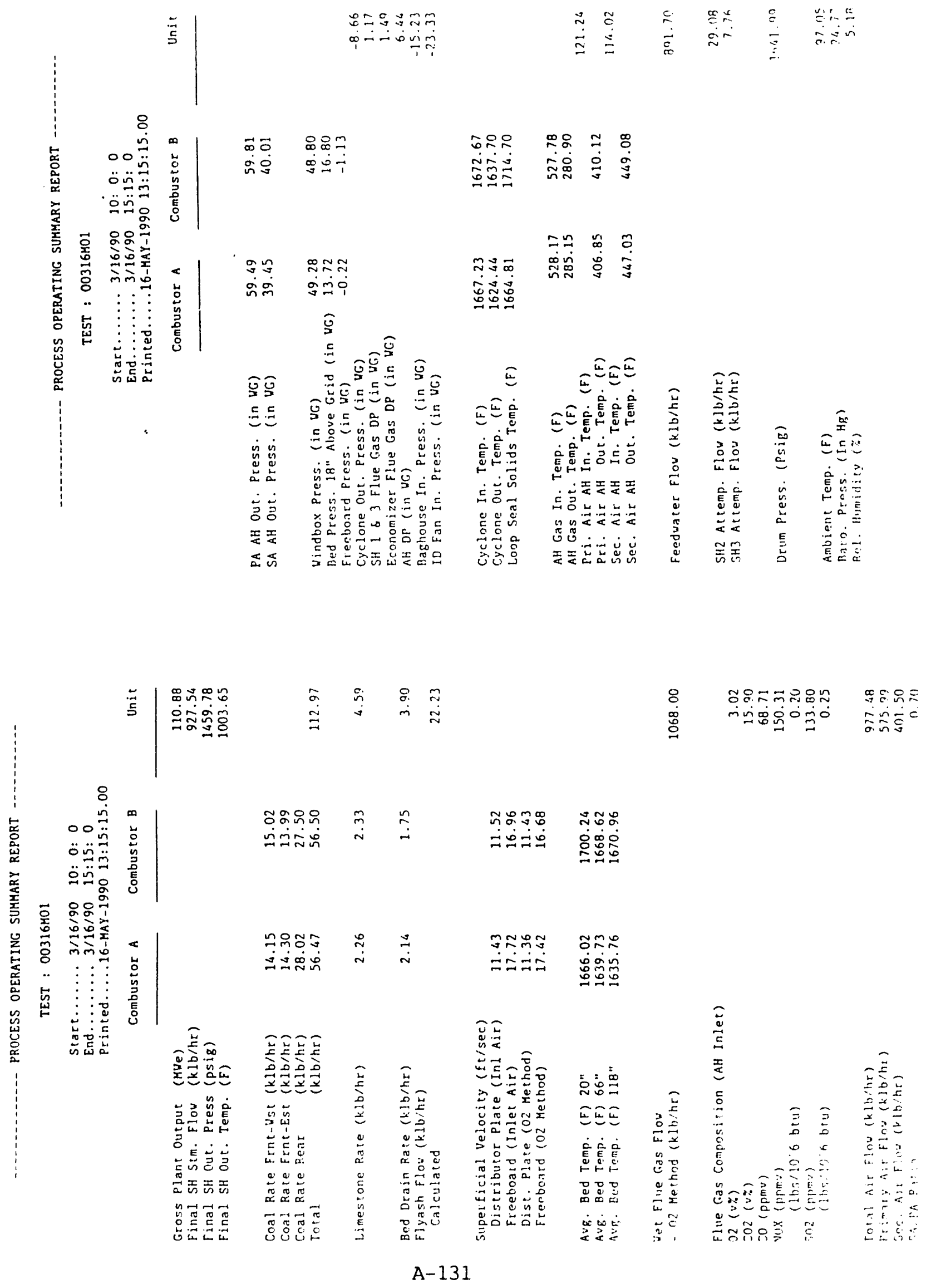


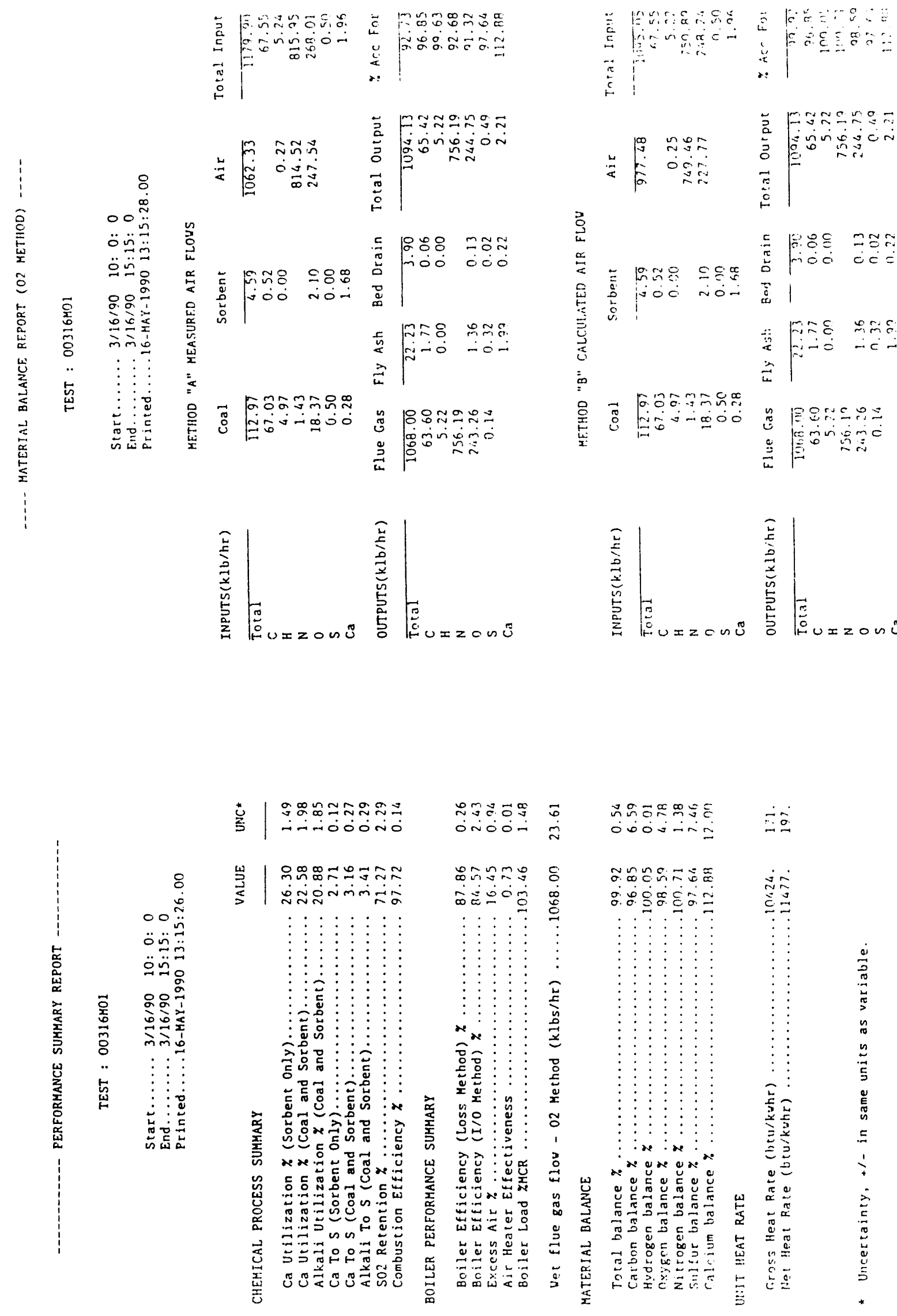



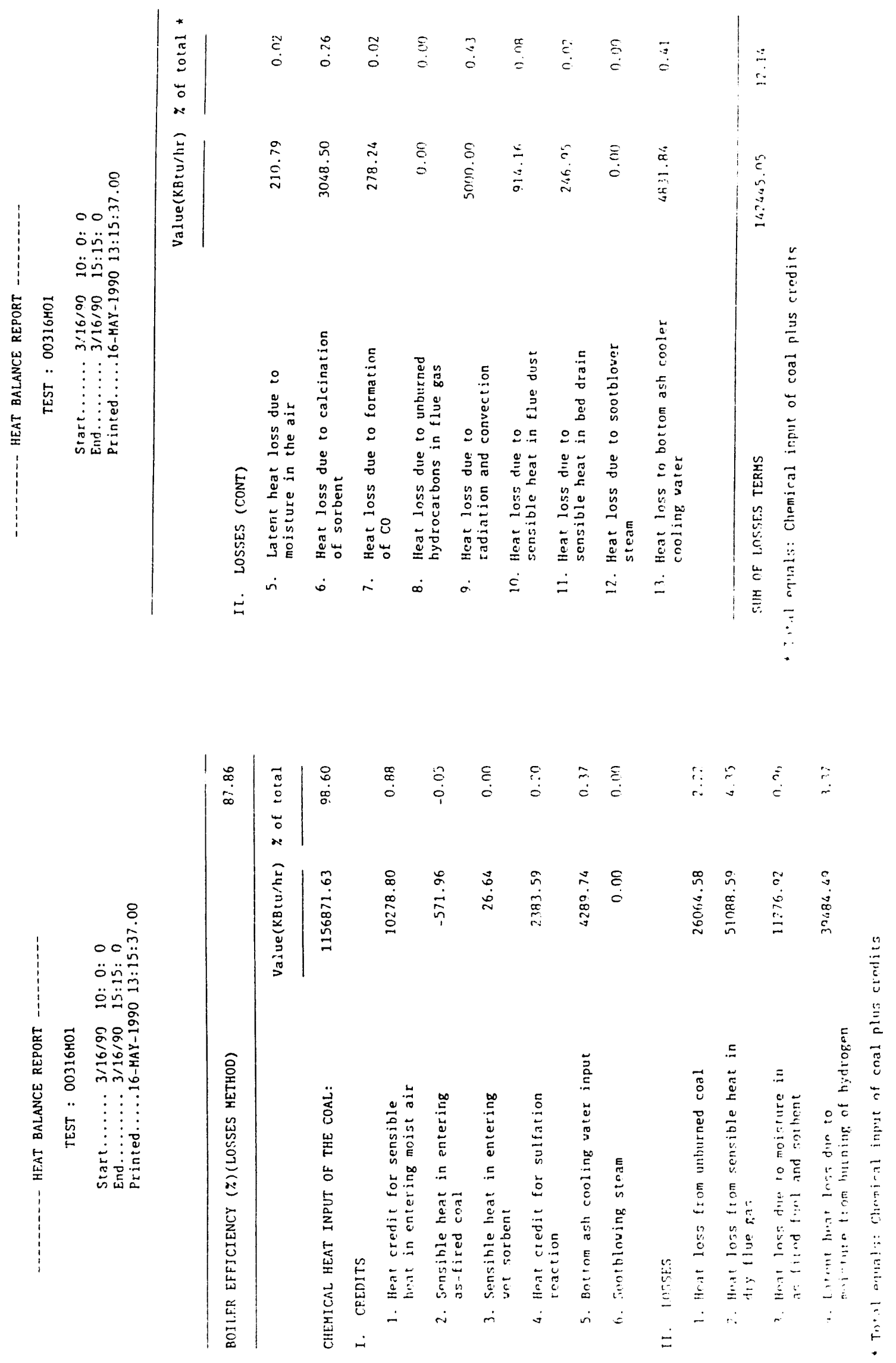

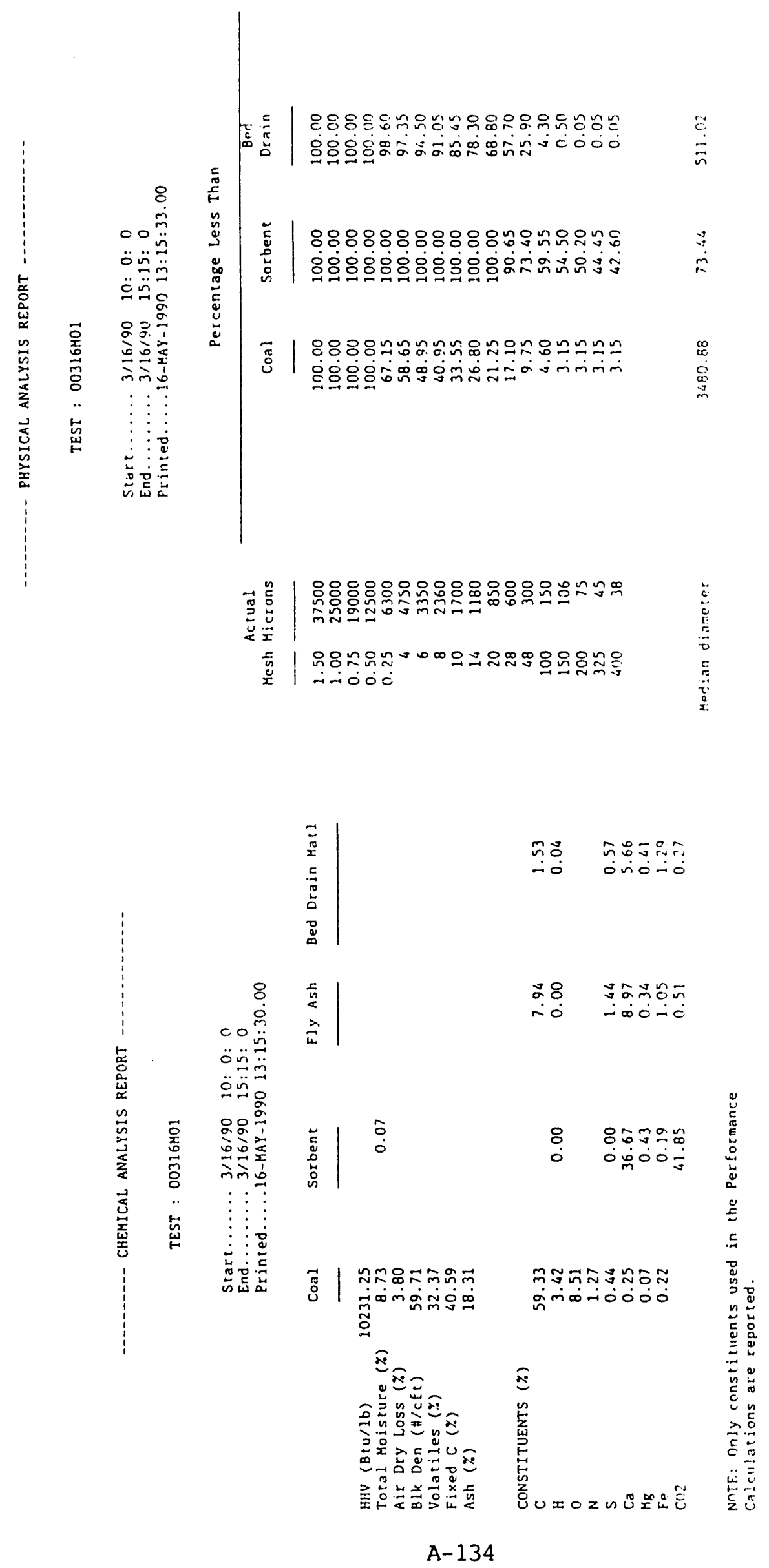

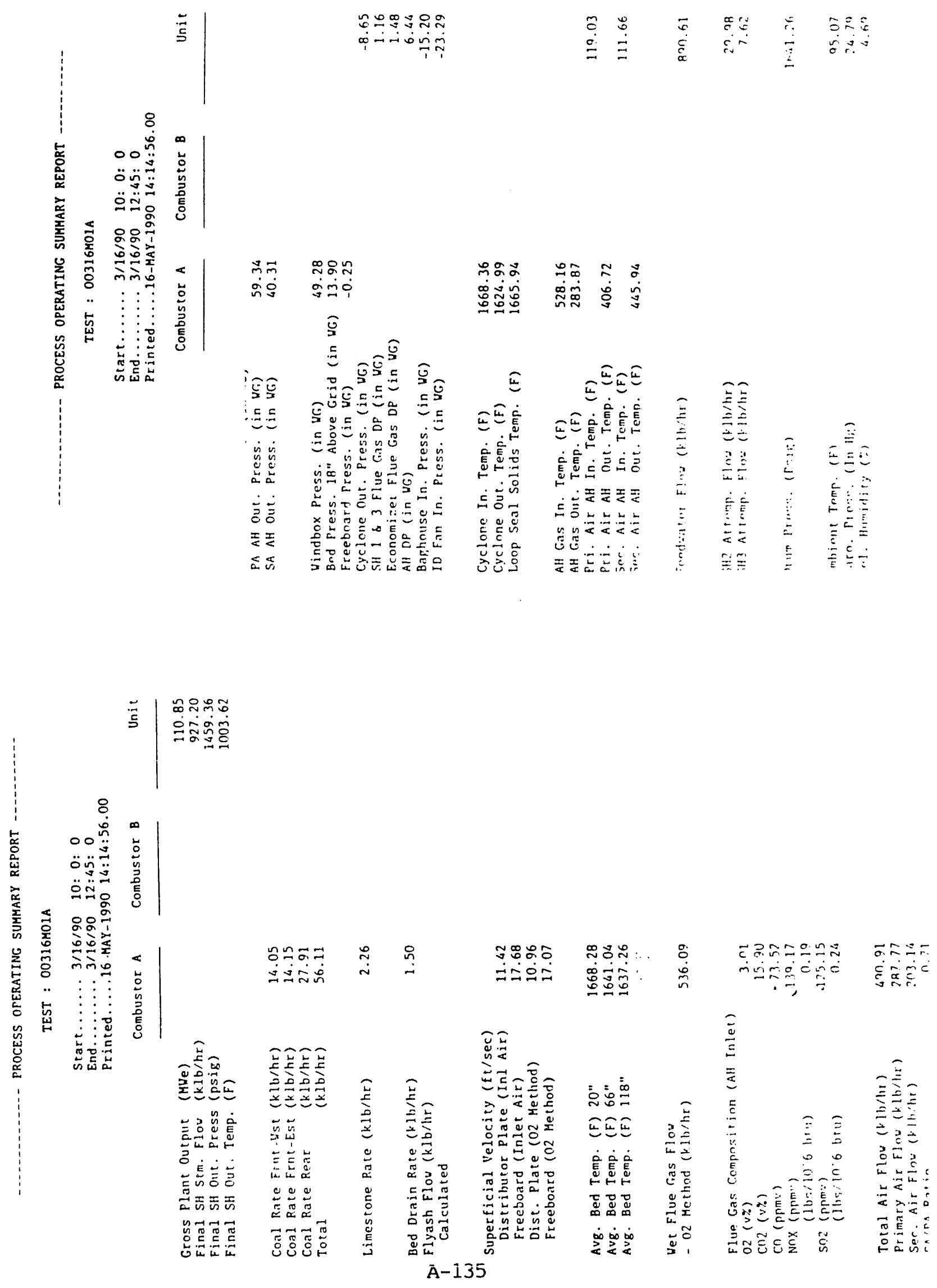


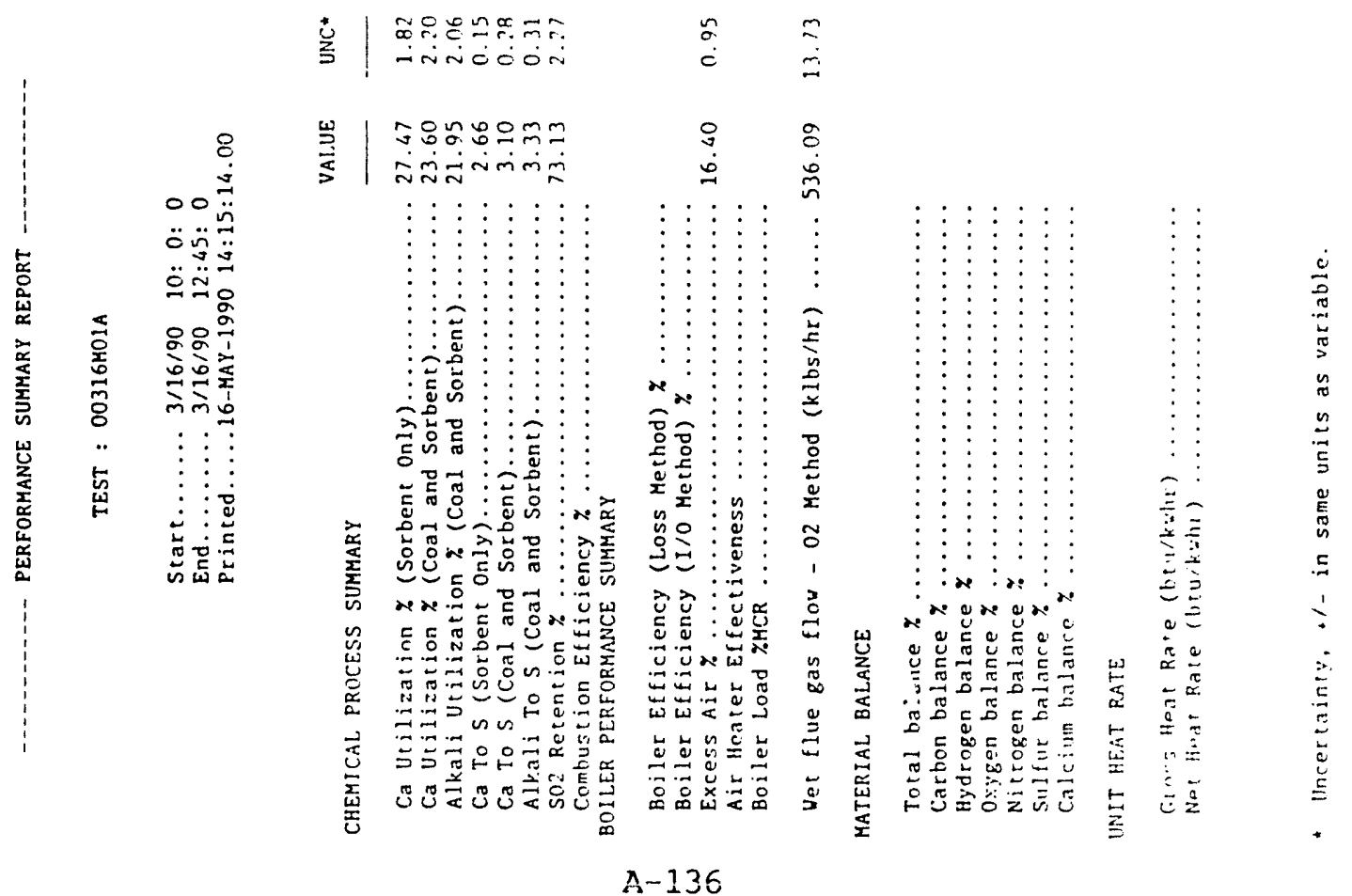



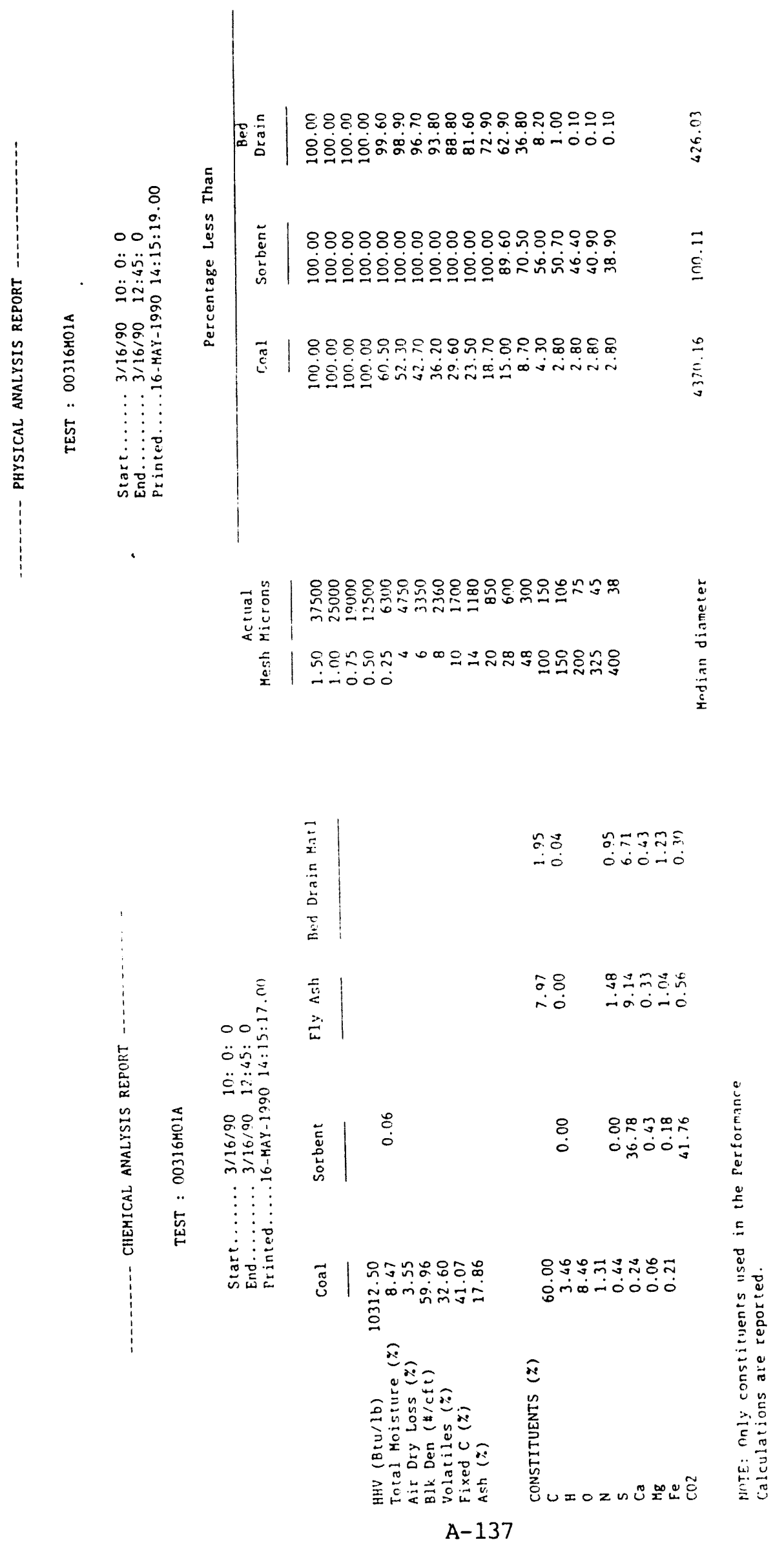

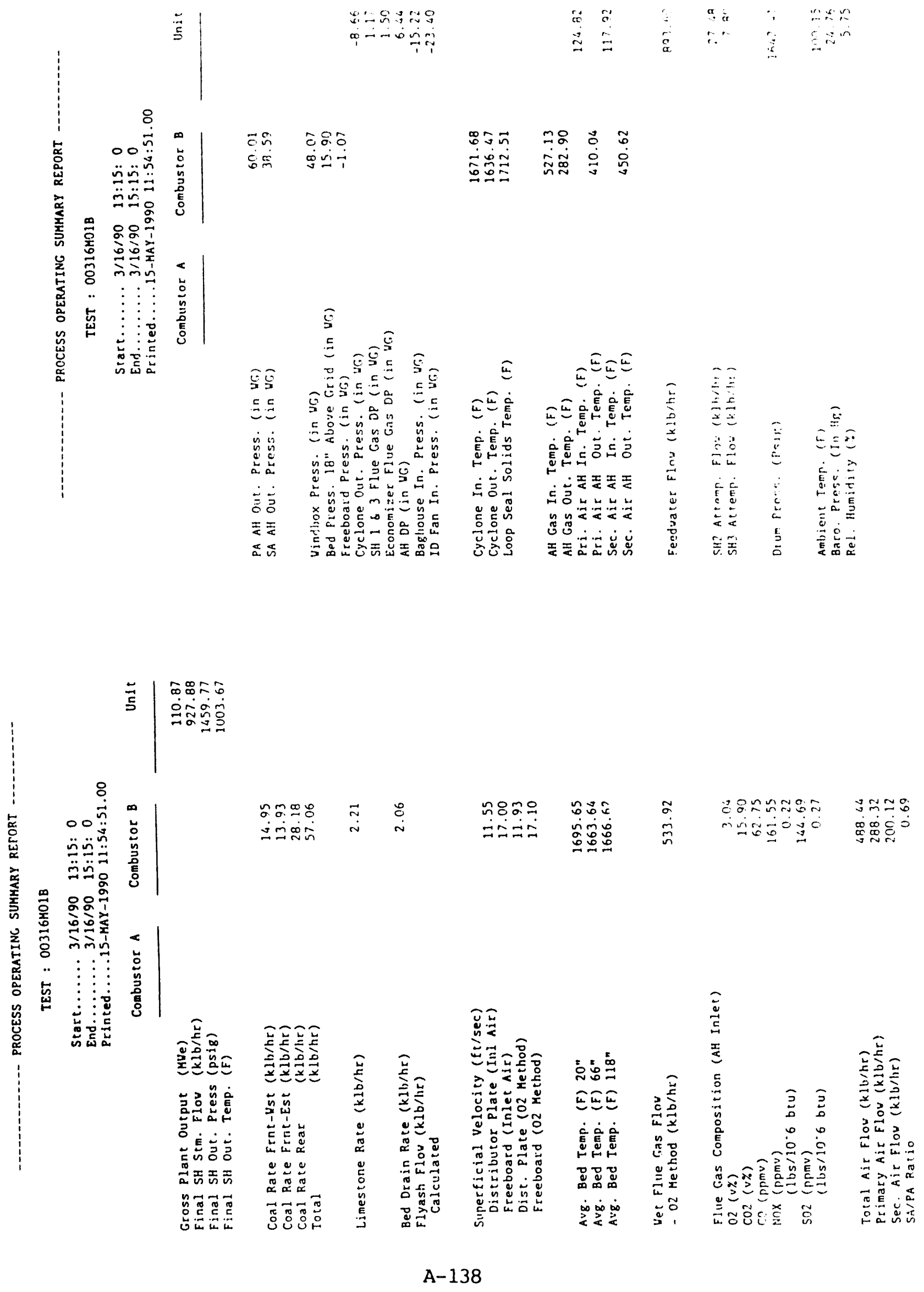


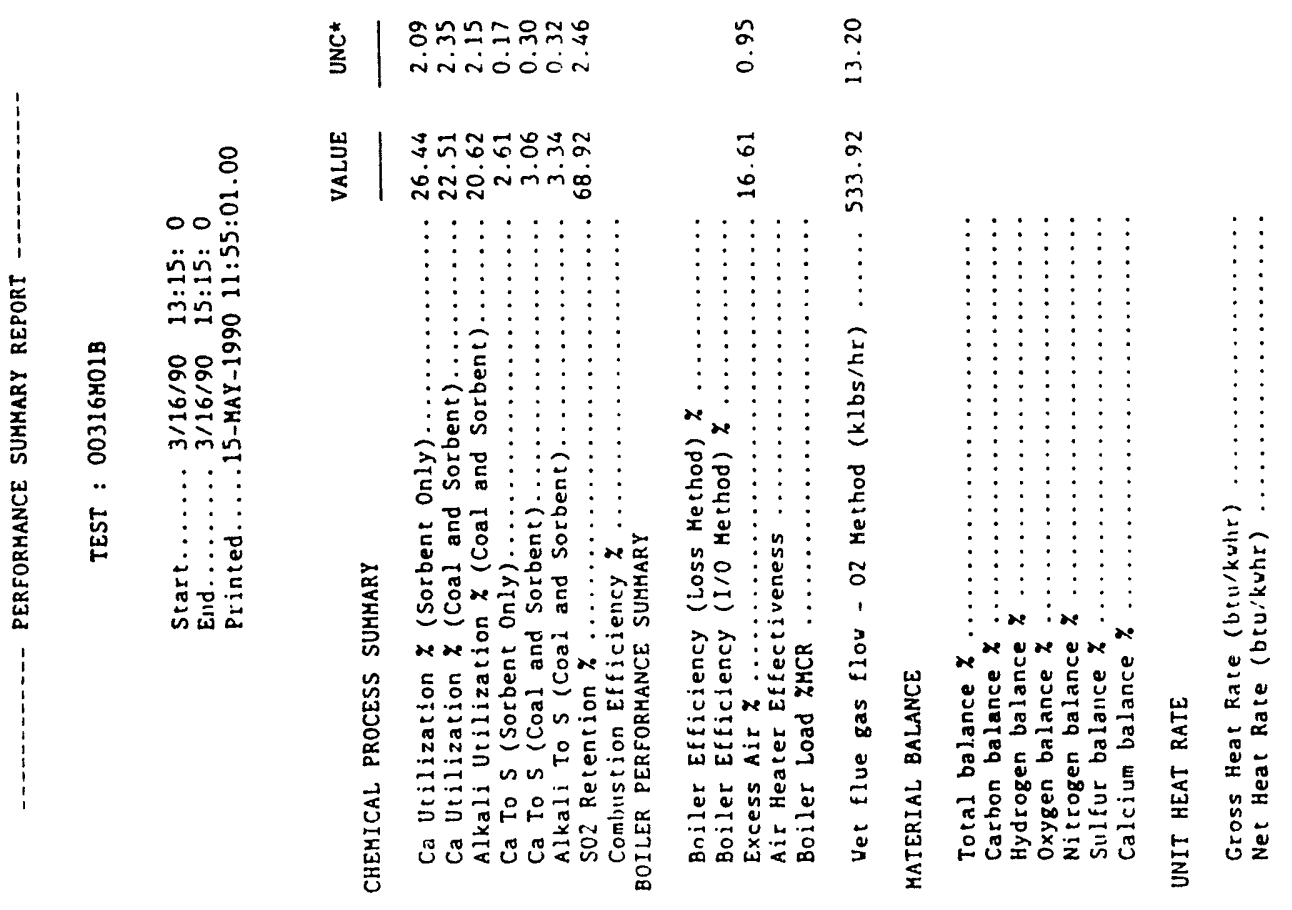

A-139 


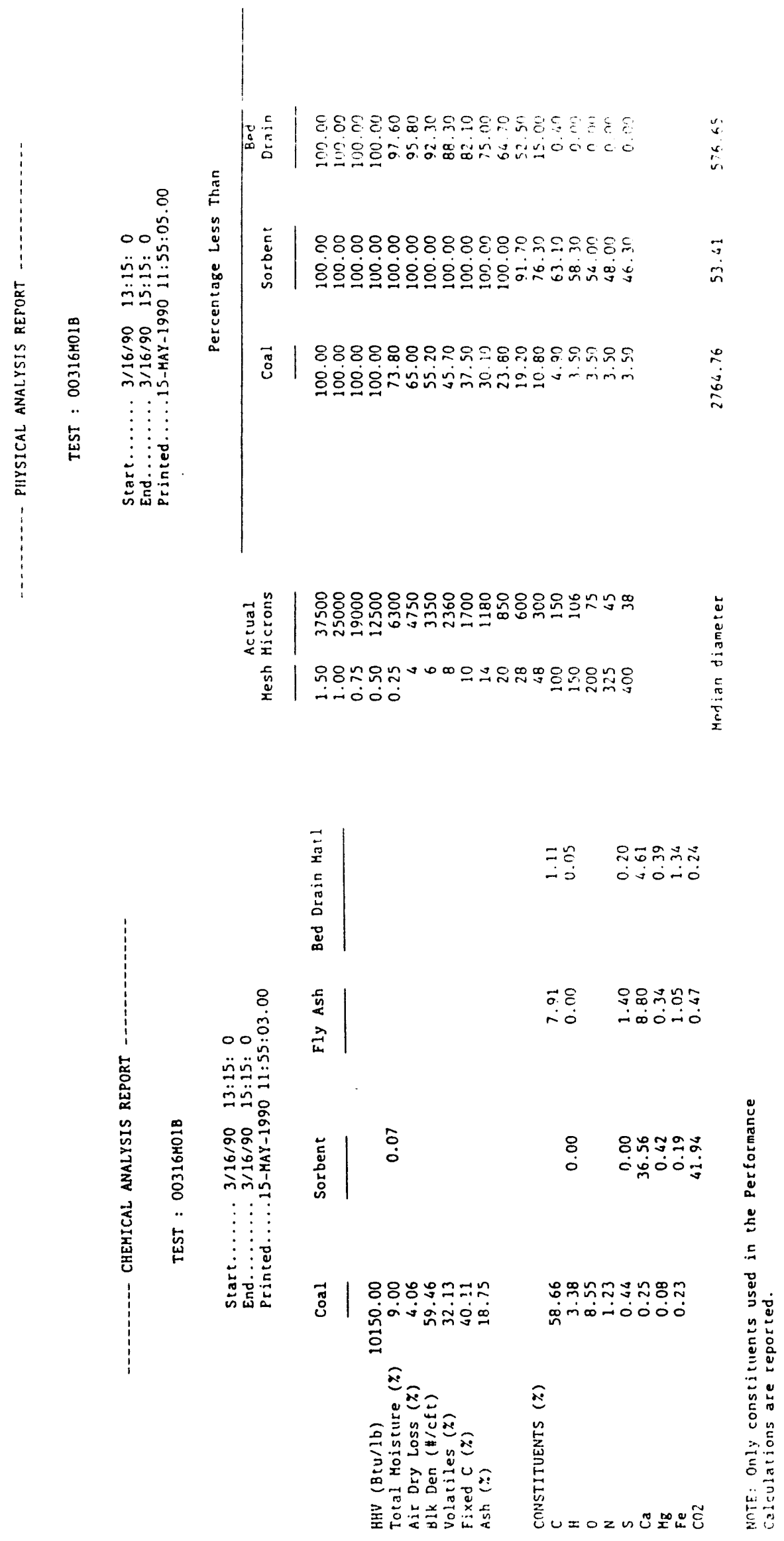

A-140 

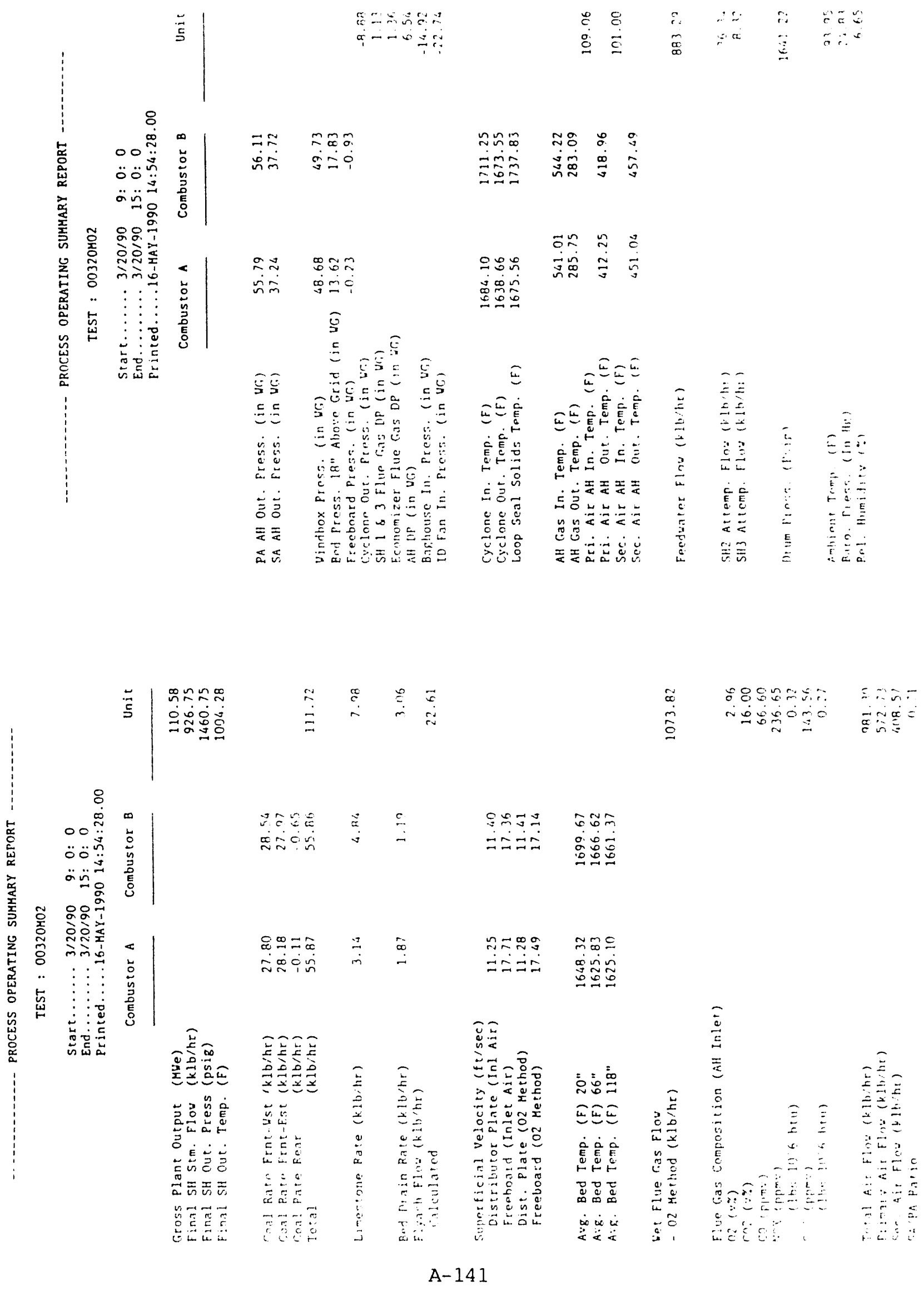


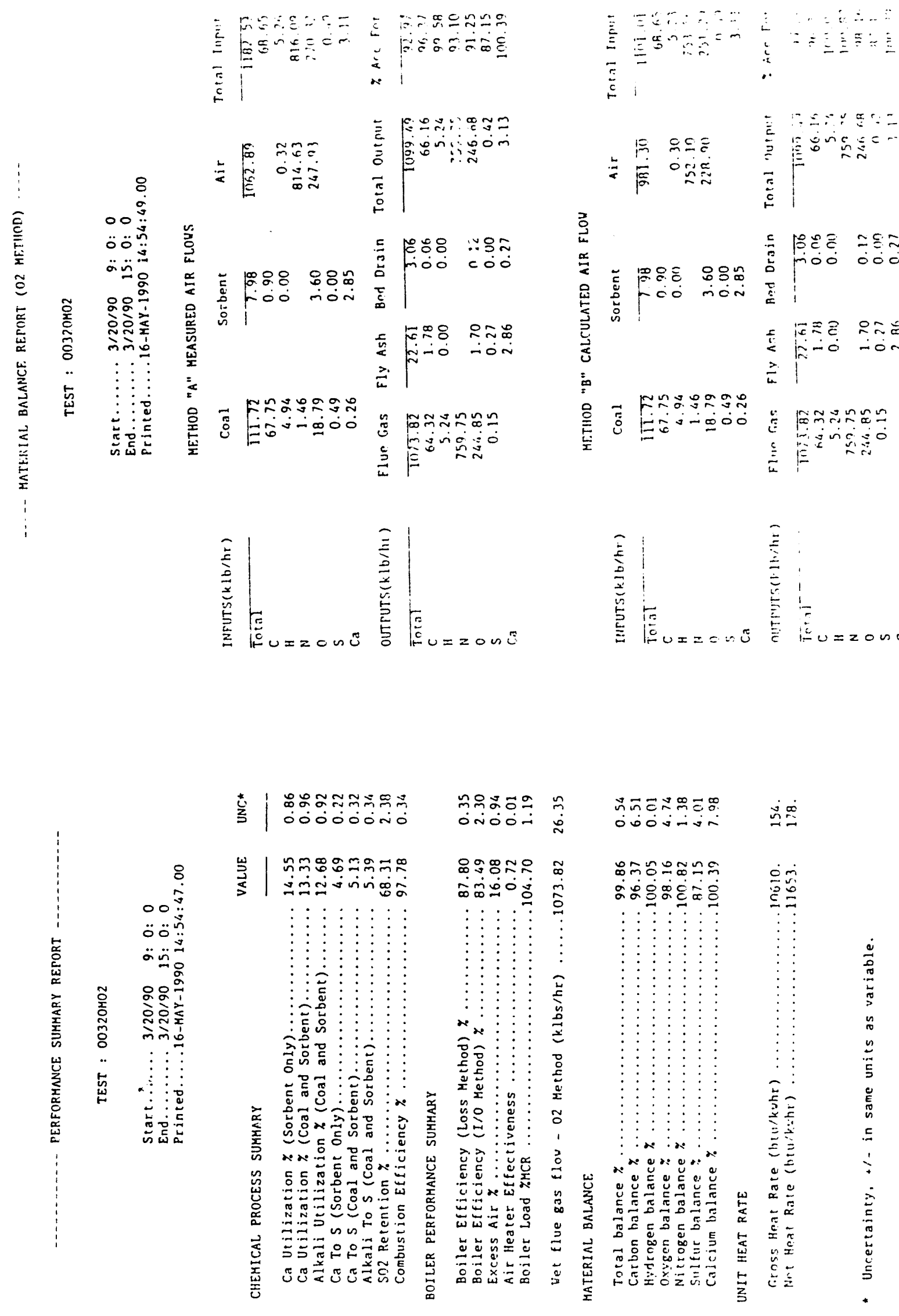




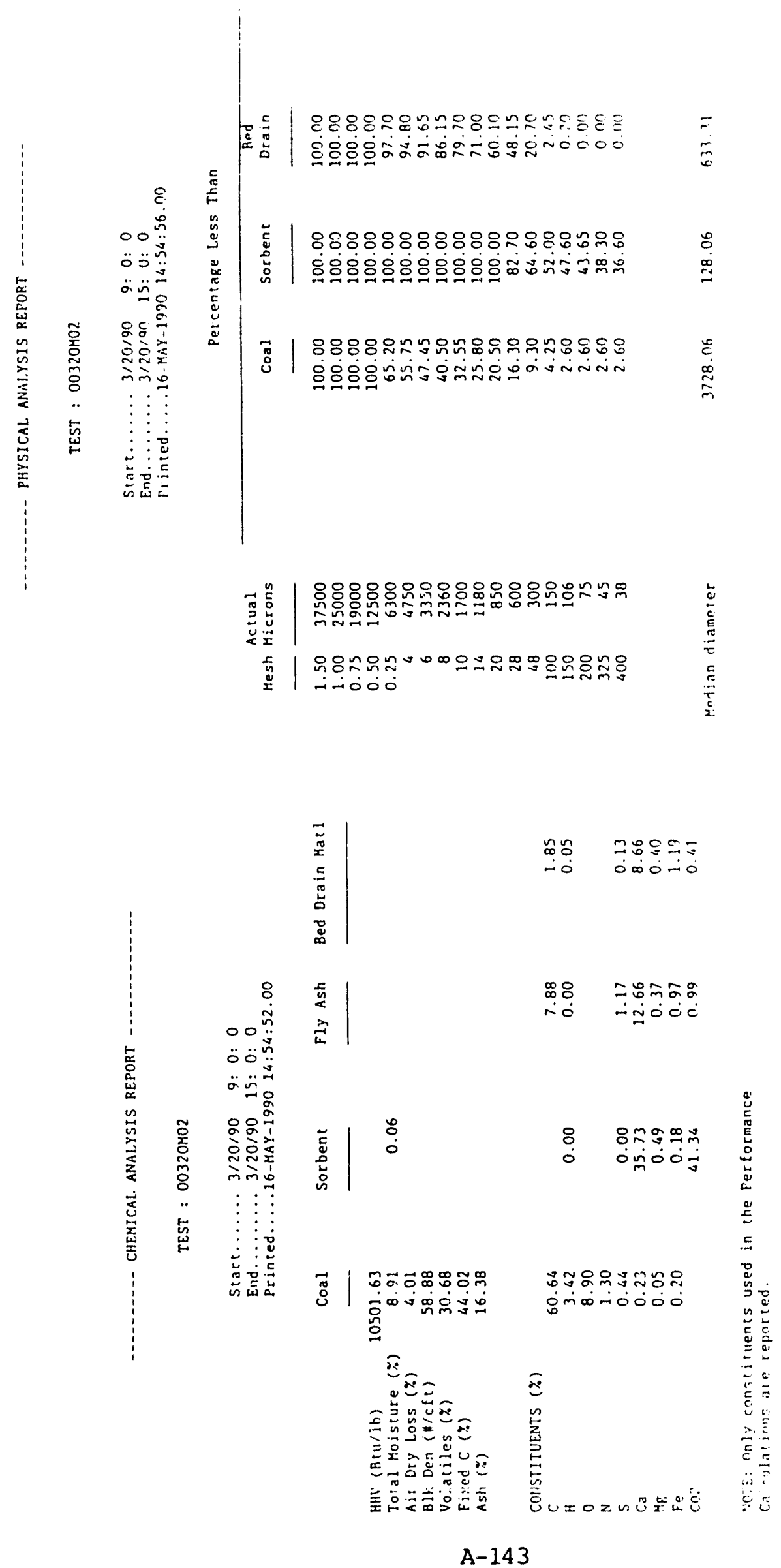



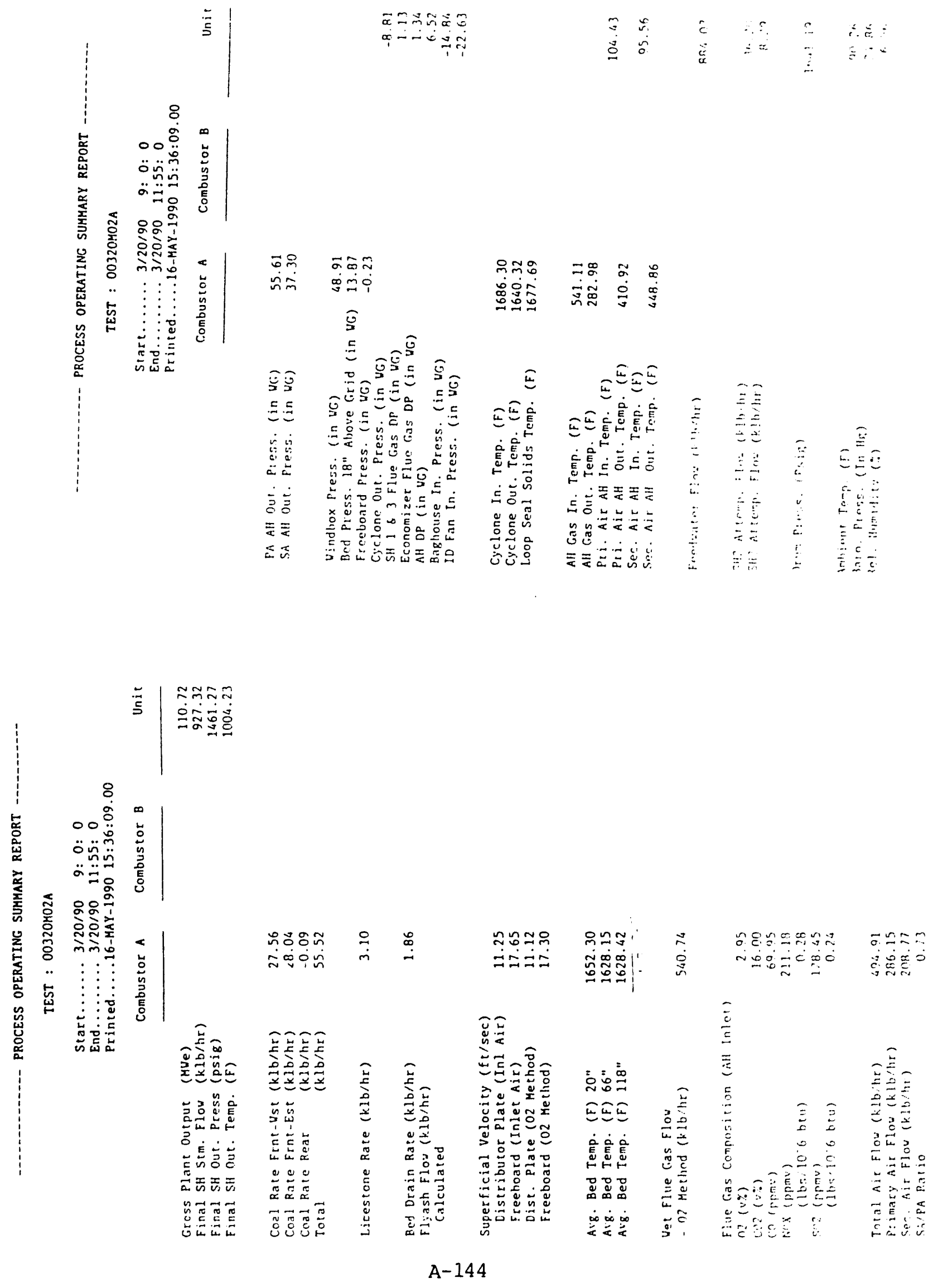


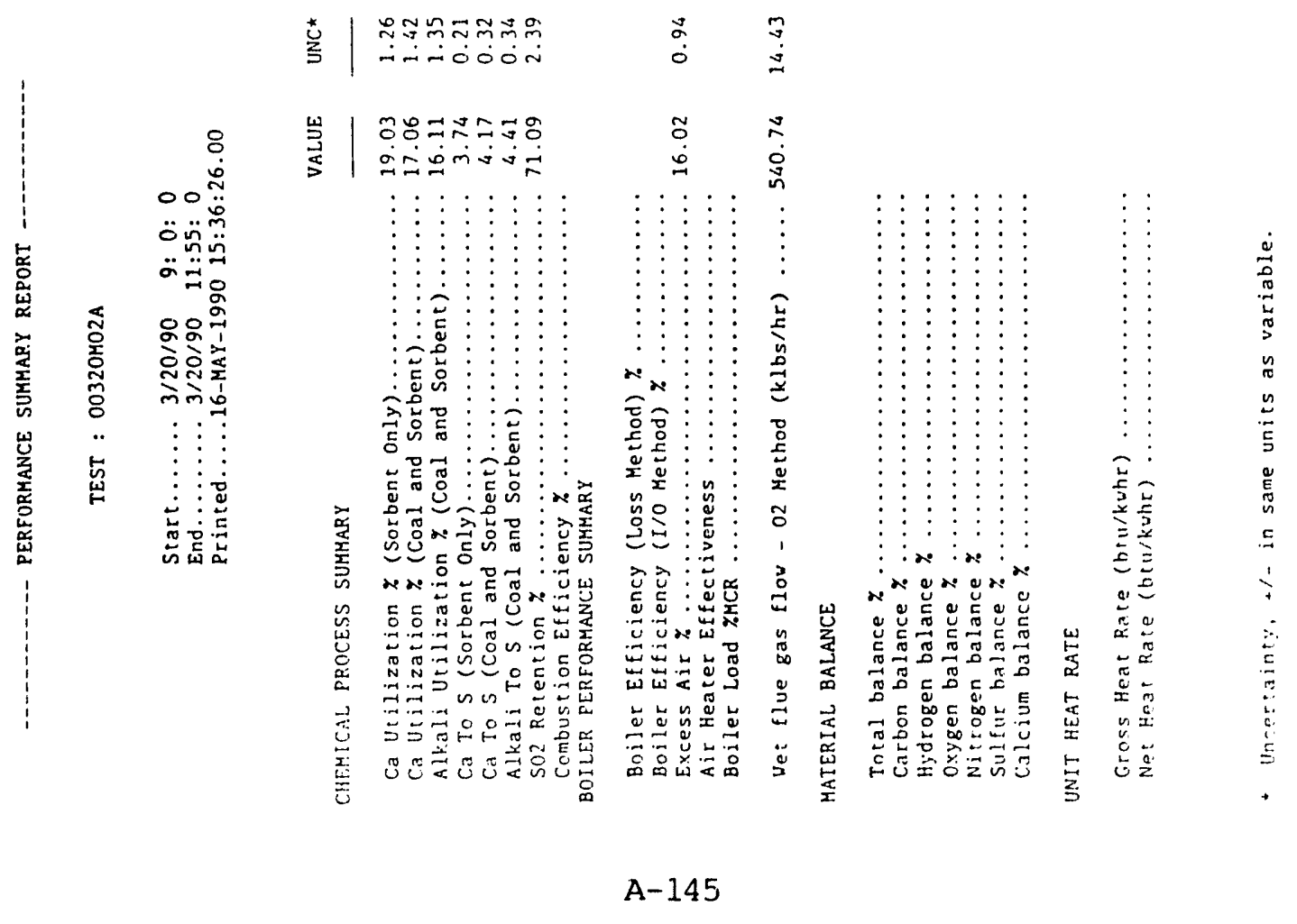



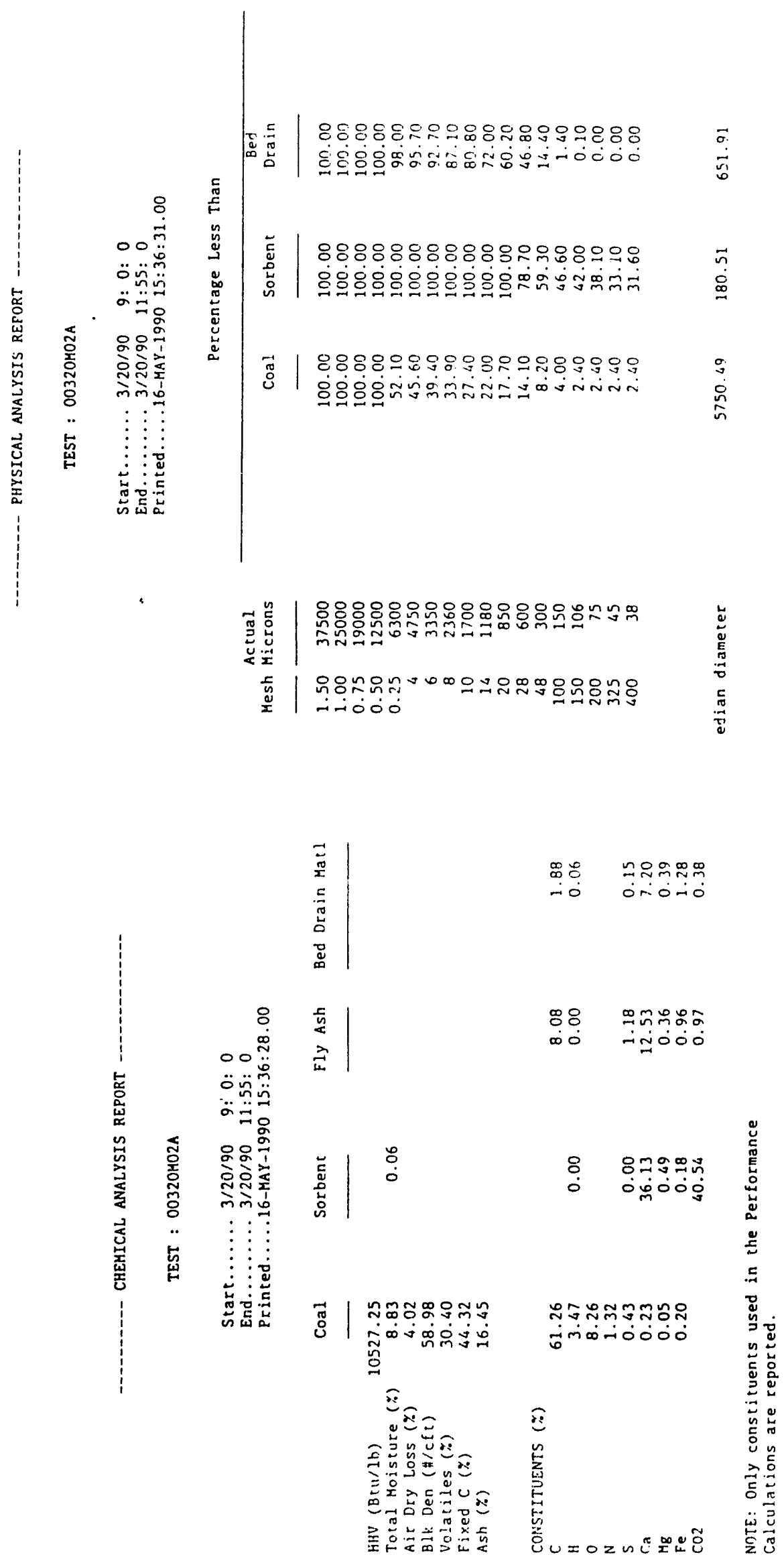

A-146 

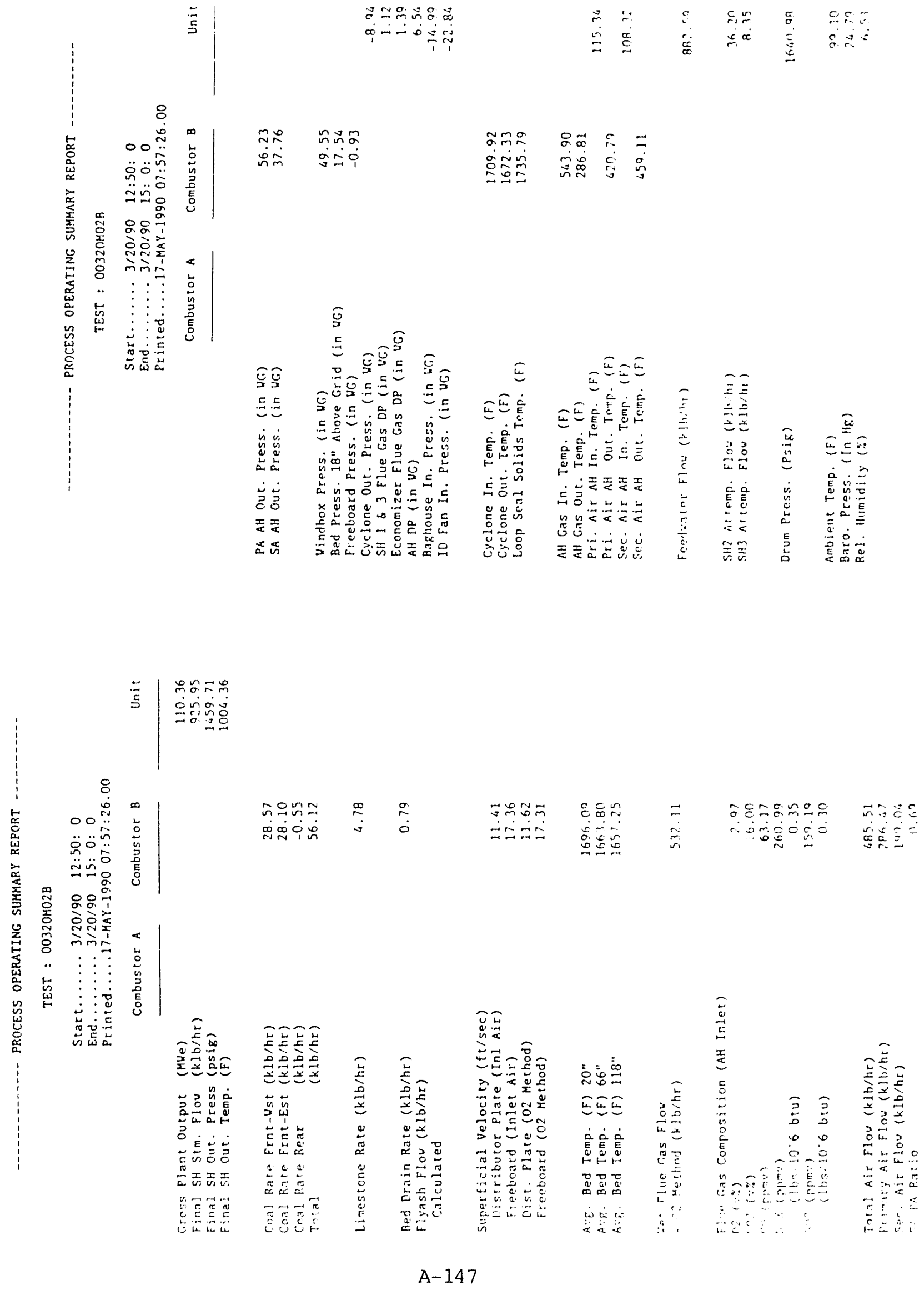


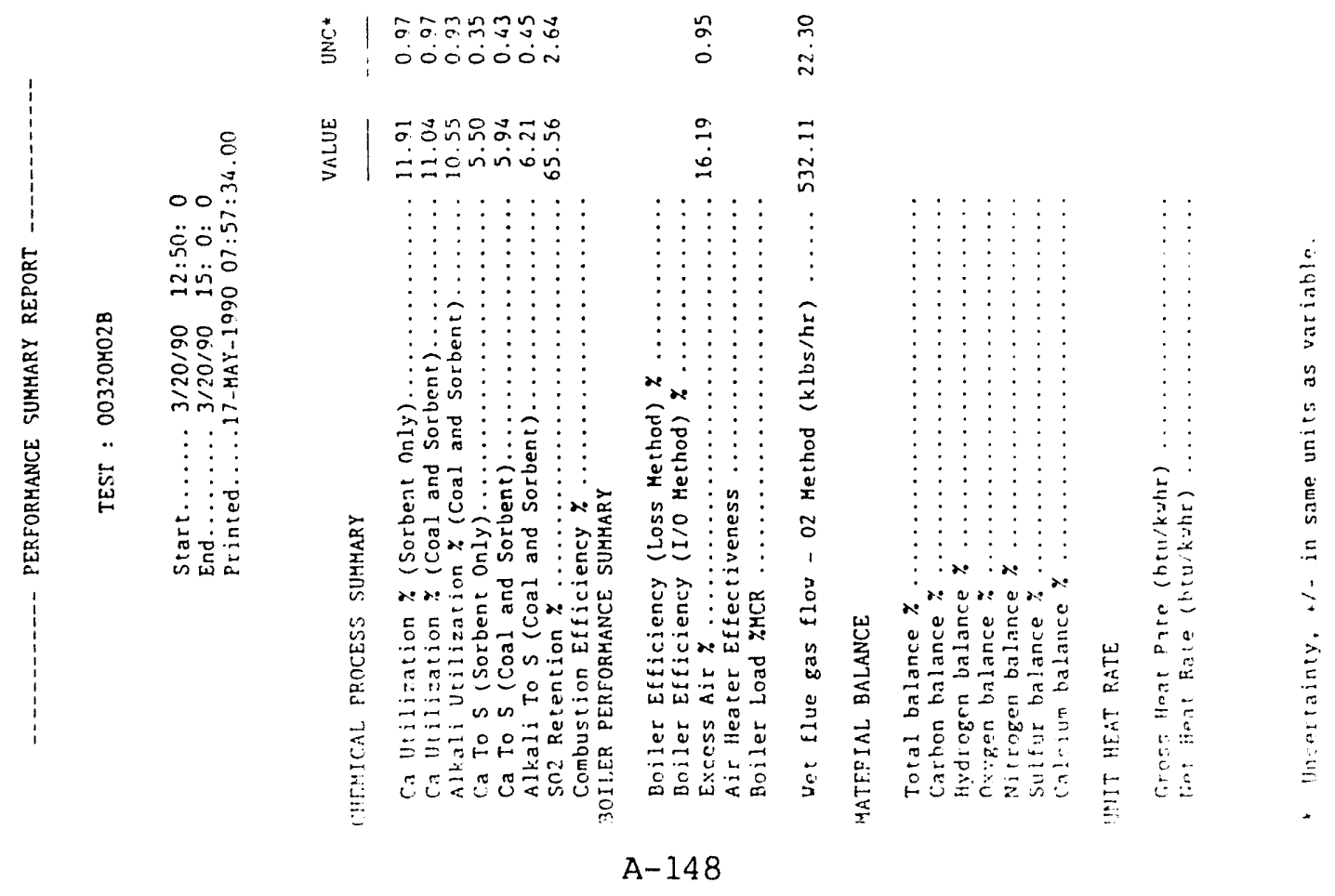



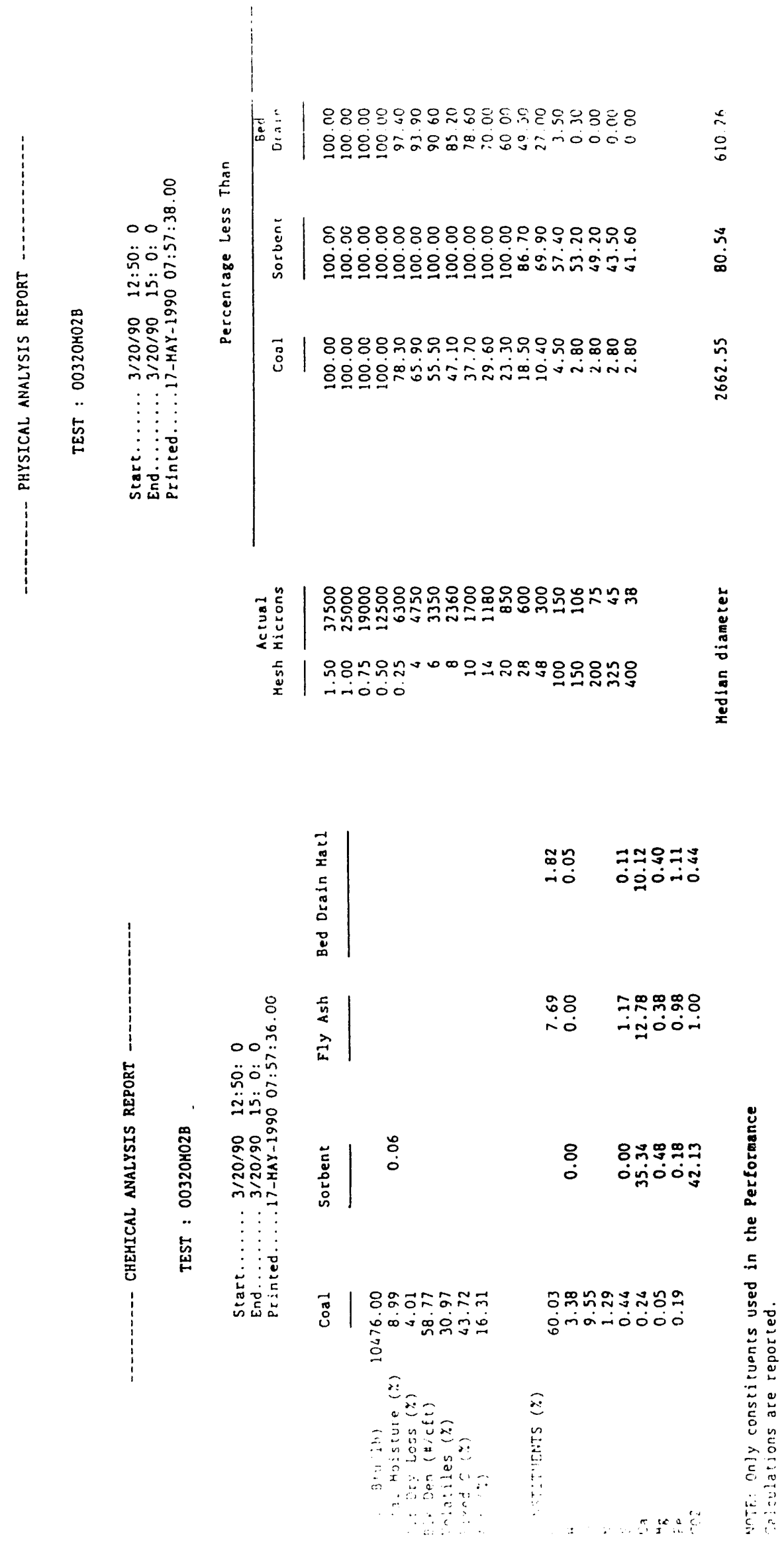


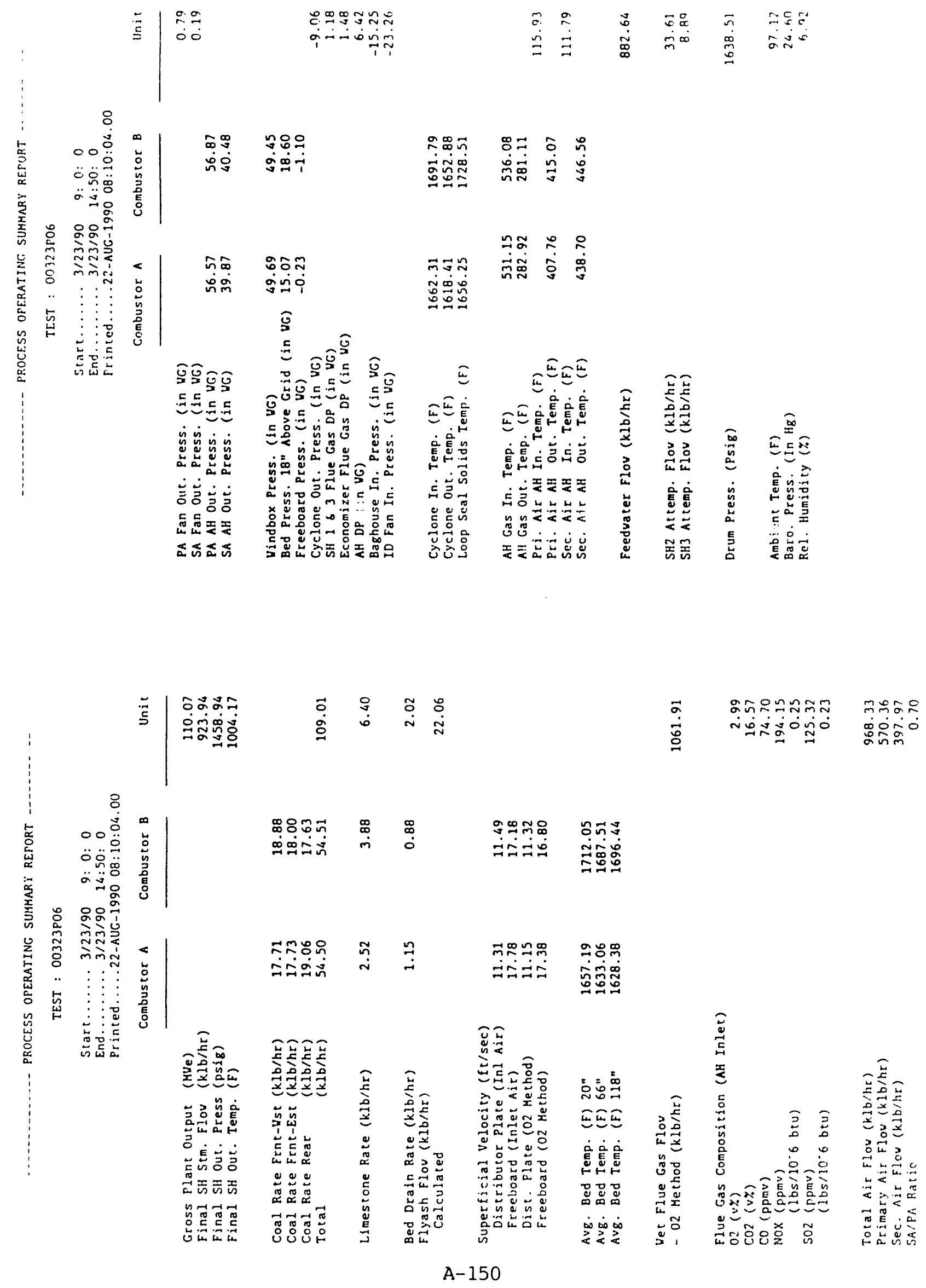




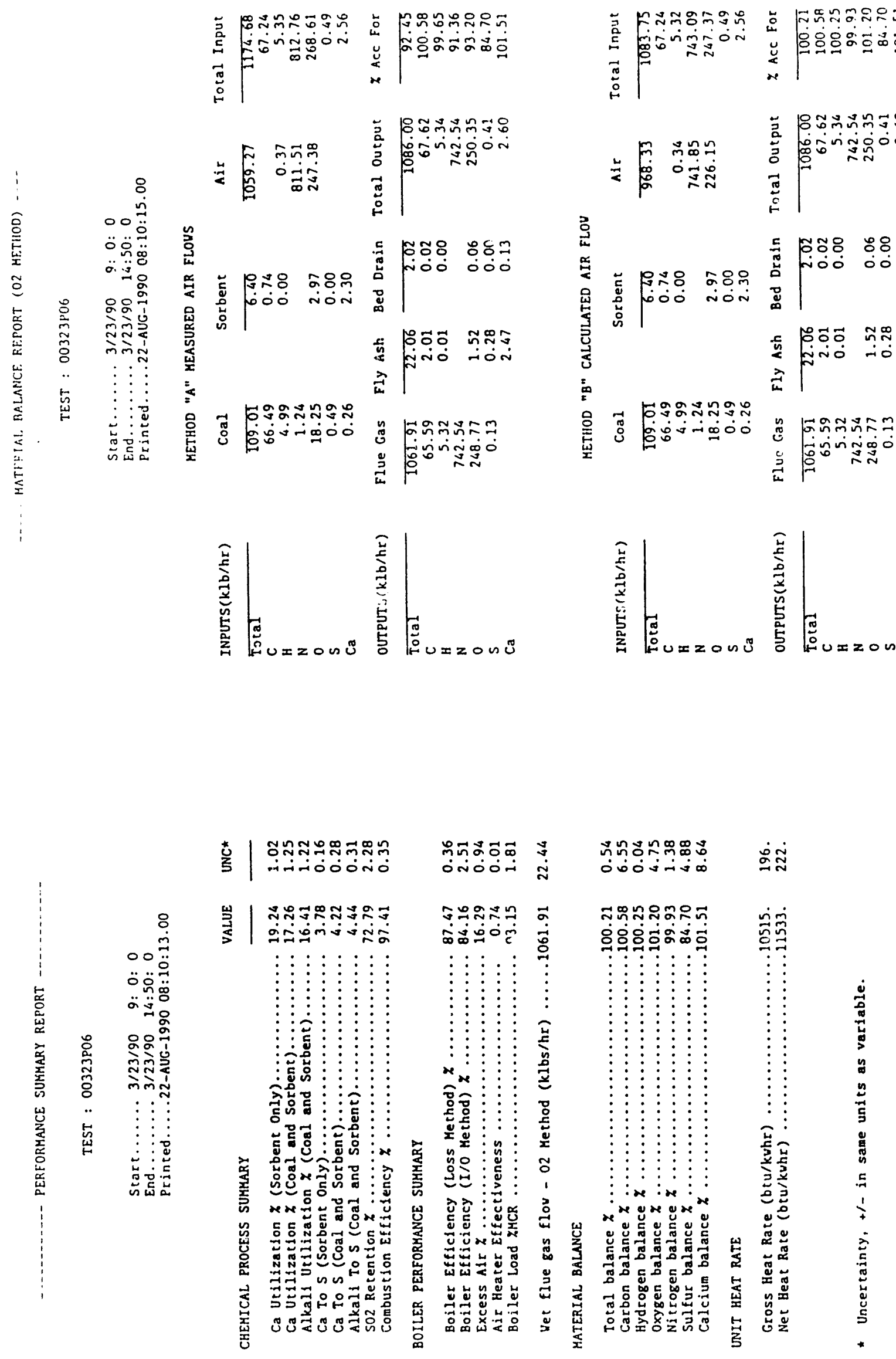



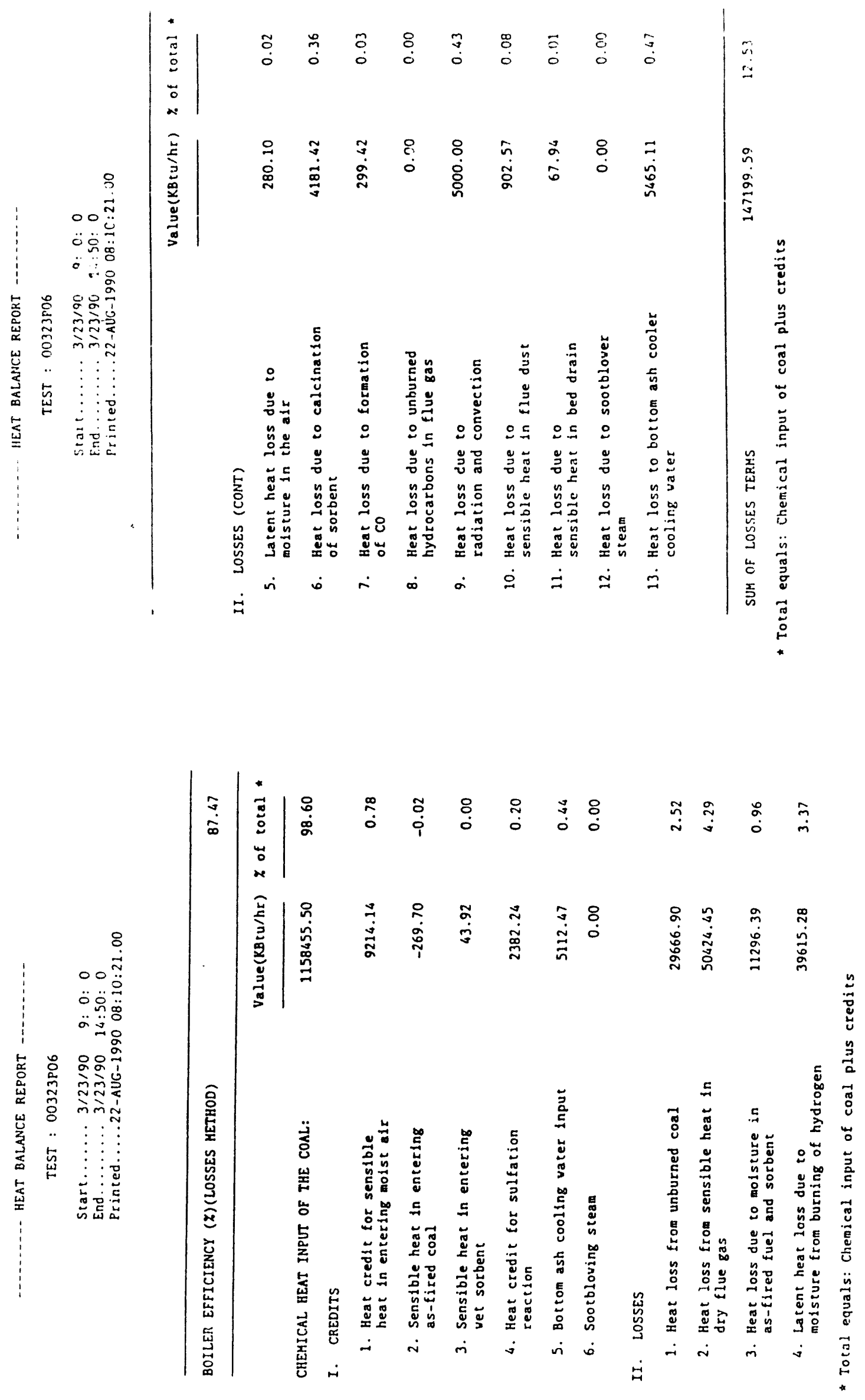

A- 152 

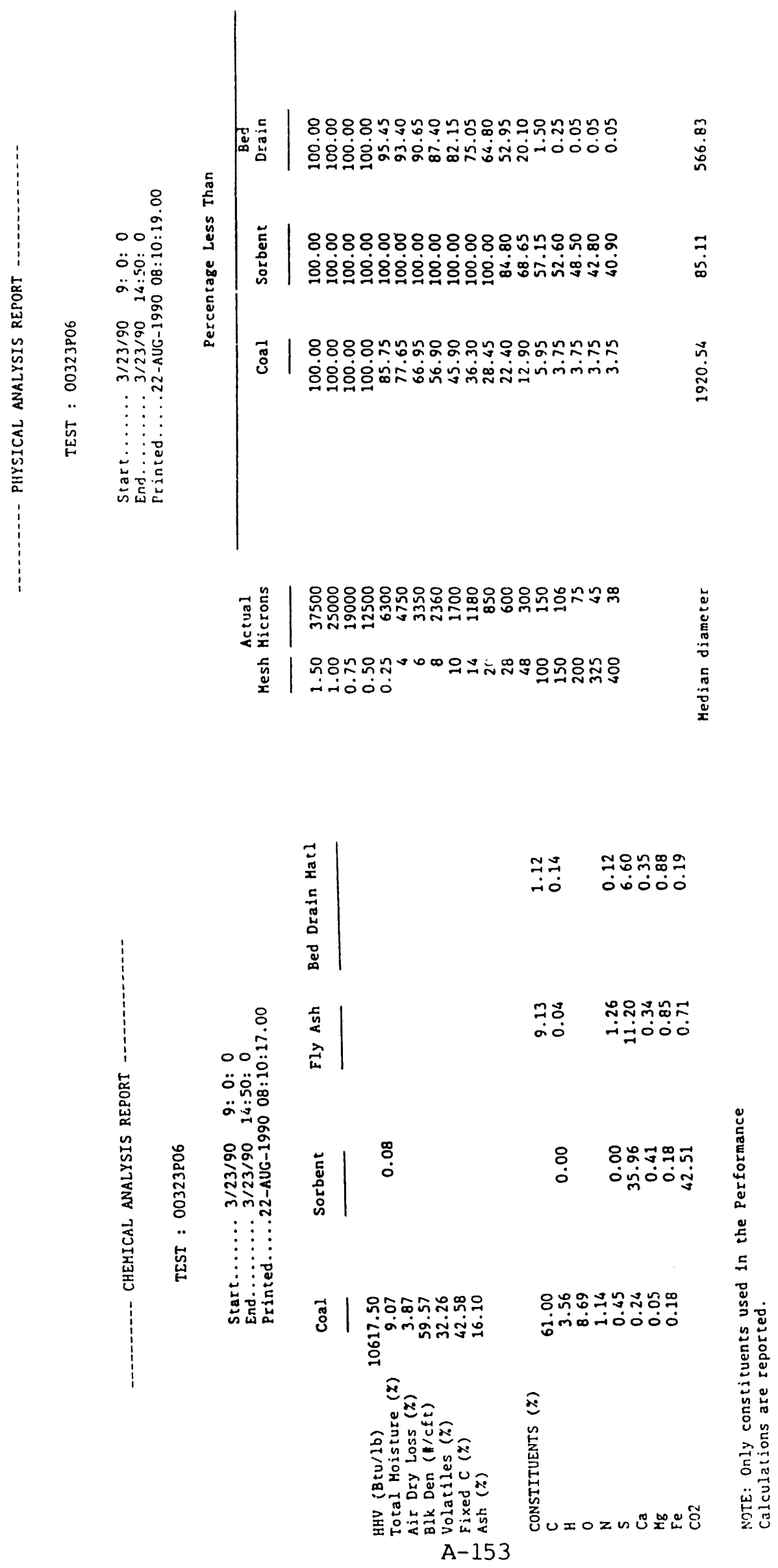

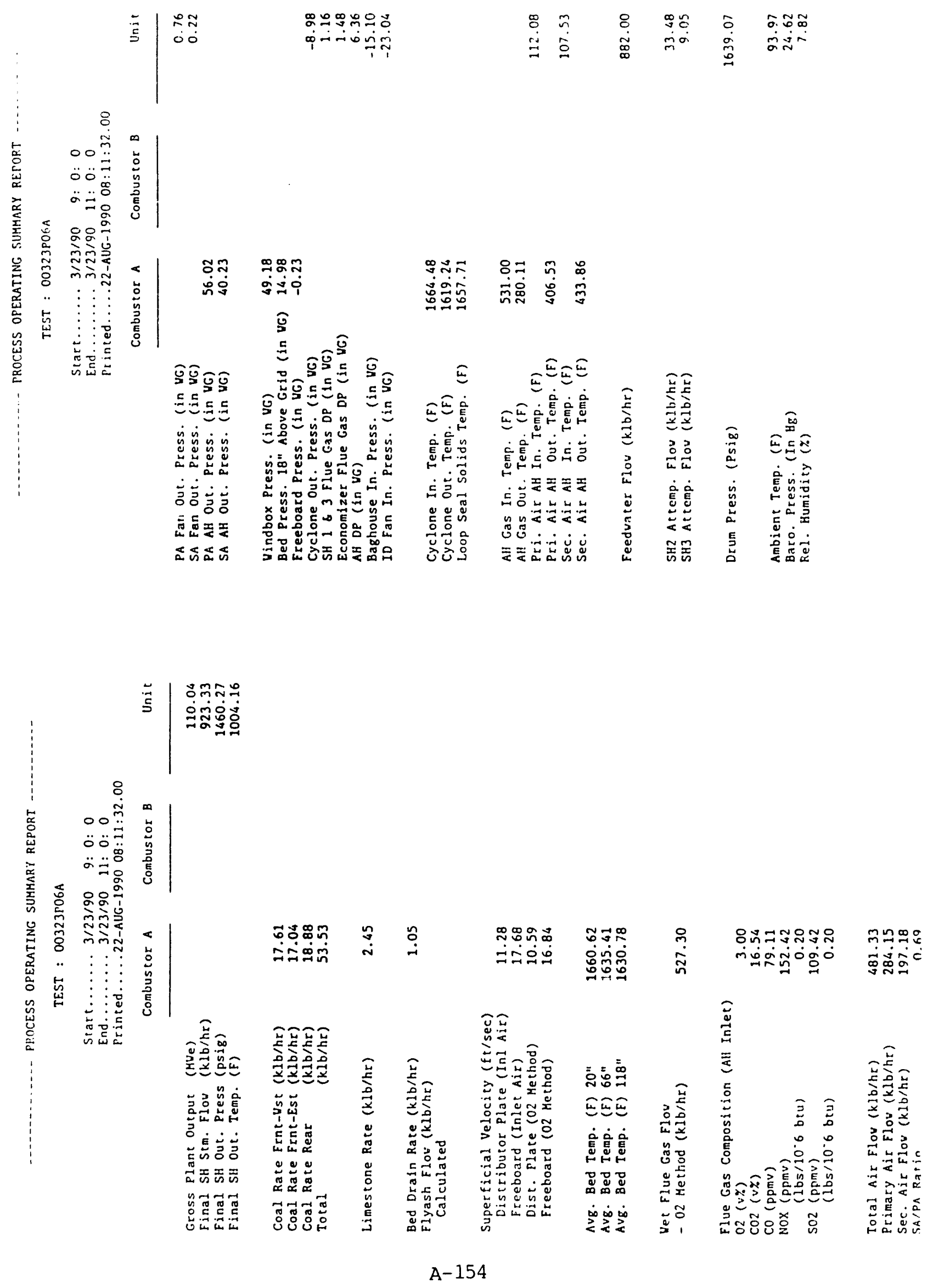


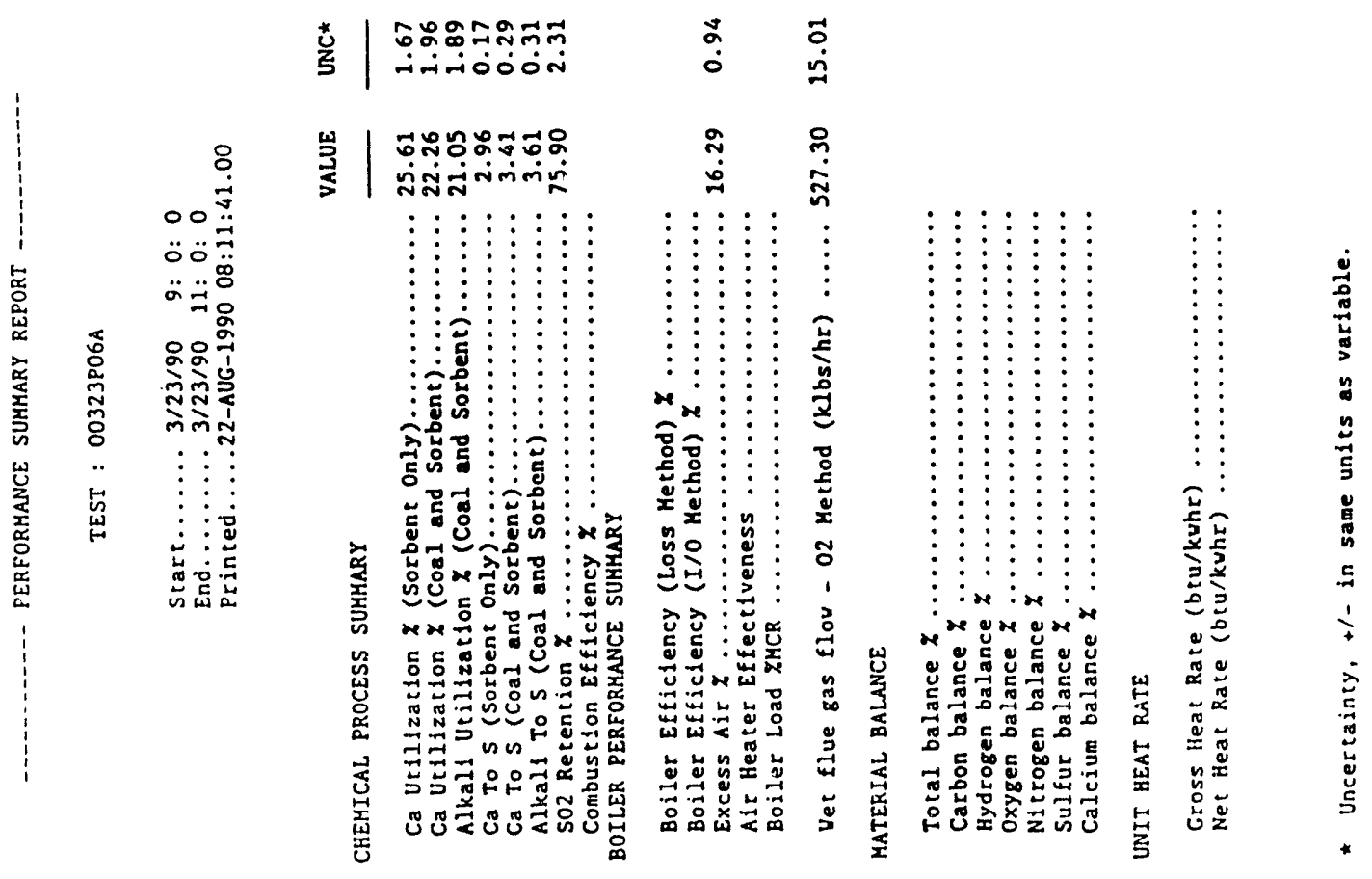



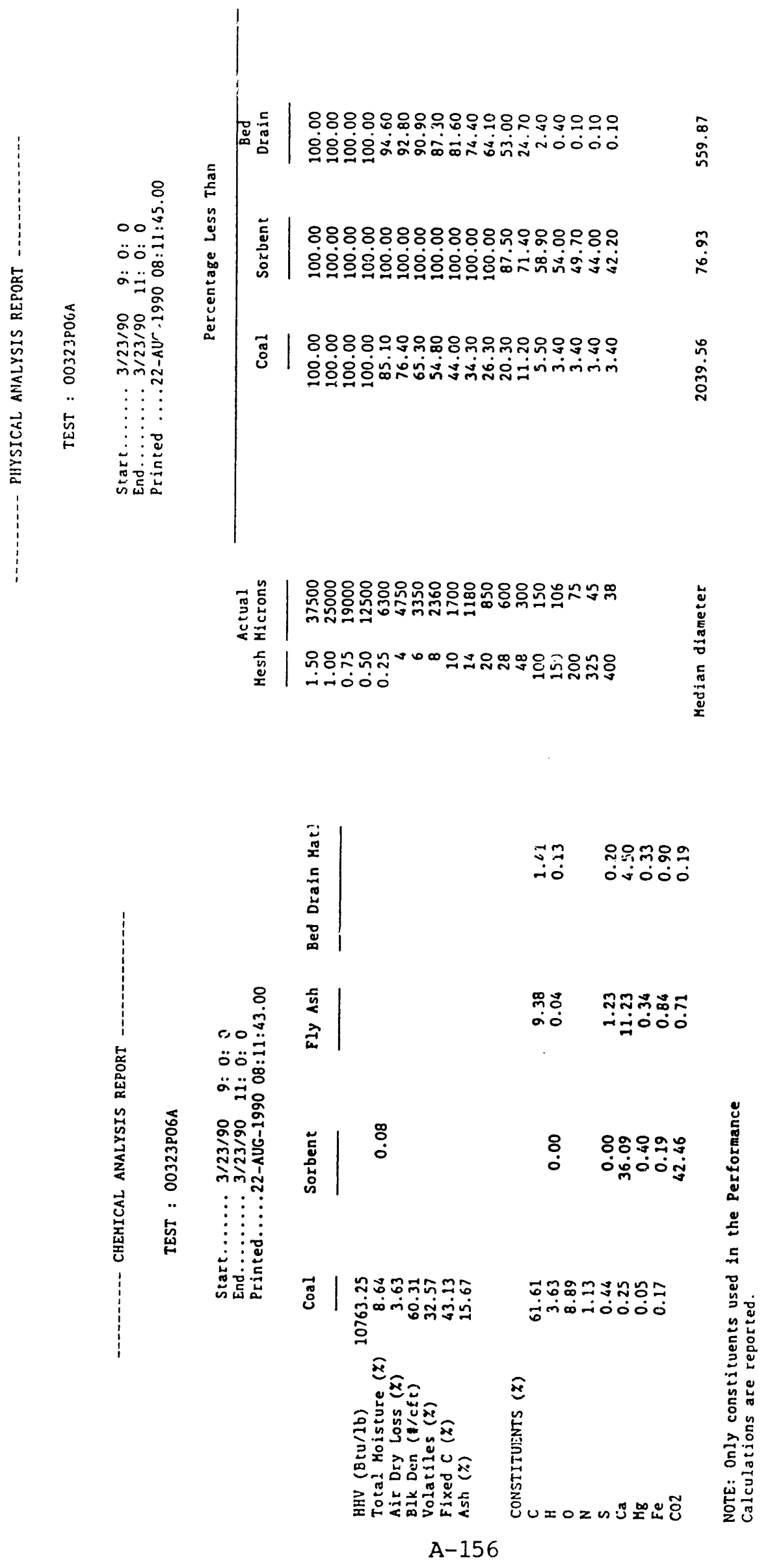


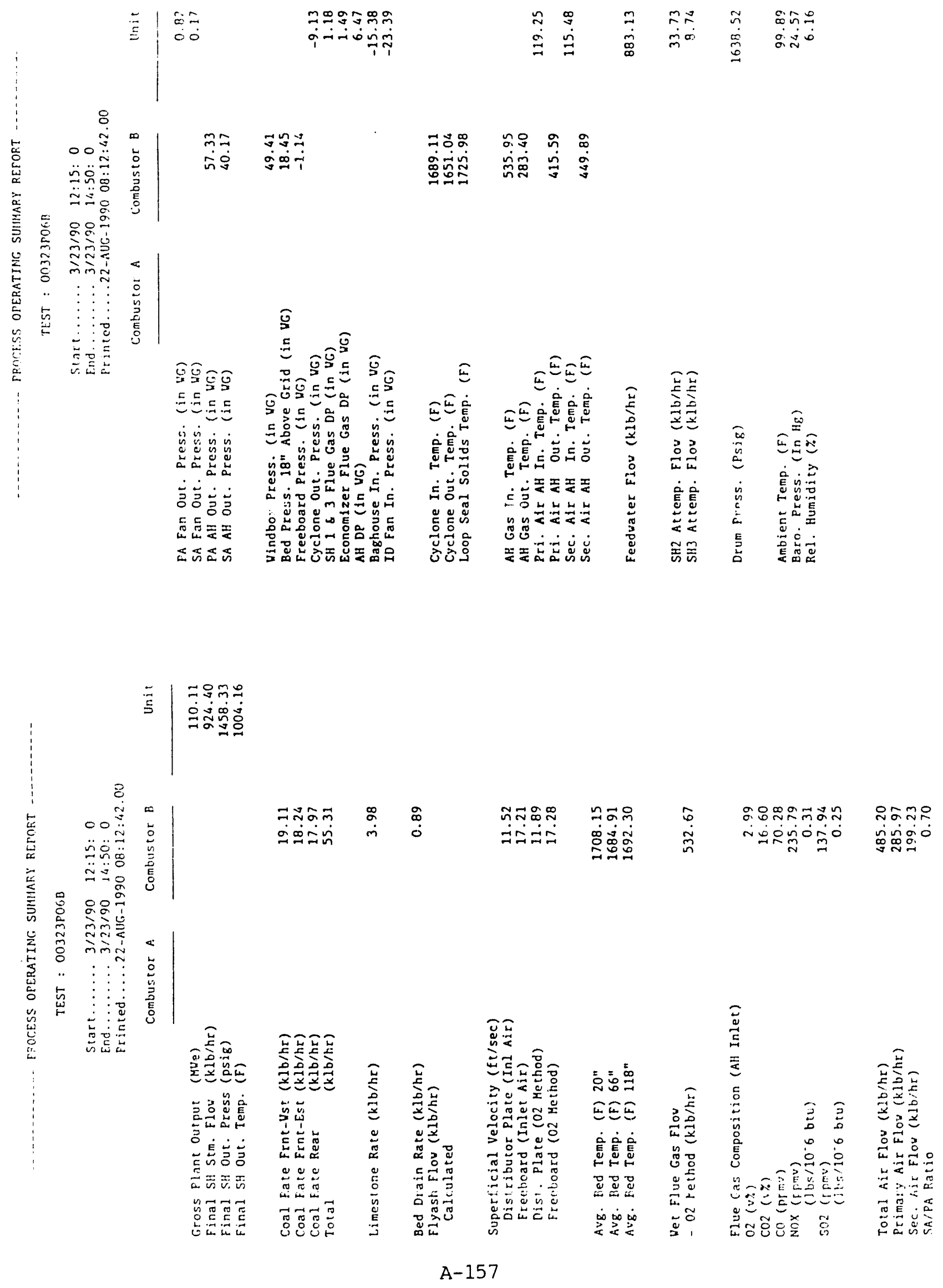




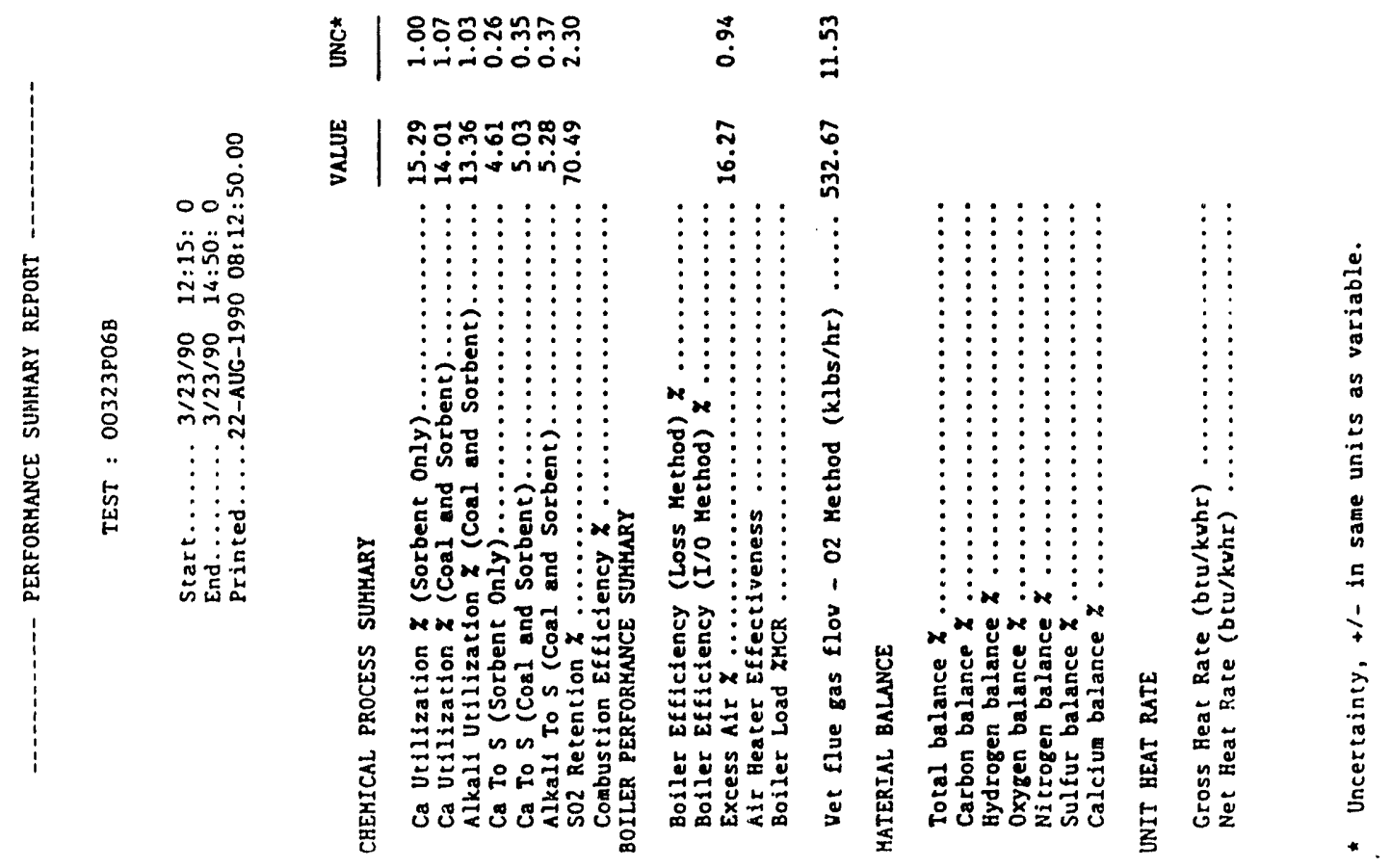

A-158 

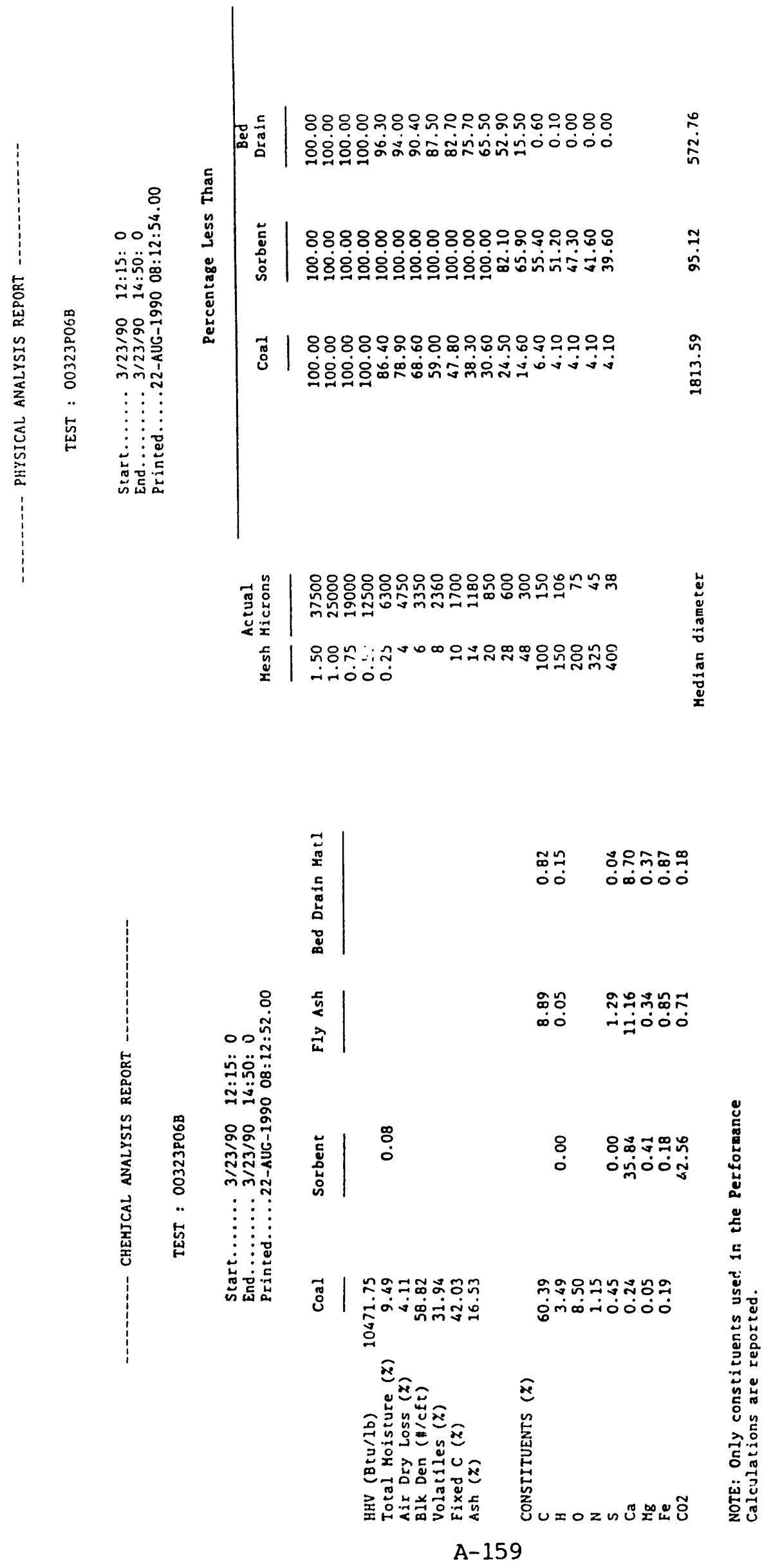


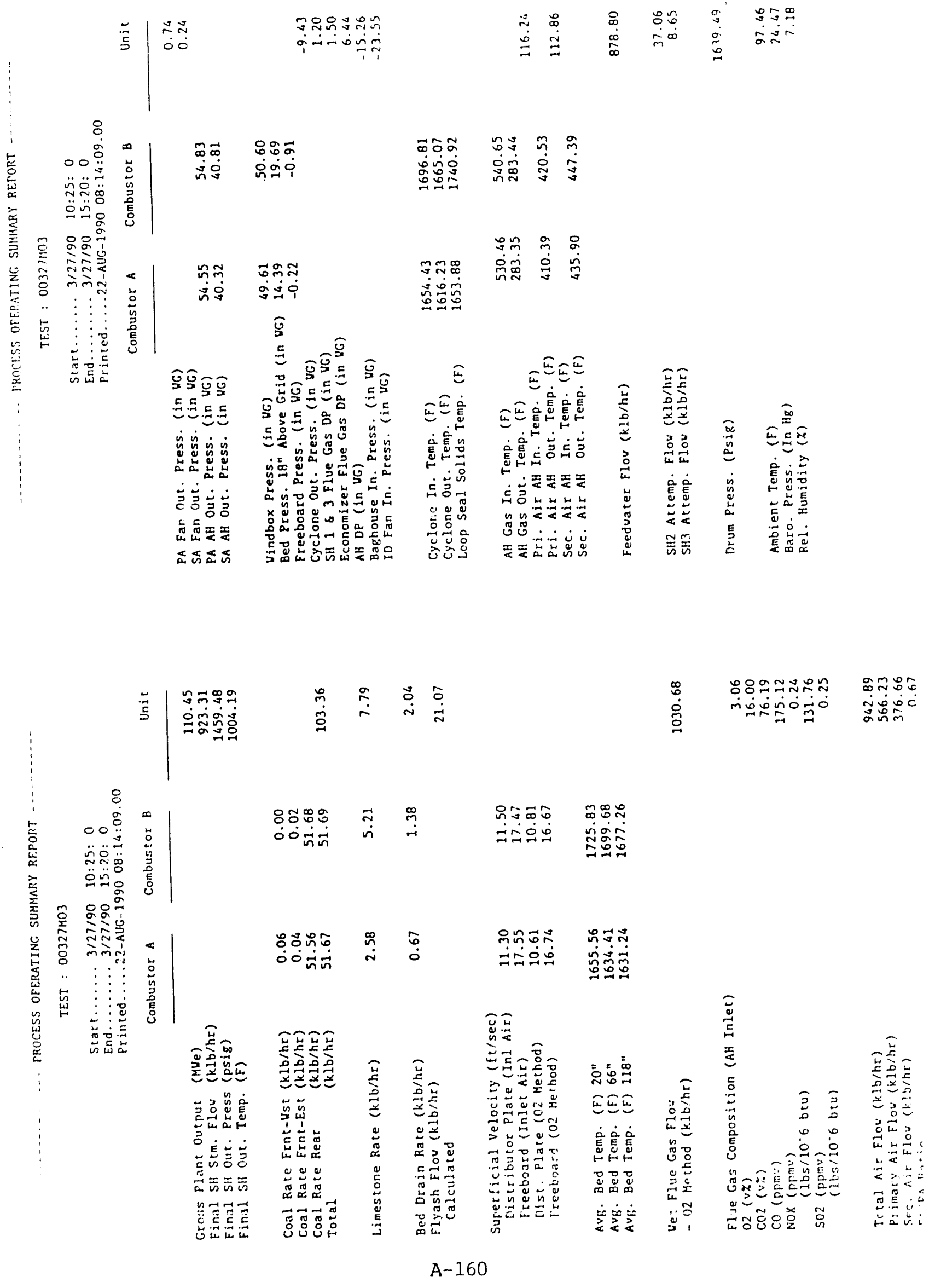




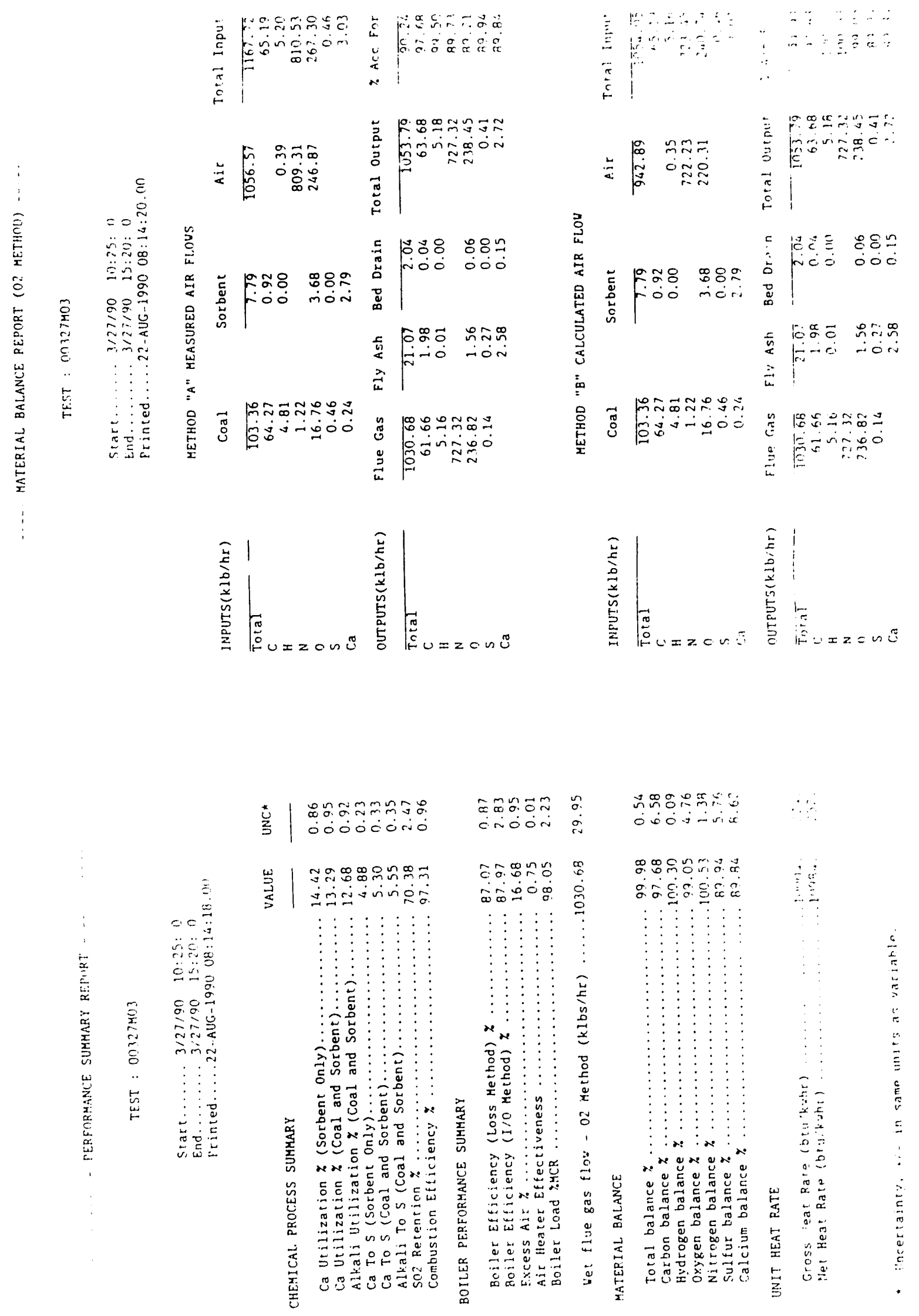




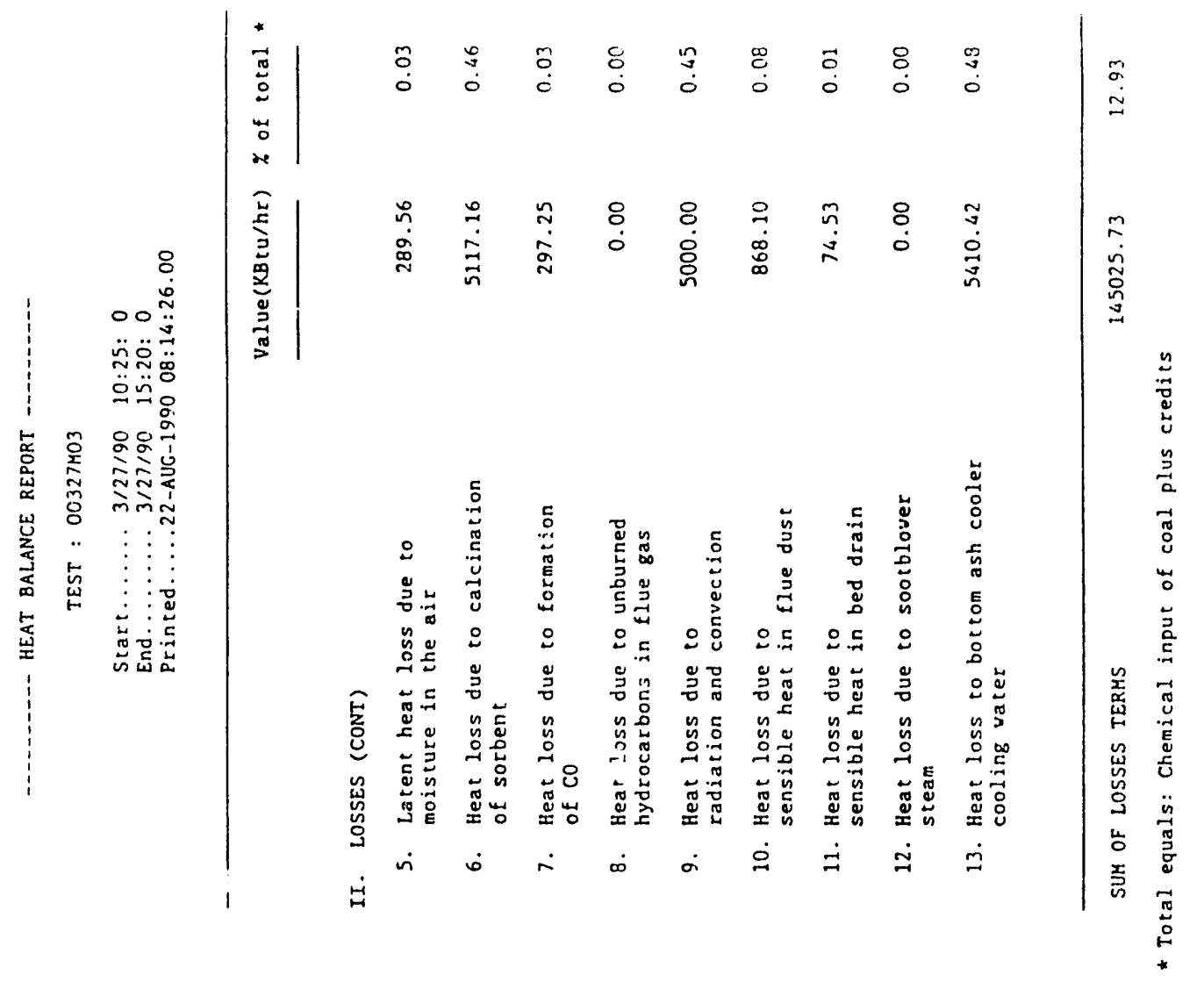

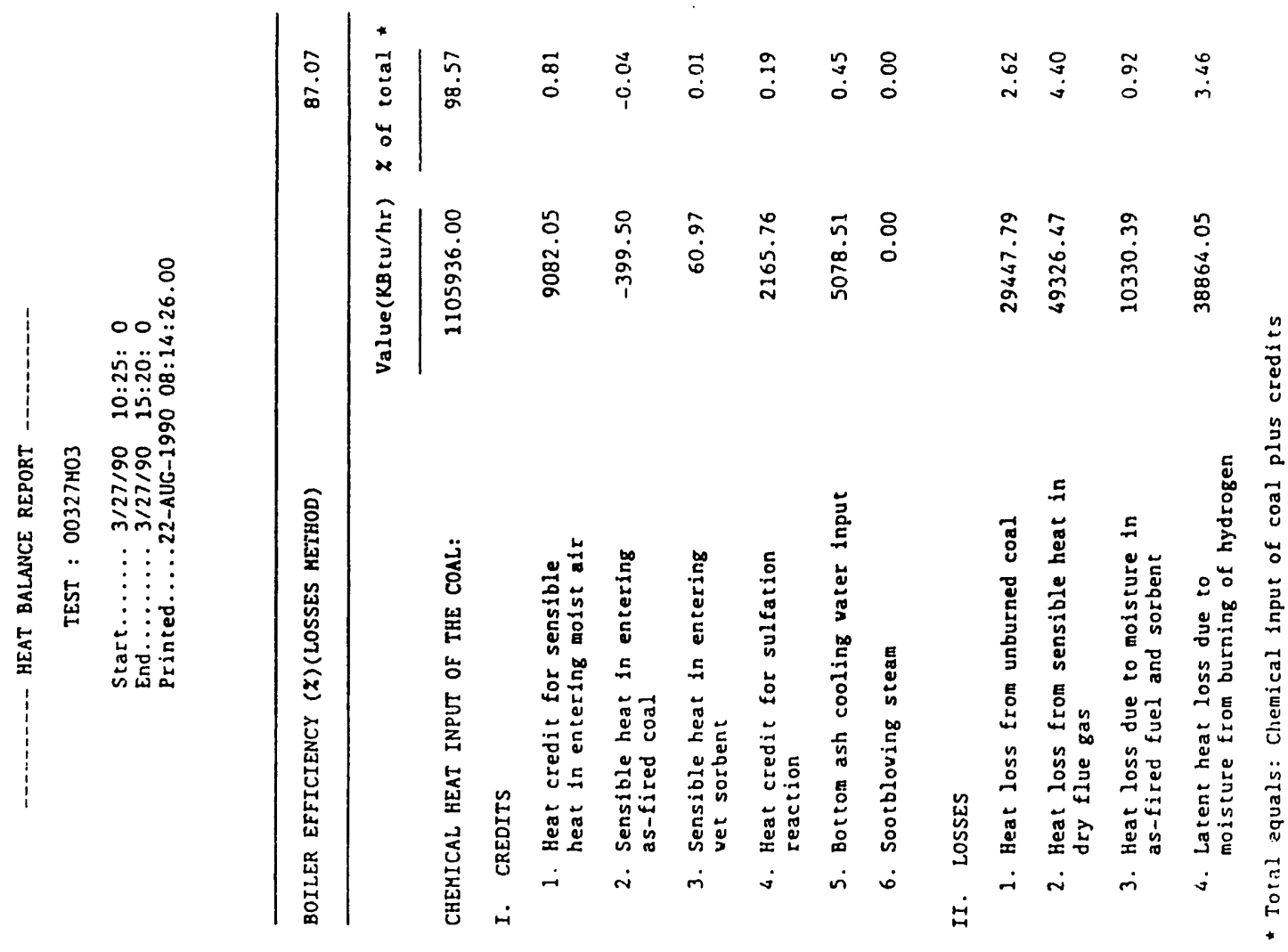



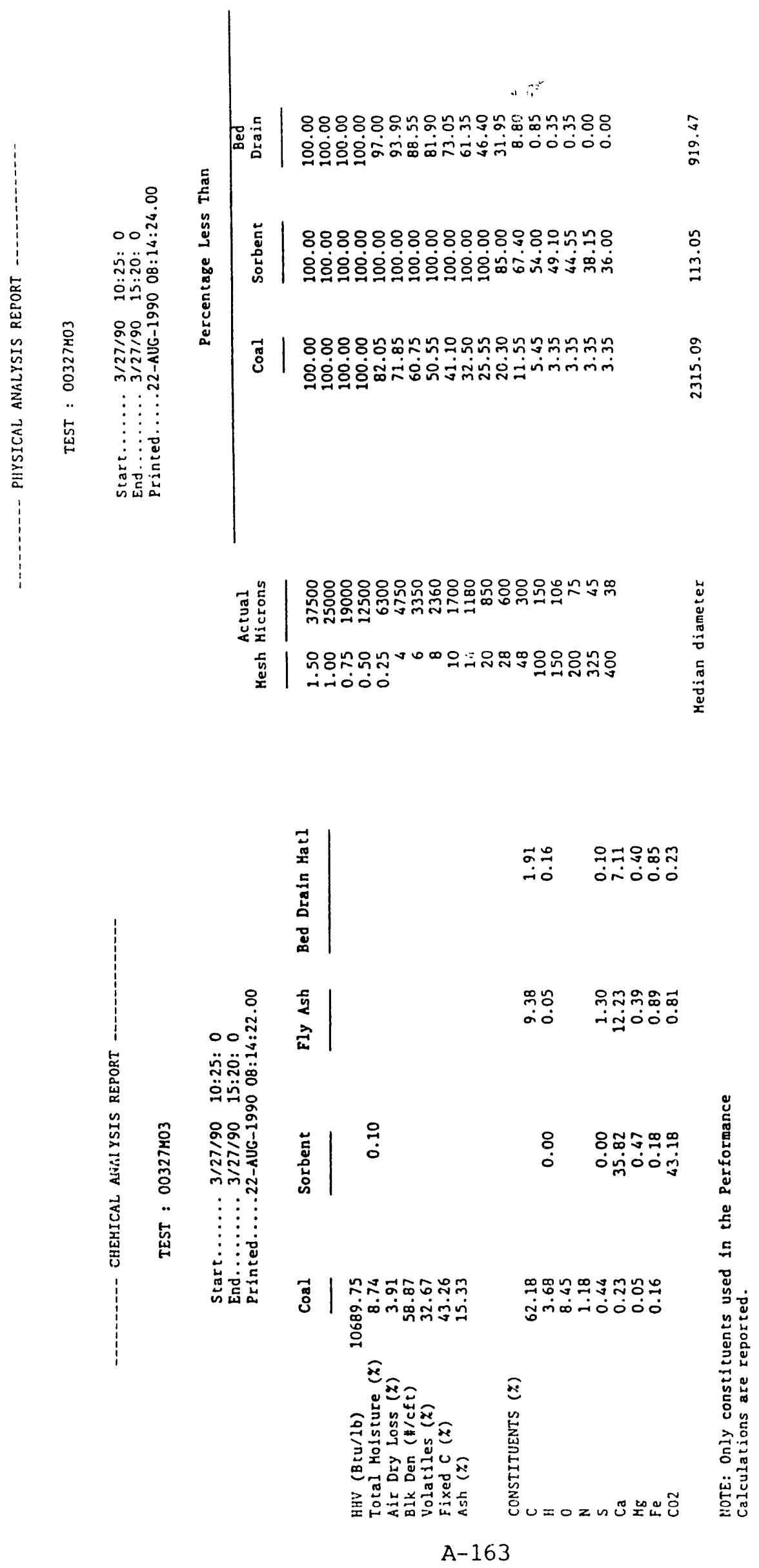


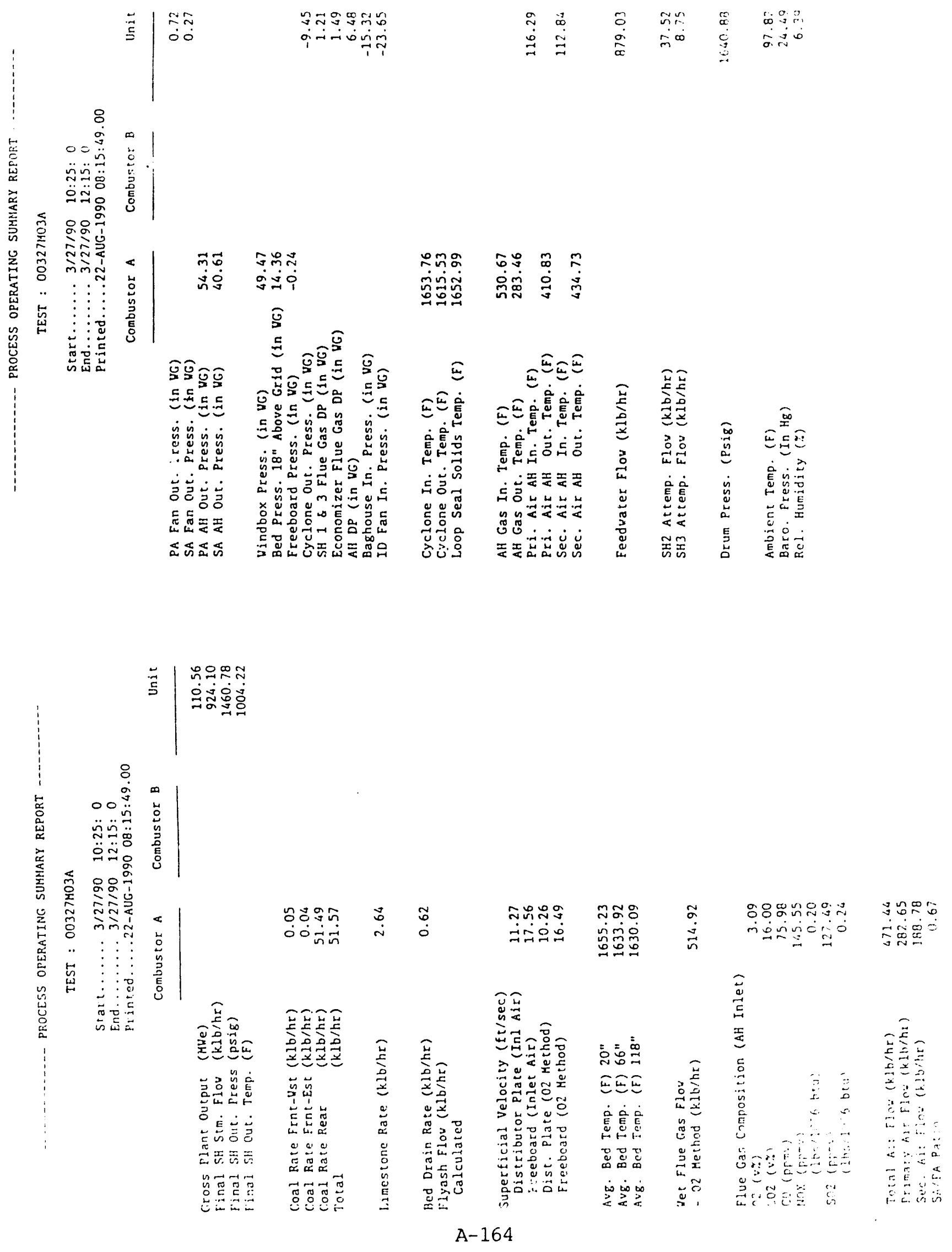




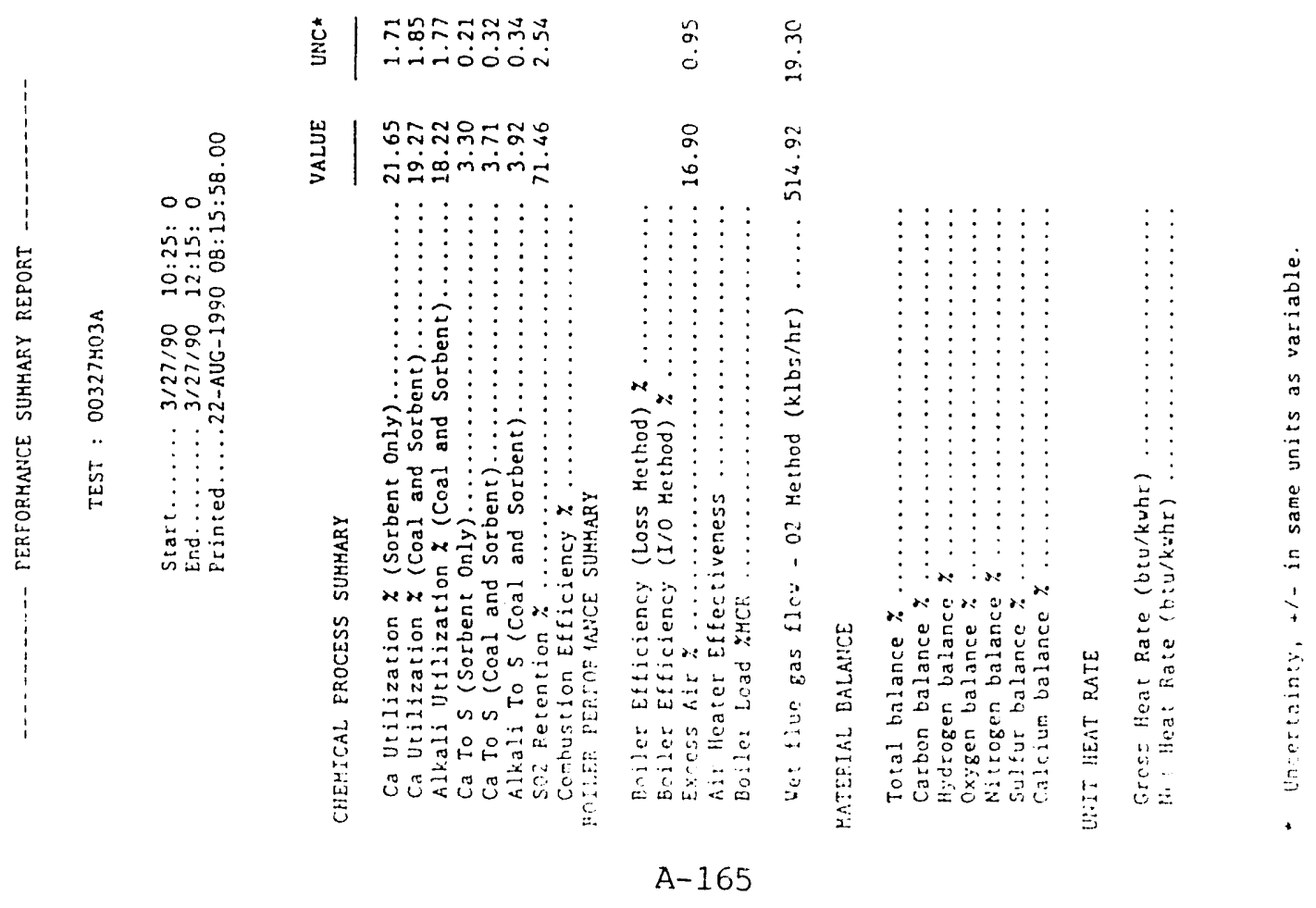




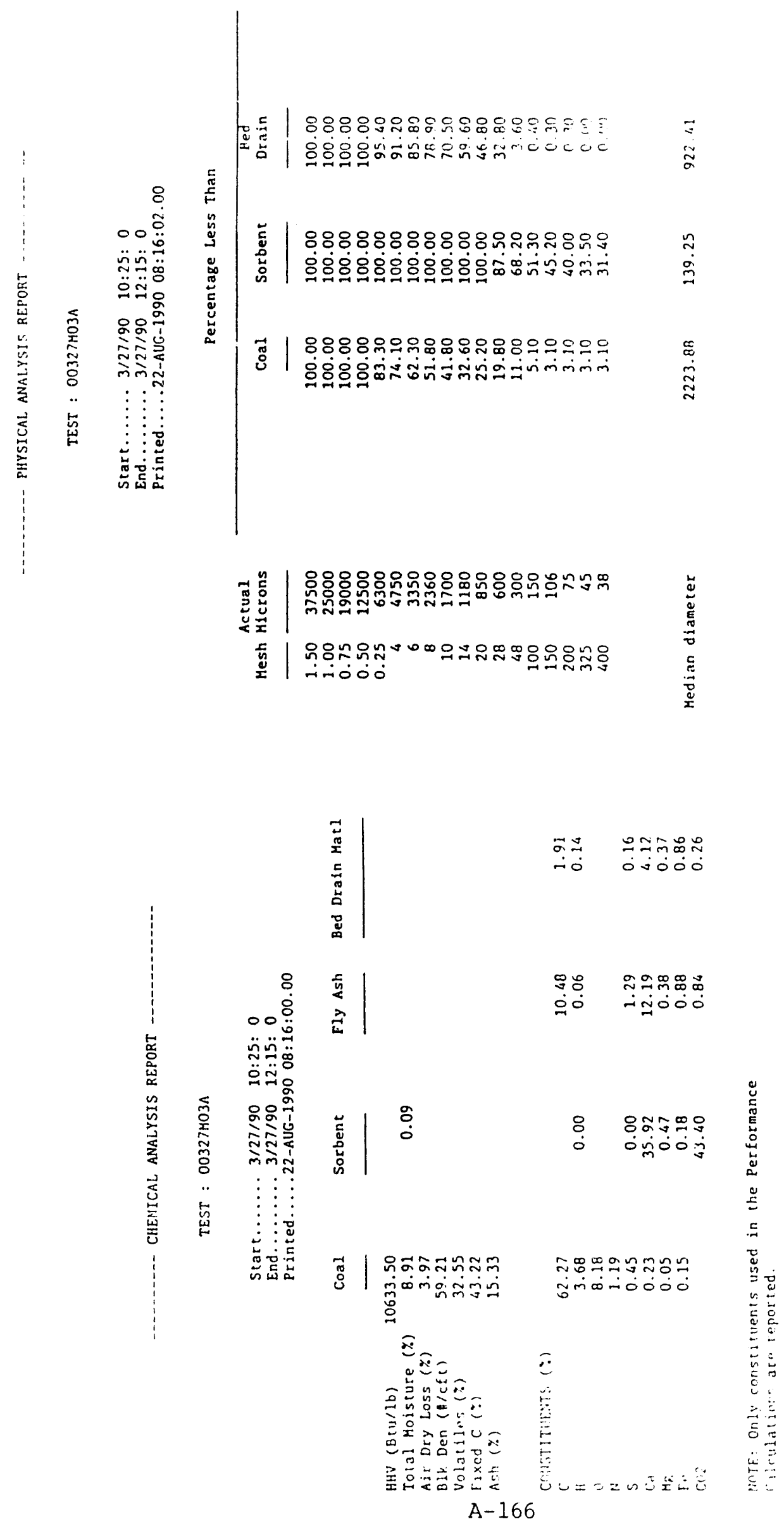



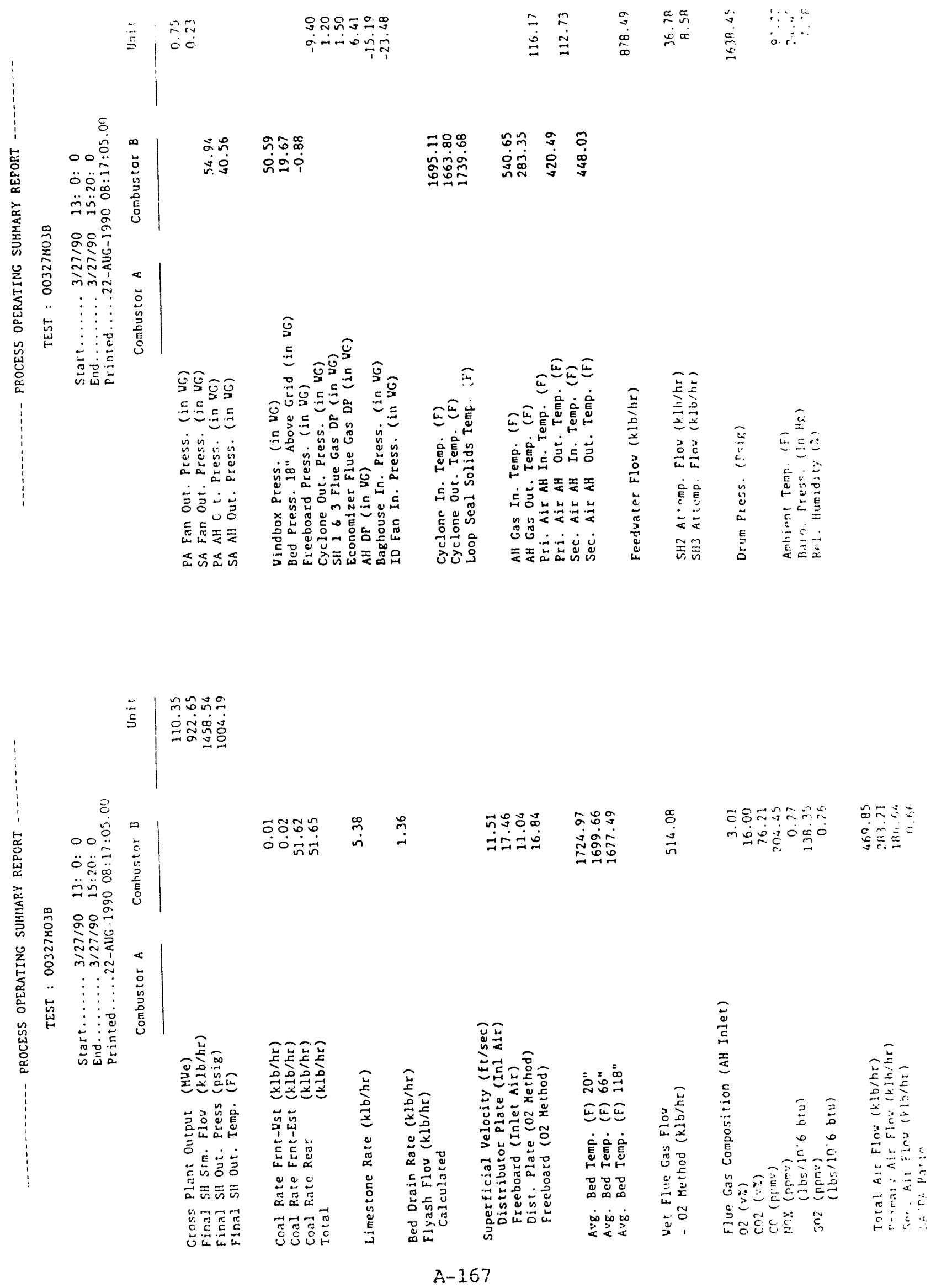


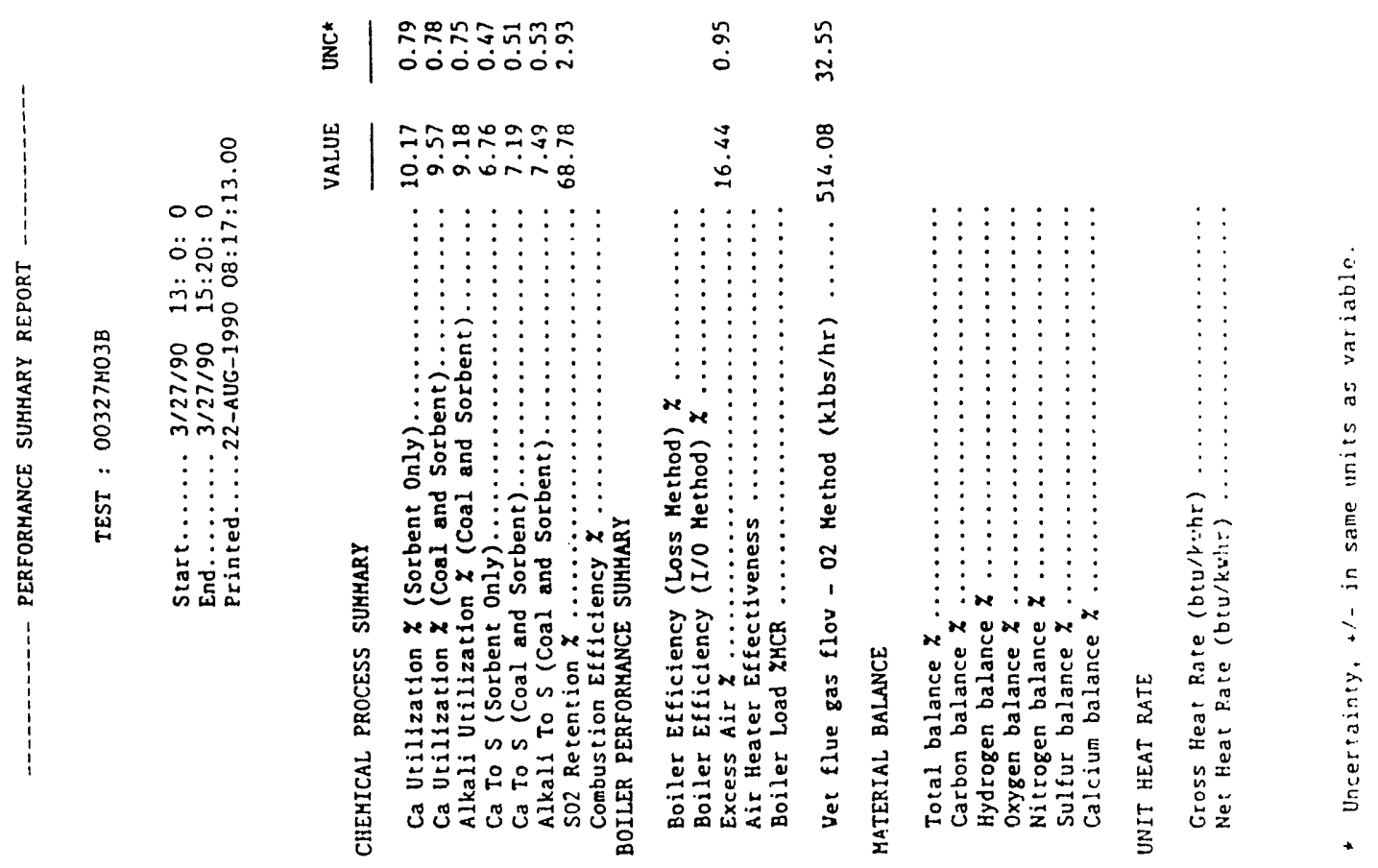




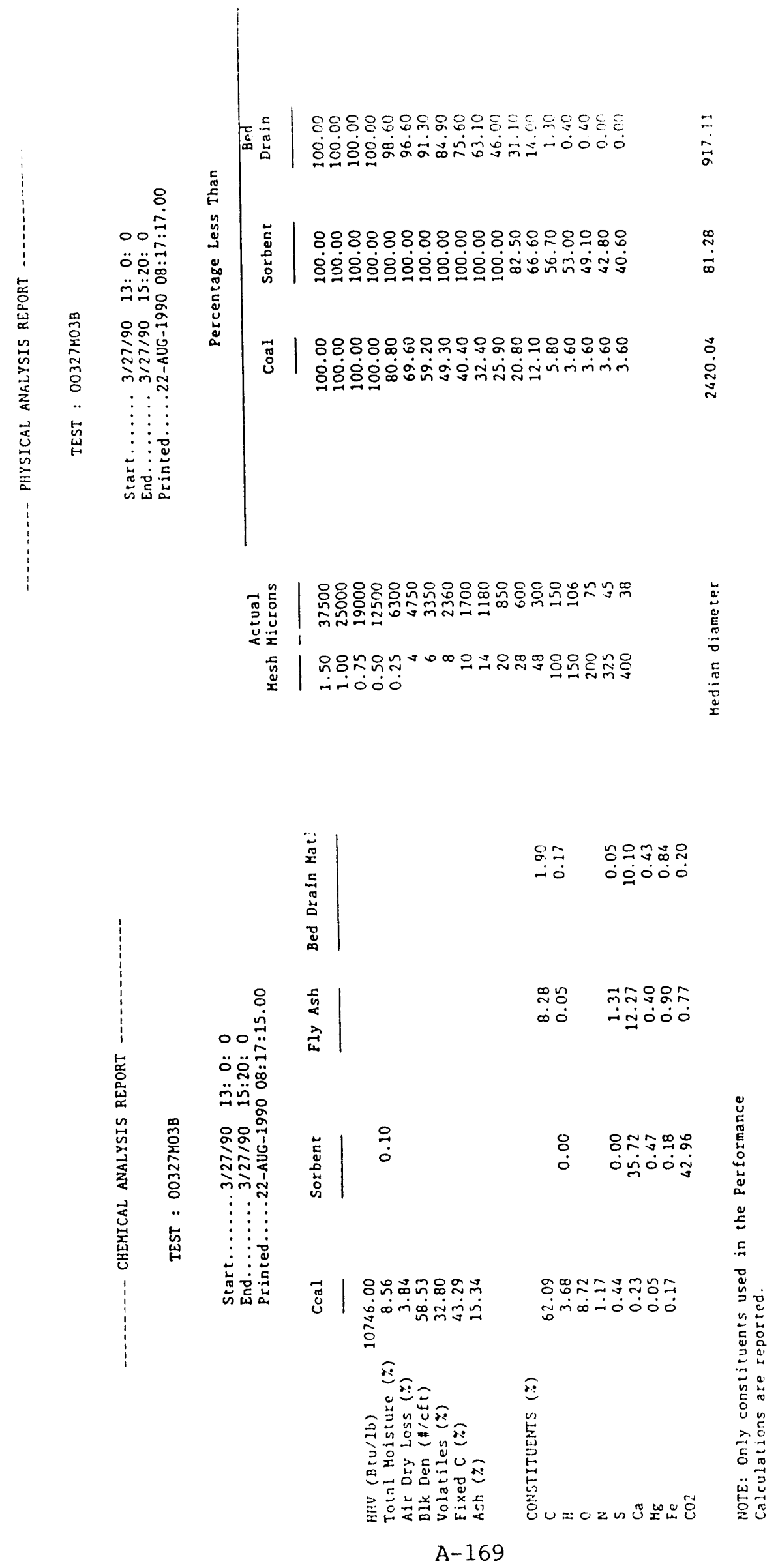




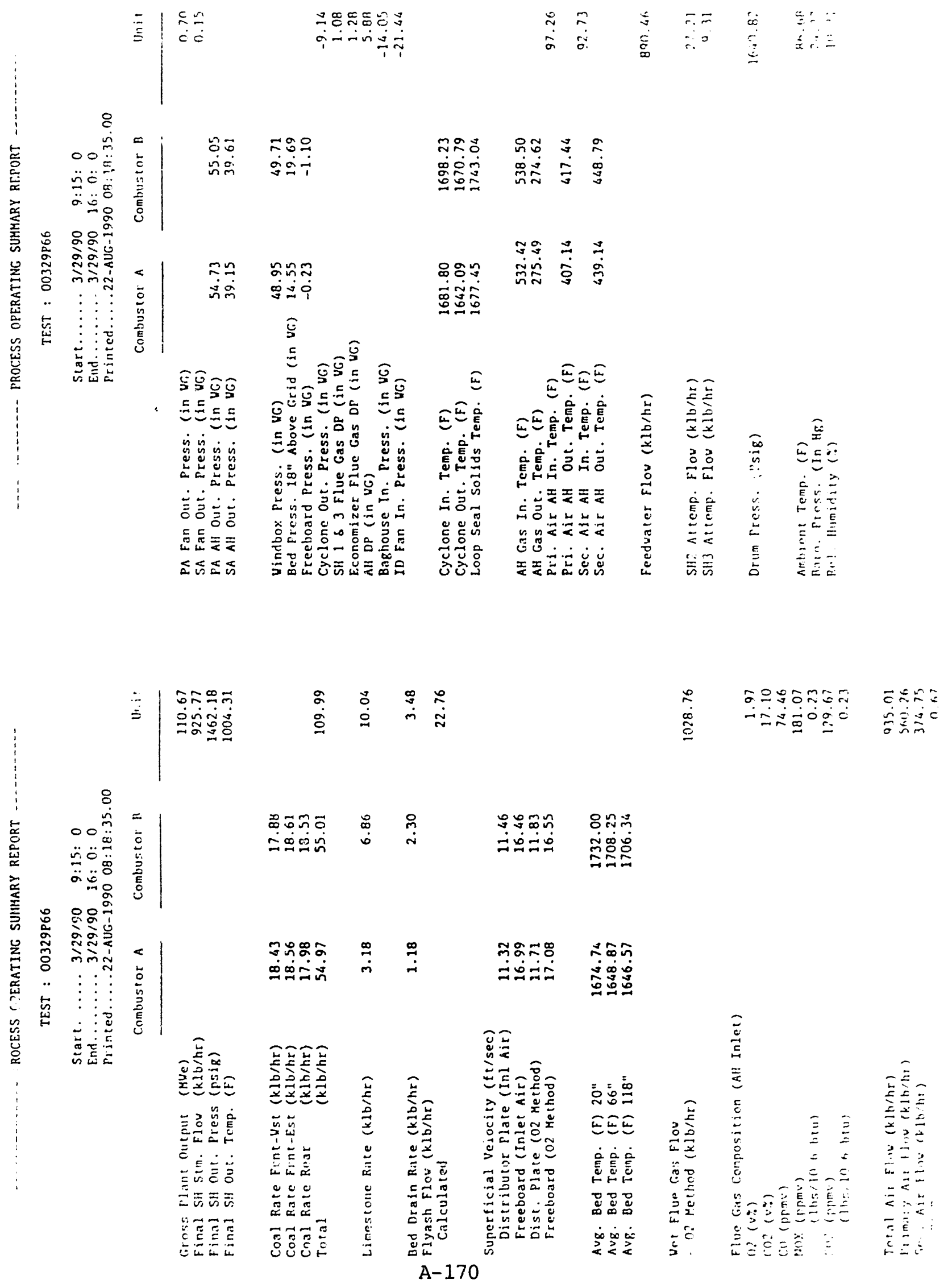




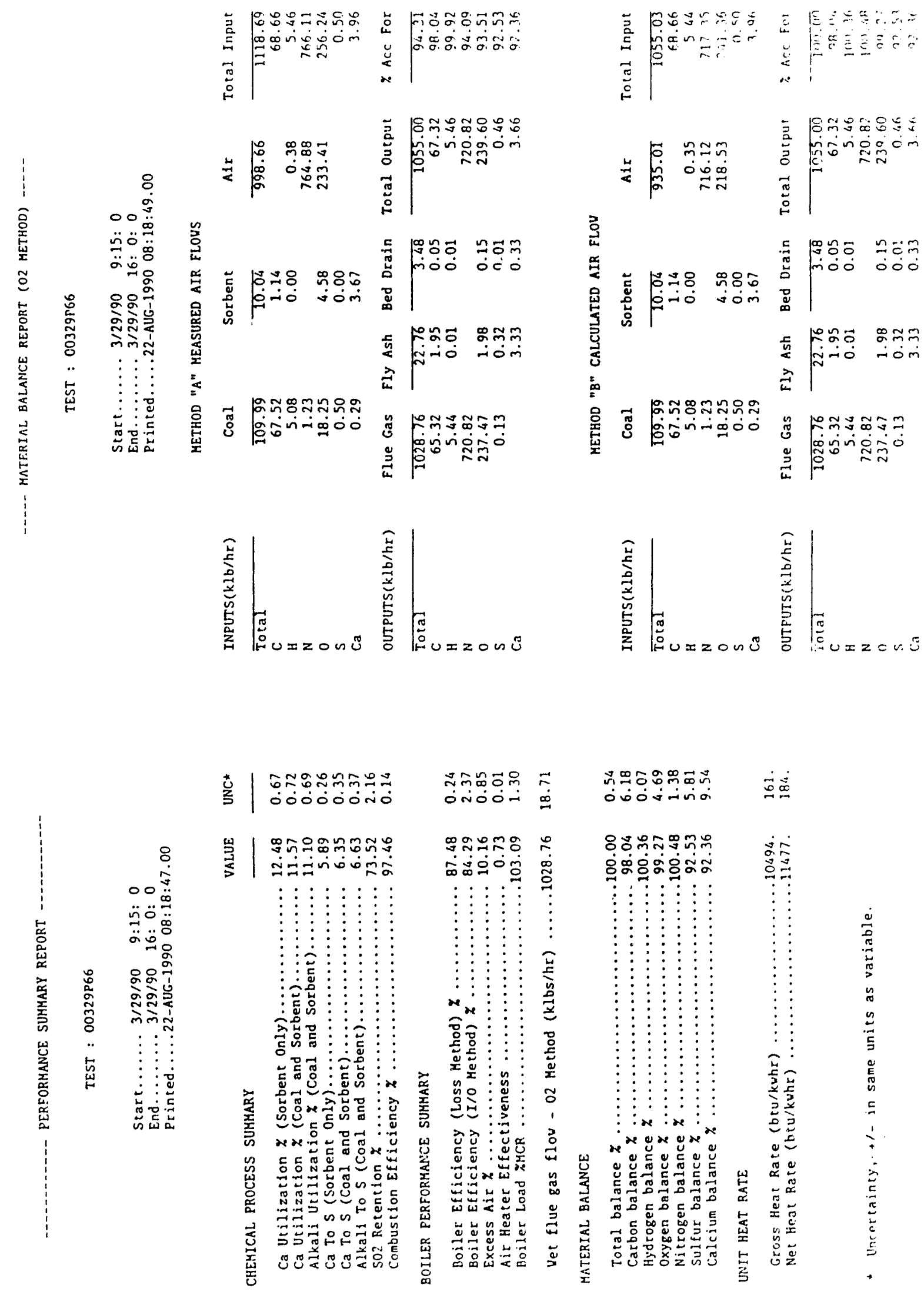



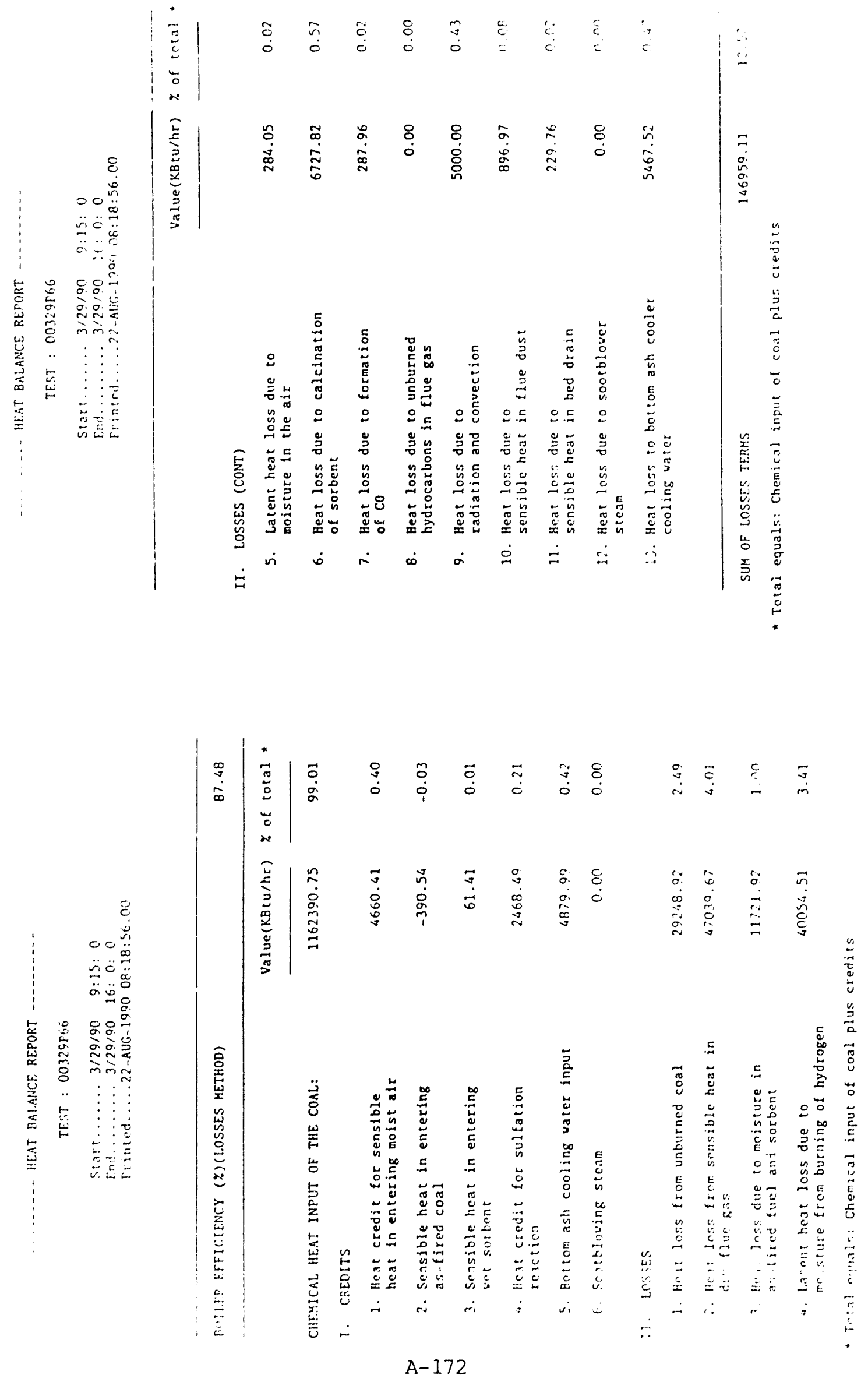

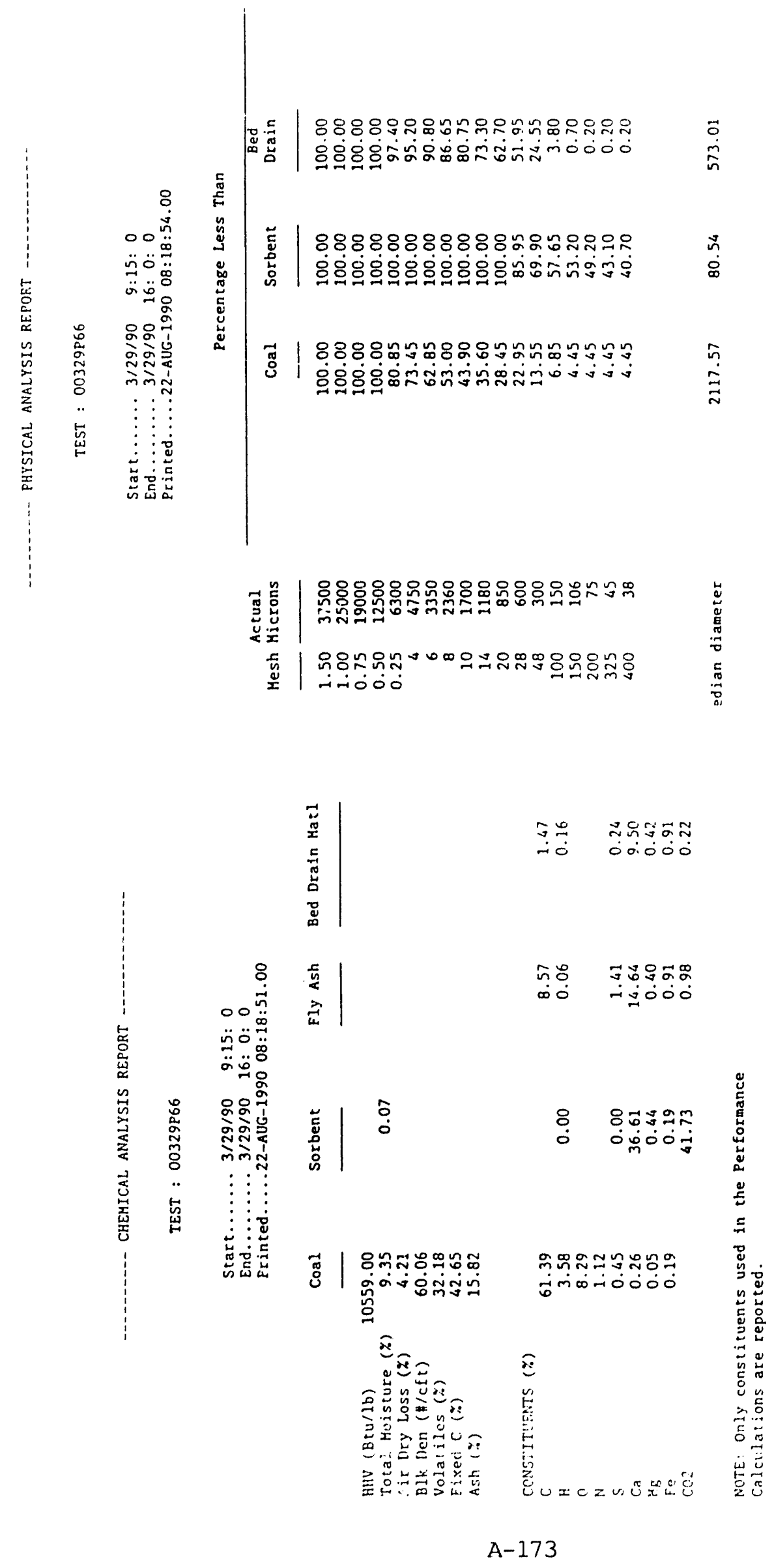

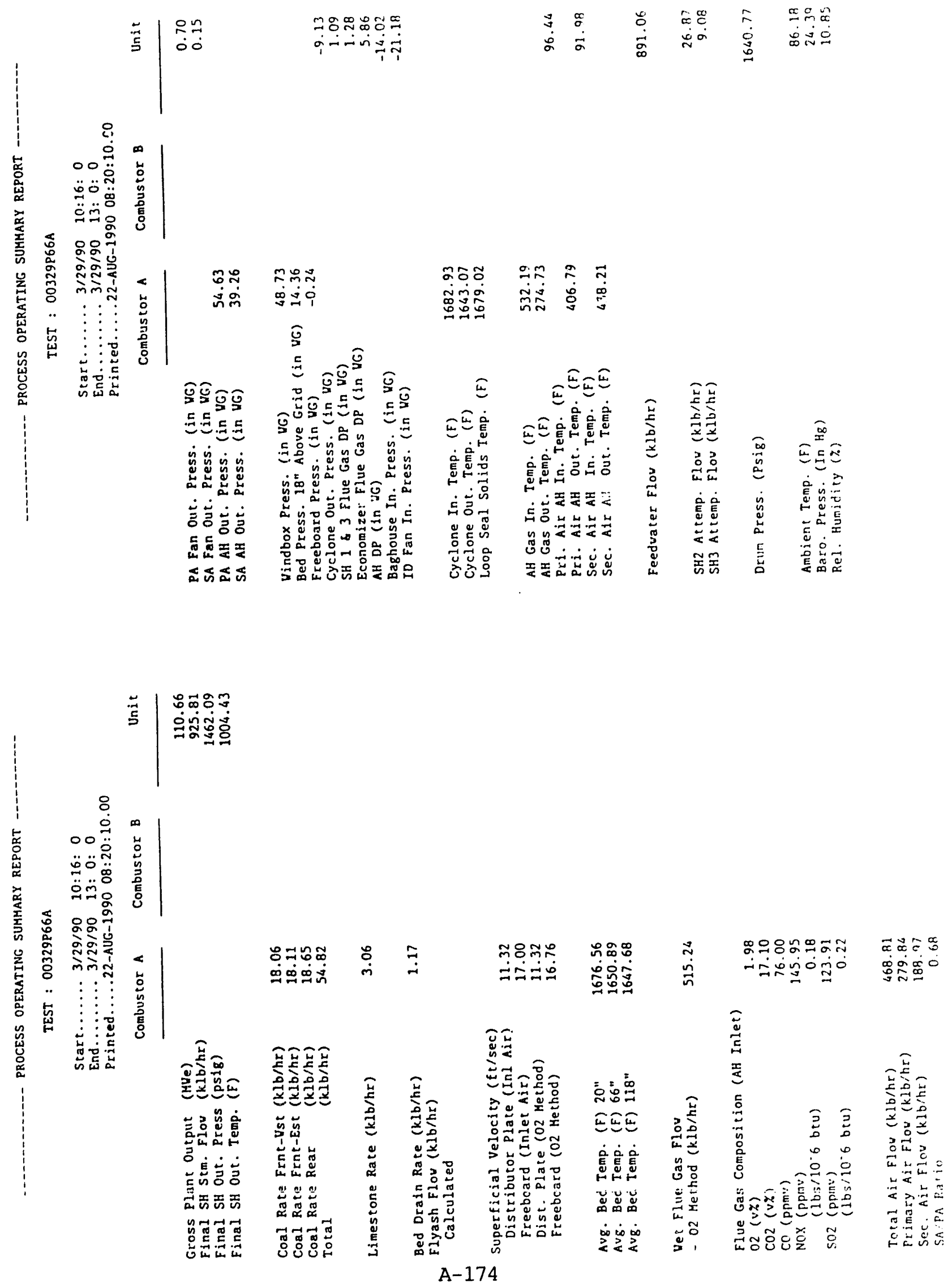


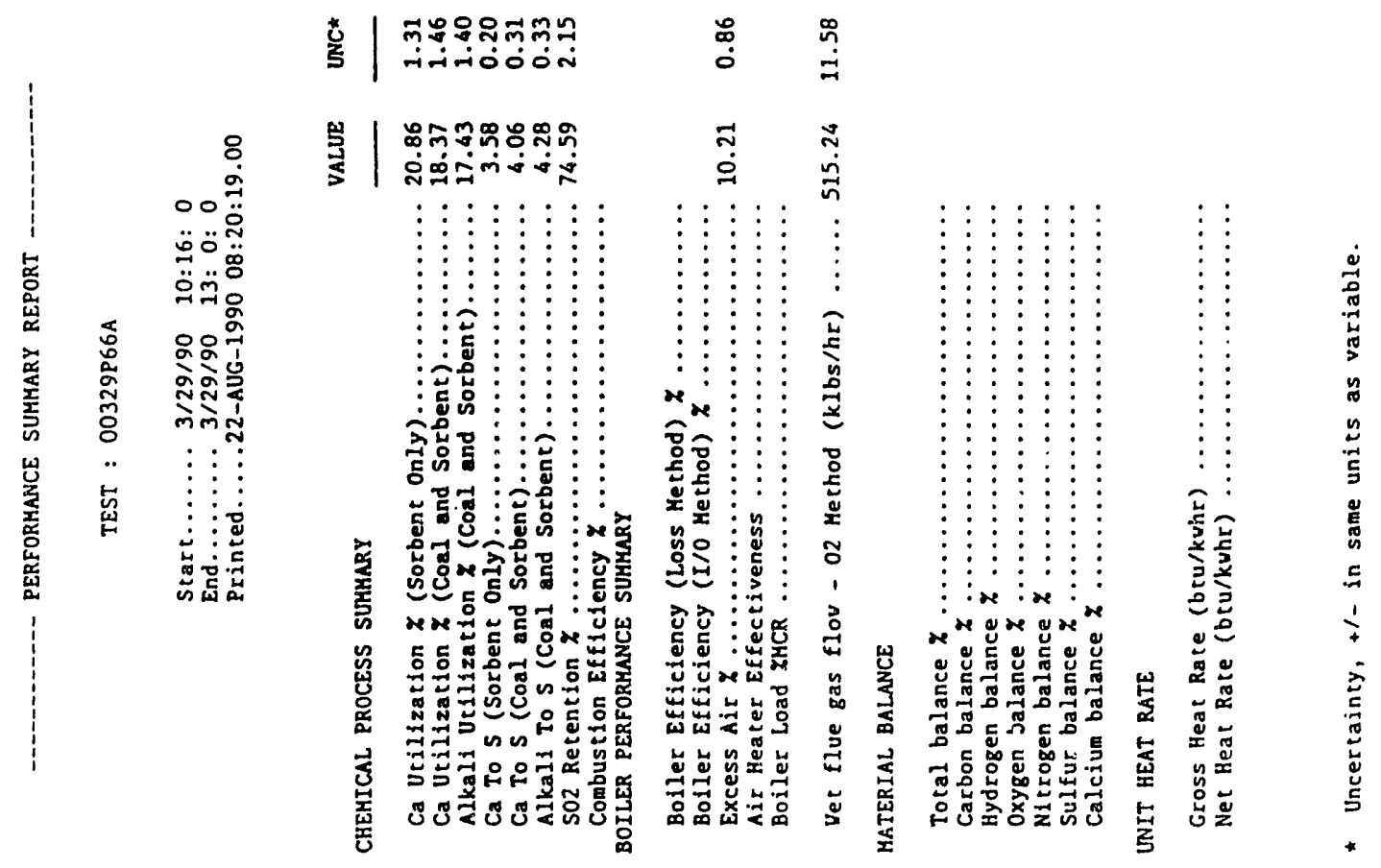

A-175 


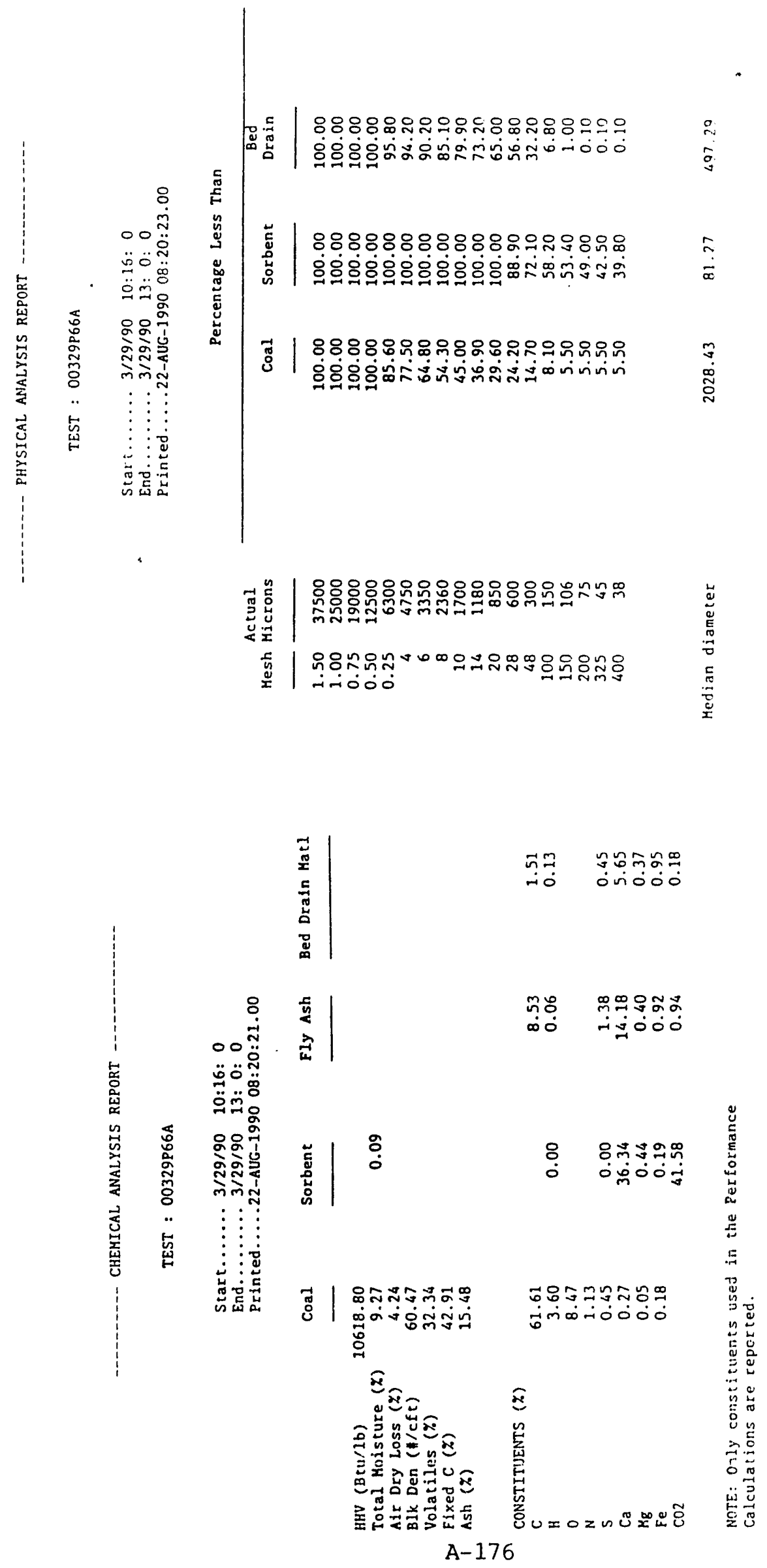



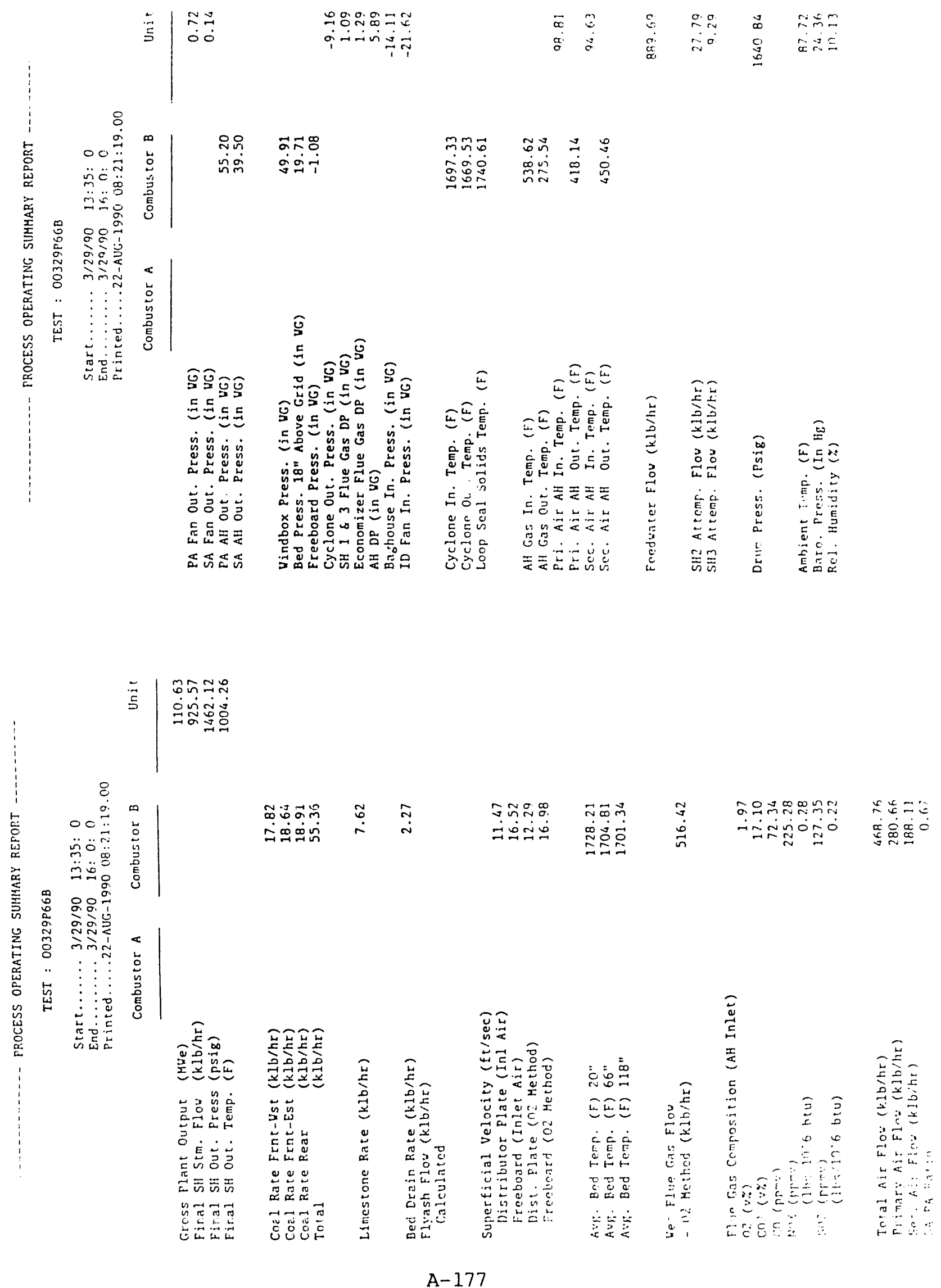


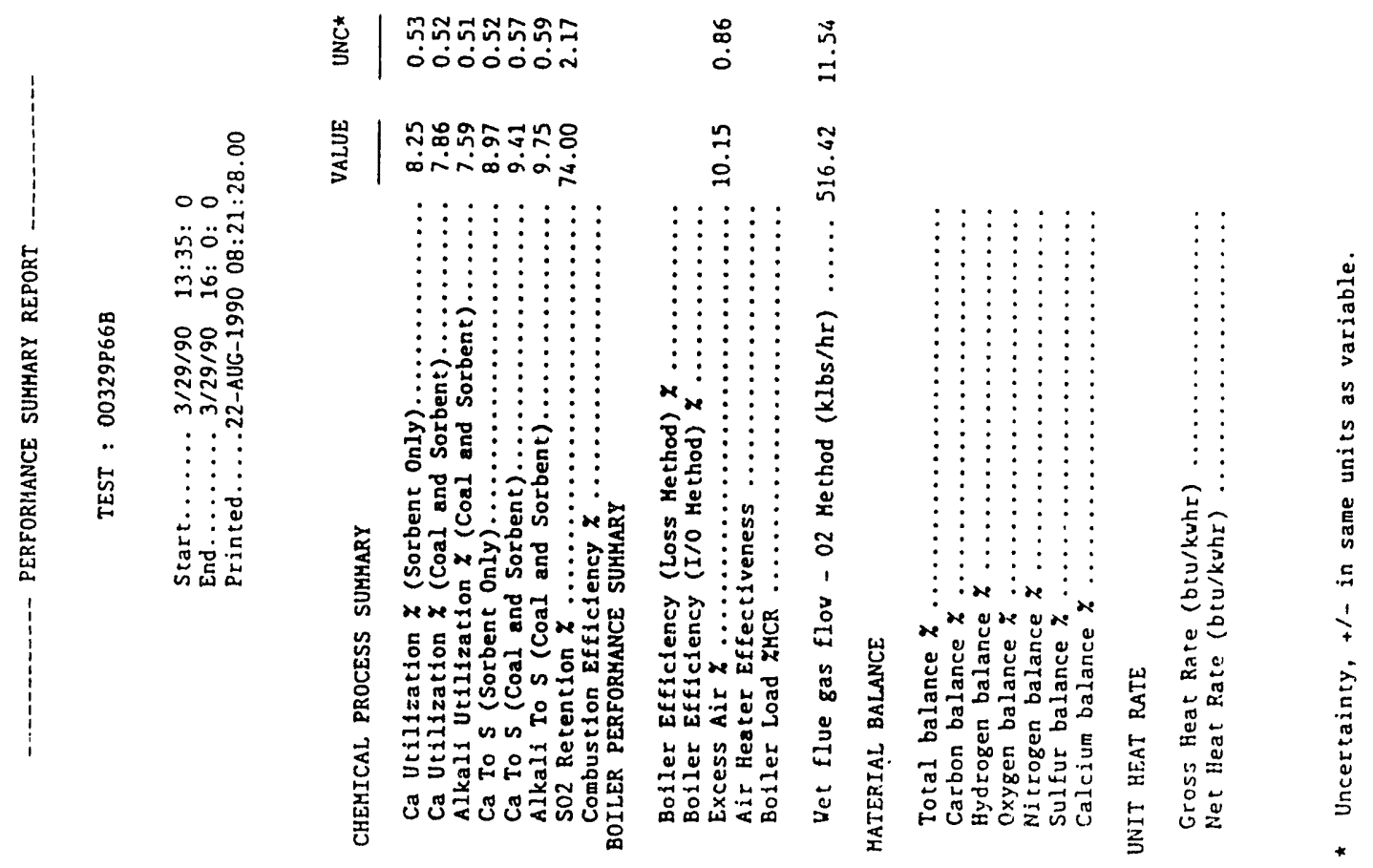

A-178 


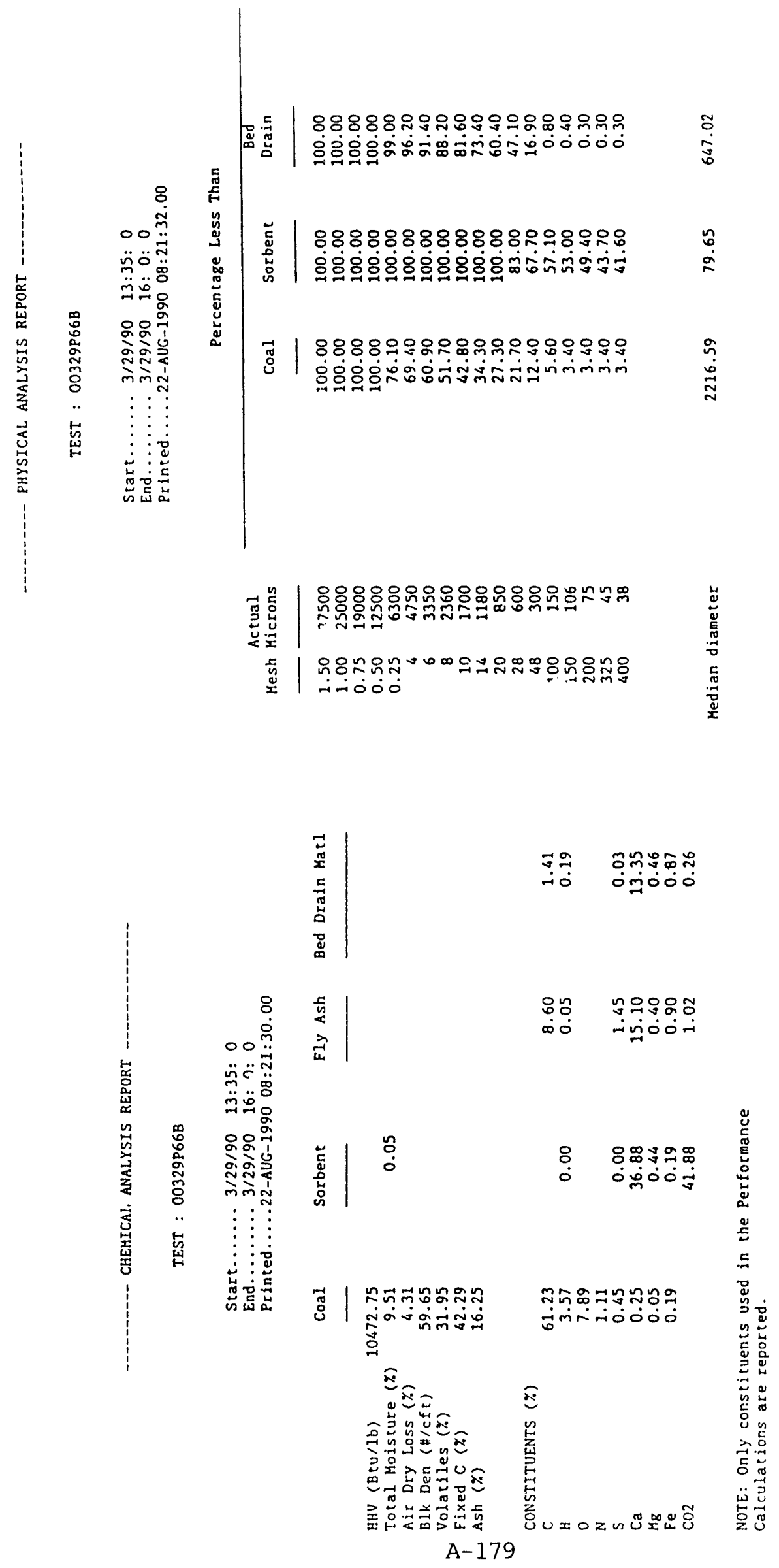




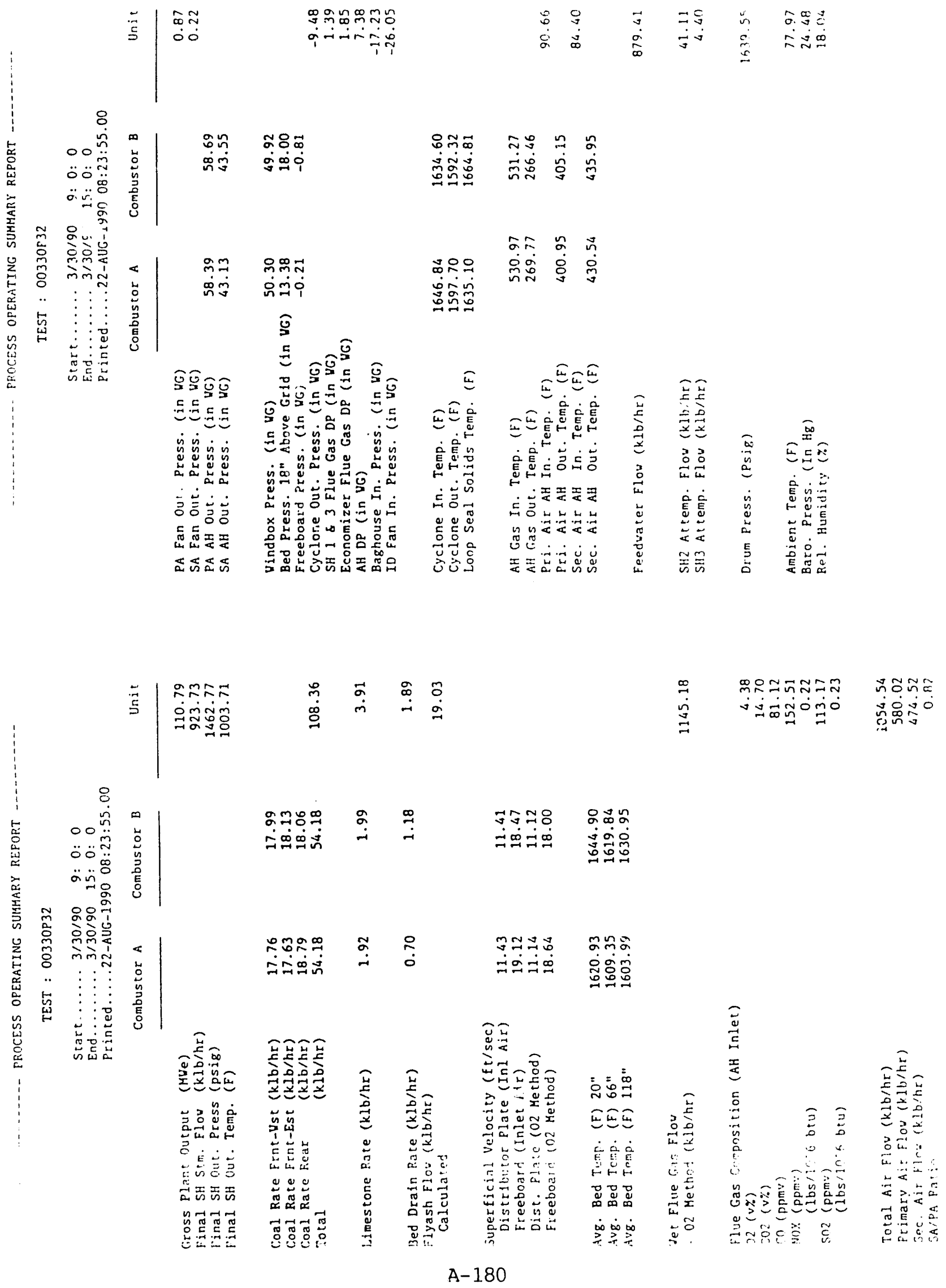




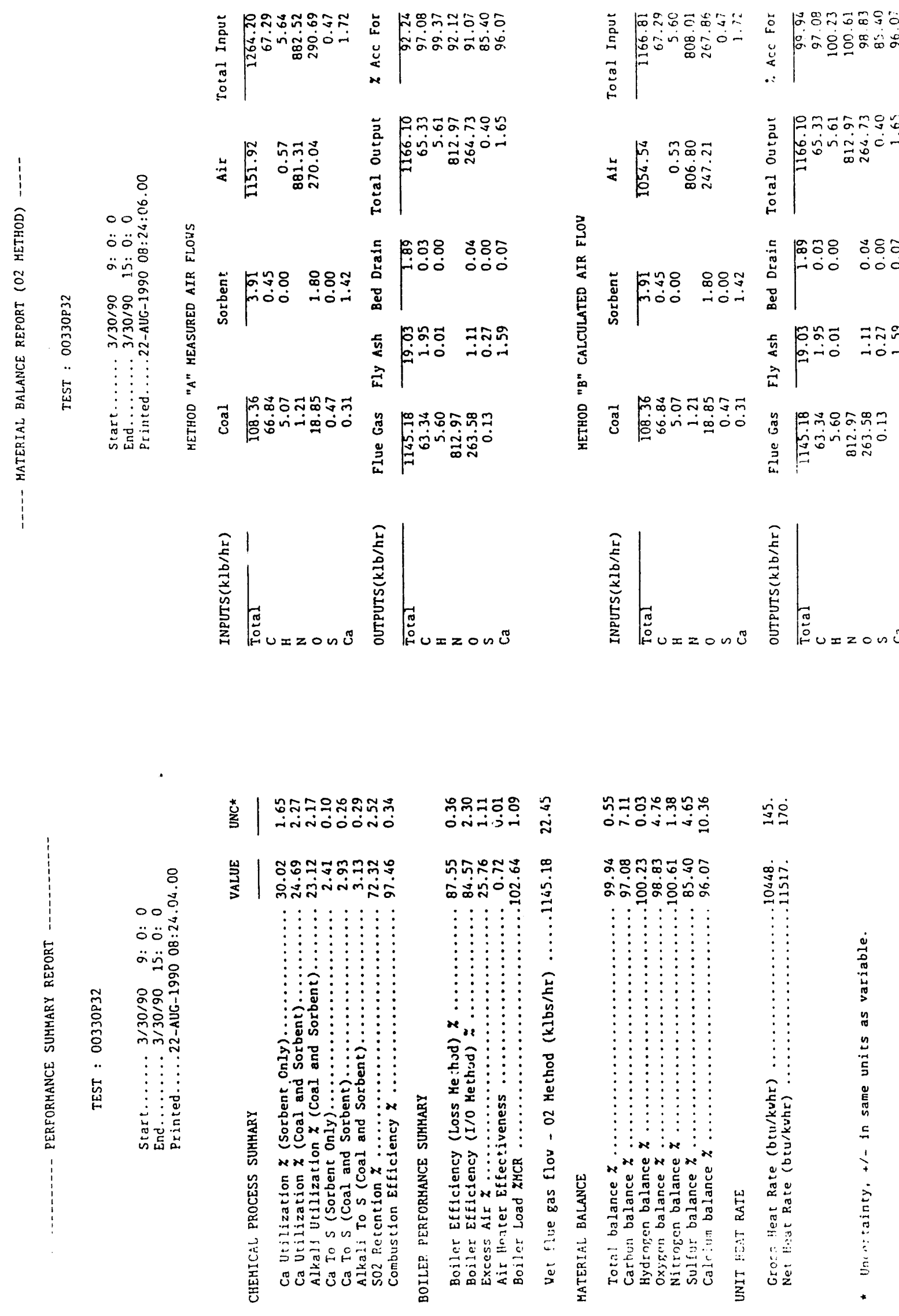



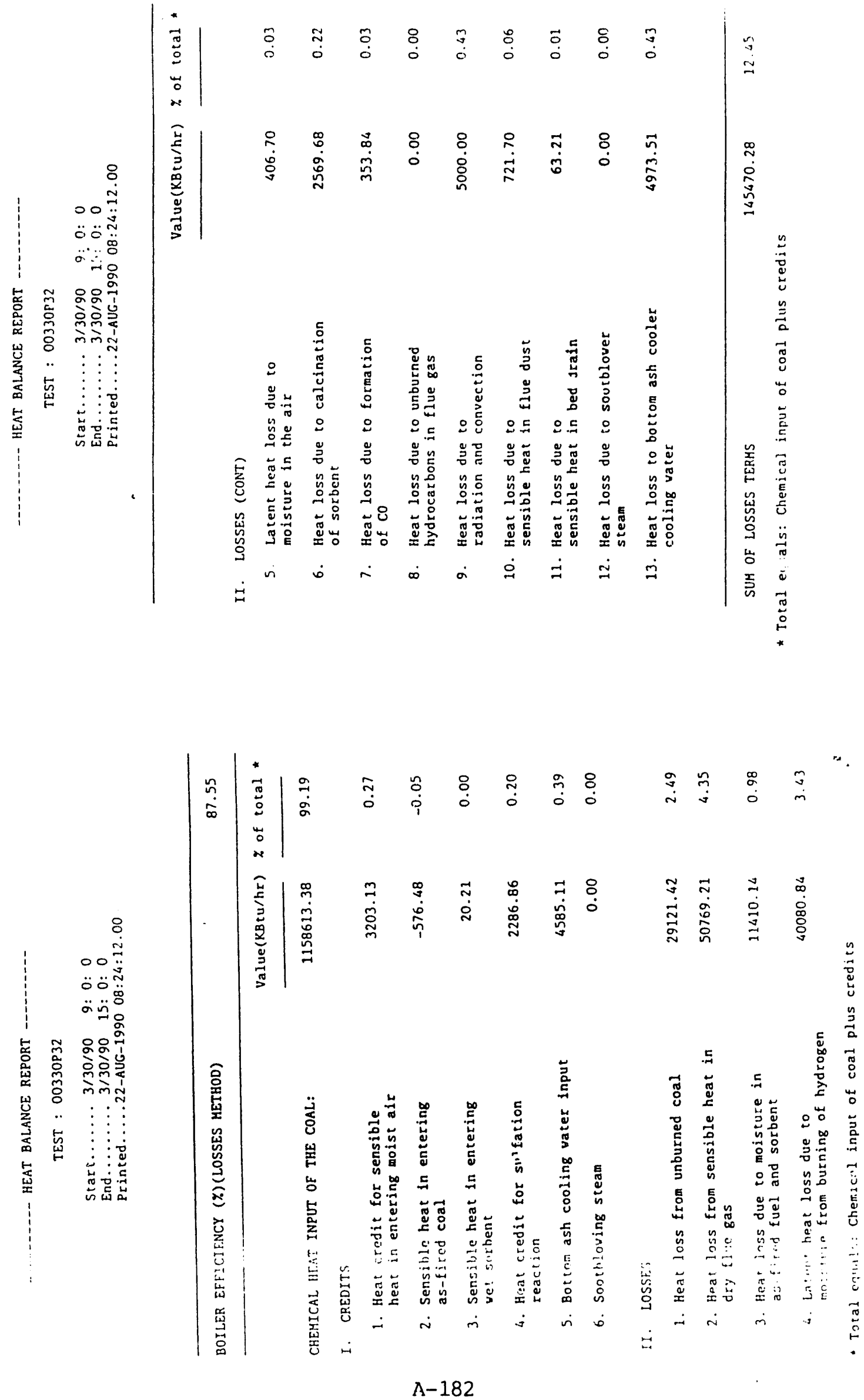


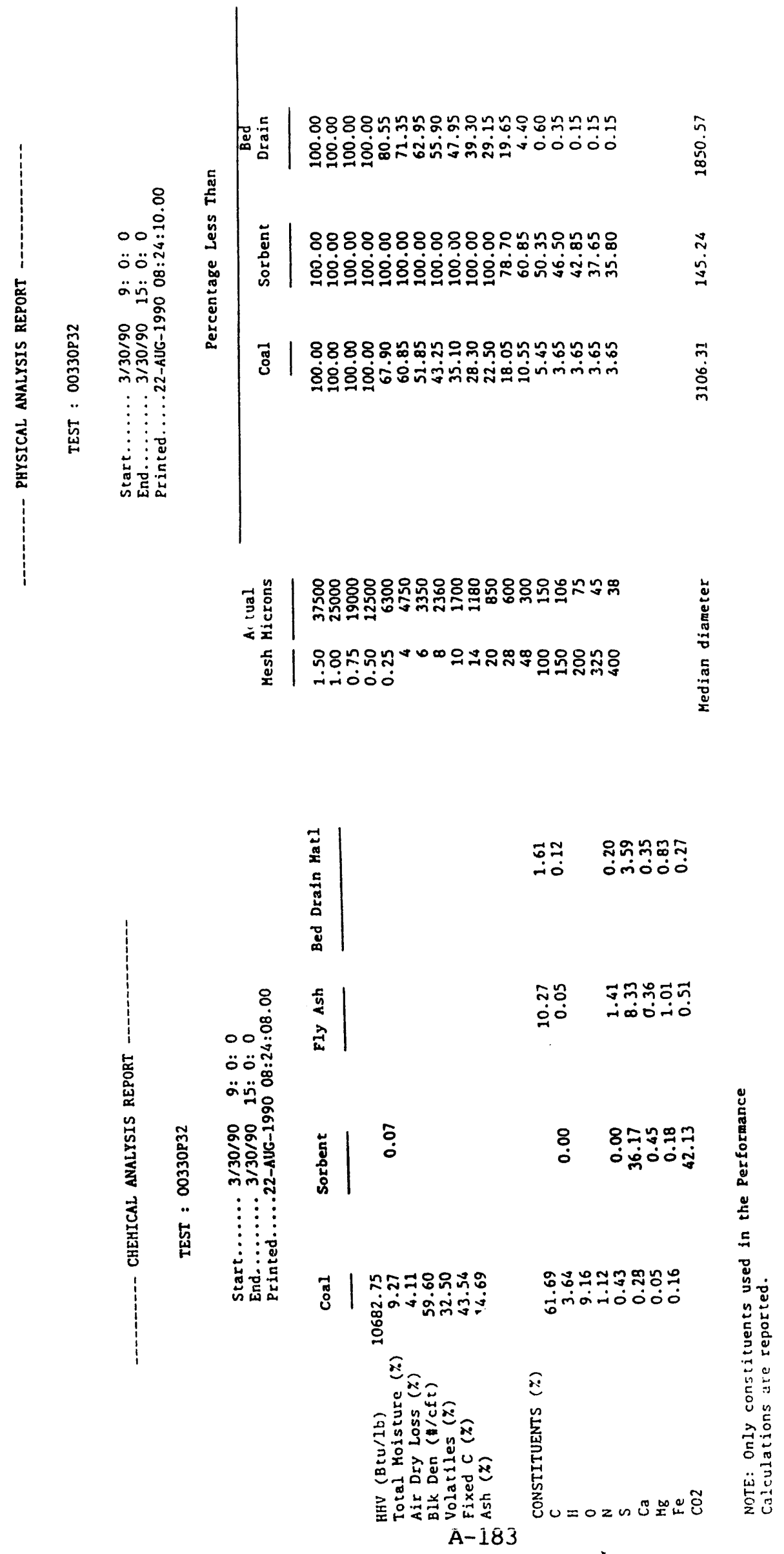



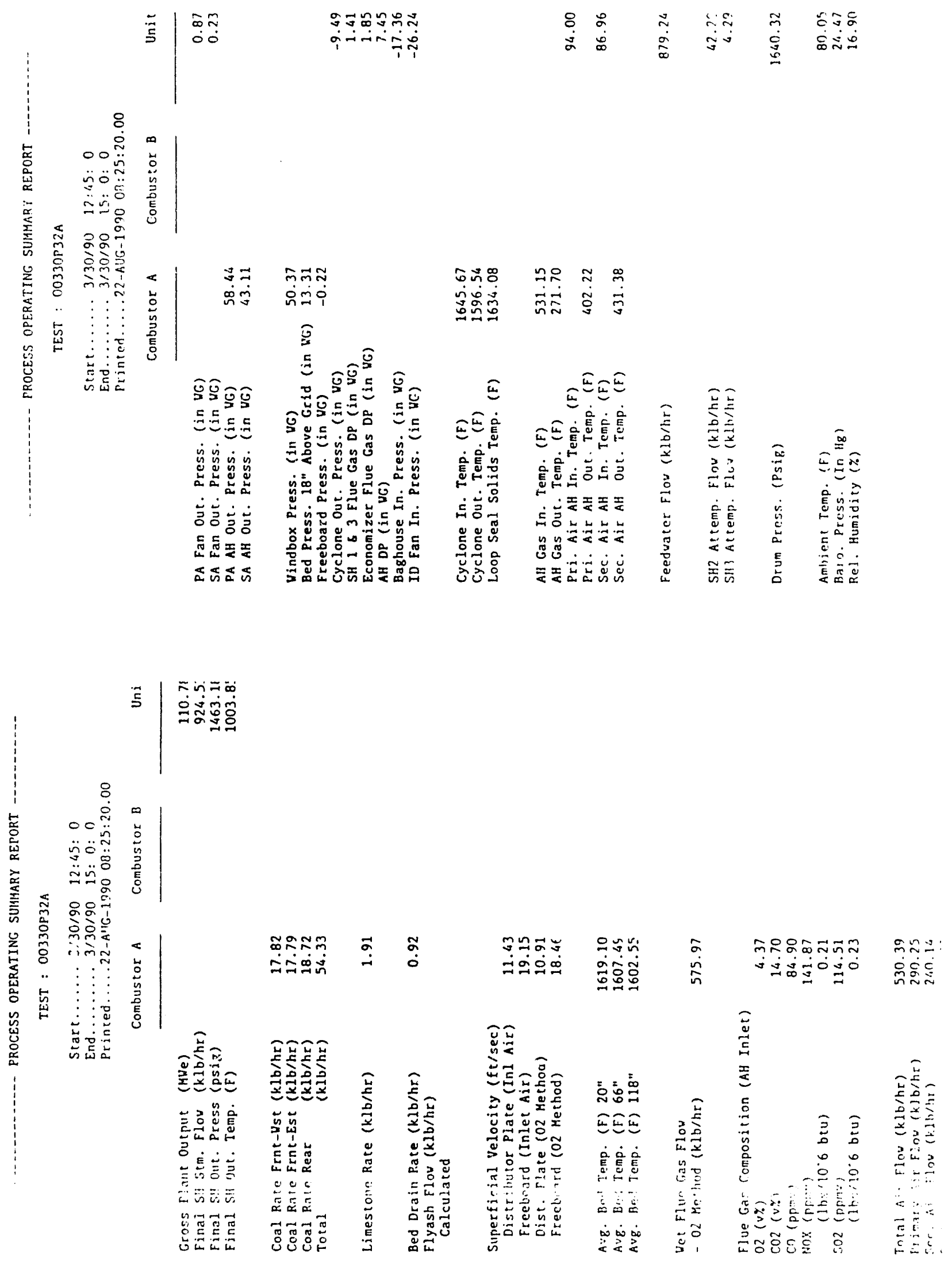


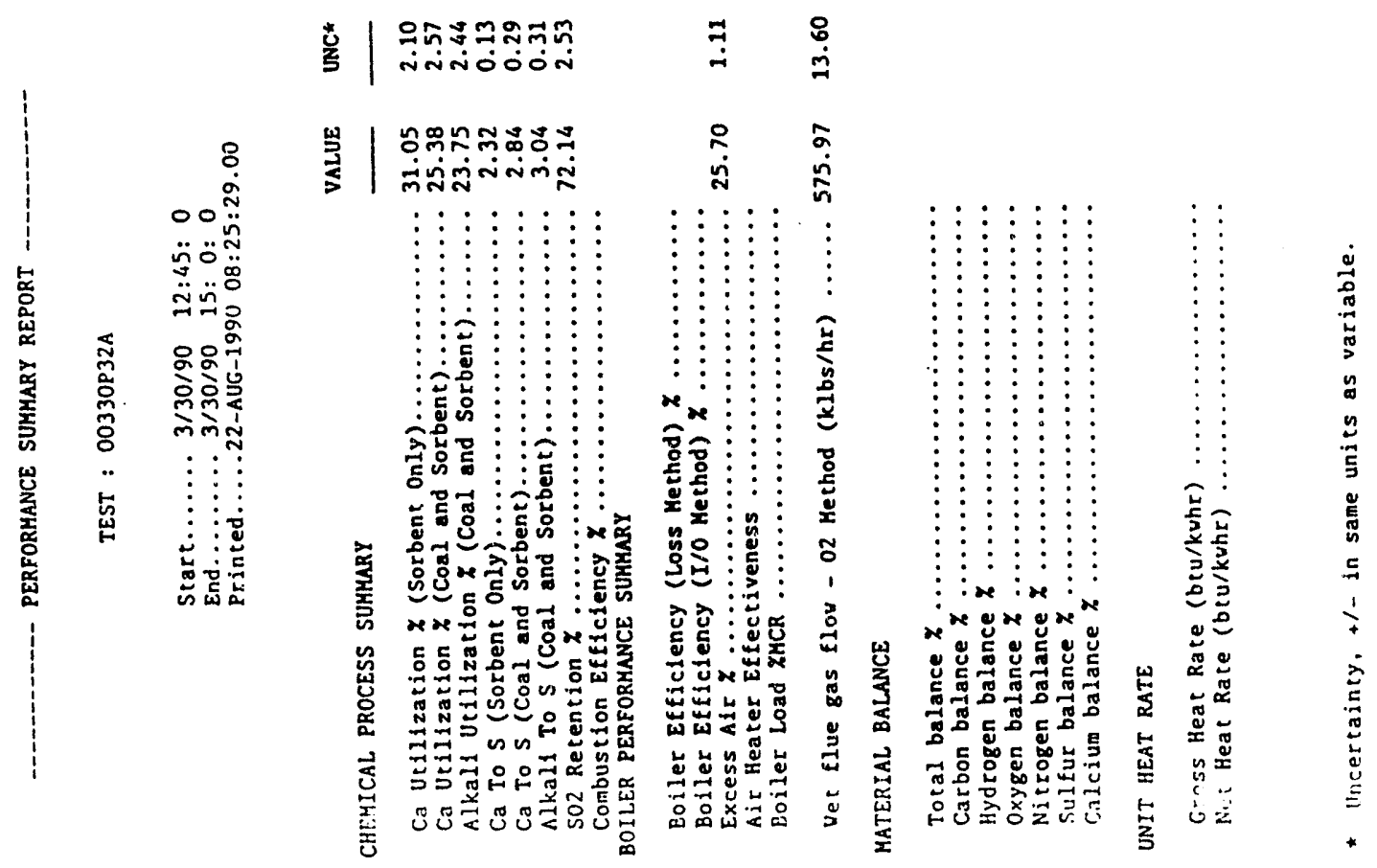

A-185 

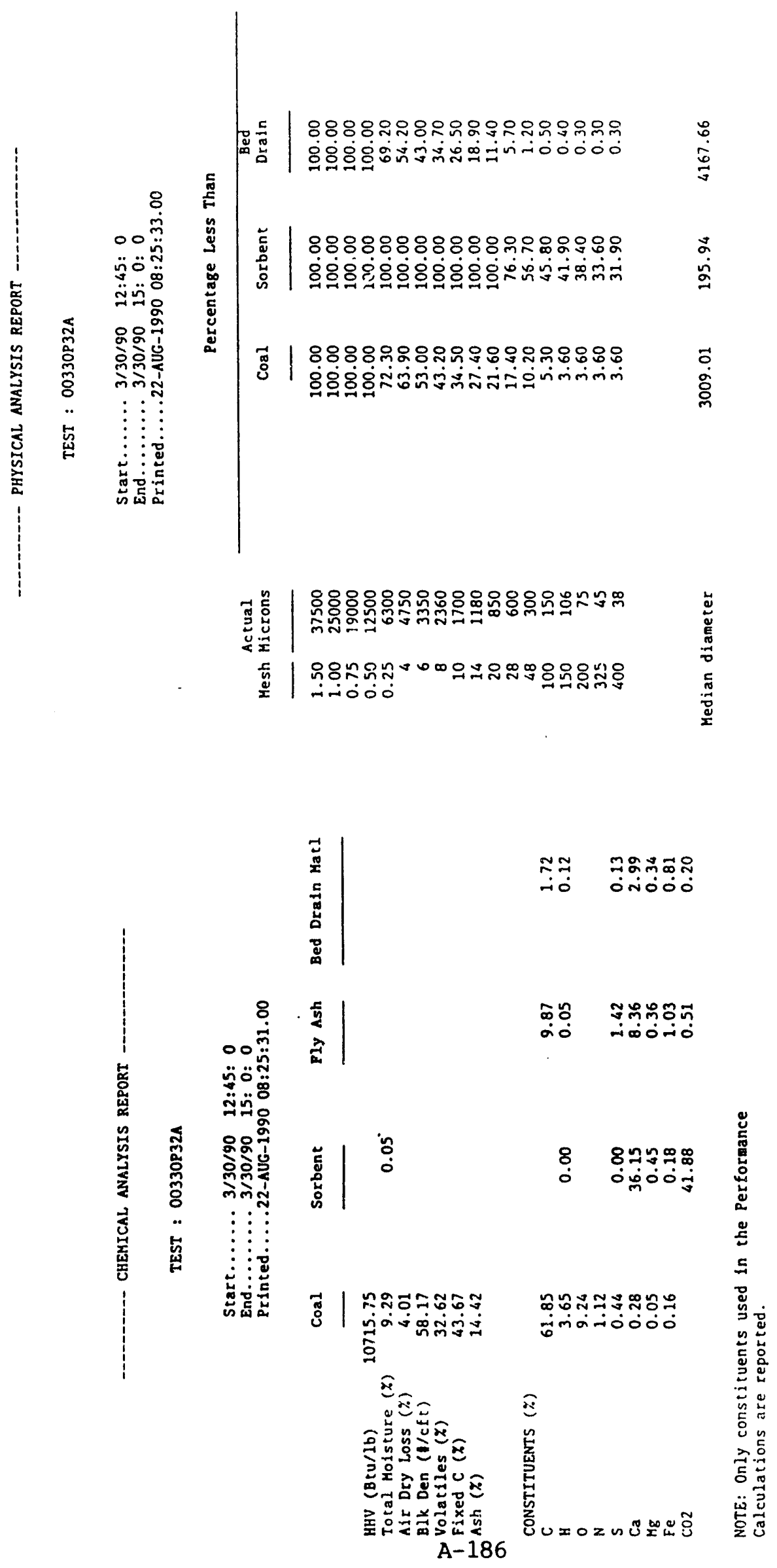

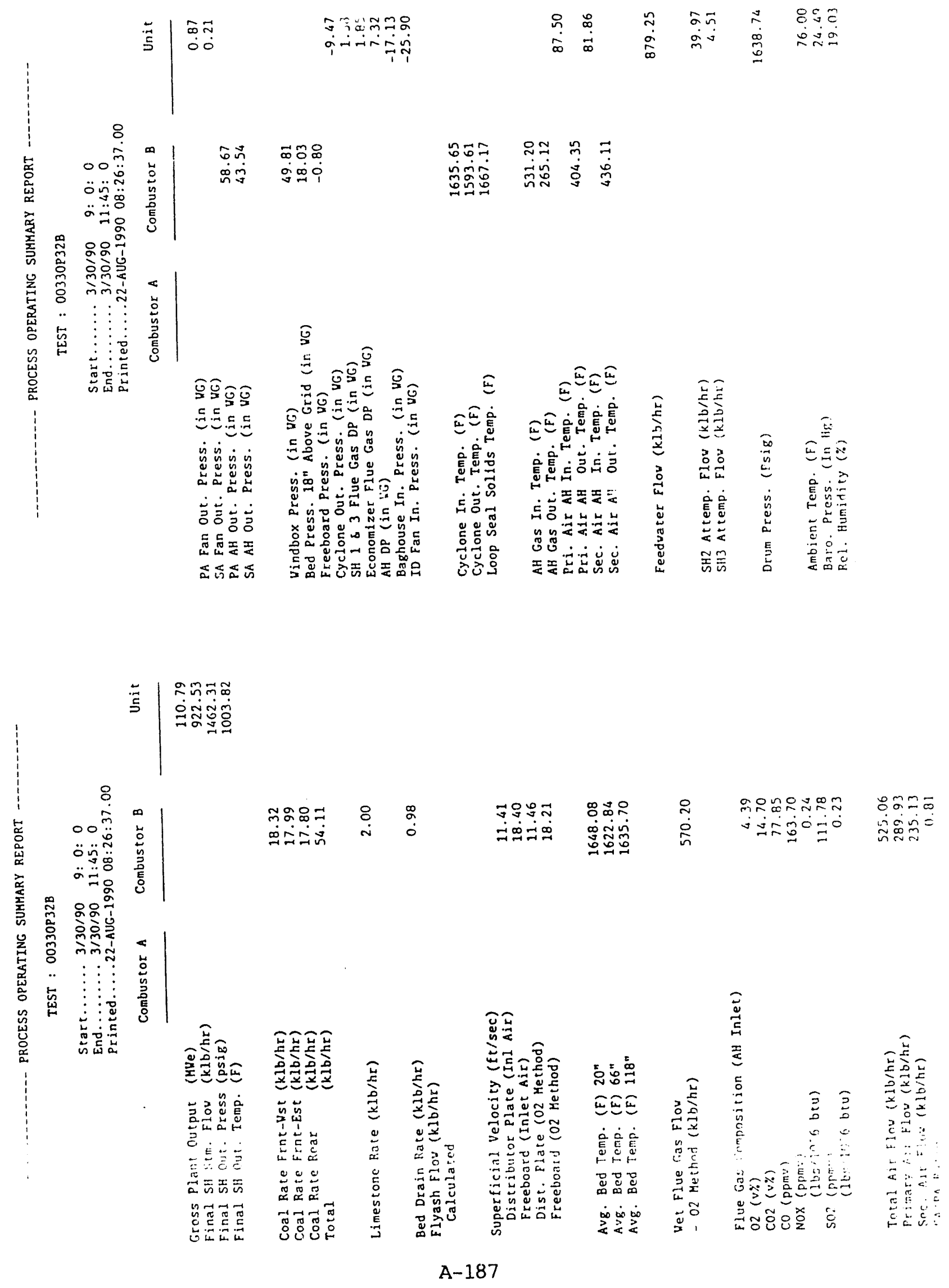


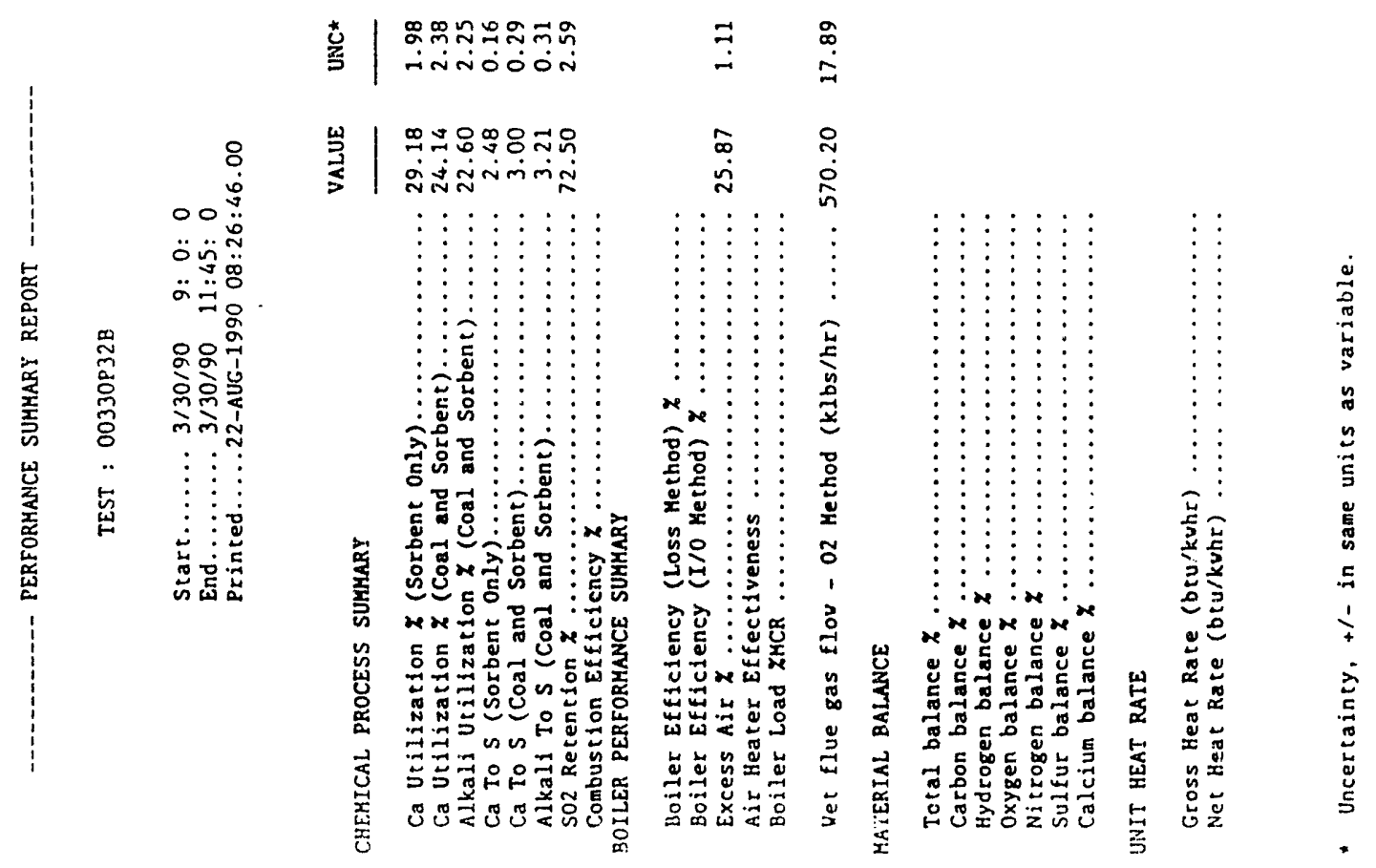



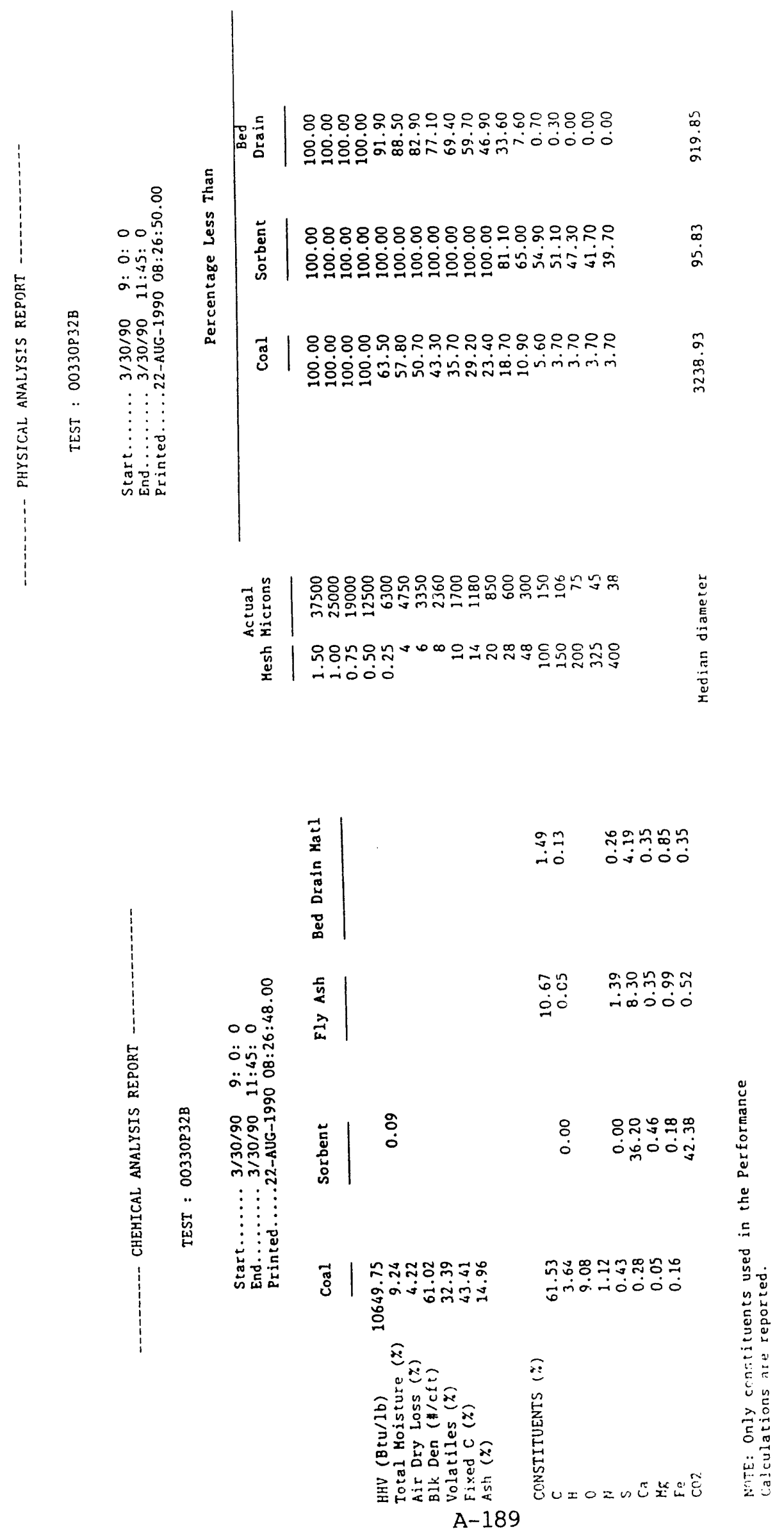

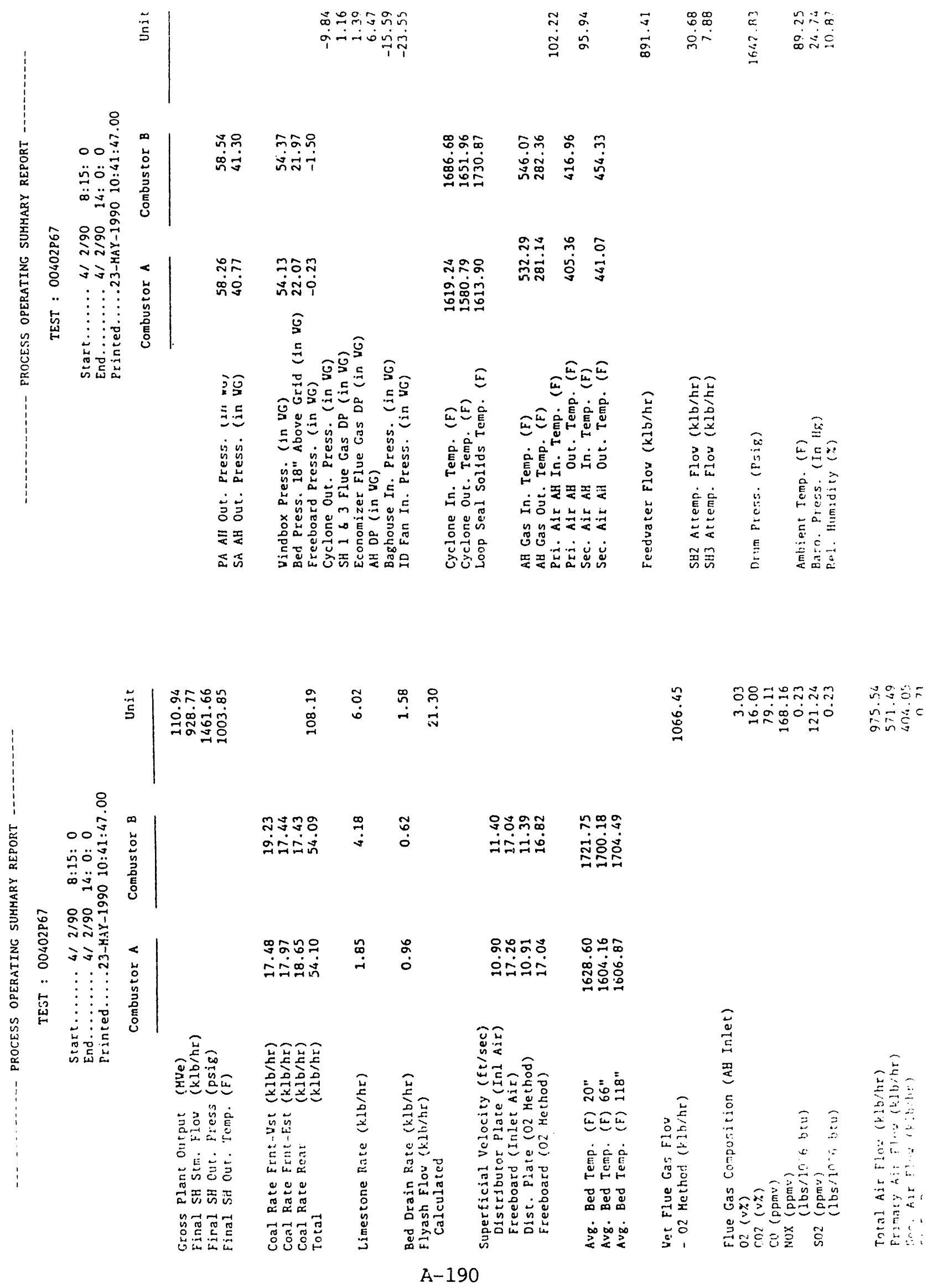


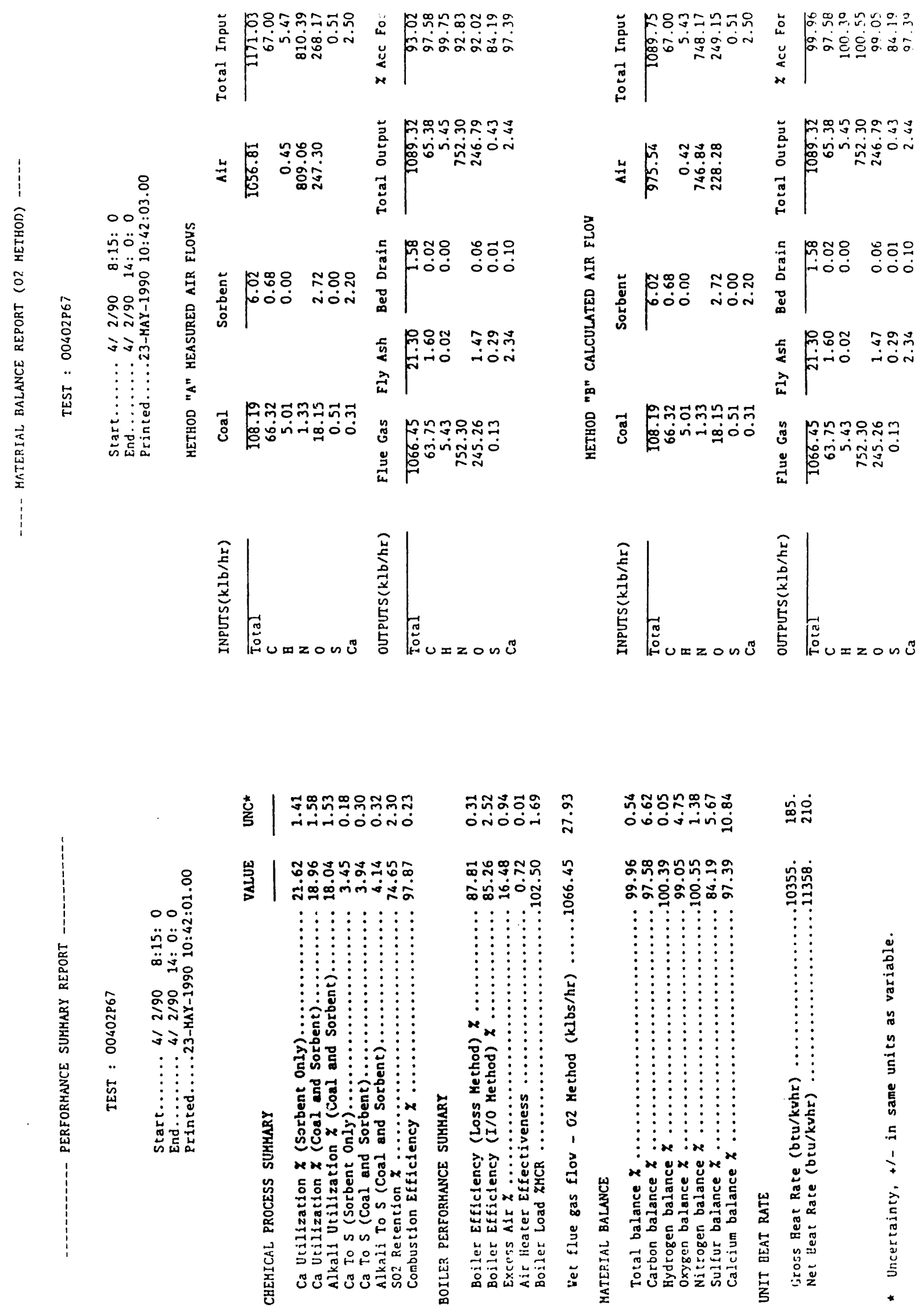



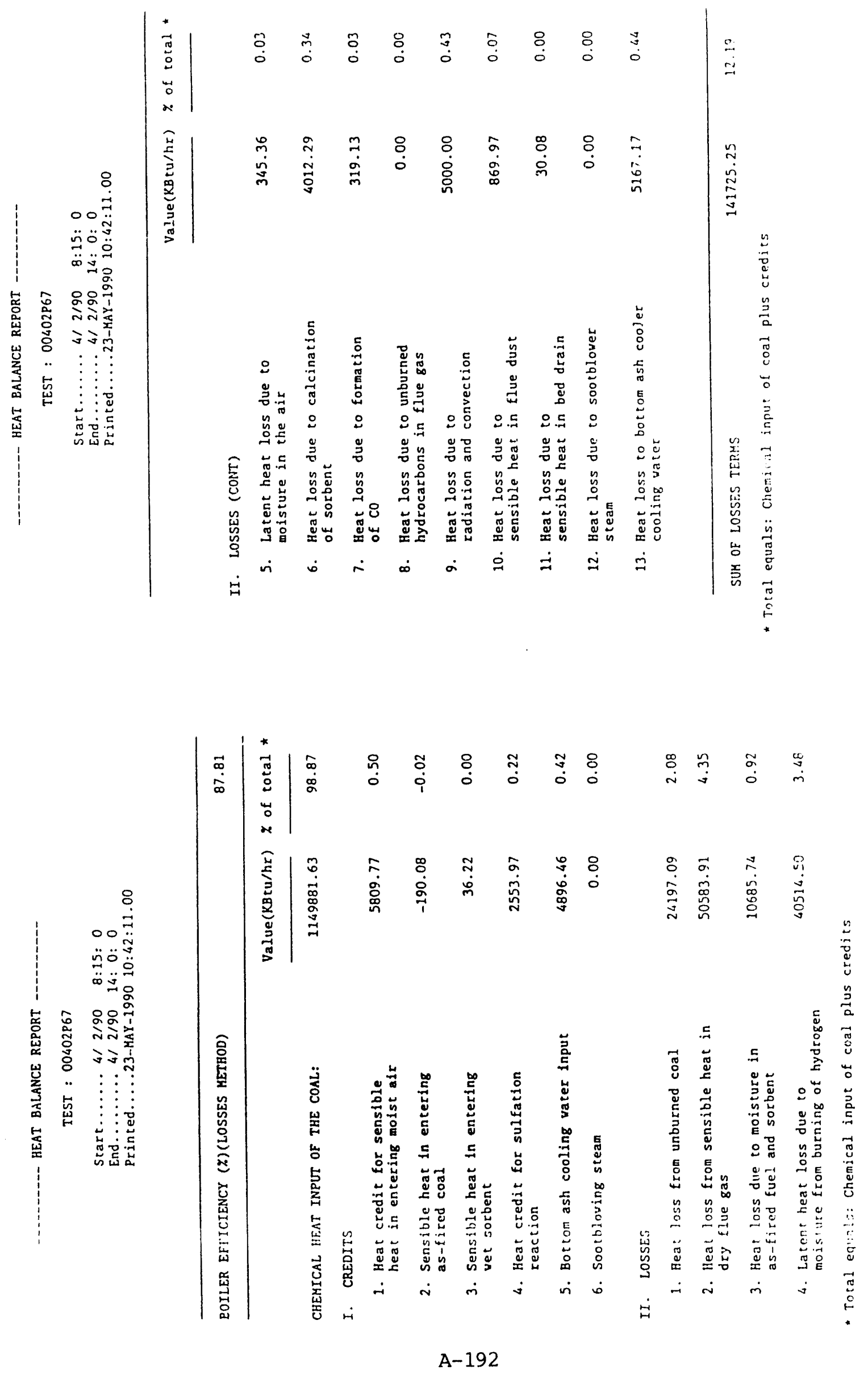


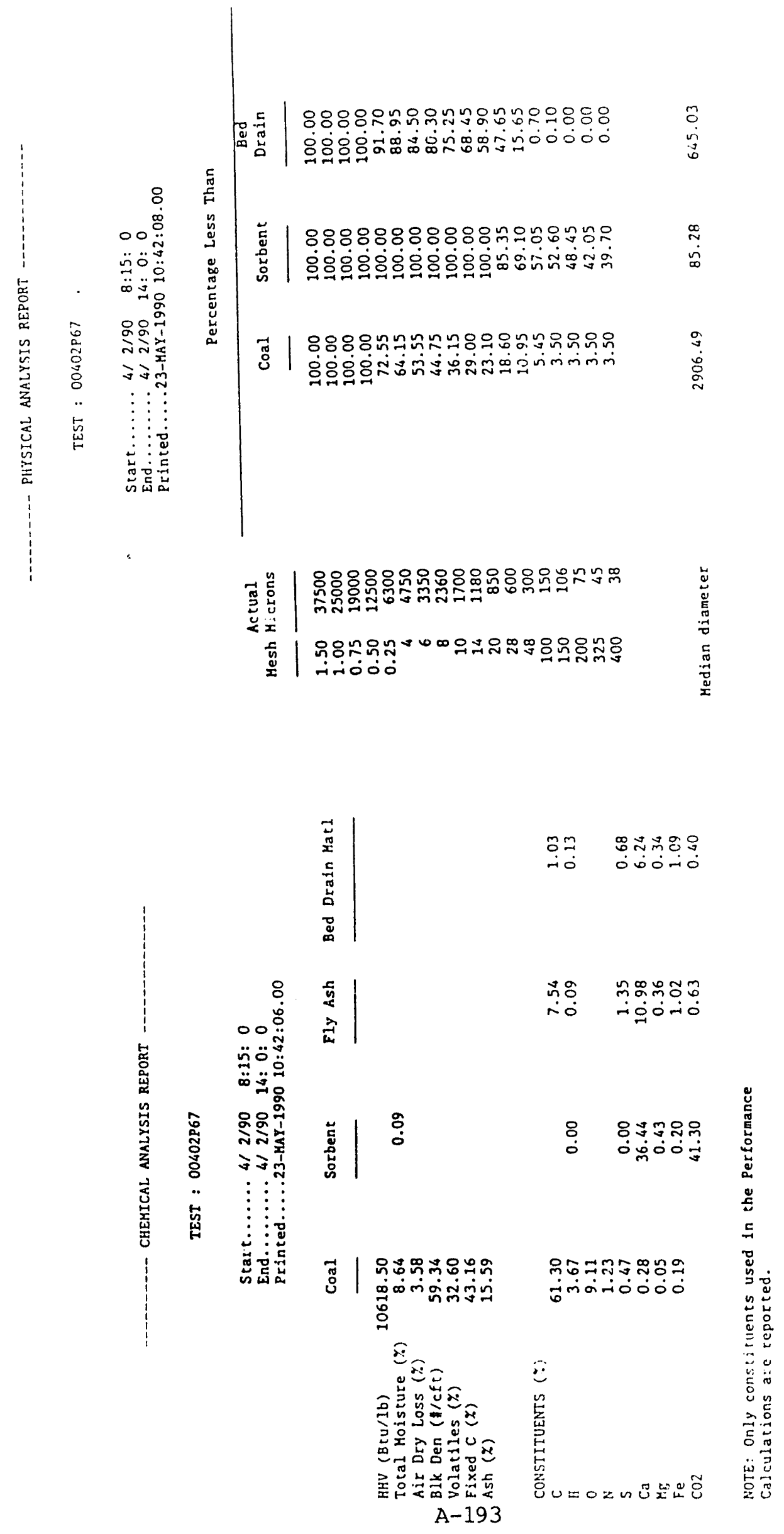



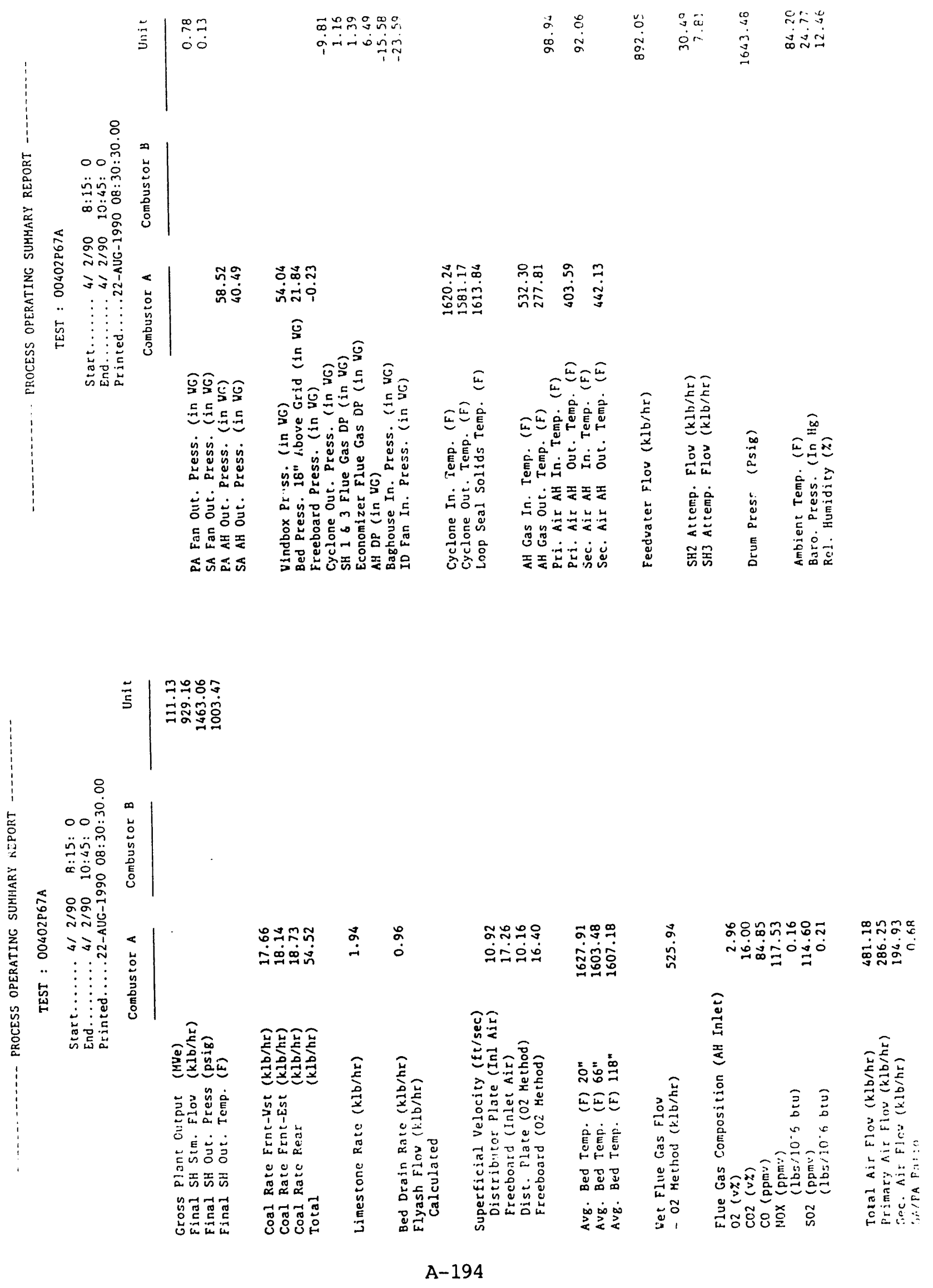


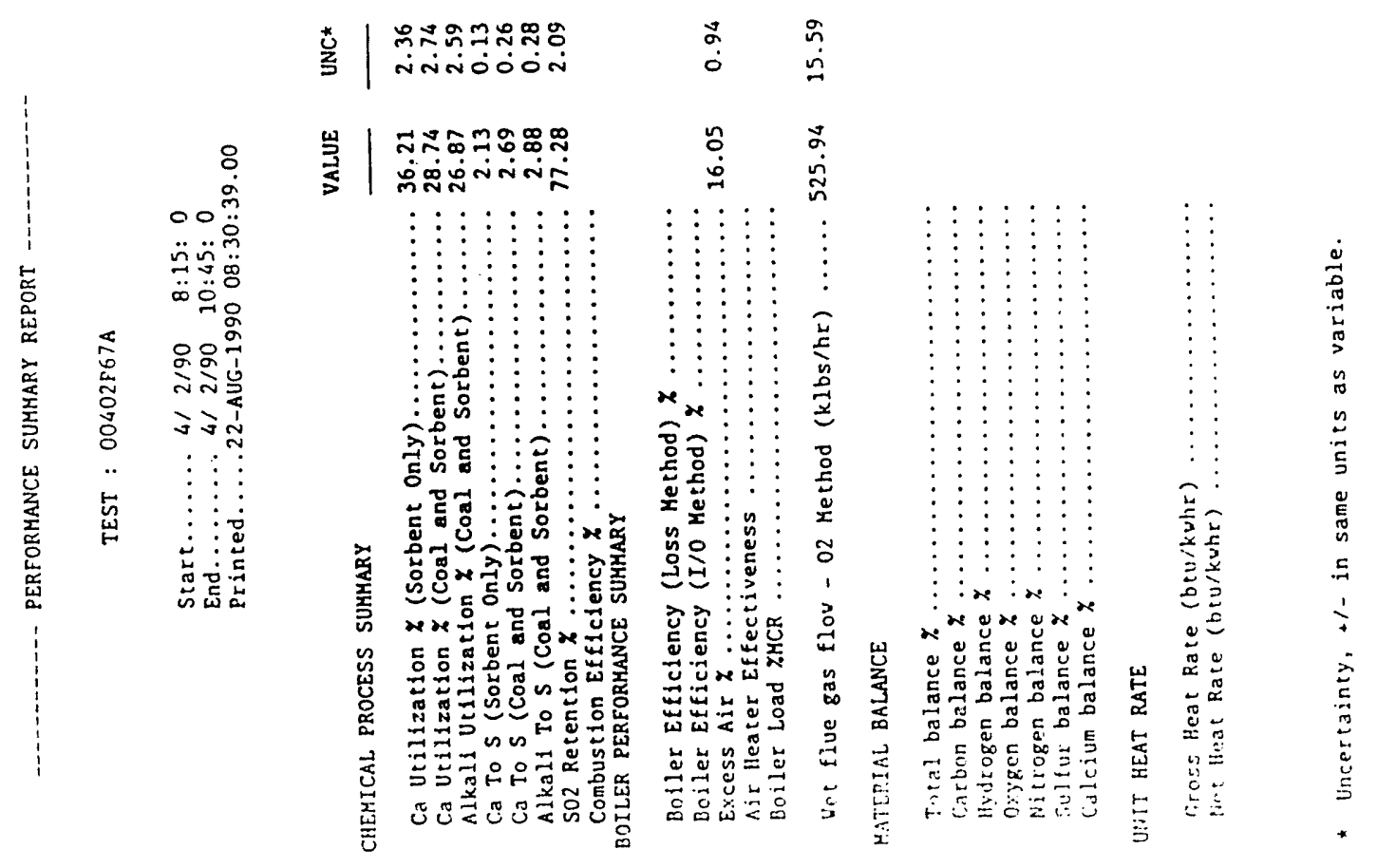



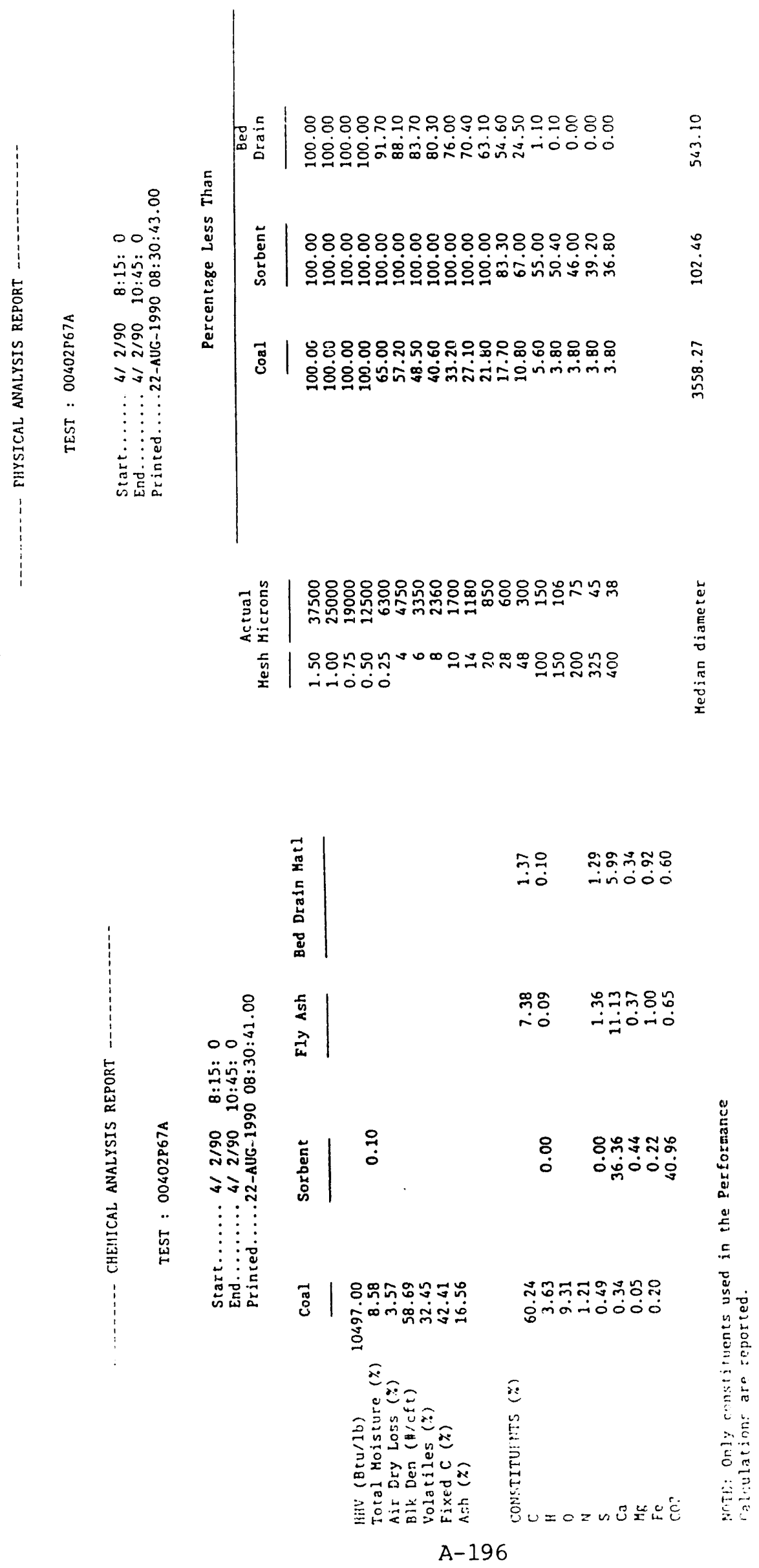


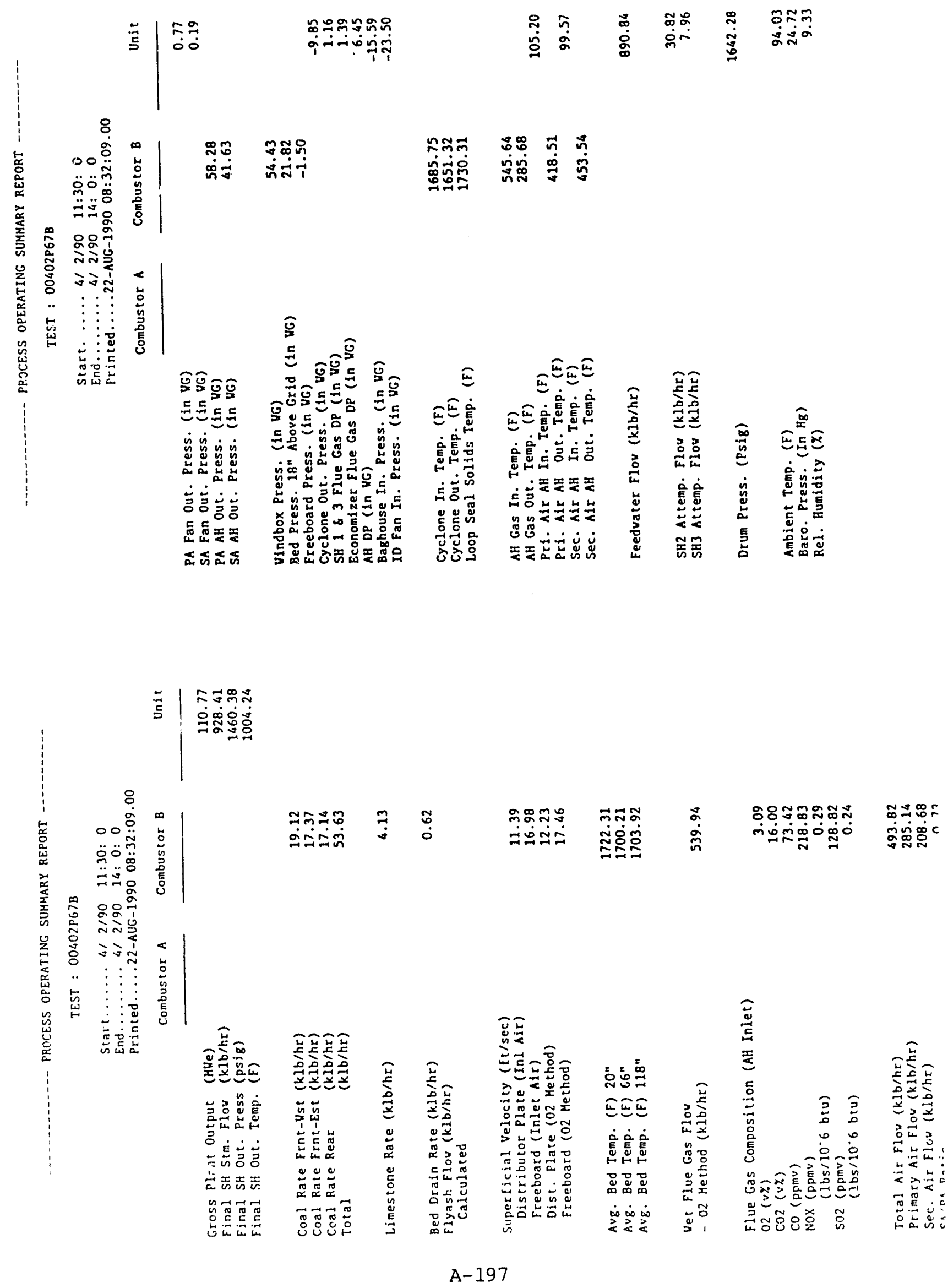




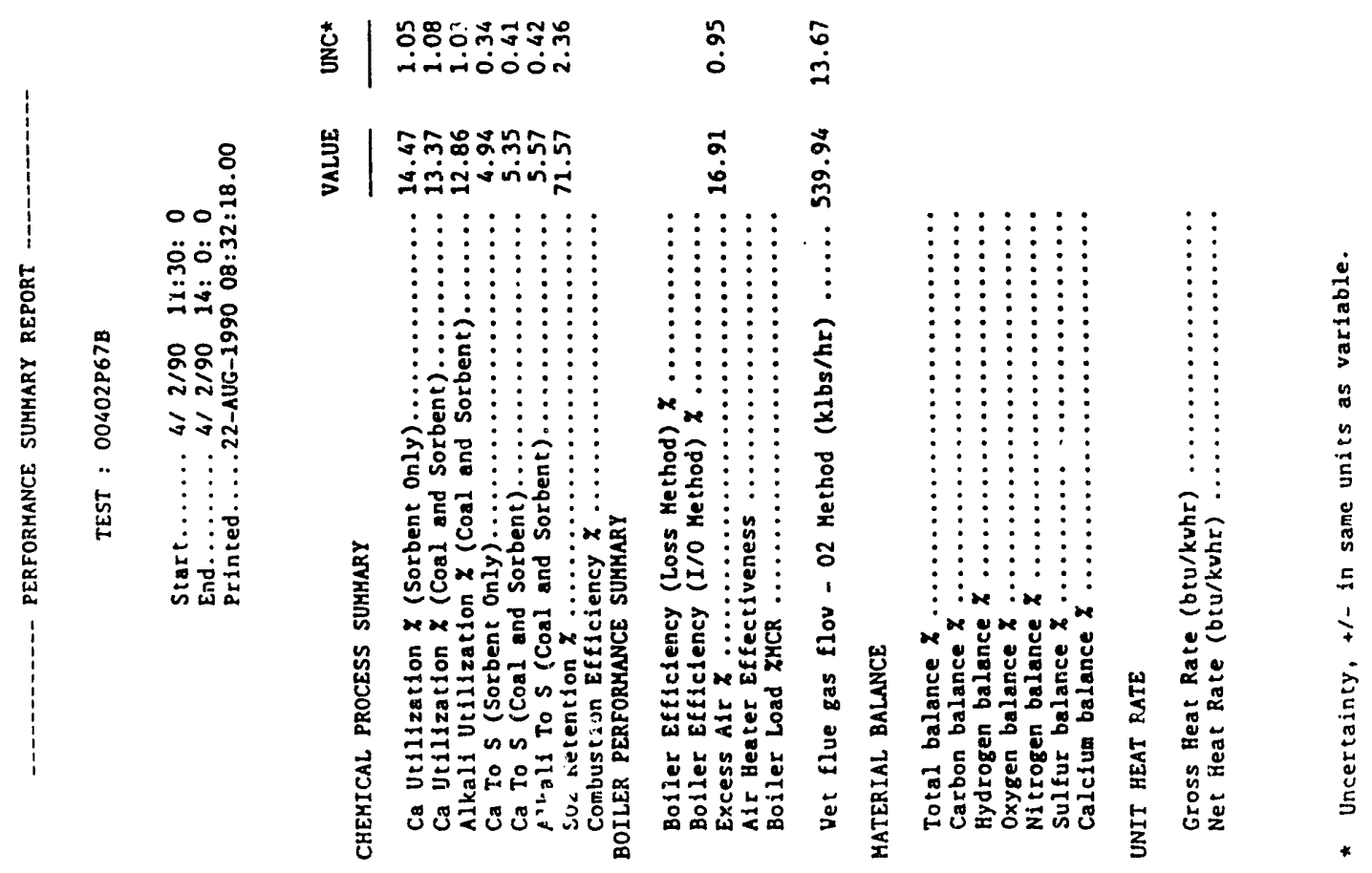

A-198 

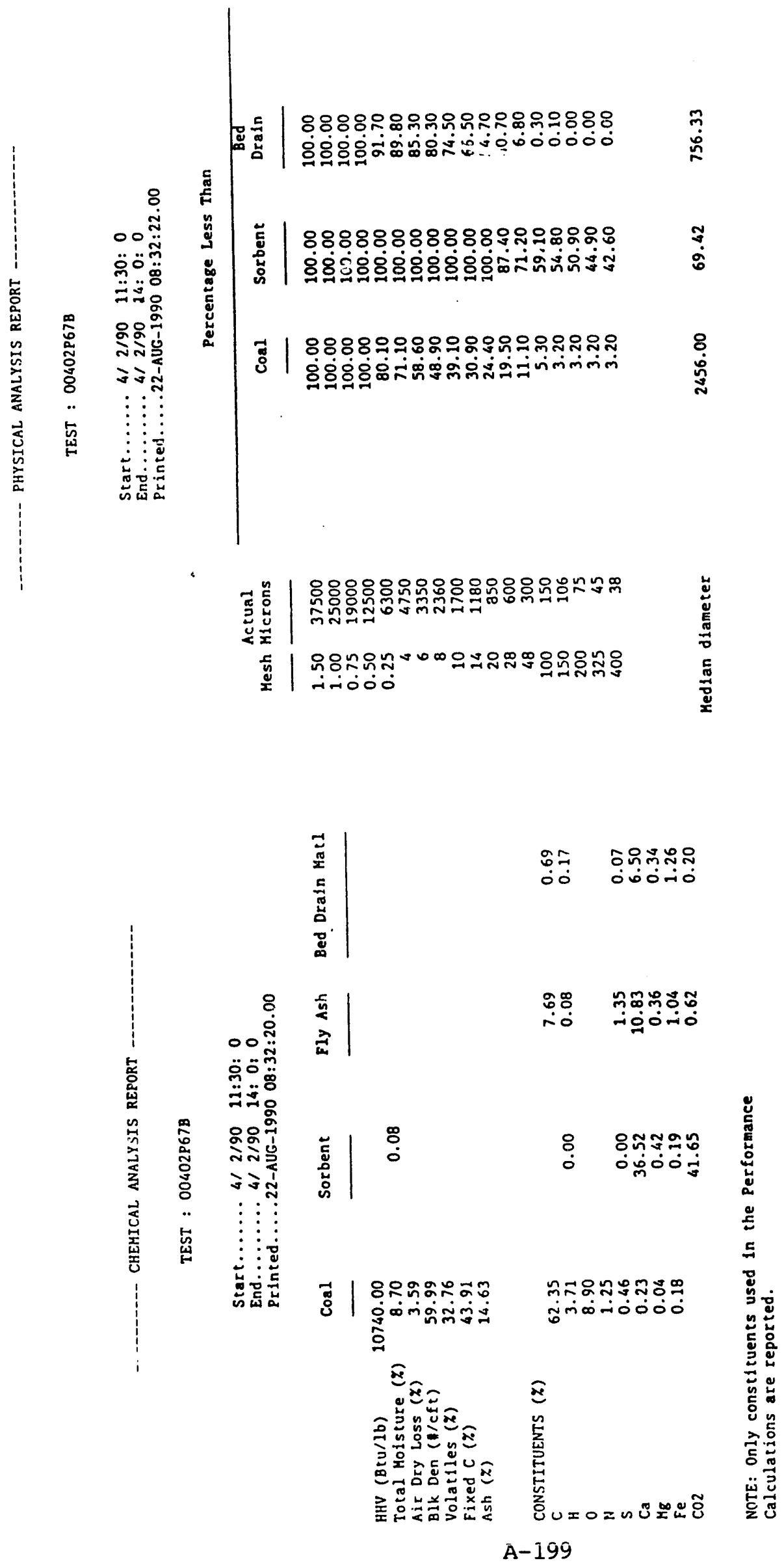


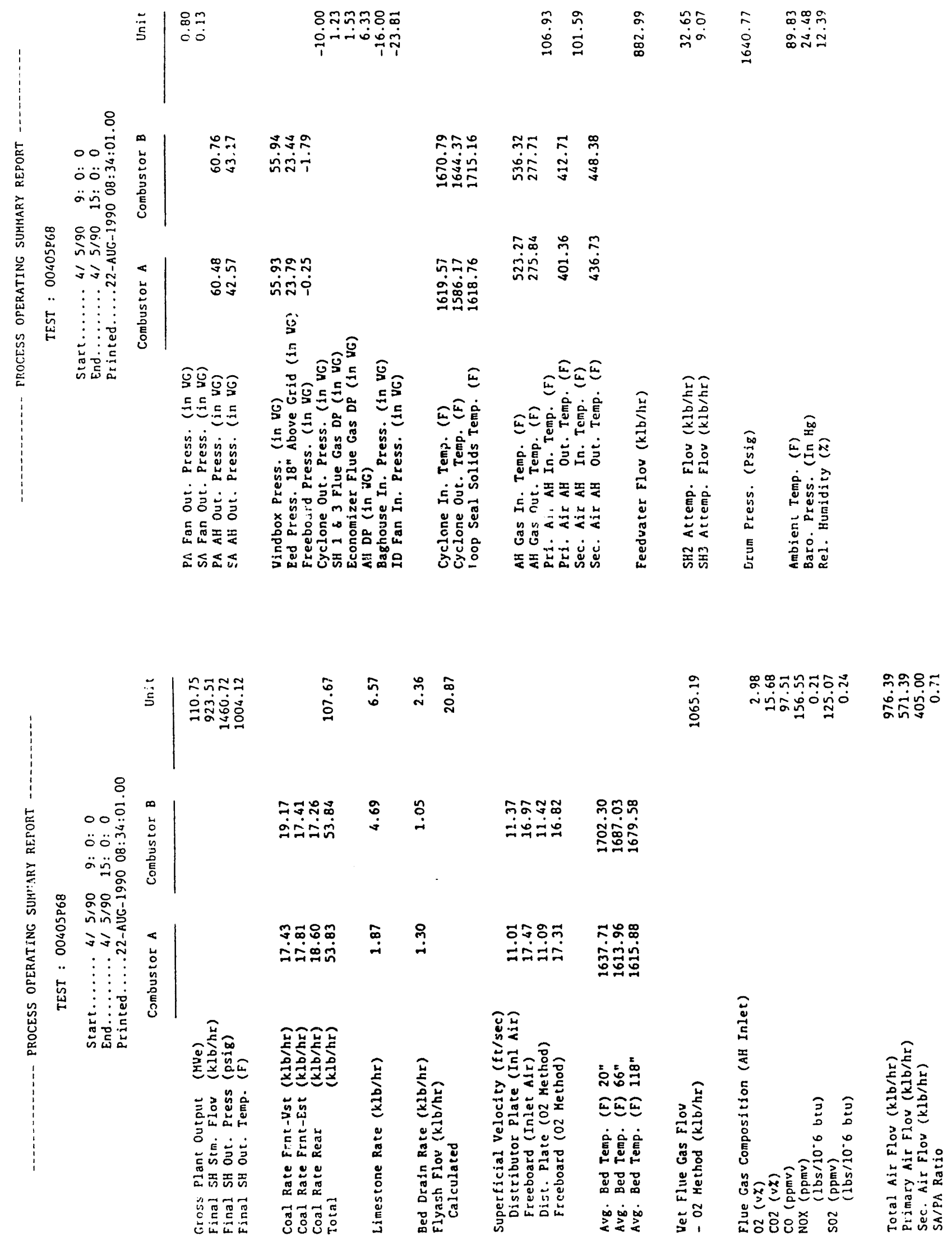




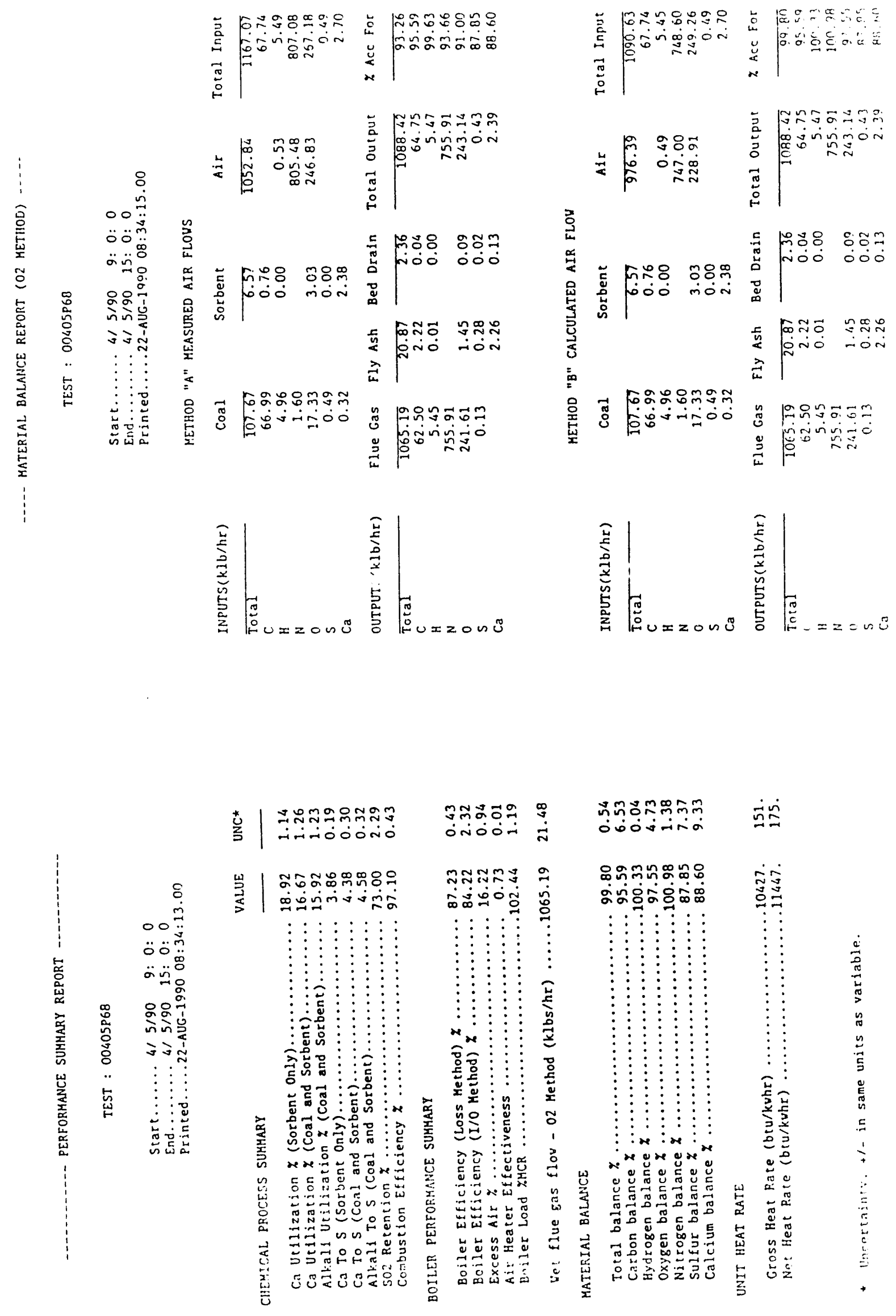




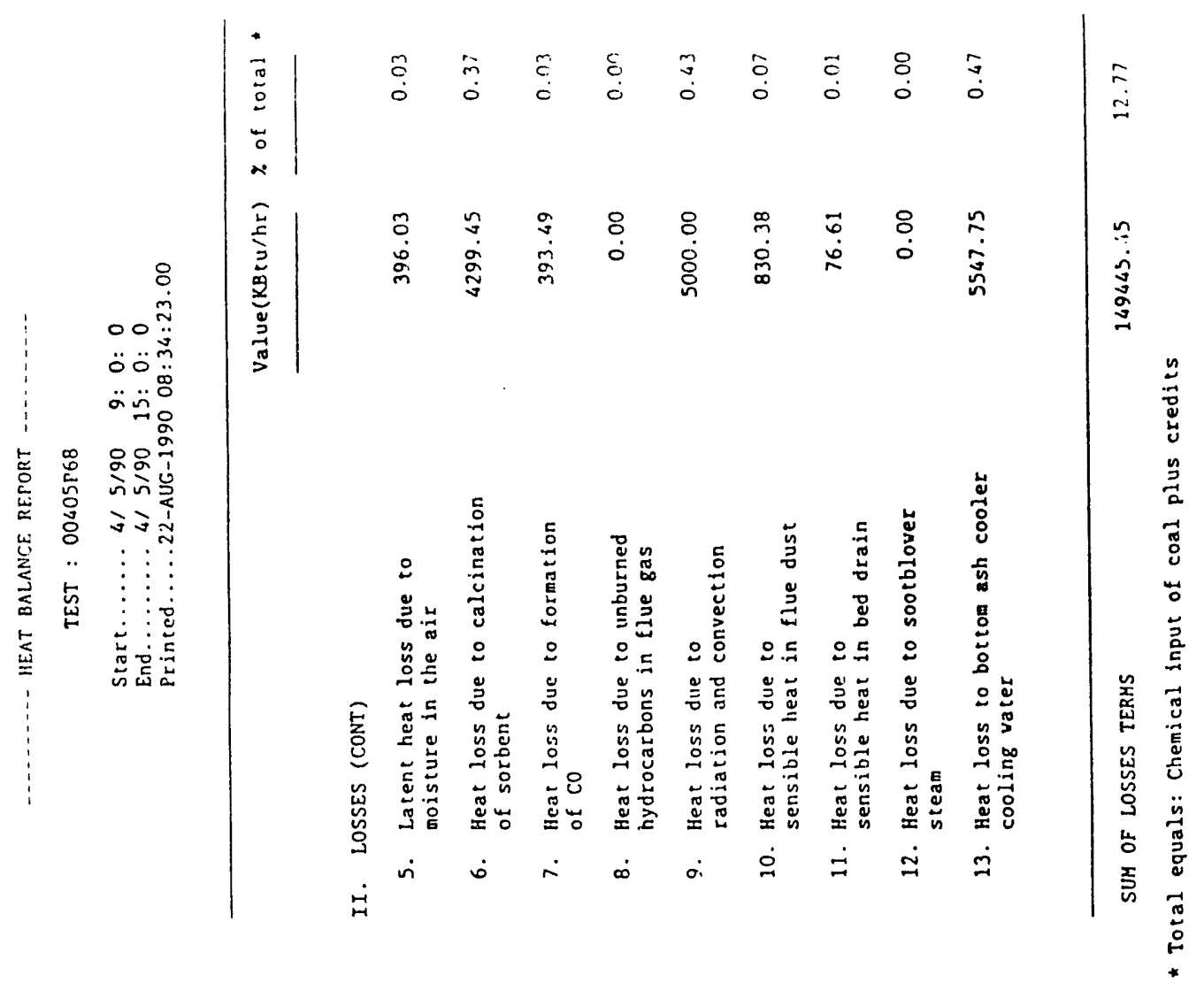

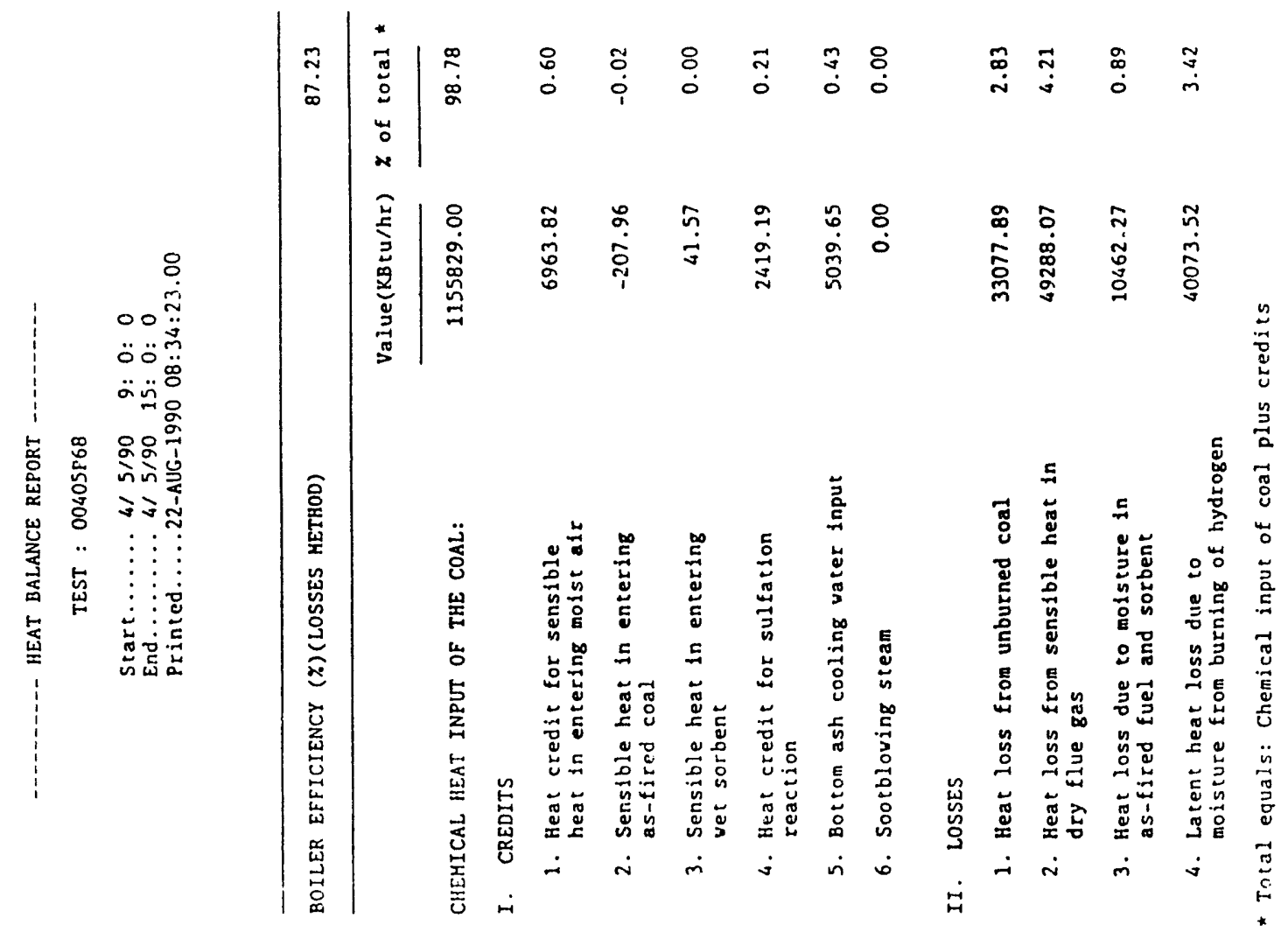



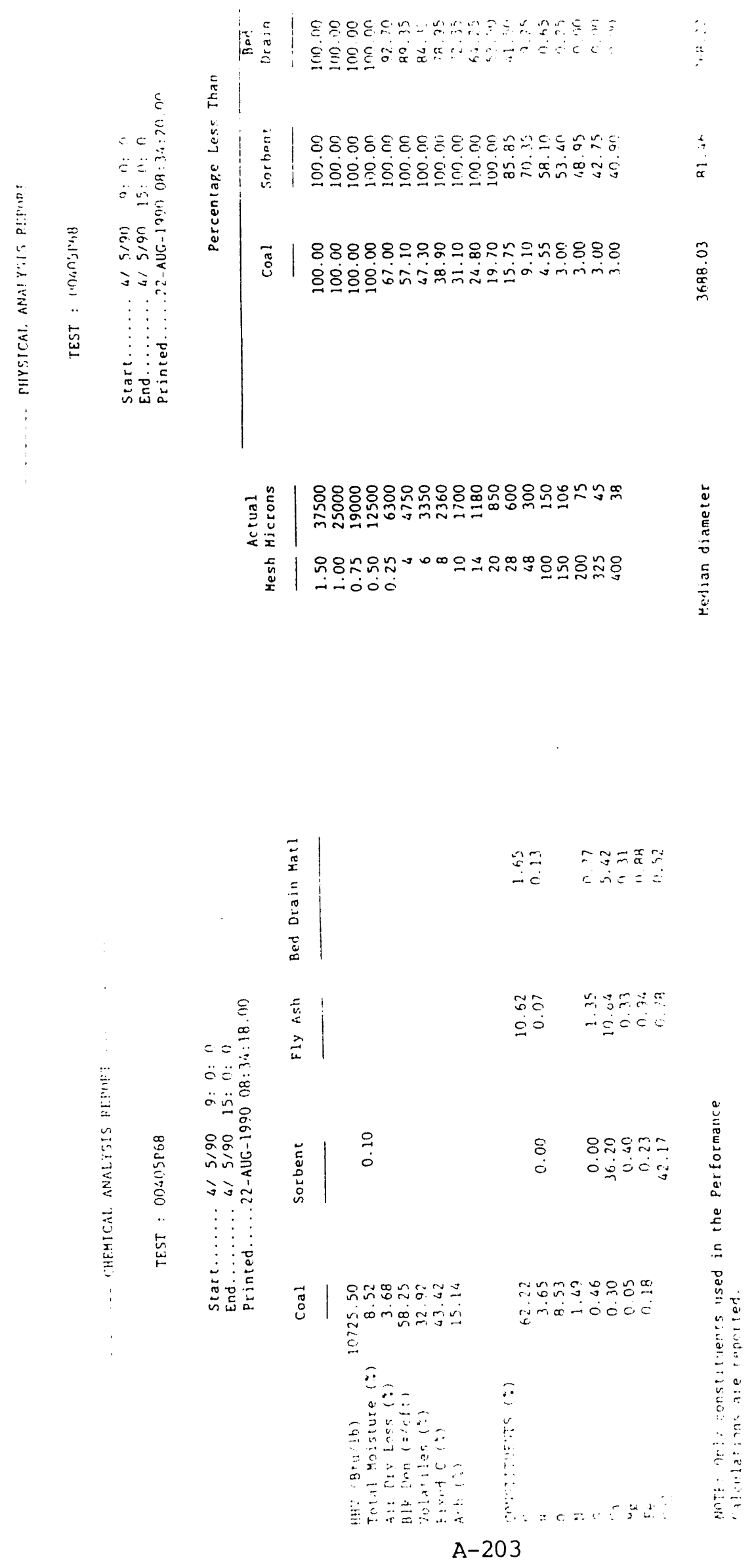

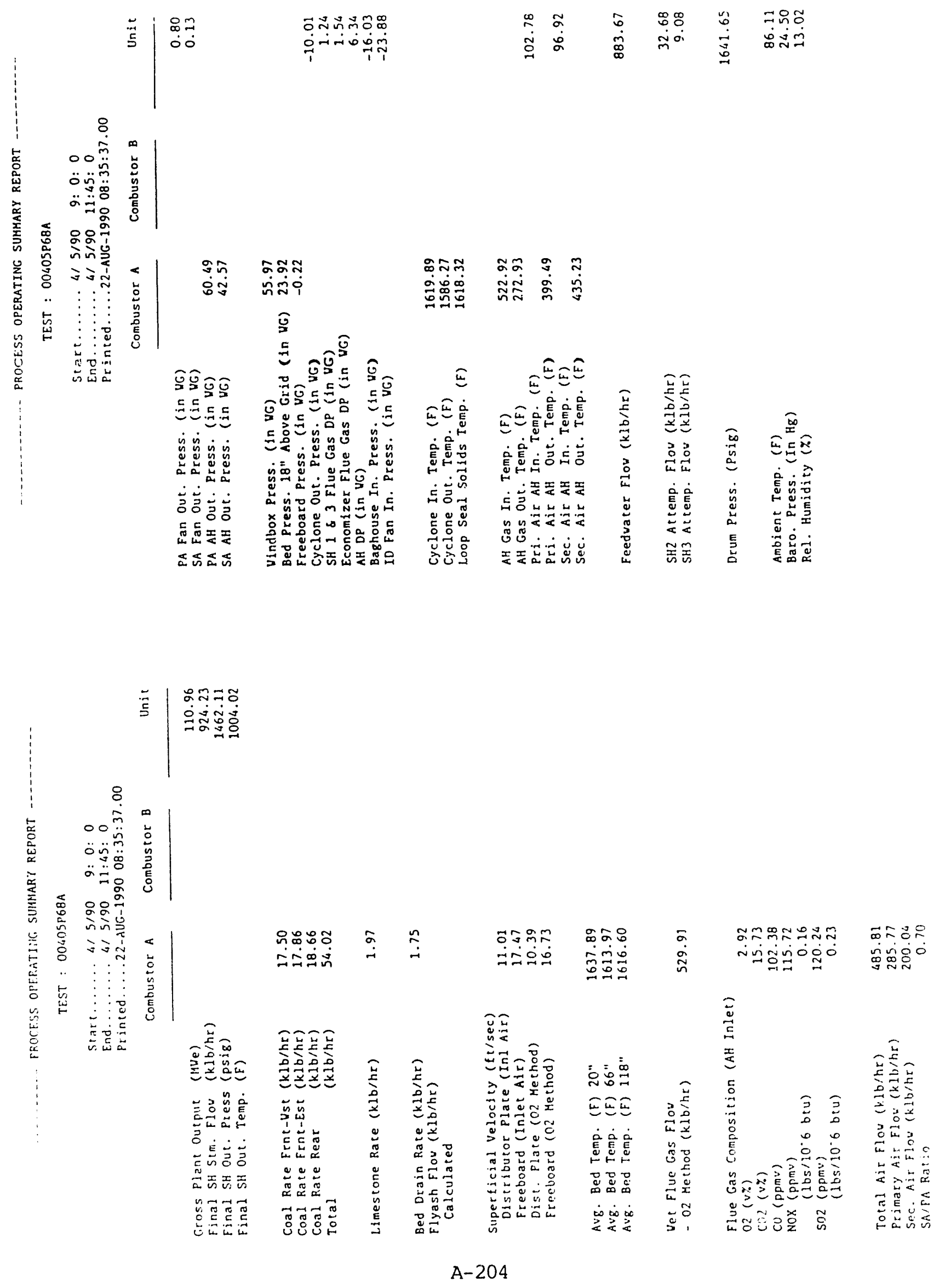


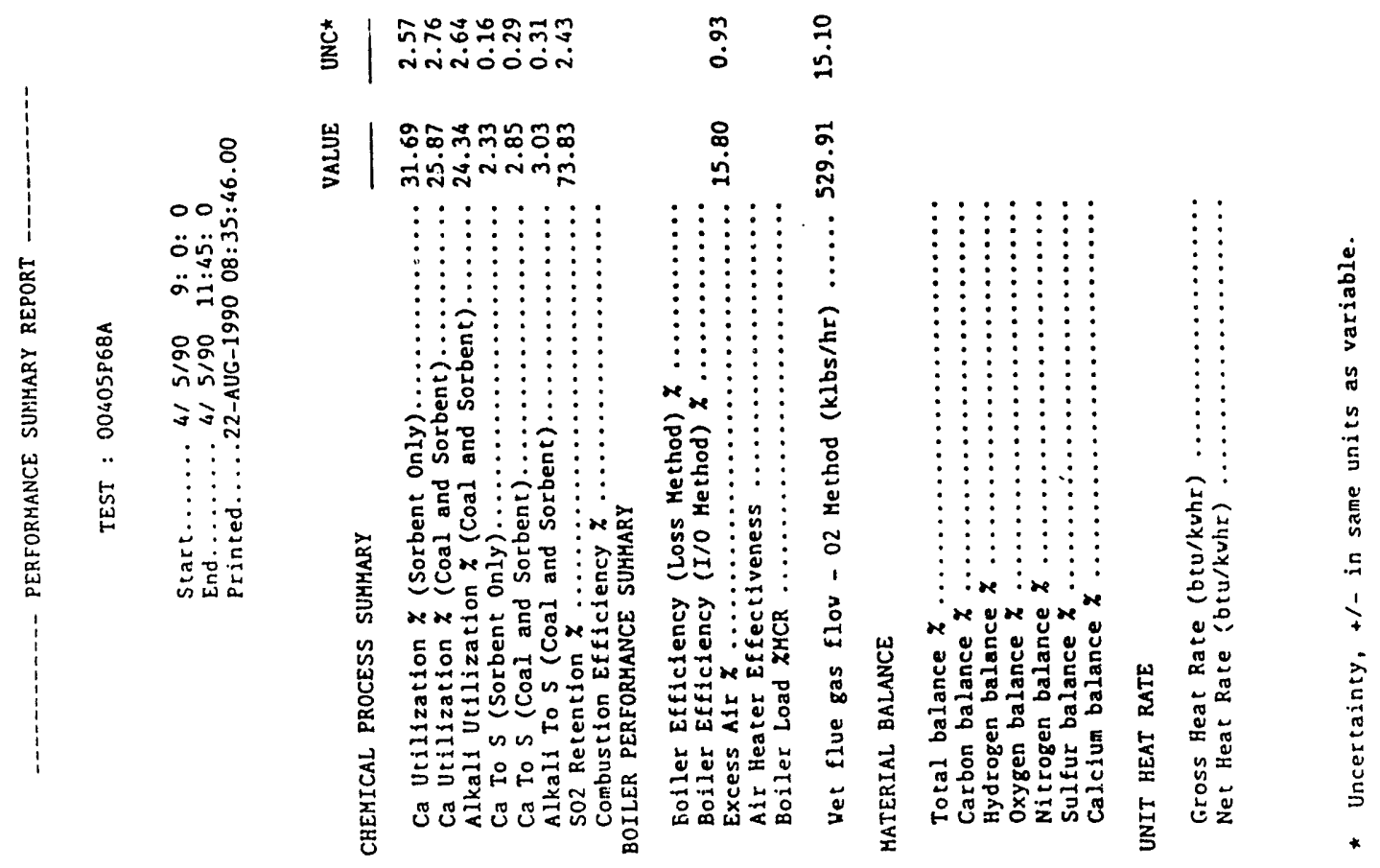

A-205 


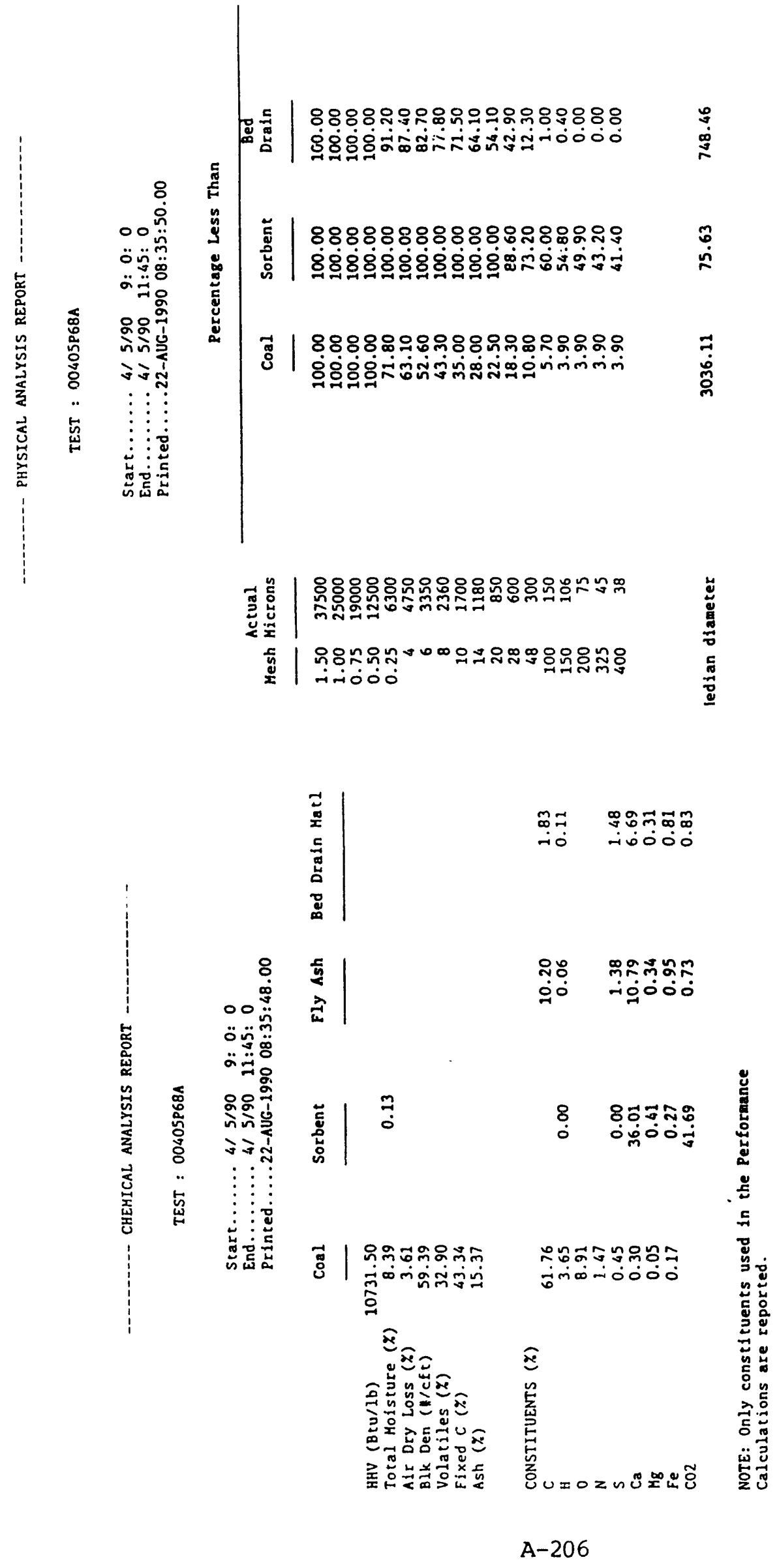



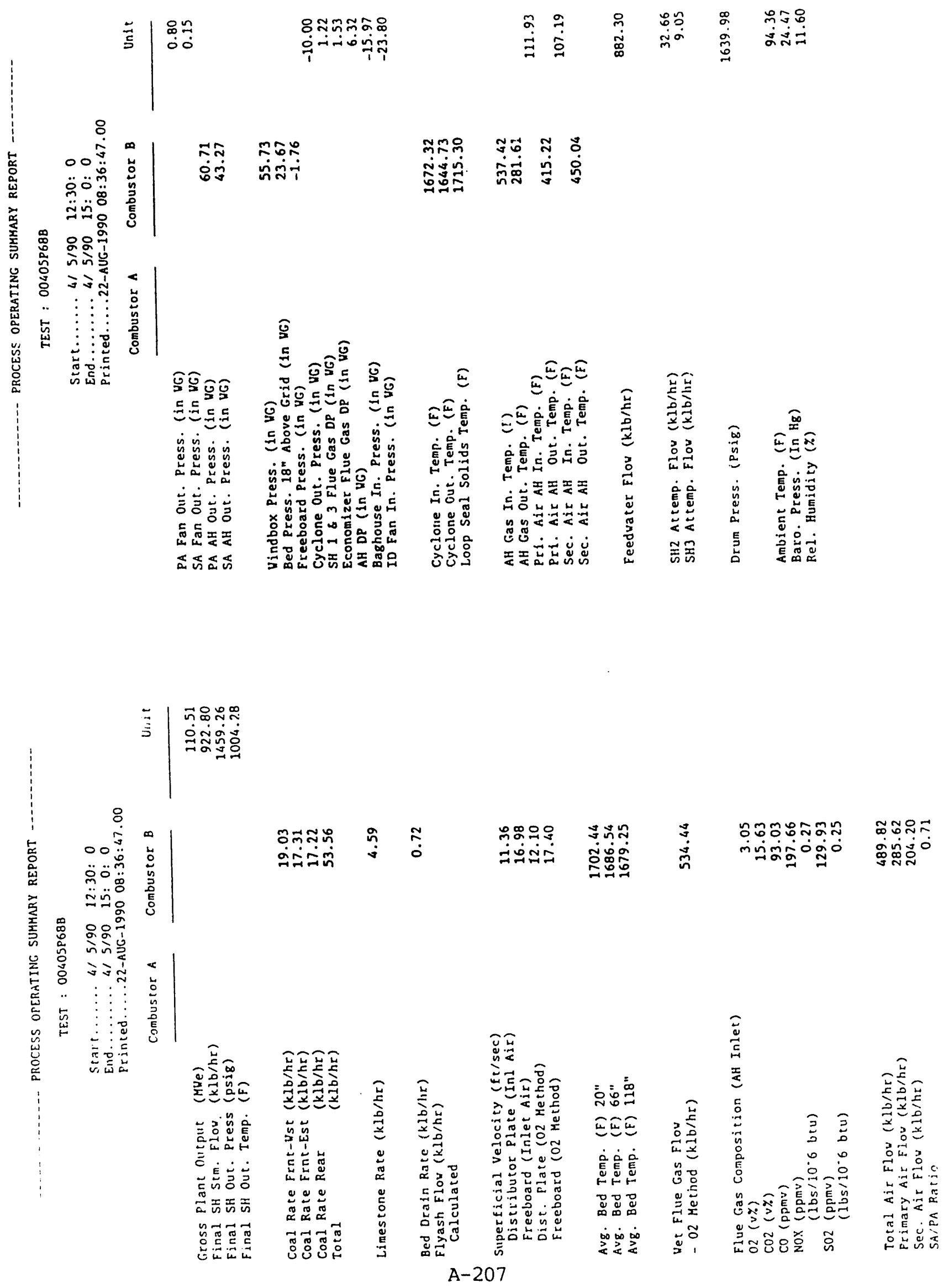


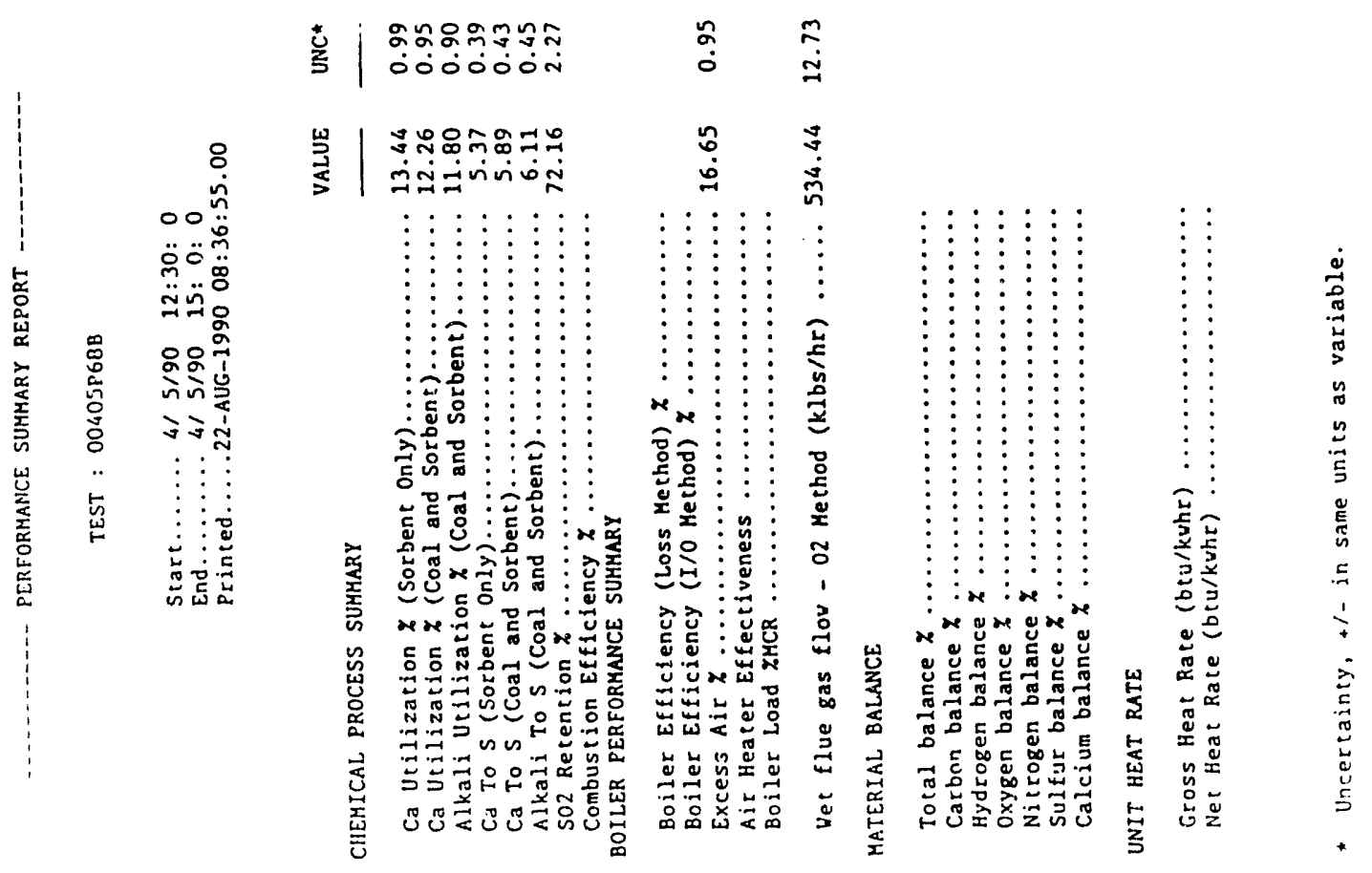

A-208 

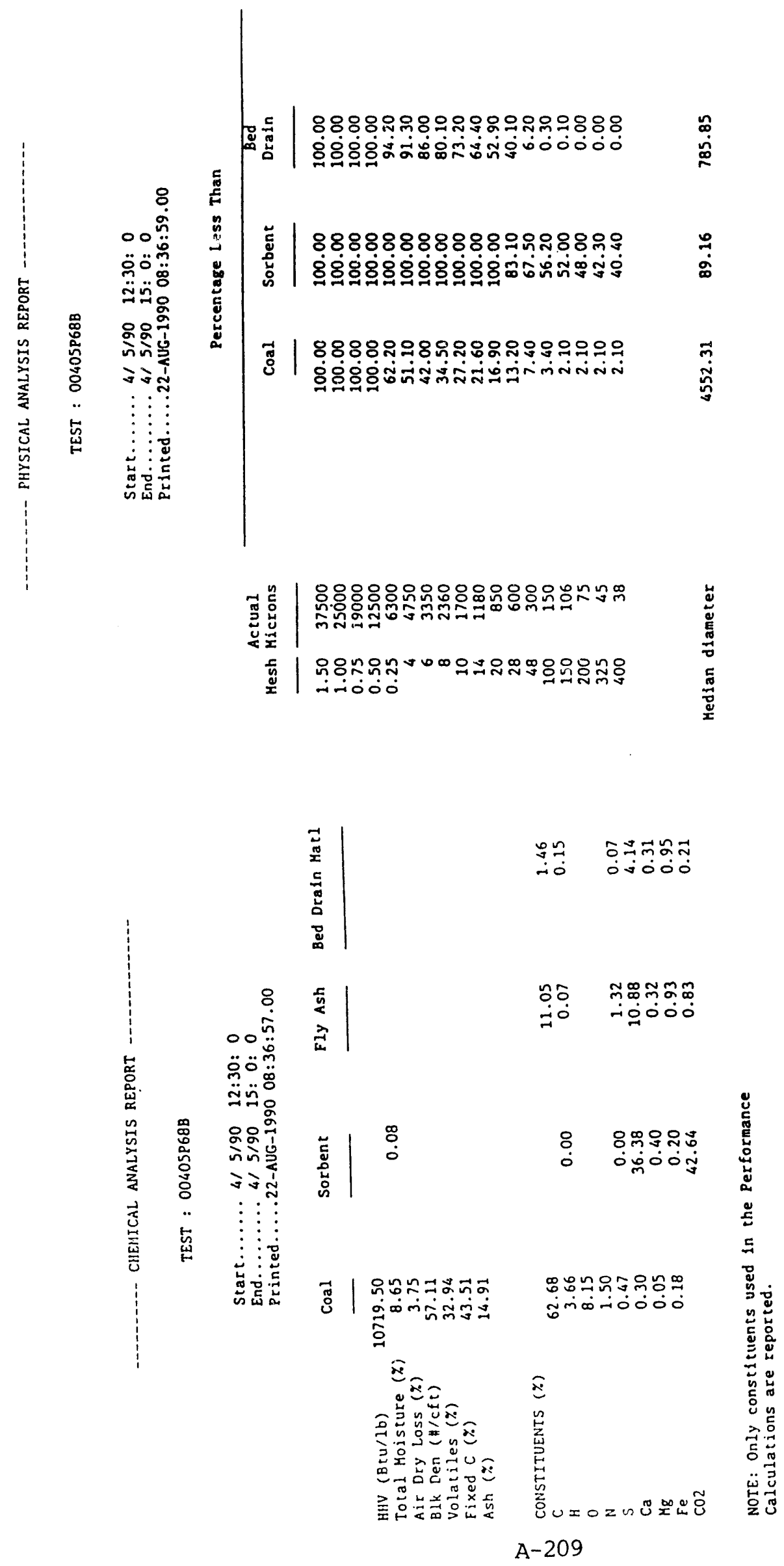


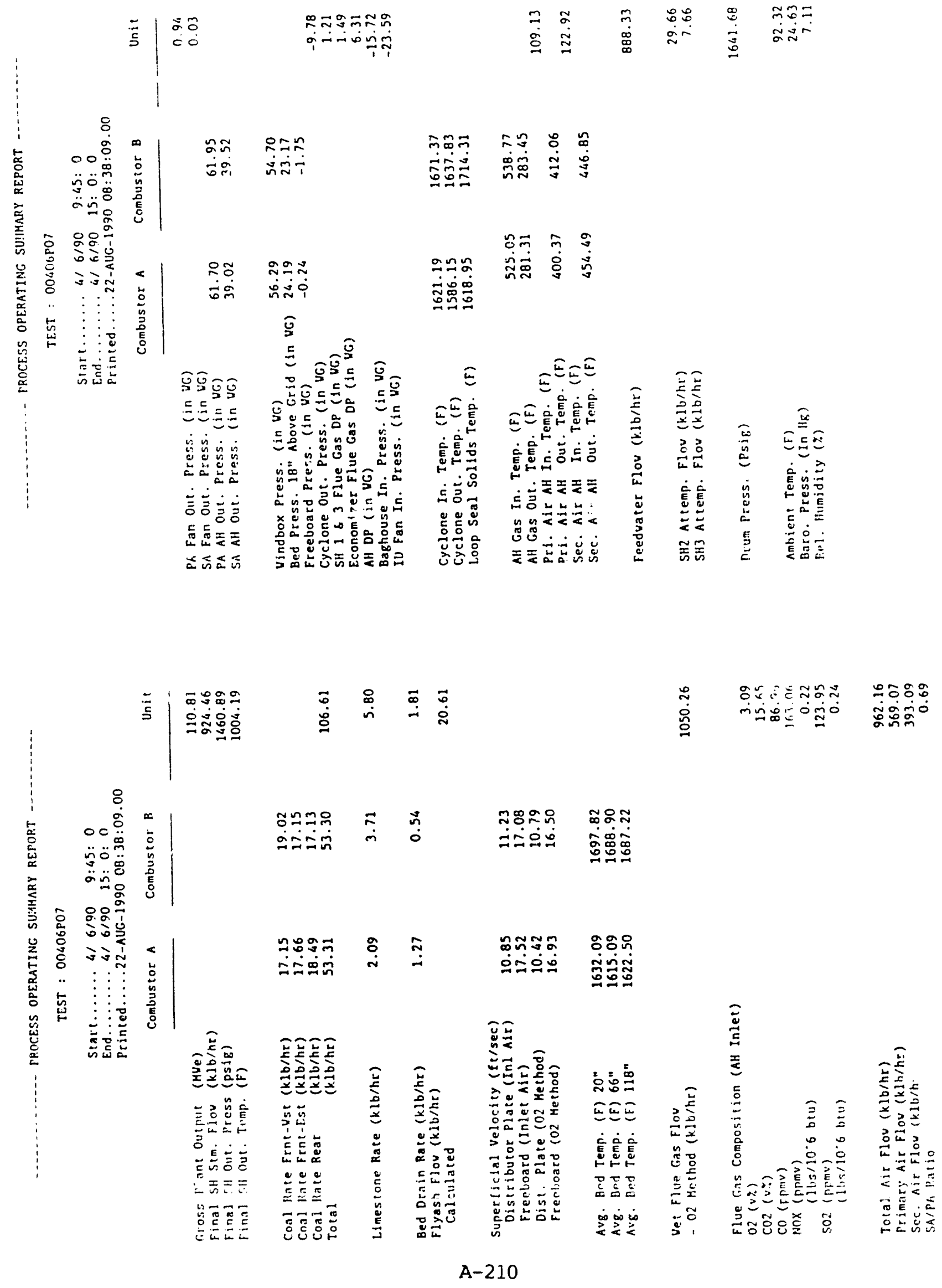




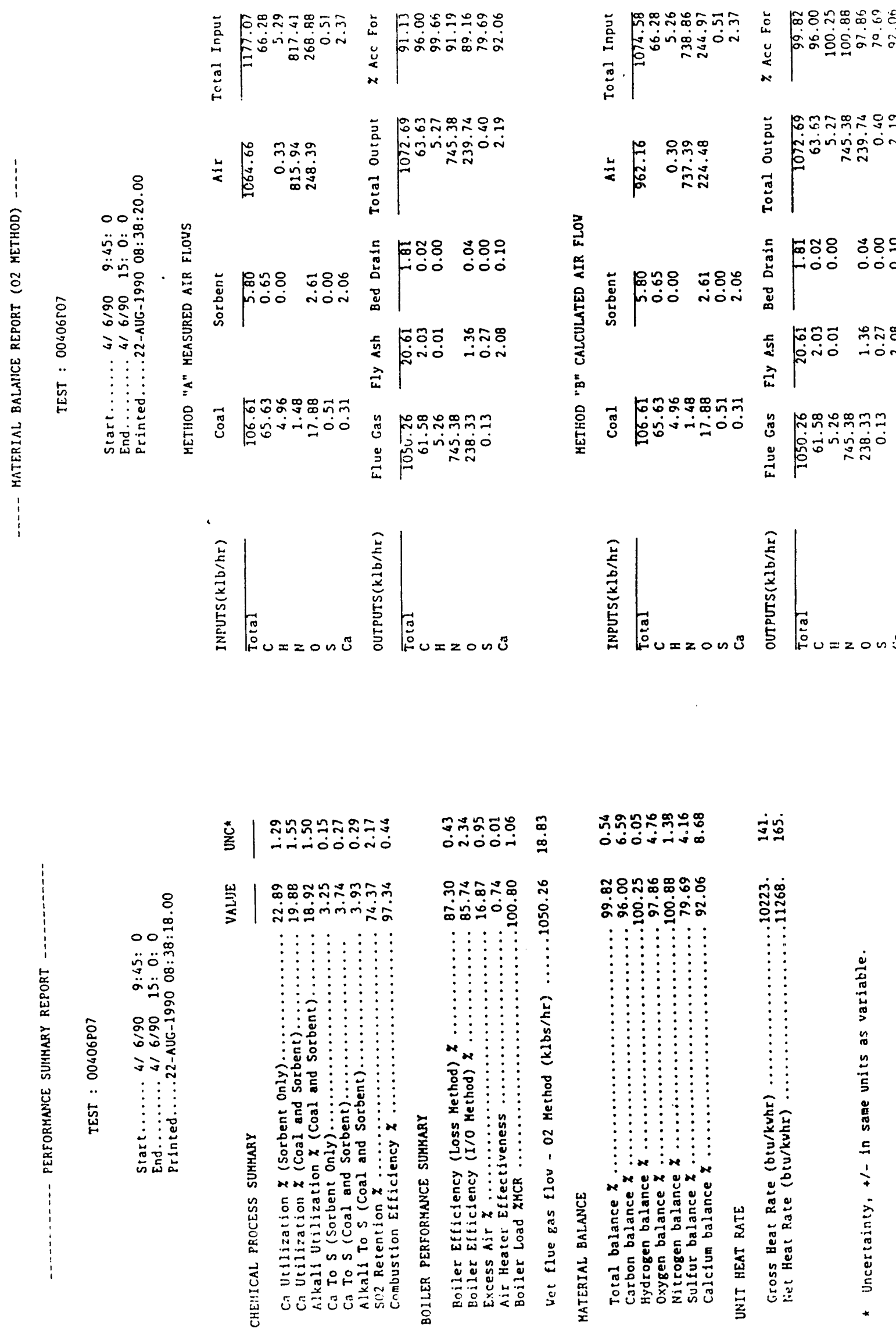



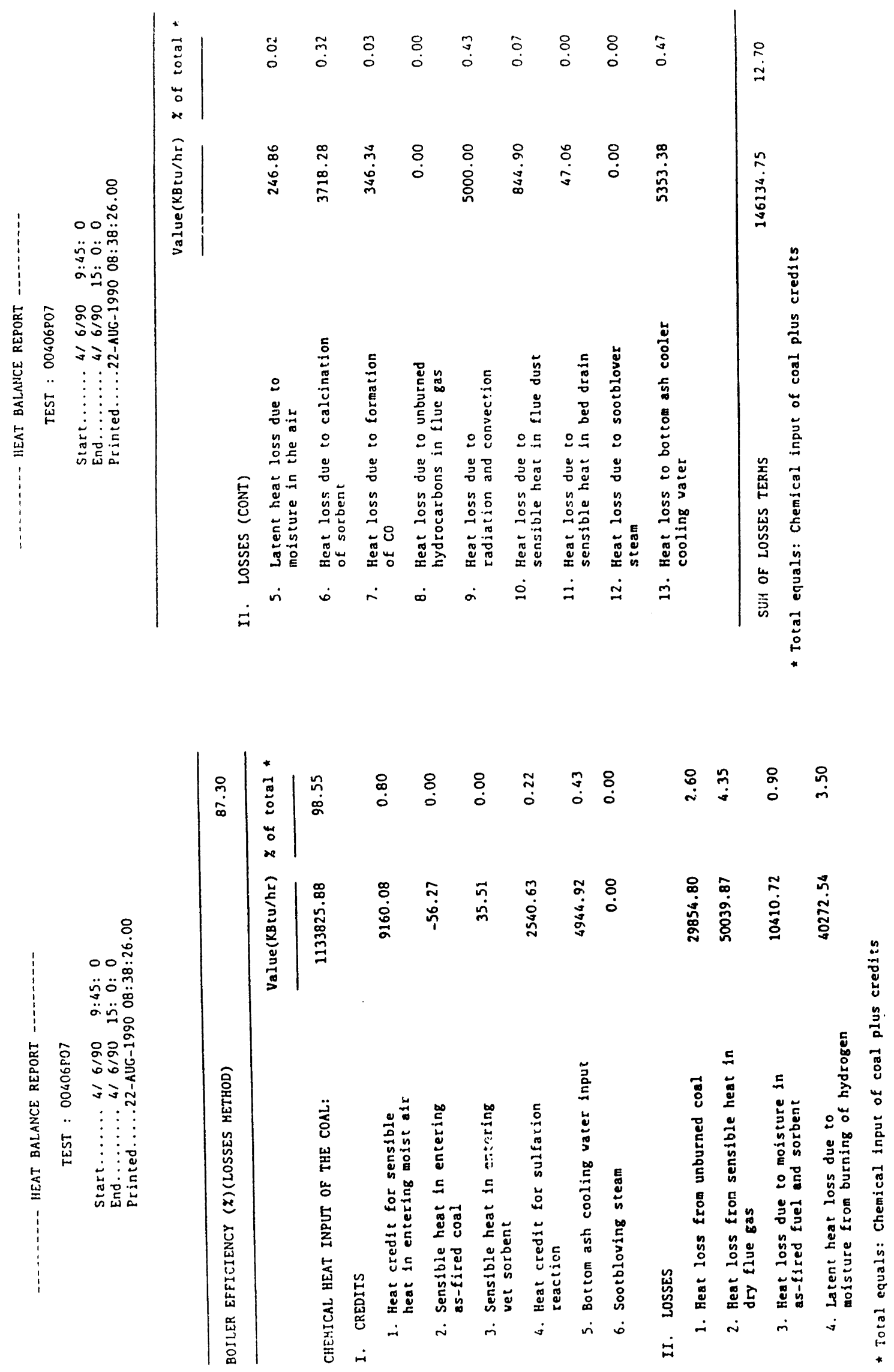


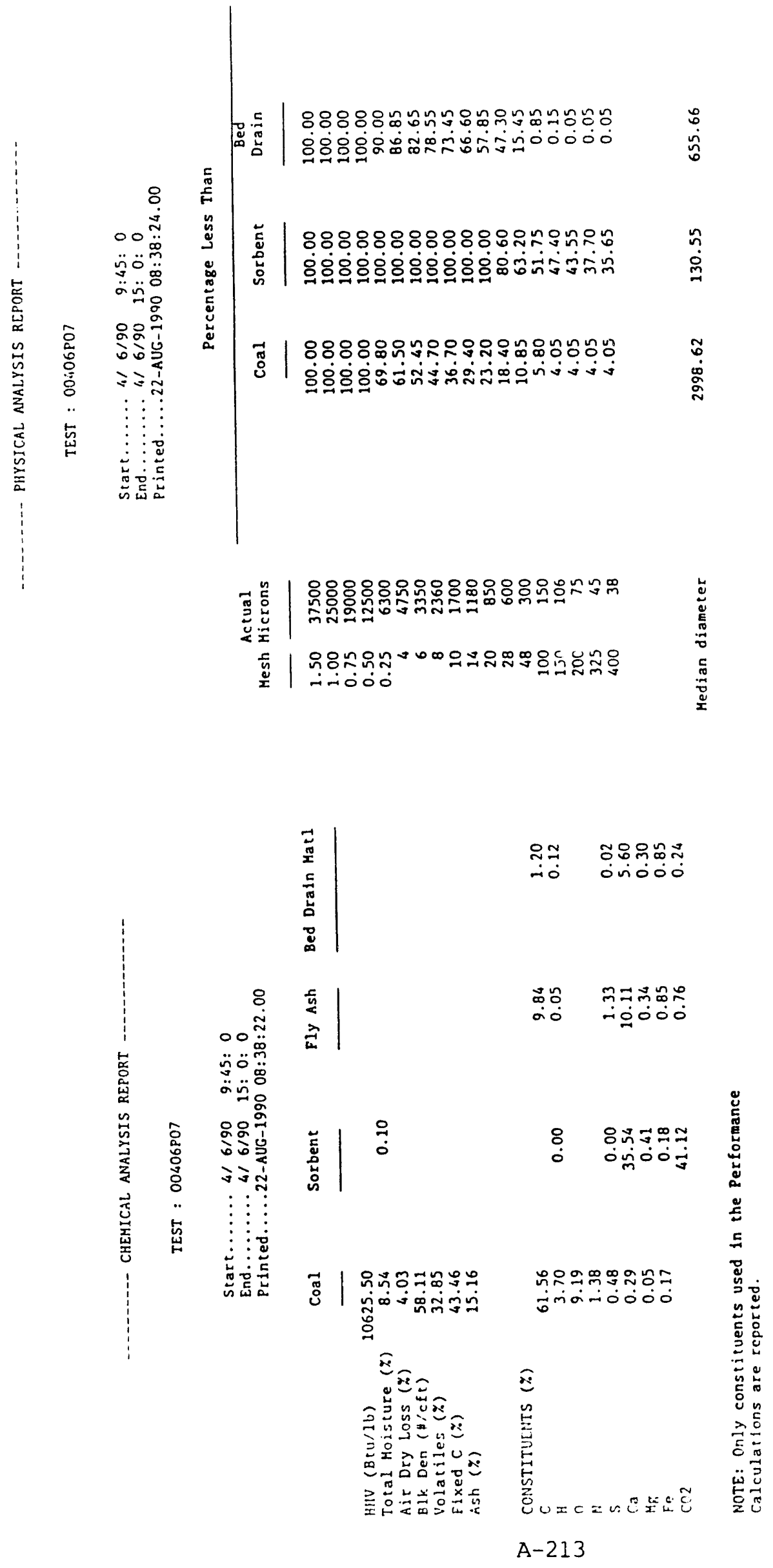



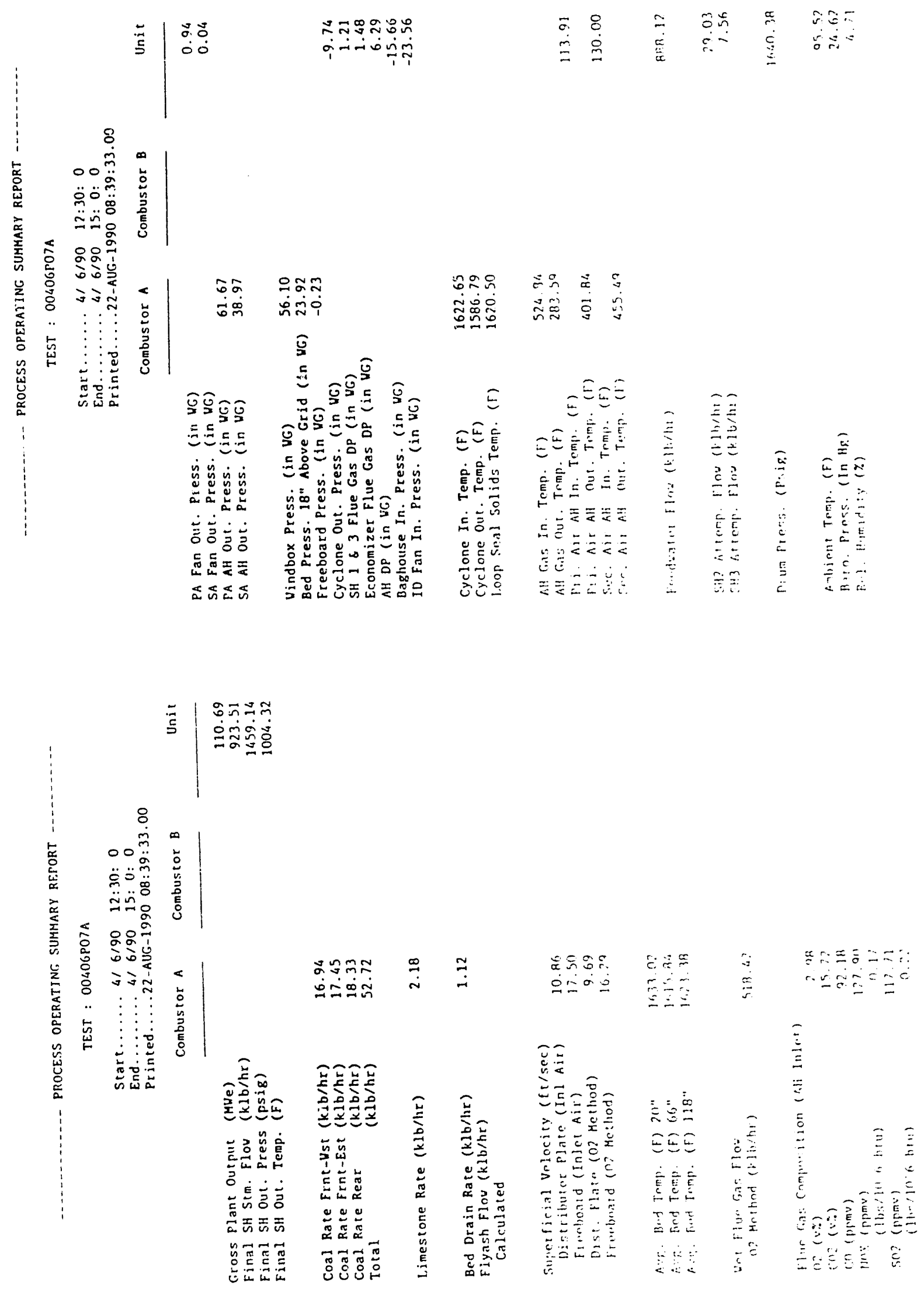


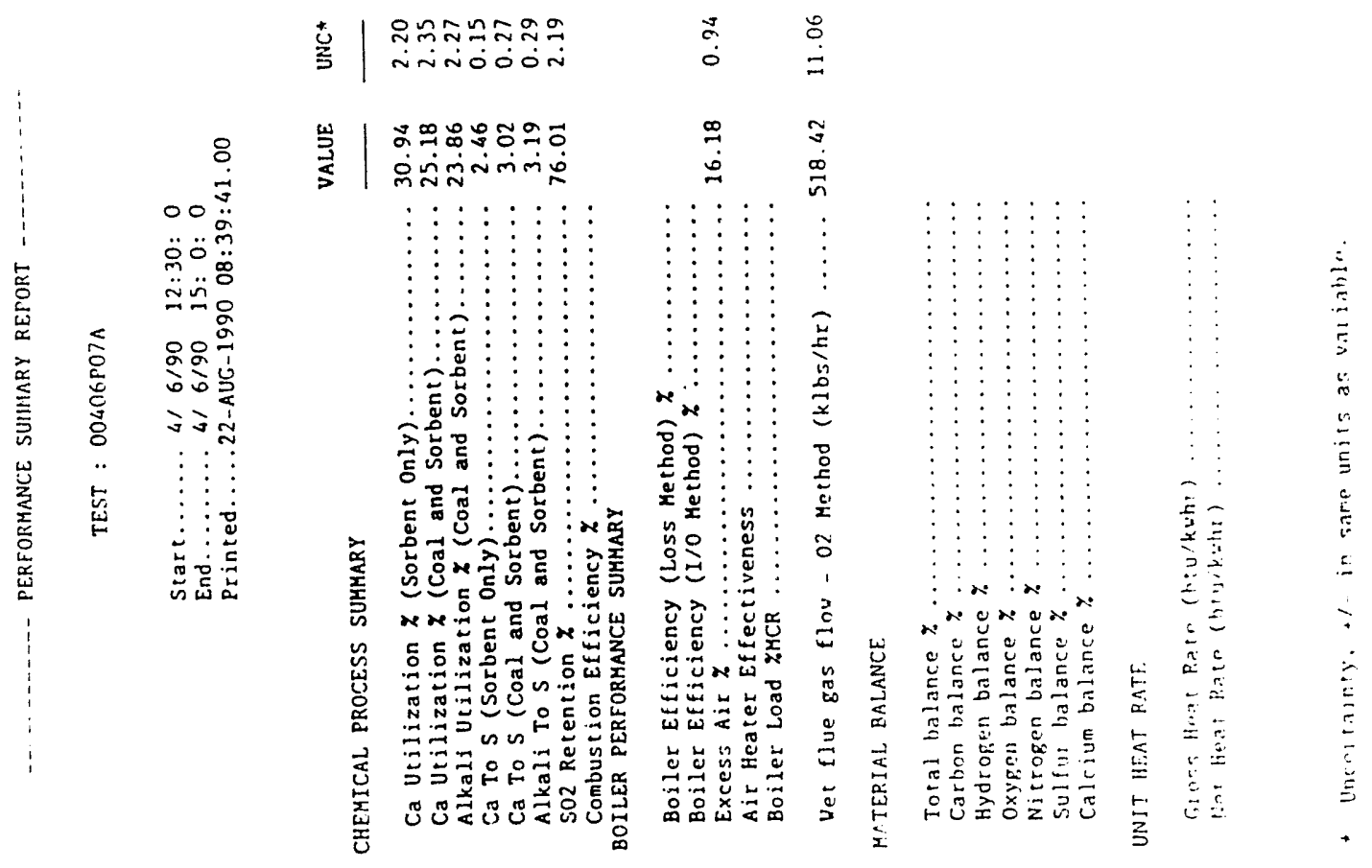

A-215 


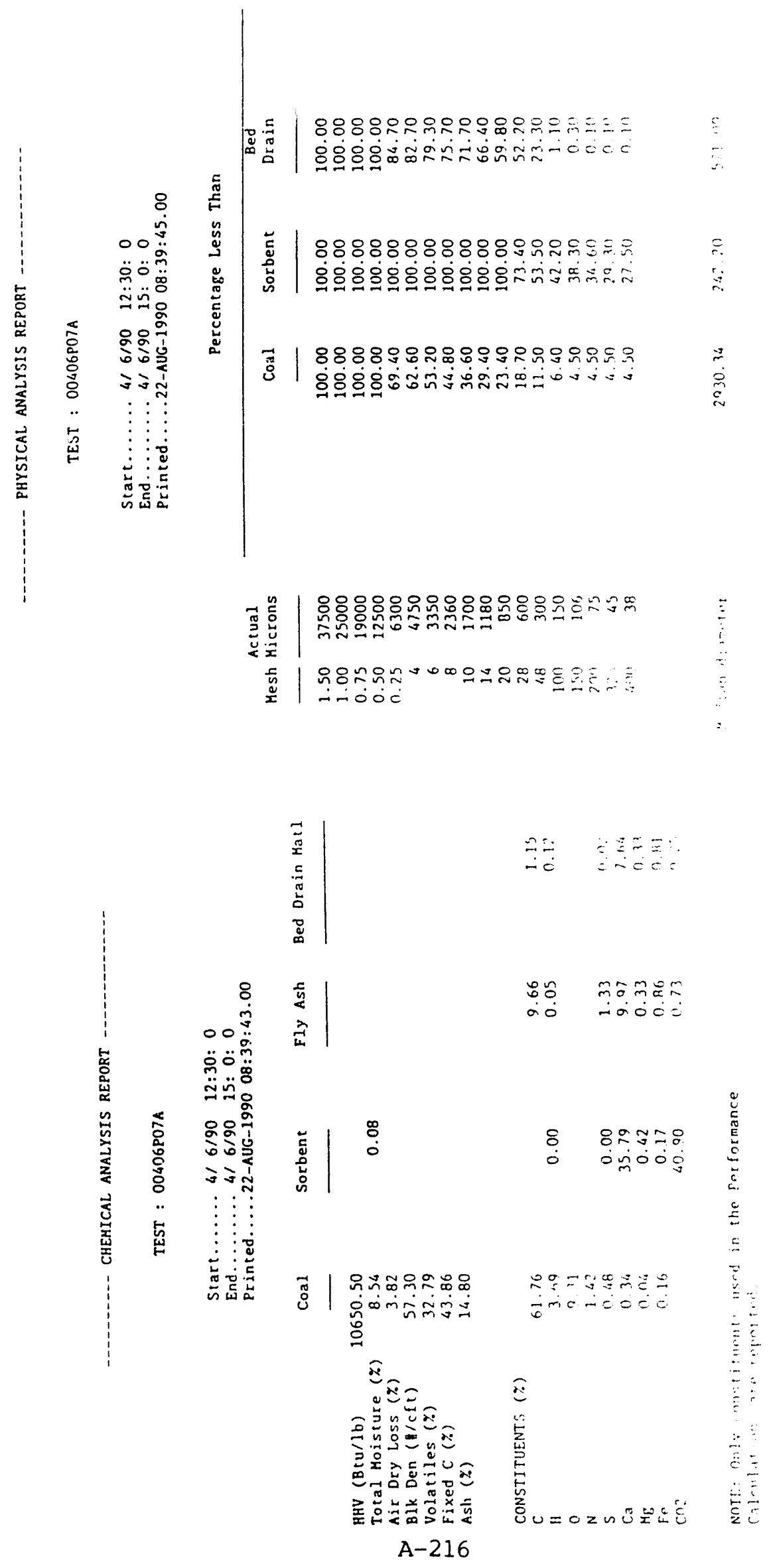



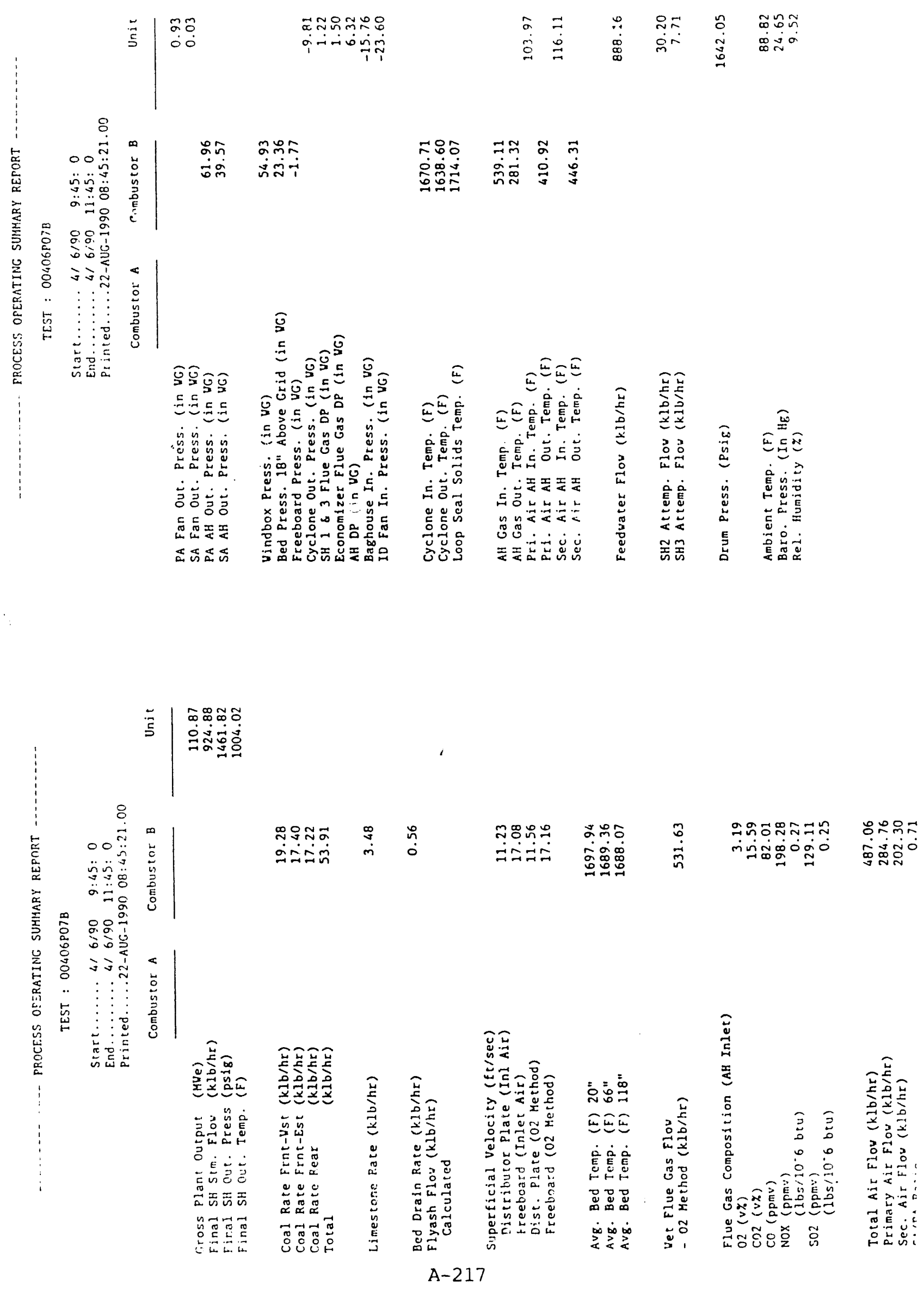


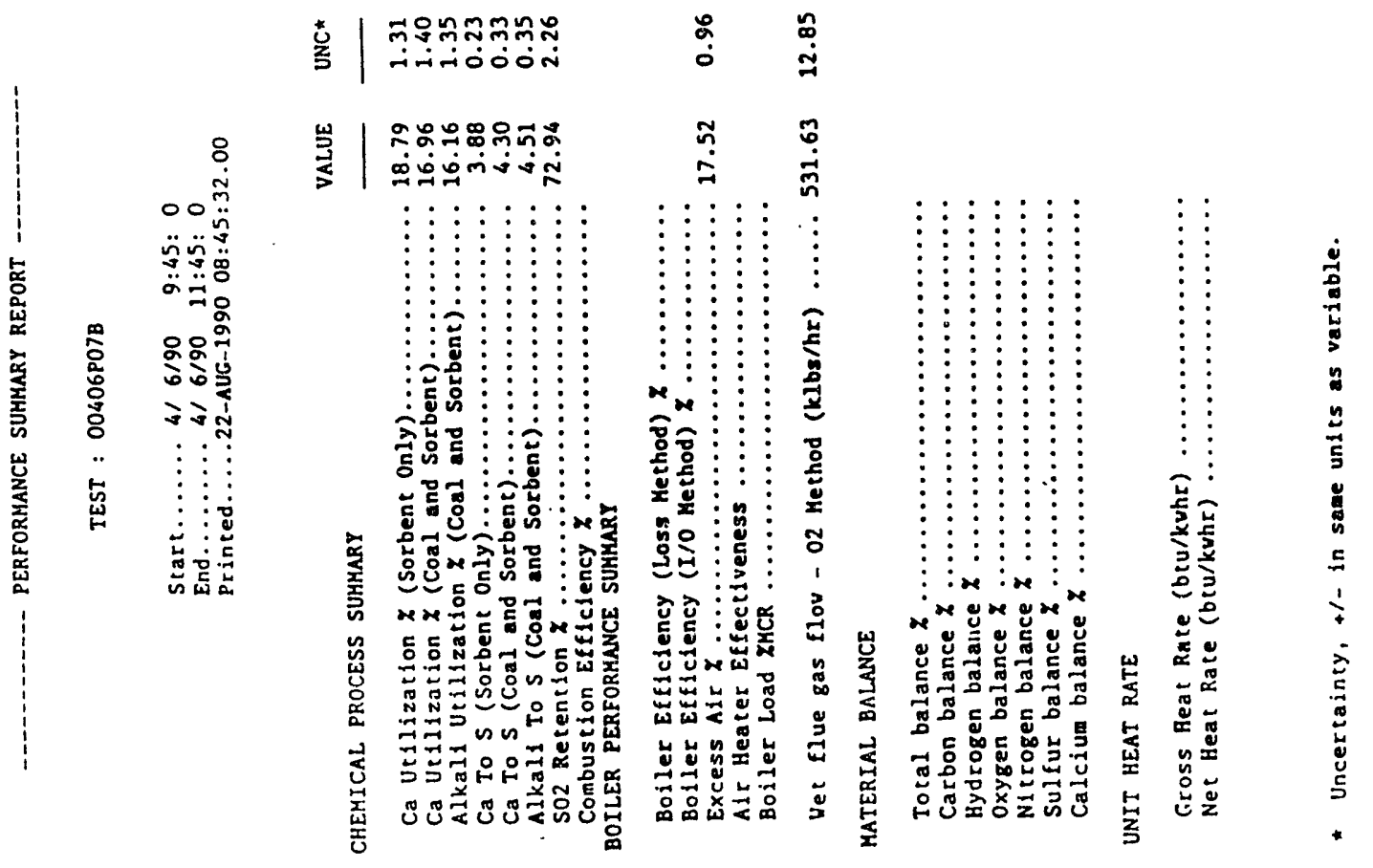

A-218 

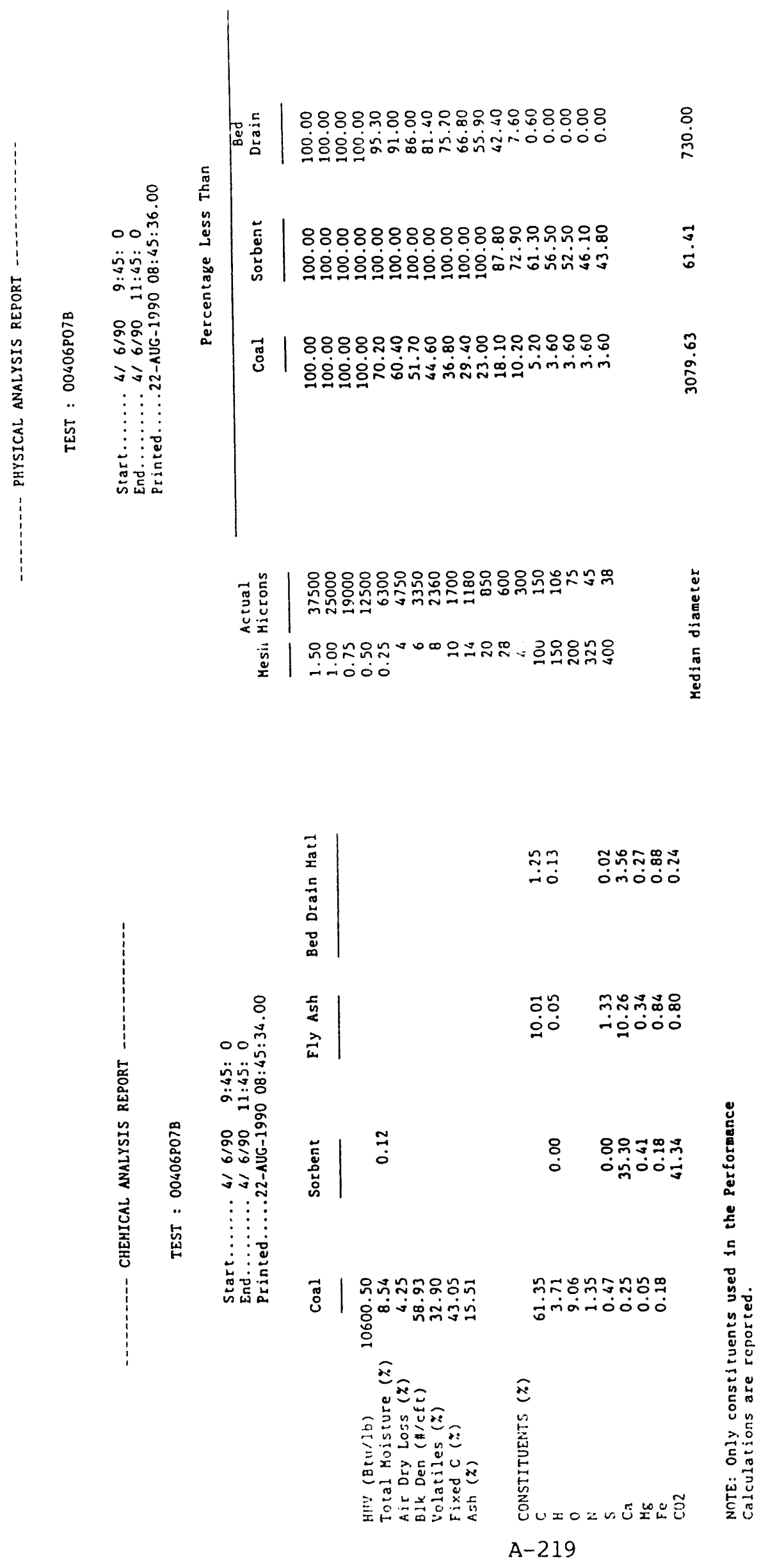


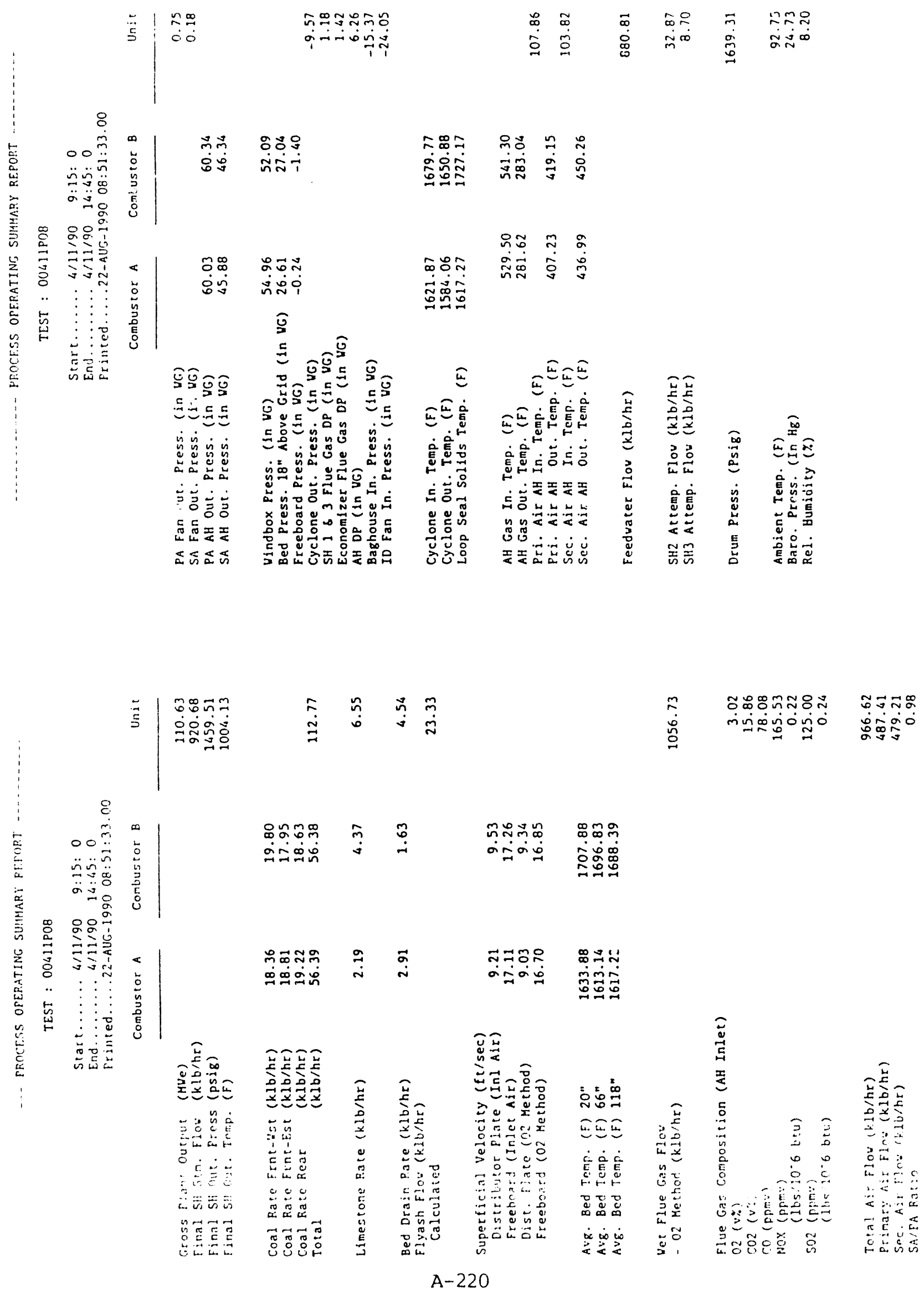




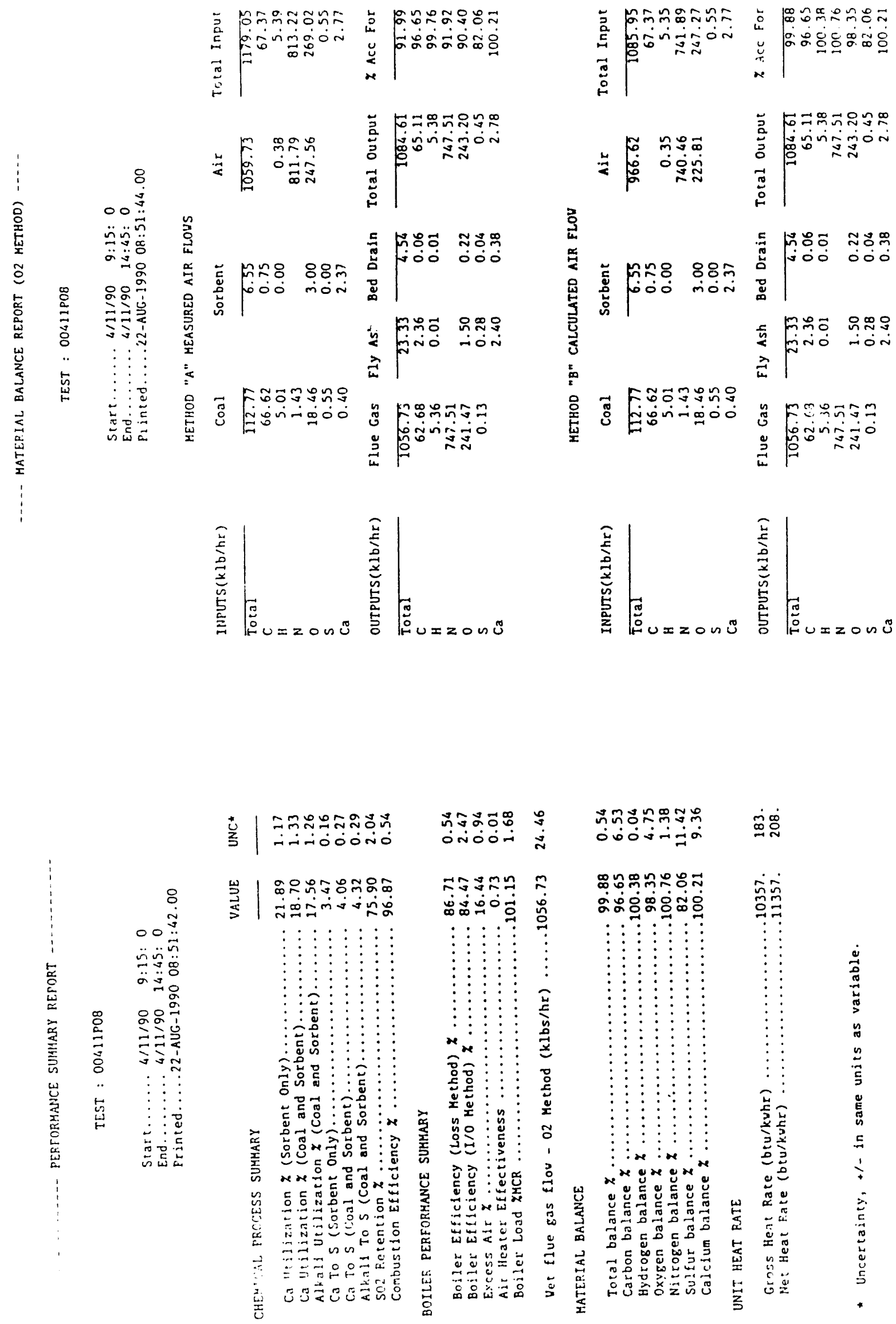




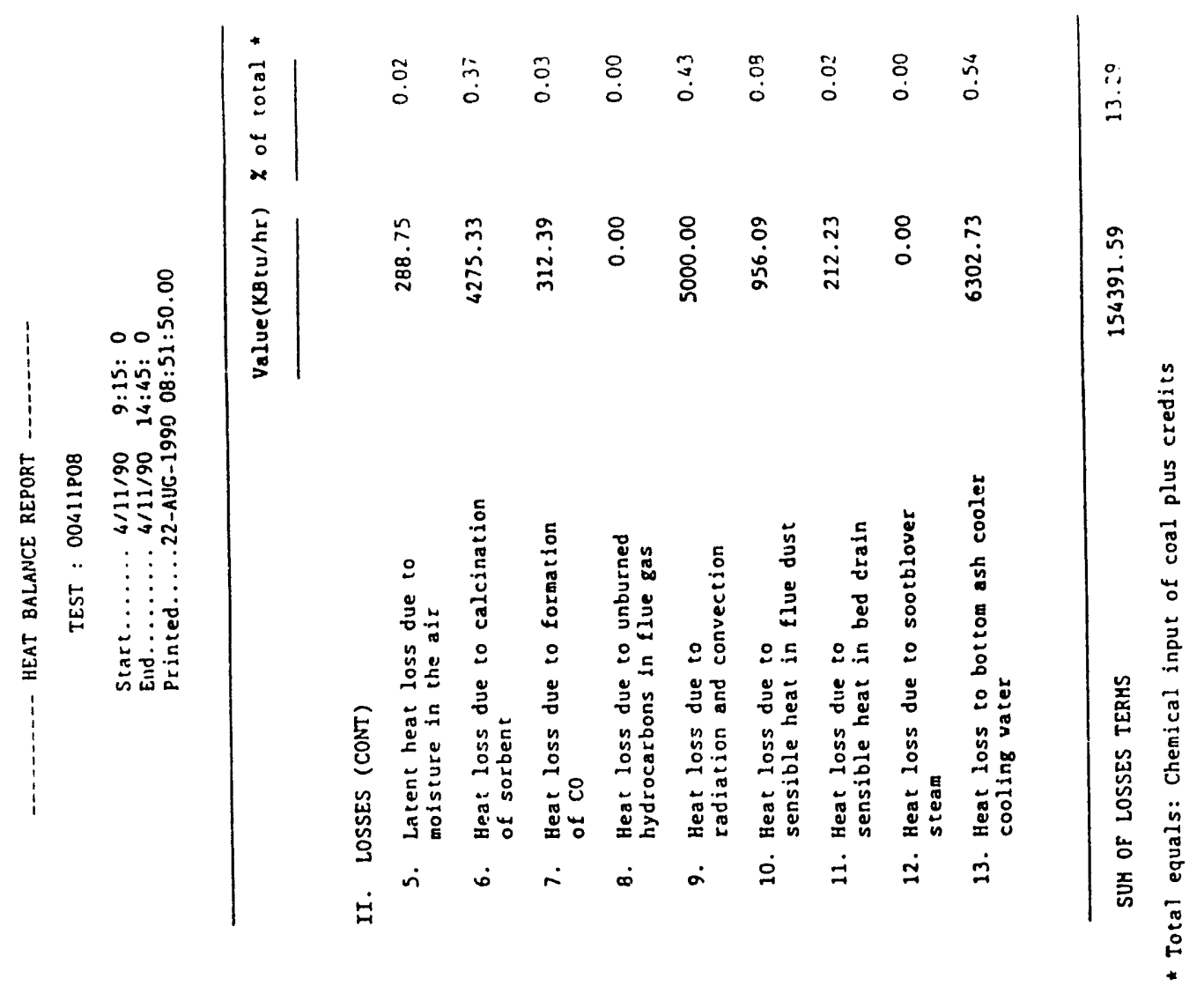

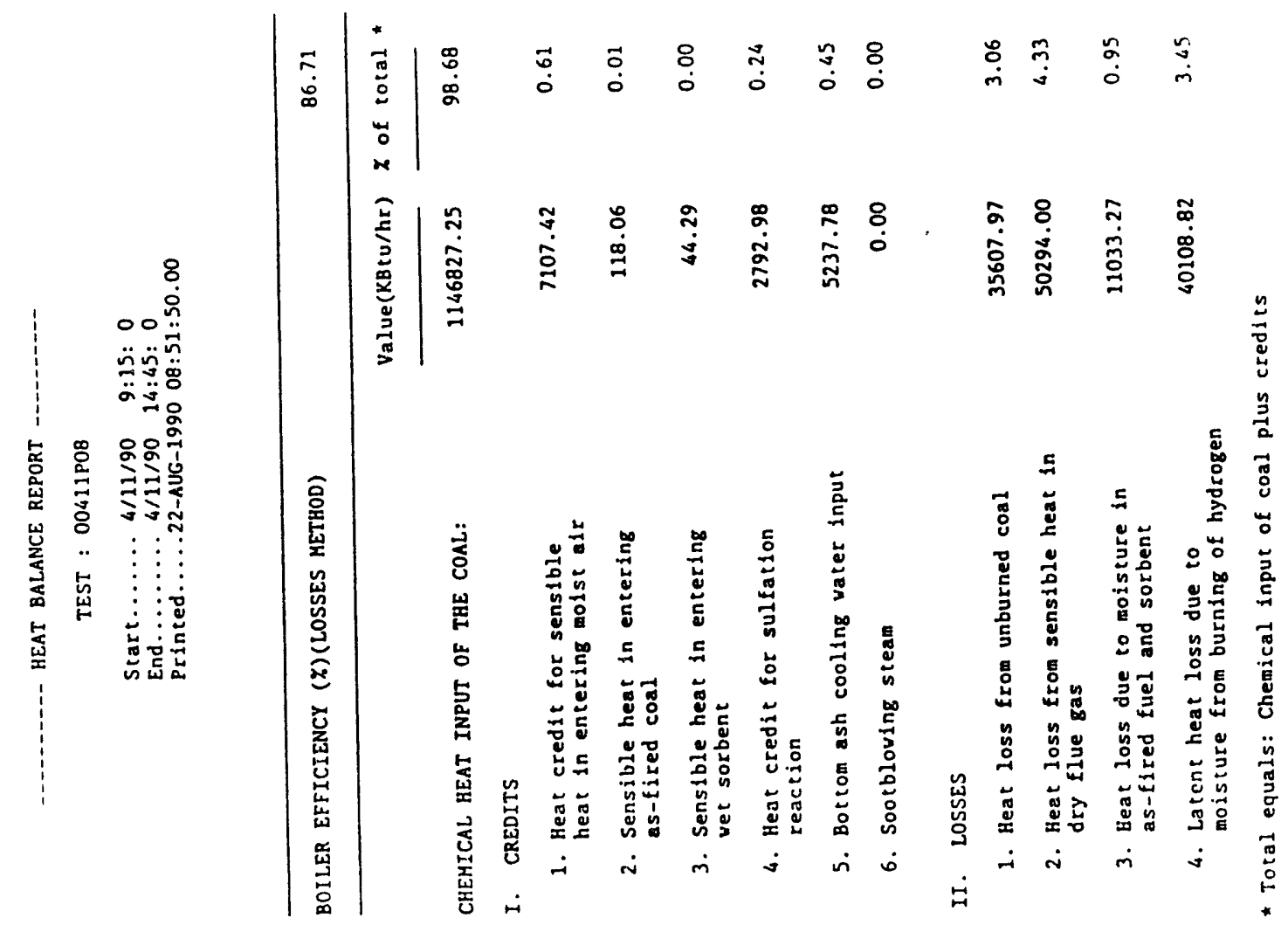



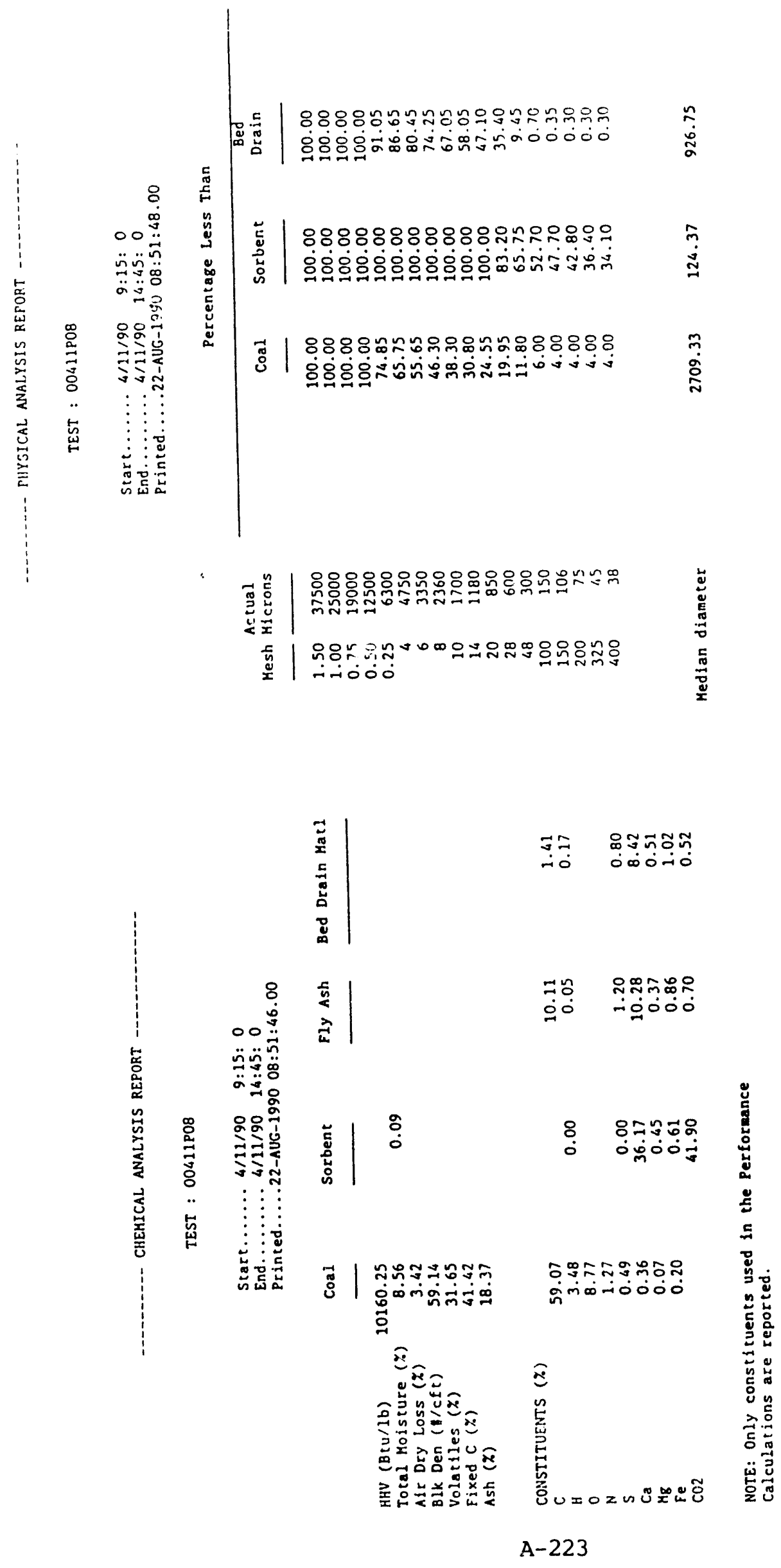

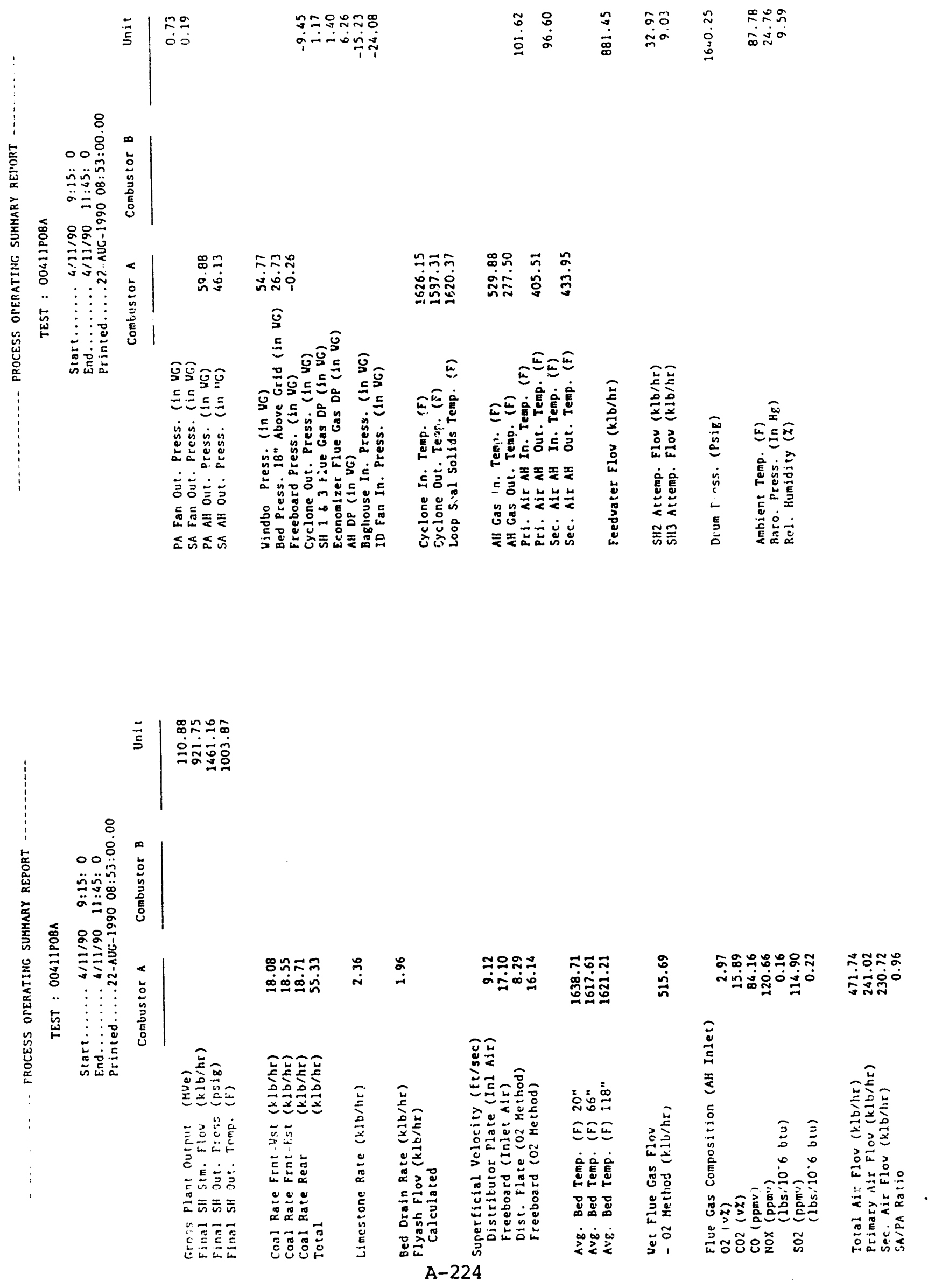


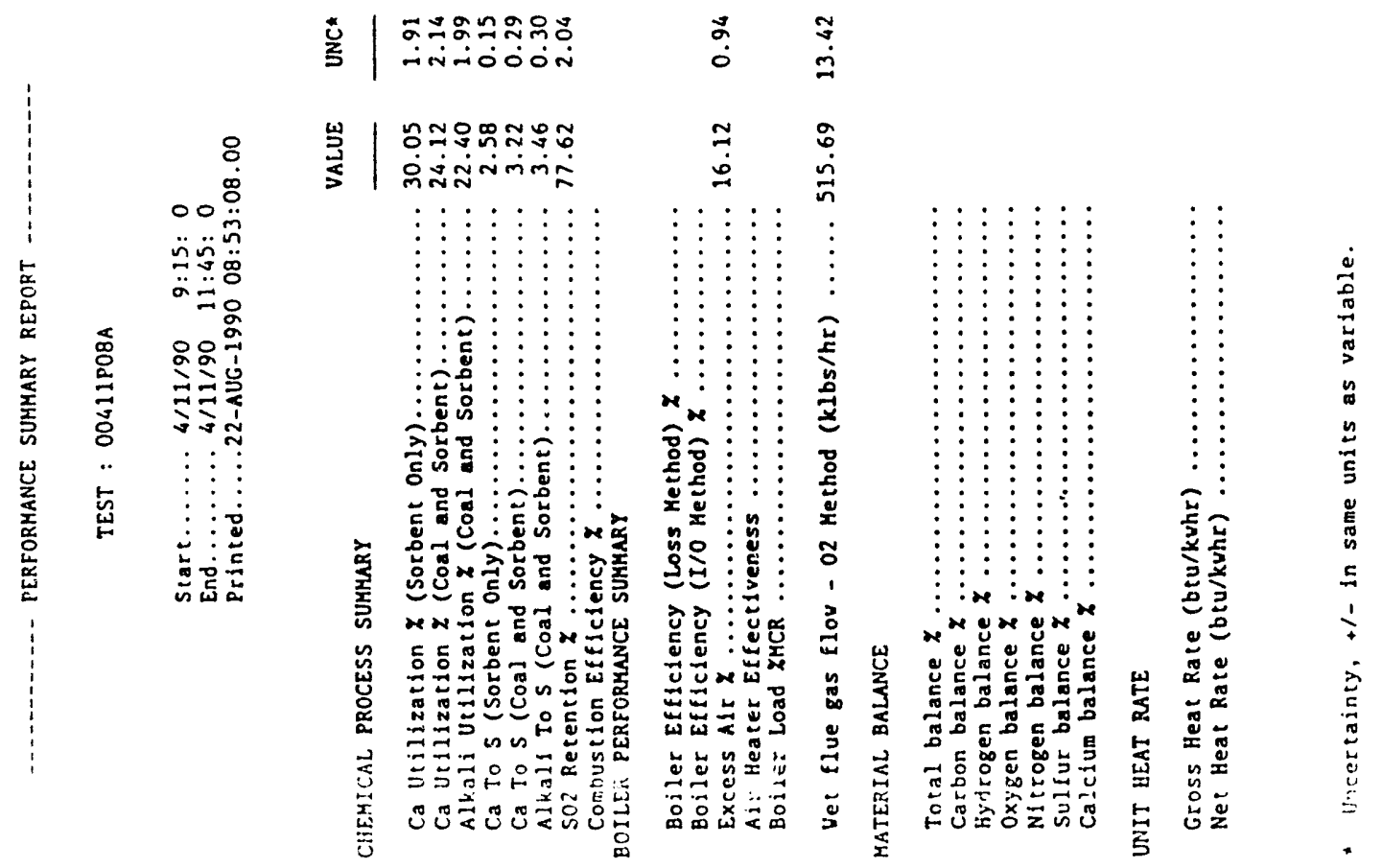



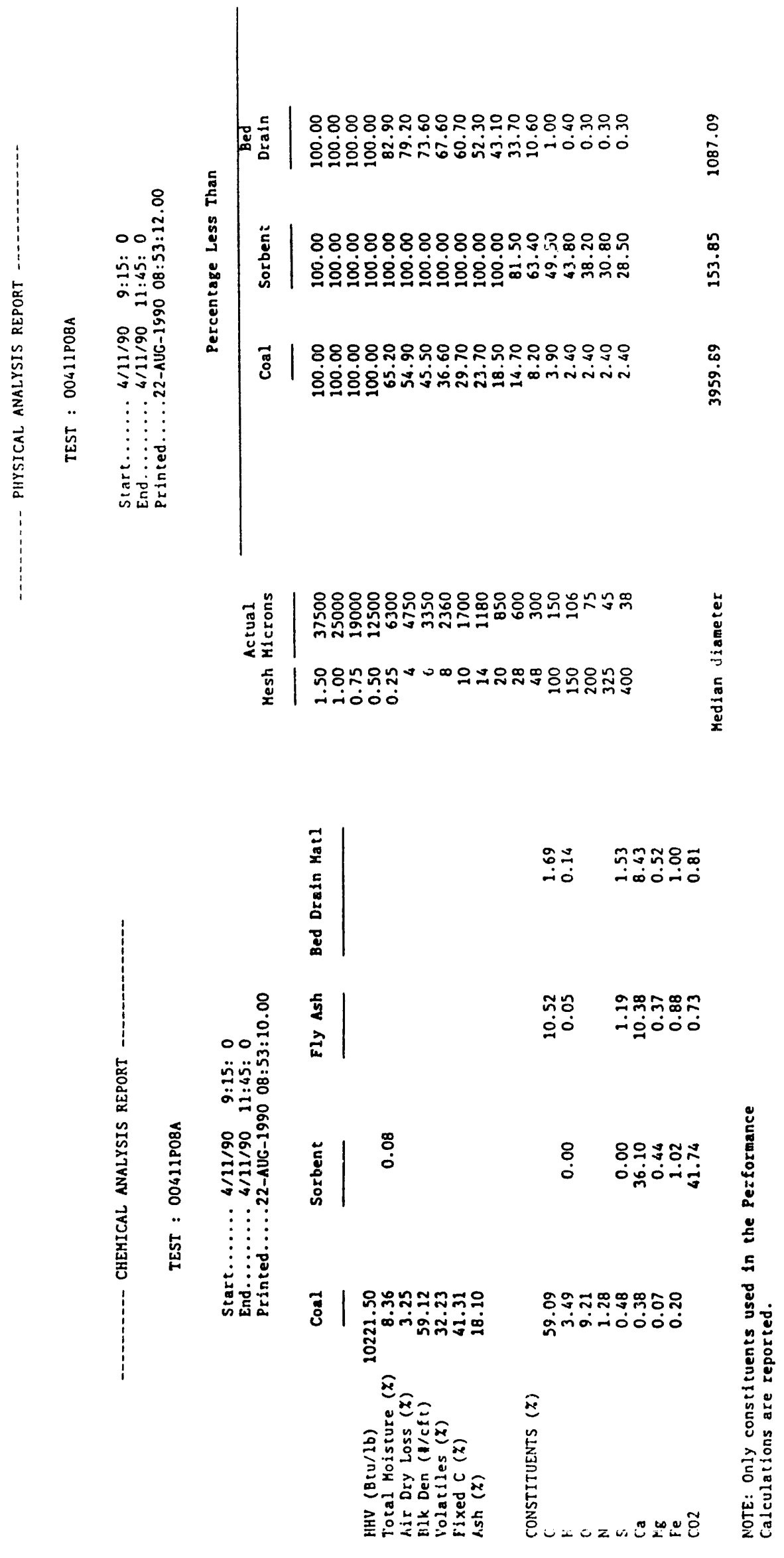

A-226 


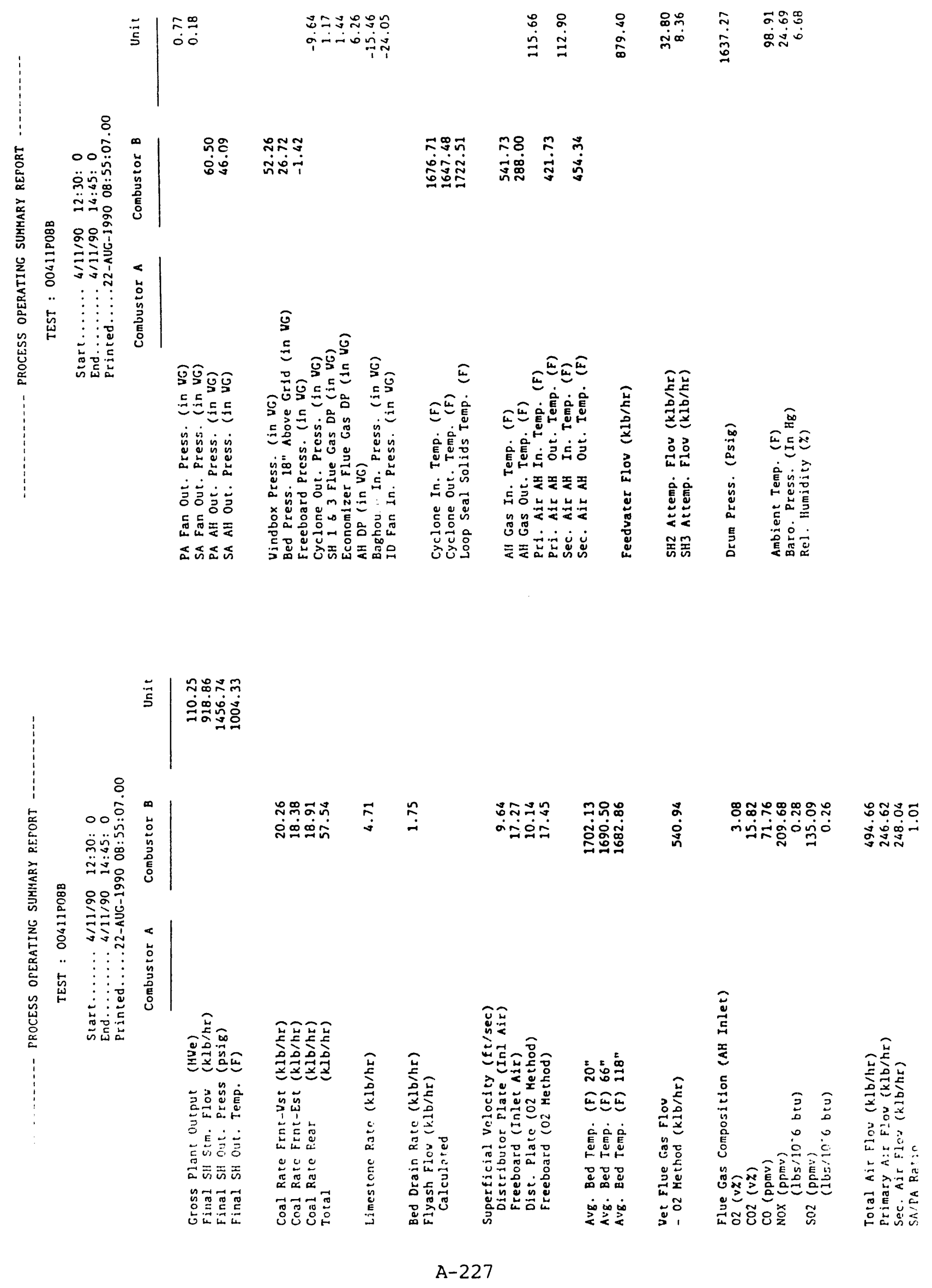




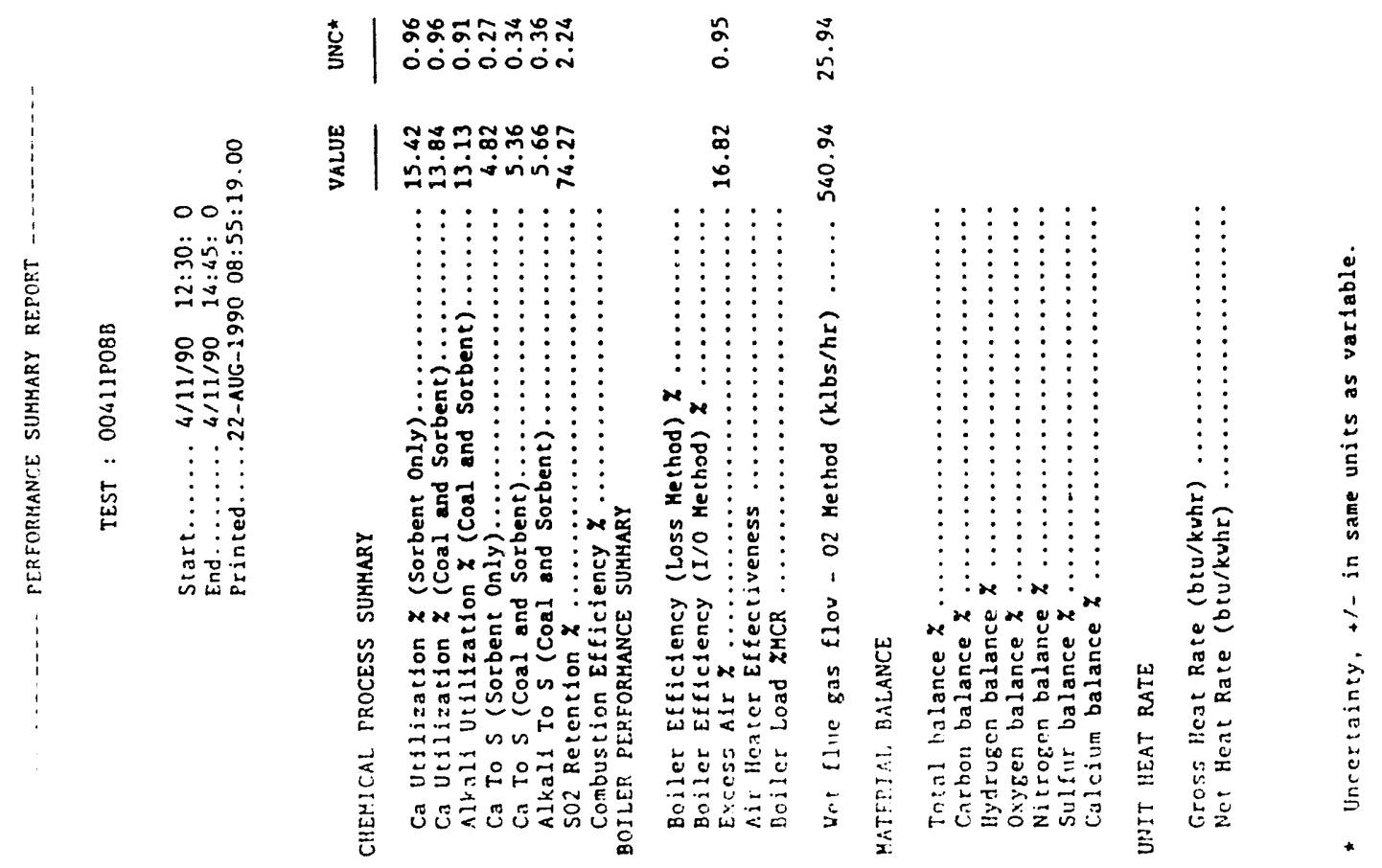

A-228 

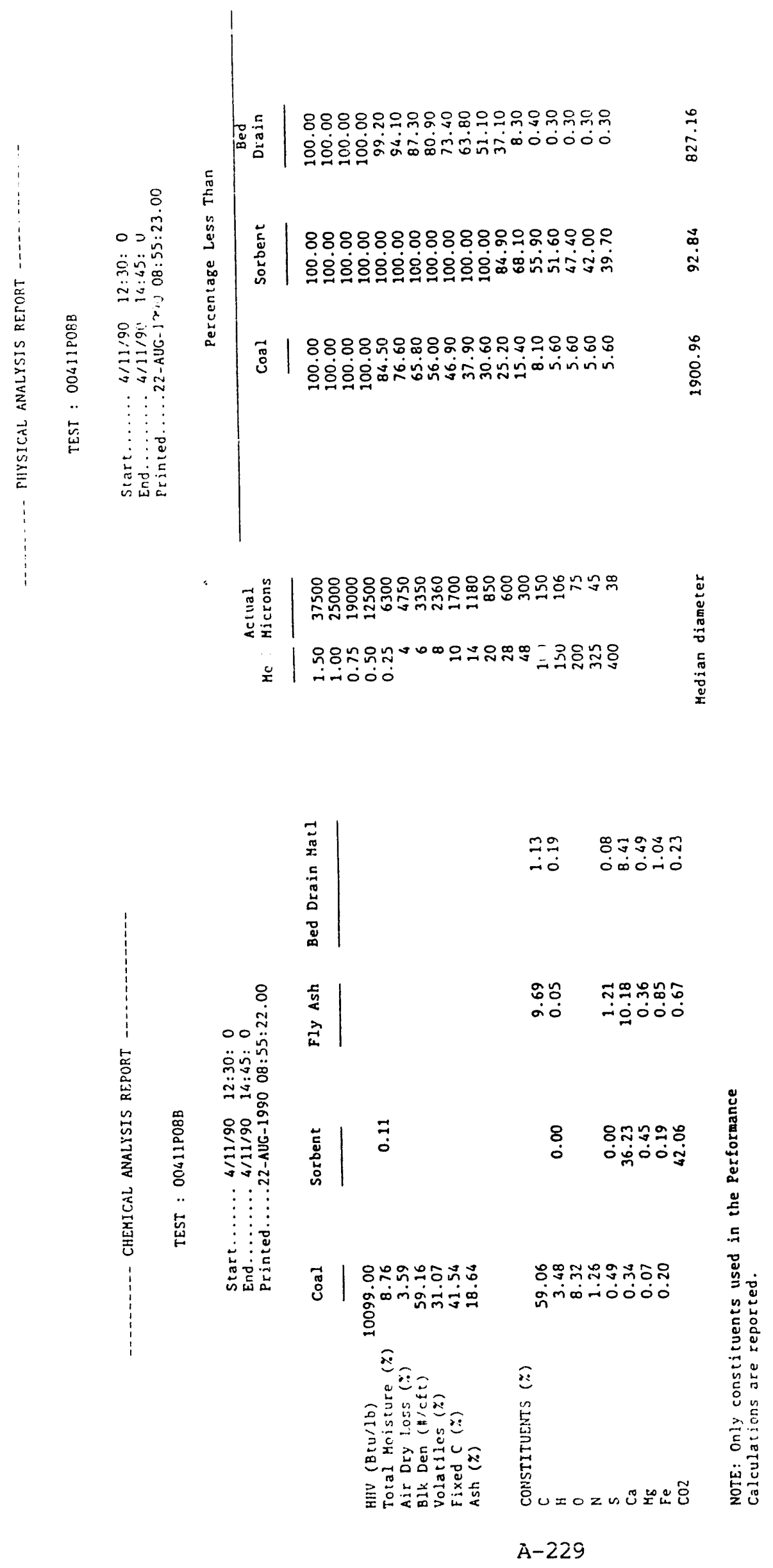

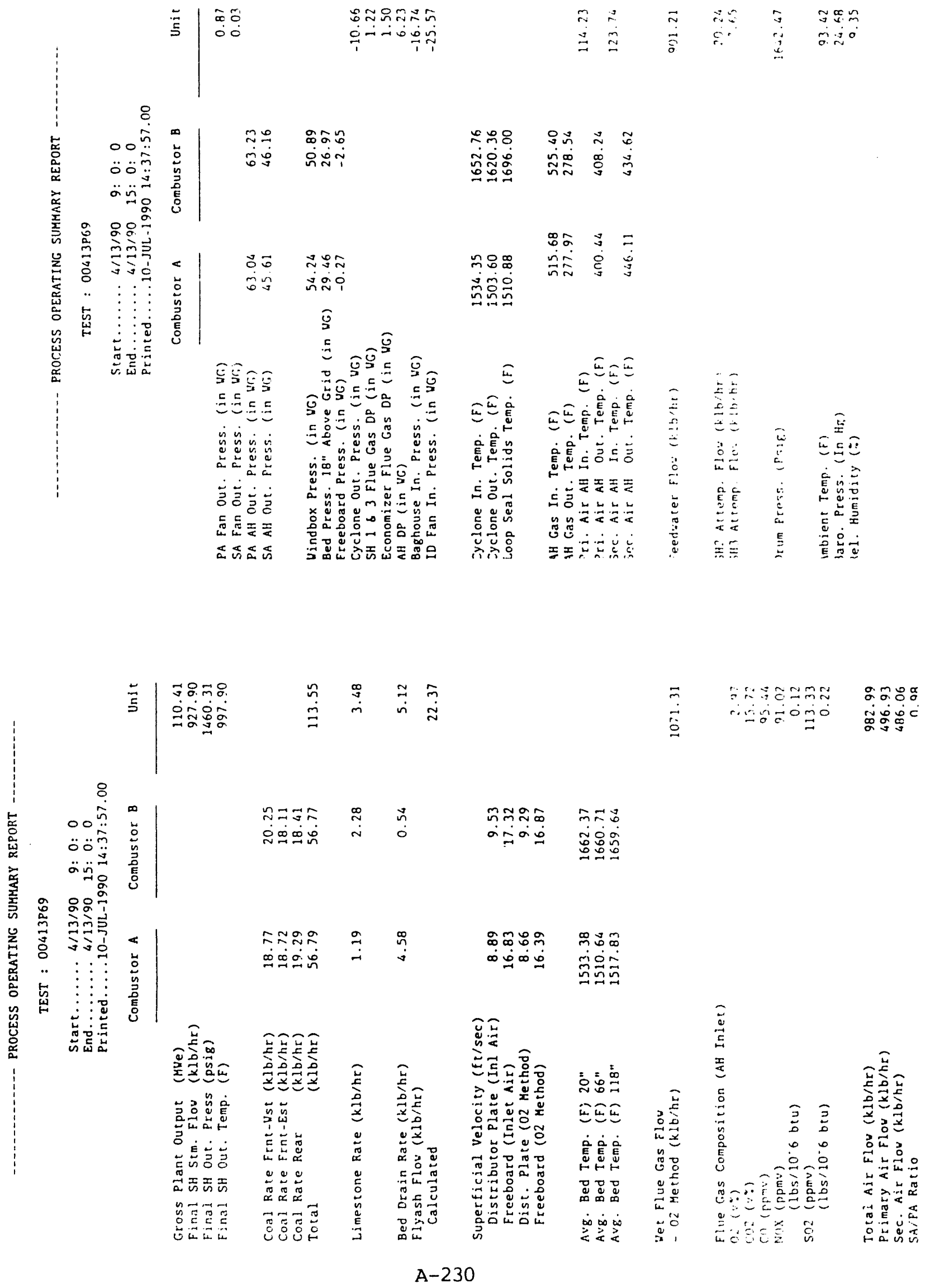


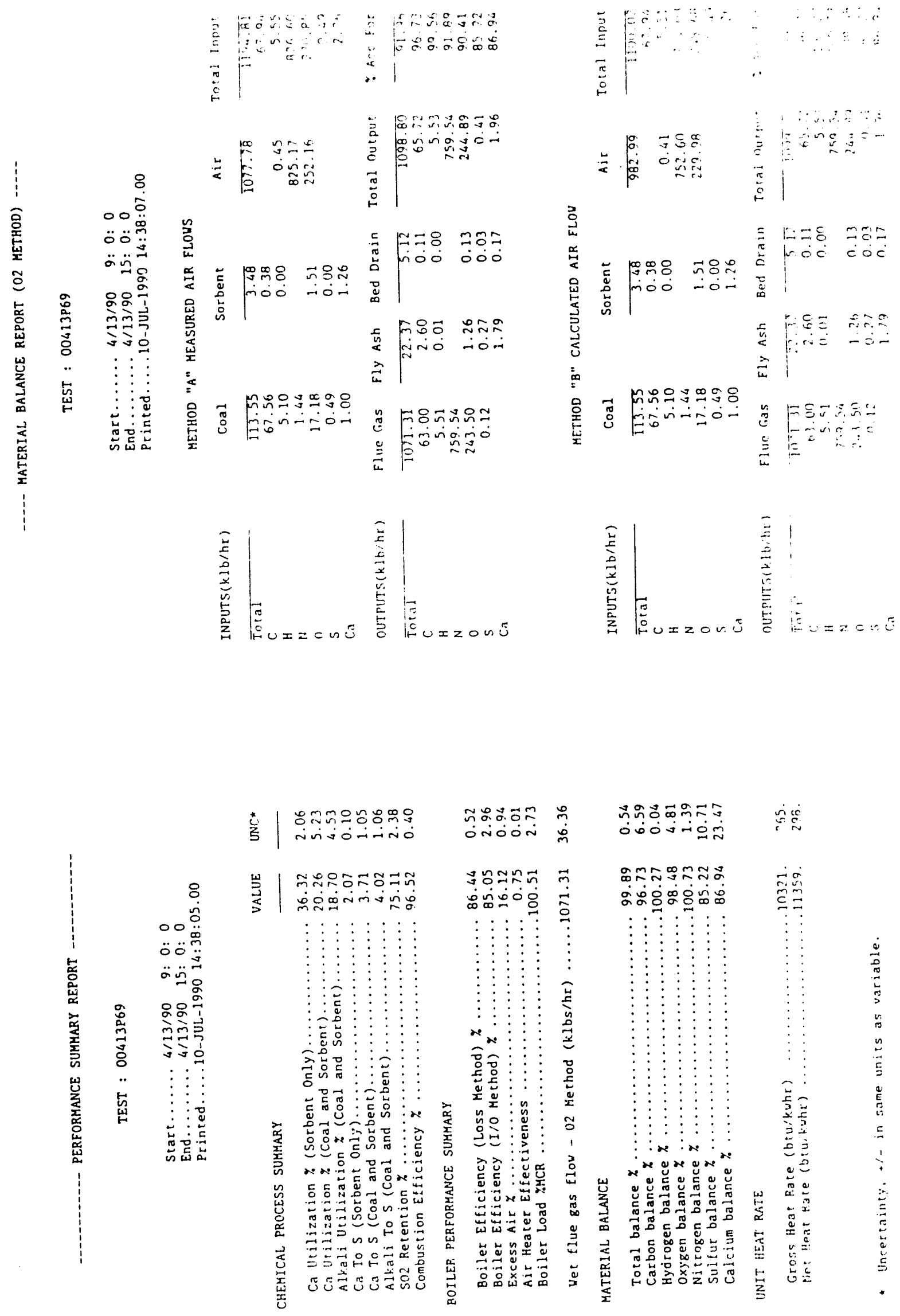



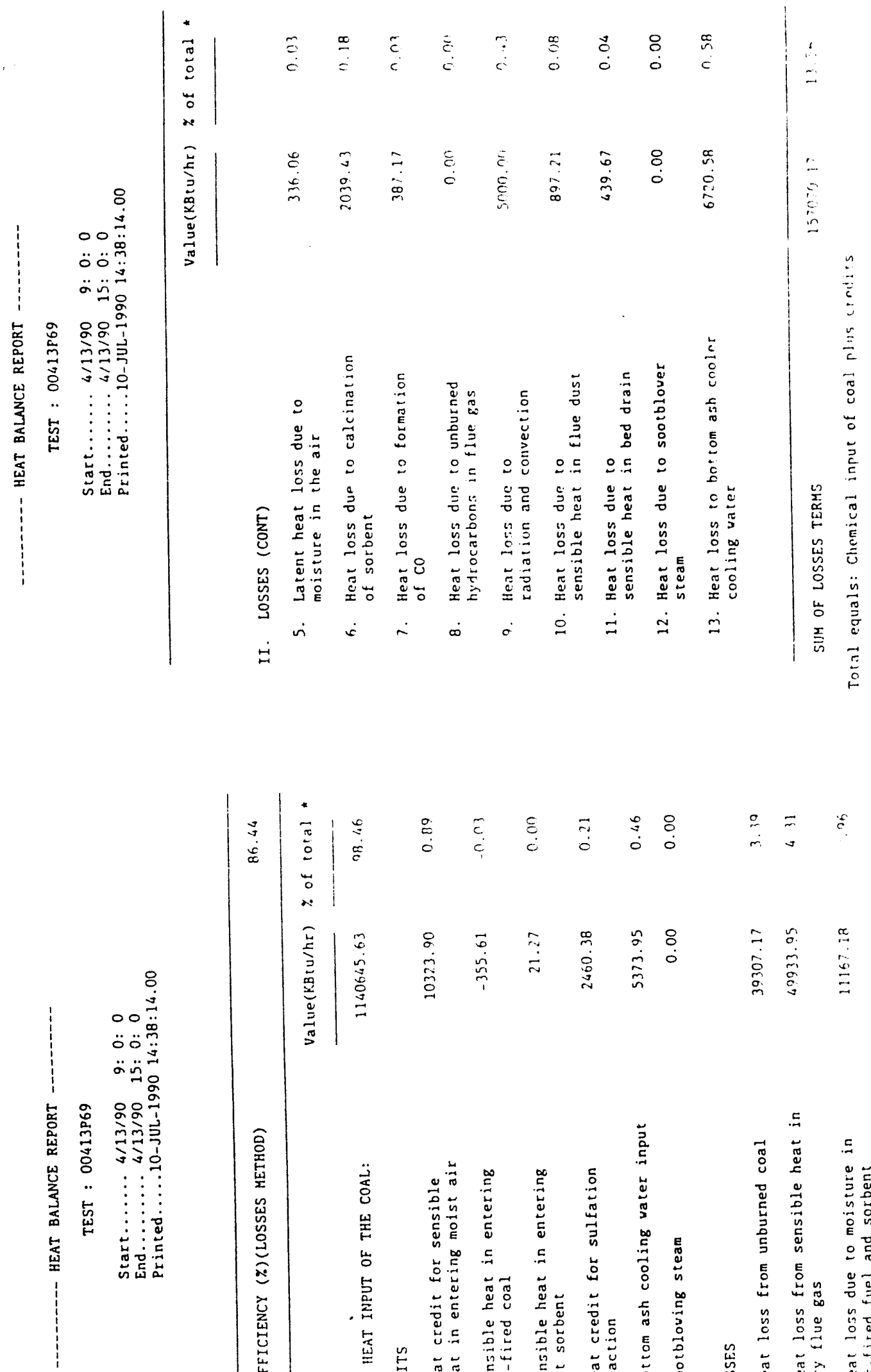

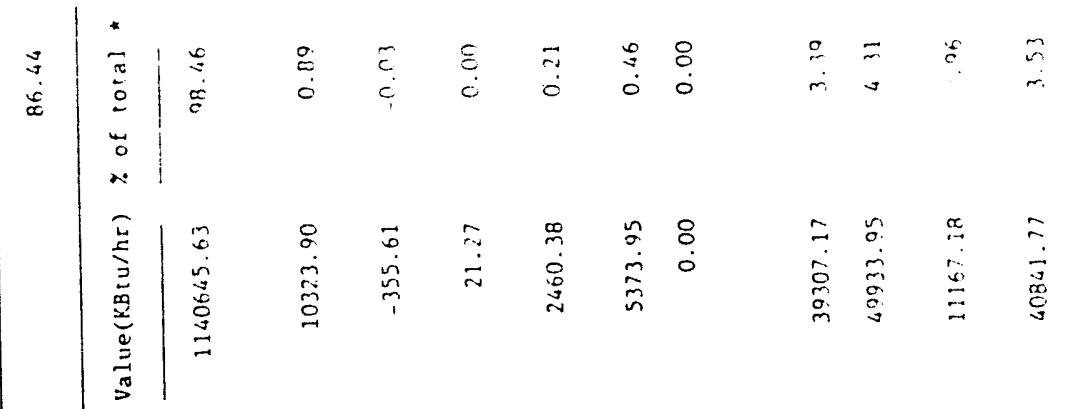

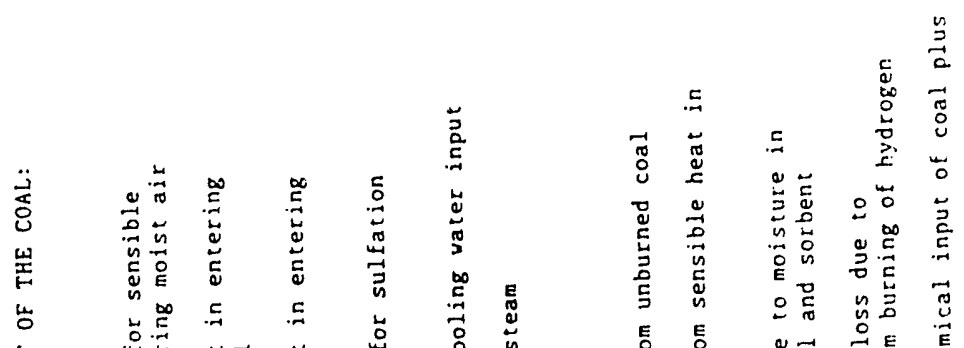

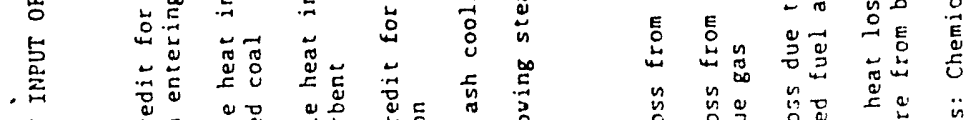

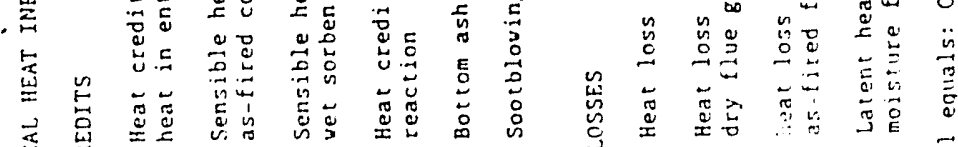

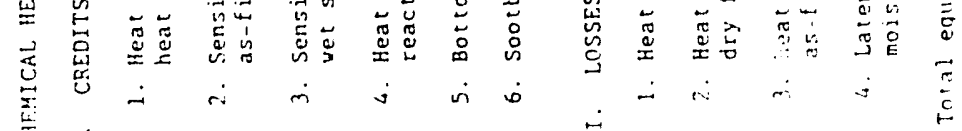$$
\text { A-232 }
$$ 

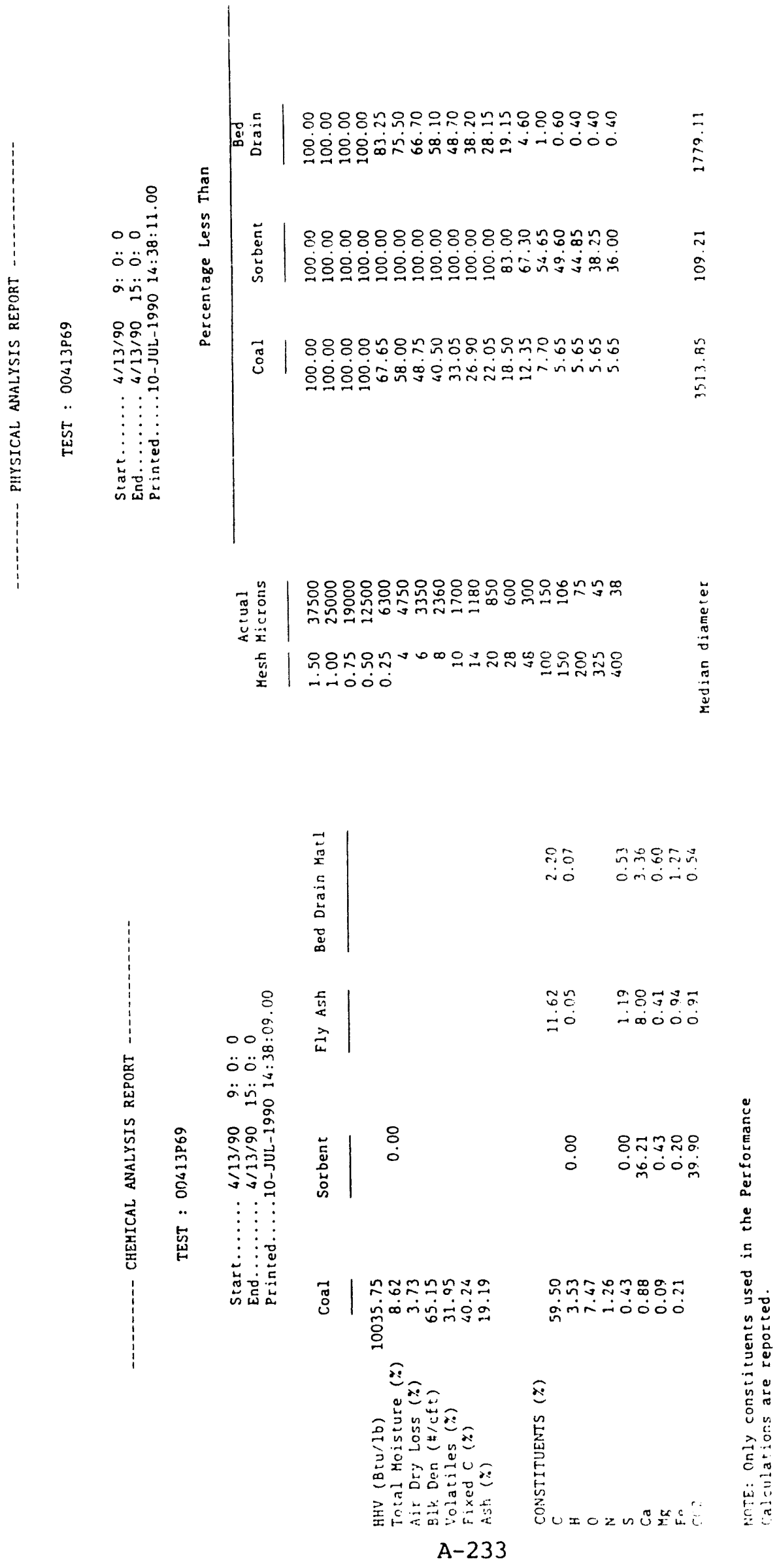

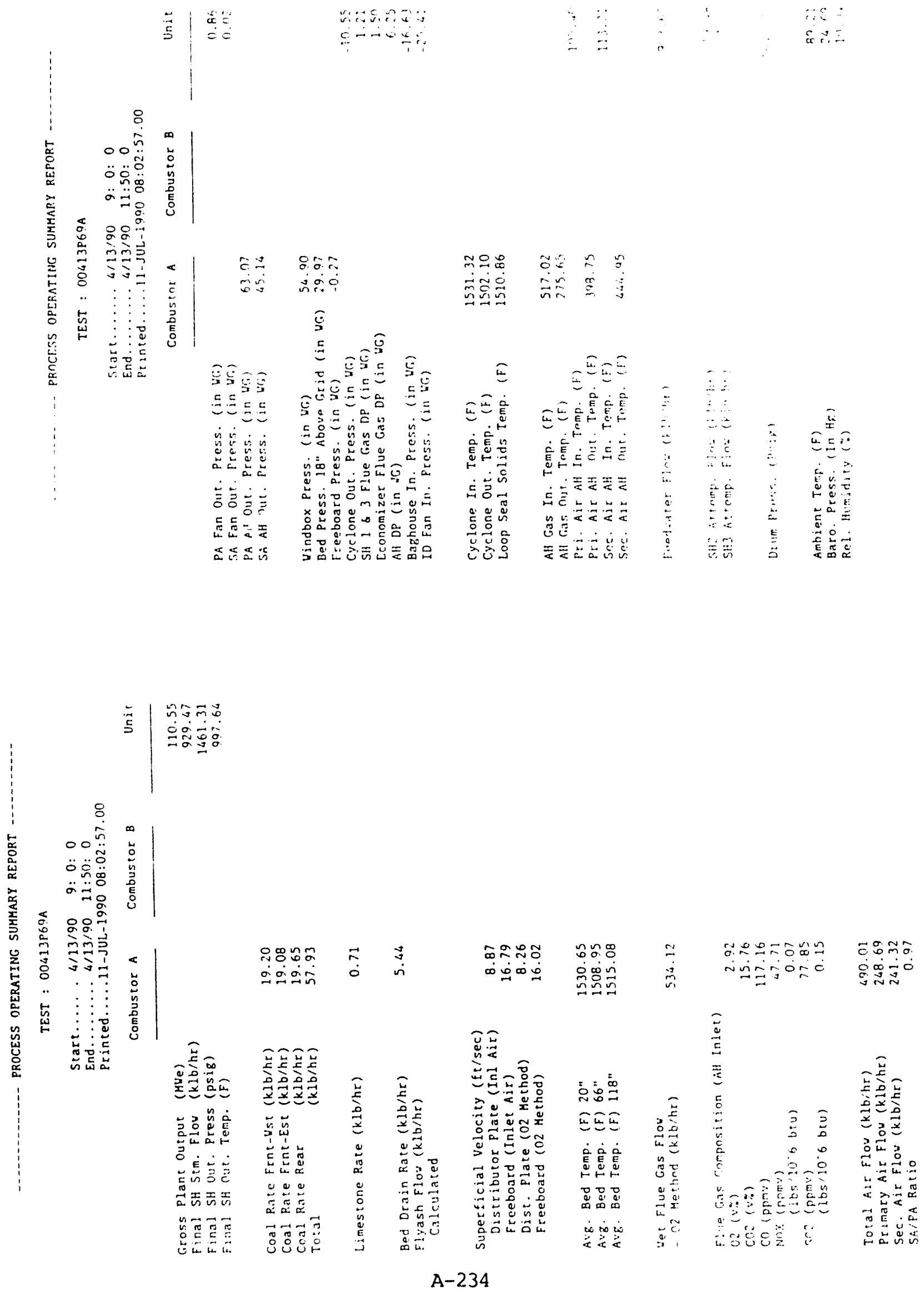


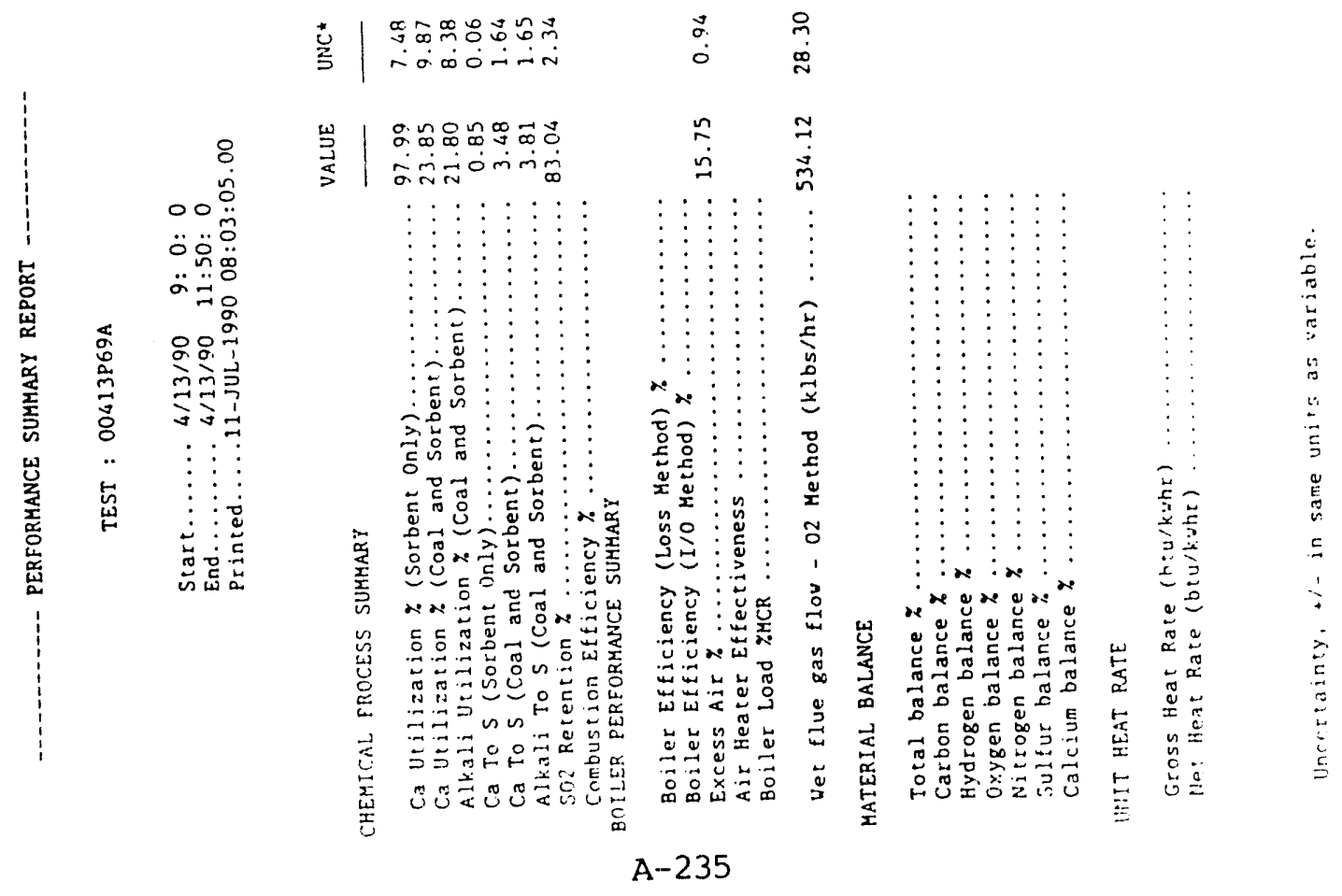




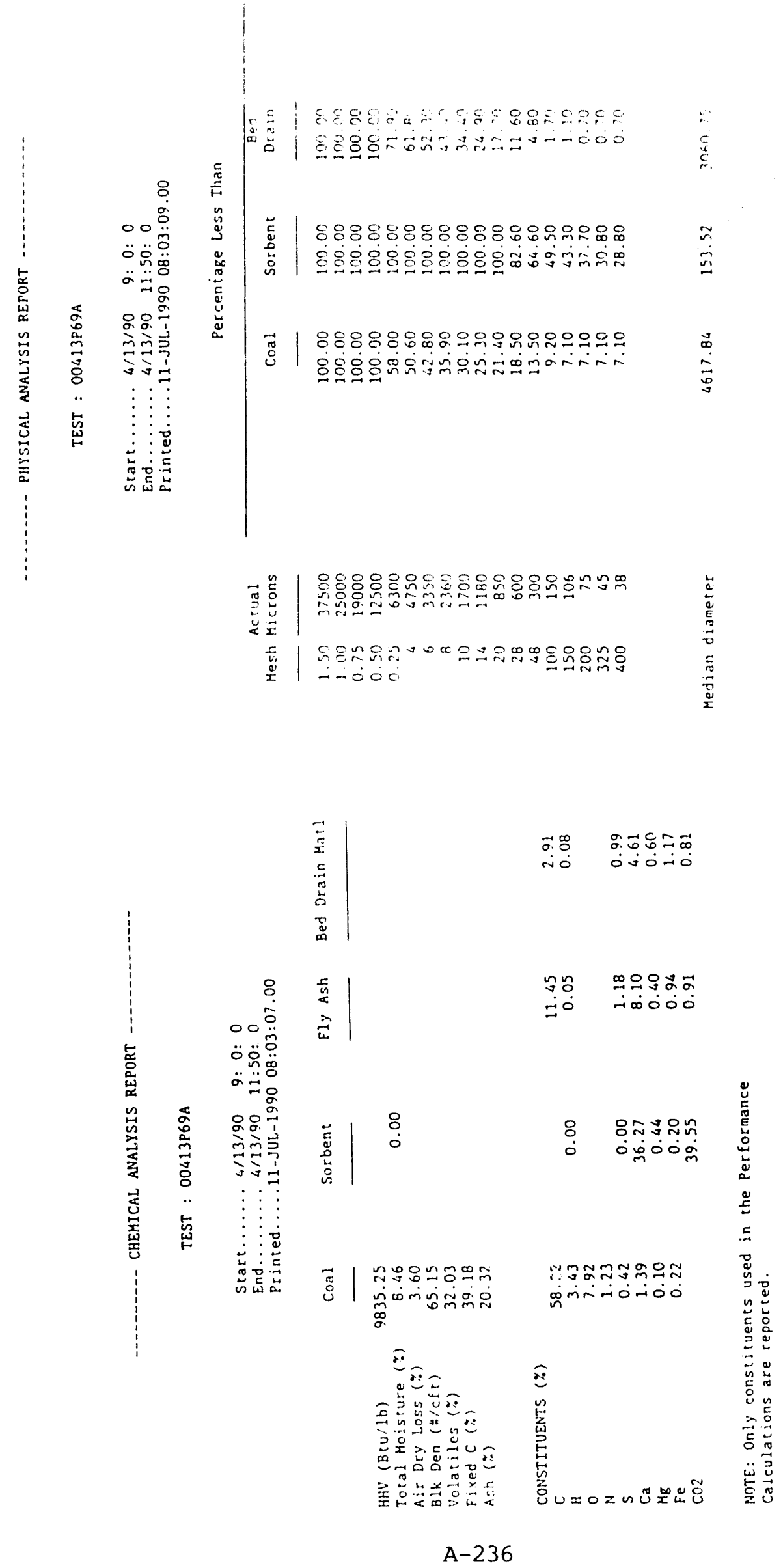




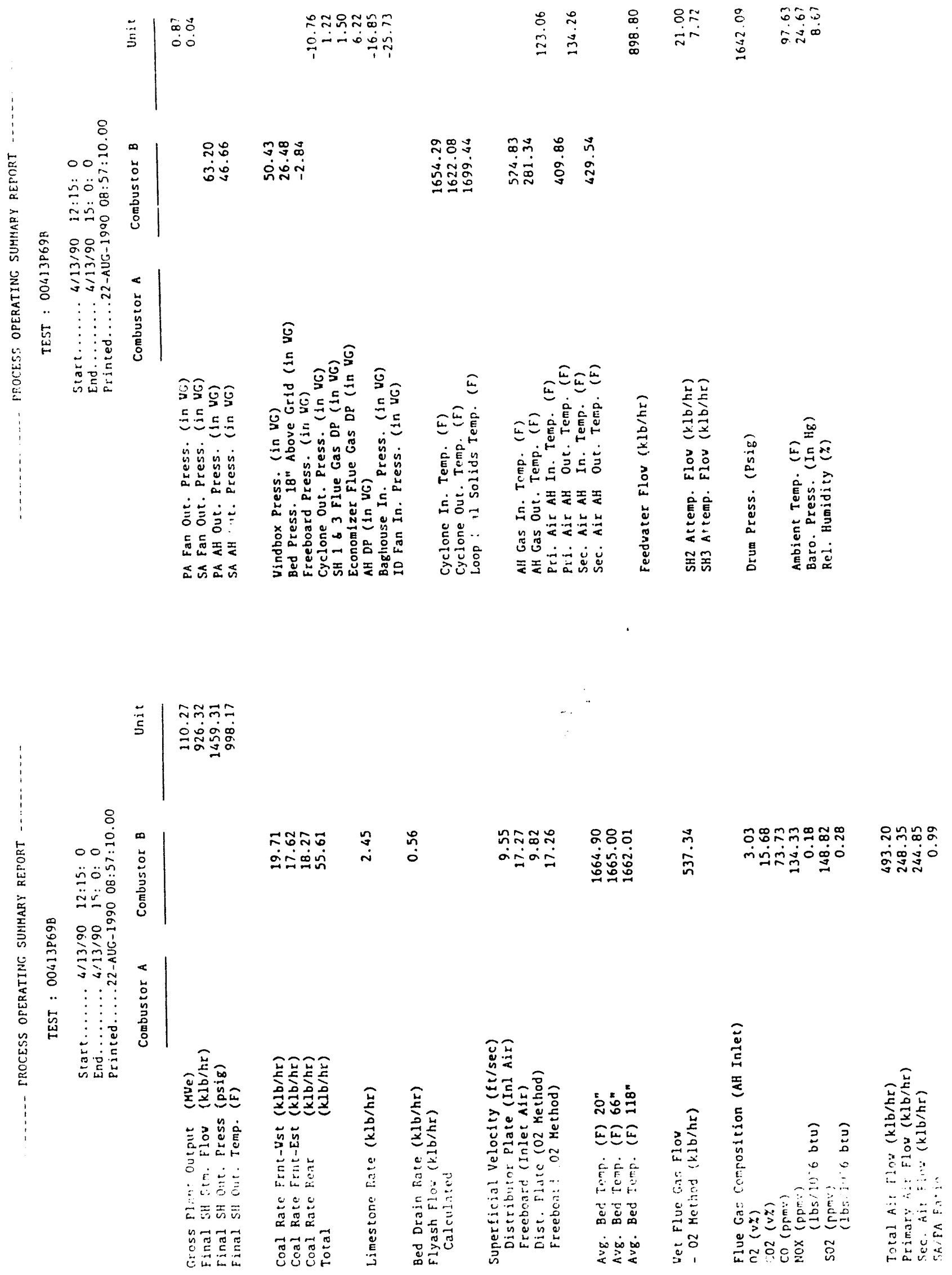




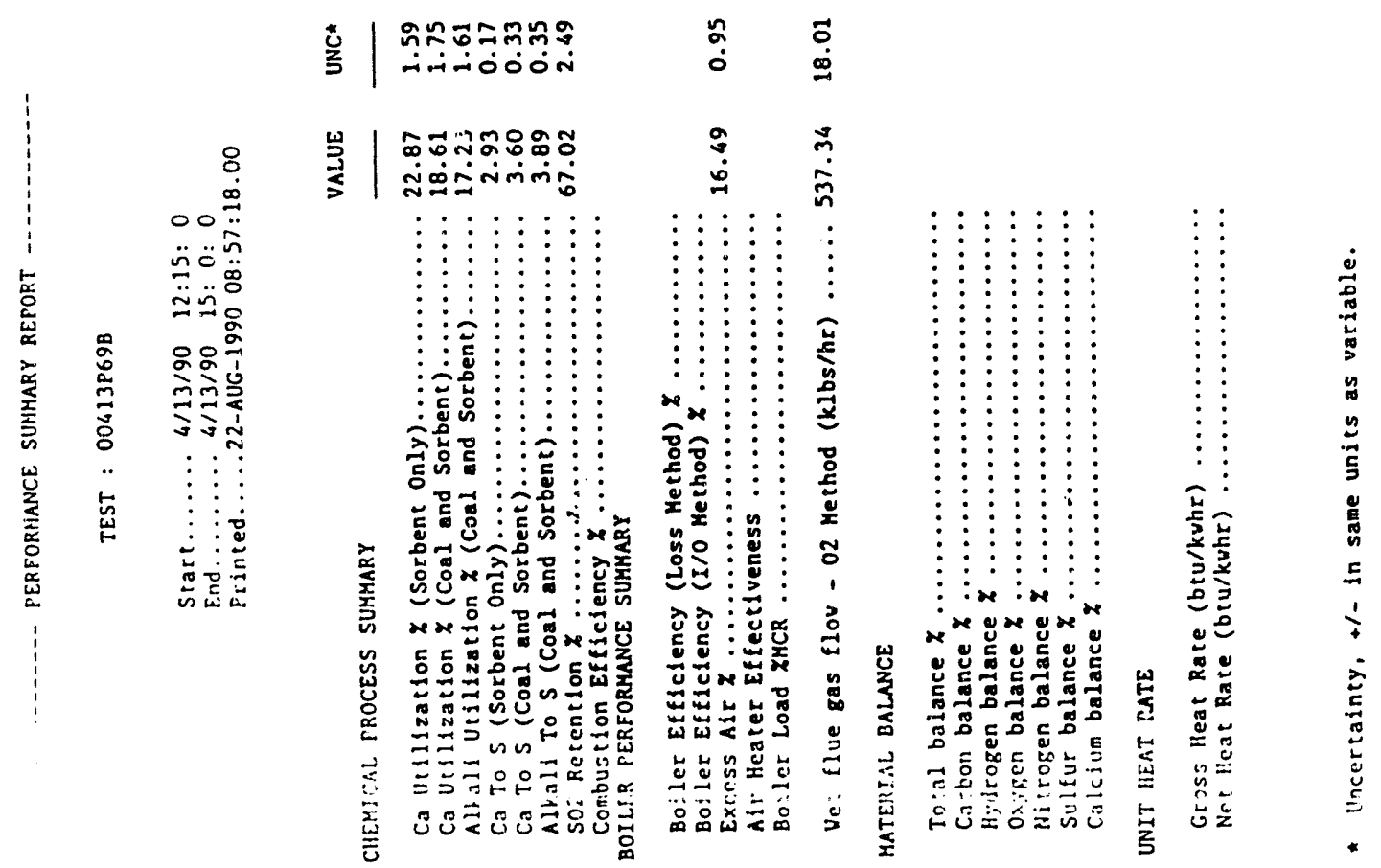




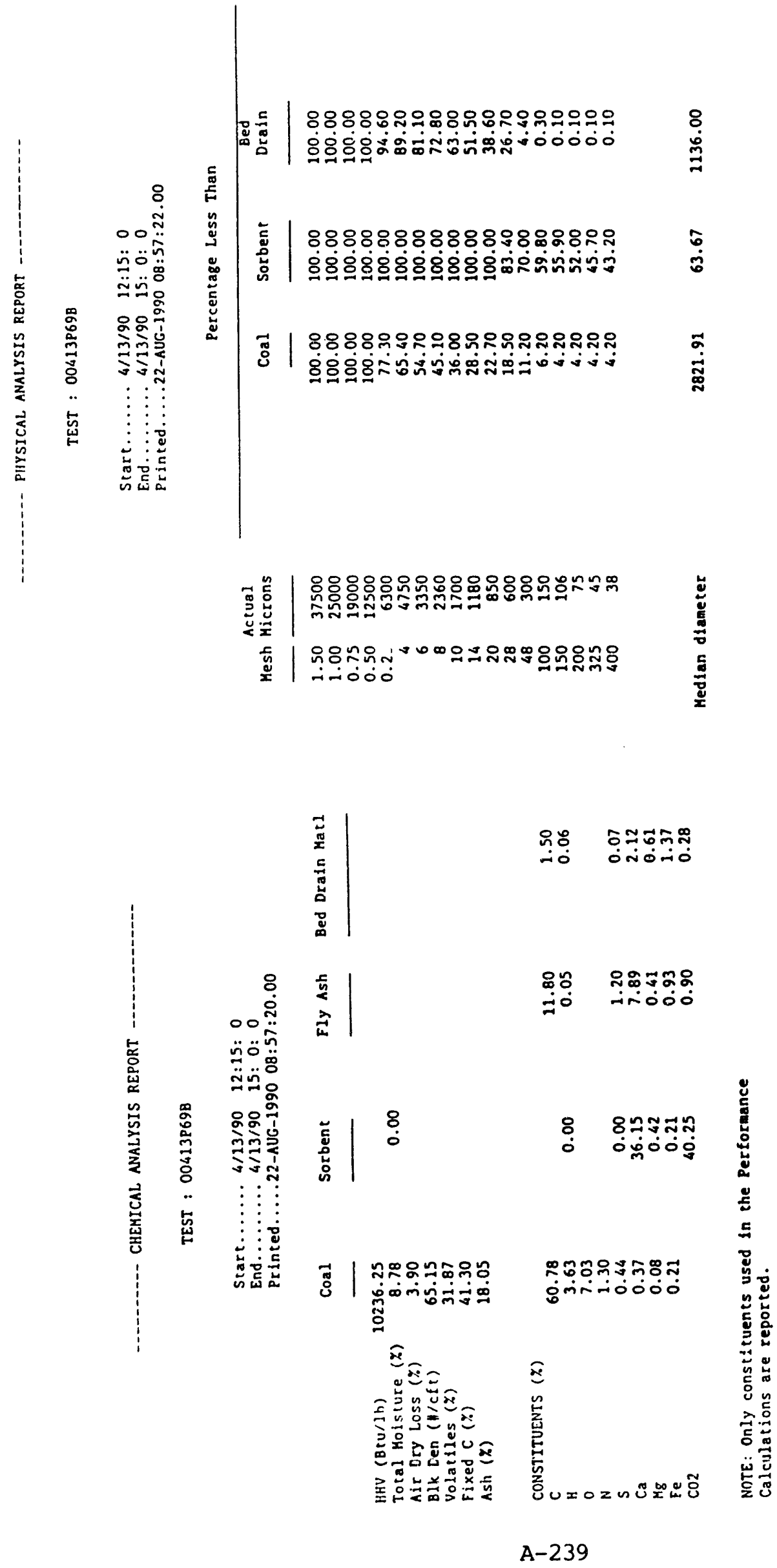




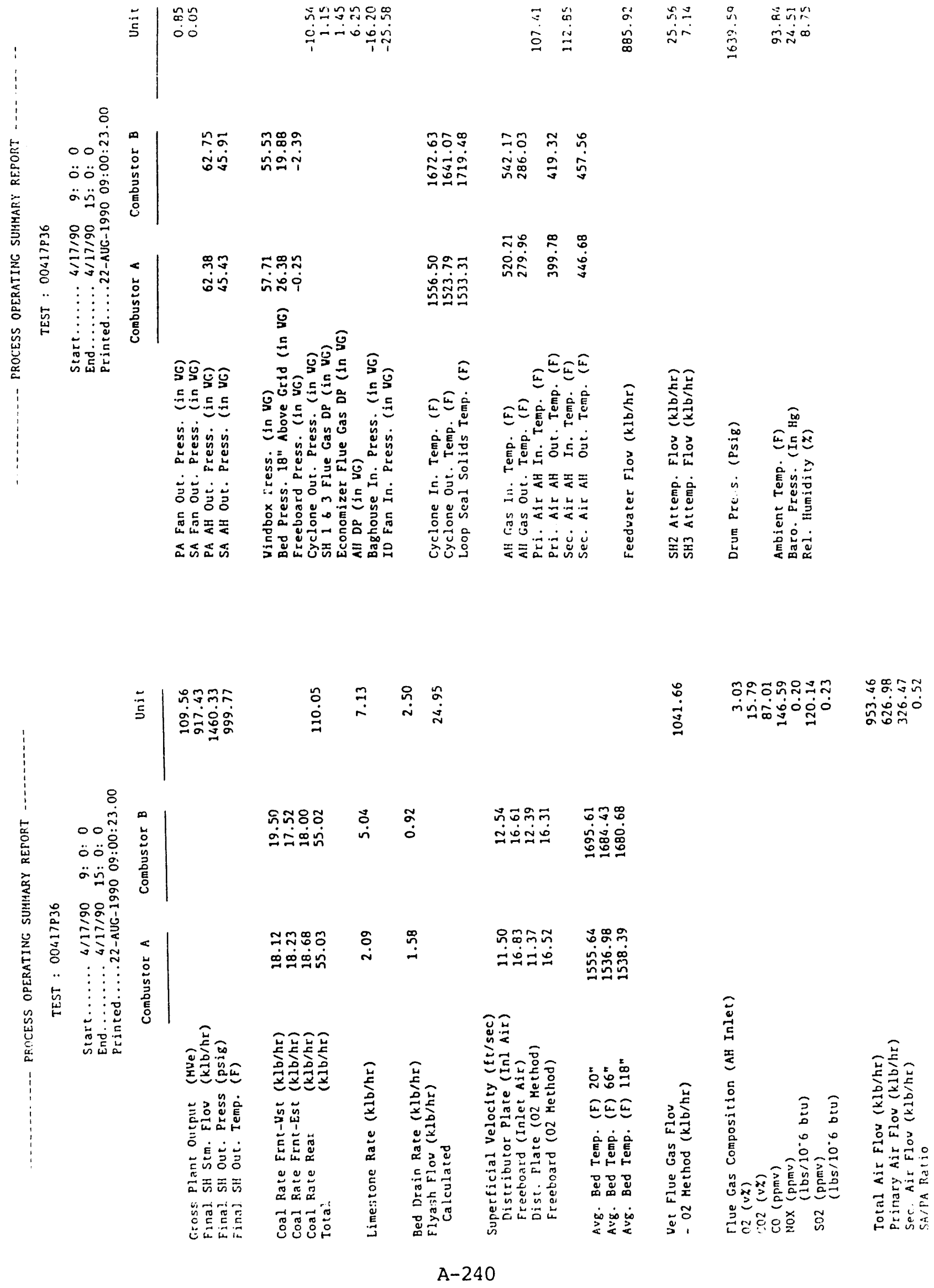




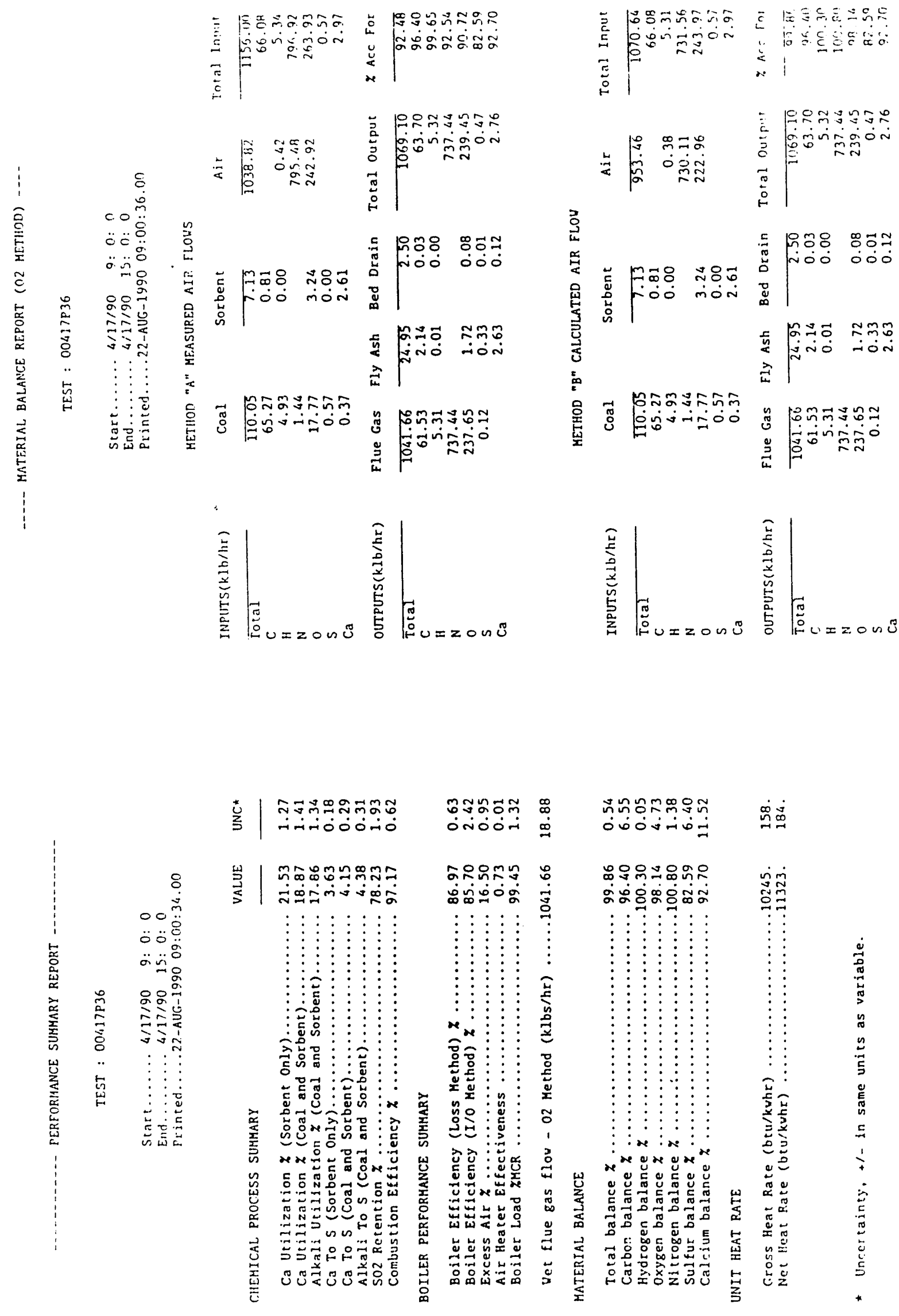




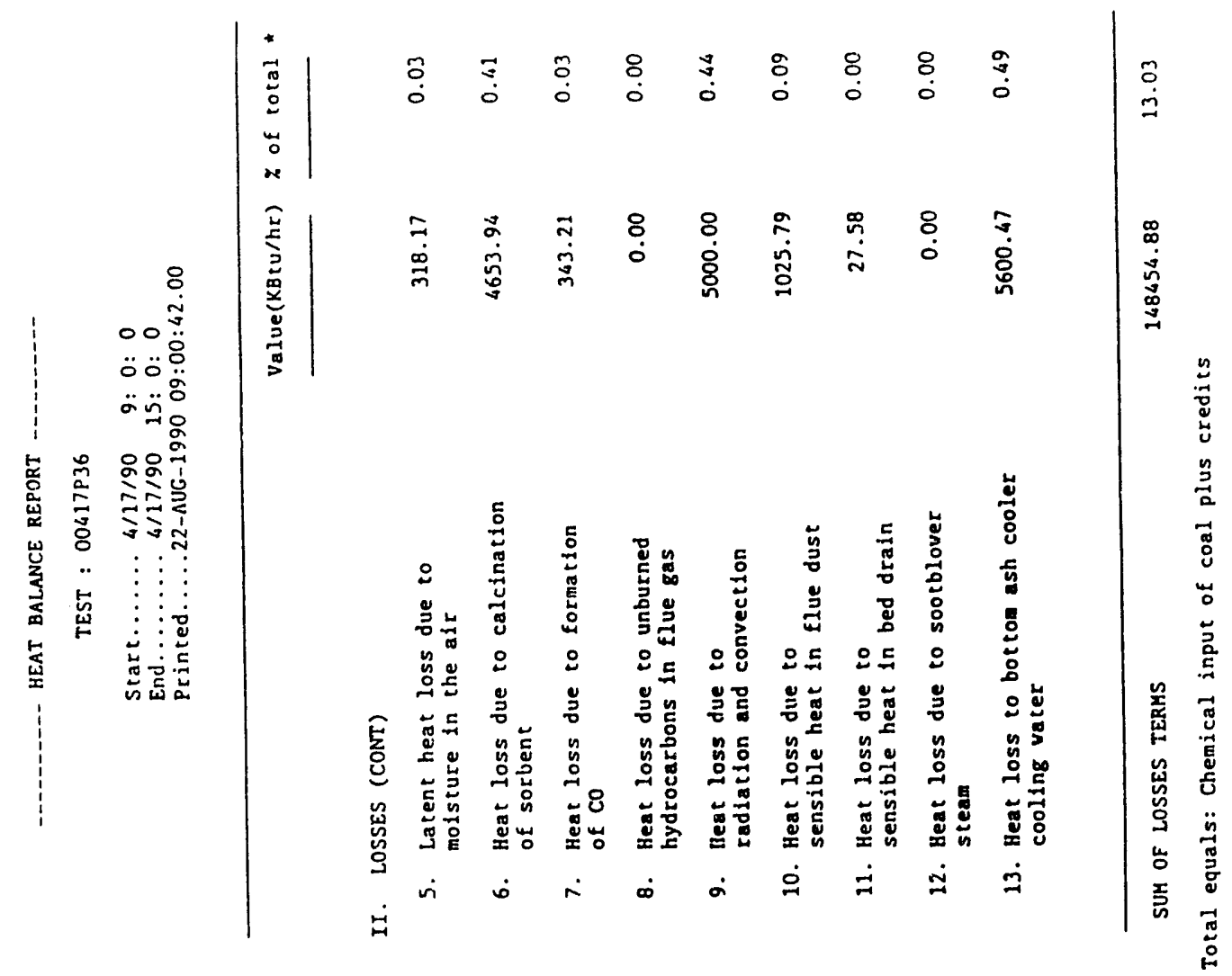

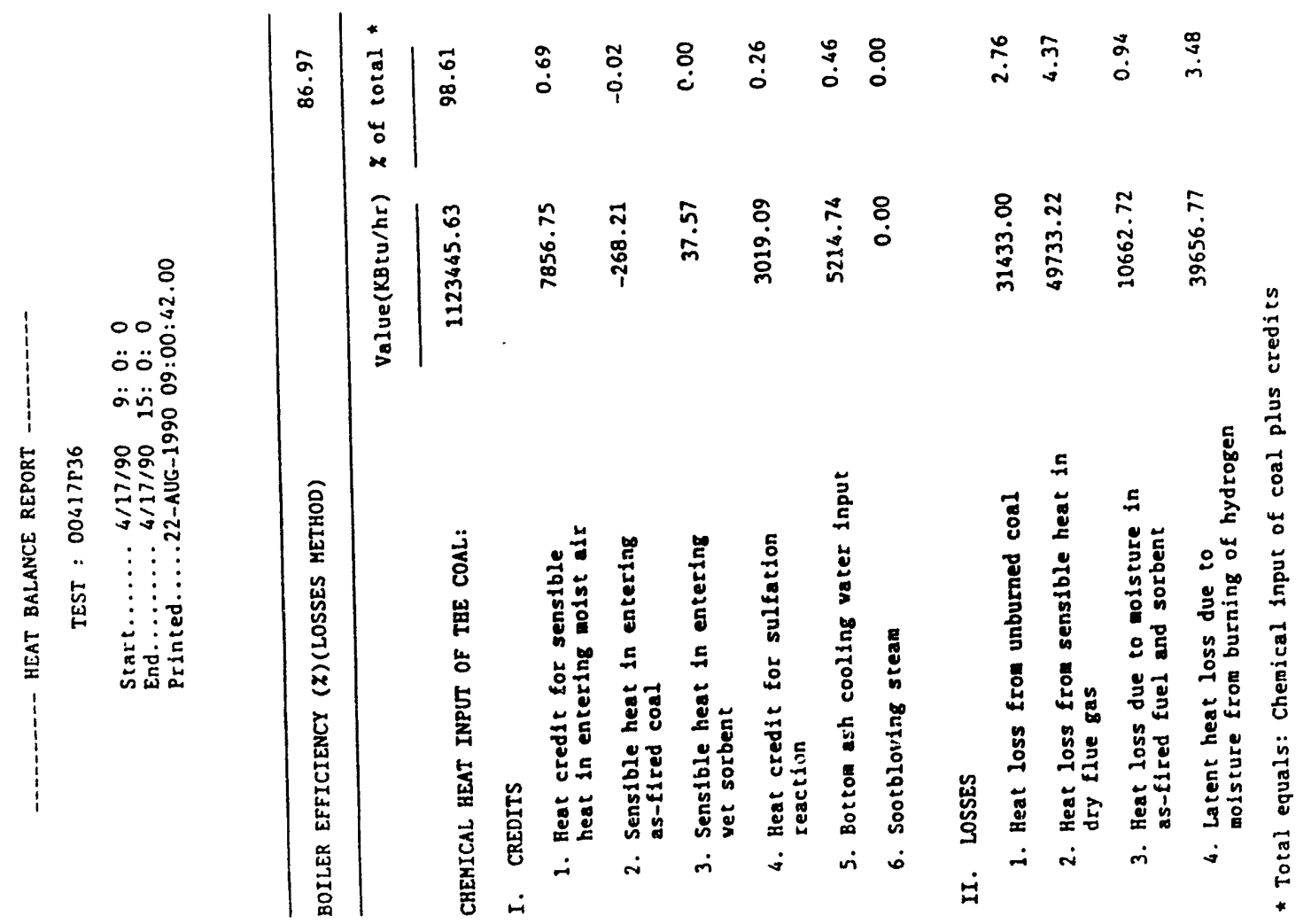



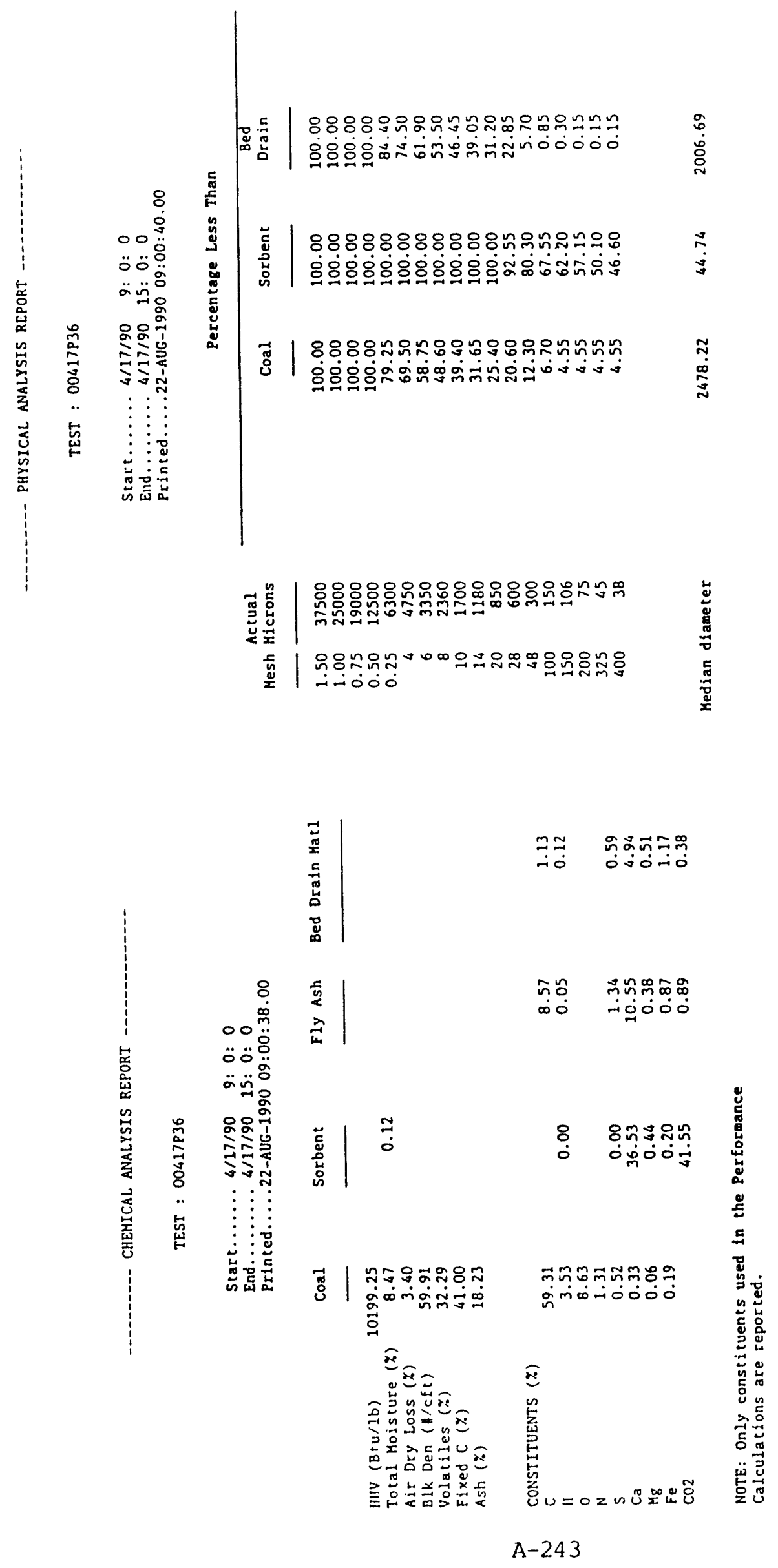

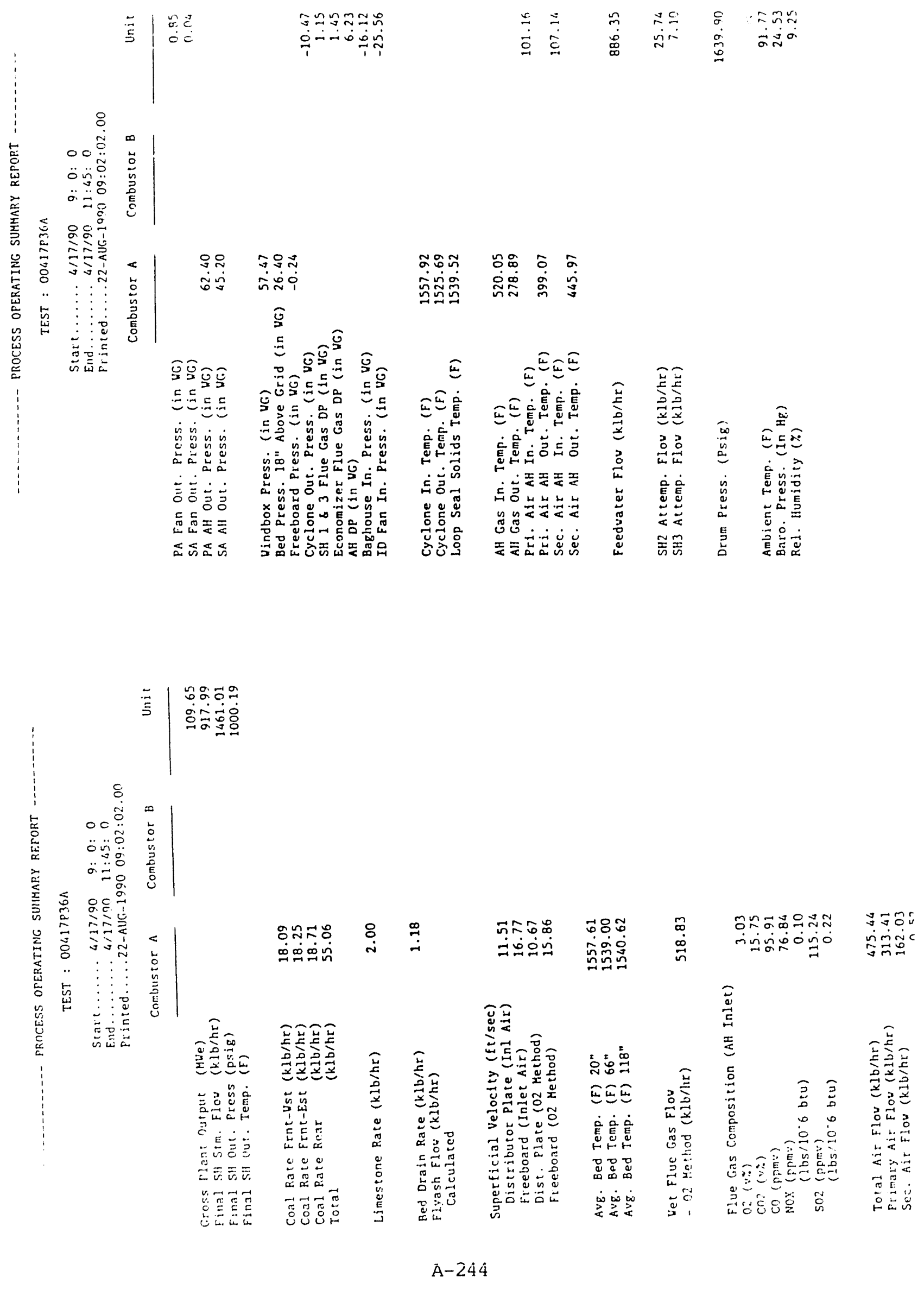


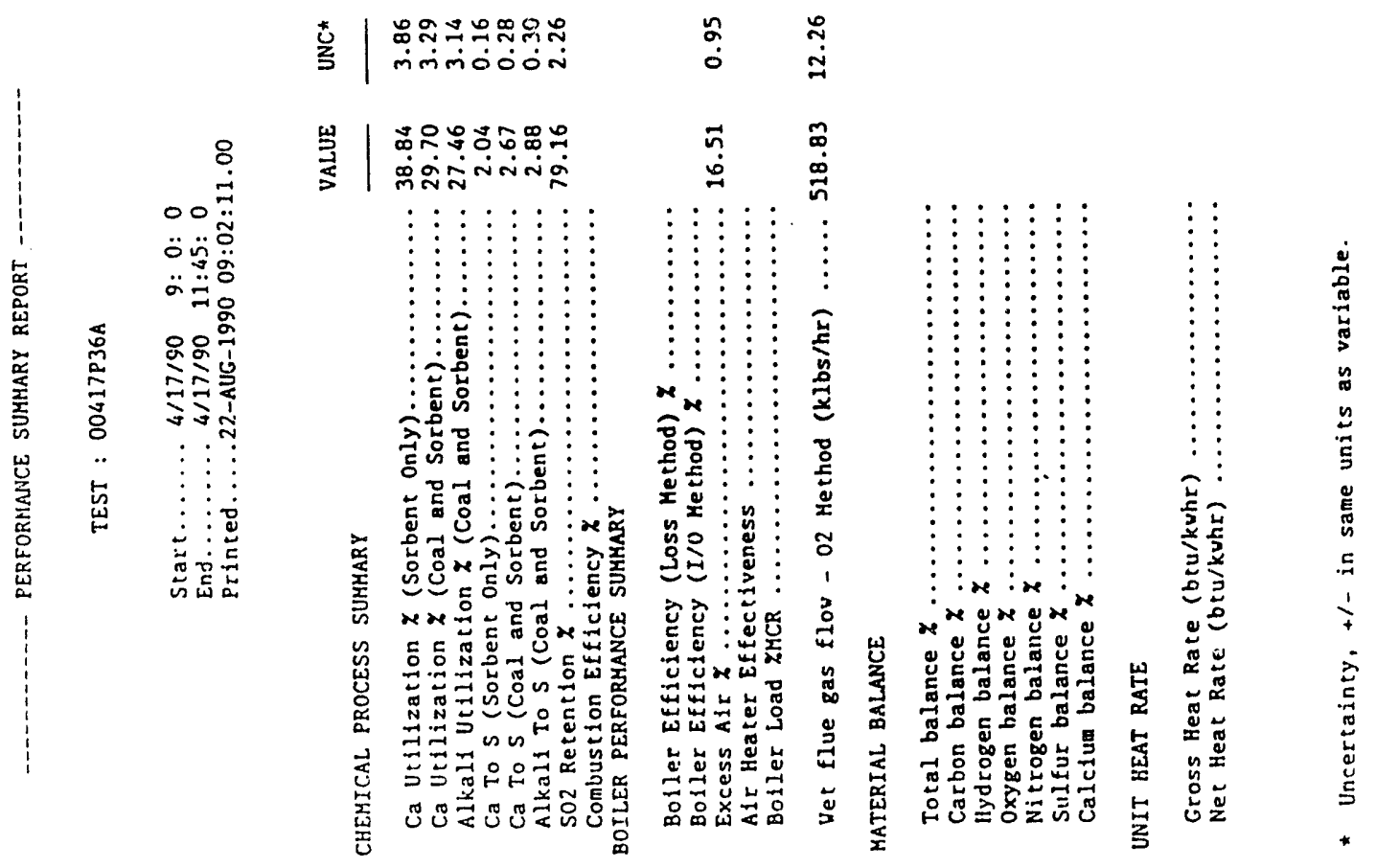



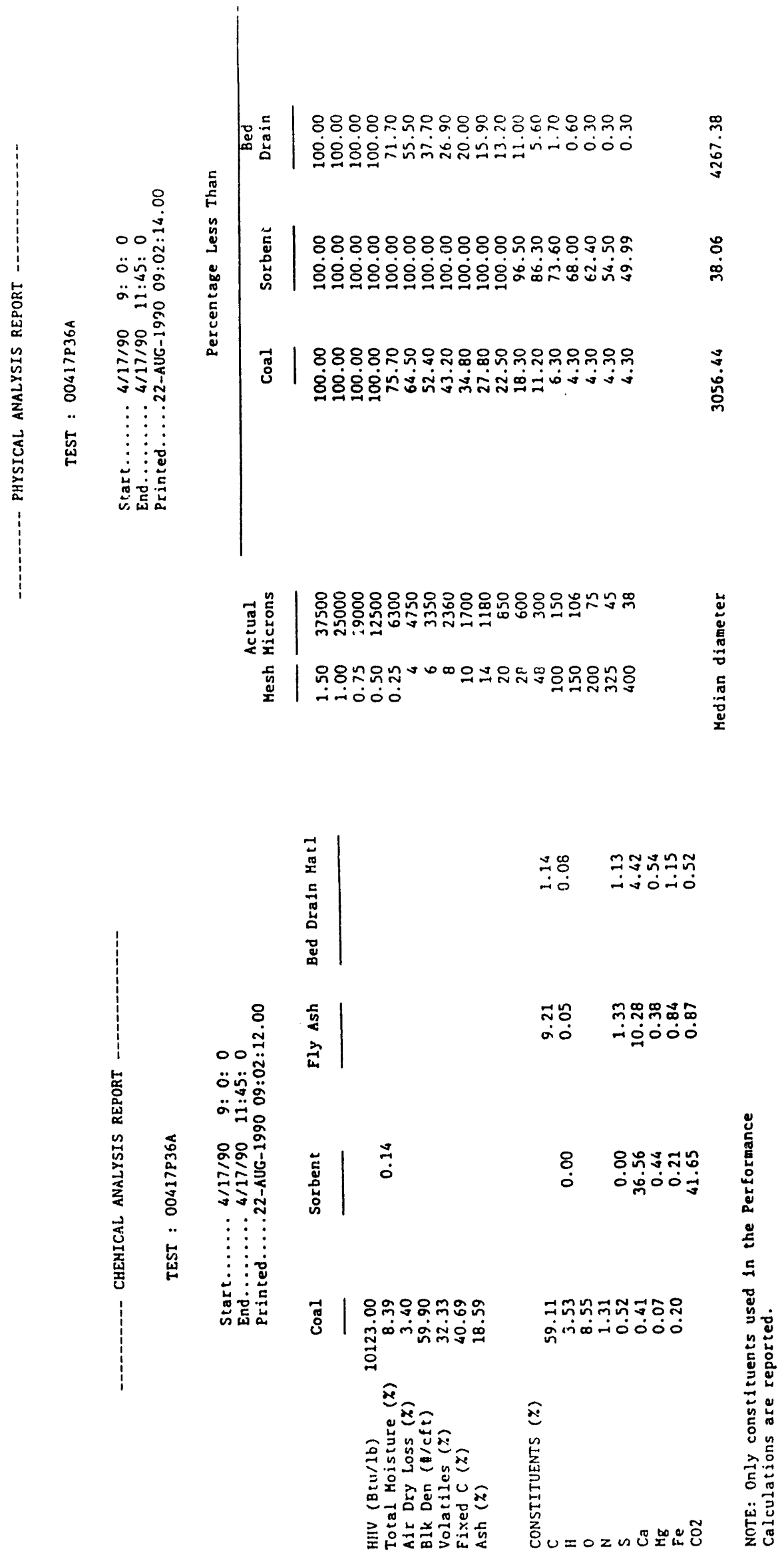

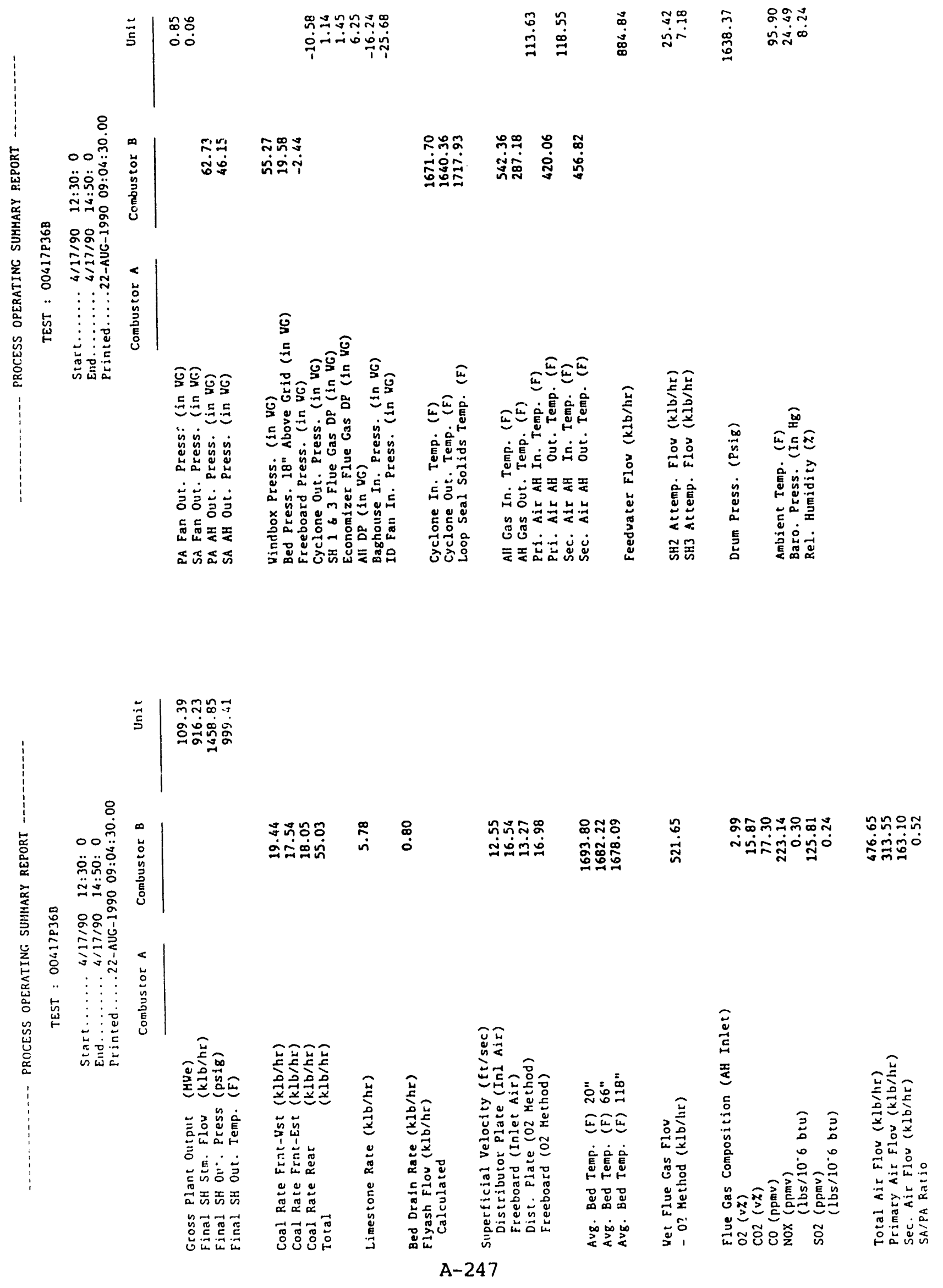


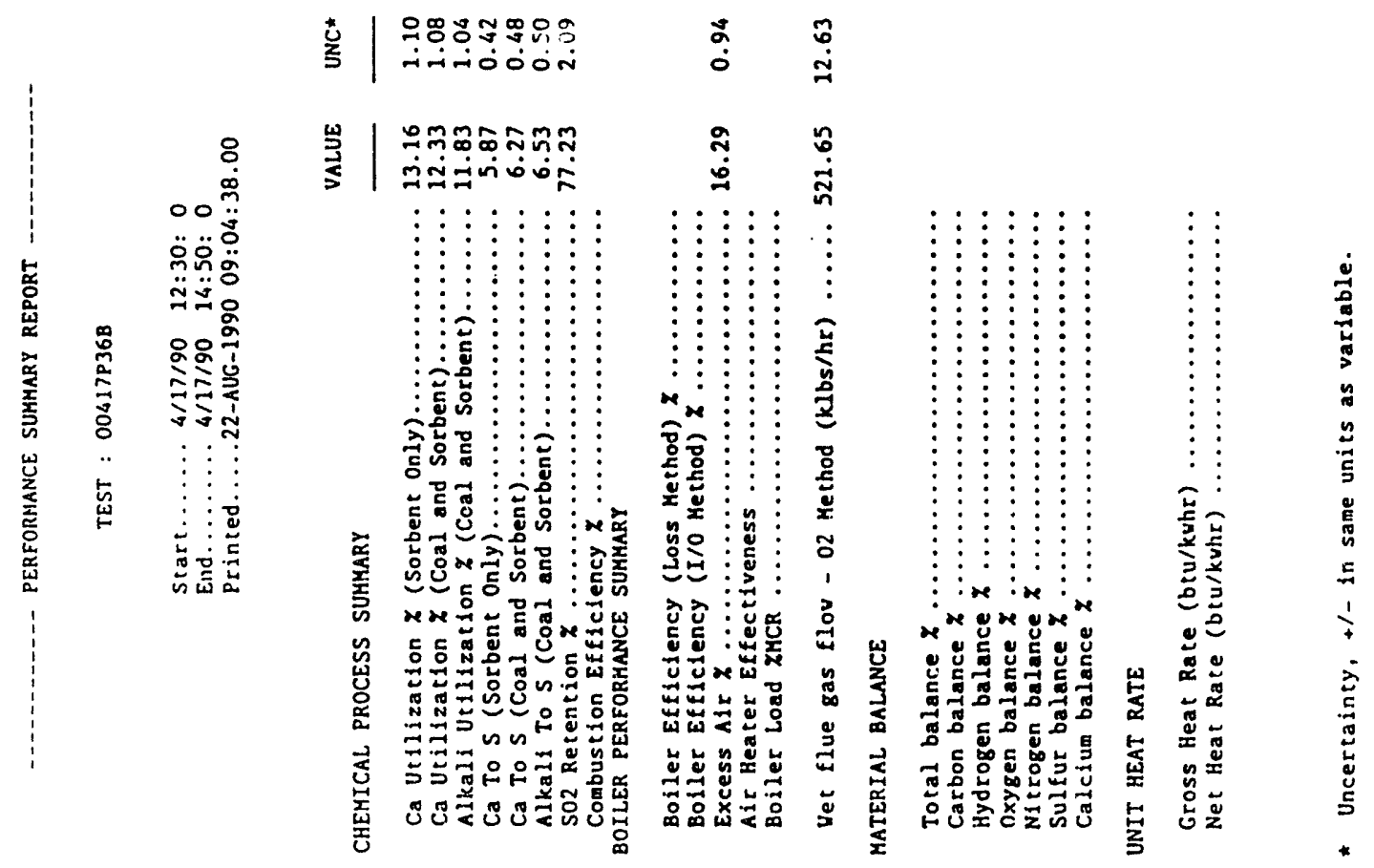



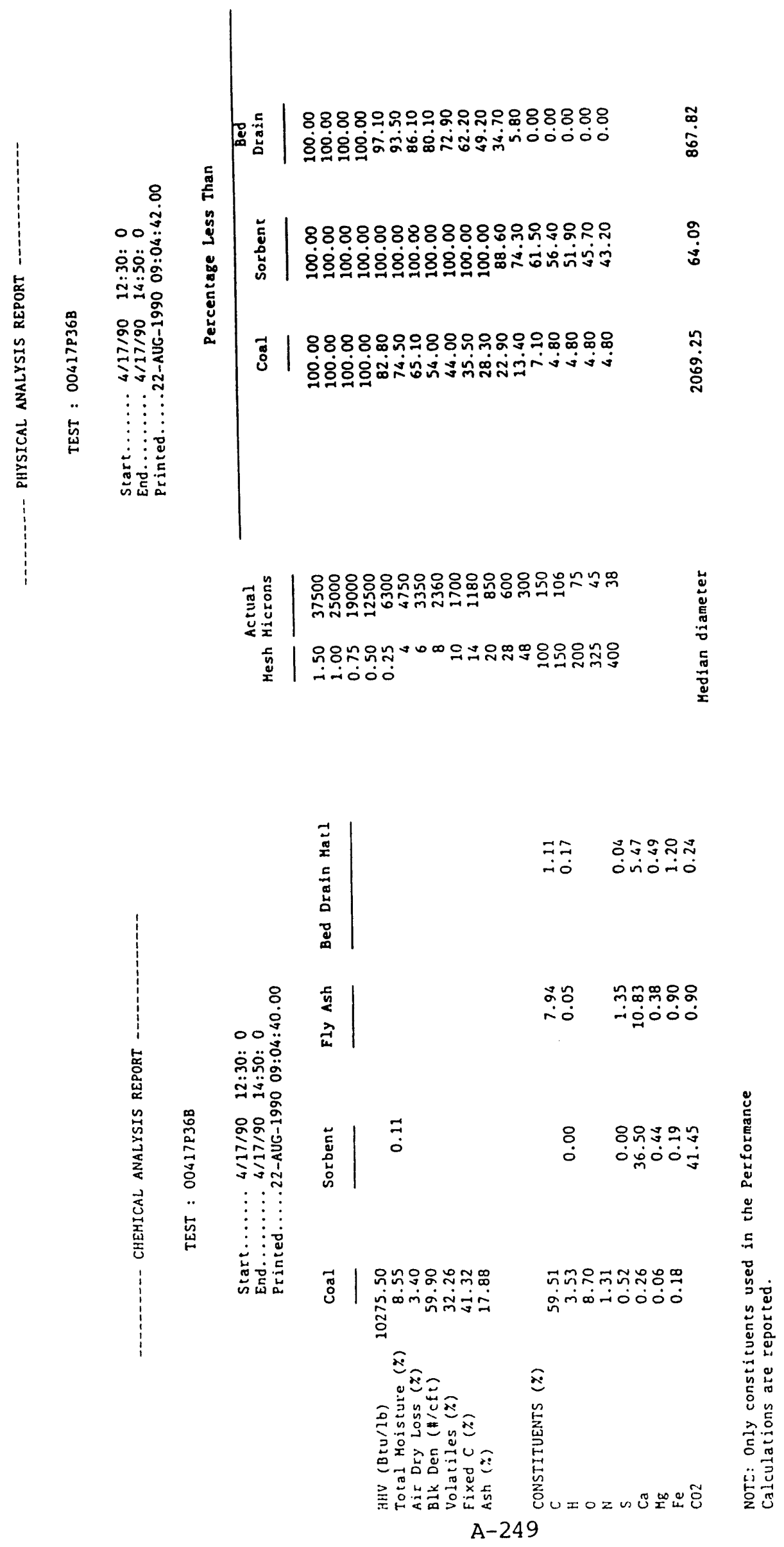

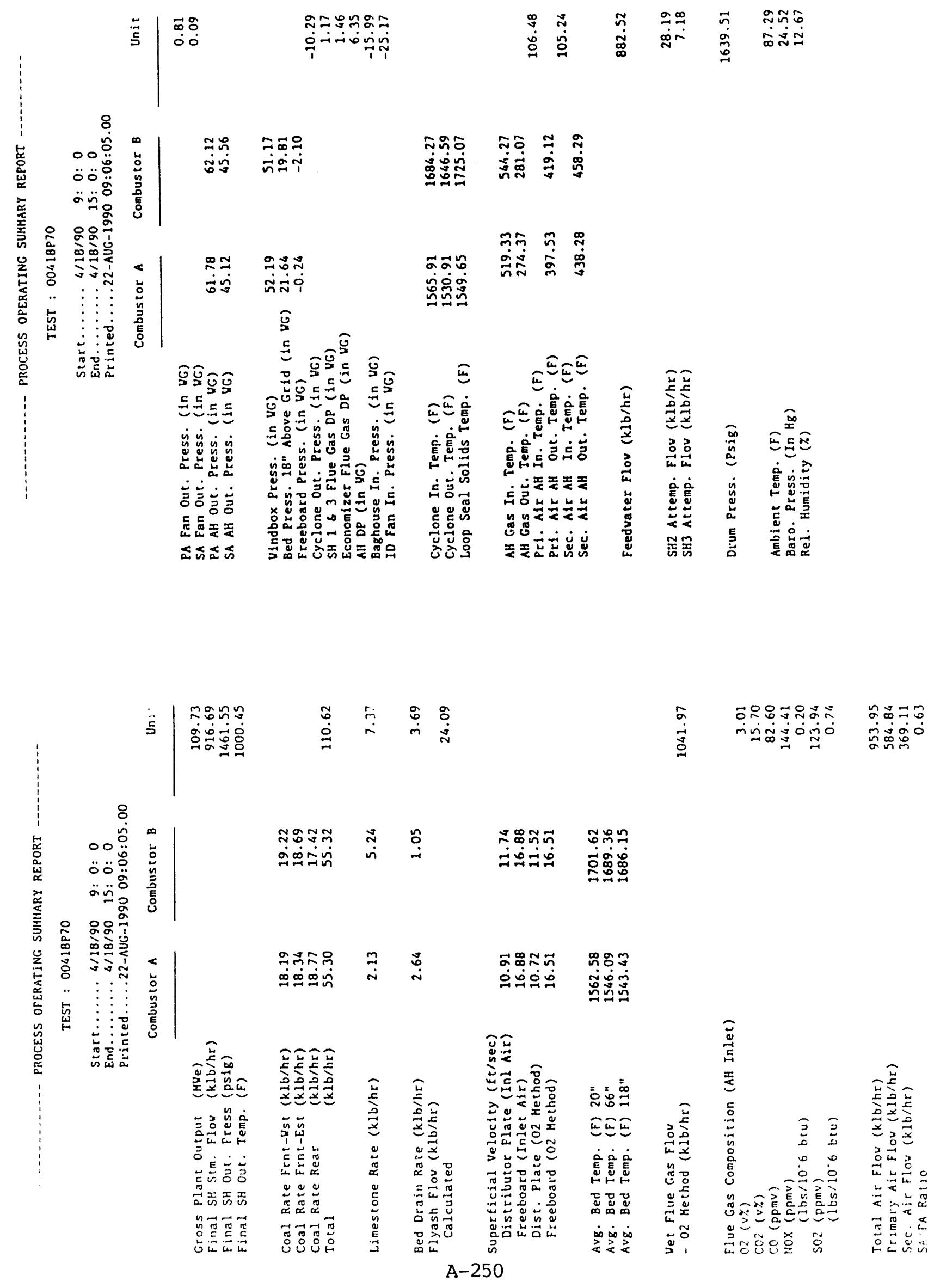


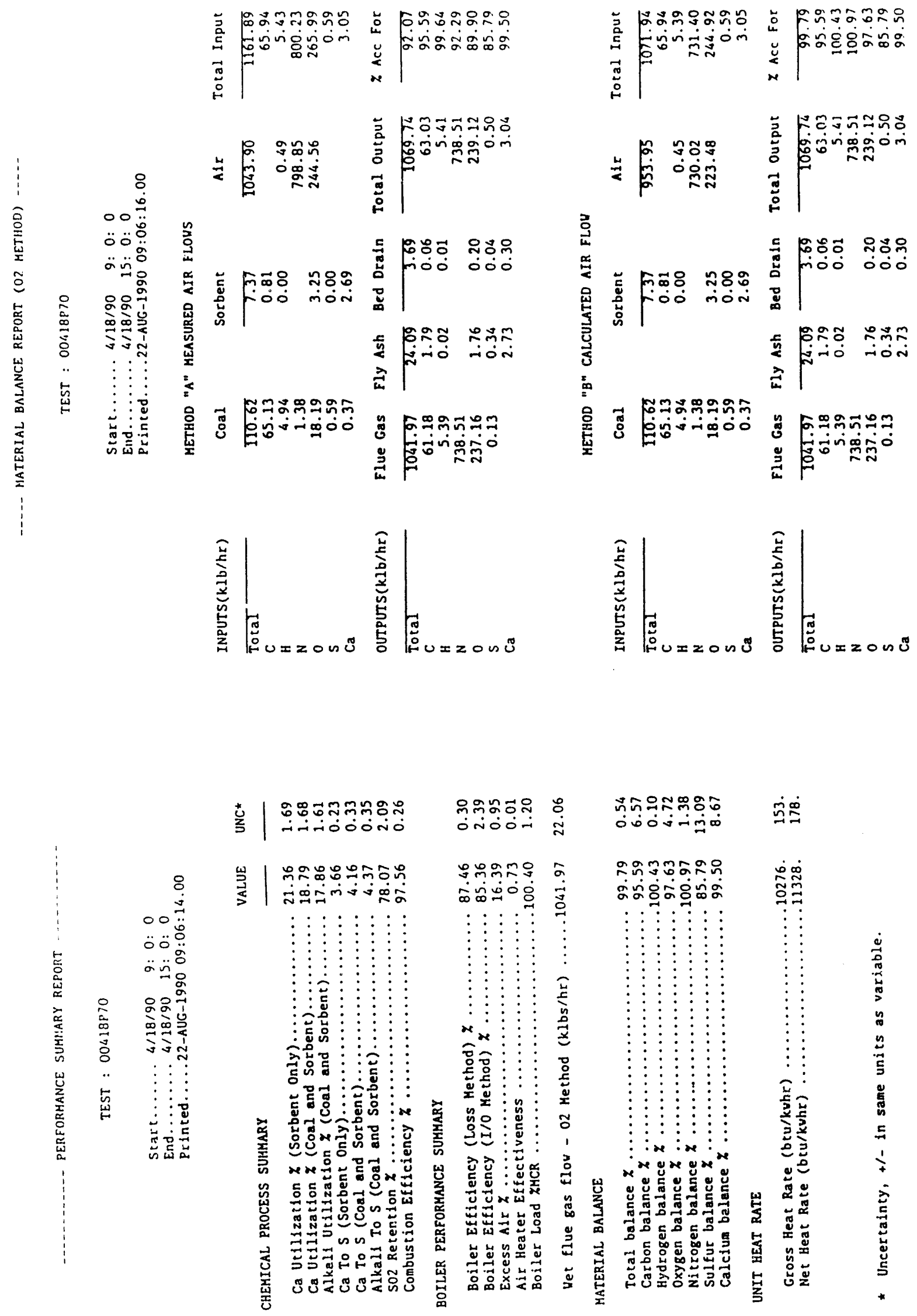




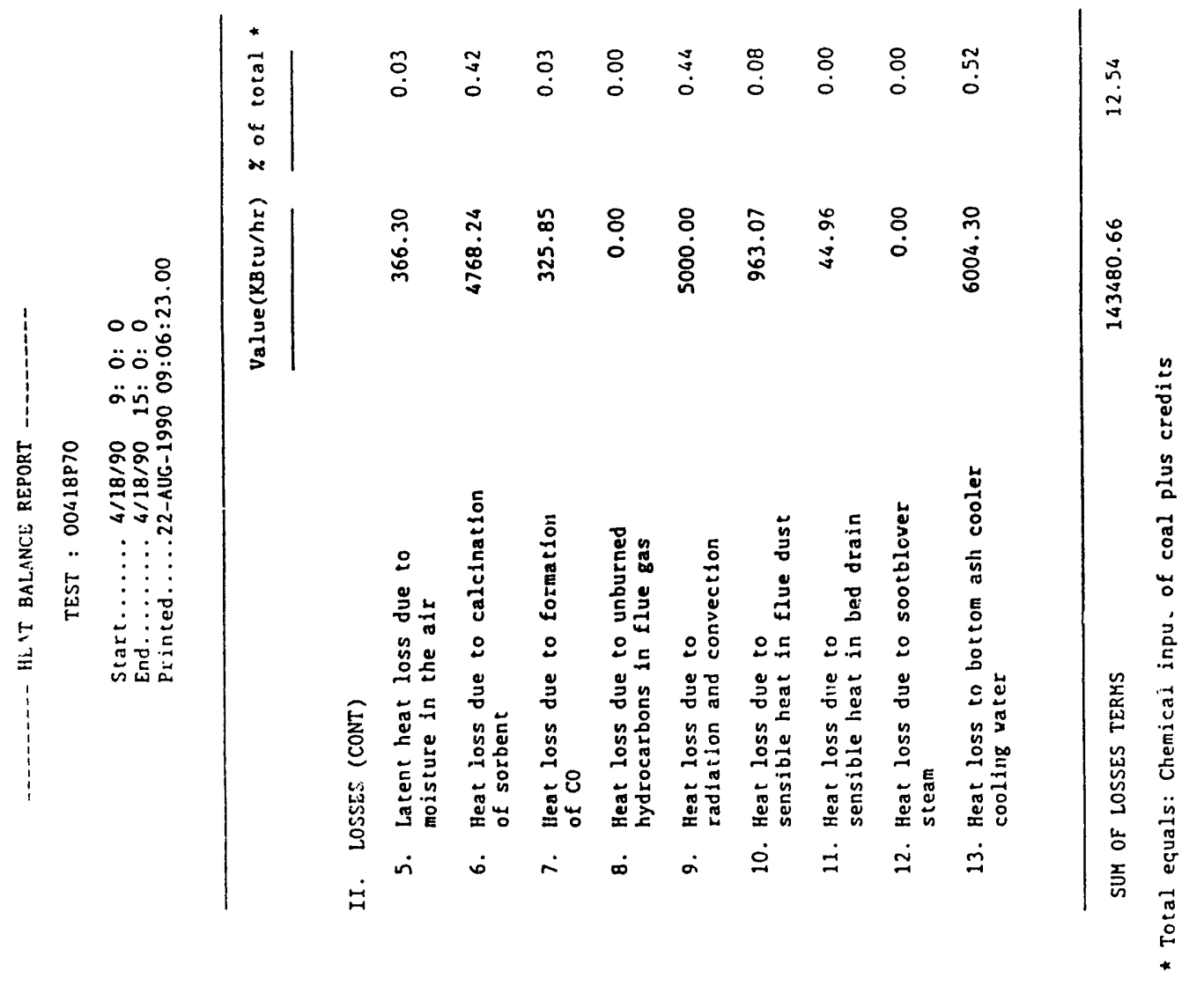

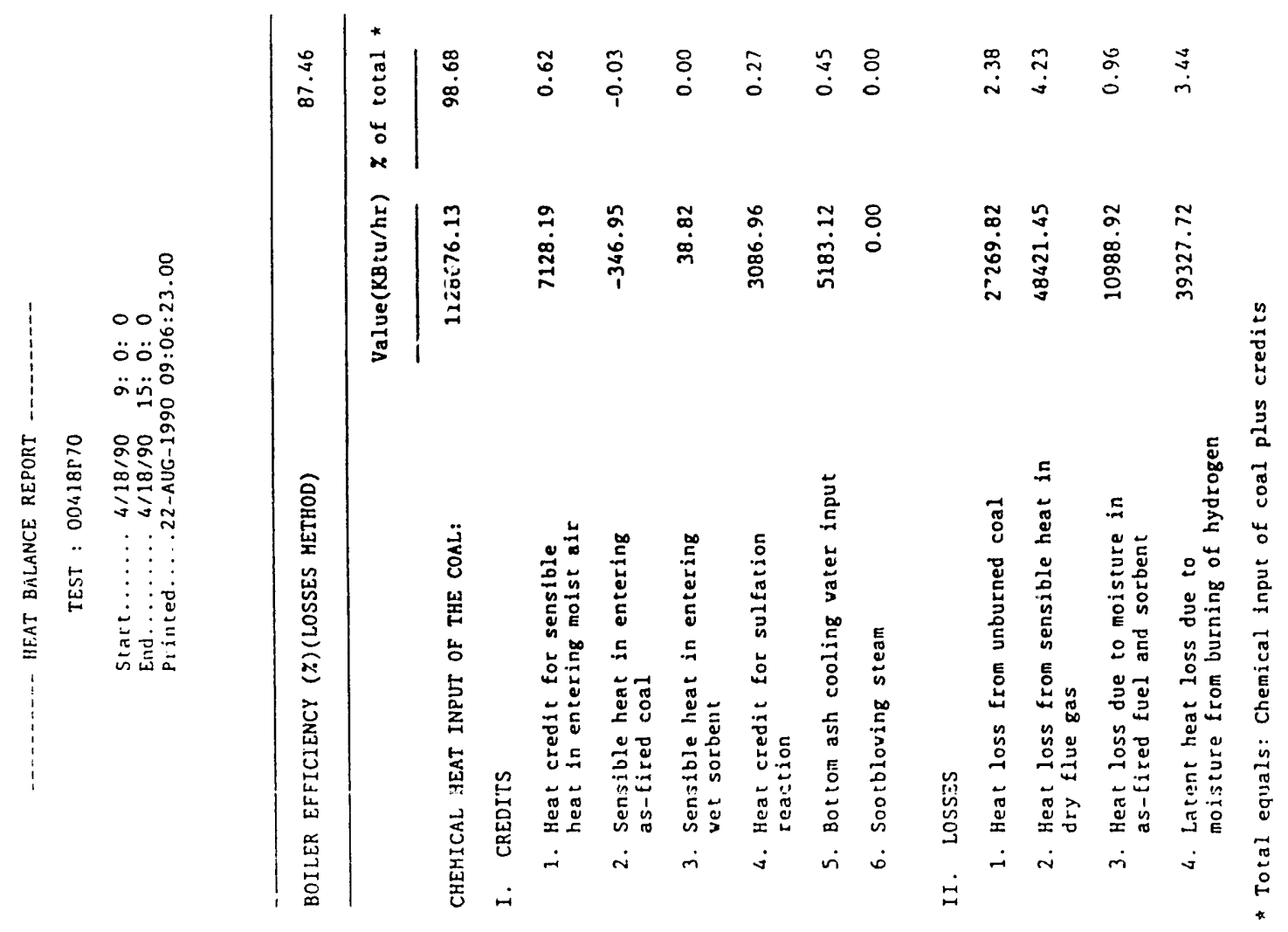



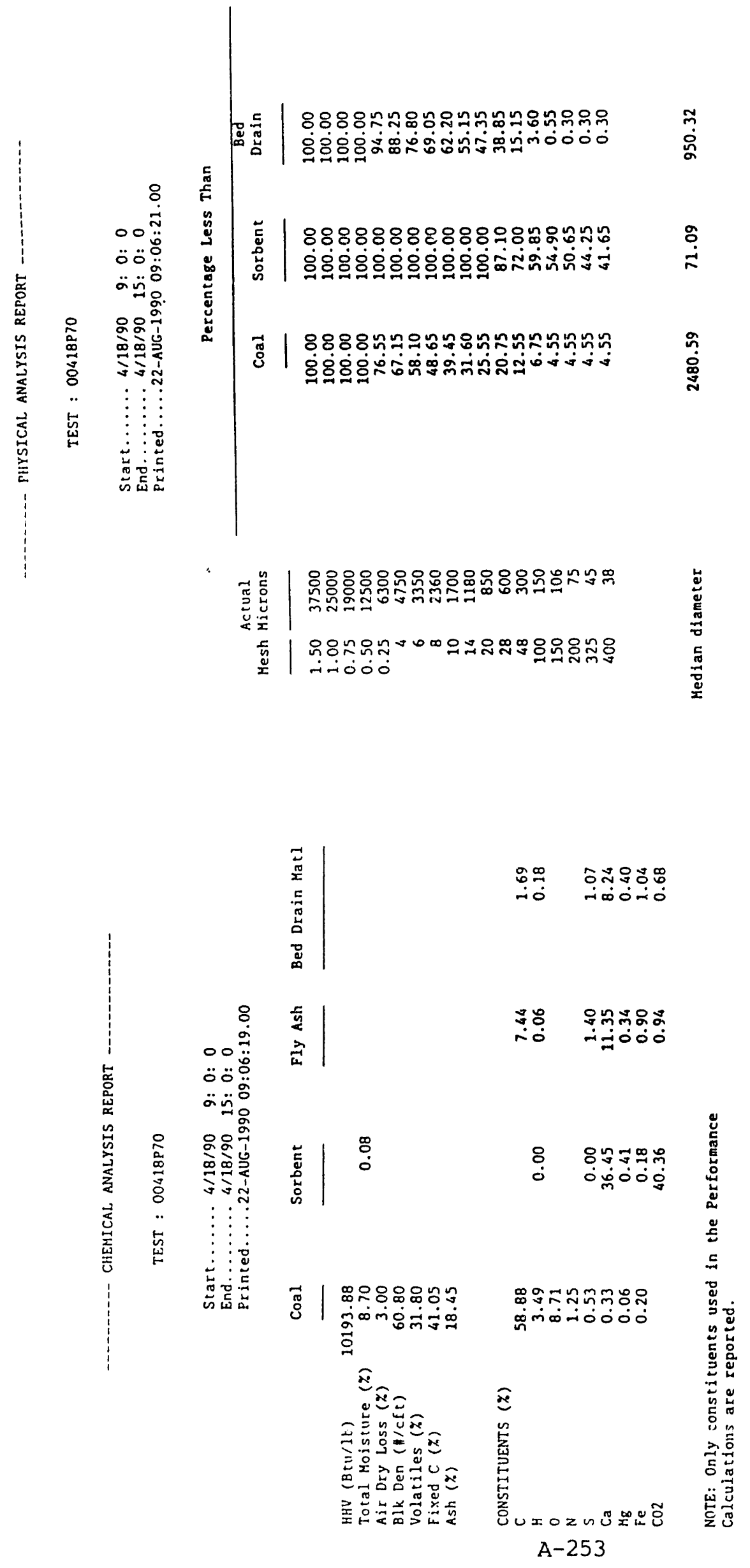

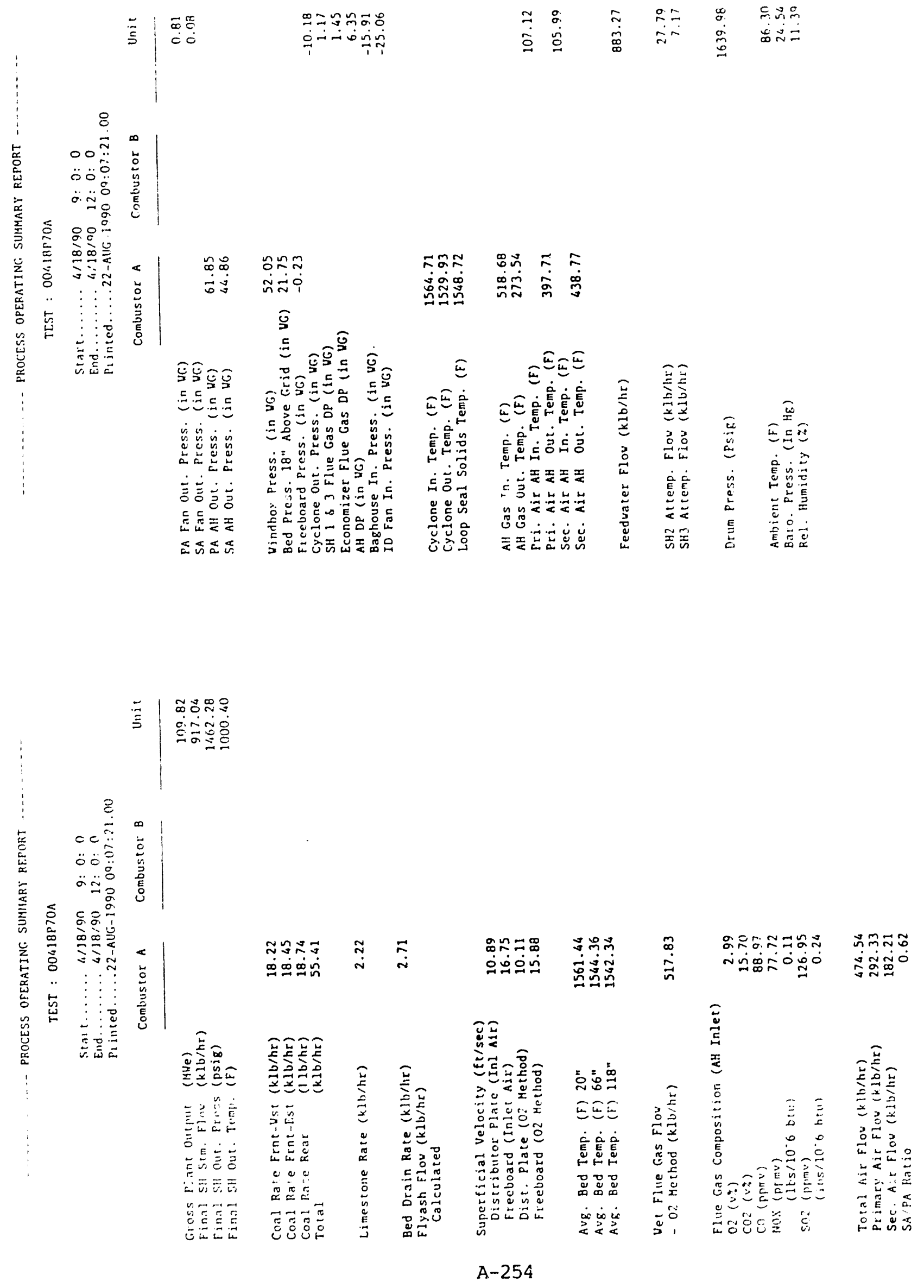


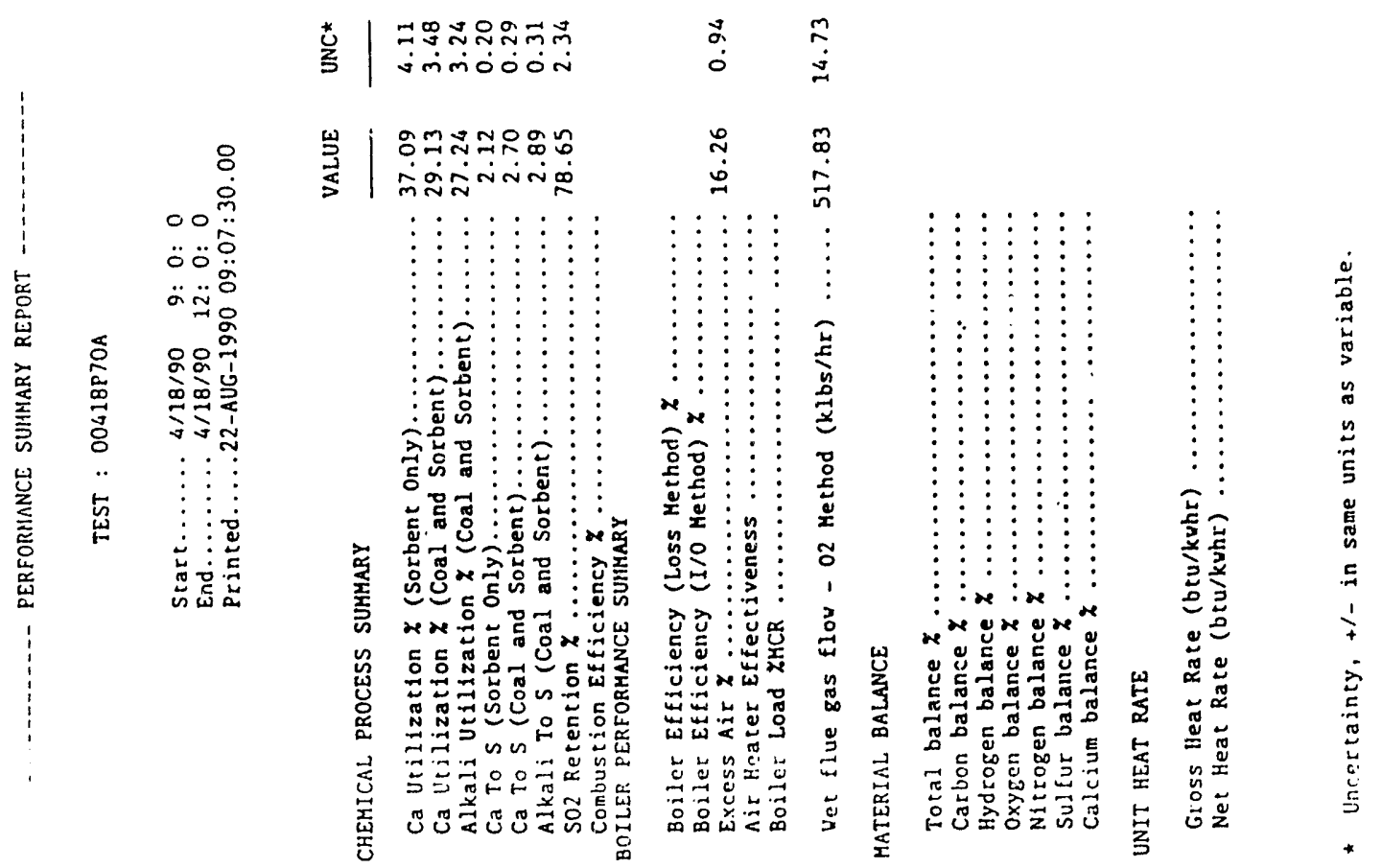



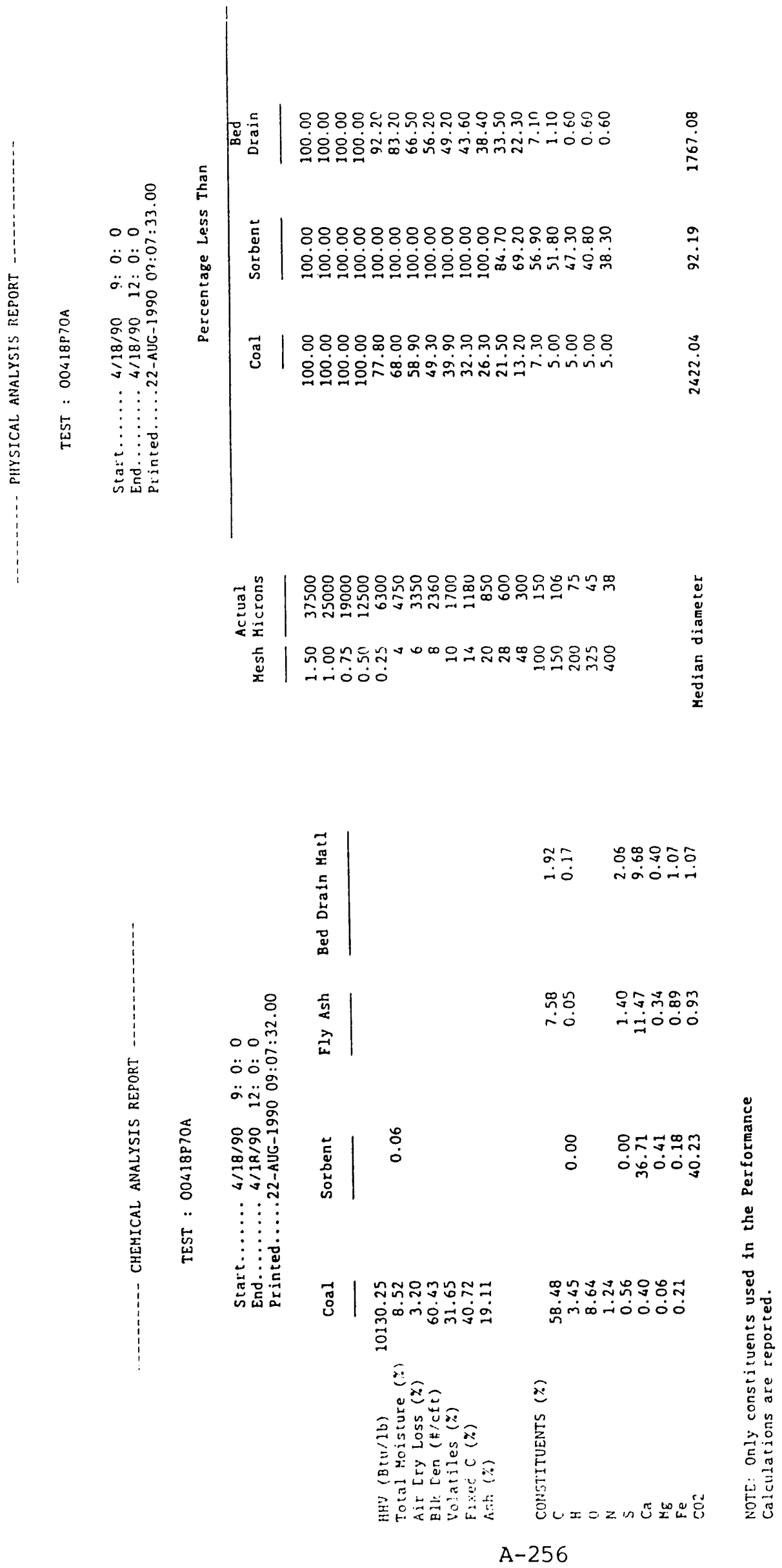

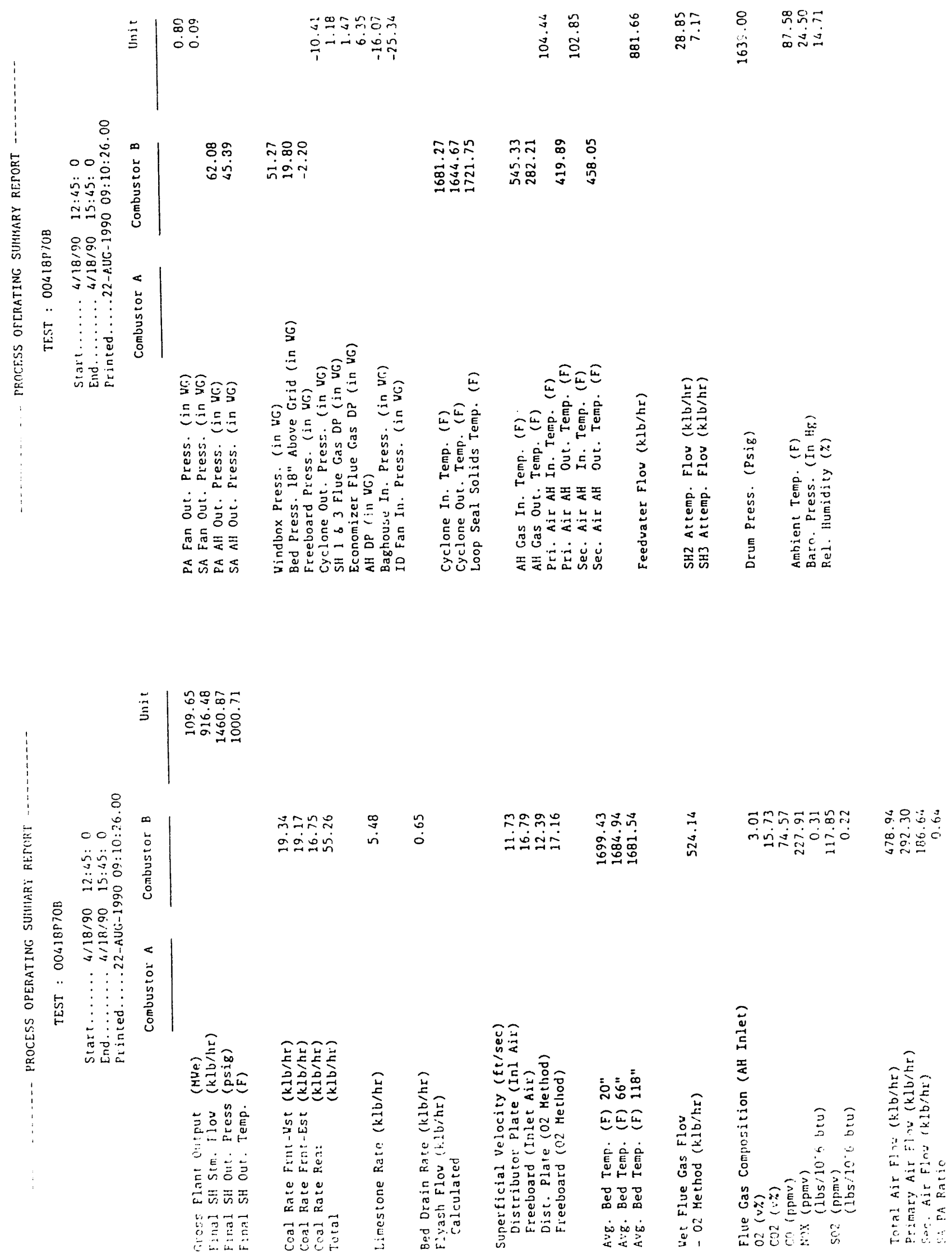


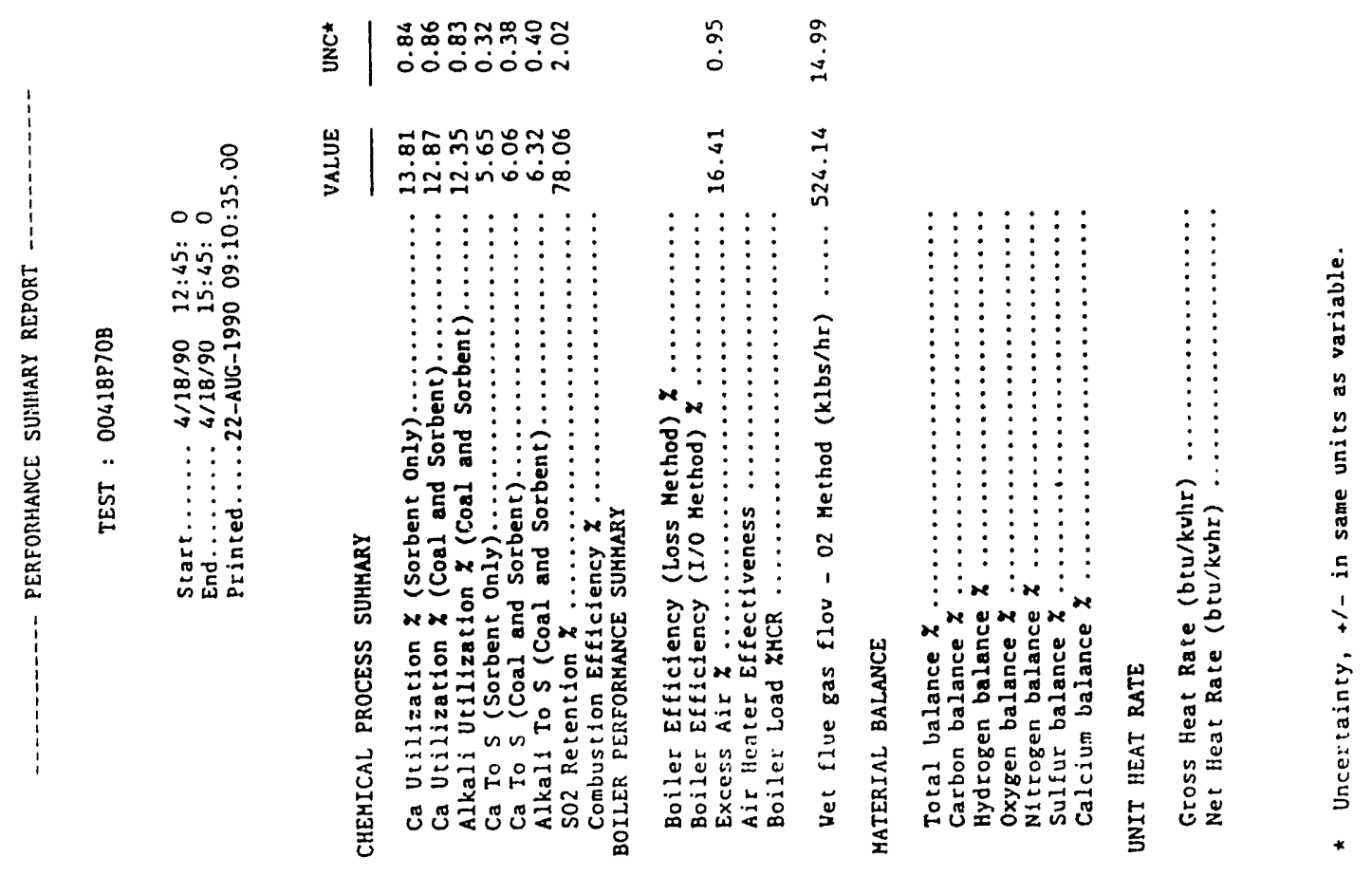

A-258 

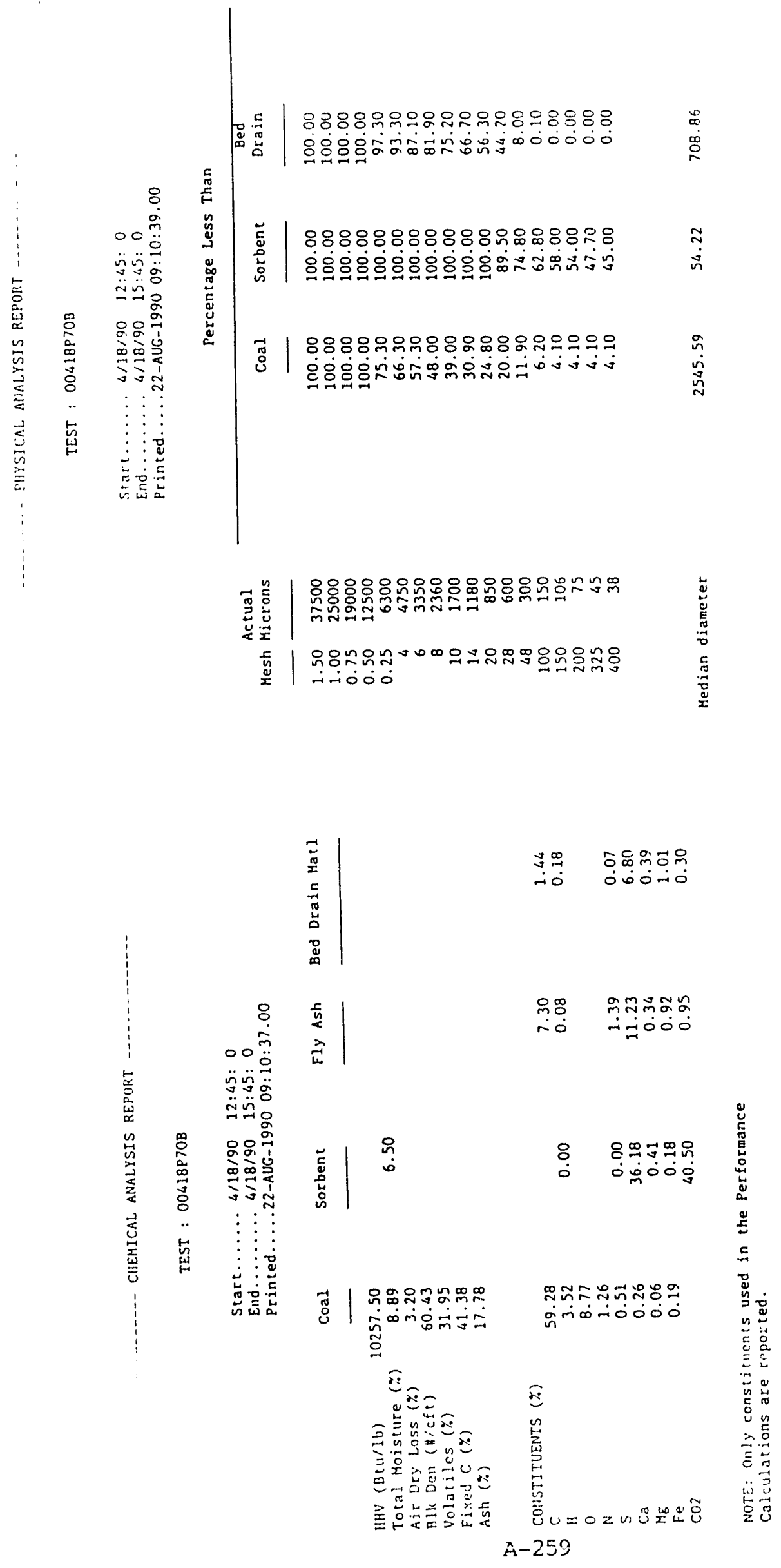


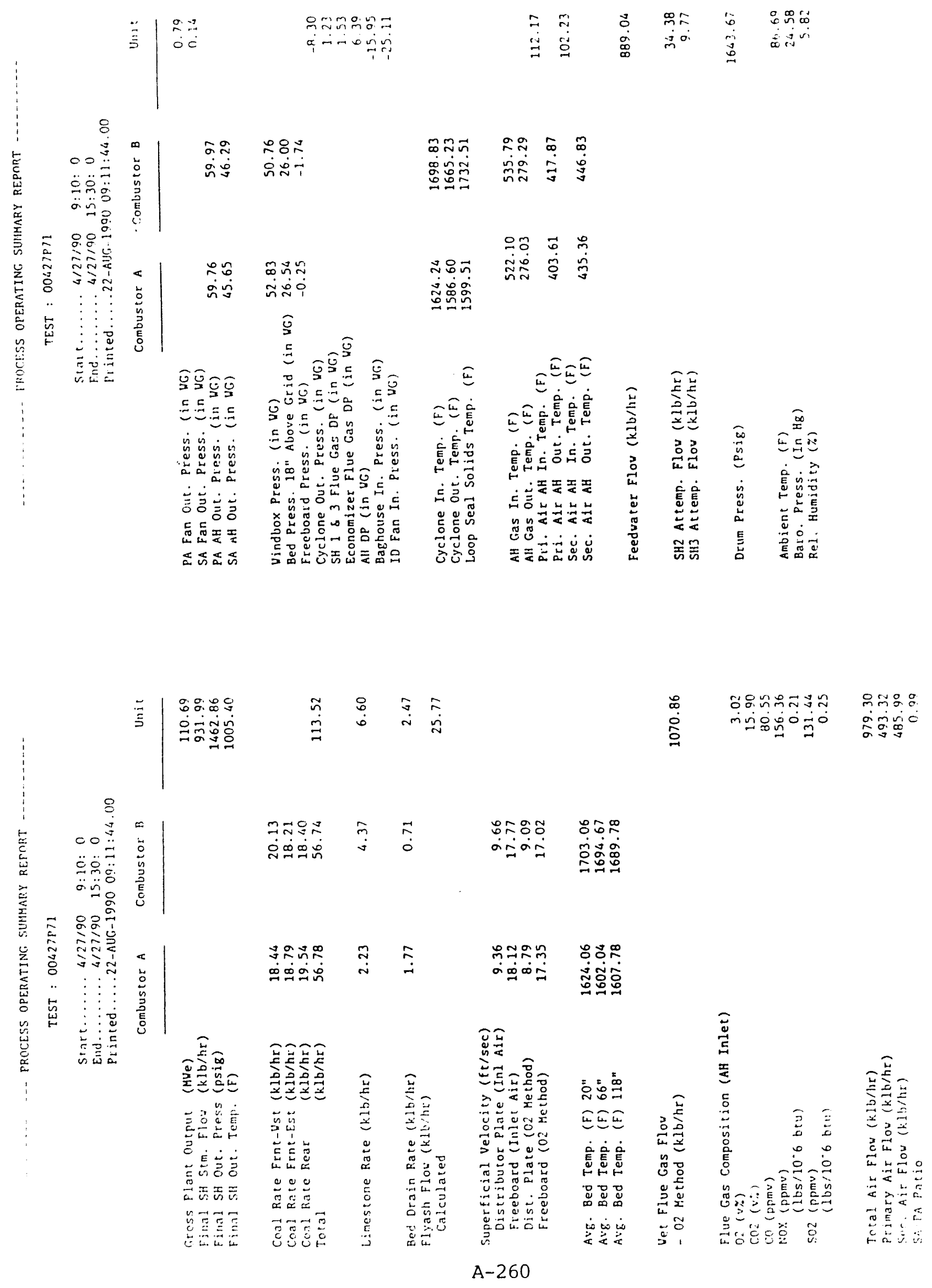




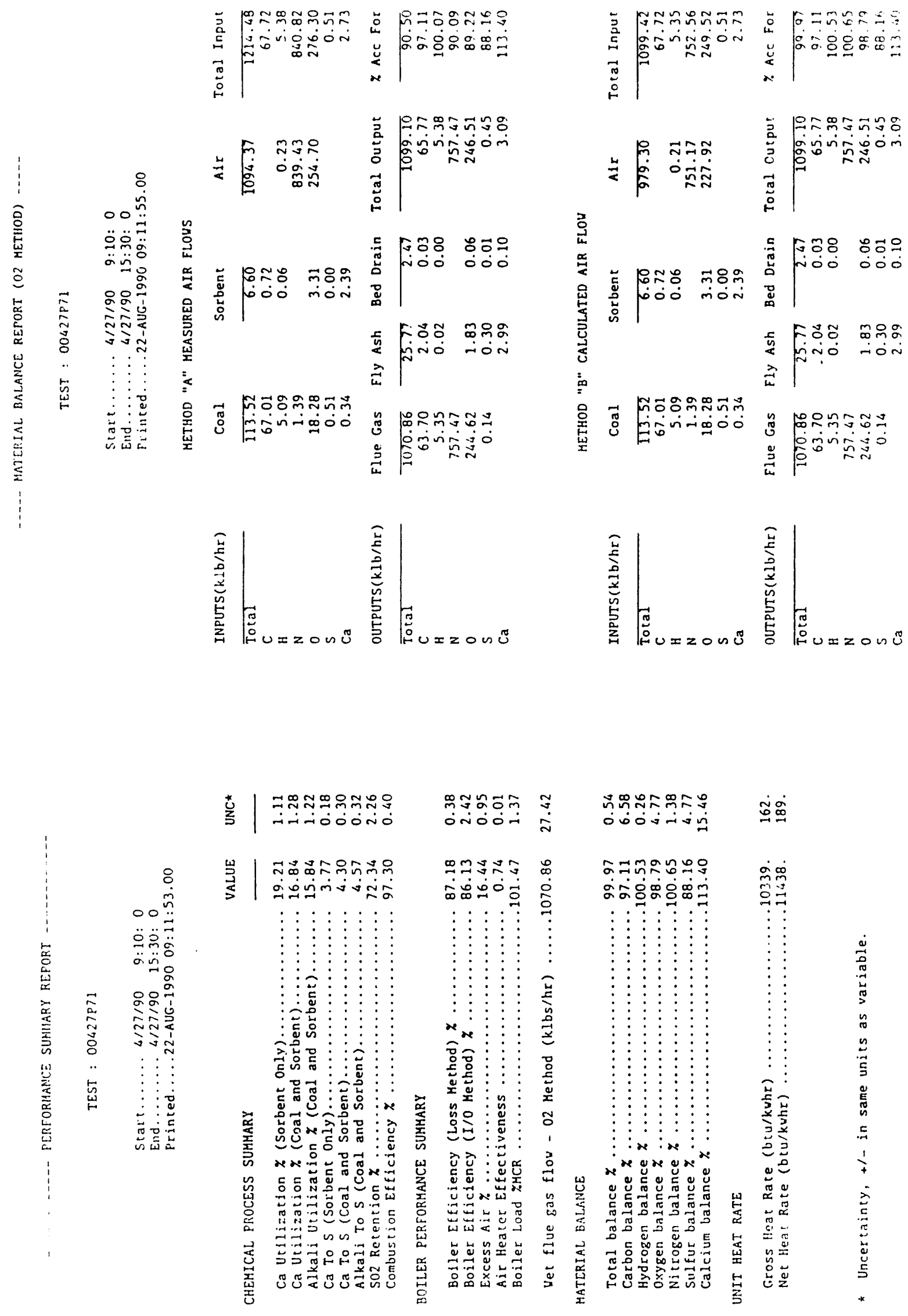



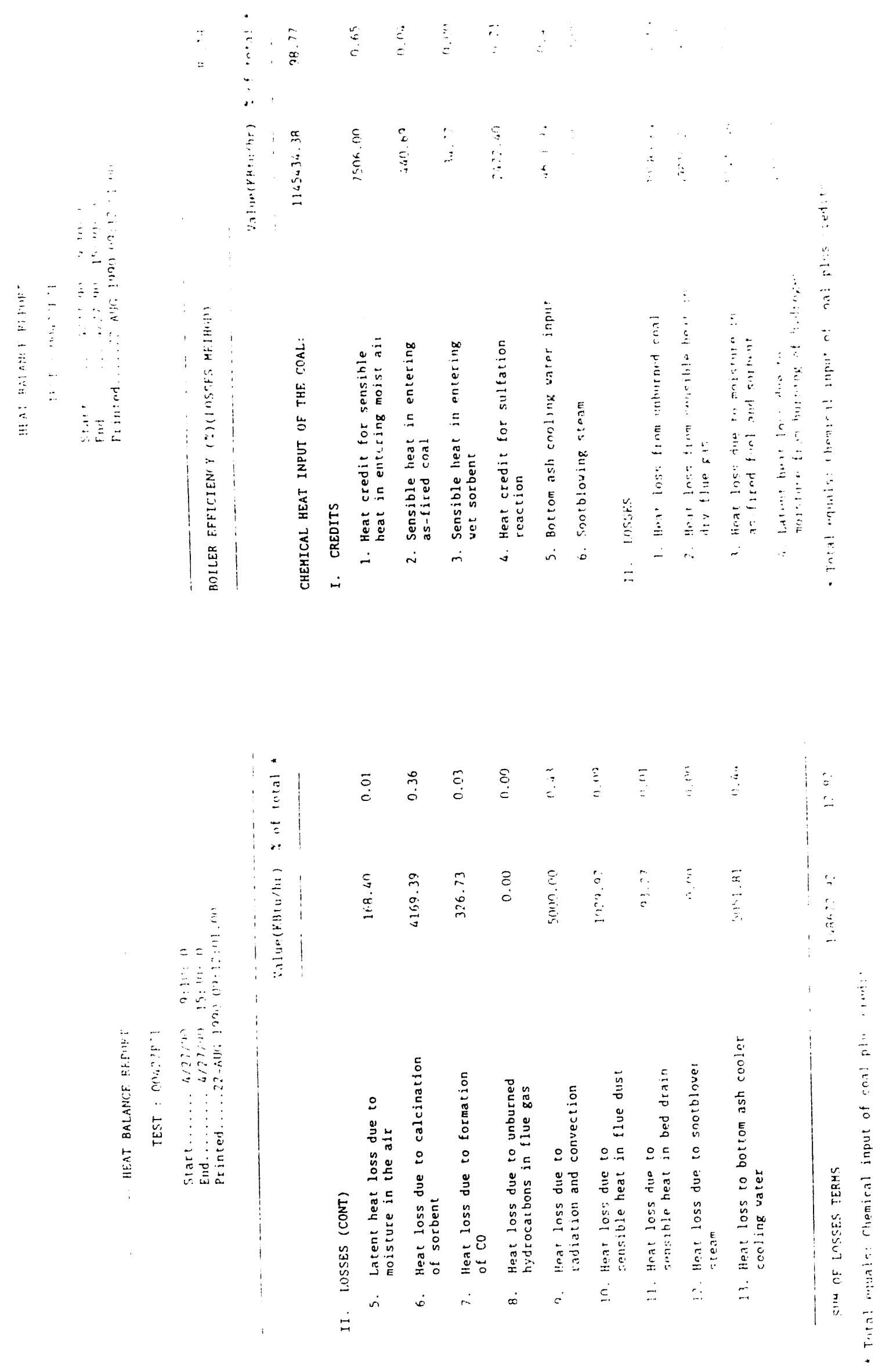

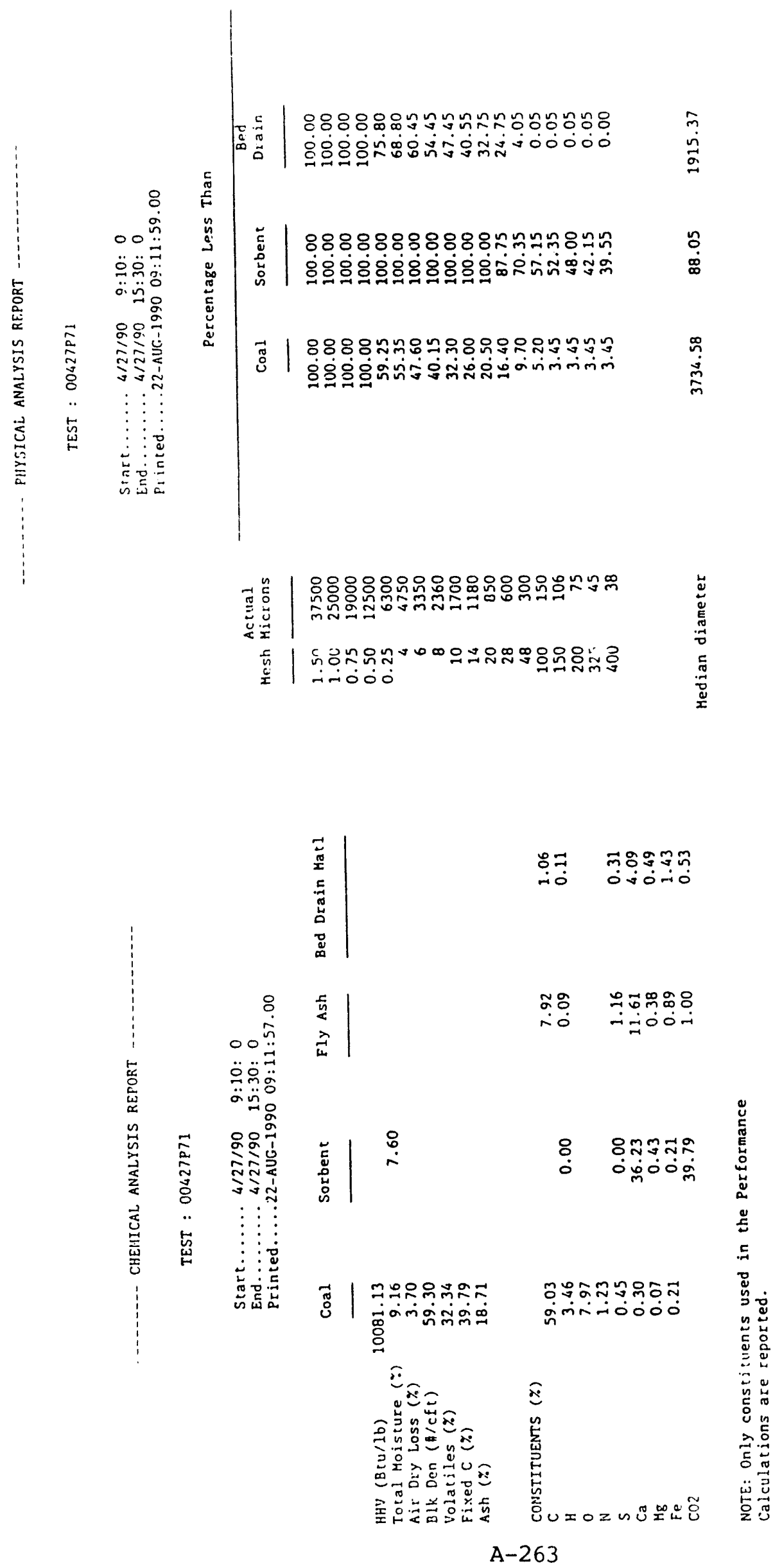

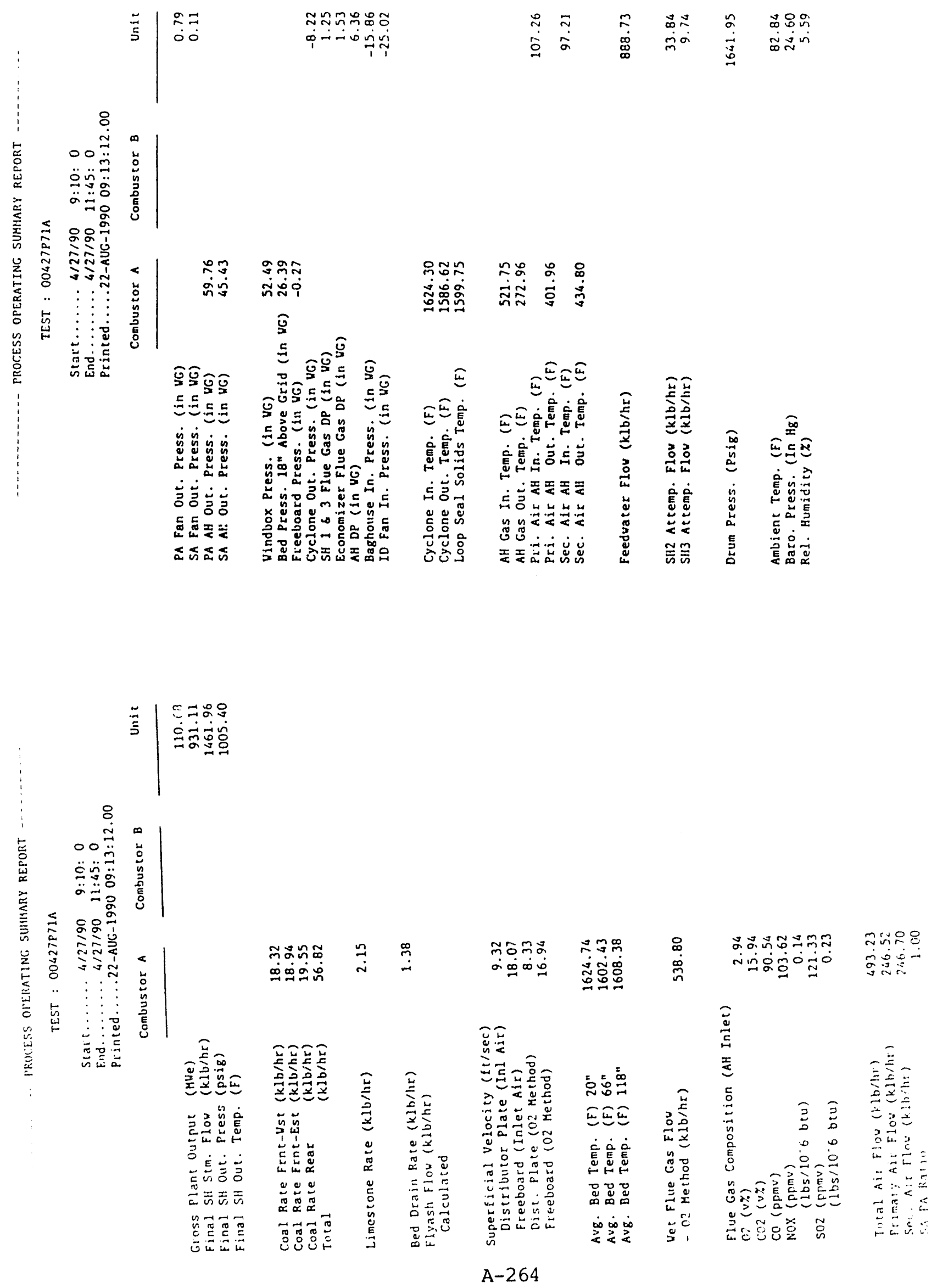


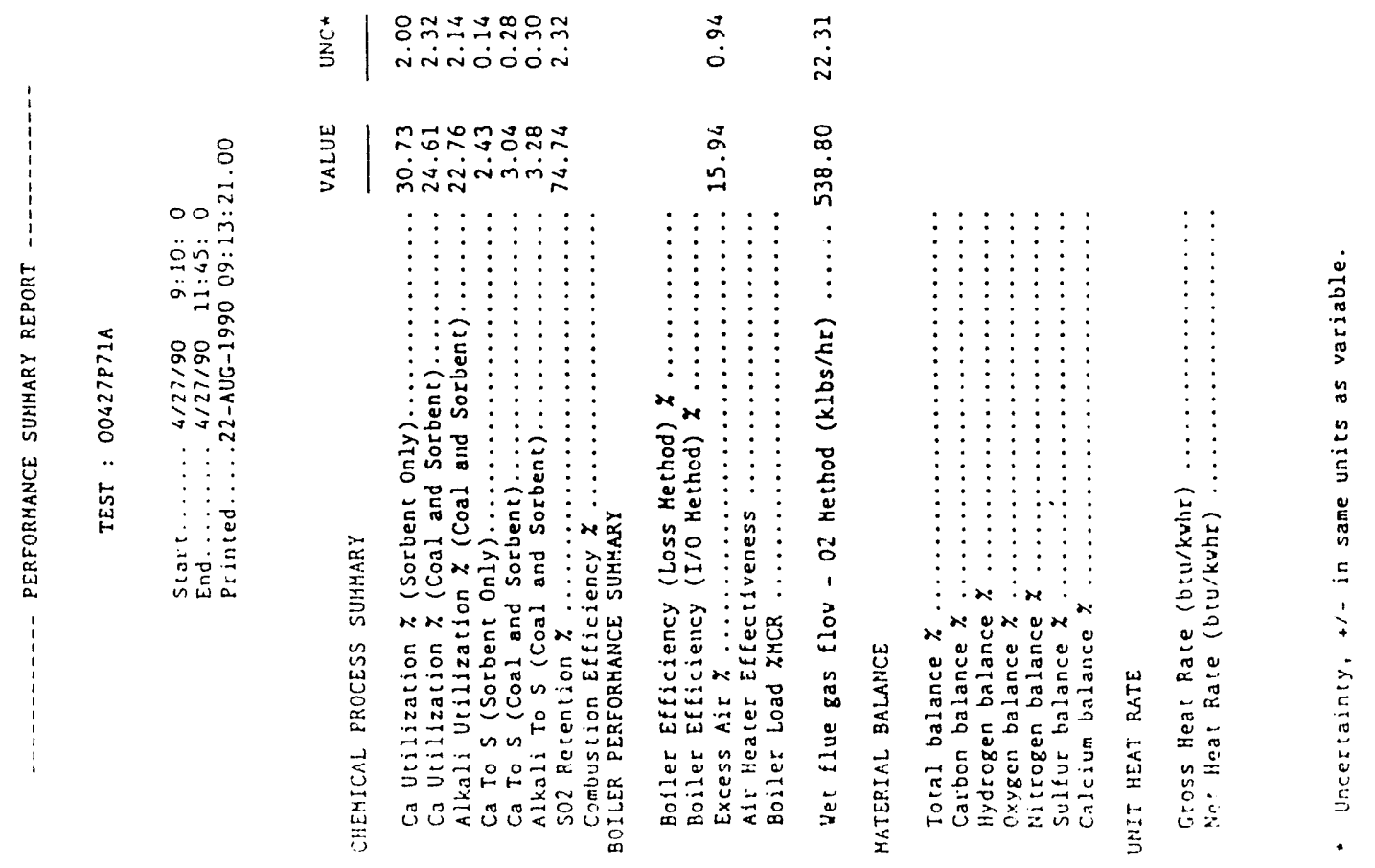

A-265 

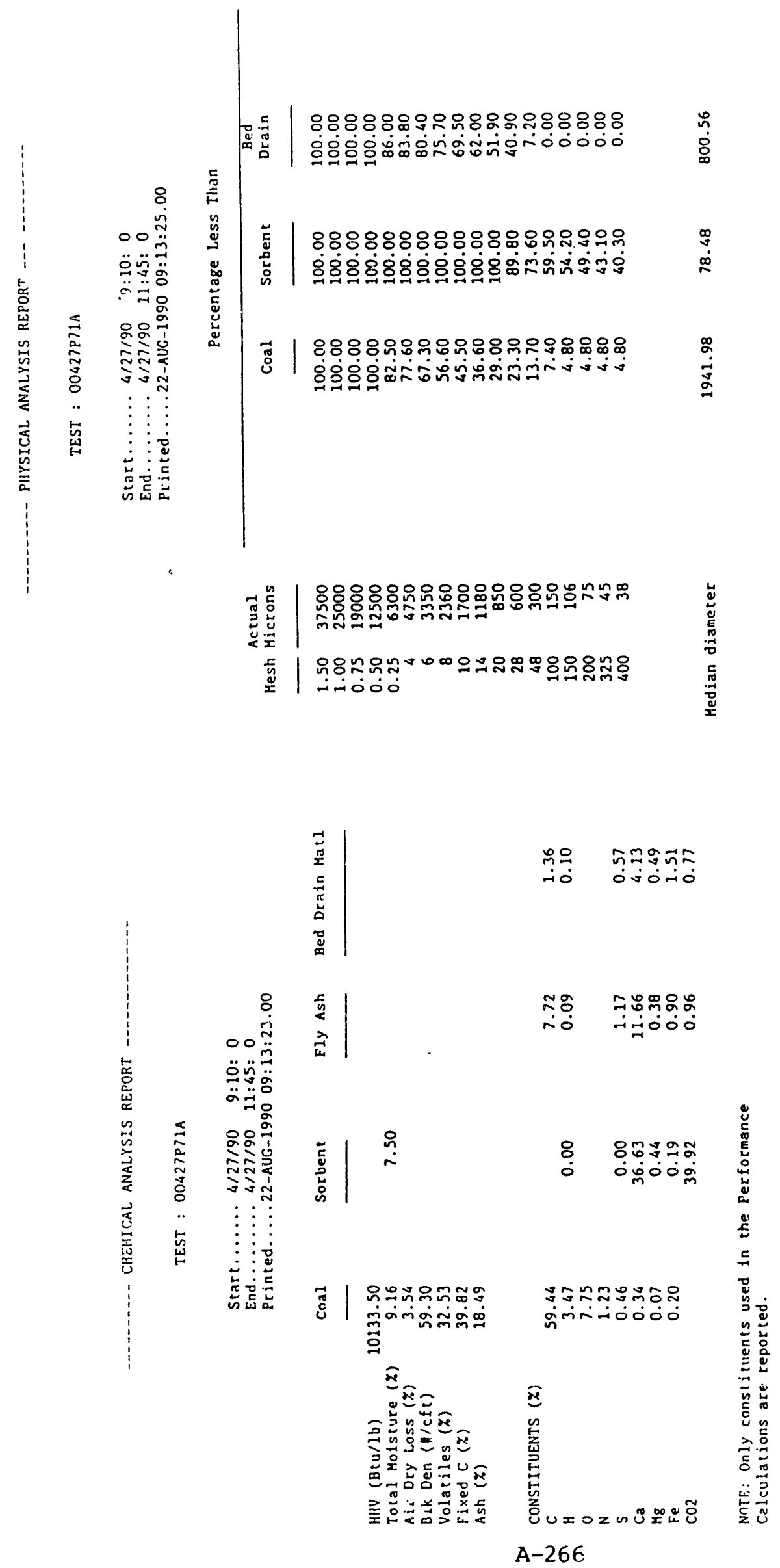

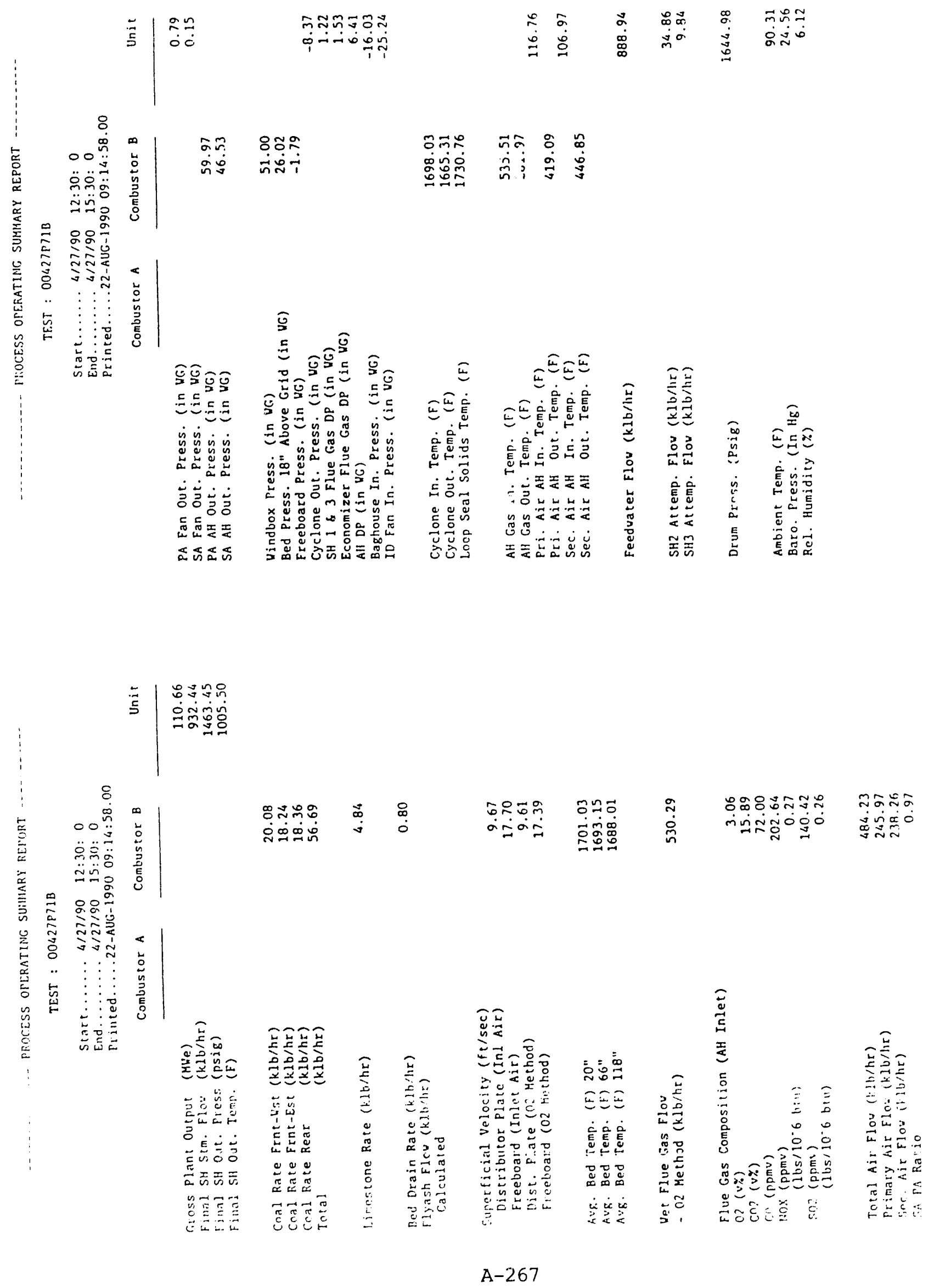


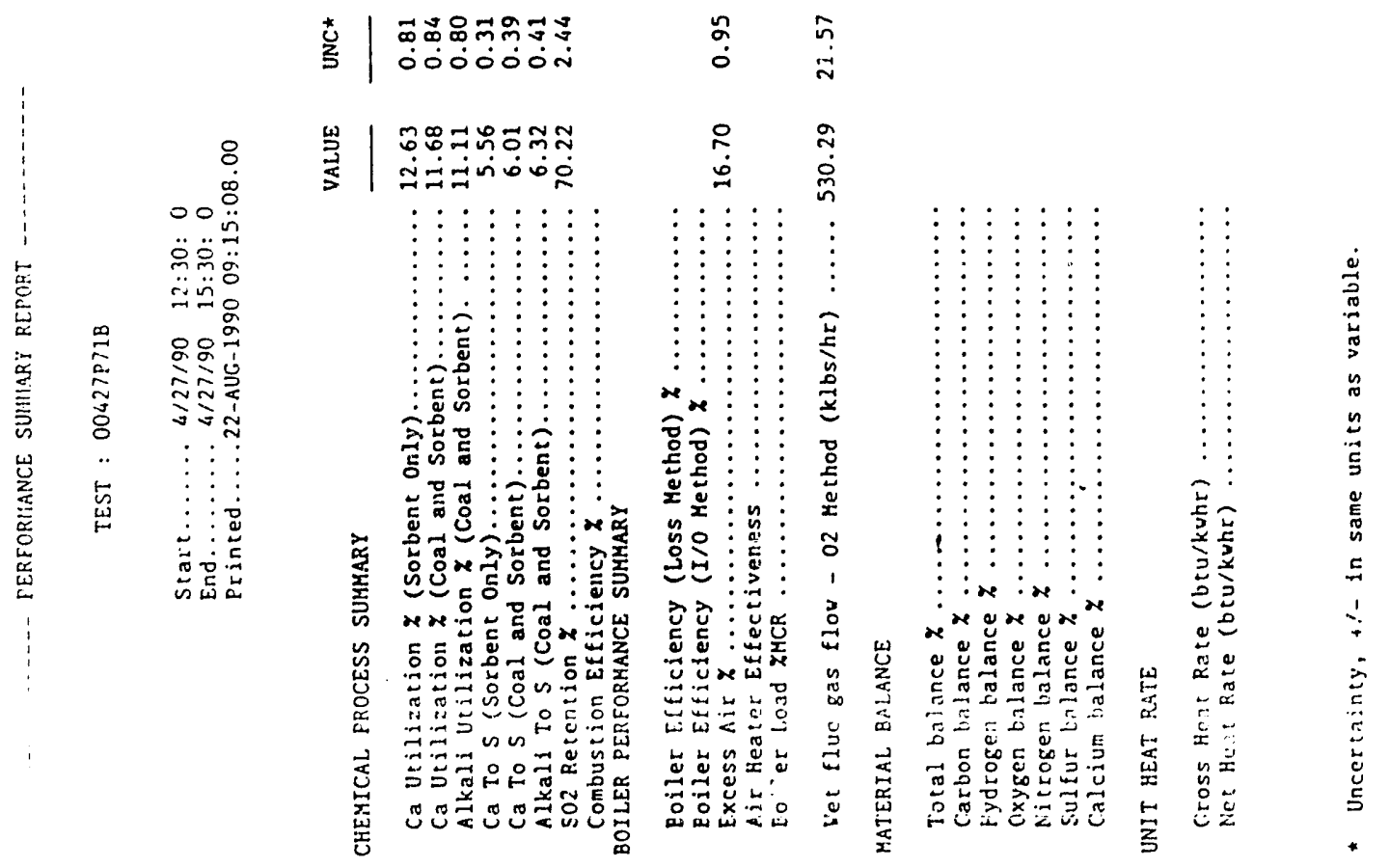



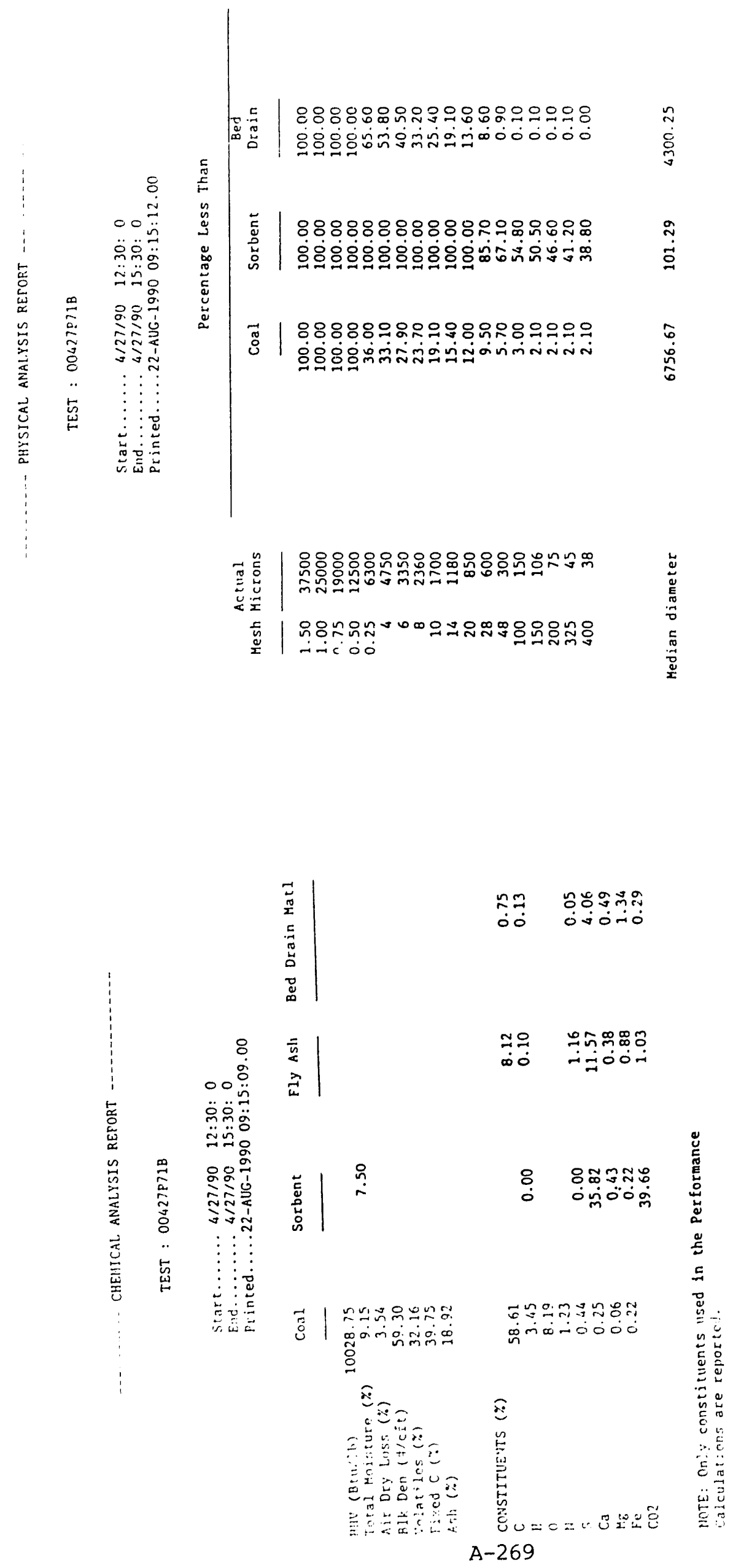


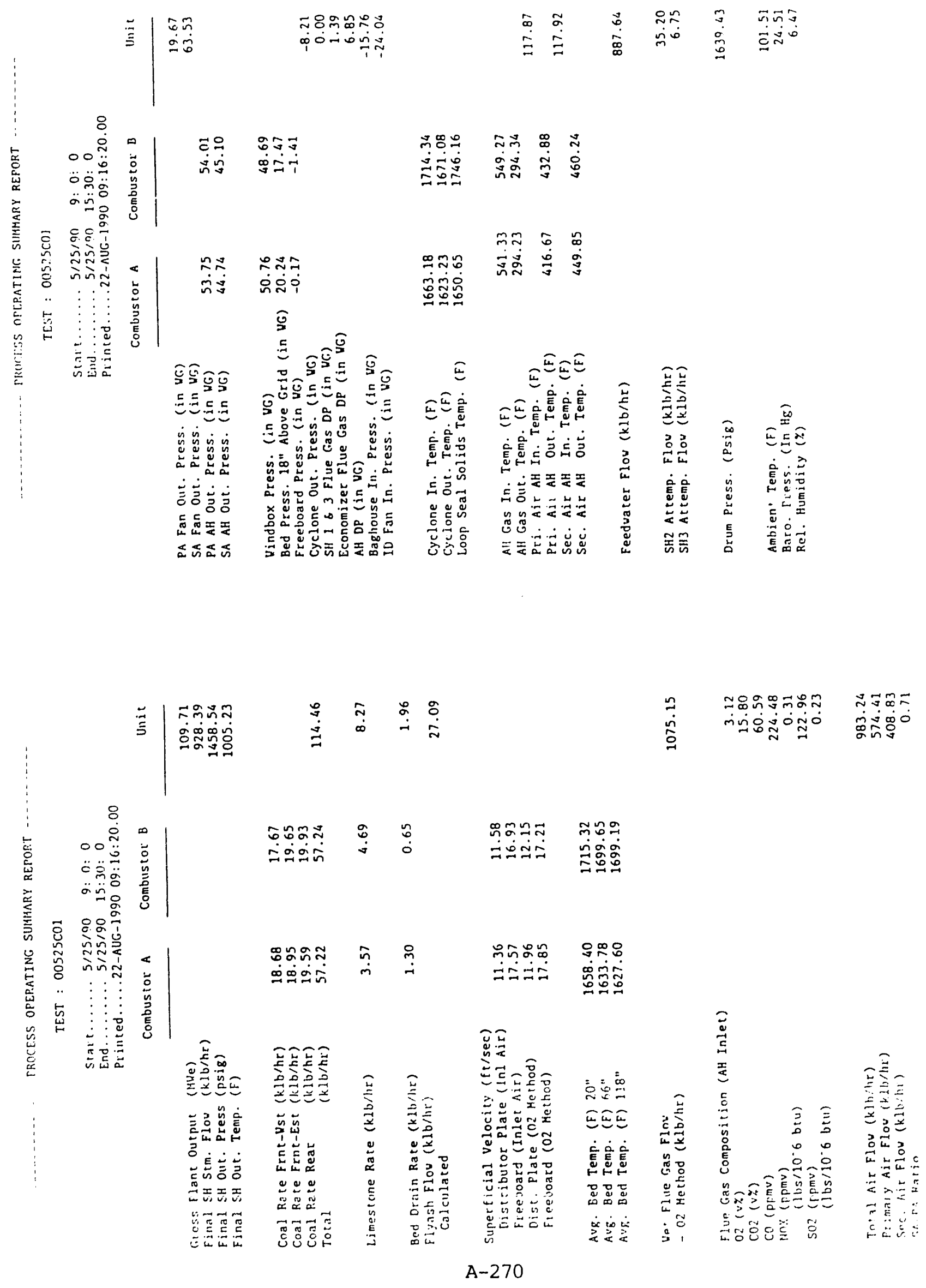




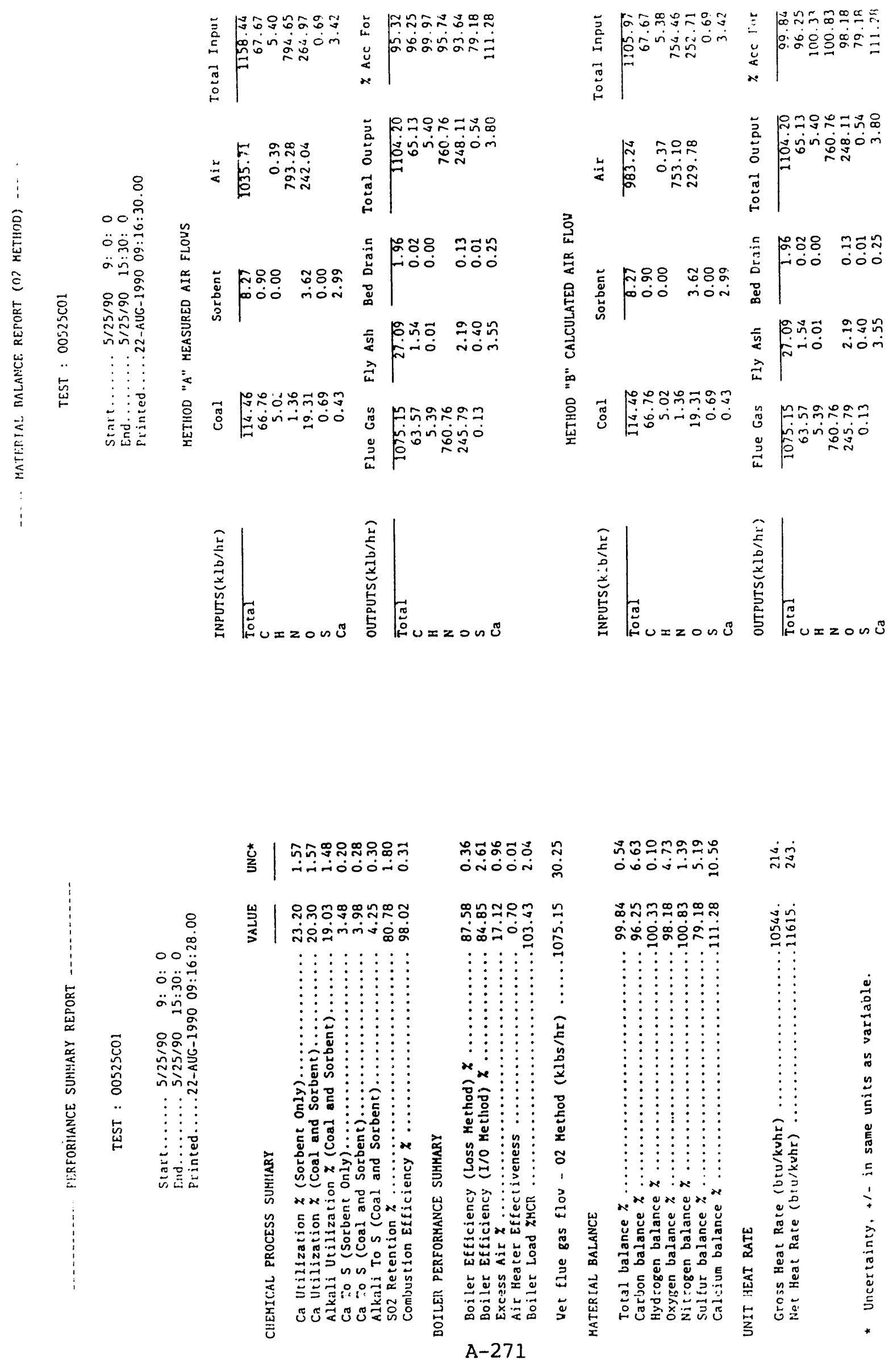




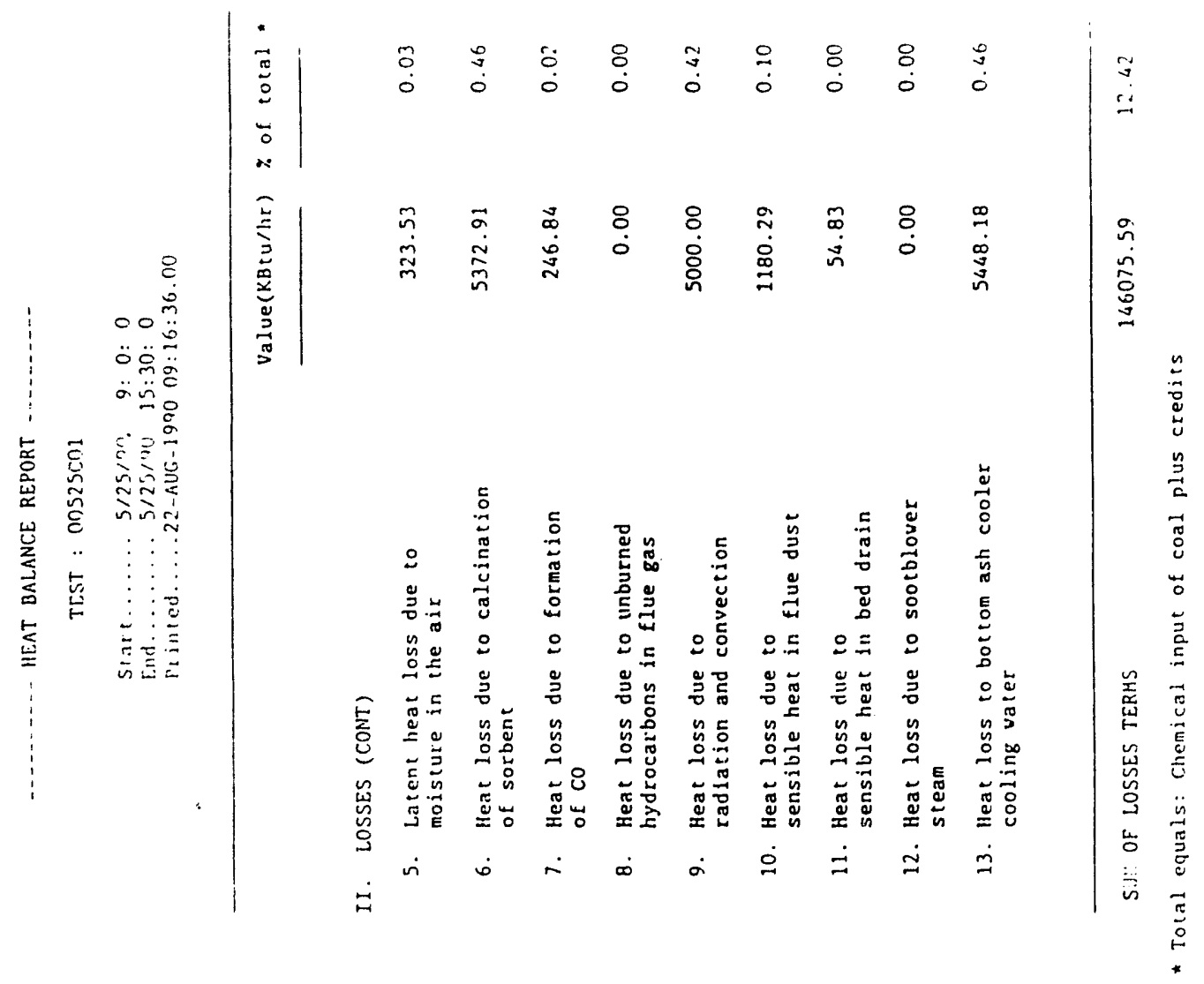

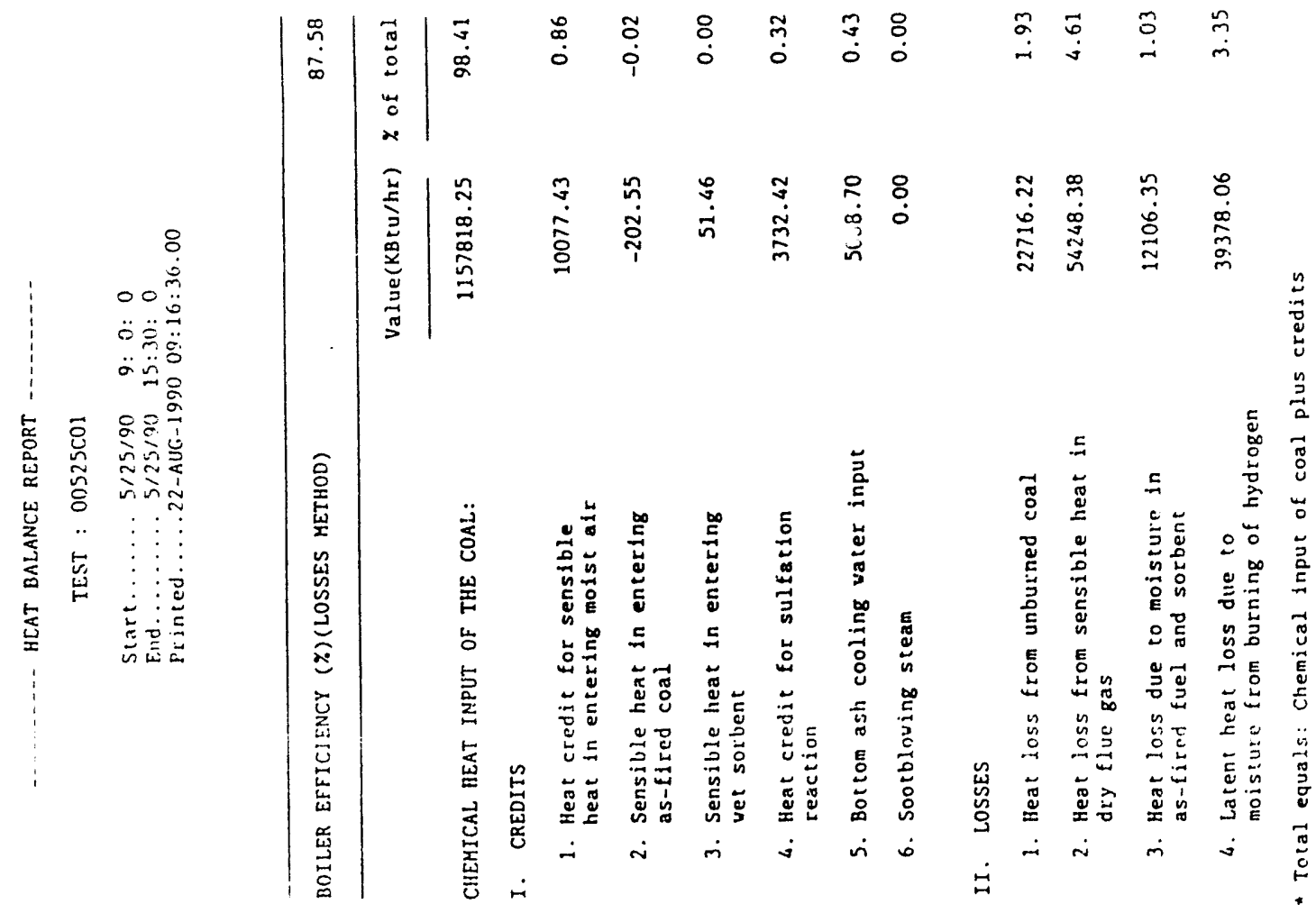



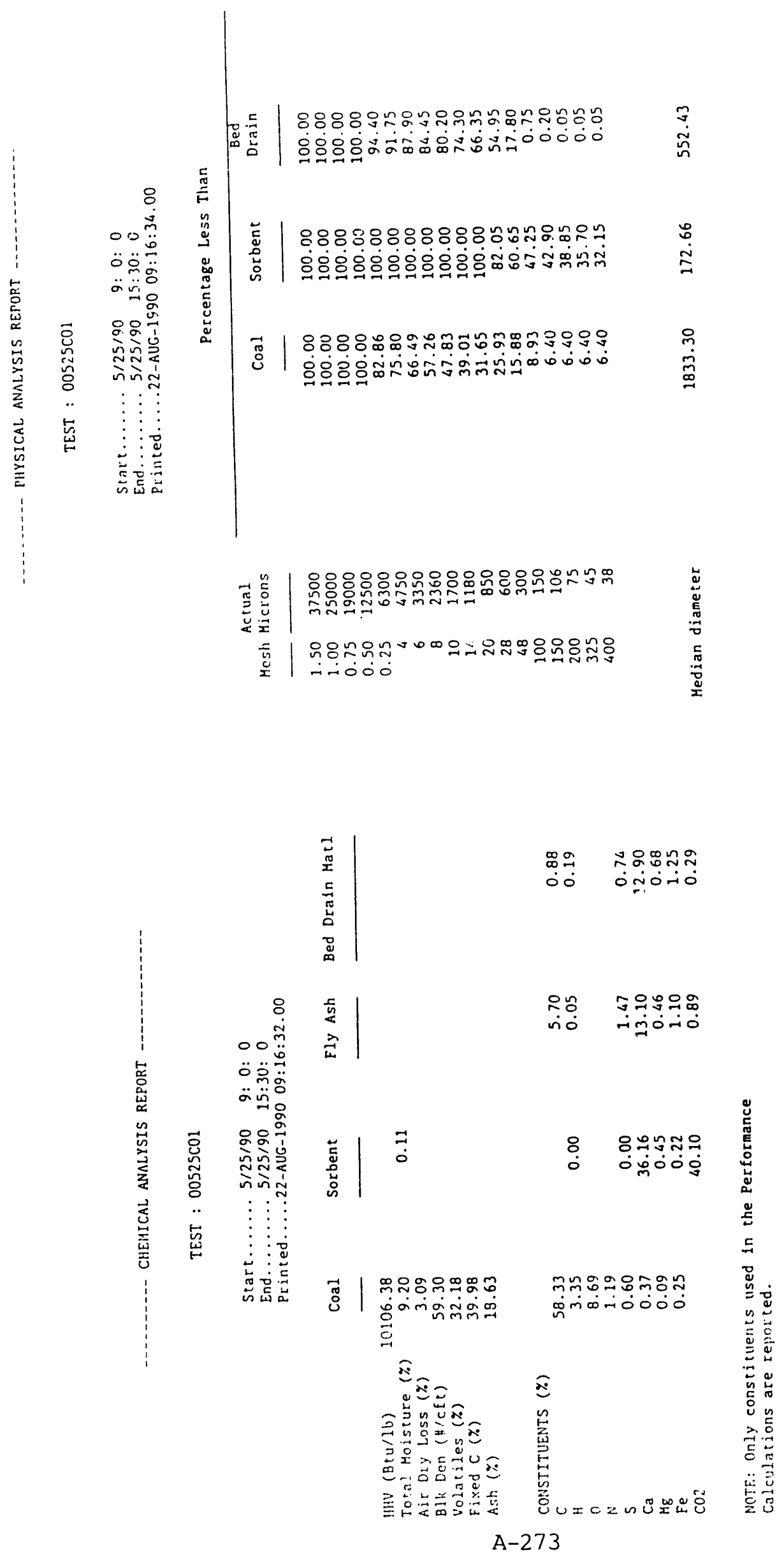

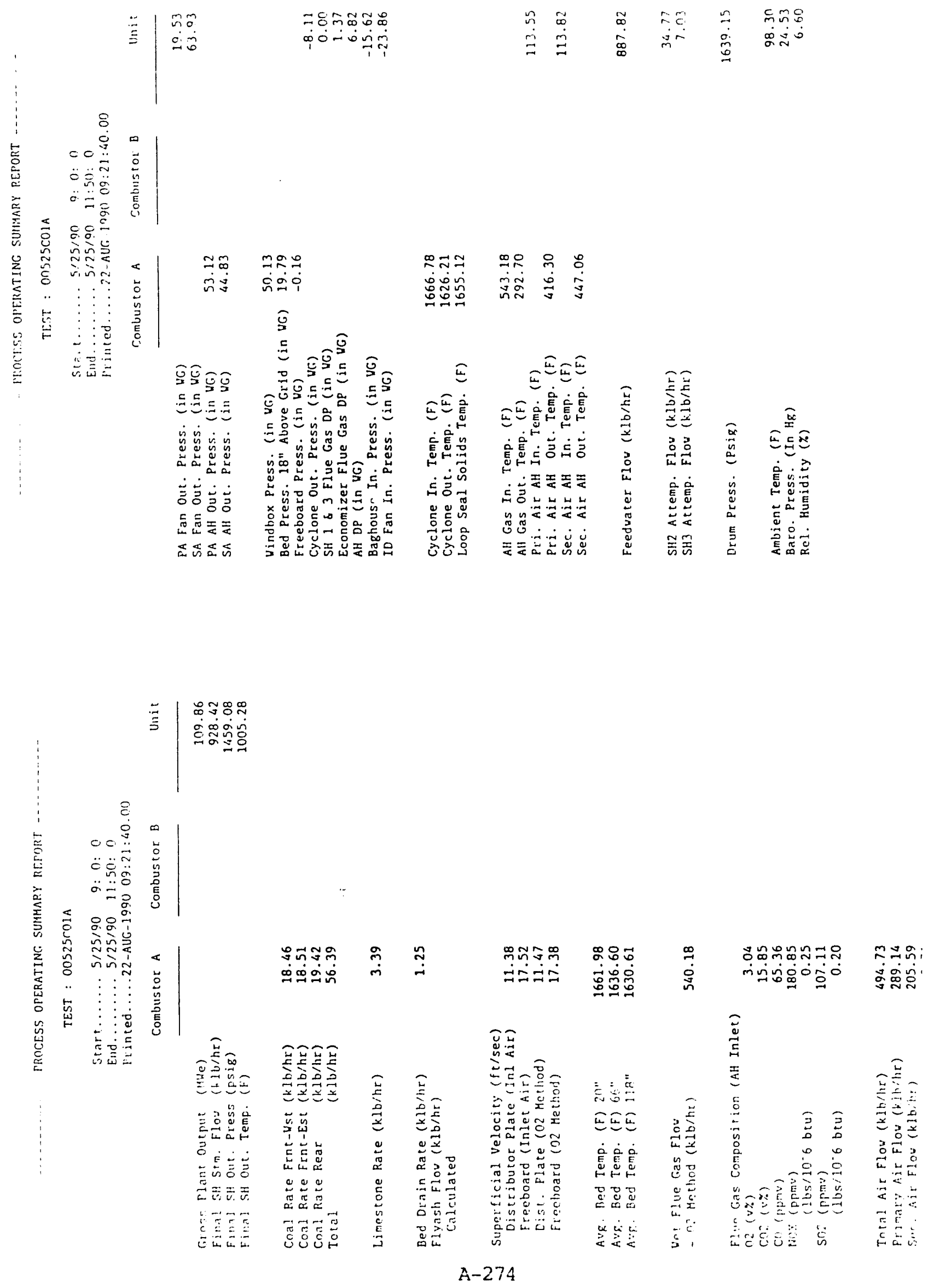


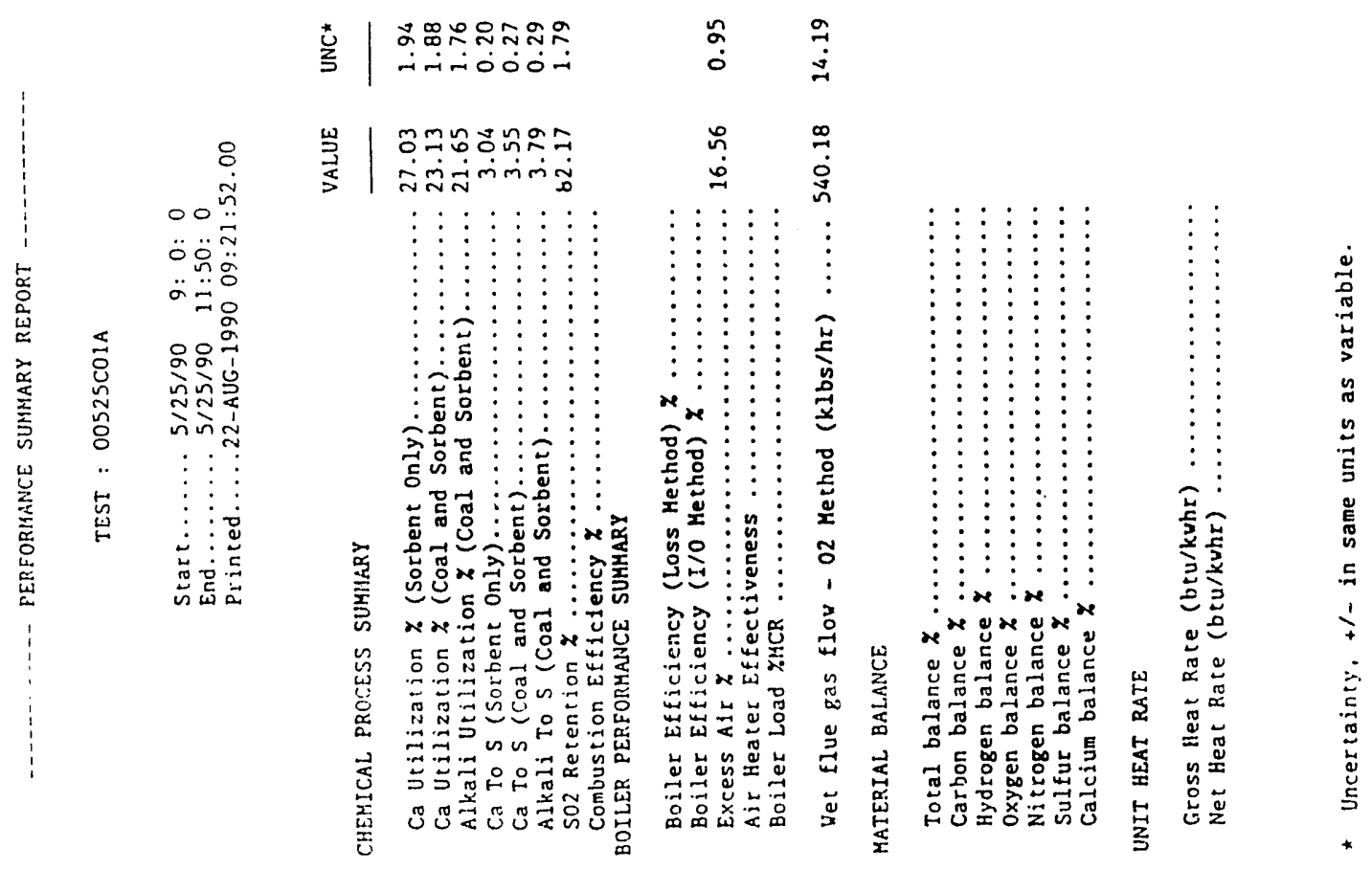



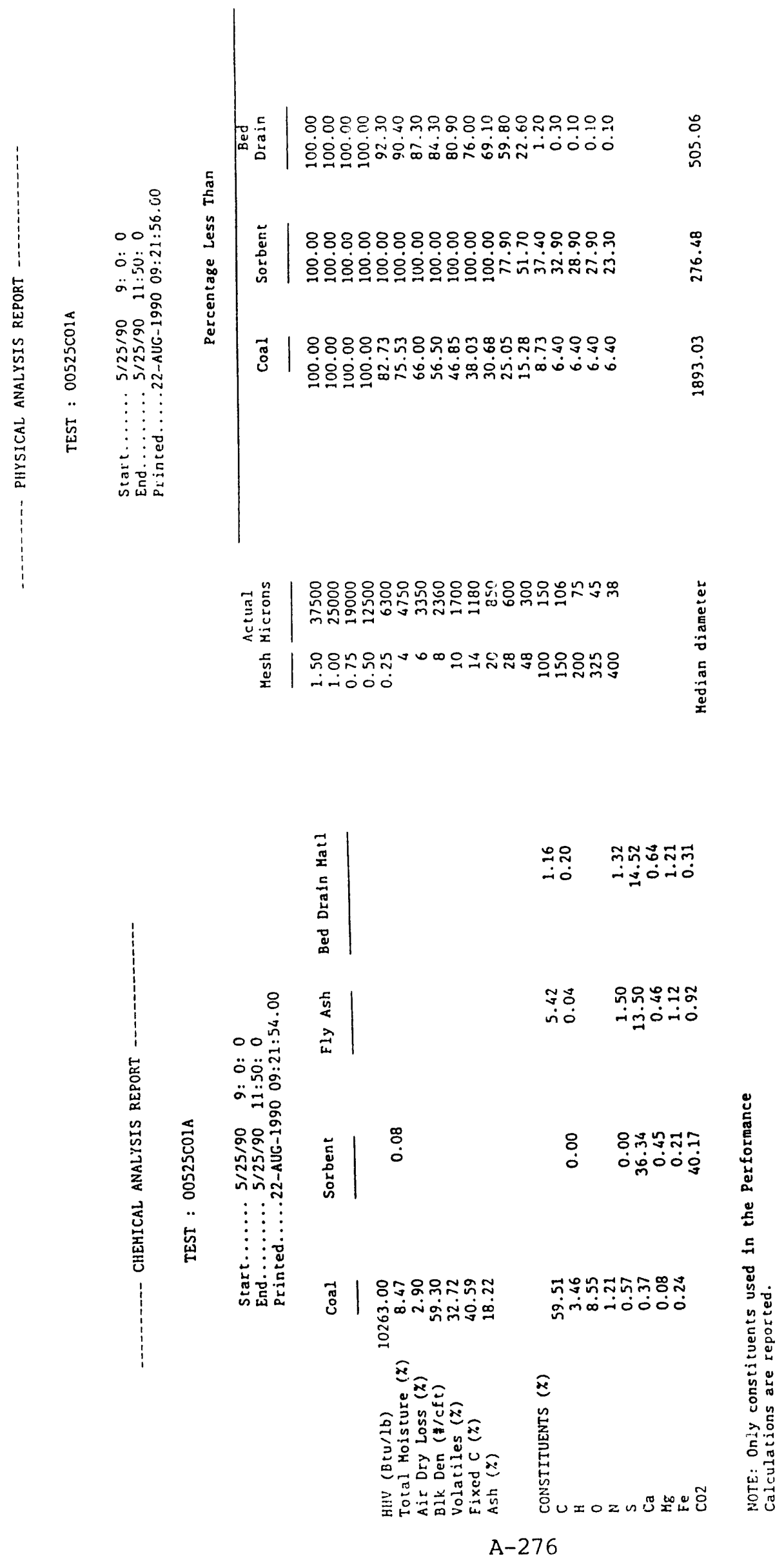

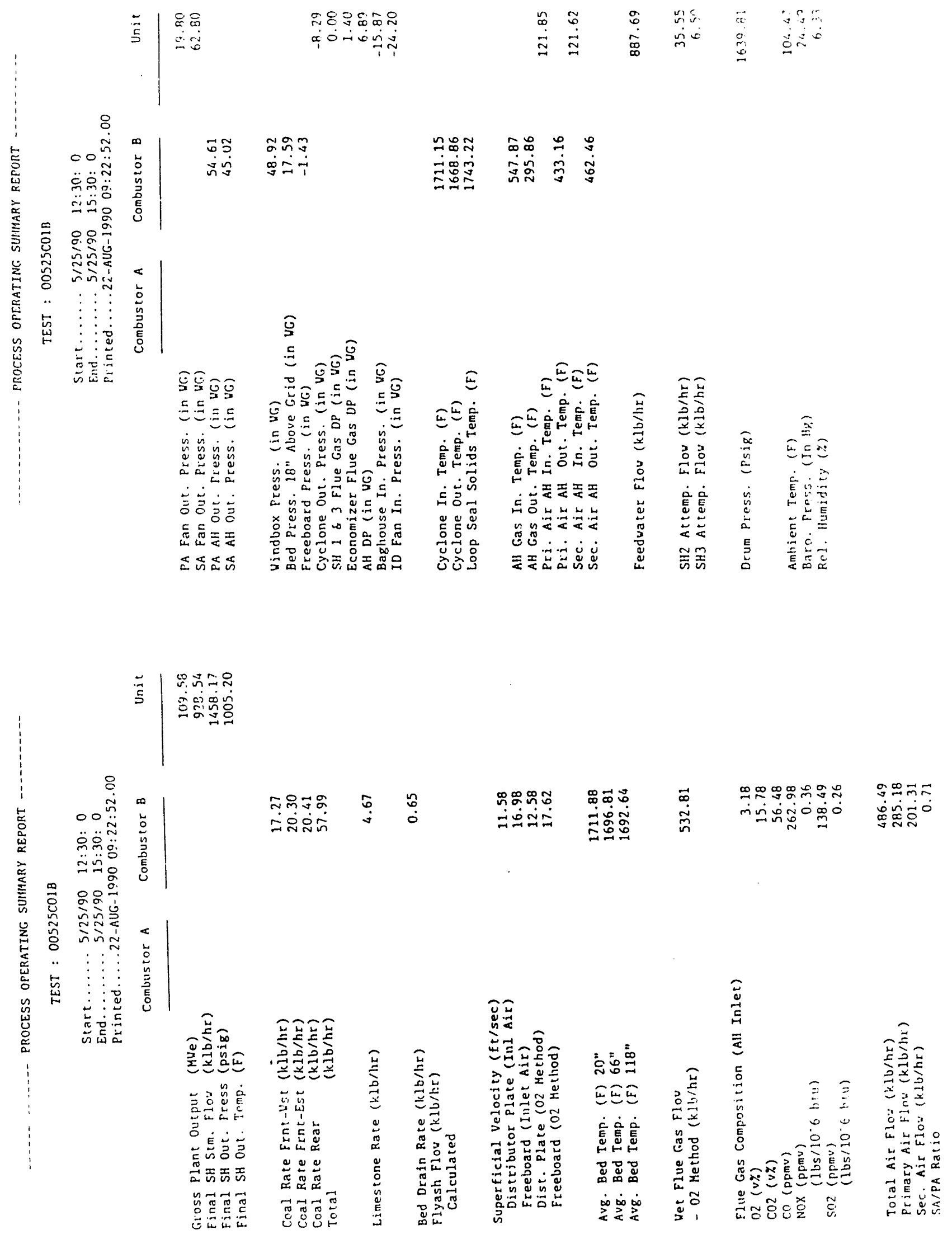


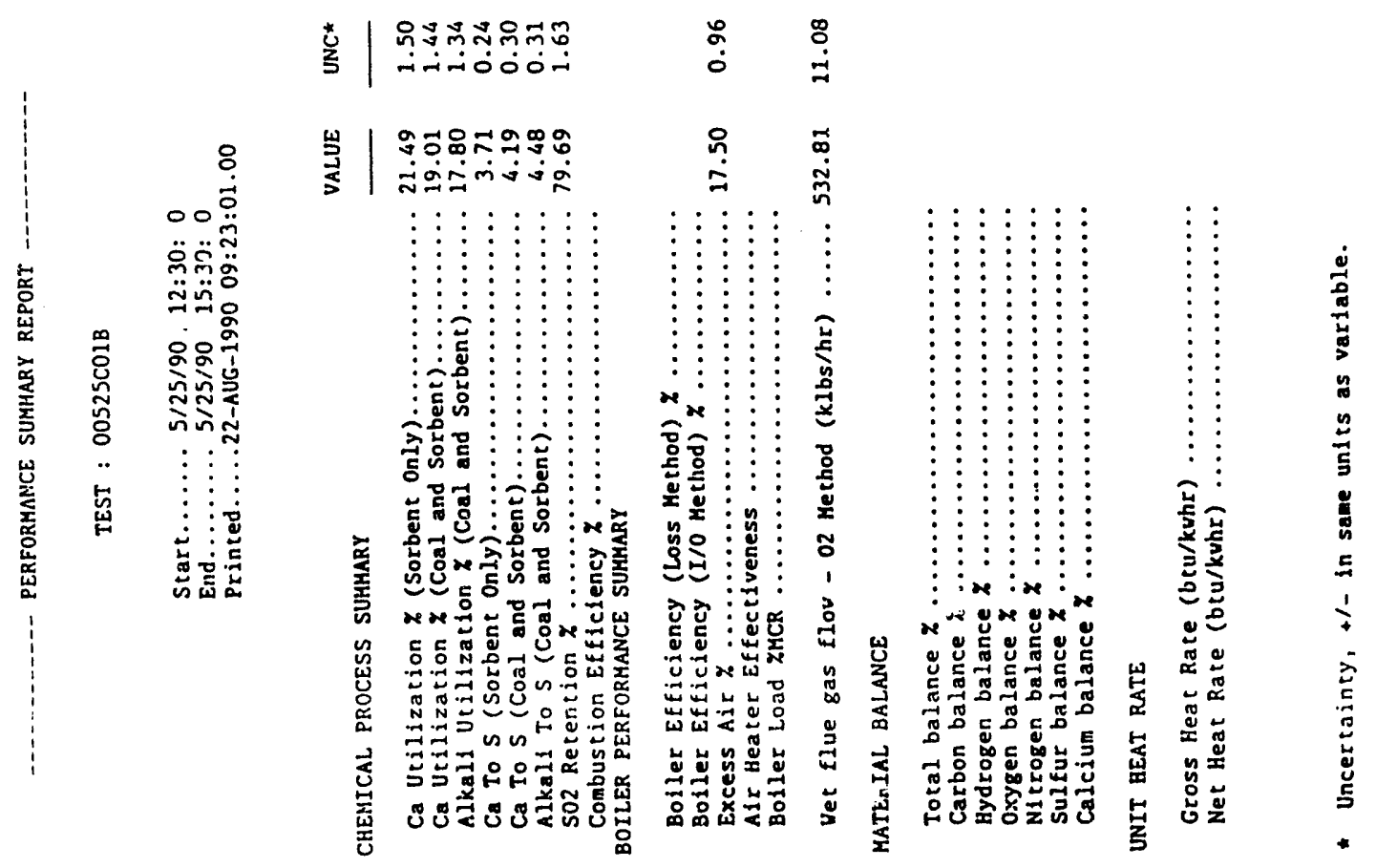




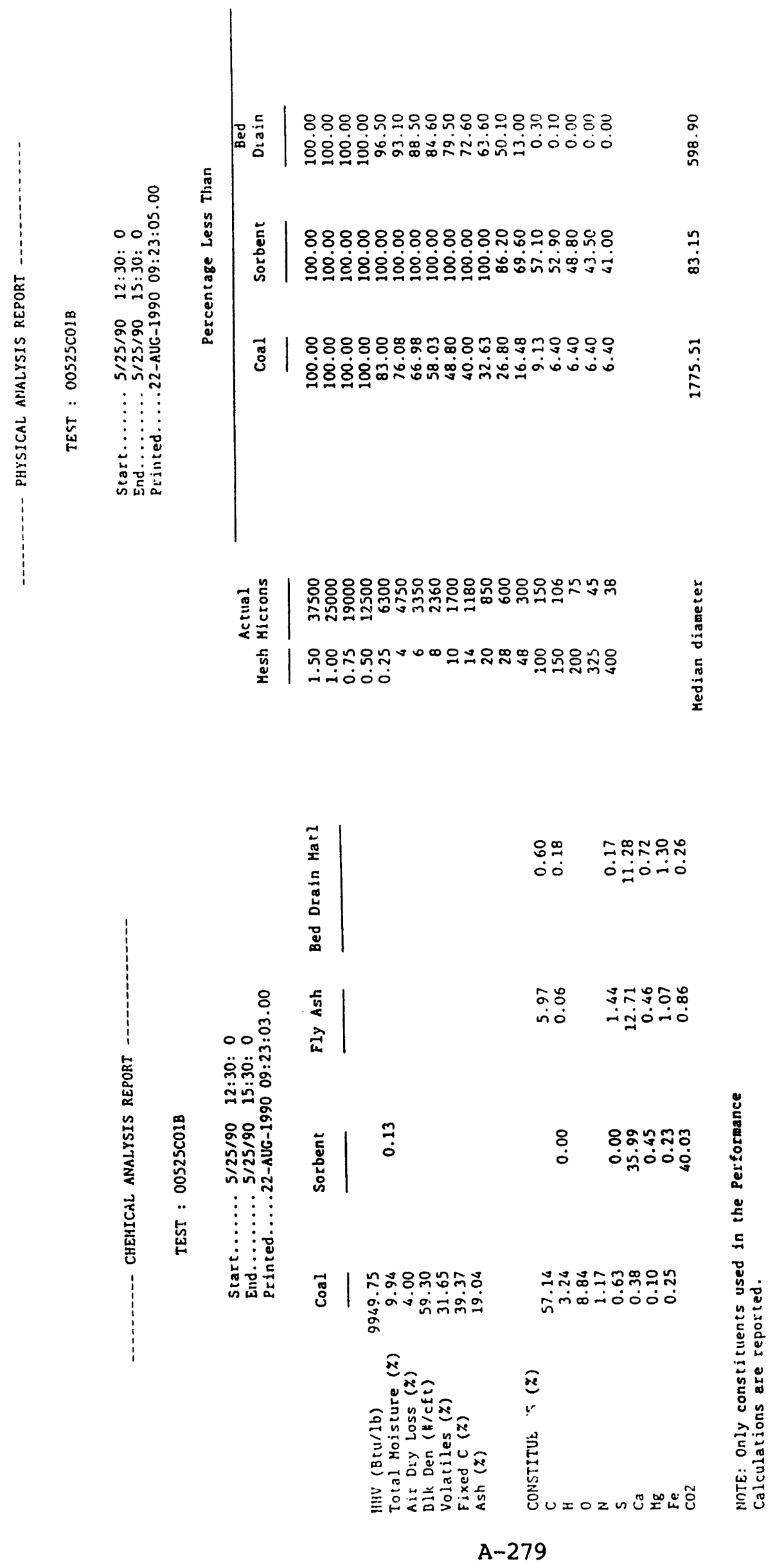



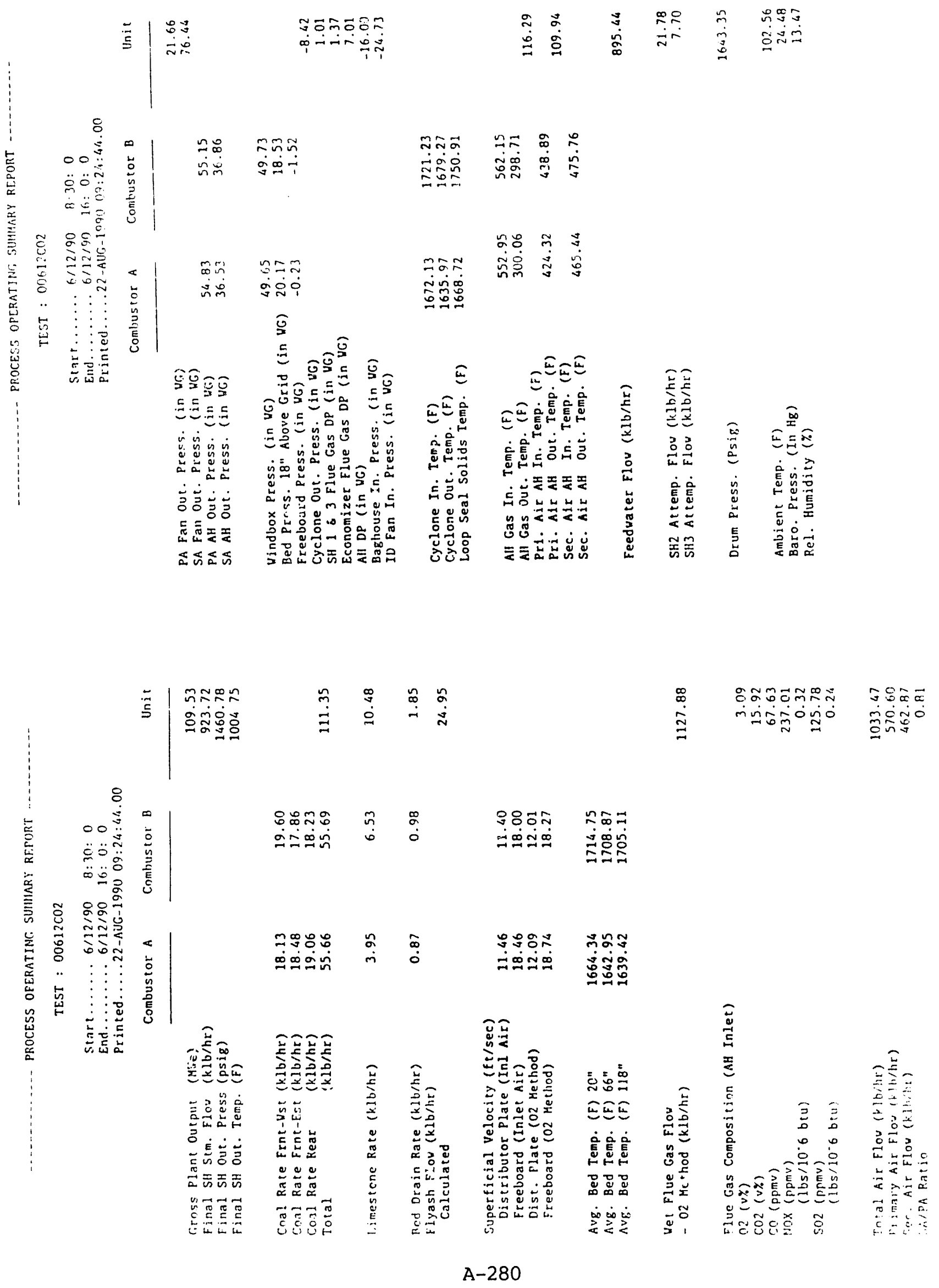


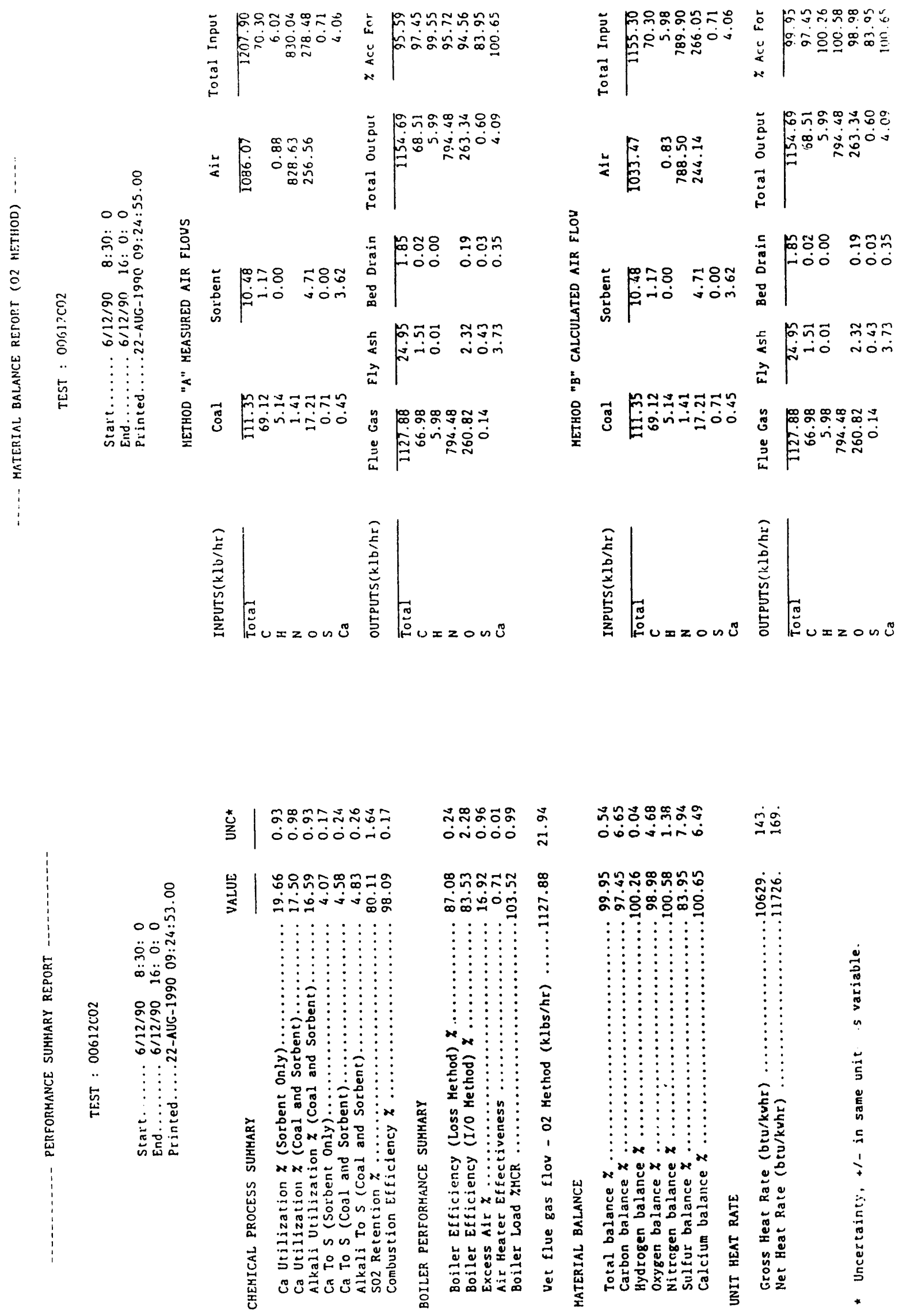

A-281 

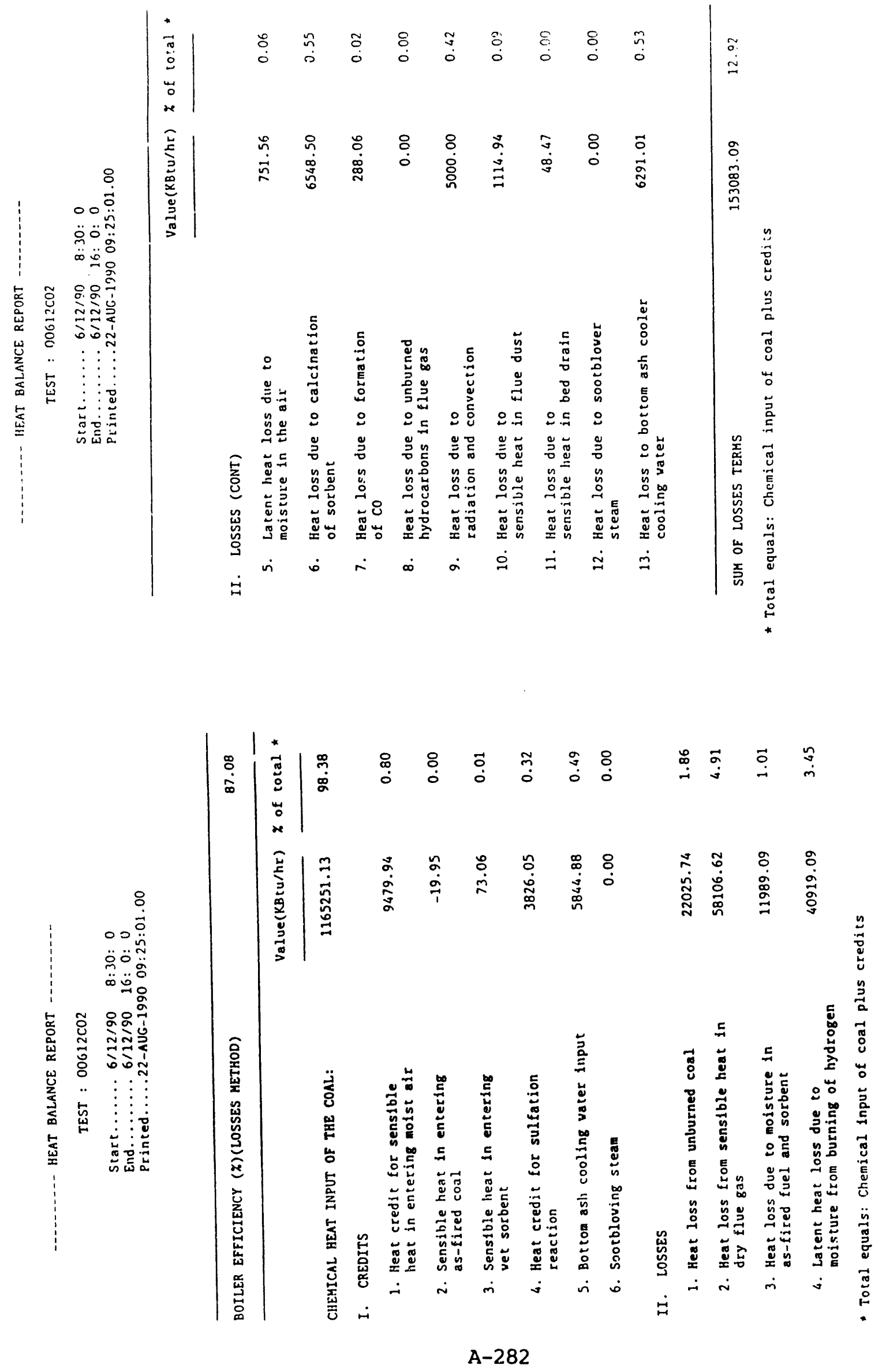

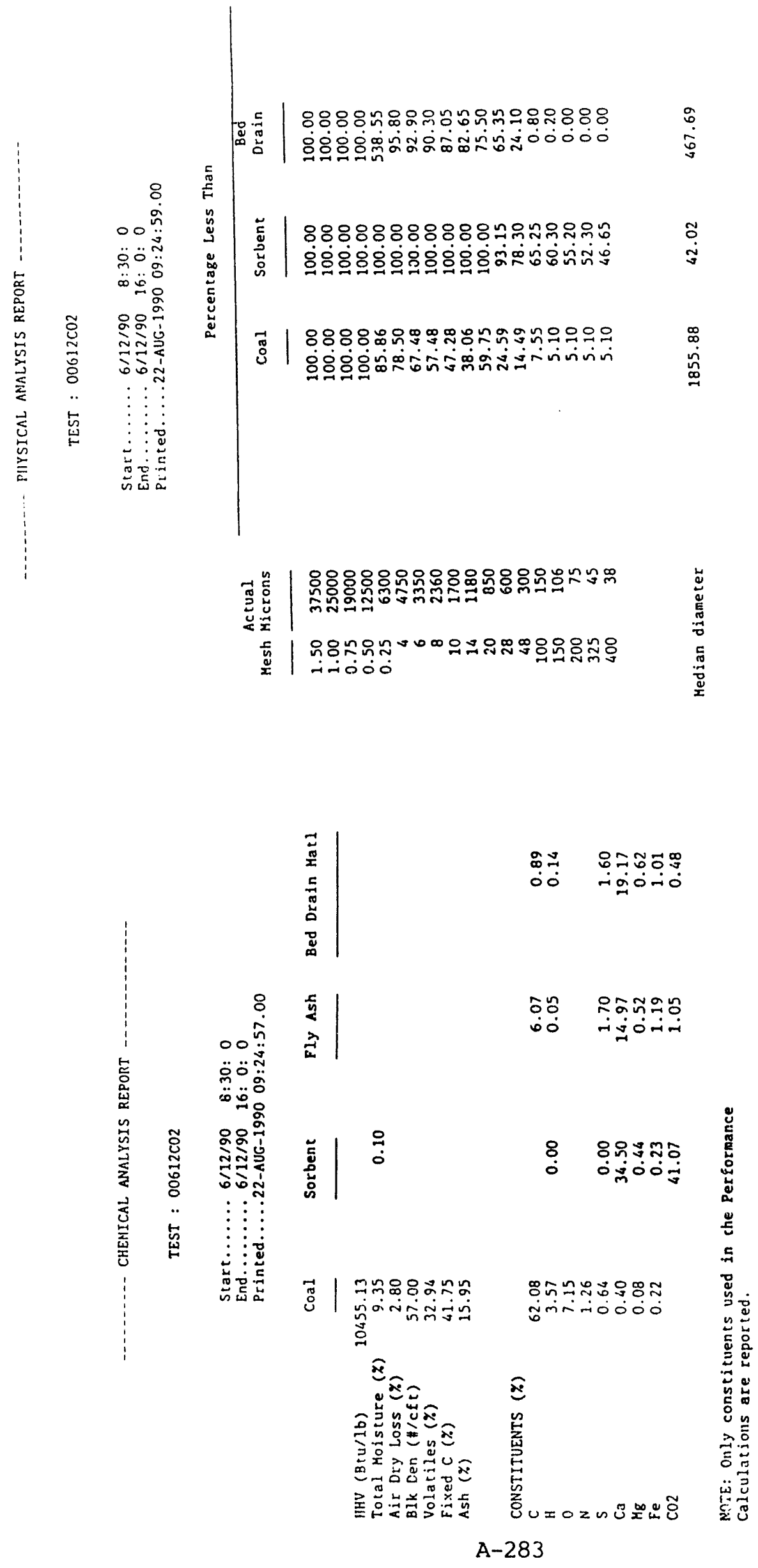

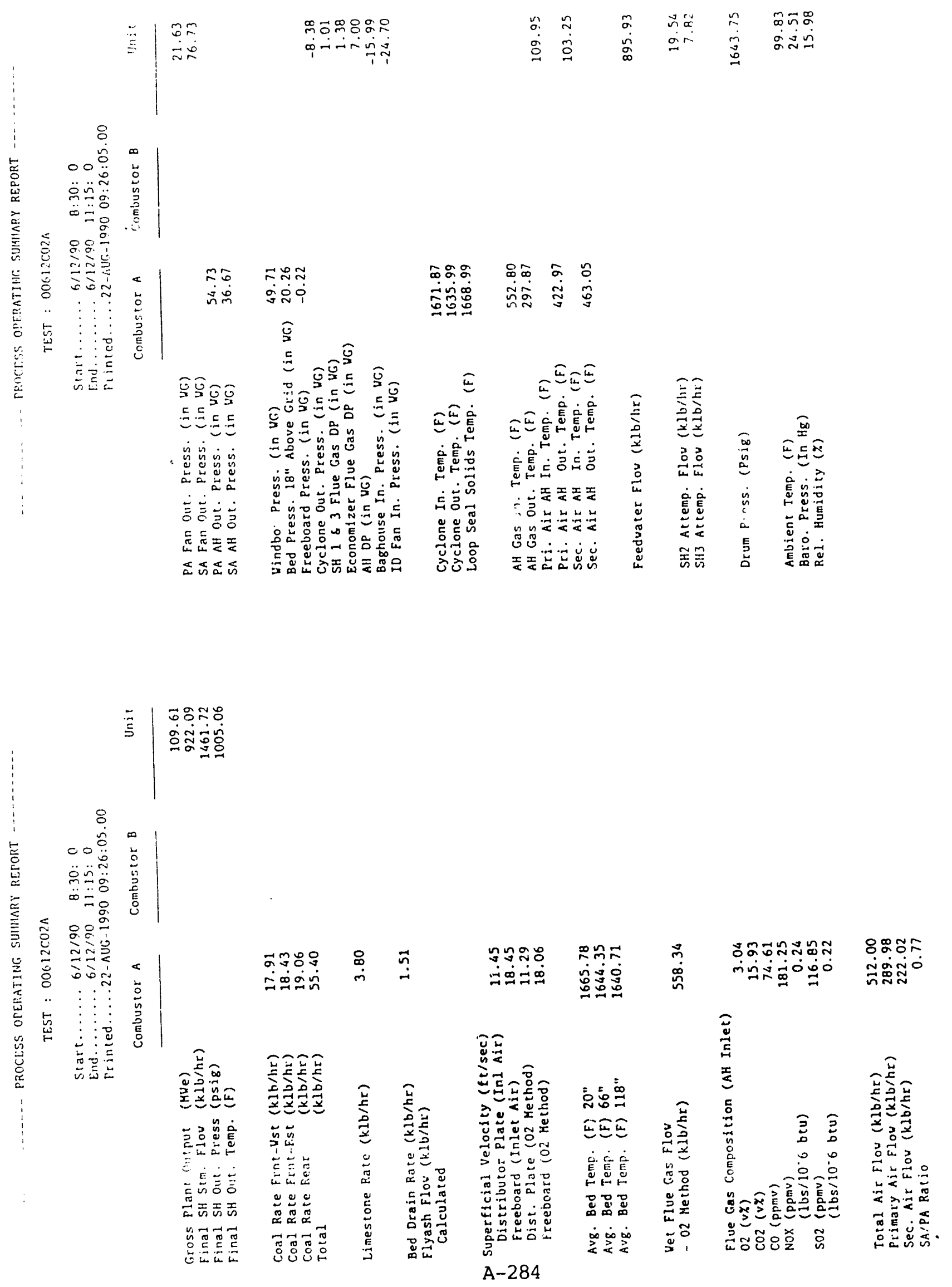


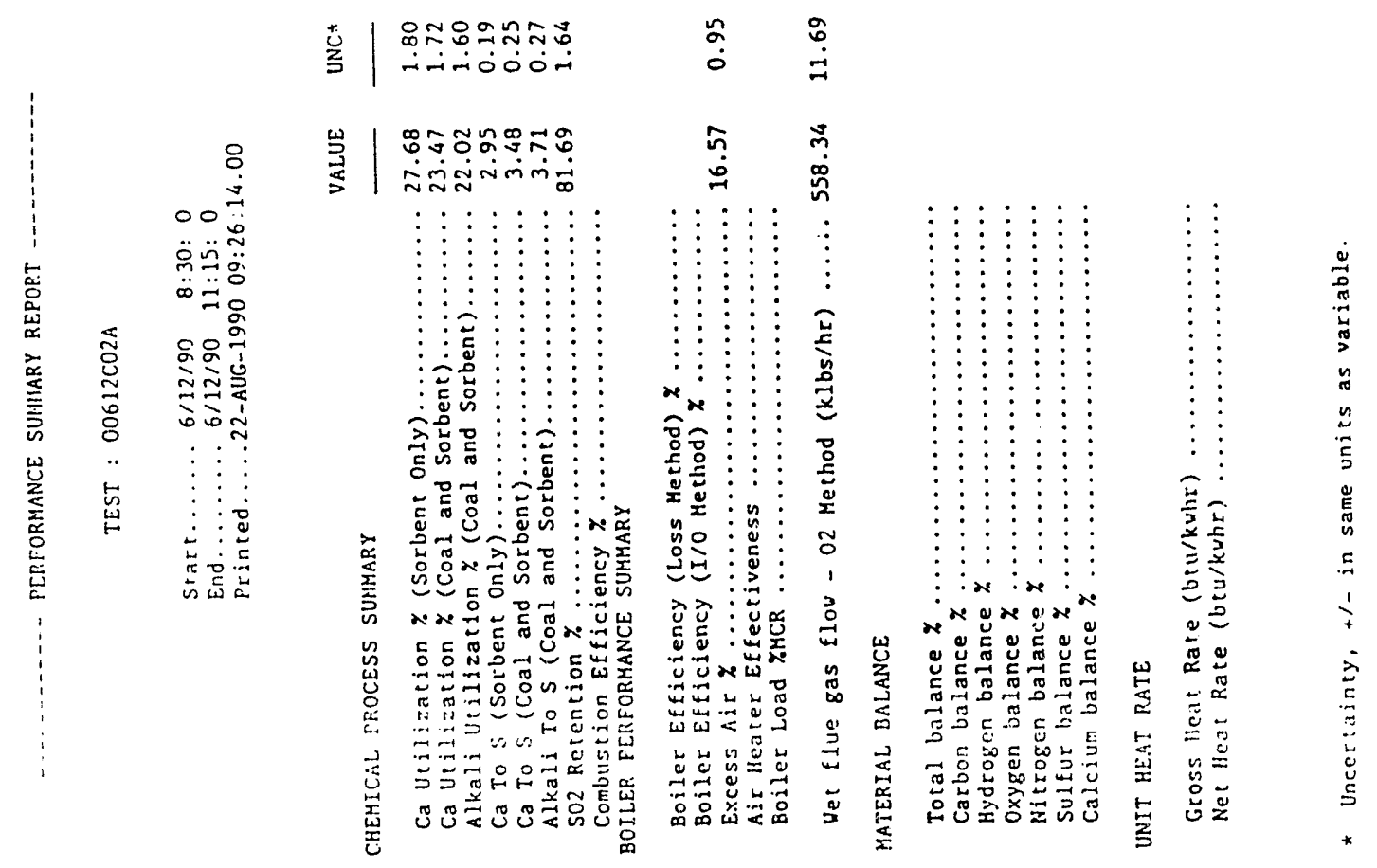

A-285 

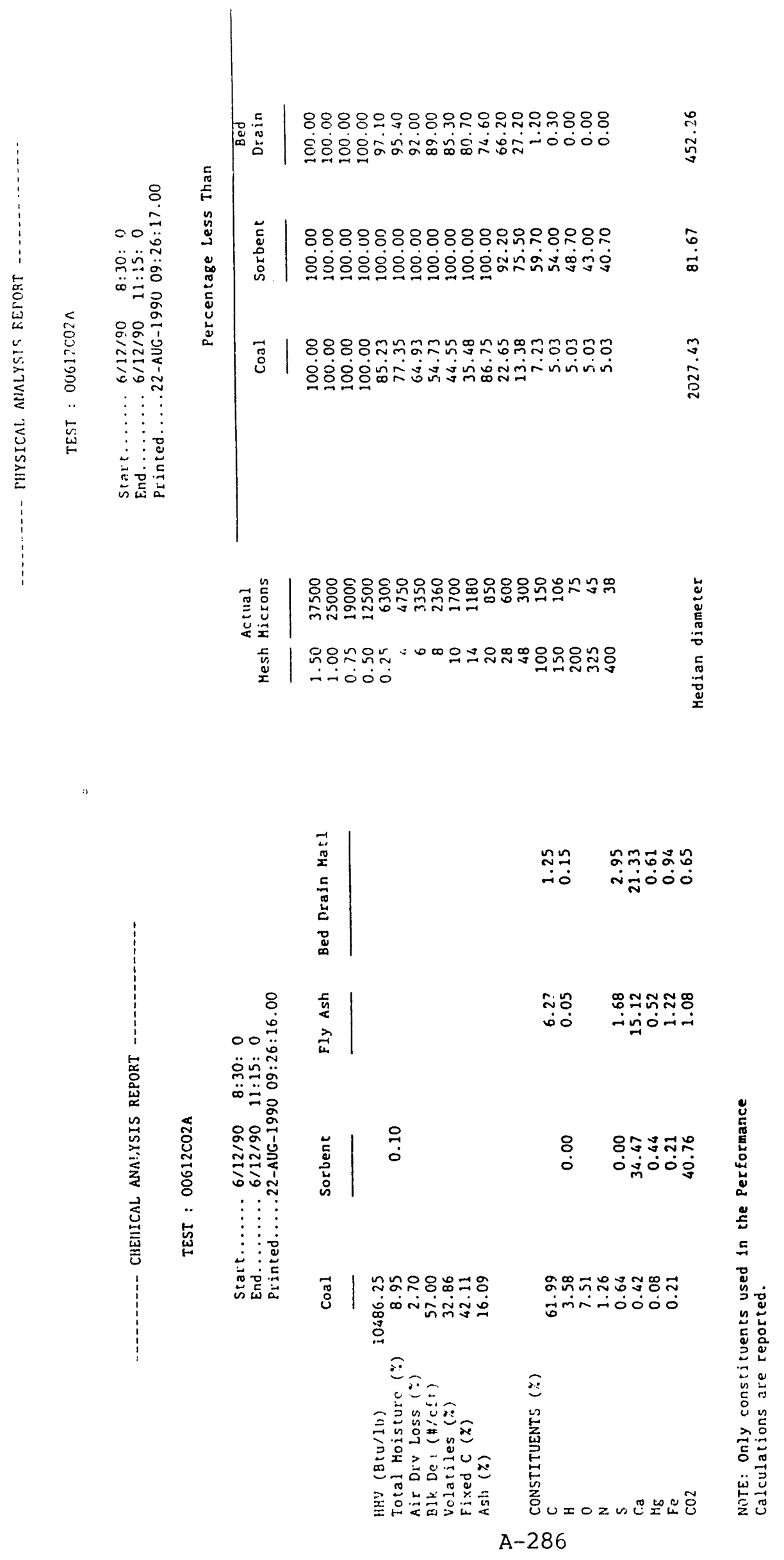

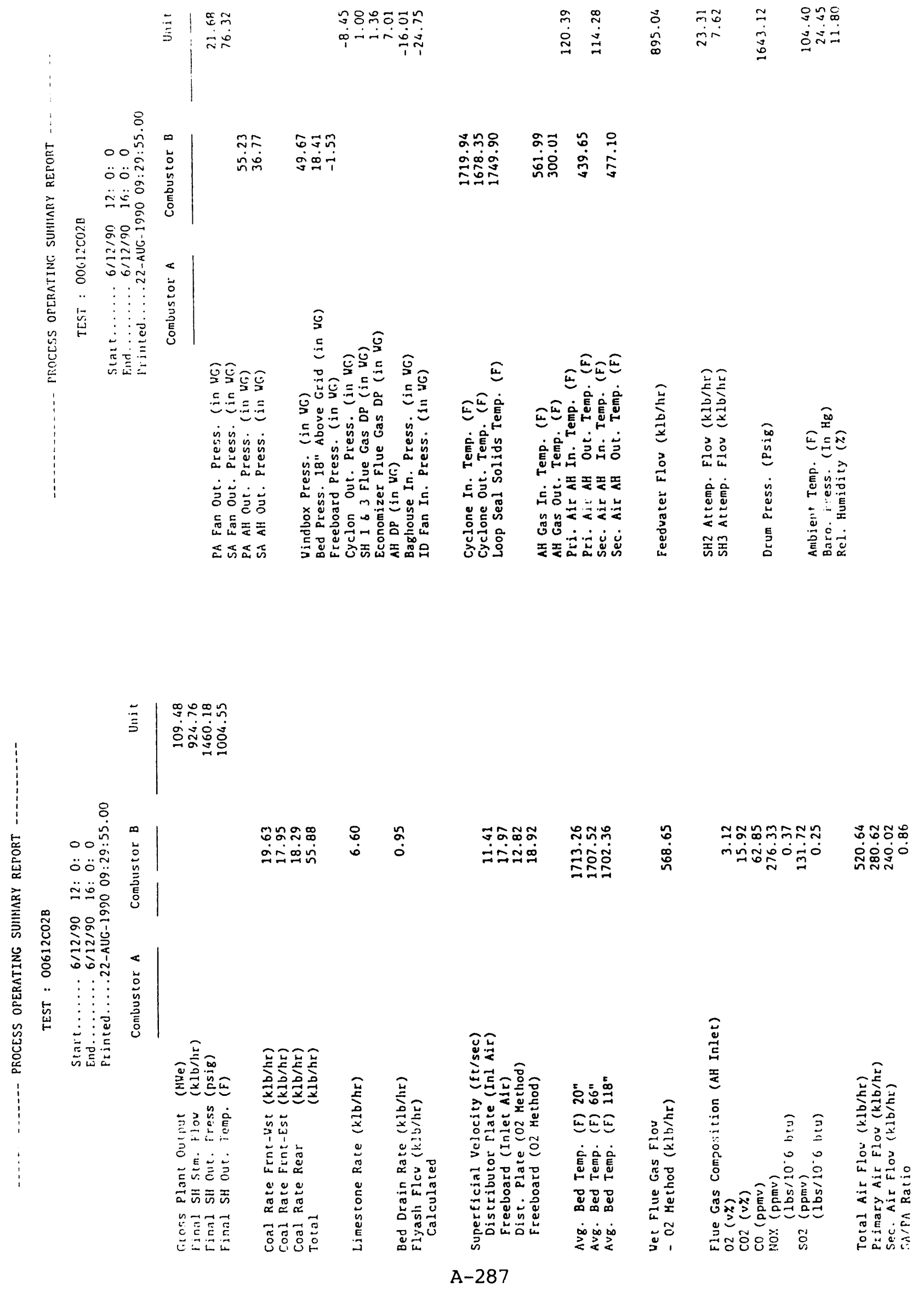


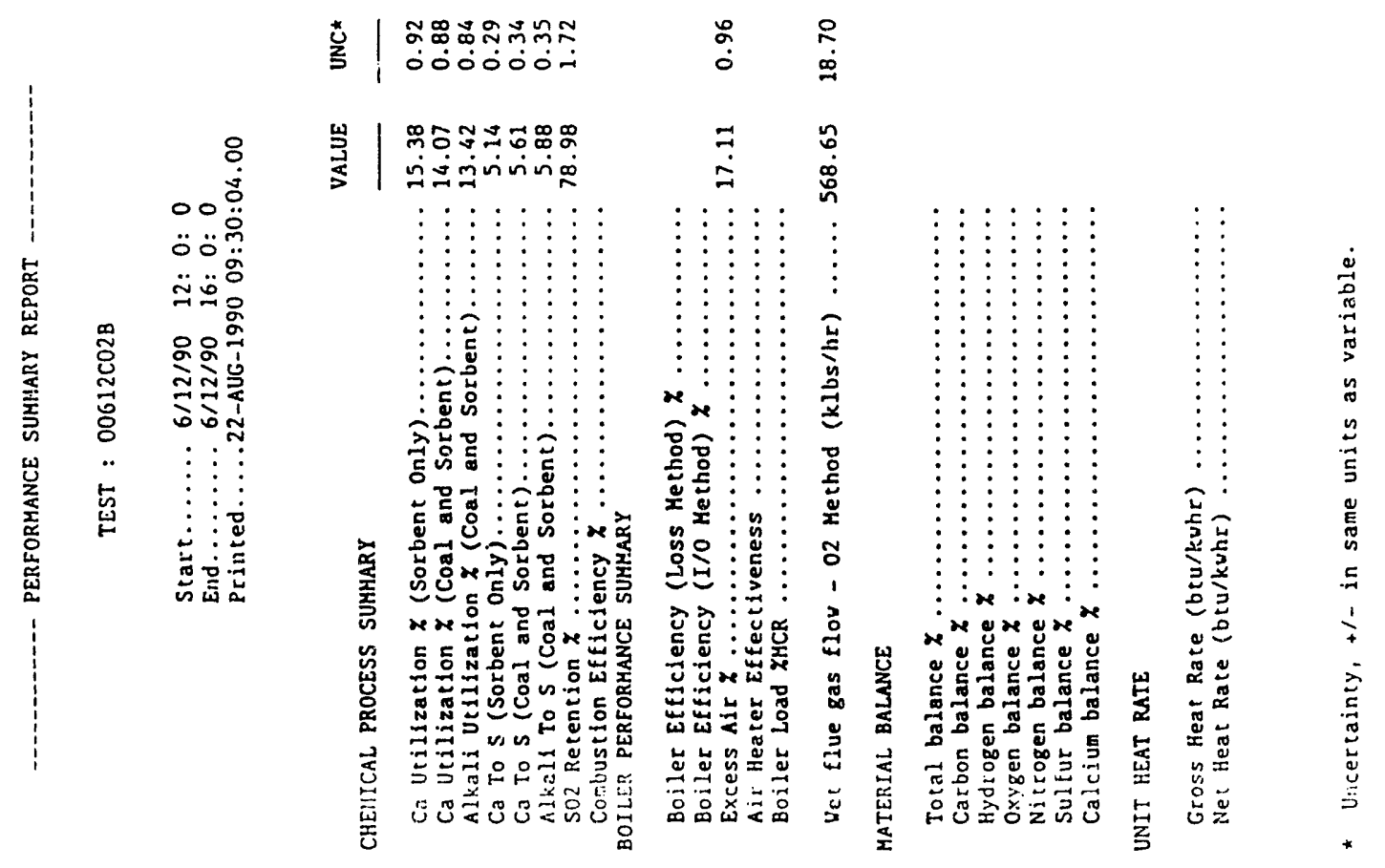




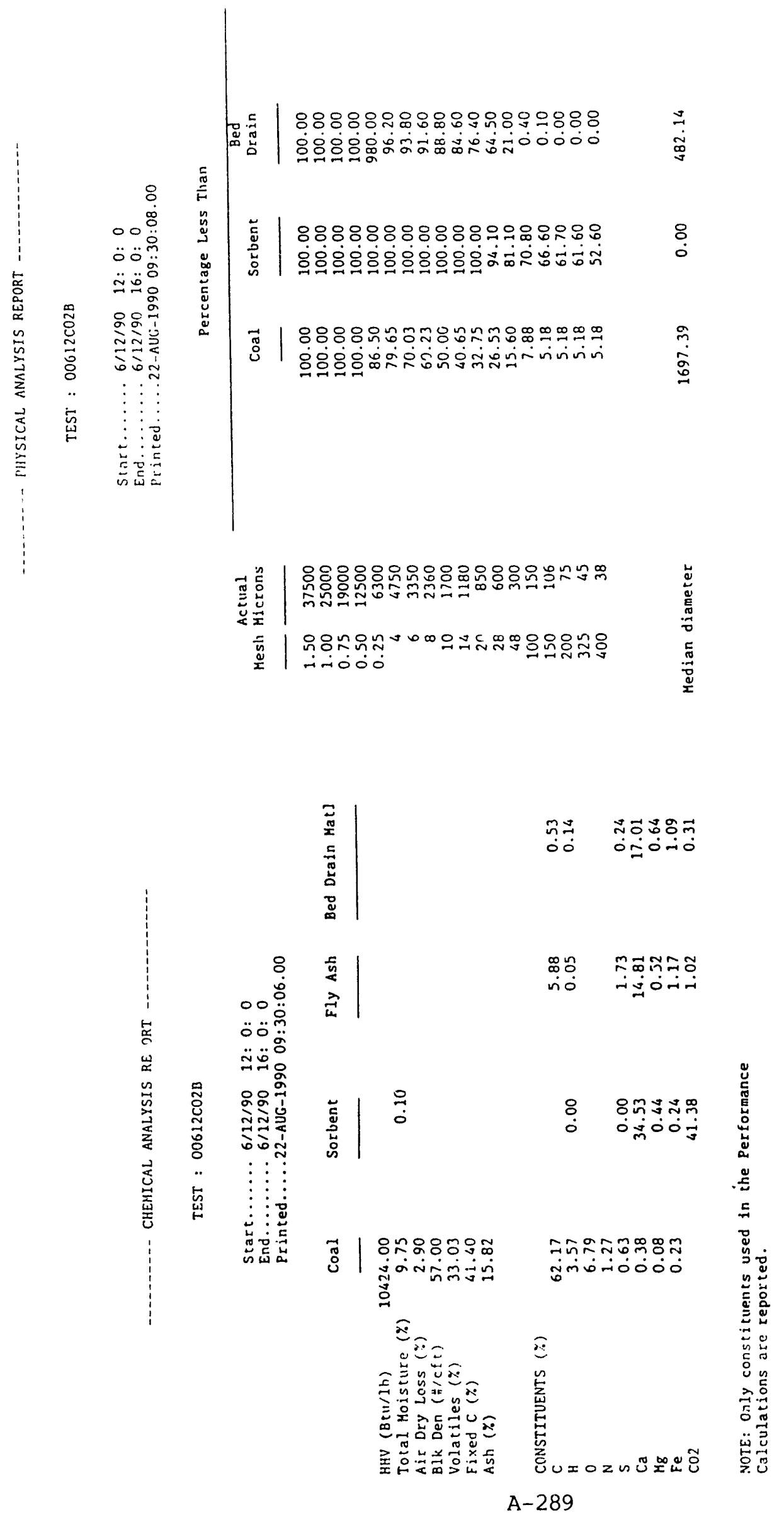




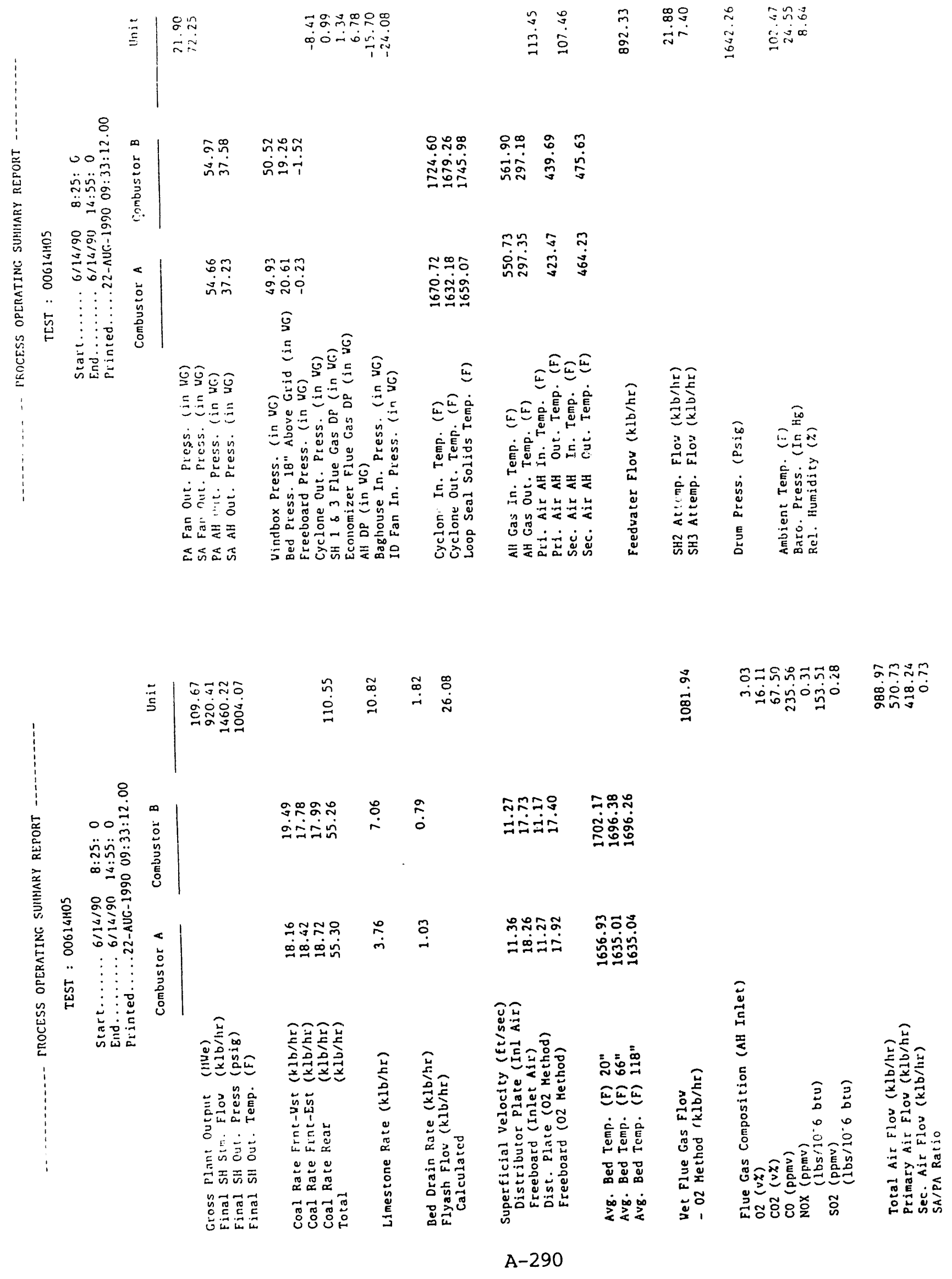




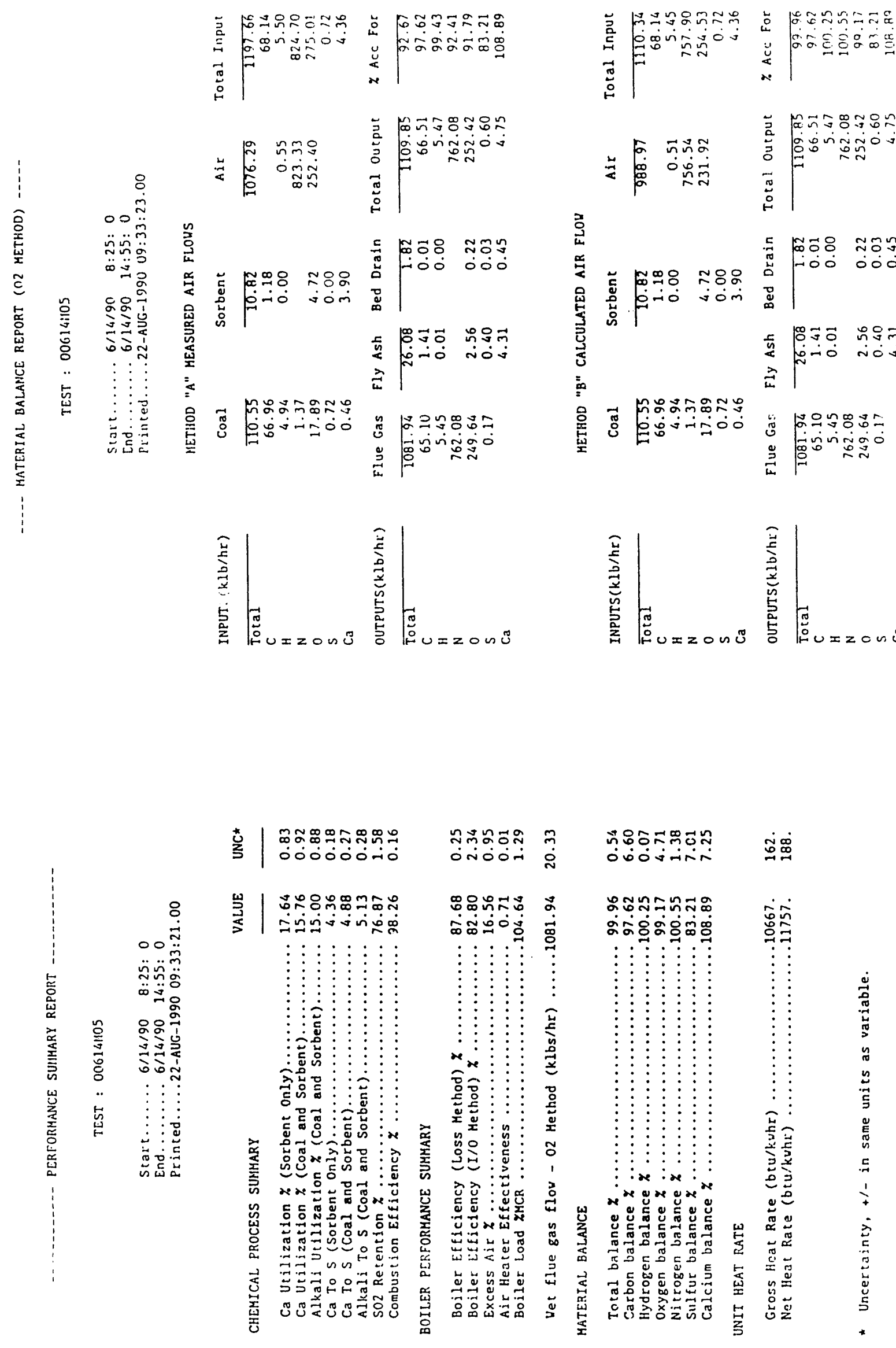



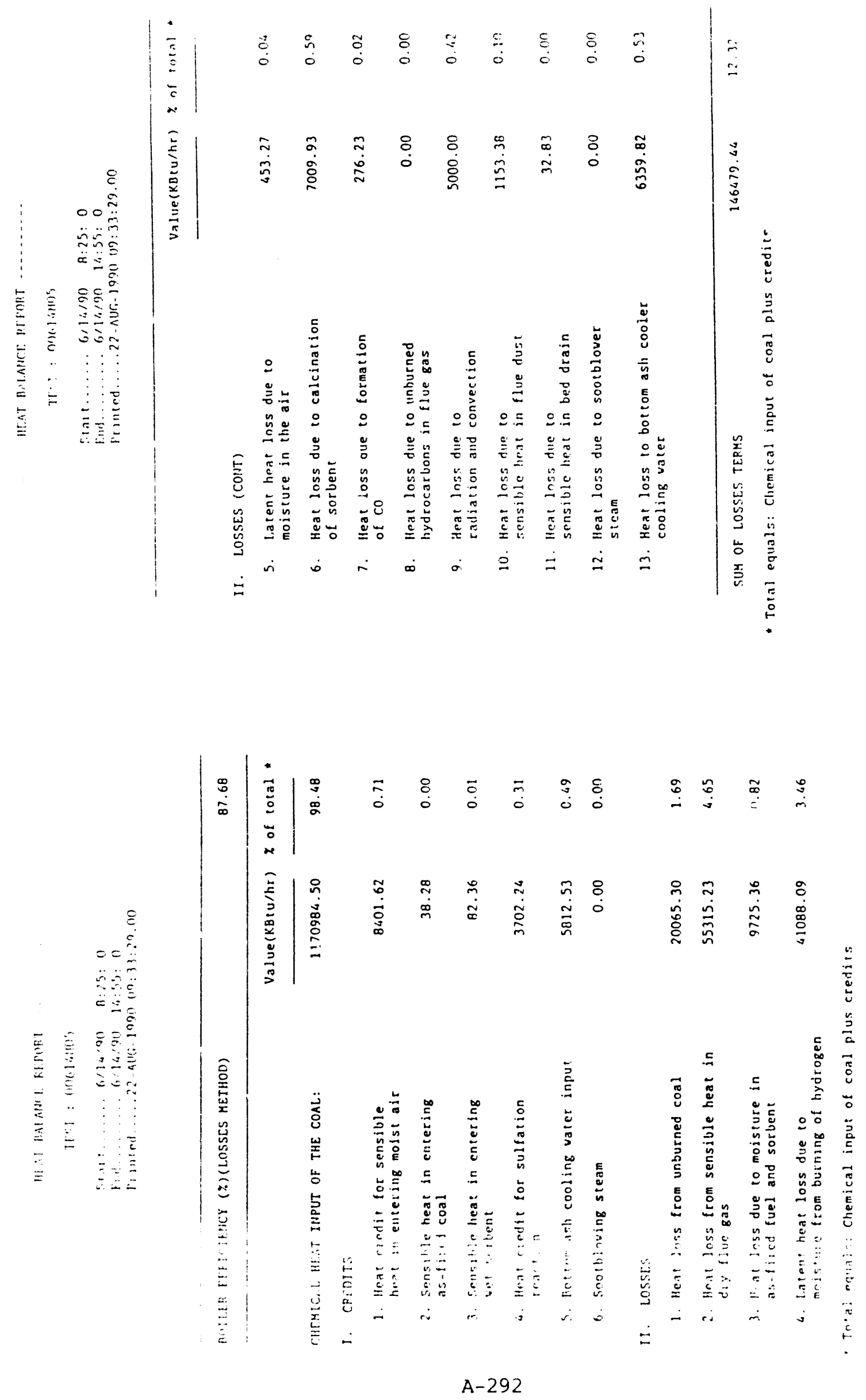

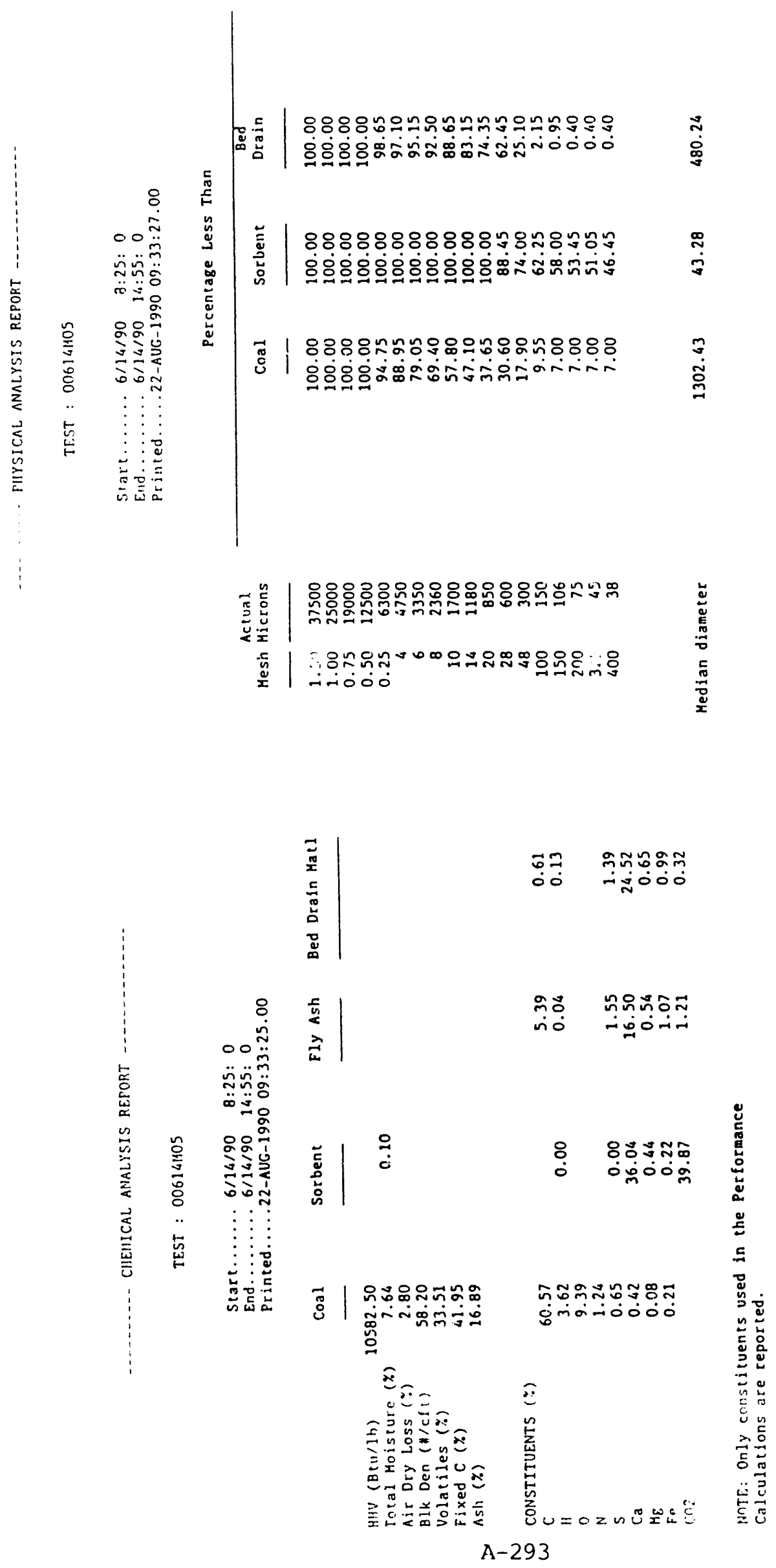


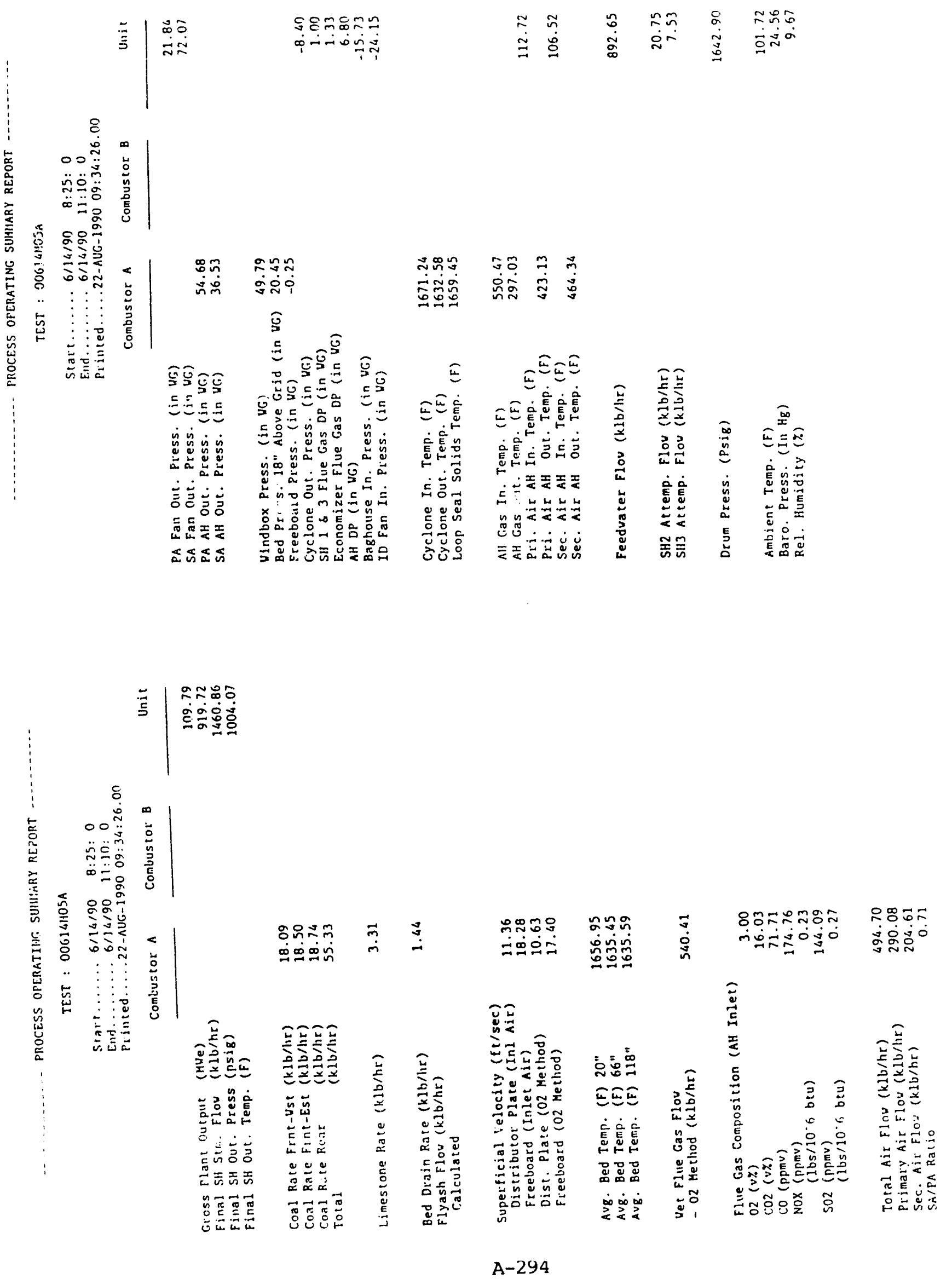




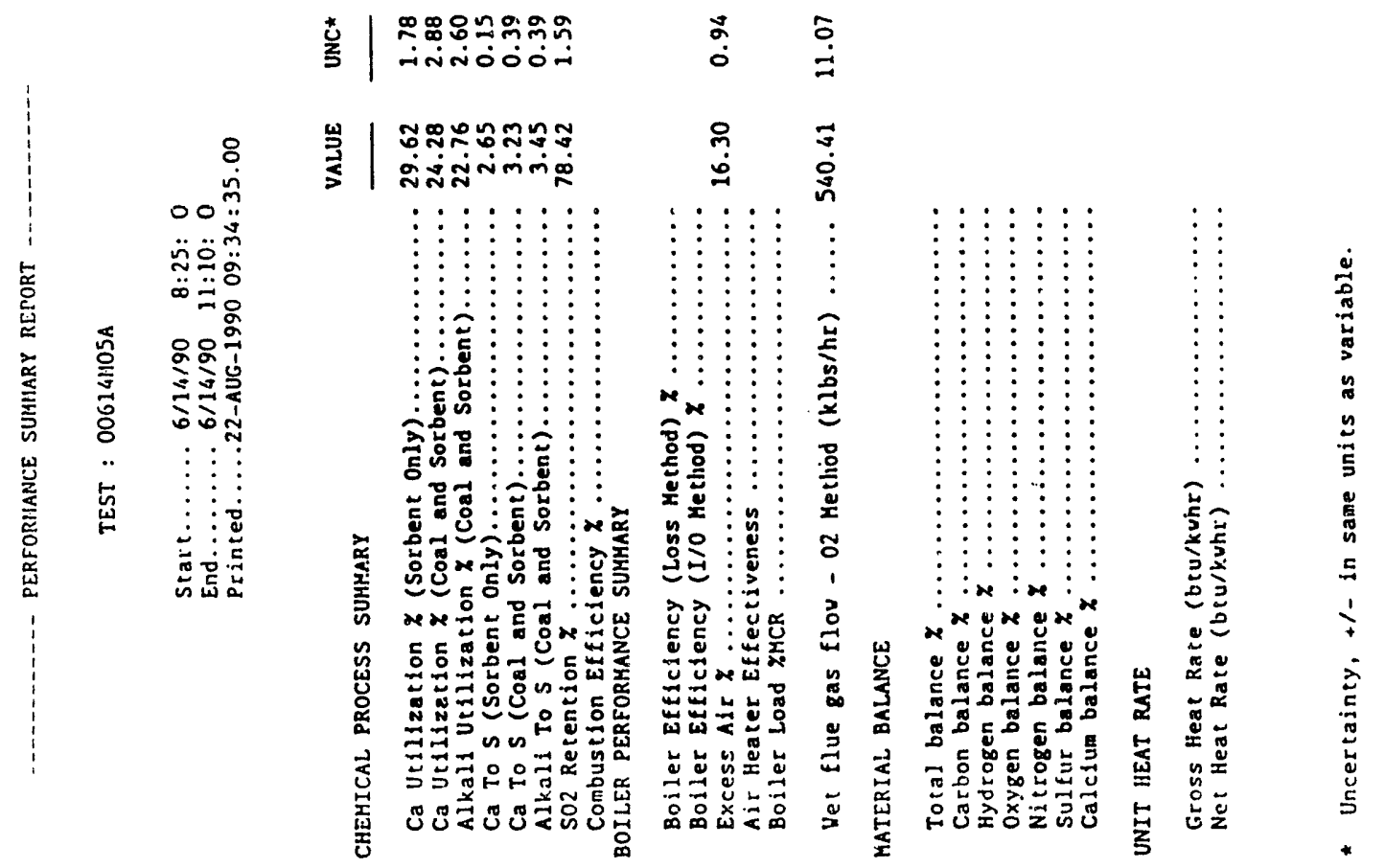

A-295 


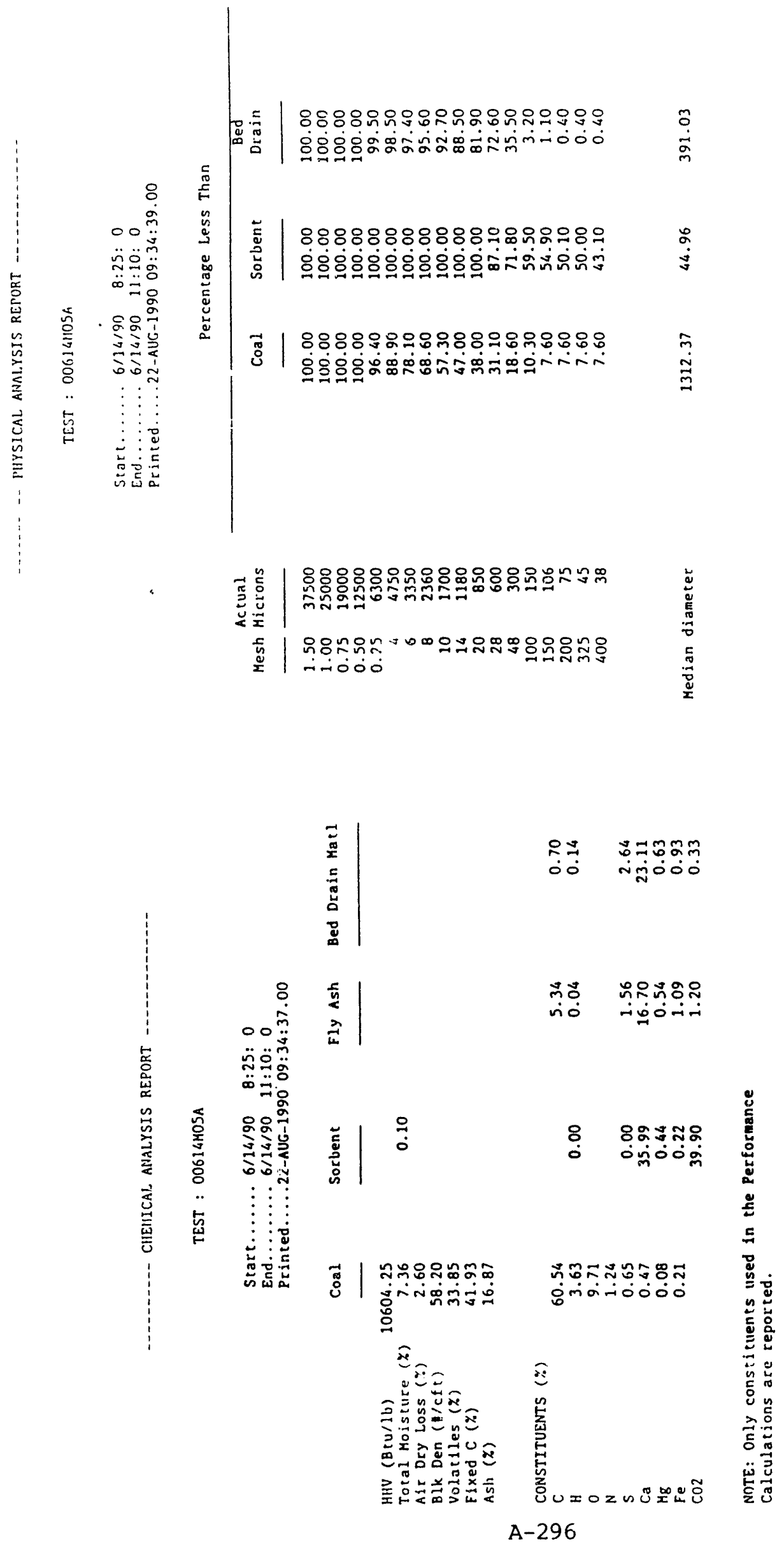




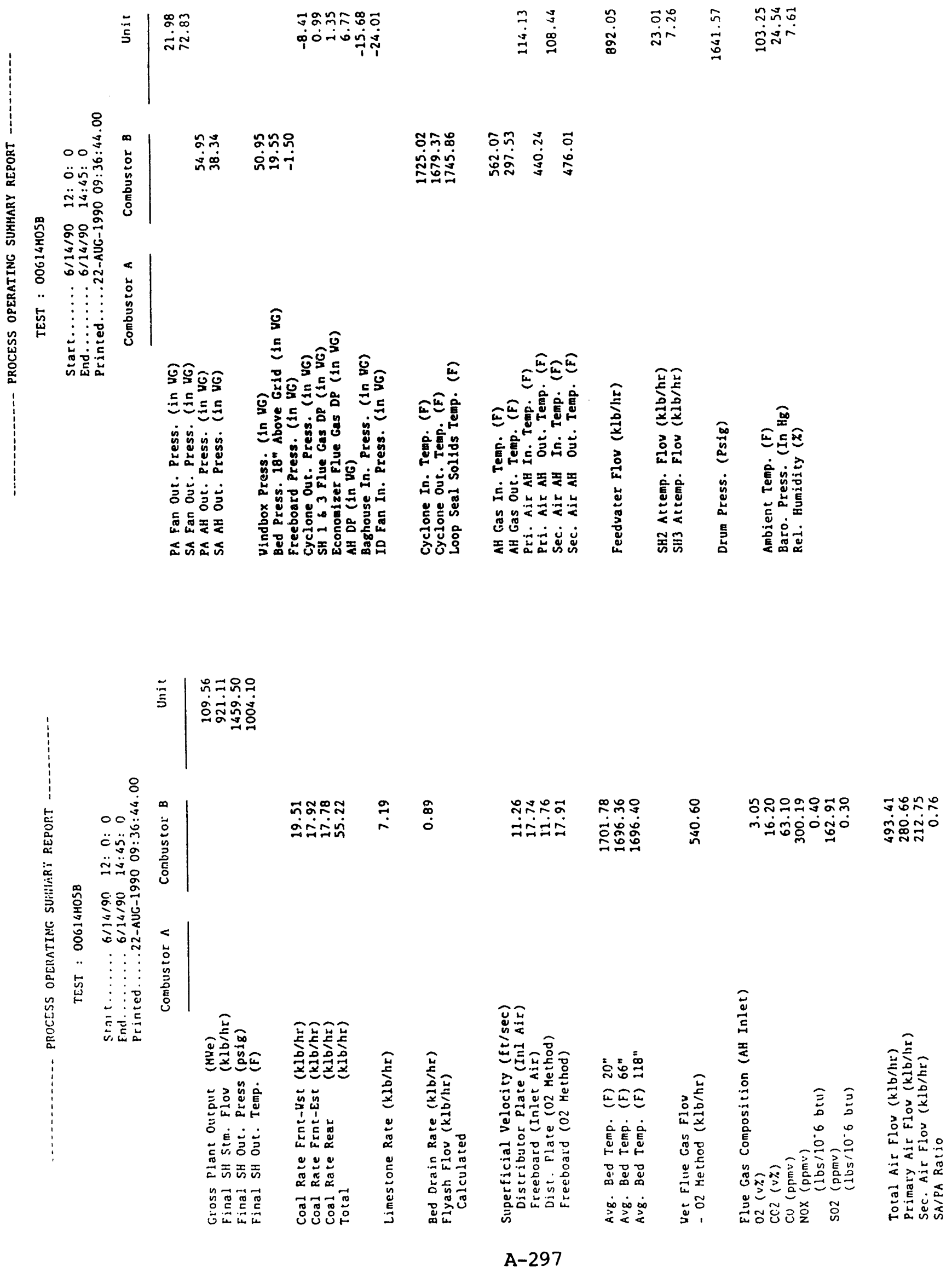




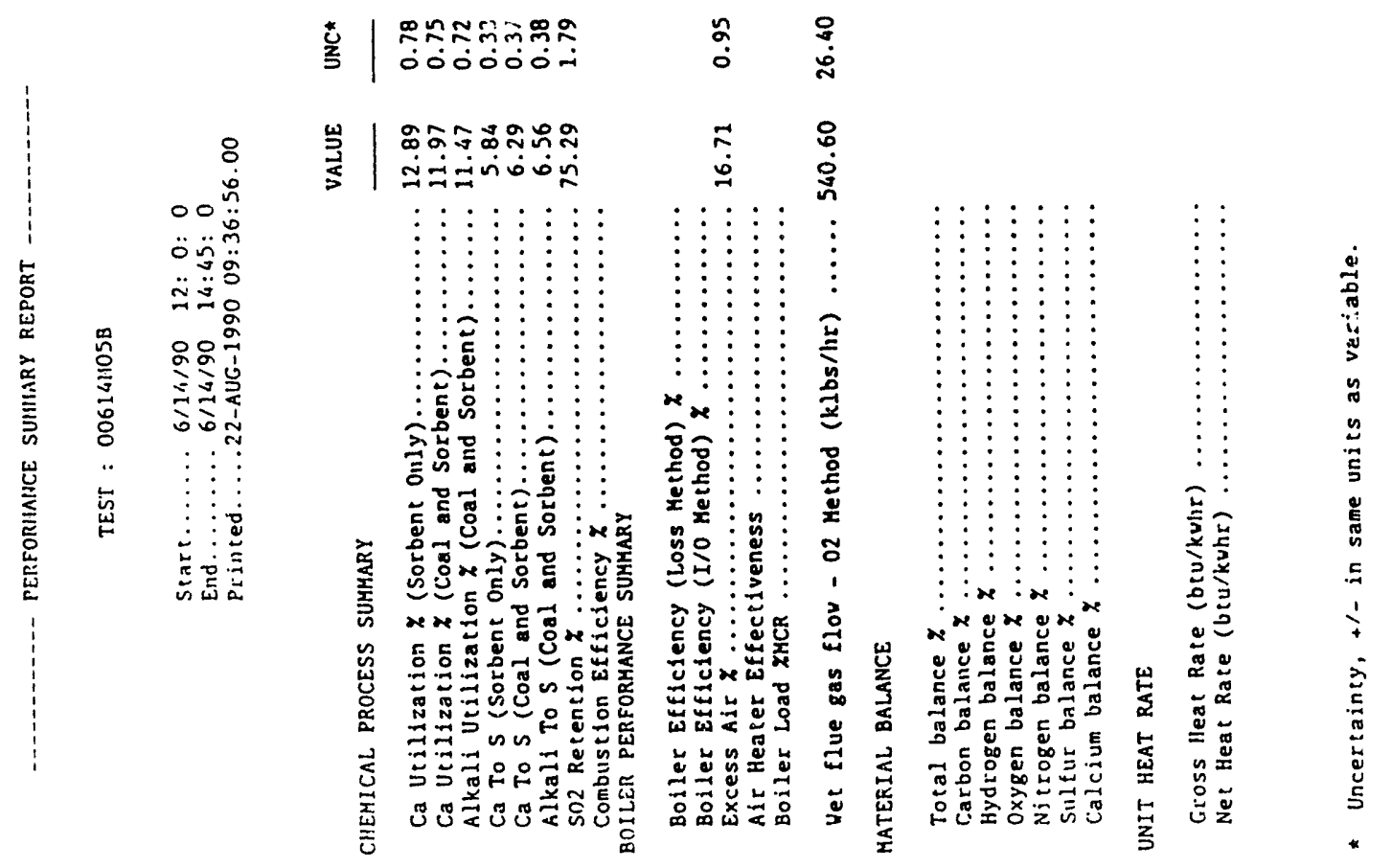



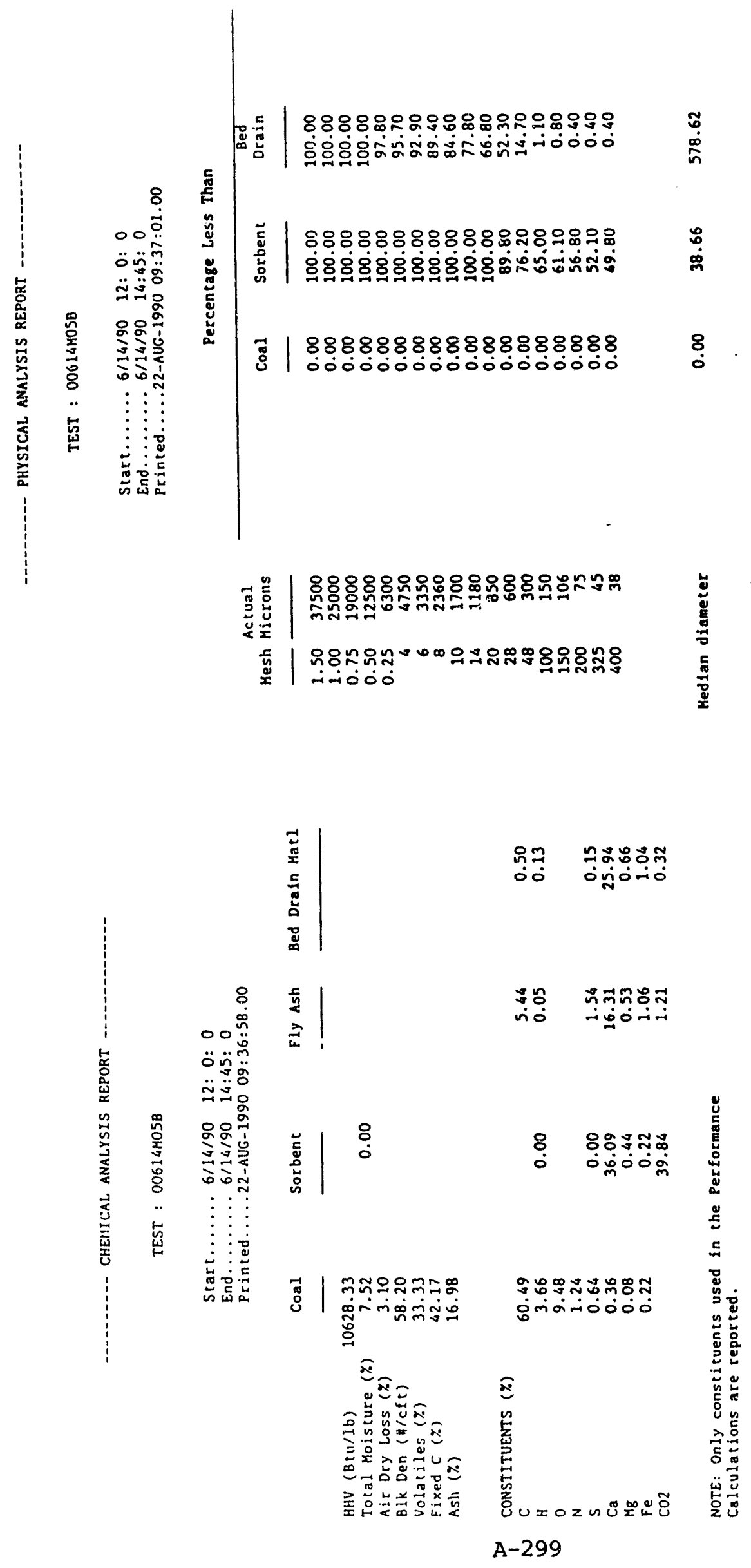

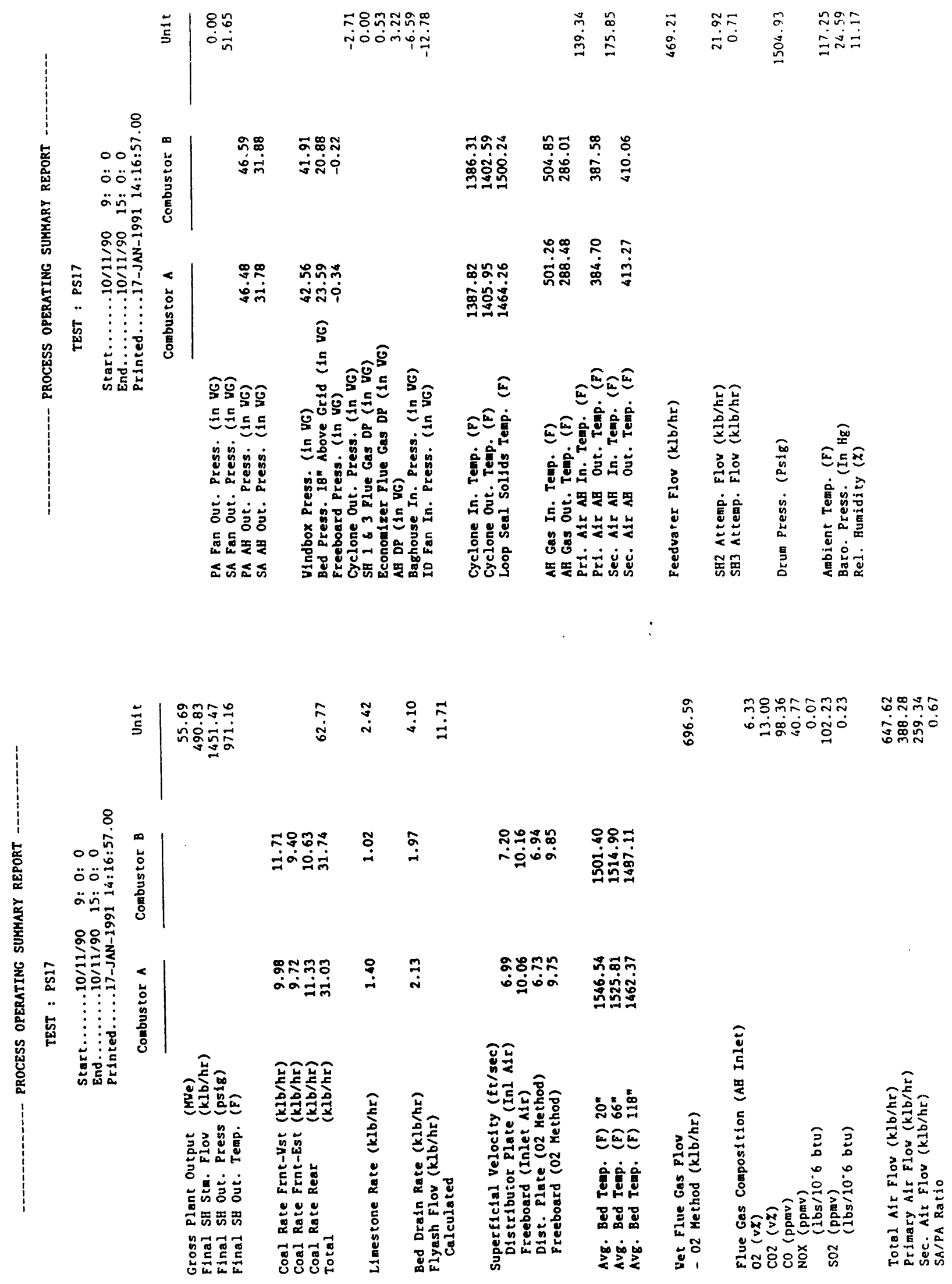


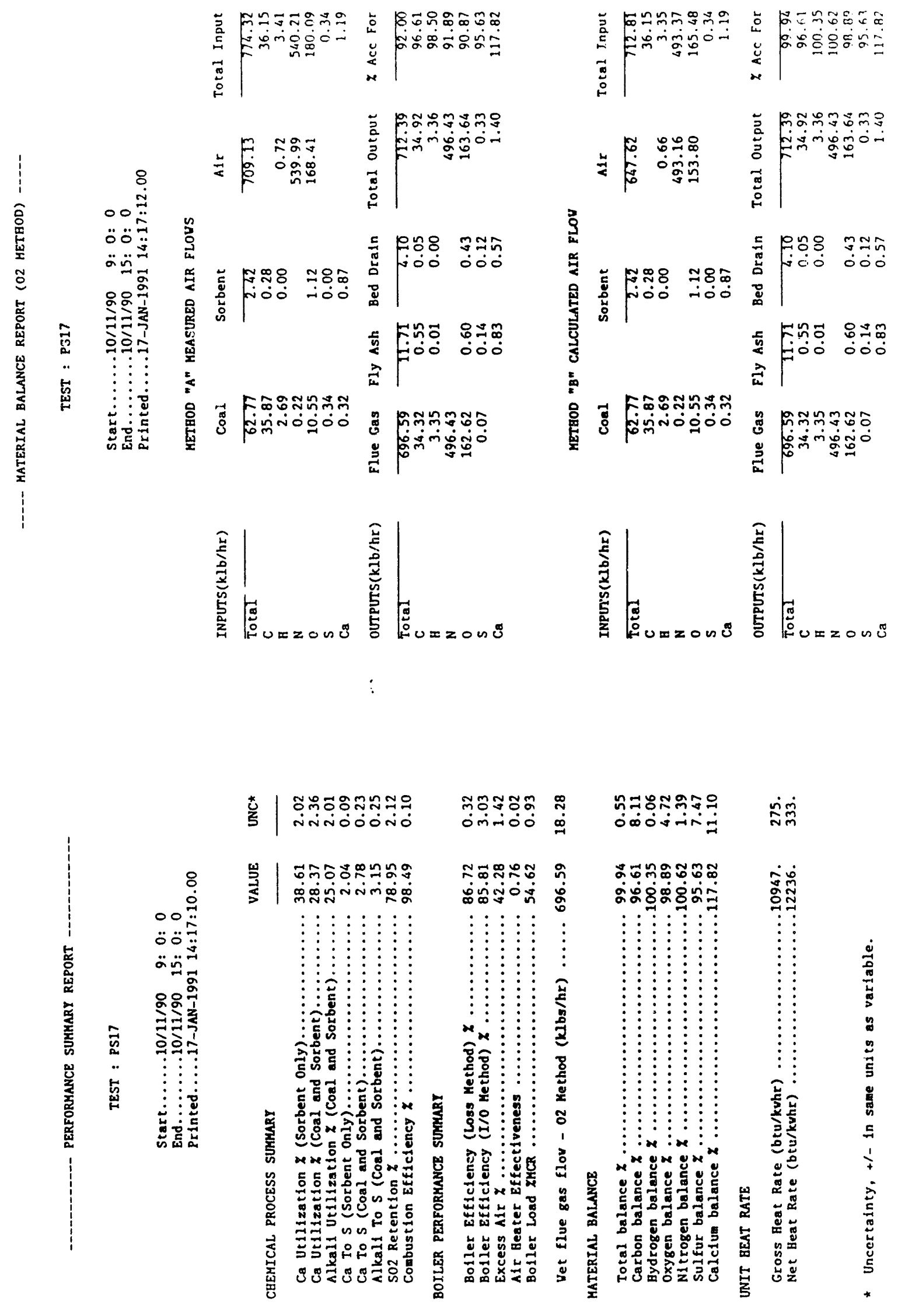




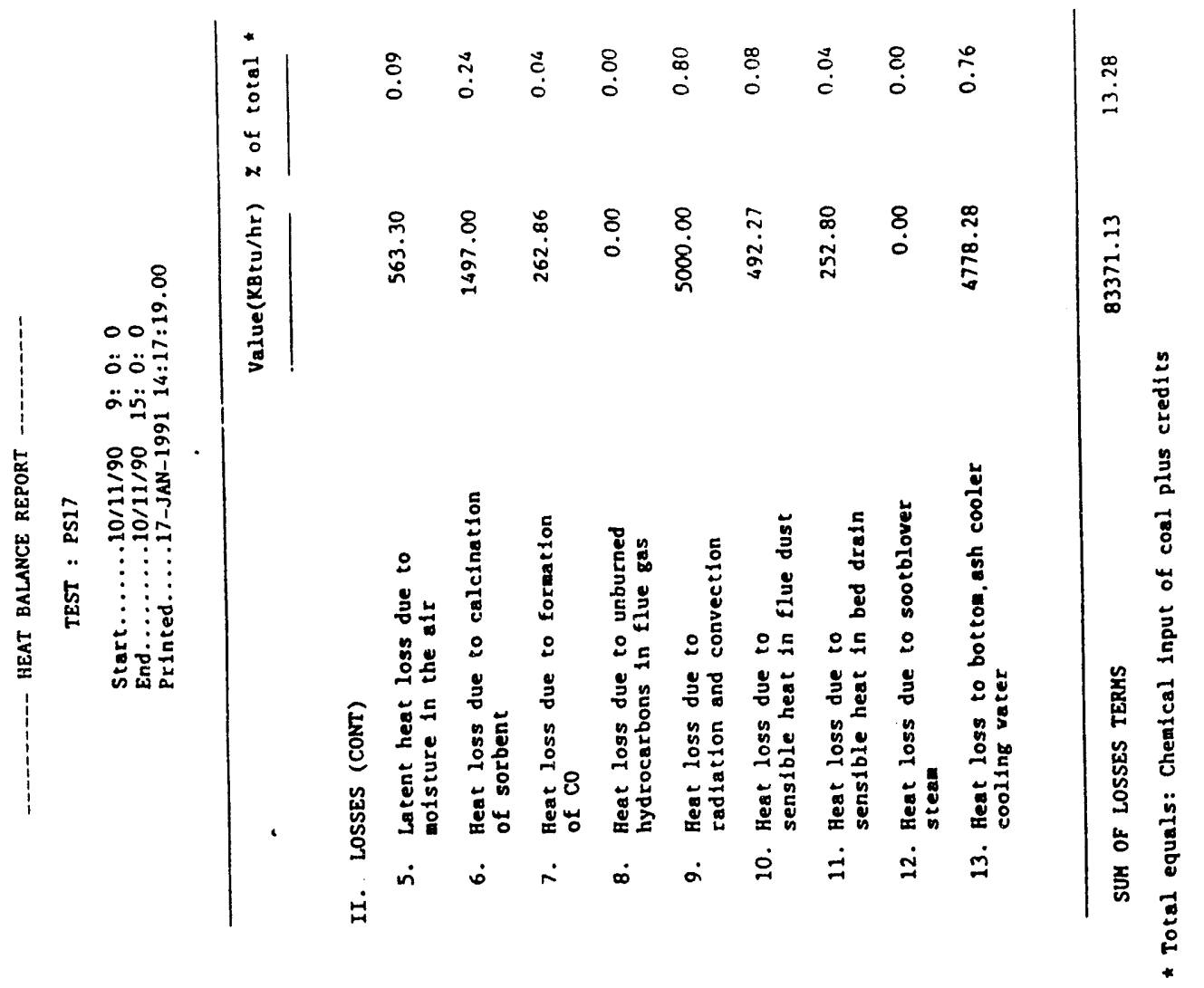

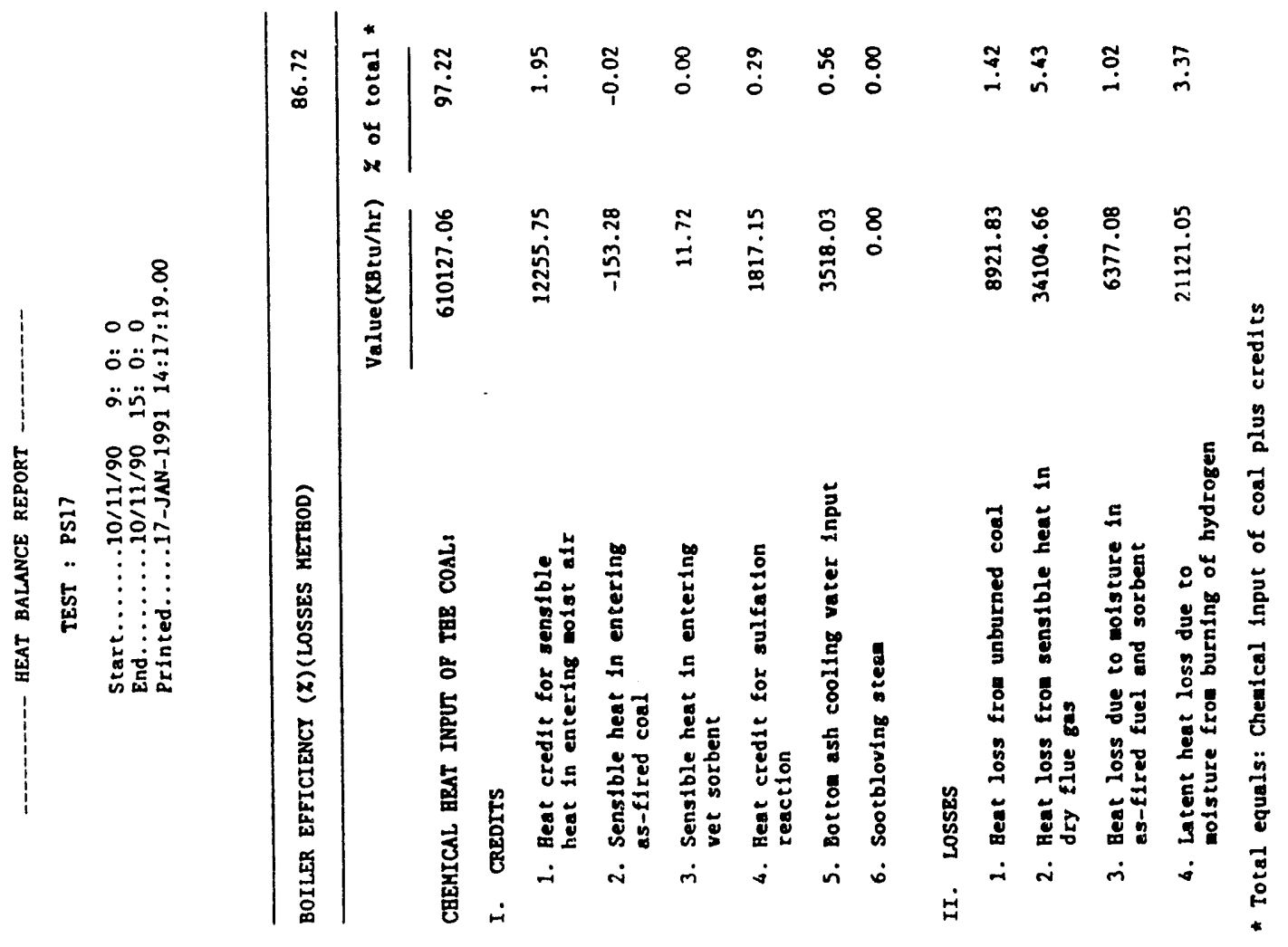




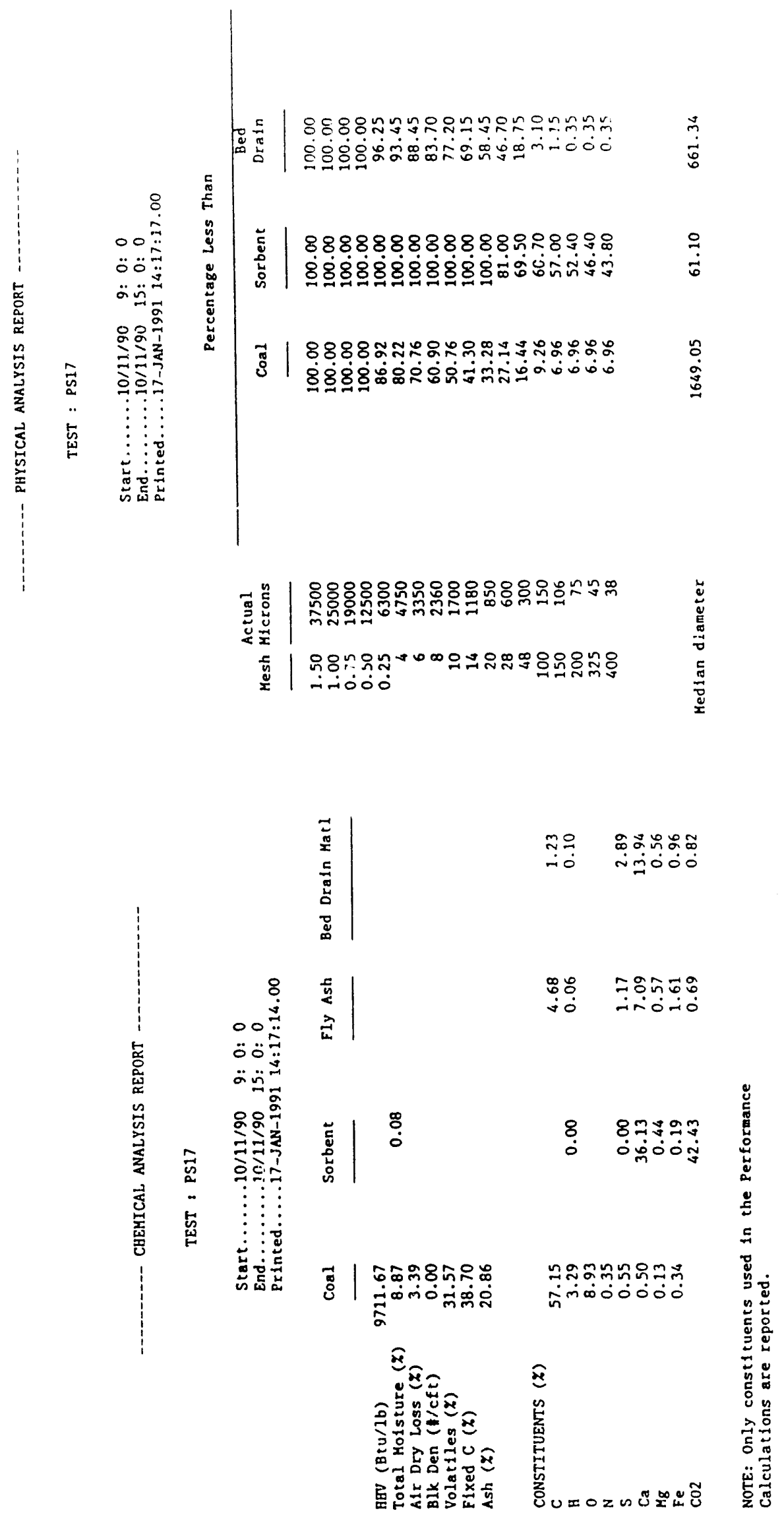



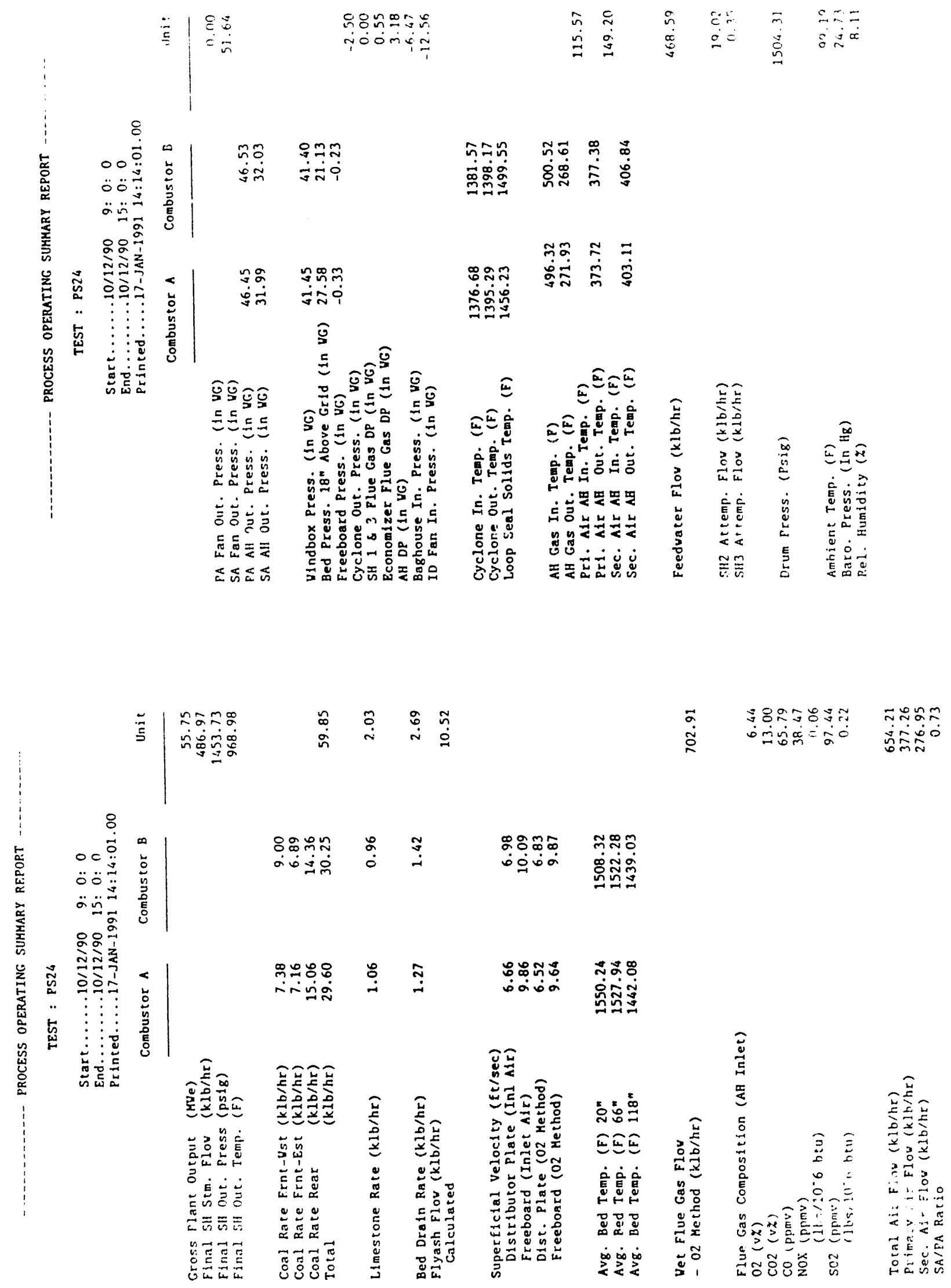


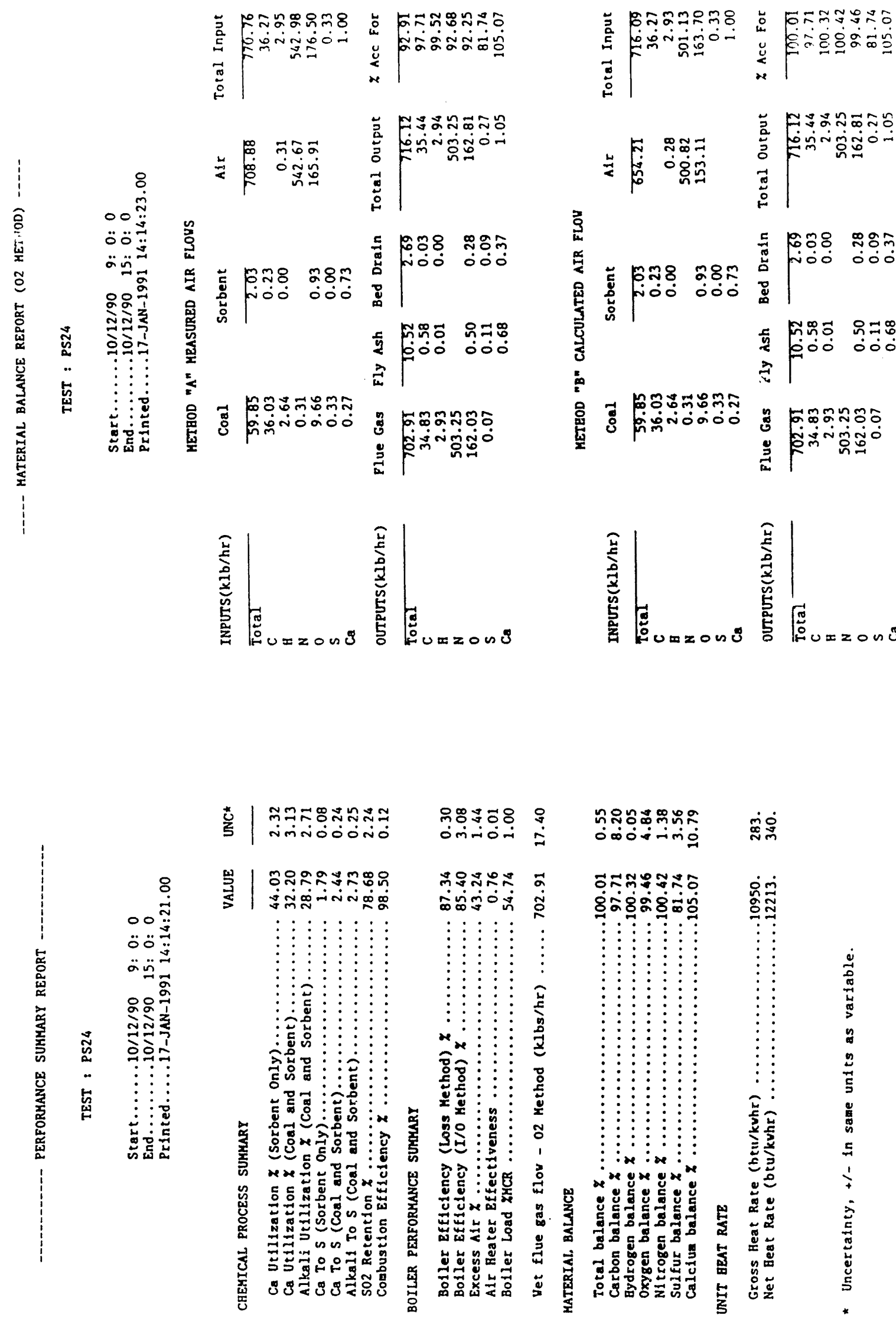




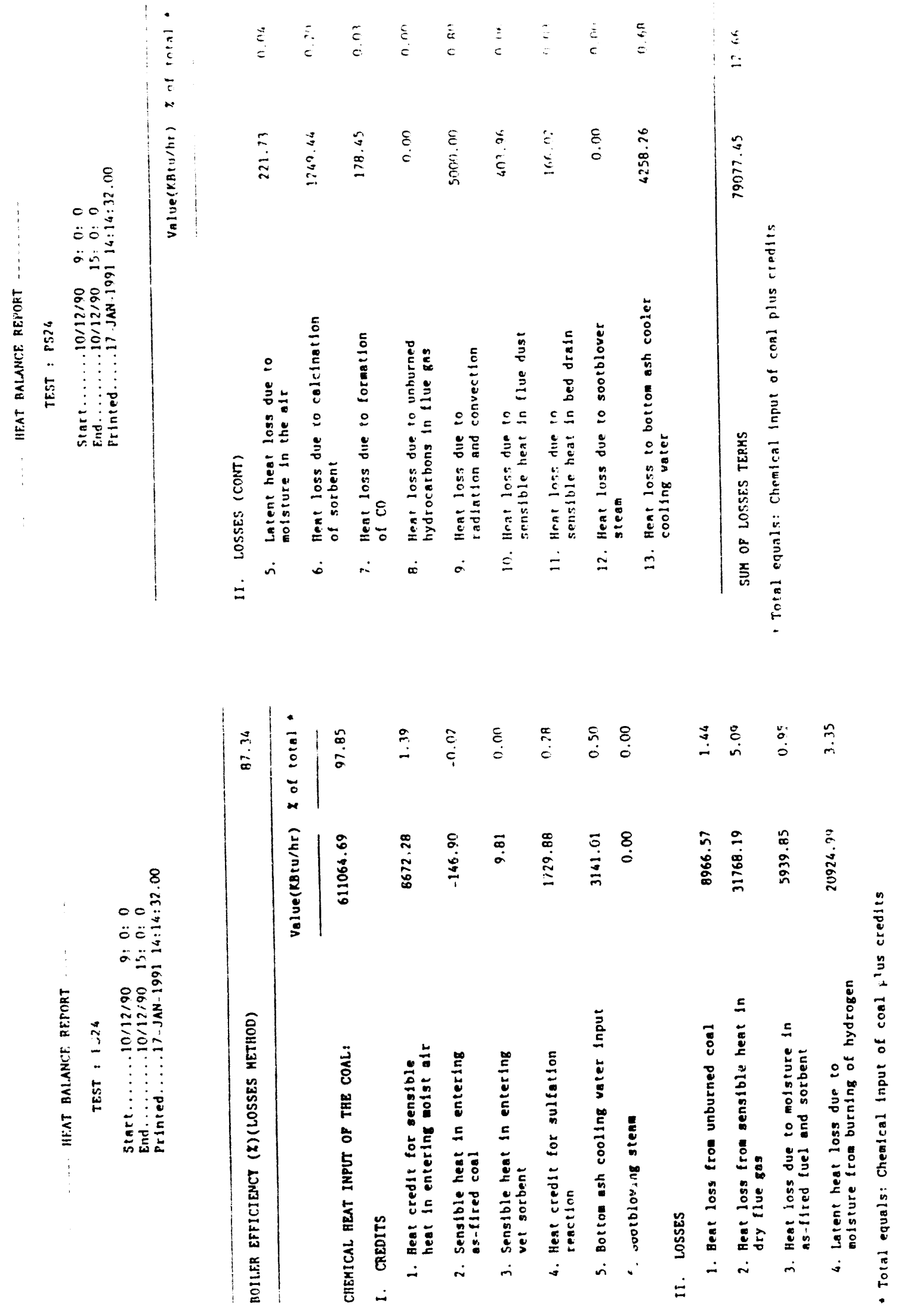



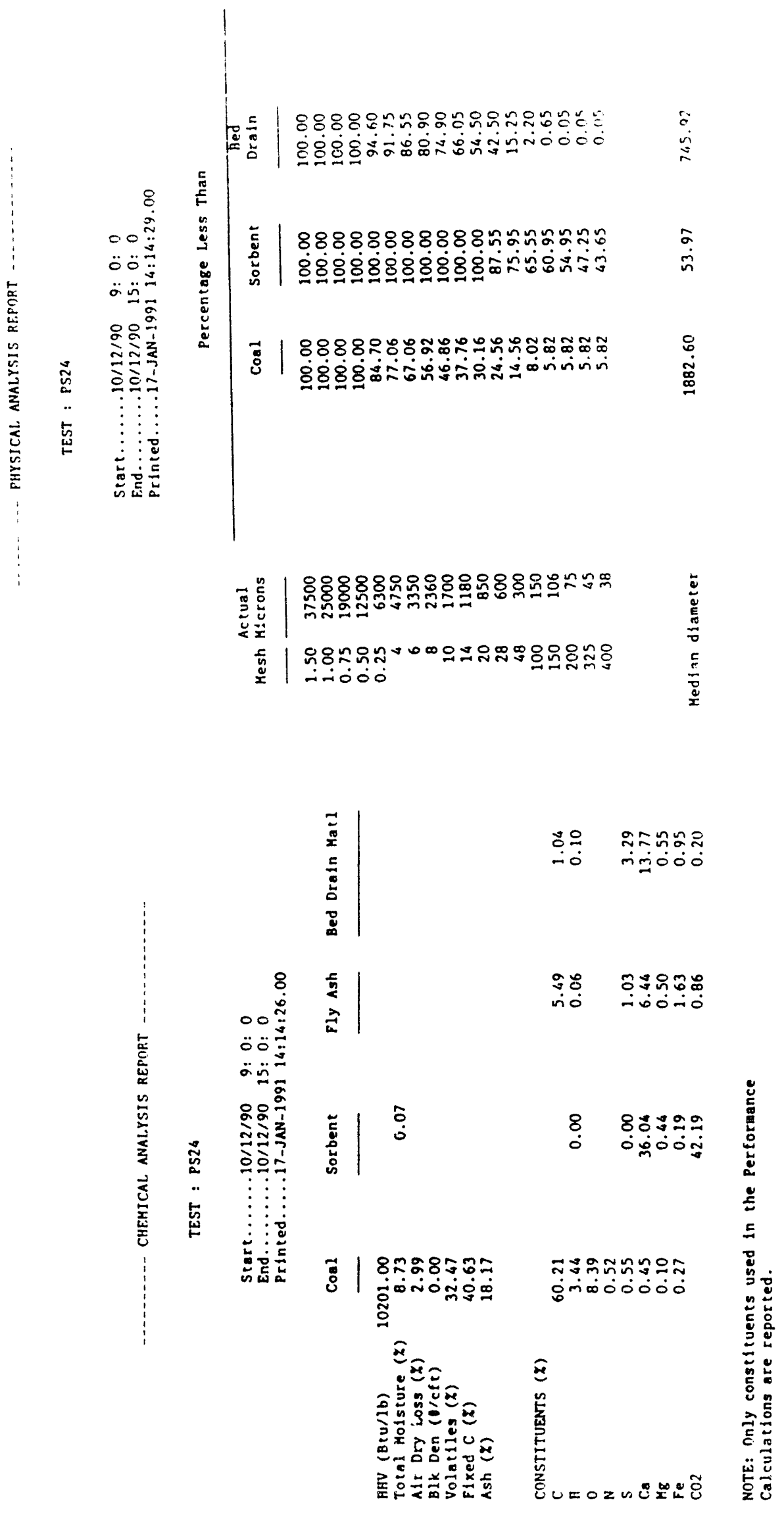

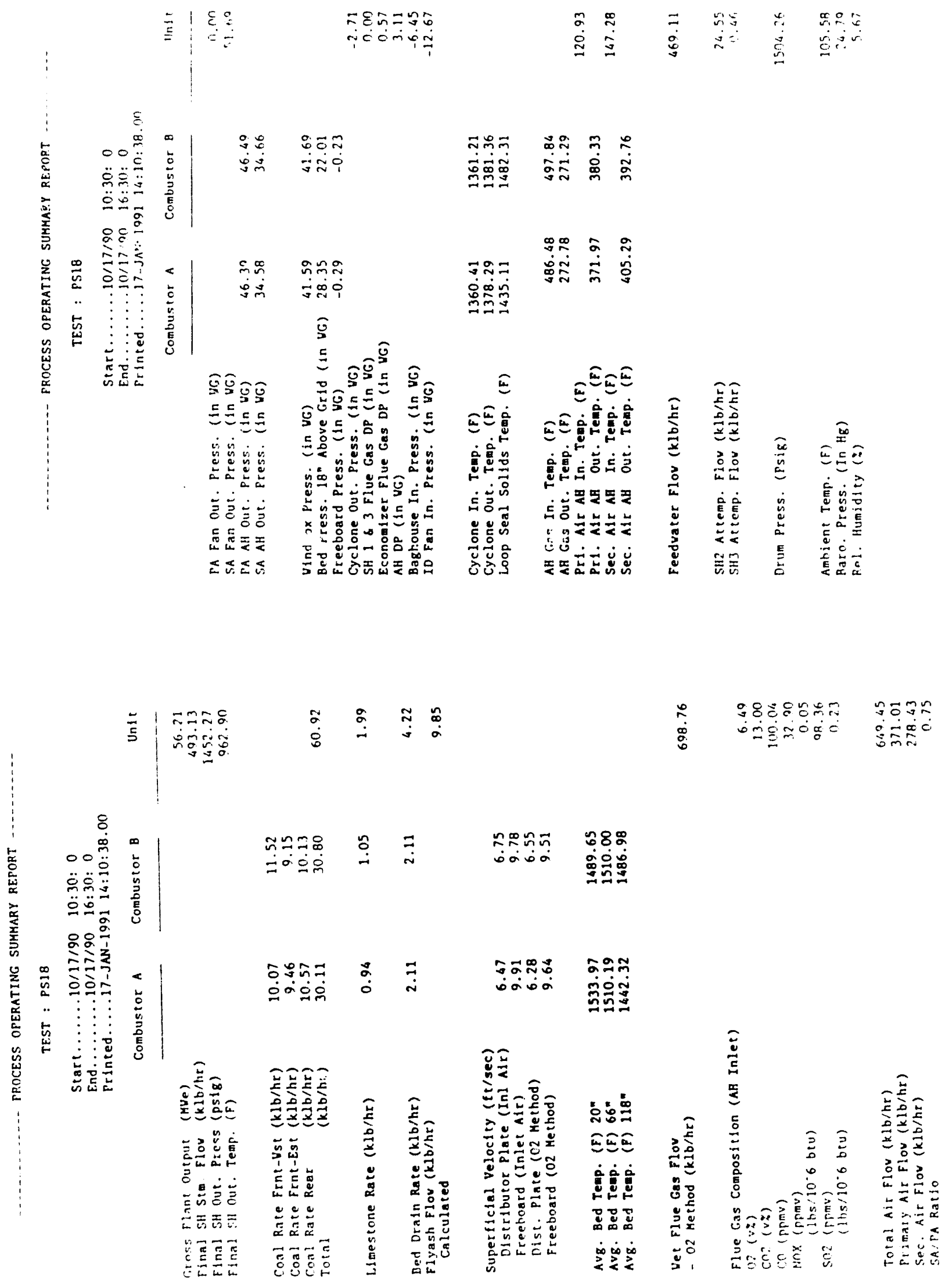


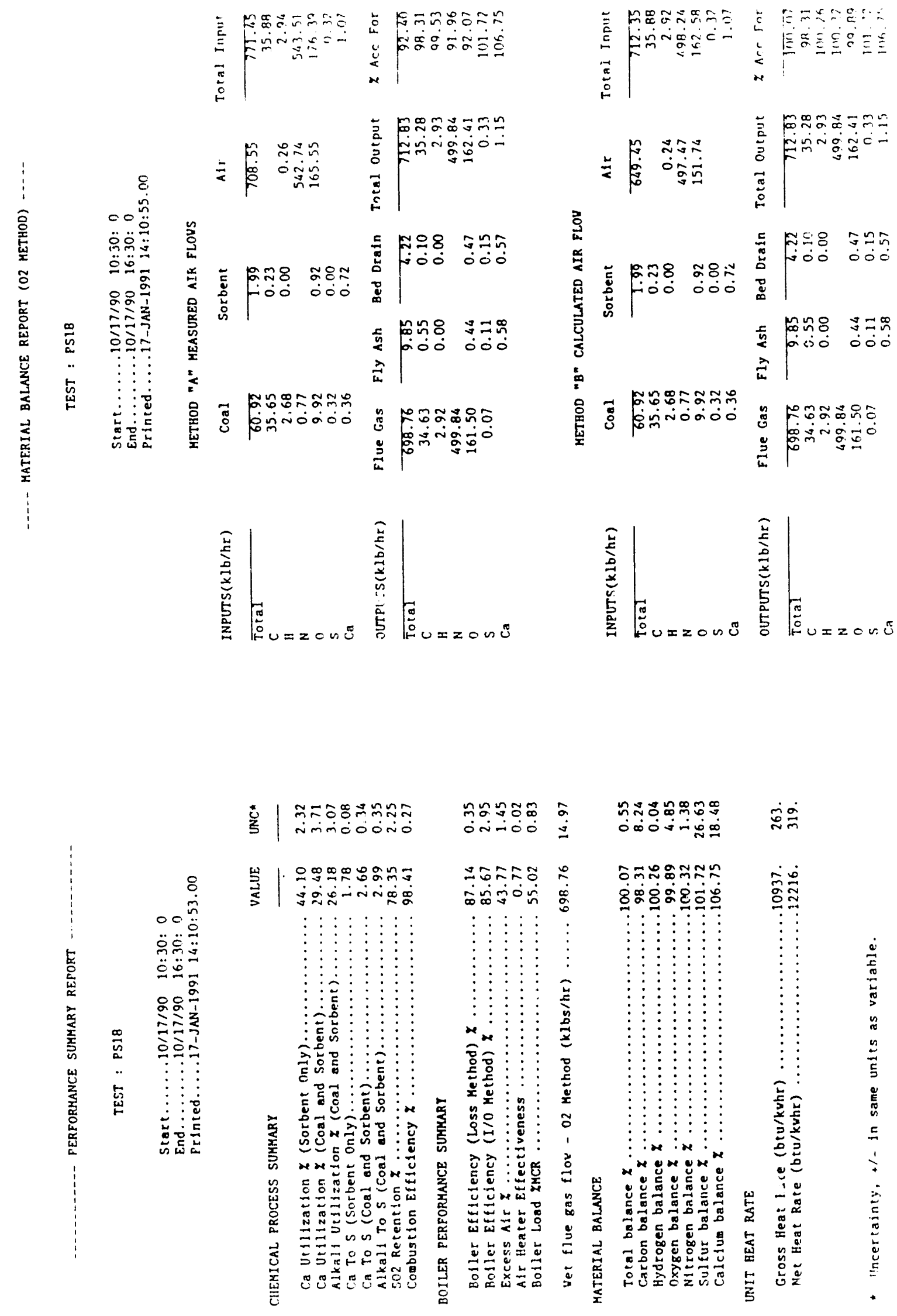




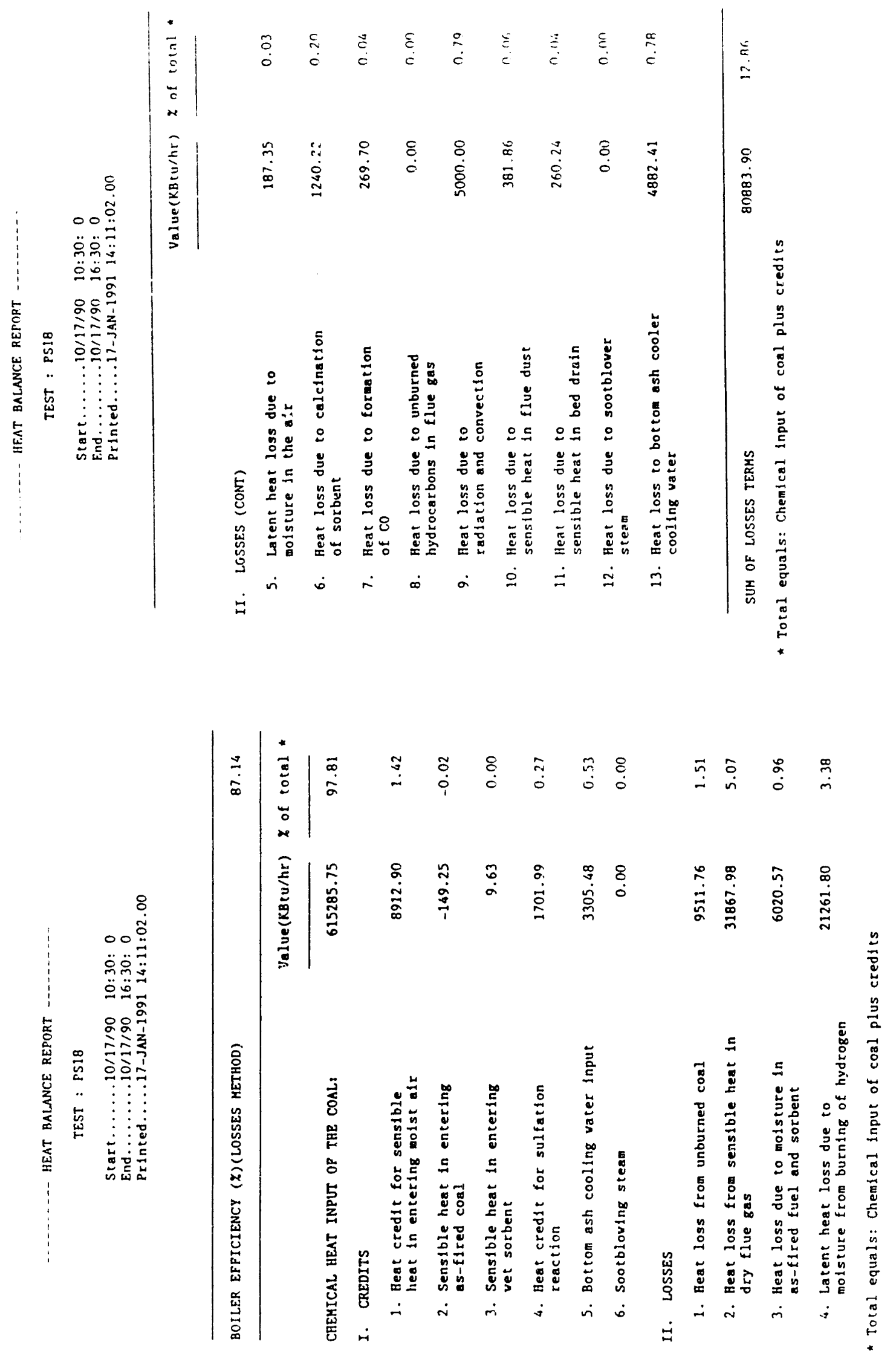



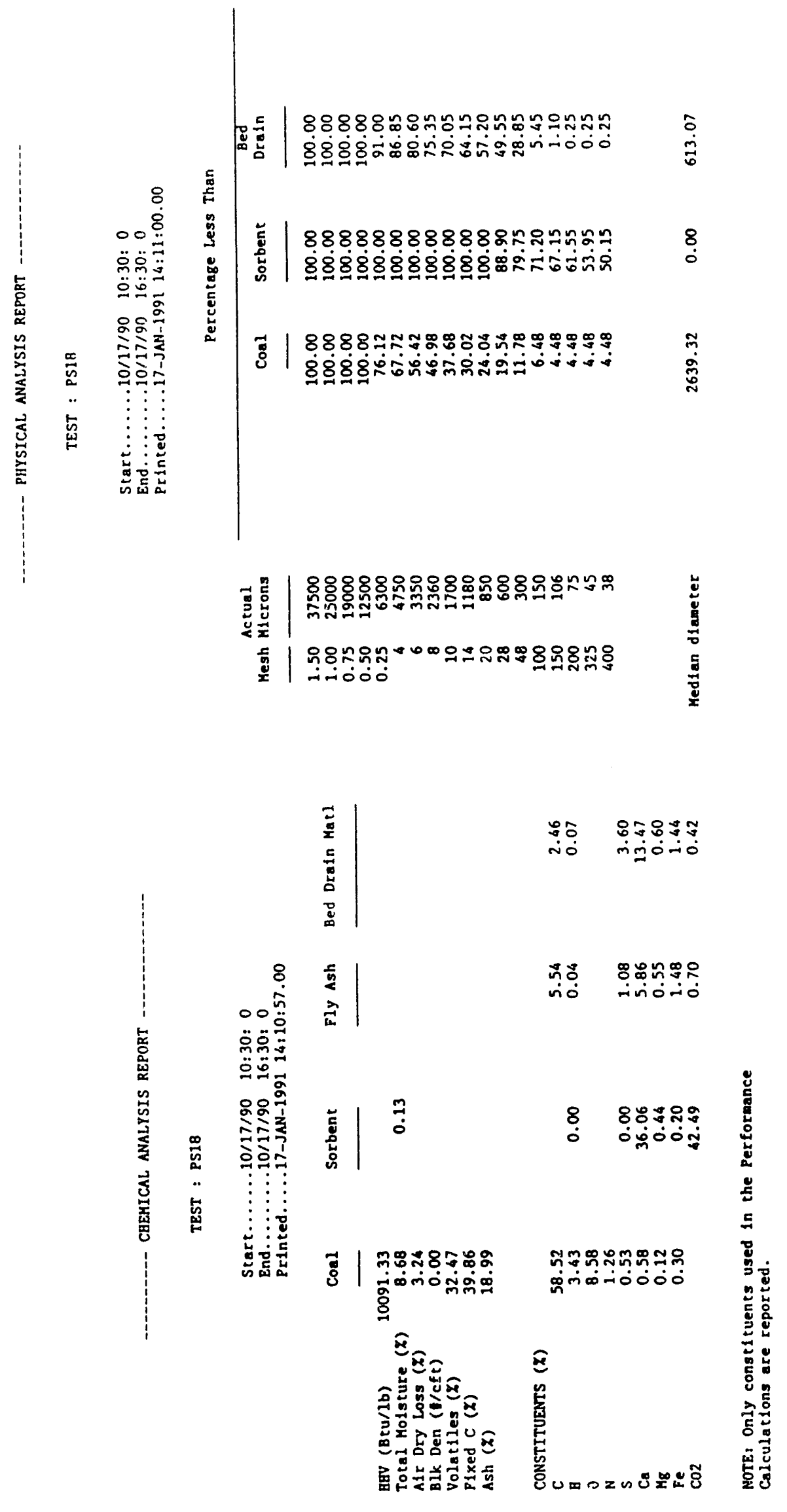

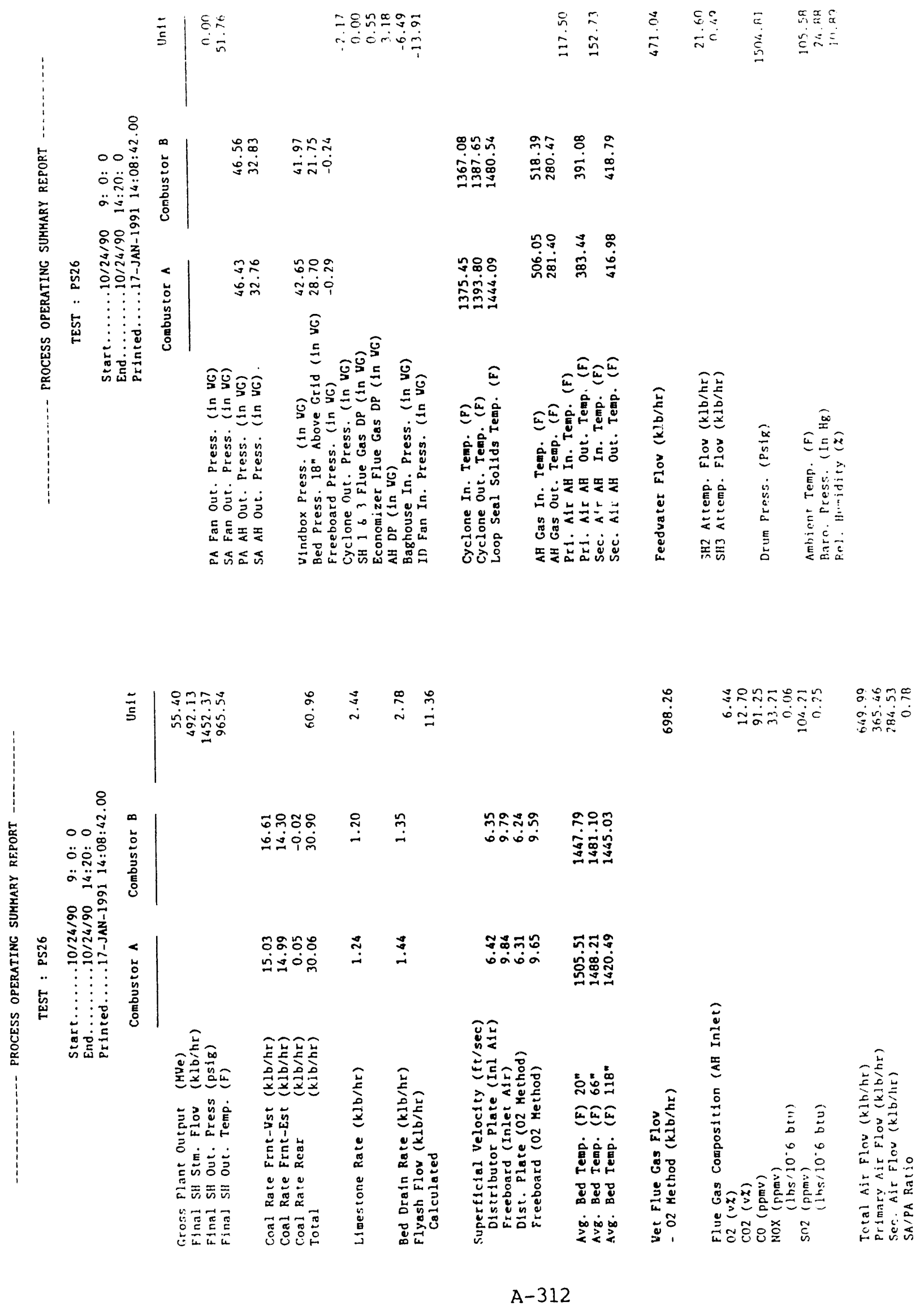


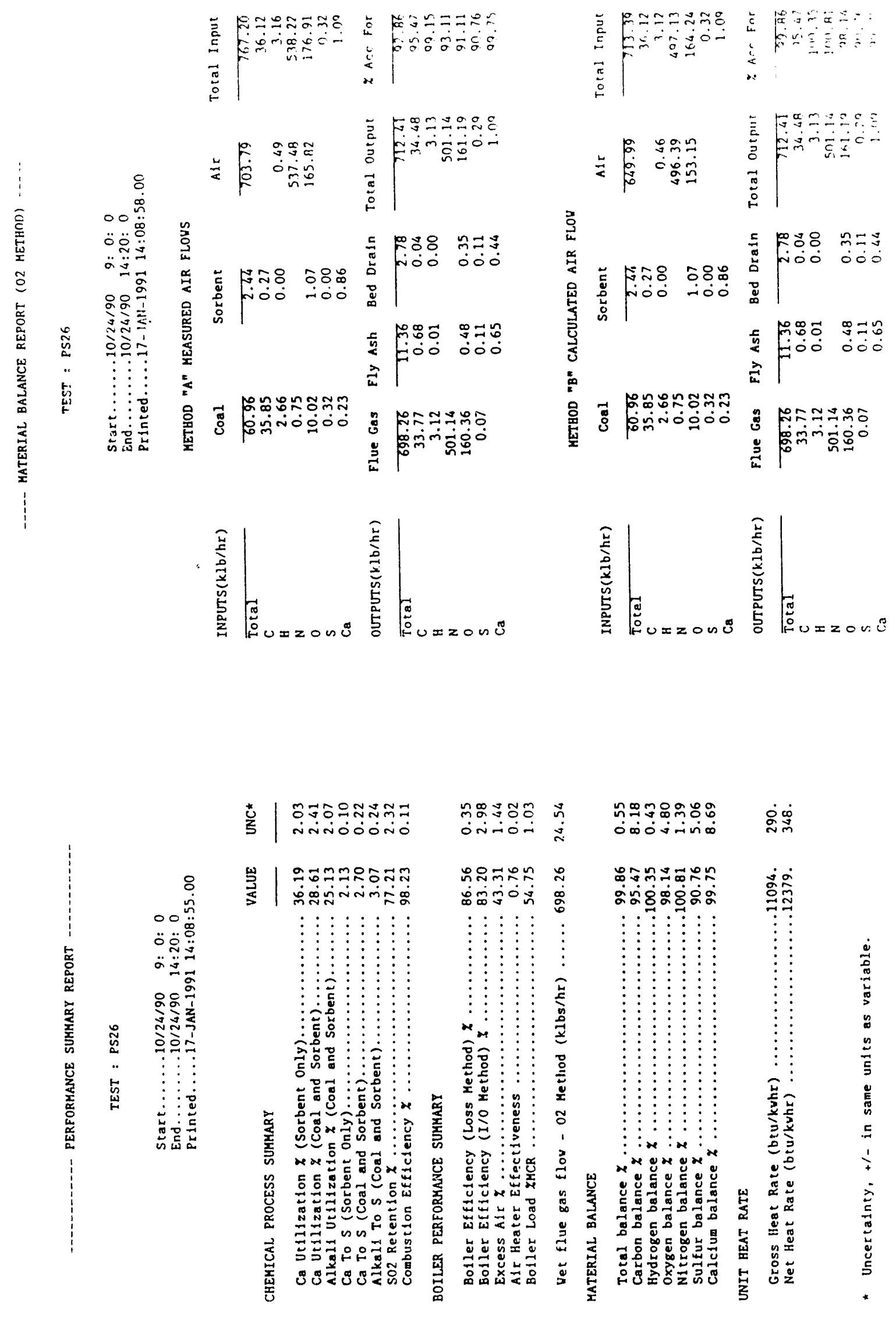




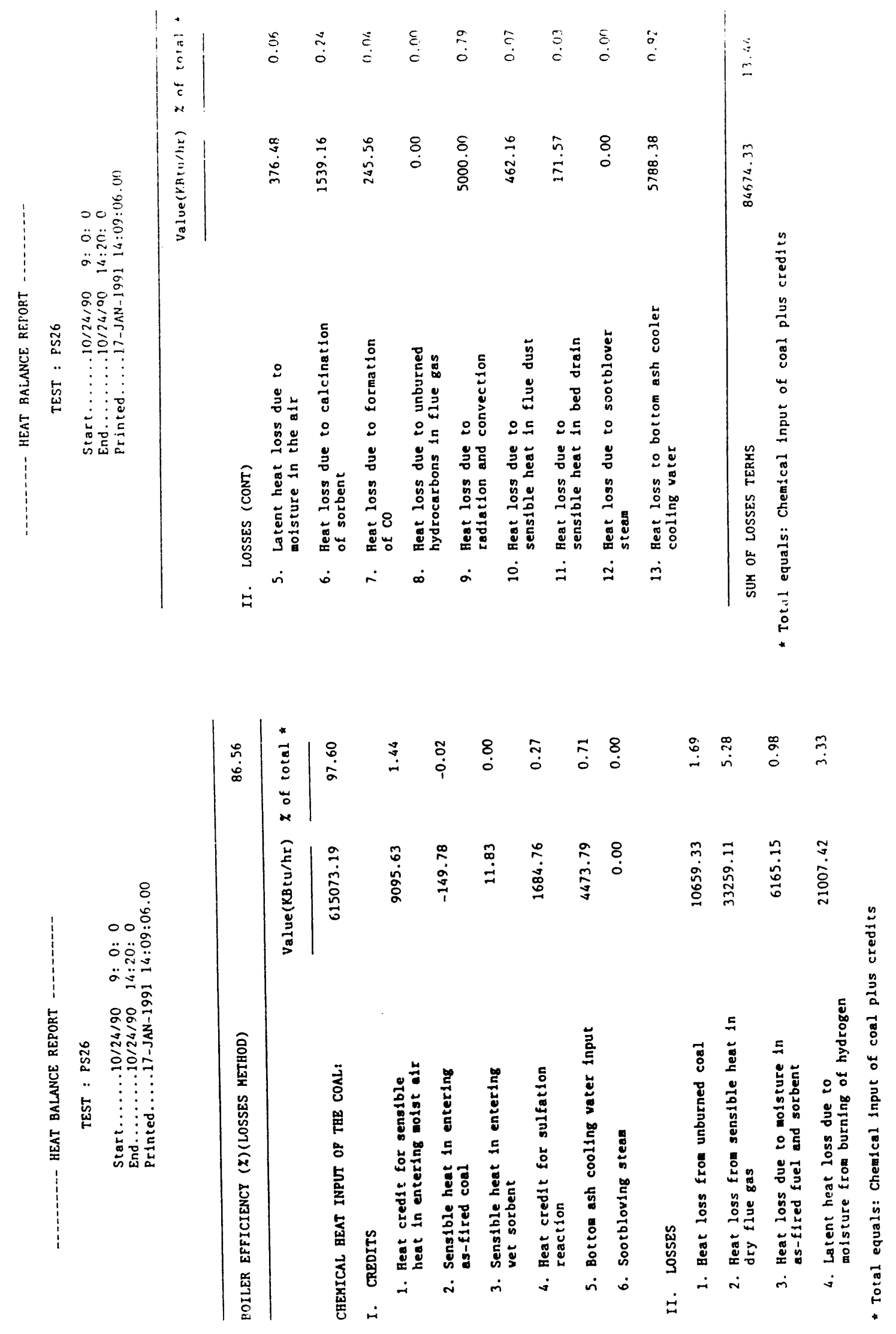




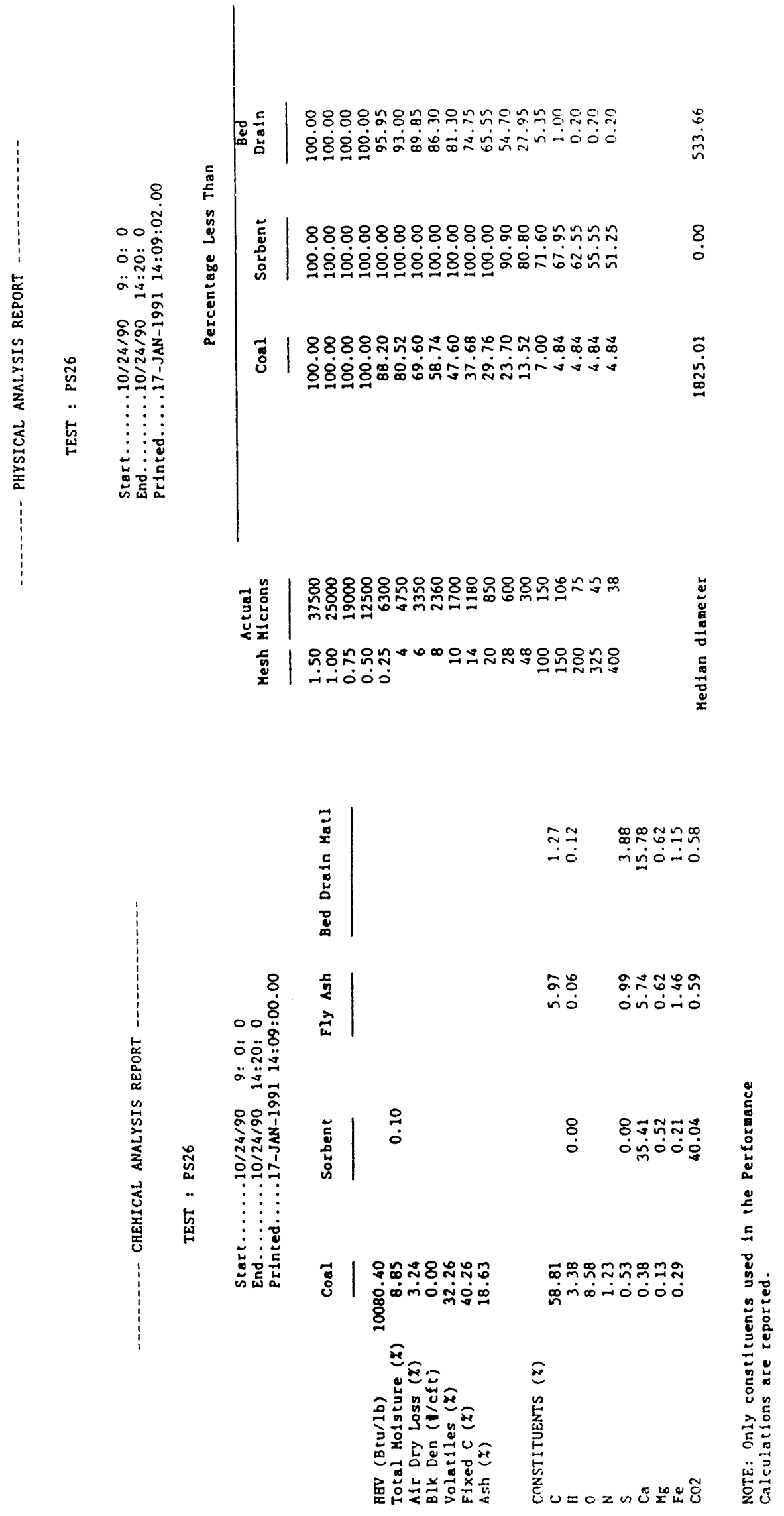



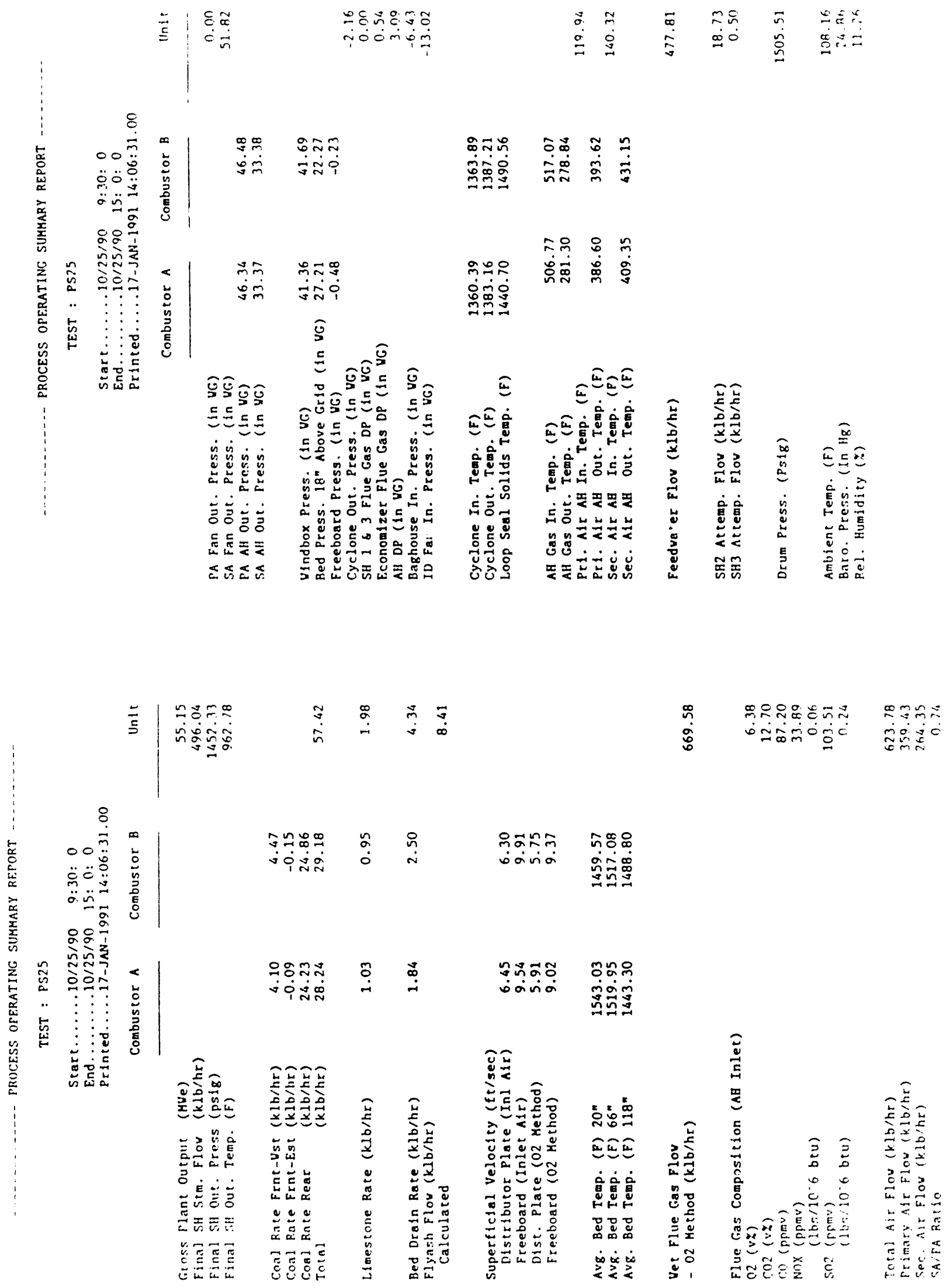


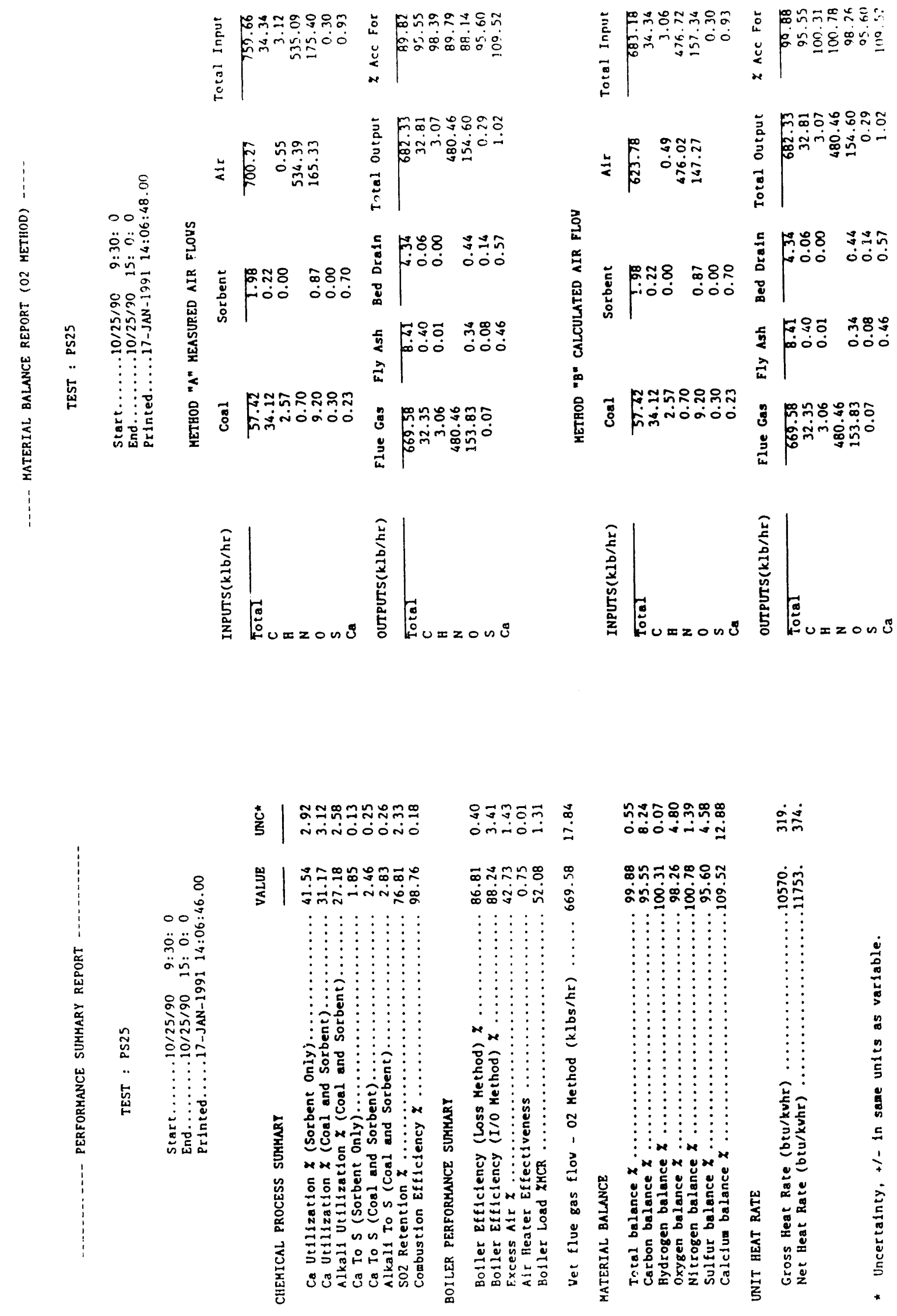




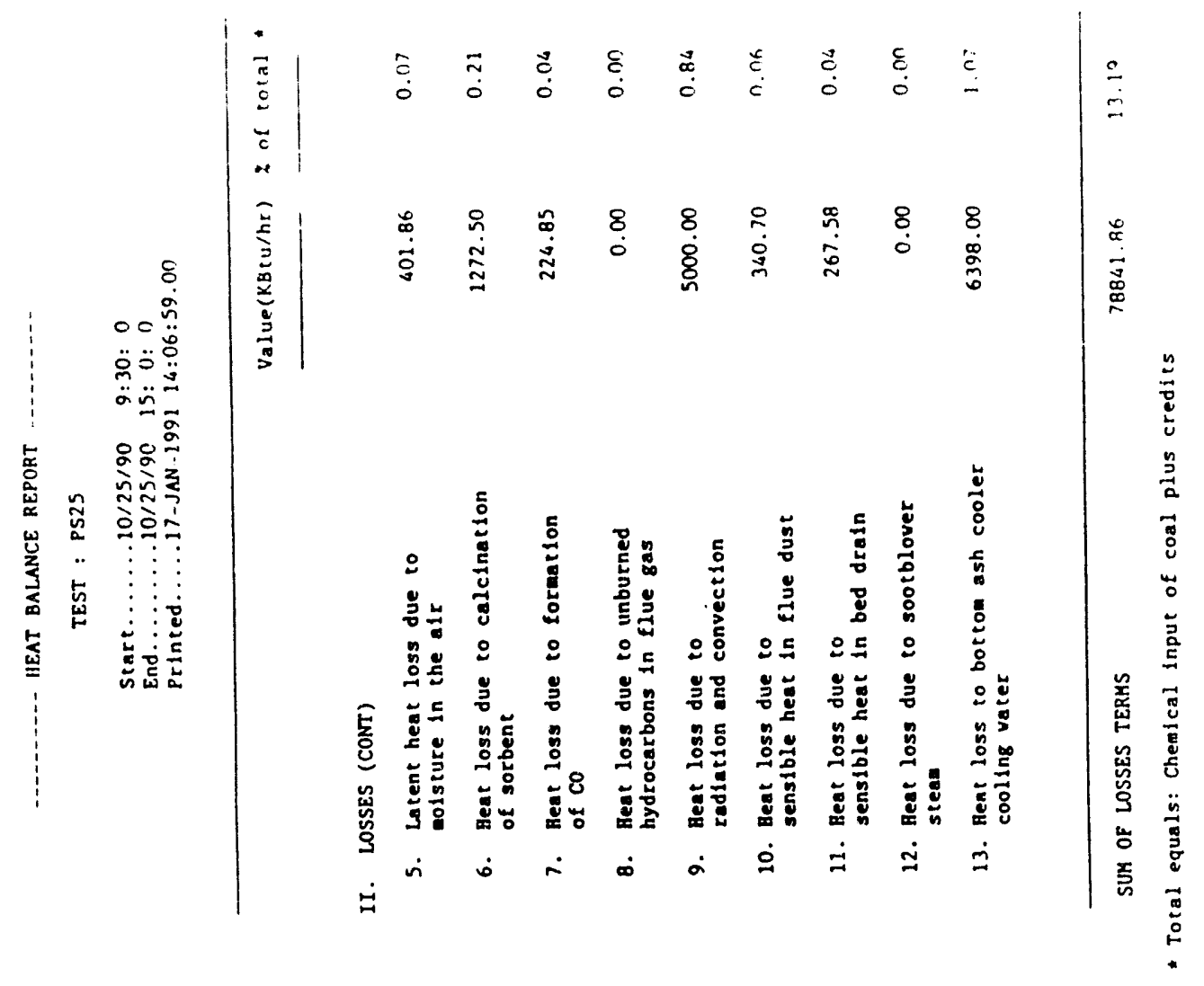

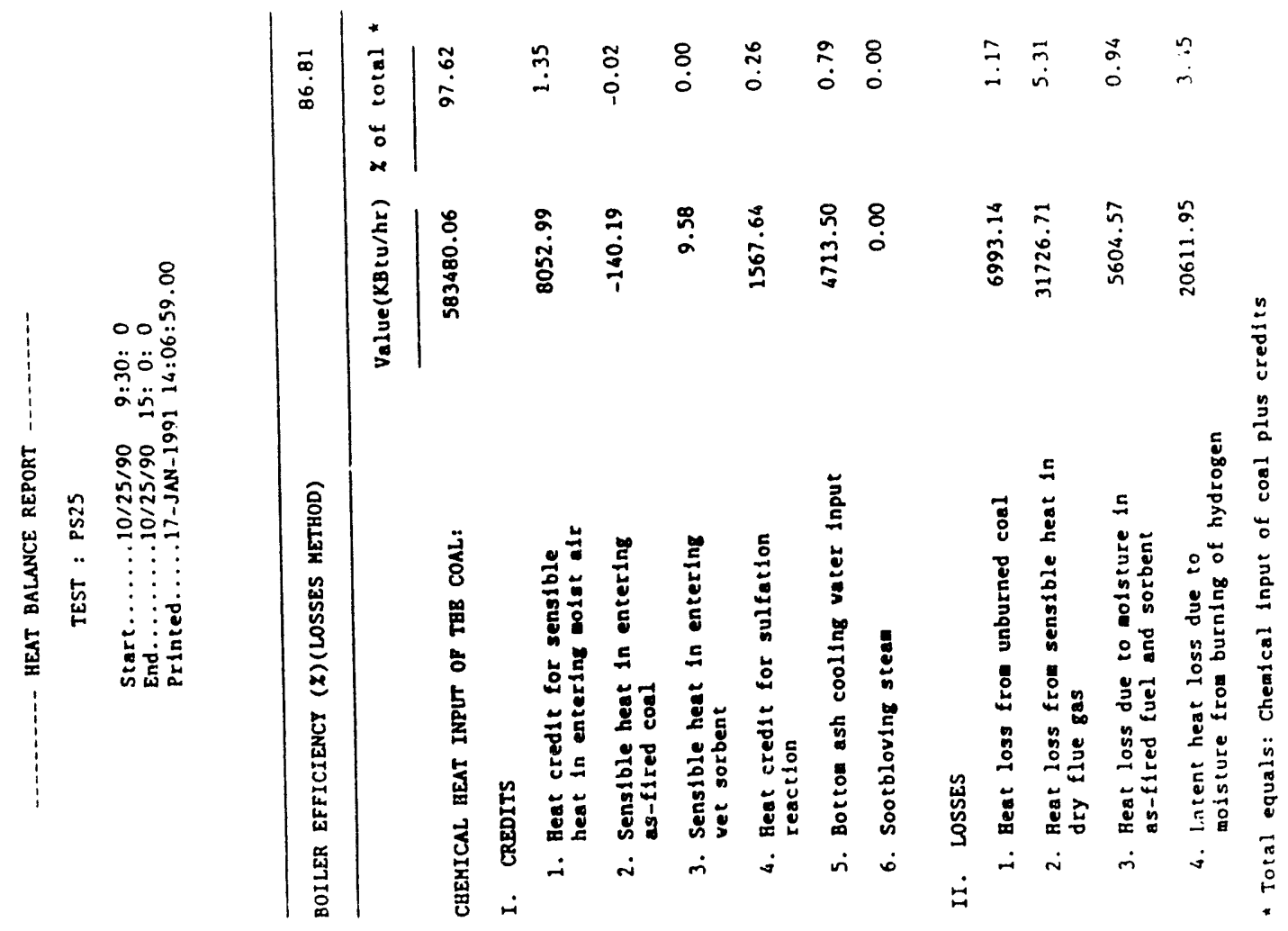



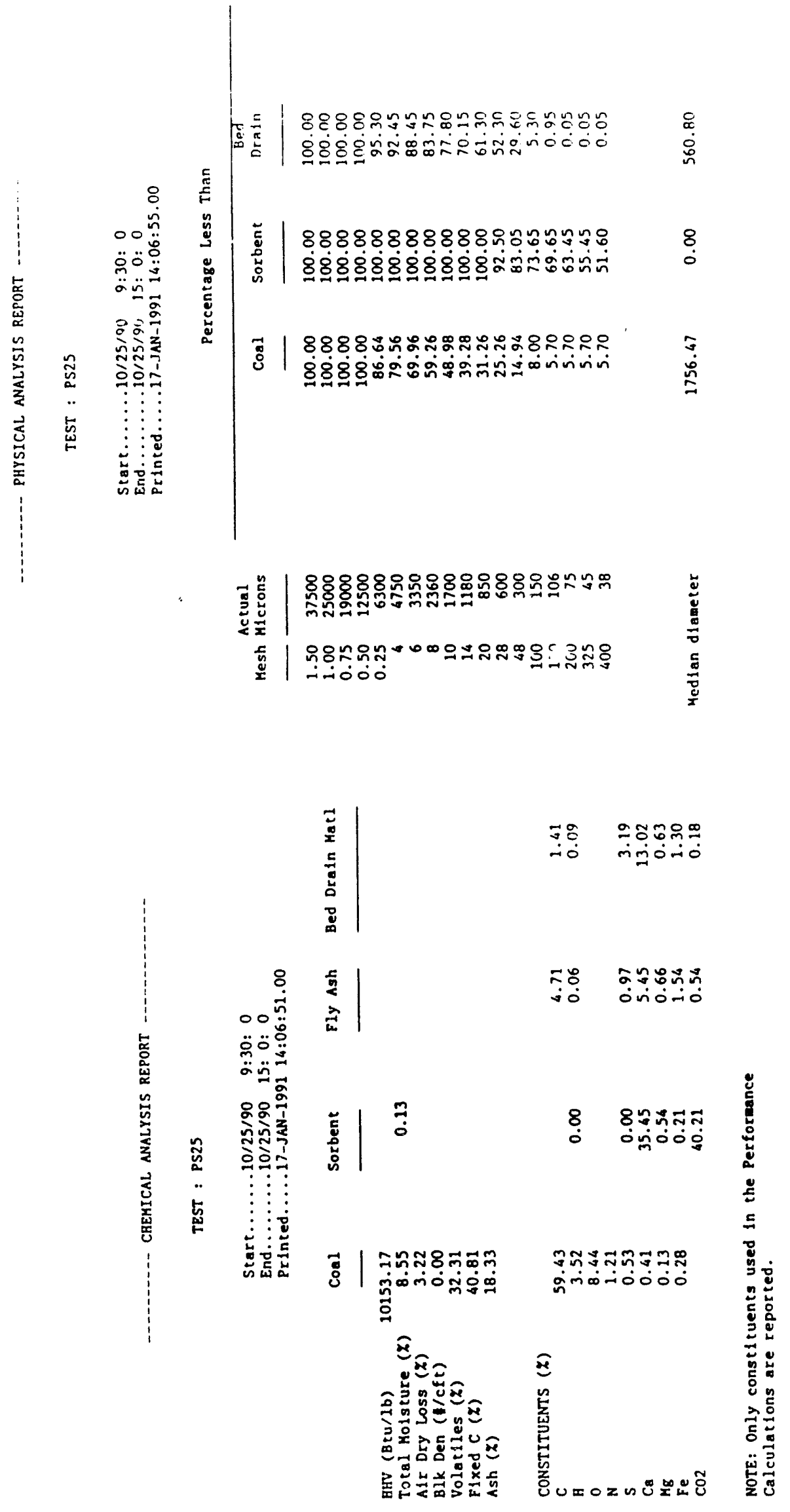

A-319 

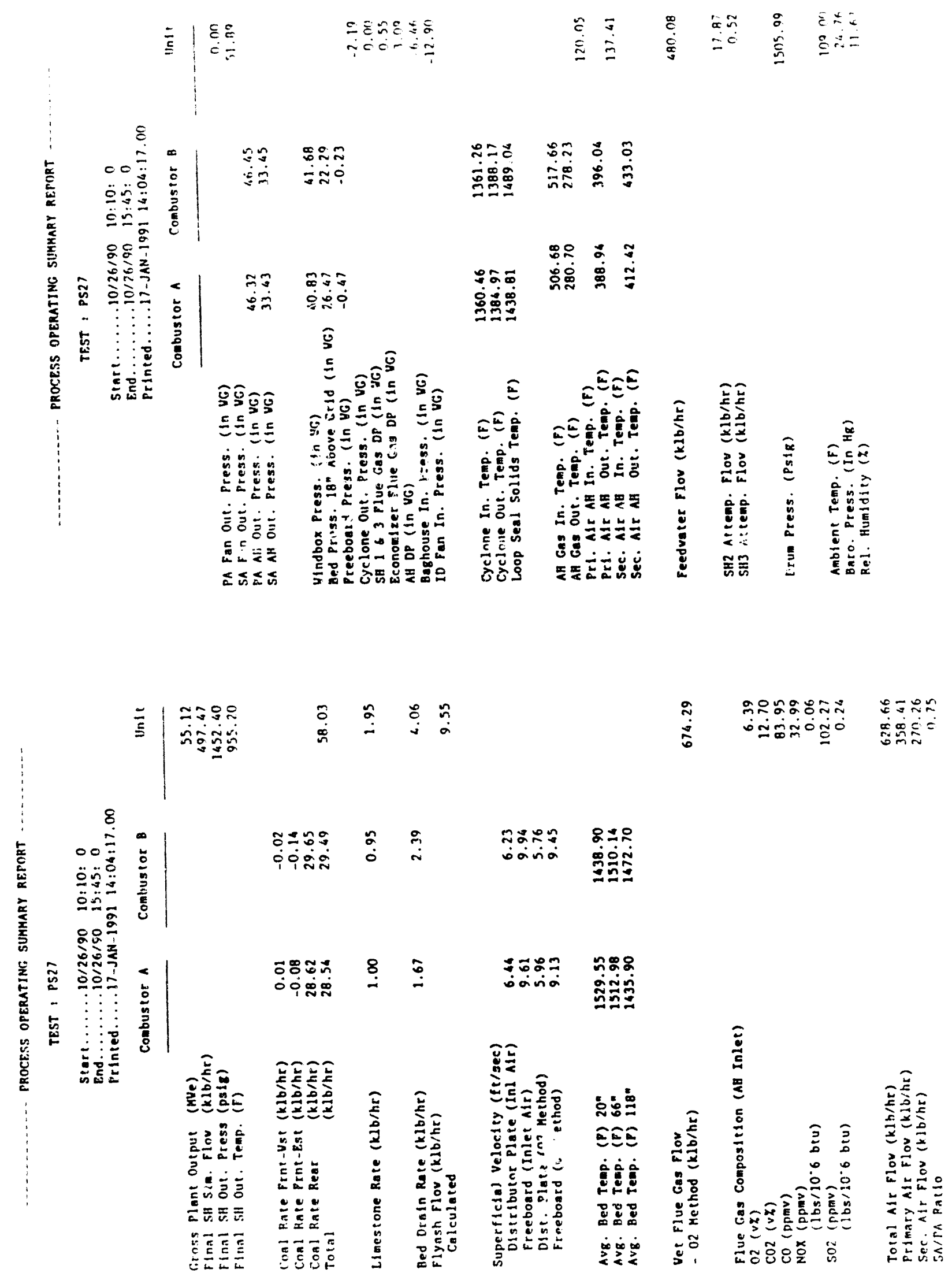


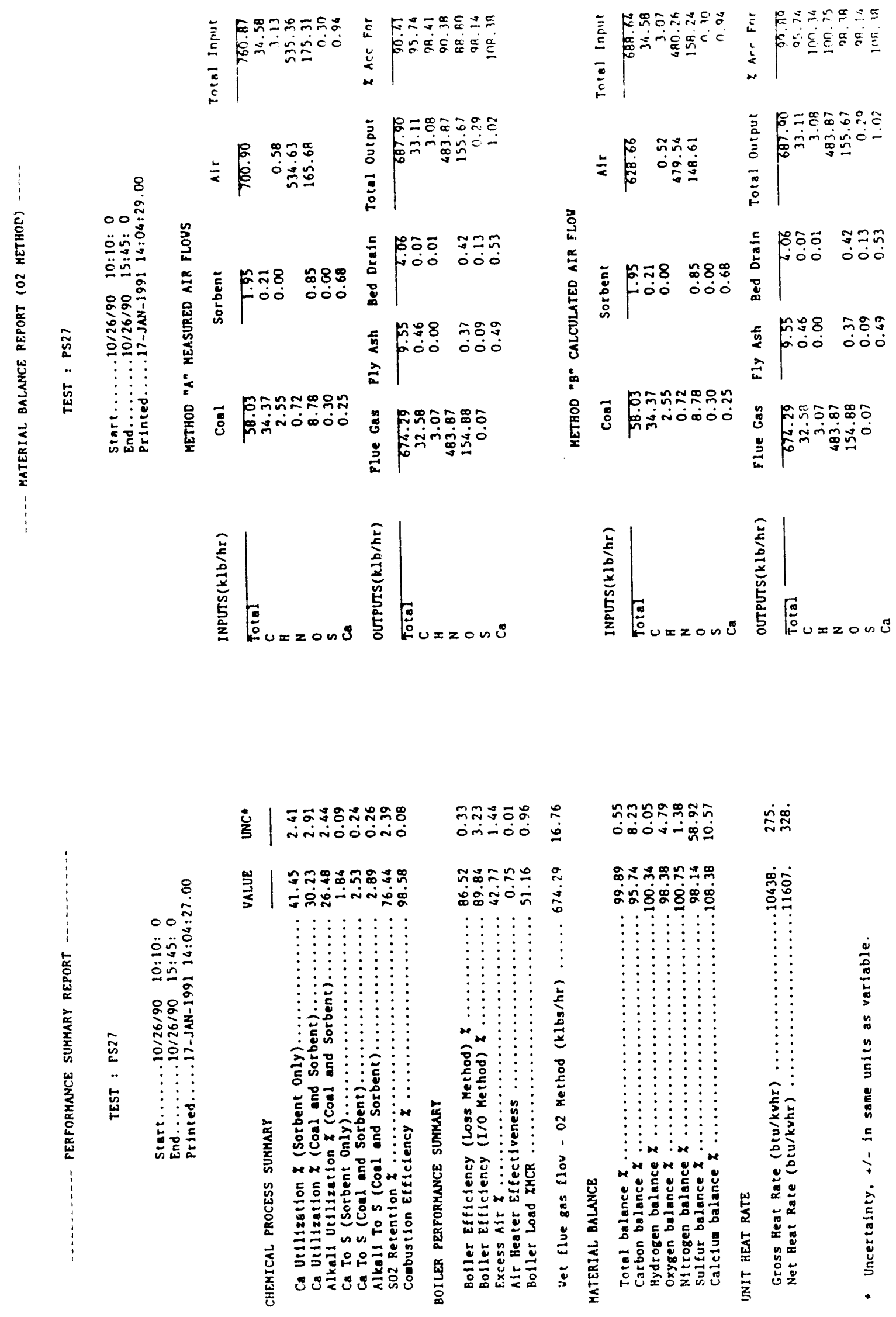




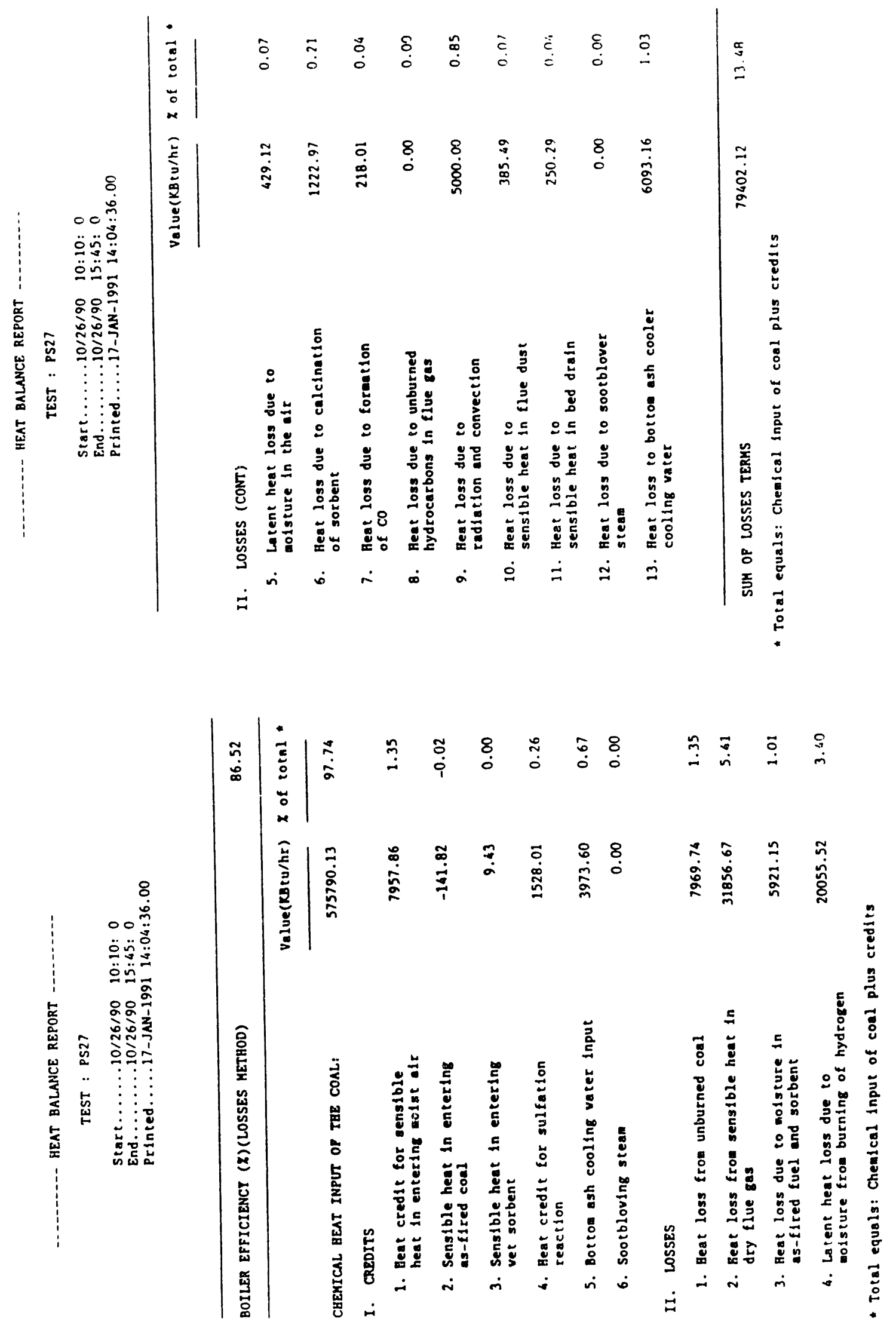




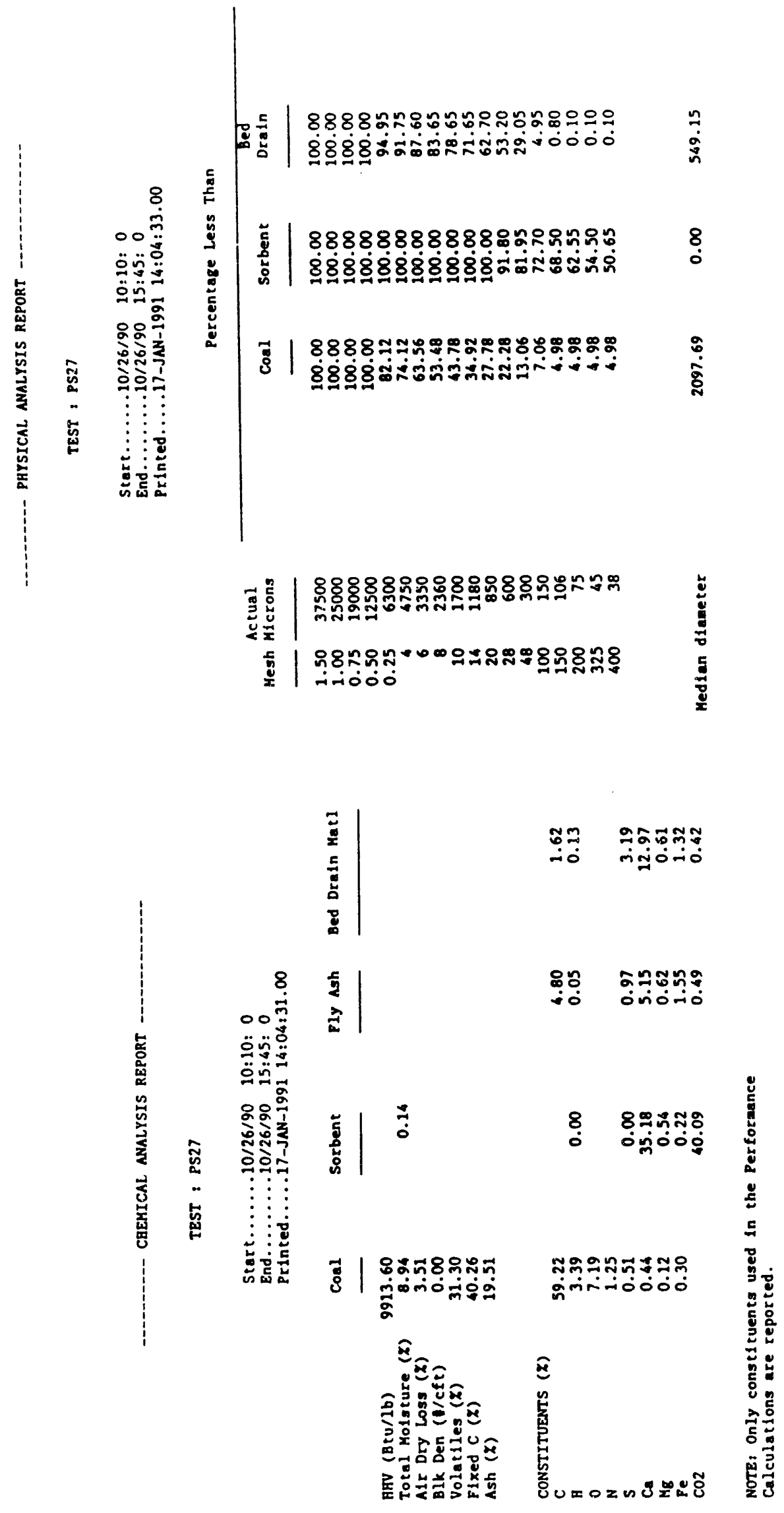

A -323 

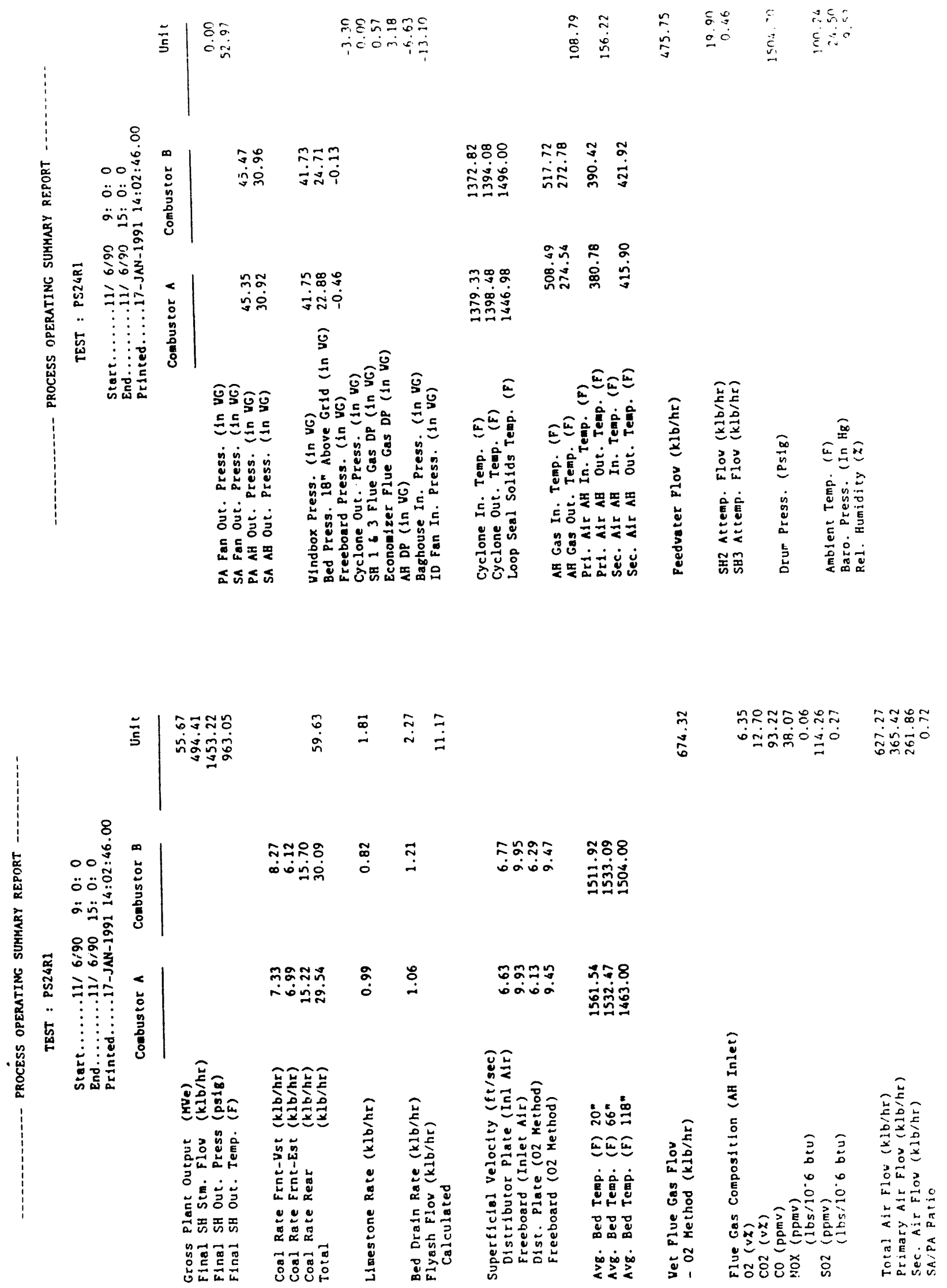


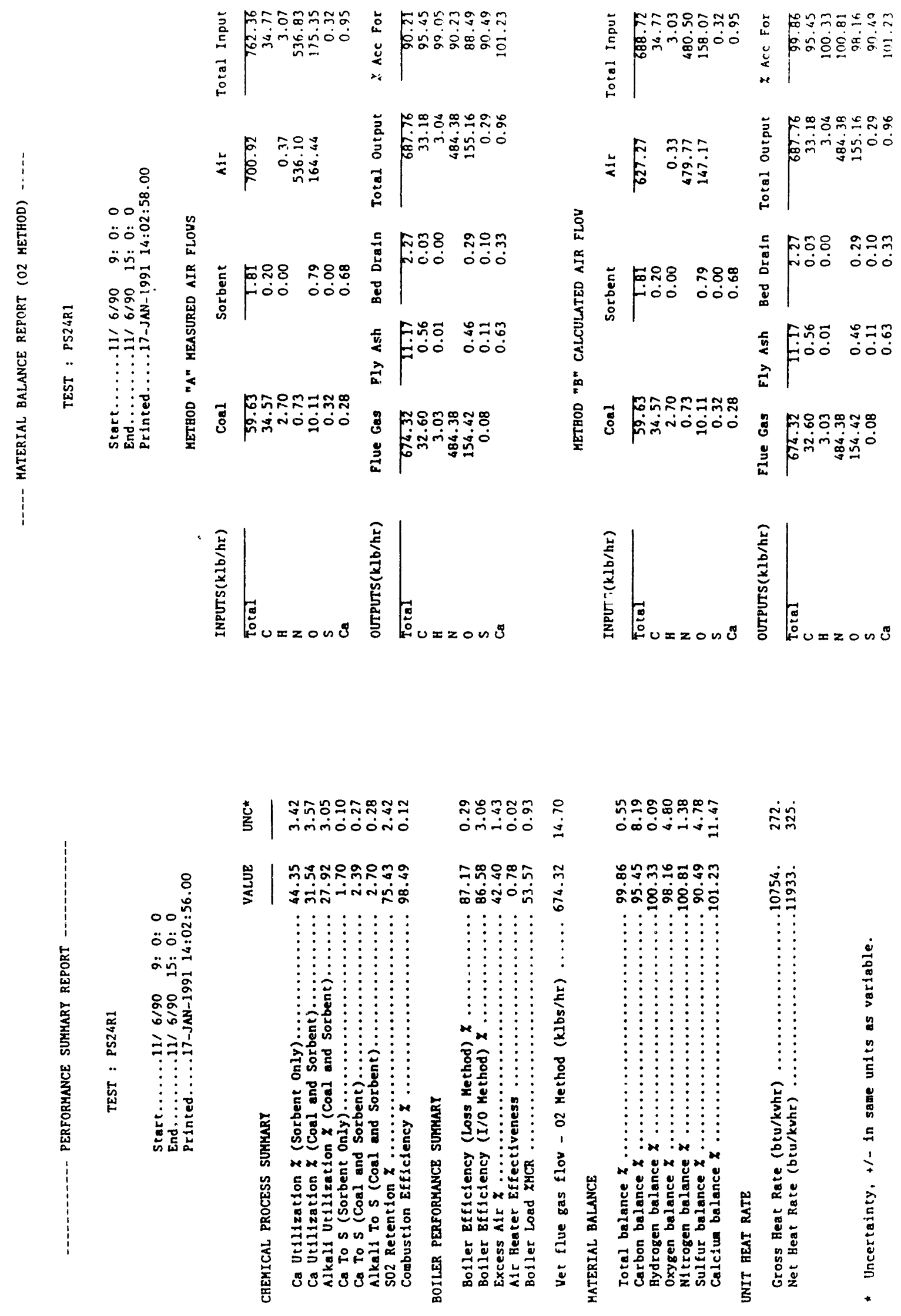




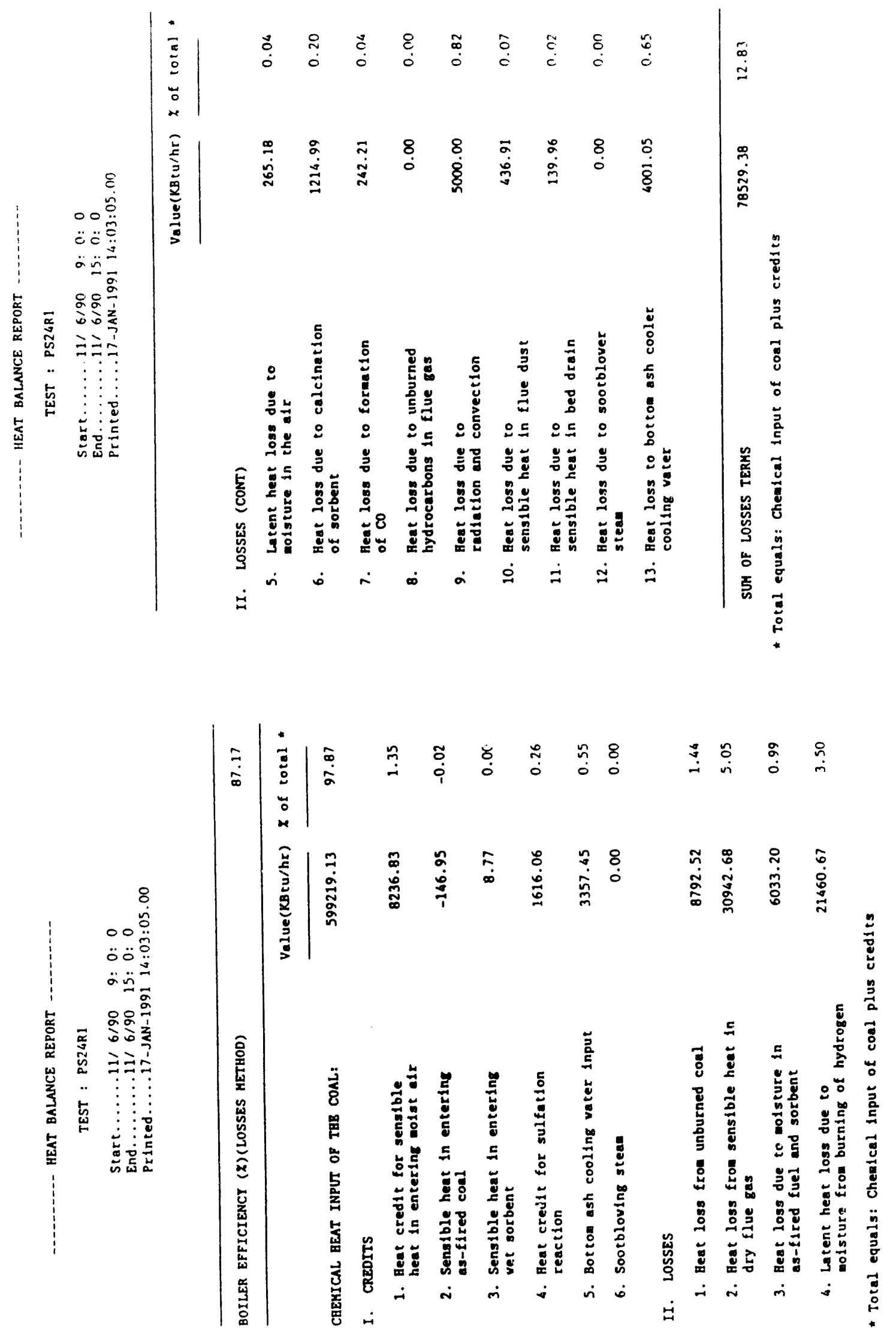



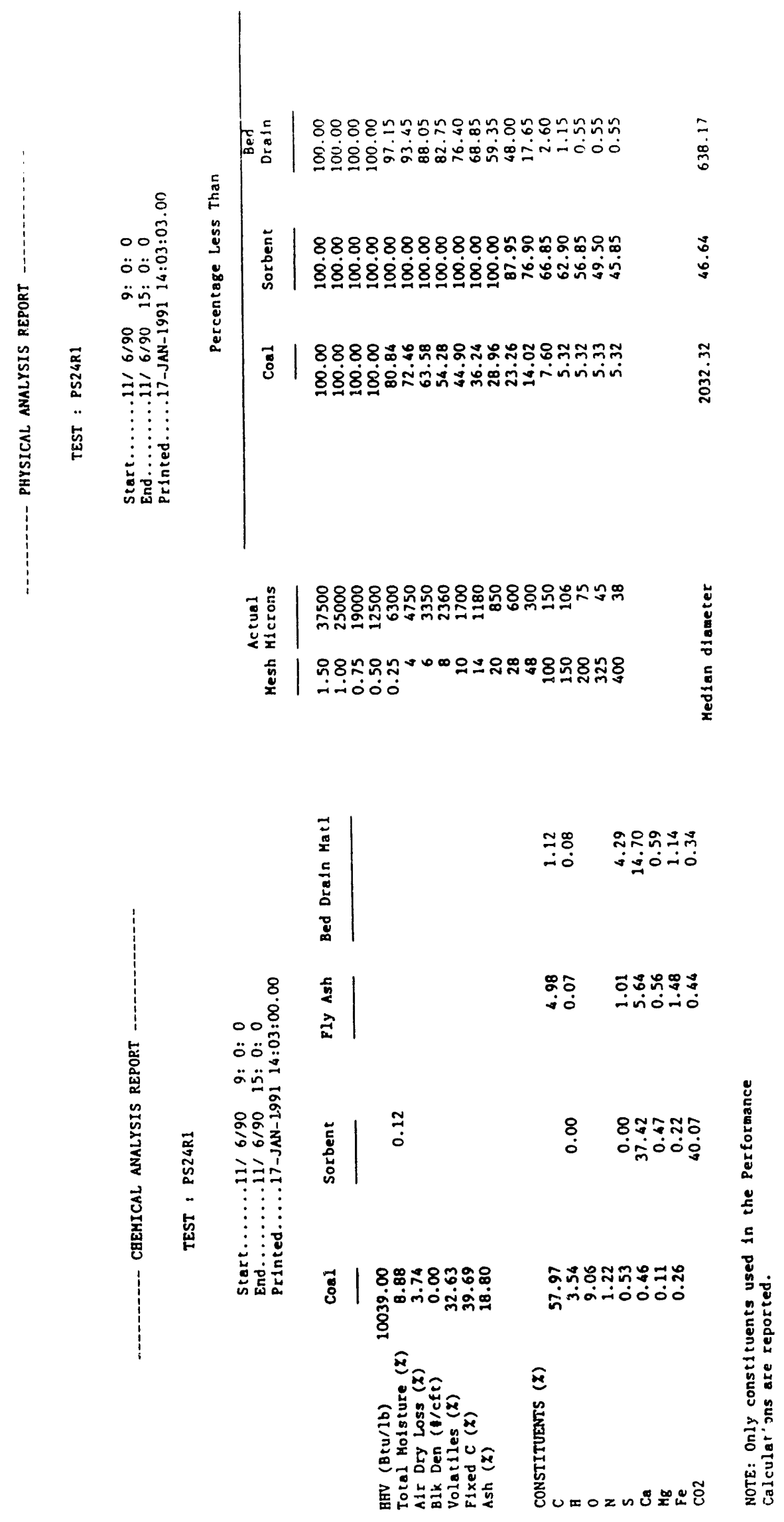

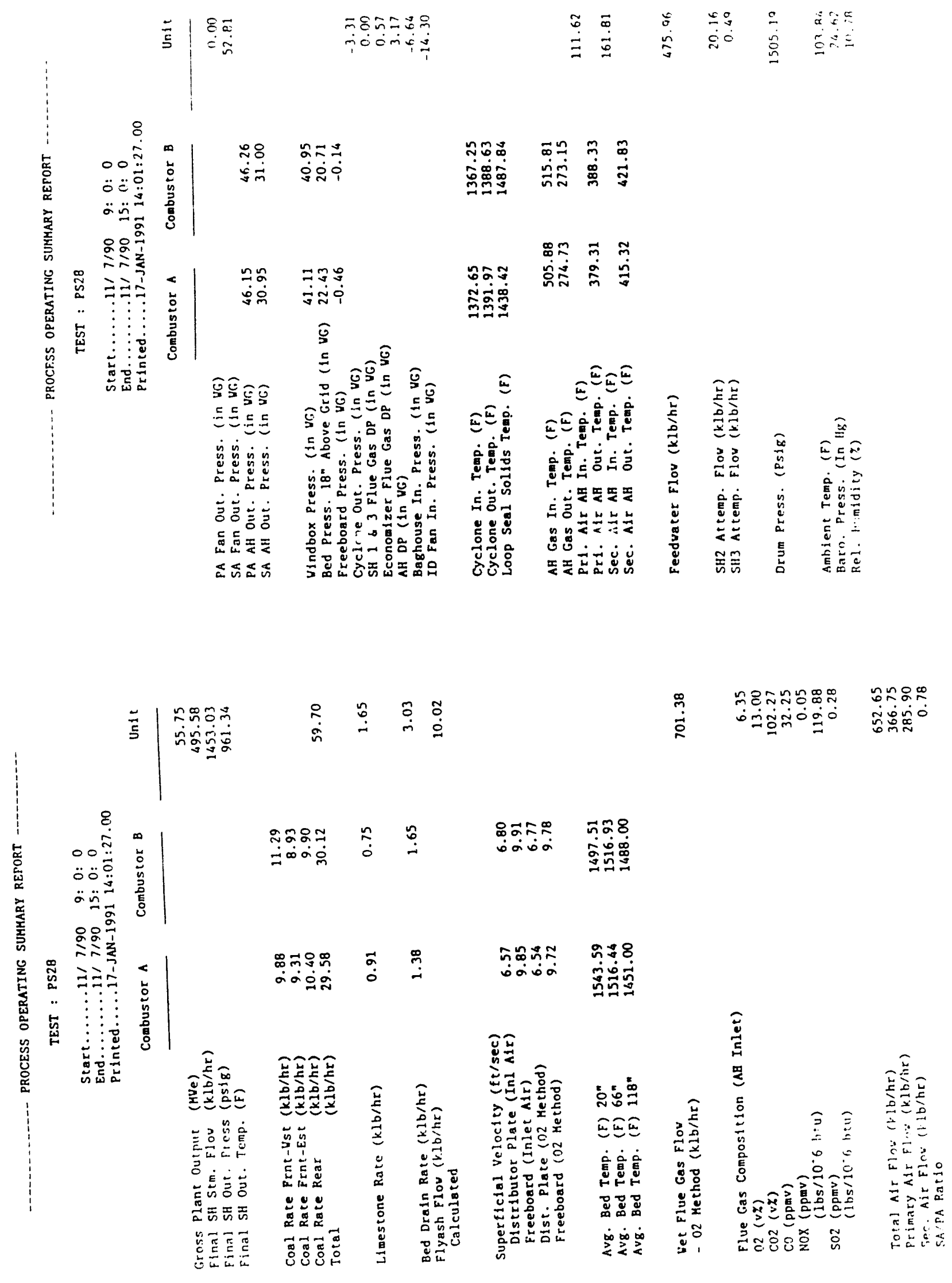


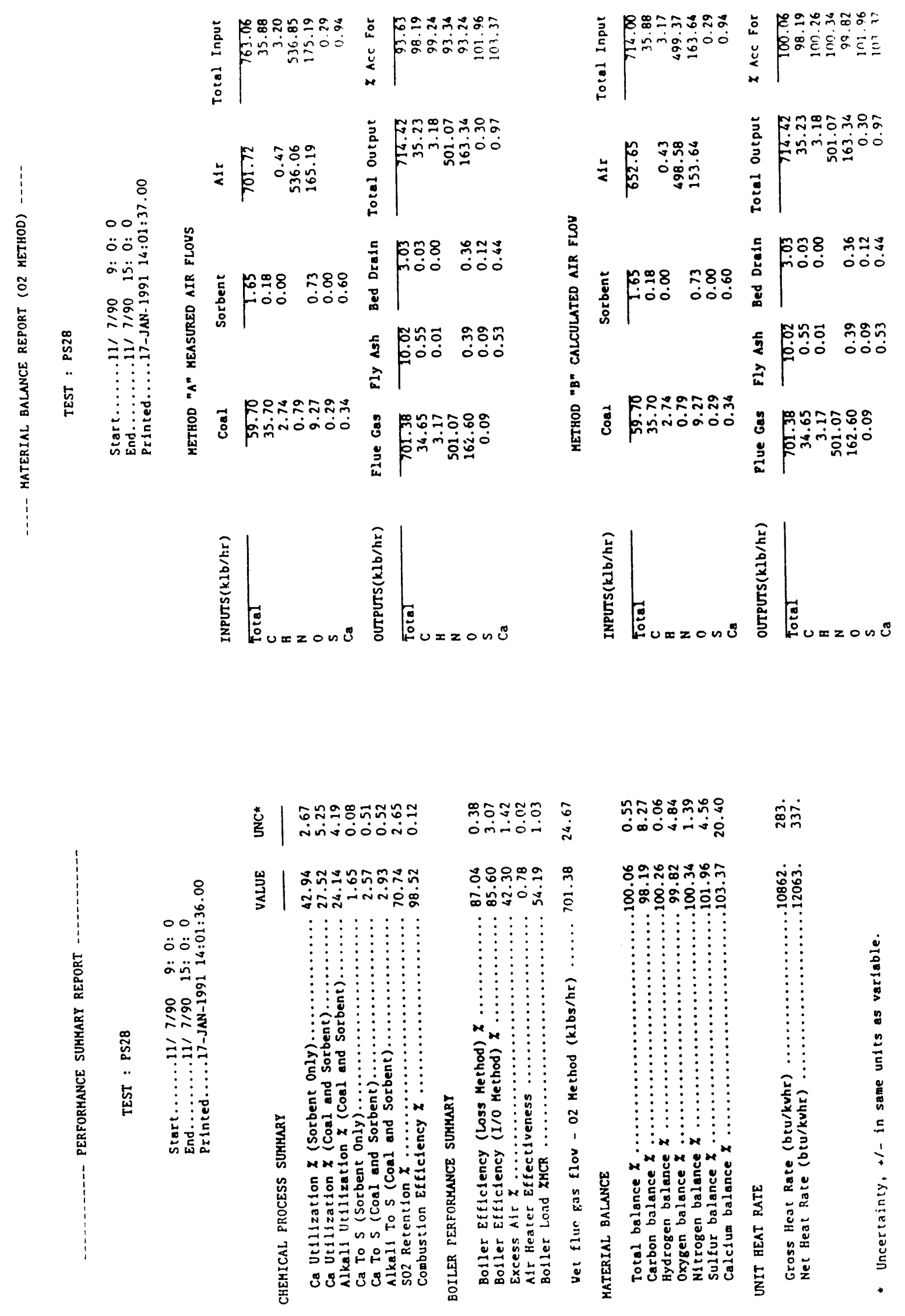




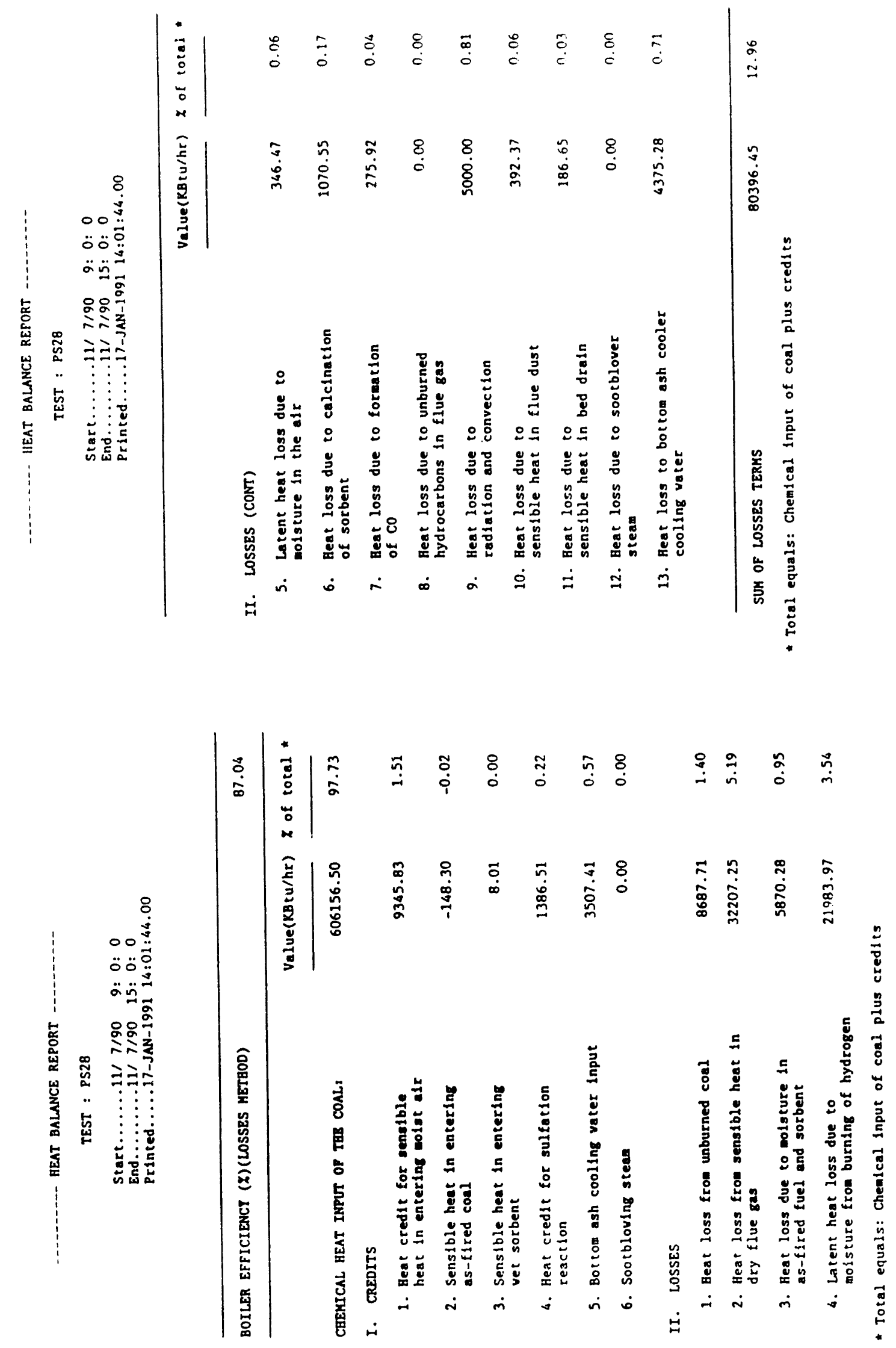



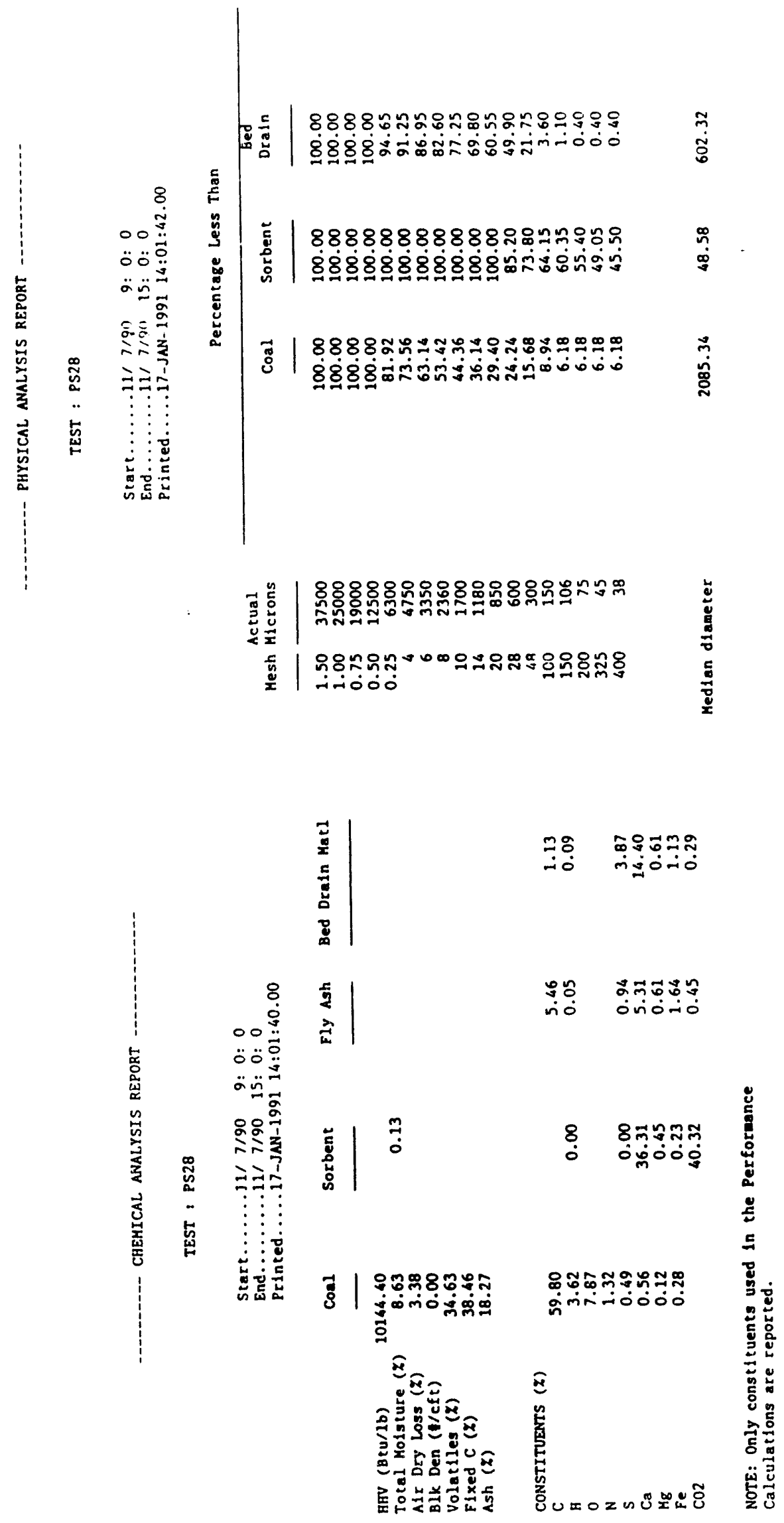

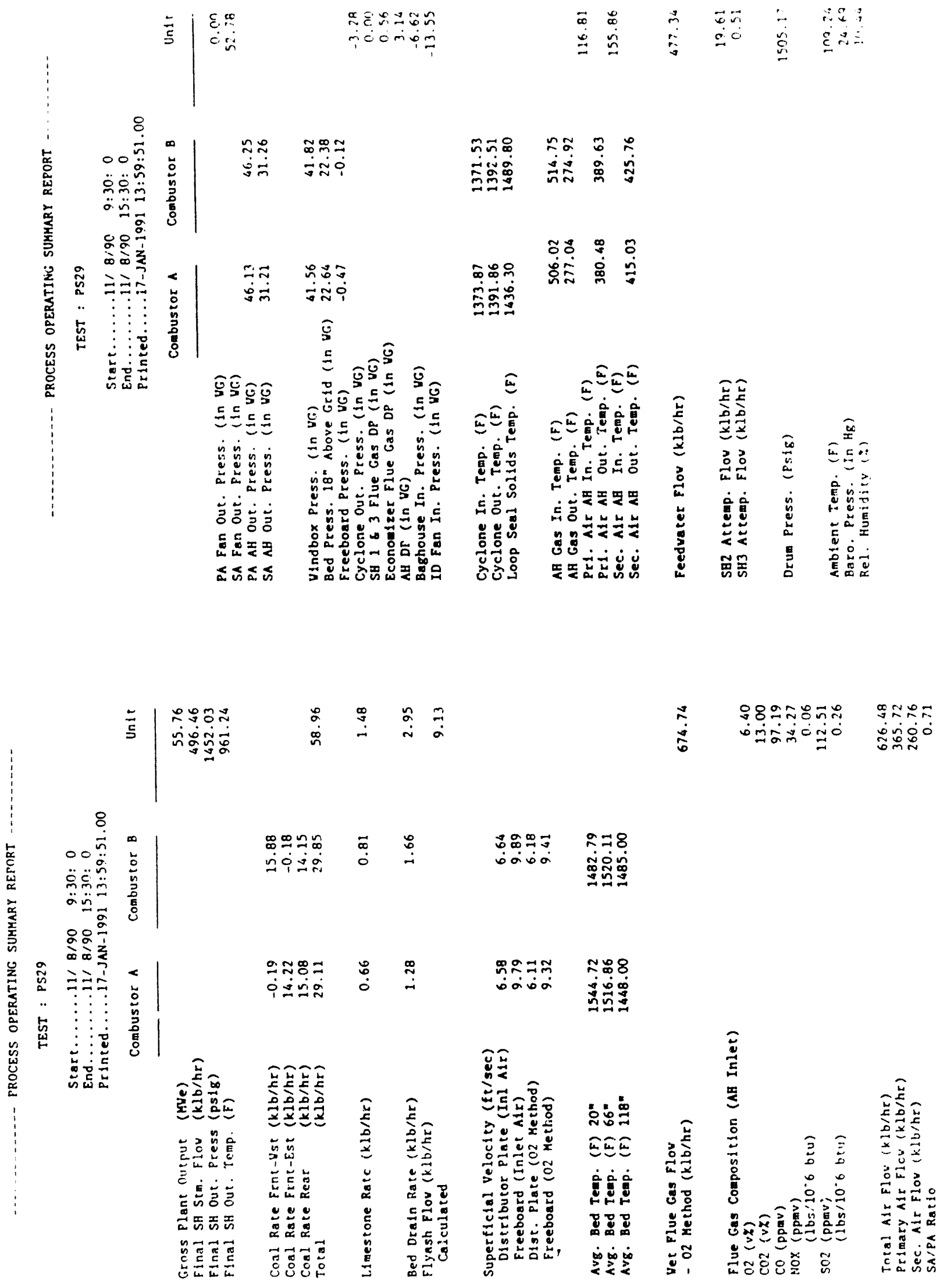


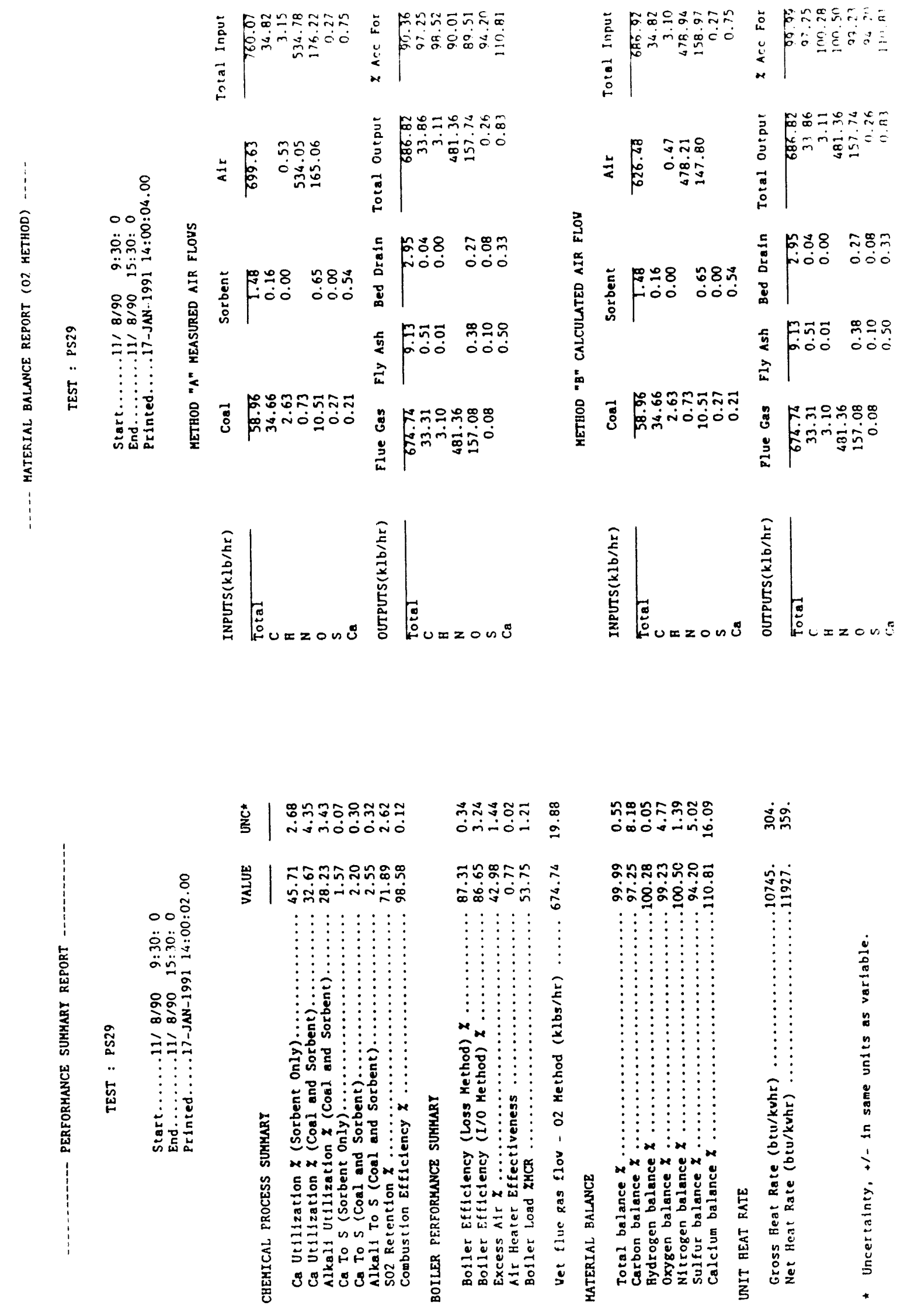




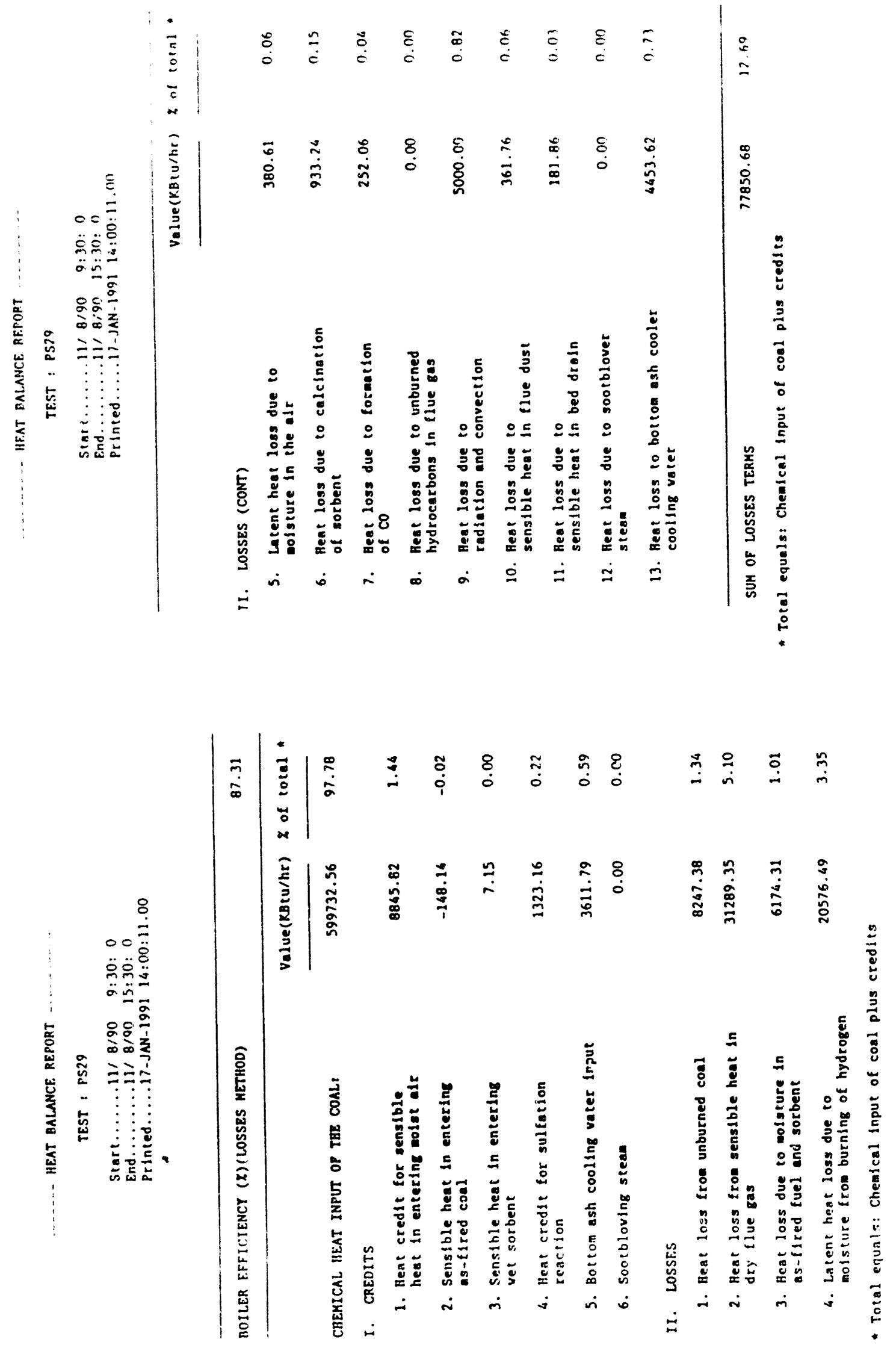



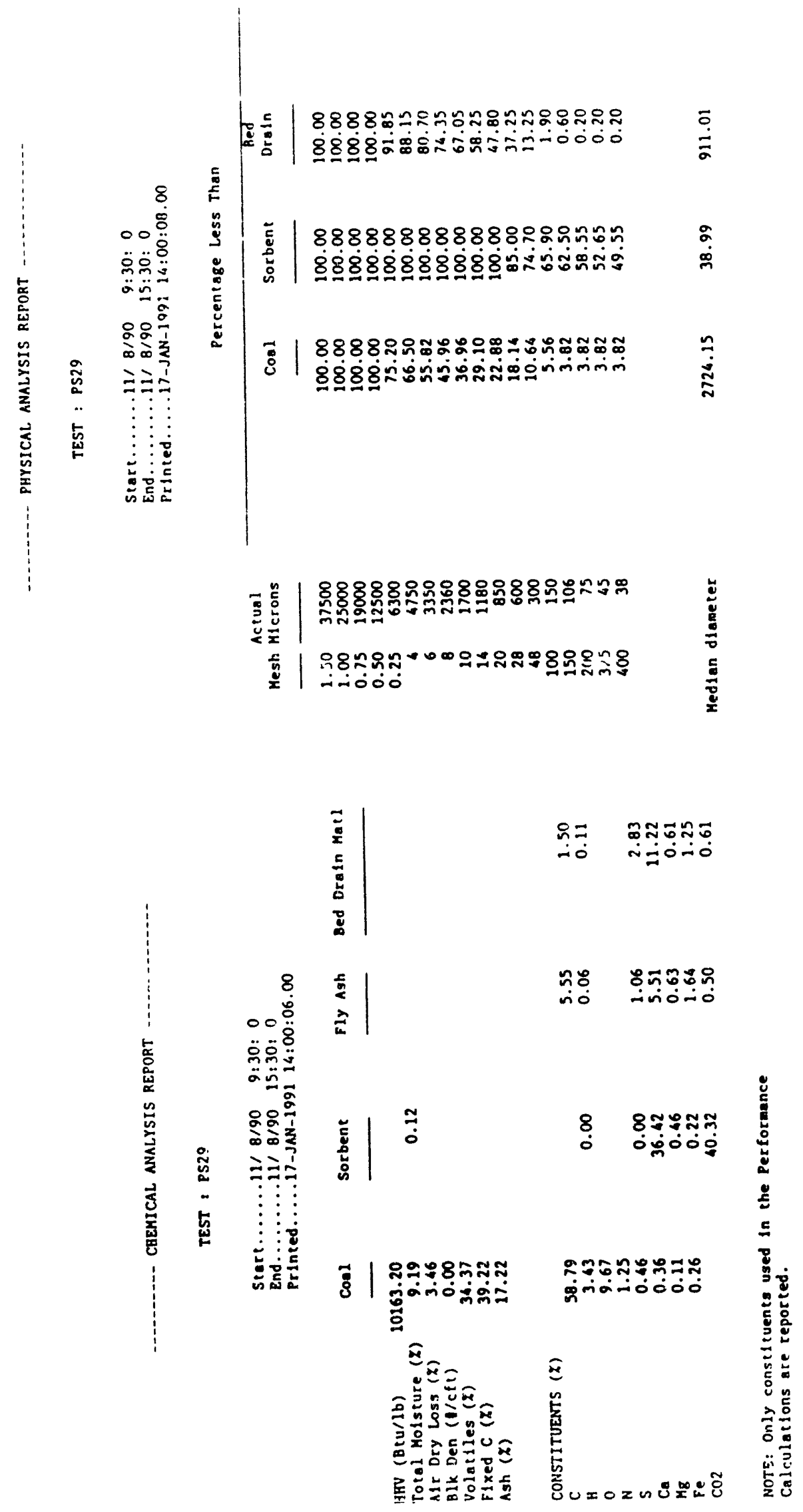

A-335 

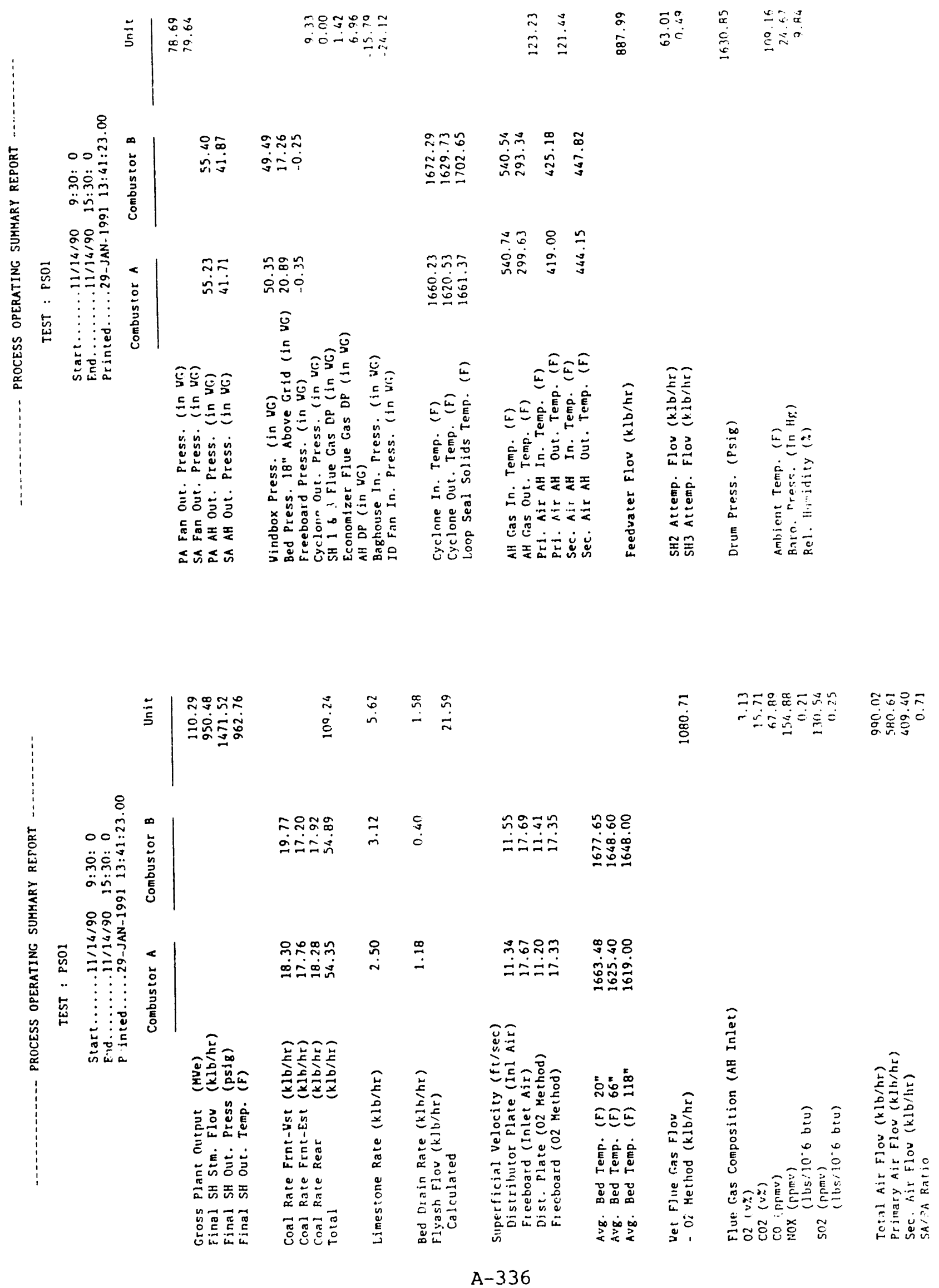


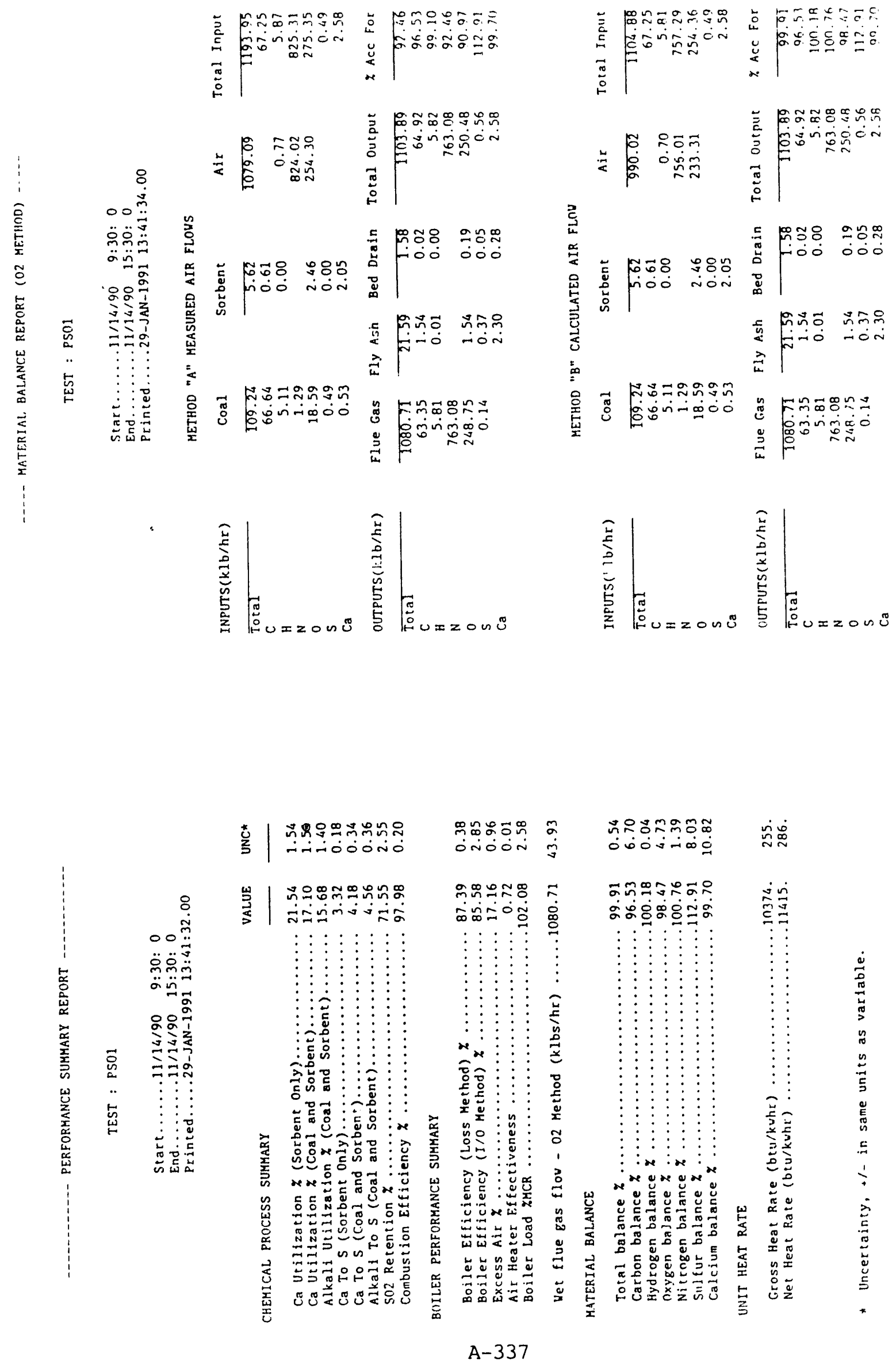



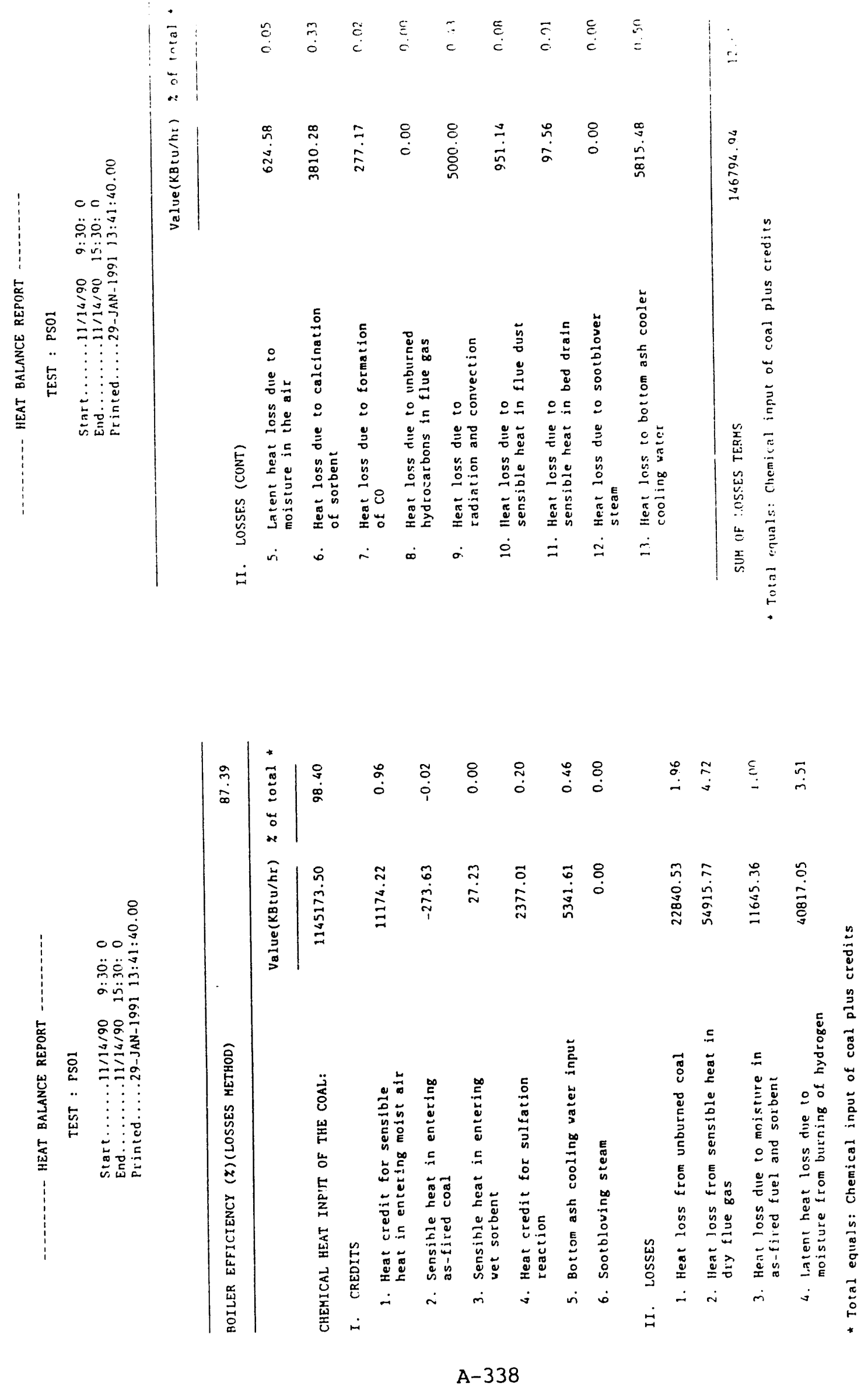


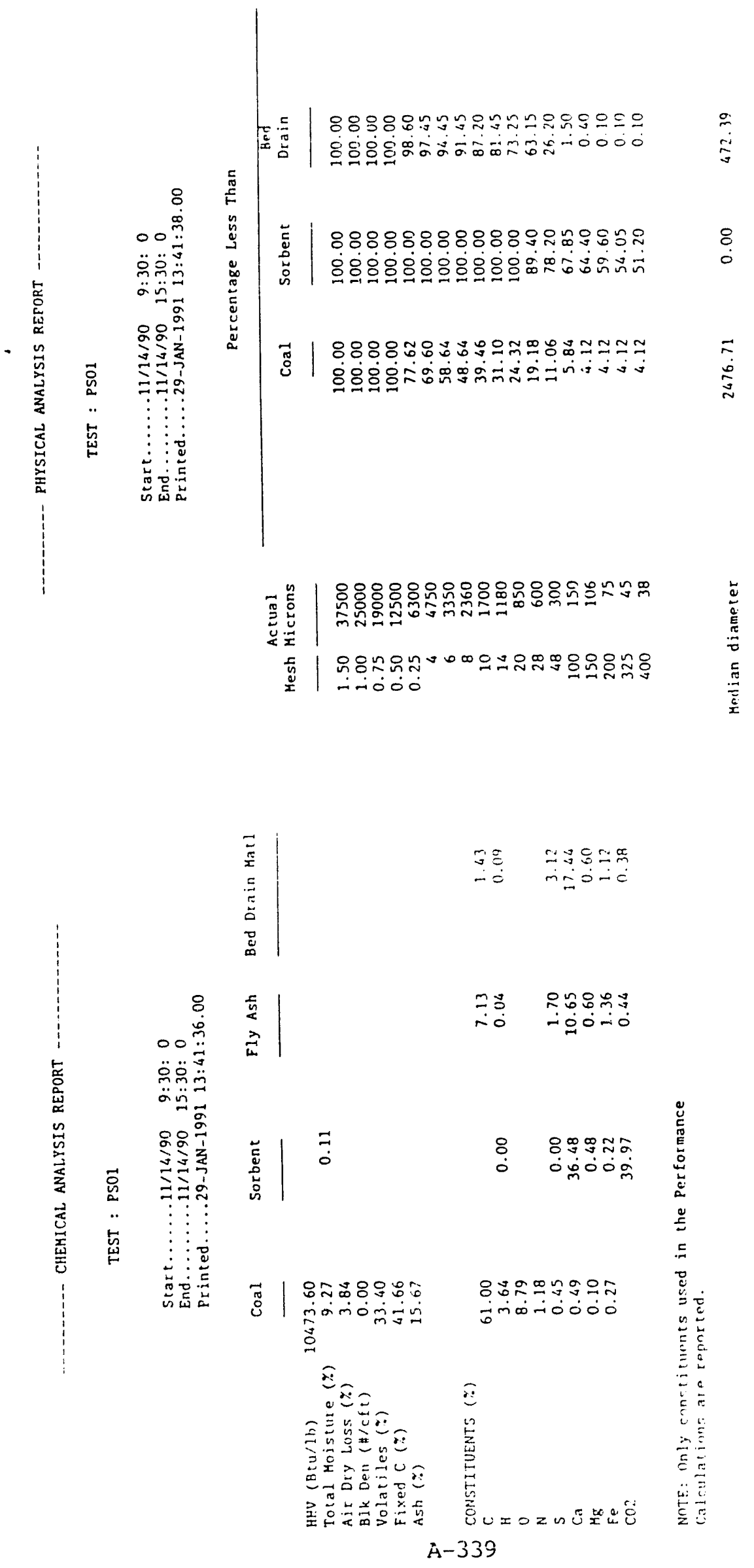



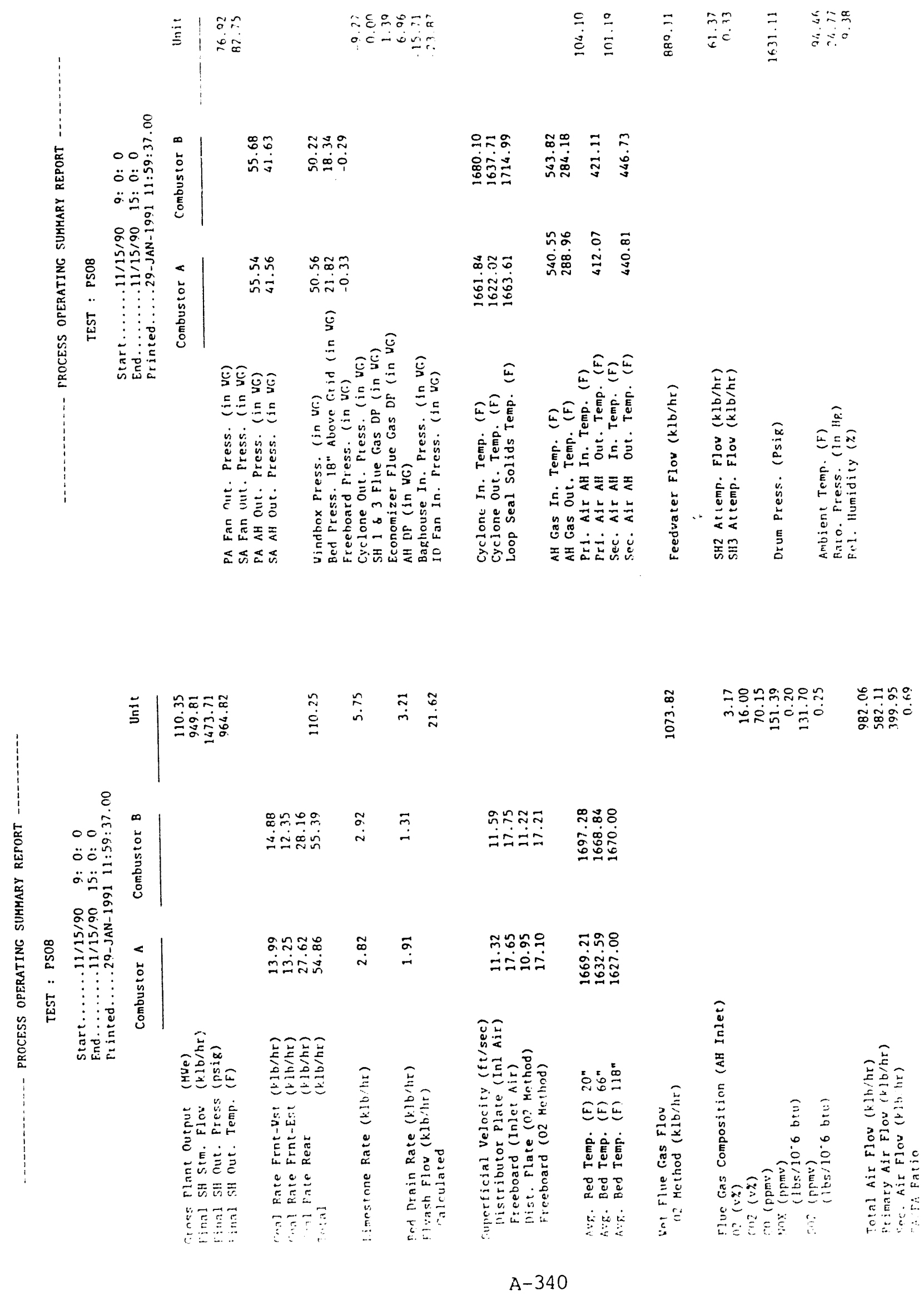


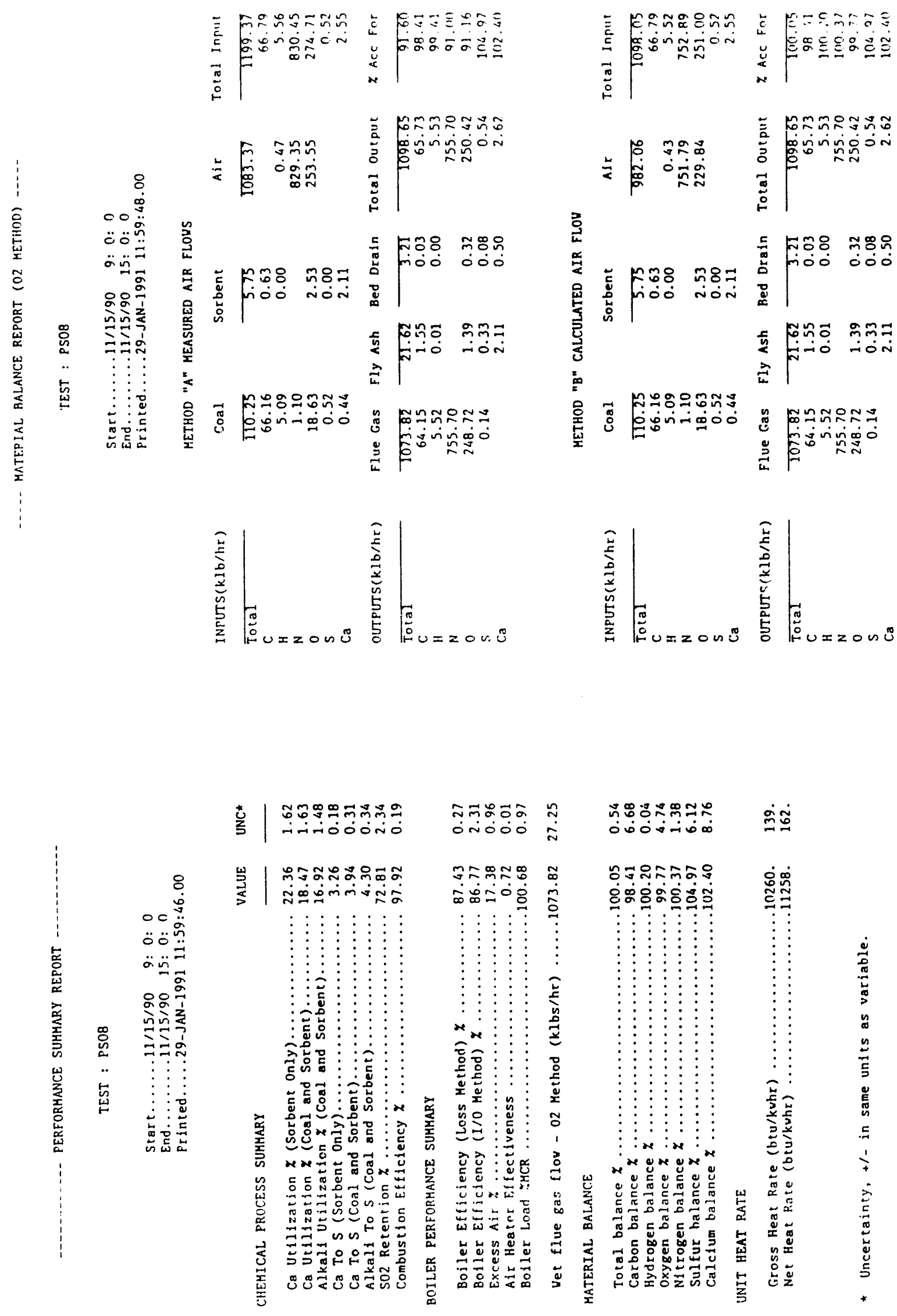




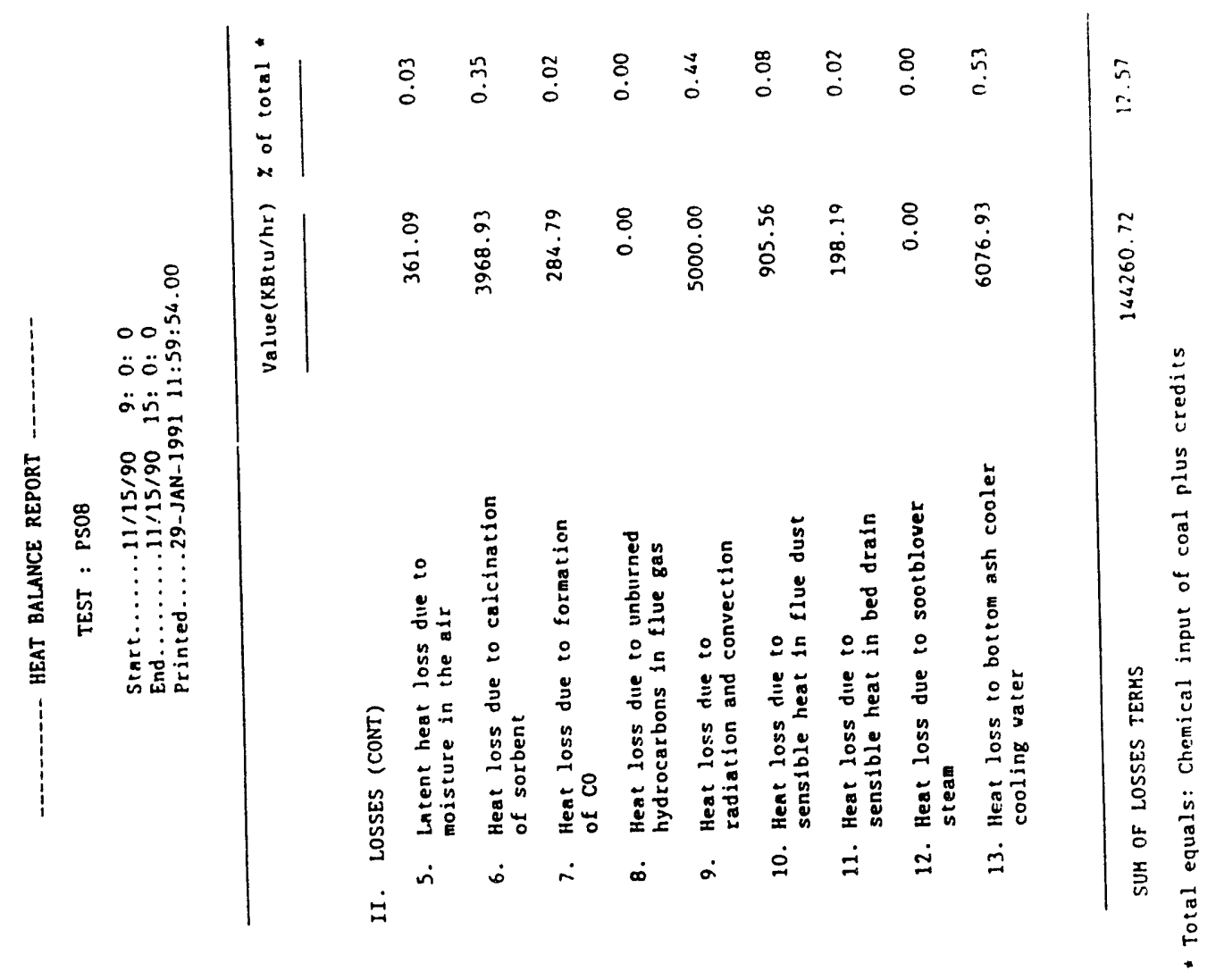

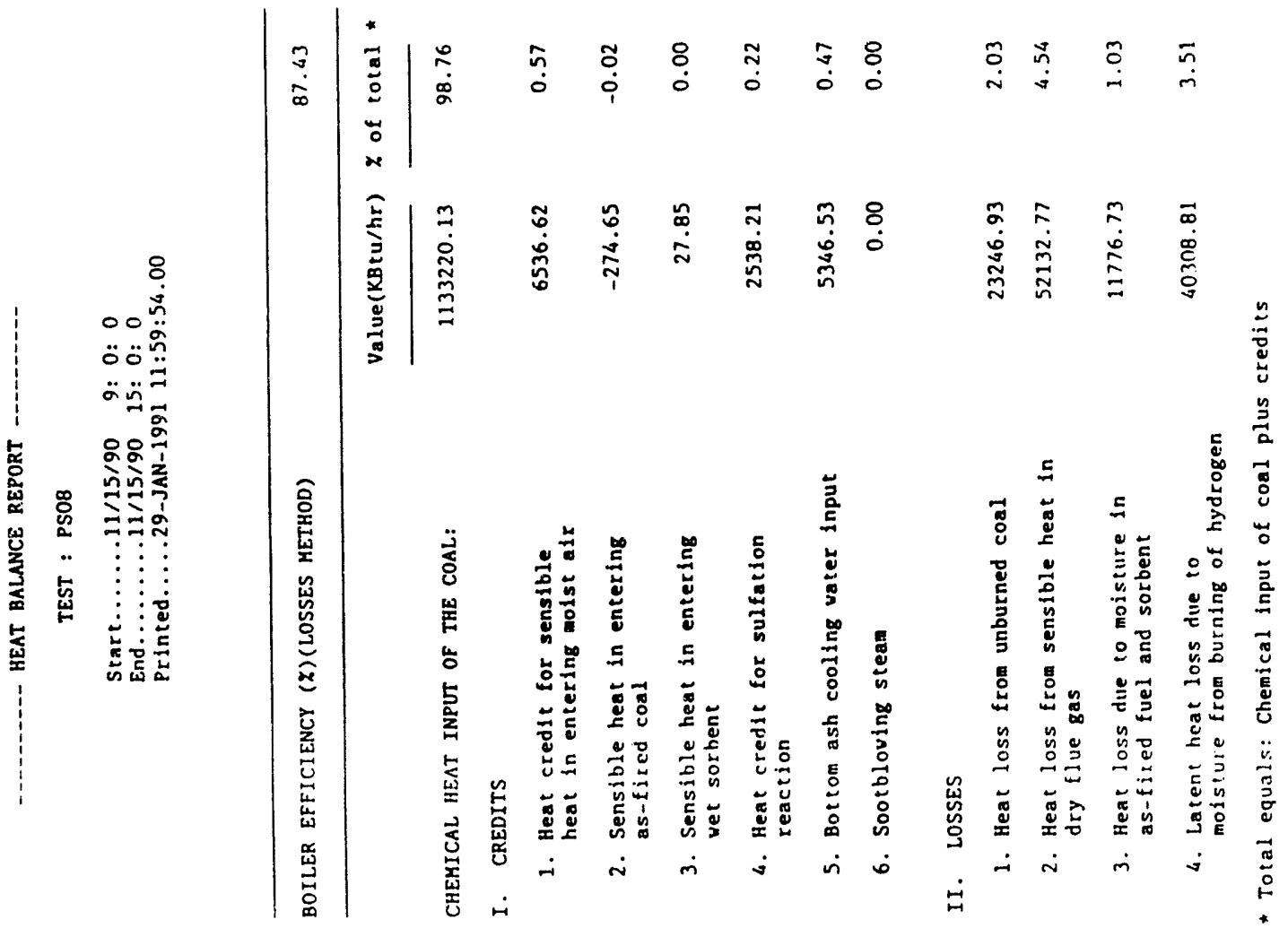



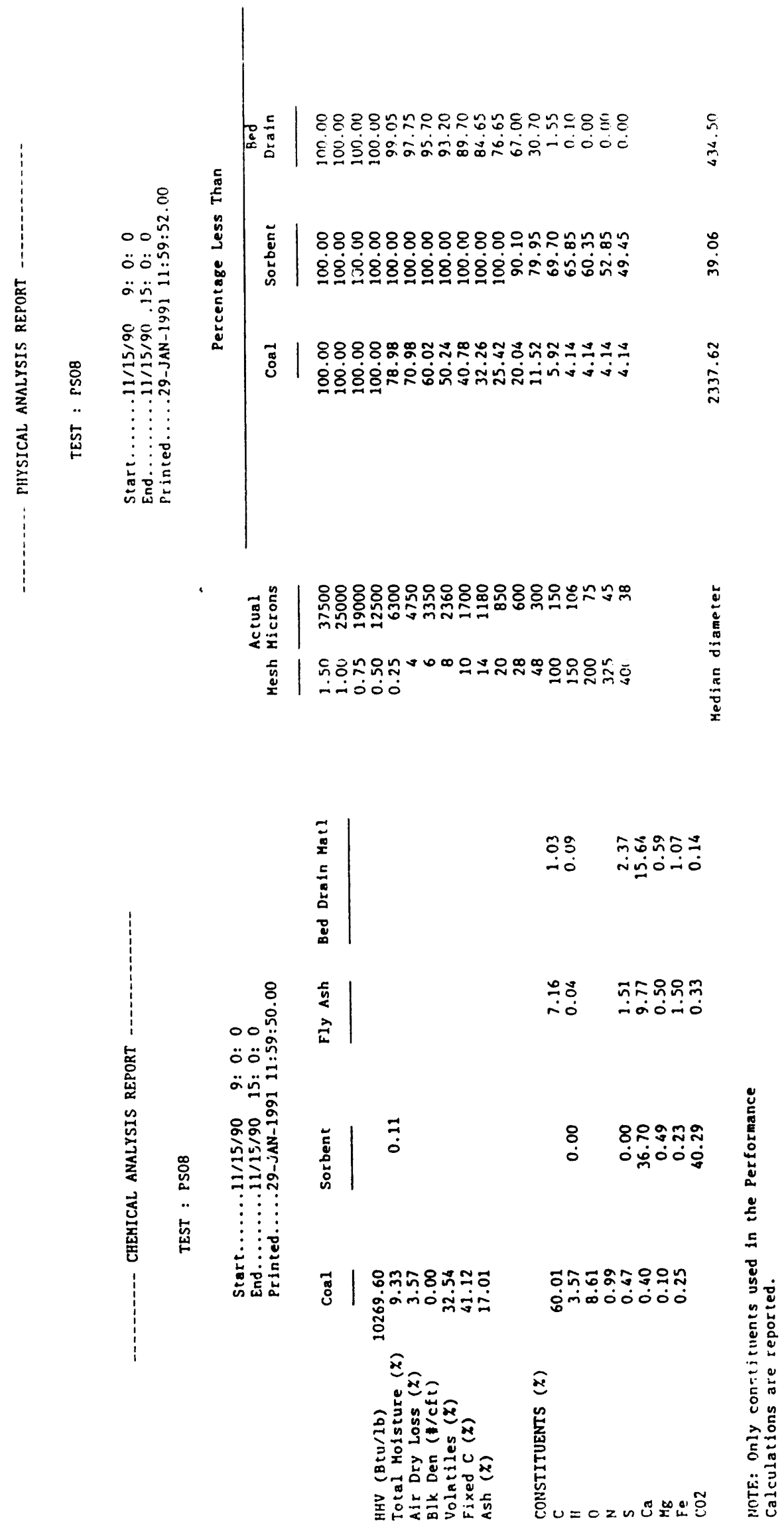

A -343 

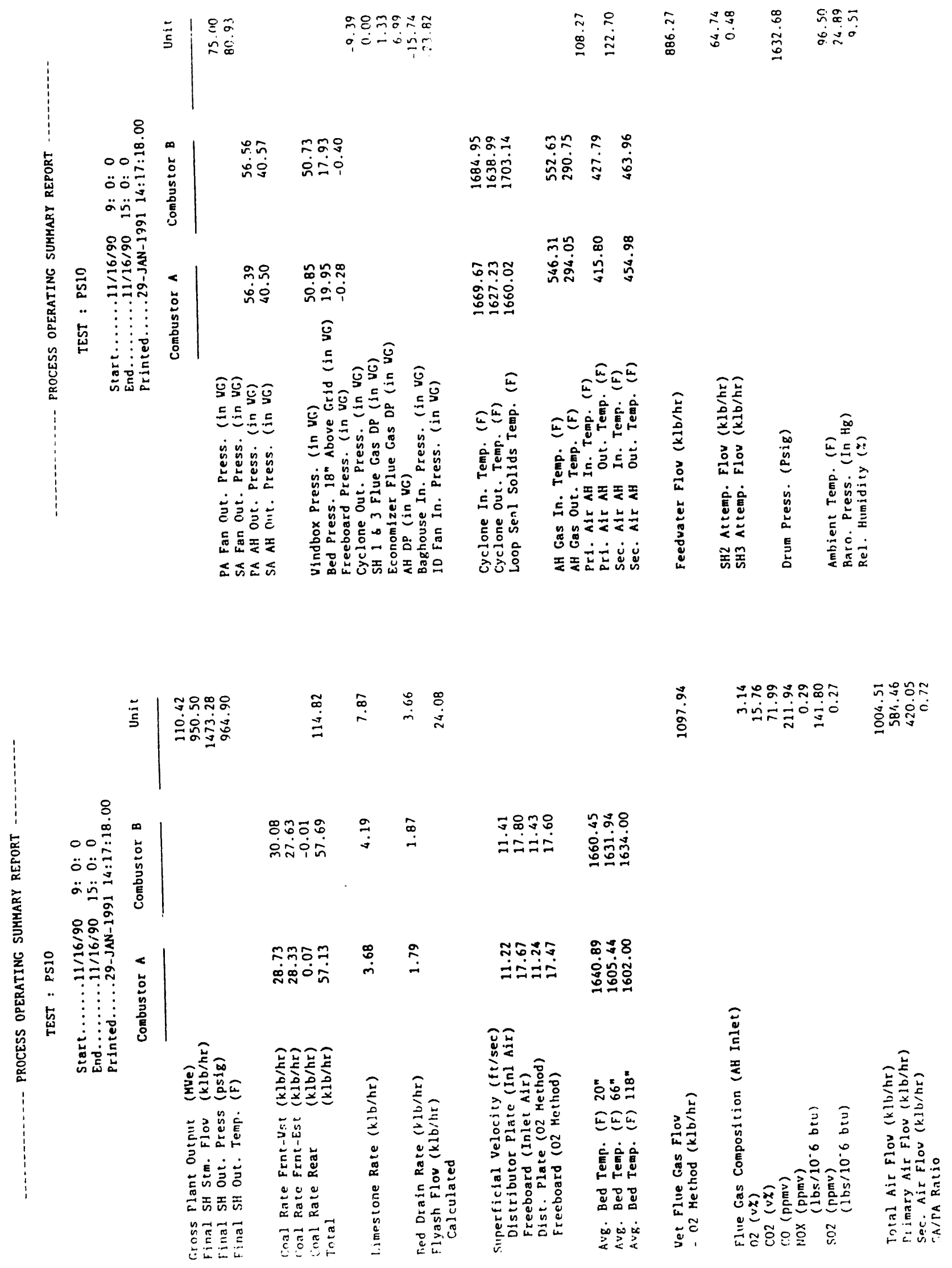


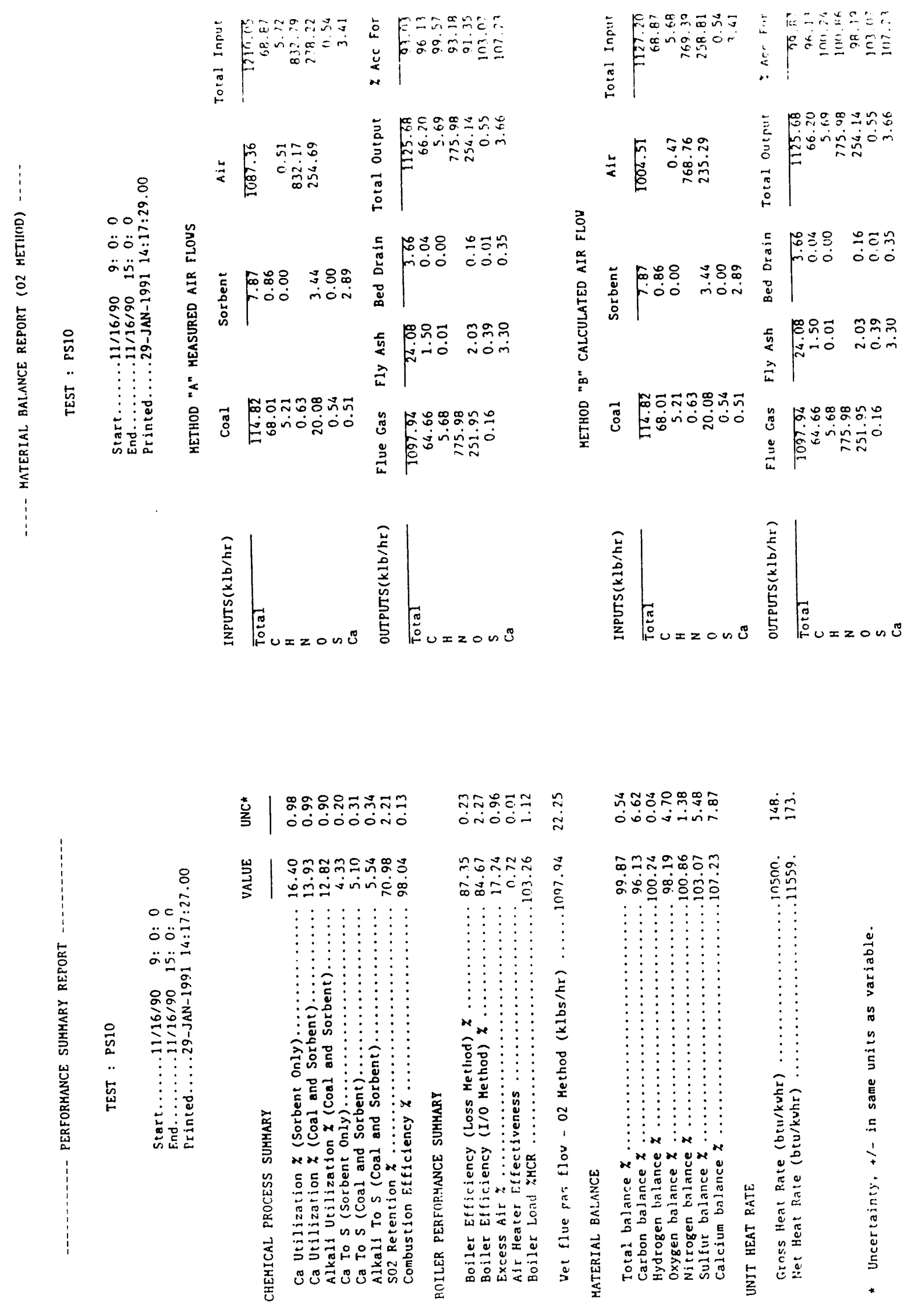



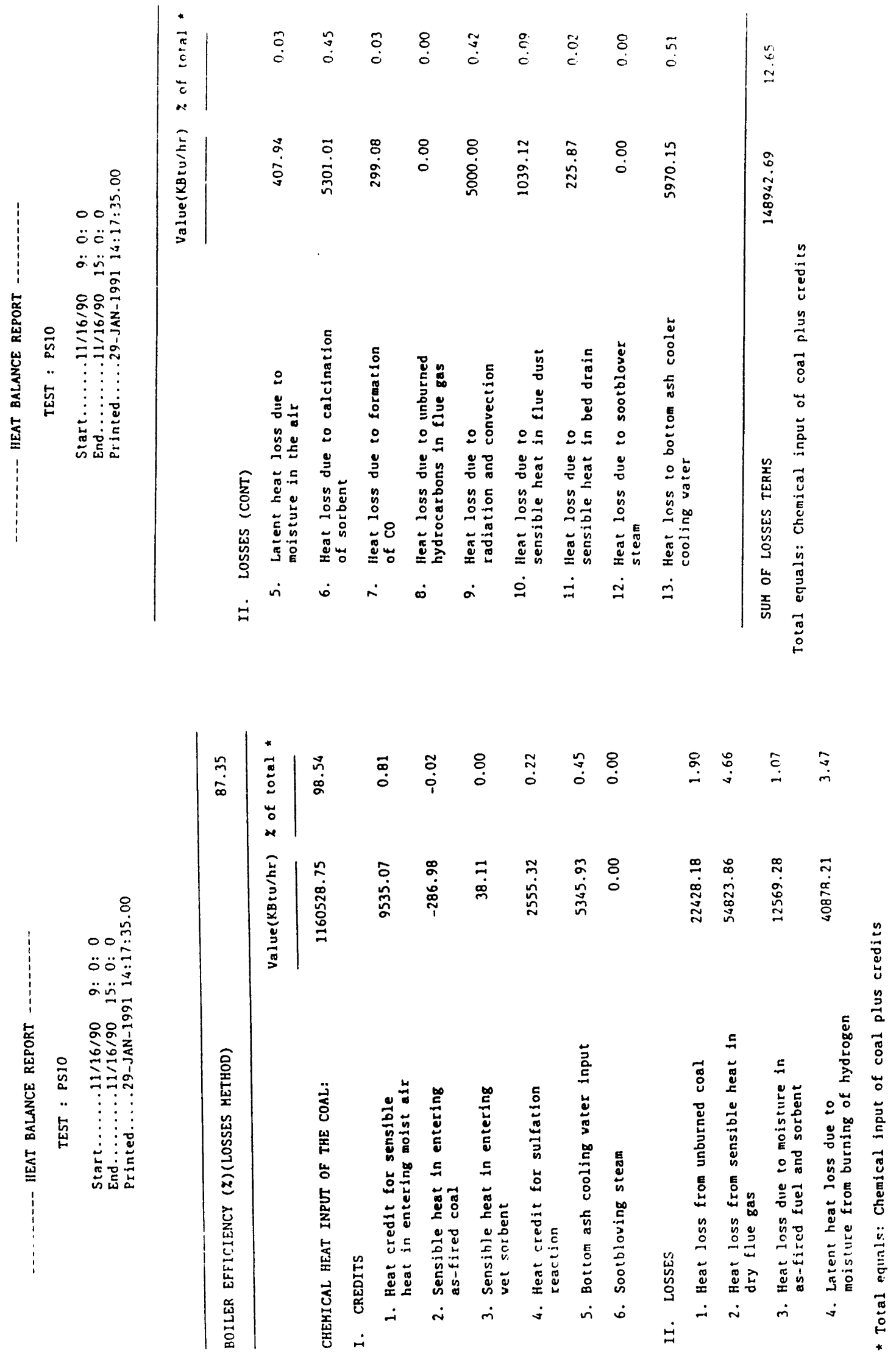


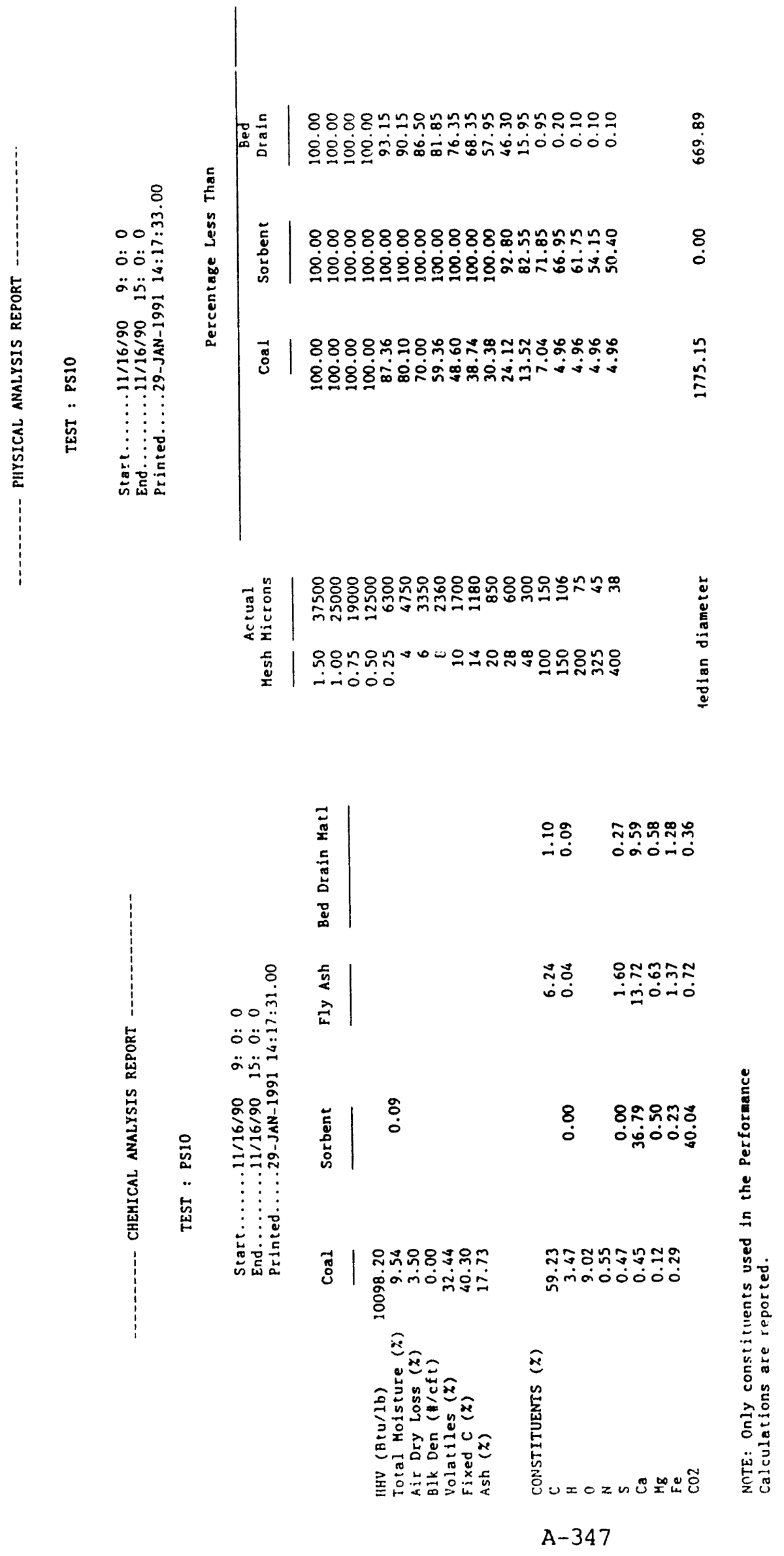




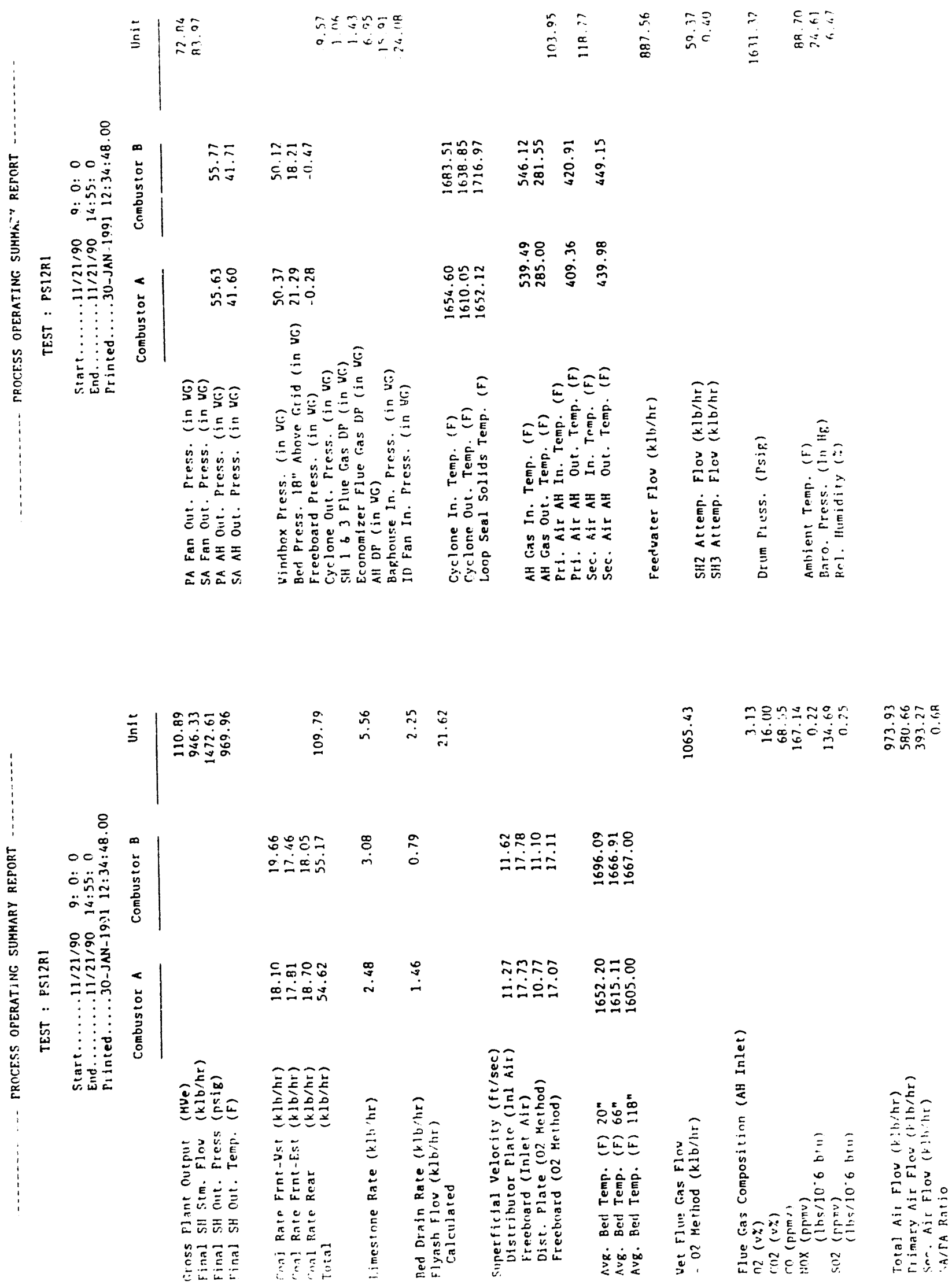




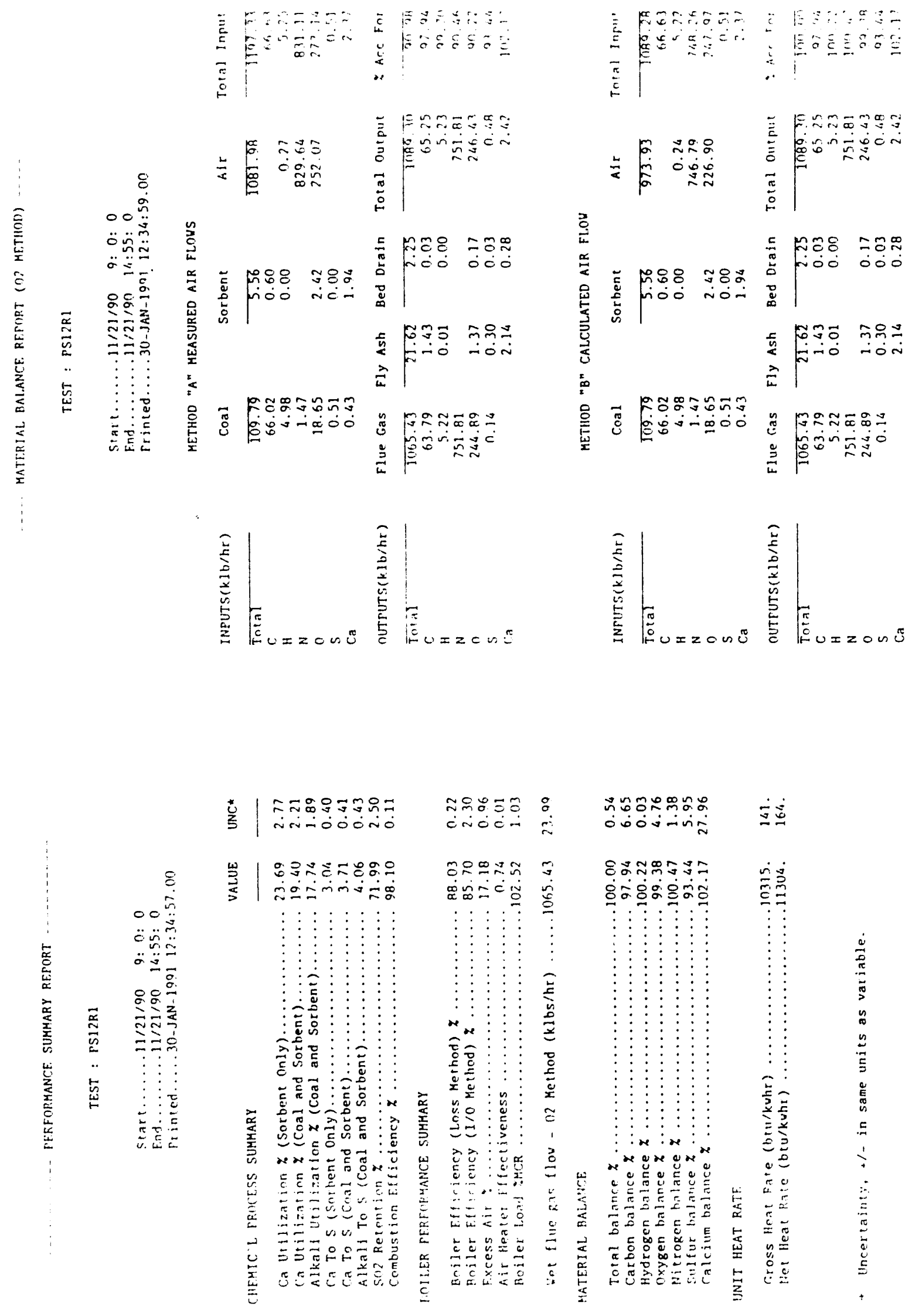



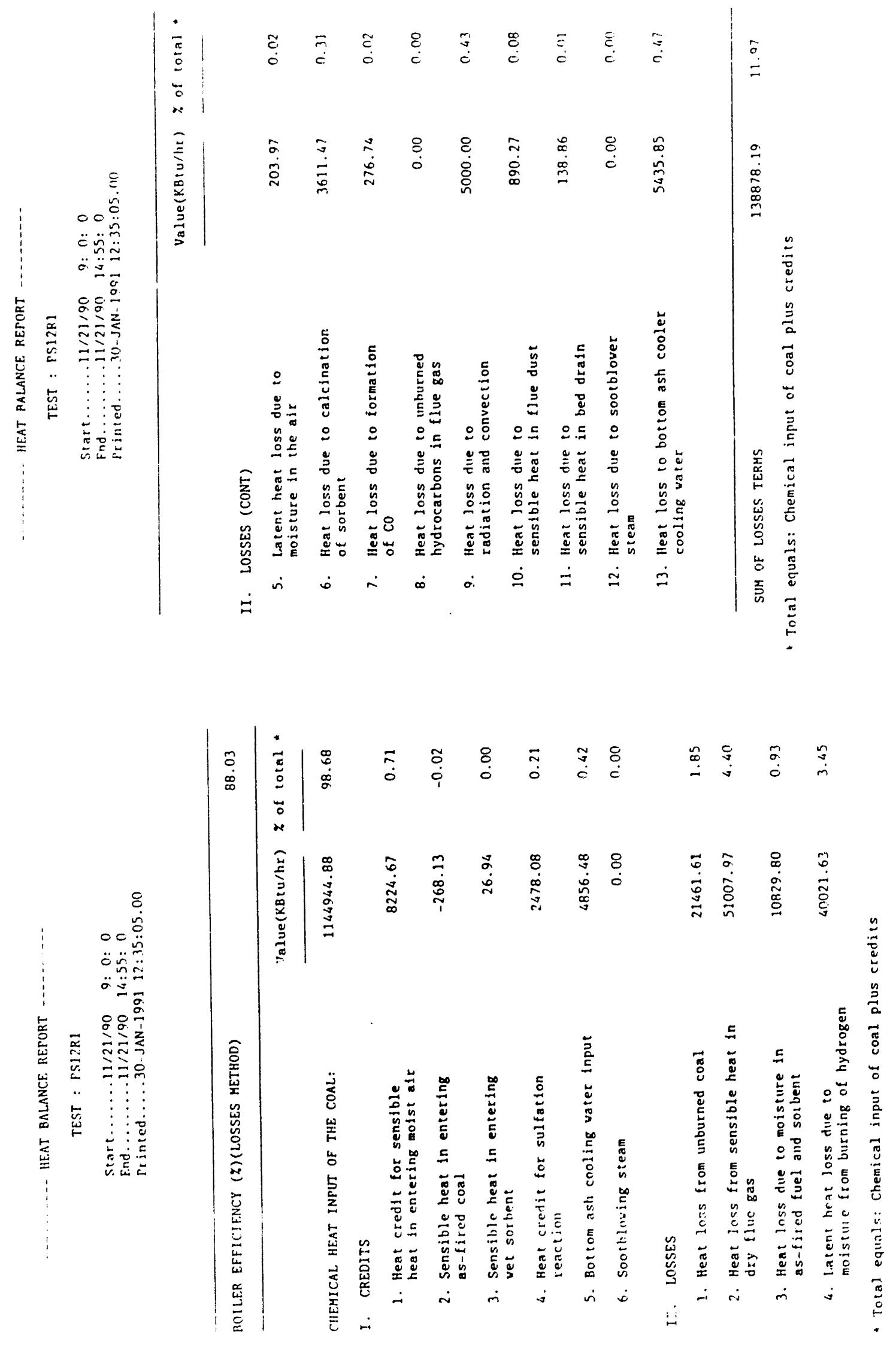

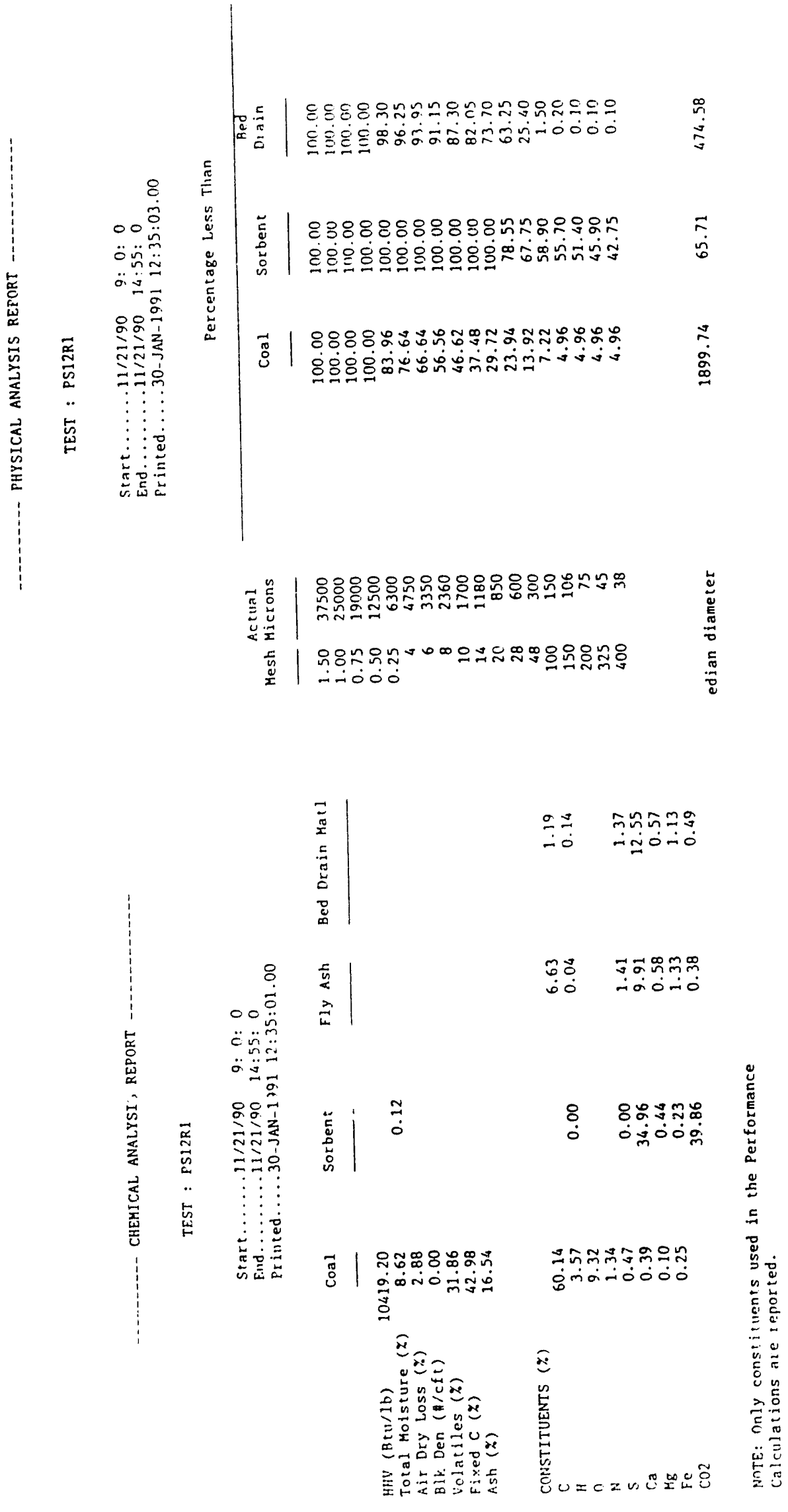

A-351 

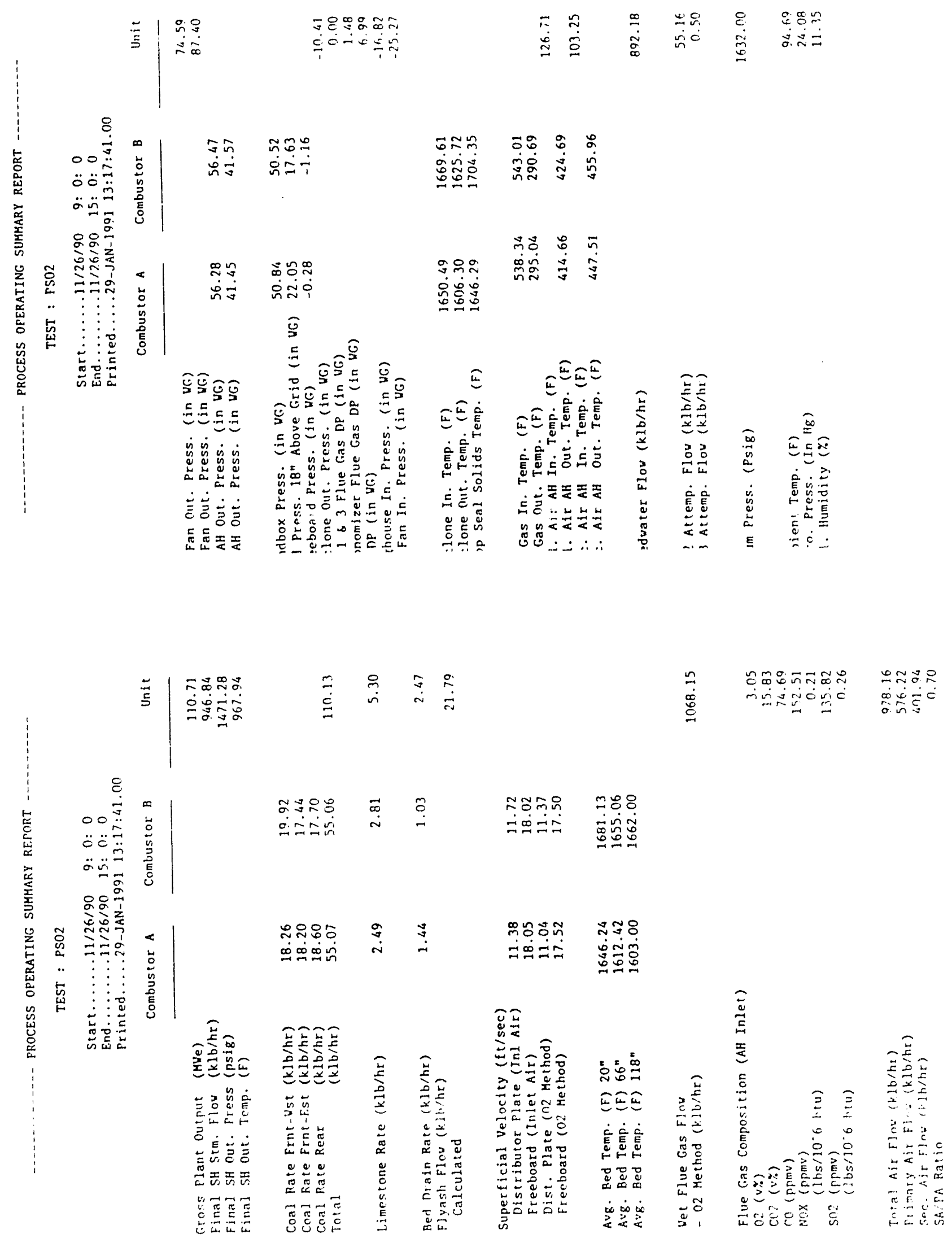


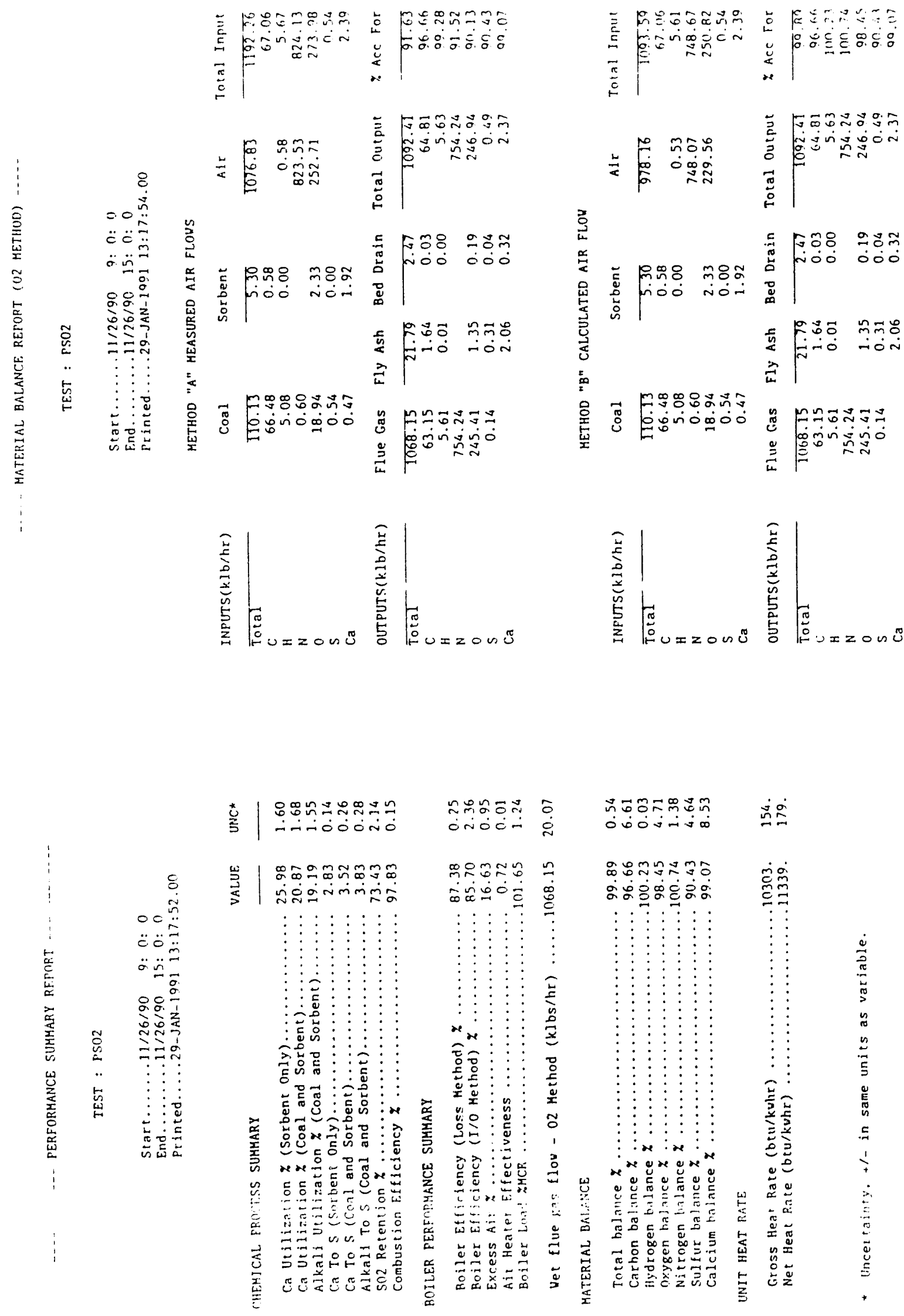



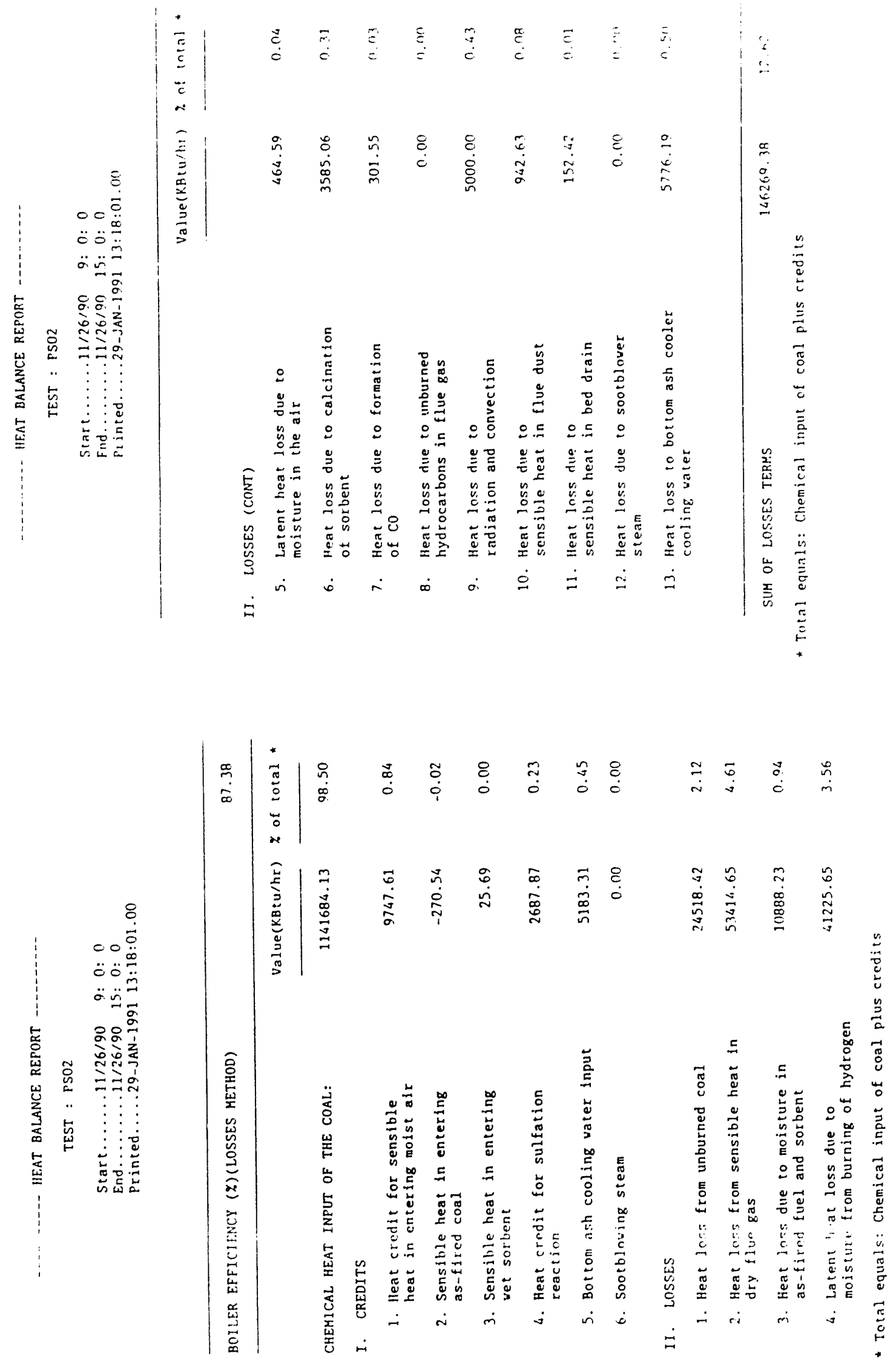

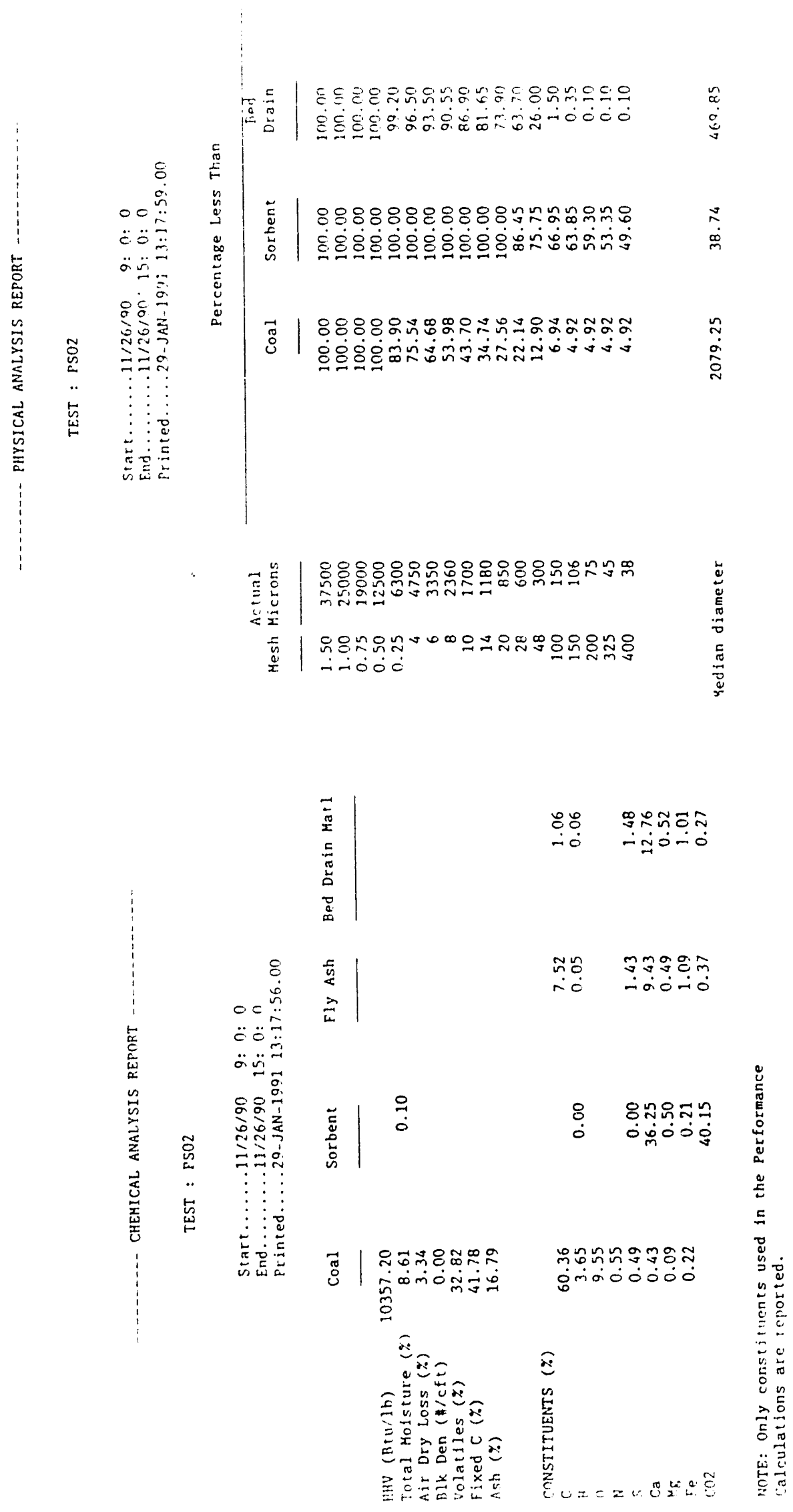

A-355 


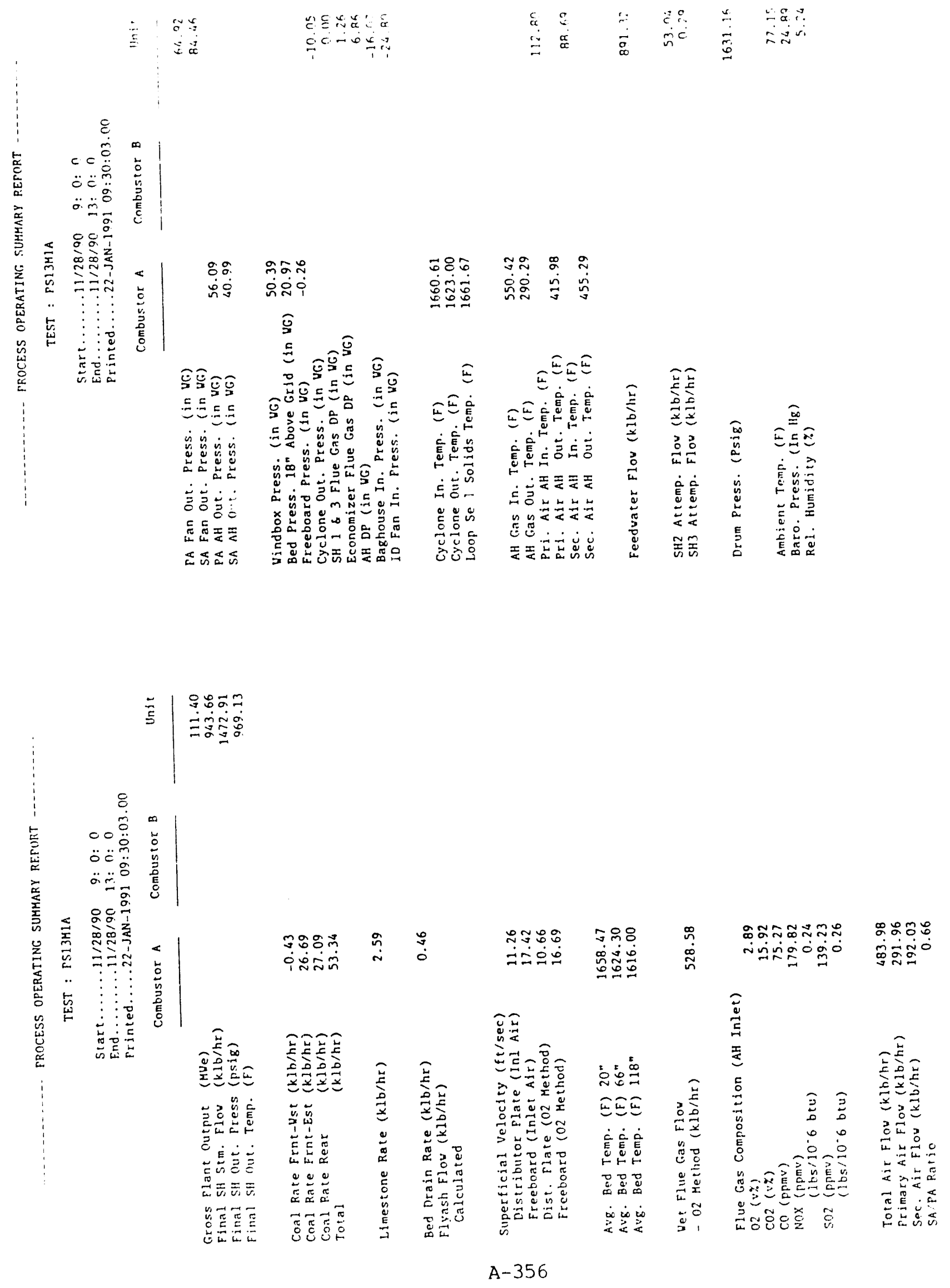




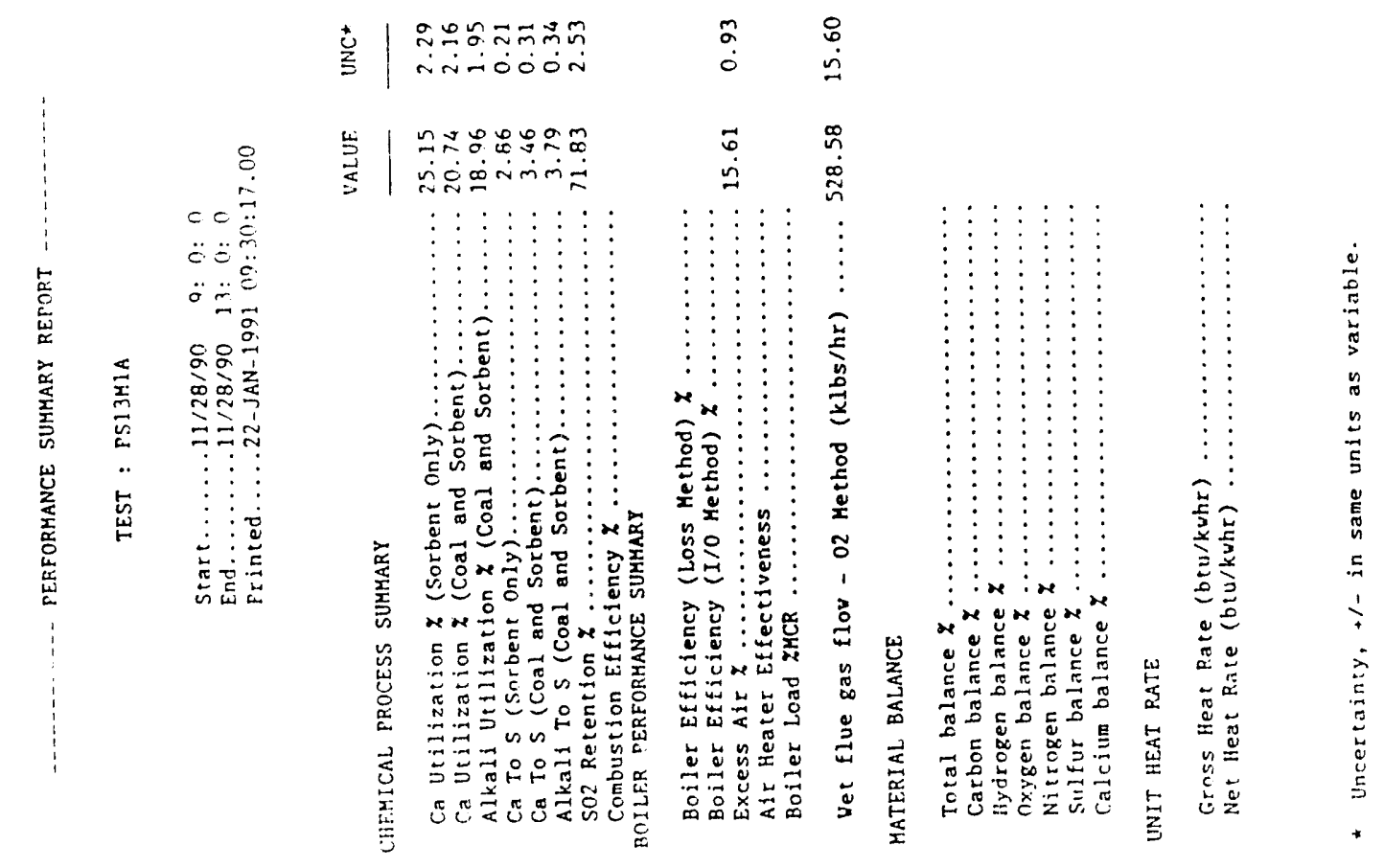



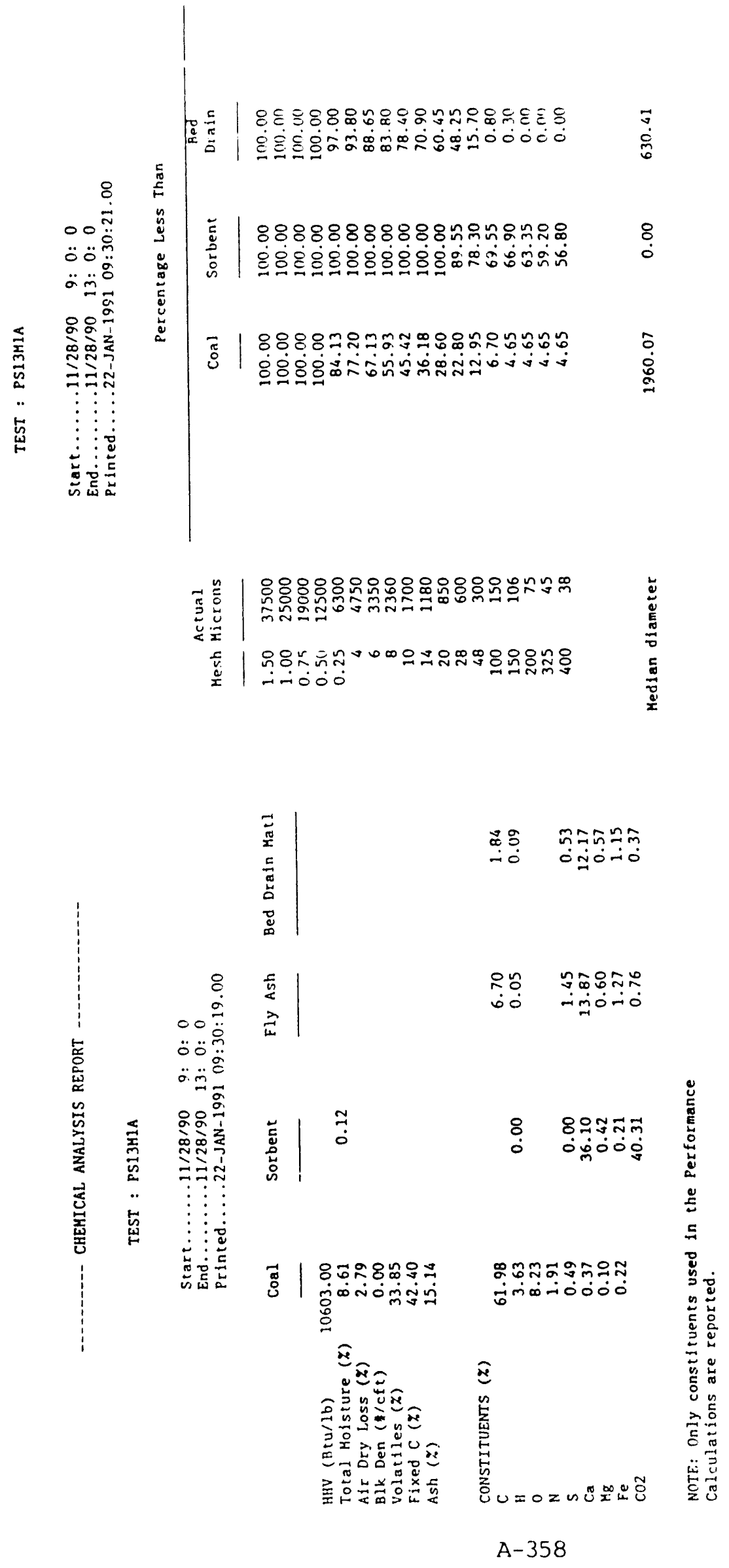


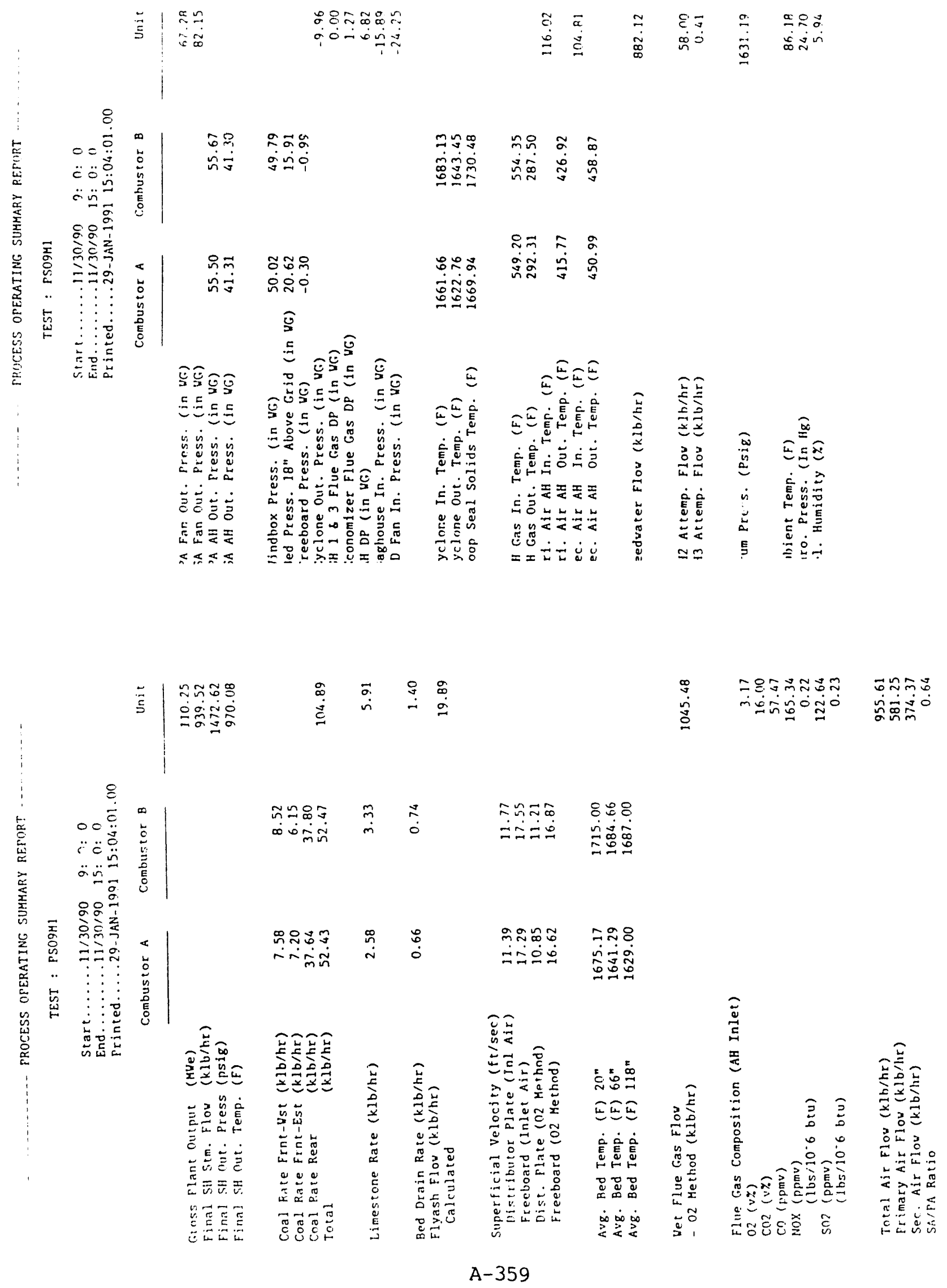




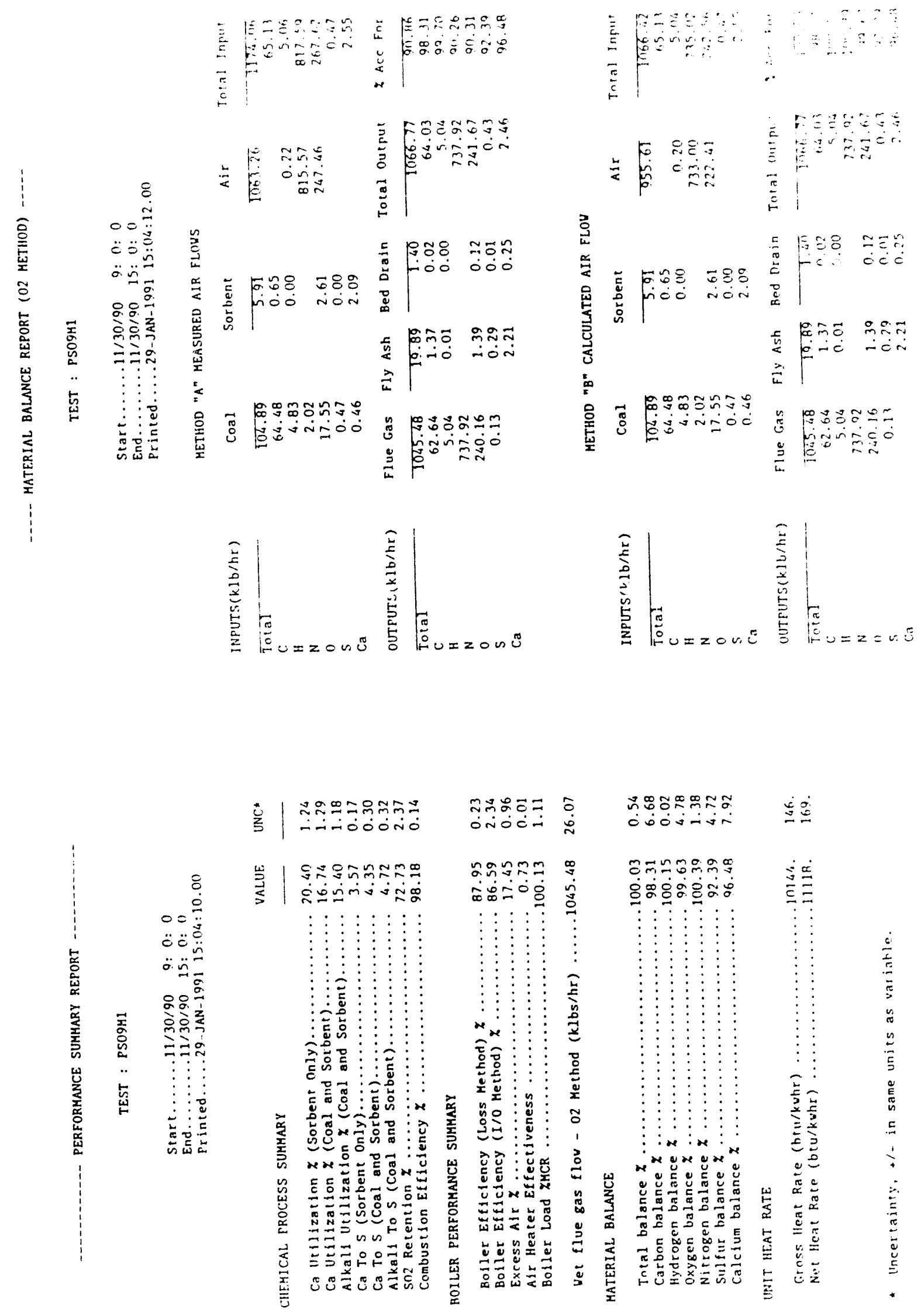




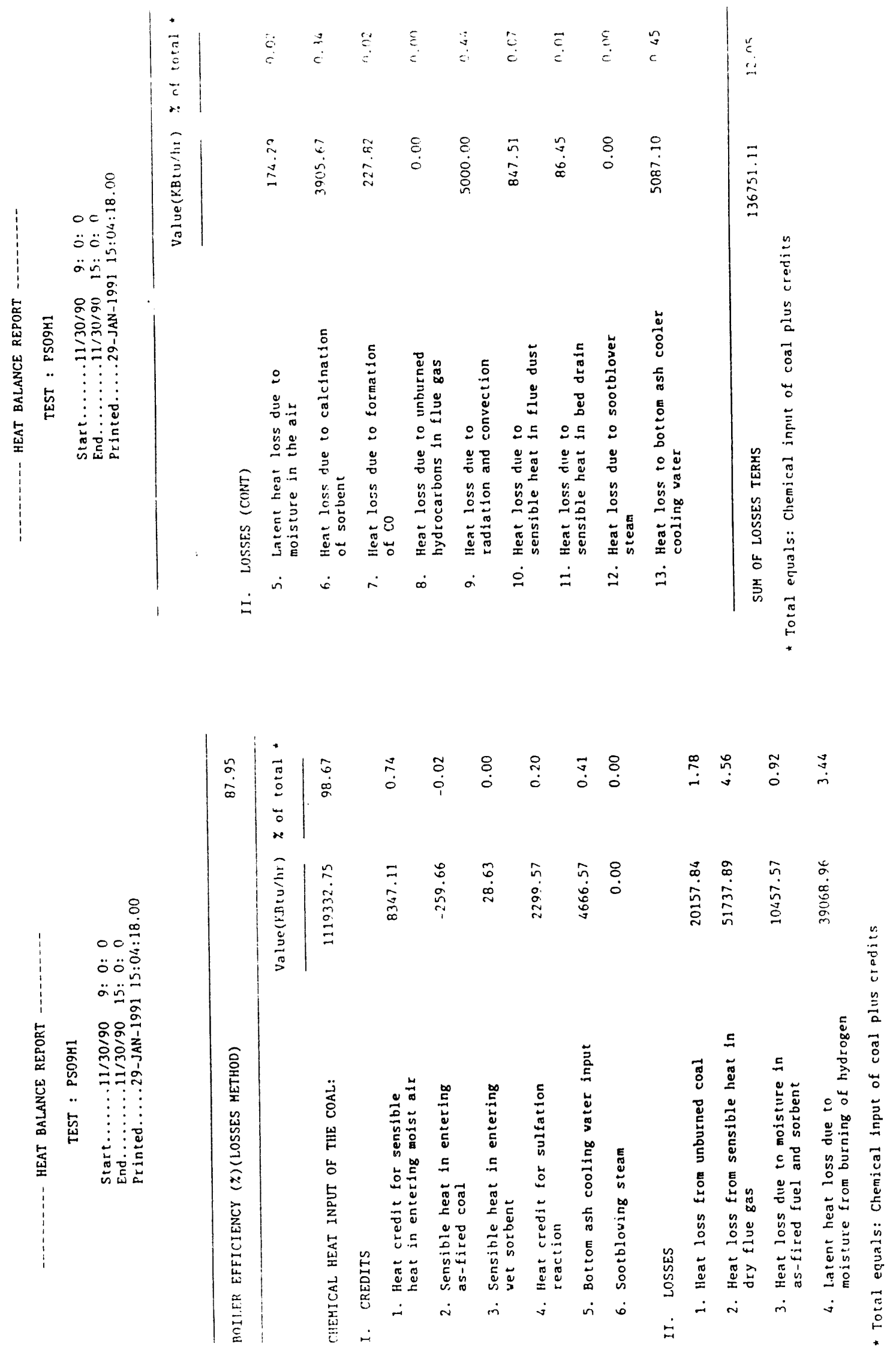



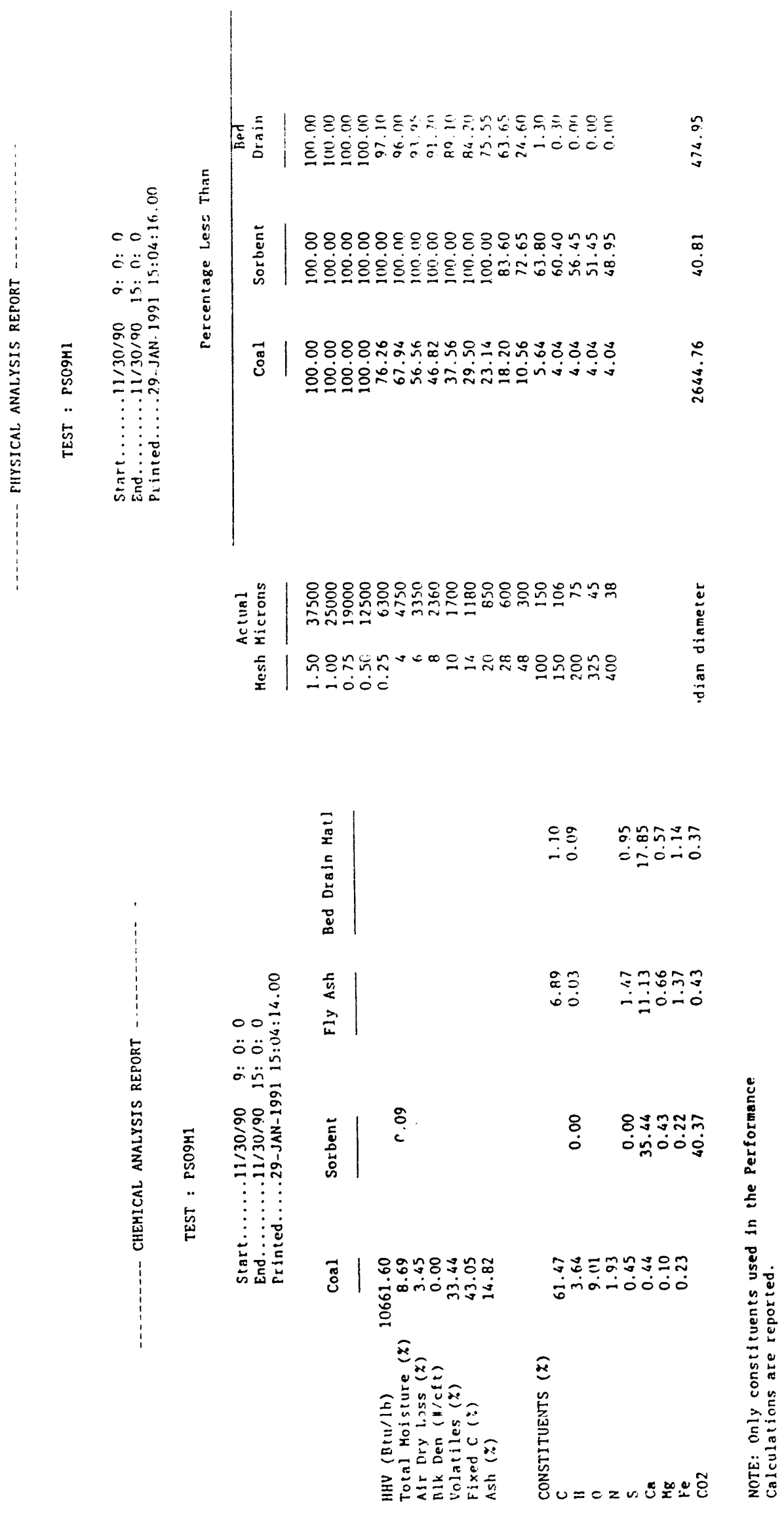


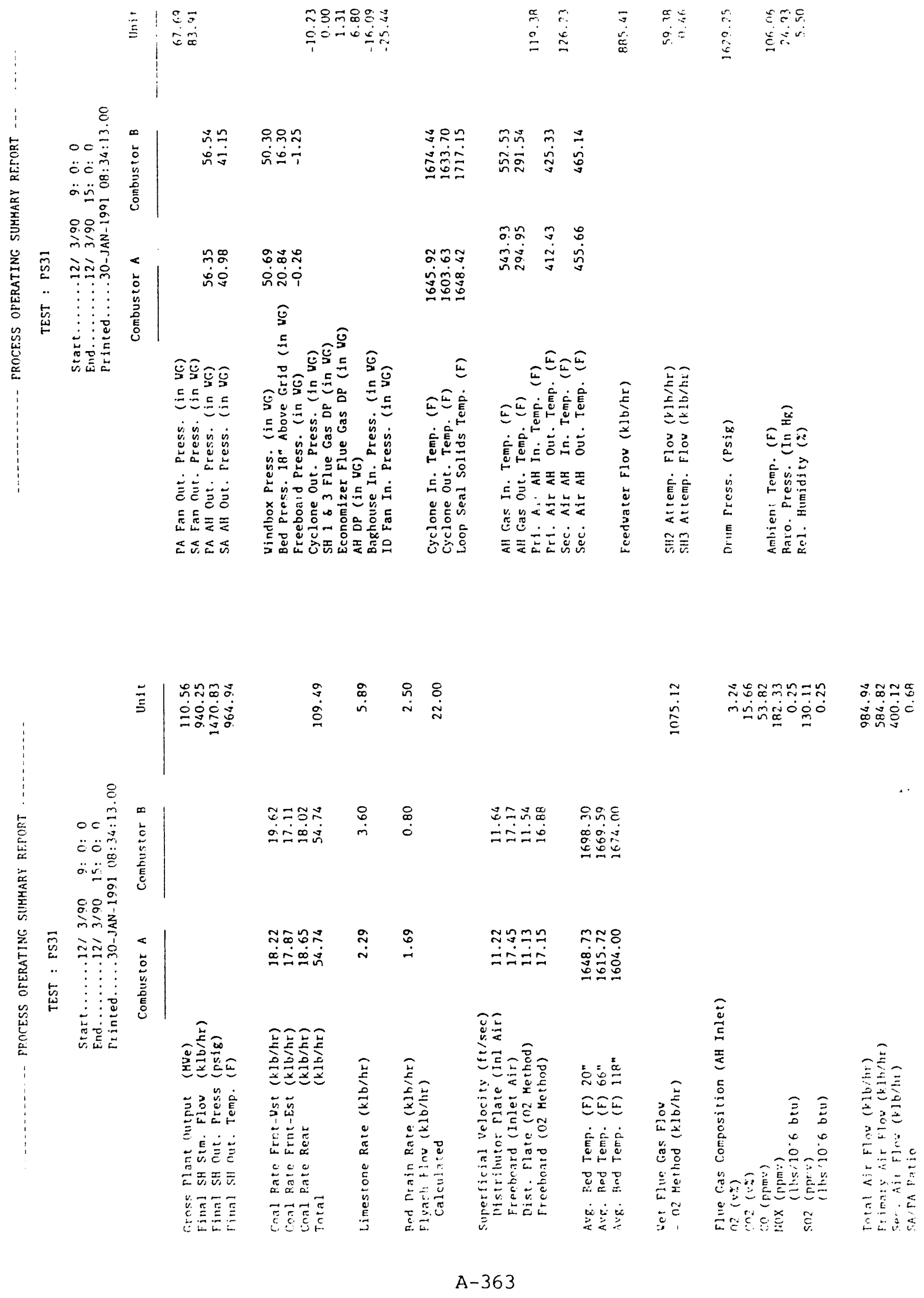




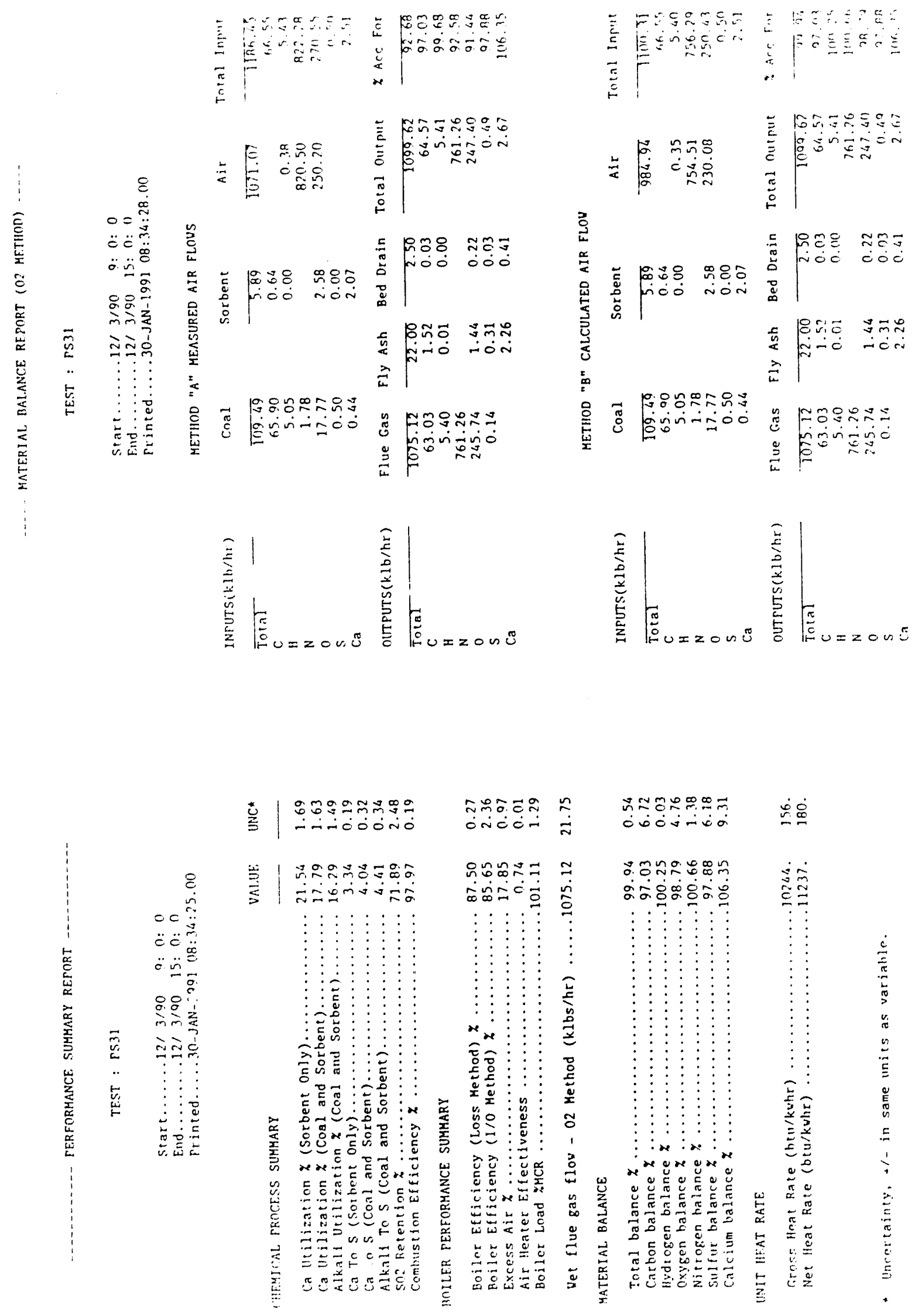




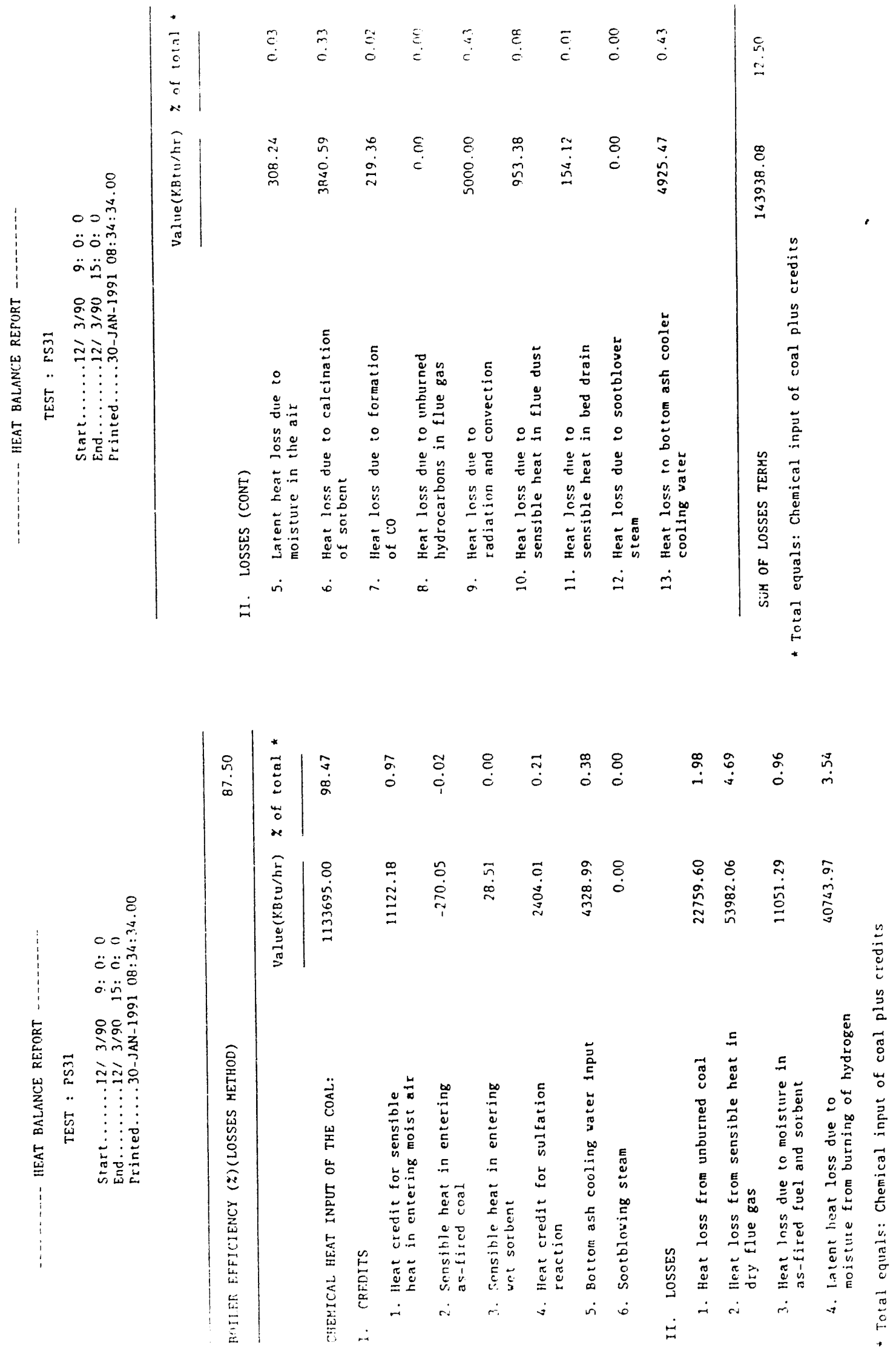




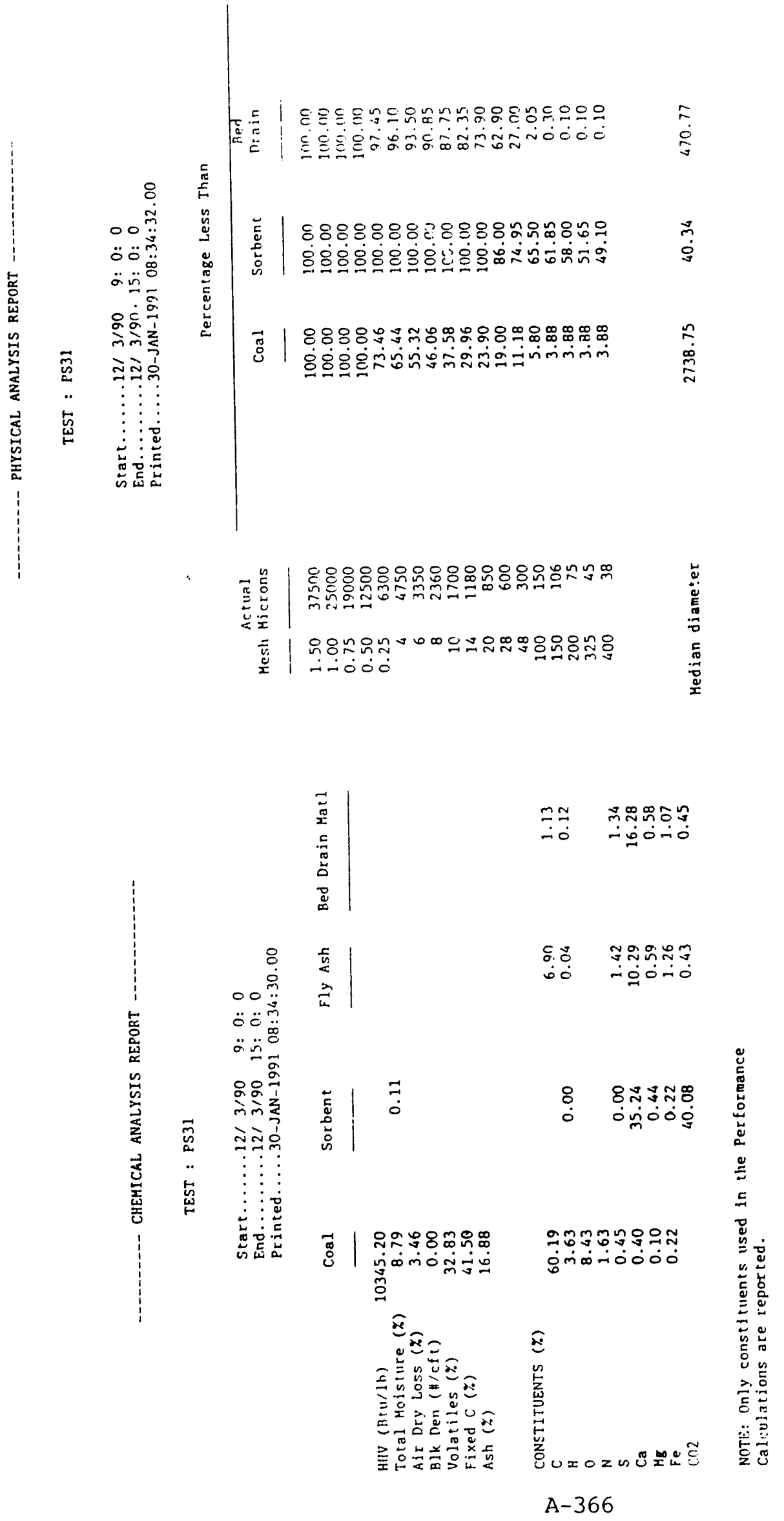




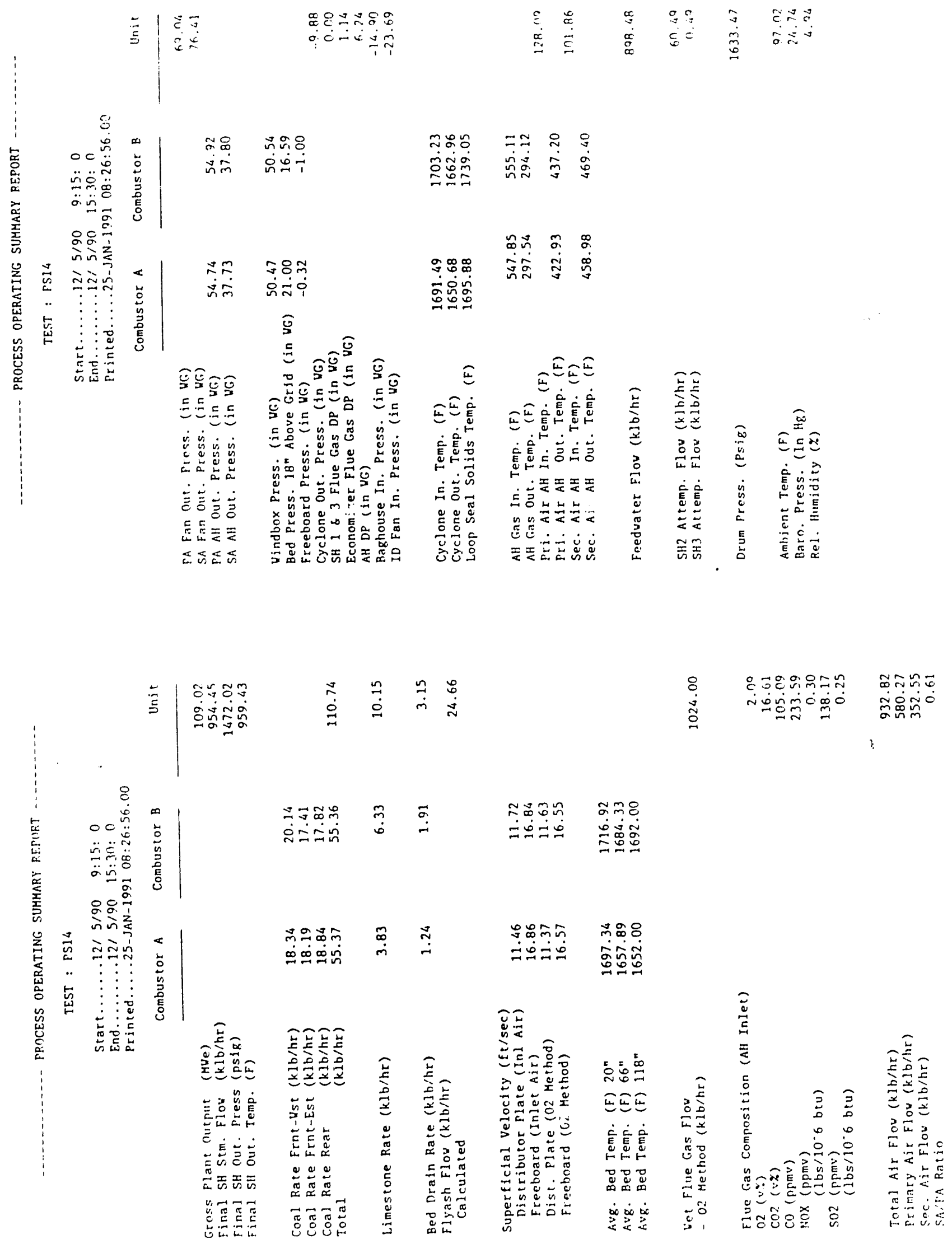




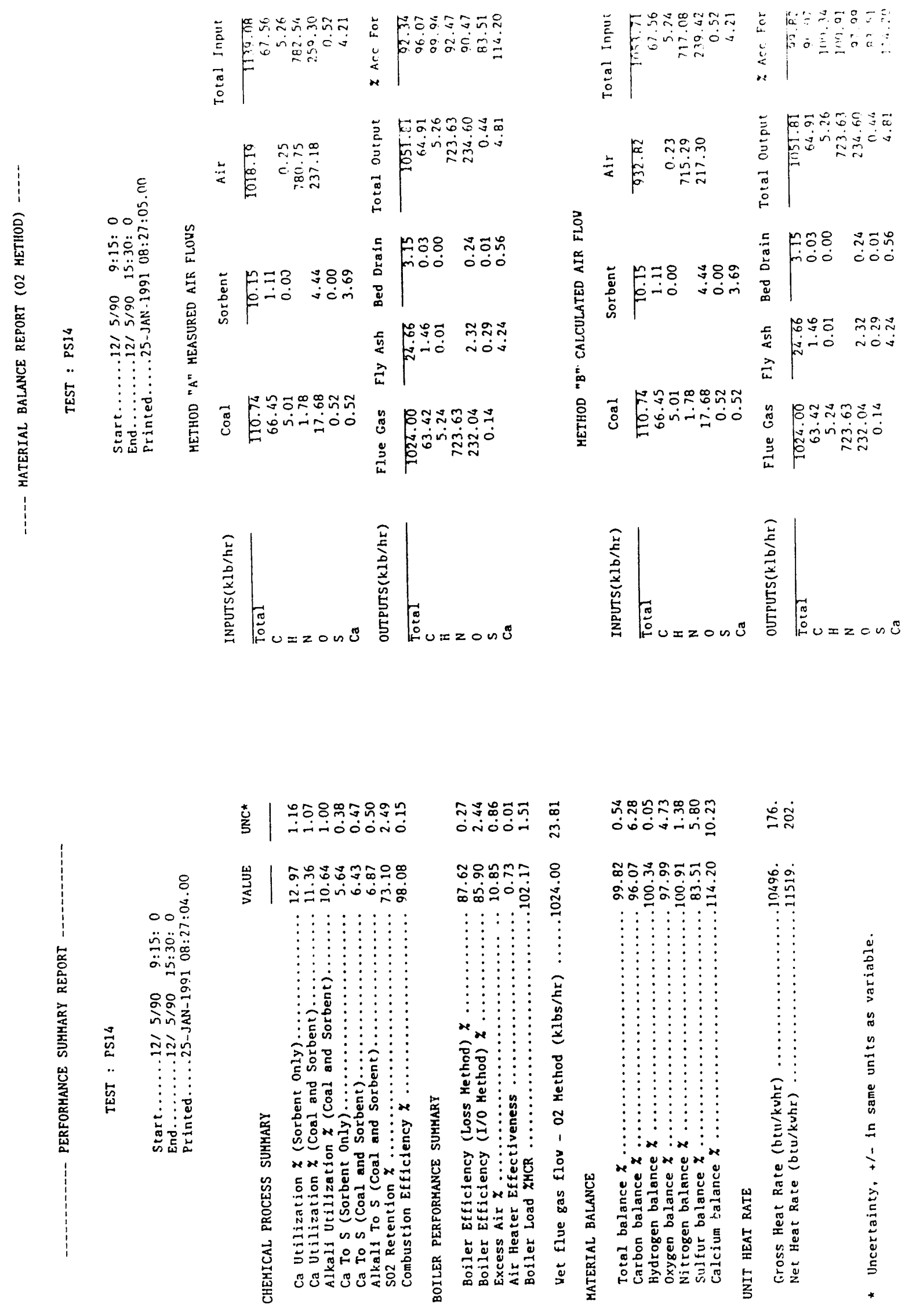




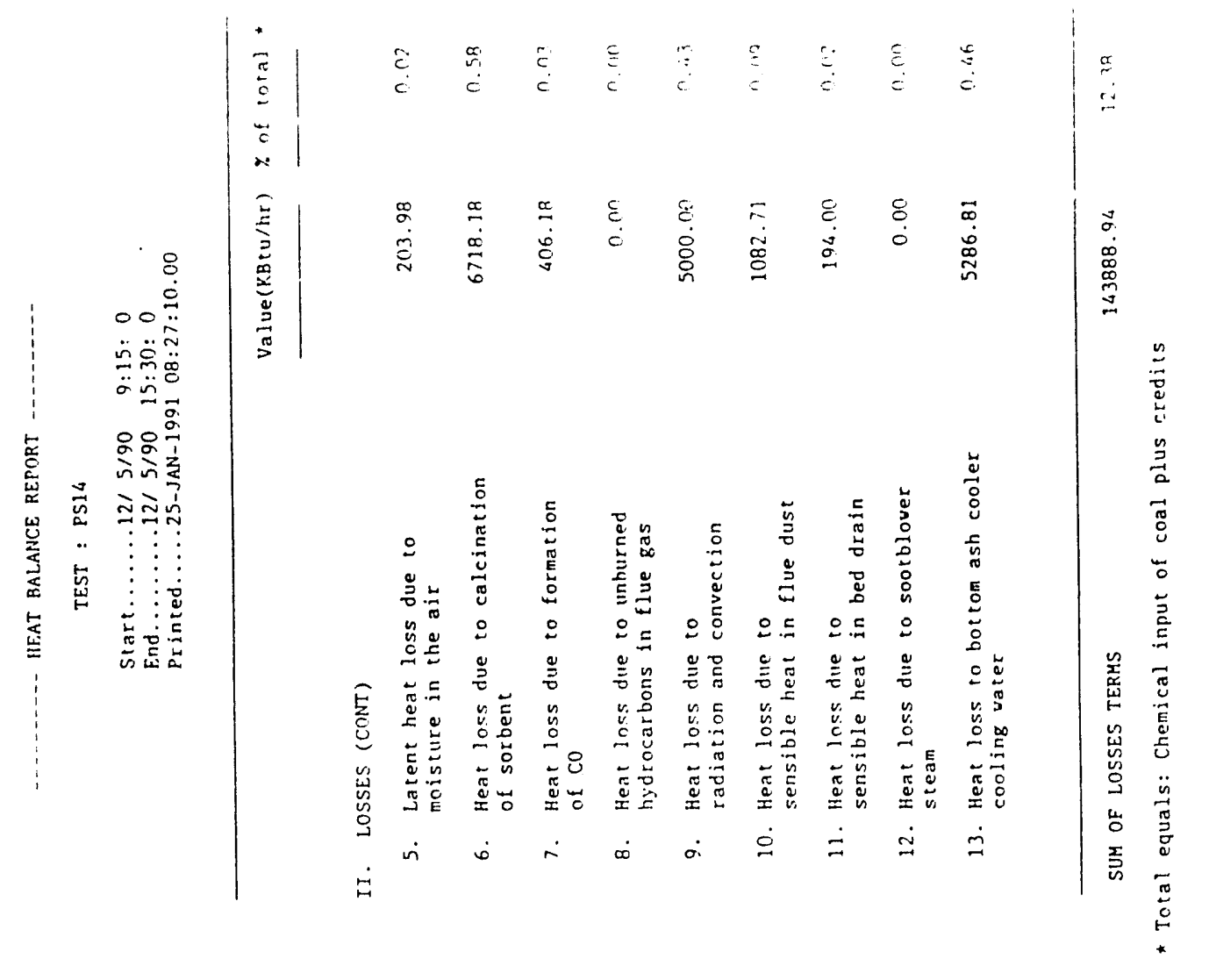

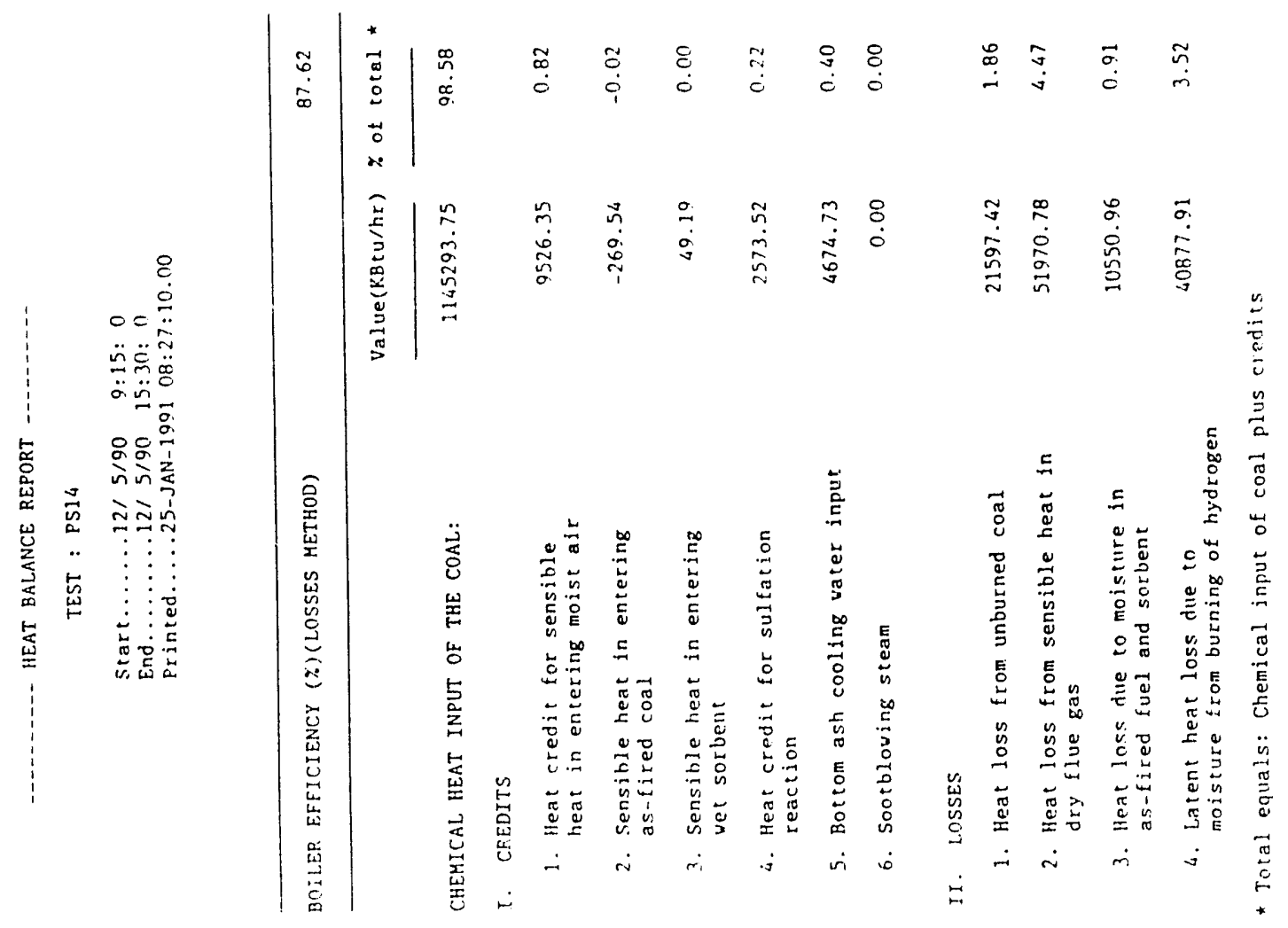



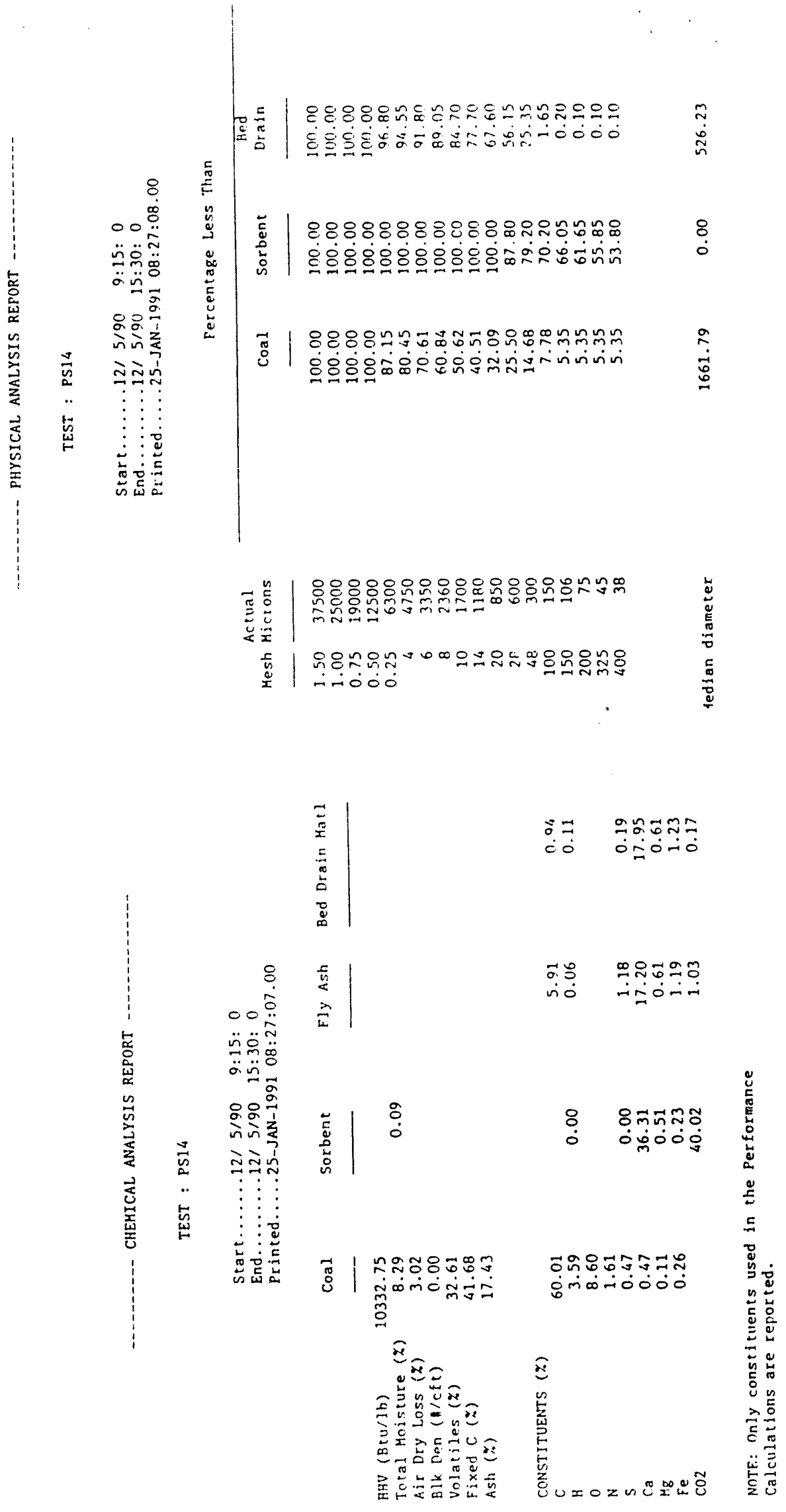

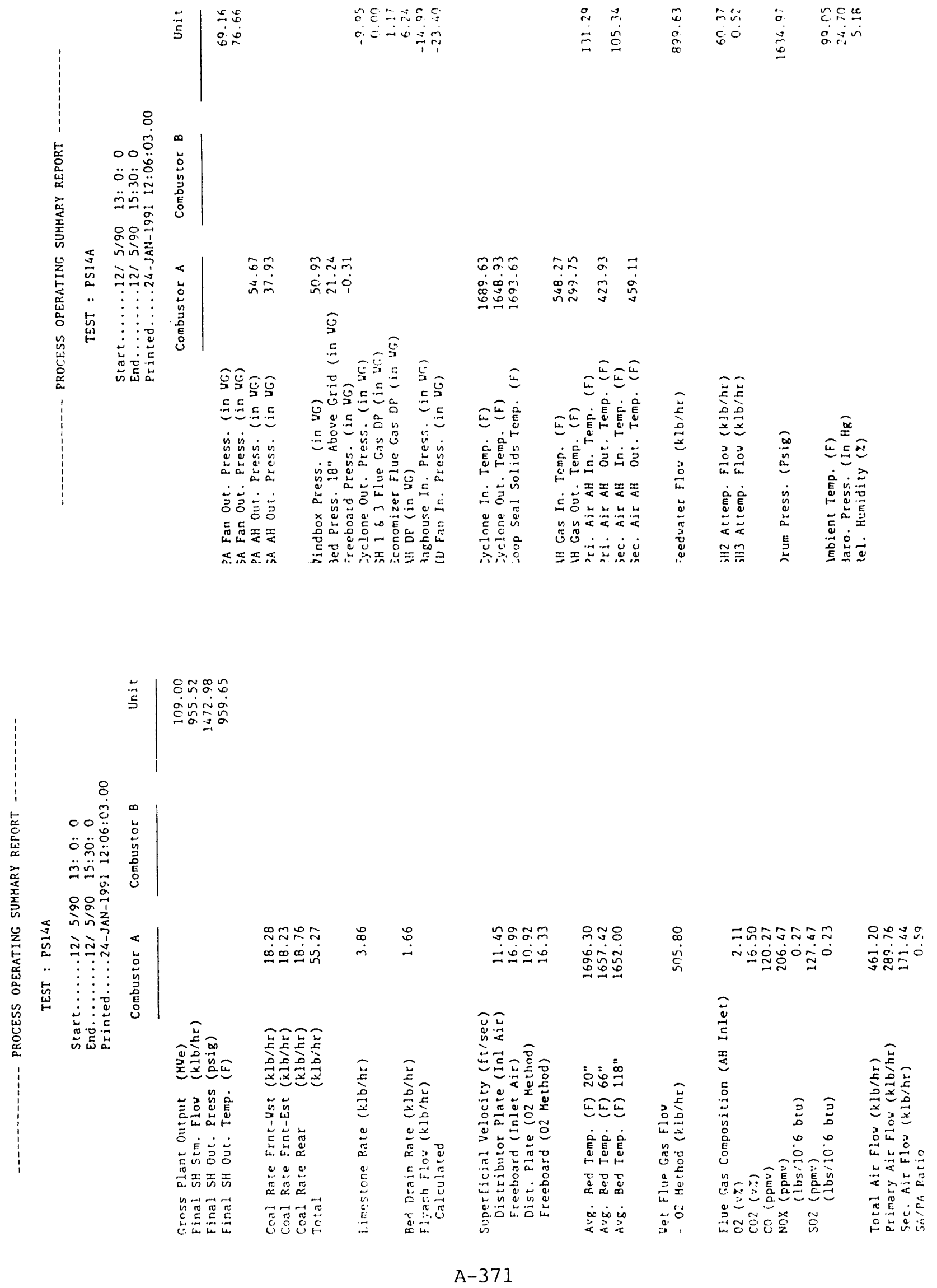


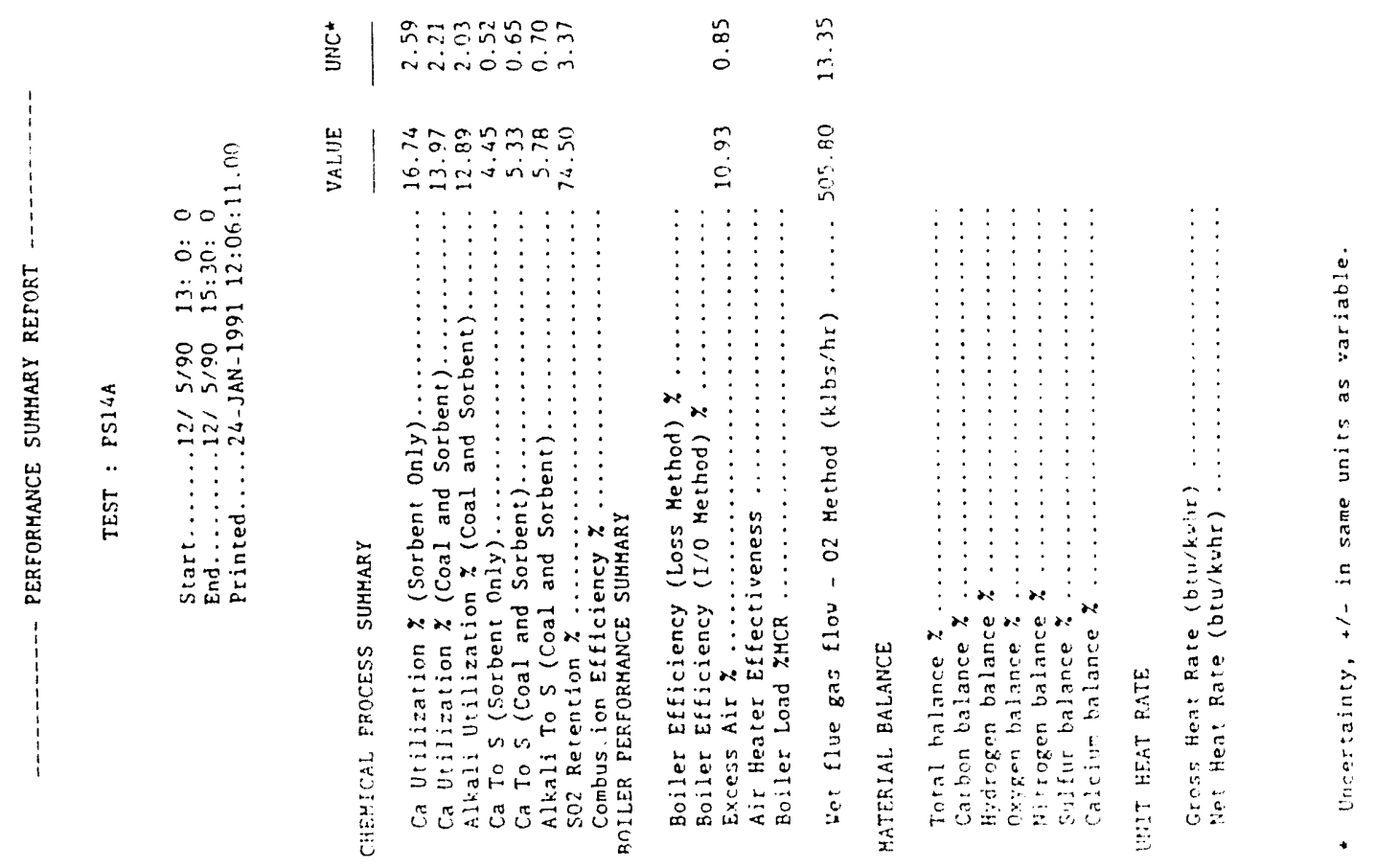

A -372 


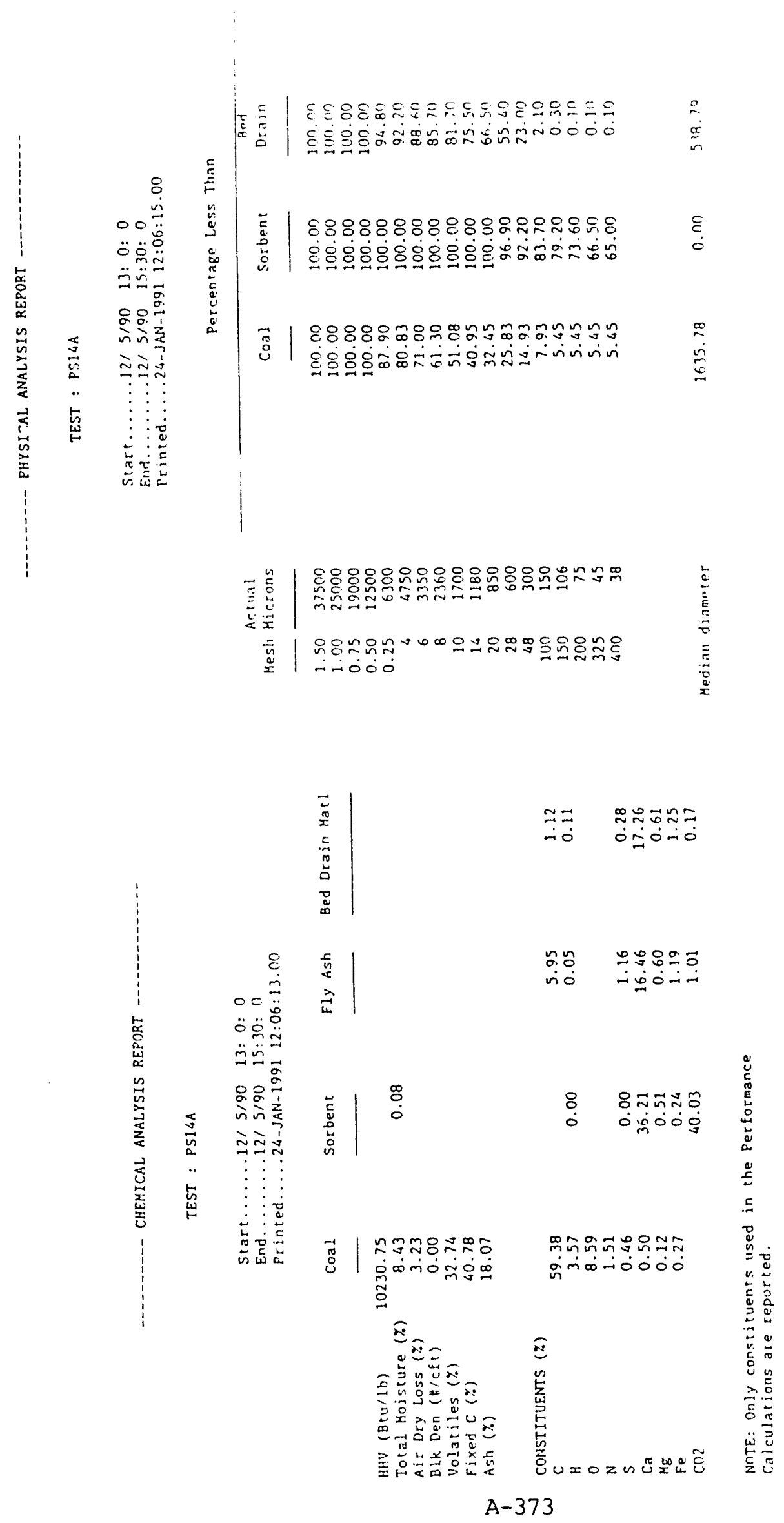



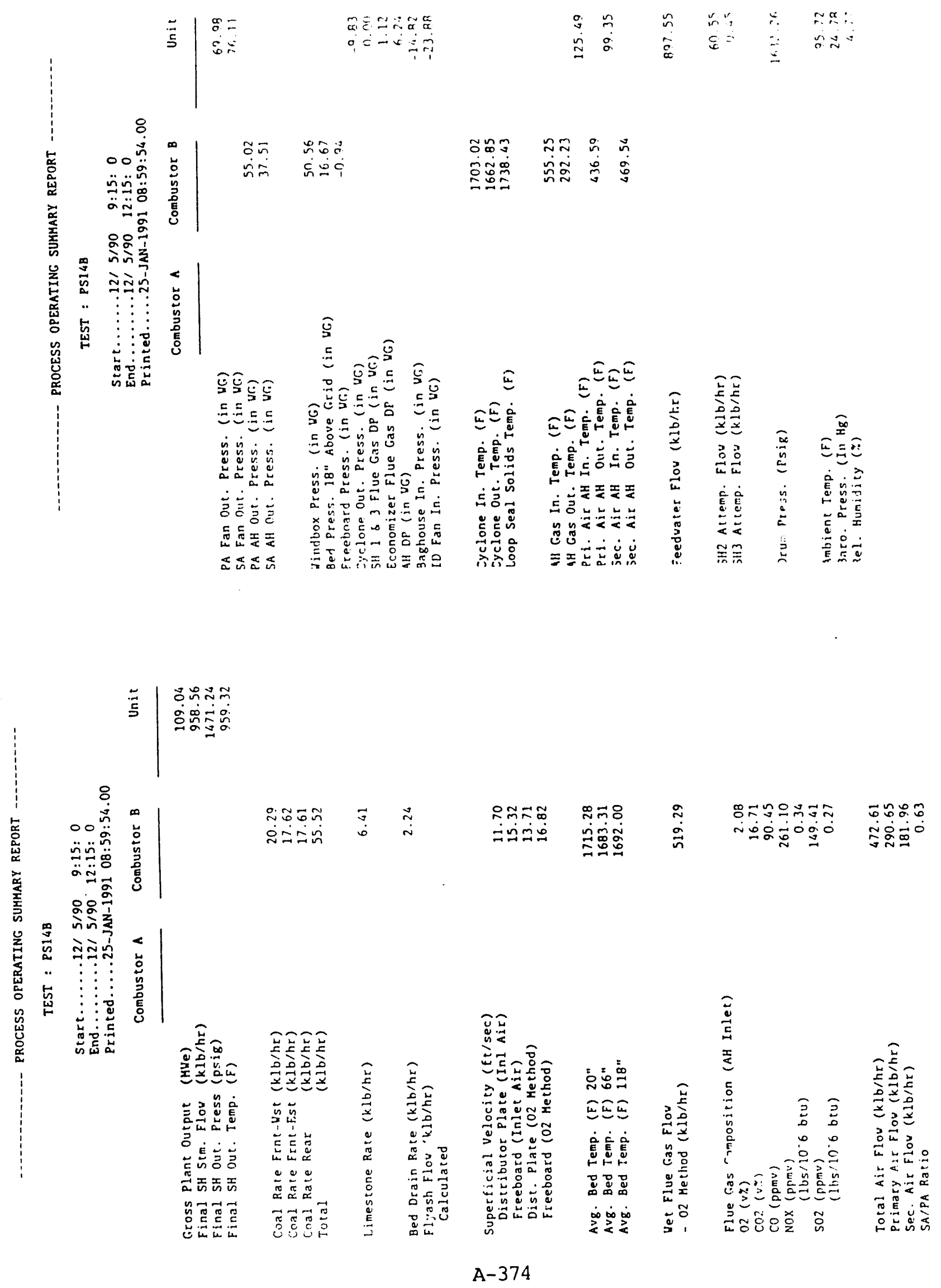


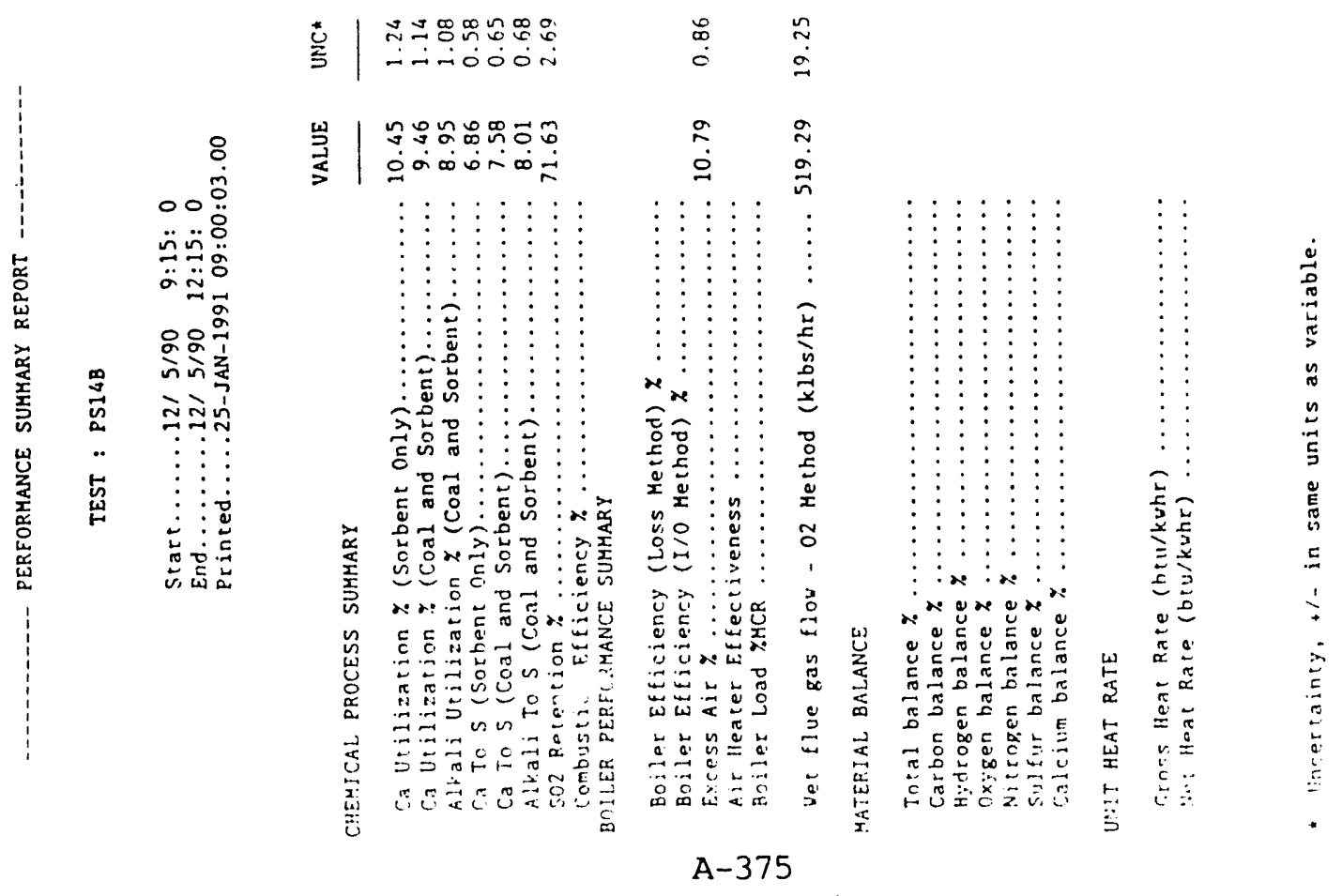



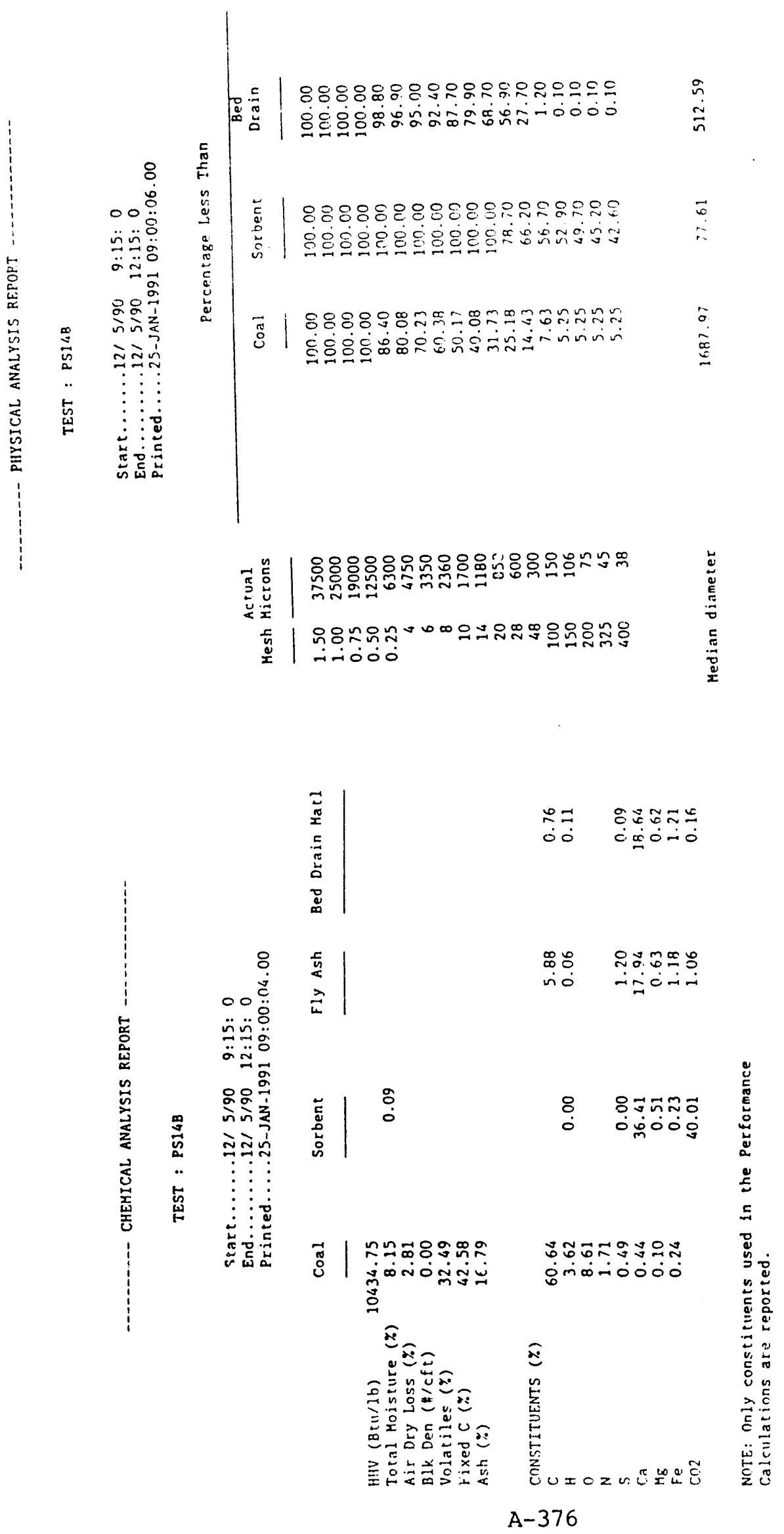


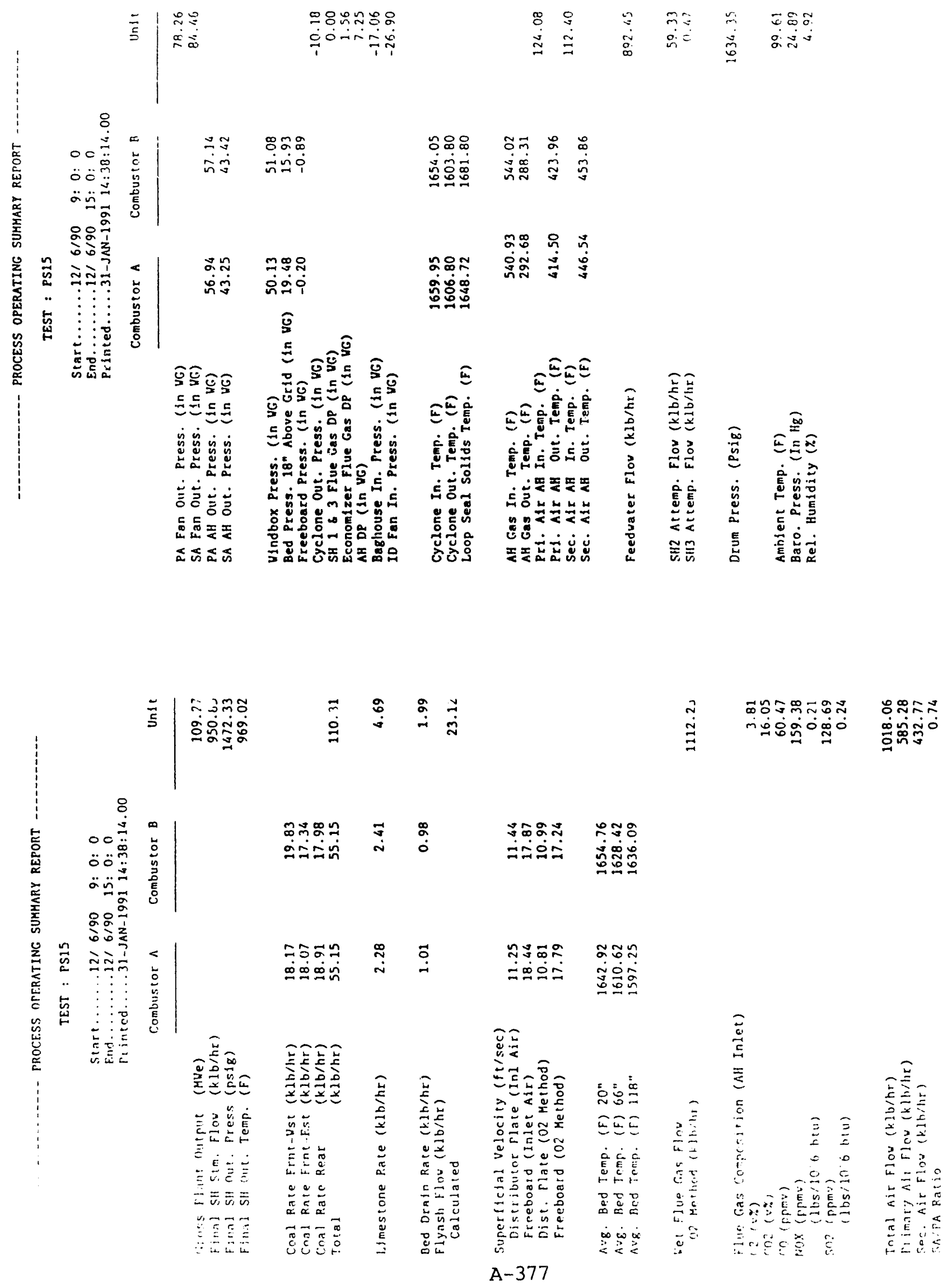



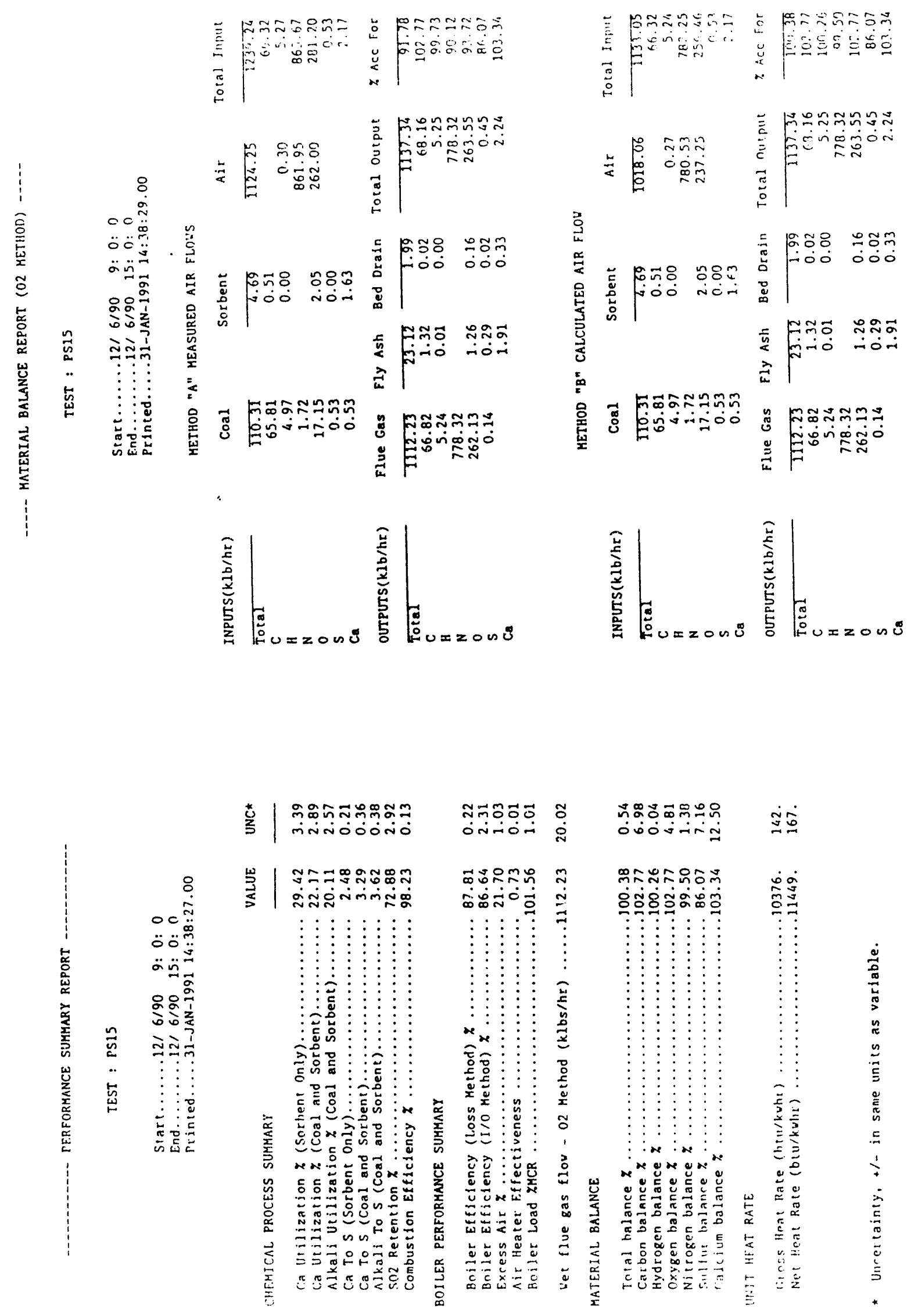

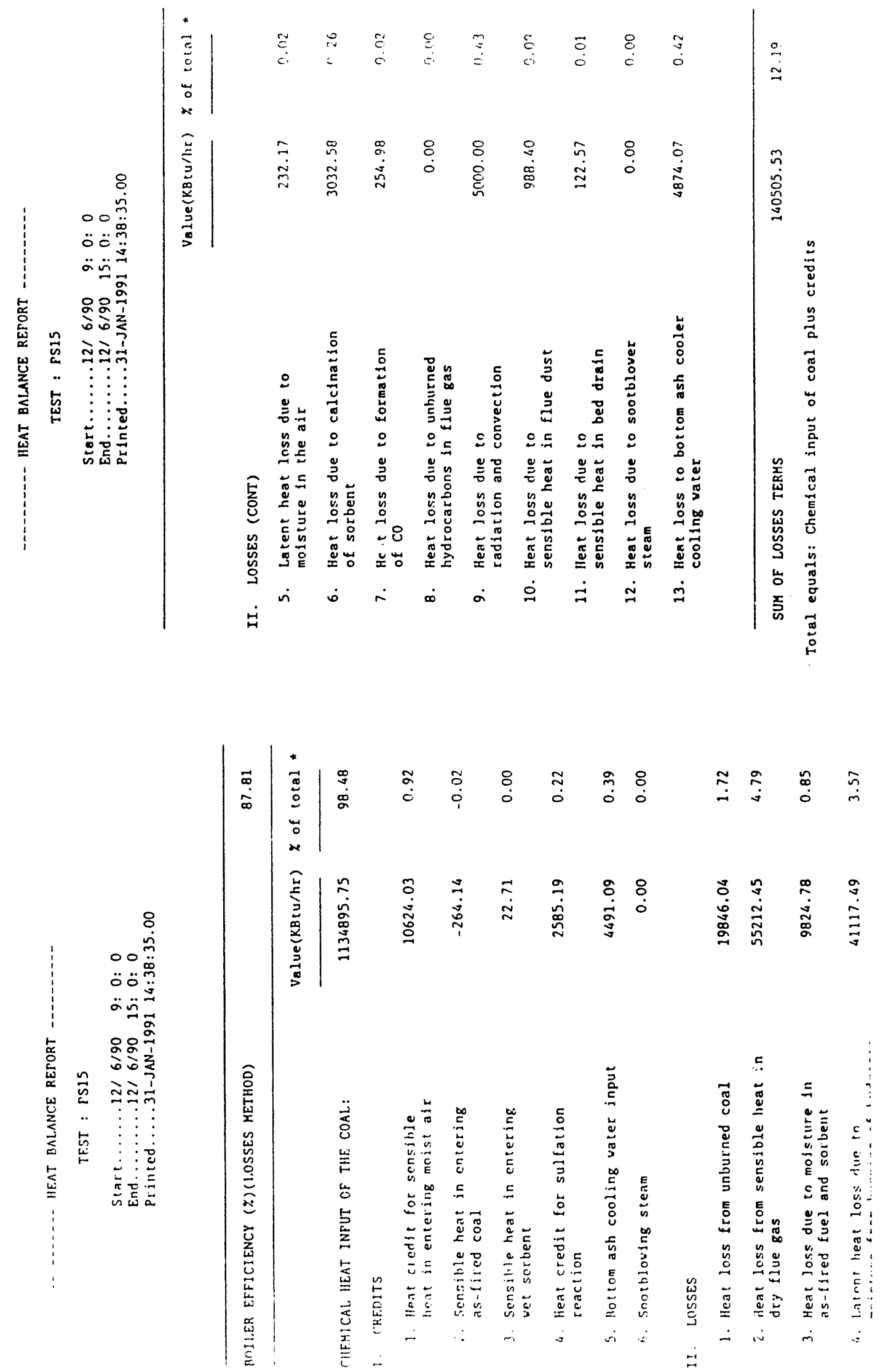


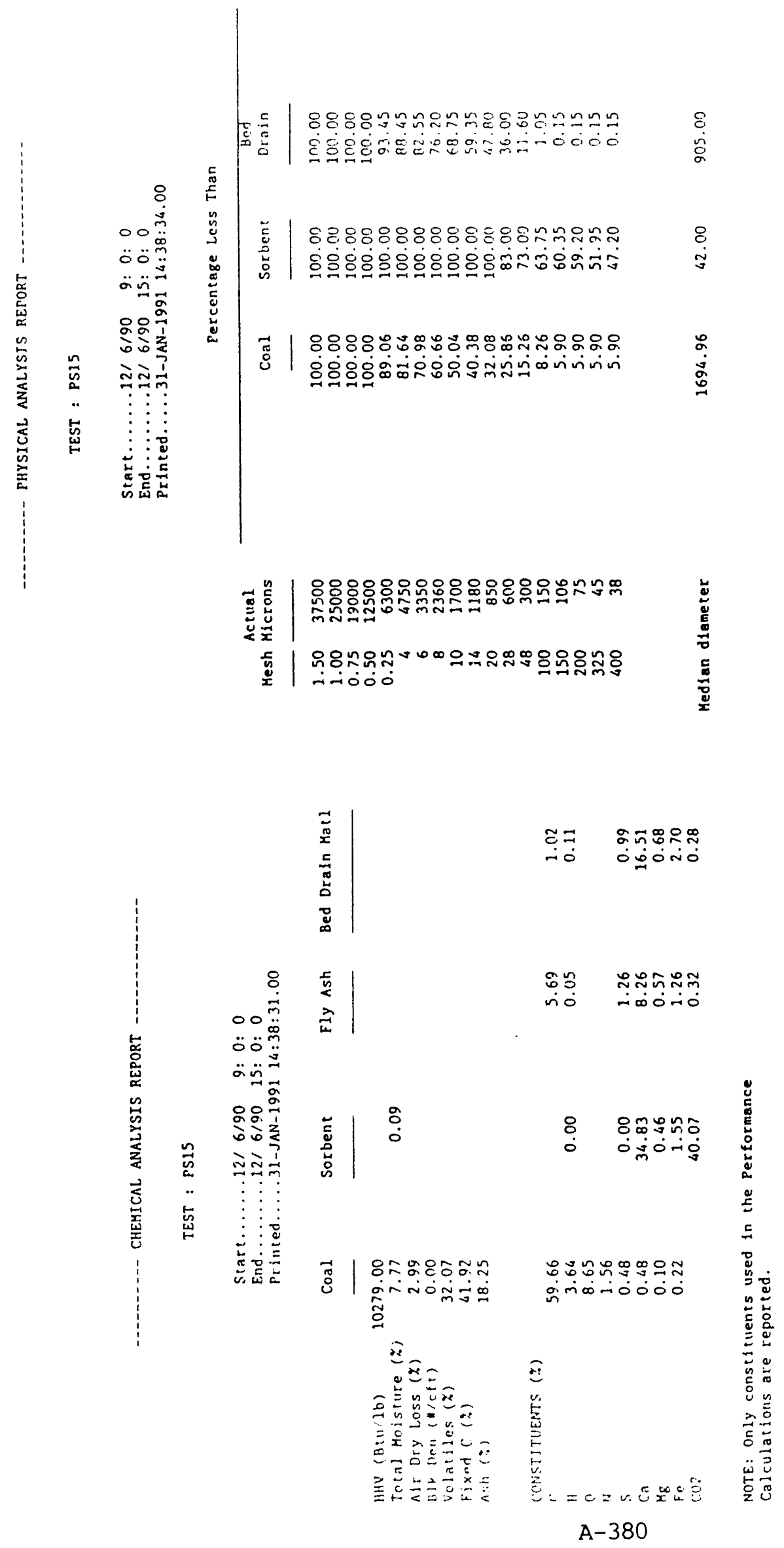




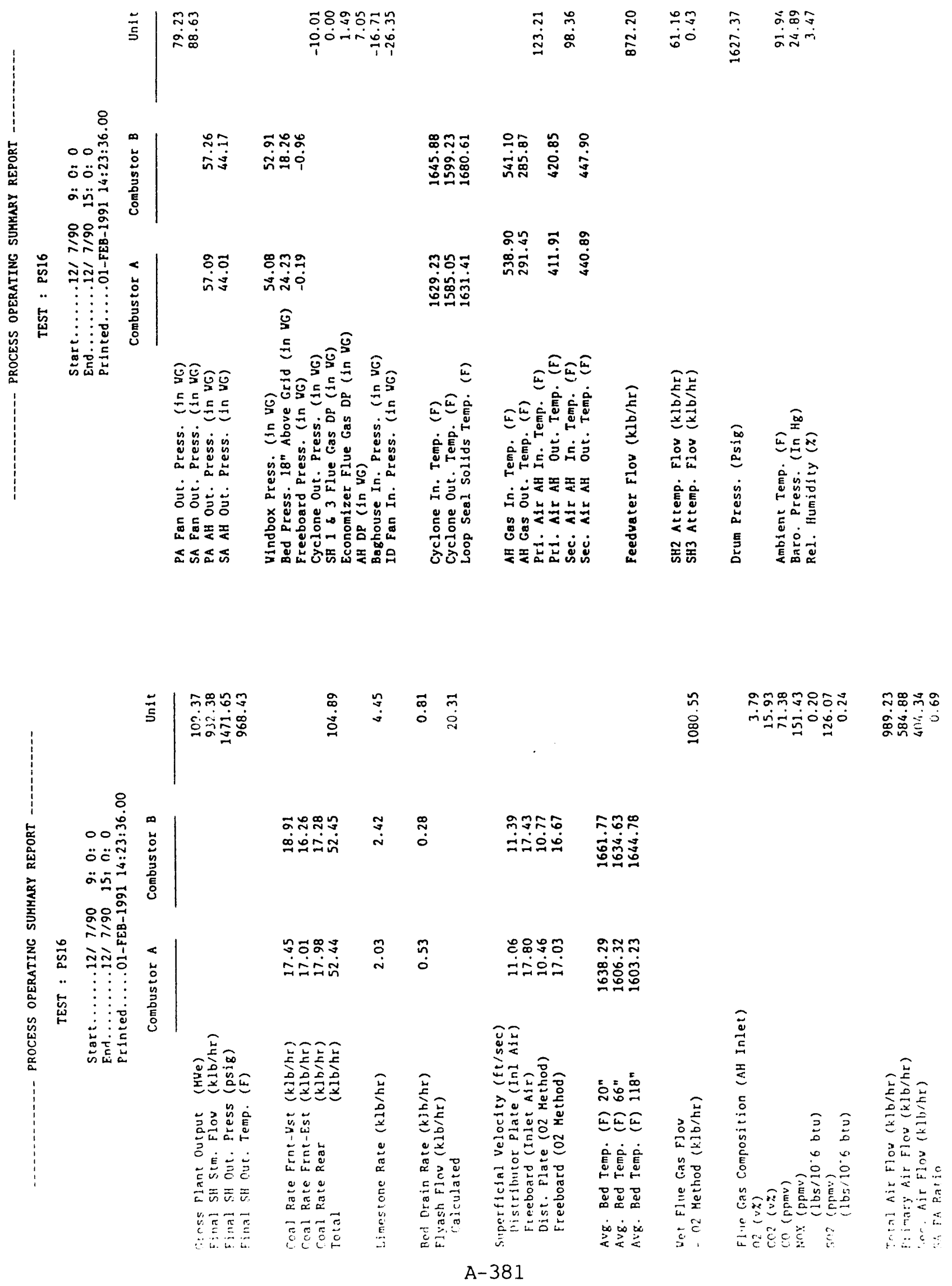




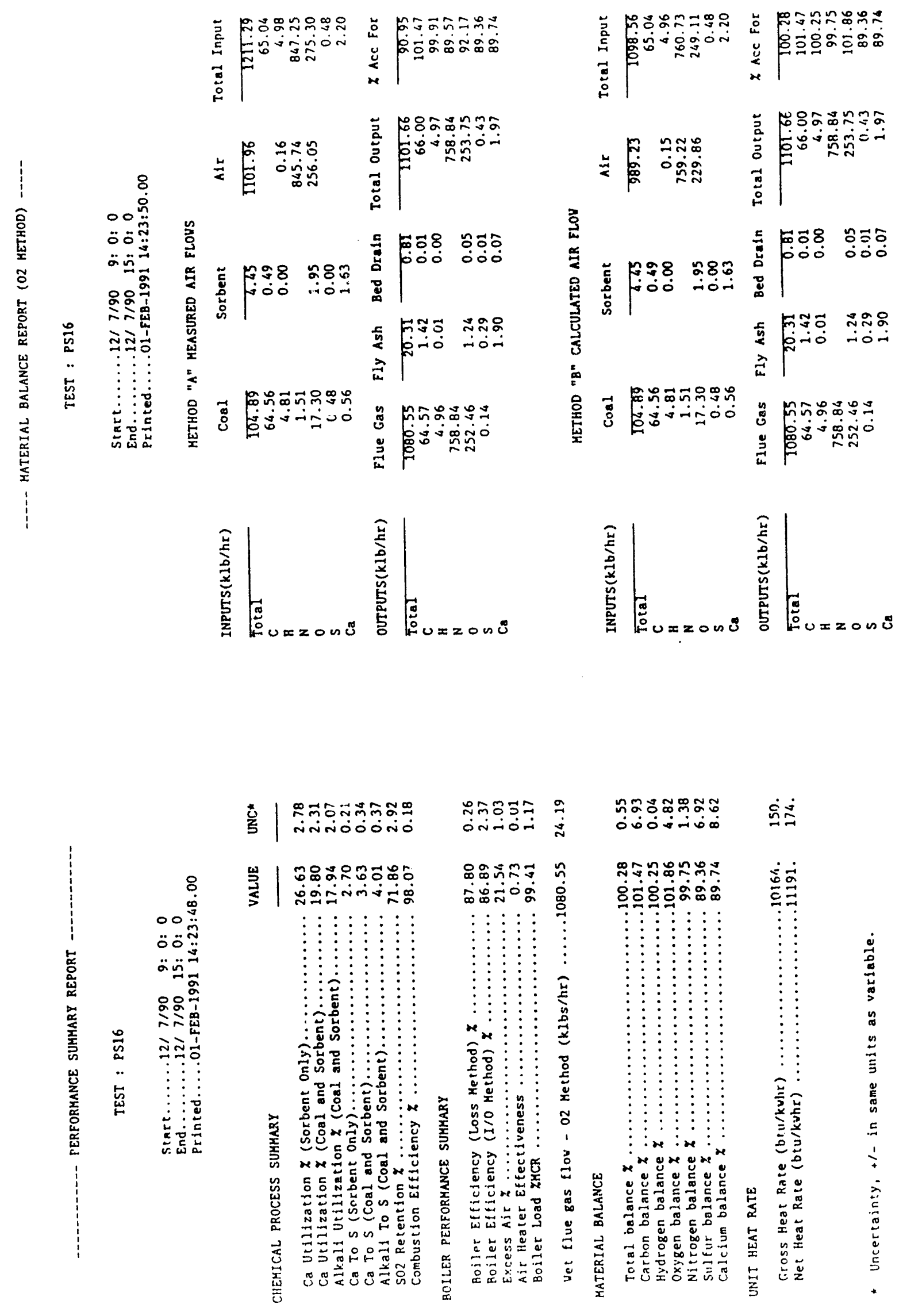




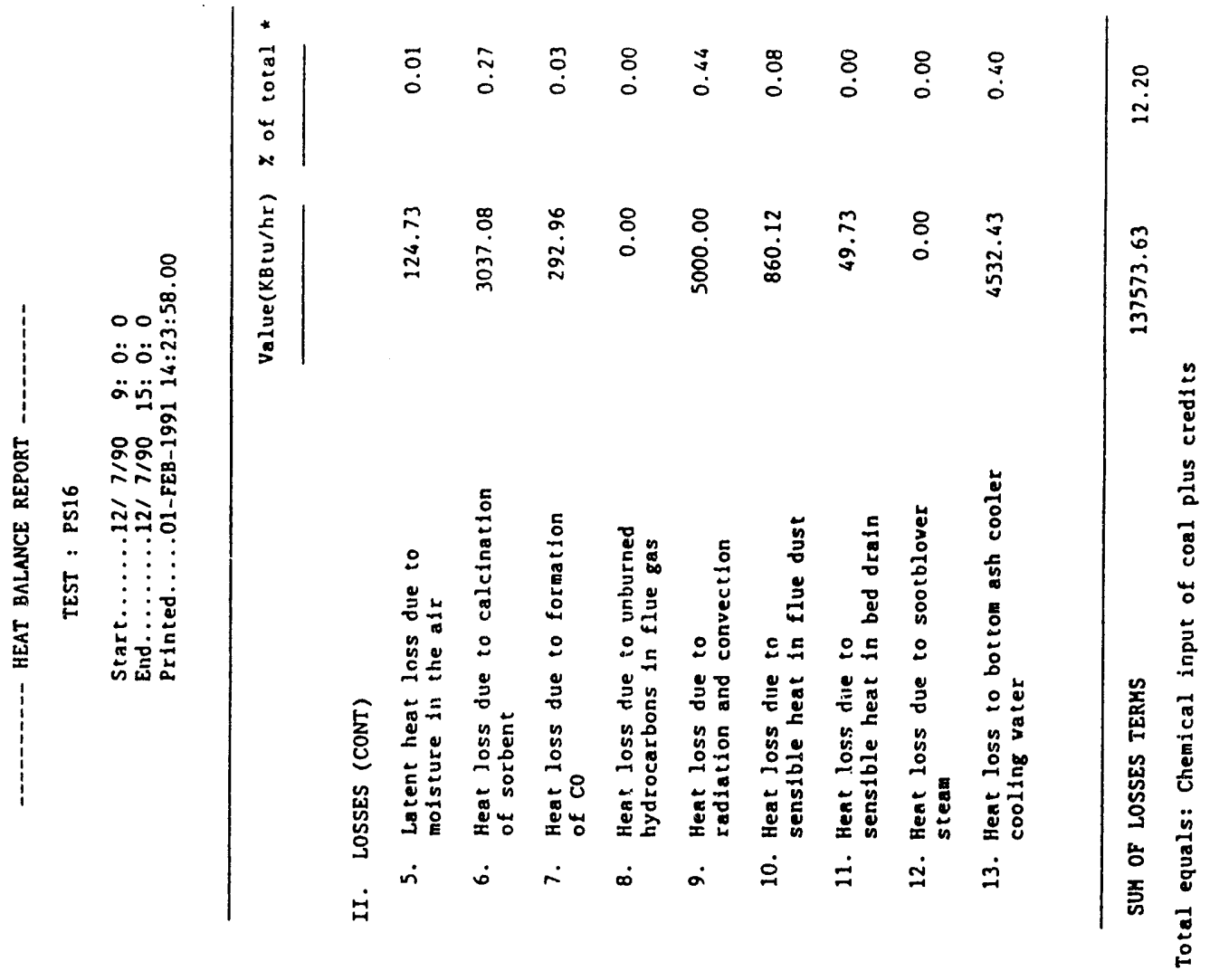

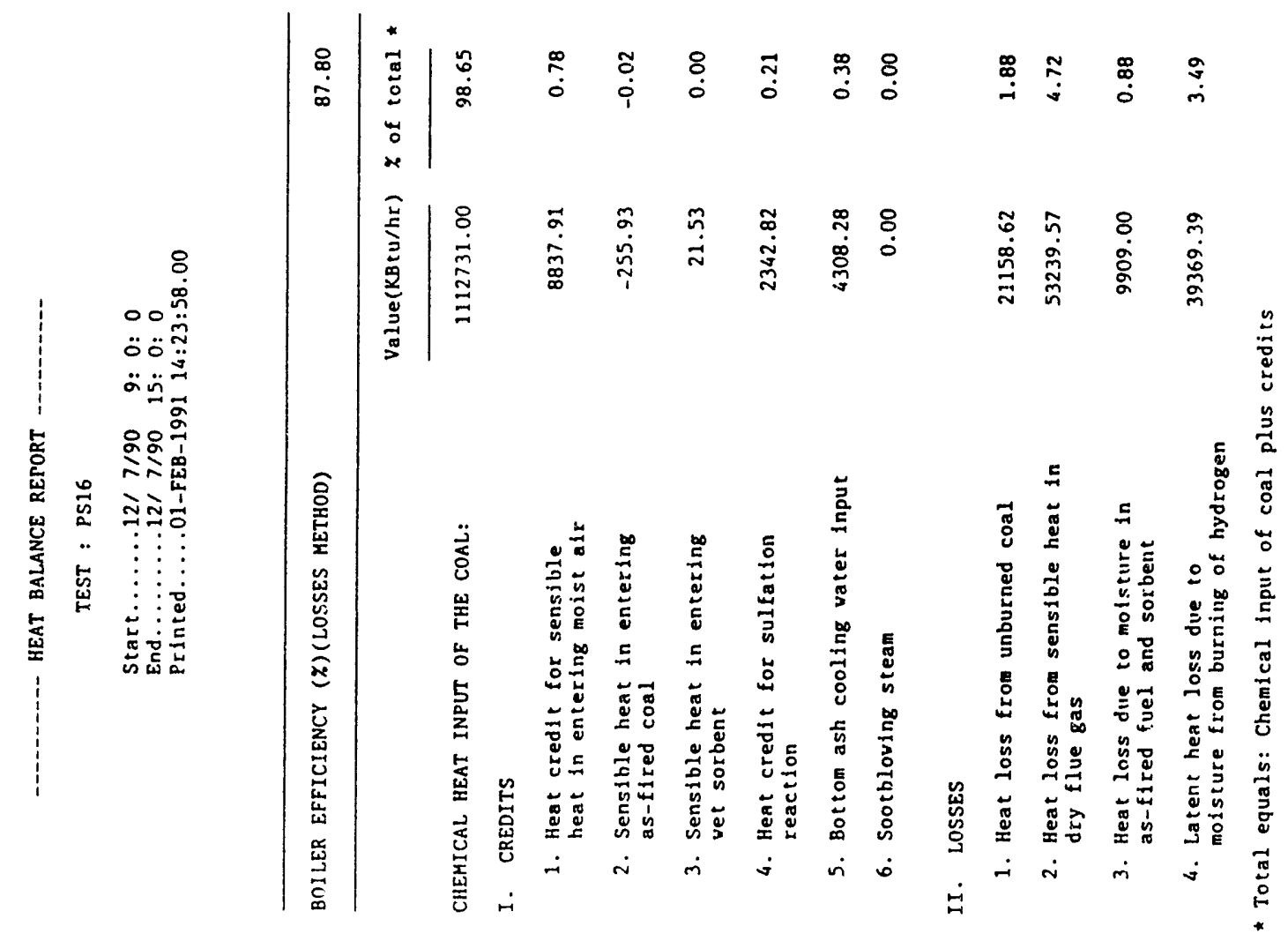



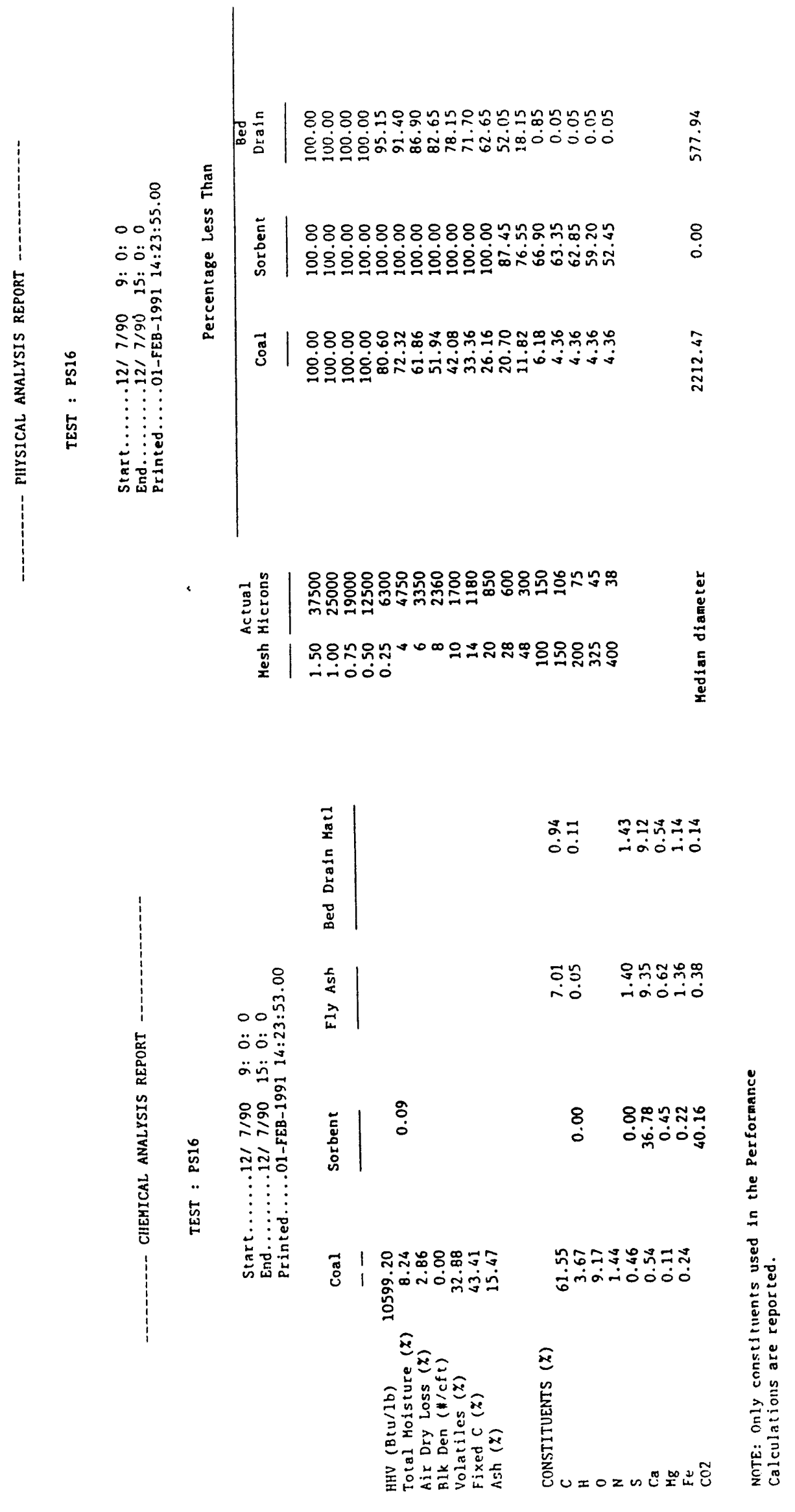


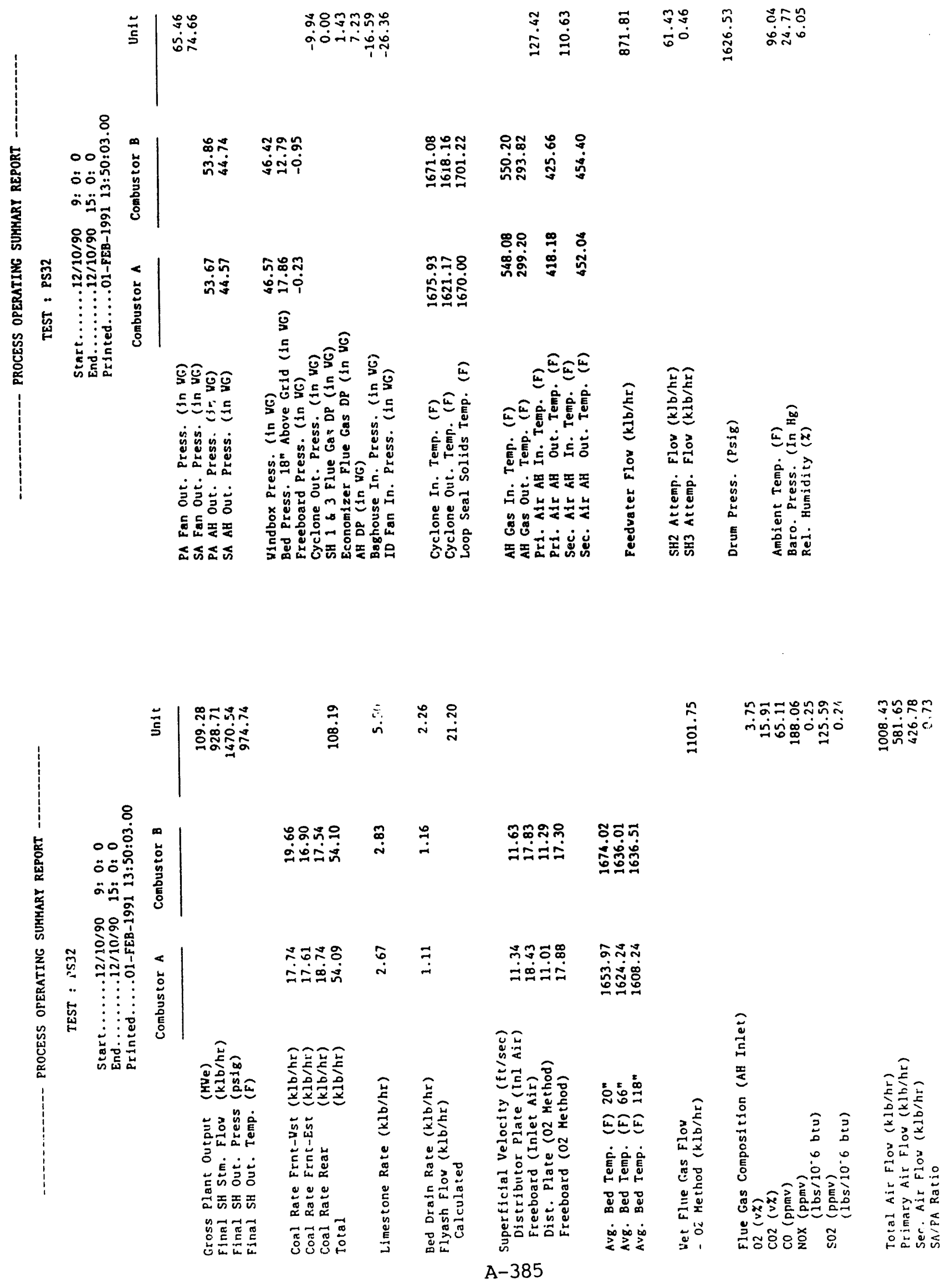




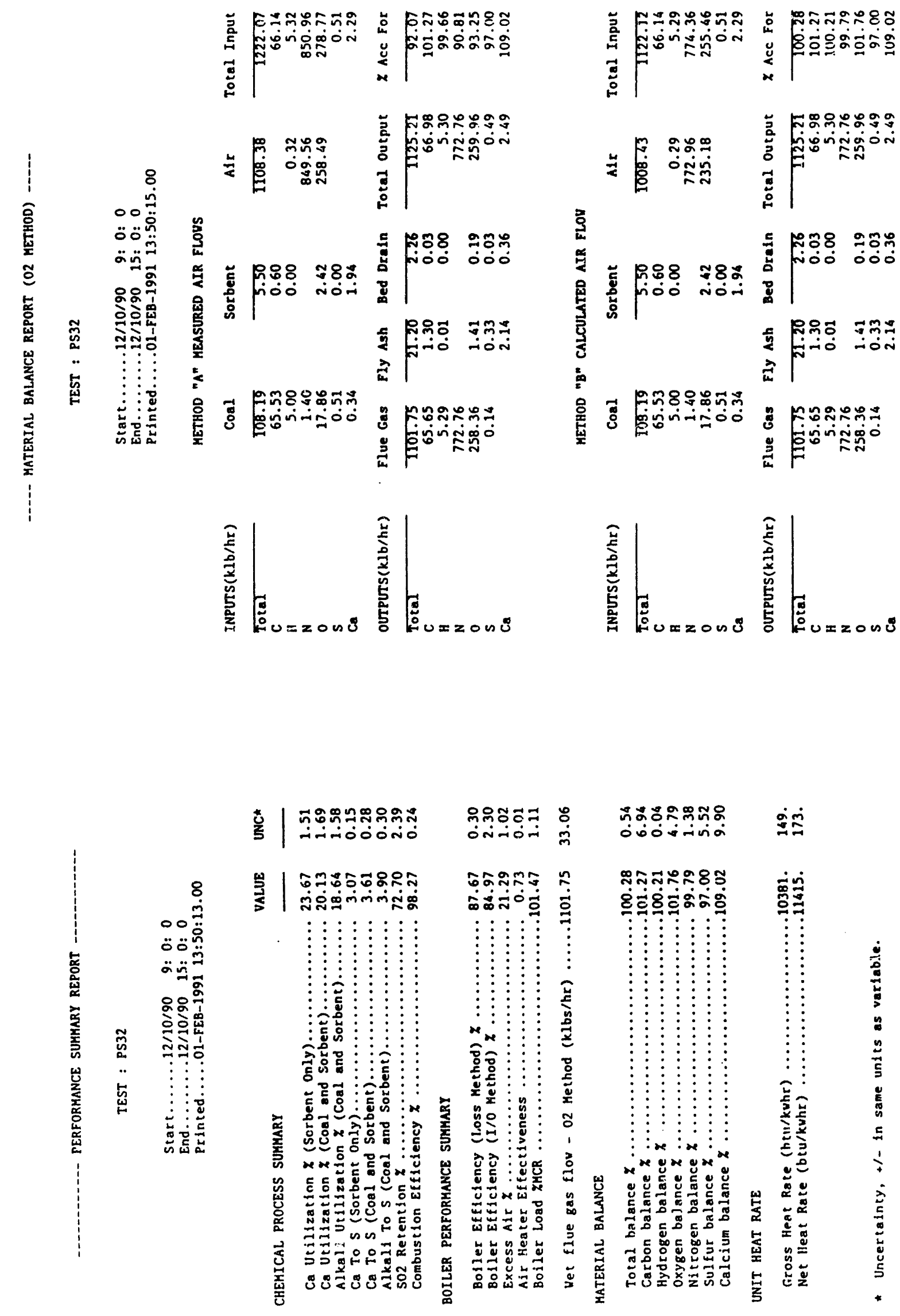



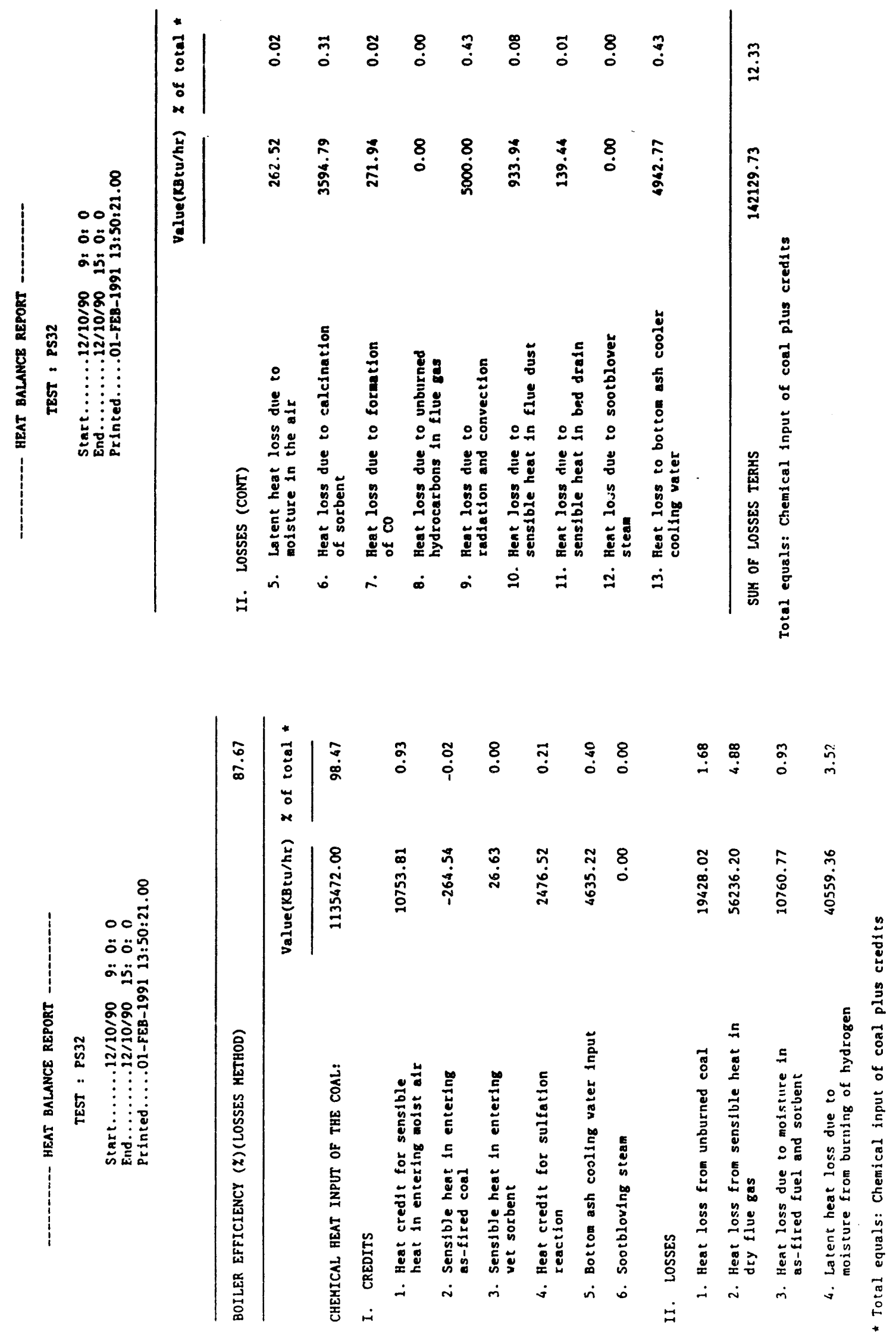


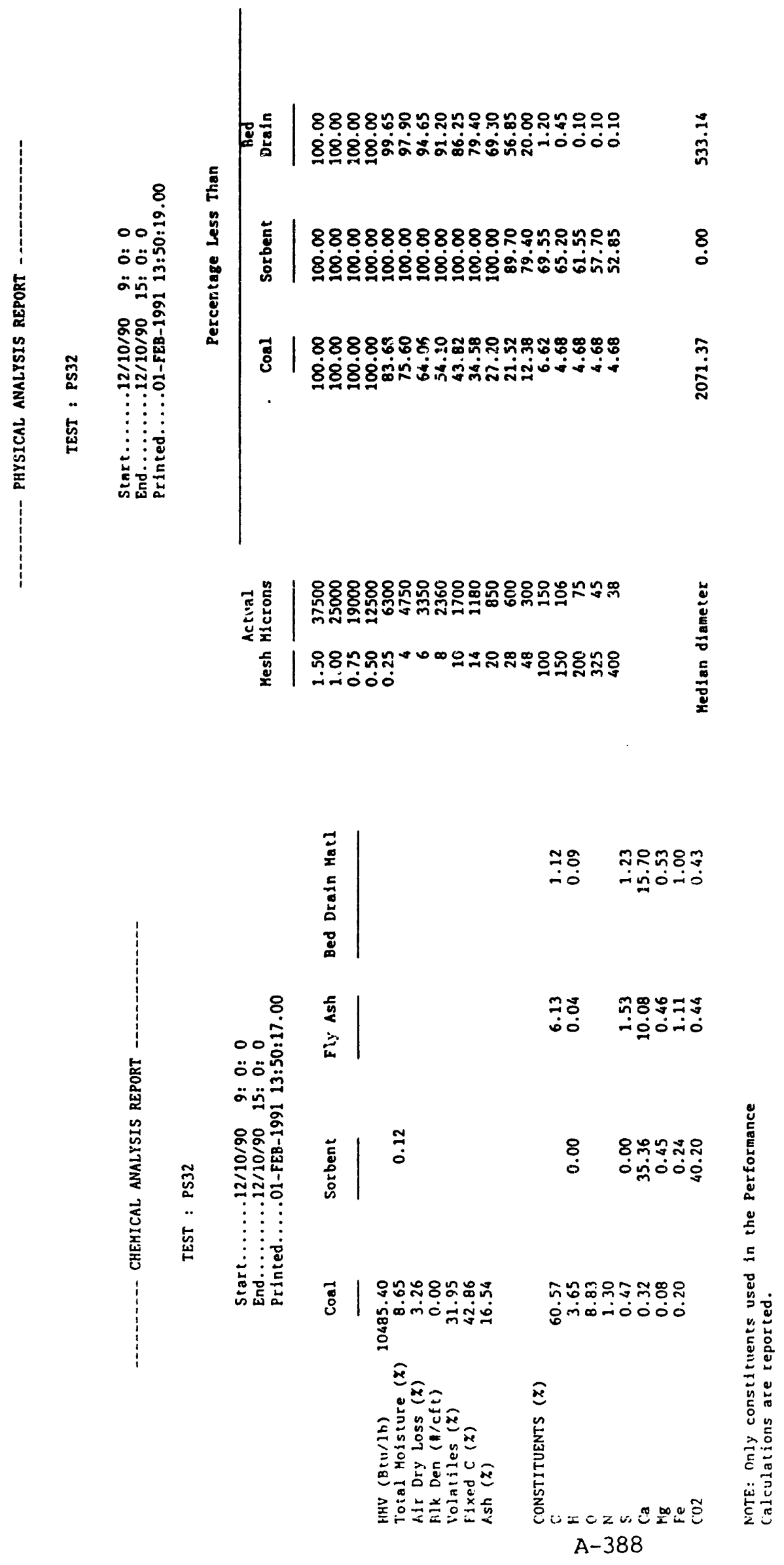




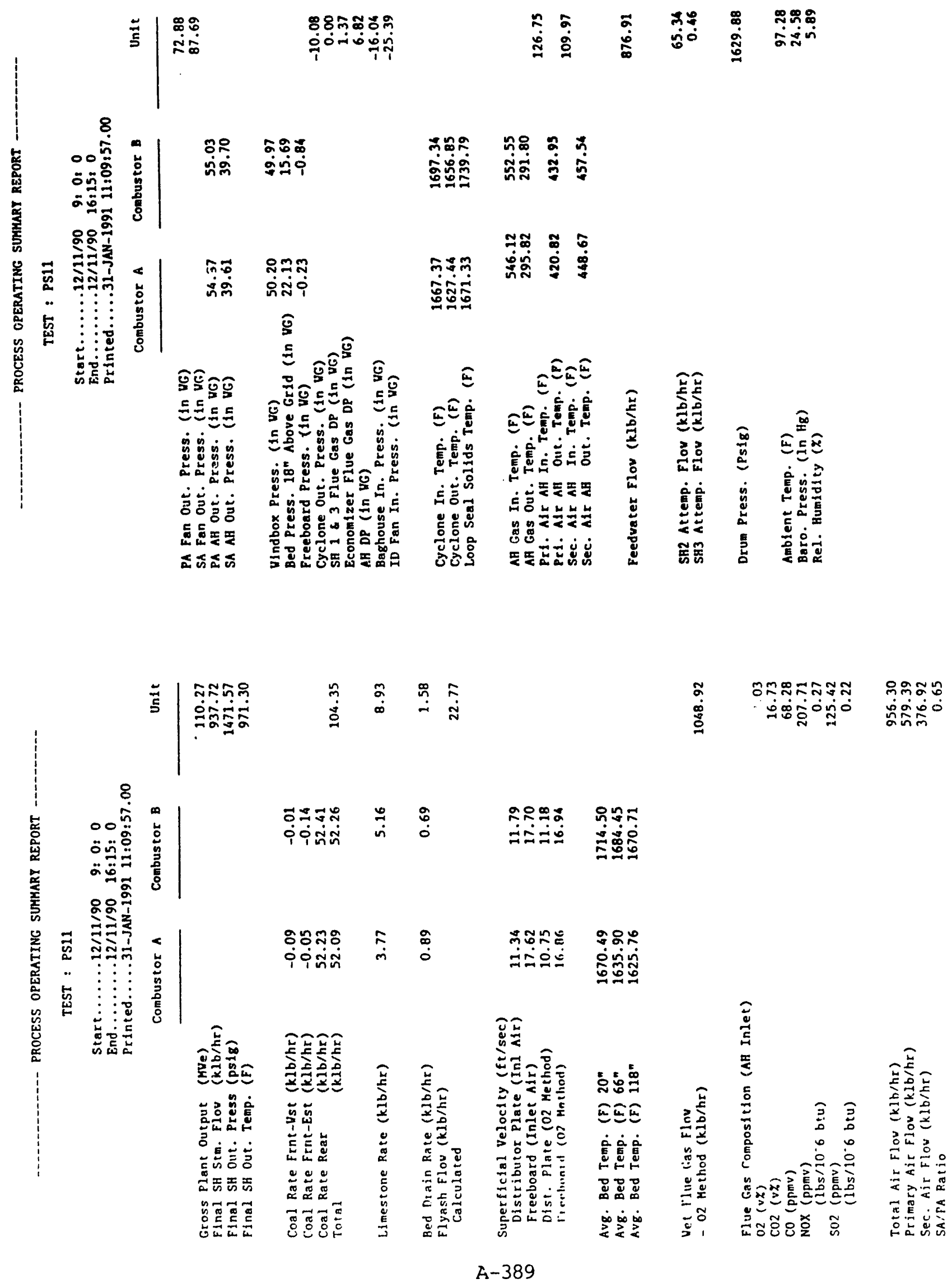



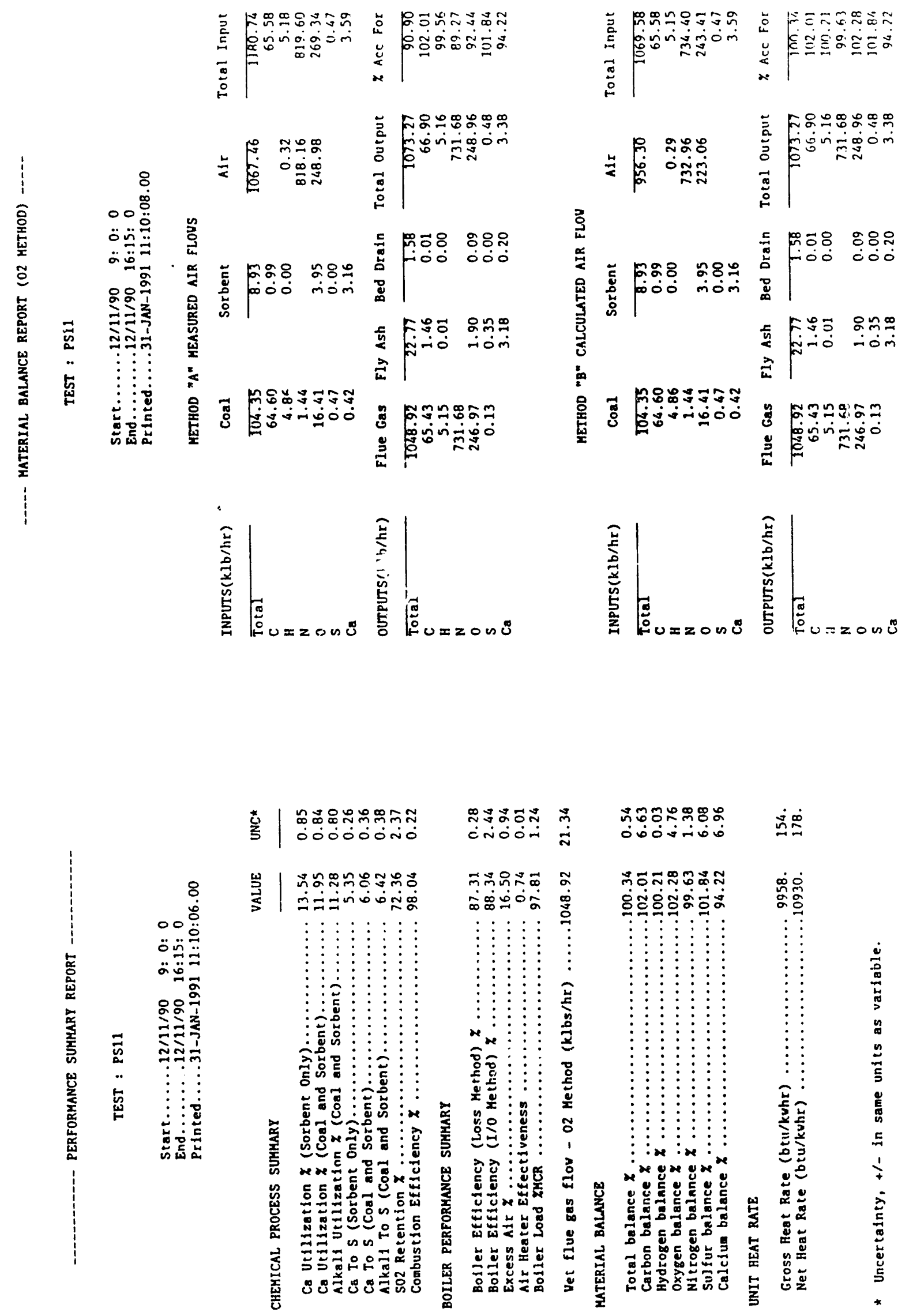


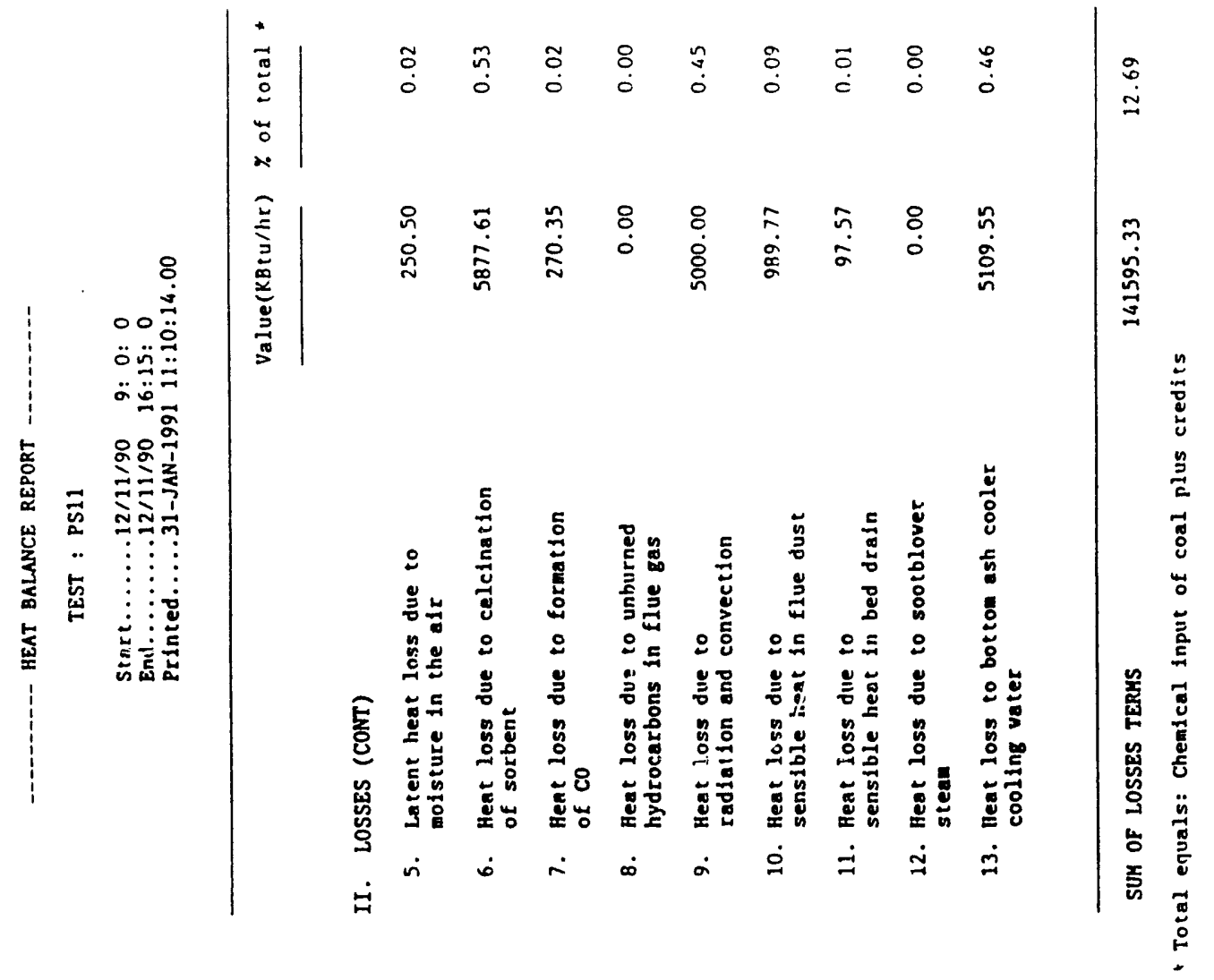

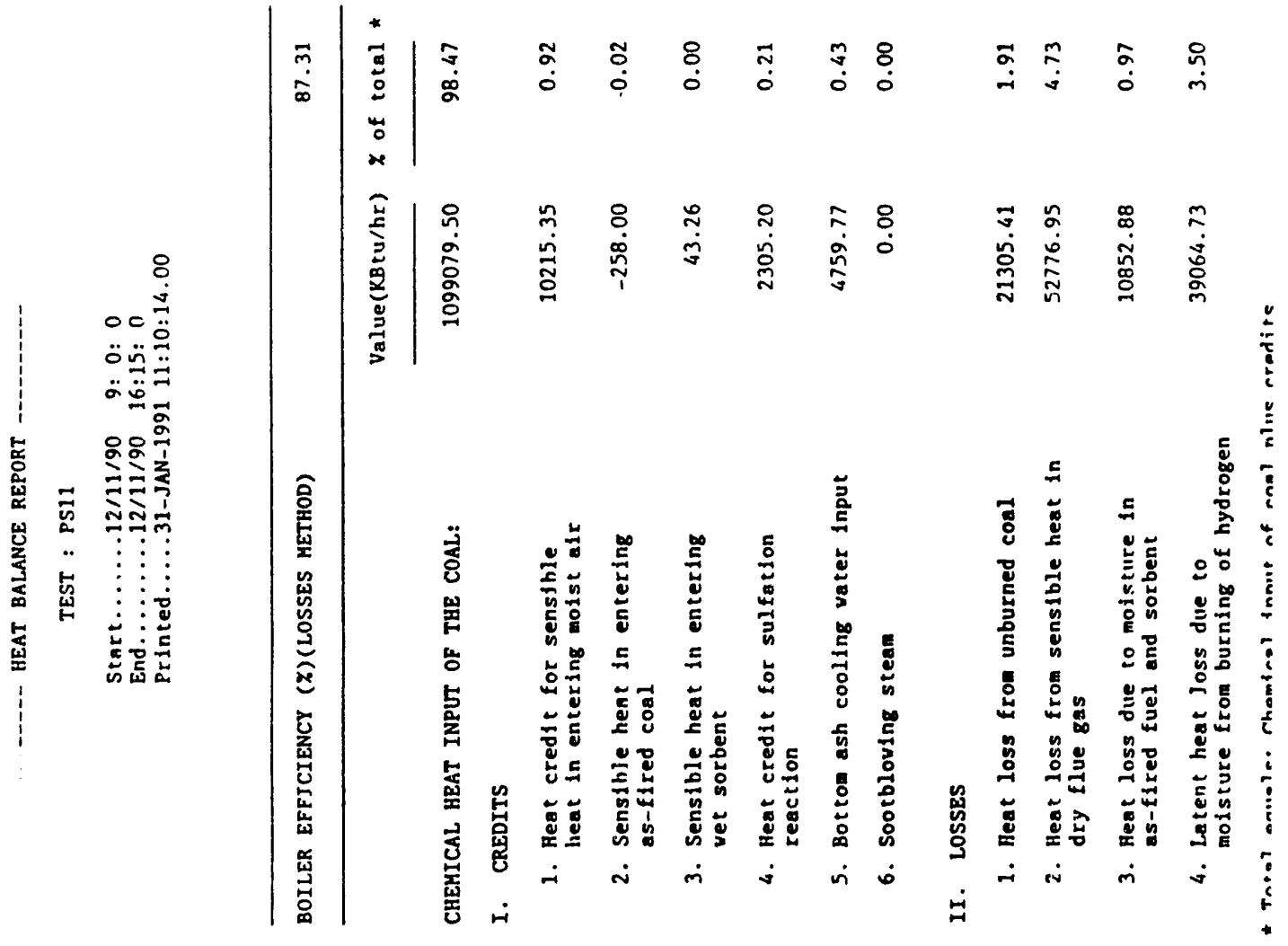



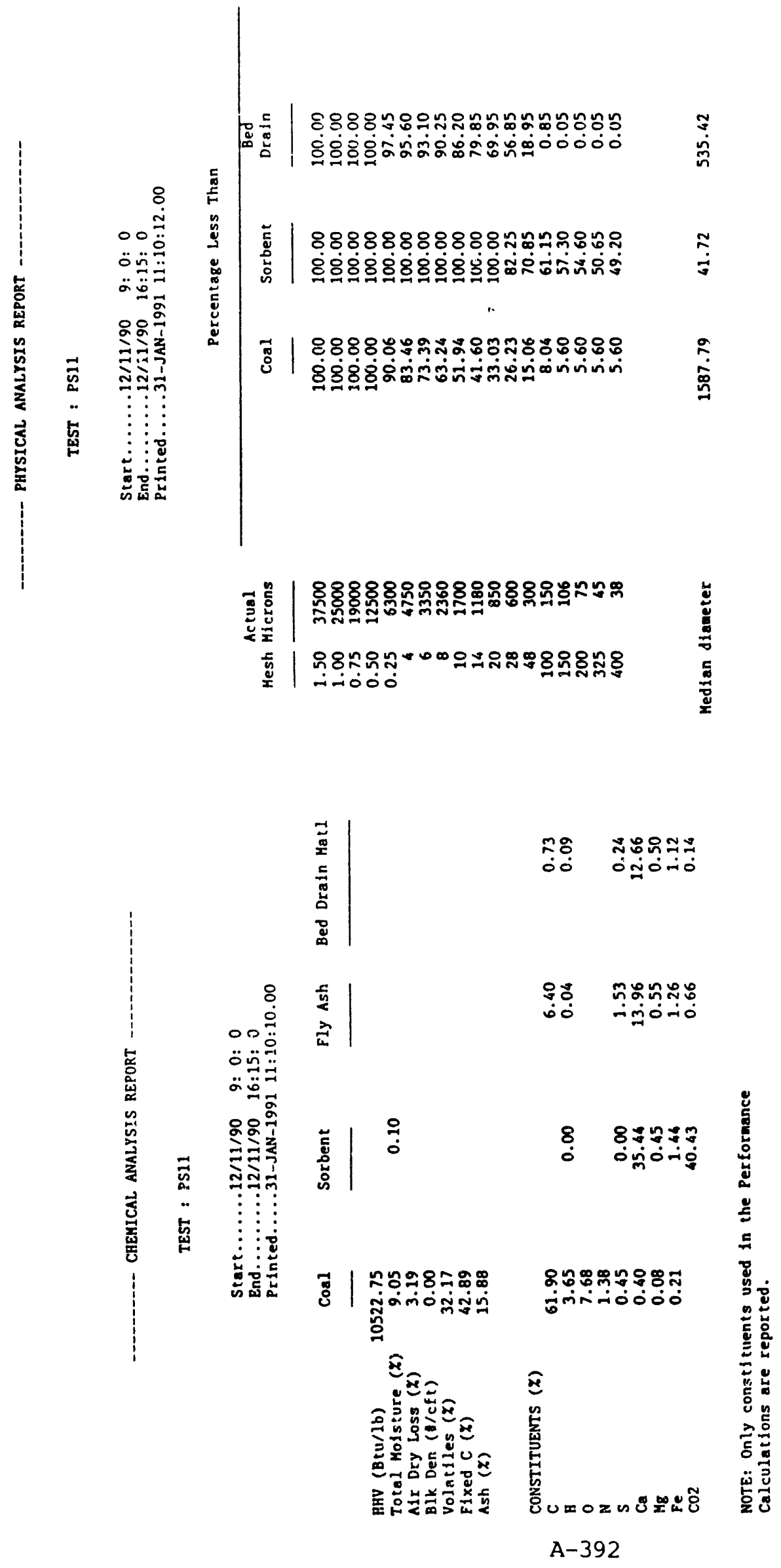


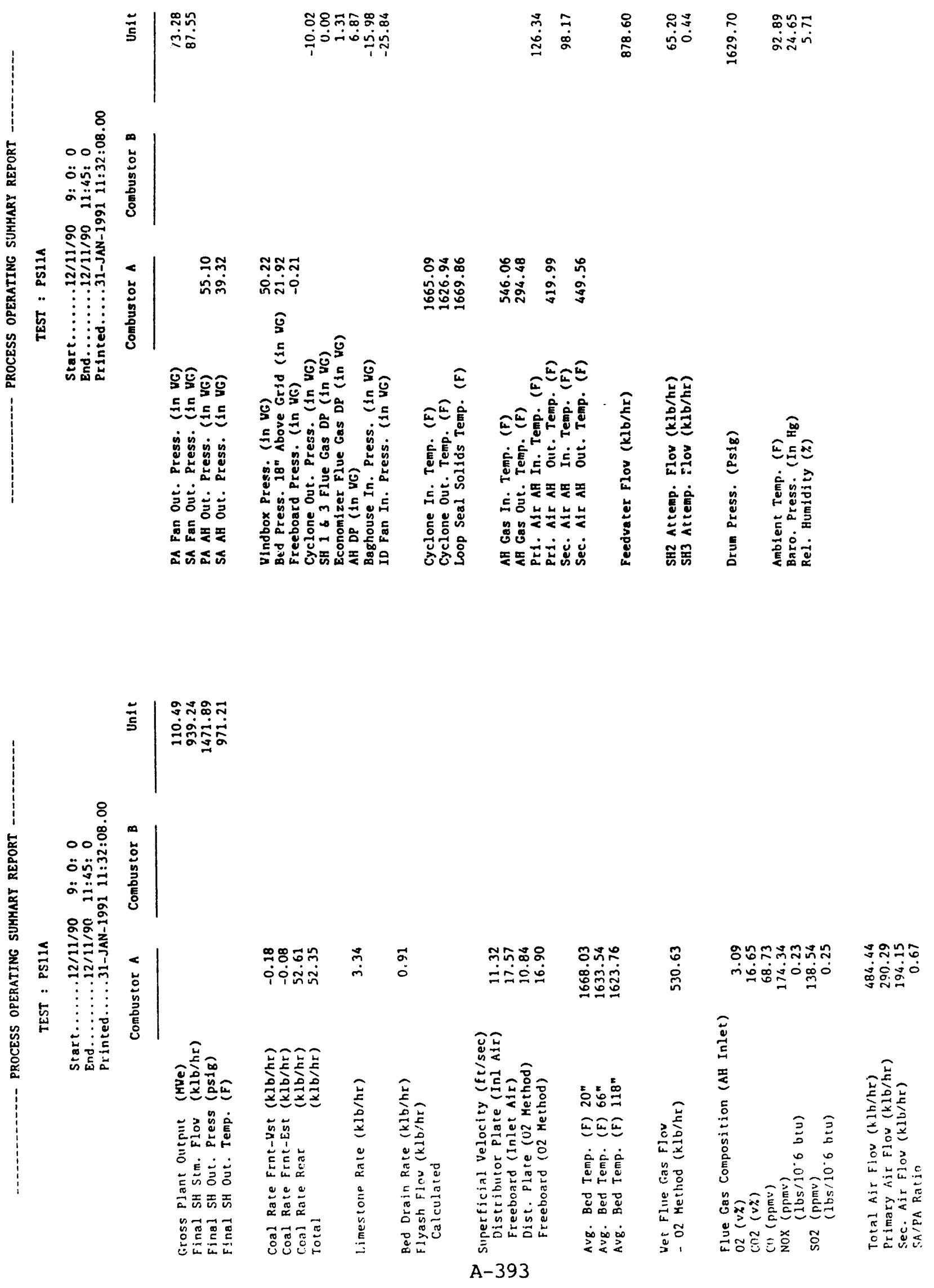




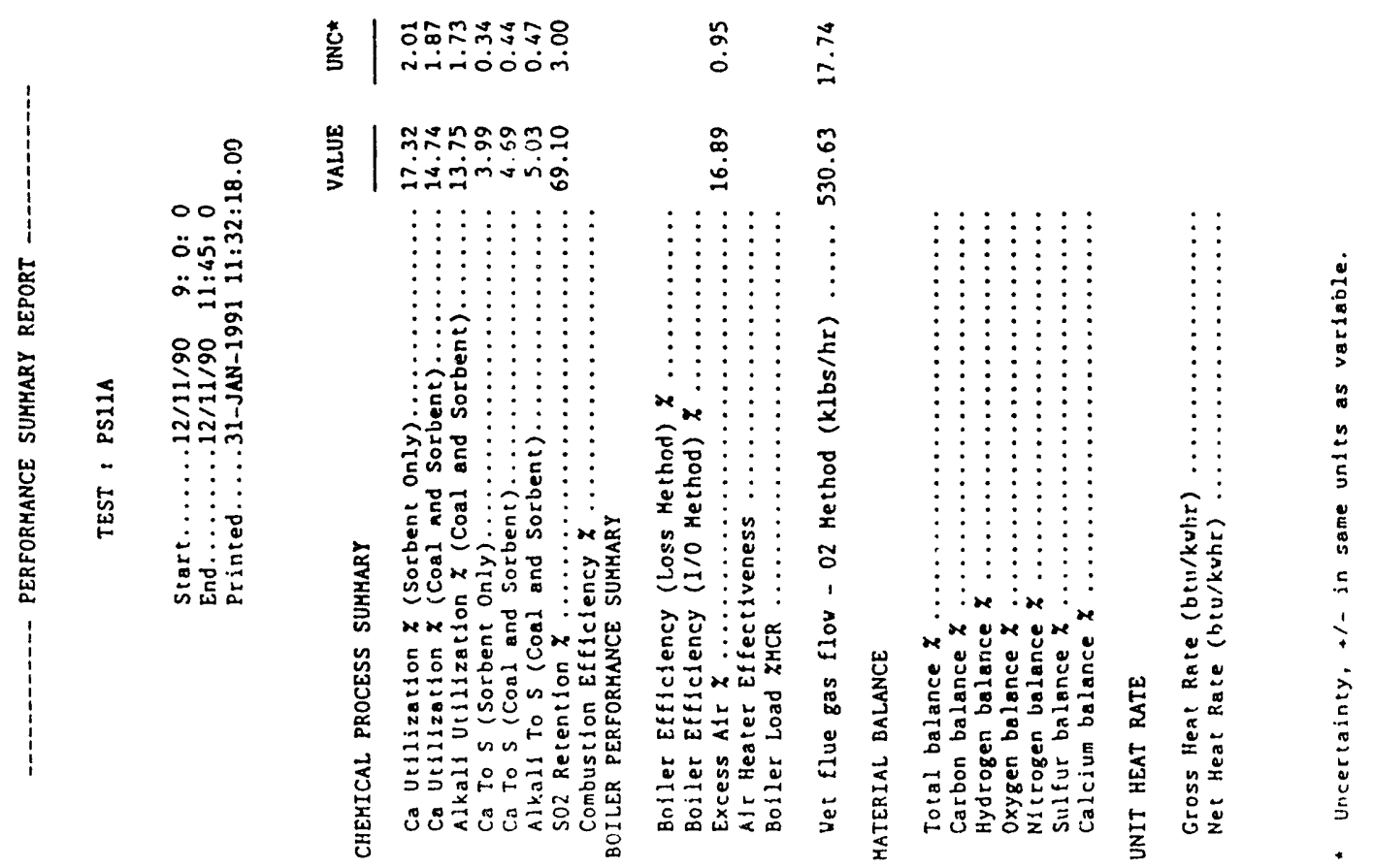



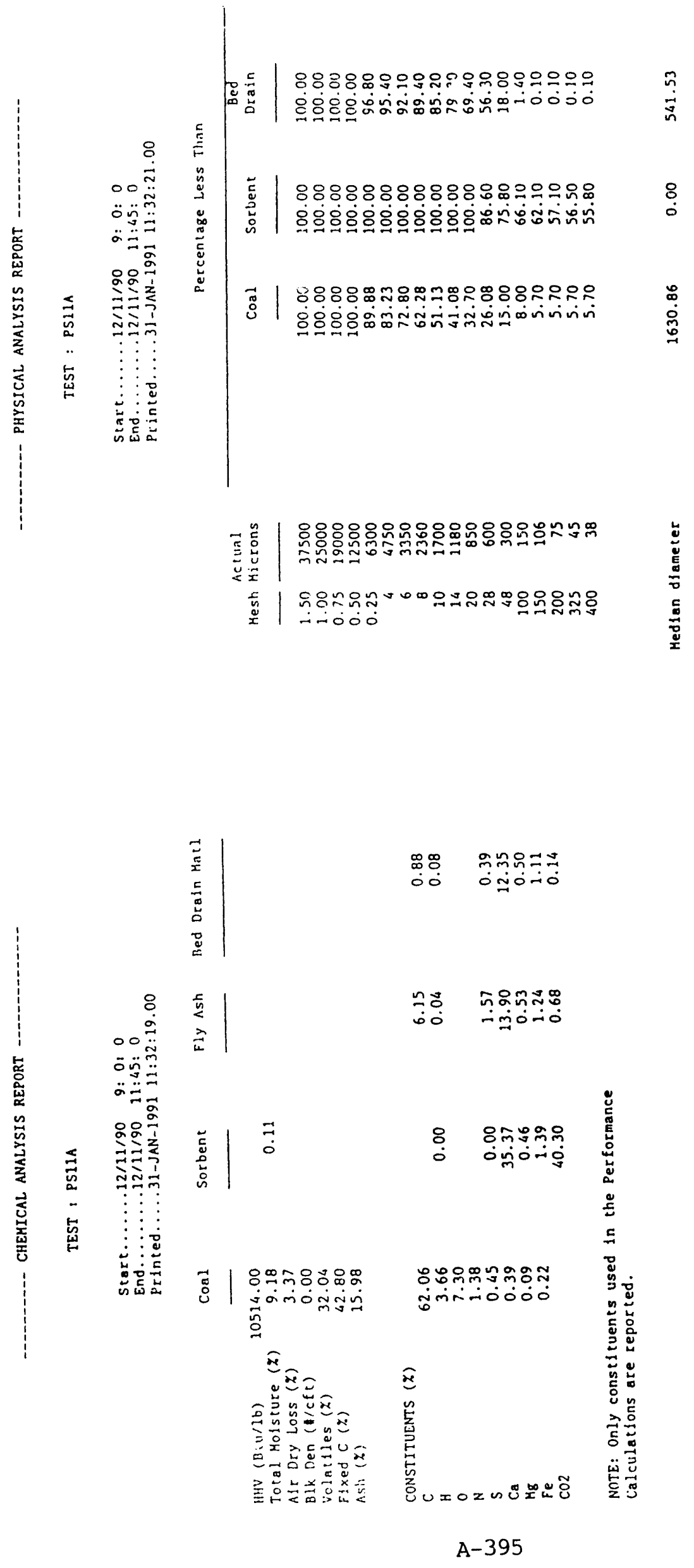

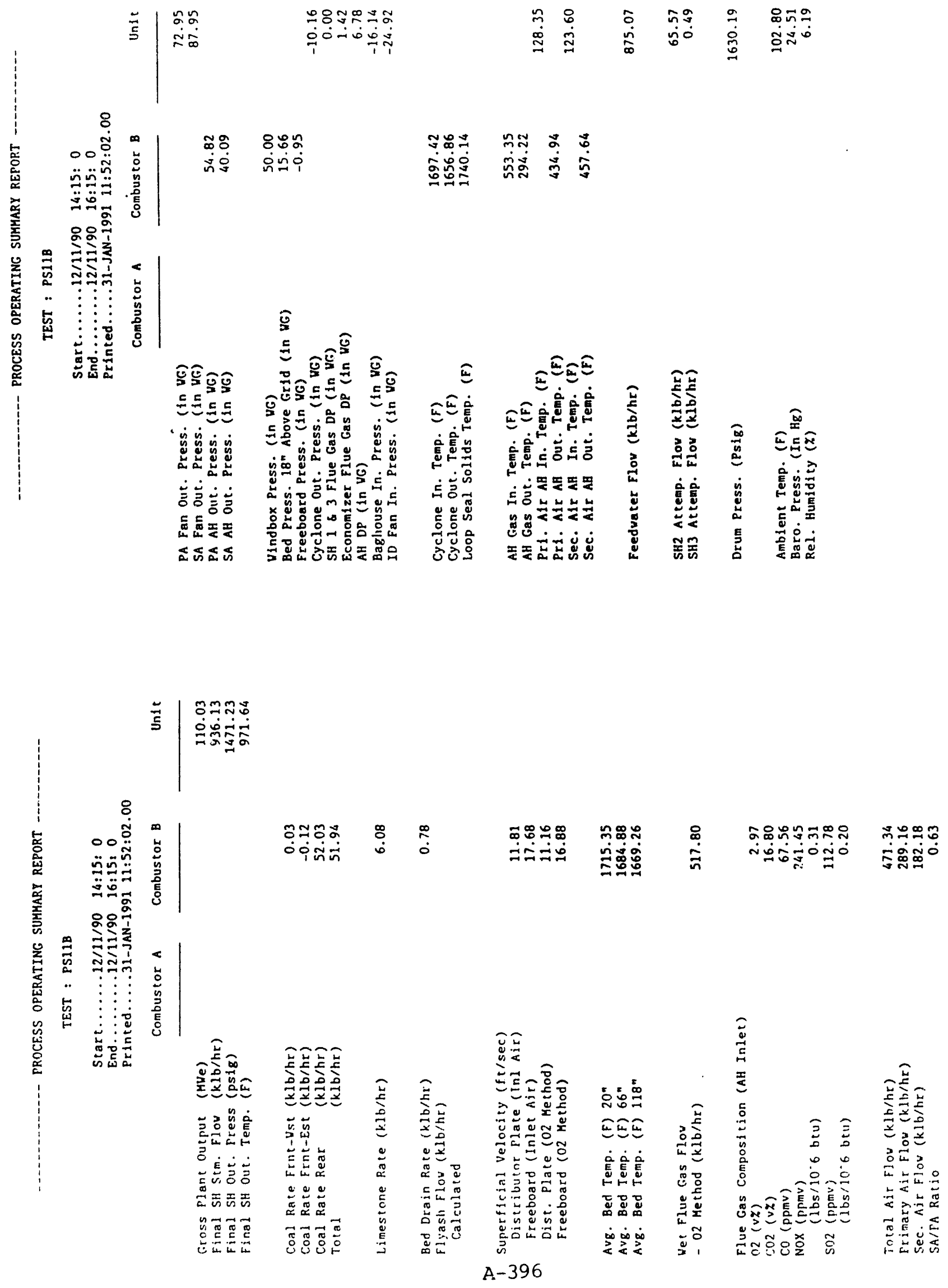


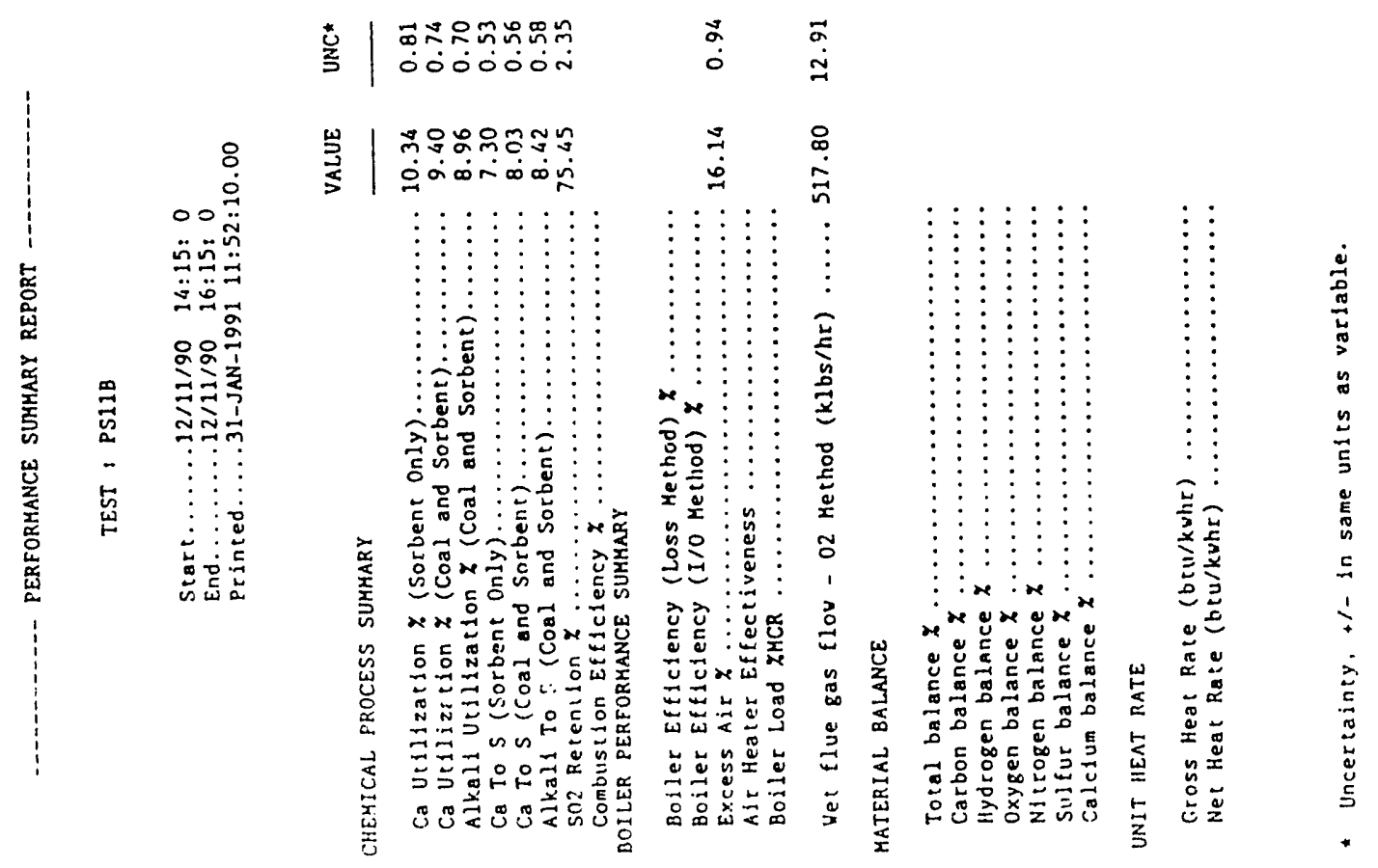



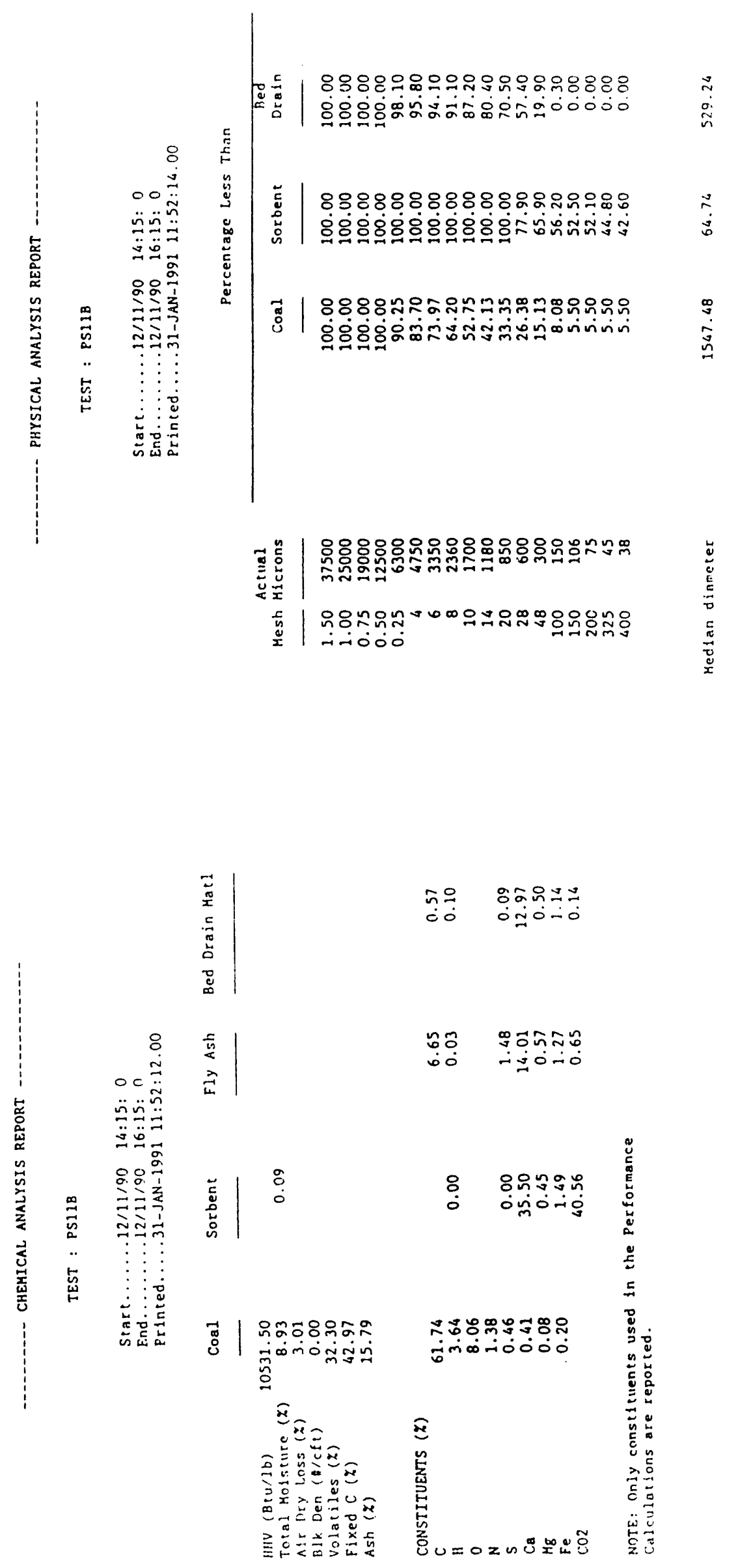

A-398 


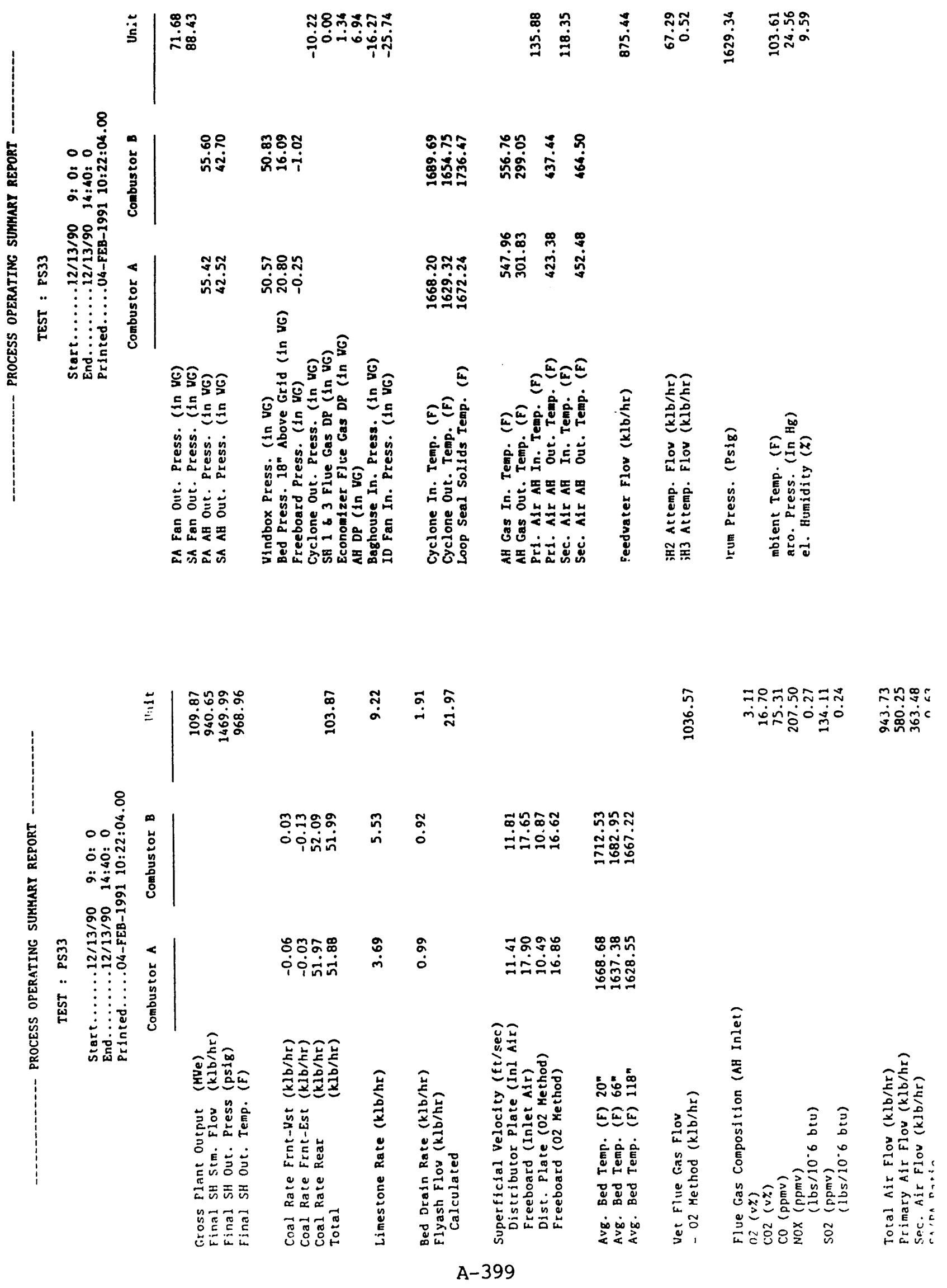




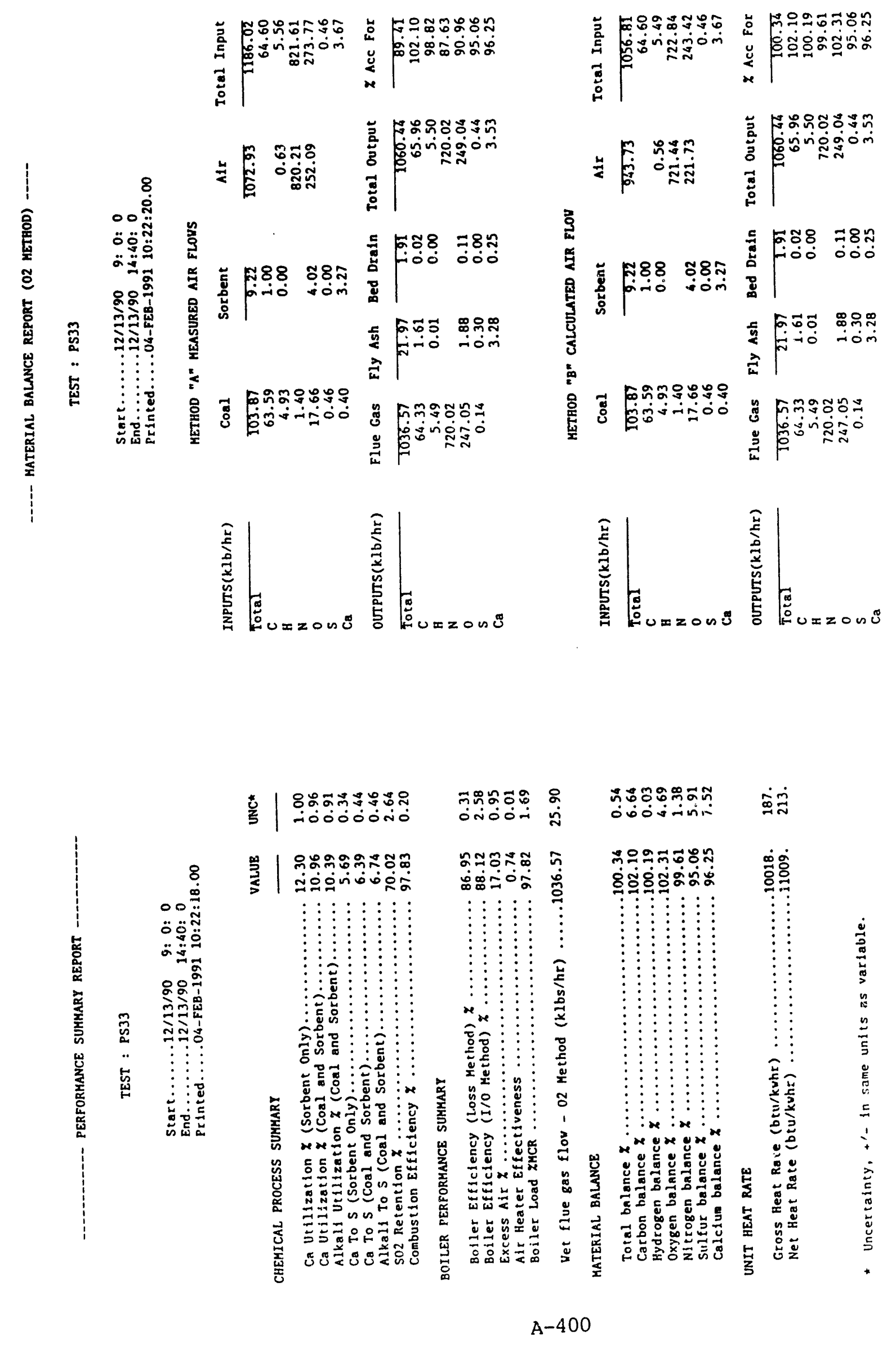




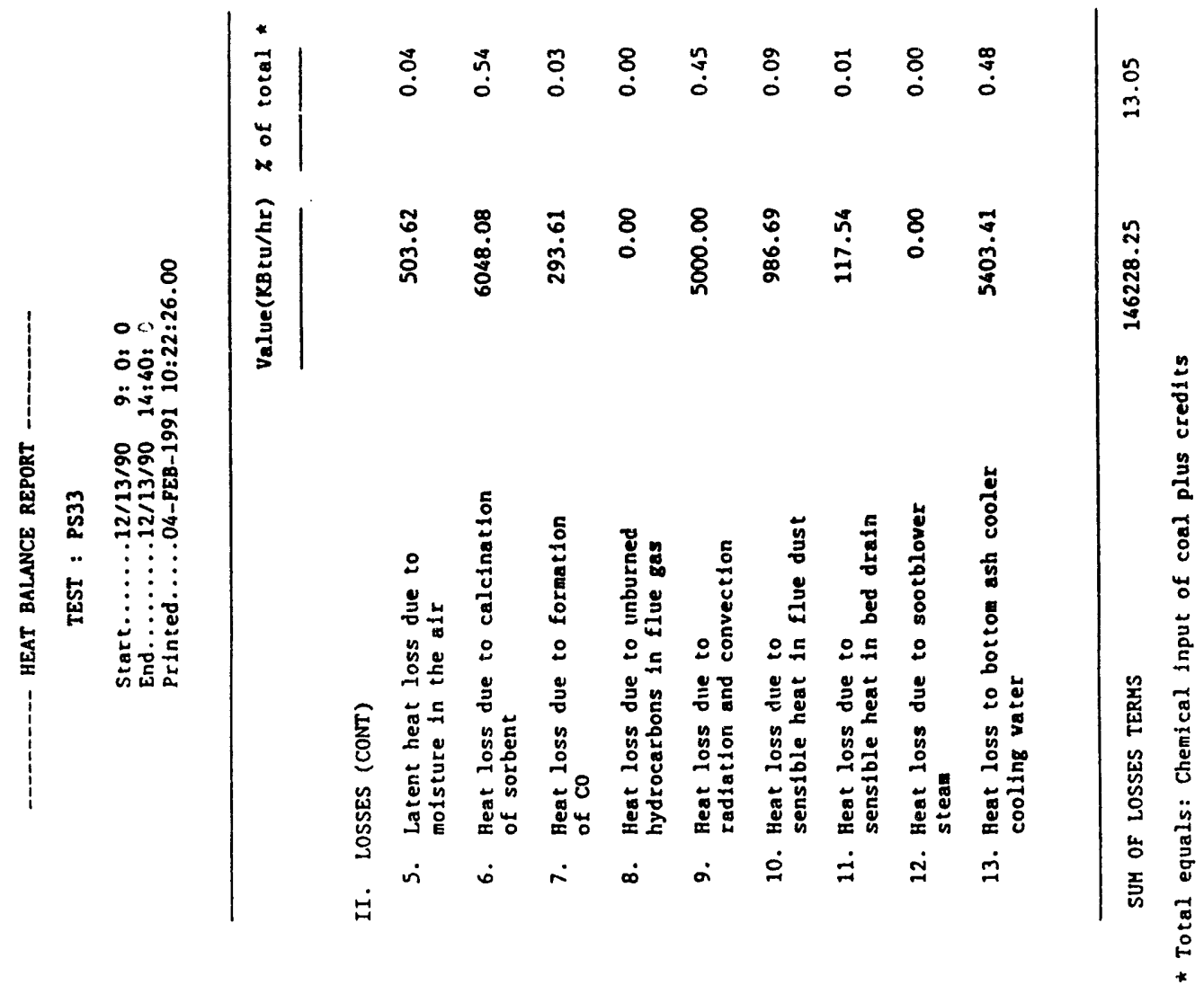

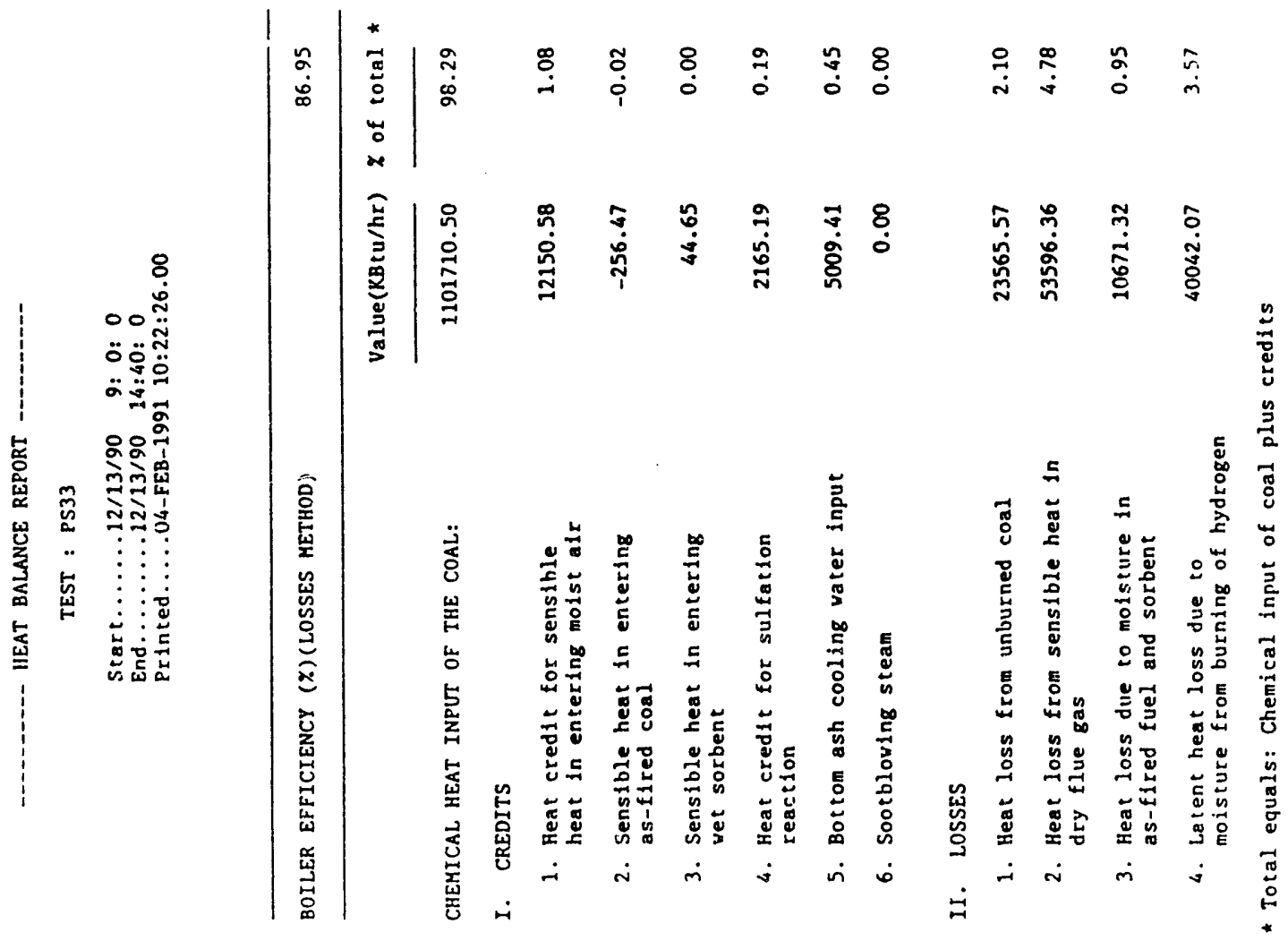



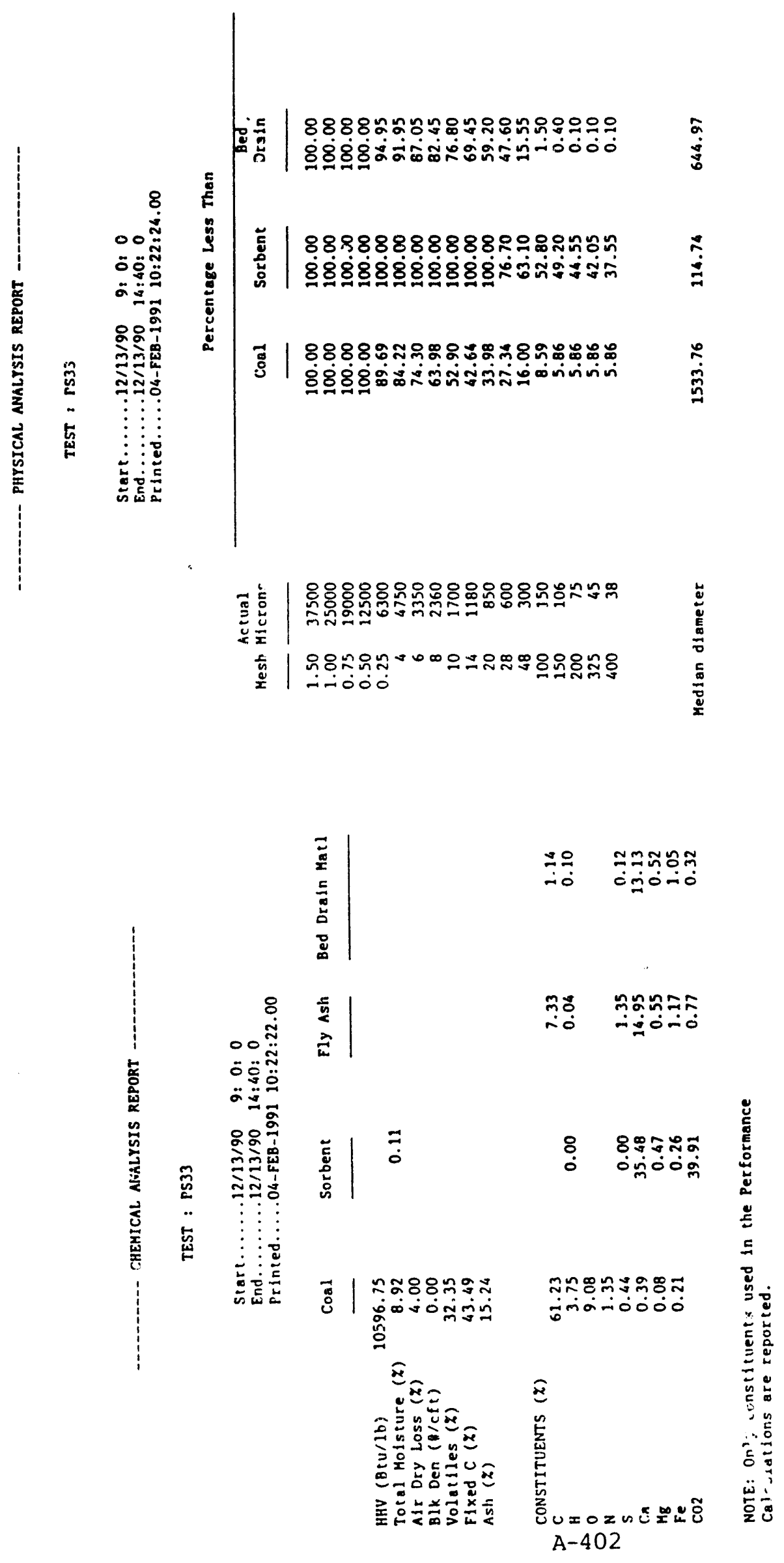

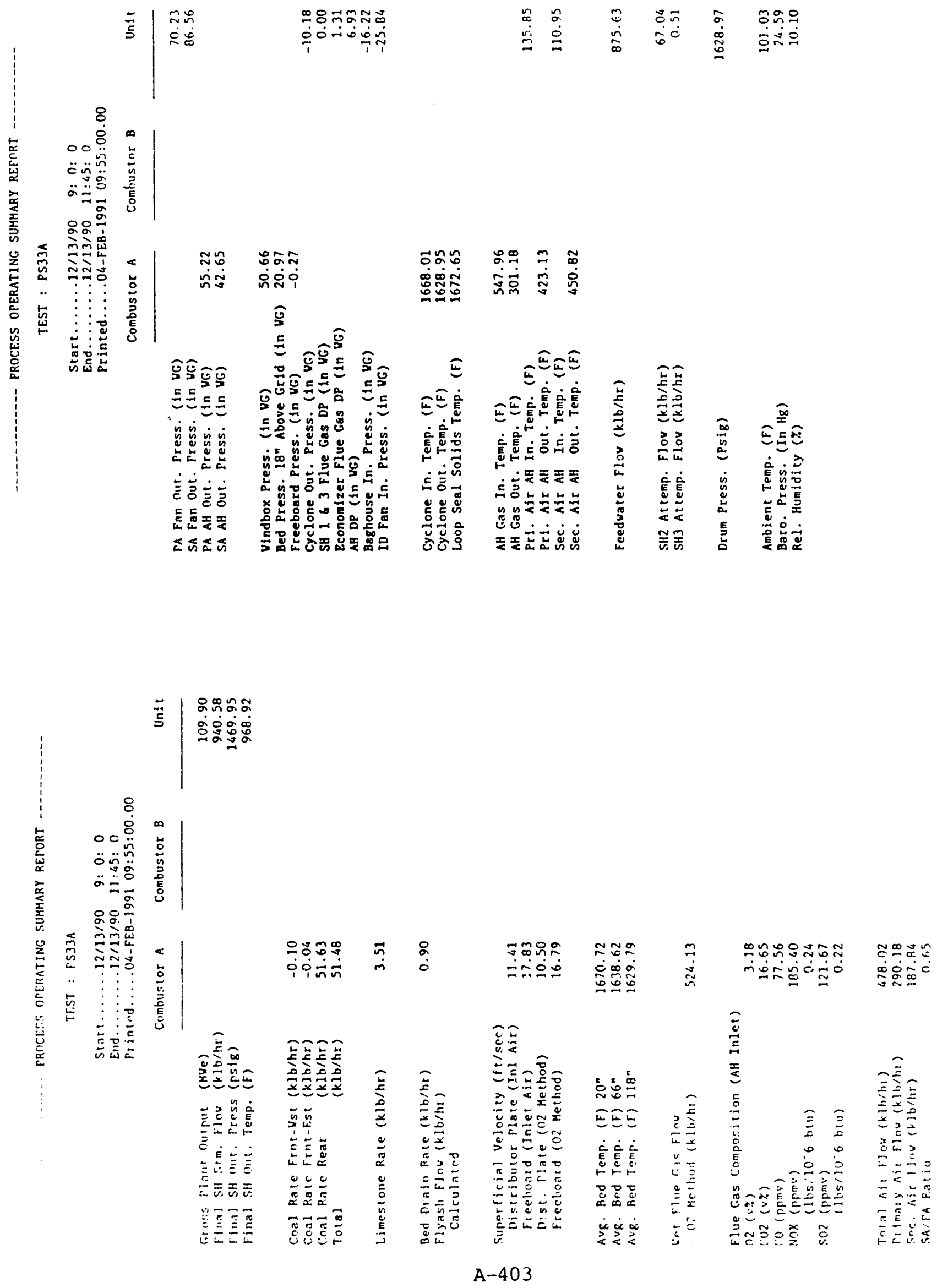


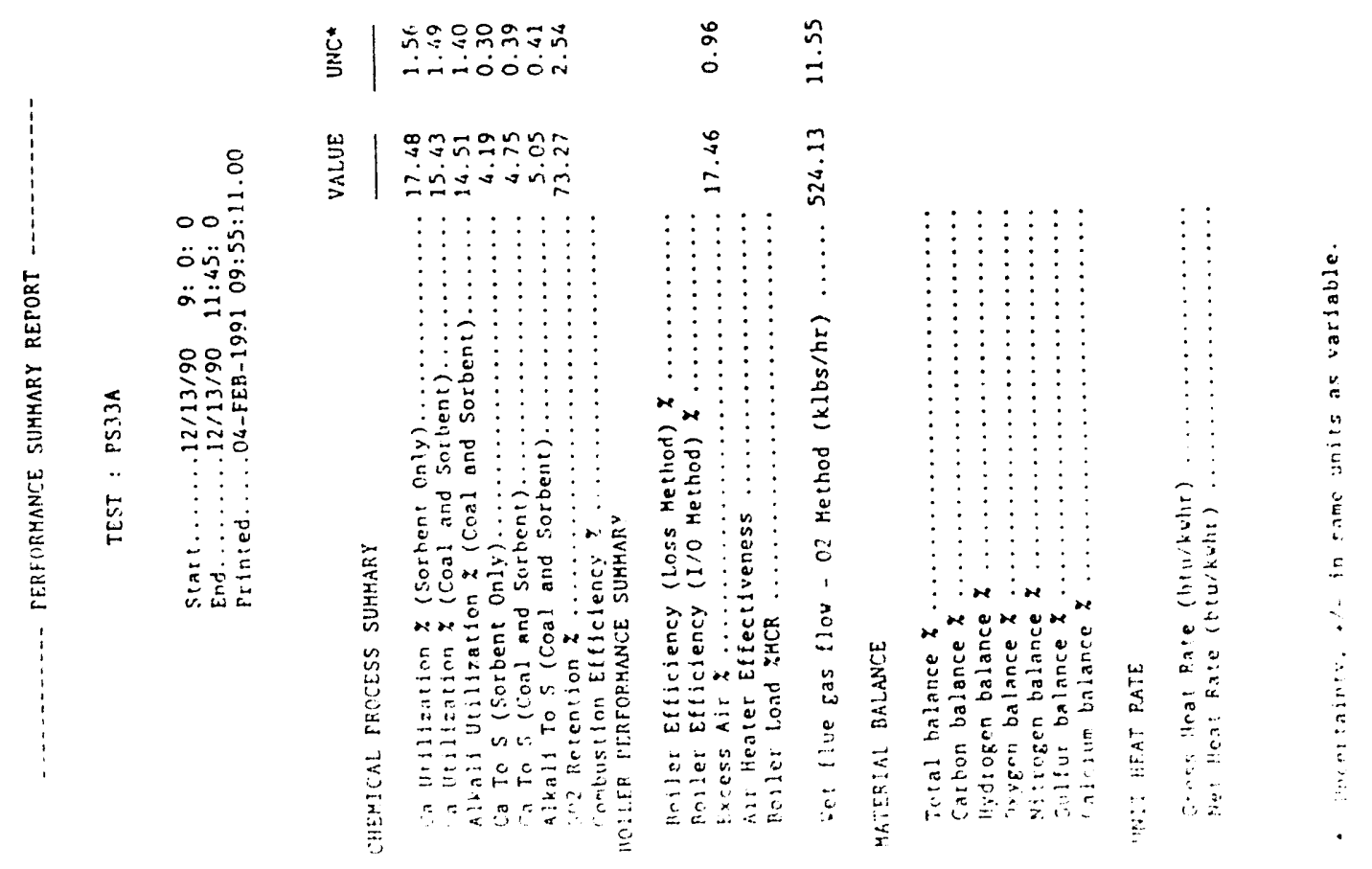

A-404 

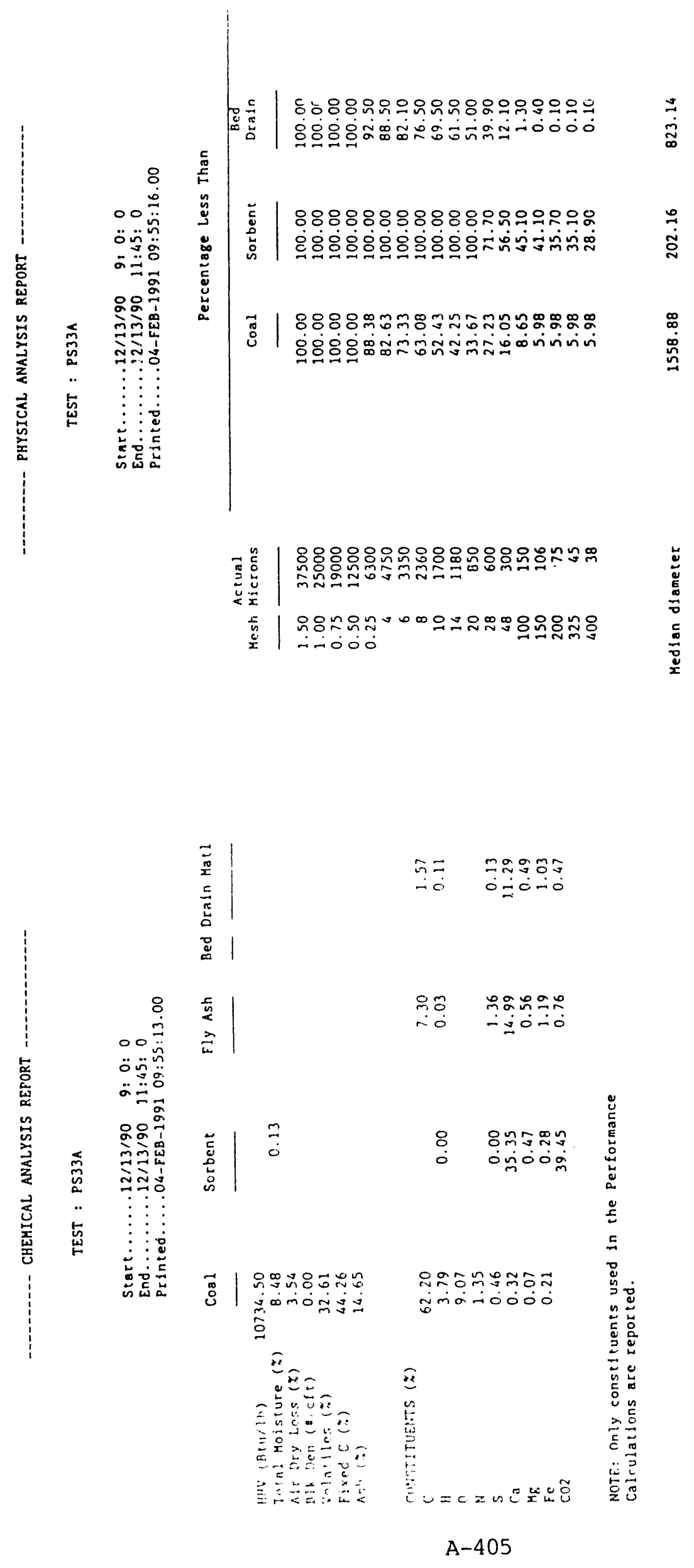


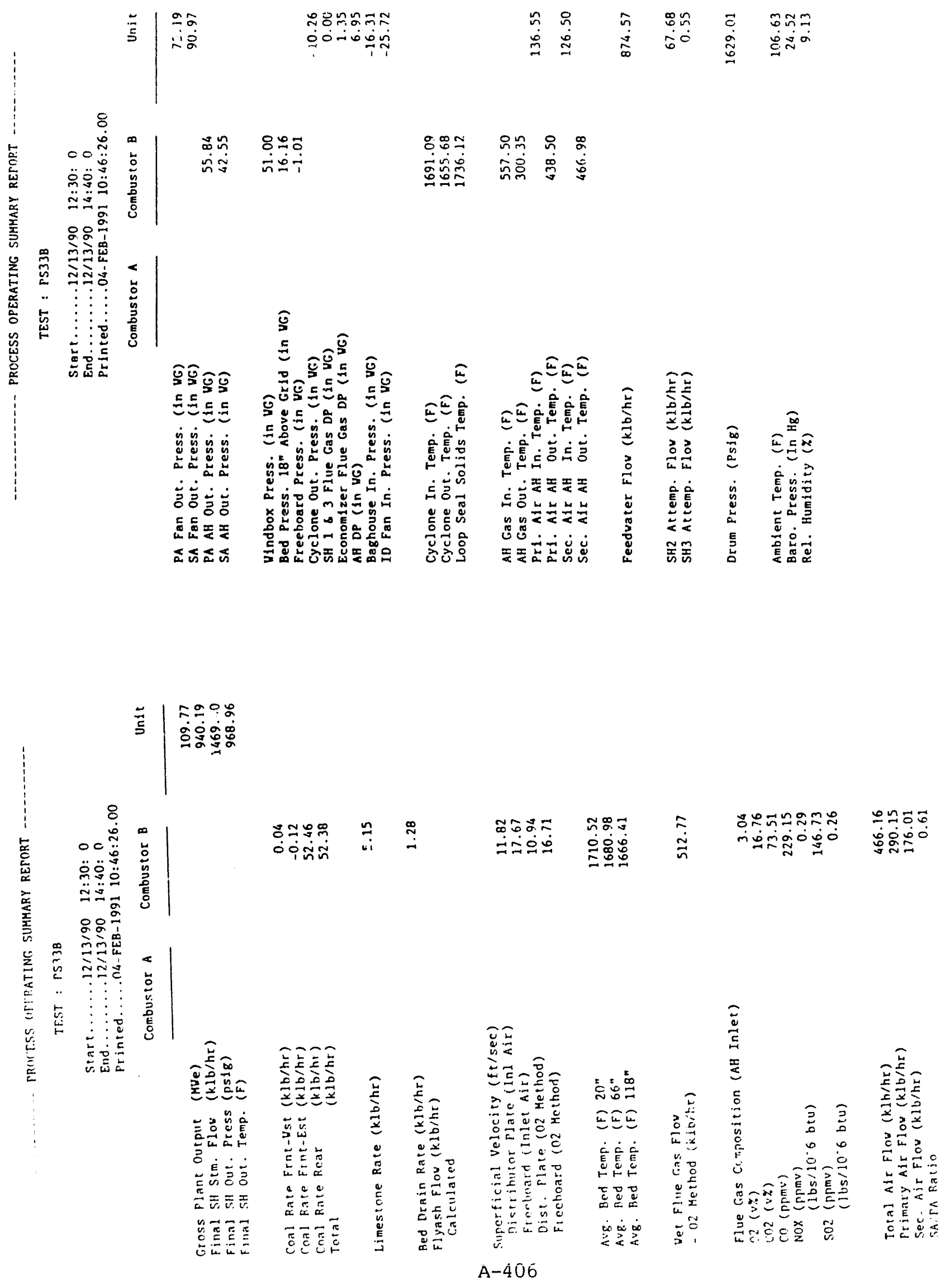




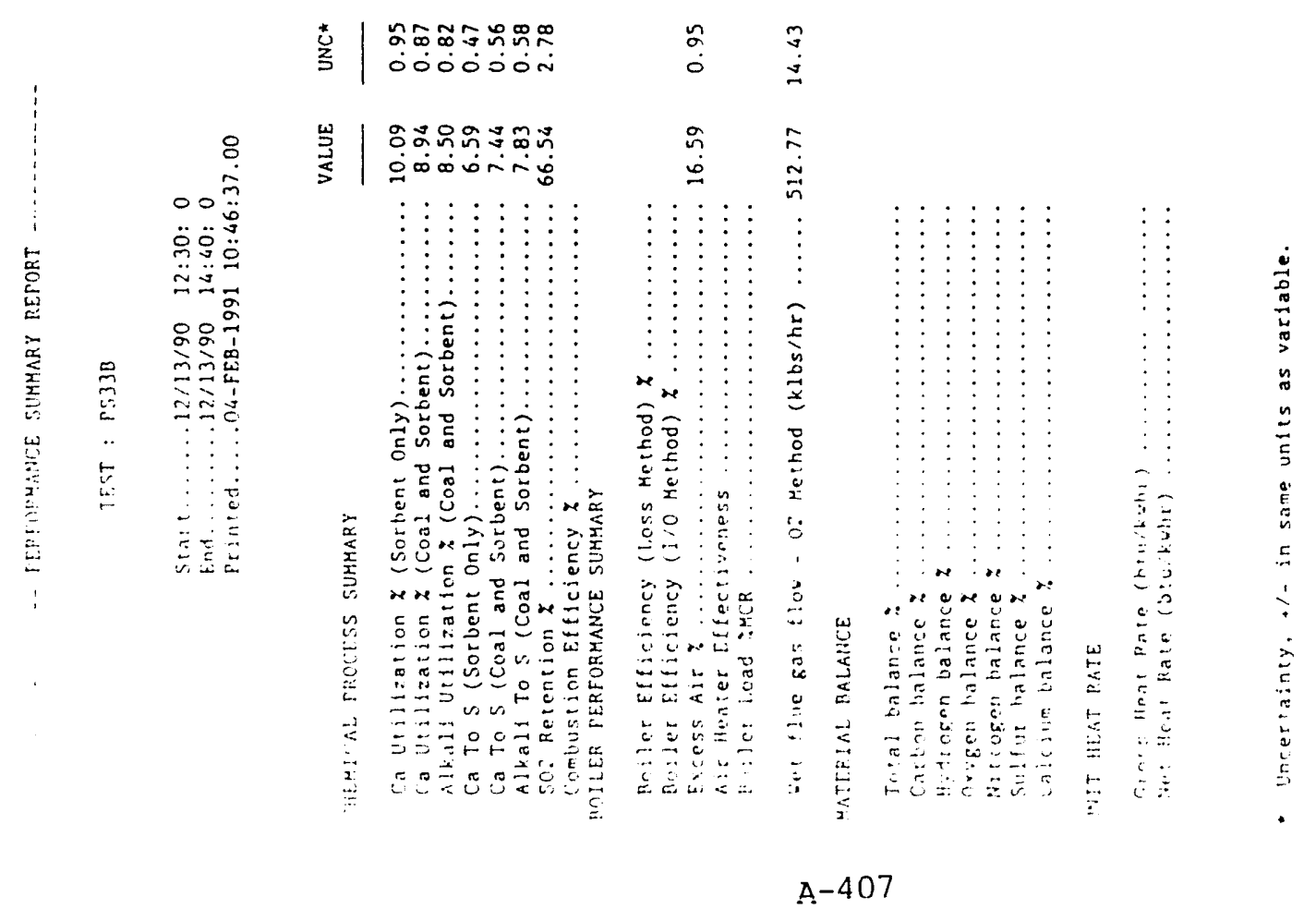



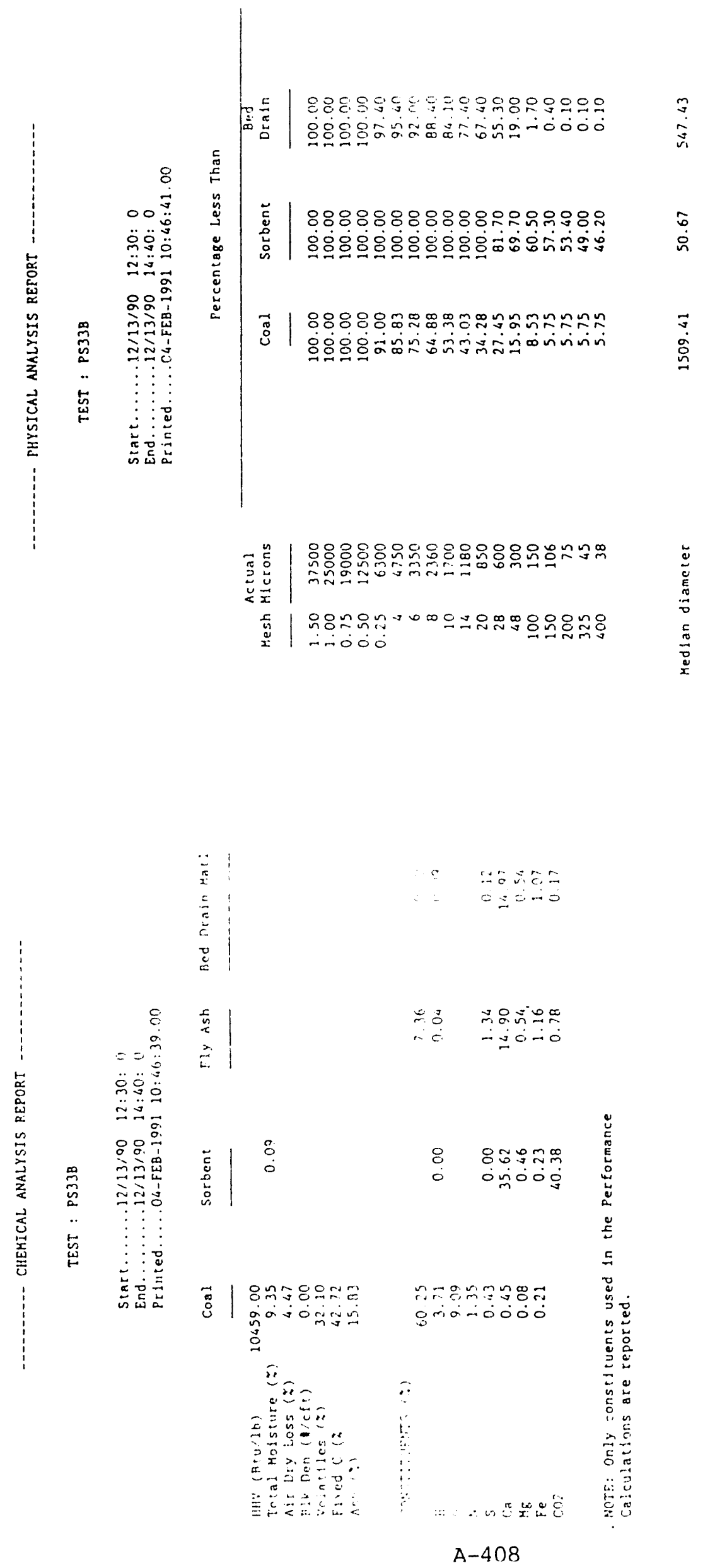

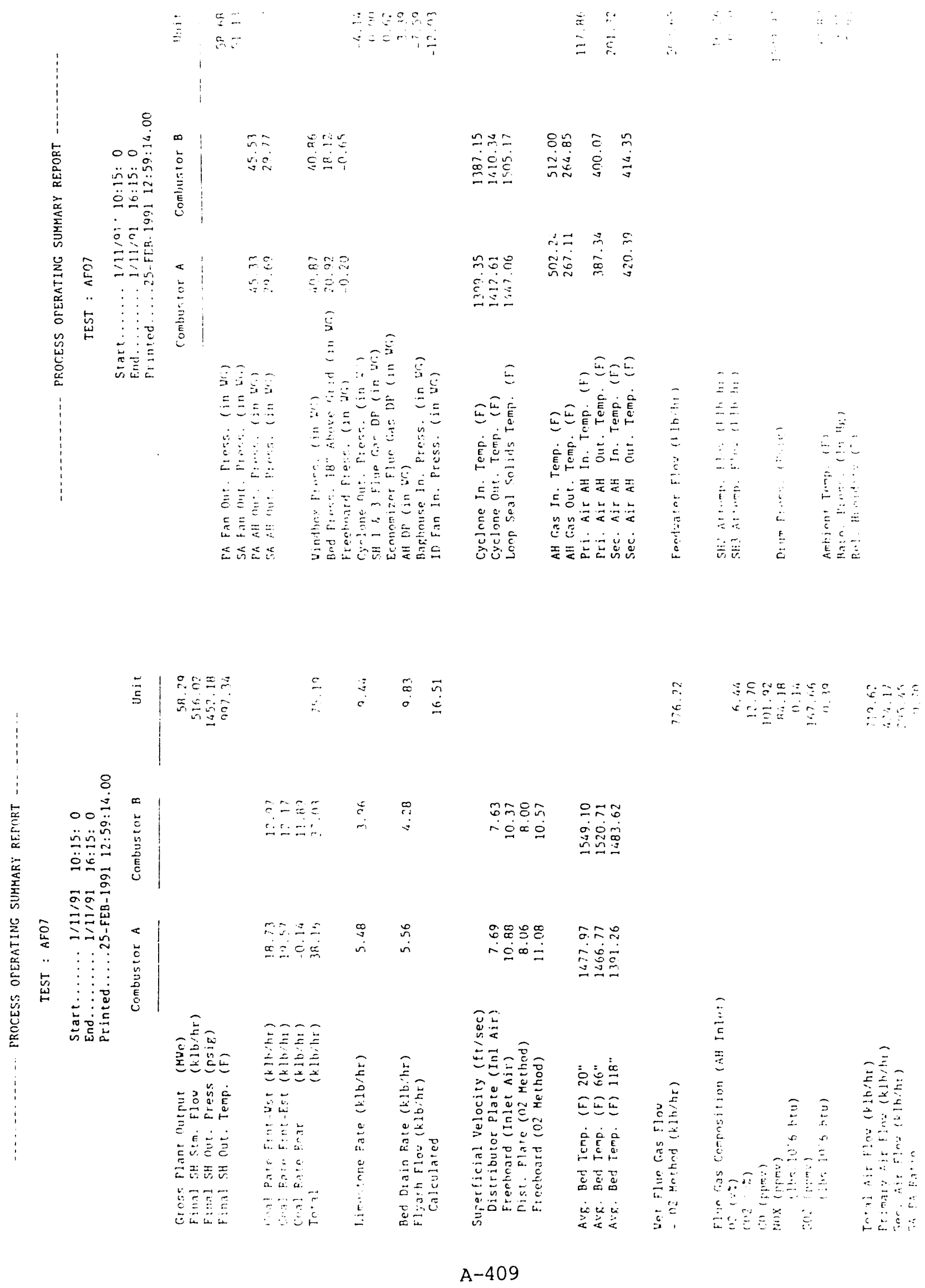


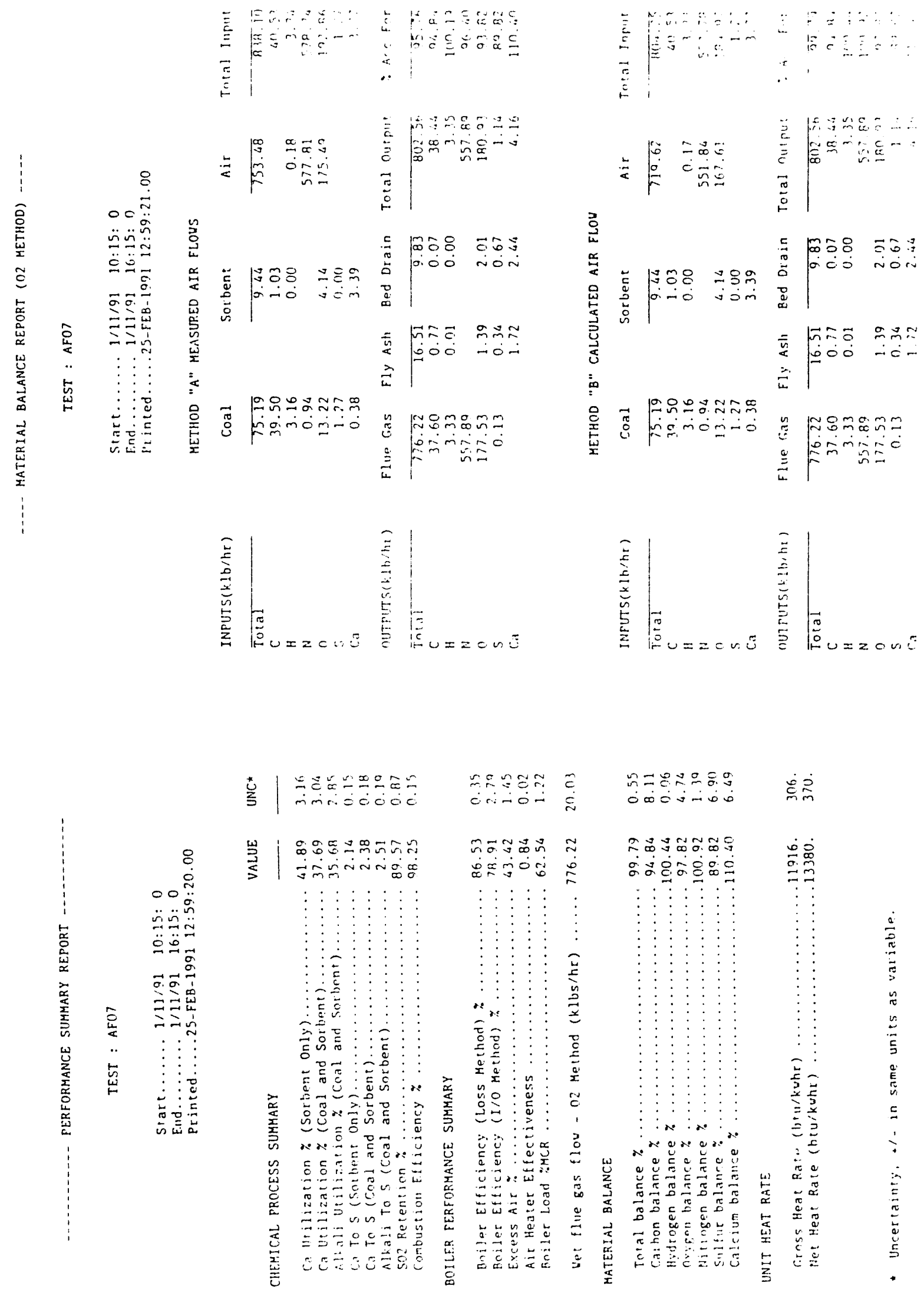




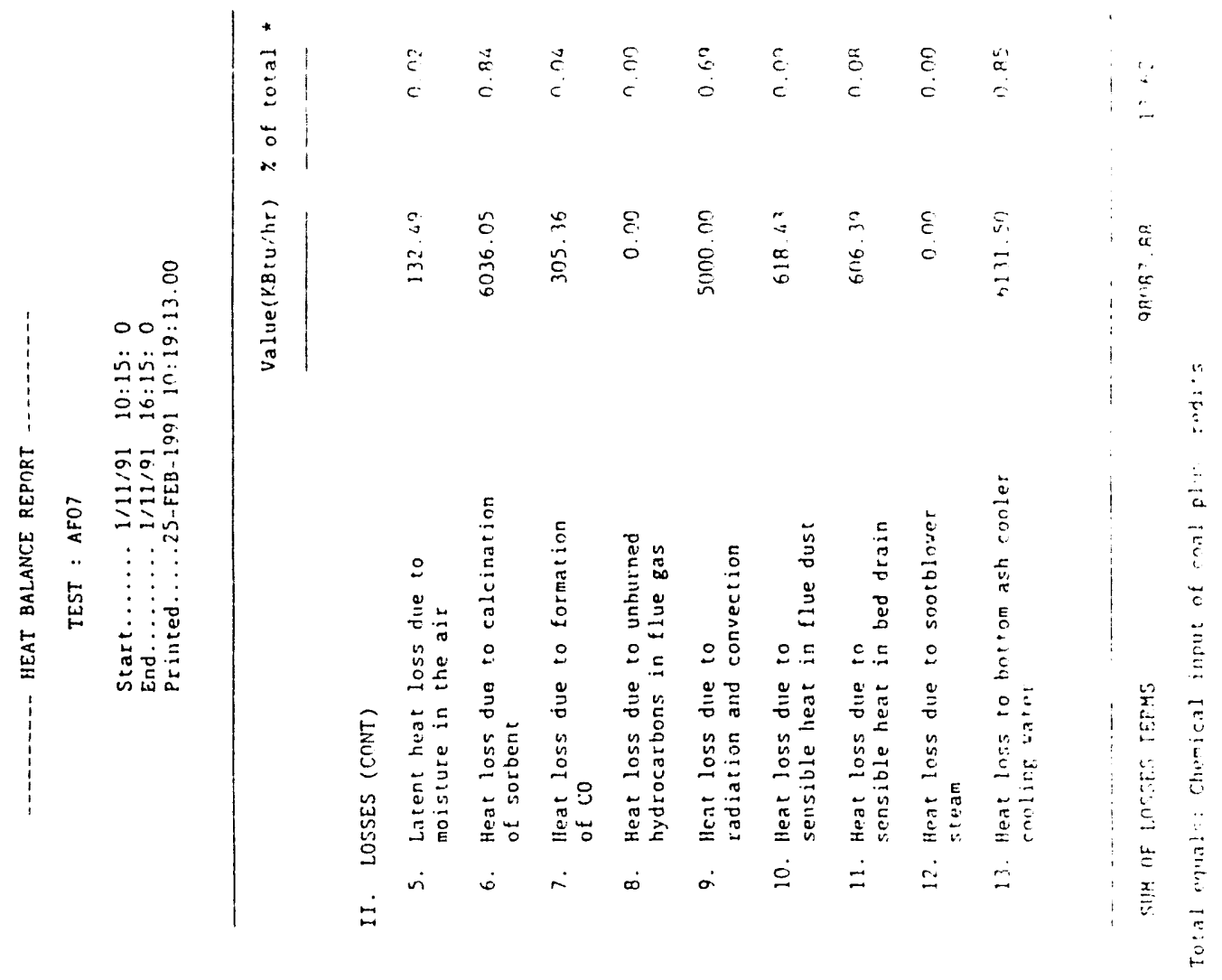

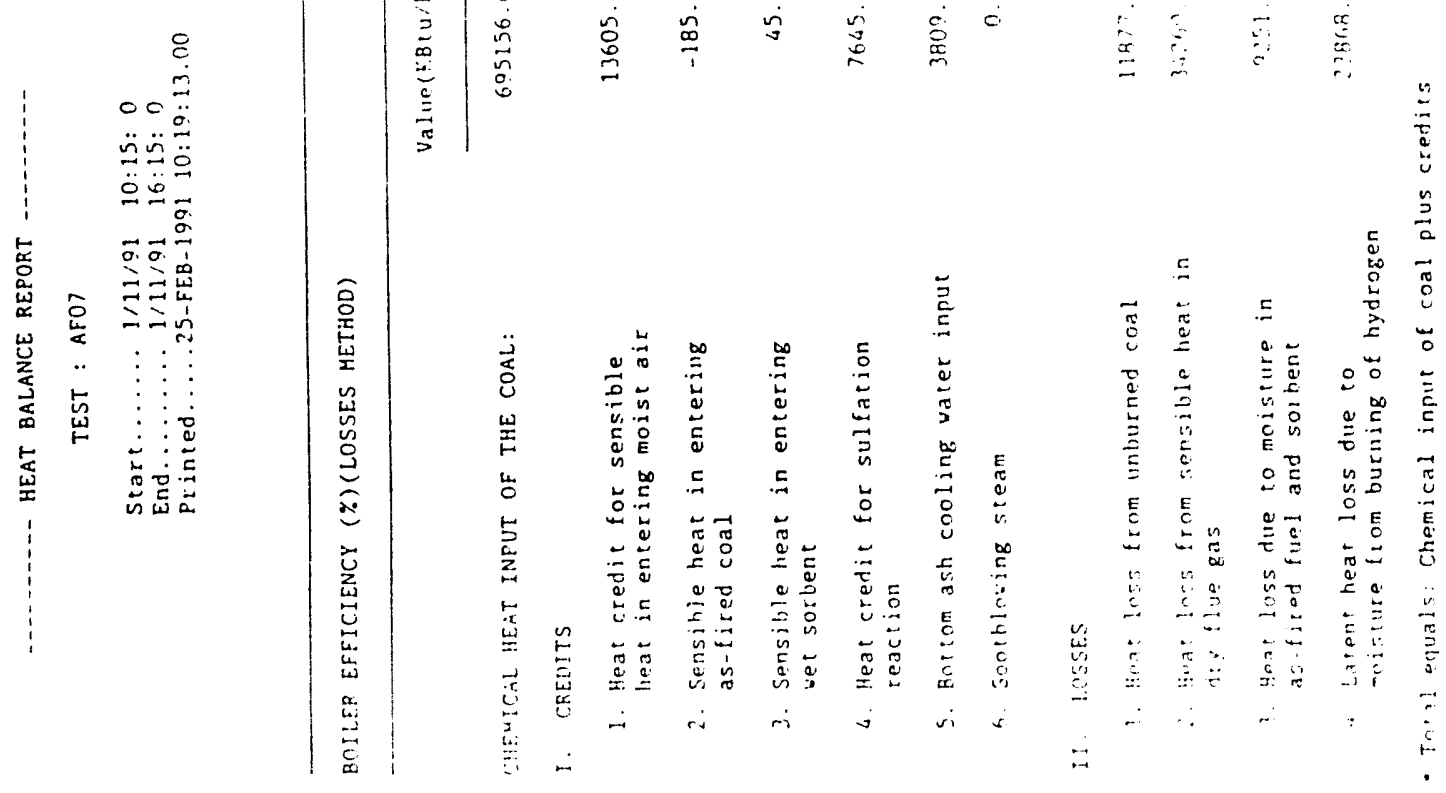




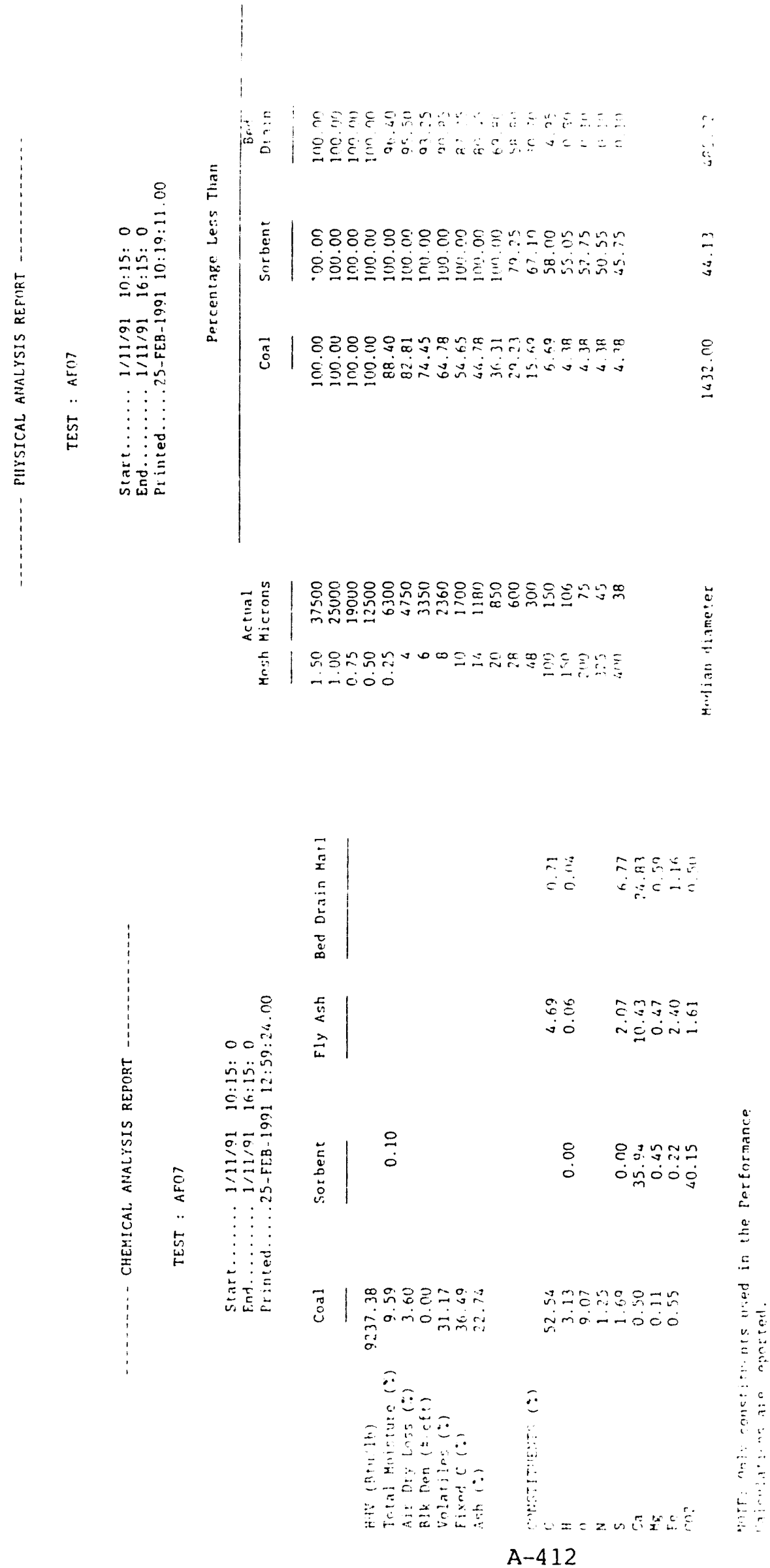



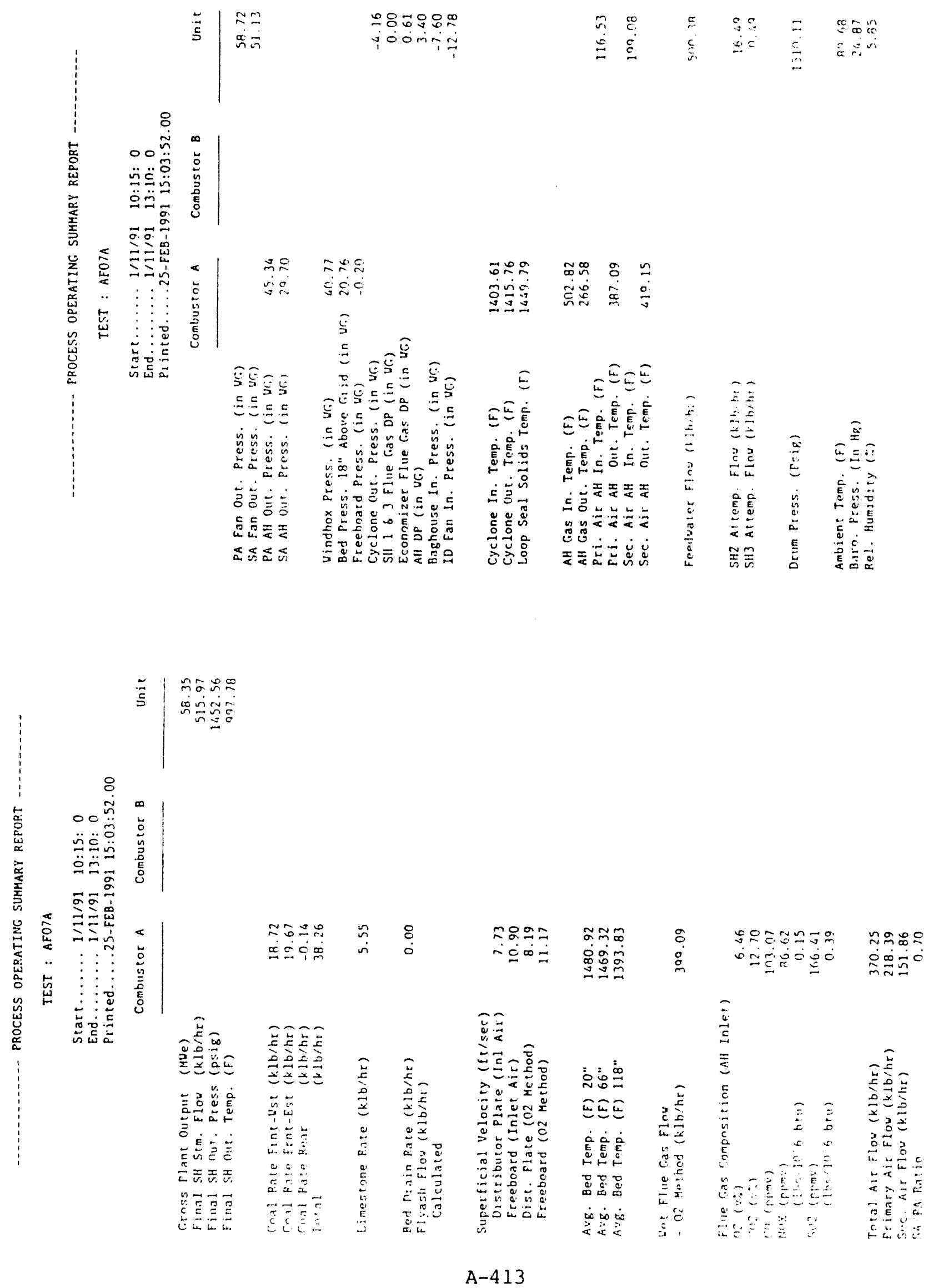


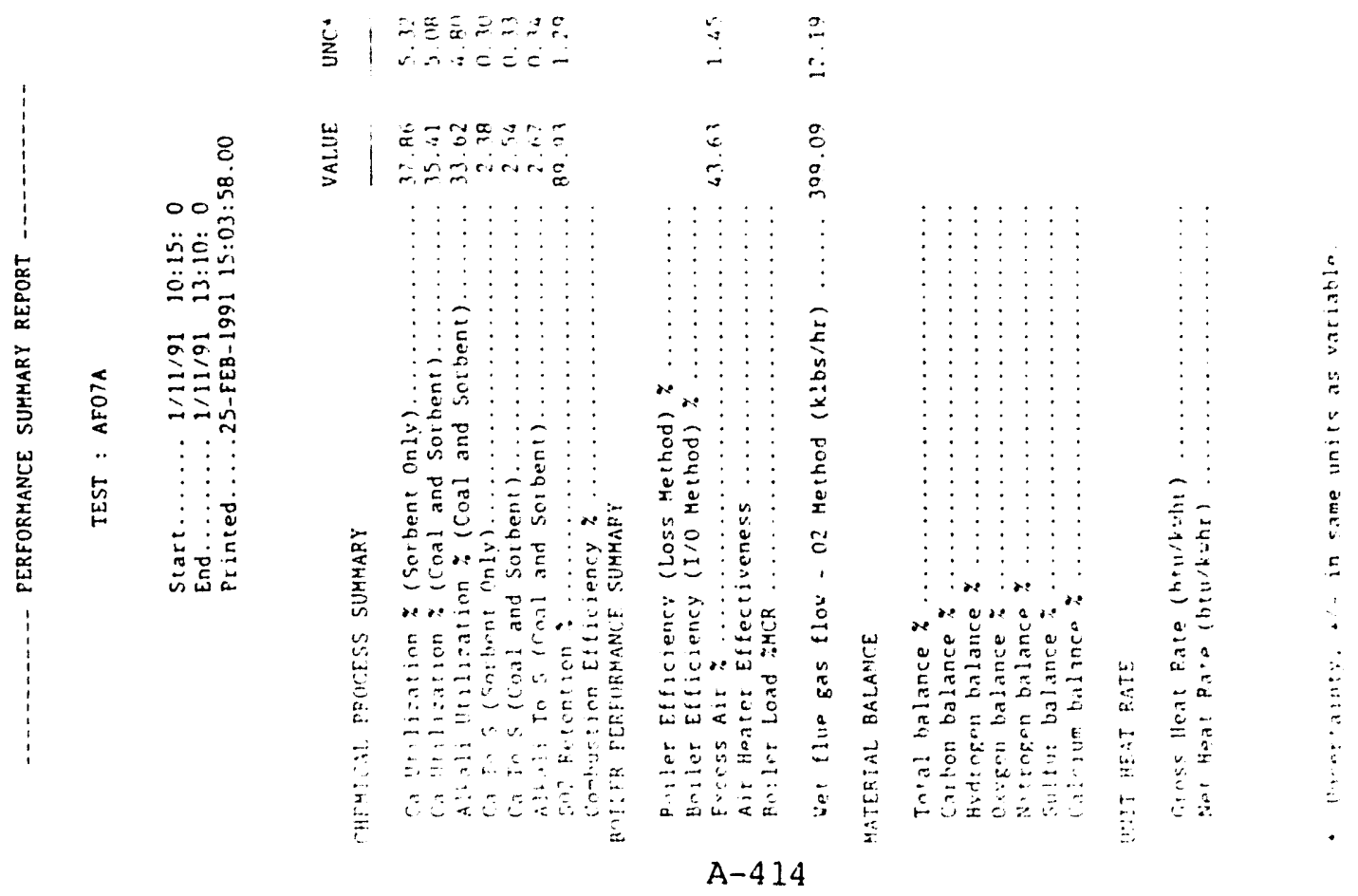




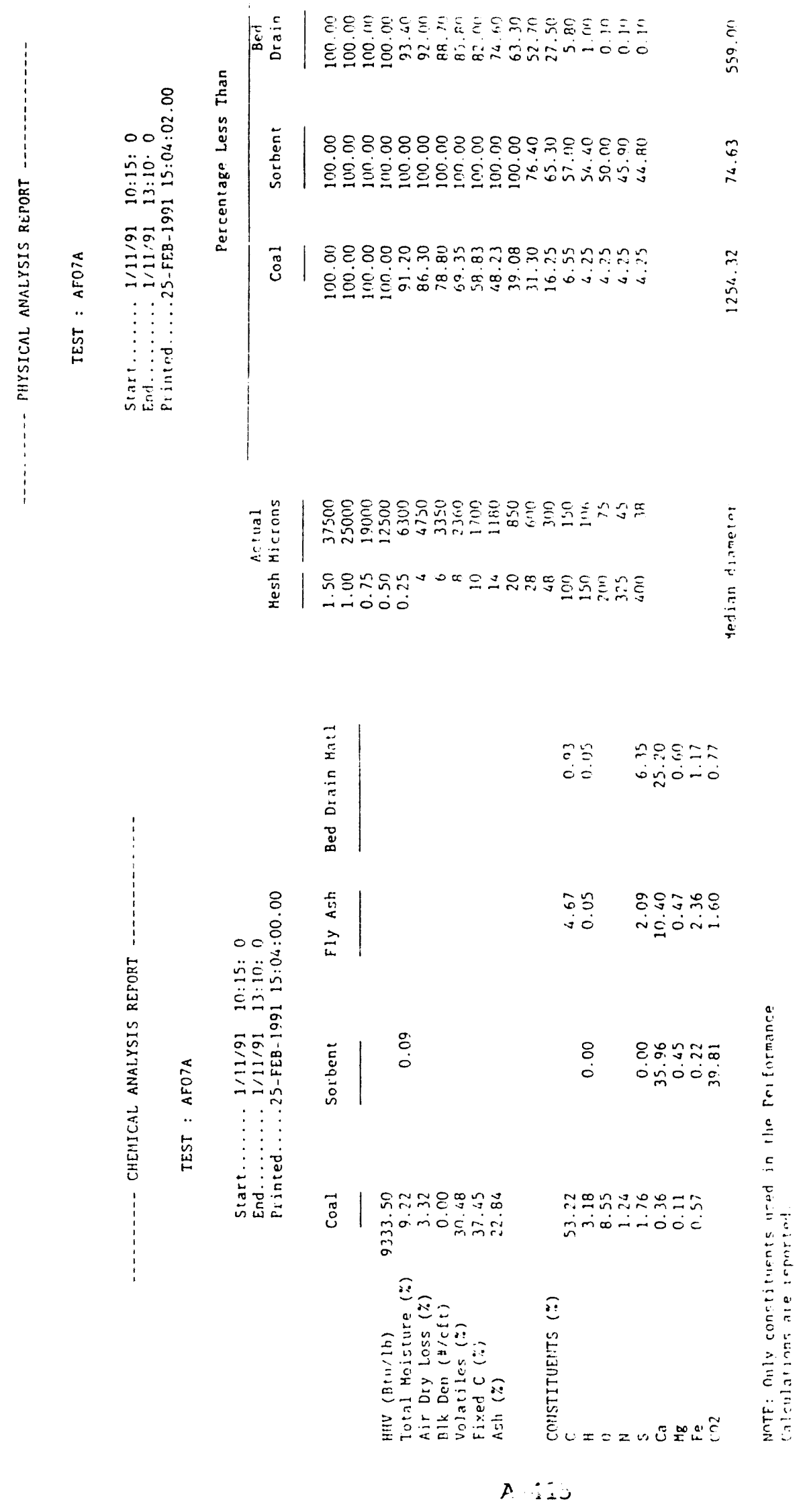



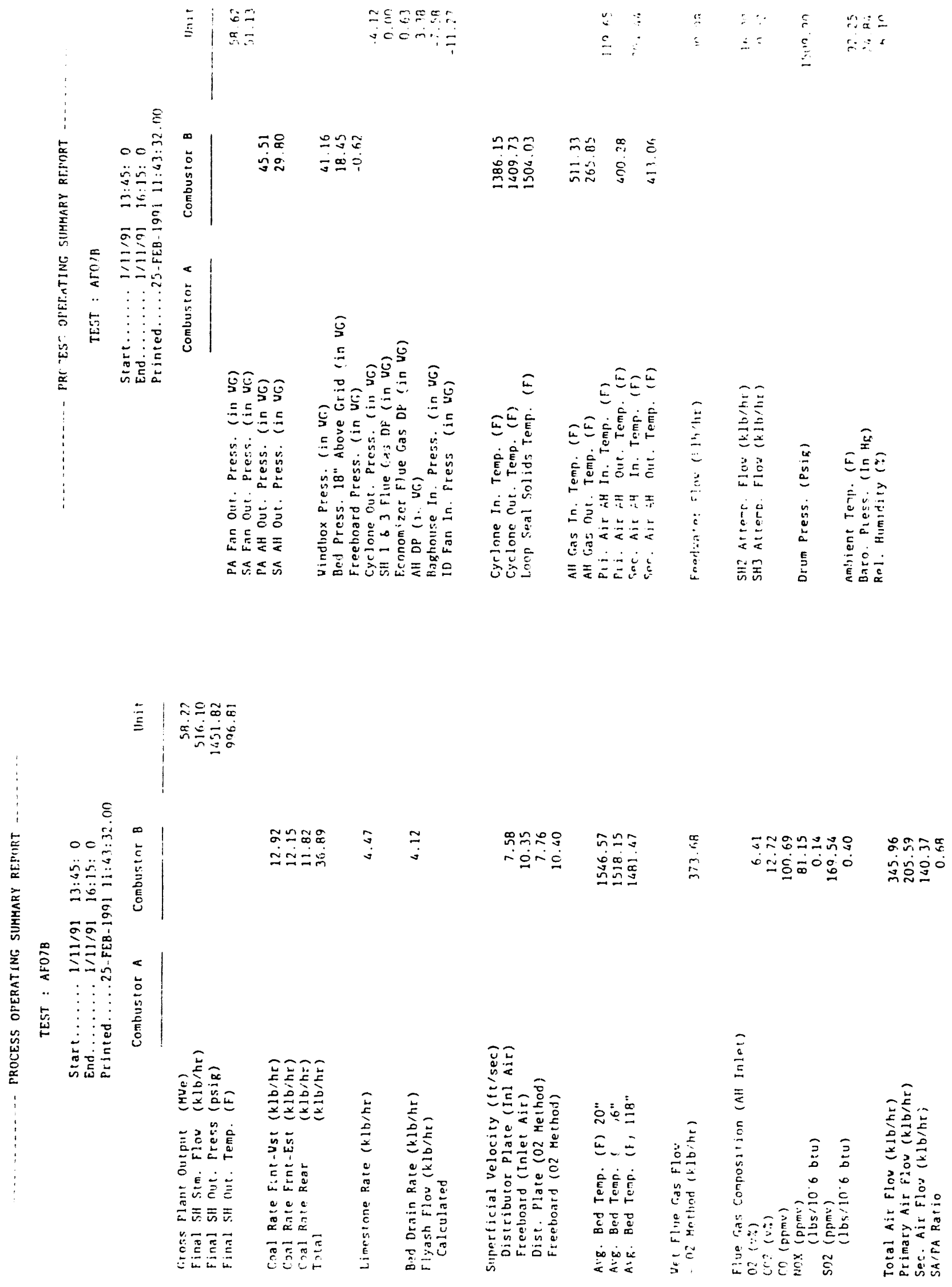


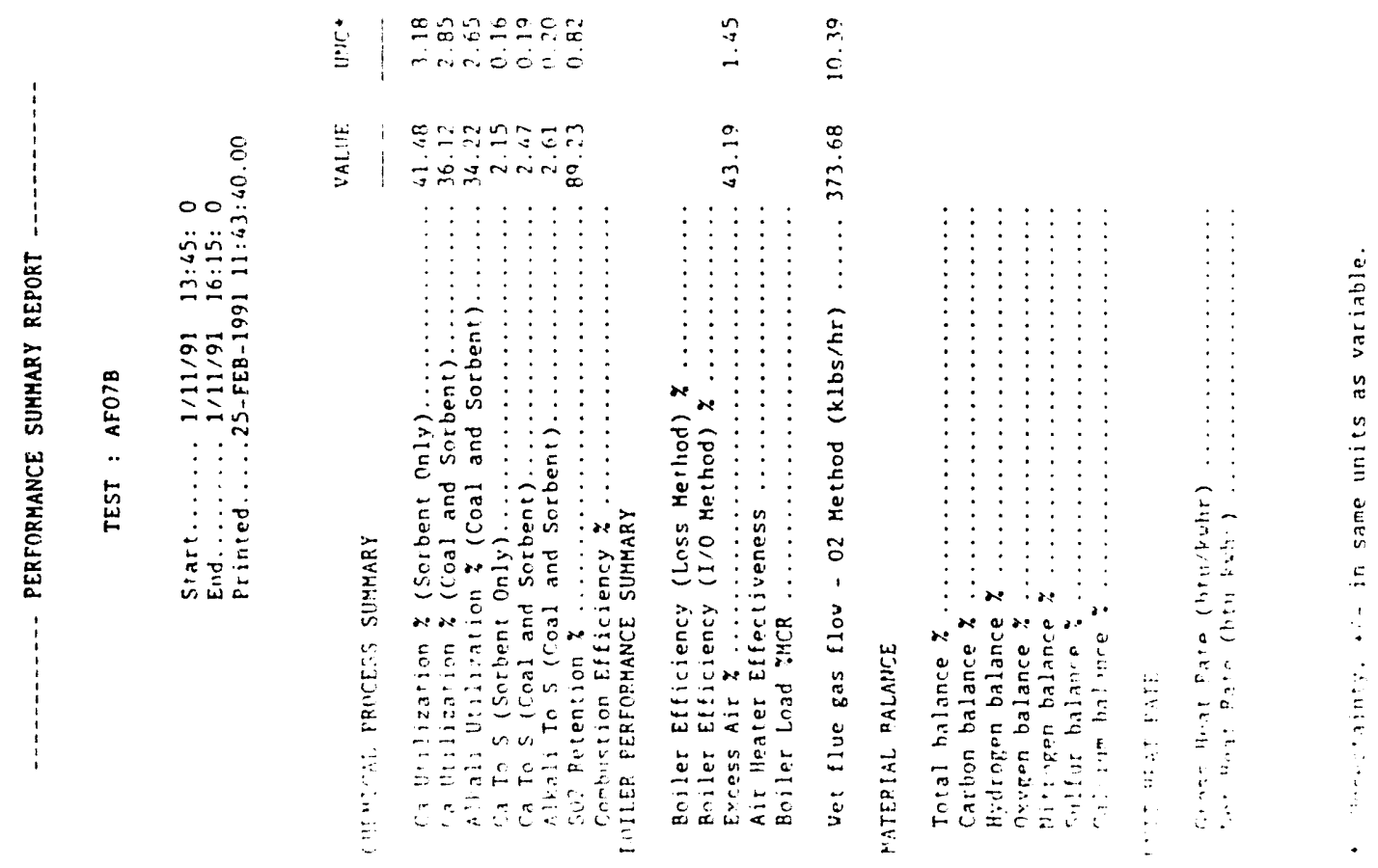

A -417 

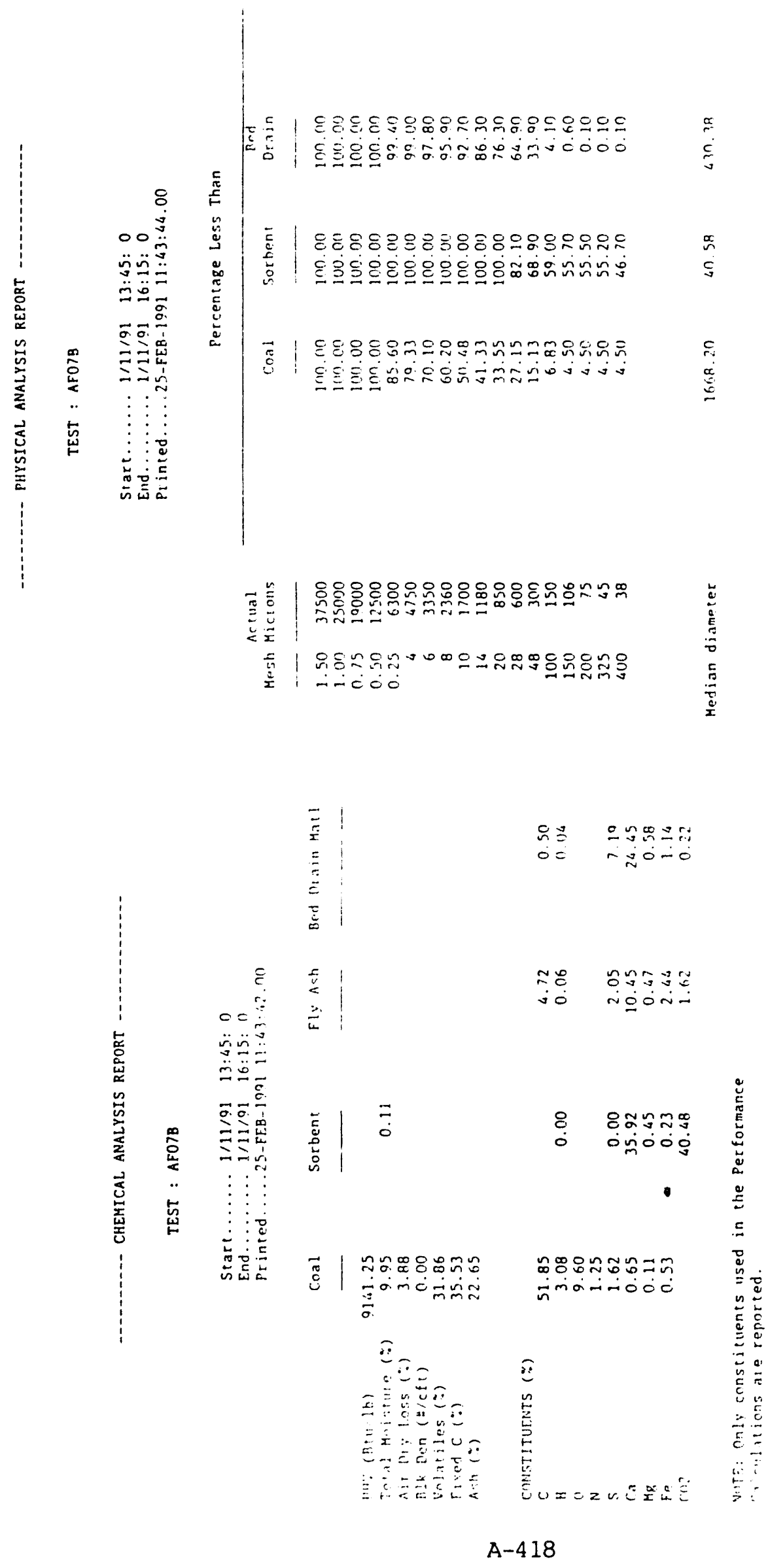

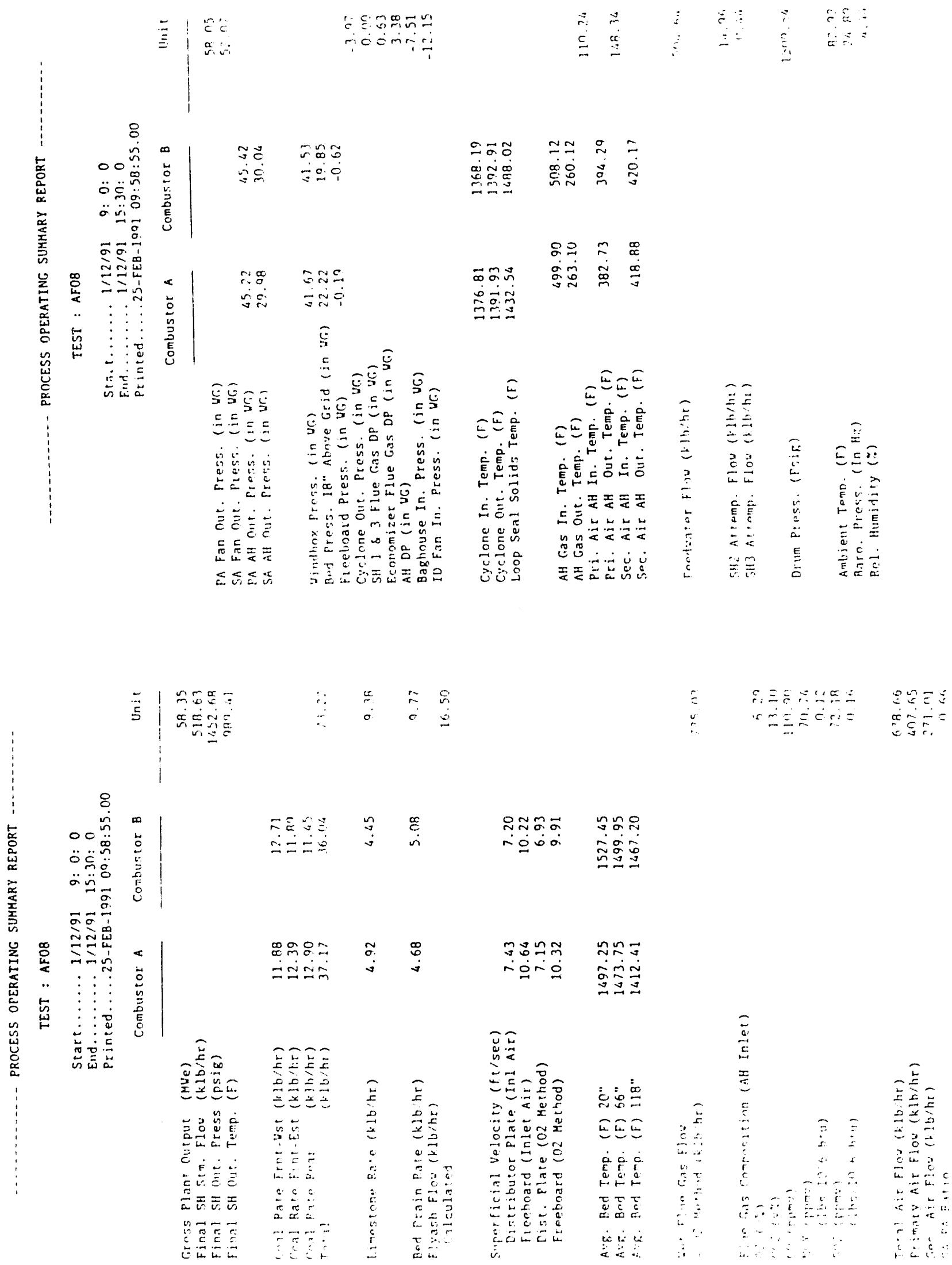


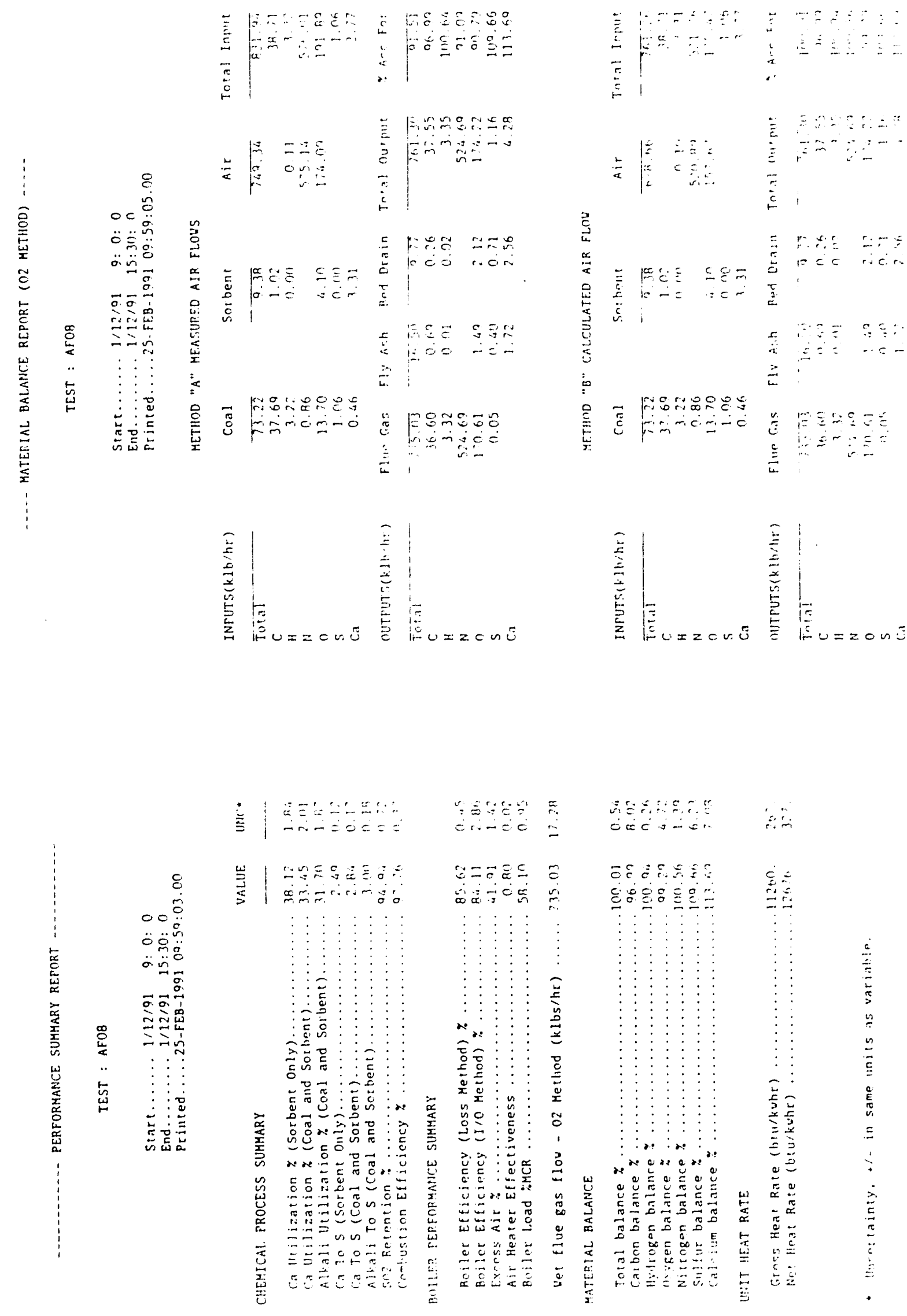




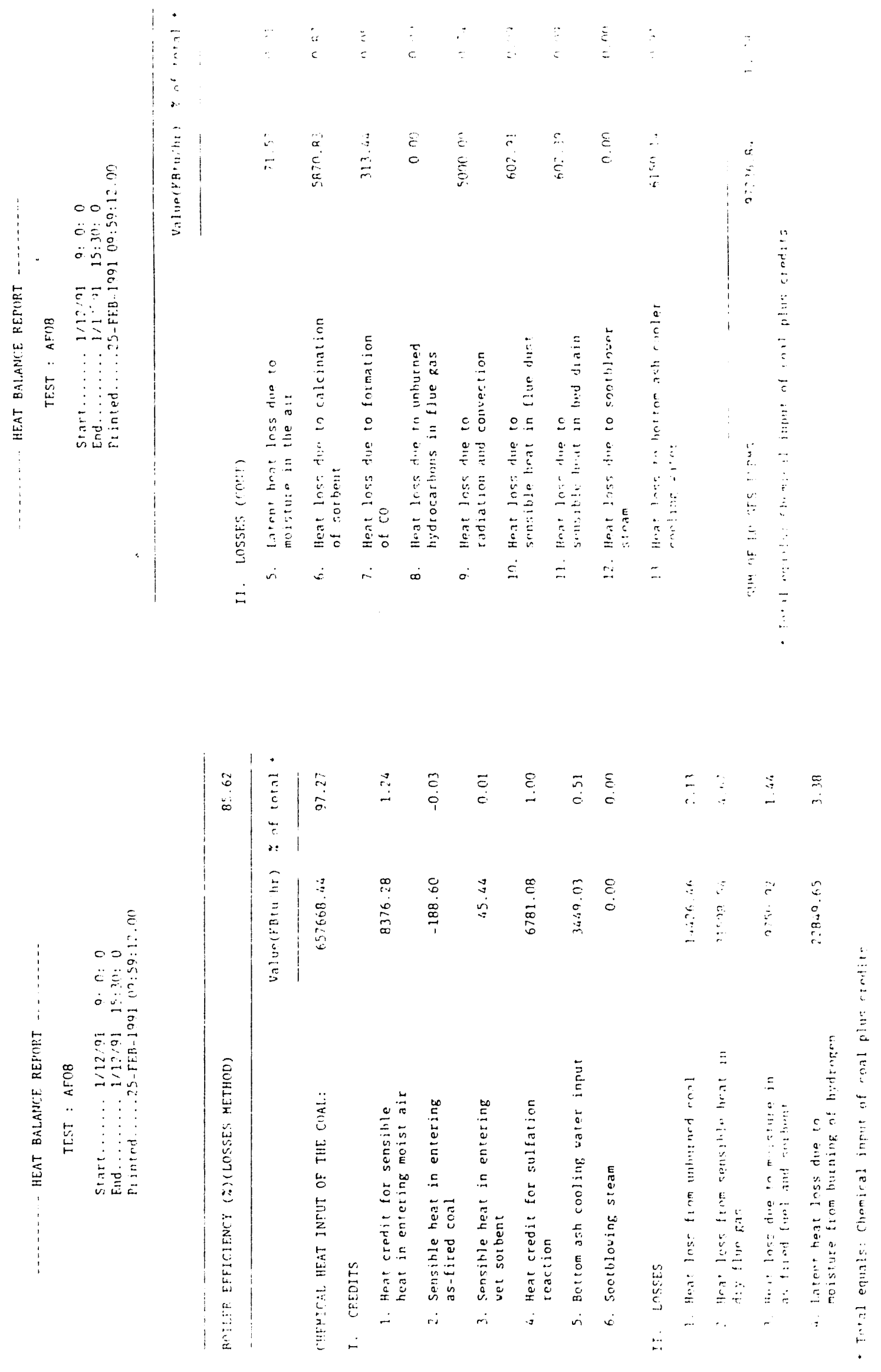




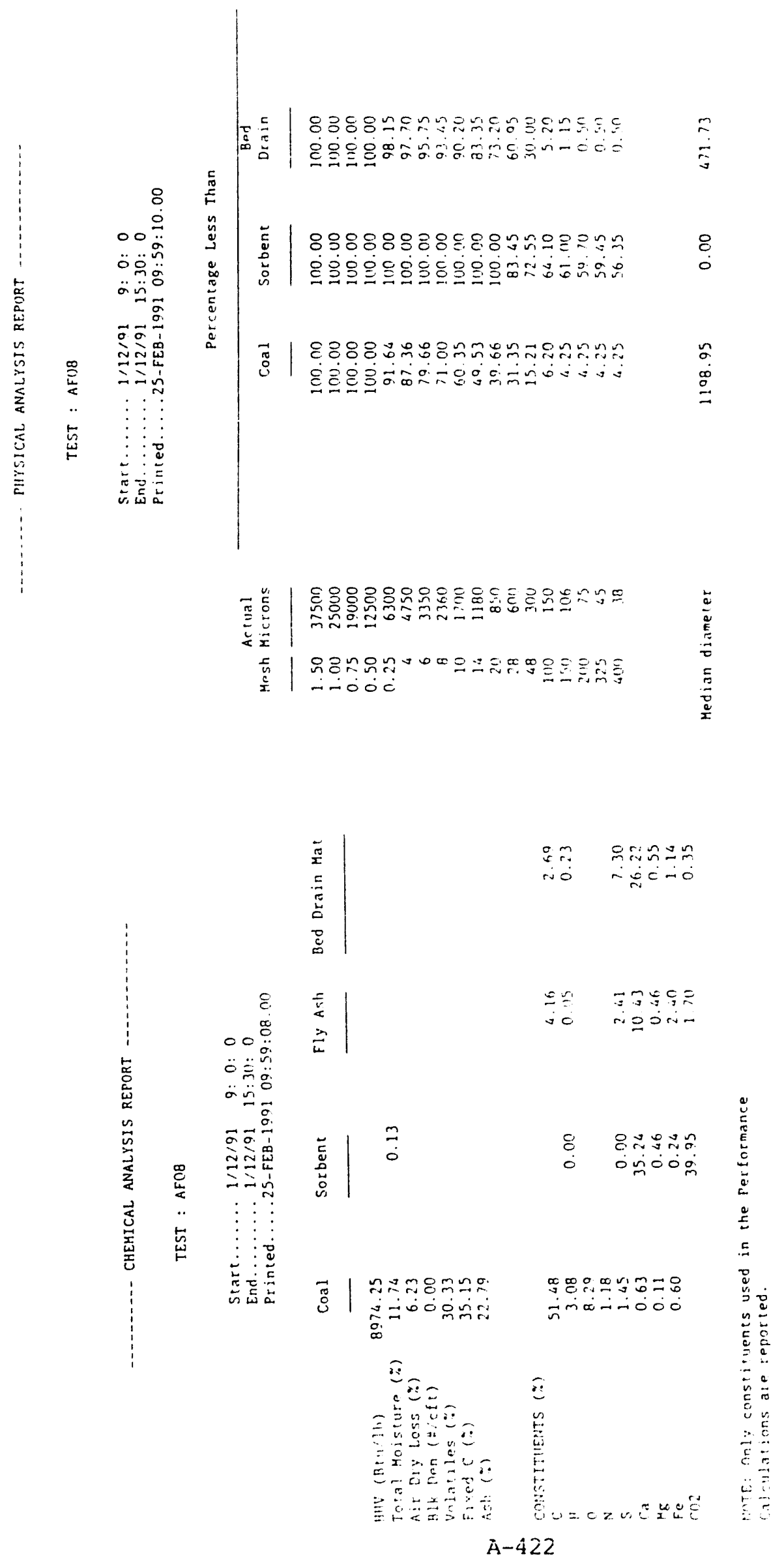



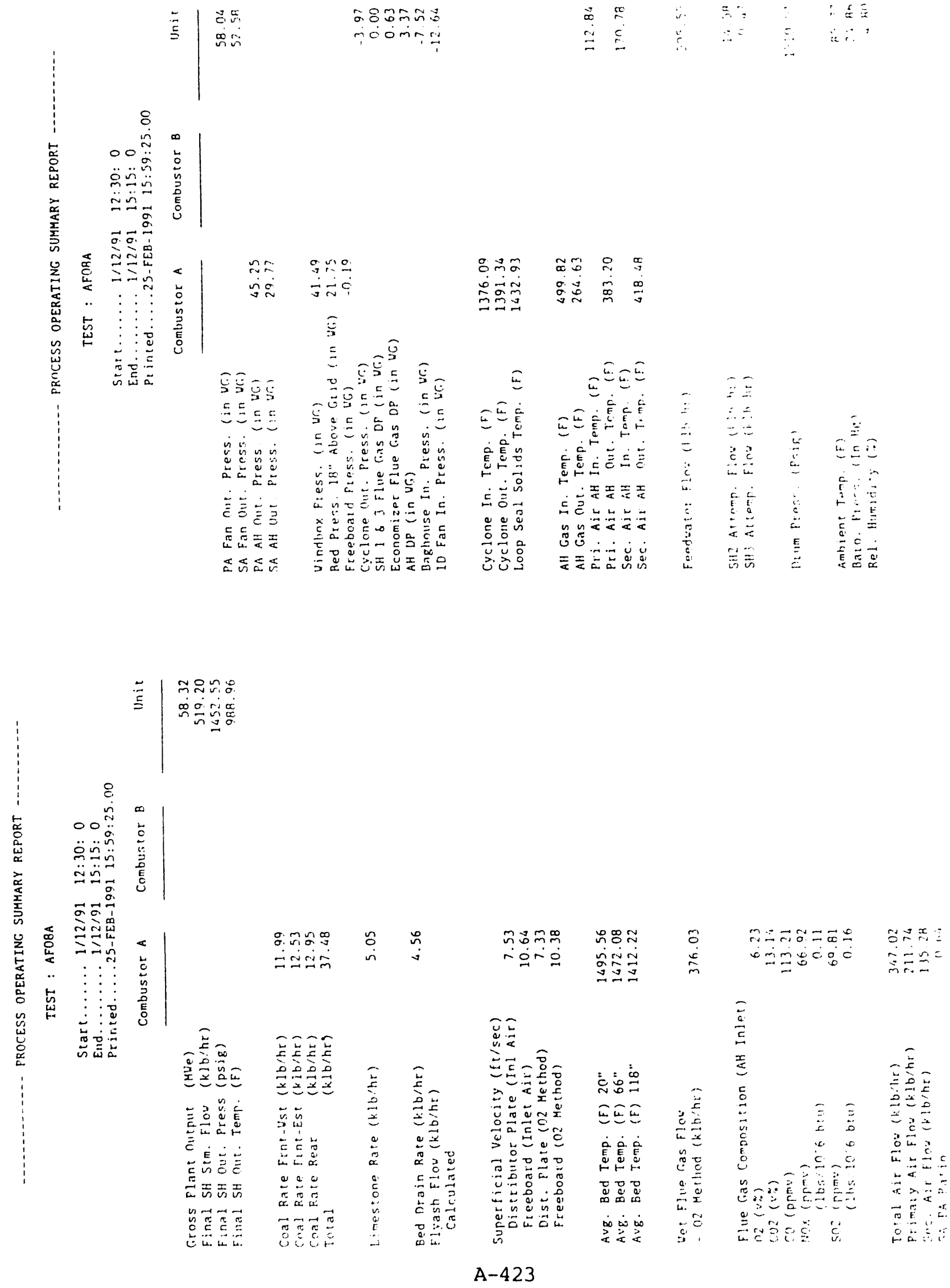


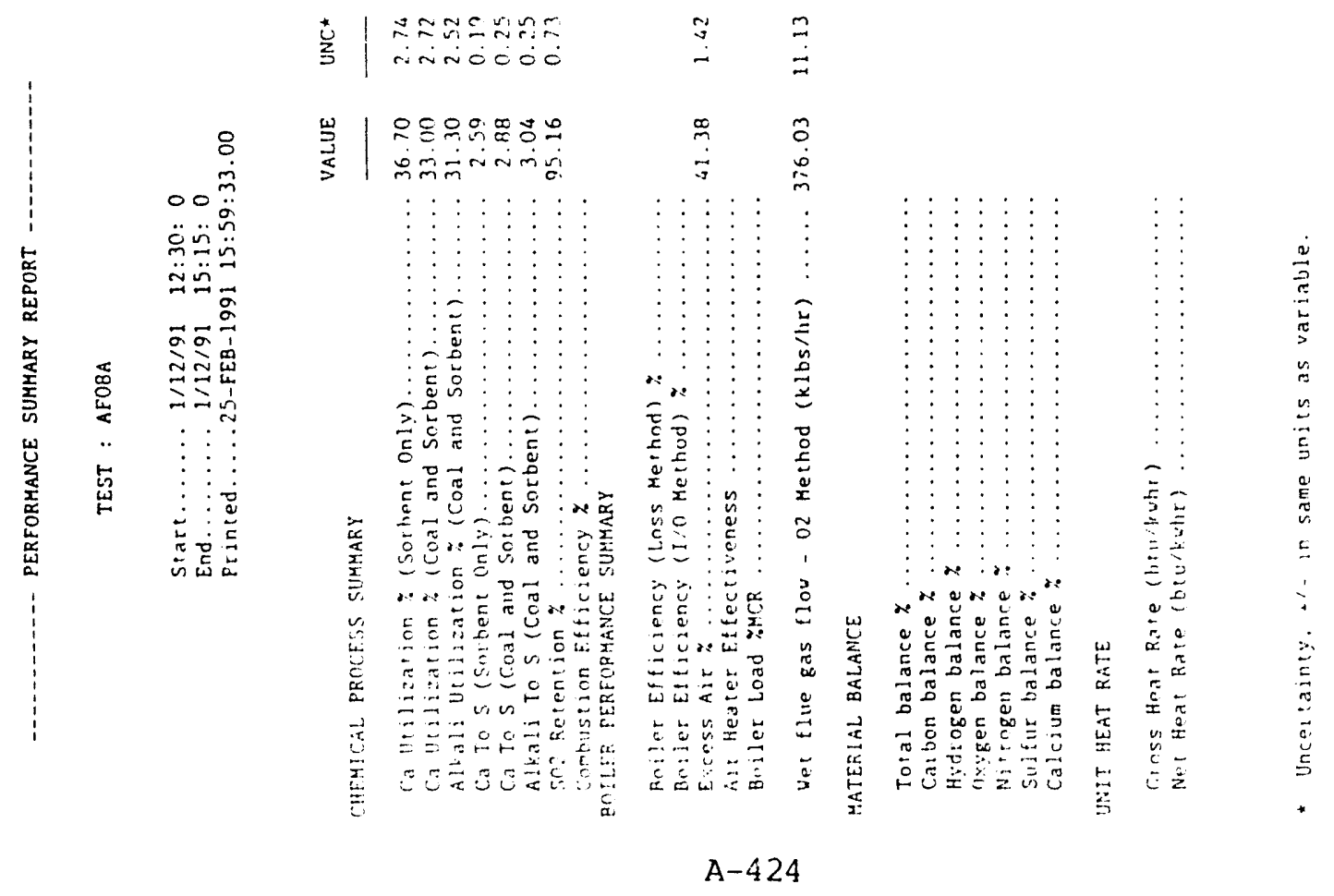



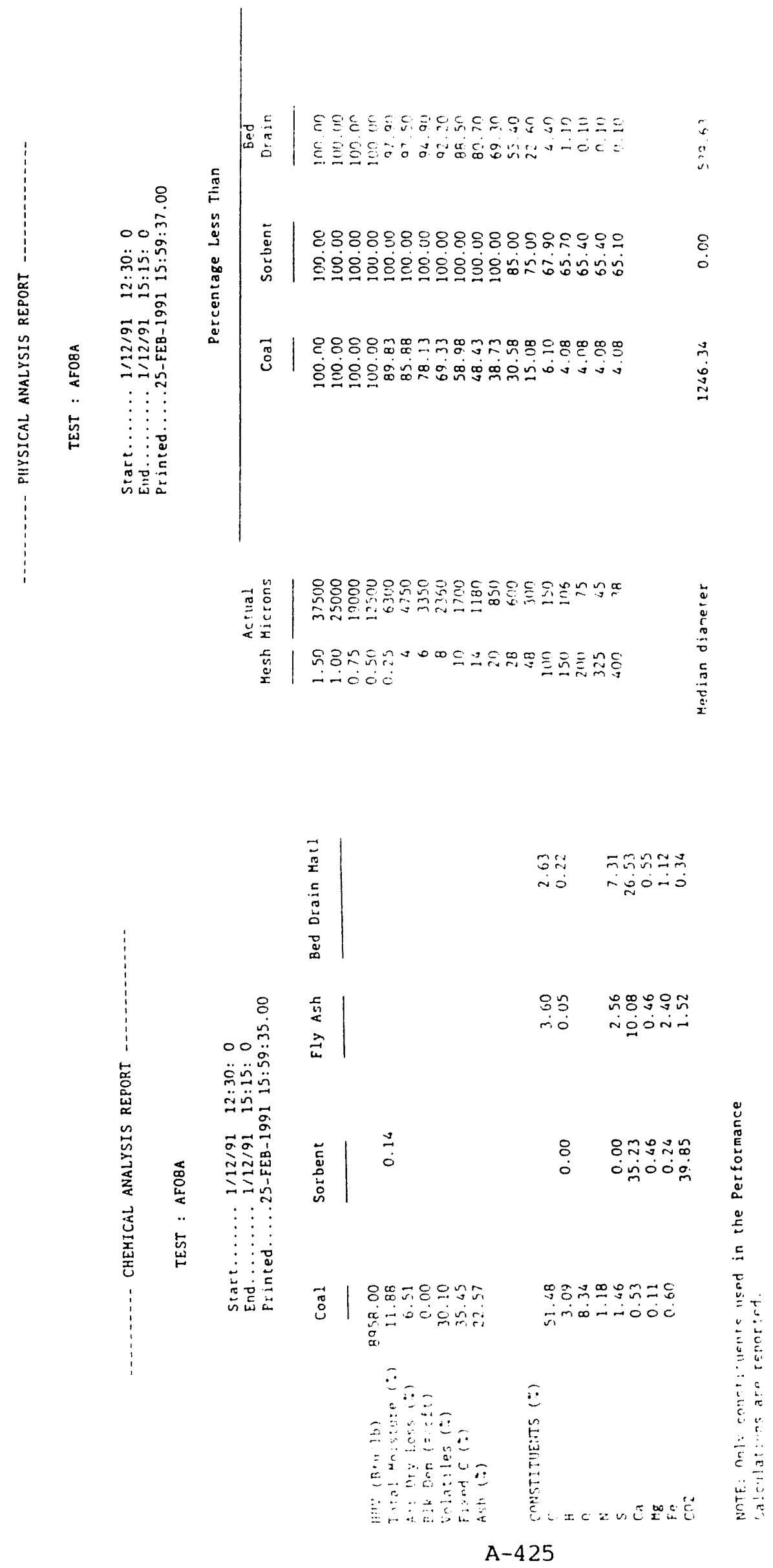

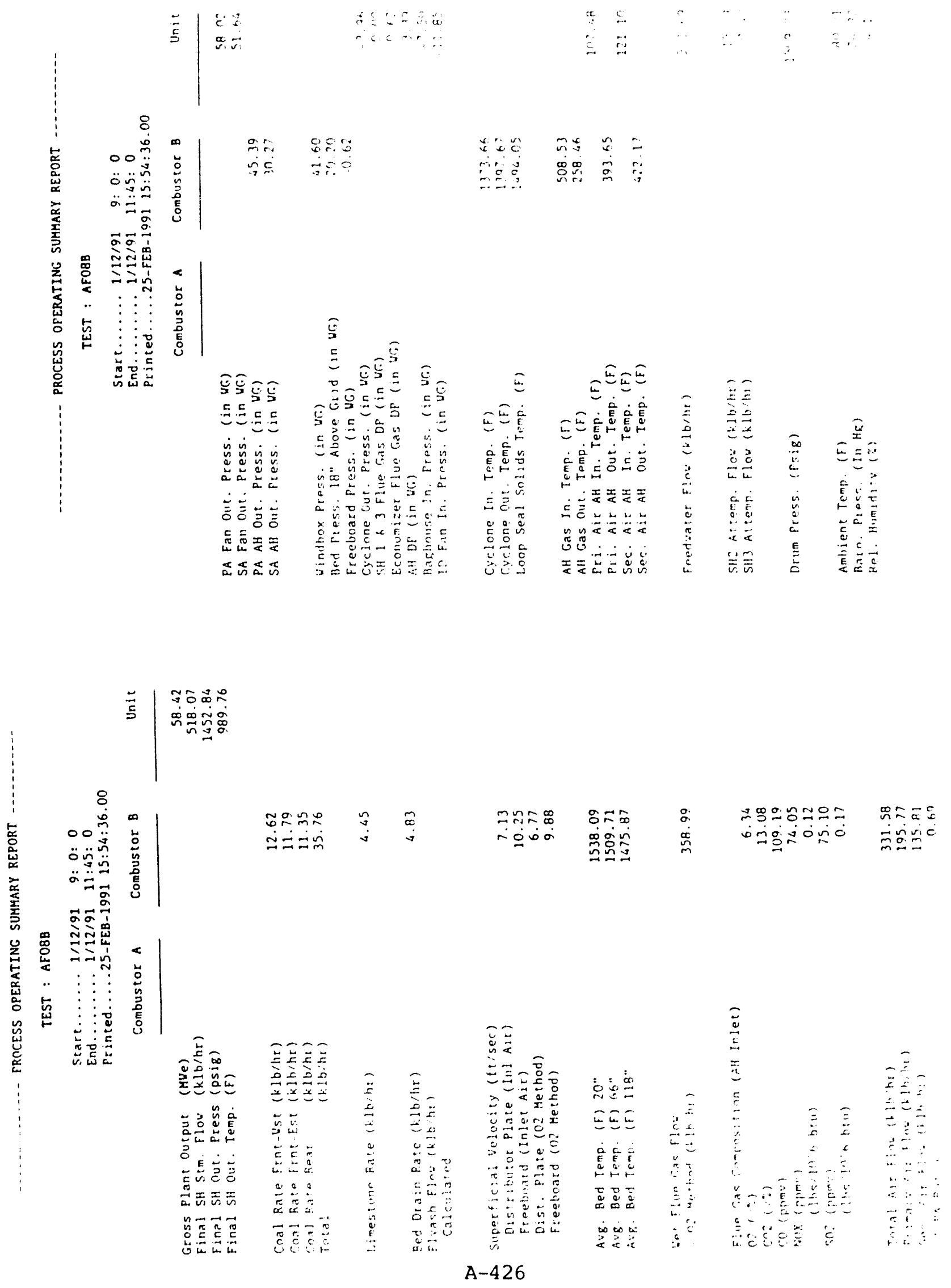


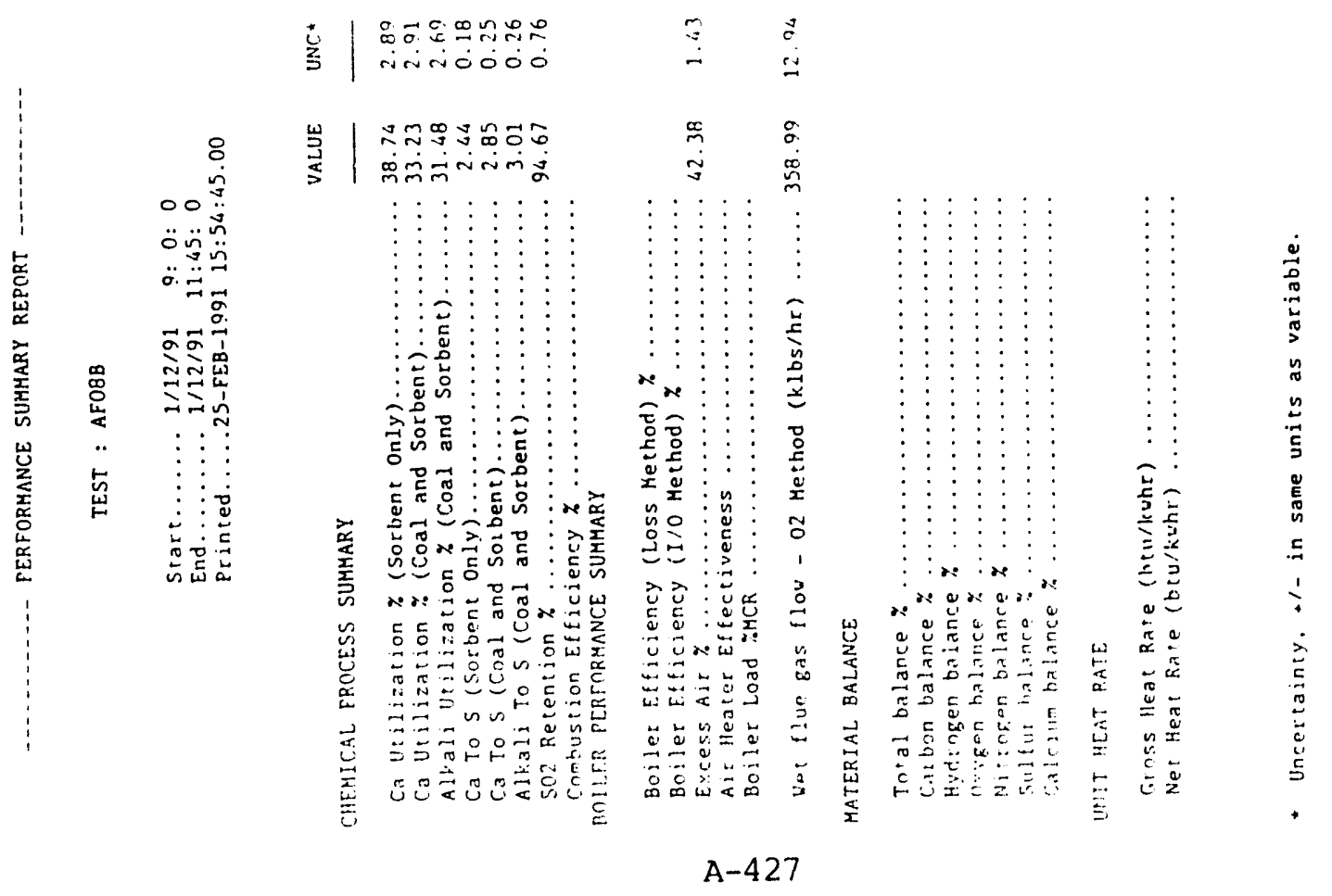



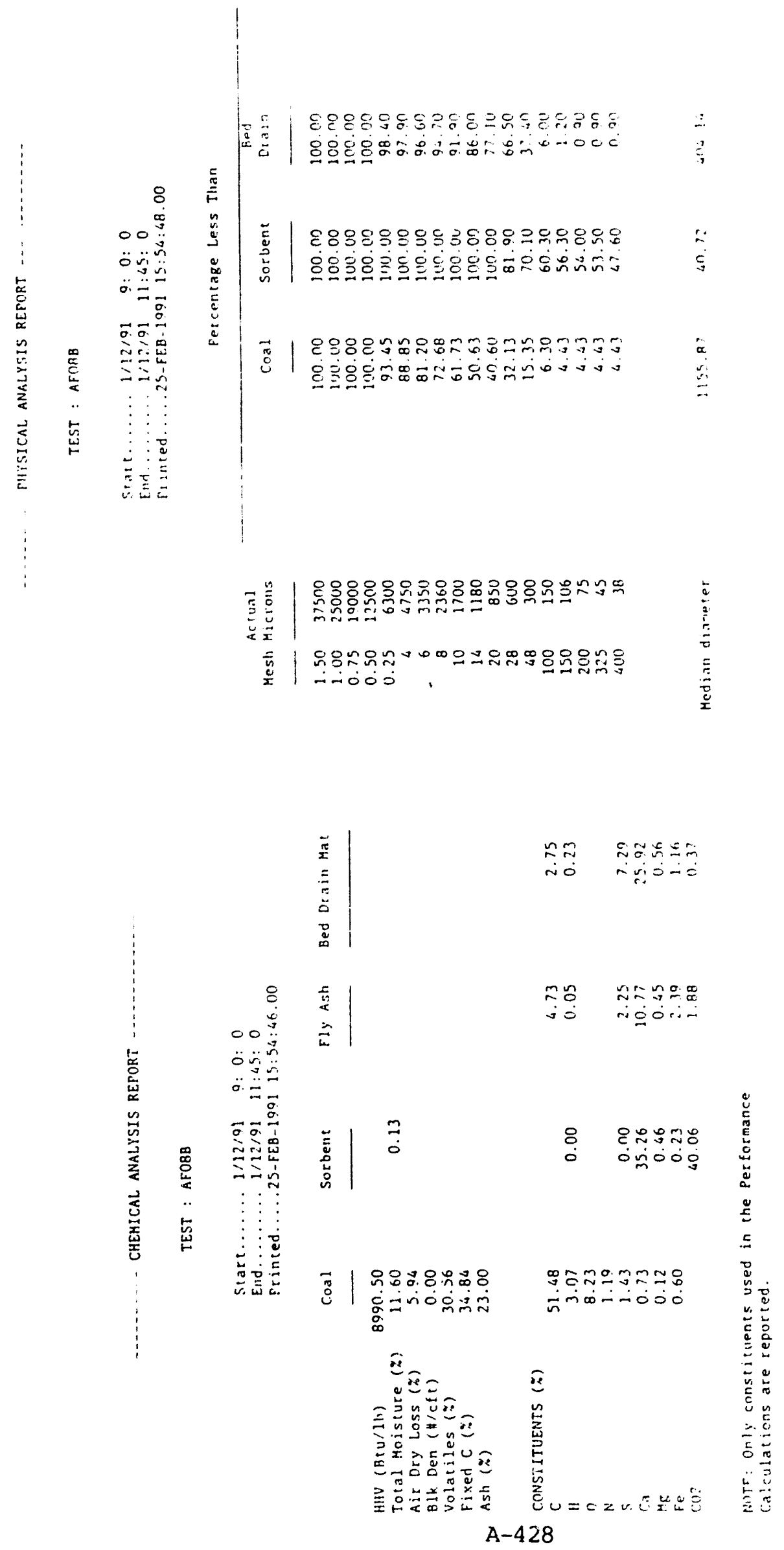

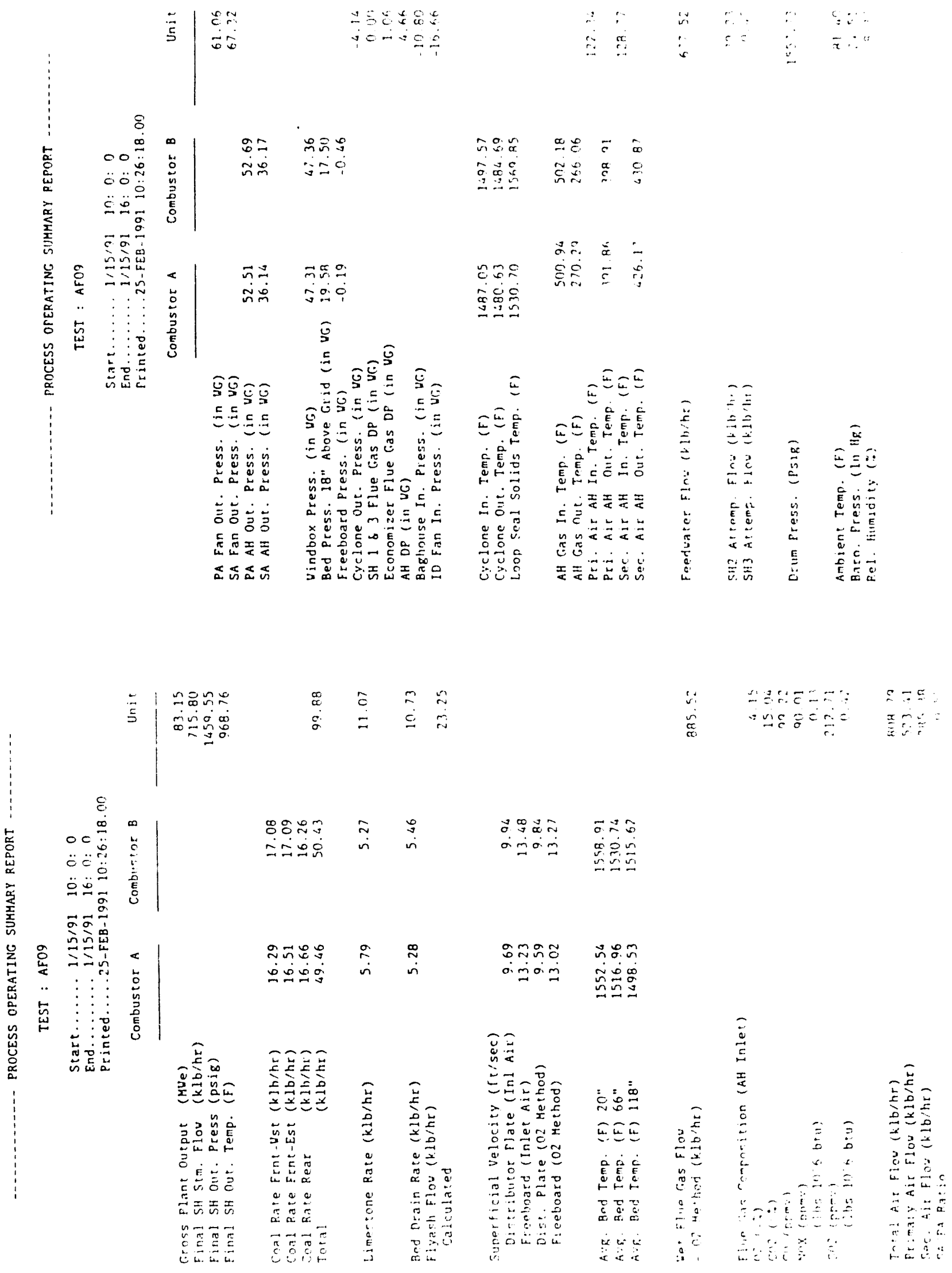


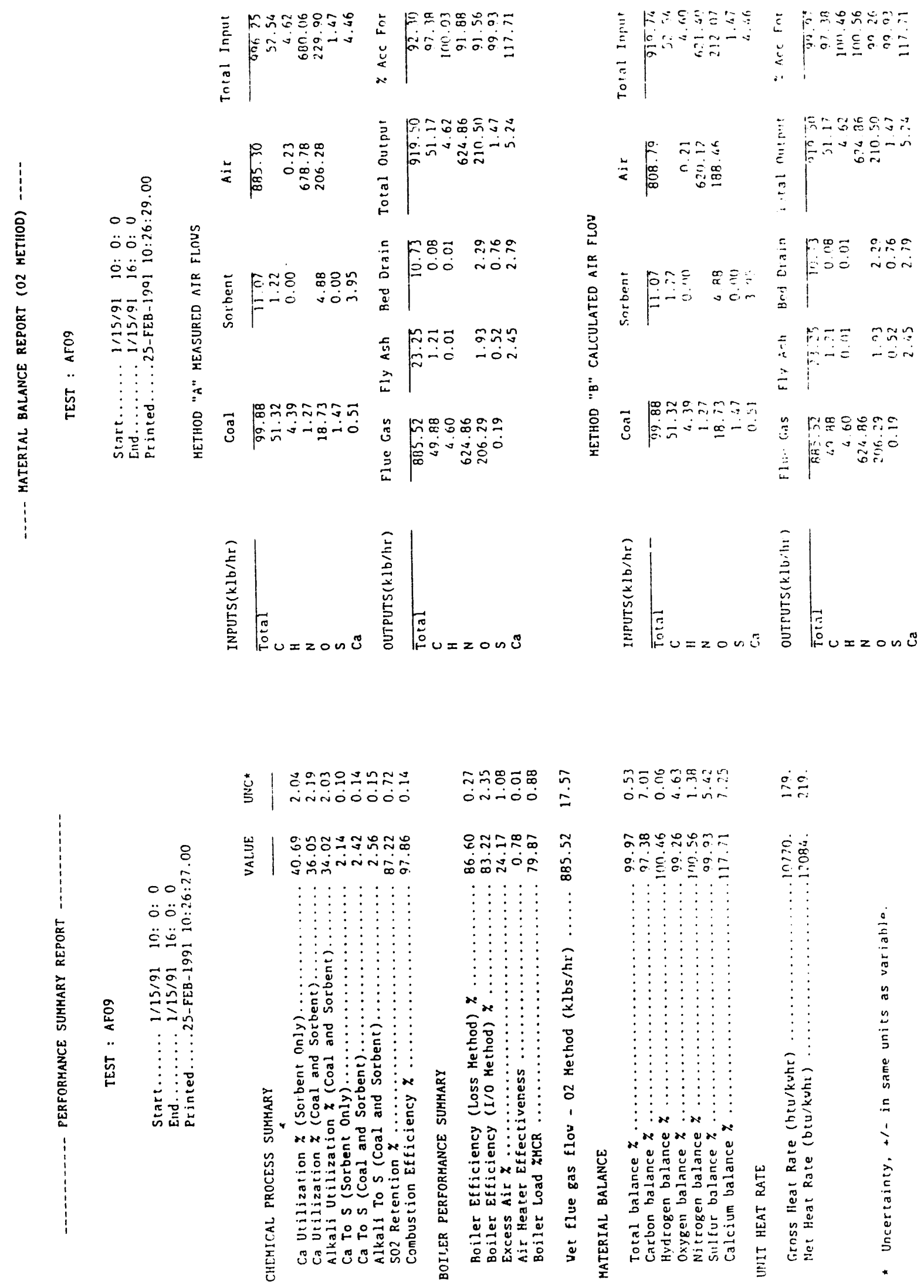



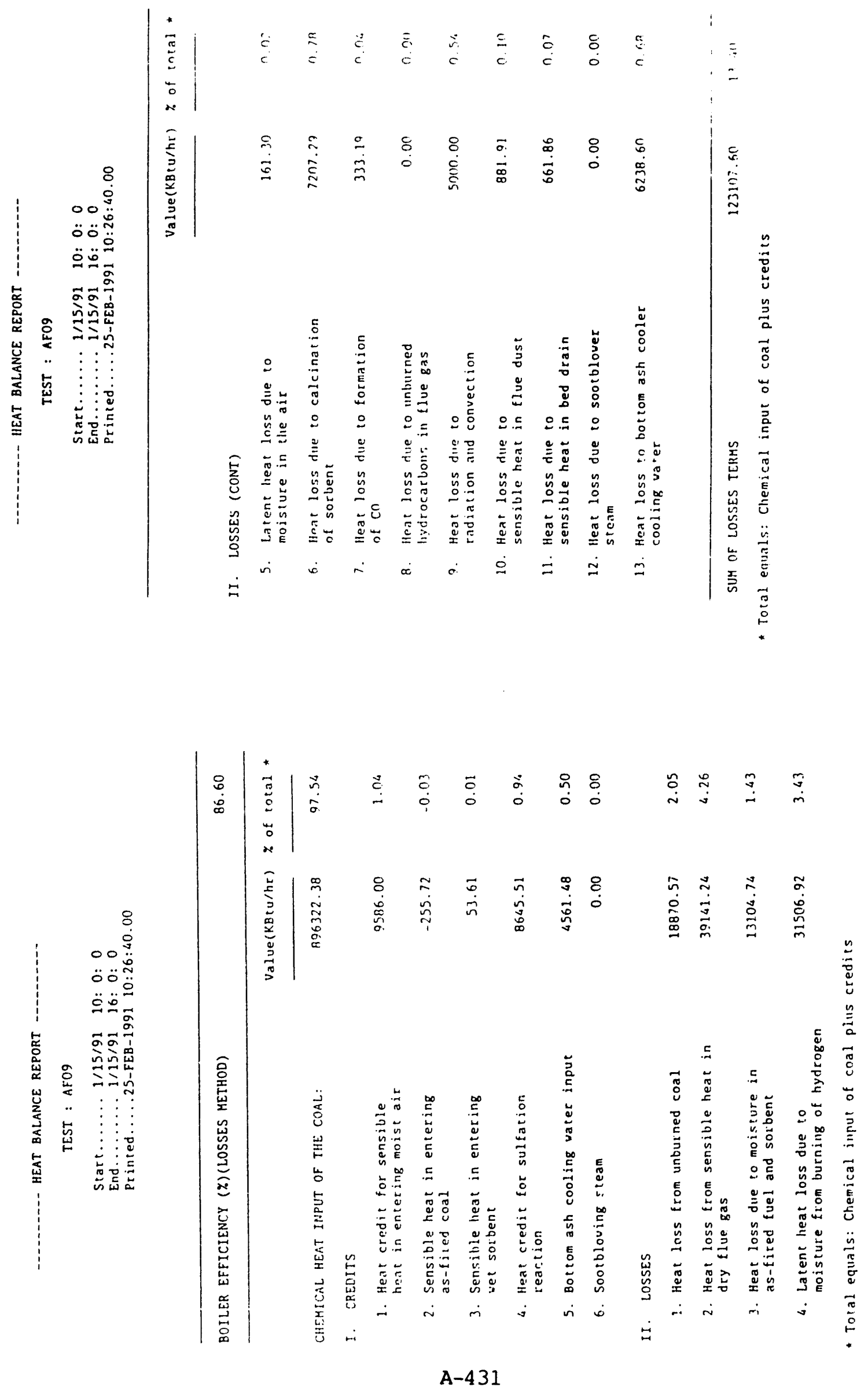

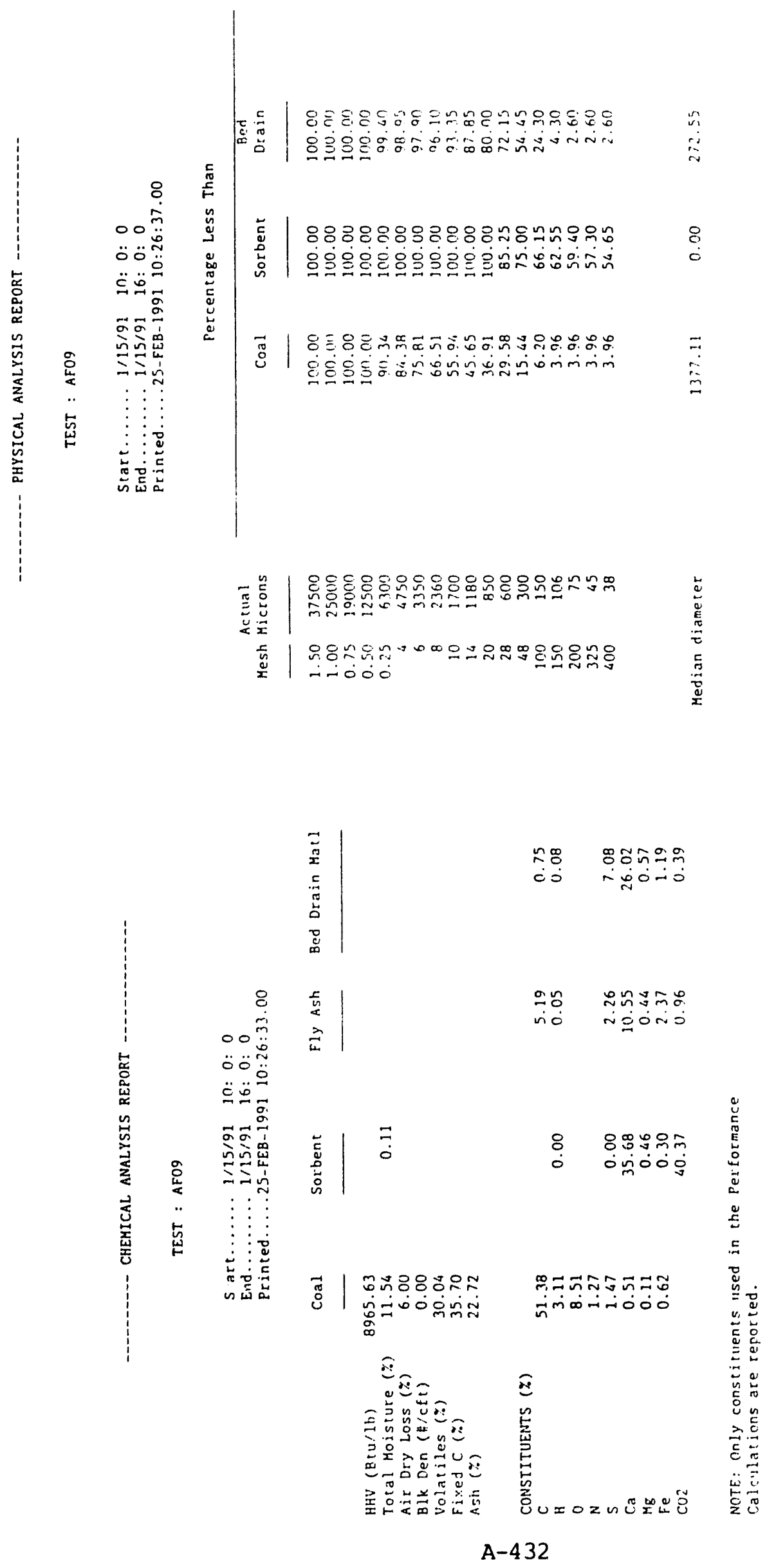

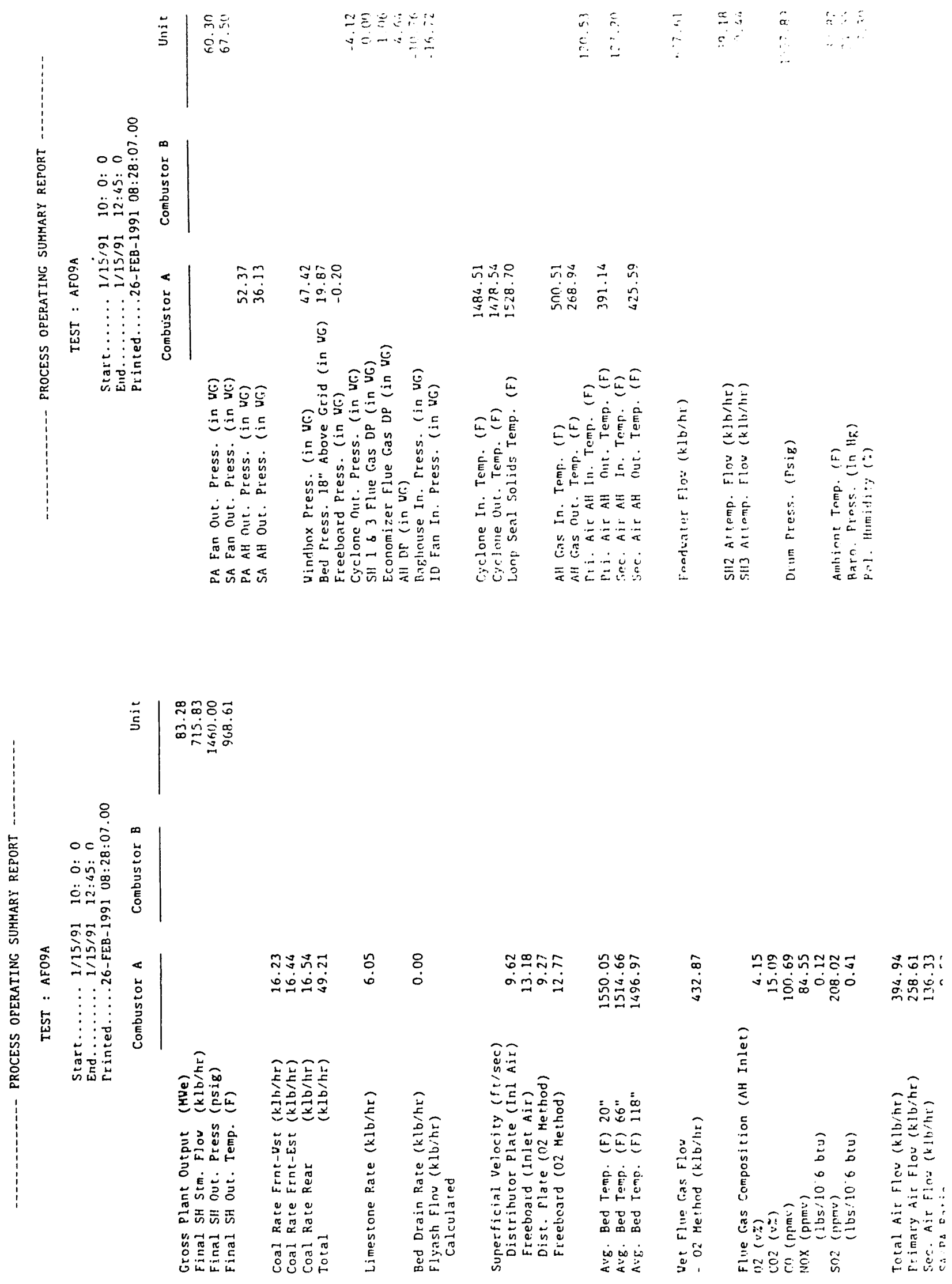


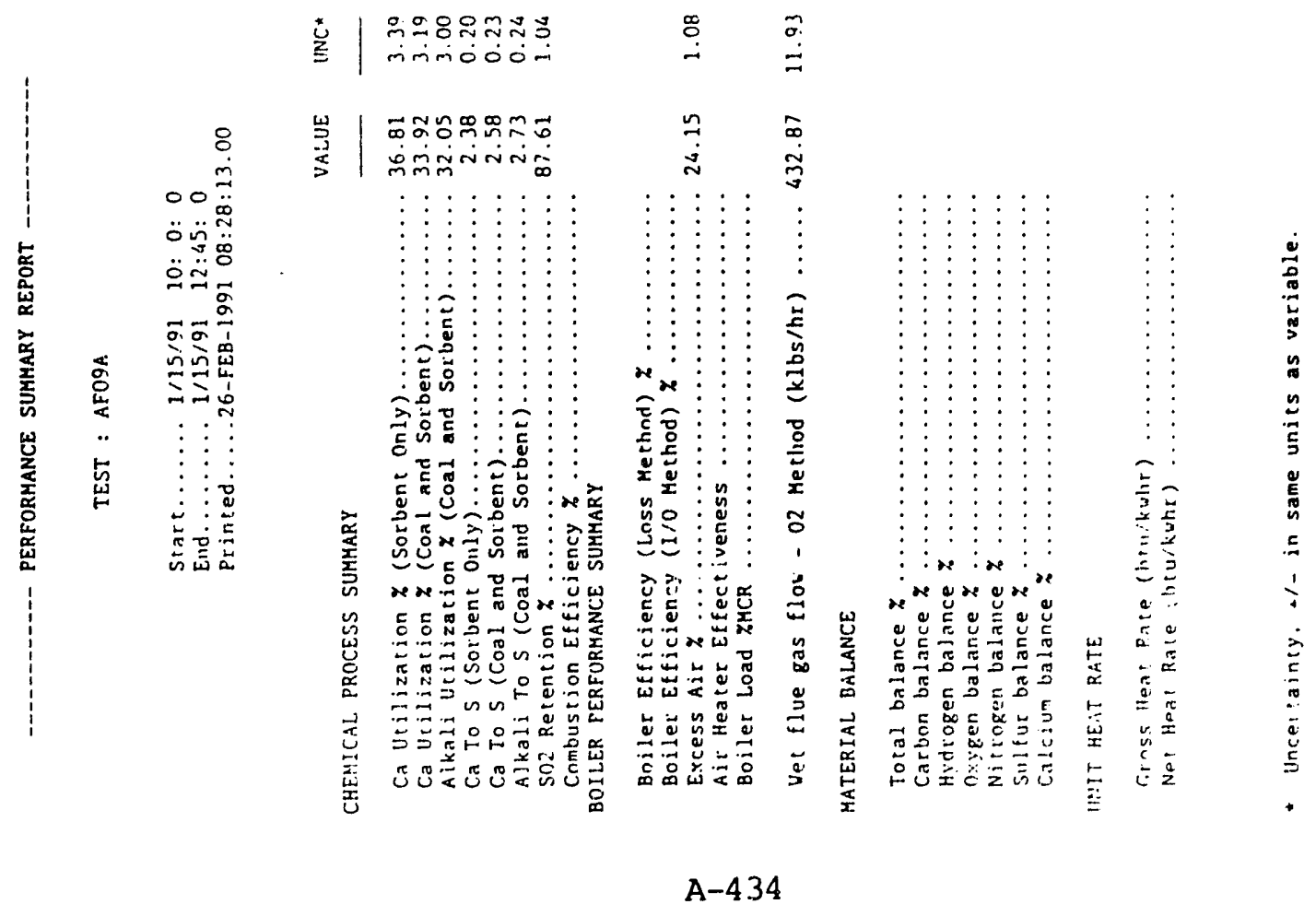



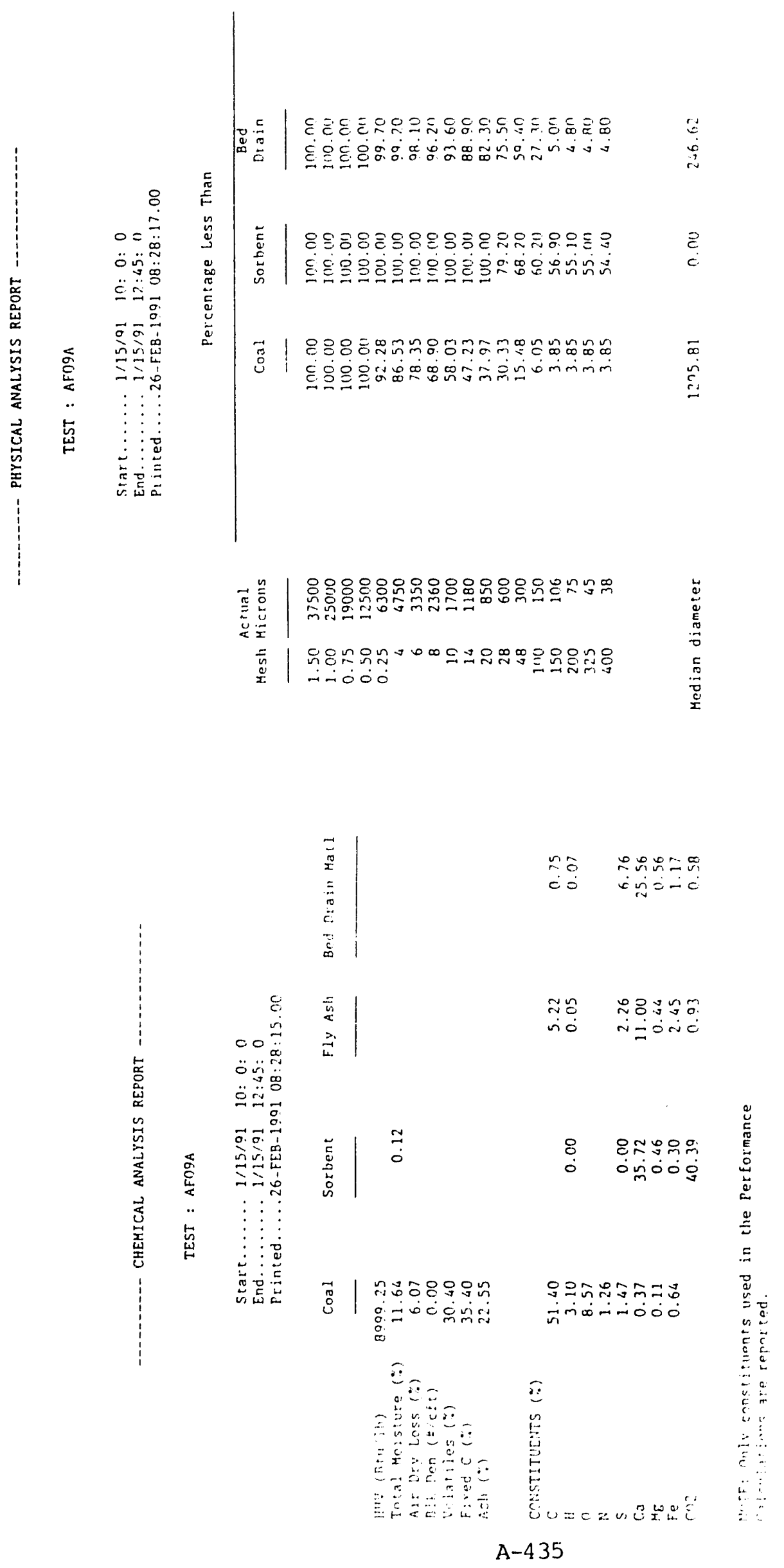

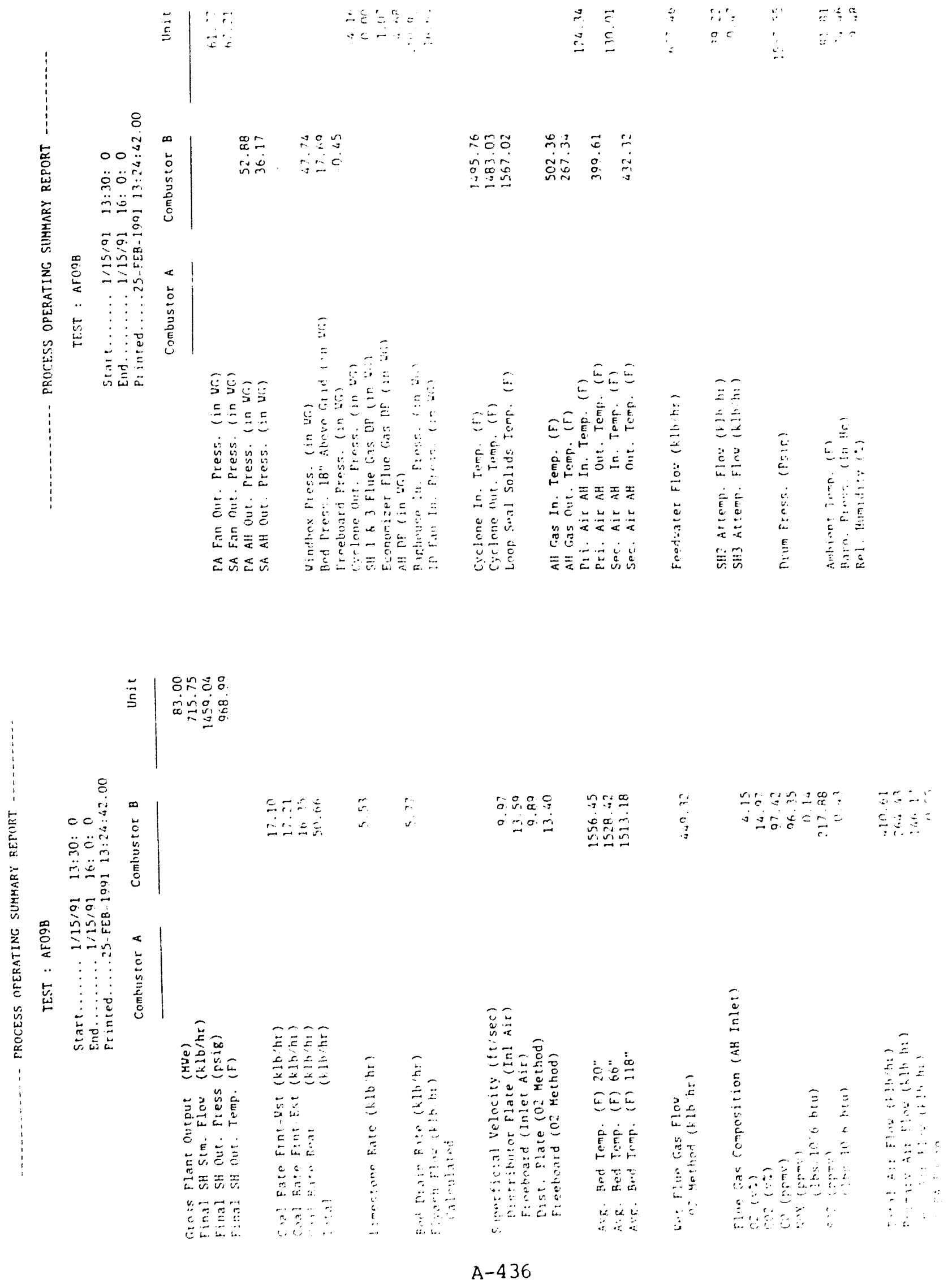


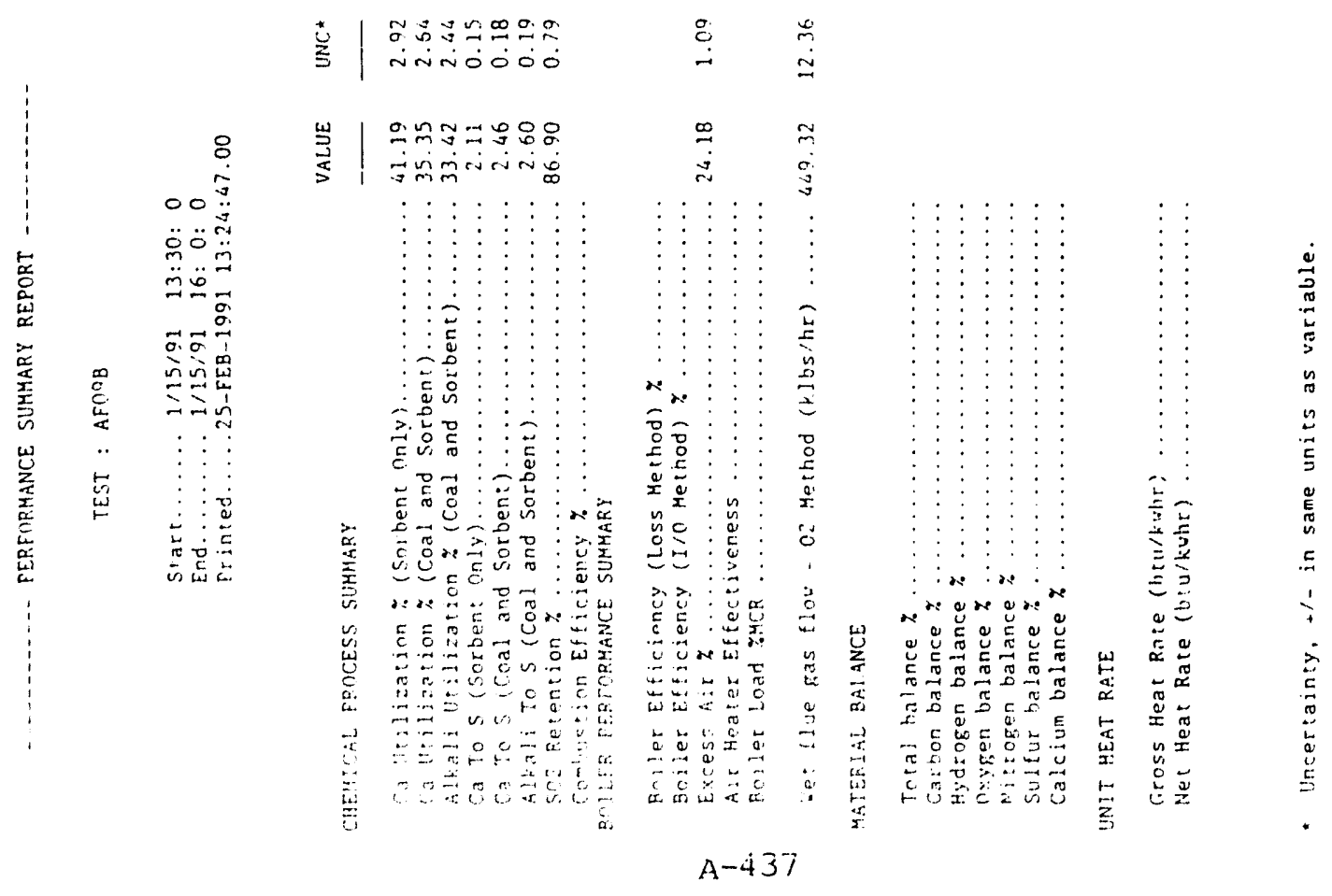



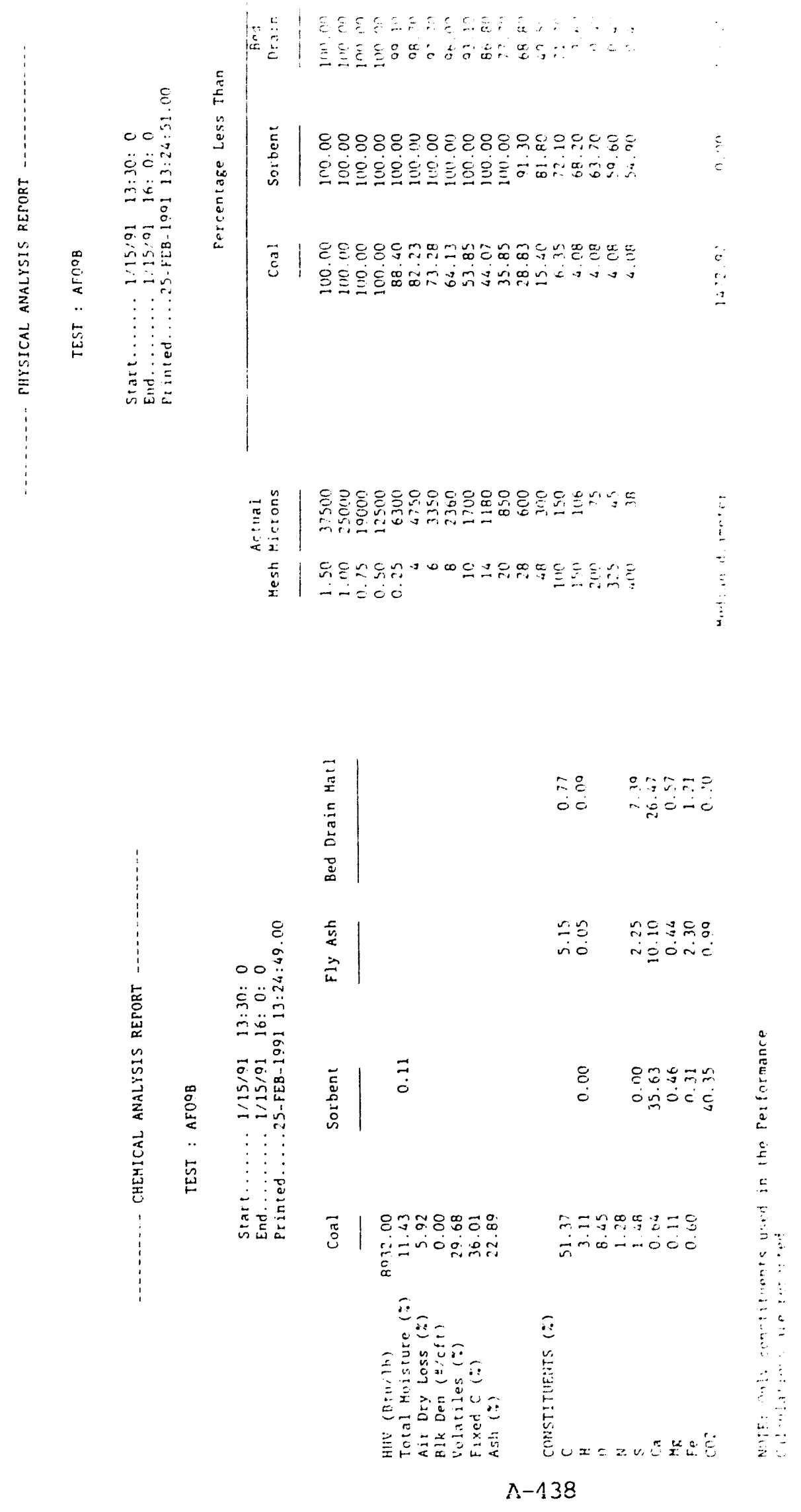

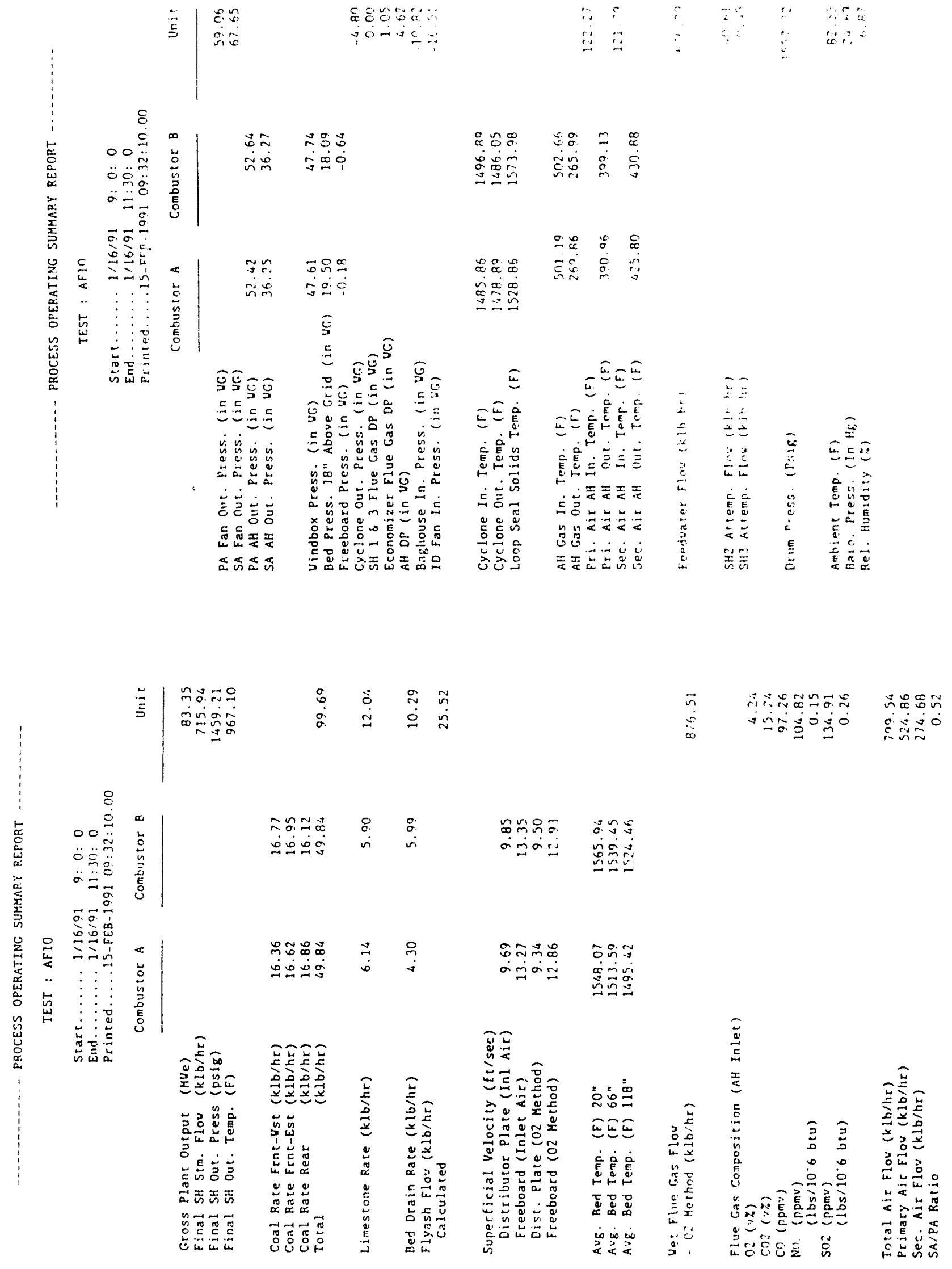


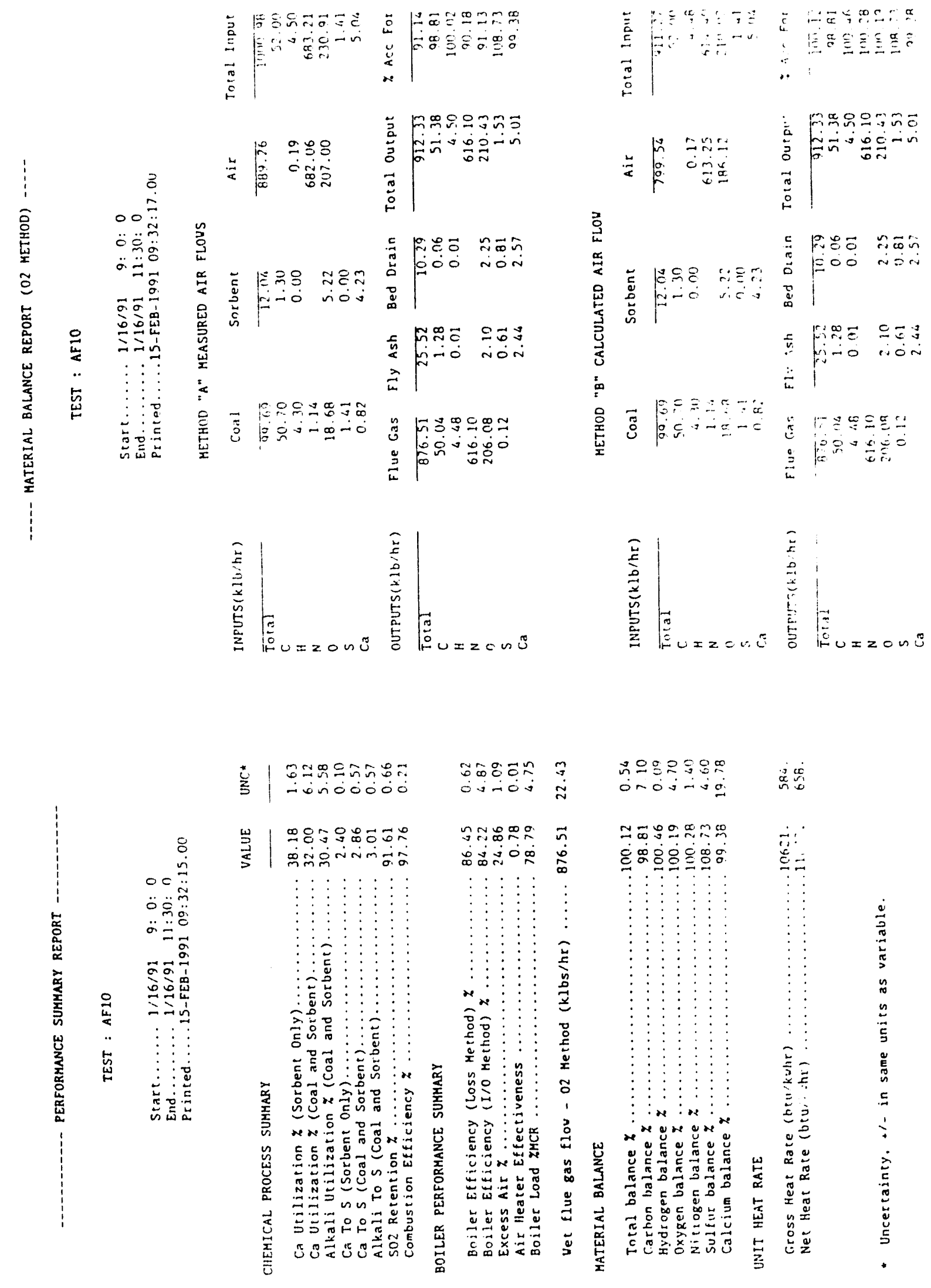




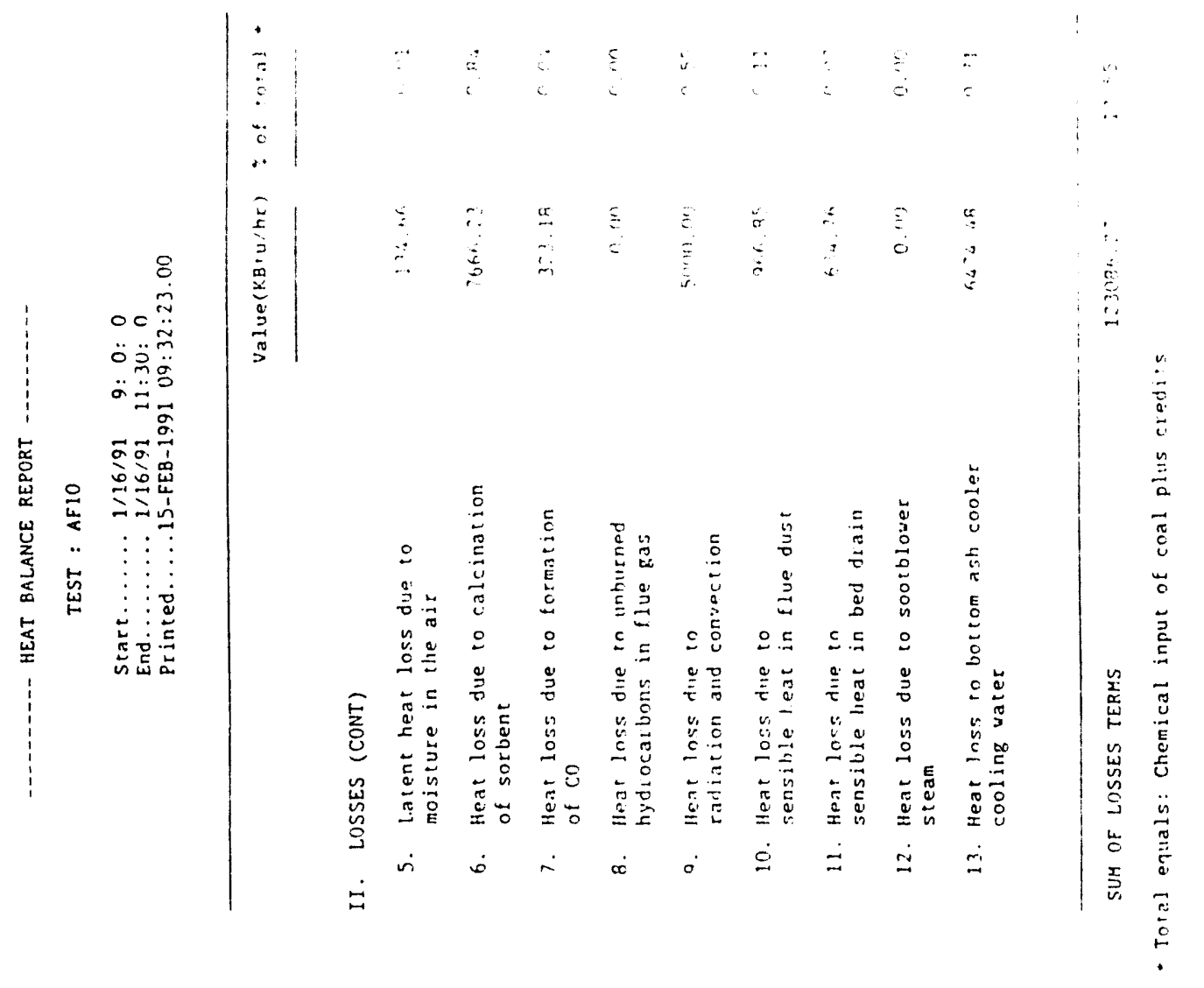

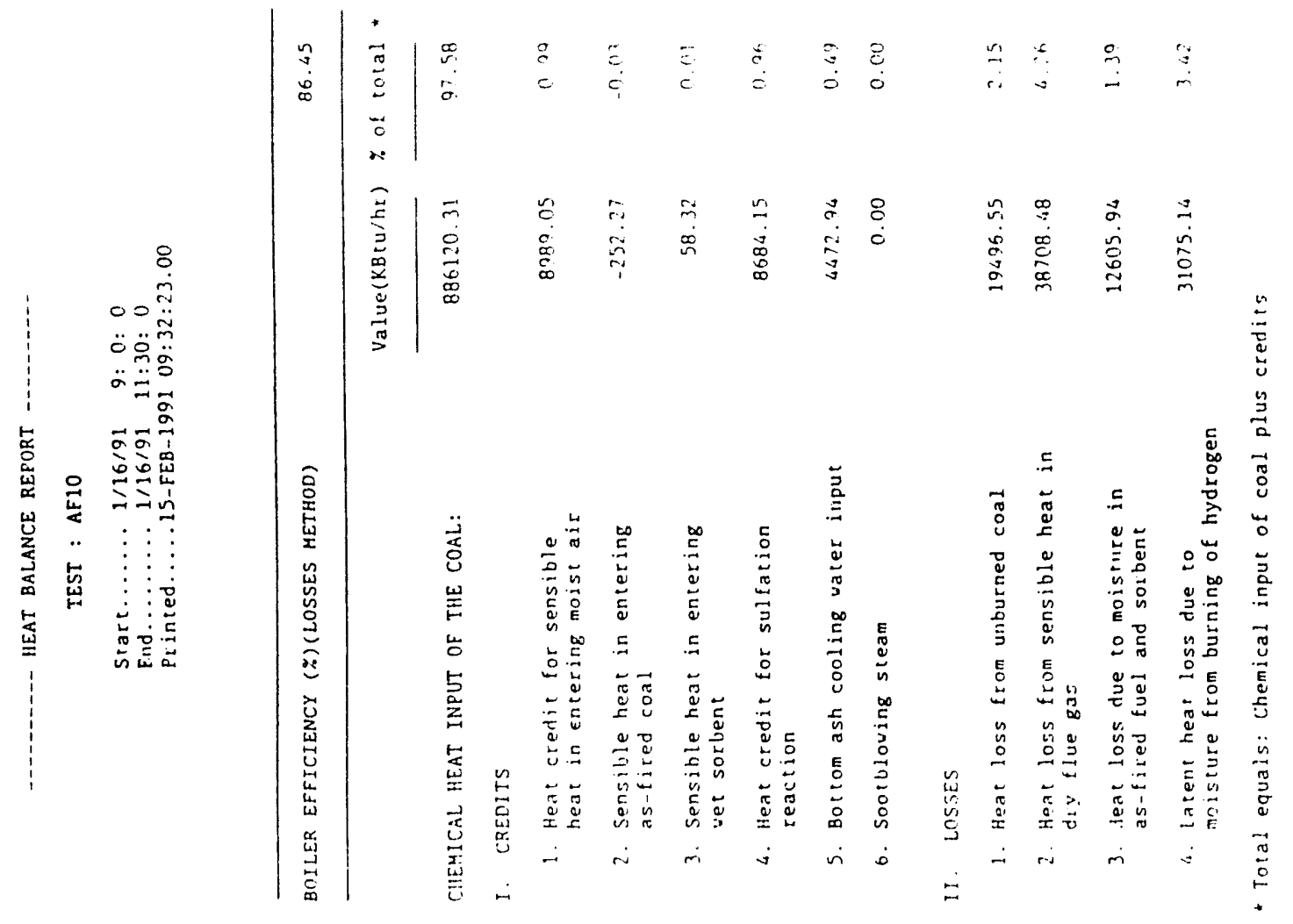




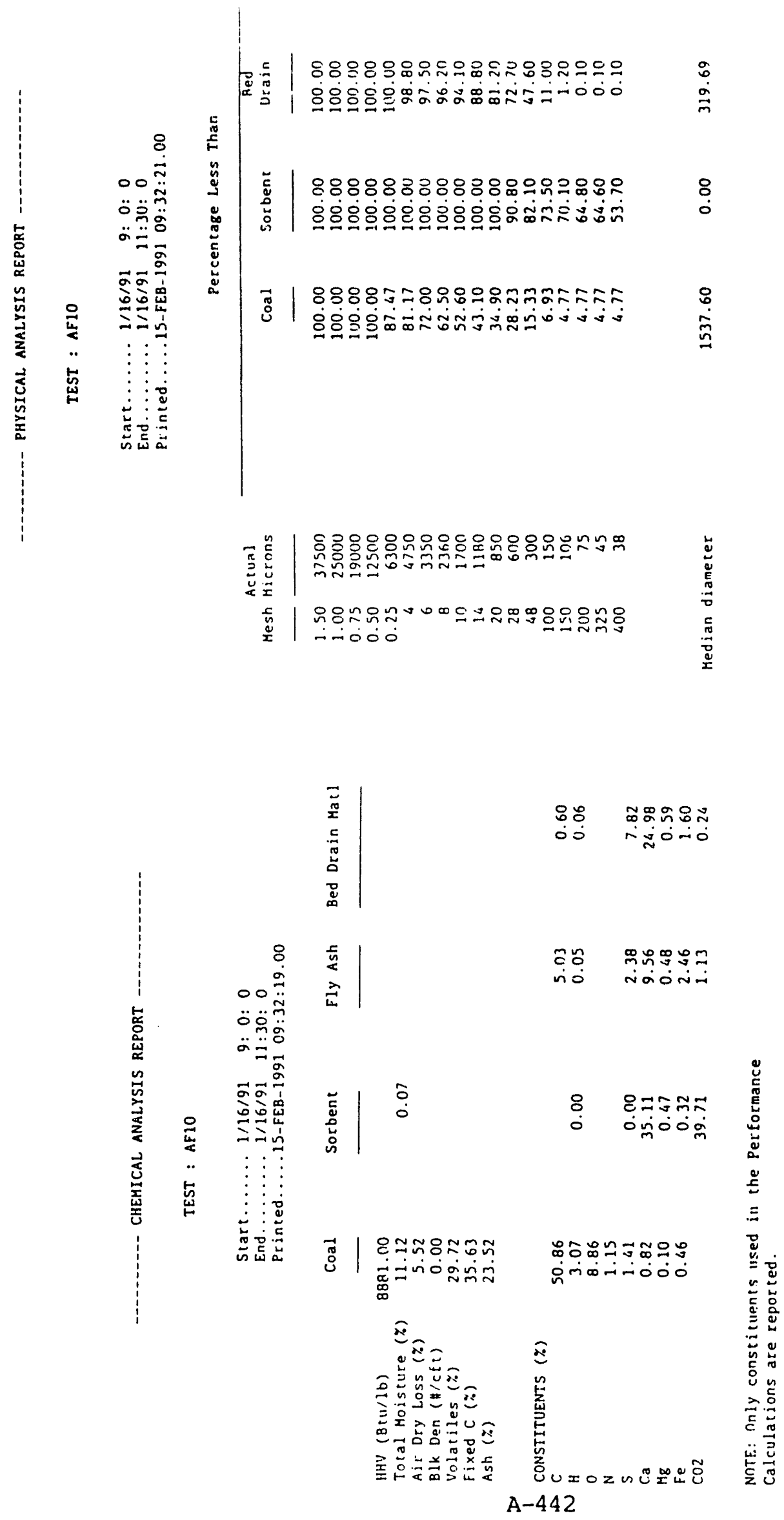



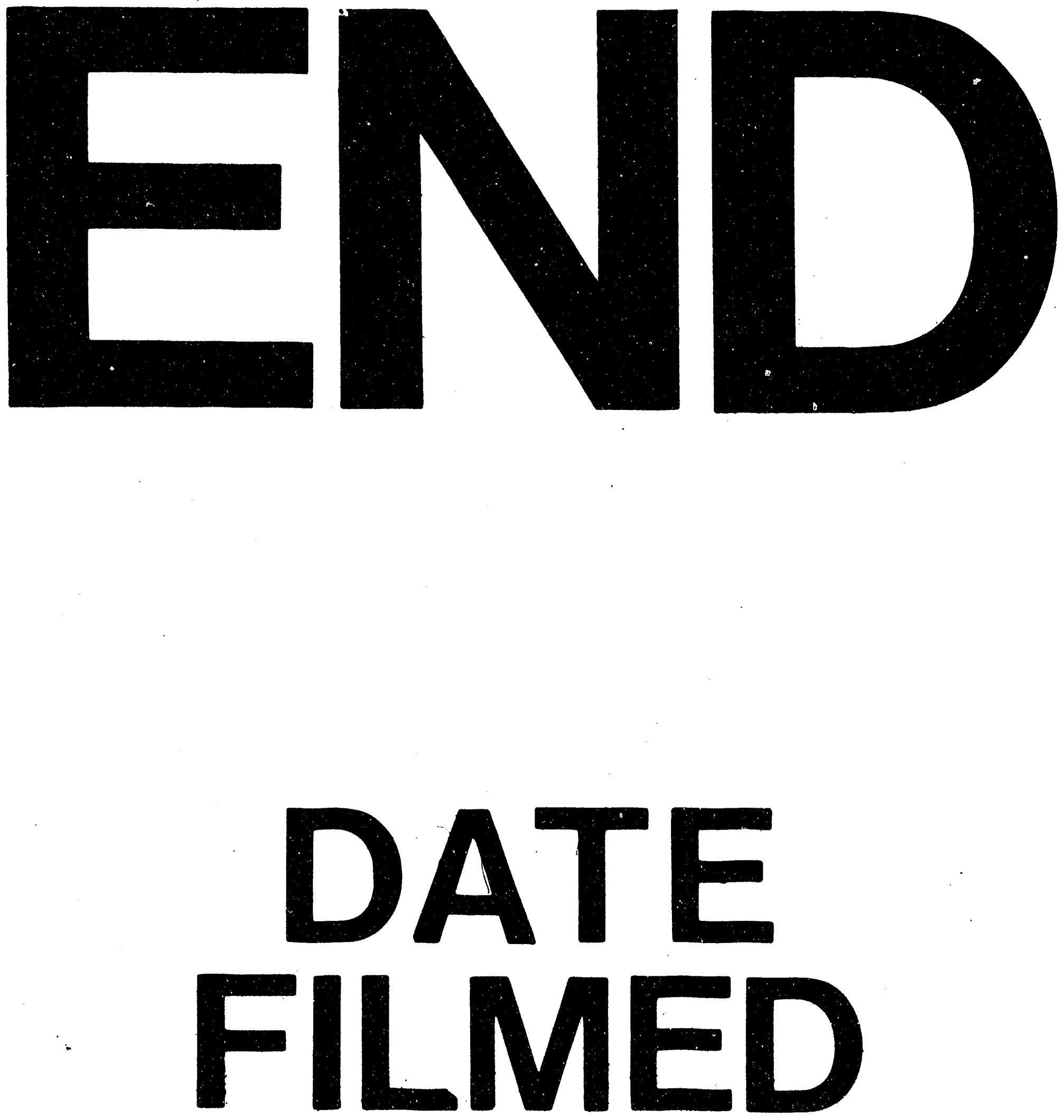

f

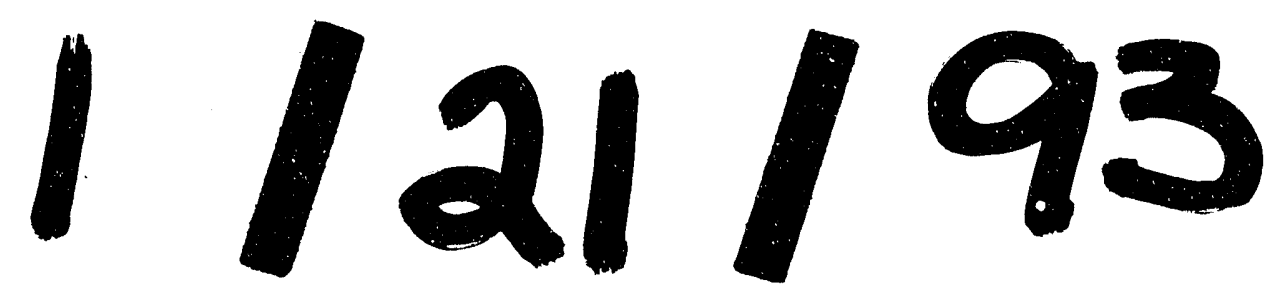

